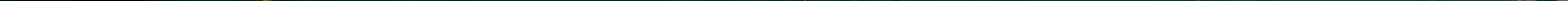






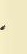


UNITED STATES DEPARTMENT OF COMMERCE • Luther H. Hodges, Secretary NATIONAL BUREAU OF STANDARDS - A. V. Astin, Director

\section{Ideal Gas Thermodynamic Functions and Isotope Exchange Functions for the Diatomic Hydrides, Deuterides, and Tritides}

Lester Haar, Abraham S. Friedman, and Charles W. Beckett

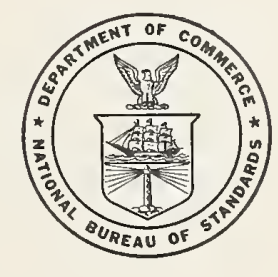

U. `. National Bureau of Standards, Monograph 20

Issued May 29, 1961

For sale by the Superintendent of Documents, U.S. Government Printing Office, Washington 25, D C. - Price $\$ 2.75$ 
National Bureau of Standards

AUG 181961
106,369

QC 100

$45 \div 6$

961

Copy 


\section{Preface}

In recent years the isotopes of hydrogen have assumed a significant role as research tools in chemistry, physics, biology, and nuclear engineering. In view of the importance of the isotopes of hydrogen in science and industry, the Thermodynamics Section of the National Bureau of Standards in 1951 undertook a research program, sponsored in part by the Division of Research of the United States Atomic Energy Commission, to investigate, both theoretically and experimentally, isotope effects in hydrogen compounds. The study has involved the isotope effects on the data of state, reaction kinetics, thermodynamic functions, and a variety of other physico-chemical phenomena. One phase of this program has been the calculation and tabulation of thermodynamic functions and equilibrium exchange functions of compounds and reactions involving hydrogen, deuterium, and tritium. This Monograph contains such tables for diatomic hydrides, deuterides, and tritides, and includes nearly all species for which adequate structural data have been experimentally reported or can be derived.

A. V. Astin, Director. 



\section{Contents}

Preface

Introduction

Molecular constants

Thermodynamic functions

Isotope exchange reactions

Tables.

Estimated uncertainties

References

Table 1. Molecular constants

Table 2. Ideal gas thermodynamic functions; heat capacity, enthalpy, free energy, entropy:

$2.001-\mathrm{H}_{2}$ $2.002-\mathrm{H}_{2}$ $2.003-\mathrm{H}_{2}$

normal mixture equilibrium mixture $\ldots \ldots \ldots$ para (includes only the even numbered rotational states)

$2.004-\mathrm{D}_{2}$ $2.005-\mathrm{D}_{2}$ $2.006-\mathrm{D}_{2}$

$2.007-\mathrm{T}_{2}$ $2.008-\mathrm{T}_{2}$ $2.009-\mathrm{T}_{2}$ normal mixture equilibrium mixture ortho (includes only the even numbered rotational states) normal mixture $\ldots \ldots \ldots$ equilibrium mixture

para (includes only the even numbered rotational states)

$2.010-\mathrm{HD}$

2.011-HT

2.012-DT

2.013- $\mathrm{LiH}$

$2.014-\mathrm{LiD}$

2.015-LiT

$2.016-\mathrm{NaH}$

2.017-NaD

$2.018-\mathrm{NaT}$

2.019-KH

2.020-KD

2.021-KT

2.022- $\mathrm{RbH}$

2.023-RbD

2.024-RbT

2.025- $\mathrm{CsH}$

2.026-CsD

2.027-CsT

$2.028-\mathrm{BeH}$

2.029-BeD

2.030-BeT

2.031-MgH

2.032- MgD

2.033-MgT

$2.034-\mathrm{CaI}$

2.035-CaD

2.036-Ca'

2.037-SrI

2.038-SrD

2.039-SrT

$2.040-\mathrm{BaH}$

2.041-BaD

2.042-BaT

2.043-CuI

2.044-CuD

2.045-CuT

2.046-AgH

2.047-AgD

2.048-AgT
Page

II

1

2

3

4

4

6

6

9

11

12

13

15

16

19

19

20

22

24

26

28

30

32

34

36

38

40

42

44

46

48

50

52
Table 2-Continued

2.049-AuH _............. 98

$2.050-\mathrm{AuD} \ldots 100$

2.051-A $\mathrm{U}$ T $\ldots \ldots \ldots \ldots . \ldots \ldots$

$2.052-\mathrm{ZnH}$

$2.053-\mathrm{ZnD} \ldots \ldots \ldots \ldots$

$2.054-Z n T$ T

$2.055-\mathrm{CdH}$

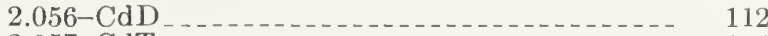

2.057-CdT

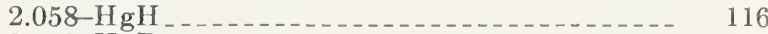

$2.059-\mathrm{HgD} \ldots \ldots \ldots \ldots \ldots \ldots \ldots$

$2.060-\mathrm{HgT}_{\ldots} \ldots \ldots$

$2.061-\mathrm{BH}$

2.062-BD _ . . .

$2.063-\mathrm{BT}_{-}$

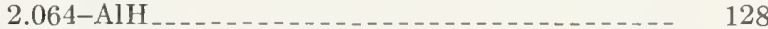

$2.065-\mathrm{AlD} \ldots$

2.066-AlT

2.067-InH_...

2.068-InD _

2.069-InT

$2.070-\mathrm{TlH}_{\ldots} \ldots \ldots \ldots \ldots \ldots$

2.071-TID

2.072-TIT $\ldots \ldots \ldots \ldots$

2.073-CH _._.

2.074-CD $\ldots$

2.075-CT

2.076- $\mathrm{SiH} \ldots \ldots \ldots \ldots$

2.077-SiD

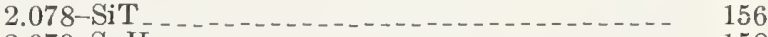

$2.079-\mathrm{SnH}_{\ldots} \ldots$

2.080-SnD

2.081-SnT

$2.082-\mathrm{PbH}_{\ldots} \ldots \ldots \ldots \ldots$

$2.083-\mathrm{PbD} \ldots$

2.084-PbT

$2.085-\mathrm{NH}$

2.086-ND -172

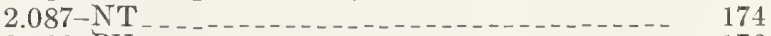

2.088-PH -PH 176

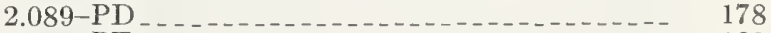

$2.090-\mathrm{PT}$

2.091-BiH

$2.092-\mathrm{BiD} \ldots$

2.093-BiT _._.

$2.094-\mathrm{OH} \ldots \ldots$

2.095-OD

2.096-OT

$2.097-\mathrm{SH}$

2.098-SD

$2.099-\mathrm{ST}$

$2.100-H F \ldots \ldots \ldots 200$

$2.101-D F$

2.102-TF

$2.103-\mathrm{ClH}_{-}$

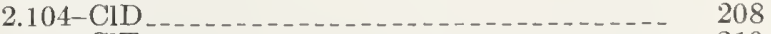

$2.105-\mathrm{ClT}$

2.106-BrH

2.107-BrD

$2.108-\mathrm{BrT}$

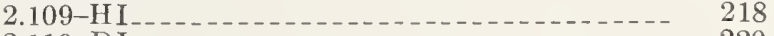

$2.110-\mathrm{DI} \ldots \ldots \ldots \ldots \ldots \ldots$

$2.111-\mathrm{TI}_{\ldots} \ldots \ldots \ldots 222$ 
Table 3. Heat capacity, enthalpy, free energy, and entropy for the isotope exchange reactions: tabulated data refer to the half-reactions, see text eqs $[3,4,5,6]$ :

3.01 Increment in thermal functions for $\mathrm{H}_{2}$ and $\mathrm{HD}$ Increment in thermal functions for $\mathrm{H}_{2}$ and $\mathrm{HT}^{--}$ Increment in thermal functions for $\mathrm{H}_{2}$ and $\mathrm{D}_{2}$

3.02 Increment in thermal functions for $\mathrm{H}_{2}$ and $\mathrm{DT}_{-}$ Inerement in thermal functions for $\mathrm{H}_{2}$ and $\mathrm{T}_{2}$ Increment in thermal functions for HD and $\mathrm{HT}$

3.03 Increment in thermal functions for $\mathrm{HD}$ and $\mathrm{D}_{2}$ Increment in thermal functions for HD and DT. Increment in thermal functions for $\mathrm{HD}$ and $\mathrm{T}_{2}$

3.04 Increment in thermal functions for HT and $D_{2}$ Increment in thermal functions for HT and DT Increment in thermal functions for $\mathrm{HT}$ and $\mathrm{T}_{2}$

3.05 Increment in thermal functions for $\mathrm{D}_{2}$ and $\mathrm{DT}^{2}$ Increment in thermal functions for $\mathrm{D}_{2}$ and $\mathrm{T}_{2}$ Increment in thermal functions for DT and $\mathrm{T}_{2}$

3.06 Increment in thermal functions for $\mathrm{LiH}$ and $\mathrm{LiD}$ Increment in thermal functions for $\mathrm{LiH}$ and LiT Increment in thermal functions for LiD and $\mathrm{LiT}$

3.07 Increment in thermal functions for $\mathrm{NaH}$ and $\mathrm{NaD}$

Increment in thermal functions for $\mathrm{NaH}$ and $\mathrm{NaT}_{-}$

Increment in thermal functions for $\mathrm{NaD}$ and $\mathrm{NaT}$

3.08 Increment in thermal functions for KI and $\mathrm{KD}-$ Increment in thermal functions for $\mathrm{KH}$ and $\mathrm{KT}$ Increment in thermal functions for $K D$ and $K^{\prime} T$.

3.09 Increment in thermal functions for $\mathrm{RbH}$ and $\mathrm{RbD}$

Increment in thermal functions for $\mathrm{RbH}$ and RbT

Inerement in thermal functions for $\mathrm{RbD}$ and RbT.

3.10 Increment in thermal functions for $\mathrm{CsH}$ and $\mathrm{CsD}$ Increment in thermal functions for $\mathrm{CsH}$ and $\mathrm{Cs} \mathrm{T}$ Increment in thermal functions for CsD and CsT.

3.11 Increment in thermal functions for $\mathrm{BeH}$ and BeD Increment in thermal functions for $\mathrm{BeH}$ and $\mathrm{Be} \mathrm{T}$ Increment in thermal functions for BeD and BeT

3.12 Increment in thermal functions for $\mathrm{MgH}$ and $\mathrm{MgD}$

Increment in thermal functions for $\mathrm{MgH}$ and $\mathrm{Mg} \mathrm{T}$

Increment in thermal functions for $\mathrm{MgD}$ and $\mathrm{MgT}$.

3.13 Increment in thermal functions for $\mathrm{CaH}$ and $\mathrm{CaD}$ Increment in thermal functions for $\mathrm{CaH}$ and $\mathrm{CaT}$ Increment in thermal functions for $\mathrm{CaD}$ and $\mathrm{CaT}$.

3.14 Increment in thermal functions for SrH and SrD Increment in thermal functions for $\mathrm{SrH}$ and $\mathrm{SrT}$ Increment in thermal functions for $\mathrm{SrD}$ and $\mathrm{SrT}$

3.15 Increment in thermal functions for Bayd and BaD Increment in thermal functions for $\mathrm{BaH}$ and $\mathrm{BaT}$. Increment in thermal functions for $\mathrm{BaD}$ and $\mathrm{BaT}$

3.16 Increment in thermal functions for $\mathrm{CuH}$ and $\mathrm{CuD}$ Increment in thermal functions for $\mathrm{CuH}$ and $\mathrm{CuT}$ Inerement in thermal functions for $\mathrm{CuD}$ and $\mathrm{Cu} T$

3.17 Increment in thermal functions for $\mathrm{AgH}$ and $\mathrm{AgD}$ Increment in thermal functions for $\mathrm{AgH}$ and $\mathrm{AgT}$ Increment in thermal functions for $\mathrm{AgD}$ and $\mathrm{AgT}$

3.18 Increment in thermal functions for AuH and AuD Increment in thermal functions for $\mathrm{AuH}$ and $\mathrm{Au} T$ Increment in thermal functions for $A u D$ and $A u T$

3.19 Increment in thermal functions for $\mathrm{ZnH}$ and $\mathrm{ZnD}$ Increment in thermal functions for $\mathrm{ZnH}$ and $\mathrm{ZnT}$. Increment in thermal functions for $\mathrm{ZnD}$ and $\mathrm{ZnT}$

3.20 Increment in thermal functions for $\mathrm{CdH}$ and $\mathrm{CdD}$ Increment in thermal functions for $\mathrm{CdH}$ and $\mathrm{CdT}$ Increment in thermal functions for $\mathrm{CdD}$ and $\mathrm{CdT}$

3.21 Increment in thermal functions for $\mathrm{HgH}$ and $\mathrm{HgD}$ Increment in thermal functions for $\mathrm{HgH}$ and $\mathrm{HgT}$ Increment in thermal functions for $\mathrm{HgD}$ and $\mathrm{HgT}$.

3.22 Increment in thermal functions for $\mathrm{BH}$ and $\mathrm{BD}$ Increment in thermal functions for $\mathrm{BH}$ and $\mathrm{BT}_{\text {- }}$ Increment in thermal functions for $\mathrm{BD}$ and $\mathrm{BT}$.

Table 3-Continued

3.23 Increment in thermal functions for $\mathrm{AlH}$ and AlD Increment in thermal functions for $\mathrm{AlH}$ and $\mathrm{AlT}$

224

224

225

225

226

226

227

227

227

228
$2 \cdot 28$

228

229

229

229

230

230

230

231

231

231

232

232

232

233

233

233

234

234

234

235

235

235

236

236

236

237

237

237

238

238

238

239

239

239

240

240

240

241

241

241

$2+2$

242

242

243

243

243

244

244

244

245

245

245
Increment in thermal functions for AlD and AlT

3.24 Increment in thermal functions for In $\mathrm{H}$ and InD Increment in thermal functions for In $\mathrm{H}$ and In T Increment in thermal functions for In D and In T

3.25 Increment in thermal functions for TIH and TID Inerement in thermal functions for TIH and TIT Increment in thermal functions for TID and TlT

3.26 Increment in thermal functions for CH and CD Increment in thermal functions for $\mathrm{CH}$ and $\mathrm{CT}$ Increment in thermal functions for CD and CT

3.27 Increment in thermal functions for $\mathrm{SiH}$ and $\mathrm{SiD}$ Increment in thermal functions for $\mathrm{SiH}$ and SiT Increment in thermal functions for SiD and SiT

3.28 Increment in thermal functions for $\mathrm{SnH}$ and $\mathrm{SnD}$ Increment in thermal functions for $\mathrm{SnH}$ and $\mathrm{SnT}$. Increment in thermal functions for Sn D and SnT.

3.29 Increment in thermal functions for $\mathrm{PbH}$ and $\mathrm{PbD}$ Increment in thermal functions for $\mathrm{PbH}$ and $\mathrm{PbT}$. Increment in thermal functions for $\mathrm{PbD}$ and $\mathrm{PbT}$.

3.30 Increment in thermal functions for NH and ND Increment in thermal functions for $\mathrm{NH}$ and $\mathrm{N} T$. Increment in thermal functions for ND and NT

3.31 Increment in thermal functions for PH and PD Increment in thermal functions for PH and PT Increment in thermal functions for PD and PT

3.32 Increment in thermal functions for $\mathrm{BiH}$ and $\mathrm{BiD}$ Increment in thermal functions for $\mathrm{BiH}$ and $\mathrm{BiT}$ Increment in thermal functions for $\mathrm{BiD}$ and $\mathrm{BiT}$

3.33 Increment in thermal functions for OH and OD Increment in thermal functions for $\mathrm{OH}$ and $\mathrm{OT}$ Increment in thermal functions for OD and OT

3.34 Increment in thermal functions for SH and SD.Increment in thermal functions for SH and ST Increment in thermal functions for SD and ST

3.35 Increment in thermal functions for FH and FD Increment in thermal functions for FH and FT Increment in thermal functions for FD and FT

3.36 Increment in thermal functions for $\mathrm{ClH}$ and $\mathrm{ClD}$ Increment in thermal functions for $\mathrm{ClH}$ and $\mathrm{ClT}$ Increment in thermal functions for $\mathrm{ClD}$ and $\mathrm{ClT}$

3.37 Increment in therinal functions for $\mathrm{BrH}$ and $\mathrm{BrD}$ Increment in thermal functions for $\mathrm{BrH}$ and $\mathrm{BrT}$ Increment in thermal functions for BrD and BrT

3.38 Increment in thermal functions for $\mathrm{HI}$ and DI Increment in thermal functions for HI and TI Increment in thermal functions for DI and T'I

Table 4. Normalized free energy for hydroges isotope exchange [see text, eq (7)]:

4.01 Normalized free energy difference for $\mathrm{H}_{2}$ and $\mathrm{HD}$ Normalized free cnergy difference for $\mathrm{H}_{2}$ and $\mathrm{HT}$ Normalized free energy difference for $\mathrm{HD}$ and $\mathrm{D}_{2}$ Normalized free energy difference for $\mathrm{HD}$ and

Normalized free energy difference for $\mathrm{HD}$ and DT

Normalized free energy difference for $\mathrm{D}_{2}$ and $\mathrm{DT}$.

Normalized free energy difference for $\mathrm{HT}$ and $\mathrm{DT}$.

Normalized free energy difference for $\mathrm{HT}$ and $\mathrm{T}_{2}$

Normalized free energy difference for DT and $T_{2}$

4.02 Normalized free energy difference for $\mathrm{LiH}$ and LiD

Normalized free energy difference for $\mathrm{LiH}$ and LiT . . . . _...

Normalized free energy difference for $\mathrm{LiD}$ and LiT

Normalized free energy difference for $\mathrm{NaH}$ and $\mathrm{NaD}$ Normalized free energy difference for $\mathrm{NaH}$ and TaT

Normalized free energy difference for $\mathrm{NaD}$ and $\mathrm{NaT}$ Normalized free energy difference for $\mathrm{KH}$ and KD . . . . .
Page 
Table 4-Continued

Normalized free energy difference for $\mathrm{KH}$ and KT

Normalized free energy difference for KD and

Normalized free energy difference for $\mathrm{RbH}$ and

$\mathrm{RbD}$
Normalized free energy difference for $\mathrm{Rb} \mathrm{H}$ and $\mathrm{RbT}_{\text {ormalized frec energy difference for } \mathrm{RbD} \text { and }}$ RbT

4.03 Normalized free energy difference for CsH and $\mathrm{CsD}$ CsT CsT

Normalized free energy difference for $\mathrm{BeH}$ and

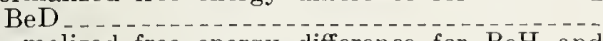

Normalized free energy difference for $\mathrm{BeH}$ and

BeT
Normalized free energy difference for $\mathrm{BeD}$ and ormalized free energy difference for $\mathrm{MgH}$ and $\mathrm{MgD} \ldots$

Normalized free energy difference for $\mathrm{MgH}$ and $\mathrm{MgT}_{\text {ormalized free energy difference for } \mathrm{MgD} \text { and }}$ MgT.

Normalized free energy difference for $\mathrm{CaH}$ and $\mathrm{CaD}$

Normalized free energy difference for $\mathrm{CaH}$ and

Normalized free energy difference for $\mathrm{CaD}$ and ormalized free energy difference for $\mathrm{SrH}$ and $\mathrm{SrD}$

Normalized free energy difference for $\mathrm{SrH}$ and $\mathrm{SrT}_{\mathrm{T}}$

Normalized free energy difference for $\mathrm{SrD}$ and
SrT. Srmalized free energy difference for $\mathrm{BaH}$ and
Normats Normalized free energy difference for $\mathrm{BaH}$ and

Normalized free energy difference for $\mathrm{BaH}$ and

Normalized free energy difference for $\mathrm{BaD}$ and $\mathrm{BaT}$
Normalized free energy difference for $\mathrm{CuH}$ and CuDalized free energy difference for $\mathrm{CuH}$ and CuT.

Normalized free energy difference for $\mathrm{CuD}$ and CuT

Normalized free energy difference for $\mathrm{AgH}$ and
$\mathrm{AgD}$ Normalized free energy difference for $\mathrm{AgH}$ and $\mathrm{AgT}$. . .

Normalized free energy difference for $\mathrm{AgD}$ and $\mathrm{AgT}$ $\mathrm{AuD} \ldots \ldots$

Normalized free energy difference for $\mathrm{AuH}$ and

Normalized free energy difference for $\mathrm{AuD}$ and Normalized free energy difference for $\mathrm{ZnH}$ and $\mathrm{ZnD}$

Normalized free energy difference for $\mathrm{ZnH}$ and ZnT

Normalized free energy difference for $\mathrm{ZnD}$ and ZnT

Normalized free energy difference for $\mathrm{CdH}$ and Normalized free energy difference for $\mathrm{CdH}$ and Normalized free energy difference for $\mathrm{CdD}$ and $\mathrm{CdT}$
Table 4 - Continued

Normalized free cnergy difference for $\mathrm{HgH}$ and IIgD . . . . . . . . . . . . . .

Normalized free encrgy difference for $\mathrm{HgH}$ and HgT

Normalized free energy diffcrence for $\mathrm{H} g \mathrm{D}$ and

4.06 Normalized free energy difference for $\mathrm{BH}$ and Normalized free energy differcnee for BH and BT _...............................

Normalized free energy difference for $B \bar{D}$ and orinalized free energy difference for $\mathrm{AlH}$ and Normalized free energy difference for $\mathrm{AlH}$ and
AlD Normalized free cnergy difference for AlH and Normalized free energy difference for AlD and AlT Normalized free encrgy differcnce for In $\mathrm{H}$ and InD Normalized free energy difference for $\operatorname{In} H$ and
InT

Normalized free energy difference for In $\mathrm{D}$ and In $\mathrm{T}$ Normalized free energy difference for $\mathrm{TlH}$ and ormalized free energy difference for TlH and $\mathrm{TlT}$.

Normalized free energy difference for TID and

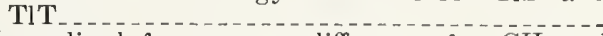

4.07 Normalized free energy difference for $\mathrm{CH}$ and $\mathrm{CD}$

Normalized free energy difference for $\mathrm{CH}$ and
CT Normalized free energy difference for $\mathrm{CD}$ and CT

Normalized free energy difference for $\mathrm{SiH}$ and $\mathrm{SiD} \ldots$

Normalized free energy difference for $\mathrm{SiH}$ and
SiT Normalized free energy difference for $\mathrm{SiD}$ and SiT . . . . . . . . . . . . . . . . . . .

Normalized free energy difference for $\mathrm{SnH}$ and Sn D

Normalized free energy difference for $\mathrm{SnH}$ and
SnT Normalized free energy difference for $\mathrm{SnD}$ and SnT

Normalized free energy difference for $\mathrm{PbH}$ and $\mathrm{PbD}_{\ldots} \ldots$

Normalized free energy difference for $\mathrm{PbH}$ and $\mathrm{PbT}$
ormalized free energy difference for $\mathrm{PbD}$ and $\mathrm{PbT}$.

4.08 Normalized free energy difference for $\mathrm{NH}$ and ND_........................

Normalized free energy difference for $\mathrm{NH}$ and NT Normalized free energy difference for $\mathrm{ND}$ and
$\mathrm{NT}$ Normalized free energy difference for PH and PD

Normalized free energy difference for PH and Normalized free energy difference for PD and PT

Normalized free energy difference for $\mathrm{BiH}$ and

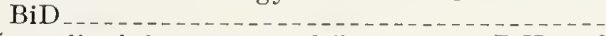

Normalized free energy difference for $\mathrm{BiH}$ and BiT _..................................

Normalized free energy difference for $\mathrm{BiD}$ and

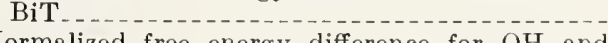
Normalized free energy difference for $\mathrm{OH}$ and

Normalized free energy difference for $O H$ and OT 
Table 4-Continued

Normalized free energy difference for $O D$ and OT

4.09 Normalized free energy difference for $\mathrm{SH}$ and SD Normalized free energy difference for $\mathrm{SH}$ and ormalized free energy difference for $\mathrm{SD}$ and ST _._.

Normalized free energy difference for $\mathrm{FH}$ and

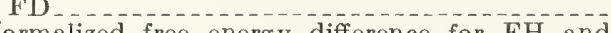
Normalized free energy difference for $\mathrm{FH}$ and FT FT Normalized free energy difference for $\mathrm{ClH}$ and CID
Table 4 -Continued

Normalized free energy difference for $\mathrm{ClH}$ and CIT _...............................

Normalized free energy difference for CID and

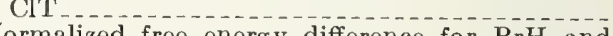
Normalized free energy difference for $\mathrm{BrH}$ and BrD _................................. Normalized free energy difference for $\mathrm{BrH}$ and BrT

Normalized free energy difference for $\mathrm{BrD}$ and

4.10 Normalized free energy difference for HI and Normalized free energy difference for HI and TI TI

IA

Figure 1. The molecular hydrides included in this Monograph

\begin{tabular}{|c|c|c|c|c|c|c|c|c|}
\hline $\begin{array}{ll}\mathrm{H}_{1} & \end{array}$ & IIA & IB & IIB & IIIA & IVA & VA & VIA & VIIA \\
\hline${ }_{2}^{\mathrm{Li}_{2}}$ & ${ }^{\mathrm{Be}}$ & & & $\mathrm{B}_{18}$ & $\mathrm{C}_{22}$ & $\mathrm{~N}_{26}$ & $\mathrm{O}_{29}$ & $\mathrm{~F}_{31}$ \\
\hline $\mathrm{Na}_{3}$ & $\mathrm{Mg}_{8}$ & & & $\mathrm{Al}_{19}$ & ${ }_{23}$ & $\mathrm{P}_{27}$ & $\mathrm{~S}_{30}$ & $\mathrm{Cl}_{32}$ \\
\hline $\mathrm{K}_{4}$ & $\mathrm{Ca}_{9}$ & $\mathrm{Cu}_{12}$ & $\mathrm{Zn}_{15}$ & & & & & ${ }_{33}{ }_{33}$ \\
\hline $\mathrm{Rb}_{5}$ & ${ }_{10}$ & $\mathrm{Ag}_{13}$ & ${ }_{16}$ & ${ }^{\text {In }} 20$ & $\mathrm{Sn}_{24}$ & & & $\mathrm{I}_{34}$ \\
\hline${ }^{\mathrm{Cs}}{ }_{6}$ & $\mathrm{Ba}_{11}$ & $\mathrm{Au}_{14}$ & $\mathrm{Hg}_{17}$ & $\mathrm{TI}_{21}$ & $\begin{array}{l}\mathrm{Pb} \\
25\end{array}$ & ${ }_{28}{ }^{B}$ & & \\
\hline
\end{tabular}

The numbers in this chart indicate the order of the tables. 


\title{
Ideal Gas Thermodynamic Functions and Isotope Exchange Functions for the Diatomic Hydrides, Deuterides, and Tritides
}

\author{
Lester Haar, Abraham S. Friedman, and Charles W. Beckett
}

\begin{abstract}
This Monograph contains a consistent set of tables of thermodynamic properties of a large number of diatomic hydrides, deuterides, and tritides, for the ideal gas state at one atmosphere pressure. In addition to the thermodynamic properties of the molecular gases, the tables also include thermodynamic properties for chemical reactions involving the isotopic exchange of hydrogen. The thermodynamic properties tabulated are the heat capacity. enthalpy, Gibbs free energy, and entropy.
\end{abstract}

\section{Introduction}

This Monograph contains a consistent set of tables of thermodynamic properties of a large number of diatomic hydrides, deuterides, and tritides, for the ideal gas state and at a pressure of one atmosphere. The entries are tabulated at close intervals for a wide temperature range (from 50 to $5,000^{\circ} \mathrm{K}$ in most cases). In addition to the thermodynamic properties of the molecular gases, the tables also include thermodynamic properties for chemical reactions involving the isotopic exchange of hydrogen. The tables are obtained from statistical calculations based on the internal vibrational and rotational structure of the individual molecules, and the calculations apply to the ideal gas under a pressure of one atmosphere. The large number of molecular species and the density of the tabulated values suggested the use of high speed machine techniques for the detailed statistical calculations, and, by use of the National Bureau of Standards Electronic Automatic Computer (SEAC), a great amount of labor was saved. The thermodynamic properties tabulated are the heat capacity, enthalpy, Gibbs free energy, and entropy. The hydrides included in this Monograph are indicated in the abbreviated periodic table given in figure 1 , and the numbers indicate the order of the tables. For each element listed in figure 1, there is a complete set of tables for the ideal gas molecular properties for the hydride, deuteride, and tritide, together with thermodynamic data for the isotopic hydrogen exchange reactions.

\section{Molecular Constants}

Table 1 lists the molecular constants from which thermodynamic functions have been calculated. These constants were obtained from the data reported in the literature up to the end of August 1958. In this list extensive use was made of the selected list of constants tabulated by Herzberg [1], and, except where otherwise noted, the constants

\footnotetext{
2 Figures in brackets indicate the literature references on page 6.
}

in table 1 were obtained from this source. In some cases the reference [1] data were used even when more recent spectroscopic data were available, the criteria for selection being whether the more recent data would result in significant differences in calculated thermodynamic properties.

The usual notation for the molecular constants is employed, in which the molecular rotational and vibrational energies are represented by polynomials in the quantum numbers. A two-constant expression was used for the vibrational energy; for those molecules where more extensive molecular data were available, these were refitted to two-constant expressions. The fundamental vibrational frequency $\nu$ in wave numbers and the first order anharmonic constant $\omega_{e} X_{e} / \nu$ as used here are defined by the equations

$$
\begin{gathered}
G(V)=\omega_{e}\left(V+\frac{1}{2}\right)-\omega_{e} X_{e}\left(V+\frac{1}{2}\right)^{2} \mathrm{~cm}^{-1} \\
\nu=G(1)-G(0)=\omega_{e}-2 \omega_{e} X_{e} \mathrm{~cm}^{-1},
\end{gathered}
$$

where $G(V)$ is the vibrational energy for the vibrational quantum integer $V$, and $G(0)$ is the zero point vibrational energy. Note that the tabulated values for $\nu$ and $G(0)$ were calculated using the complete set of vibrational data available for each molecule. The rotational constant $B_{0}$ in wave numbers, the stretching constant $\rho$ in reciprocal degrees, and the dimensionless rotationvibration interaction constant $\delta$, are defined by the following equations:

$$
\begin{aligned}
F(J) & =B_{v} J(J+1)-D J^{2}(J+1)^{2} \mathrm{~cm}^{-1} \\
B_{v} & =B_{e}\left[1-\delta\left(V+\frac{1}{2}\right)+\cdots\right] \mathrm{cm}^{-1} \\
B_{0} & =B_{e}(1-\delta / 2), \text { where } \delta=\frac{\alpha_{e}}{B_{e}} \\
\rho & =\frac{2 k}{h c} \frac{D}{B_{0}^{2}}{ }^{\circ} K^{-1},
\end{aligned}
$$


where $F(J)$ here is the rotational energy in wave numbers for molecules with ${ }^{1} \Sigma$ electronic ground states, and where $J$ is the rotational quantum integer.

Also treated in this Monograph are molecules with multiplet electronic ground states, including molecules with ${ }^{2} \Sigma,{ }^{3} \Sigma$, and ${ }^{2} \Pi$ states. The rotational energy for these includes the effect of a coupling between the nuclear rotational motion and the angular inomentum and spin of the electrons, and as a consequence of this coupling the rotational levels are split. This splitting is somewhat complicated when it is nearly equal in magnitude to the rotational quantization, which occurs for most of the ${ }^{2} \Pi$ state molecules we treat. A description of the rotational energy for ${ }^{2} \Pi$ state molecules has been given by Hill and Van Vleck [2] and is also discussed in reference [1]. As listed in reference [1], the energy expressions for the two rotational branches for these molecules are given by

$$
\begin{aligned}
F_{1}(J)=B_{v}\left\{\left(J+\frac{1}{2}\right)^{2}-1-\frac{1}{2}\left[4\left(J+\frac{1}{2}\right)^{2}\right.\right. \\
\left.+Y(Y-4)]^{1 / 2}\right\}-D_{v} J^{4} \mathrm{~cm}^{-1}
\end{aligned}
$$

$$
\begin{aligned}
F_{2}(J)=B_{v}\left\{\left(J+\frac{1}{2}\right)^{2}-1+\frac{1}{2}\left[4\left(J+\frac{1}{2}\right)^{2}\right.\right. \\
\left.+Y(Y-4)]^{1 / 2}\right\}-D_{v}(J+1)^{4} \mathrm{~cm}^{-1},
\end{aligned}
$$

where $Y=A / B_{t}$, and where $A$ is a measure of the electronic splitting and is listed in table 1. For the hydrides with ${ }^{2} \Sigma$ and ${ }^{3} \Sigma$ electronic ground states, the splitting of the rotational states is small, and it is adequate to employ the energy expressions for ${ }^{1} \Sigma$ molecules with appropriate degeneracy factor's.

The rotation-vibration energy is referred to the lowest energy state as zero of energy for the molecule. For ${ }^{1} \Sigma$ molecules this is accomplished by subtracting $G(0)$, the zero point vibrational energy. For the species with multiplet ground states, in addition to $G(0)$, a small residual rotational energy must be subtracted also.

For many of the deuterated compounds and for all of the tritium substituted compounds except $H T$ and $T_{2}$, there are no experimental spectroscopic data of sufficient accuracy to use directly in the partition function. In these cases, therefore, the molecular data were calculated with the aid of isotope relations. That is, the relations $\omega_{e}^{\prime} / \omega_{e}=$ $\left(\mu / \mu^{\prime}\right)^{1 / 2}, \quad \omega_{e}^{\prime} X_{e}^{\prime} / \omega_{e} X_{e}=\mu / \mu^{\prime}, \quad B_{e}^{\prime} / B_{e}=\mu / \mu^{\prime}, \quad \delta^{\prime} / \delta=$ $\left(\mu / \mu^{\prime}\right)^{1 / 2}$, and $\rho=\rho^{\prime}$ were used, where the primed quantities refer to the isotope whose molecular constants are to be calculated and the unprimed constants are those of the reference isotope; $\mu$ is the molecular reduced mass. In those cascs where molecular data were available for both the protium and deuterium isotopic modifications, data for the tritide were obtained by averaging the results from applying the isotope relations to each. For the six isotopic modifications of molecular hydrogen, molecular data were missing only for $D T$, and in this case molecular data were obtained by averaging the l'esults from the other five modifications.

In several cases some of the data were not available for any of the isotopic species, and for these, the missing data were estimated empirically. For those lacking either a vibrational or rotational constant, the missing value was estimated by averaging the results from Badger's rule and the Morse-Clark relation, both of which are discussed in reference [1]. Those molecular species which did not have at least one experimentally obtained vibrational or rotational constant among their three isotopic modifications were not included in this Monograph. The anharmonicity constant $\omega_{e} X_{e} / \nu$ was found to be nearly the same for those hydrides of nearly equal reduced mass; this was also the case for the vibrational rotational coupling constant, $\delta$. A missing rotational stretching constant, $\rho$, was estimated using the Morse potential function.

The chemical atomic weights used in the calculations of the translational partition functions were obtained from the values listed by Wichers [3]. The isotopic masses used in the calculation of the molecular constants are the values listed by Mattauch and Fluegge [4]. The translation contributions for the isotopic hydrogen molecules were calculated using the nuclear masses in reference [4] reduced by the ratio of the chemical [3] to physical mass. The values for the other physical constants employed are those listed in the National Bureau of Standards Circular 500 [5].

\section{Thermodynamic Functions}

The tabulated properties are derived in the usual manner from a partition function which is written

$$
Q=\Sigma e^{-\epsilon_{i} / k T},
$$

where the $\epsilon_{i}$ refer to the molecular energy levels. Equation (1) is limited here to the rotational and vibrational levels of the electronic ground state and the molecular translational contributions. The ideal gas thermodynamic functions in dimensionless units are related to $Q$ in the following way: The Gibbs free energy per mole per degree Kelvin is given by

$$
-\left(\frac{F^{\circ}-E_{0}^{\circ}}{R T}\right)=\ln Q-\ln \mathrm{N},
$$

where $N$ is Avogadro's number.

The enthalpy per mole per degree Kelvin is

$$
\left(\frac{H^{\circ}-E_{0}^{\circ}}{R T}\right)=-\frac{d \ln Q}{d(1 / T)}
$$


The constant pressure heat capacity per mole is

$$
\frac{C_{p}^{\circ}}{R}=\frac{d}{d T}\left(\frac{H^{\circ}-E_{0}^{\circ}}{R}\right)
$$

The entropy per mole is

$$
\frac{S^{\circ}}{R}=\frac{H^{\circ}-E_{0}^{\circ}}{R T}-\frac{F^{\circ}-E_{0}^{\circ}}{R T} .
$$

The translational contribution to the partition function, eq (1), is evaluated in the usual way; see, for example, Mayer and Mayer [6]. The tedious task of performing the partition function summations for the rotational and vibrational contribution is considerably simplified by employing closed form approximations. These approximations include, in addition to the harmonic oscillator-rigid rotator contributions, first order contributions due to anharmonicity, rotation-vibration coupling, and rotational stretching, all of which increase with temperature. They also include low temperature quantum contributions due to the finite spacing of the rotational energy states. However, at very low teniperatures the closed form approximations are not sufficiently accurate for the molecular hydrogen species and for these the term by term summation of the unexpanded partition function was employed in this temperature region.

Most of the species have ${ }^{1} \Sigma$ electronic ground states. The closed form approximation employed for the molecular rotational and vibrational contribution to the partition function for these (except at the low temperatures for molecular hydrogen) may be written

$$
\begin{array}{r}
Q_{\ln \mathrm{t}=\frac{1}{\sigma_{0}}\left(1-e^{-u}\right)^{-1}}\left(1+\frac{\delta}{e^{u}-1}\right)\left(1+\frac{2 \theta}{\left(e^{u}-1\right)^{2}}\right) \\
(1+\rho T)\left(1+\frac{\sigma_{0}}{3}+\frac{\sigma_{0}^{2}}{15}+\frac{4 \sigma_{0}^{3}}{315}\right),
\end{array}
$$

where $\sigma_{0}$ and $u$ are the rotational and vibrational fundamentals in dimensionless units, $\sigma_{0}=\frac{h c}{k T} B_{0}$, $u=\frac{h c}{k T} \nu$, and $\theta=\frac{h c}{k T} \omega_{e} X_{e}$. For the heteronuclear molecular hydrogen isotopes the factor

$$
\frac{1}{\sigma_{0}}\left(1+\frac{\sigma_{0}}{3}+\frac{\sigma_{0}^{2}}{15}+\frac{4}{315} \sigma_{0}^{3}\right)(1+\rho T)
$$

is replaced at low temperatures by the unexpanded expression

$$
Q_{j} \equiv \sum_{J}(2 J+1) e^{-\frac{h c}{k T} F(J)}
$$

The procedure employed for the isotopic hydrides of carbon, silicon, oxygen, and sulfur, which have ${ }^{2} \Pi$ electronic ground states, is given by Haar and
Friedman [7], where the somewhat complicated rotational contribution is obtained as a power series expansion in $\sigma_{0}$ and $Y$. For the remaining ${ }^{2} \Pi$ hydrides, those containing $\mathrm{Sn}$ and $\mathrm{Pb}$, the ground state splitting is comparatively large, with a consequent smaller coupling between the electronic and molecular rotational motion. For these it was adequate to employ the partitition function for ${ }^{1} \Sigma$ molecules, with the splitting accounted for by the factor

$$
2\left(1+e^{-(h c / k T)\left(A-2 B_{0}\right)}\right) .
$$

For the species with ${ }^{2} \Sigma$ and ${ }^{3} \Sigma$ ground states, the treatment is identical with that for ${ }^{1} \Sigma$ molecules, except that the rotational energy includes the appropriate degeneracy factors. For ${ }^{2} \Sigma$ molecules, the degeneracy factor is two, and for ${ }^{3} \Sigma$ molecules, it is three.

The homonuclear molecular species, $\mathrm{H}_{2}, \mathrm{D}_{2}$, and $\mathrm{T}_{2}$ exist in both ortho and para modifications. The normal composition corresponds to a weighted average of the contributions of these two noninteracting modifications. For $\mathrm{H}_{2}$ and $\mathrm{T}_{2}$ the ortho and para modifications are weighted $3 / 4$ and $1 / 4$, respectively, and for $D_{2}, 2 / 3$ and $1 / 3$, respectively. Writing

and

$$
Q_{j(\text { odd })} \equiv \sum_{J(\text { odd values })}(2 J+1) e^{-\frac{h c}{k T} F(J)}
$$

$$
Q_{j(\text { even })} \equiv \sum_{J(\text { even values })}(2 J+1) e^{-\frac{h c}{k T}} F(J)
$$

the rotational contribution to the partition functions for the normal mixtures are given by

$$
\begin{aligned}
& Q_{j}=\left(Q_{j(\text { odd })}\right)^{3 / 4}\left(Q_{j(\text { even })}\right)^{1 / 4} \text { for } H_{2} \text { and } T_{2}, \\
& Q_{j}=\left(Q_{j(\text { odd })}\right)^{1 / 3}\left(Q_{j(\text { even })}\right)^{2 / 3} \text { for } D_{2} .
\end{aligned}
$$

Should a catalyst be present the two modifications would interact to yield the equilibrium composition. The equilibrium expressions for $Q_{j}$ are given by

$$
\begin{aligned}
Q_{j} & =\frac{3}{4} Q_{j(\mathrm{odd})}+\frac{1}{4} Q_{j(\text { even })} \text { for } H_{2} \text { and } T_{2}, \\
Q_{j} & =\frac{1}{3} Q_{j(\mathrm{odd})}+\frac{2}{3} Q_{j(\text { even })} \text { for } D_{2} .
\end{aligned}
$$

\section{Isotope Exchange Reactions}

The equilibrium constants and thermodynamic functions for isotopic exchange reactions involving the diatomic hydrides can be derived readily from the data of table 2 . The equilibrium constant for the isotope exchange reaction

$$
A_{1}+B_{1} \rightarrow A_{2}+B_{2}
$$


where molecule $A_{1}$ differs from $A_{2}$ and $B_{1}$ from $B_{2}$ by the isotope of hydrogen involved, is given by

$$
K=\frac{Q_{A_{2}} \times Q_{B_{2}}}{Q_{A_{1}} \times Q_{B_{1}}}
$$

The equilibrium constant is related to the change in the Gibbs free energy for the reaction by

$$
\frac{\Delta F^{\circ}}{R T}=-\ln K \text {. }
$$

The change in free energy for the reaction may be written in terms of the half-reactions $A_{1} \rightarrow A_{2}$ $B_{1} \rightarrow B_{2}$. Thus,

$$
\frac{\Delta F^{\circ}}{R T}=\left(\frac{\Delta F^{\circ}}{R T}\right)_{A_{1} \rightarrow A_{2}}+\left(\frac{\Delta F^{\circ}}{R T}\right)_{B_{1} \rightarrow B_{2}},
$$

where

$$
\begin{aligned}
\left(\frac{\Delta F^{\circ}}{R T}\right)_{A_{1} \rightarrow A_{2}}= & \left(\frac{F^{\circ}-E_{0}^{\circ}}{R T}\right)_{A_{2}} \\
& -\left(\frac{F^{\circ}-E_{0}^{\circ}}{R T}\right)_{A_{1}}+\frac{\left(\epsilon_{0}\right)_{A_{2}}-\left(\epsilon_{0}\right)_{A_{1}}}{k T} \\
= & \frac{F_{A_{2}}^{\circ}-F_{A_{1}}^{\circ}}{R T},
\end{aligned}
$$

and

$\left(\frac{\Delta F^{\circ}}{R T}\right)_{B_{1} \rightarrow B_{2}}=\frac{F_{B_{2}}^{\circ}-F_{B_{1}}^{\circ}}{R T}$

respectively, where $\epsilon_{0}$ is the zero point energy $\left(\epsilon_{0}=h c G(\mathrm{O})\right)$. The changes in the enthalpy, heat capacity, and entropy for the half-reaction $A_{1} \rightarrow A_{2}$ are given by

$$
\begin{gathered}
\left(\frac{\Delta H^{\circ}}{R T}\right)_{A_{1} \rightarrow A_{2}}=\frac{H_{A_{2}}^{\circ}-H_{A_{1}}^{\circ}}{R T} \\
\left(\frac{\Delta C_{p}^{\circ}}{R}\right)_{A_{1} \rightarrow A_{2}}=\frac{C_{p A_{2}}^{\circ}-C_{p A_{1}}^{\circ}}{R} \\
\left(\frac{\Delta S^{\circ}}{R}\right)_{A_{1} \rightarrow A_{2}}=\left\{\frac{\left.H_{A_{2}}^{\circ}-F_{A_{2}}^{\circ}-\frac{H_{A_{1}}^{\circ}-F_{A_{1}}^{\circ}}{R T}\right\}}{R T}-\right.
\end{gathered}
$$

and similarly for the half-reaction $B_{1} \rightarrow B_{2}$.

Another property of the half-reaction of some interest is

$$
\left(\frac{\Delta \mathscr{F}}{R T}\right)_{A_{1} \rightarrow A_{2}}=\left(\frac{\Delta F^{\circ}}{R T}\right)_{A_{1} \rightarrow A_{2}}-\ln \frac{S_{A_{2}}}{S_{A_{1}}}+\frac{3}{2} \ln \frac{m_{A_{2}}}{m_{A_{1}}}
$$

where $S_{A_{2}}$ is the symmetry number of the molecule $A_{2}$, and $m_{A_{2}}$ the mass of the hydrogen isotope; similarly, for $S_{A_{1}}$ and $m_{A_{1}}$ of molecule $A_{1} . \quad \Delta \mathscr{F} \circ / R T$ is the free energy difference less the effect of the mass difference on the classical contributions to the free energy of rigid rotation, harmonic oscillation, and translation, and less the contributions of molecular symmetry, so that it is a measure of the quantum mechanical contribution to the free energy difference of the half-reaction. ${ }^{2}$ It is analogous to the quantity tabulated by Bigeleisen and Mayer [8].

\section{Tables}

The thermodynamic properties are all tabulated in dimensionless units and refer to the ideal gas at a pressure of one atmosphere. In all cases, the entropy of nuclear spin is omitted, though as discussed in the text the effect of the low temperature spin weighting of the rotational states for the homonuclear hydrogen molecules is included. Many of the elements (other than hydrogen) have isotopic modifications, and the thermal functions are calculated for the naturally occurring mixture of these. In all cases the entropy due to isotope mixing is omitted.

The ideal gas heat capacity, enthalpy, Gibbs free energy, and entropy are tabulated in table 2 at closely spaced temperature intervals. In most cases the tables extend from 50 to $5000{ }^{\circ} \mathrm{K}$. The molecular hydrogen group extends at the low temperature to $10{ }^{\circ} \mathrm{K}$. Table 3 contains the free energy, difference for the half-reactions defined by eqs $(3,4,5,6)$. Table 4 includes the quantity $\Delta \mathscr{F} / R T$ defined by eq (7). Tables 3 and 4 are somewhat more abbreviated than table 2 and the entries are tabulated, in most cases, from 100 to $5000^{\circ} \mathrm{K}$.

The thermodynamic functions in table 2 for the species $H_{2}, D_{2}$, and $T_{2}$ are tabulated for the normal and equilibrium modifications and also for para $H_{2}$ and $T_{2}$, and or tho $D_{2}$. The last group would apply to the low temperature gas where the molecular states having odd rotational quantum integers have been frozen out. Since significant differences between the contributions per mole of the normal and equilibrium modifications exist only at the low temperatures, it is sufficient to include separate tables only at the low temperatures for the equilibrium modifications. The tabulated values involving $H_{2}, D_{2}$, and $T_{2}$ in tables 3 and 4 refer only to the normal niodifications.

\section{Estimated Uncertainties}

The accuracy of table 2 up to temperatures of about $100^{\circ} \mathrm{K}$ depends primarily on the accuracy of the rotational and vibrational fundamentals. At higher temperatures the tables are sensitive to the values of the first order corrections for anharmonicity, vibration-rotation coupling and rotational stretching (all of which were included

2 Thus, in the partition function for the molecular isotope $H X$, the mass appears in the translational contribution as the factor $\left(m_{H}+m_{X}\right)^{3 / 2}$. It appears in the rotational contribution as the reduced mass factor $\mu$, and finally, on expanding the harmonic oscillator vibrational factor for high temperatures, it appears as the factor $\mu^{1 / 2}$. Combining these, and deleting this quantity together with the molecular symmetry from the free energy differences between isotopes leads to eq (7). 
in this treatment), and at the very highest temperatures, to second order rotational and vibrational corrections, not included in this treatment. In addition, since only the ground electronic states were included, table 2 for those species having low-lying excited electronic states such as $\mathrm{BiH}$, $\mathrm{BiD}$, and $\mathrm{BiT}$ have additional large uncertainties at the high temperatures. (See footnote on page 8 below table 1 on $\mathrm{BiH}, \mathrm{BiD}$, and $\mathrm{BiT}^{\prime}$.)

The electronic structure is nearly identical for isotopic molecules, so that tables 3 and 4 which contain differences between the thermal functions for isotopes should not be significantly affected by the omission of the excited electronic states. The tables primarily are sensitive to vibrational and rotational quantum effects, so that higher order ground state corrections should also cancel. Tables 3 and 4 thus are sensitive primarily to the vibrational fundamental (and, at low temperatures, somewhat to the rotational fundamental), and at the very high temperatures to the anharmonicity of the vibrations.

The approximations used in the statistical method lead to an uncertainty in the thermodynamic functions (table 2 ) in the third place to the right of the decimal below $1000^{\circ} \mathrm{K}$, provided the uncertainty in the values used for the rotational and vibrational fundamentals do not exceed 1 percent. In nearly all cases, experimentally determined values are more accurate than 1 percent. In the few cases where the fundamentals were estimated (empirically) they could be in error by as much as 5 percent and the uncertainty in the thermodynamic functions might then extend to the second digit to the right of the decimal: at the very low temperatures, if the rotational fundamental were estimated; or at temperatures above $500^{\circ} \mathrm{K}$, if the estimate were made in the vibrational fundamental. The isotope relations employed for calculating the molecular constants of the deuterides and tritides should not contribute significantly to the uncertainties, so that the same error estimates should apply for all three isotopic modifications. At higher temperatures the uncertainty due to neglected higher order terms in the energy level expressions and the omission of the effect of some sort of rotational cut-off (see ref. [9] for a procedure for including this effect) is somewhat larger, perhaps extending to the second or even the first place to the right of the decimal. (The group IIB hydrides $\mathrm{ZnH}, \mathrm{CdH}, \mathrm{HgH}$, all have shallow potential energy wells-less than 1 ev in depth. Thus for these, the high temperature calculations have even larger uncertainties. Tables 3 and 4 for this group are therefore abbreviated at the higher temperatures). ${ }^{3}$ In most

For these a virial treatment such as used in reference [9] might be more appropriate at the higher temperatures. instances the internal consistency of the tables is such that differences between the thermal functions of isotopic species are significant to the fourth (or fifth) figure to the right of the decimal (particularly for the heat capacity and entropy differences). The contributions of excited electronic states omitted from these calculations depend largely upon their excitation energies. A simple metliod for estimating their contribution is given by Beckett and Haar [9].

The free energy (and enthalpy) differences in tables 3 and 4 are more sensitive to errors in the vibrational fundamental than table 2 through their dependence on the zero point energy. For instance, the uncertainty in the free energy differences may be about 0.1 percent if $G(0)$ is in error by about 5 percent.

The tabulated properties are given to five decimal places. This was done to facilitate their use in certain applications, such as in repeated operations involving small differences, where additional digits are needed to retain significance in the final result. As indicated earlier, up to $1000^{\circ} \mathrm{K}$, the entries at best are accurate to only the third or, possibly, the fourth decimal place, and at the higher temperatures, the uncertainty is appreciably greater.

We acknowledge the assistance of a number of members of the staff of the Thermodynamics Section and Applied Mathematics Laboratory; in particular, Dr. Harold W. Woolley, Dr. Irwin Oppenheim, and Mr. Joseph Hilsenrath for helpful advice and discussions; Messrs. John Park, Joe C. Bradley, and Charles M. Sommerfield for assistance in the assembly of the data; Mrs. Bertha H. Walter and Miss Mary M. Dunlap for performing many of the tedious desk calculations; Mrs. Ethel Marden and Mr. Peter O'Hara for programing the machine calculations, and Miss Hattie M. Napier for assistance in the preparation of the manuscript and tables.

Since the compilation of the bulk of the calculations on which these tables are based, two of the authors have left the National Bureau of Standards and carried on incidental calculations and manuscript preparation from their present positions. We wish, therefore, to thank CONVAIR, A Division of General Dynamics, with whom Mr. Haar is now associated, for making $\mathrm{Mr}$. Lester Haar available for this task, and the U.S. Atomic Energy Commission, with whom Dr. Abraham S. Friedman is now associated. Thanks are also due to the Johns Hopkins University Applied Physics Laboratory for their cooperation during a period prior to 1959 when Mr. Haar was associated with that laboratory. 


\section{References}

[1] G. Herzberg, Molecular spectra and molecular structure. I. "Spectra of Diatomic Molecules." Second Edition (D. Van Nostrand Co., Inc., New York, N.Y., 1951).

[2] E. L. Hill and J. H. Van Vleck, Phys. Rev. 32, 250 (1923).

[3] E. Wichers, J. Am. Chem. Soc. 74, 2447 (1952).

[4] J. Mattauch and S. Fluegge, Nuclear physics tables (Interscience Publishers, Inc., New York, N.Y., 1946).
[5] F. D. Rossini, D. D. Wagman, W. H. Evans, S. Levine, and I. Jaffe, Selected values of chemical thermodynamic properties, NBS Circ. 500 (U.S. Government Printing Office, Washington, D.C., 1952).

[6] J. E. Mayer and M. G. Mayer, Statistical mechanics (John Wiley \& Sons, Inc., New York, N.Y., 1940).

[7] L. Haar and A. S. Friedman, J. Chem. Phys. 23, 869 (1955).

[8] J. Bigeleisen and M. G. Mayer, J. Chem. Phys. 15, $261(1947)$.

[9] C. W. Beckett and L. Haar, Proceedings of the Conference on Thermodynamic and Transport Properties of Fluids, London, pp. 27-33, Institution of Mechanical Engineers, London (1958).

TABLE 1. Molecular constants

\begin{tabular}{|c|c|c|c|c|c|c|}
\hline Compound & $\nu$ & $\omega_{e} X_{e} / \nu$ & $\delta$ & $B_{0}$ & $\rho \times 10^{5}$ & $\mathrm{G}(0)$ \\
\hline $\begin{array}{l}\mathrm{H}_{2}{ }^{1} \Sigma \\
\mathrm{HD}^{1} \Sigma \\
\mathrm{HT}^{1}{ }^{1} \\
\mathrm{D}_{2}{ }^{1} \Sigma \\
\mathrm{DT}^{1}{ }^{1} \Sigma \\
\mathrm{T}_{2}{ }^{1} \Sigma\end{array}$ & $\begin{array}{l}4160.20 \\
3631.52 \\
3433.1 \\
2993.82 \\
2744.0 \\
2466.1\end{array}$ & $\begin{array}{l}0.0283 \\
0.0261 \\
0.0255 \\
0.0214 \\
0.0190 \\
0.0178\end{array}$ & $\begin{array}{l}0.0504 \\
0.0446 \\
0.0420 \\
0.0350 \\
0.0320 \\
0.0296\end{array}$ & $\begin{array}{l}\text { 59. } 319 \\
44.669 \\
\text { 39. } 7468 \\
29.905 \\
24.970 \\
20.0300\end{array}$ & $\begin{array}{l}\text { 1. } 810 \\
\text { 1. } 790 \\
\text { 1. } 802 \\
\text { 1. } 792 \\
\text { 1. } 790 \\
\text { 1. } 788\end{array}$ & $\begin{array}{l}2168.16 \\
1884.98 \\
1782.2 \\
1543.36 \\
1413.2 \\
1265.9\end{array}$ \\
\hline $\begin{array}{l}\mathrm{LiH}^{1}{ }^{1} \\
\mathrm{LiD}^{1} \\
\mathrm{LiT}^{1}{ }_{\Sigma}\end{array}$ & $\begin{array}{l}1359.78 \\
1029.05 \\
889\end{array}$ & $\begin{array}{l}0.0171 \\
0.0129 \\
0.011\end{array}$ & $\begin{array}{l}0.0288 \\
0.0220 \\
0.019\end{array}$ & $\begin{array}{l}\text { 7. } 4067 \\
\text { 4. } 1879 \\
\text { 3. } 112\end{array}$ & $\begin{array}{l}1.08 \\
1.08 \\
1.08\end{array}$ & $\begin{array}{l}697.05 \\
524.27 \\
452\end{array}$ \\
\hline $\begin{array}{l}\mathrm{NaH}^{1} \Sigma \\
\mathrm{NaD}^{1}{ }^{2} \\
\mathrm{NaT}^{1} \Sigma\end{array}$ & $\begin{array}{c}1133.3 \\
826.1 \\
691\end{array}$ & $\begin{array}{l}0.0174 \\
0.0124 \\
0.010\end{array}$ & $\begin{array}{l}0.0280 \\
0.0205 \\
0.017\end{array}$ & $\begin{array}{l}\text { 4. } 8335 \\
\text { 2. } 5315 \\
\text { 1. } 762\end{array}$ & $\begin{array}{l}\text { 1. } 96 \\
\text { 1. } 96 \\
1.96\end{array}$ & $\begin{array}{l}581.2 \\
420.8 \\
351\end{array}$ \\
\hline $\begin{array}{l}\mathrm{KH}^{1}{ }^{1 \Sigma} \\
\mathrm{KD}^{1}{ }^{1 \Sigma} \\
\mathrm{KT}^{1}{ }^{1}\end{array}$ & $\begin{array}{l}955.7 \\
690.7 \\
574\end{array}$ & $\begin{array}{l}0.0153 \\
0.0110 \\
0.0091\end{array}$ & $\begin{array}{l}0.0200 \\
0.0169 \\
0.014\end{array}$ & $\begin{array}{l}\text { 3. } 373 \\
\text { 1. } 6524 \\
\text { 1. } 132\end{array}$ & $\begin{array}{l}\text { 1. } 60 \\
\text { 1. } 60 \\
1.60\end{array}$ & $\begin{array}{l}488.8 \\
351.1 \\
291\end{array}$ \\
\hline $\begin{array}{l}\text { RbH }{ }^{1} \Sigma \\
\mathrm{RbD}^{1} \Sigma \\
\mathrm{RbT}^{1} \Sigma\end{array}$ & $\begin{array}{l}908.71 \\
652 \\
538\end{array}$ & $\begin{array}{l}0.0156 \\
0.0110 \\
0.0090\end{array}$ & $\begin{array}{l}0.0242 \\
0.017 \\
0.014\end{array}$ & $\begin{array}{l}\text { 2. } 984 \\
1.516 \\
1.025\end{array}$ & $\begin{array}{l}\text { 1. } 90 \\
1.90 \\
1.90\end{array}$ & $\begin{array}{l}464.86 \\
331 \\
273\end{array}$ \\
\hline $\begin{array}{l}\mathrm{CsH}{ }^{1 \Sigma} \\
\mathrm{CsD}{ }^{1 \Sigma} \\
\mathrm{CsT}^{1 \Sigma}\end{array}$ & $\begin{array}{l}865.5 \\
620 \\
510\end{array}$ & $\begin{array}{l}0.0146 \\
0.010 \\
0.008\end{array}$ & $\begin{array}{l}0.0213 \\
0.015 \\
0.012\end{array}$ & $\begin{array}{l}2.680 \\
1.356 \\
0.913\end{array}$ & $\begin{array}{l}1.94 \\
1.94 \\
1.94\end{array}$ & $\begin{array}{l}442.2 \\
315 \\
258\end{array}$ \\
\hline $\begin{array}{l}\mathrm{CuH}{ }^{1} \Sigma^{+} \\
\mathrm{CuD} 1 \Sigma^{+} \\
\mathrm{CuT}{ }^{1} \Sigma^{+}\end{array}$ & $\begin{array}{l}1866.4 \\
1346.2 \\
1114\end{array}$ & $\begin{array}{l}0.0198 \\
0.0142 \\
0.012\end{array}$ & $\begin{array}{l}0.0319 \\
0.0229 \\
0.019\end{array}$ & $\begin{array}{l}\text { 7. } 813 \\
\text { 3. } 992 \\
\text { 2. } 710\end{array}$ & $\begin{array}{l}\text { 1. } 18 \\
\text { 1. } 18 \\
1.18\end{array}$ & $\begin{array}{l}961.0 \\
687.4 \\
563\end{array}$ \\
\hline $\begin{array}{l}\mathrm{AgH} 1 \Sigma^{+} \\
\mathrm{AgD} 1 \Sigma^{+} \\
\operatorname{AgT} 1 \Sigma^{+}\end{array}$ & $\begin{array}{l}1691.9 \\
1216 \\
1004\end{array}$ & $\begin{array}{l}0.0202 \\
0.015 \\
0.012\end{array}$ & $\begin{array}{l}0.0554 \\
0.0227 \\
0.019\end{array}$ & $\begin{array}{l}\text { 6. } 279 \\
\text { 3. } 223 \\
\text { 2. } 17\end{array}$ & $\begin{array}{l}\text { 1. } 20 \\
\text { 1. } 20 \\
\text { 1. } 20\end{array}$ & $\begin{array}{l}871.5 \\
621 \\
511\end{array}$ \\
\hline $\begin{array}{l}\text { AuH }{ }^{1} \Sigma^{+} \\
\text {AuD }{ }^{1} \Sigma^{+} \\
\text {AuT }{ }^{1} \Sigma^{+}\end{array}$ & $\begin{array}{l}2218.8 \\
1591.6 \\
1310\end{array}$ & $\begin{array}{l}0.0195 \\
0.0136 \\
0.011\end{array}$ & $\begin{array}{l}0.0299 \\
0.0211 \\
0.0085\end{array}$ & $\begin{array}{l}\text { 7. } 1333 \\
\text { 3. } 6034 \\
\text { 2. } 43\end{array}$ & $\begin{array}{l}0.76 \\
0.76 \\
0.76\end{array}$ & $\begin{array}{l}\text { 1141. } 7 \\
812.1 \\
666\end{array}$ \\
\hline $\begin{array}{l}\text { BeH }{ }^{2} \Sigma \\
\text { BeD }^{2} \Sigma \\
\text { BeT }^{2} \Sigma\end{array}$ & $\begin{array}{l}1986.0 \\
1488 \\
1275\end{array}$ & $\begin{array}{l}0.0179 \\
0.013 \\
0.011\end{array}$ & $\begin{array}{l}0.0295 \\
0.022 \\
0.019\end{array}$ & $\begin{array}{c}10.158 \\
5.62 \\
4.10\end{array}$ & $\begin{array}{l}1.32 \\
1.32 \\
1.32\end{array}$ & $\begin{array}{c}1020.4 \\
759 \\
648\end{array}$ \\
\hline $\begin{array}{l}\operatorname{MgH}^{2} \Sigma \\
\operatorname{MgD}^{2} \Sigma \\
\operatorname{MgT}^{2} \Sigma\end{array}$ & $\begin{array}{c}1432.2 \\
1045.6 \\
875\end{array}$ & $\begin{array}{l}0.0220 \\
0.0154 \\
0.013\end{array}$ & $\begin{array}{l}0.0291 \\
0.0218 \\
0.018\end{array}$ & $\begin{array}{l}\text { 5. } 7329 \\
\text { 2. } 9980 \\
\text { 2. } 080\end{array}$ & $\begin{array}{l}\text { 1. } 39 \\
\text { 1. } 39 \\
\text { 1. } 39\end{array}$ & $\begin{array}{l}740.0 \\
534.9 \\
446\end{array}$ \\
\hline $\begin{array}{l}\mathrm{CaH}^{2} \Sigma \\
\mathrm{CaD}^{2} \Sigma \\
\mathrm{CaT}^{2} \Sigma\end{array}$ & $\begin{array}{r}1260 \\
910 \\
755\end{array}$ & $\begin{array}{l}0.0155 \\
0.011 \\
0.009\end{array}$ & $\begin{array}{l}0.0228 \\
0.0161 \\
0.013\end{array}$ & $\begin{array}{l}\text { 4. } 2297 \\
\text { 2. } 178 \\
\text { 1. } 489\end{array}$ & $\begin{array}{l}\text { 1. } 43 \\
\text { 1. } 43 \\
\text { 1. } 43\end{array}$ & $\begin{array}{l}645 \\
463 \\
383\end{array}$ \\
\hline $\begin{array}{l}\text { SrH }{ }^{2} \Sigma \\
\text { SrD }^{2} \Sigma \\
\text { SrT }^{2} \Sigma\end{array}$ & $\begin{array}{c}1172.2 \\
841 \\
694\end{array}$ & $\begin{array}{l}0.0145 \\
0.010 \\
0.008\end{array}$ & $\begin{array}{l}0.0223 \\
0.0158 \\
0.013\end{array}$ & $\begin{array}{l}\text { 3. } 6344 \\
\text { 1. } 8463 \\
\text { 1. } 248\end{array}$ & $\begin{array}{l}\text { 1. } 42 \\
\text { 1. } 42 \\
\text { 1. } 42\end{array}$ & $\begin{array}{l}598.9 \\
427 \\
351\end{array}$ \\
\hline
\end{tabular}


TaBle 1. Molecular constants-Continued

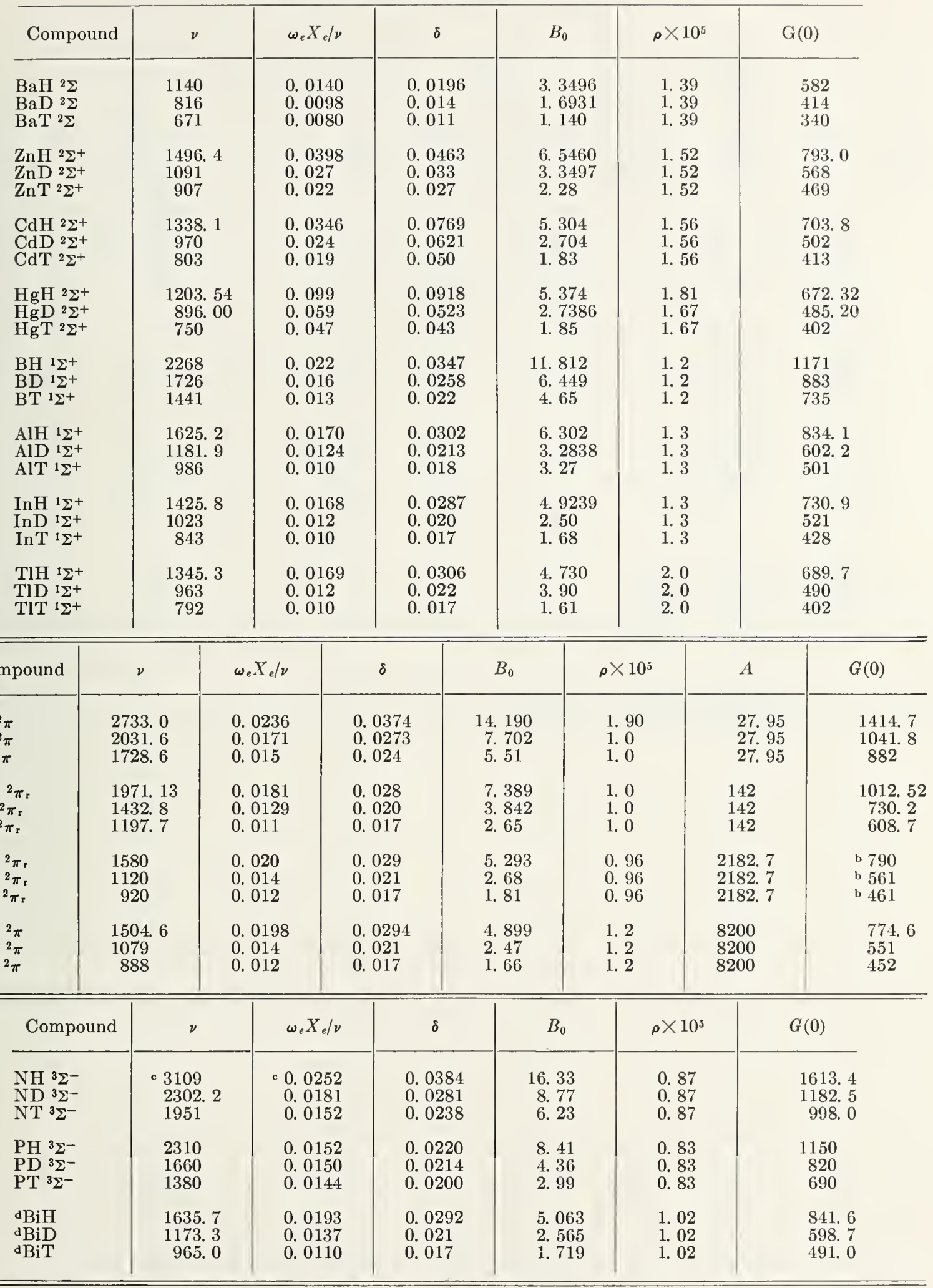


TABLE 1. Molecular constants-Continued

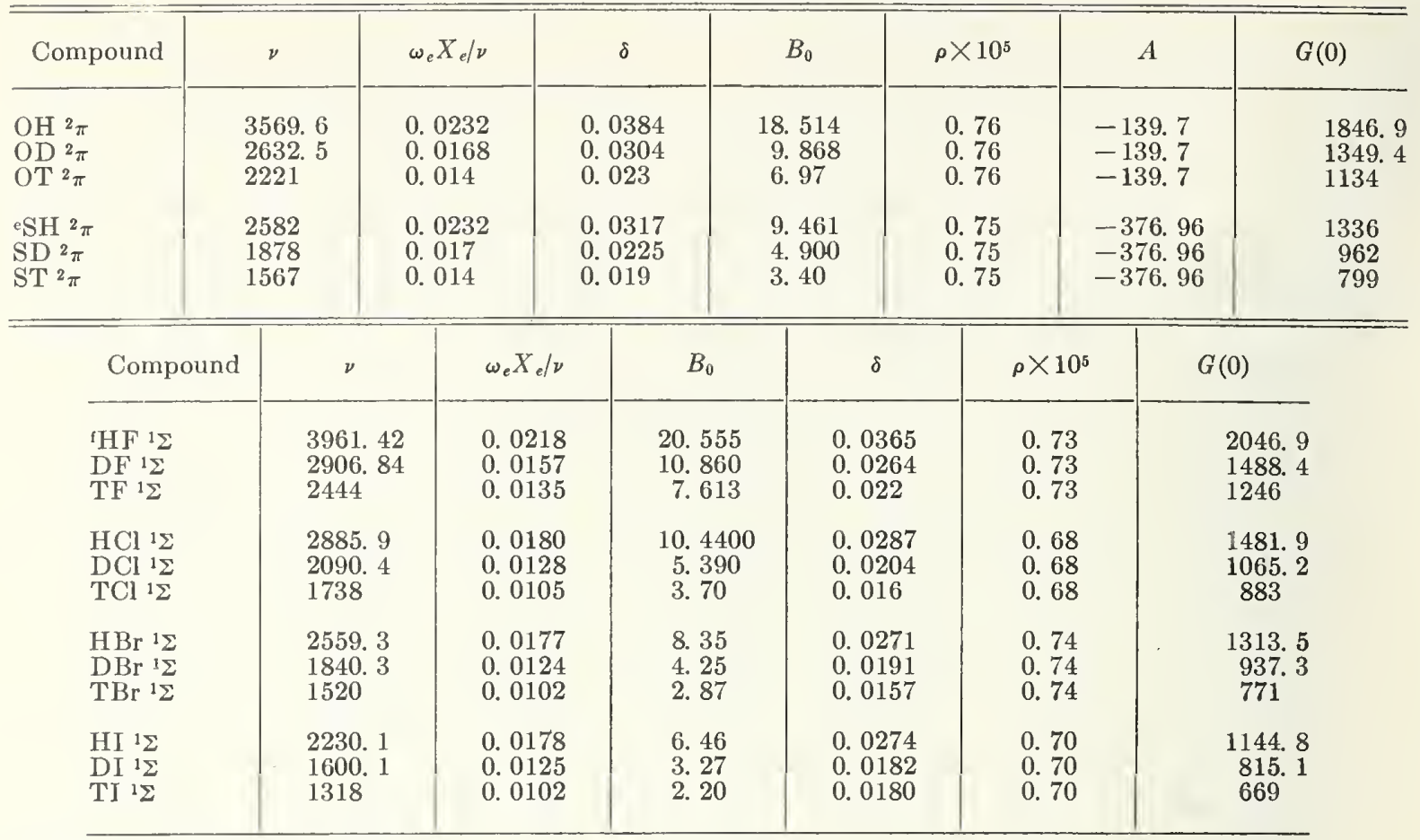

\& A. E. Douglas, Can. J. Phys. 35, 71 (1957).

b Taken as one-half the estimated fundamental.

c Vibrational data from Pannetier and Gaydon, J chim, phys, 48, 221 (1951).

d The lowest state is a singlet state $O^{+}$Herzberg [1], and the tables are computed for this state. An excited doublet state at $4923 \mathrm{~cm}-1$ occurs but is not included in the thermal functions. If this doublet state were included in the thermal functions, it would give a factor of

$$
Q_{0}=1+2 e^{-7082 / T}
$$

In the partition function of all three isotopic species ( $\mathrm{BiH}, \mathrm{BiD}, \mathrm{Bi} \mathrm{T})$ to a very close approximation.

e Molecular constants by Ramsey, J. Chem. Phys. 20, 1920 (1952). A force constant has since been reported by Leach, J. Chem. Phys. 22, 1261 (1954), which is in good agreement, within 0.1 percent, of that obtained by Ramsey.

Data from Talley, Kaylor, Nielson, Phys. Rev. 7\%, 529 (1950) 
Table 2.001. $\mathrm{H}_{2}$ - Normal Mixture

\begin{tabular}{|l|l|l|l|l|}
\hline${ }^{\circ} K$ & $\frac{C_{p}^{0}}{R}$ & $\frac{\left(H^{0}-E_{0}^{0}\right)}{R T}$ & $\frac{-\left(F^{0}-E_{0}^{0}\right)}{R T}$ & $\frac{S^{0}}{R}$ \\
\hline
\end{tabular}

\begin{tabular}{|c|c|c|c|c|c|c|c|c|}
\hline $\begin{array}{l}10 \\
20 \\
30 \\
40\end{array}$ & $\begin{array}{l}2.50000 \\
2.50000 \\
2.50002 \\
2.50059\end{array}$ & $\begin{array}{r}2 \\
57 \\
429\end{array}$ & $\begin{array}{r}15.28092 \\
8.89040 \\
6.76031 \\
5.69528\end{array}$ & $\begin{array}{l}-639052 \\
-213009 \\
-106503 \\
-63862\end{array}$ & $\begin{array}{r}-8.81350 \\
-\quad .69019 \\
2.45362 \\
4.23789\end{array}$ & $\begin{array}{l}812331 \\
314381 \\
178427 \\
119695\end{array}$ & $\begin{array}{l}6.46742 \\
8.20027 \\
9.21393 \\
9.93317\end{array}$ & $\begin{array}{r}173285 \\
101366 \\
71924 \\
55833\end{array}$ \\
\hline $\begin{array}{l}50 \\
60 \\
70 \\
80 \\
90\end{array}$ & $\begin{array}{l}2.50488 \\
2.51868 \\
2.54678 \\
2.59041 \\
2.64746\end{array}$ & $\begin{array}{l}1380 \\
2810 \\
4363 \\
5705 \\
6642\end{array}$ & $\begin{array}{l}5.05666 \\
4.63234 \\
4.33221 \\
4.11160 \\
3.94564\end{array}$ & $\begin{array}{l}-42432 \\
-30013 \\
-22061 \\
-16596 \\
-12655\end{array}$ & $\begin{array}{l}5.43484 \\
6.31688 \\
7.00717 \\
7.57054 \\
8.04478\end{array}$ & $\begin{array}{l}88204 \\
69029 \\
56337 \\
47424 \\
40887\end{array}$ & $\begin{array}{l}10.49150 \\
10.94922 \\
11.33938 \\
11.68214 \\
11.99042\end{array}$ & $\begin{array}{l}45772 \\
39016 \\
34276 \\
30828 \\
28232\end{array}$ \\
\hline $\begin{array}{l}100 \\
110 \\
120 \\
130 \\
140\end{array}$ & $\begin{array}{l}2.71388 \\
2.78512 \\
2.85715 \\
2.92697 \\
2.99268\end{array}$ & $\begin{array}{l}7124 \\
7203 \\
6982 \\
6571 \\
6964\end{array}$ & $\begin{array}{l}3.81909 \\
3.72183 \\
3.64678 \\
3.58875 \\
3.54385\end{array}$ & $\begin{array}{l}-\quad 9726 \\
-\quad 7505 \\
-\quad 5803 \\
-\quad 4490 \\
-\quad 3469\end{array}$ & $\begin{array}{l}8.45365 \\
8.81288 \\
9.13336 \\
9.42286 \\
9.68711\end{array}$ & $\begin{array}{l}35923 \\
32048 \\
28950 \\
26425 \\
24326\end{array}$ & $\begin{array}{l}12.27274 \\
12.53471 \\
12.78014 \\
13.01161 \\
13.23096\end{array}$ & $\begin{array}{l}26197 \\
24543 \\
23147 \\
21935 \\
20857\end{array}$ \\
\hline $\begin{array}{l}150 \\
160 \\
170 \\
180 \\
190\end{array}$ & $\begin{array}{l}3.05332 \\
3.10860 \\
3.15860 \\
3.20360 \\
3.24394\end{array}$ & $\begin{array}{l}5528 \\
5000 \\
4500 \\
4034 \\
3604\end{array}$ & $\begin{array}{l}3.50916 \\
3.48242 \\
3.46193 \\
3.44635 \\
3.43465\end{array}$ & $\begin{array}{l}-\quad 2674 \\
-\quad 2049 \\
-\quad 1558 \\
-\quad 1170 \\
-\quad 861\end{array}$ & $\begin{array}{r}9.93037 \\
10.15595 \\
10.36643 \\
10.56384 \\
10.74985\end{array}$ & $\begin{array}{l}22558 \\
21048 \\
19741 \\
18601 \\
17594\end{array}$ & $\begin{array}{l}13.43953 \\
13.63837 \\
13.82836 \\
14.01019 \\
14.18450\end{array}$ & $\begin{array}{l}19884 \\
18999 \\
18183 \\
17431 \\
16733\end{array}$ \\
\hline $\begin{array}{l}200 \\
210 \\
220 \\
230 \\
240\end{array}$ & $\begin{array}{l}3.27998 \\
3.31206 \\
3.34048 \\
3.36555 \\
3.38754\end{array}$ & $\begin{array}{l}3208 \\
2842 \\
2507 \\
2199 \\
1919\end{array}$ & $\begin{array}{l}3.42604 \\
3.41986 \\
3.41562 \\
3.41291 \\
3.41140\end{array}$ & $\begin{array}{l}-\quad 618 \\
-\quad 424 \\
-\quad 271 \\
-\quad 151 \\
-\quad 56\end{array}$ & $\begin{array}{l}10.92579 \\
11.09279 \\
11.25178 \\
11.40354 \\
11.54875\end{array}$ & $\begin{array}{l}16700 \\
15899 \\
15176 \\
14521 \\
13925\end{array}$ & $\begin{array}{l}14.35183 \\
14.51265 \\
14.66740 \\
14.81645 \\
14.96015\end{array}$ & $\begin{array}{l}16082 \\
15475 \\
14905 \\
14370 \\
13869\end{array}$ \\
\hline $\begin{array}{l}250 \\
260 \\
270 \\
280 \\
290\end{array}$ & $\begin{array}{l}3.40673 \\
3.42339 \\
3.43778 \\
3.45016 \\
3.46074\end{array}$ & $\begin{array}{r}1656 \\
1439 \\
1238 \\
1058 \\
903\end{array}$ & $\begin{array}{l}3.41084 \\
3.41101 \\
3.41174 \\
3.41290 \\
3.41437\end{array}$ & $\begin{array}{r}17 \\
73 \\
116 \\
147 \\
170\end{array}$ & $\begin{array}{l}11.68800 \\
11.82178 \\
11.95052 \\
12.07461 \\
12.19440\end{array}$ & $\begin{array}{l}13378 \\
12874 \\
12409 \\
11979 \\
11579\end{array}$ & $\begin{array}{l}15.09884 \\
15.23279 \\
15.36226 \\
15.48751 \\
15.60877\end{array}$ & $\begin{array}{l}13395 \\
12947 \\
12525 \\
12126 \\
11749\end{array}$ \\
\hline $\begin{array}{l}300 \\
310 \\
320 \\
330 \\
340\end{array}$ & $\begin{array}{l}3.46977 \\
3.47744 \\
3.48394 \\
3.48944 \\
3.49408\end{array}$ & $\begin{array}{l}767 \\
650 \\
550 \\
454 \\
391\end{array}$ & $\begin{array}{l}3.41607 \\
3.41793 \\
3.41990 \\
3.42192 \\
3.42398\end{array}$ & $\begin{array}{l}186 \\
197 \\
202 \\
206 \\
206\end{array}$ & $\begin{array}{l}12.31019 \\
12.42223 \\
12.53077 \\
12.63604 \\
12.73822\end{array}$ & $\begin{array}{r}11204 \\
10854 \\
10527 \\
10218 \\
9928\end{array}$ & $\begin{array}{l}15.72626 \\
15.84016 \\
15.95067 \\
16.05796 \\
16.16220\end{array}$ & $\begin{array}{l}11390 \\
11051 \\
10729 \\
10424 \\
10134\end{array}$ \\
\hline $\begin{array}{l}350 \\
360 \\
370 \\
380 \\
390\end{array}$ & $\begin{array}{l}3.49799 \\
3.50129 \\
3.50439 \\
3.50645 \\
3.50846\end{array}$ & $\begin{array}{l}330 \\
280 \\
236 \\
201 \\
171\end{array}$ & $\begin{array}{l}3.42604 \\
3.42808 \\
3.43010 \\
3.43208 \\
3.43401\end{array}$ & $\begin{array}{l}204 \\
202 \\
198 \\
193 \\
189\end{array}$ & $\begin{array}{l}12.83750 \\
12.93405 \\
13.02799 \\
13.11950 \\
13.20867\end{array}$ & $\begin{array}{l}9655 \\
9394 \\
9151 \\
8917 \\
8696\end{array}$ & $\begin{array}{l}16.26354 \\
16.36213 \\
16.45809 \\
16.55158 \\
16.64268\end{array}$ & $\begin{array}{l}9859 \\
9596 \\
9349 \\
9110 \\
8885\end{array}$ \\
\hline $\begin{array}{l}400 \\
450 \\
500 \\
550 \\
600\end{array}$ & $\begin{array}{l}3.51017 \\
3.51584 \\
3.51916 \\
3.52248 \\
3.52672\end{array}$ & $\begin{array}{l}567 \\
332 \\
332 \\
424 \\
572\end{array}$ & $\begin{array}{l}3.43590 \\
3.44450 \\
3.45181 \\
3.45794 \\
3.46335\end{array}$ & $\begin{array}{l}860 \\
731 \\
613 \\
541 \\
508\end{array}$ & $\begin{array}{l}13.29563 \\
13.70084 \\
14.06414 \\
14.39367 \\
14.69493\end{array}$ & $\begin{array}{l}40521 \\
36330 \\
32953 \\
30126 \\
27742\end{array}$ & $\begin{array}{l}16.73153 \\
17.14534 \\
17.51595 \\
17.85162 \\
18.15829\end{array}$ & $\begin{array}{l}41381 \\
37061 \\
33567 \\
30567 \\
28250\end{array}$ \\
\hline $\begin{array}{l}650 \\
700 \\
750 \\
800 \\
850\end{array}$ & $\begin{array}{l}3.53244 \\
3.54012 \\
3.54985 \\
3.56183 \\
3.57604\end{array}$ & $\begin{array}{r}768 \\
973 \\
1198 \\
1421 \\
1637\end{array}$ & $\begin{array}{l}3.46843 \\
3.47327 \\
3.47804 \\
3.48289 \\
3.48794\end{array}$ & $\begin{array}{l}484 \\
477 \\
485 \\
505 \\
534\end{array}$ & $\begin{array}{l}14.97235 \\
15.22957 \\
15.46936 \\
15.69399 \\
15.90529\end{array}$ & $\begin{array}{l}25722 \\
23979 \\
22463 \\
21130 \\
19951\end{array}$ & $\begin{array}{l}18.44079 \\
18.70285 \\
18.94741 \\
19.17688 \\
19.39323\end{array}$ & $\begin{array}{l}26206 \\
24456 \\
22947 \\
21635 \\
20485\end{array}$ \\
\hline $\begin{array}{r}900 \\
950 \\
1000 \\
1050 \\
1100\end{array}$ & $\begin{array}{l}3.59241 \\
3.61076 \\
3.63089 \\
3.65255 \\
3.67548\end{array}$ & $\begin{array}{l}1835 \\
2013 \\
2166 \\
2293 \\
2396\end{array}$ & $\begin{array}{l}3.49328 \\
3.49897 \\
3.50506 \\
3.51156 \\
3.51849\end{array}$ & $\begin{array}{l}569 \\
609 \\
650 \\
693 \\
734\end{array}$ & $\begin{array}{l}16.10480 \\
16.29382 \\
16.47345 \\
16.64462 \\
16.80813\end{array}$ & $\begin{array}{l}18902 \\
17963 \\
17117 \\
16351 \\
15657\end{array}$ & $\begin{array}{l}19.59808 \\
19.79280 \\
19.97851 \\
20.15618 \\
20.32662\end{array}$ & $\begin{array}{l}19472 \\
18571 \\
17767 \\
17044 \\
16391\end{array}$ \\
\hline 1150 & 3.69944 & 2473 & 3.52583 & 775 & 16.96470 & 15022 & 20.49053 & 15797 \\
\hline
\end{tabular}


Table 2.001. $\mathrm{H}_{2}$ - Normal Mixture (Cont.)

\begin{tabular}{|c|c|c|c|c|c|c|c|c|}
\hline${ }^{\circ} \mathrm{K}$ & $\frac{C_{p}^{0}}{R}$ & & $\frac{\left(H^{0}-E_{0}^{0}\right)}{R T}$ & & $\frac{-\left(F^{0}-E\right.}{R T}$ & & $\frac{S^{0}}{R}$ & \\
\hline $\begin{array}{l}1200 \\
1300 \\
1400 \\
1500 \\
1600\end{array}$ & $\begin{array}{l}3.72417 \\
3.77513 \\
3.82681 \\
3.87808 \\
3.92809\end{array}$ & $\begin{array}{l}5096 \\
5168 \\
5127 \\
5001 \\
4821\end{array}$ & $\begin{array}{l}3.53358 \\
3.55019 \\
3.56810 \\
3.58706 \\
3.60682\end{array}$ & $\begin{array}{l}1661 \\
1791 \\
1896 \\
1976 \\
2033\end{array}$ & $\begin{array}{l}17.11492 \\
17.39840 \\
17.66215 \\
17.90896 \\
18.14109\end{array}$ & $\begin{array}{l}28348 \\
26375 \\
24681 \\
23213 \\
21927\end{array}$ & $\begin{array}{l}20.64850 \\
20.94859 \\
21.23025 \\
21.49603 \\
21.74792\end{array}$ & $\begin{array}{l}30009 \\
28166 \\
26578 \\
25189 \\
23960\end{array}$ \\
\hline $\begin{array}{l}1700 \\
1800 \\
1900 \\
2000 \\
2100\end{array}$ & $\begin{array}{l}3.97630 \\
4.02239 \\
4.0661 .7 \\
4.10760 \\
4.14668\end{array}$ & $\begin{array}{l}4609 \\
4378 \\
4143 \\
3908 \\
3682\end{array}$ & $\begin{array}{l}3.62715 \\
3.64783 \\
3.66871 \\
3.68963 \\
3.71047\end{array}$ & $\begin{array}{l}2068 \\
2088 \\
2092 \\
2084 \\
2067\end{array}$ & $\begin{array}{l}18.36036 \\
18.56827 \\
18.76606 \\
18.95477 \\
19.13529\end{array}$ & $\begin{array}{l}20791 \\
19779 \\
18871 \\
18052 \\
17309\end{array}$ & $\begin{array}{l}21.98752 \\
22.21611 \\
22.43477 \\
22.64440 \\
22.84577\end{array}$ & $\begin{array}{l}22859 \\
21866 \\
20963 \\
20137 \\
19376\end{array}$ \\
\hline $\begin{array}{l}2200 \\
2300 \\
2400 \\
2500 \\
2600\end{array}$ & $\begin{array}{l}4.18350 \\
4.21815 \\
4.25076 \\
4.28146 \\
4.31038\end{array}$ & $\begin{array}{l}3465 \\
3261 \\
3070 \\
2892 \\
2728\end{array}$ & $\begin{array}{l}3.73114 \\
3.75157 \\
3.77170 \\
3.79148 \\
3.81089\end{array}$ & $\begin{array}{l}2043 \\
2013 \\
1978 \\
1941 \\
1901\end{array}$ & $\begin{array}{l}19.30838 \\
19.47469 \\
19.63478 \\
19.78915 \\
19.93824\end{array}$ & $\begin{array}{l}16631 \\
16009 \\
15437 \\
14909 \\
14418\end{array}$ & $\begin{array}{l}23.03953 \\
23.22627 \\
23.40649 \\
23.58064 \\
23.74913\end{array}$ & $\begin{array}{l}18674 \\
18022 \\
17415 \\
16849 \\
16319\end{array}$ \\
\hline $\begin{array}{l}2700 \\
2800 \\
2900 \\
3000\end{array}$ & $\begin{array}{l}4.33766 \\
4.36341 \\
4.38775 \\
4.41080\end{array}$ & $\begin{array}{l}2575 \\
2434 \\
2305 \\
4263\end{array}$ & $\begin{array}{l}3.82990 \\
3.84850 \\
3.86668 \\
3.88443\end{array}$ & $\begin{array}{l}1860 \\
1818 \\
1775 \\
3426\end{array}$ & $\begin{array}{l}20.08242 \\
20.22204 \\
20.35741 \\
20.48880\end{array}$ & $\begin{array}{l}13962 \\
13537 \\
13139 \\
25179\end{array}$ & $\begin{array}{l}23.91232 \\
24.07054 \\
24.22409 \\
24.37323\end{array}$ & $\begin{array}{l}15822 \\
15355 \\
14914 \\
28606\end{array}$ \\
\hline $\begin{array}{l}3200 \\
3400 \\
3600 \\
3800 \\
4000\end{array}$ & $\begin{array}{l}4.45343 \\
4.49201 \\
4.52718 \\
4.55943 \\
4.58920\end{array}$ & $\begin{array}{l}3858 \\
3517 \\
3225 \\
2977 \\
2765\end{array}$ & $\begin{array}{l}3.91869 \\
3.95129 \\
3.98233 \\
4.01186 \\
4.04000\end{array}$ & $\begin{array}{l}3260 \\
3104 \\
2953 \\
2814 \\
2681\end{array}$ & $\begin{array}{l}20.74059 \\
20.97915 \\
21.20589 \\
21.42200 \\
21.62850\end{array}$ & $\begin{array}{l}23856 \\
22674 \\
21611 \\
20650 \\
19777\end{array}$ & $\begin{array}{l}24.65929 \\
24.93045 \\
25.18822 \\
25.43387 \\
25.66850\end{array}$ & $\begin{array}{l}27116 \\
25777 \\
24565 \\
23463 \\
22459\end{array}$ \\
\hline $\begin{array}{l}4200 \\
4400 \\
4600 \\
4800 \\
5000\end{array}$ & $\begin{array}{l}4.61685 \\
4.64266 \\
4.66689 \\
4.68975 \\
4.71140\end{array}$ & $\begin{array}{l}2581 \\
2423 \\
2286 \\
2165\end{array}$ & $\begin{array}{l}4.06681 \\
4.09241 \\
4.11687 \\
4.14026 \\
4.16268\end{array}$ & $\begin{array}{l}2560 \\
2446 \\
2339 \\
2242\end{array}$ & $\begin{array}{l}21.82627 \\
22.01605 \\
22.19851 \\
22.37422 \\
22.54369\end{array}$ & $\begin{array}{l}18978 \\
18246 \\
17571 \\
16947\end{array}$ & $\begin{array}{l}25.89309 \\
26.10846 \\
26.31538 \\
26.51449 \\
26.70638\end{array}$ & $\begin{array}{l}21537 \\
20692 \\
19911 \\
19189\end{array}$ \\
\hline
\end{tabular}


Table 2.002. $\mathrm{H}_{2}$ - Equilibrium Mixture

\begin{tabular}{|l|l|l|l|l|}
\hline$K$ & $\frac{C_{p}^{0}}{R}$ & $\frac{\left(H^{0}-E_{0}^{0}\right)}{R T}$ & $\frac{-\left(F^{0}-E_{0}^{0}\right)}{R T}$ & $\frac{S^{0}}{R}$ \\
\hline
\end{tabular}

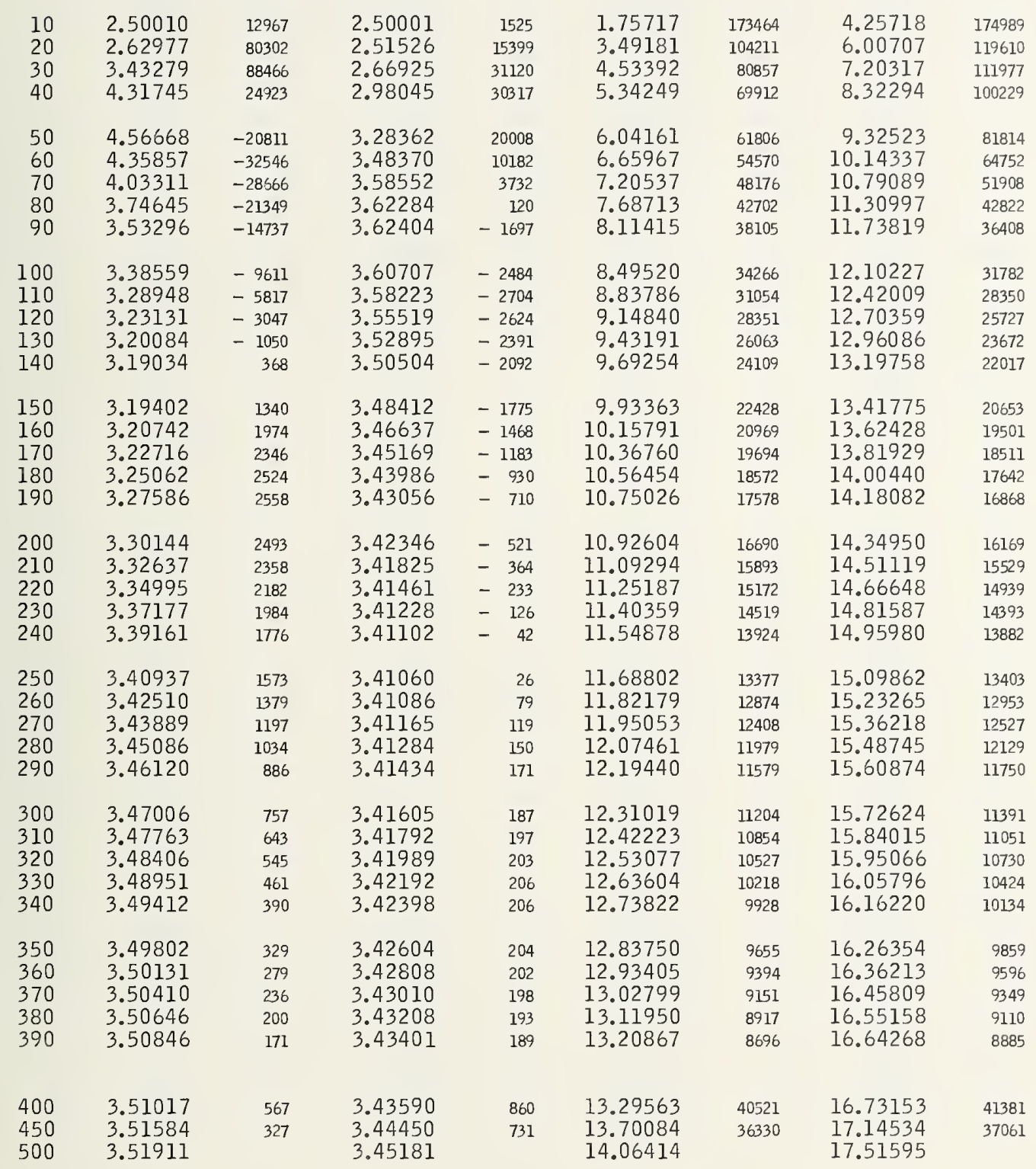


Table 2.003. $\mathrm{H}_{2}$ - Para (includes only the even numbered rotational states)

\begin{tabular}{|c|c|c|c|c|c|c|c|c|}
\hline${ }^{\circ} \mathrm{K}$ & $\frac{C_{p}^{0}}{R}$ & & $\frac{\left(H^{\circ}-E_{0}^{0}\right)}{R T}$ & & $\frac{-\left(F^{\circ}-E\right.}{R T}$ & & $\frac{S^{0}}{R}$ & \\
\hline $\begin{array}{l}10 \\
20 \\
30 \\
40\end{array}$ & $\begin{array}{l}2.50000 \\
2.50000 \\
2.50006 \\
2.50238\end{array}$ & $\begin{array}{r}6 \\
232 \\
1705\end{array}$ & $\begin{array}{l}2.50000 \\
2.50000 \\
2.50000 \\
2.50019\end{array}$ & $\begin{array}{r}19 \\
172\end{array}$ & $\begin{array}{l}1.75717 \\
3.49002 \\
4.50368 \\
5.22288\end{array}$ & $\begin{array}{r}173285 \\
101366 \\
71920 \\
55804\end{array}$ & $\begin{array}{l}4.25717 \\
5.99002 \\
7.00368 \\
7.72307\end{array}$ & $\begin{array}{r}173285 \\
101366 \\
71939 \\
55976\end{array}$ \\
\hline $\begin{array}{l}50 \\
60 \\
70 \\
80 \\
90\end{array}$ & $\begin{array}{l}2.51943 \\
2.57365 \\
2.68123 \\
2.84128 \\
3.03790\end{array}$ & $\begin{array}{r}5422 \\
10758 \\
16005 \\
19662 \\
21020\end{array}$ & $\begin{array}{l}2.50191 \\
2.50868 \\
2.52498 \\
2.55403 \\
2.59663\end{array}$ & $\begin{array}{r}677 \\
1630 \\
2905 \\
4260 \\
5462\end{array}$ & $\begin{array}{l}5.78092 \\
6.23755 \\
6.62534 \\
6.96425 \\
7.26739\end{array}$ & $\begin{array}{l}45663 \\
38779 \\
33891 \\
30314 \\
27631\end{array}$ & $\begin{array}{l}8.28283 \\
8.74623 \\
9.15032 \\
9.51828 \\
9.86402\end{array}$ & $\begin{array}{l}46340 \\
40409 \\
36796 \\
34574 \\
33093\end{array}$ \\
\hline 0 & $\begin{array}{l}3.24810 \\
3.44947 \\
3.62491 \\
3.76456 \\
3.86528\end{array}$ & $\begin{array}{r}20137 \\
17544 \\
13965 \\
10072 \\
6372\end{array}$ & $\begin{array}{l}2.65125 \\
2.71480 \\
2.78356 \\
2.85390 \\
2.92277\end{array}$ & $\begin{array}{l}6355 \\
6876 \\
7034 \\
6887 \\
6515\end{array}$ & $\begin{array}{l}7.54370 \\
7.79931 \\
8.03846 \\
8.26403 \\
8.47807\end{array}$ & $\begin{array}{l}25561 \\
23915 \\
22557 \\
21404 \\
20389\end{array}$ & $\begin{array}{l}10.19495 \\
10.51411 \\
10.82202 \\
11.11793 \\
11.40084\end{array}$ & $\begin{array}{l}31916 \\
30791 \\
29591 \\
28291 \\
26904\end{array}$ \\
\hline 0 & $\begin{array}{l}3.92900 \\
3.96082 \\
3.96719 \\
3.95470 \\
3.92931\end{array}$ & $\begin{array}{r}3182 \\
637 \\
-\quad 1249 \\
-2539 \\
-3331\end{array}$ & $\begin{array}{l}2.98792 \\
3.04789 \\
3.10188 \\
3.14968 \\
3.19143\end{array}$ & $\begin{array}{l}5997 \\
5399 \\
4780 \\
4175 \\
3608\end{array}$ & $\begin{array}{l}8.68196 \\
8.87675 \\
9.06317 \\
9.24185 \\
9.41329\end{array}$ & $\begin{array}{l}19479 \\
18642 \\
17868 \\
17144 \\
16464\end{array}$ & $\begin{array}{l}11.66988 \\
11.92464 \\
12.16505 \\
12.39153 \\
12.60472\end{array}$ & $\begin{array}{l}25476 \\
24041 \\
22648 \\
21319 \\
20072\end{array}$ \\
\hline 0 & $\begin{array}{l}3.89600 \\
3.85865 \\
3.82013 \\
3.78243 \\
3.74686\end{array}$ & $\begin{array}{l}-3735 \\
-3852 \\
-3770 \\
-3557 \\
-3267\end{array}$ & $\begin{array}{l}3.22751 \\
3.25847 \\
3.28487 \\
3.30732 \\
3.32637\end{array}$ & $\begin{array}{l}3096 \\
2640 \\
2245 \\
1905 \\
1615\end{array}$ & $\begin{array}{r}9.57793 \\
9.73617 \\
9.88838 \\
10.03490 \\
10.17607\end{array}$ & $\begin{array}{l}15824 \\
15221 \\
14652 \\
14117 \\
13613\end{array}$ & $\begin{array}{l}12.80544 \\
12.99464 \\
13.17325 \\
13.34222 \\
13.50244\end{array}$ & $\begin{array}{l}18920 \\
17861 \\
16897 \\
16022 \\
15228\end{array}$ \\
\hline 0 & $\begin{array}{l}3.71419 \\
3.68480 \\
3.65877 \\
3.63605 \\
3.61642\end{array}$ & $\begin{array}{l}-2939 \\
-2603 \\
-2272 \\
-1963 \\
-1680\end{array}$ & $\begin{array}{l}3.34252 \\
3.35624 \\
3.36792 \\
3.37789 \\
3.38644\end{array}$ & $\begin{array}{r}1372 \\
1168 \\
997 \\
855 \\
738\end{array}$ & $\begin{array}{l}10.31220 \\
10.44357 \\
10.57046 \\
10.69312 \\
10.81181\end{array}$ & $\begin{array}{l}13137 \\
12689 \\
12266 \\
11869 \\
11494\end{array}$ & $\begin{array}{l}13.65472 \\
13.79981 \\
13.93838 \\
14.07101 \\
14.19825\end{array}$ & $\begin{array}{l}14509 \\
13857 \\
13263 \\
12724 \\
12232\end{array}$ \\
\hline $\begin{array}{l}0 \\
0 \\
10\end{array}$ & $\begin{array}{l}3.59962 \\
3.58537 \\
3.57336 \\
3.56331 \\
3.55496\end{array}$ & $\begin{array}{l}-1425 \\
-1201 \\
-\quad 1005 \\
-\quad 835 \\
-\quad 691\end{array}$ & $\begin{array}{l}3.39382 \\
3.40022 \\
3.40582 \\
3.41074 \\
3.41510\end{array}$ & $\begin{array}{l}640 \\
560 \\
492 \\
436 \\
389\end{array}$ & $\begin{array}{l}10.92675 \\
11.03813 \\
11.14617 \\
11.25106 \\
11.35294\end{array}$ & $\begin{array}{r}11138 \\
10804 \\
10489 \\
10188 \\
9905\end{array}$ & $\begin{array}{l}14.32057 \\
14.43835 \\
14.55199 \\
14.66180 \\
14.76804\end{array}$ & $\begin{array}{l}11778 \\
11364 \\
10981 \\
10624 \\
10294\end{array}$ \\
\hline $\begin{array}{l}0 \\
0\end{array}$ & $\begin{array}{l}3.54805 \\
3.54237 \\
3.53773 \\
3.53396 \\
3.53092\end{array}$ & $\begin{array}{l}-\quad 568 \\
-\quad 464 \\
-\quad 377 \\
-\quad 304 \\
-\quad 243\end{array}$ & $\begin{array}{l}3.41899 \\
3.42250 \\
3.42567 \\
3.42857 \\
3.43123\end{array}$ & $\begin{array}{l}351 \\
317 \\
290 \\
266 \\
246\end{array}$ & $\begin{array}{l}11.45199 \\
11.54836 \\
11.64217 \\
11.73357 \\
11.82266\end{array}$ & $\begin{array}{l}9637 \\
9381 \\
9140 \\
8909 \\
8690\end{array}$ & $\begin{array}{l}14.87098 \\
14.97086 \\
15.06784 \\
15.16214 \\
15.25389\end{array}$ & $\begin{array}{l}9988 \\
9698 \\
9430 \\
9175 \\
8936\end{array}$ \\
\hline DO & $\begin{array}{l}3.52849 \\
3.52236 \\
3.52139\end{array}$ & $\begin{array}{l}-\quad 613 \\
-\quad 97\end{array}$ & $\begin{array}{l}3.43369 \\
3.44381 \\
3.45159\end{array}$ & $\begin{array}{r}1012 \\
778\end{array}$ & $\begin{array}{l}11.90956 \\
12.31461 \\
12.67786\end{array}$ & $\begin{array}{l}40505 \\
36325\end{array}$ & $\begin{array}{l}15.34325 \\
15.75842 \\
16.12945\end{array}$ & $\begin{array}{l}41517 \\
37103\end{array}$ \\
\hline
\end{tabular}


Table 2.004. $\mathrm{D}_{2}$ - Normal Mixture

\begin{tabular}{|l|l|l|l|l|}
\hline$K$ & $\frac{C_{p}^{0}}{R}$ & $\frac{\left(H^{\circ}-E_{0}^{0}\right)}{R T}$ & $\frac{-\left(F^{\circ}-E_{0}^{0}\right)}{R T}$ & $\frac{S^{\circ}}{R}$ \\
\hline
\end{tabular}

\begin{tabular}{|c|c|c|c|c|c|c|c|c|}
\hline $\begin{array}{l}10 \\
20 \\
30 \\
40\end{array}$ & $\begin{array}{l}2.50000 \\
2.50142 \\
2.54598 \\
2.71957\end{array}$ & $\begin{array}{r}142 \\
4456 \\
17359 \\
27562\end{array}$ & $\begin{array}{l}5.36562 \\
3.93292 \\
3.46056 \\
3.25066\end{array}$ & $\begin{array}{l}-143270 \\
-\quad 47236 \\
-20990 \\
-\quad 7951\end{array}$ & $\begin{array}{l}1.68258 \\
4.84824 \\
6.34012 \\
7.30282\end{array}$ & $\begin{array}{r}316566 \\
149188 \\
96270 \\
71501\end{array}$ & $\begin{array}{r}7.04820 \\
8.78116 \\
9.80068 \\
10.55348\end{array}$ & $\begin{array}{r}173296 \\
101952 \\
75280 \\
63550\end{array}$ \\
\hline $\begin{array}{l}50 \\
60 \\
70 \\
80 \\
90\end{array}$ & $\begin{array}{l}2.99519 \\
3.26809 \\
3.46556 \\
3.57472 \\
3.61698\end{array}$ & $\begin{array}{r}27290 \\
19747 \\
10916 \\
4226 \\
317\end{array}$ & $\begin{array}{l}3.17115 \\
3.16526 \\
3.19515 \\
3.23661 \\
3.27701\end{array}$ & $\begin{array}{r}589 \\
2989 \\
4146 \\
4040 \\
3438\end{array}$ & $\begin{array}{l}8.01783 \\
8.59469 \\
9.08458 \\
9.51390 \\
9.89749\end{array}$ & $\begin{array}{l}57686 \\
48989 \\
42932 \\
38359 \\
34710\end{array}$ & $\begin{array}{l}11.18898 \\
11.75995 \\
12.27973 \\
12.75051 \\
13.17450\end{array}$ & $\begin{array}{l}57097 \\
51978 \\
47078 \\
42399 \\
38148\end{array}$ \\
\hline $\begin{array}{l}100 \\
110 \\
120 \\
130 \\
140\end{array}$ & $\begin{array}{l}3.62015 \\
3.60538 \\
3.58527 \\
3.56599 \\
3.54993\end{array}$ & $\begin{array}{l}-1477 \\
-2011 \\
-1928 \\
-1606 \\
-1238\end{array}$ & $\begin{array}{l}3.31139 \\
3.33887 \\
3.36025 \\
3.37680 \\
3.38972\end{array}$ & $\begin{array}{l}2748 \\
2138 \\
1655 \\
1292 \\
1025\end{array}$ & $\begin{array}{l}10.24459 \\
10.56156 \\
10.85302 \\
11.12265 \\
11.37339\end{array}$ & $\begin{array}{l}31697 \\
29146 \\
26963 \\
25074 \\
23423\end{array}$ & $\begin{array}{l}13.55598 \\
13.90043 \\
14.21327 \\
14.49945 \\
14.76311\end{array}$ & $\begin{array}{l}34445 \\
31284 \\
28618 \\
26366 \\
24448\end{array}$ \\
\hline $\begin{array}{l}150 \\
160 \\
170 \\
180 \\
190\end{array}$ & $\begin{array}{l}3.53755 \\
3.52846 \\
3.52203 \\
3.51761 \\
3.51467\end{array}$ & $\begin{array}{l}-\quad 909 \\
-\quad 643 \\
-\quad 442 \\
-\quad 294 \\
-\quad 190\end{array}$ & $\begin{array}{l}3.39997 \\
3.40826 \\
3.41513 \\
3.42094 \\
3.42595\end{array}$ & $\begin{array}{l}829 \\
687 \\
581 \\
501 \\
438\end{array}$ & $\begin{array}{l}11.60762 \\
11.82733 \\
12.03416 \\
12.22953 \\
12.41463\end{array}$ & $\begin{array}{l}21971 \\
20693 \\
19537 \\
18510 \\
17584\end{array}$ & $\begin{array}{l}15.00759 \\
15.23559 \\
15.44929 \\
15.65047 \\
15.84058\end{array}$ & $\begin{array}{l}22800 \\
21370 \\
20118 \\
19011 \\
18022\end{array}$ \\
\hline $\begin{array}{l}200 \\
210 \\
220 \\
230 \\
240\end{array}$ & $\begin{array}{l}3.51277 \\
3.51159 \\
3.51092 \\
3.51058 \\
3.51047\end{array}$ & $\begin{array}{r}-\quad 118 \\
-\quad 67 \\
-\quad 34 \\
-\quad 11 \\
5\end{array}$ & $\begin{array}{l}3.43033 \\
3.43423 \\
3.43773 \\
3.44090 \\
3.44380\end{array}$ & $\begin{array}{l}390 \\
350 \\
317 \\
290 \\
267\end{array}$ & $\begin{array}{l}12.59047 \\
12.75793 \\
12.91778 \\
13.07065 \\
13.21716\end{array}$ & $\begin{array}{l}16746 \\
15985 \\
15287 \\
14651 \\
14064\end{array}$ & $\begin{array}{l}16.02080 \\
16.19216 \\
16.35551 \\
16.51155 \\
16.66096\end{array}$ & $\begin{array}{l}17136 \\
16335 \\
15604 \\
14941 \\
14331\end{array}$ \\
\hline $\begin{array}{l}250 \\
260 \\
270 \\
280 \\
290\end{array}$ & $\begin{array}{l}3.51052 \\
3.51067 \\
3.51089 \\
3.51115 \\
3.51145\end{array}$ & $\begin{array}{l}15 \\
22 \\
26 \\
30 \\
32\end{array}$ & $\begin{array}{l}3.44647 \\
3.44893 \\
3.45123 \\
3.45336 \\
3.45536\end{array}$ & $\begin{array}{l}246 \\
230 \\
213 \\
200 \\
187\end{array}$ & $\begin{array}{l}13.35780 \\
13.49302 \\
13.62323 \\
13.74877 \\
13.86999\end{array}$ & $\begin{array}{l}13522 \\
13021 \\
12554 \\
12122 \\
11718\end{array}$ & $\begin{array}{l}16.80427 \\
16.94195 \\
17.07446 \\
17.20213 \\
17.32535\end{array}$ & $\begin{array}{l}13768 \\
13251 \\
12767 \\
12322 \\
11905\end{array}$ \\
\hline $\begin{array}{l}300 \\
310 \\
320 \\
330 \\
340\end{array}$ & $\begin{array}{l}3.51177 \\
3.51200 \\
3.51217 \\
3.51250 \\
3.51298\end{array}$ & $\begin{array}{l}23 \\
17 \\
33 \\
48 \\
51\end{array}$ & $\begin{array}{l}3.45723 \\
3.45900 \\
3.46055 \\
3.46206 \\
3.46355\end{array}$ & $\begin{array}{l}177 \\
155 \\
151 \\
149 \\
142\end{array}$ & $\begin{array}{l}13.98717 \\
14.10056 \\
14.21056 \\
14.31716 \\
14.42054\end{array}$ & $\begin{array}{l}11339 \\
11000 \\
10660 \\
10338 \\
10042\end{array}$ & $\begin{array}{l}17.44440 \\
17.55956 \\
17.67111 \\
17.77922 \\
17.88409\end{array}$ & $\begin{array}{l}11516 \\
11155 \\
10811 \\
10487 \\
10184\end{array}$ \\
\hline $\begin{array}{l}350 \\
360 \\
370 \\
380 \\
390\end{array}$ & $\begin{array}{l}3.51349 \\
3.51406 \\
3.51468 \\
3.51537 \\
3.51614\end{array}$ & $\begin{array}{l}57 \\
62 \\
69 \\
77 \\
84\end{array}$ & $\begin{array}{l}3.46497 \\
3.46632 \\
3.46762 \\
3.46887 \\
3.47007\end{array}$ & $\begin{array}{l}135 \\
130 \\
125 \\
120 \\
116\end{array}$ & $\begin{array}{l}14.52096 \\
14.61859 \\
14.71358 \\
14.80607 \\
14.89619\end{array}$ & $\begin{array}{l}9763 \\
9499 \\
9249 \\
9012 \\
8787\end{array}$ & $\begin{array}{l}17.98593 \\
18.08492 \\
18.18121 \\
18.27495 \\
18.36627\end{array}$ & $\begin{array}{l}9899 \\
9629 \\
9374 \\
9132 \\
8903\end{array}$ \\
\hline $\begin{array}{l}400 \\
450 \\
500 \\
550 \\
600\end{array}$ & $\begin{array}{l}3.51698 \\
3.52277 \\
3.53186 \\
3.54483 \\
3.56209\end{array}$ & $\begin{array}{r}579 \\
909 \\
1297 \\
1726 \\
2140\end{array}$ & $\begin{array}{l}3.47123 \\
3.47661 \\
3.48165 \\
3.48677 \\
3.49230\end{array}$ & $\begin{array}{l}538 \\
504 \\
512 \\
553 \\
616\end{array}$ & $\begin{array}{l}14.98406 \\
15.39323 \\
15.75979 \\
16.09187 \\
16.39549\end{array}$ & $\begin{array}{l}40917 \\
36656 \\
33208 \\
30362 \\
27977\end{array}$ & $\begin{array}{l}18.45530 \\
18.86985 \\
19.24145 \\
19.57864 \\
19.88779\end{array}$ & $\begin{array}{l}41455 \\
37160 \\
33719 \\
30915 \\
28594\end{array}$ \\
\hline $\begin{array}{l}650 \\
700 \\
750 \\
800 \\
850\end{array}$ & $\begin{array}{l}3.58349 \\
3.60863 \\
3.63692 \\
3.66769 \\
3.70028\end{array}$ & $\begin{array}{l}2514 \\
2829 \\
3077 \\
3259 \\
3378\end{array}$ & $\begin{array}{l}3.49846 \\
3.50541 \\
3.51322 \\
3.52190 \\
3.53143\end{array}$ & $\begin{array}{r}695 \\
781 \\
868 \\
953 \\
1031\end{array}$ & $\begin{array}{l}16.67526 \\
16.93477 \\
17.17688 \\
17.40390 \\
17.61769\end{array}$ & $\begin{array}{l}25951 \\
24211 \\
22702 \\
21379 \\
20214\end{array}$ & $\begin{array}{l}20.17373 \\
20.44019 \\
20.69011 \\
20.92580 \\
21.14913\end{array}$ & $\begin{array}{l}26646 \\
24992 \\
23569 \\
22333 \\
21245\end{array}$ \\
\hline $\begin{array}{r}900 \\
950 \\
1000 \\
1050 \\
1100\end{array}$ & $\begin{array}{l}3.73406 \\
3.76850 \\
3.80311 \\
3.83754 \\
3.87148\end{array}$ & $\begin{array}{l}3444 \\
3461 \\
3443 \\
3394 \\
3322\end{array}$ & $\begin{array}{l}3.54174 \\
3.55277 \\
3.56442 \\
3.57661 \\
3.58924\end{array}$ & $\begin{array}{l}1103 \\
1165 \\
1219 \\
1263 \\
1300\end{array}$ & $\begin{array}{l}17.81983 \\
18.01162 \\
18.19414 \\
18.36834 \\
18.53502\end{array}$ & $\begin{array}{l}19179 \\
18252 \\
17420 \\
16668 \\
15983\end{array}$ & $\begin{array}{l}21.36158 \\
21.56439 \\
21.75857 \\
21.94496 \\
22.12427\end{array}$ & $\begin{array}{l}20281 \\
19418 \\
18639 \\
17931 \\
17283\end{array}$ \\
\hline 1150 & 3.90470 & 3233 & 3.60224 & 1328 & 18.69485 & 15359 & 22.29710 & 16687 \\
\hline
\end{tabular}


Table 2.004. $\quad D_{2}-$ Normal Mixture (Cont.)

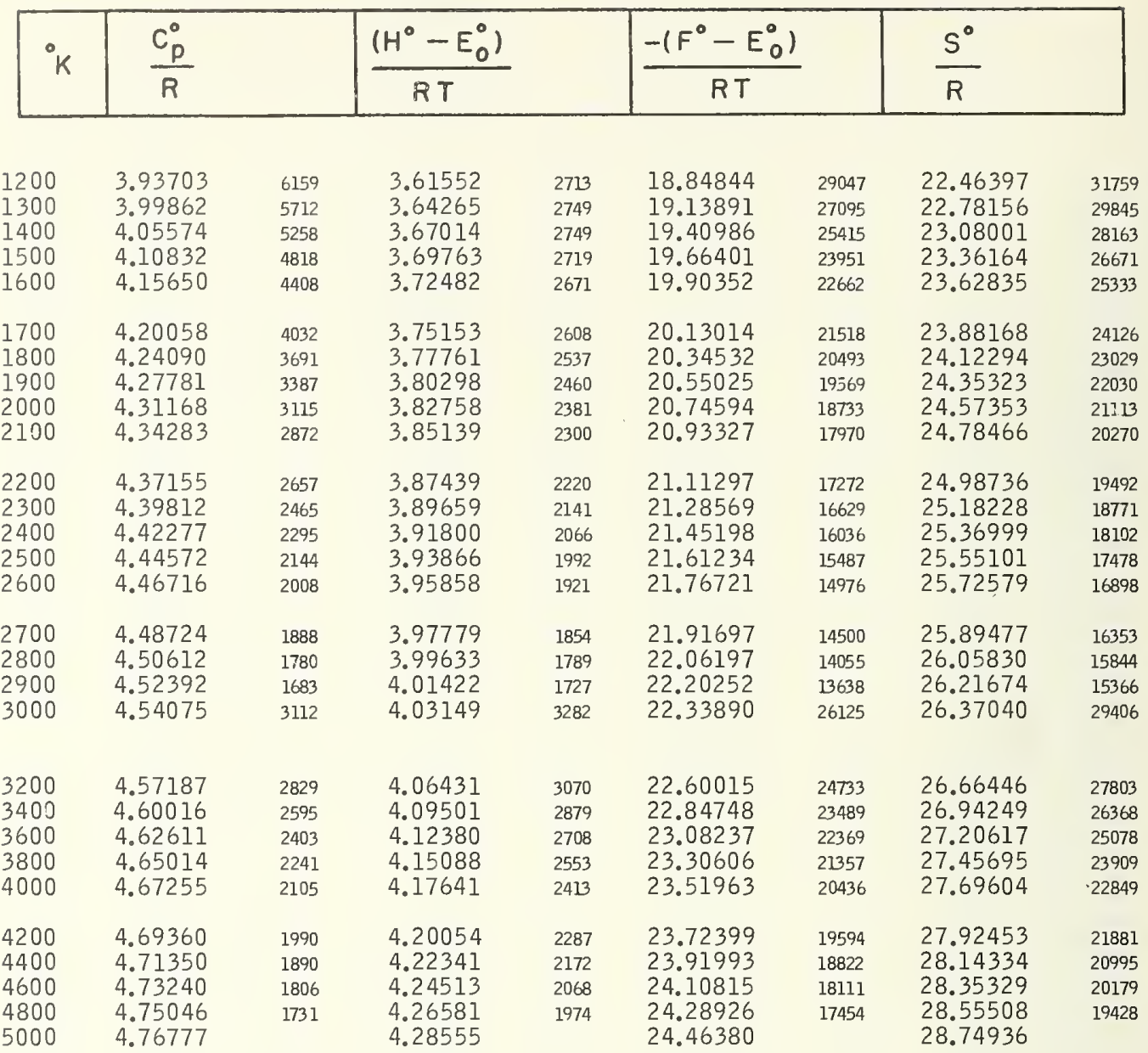


Table 2.005. $\mathrm{D}_{2}$ - Equilibrium Mixture

\begin{tabular}{|c|c|c|c|c|c|c|c|c|}
\hline${ }^{\circ} \mathrm{K}$ & $\frac{C_{p}^{0}}{R}$ & & $\frac{\left(H^{0}-E_{0}^{0}\right)}{R T}$ & & $\frac{-\left(F^{0}-\right.}{R T}$ & & $\frac{S^{0}}{R}$ & \\
\hline $\begin{array}{l}10 \\
20 \\
30 \\
40\end{array}$ & $\begin{array}{l}2.52046 \\
2.86379 \\
3.15504 \\
3.33386\end{array}$ & $\begin{array}{l}34333 \\
29125 \\
17882 \\
15788\end{array}$ & $\begin{array}{l}2.50238 \\
2.58603 \\
2.73272 \\
2.86136\end{array}$ & $\begin{array}{r}8365 \\
14669 \\
12864 \\
11070\end{array}$ & $\begin{array}{l}3.77680 \\
5.52957 \\
6.60587 \\
7.41017\end{array}$ & $\begin{array}{r}175277 \\
107630 \\
80430 \\
65065\end{array}$ & $\begin{array}{r}6.27918 \\
8.11560 \\
9.33859 \\
10.27153\end{array}$ & $\begin{array}{r}183642 \\
122299 \\
93294 \\
76135\end{array}$ \\
\hline $\begin{array}{l}50 \\
60 \\
70 \\
80 \\
90\end{array}$ & $\begin{array}{l}3.49174 \\
3.60906 \\
3.67064 \\
3.68586 \\
3.67261\end{array}$ & $\begin{array}{r}11732 \\
6158 \\
1522 \\
-1325 \\
-2624\end{array}$ & $\begin{array}{l}2.97206 \\
3.06919 \\
3.15136 \\
3.21761 \\
3.26909\end{array}$ & $\begin{array}{l}9713 \\
8217 \\
6625 \\
5148 \\
3910\end{array}$ & $\begin{array}{l}8.06082 \\
8.61148 \\
9.09097 \\
9.51627 \\
9.89835\end{array}$ & $\begin{array}{l}55066 \\
47949 \\
42530 \\
38208 \\
34656\end{array}$ & $\begin{array}{l}11.03288 \\
11.68067 \\
12.24233 \\
12.73388 \\
13.16744\end{array}$ & $\begin{array}{l}64779 \\
56166 \\
49155 \\
43356 \\
38566\end{array}$ \\
\hline $\begin{array}{l}100 \\
110 \\
120 \\
130 \\
140\end{array}$ & $\begin{array}{l}3.64637 \\
3.61719 \\
3.59040 \\
3.56816 \\
3.55083\end{array}$ & $\begin{array}{l}-2918 \\
-2679 \\
-2224 \\
-1733 \\
-1292\end{array}$ & $\begin{array}{l}3.30819 \\
3.33760 \\
3.35976 \\
3.37661 \\
3.38965\end{array}$ & $\begin{array}{l}2941 \\
2216 \\
1685 \\
1304 \\
1029\end{array}$ & $\begin{array}{l}10.24491 \\
10.56167 \\
10.85306 \\
11.12267 \\
11.37340\end{array}$ & $\begin{array}{l}31676 \\
29139 \\
26961 \\
25073 \\
23422\end{array}$ & $\begin{array}{l}13.55310 \\
13.89927 \\
14.21282 \\
14.49928 \\
14.76305\end{array}$ & $\begin{array}{l}34617 \\
31355 \\
28646 \\
26377 \\
24451\end{array}$ \\
\hline $\begin{array}{l}150 \\
160 \\
170 \\
180 \\
190\end{array}$ & $\begin{array}{l}3.53791 \\
3.52861 \\
3.52209 \\
3.51764 \\
3.51468\end{array}$ & $\begin{array}{l}-\quad 930 \\
-\quad 652 \\
-\quad 445 \\
-\quad 296 \\
-\quad 191\end{array}$ & $\begin{array}{l}3.39994 \\
3.40826 \\
3.41513 \\
3.42094 \\
3.42595\end{array}$ & $\begin{array}{l}832 \\
687 \\
581 \\
501 \\
438\end{array}$ & $\begin{array}{l}11.60762 \\
11.82733 \\
12.03416 \\
12.22953 \\
12.41463\end{array}$ & $\begin{array}{l}21971 \\
20683 \\
19537 \\
18510 \\
17584\end{array}$ & $\begin{array}{l}15.00756 \\
15.23559 \\
15.44929 \\
15.65047 \\
15.84058\end{array}$ & $\begin{array}{l}22803 \\
21370 \\
20118 \\
19011 \\
18022\end{array}$ \\
\hline $\begin{array}{l}200 \\
210 \\
220 \\
230 \\
240\end{array}$ & $\begin{array}{l}3.51277 \\
3.51159 \\
3.51092 \\
3.51058 \\
3.51047\end{array}$ & $\begin{array}{rr}- & 118 \\
- & 67 \\
- & 34 \\
-\quad 11 \\
& 5\end{array}$ & $\begin{array}{l}3.43033 \\
3.43423 \\
3.43773 \\
3.44090 \\
3.44380\end{array}$ & $\begin{array}{l}390 \\
350 \\
317 \\
290 \\
267\end{array}$ & $\begin{array}{l}12.59047 \\
12.75793 \\
12.91778 \\
13.07065 \\
13.21716\end{array}$ & $\begin{array}{l}16746 \\
15985 \\
15287 \\
14651 \\
14064\end{array}$ & $\begin{array}{l}16.02080 \\
16.19216 \\
16.35551 \\
16.51155 \\
16.66096\end{array}$ & $\begin{array}{l}17136 \\
16335 \\
15604 \\
14941 \\
14331\end{array}$ \\
\hline $\begin{array}{l}250 \\
260 \\
270 \\
280 \\
290\end{array}$ & $\begin{array}{l}3.51052 \\
3.51067 \\
3.51089 \\
3.51115 \\
3.51145\end{array}$ & $\begin{array}{l}15 \\
22 \\
26 \\
30 \\
32\end{array}$ & $\begin{array}{l}3.44647 \\
3.44893 \\
3.45123 \\
3.45336 \\
3.45536\end{array}$ & $\begin{array}{l}246 \\
230 \\
213 \\
200 \\
187\end{array}$ & $\begin{array}{l}13.35780 \\
13.49302 \\
13.62323 \\
13.74877 \\
13.86999\end{array}$ & $\begin{array}{l}13522 \\
13021 \\
12554 \\
12122 \\
11718\end{array}$ & $\begin{array}{l}16.80427 \\
16.94195 \\
17.07446 \\
17.20213 \\
17.32535\end{array}$ & $\begin{array}{l}13768 \\
13251 \\
12767 \\
12322 \\
11905\end{array}$ \\
\hline 300 & 3.51177 & & 3.45723 & & 13.98717 & & 17.44440 & \\
\hline
\end{tabular}


Table 2.006. $\mathrm{D}_{2}$ - Ortho (includes only the even numbered rotational states)

\begin{tabular}{|l|l|l|l|l|}
\hline${ }^{\circ} K$ & $\frac{C_{p}^{\circ}}{R}$ & $\frac{\left(H^{0}-E_{0}^{0}\right)}{R T}$ & $\frac{-\left(F^{\circ}-E_{0}^{\circ}\right)}{R T}$ & $\frac{S^{\circ}}{R}$ \\
\hline
\end{tabular}

\begin{tabular}{|c|c|c|c|c|c|c|c|c|}
\hline $\begin{array}{l}10 \\
20 \\
30 \\
40\end{array}$ & $\begin{array}{l}2.50000 \\
2.50212 \\
2.56882 \\
2.82633\end{array}$ & $\begin{array}{r}212 \\
6670 \\
25751 \\
40004\end{array}$ & $\begin{array}{l}2.50000 \\
2.50016 \\
2.50802 \\
2.55110\end{array}$ & $\begin{array}{r}16 \\
786 \\
4308 \\
9402\end{array}$ & $\begin{array}{l}3.77652 \\
5.50938 \\
6.52397 \\
7.25019\end{array}$ & $\begin{array}{r}173286 \\
101459 \\
72622 \\
57847\end{array}$ & $\begin{array}{l}6.27652 \\
8.00954 \\
9.03199 \\
9.80129\end{array}$ & $\begin{array}{r}173302 \\
102245 \\
76930 \\
67249\end{array}$ \\
\hline $\begin{array}{l}50 \\
60 \\
70 \\
80 \\
90\end{array}$ & $\begin{array}{l}3.22637 \\
3.60484 \\
3.85274 \\
3.95667 \\
3.95656\end{array}$ & $\begin{array}{r}37847 \\
24790 \\
10393 \\
-\quad 11 \\
-\quad 5581\end{array}$ & $\begin{array}{l}2.64512 \\
2.77485 \\
2.91294 \\
3.03825 \\
3.14102\end{array}$ & $\begin{array}{r}12973 \\
13809 \\
12531 \\
10277 \\
7905\end{array}$ & $\begin{array}{l}7.82866 \\
8.32205 \\
8.76021 \\
9.15758 \\
9.52160\end{array}$ & $\begin{array}{l}49339 \\
43816 \\
39737 \\
36402 \\
33523\end{array}$ & $\begin{array}{l}10.47378 \\
11.09690 \\
11.67315 \\
12.19583 \\
12.66262\end{array}$ & $\begin{array}{l}62312 \\
57625 \\
52268 \\
46679 \\
41428\end{array}$ \\
\hline $\begin{array}{l}100 \\
110 \\
120 \\
130 \\
140\end{array}$ & $\begin{array}{l}3.90075 \\
3.82524 \\
3.75081 \\
3.68688 \\
3.63612\end{array}$ & $\begin{array}{l}-7551 \\
-7443 \\
-\quad 6393 \\
-5076 \\
-3831\end{array}$ & $\begin{array}{l}3.22007 \\
3.27857 \\
3.32097 \\
3.35150 \\
3.37356\end{array}$ & $\begin{array}{l}5850 \\
4240 \\
3053 \\
2206 \\
1616\end{array}$ & $\begin{array}{r}9.85683 \\
10.16664 \\
10.45381 \\
10.72089 \\
10.97012\end{array}$ & $\begin{array}{l}30981 \\
28717 \\
26708 \\
24923 \\
23333\end{array}$ & $\begin{array}{l}13.07690 \\
13.44521 \\
13.77478 \\
14.07239 \\
14.34368\end{array}$ & $\begin{array}{l}36831 \\
32957 \\
29761 \\
27129 \\
24949\end{array}$ \\
\hline $\begin{array}{l}150 \\
160 \\
170 \\
180 \\
190\end{array}$ & $\begin{array}{l}3.59781 \\
3.56995 \\
3.55022 \\
3.53657 \\
3.52730\end{array}$ & $\begin{array}{l}-2786 \\
-1973 \\
-\quad 1365 \\
-\quad 927 \\
-\quad 618\end{array}$ & $\begin{array}{l}3.38972 \\
3.40181 \\
3.41109 \\
3.41841 \\
3.42437\end{array}$ & $\begin{array}{r}1209 \\
928 \\
732 \\
596 \\
498\end{array}$ & $\begin{array}{l}11.20345 \\
11.42262 \\
11.62914 \\
11.82433 \\
12.00932\end{array}$ & $\begin{array}{l}21917 \\
20652 \\
19519 \\
18499 \\
17577\end{array}$ & $\begin{array}{l}14.59317 \\
14.82443 \\
15.04023 \\
15.24274 \\
15.43369\end{array}$ & $\begin{array}{l}23126 \\
21580 \\
20251 \\
19095 \\
18075\end{array}$ \\
\hline $\begin{array}{l}200 \\
210 \\
220 \\
230 \\
240\end{array}$ & $\begin{array}{l}3.52112 \\
3.51708 \\
3.51451 \\
3.51292 \\
3.51199\end{array}$ & $\begin{array}{l}-\quad 404 \\
-\quad 257 \\
-\quad 159 \\
-\quad 93 \\
-\quad 49\end{array}$ & $\begin{array}{l}3.42935 \\
3.43362 \\
3.43735 \\
3.44067 \\
3.44366\end{array}$ & $\begin{array}{l}427 \\
373 \\
332 \\
299 \\
272\end{array}$ & $\begin{array}{l}12.18509 \\
12.35252 \\
12.51234 \\
12.66520 \\
12.81170\end{array}$ & $\begin{array}{l}16743 \\
15982 \\
15286 \\
14650 \\
14064\end{array}$ & $\begin{array}{l}15.61444 \\
15.78614 \\
15.94969 \\
16.10587 \\
16.25536\end{array}$ & $\begin{array}{l}17170 \\
16355 \\
15618 \\
14949 \\
14336\end{array}$ \\
\hline $\begin{array}{l}250 \\
260 \\
270 \\
280 \\
290\end{array}$ & $\begin{array}{l}3.51150 \\
3.51130 \\
3.51129 \\
3.51141 \\
3.51162\end{array}$ & $\begin{array}{rr}-\quad 20 \\
-\quad 1 \\
12 \\
21 \\
26\end{array}$ & $\begin{array}{l}3.44638 \\
3.44888 \\
3.45119 \\
3.45334 \\
3.45535\end{array}$ & $\begin{array}{l}250 \\
231 \\
215 \\
201 \\
188\end{array}$ & $\begin{array}{l}12.95234 \\
13.08756 \\
13.21776 \\
13.34330 \\
13.46452\end{array}$ & $\begin{array}{l}13522 \\
13020 \\
12554 \\
12122 \\
11718\end{array}$ & $\begin{array}{l}16.39872 \\
16.53644 \\
16.66895 \\
16.79664 \\
16.91987\end{array}$ & $\begin{array}{l}13772 \\
13251 \\
12769 \\
12323 \\
11906\end{array}$ \\
\hline & 3.51188 & & 3.45723 & & 13.58170 & & 17.03893 & \\
\hline
\end{tabular}


Table 2.007. $T_{2}$ - Normal Mixture

\begin{tabular}{|l|l|l|l|l|}
\hline$i^{\circ}$ & $\frac{C_{p}^{0}}{R}$ & $\frac{\left(H^{\circ}-E_{0}^{0}\right)}{R T}$ & $\frac{-\left(F^{0}-E_{0}^{0}\right)}{R T}$ & $\frac{S^{0}}{R}$ \\
\hline
\end{tabular}

\begin{tabular}{|c|c|c|c|c|c|c|c|c|}
\hline $\begin{array}{l}10 \\
20 \\
30 \\
40\end{array}$ & $\begin{array}{l}2.50001 \\
2.51681 \\
2.63835 \\
2.84216\end{array}$ & $\begin{array}{r}1680 \\
12154 \\
20381 \\
19440\end{array}$ & $\begin{array}{l}6.81972 \\
4.66180 \\
3.96353 \\
3.65709\end{array}$ & $\begin{array}{l}-215792 \\
-69827 \\
-30644 \\
-14291\end{array}$ & $\begin{array}{l}1.29189 \\
5.18482 \\
6.92227 \\
8.01489\end{array}$ & $\begin{array}{r}389293 \\
173745 \\
109262 \\
79870\end{array}$ & $\begin{array}{r}8.11161 \\
9.84662 \\
10.88580 \\
11.67198\end{array}$ & $\begin{array}{r}173501 \\
103918 \\
78618 \\
65579\end{array}$ \\
\hline $\begin{array}{l}50 \\
60 \\
70 \\
80 \\
90\end{array}$ & $\begin{array}{l}3.03656 \\
3.18727 \\
3.29707 \\
3.37425 \\
3.42609\end{array}$ & $\begin{array}{r}15071 \\
10980 \\
7718 \\
5184 \\
3310\end{array}$ & $\begin{array}{l}3.51418 \\
3.44776 \\
3.41882 \\
3.40873 \\
3.40798\end{array}$ & $\begin{array}{r}6642 \\
-\quad 2894 \\
-\quad 1009 \\
-\quad 75 \\
\quad 360\end{array}$ & $\begin{array}{r}8.81359 \\
9.44764 \\
9.97661 \\
10.43229 \\
10.83370\end{array}$ & $\begin{array}{l}63405 \\
52897 \\
45568 \\
40141 \\
35923\end{array}$ & $\begin{array}{l}12.32777 \\
12.89540 \\
13.39543 \\
13.84102 \\
14.24168\end{array}$ & $\begin{array}{l}56763 \\
50003 \\
44559 \\
40066 \\
36283\end{array}$ \\
\hline $\begin{array}{l}100 \\
110 \\
120 \\
130 \\
140\end{array}$ & $\begin{array}{l}3.45919 \\
3.47940 \\
3.49130 \\
3.49812 \\
3.50200\end{array}$ & $\begin{array}{r}2021 \\
1190 \\
682 \\
388 \\
222\end{array}$ & $\begin{array}{l}3.41158 \\
3.41690 \\
3.42265 \\
3.42822 \\
3.43336\end{array}$ & $\begin{array}{l}532 \\
575 \\
557 \\
514 \\
466\end{array}$ & $\begin{array}{l}11.19293 \\
11.51834 \\
11.81588 \\
12.09006 \\
12.34431\end{array}$ & $\begin{array}{l}32541 \\
29754 \\
27418 \\
25425 \\
23703\end{array}$ & $\begin{array}{l}14.60451 \\
14.93524 \\
15.23853 \\
15.51828 \\
15.77767\end{array}$ & $\begin{array}{l}33073 \\
30329 \\
27975 \\
25939 \\
24169\end{array}$ \\
\hline $\begin{array}{l}150 \\
160 \\
170 \\
180 \\
190\end{array}$ & $\begin{array}{l}3.50422 \\
3.50553 \\
3.50636 \\
3.50694 \\
3.50739\end{array}$ & $\begin{array}{r}131 \\
83 \\
58 \\
45 \\
40\end{array}$ & $\begin{array}{l}3.43802 \\
3.44220 \\
3.44595 \\
3.44933 \\
3.45237\end{array}$ & $\begin{array}{l}418 \\
375 \\
338 \\
304 \\
276\end{array}$ & $\begin{array}{l}12.58134 \\
12.80337 \\
13.01216 \\
13.20922 \\
13.39580\end{array}$ & $\begin{array}{l}22203 \\
20879 \\
19706 \\
18658 \\
17715\end{array}$ & $\begin{array}{l}16.01936 \\
16.24557 \\
16.45811 \\
16.65855 \\
16.84817\end{array}$ & $\begin{array}{l}22621 \\
21254 \\
20044 \\
18962 \\
17991\end{array}$ \\
\hline $\begin{array}{l}200 \\
210 \\
220 \\
230 \\
240\end{array}$ & $\begin{array}{l}3.50779 \\
3.50800 \\
3.50823 \\
3.50855 \\
3.50895\end{array}$ & $\begin{array}{l}21 \\
23 \\
32 \\
40 \\
38\end{array}$ & $\begin{array}{l}3.45513 \\
3.45761 \\
3.45986 \\
3.46197 \\
3.46392\end{array}$ & $\begin{array}{l}248 \\
225 \\
211 \\
195 \\
181\end{array}$ & $\begin{array}{l}13.57295 \\
13.74168 \\
13.90267 \\
14.05651 \\
14.20389\end{array}$ & $\begin{array}{l}16873 \\
16099 \\
15384 \\
14738 \\
14145\end{array}$ & $\begin{array}{l}17.02808 \\
17.19929 \\
17.36253 \\
17.51849 \\
17.66782\end{array}$ & $\begin{array}{l}17121 \\
16324 \\
15596 \\
14933 \\
14325\end{array}$ \\
\hline $\begin{array}{l}250 \\
260 \\
270 \\
280 \\
290\end{array}$ & $\begin{array}{l}3.50933 \\
3.50975 \\
3.51020 \\
3.51070 \\
3.51127\end{array}$ & $\begin{array}{l}42 \\
45 \\
50 \\
57 \\
64\end{array}$ & $\begin{array}{l}3.46573 \\
3.46742 \\
3.46899 \\
3.47047 \\
3.47187\end{array}$ & $\begin{array}{l}169 \\
157 \\
148 \\
140 \\
132\end{array}$ & $\begin{array}{l}14.34534 \\
14.48130 \\
14.61219 \\
14.73837 \\
14.86018\end{array}$ & $\begin{array}{l}13596 \\
13089 \\
12618 \\
12181 \\
11773\end{array}$ & $\begin{array}{l}17.81107 \\
17.94872 \\
18.08119 \\
18.20885 \\
18.33206\end{array}$ & $\begin{array}{l}13765 \\
13247 \\
12766 \\
12321 \\
11905\end{array}$ \\
\hline $\begin{array}{l}300 \\
310 \\
320 \\
330 \\
340\end{array}$ & $\begin{array}{l}3.51191 \\
3.51264 \\
3.51347 \\
3.51442 \\
3.51551\end{array}$ & $\begin{array}{r}73 \\
83 \\
95 \\
109 \\
124\end{array}$ & $\begin{array}{l}3.47319 \\
3.47445 \\
3.47566 \\
3.47682 \\
3.47794\end{array}$ & $\begin{array}{l}126 \\
121 \\
116 \\
112 \\
109\end{array}$ & $\begin{array}{l}14.97791 \\
15.09181 \\
15.20214 \\
15.30911 \\
15.41292\end{array}$ & $\begin{array}{l}11390 \\
11033 \\
10697 \\
10381 \\
10084\end{array}$ & $\begin{array}{l}18.45111 \\
18.56627 \\
18.67781 \\
18.78594 \\
18.89087\end{array}$ & $\begin{array}{l}11516 \\
11154 \\
10813 \\
10493 \\
10192\end{array}$ \\
\hline $\begin{array}{l}350 \\
360 \\
370 \\
380 \\
390\end{array}$ & $\begin{array}{l}3.51675 \\
3.51816 \\
3.51972 \\
3.52152 \\
3.52351\end{array}$ & $\begin{array}{l}141 \\
156 \\
180 \\
199 \\
218\end{array}$ & $\begin{array}{l}3.47903 \\
3.48010 \\
3.48115 \\
3.48219 \\
3.48322\end{array}$ & $\begin{array}{l}107 \\
105 \\
104 \\
103 \\
104\end{array}$ & $\begin{array}{l}15.51376 \\
15.61178 \\
15.70714 \\
15.79999 \\
15.89046\end{array}$ & $\begin{array}{l}9802 \\
9536 \\
9285 \\
9047 \\
8820\end{array}$ & $\begin{array}{l}18.99279 \\
19.09188 \\
19.18830 \\
19.28219 \\
19.37369\end{array}$ & $\begin{array}{l}9907 \\
9642 \\
9389 \\
9150 \\
8923\end{array}$ \\
\hline $\begin{array}{l}400 \\
450 \\
500 \\
550 \\
600\end{array}$ & $\begin{array}{l}3.52569 \\
3.54014 \\
3.56080 \\
3.58745 \\
3.61927\end{array}$ & $\begin{array}{l}1445 \\
2066 \\
2665 \\
3182 \\
3588\end{array}$ & $\begin{array}{l}3.48426 \\
3.48960 \\
3.49564 \\
3.50273 \\
3.51108\end{array}$ & $\begin{array}{l}534 \\
604 \\
709 \\
835 \\
968\end{array}$ & $\begin{array}{l}15.97866 \\
16.38935 \\
16.75732 \\
17.09082 \\
17.39594\end{array}$ & $\begin{array}{l}41069 \\
36797 \\
33350 \\
30512 \\
28141\end{array}$ & $\begin{array}{l}19.46292 \\
19.87896 \\
20.25296 \\
20.59355 \\
20.90703\end{array}$ & $\begin{array}{l}41604 \\
37400 \\
34059 \\
31348 \\
29109\end{array}$ \\
\hline $\begin{array}{l}650 \\
700 \\
750 \\
800 \\
850\end{array}$ & $\begin{array}{l}3.65515 \\
3.69388 \\
3.73435 \\
3.77560 \\
3.81686\end{array}$ & $\begin{array}{l}3873 \\
4047 \\
4125 \\
4126 \\
4066\end{array}$ & $\begin{array}{l}3.52076 \\
3.53173 \\
3.54388 \\
3.55708 \\
3.57115\end{array}$ & $\begin{array}{l}1097 \\
1215 \\
1320 \\
1407 \\
1478\end{array}$ & $\begin{array}{l}17.67735 \\
17.93866 \\
18.18274 \\
18.41187 \\
18.62793\end{array}$ & $\begin{array}{l}26131 \\
24408 \\
22913 \\
21606 \\
20454\end{array}$ & $\begin{array}{l}21.19812 \\
21.47040 \\
21.72662 \\
21.96895 \\
22.19908\end{array}$ & $\begin{array}{l}27228 \\
25622 \\
24233 \\
23013 \\
21932\end{array}$ \\
\hline $\begin{array}{r}900 \\
950 \\
1000 \\
1050 \\
1100\end{array}$ & $\begin{array}{l}3.85752 \\
3.89715 \\
3.93546 \\
3.97224 \\
4.00739\end{array}$ & $\begin{array}{l}3963 \\
3831 \\
3678 \\
3515 \\
3347\end{array}$ & $\begin{array}{l}3.58593 \\
3.60127 \\
3.61703 \\
3.63307 \\
3.64930\end{array}$ & $\begin{array}{l}1534 \\
1576 \\
1604 \\
1623 \\
1630\end{array}$ & $\begin{array}{l}18.83247 \\
19.02676 \\
19.21188 \\
19.38874 \\
19.55813\end{array}$ & $\begin{array}{l}19429 \\
18512 \\
17686 \\
16939 \\
16258\end{array}$ & $\begin{array}{l}22.41840 \\
22.62804 \\
22.82891 \\
23.02182 \\
23.20743\end{array}$ & $\begin{array}{l}20964 \\
20087 \\
19291 \\
18561 \\
17888\end{array}$ \\
\hline 1150 & 4.04086 & 3178 & 3.66560 & 1630 & 19.72071 & 15635 & 23.38631 & 17266 \\
\hline
\end{tabular}


Table 2.007. $\quad \mathrm{T}_{2}$ - Normal Mixture (Cont.)

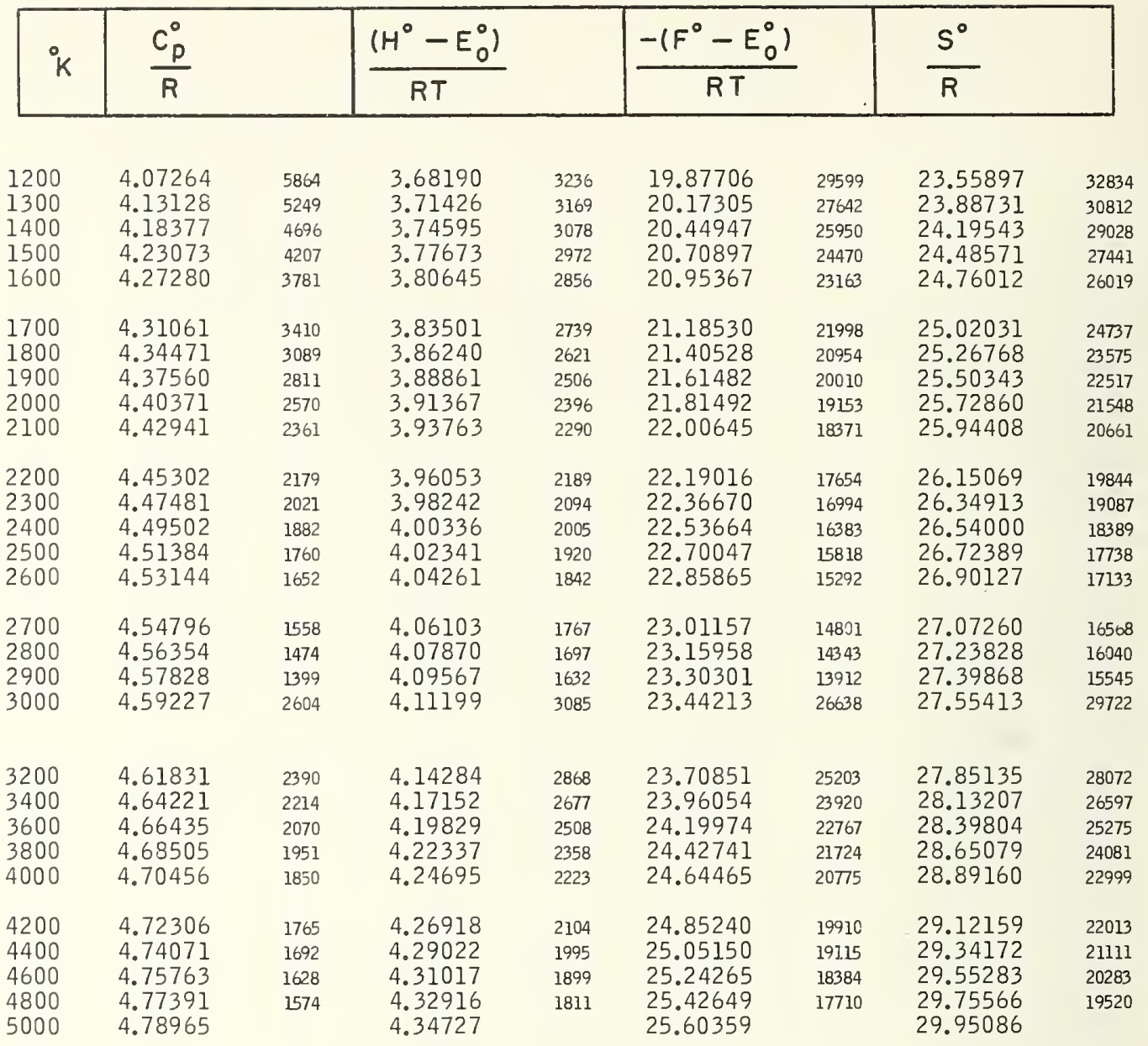


Table 2.008. $\mathrm{T}_{2}-$ Equilibrium Mixture

\begin{tabular}{|c|c|c|c|c|c|c|c|c|}
\hline${ }^{\circ} \mathrm{K}$ & $\frac{C_{p}^{0}}{R}$ & & $\frac{\left(H^{\circ}-E\right.}{R T}$ & & $\frac{-\left(F^{0}-\right.}{R T}$ & & $\frac{S^{0}}{R}$ & \\
\hline $\begin{array}{l}10 \\
20 \\
30 \\
40\end{array}$ & $\begin{array}{l}3.38998 \\
4.38323 \\
3.55416 \\
3.23596\end{array}$ & $\begin{array}{r}99325 \\
-82907 \\
-31820 \\
-4816\end{array}$ & $\begin{array}{l}2.65890 \\
3.47126 \\
3.62481 \\
3.55890\end{array}$ & $\begin{array}{r}81236 \\
15355 \\
-\quad 6591 \\
-7207\end{array}$ & $\begin{array}{l}3.42933 \\
5.54380 \\
6.99618 \\
8.03121\end{array}$ & $\begin{array}{r}211447 \\
145238 \\
103503 \\
78599\end{array}$ & $\begin{array}{r}6.08823 \\
9.01506 \\
10.62099 \\
11.59011\end{array}$ & $\begin{array}{r}292683 \\
160593 \\
96912 \\
71392\end{array}$ \\
\hline $\begin{array}{l}50 \\
60 \\
70 \\
80 \\
90\end{array}$ & $\begin{array}{l}3.18780 \\
3.23885 \\
3.31304 \\
3.37884 \\
3.42733\end{array}$ & $\begin{array}{l}5105 \\
7419 \\
6580 \\
4849 \\
3219\end{array}$ & $\begin{array}{l}3.48683 \\
3.44055 \\
3.41702 \\
3.40829 \\
3.40788\end{array}$ & $\begin{array}{r}-4628 \\
-\quad 2353 \\
-\quad 873 \\
-\quad 41 \\
367\end{array}$ & $\begin{array}{r}8.81720 \\
9.44843 \\
9.97677 \\
10.43236 \\
10.83371\end{array}$ & $\begin{array}{l}63123 \\
52834 \\
45559 \\
40135 \\
35922\end{array}$ & $\begin{array}{l}12.30403 \\
12.88898 \\
13.39379 \\
13.84065 \\
14.24159\end{array}$ & $\begin{array}{l}58495 \\
50481 \\
44686 \\
40094 \\
36289\end{array}$ \\
\hline $\begin{array}{l}100 \\
110 \\
120 \\
130 \\
140\end{array}$ & $\begin{array}{l}3.45952 \\
3.47948 \\
3.49132 \\
3.49813 \\
3.50200\end{array}$ & $\begin{array}{r}1996 \\
1184 \\
681 \\
387 \\
222\end{array}$ & $\begin{array}{l}3.41155 \\
3.41690 \\
3.42265 \\
3.42822 \\
3.43336\end{array}$ & $\begin{array}{l}535 \\
575 \\
557 \\
514 \\
466\end{array}$ & $\begin{array}{l}11.19293 \\
11.51834 \\
11.81588 \\
12.09006 \\
12.34431\end{array}$ & $\begin{array}{l}3254] \\
29754 \\
27418 \\
25425 \\
23703\end{array}$ & $\begin{array}{l}14.60448 \\
14.93524 \\
15.23853 \\
15.51828 \\
15.77767\end{array}$ & $\begin{array}{l}33076 \\
30329 \\
27975 \\
25939 \\
24169\end{array}$ \\
\hline $\begin{array}{l}150 \\
160 \\
170 \\
180 \\
190\end{array}$ & $\begin{array}{l}3.50422 \\
3.50553 \\
3.50636 \\
3.50694 \\
3.50739\end{array}$ & $\begin{array}{r}131 \\
83 \\
58 \\
45 \\
40\end{array}$ & $\begin{array}{l}3.43802 \\
3.44220 \\
3.44595 \\
3.44933 \\
3.45237\end{array}$ & $\begin{array}{l}418 \\
375 \\
338 \\
304 \\
276\end{array}$ & $\begin{array}{l}12.58134 \\
12.80337 \\
13.01216 \\
13.20922 \\
13.39580\end{array}$ & $\begin{array}{l}22203 \\
20879 \\
19706 \\
18658 \\
17715\end{array}$ & $\begin{array}{l}16.01936 \\
16.24557 \\
16.45811 \\
16.65855 \\
16.84817\end{array}$ & $\begin{array}{l}22621 \\
21254 \\
20044 \\
18962 \\
17991\end{array}$ \\
\hline 00 & 3.50779 & & 3.45513 & & 13.57295 & & 17.02808 & \\
\hline
\end{tabular}

Table 2.009. $\mathrm{T}_{2}$ - Para (includes only the even numbered rotational states)

\begin{tabular}{|l|l|l|l|l|}
\hline$k$ & $\frac{C_{p}^{0}}{R}$ & $\frac{\left(H^{0}-E_{0}^{0}\right)}{R T}$ & $\frac{-\left(F^{0}-E_{0}^{0}\right)}{R T}$ & $\frac{S^{0}}{R}$ \\
\hline
\end{tabular}

\begin{tabular}{|c|c|c|c|c|c|c|c|c|}
\hline $\begin{array}{l}10 \\
20 \\
30 \\
40\end{array}$ & $\begin{array}{l}2.50005 \\
2.56639 \\
3.00865 \\
3.59421\end{array}$ & $\begin{array}{r}6634 \\
44226 \\
58556 \\
32217\end{array}$ & $\begin{array}{l}2.50000 \\
2.50770 \\
2.58980 \\
2.77034\end{array}$ & $\begin{array}{r}770 \\
8210 \\
18054 \\
20255\end{array}$ & $\begin{array}{l}3.40136 \\
5.13510 \\
6.16360 \\
6.93175\end{array}$ & $\begin{array}{r}173374 \\
102850 \\
76815 \\
64027\end{array}$ & $\begin{array}{l}5.90136 \\
7.64280 \\
8.75340 \\
9.70209\end{array}$ & $\begin{array}{r}174144 \\
111060 \\
94869 \\
84282\end{array}$ \\
\hline $\begin{array}{l}50 \\
60 \\
70 \\
80 \\
90\end{array}$ & $\begin{array}{l}3.91638 \\
3.95770 \\
3.86716 \\
3.75388 \\
3.66167\end{array}$ & $\begin{array}{r}4132 \\
-\quad 9054 \\
-11328 \\
-\quad 9221 \\
-\quad 6339\end{array}$ & $\begin{array}{l}2.97289 \\
3.13648 \\
3.24809 \\
3.31830 \\
3.36131\end{array}$ & $\begin{array}{r}16359 \\
11161 \\
7021 \\
4301 \\
2664\end{array}$ & $\begin{array}{l}7.57202 \\
8.12930 \\
8.62179 \\
9.06047 \\
9.45399\end{array}$ & $\begin{array}{l}55728 \\
49249 \\
43868 \\
39352 \\
35564\end{array}$ & $\begin{array}{l}10.54491 \\
11.26578 \\
11.86988 \\
12.37877 \\
12.81530\end{array}$ & $\begin{array}{l}72087 \\
60410 \\
50889 \\
43653 \\
38228\end{array}$ \\
\hline $\begin{array}{l}100 \\
110 \\
120 \\
130 \\
140\end{array}$ & $\begin{array}{l}3.59828 \\
3.55856 \\
3.53511 \\
3.52186 \\
3.51464\end{array}$ & $\begin{array}{l}-3972 \\
-2345 \\
-\quad 1325 \\
-\quad 722 \\
-\quad 378\end{array}$ & $\begin{array}{l}3.38795 \\
3.40511 \\
3.41683 \\
3.42537 \\
3.43198\end{array}$ & $\begin{array}{r}1716 \\
1172 \\
854 \\
661 \\
537\end{array}$ & $\begin{array}{r}9.80963 \\
10.13340 \\
10.43020 \\
10.70404 \\
10.95814\end{array}$ & $\begin{array}{l}32377 \\
29680 \\
27384 \\
25410 \\
23697\end{array}$ & $\begin{array}{l}13.19758 \\
13.53851 \\
13.84703 \\
14.12941 \\
14.39012\end{array}$ & $\begin{array}{l}34093 \\
30852 \\
28238 \\
26071 \\
24234\end{array}$ \\
\hline $\begin{array}{l}150 \\
160 \\
170 \\
180 \\
190\end{array}$ & $\begin{array}{l}3.51086 \\
3.50898 \\
3.50814 \\
3.50785 \\
3.50786\end{array}$ & $\begin{array}{r}-\quad 188 \\
-\quad 84 \\
-\quad 29 \\
1 \\
16\end{array}$ & $\begin{array}{l}3.43735 \\
3.44188 \\
3.44580 \\
3.44925 \\
3.45233\end{array}$ & $\begin{array}{l}453 \\
392 \\
345 \\
308 \\
278\end{array}$ & $\begin{array}{l}11.19511 \\
11.41710 \\
11.62589 \\
11.82294 \\
12.00951\end{array}$ & $\begin{array}{l}22199 \\
20879 \\
19705 \\
18657 \\
17715\end{array}$ & $\begin{array}{l}14.63246 \\
14.85898 \\
15.07169 \\
15.27219 \\
15.46184\end{array}$ & $\begin{array}{l}22652 \\
21271 \\
20050 \\
18965 \\
17993\end{array}$ \\
\hline 200 & 3.50802 & & 3.45511 & & 12.18666 & & 15.64177 & \\
\hline
\end{tabular}


Table 2.010. HD

\begin{tabular}{|l|l|l|l|l|}
\hline$K$ & $\frac{C_{p}^{0}}{R}$ & $\frac{\left(H^{0}-E_{0}^{0}\right)}{R T}$ & $\frac{-\left(F^{0}-E_{0}^{0}\right)}{R T}$ & $\frac{S^{0}}{R}$ \\
\hline
\end{tabular}

\begin{tabular}{|c|c|c|c|c|c|c|c|c|}
\hline $\begin{array}{l}50 \\
60 \\
70 \\
80 \\
90\end{array}$ & $\begin{array}{l}3.52863 \\
3.52466 \\
3.52081 \\
3.51773 \\
3.51541\end{array}$ & $\begin{array}{l}-397 \\
-385 \\
-308 \\
-232 \\
-173\end{array}$ & $\begin{array}{l}3.03493 \\
3.11690 \\
3.17487 \\
3.21791 \\
3.25109\end{array}$ & $\begin{array}{l}8197 \\
5797 \\
4304 \\
3318 \\
2634\end{array}$ & $\begin{array}{l}7.97262 \\
8.53366 \\
9.01873 \\
9.44561 \\
9.82662\end{array}$ & $\begin{array}{l}56104 \\
48507 \\
42688 \\
33101 \\
34395\end{array}$ & $\begin{array}{l}11.00756 \\
11.65057 \\
12.19360 \\
12.66353 \\
13.07772\end{array}$ & $\begin{array}{l}64301 \\
54303 \\
46993 \\
41419 \\
37029\end{array}$ \\
\hline $\begin{array}{l}100 \\
110 \\
120 \\
130 \\
140\end{array}$ & $\begin{array}{l}3.51368 \\
3.51240 \\
3.51147 \\
3.51080 \\
3.51032\end{array}$ & $\begin{array}{l}-128 \\
-93 \\
-67 \\
-48 \\
-32\end{array}$ & $\begin{array}{l}3.27743 \\
3.29884 \\
3.31660 \\
3.33156 \\
3.34435\end{array}$ & $\begin{array}{l}2141 \\
1776 \\
1496 \\
1279 \\
1105\end{array}$ & $\begin{array}{l}10.17057 \\
10.48398 \\
10.77181 \\
11.03788 \\
11.28526\end{array}$ & $\begin{array}{l}31341 \\
28783 \\
26607 \\
24738 \\
23112\end{array}$ & $\begin{array}{l}13.44801 \\
13.78283 \\
14.08841 \\
14.36945 \\
14.62961\end{array}$ & $\begin{array}{l}33482 \\
30558 \\
28104 \\
26016 \\
24218\end{array}$ \\
\hline $\begin{array}{l}150 \\
160 \\
170 \\
180 \\
190\end{array}$ & $\begin{array}{l}3.51000 \\
3.50979 \\
3.50968 \\
3.50964 \\
3.50966\end{array}$ & $\begin{array}{r}-21 \\
-\quad 11 \\
-\quad 4 \\
2 \\
7\end{array}$ & $\begin{array}{l}3.35540 \\
3.36506 \\
3.37357 \\
3.38113 \\
3.38789\end{array}$ & $\begin{array}{l}966 \\
851 \\
756 \\
676 \\
609\end{array}$ & $\begin{array}{l}11.51638 \\
11.73325 \\
11.93752 \\
12.13056 \\
12.31355\end{array}$ & $\begin{array}{l}21697 \\
20427 \\
19304 \\
18299 \\
17394\end{array}$ & $\begin{array}{l}14.87179 \\
15.09831 \\
15.31109 \\
15.51169 \\
15.70145\end{array}$ & $\begin{array}{l}22652 \\
21278 \\
20060 \\
18976 \\
18002\end{array}$ \\
\hline $\begin{array}{l}200 \\
210 \\
220 \\
230 \\
240\end{array}$ & $\begin{array}{l}3.50973 \\
3.50983 \\
3.50997 \\
3.51014 \\
3.51033\end{array}$ & $\begin{array}{l}10 \\
14 \\
17 \\
19 \\
21\end{array}$ & $\begin{array}{l}3.39398 \\
3.39949 \\
3.40451 \\
3.40910 \\
3.41332\end{array}$ & $\begin{array}{l}551 \\
502 \\
459 \\
422 \\
388\end{array}$ & $\begin{array}{l}12.48749 \\
12.65322 \\
12.81148 \\
12.96292 \\
13.10810\end{array}$ & $\begin{array}{l}16573 \\
15826 \\
15144 \\
14518 \\
13942\end{array}$ & $\begin{array}{l}15.88147 \\
16.05272 \\
16.21600 \\
16.37203 \\
16.52142\end{array}$ & $\begin{array}{l}17125 \\
16328 \\
15603 \\
14939 \\
14330\end{array}$ \\
\hline $\begin{array}{l}250 \\
260 \\
270 \\
280 \\
290\end{array}$ & $\begin{array}{l}3.51054 \\
3.51076 \\
3.51100 \\
3.51125 \\
3.51151\end{array}$ & $\begin{array}{l}22 \\
24 \\
25 \\
26 \\
27\end{array}$ & $\begin{array}{l}3.41720 \\
3.42079 \\
3.42413 \\
3.42724 \\
3.43014\end{array}$ & $\begin{array}{l}359 \\
334 \\
311 \\
290 \\
272\end{array}$ & $\begin{array}{l}13.24752 \\
13.38161 \\
13.51078 \\
13.63536 \\
13.75568\end{array}$ & $\begin{array}{l}13409 \\
12917 \\
12458 \\
12032 \\
11633\end{array}$ & $\begin{array}{l}16.66472 \\
16.80241 \\
16.93492 \\
17.06261 \\
17.18583\end{array}$ & $\begin{array}{l}13769 \\
13251 \\
12769 \\
12322 \\
11905\end{array}$ \\
\hline $\begin{array}{l}300 \\
310 \\
320 \\
330 \\
340\end{array}$ & $\begin{array}{l}3.51178 \\
3.51206 \\
3.51235 \\
3.51265 \\
3.51297\end{array}$ & $\begin{array}{l}28 \\
29 \\
30 \\
32 \\
34\end{array}$ & $\begin{array}{l}3.43286 \\
3.43541 \\
3.43781 \\
3.44007 \\
3.44221\end{array}$ & $\begin{array}{l}255 \\
240 \\
226 \\
214 \\
203\end{array}$ & $\begin{array}{l}13.87201 \\
13.98462 \\
14.09373 \\
14.19955 \\
14.30228\end{array}$ & $\begin{array}{r}11261 \\
10911 \\
10582 \\
10273 \\
9981\end{array}$ & $\begin{array}{l}17.30488 \\
17.42003 \\
17.53154 \\
17.63963 \\
17.74449\end{array}$ & $\begin{array}{l}11515 \\
11151 \\
10809 \\
10486 \\
10184\end{array}$ \\
\hline $\begin{array}{l}350 \\
360 \\
370 \\
380 \\
390\end{array}$ & $\begin{array}{l}3.51331 \\
3.51365 \\
3.51400 \\
3.51437 \\
3.51476\end{array}$ & $\begin{array}{l}34 \\
35 \\
37 \\
39 \\
41\end{array}$ & $\begin{array}{l}3.44424 \\
3.44616 \\
3.44799 \\
3.44973 \\
3.45140\end{array}$ & $\begin{array}{l}192 \\
183 \\
174 \\
167 \\
158\end{array}$ & $\begin{array}{l}14.40209 \\
14.49914 \\
14.59359 \\
14.68557 \\
14.77520\end{array}$ & $\begin{array}{l}9705 \\
9445 \\
9198 \\
8963 \\
8740\end{array}$ & $\begin{array}{l}17.84633 \\
17.94531 \\
18.04159 \\
18.13530 \\
18.22660\end{array}$ & $\begin{array}{l}9898 \\
9628 \\
9371 \\
9130 \\
8899\end{array}$ \\
\hline $\begin{array}{l}400 \\
450 \\
500 \\
550 \\
600\end{array}$ & $\begin{array}{l}3.51517 \\
3.51768 \\
3.52137 \\
3.52680 \\
3.53450\end{array}$ & $\begin{array}{r}251 \\
369 \\
543 \\
770 \\
1040\end{array}$ & $\begin{array}{l}3.45298 \\
3.46003 \\
3.46596 \\
3.47123 \\
3.47616\end{array}$ & $\begin{array}{l}705 \\
593 \\
527 \\
493 \\
488\end{array}$ & $\begin{array}{l}14.86260 \\
15.26972 \\
15.63459 \\
15.96518 \\
16.26743\end{array}$ & $\begin{array}{l}40712 \\
36487 \\
33059 \\
30225 \\
27843\end{array}$ & $\begin{array}{l}18.31559 \\
18.72975 \\
19.10056 \\
19.43642 \\
19.74359\end{array}$ & $\begin{array}{l}41416 \\
37081 \\
33586 \\
30717 \\
28332\end{array}$ \\
\hline $\begin{array}{l}650 \\
700 \\
750 \\
800 \\
850\end{array}$ & $\begin{array}{l}3.54490 \\
3.55823 \\
3.57444 \\
3.59342 \\
3.61491\end{array}$ & $\begin{array}{l}1333 \\
16 ? 1 \\
1898 \\
2149 \\
2364\end{array}$ & $\begin{array}{l}3.48104 \\
3.48606 \\
3.49139 \\
3.49716 \\
3.50344\end{array}$ & $\begin{array}{l}502 \\
533 \\
577 \\
628 \\
684\end{array}$ & $\begin{array}{l}16.54586 \\
16.80402 \\
17.04471 \\
17.27022 \\
17.48242\end{array}$ & $\begin{array}{l}25816 \\
24069 \\
22551 \\
21220 \\
20045\end{array}$ & $\begin{array}{l}20.02691 \\
20.29008 \\
20.53611 \\
20.76739 \\
20.98587\end{array}$ & $\begin{array}{l}26317 \\
24603 \\
23128 \\
21848 \\
20728\end{array}$ \\
\hline $\begin{array}{r}900 \\
950 \\
1000 \\
1050 \\
1100\end{array}$ & $\begin{array}{l}3.63855 \\
3.66398 \\
3.69081 \\
3.71869 \\
3.74728\end{array}$ & $\begin{array}{l}2543 \\
2683 \\
2788 \\
2859 \\
2899\end{array}$ & $\begin{array}{l}3.51028 \\
3.51770 \\
3.52568 \\
3.53420 \\
3.54323\end{array}$ & $\begin{array}{l}742 \\
798 \\
852 \\
903 \\
951\end{array}$ & $\begin{array}{l}17.68287 \\
17.87285 \\
18.05349 \\
18.22571 \\
18.39033\end{array}$ & $\begin{array}{l}18998 \\
18064 \\
17222 \\
16462 \\
15771\end{array}$ & $\begin{array}{l}21.19315 \\
21.39056 \\
21.57917 \\
21.75992 \\
21.93357\end{array}$ & $\begin{array}{l}19741 \\
18861 \\
18075 \\
17365 \\
16721\end{array}$ \\
\hline 115 & 3.77627 & 2915 & 3.55274 & 992 & 18.54804 & 15141 & 22.10078 & 16133 \\
\hline
\end{tabular}


Table 2.010. HD (Cont.)

\begin{tabular}{|l|l|l|l|l|}
\hline$K$ & $\frac{C_{p}^{0}}{R}$ & $\frac{\left(H^{0}-E_{0}^{0}\right)}{R T}$ & $\frac{-\left(F^{0}-E_{0}^{0}\right)}{R T}$ & $\frac{S^{\circ}}{R}$ \\
\hline
\end{tabular}

\begin{tabular}{|c|c|c|c|c|c|c|c|c|}
\hline 1200 & 3.80542 & 5791 & 3.56266 & 2090 & 18.69945 & 28598 & 22.26211 & 30689 \\
\hline 1300 & 3.86333 & 5636 & 3.58356 & 2201 & 18.98543 & 26637 & 22.56900 & 28838 \\
\hline 1400 & 3.91969 & 5398 & 3.60557 & 2276 & 19.25180 & 24954 & 22.85738 & 27229 \\
\hline 1500 & 3.97367 & 5113 & 3.62833 & 2319 & 19.50134 & 23490 & 23.12967 & 25810 \\
\hline 1600 & 4.02480 & 4807 & 3.65152 & 2339 & 19.73624 & 22208 & 23.38777 & 24546 \\
\hline 1700 & 4.07287 & 4499 & 3.67491 & 2337 & 19.95832 & 21071 & 23.63323 & 23409 \\
\hline 1800 & 4.11786 & 4197 & 3.69828 & 2320 & 20.16903 & 20058 & 23.86732 & 22378 \\
\hline 1900 & 4.15983 & 3911 & 3.72148 & 2291 & 20.36961 & 19147 & 24.09110 & 21438 \\
\hline 2000 & 4.19894 & 3643 & 3.74439 & 2252 & 20.56108 & 18324 & 24.30548 & 20576 \\
\hline 2100 & 4.23537 & 3394 & 3.76691 & 2208 & 20.74432 & 17575 & 24.51124 & 19782 \\
\hline 2200 & 4.26931 & 3164 & 3.78899 & 2158 & 20.92007 & 16890 & 24.70906 & 19048 \\
\hline 2300 & 4.30095 & 2956 & 3.81057 & 2105 & 21.08897 & 16262 & 24.89954 & 18368 \\
\hline 2400 & 4.33051 & 2764 & 3.83162 & 2052 & 21.25159 & 15684 & 25.08322 & 17735 \\
\hline 2500 & 4.35815 & 2591 & 3.85214 & 1996 & 21.40843 & 15147 & 25.26057 & 17144 \\
\hline 2600 & 4.38406 & 2433 & 3.87210 & 1942 & 21.55990 & 14650 & 25.43201 & 16592 \\
\hline 2700 & 4.40839 & 2290 & 3.89152 & 1887 & 21.70640 & 14187 & 25.59793 & 16074 \\
\hline 2800 & 4.43129 & 2159 & 3.91039 & 1834 & 21.84827 & 13754 & 25.75867 & 15588 \\
\hline 2900 & 4.45288 & 2041 & 3.92873 & 1781 & 21.98581 & 13349 & 1455 & 15130 \\
\hline 3000 & 4.47329 & 3768 & 3.94654 & 3412 & 22.11930 & 25581 & 26.06585 & 28993 \\
\hline 3200 & 4.51097 & 3410 & 3.98066 & 3222 & 22.37511 & 24230 & 26.35578 & 27451 \\
\hline 10 & 4.54507 & 3111 & 4.01288 & 3044 & 22.61741 & 23024 & 029 & 26368 \\
\hline 3600 & 4.57618 & 2860 & 4.04332 & 2881 & 22.84765 & 21939 & 39097 & 24820 \\
\hline 3800 & 4.60478 & 2649 & 4.07213 & 2730 & 23.06704 & 20957 & 917 & 23688 \\
\hline 4000 & 4.63127 & 2470 & 4.09943 & 2592 & 23.27661 & 20065 & 27.37605 & 22656 \\
\hline 4200 & 4.65597 & 2316 & 4.12535 & 2465 & 23.47726 & 19248 & 27.60261 & 21714 \\
\hline 4400 & 4.67913 & 2184 & 4.15000 & 2349 & 23.66974 & 18500 & 27.81975 & 20848 \\
\hline 4600 & 4.70097 & 2069 & 4.17349 & 2241 & 23.85474 & 17810 & 28.02823 & 20052 \\
\hline 4800 & 4.72166 & 1970 & 4.19590 & 2143 & 24.03284 & 17172 & 28.22875 & 19315 \\
\hline 500 & 4.74136 & & 4.21733 & & 24.20456 & & 28.42190 & \\
\hline
\end{tabular}


Table 2.011. HT

\begin{tabular}{|l|l|l|l|l|}
\hline$K$ & $\frac{C_{p}^{0}}{R}$ & $\frac{\left(H^{0}-E_{0}^{0}\right)}{R T}$ & $\frac{-\left(F^{0}-E_{0}^{0}\right)}{R T}$ & $\frac{S^{0}}{R}$ \\
\hline
\end{tabular}

\begin{tabular}{|c|c|c|c|c|c|c|c|c|}
\hline $\begin{array}{l}50 \\
60 \\
70 \\
80 \\
90\end{array}$ & $\begin{array}{l}3.52604 \\
3.52148 \\
3.51781 \\
3.51509 \\
3.51314\end{array}$ & $\begin{array}{l}-456 \\
-367 \\
-272 \\
-195 \\
-140\end{array}$ & $\begin{array}{l}3.08910 \\
3.16154 \\
3.21268 \\
3.25065 \\
3.27991\end{array}$ & $\begin{array}{l}7244 \\
5114 \\
3797 \\
2926 \\
2325\end{array}$ & $\begin{array}{r}8.46785 \\
9.03788 \\
9.52928 \\
9.96087 \\
10.34550\end{array}$ & $\begin{array}{l}57003 \\
49140 \\
43159 \\
38463 \\
34683\end{array}$ & $\begin{array}{l}11.55696 \\
12.19942 \\
12.74197 \\
13.21152 \\
13.62542\end{array}$ & $\begin{array}{l}64246 \\
54255 \\
46955 \\
41390 \\
37007\end{array}$ \\
\hline $\begin{array}{l}100 \\
110 \\
120 \\
130 \\
140\end{array}$ & $\begin{array}{l}3.51174 \\
3.51074 \\
3.51005 \\
3.50957 \\
3.50926\end{array}$ & $\begin{array}{l}-100 \\
-\quad 69 \\
-48 \\
-\quad 31 \\
-19\end{array}$ & $\begin{array}{l}3.30316 \\
3.32208 \\
3.33777 \\
3.35100 \\
3.36231\end{array}$ & $\begin{array}{r}1892 \\
1569 \\
1323 \\
1131 \\
979\end{array}$ & $\begin{array}{l}10.69233 \\
11.00807 \\
11.29782 \\
11.56552 \\
11.81428\end{array}$ & $\begin{array}{l}31574 \\
28975 \\
26770 \\
24876 \\
23232\end{array}$ & $\begin{array}{l}13.99549 \\
14.33015 \\
14.63559 \\
14.91653 \\
15.17660\end{array}$ & $\begin{array}{l}33465 \\
30544 \\
28094 \\
26007 \\
24211\end{array}$ \\
\hline $\begin{array}{l}150 \\
160 \\
170 \\
180 \\
190\end{array}$ & $\begin{array}{l}3.50907 \\
3.50897 \\
3.50896 \\
3.50900 \\
3.50909\end{array}$ & $\begin{array}{r}-10 \\
-\quad 1 \\
4 \\
9 \\
13\end{array}$ & $\begin{array}{l}3.37210 \\
3.38066 \\
3.38821 \\
3.39492 \\
3.40092\end{array}$ & $\begin{array}{l}856 \\
755 \\
671 \\
600 \\
541\end{array}$ & $\begin{array}{l}12.04660 \\
12.26451 \\
12.46969 \\
12.66355 \\
12.84727\end{array}$ & $\begin{array}{l}21791 \\
20518 \\
19386 \\
18372 \\
17458\end{array}$ & $\begin{array}{l}15.41871 \\
15.64517 \\
15.85790 \\
16.05847 \\
16.24 .820\end{array}$ & $\begin{array}{l}22646 \\
21273 \\
20057 \\
18973 \\
17999\end{array}$ \\
\hline $\begin{array}{l}200 \\
210 \\
220 \\
230 \\
240\end{array}$ & $\begin{array}{l}3.50922 \\
3.50938 \\
3.50957 \\
3.50978 \\
3.51000\end{array}$ & $\begin{array}{l}16 \\
19 \\
21 \\
22 \\
24\end{array}$ & $\begin{array}{l}3.40633 \\
3.41124 \\
3.41570 \\
3.41979 \\
3.42354\end{array}$ & $\begin{array}{l}491 \\
446 \\
409 \\
375 \\
347\end{array}$ & $\begin{array}{l}13.02185 \\
13.18817 \\
13.34696 \\
13.49889 \\
13.64452\end{array}$ & $\begin{array}{l}16632 \\
15879 \\
15193 \\
14563 \\
13982\end{array}$ & $\begin{array}{l}16.42819 \\
16.59941 \\
16.76267 \\
16.91868 \\
17.06806\end{array}$ & $\begin{array}{l}17122 \\
16326 \\
15601 \\
14938 \\
14329\end{array}$ \\
\hline $\begin{array}{l}250 \\
260 \\
270 \\
280 \\
290\end{array}$ & $\begin{array}{l}3.51024 \\
3.51050 \\
3.51076 \\
3.51104 \\
3.51132\end{array}$ & $\begin{array}{l}26 \\
26 \\
28 \\
28 \\
29\end{array}$ & $\begin{array}{l}3.42701 \\
3.43021 \\
3.43319 \\
3.43597 \\
3.43856\end{array}$ & $\begin{array}{l}320 \\
298 \\
278 \\
259 \\
243\end{array}$ & $\begin{array}{l}13.78434 \\
13.91882 \\
14.04833 \\
14.17324 \\
14.29386\end{array}$ & $\begin{array}{l}13448 \\
12951 \\
12491 \\
12062 \\
11651\end{array}$ & $\begin{array}{l}17.21135 \\
17.34903 \\
17.48153 \\
17.60921 \\
17.73242\end{array}$ & $\begin{array}{l}13768 \\
13250 \\
12768 \\
12321 \\
11905\end{array}$ \\
\hline $\begin{array}{l}300 \\
310 \\
320 \\
330 \\
340\end{array}$ & $\begin{array}{l}3.51161 \\
3.51191 \\
3.51224 \\
3.51258 \\
3.51293\end{array}$ & $\begin{array}{l}30 \\
33 \\
34 \\
35 \\
36\end{array}$ & $\begin{array}{l}3.44099 \\
3.44327 \\
3.44542 \\
3.44746 \\
3.44938\end{array}$ & $\begin{array}{l}228 \\
215 \\
204 \\
192 \\
182\end{array}$ & $\begin{array}{l}14.41047 \\
14.52334 \\
14.63269 \\
14.73874 \\
14.84169\end{array}$ & $\begin{array}{l}11287 \\
10935 \\
10605 \\
10295 \\
10002\end{array}$ & $\begin{array}{l}17.85147 \\
17.96662 \\
18.07812 \\
18.18621 \\
18.29107\end{array}$ & $\begin{array}{l}11515 \\
11150 \\
10809 \\
10486 \\
10184\end{array}$ \\
\hline $\begin{array}{l}350 \\
360 \\
370 \\
380 \\
390\end{array}$ & $\begin{array}{l}3.51329 \\
3.51367 \\
3.51407 \\
3.51450 \\
3.51495\end{array}$ & $\begin{array}{l}38 \\
40 \\
43 \\
45 \\
49\end{array}$ & $\begin{array}{l}3.45120 \\
3.45293 \\
3.45457 \\
3.45615 \\
3.45765\end{array}$ & $\begin{array}{l}173 \\
164 \\
158 \\
150 \\
144\end{array}$ & $\begin{array}{l}14.94171 \\
15.03895 \\
15.13358 \\
15.22573 \\
15.31553\end{array}$ & $\begin{array}{l}9724 \\
9463 \\
9215 \\
8980 \\
8756\end{array}$ & $\begin{array}{l}18.39291 \\
18.49189 \\
18.58816 \\
18.68188 \\
18.77318\end{array}$ & $\begin{array}{l}9898 \\
9627 \\
9372 \\
9130 \\
8900\end{array}$ \\
\hline $\begin{array}{l}400 \\
450 \\
500 \\
550 \\
600\end{array}$ & $\begin{array}{l}3.51544 \\
3.51855 \\
3.52331 \\
3.53040 \\
3.54033\end{array}$ & $\begin{array}{r}311 \\
476 \\
709 \\
993 \\
1310\end{array}$ & $\begin{array}{l}3.45909 \\
3.46551 \\
3.47104 \\
3.47609 \\
3.48102\end{array}$ & $\begin{array}{l}642 \\
553 \\
505 \\
493 \\
503\end{array}$ & $\begin{array}{l}15.40309 \\
15.81089 \\
16.17631 \\
16.50737 \\
16.81005\end{array}$ & $\begin{array}{l}40780 \\
36542 \\
33106 \\
30268 \\
27882\end{array}$ & $\begin{array}{l}18.86218 \\
19.27640 \\
19.64735 \\
19.98347 \\
20.29107\end{array}$ & $\begin{array}{l}41422 \\
37095 \\
33612 \\
30760 \\
28386\end{array}$ \\
\hline $\begin{array}{l}650 \\
700 \\
750 \\
800 \\
850\end{array}$ & $\begin{array}{l}3.55343 \\
3.56980 \\
3.58931 \\
3.61166 \\
3.63645\end{array}$ & $\begin{array}{l}1637 \\
1951 \\
2235 \\
2479 \\
2679\end{array}$ & $\begin{array}{l}3.48605 \\
3.49143 \\
3.49729 \\
3.50372 \\
3.51079\end{array}$ & $\begin{array}{l}538 \\
586 \\
643 \\
707 \\
772\end{array}$ & $\begin{array}{l}17.08887 \\
17.34741 \\
17.58849 \\
17.81440 \\
18.02702\end{array}$ & $\begin{array}{l}25854 \\
24108 \\
22591 \\
21262 \\
20089\end{array}$ & $\begin{array}{l}20.57493 \\
20.83884 \\
21.08578 \\
21.31813 \\
21.53782\end{array}$ & $\begin{array}{l}26391 \\
24694 \\
23235 \\
21969 \\
20860\end{array}$ \\
\hline $\begin{array}{r}900 \\
950 \\
1000 \\
1050 \\
1100\end{array}$ & $\begin{array}{l}3.66324 \\
3.69158 \\
3.72105 \\
3.75125 \\
3.78185\end{array}$ & $\begin{array}{l}2834 \\
2947 \\
3020 \\
3060 \\
3069\end{array}$ & $\begin{array}{l}3.51851 \\
3.52686 \\
3.53583 \\
3.54537 \\
3.55542\end{array}$ & $\begin{array}{r}835 \\
897 \\
954 \\
1005 \\
1052\end{array}$ & $\begin{array}{l}18.22791 \\
18.41837 \\
18.59950 \\
18.77224 \\
18.93740\end{array}$ & $\begin{array}{l}19046 \\
18113 \\
17274 \\
16516 \\
15828\end{array}$ & $\begin{array}{l}21.74642 \\
21.94524 \\
22.13534 \\
22.31762 \\
22.49283\end{array}$ & $\begin{array}{l}19882 \\
19010 \\
18228 \\
17521 \\
16879\end{array}$ \\
\hline 1150 & 3.81254 & 3054 & 3.56594 & 1091 & 19.09568 & 15199 & 22.66162 & 16290 \\
\hline
\end{tabular}


Table 2.011. HT (Cont.)

\begin{tabular}{|l|l|l|l|l|}
\hline${ }^{\circ} K$ & $\frac{C_{p}^{0}}{R}$ & $\frac{\left(H^{0}-E_{0}^{0}\right)}{R T}$ & $\frac{-\left(F^{0}-E_{0}^{0}\right)}{R T}$ & $\frac{S^{0}}{R}$ \\
\hline
\end{tabular}

\begin{tabular}{|c|c|c|c|c|c|c|c|c|}
\hline $\begin{array}{l}1200 \\
1300 \\
1400 \\
1500 \\
1600\end{array}$ & $\begin{array}{l}3.84308 \\
3.90297 \\
3.96037 \\
4.01467 \\
4.06557\end{array}$ & $\begin{array}{l}5989 \\
5740 \\
5430 \\
5090 \\
4744\end{array}$ & $\begin{array}{l}3.57685 \\
3.59964 \\
3.62338 \\
3.64767 \\
3.67222\end{array}$ & $\begin{array}{l}2279 \\
2374 \\
2429 \\
2455 \\
2455\end{array}$ & $\begin{array}{l}19.24767 \\
19.53487 \\
19.80249 \\
20.05331 \\
20.28950\end{array}$ & $\begin{array}{l}28720 \\
26762 \\
25082 \\
23619 \\
22337\end{array}$ & $\begin{array}{l}22.82452 \\
23.13451 \\
23.42587 \\
23.70098 \\
23.96173\end{array}$ & $\begin{array}{l}30999 \\
29136 \\
27511 \\
26075 \\
24791\end{array}$ \\
\hline $\begin{array}{l}1700 \\
1800 \\
1900 \\
2000 \\
2100\end{array}$ & $\begin{array}{l}4.11301 \\
4.15708 \\
4.19795 \\
4.23585 \\
4.27100\end{array}$ & $\begin{array}{l}4407 \\
4087 \\
3790 \\
3515 \\
3264\end{array}$ & $\begin{array}{l}3.69677 \\
3.72113 \\
3.74517 \\
3.76876 \\
3.79185\end{array}$ & $\begin{array}{l}2436 \\
2404 \\
2359 \\
2309 \\
2253\end{array}$ & $\begin{array}{l}20.51287 \\
20.72486 \\
20.92670 \\
21.11940 \\
21.30384\end{array}$ & $\begin{array}{l}21199 \\
20184 \\
19270 \\
18444 \\
17692\end{array}$ & $\begin{array}{l}24.20964 \\
24.44600 \\
24.67187 \\
24.88817 \\
25.09570\end{array}$ & $\begin{array}{l}23636 \\
22587 \\
21630 \\
20753 \\
19945\end{array}$ \\
\hline $\begin{array}{l}2200 \\
2300 \\
2400 \\
2500 \\
2600\end{array}$ & $\begin{array}{l}4.30364 \\
4.33399 \\
4.36228 \\
4.38870 \\
4.41343\end{array}$ & $\begin{array}{l}3035 \\
2829 \\
2642 \\
2473 \\
2320\end{array}$ & $\begin{array}{l}3.81438 \\
3.83632 \\
3.85766 \\
3.87837 \\
3.89848\end{array}$ & $\begin{array}{l}2194 \\
2134 \\
2071 \\
2011 \\
1951\end{array}$ & $\begin{array}{l}21.48076 \\
21.65080 \\
21.81453 \\
21.97243 \\
22.12494\end{array}$ & $\begin{array}{l}17004 \\
16373 \\
15790 \\
15251 \\
14749\end{array}$ & $\begin{array}{l}25.29515 \\
25.48713 \\
25.67219 \\
25.85081 \\
26.02342\end{array}$ & $\begin{array}{l}19198 \\
18506 \\
17862 \\
17261 \\
16701\end{array}$ \\
\hline $\begin{array}{l}2700 \\
2800 \\
2900 \\
3000\end{array}$ & $\begin{array}{l}4.43663 \\
4.45845 \\
4.47903 \\
4.49848\end{array}$ & $\begin{array}{l}2182 \\
2058 \\
1945 \\
3593\end{array}$ & $\begin{array}{l}3.91799 \\
3.93691 \\
3.95525 \\
3.97303\end{array}$ & $\begin{array}{l}1892 \\
1834 \\
1778 \\
3399\end{array}$ & $\begin{array}{l}22.27243 \\
22.41527 \\
22.55374 \\
22.68813\end{array}$ & $\begin{array}{l}14284 \\
13847 \\
13439 \\
25751\end{array}$ & $\begin{array}{l}26.19043 \\
26.35218 \\
26.50899 \\
26.66117\end{array}$ & $\begin{array}{l}16175 \\
15681 \\
15218 \\
29149\end{array}$ \\
\hline $\begin{array}{l}3200 \\
3400 \\
3600 \\
3800 \\
4000\end{array}$ & $\begin{array}{l}4.53441 \\
4.56696 \\
4.59671 \\
4.62412 \\
4.64956\end{array}$ & $\begin{array}{l}3255 \\
2975 \\
2741 \\
2544 \\
2378\end{array}$ & $\begin{array}{l}4.00702 \\
4.03901 \\
4.06918 \\
4.09768 \\
4.12464\end{array}$ & $\begin{array}{l}3199 \\
3017 \\
2850 \\
2696 \\
2557\end{array}$ & $\begin{array}{l}22.94564 \\
23.18953 \\
23.42126 \\
23.64204 \\
23.85291\end{array}$ & $\begin{array}{l}24389 \\
23173 \\
22078 \\
21087 \\
20187\end{array}$ & $\begin{array}{l}26.95266 \\
27.22855 \\
27.49045 \\
27.73972 \\
27.97756\end{array}$ & $\begin{array}{l}27589 \\
26190 \\
24727 \\
23784 \\
22744\end{array}$ \\
\hline $\begin{array}{l}4200 \\
4400 \\
4600 \\
4800 \\
5000\end{array}$ & $\begin{array}{l}4.67334 \\
4.69569 \\
4.71682 \\
4.73690 \\
4.75605\end{array}$ & $\begin{array}{l}2235 \\
2113 \\
2008 \\
1915\end{array}$ & $\begin{array}{l}4.15021 \\
4.17450 \\
4.19763 \\
4.21968 \\
4.24076\end{array}$ & $\begin{array}{l}2429 \\
2313 \\
2205 \\
2108\end{array}$ & $\begin{array}{l}24.05478 \\
24.24841 \\
24.43449 \\
24.61361 \\
24.78630\end{array}$ & $\begin{array}{l}19363 \\
18608 \\
17912 \\
17269\end{array}$ & $\begin{array}{l}28.20500 \\
28.42292 \\
28.63212 \\
28.83330 \\
29.02706\end{array}$ & $\begin{array}{l}21792 \\
20920 \\
20118 \\
19376\end{array}$ \\
\hline
\end{tabular}


Table 2.012. DT

\begin{tabular}{|c|c|c|c|c|c|c|c|c|}
\hline${ }^{\circ} \mathrm{K}$ & $\frac{C_{p}^{0}}{R}$ & & $\frac{\left(H^{0}-E_{0}^{0}\right)}{R T}$ & & $\frac{-1 F^{\circ}-E}{R T}$ & & $\frac{S^{0}}{R}$ & \\
\hline $\begin{array}{l}50 \\
60 \\
70 \\
80 \\
90\end{array}$ & $\begin{array}{l}3.51411 \\
3.51102 \\
3.50913 \\
3.50796 \\
3.50725\end{array}$ & $\begin{array}{l}-309 \\
-189 \\
-117 \\
-71 \\
-41\end{array}$ & $\begin{array}{l}3.24874 \\
3.29269 \\
3.32374 \\
3.34683 \\
3.36469\end{array}$ & $\begin{array}{l}4395 \\
3105 \\
2309 \\
1786 \\
1423\end{array}$ & $\begin{array}{r}9.11621 \\
9.71266 \\
10.22269 \\
10.66809 \\
11.06337\end{array}$ & $\begin{array}{l}59645 \\
51003 \\
44540 \\
39528 \\
35527\end{array}$ & $\begin{array}{l}12.36496 \\
13.00536 \\
13.54643 \\
14.01493 \\
14.42806\end{array}$ & $\begin{array}{l}64040 \\
54107 \\
46850 \\
41313 \\
36951\end{array}$ \\
\hline $\begin{array}{l}100 \\
110 \\
120 \\
130 \\
140\end{array}$ & $\begin{array}{l}3.50684 \\
3.50662 \\
3.50654 \\
3.50655 \\
3.50663\end{array}$ & $\begin{array}{r}-\quad 22 \\
-\quad 8 \\
1 \\
8 \\
14\end{array}$ & $\begin{array}{l}3.37892 \\
3.39054 \\
3.40021 \\
3.40839 \\
3.41540\end{array}$ & $\begin{array}{r}1162 \\
967 \\
818 \\
701 \\
609\end{array}$ & $\begin{array}{l}11.41864 \\
11.74125 \\
12.03669 \\
12.30918 \\
12.56204\end{array}$ & $\begin{array}{l}32261 \\
29544 \\
27247 \\
25286 \\
23585\end{array}$ & $\begin{array}{l}14.79757 \\
15.13179 \\
15.43690 \\
15.71758 \\
15.97744\end{array}$ & $\begin{array}{l}33422 \\
30511 \\
28068 \\
25986 \\
24194\end{array}$ \\
\hline $\begin{array}{l}150 \\
160 \\
170 \\
180 \\
190\end{array}$ & $\begin{array}{l}3.50677 \\
3.50695 \\
3.50716 \\
3.50739 \\
3.50764\end{array}$ & $\begin{array}{l}18 \\
21 \\
23 \\
25 \\
27\end{array}$ & $\begin{array}{l}3.42149 \\
3.42683 \\
3.43154 \\
3.43575 \\
3.43953\end{array}$ & $\begin{array}{l}534 \\
471 \\
421 \\
378 \\
341\end{array}$ & $\begin{array}{l}12.79789 \\
13.01888 \\
13.22677 \\
13.42304 \\
13.60890\end{array}$ & $\begin{array}{l}22099 \\
20789 \\
19627 \\
18586 \\
17651\end{array}$ & $\begin{array}{l}16.21938 \\
16.44571 \\
16.65832 \\
16.85879 \\
17.04843\end{array}$ & $\begin{array}{l}22633 \\
21261 \\
20047 \\
18964 \\
17993\end{array}$ \\
\hline $\begin{array}{l}200 \\
210 \\
220 \\
230 \\
240\end{array}$ & $\begin{array}{l}3.50791 \\
3.50818 \\
3.50847 \\
3.50877 \\
3.50907\end{array}$ & $\begin{array}{l}27 \\
29 \\
30 \\
30 \\
32\end{array}$ & $\begin{array}{l}3.44294 \\
3.44604 \\
3.44887 \\
3.45147 \\
3.45386\end{array}$ & $\begin{array}{l}310 \\
283 \\
260 \\
239 \\
222\end{array}$ & $\begin{array}{l}13.78541 \\
13.95347 \\
14.11385 \\
14.26722 \\
14.41416\end{array}$ & $\begin{array}{l}16806 \\
16038 \\
15337 \\
14694 \\
14104\end{array}$ & $\begin{array}{l}17.22836 \\
17.39952 \\
17.56273 \\
17.71869 \\
17.86803\end{array}$ & $\begin{array}{l}17116 \\
16321 \\
15596 \\
14934 \\
14325\end{array}$ \\
\hline $\begin{array}{l}250 \\
260 \\
270 \\
280 \\
290\end{array}$ & $\begin{array}{l}3.50939 \\
3.50973 \\
3.51010 \\
3.51049 \\
3.51090\end{array}$ & $\begin{array}{l}34 \\
37 \\
39 \\
41 \\
44\end{array}$ & $\begin{array}{l}3.45608 \\
3.45813 \\
3.46006 \\
3.46185 \\
3.46354\end{array}$ & $\begin{array}{l}205 \\
193 \\
179 \\
169 \\
158\end{array}$ & $\begin{array}{l}1455520 \\
14.69079 \\
14.82134 \\
14.94721 \\
15.06872\end{array}$ & $\begin{array}{l}13559 \\
13055 \\
12587 \\
12151 \\
11744\end{array}$ & $\begin{array}{l}18.01128 \\
18.14893 \\
18.28140 \\
18.40906 \\
18.53226\end{array}$ & $\begin{array}{l}13765 \\
13247 \\
12766 \\
12320 \\
11903\end{array}$ \\
\hline $\begin{array}{l}300 \\
310 \\
320 \\
330 \\
340\end{array}$ & $\begin{array}{l}3.51134 \\
3.51182 \\
3.51235 \\
3.51293 \\
3.51358\end{array}$ & $\begin{array}{l}48 \\
53 \\
58 \\
65 \\
74\end{array}$ & $\begin{array}{l}3.46512 \\
3.46662 \\
3.46804 \\
3.46939 \\
3.47068\end{array}$ & $\begin{array}{l}150 \\
142 \\
135 \\
129 \\
124\end{array}$ & $\begin{array}{l}15.18616 \\
15.29981 \\
15.40989 \\
15.51663 \\
15.62022\end{array}$ & $\begin{array}{l}11365 \\
11008 \\
10674 \\
10359 \\
10063\end{array}$ & $\begin{array}{l}18.65129 \\
18.76643 \\
18.87794 \\
18.98603 \\
19.09091\end{array}$ & $\begin{array}{l}11514 \\
11151 \\
10809 \\
10488 \\
10186\end{array}$ \\
\hline $\begin{array}{l}350 \\
360 \\
370 \\
380 \\
390\end{array}$ & $\begin{array}{l}3.51432 \\
3.51515 \\
3.51607 \\
3.51711 \\
3.51827\end{array}$ & $\begin{array}{r}83 \\
92 \\
104 \\
116 \\
129\end{array}$ & $\begin{array}{l}3.47192 \\
3.47311 \\
3.47426 \\
3.47537 \\
3.47645\end{array}$ & $\begin{array}{l}119 \\
115 \\
111 \\
103 \\
107\end{array}$ & $\begin{array}{l}15.72085 \\
15.81867 \\
15.91384 \\
16.00651 \\
16.09680\end{array}$ & $\begin{array}{l}9782 \\
9517 \\
9267 \\
9029 \\
8803\end{array}$ & $\begin{array}{l}19.19277 \\
19.29178 \\
19.38810 \\
19.48189 \\
19.57326\end{array}$ & $\begin{array}{l}9901 \\
9632 \\
9379 \\
9137 \\
8909\end{array}$ \\
\hline $\begin{array}{l}400 \\
450 \\
500 \\
550 \\
600\end{array}$ & $\begin{array}{l}3.51956 \\
3.52839 \\
3.54181 \\
3.56025 \\
3.58361\end{array}$ & $\begin{array}{r}883 \\
1342 \\
1844 \\
2336 \\
2773\end{array}$ & $\begin{array}{l}3.47752 \\
3.48264 \\
3.48785 \\
3.49355 \\
3.50005\end{array}$ & $\begin{array}{l}512 \\
521 \\
570 \\
650 \\
747\end{array}$ & $\begin{array}{l}16.18483 \\
16.59472 \\
16.96192 \\
17.29461 \\
17.59886\end{array}$ & $\begin{array}{r}40989 \\
36720 \\
-33269 \\
30425 \\
28044\end{array}$ & $\begin{array}{l}19.66235 \\
20.07736 \\
20.44977 \\
20.78817 \\
21.09892\end{array}$ & $\begin{array}{l}41501 \\
37241 \\
33840 \\
31075 \\
28791\end{array}$ \\
\hline $\begin{array}{l}650 \\
700 \\
750 \\
800 \\
850\end{array}$ & $\begin{array}{l}3.61134 \\
3.64266 \\
3.67672 \\
3.71264 \\
3.74967\end{array}$ & $\begin{array}{l}3132 \\
3406 \\
3592 \\
3703 \\
3749\end{array}$ & $\begin{array}{l}3.50752 \\
3.51603 \\
3.52560 \\
3.53616 \\
3.54763\end{array}$ & $\begin{array}{r}851 \\
957 \\
1056 \\
1147 \\
1226\end{array}$ & $\begin{array}{l}17.87930 \\
18.13954 \\
18.38245 \\
18.61032 \\
18.82503\end{array}$ & $\begin{array}{l}26024 \\
24291 \\
22787 \\
21471 \\
20312\end{array}$ & $\begin{array}{l}21.38683 \\
21.65558 \\
21.90805 \\
22.14648 \\
22.37266\end{array}$ & $\begin{array}{l}26875 \\
25247 \\
23843 \\
22618 \\
21539\end{array}$ \\
\hline $\begin{array}{l}900 \\
950 \\
000 \\
050 \\
100\end{array}$ & $\begin{array}{l}3.78716 \\
3.82455 \\
3.86145 \\
3.89753 \\
3.93257\end{array}$ & $\begin{array}{l}3739 \\
3690 \\
3608 \\
3504 \\
3385\end{array}$ & $\begin{array}{l}3.55989 \\
3.57284 \\
3.58635 \\
3.60031 \\
3.61462\end{array}$ & $\begin{array}{l}1295 \\
1351 \\
1396 \\
1431 \\
1457\end{array}$ & $\begin{array}{l}19.02815 \\
19.22097 \\
19.40458 \\
19.57989 \\
19.74771\end{array}$ & $\begin{array}{l}19282 \\
18361 \\
17531 \\
16782 \\
16097\end{array}$ & $\begin{array}{l}22.58805 \\
22.79381 \\
22.99093 \\
23.18021 \\
23.36233\end{array}$ & $\begin{array}{l}20576 \\
19712 \\
18928 \\
18212 \\
17557\end{array}$ \\
\hline 0 & 3.96642 & 3255 & 3.62919 & 1473 & 19.90870 & 15477 & 23.53790 & 16950 \\
\hline
\end{tabular}


Table 2.012. DT (Cont.)

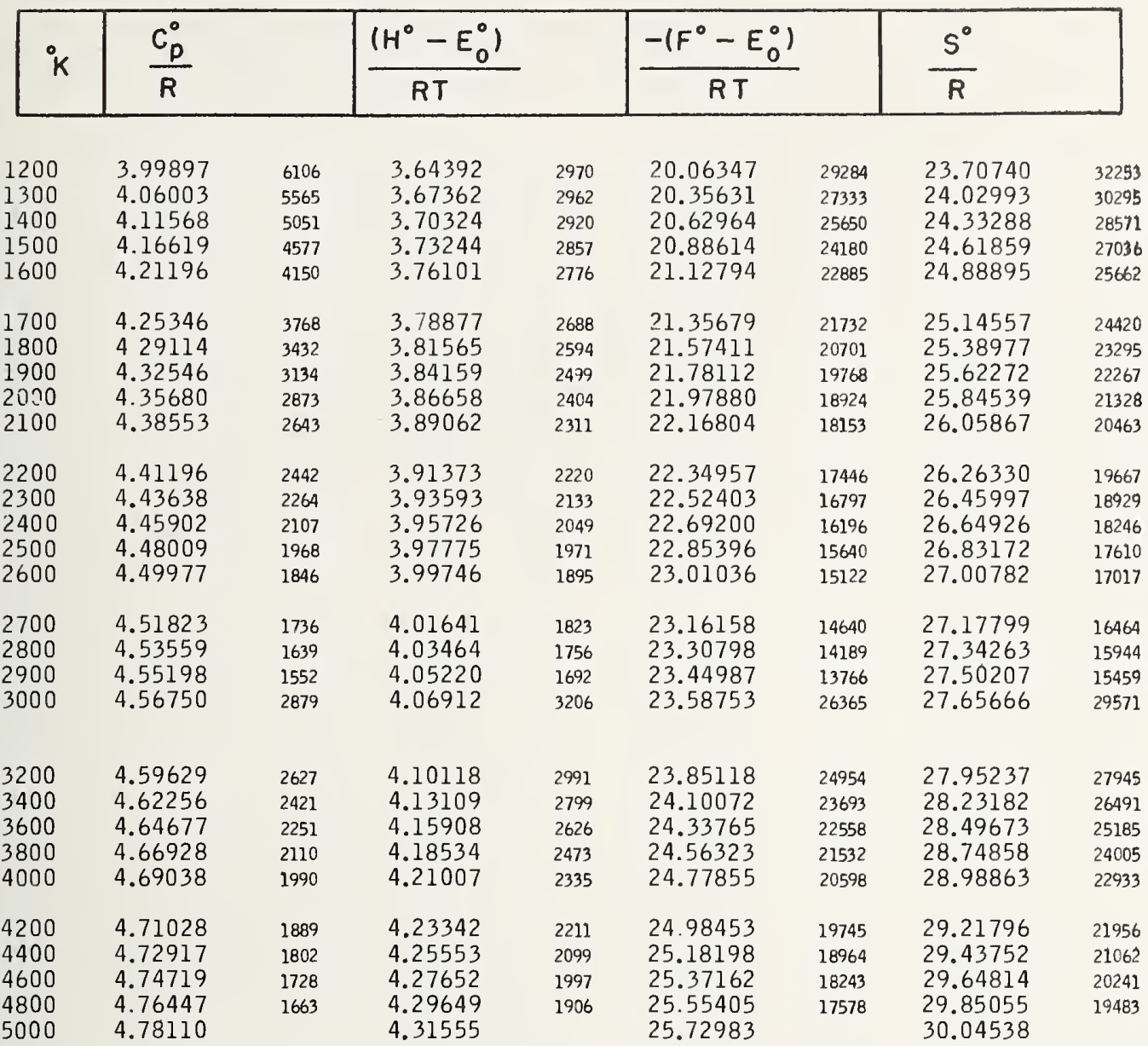


Table 2.013. LiH

\begin{tabular}{|l|l|l|l|l|}
\hline$K$ & $\frac{C_{p}^{0}}{R}$ & $\frac{\left(H^{0}-E_{0}^{0}\right)}{R T}$ & $\frac{-\left(F^{0}-E_{0}^{0}\right)}{R T}$ & $\frac{S^{0}}{R}$ \\
\hline
\end{tabular}

\begin{tabular}{|c|c|c|c|c|c|c|c|c|}
\hline $\begin{array}{l}50 \\
60 \\
70 \\
80 \\
90\end{array}$ & $\begin{array}{l}3.50220 \\
3.50206 \\
3.50207 \\
3.50215 \\
3.50227\end{array}$ & $\begin{array}{r}-14 \\
1 \\
8 \\
12 \\
16\end{array}$ & $\begin{array}{l}3.42843 \\
3.44072 \\
3.44948 \\
3.45606 \\
3.46118\end{array}$ & $\begin{array}{r}1229 \\
876 \\
658 \\
512 \\
412\end{array}$ & $\begin{array}{l}10.84307 \\
11.46930 \\
12.00038 \\
12.46145 \\
12.86882\end{array}$ & $\begin{array}{l}62623 \\
53108 \\
46107 \\
40737 \\
36489\end{array}$ & $\begin{array}{l}14.27151 \\
14.91002 \\
15.44987 \\
15.91751 \\
16.33001\end{array}$ & $\begin{array}{l}63851 \\
53985 \\
46764 \\
41250 \\
36901\end{array}$ \\
\hline $\begin{array}{l}100 \\
110 \\
120 \\
130 \\
140\end{array}$ & $\begin{array}{l}3.50243 \\
3.50260 \\
3.50280 \\
3.50303 \\
3.50332\end{array}$ & $\begin{array}{l}17 \\
20 \\
23 \\
29 \\
41\end{array}$ & $\begin{array}{l}3.46530 \\
3.46868 \\
3.47152 \\
3.47393 \\
3.47602\end{array}$ & $\begin{array}{l}338 \\
284 \\
241 \\
209 \\
183\end{array}$ & $\begin{array}{l}13.23371 \\
13.56416 \\
13.86610 \\
14.14406 \\
14.40159\end{array}$ & $\begin{array}{l}33045 \\
30194 \\
27796 \\
25753 \\
23988\end{array}$ & $\begin{array}{l}16.69902 \\
17.03284 \\
17.33762 \\
17.61800 \\
17.87761\end{array}$ & $\begin{array}{l}33382 \\
30478 \\
28038 \\
25961 \\
24172\end{array}$ \\
\hline $\begin{array}{l}150 \\
160 \\
170 \\
180 \\
190\end{array}$ & $\begin{array}{l}3.50373 \\
3.50431 \\
3.50513 \\
3.50628 \\
3.50785\end{array}$ & $\begin{array}{r}58 \\
82 \\
115 \\
157 \\
209\end{array}$ & $\begin{array}{l}3.47785 \\
3.47949 \\
3.48097 \\
3.48234 \\
3.48364\end{array}$ & $\begin{array}{l}164 \\
148 \\
137 \\
130 \\
126\end{array}$ & $\begin{array}{l}14.64147 \\
14.86598 \\
15.07697 \\
15.27598 \\
15.46429\end{array}$ & $\begin{array}{l}22451 \\
21099 \\
19901 \\
18831 \\
17872 .\end{array}$ & $\begin{array}{l}18.11933 \\
18.34548 \\
18.55795 \\
18.75832 \\
18.94794\end{array}$ & $\begin{array}{l}22615 \\
21247 \\
20037 \\
18962 \\
17998\end{array}$ \\
\hline $\begin{array}{l}200 \\
210 \\
220 \\
230 \\
240\end{array}$ & $\begin{array}{l}3.50994 \\
3.51263 \\
3.51599 \\
3.52009 \\
3.52496\end{array}$ & $\begin{array}{l}269 \\
336 \\
410 \\
487 \\
569\end{array}$ & $\begin{array}{l}3.48490 \\
3.48616 \\
3.48743 \\
3.48876 \\
3.49017\end{array}$ & $\begin{array}{l}126 \\
127 \\
133 \\
141 \\
150\end{array}$ & $\begin{array}{l}15.64301 \\
15.81307 \\
15.97528 \\
16.13033 \\
16.27884\end{array}$ & $\begin{array}{l}17006 \\
16221 \\
15505 \\
14851 \\
14251\end{array}$ & $\begin{array}{l}19.12792 \\
19.29923 \\
19.46272 \\
19.61910 \\
19.76901\end{array}$ & $\begin{array}{l}17131 \\
16349 \\
15638 \\
14991 \\
14401\end{array}$ \\
\hline $\begin{array}{l}250 \\
260 \\
270 \\
280 \\
290\end{array}$ & $\begin{array}{l}3.53065 \\
3.53715 \\
3.54448 \\
3.55262 \\
3.56154\end{array}$ & $\begin{array}{l}650 \\
733 \\
814 \\
892 \\
966\end{array}$ & $\begin{array}{l}3.49167 \\
3.49329 \\
3.49505 \\
3.49696 \\
3.49903\end{array}$ & $\begin{array}{l}162 \\
176 \\
191 \\
207 \\
224\end{array}$ & $\begin{array}{l}16.42135 \\
16.55832 \\
16.69019 \\
16.81733 \\
16.94008\end{array}$ & $\begin{array}{l}13697 \\
13187 \\
12714 \\
12275 \\
11866\end{array}$ & $\begin{array}{l}19.91302 \\
20.05162 \\
20.18525 \\
20.31429 \\
20.43911\end{array}$ & $\begin{array}{l}13860 \\
13363 \\
12904 \\
12482 \\
12091\end{array}$ \\
\hline $\begin{array}{l}300 \\
310 \\
320 \\
330 \\
340\end{array}$ & $\begin{array}{l}3.57120 \\
3.58156 \\
3.59257 \\
3.60417 \\
3.61631\end{array}$ & $\begin{array}{l}1036 \\
1101 \\
1160 \\
1214 \\
1263\end{array}$ & $\begin{array}{l}3.50127 \\
3.50369 \\
3.50629 \\
3.50908 \\
3.51206\end{array}$ & $\begin{array}{l}242 \\
260 \\
279 \\
298 \\
316\end{array}$ & $\begin{array}{l}17.05874 \\
17.17359 \\
17.28487 \\
17.39280 \\
17.49760\end{array}$ & $\begin{array}{l}11485 \\
11128 \\
10793 \\
10480 \\
10185\end{array}$ & $\begin{array}{l}20.56002 \\
20.67728 \\
20.79117 \\
20.90189 \\
21.00967\end{array}$ & $\begin{array}{l}11726 \\
11389 \\
11072 \\
10778 \\
10500\end{array}$ \\
\hline $\begin{array}{l}350 \\
360 \\
370 \\
380 \\
390\end{array}$ & $\begin{array}{l}3.62894 \\
3.64198 \\
3.65539 \\
3.66911 \\
3.68309\end{array}$ & $\begin{array}{l}1304 \\
1341 \\
1372 \\
1398 \\
1419\end{array}$ & $\begin{array}{l}3.51522 \\
3.51855 \\
3.52207 \\
3.52576 \\
3.52961\end{array}$ & $\begin{array}{l}333 \\
352 \\
369 \\
385 \\
402\end{array}$ & $\begin{array}{l}17.59945 \\
17.69853 \\
17.79498 \\
17.88896 \\
17.98059\end{array}$ & $\begin{array}{l}9908 \\
9645 \\
9398 \\
9163 \\
8941\end{array}$ & $\begin{array}{l}21.11467 \\
21.21709 \\
21.31706 \\
21.41472 \\
21.51021\end{array}$ & $\begin{array}{r}10242 \\
9997 \\
9766 \\
9549 \\
9342\end{array}$ \\
\hline $\begin{array}{l}400 \\
450 \\
500 \\
550 \\
600\end{array}$ & $\begin{array}{l}3.69728 \\
3.76976 \\
3.84167 \\
3.91012 \\
3.97364\end{array}$ & $\begin{array}{l}7248 \\
7191 \\
6845 \\
6352 \\
5805\end{array}$ & $\begin{array}{l}3.53363 \\
3.55582 \\
3.58083 \\
3.60769 \\
3.63558\end{array}$ & $\begin{array}{l}2219 \\
2501 \\
2686 \\
2789 \\
2827\end{array}$ & $\begin{array}{l}18.07000 \\
18.48745 \\
18.86337 \\
19.20591 \\
19.52101\end{array}$ & $\begin{array}{l}41745 \\
37592 \\
34254 \\
31510 \\
29212\end{array}$ & $\begin{array}{l}21.60363 \\
22.04328 \\
22.44421 \\
22.81360 \\
23.15659\end{array}$ & $\begin{array}{l}43965 \\
40093 \\
36939 \\
34299 \\
32039\end{array}$ \\
\hline $\begin{array}{l}650 \\
700 \\
750 \\
800 \\
850\end{array}$ & $\begin{array}{l}4.03169 \\
4.08426 \\
4.13164 \\
4.17428 \\
4.21266\end{array}$ & $\begin{array}{l}5257 \\
4738 \\
4264 \\
3838 \\
3458\end{array}$ & $\begin{array}{l}3.66385 \\
3.69203 \\
3.71979 \\
3.74688 \\
3.77317\end{array}$ & $\begin{array}{l}2818 \\
2776 \\
2709 \\
2629 \\
2540\end{array}$ & $\begin{array}{l}19.81313 \\
20.08568 \\
20.34136 \\
20.58229 \\
20.81024\end{array}$ & $\begin{array}{l}27255 \\
25568 \\
24093 \\
22795 \\
21639\end{array}$ & $\begin{array}{l}23.47698 \\
23.77772 \\
24.06115 \\
24.32918 \\
24.58342\end{array}$ & $\begin{array}{l}30074 \\
28343 \\
26803 \\
25424 \\
24178\end{array}$ \\
\hline $\begin{array}{r}900 \\
950 \\
1000 \\
1050 \\
1100\end{array}$ & $\begin{array}{l}4.24724 \\
4.27849 \\
4.30680 \\
4.33253 \\
4.35601\end{array}$ & $\begin{array}{l}3125 \\
2831 \\
2573 \\
2348 \\
2150\end{array}$ & $\begin{array}{l}3.79857 \\
3.82302 \\
3.84651 \\
3.86905 \\
3.89066\end{array}$ & $\begin{array}{l}2445 \\
2349 \\
2254 \\
2161 \\
2071\end{array}$ & $\begin{array}{l}21.02663 \\
21.23267 \\
21.42937 \\
21.61759 \\
21.79808\end{array}$ & $\begin{array}{l}20604 \\
19670 \\
18822 \\
18049 \\
17341\end{array}$ & $\begin{array}{l}24.82520 \\
25.05569 \\
25.27588 \\
25.48664 \\
25.68874\end{array}$ & $\begin{array}{l}23049 \\
22019 \\
21076 \\
20210 \\
19412\end{array}$ \\
\hline 1150 & 4.37751 & 1977 & 3.91137 & 2984 & 21.97149 & 16689 & 25.38286 & 18673 \\
\hline
\end{tabular}


Table 2.013. LiH (Cont.)

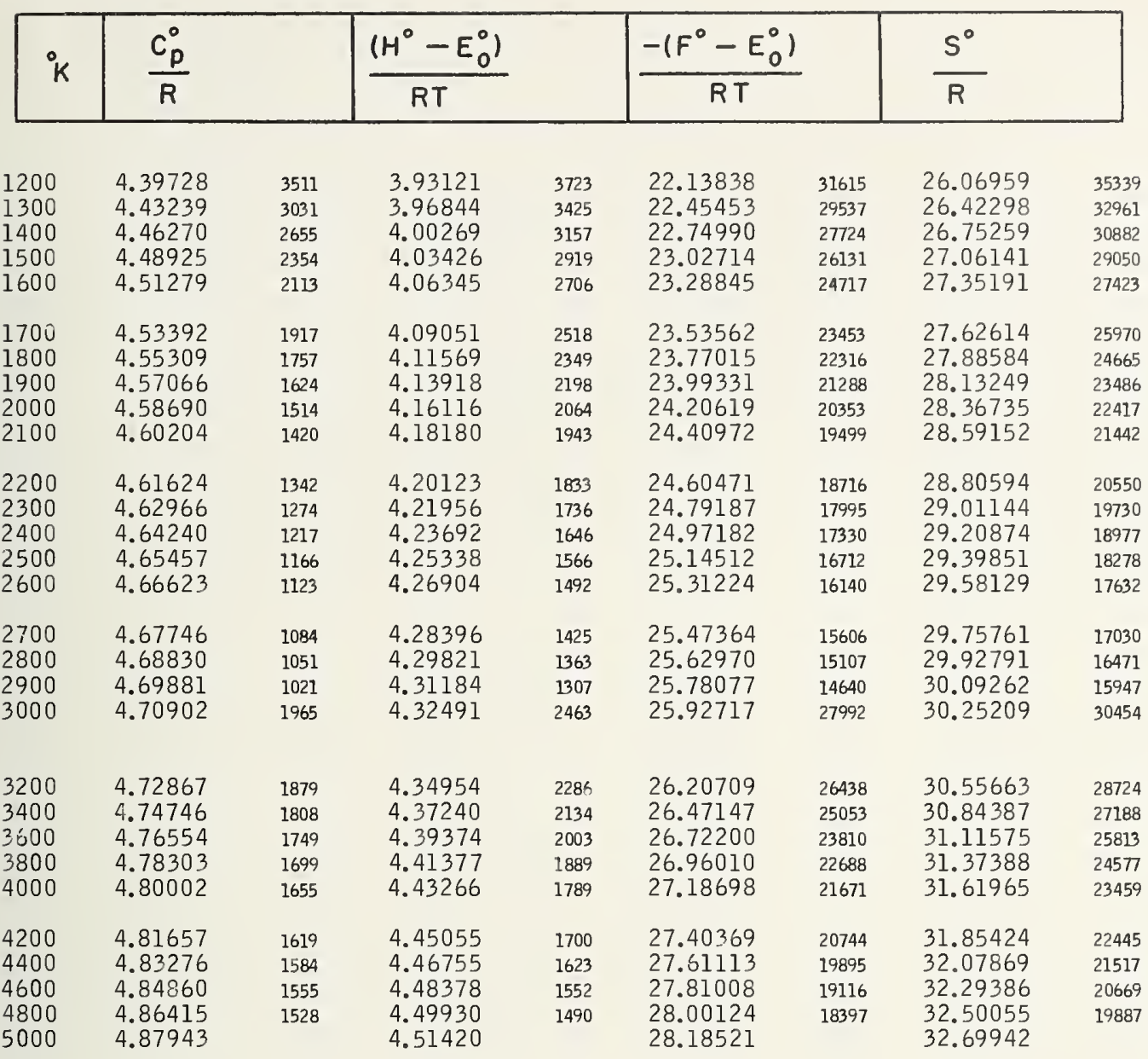


Table 2.014. LiD

\begin{tabular}{|l|l|l|l|l|}
\hline$K$ & $\frac{C_{p}^{0}}{R}$ & $\frac{\left(H^{0}-E_{0}^{0}\right)}{R T}$ & $\frac{-\left(F^{0}-E_{0}^{0}\right)}{R T}$ & $\frac{S^{0}}{R}$ \\
\hline
\end{tabular}

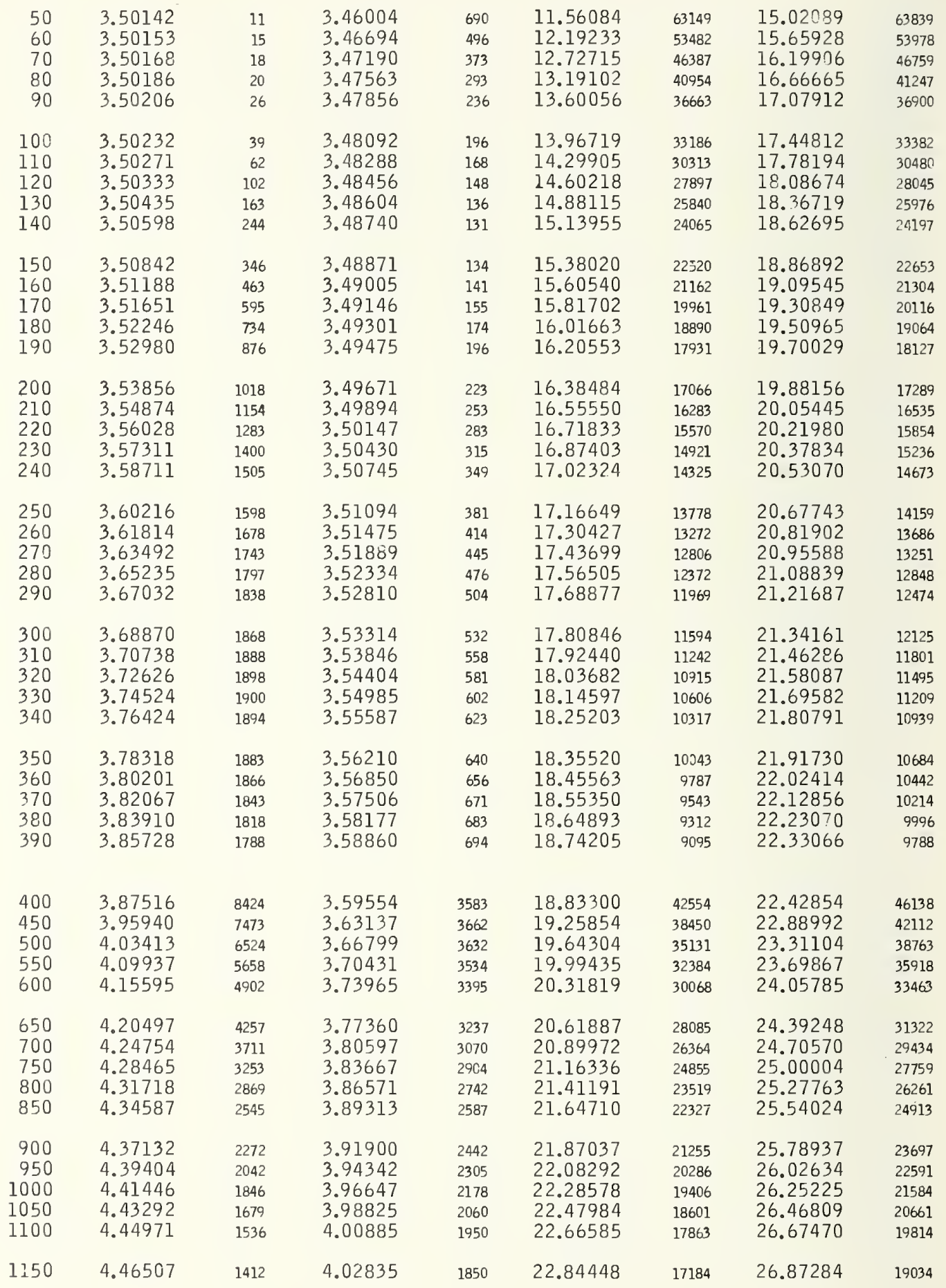


Table 2.014. LiD (Cont.)

\begin{tabular}{|c|c|c|c|c|c|c|c|c|}
\hline${ }^{\circ} \mathrm{K}$ & $\frac{C_{p}^{0}}{R}$ & & $\frac{\left(H^{0}-E_{0}^{0}\right)}{R T}$ & & $\frac{-\left(F^{0}-E_{0}^{0}\right)}{R T}$ & & $\frac{S^{\circ}}{R}$ & \\
\hline $\begin{array}{l}1200 \\
1300 \\
1400 \\
1500 \\
1600\end{array}$ & $\begin{array}{l}4.47919 \\
4.50438 \\
4.52632 \\
4.54576 \\
4.56324\end{array}$ & $\begin{array}{l}2519 \\
2194 \\
1944 \\
1748 \\
1595\end{array}$ & $\begin{array}{l}4.04685 \\
4.08110 \\
4.11213 \\
4.14040 \\
4.16629\end{array}$ & $\begin{array}{l}3425 \\
3103 \\
2827 \\
2589 \\
2383\end{array}$ & $\begin{array}{l}23.01632 \\
23.34162 \\
23.64522 \\
23.92990 \\
24.19796\end{array}$ & $\begin{array}{l}32530 \\
30360 \\
28468 \\
26806 \\
25330\end{array}$ & $\begin{array}{l}27.06318 \\
27.42272 \\
27.75735 \\
28.07031 \\
28.36426\end{array}$ & $\begin{array}{l}35954 \\
33463 \\
31296 \\
29395 \\
27713\end{array}$ \\
\hline $\begin{array}{l}1700 \\
1800 \\
1900 \\
2000 \\
2100\end{array}$ & $\begin{array}{l}4.57919 \\
4.59389 \\
4.60758 \\
4.62045 \\
4.63262\end{array}$ & $\begin{array}{l}1470 \\
1369 \\
1287 \\
1217 \\
1160\end{array}$ & $\begin{array}{l}4.19012 \\
4.21215 \\
4.23260 \\
4.25168 \\
4.26953\end{array}$ & $\begin{array}{l}2203 \\
2045 \\
1908 \\
1785 \\
1677\end{array}$ & $\begin{array}{l}24.45126 \\
24.69140 \\
24.91969 \\
25.13728 \\
25.34516\end{array}$ & $\begin{array}{l}24014 \\
22829 \\
21759 \\
20788 \\
19901\end{array}$ & $\begin{array}{l}28.64139 \\
28.90355 \\
29.15230 \\
29.38897 \\
29.61470\end{array}$ & $\begin{array}{l}26216 \\
24875 \\
23667 \\
22573 \\
21578\end{array}$ \\
\hline $\begin{array}{l}2200 \\
2300 \\
2400 \\
2500 \\
2600\end{array}$ & $\begin{array}{l}4.64422 \\
4.65533 \\
4.66603 \\
4.67637 \\
4.68640\end{array}$ & $\begin{array}{r}1111 \\
1070 \\
1034 \\
1003 \\
975\end{array}$ & $\begin{array}{l}4.28630 \\
4.30211 \\
4.31705 \\
4.33121 \\
4.34468\end{array}$ & $\begin{array}{l}1581 \\
1494 \\
1416 \\
1347 \\
1284\end{array}$ & $\begin{array}{l}25.54417 \\
25.73506 \\
25.91847 \\
26.09499 \\
26.26513\end{array}$ & $\begin{array}{l}19089 \\
18341 \\
17652 \\
17014 \\
16421\end{array}$ & $\begin{array}{l}29.83048 \\
30.03717 \\
30.23552 \\
30.42621 \\
30.60982\end{array}$ & $\begin{array}{l}20669 \\
19835 \\
19069 \\
18361 \\
17705\end{array}$ \\
\hline $\begin{array}{l}2700 \\
2800 \\
2900 \\
3000\end{array}$ & $\begin{array}{l}4.69615 \\
4.70567 \\
4.71497 \\
4.72409\end{array}$ & $\begin{array}{r}952 \\
930 \\
912 \\
1774\end{array}$ & $\begin{array}{l}4.35752 \\
4.36979 \\
4.38153 \\
4.39280\end{array}$ & $\begin{array}{l}1227 \\
1174 \\
1127 \\
2126\end{array}$ & $\begin{array}{l}26.42934 \\
26.58804 \\
26.74159 \\
26.89032\end{array}$ & $\begin{array}{l}15870 \\
15355 \\
14873 \\
28419\end{array}$ & $\begin{array}{l}30.78687 \\
30.95783 \\
31.12312 \\
31.28312\end{array}$ & $\begin{array}{l}17096 \\
16529 \\
16000 \\
30546\end{array}$ \\
\hline $\begin{array}{l}3200 \\
3400 \\
3600 \\
3800 \\
4000\end{array}$ & $\begin{array}{l}4.74183 \\
4.75902 \\
4.77574 \\
4.79207 \\
4.80806\end{array}$ & $\begin{array}{l}1719 \\
1672 \\
1633 \\
1599 \\
1569\end{array}$ & $\begin{array}{l}4.41406 \\
4.43385 \\
4.45238 \\
4.46983 \\
4.48634\end{array}$ & $\begin{array}{l}1979 \\
1853 \\
1745 \\
1651 \\
1570\end{array}$ & $\begin{array}{l}27.17451 \\
27.44271 \\
27.69667 \\
27.93787 \\
28.16757\end{array}$ & $\begin{array}{l}26820 \\
25396 \\
24120 \\
22970 \\
21927\end{array}$ & $\begin{array}{l}31.58858 \\
31.87657 \\
32.14906 \\
32.40771 \\
32.65392\end{array}$ & $\begin{array}{l}28799 \\
27249 \\
25865 \\
24621 \\
23497\end{array}$ \\
\hline $\begin{array}{l}4200 \\
4400 \\
4600 \\
4800 \\
5000\end{array}$ & $\begin{array}{l}4.82375 \\
4.83917 \\
4.85434 \\
4.86930 \\
4.88405\end{array}$ & $\begin{array}{l}1542 \\
1517 \\
1496 \\
1475\end{array}$ & $\begin{array}{l}4.50204 \\
4.51701 \\
4.53135 \\
4.54512 \\
4.55838\end{array}$ & $\begin{array}{l}1497 \\
1434 \\
1377 \\
1326\end{array}$ & $\begin{array}{l}28.38684 \\
28.59663 \\
28.79773 \\
28.99088 \\
29.17669\end{array}$ & $\begin{array}{l}20979 \\
20110 \\
19315 \\
18581\end{array}$ & $\begin{array}{l}32.88889 \\
33.11364 \\
33.32909 \\
33.53601 \\
33.73508\end{array}$ & $\begin{array}{l}22475 \\
21545 \\
20692 \\
19907\end{array}$ \\
\hline
\end{tabular}


Table 2.015. LiT

\begin{tabular}{|c|c|c|c|c|c|c|c|c|}
\hline${ }^{\circ} \mathrm{K}$ & $\frac{C_{p}^{9}}{R}$ & & $\frac{\left(H^{0}-E_{0}^{0}\right)}{R T}$ & & $\frac{-\left\langle F^{0}-\right.}{R T}$ & & $\frac{S^{0}}{R}$ & \\
\hline $\begin{array}{l}50 \\
60 \\
70 \\
80 \\
90\end{array}$ & $\begin{array}{l}3.50126 \\
3.50142 \\
3.50161 \\
3.50183 \\
3.50213\end{array}$ & $\begin{array}{l}16 \\
19 \\
22 \\
30 \\
53\end{array}$ & $\begin{array}{l}3.47051 \\
3.47565 \\
3.47935 \\
3.48214 \\
3.48434\end{array}$ & $\begin{array}{l}514 \\
370 \\
279 \\
220 \\
181\end{array}$ & $\begin{array}{l}12.00654 \\
12.63978 \\
13.17584 \\
13.64064 \\
14.05091\end{array}$ & $\begin{array}{l}63324 \\
53606 \\
46480 \\
41027 \\
36721\end{array}$ & $\begin{array}{l}15.47706 \\
16.11543 \\
16.65519 \\
17.12278 \\
17.53526\end{array}$ & $\begin{array}{l}63837 \\
53976 \\
46759 \\
41248 \\
36901\end{array}$ \\
\hline $\begin{array}{l}100 \\
110 \\
120 \\
130 \\
140\end{array}$ & $\begin{array}{l}3.50266 \\
3.50363 \\
3.50533 \\
3.50808 \\
3.51219\end{array}$ & $\begin{array}{r}97 \\
170 \\
275 \\
411 \\
572\end{array}$ & $\begin{array}{l}3.48615 \\
3.48769 \\
3.48908 \\
3.49043 \\
3.49183\end{array}$ & $\begin{array}{l}154 \\
139 \\
135 \\
140 \\
154\end{array}$ & $\begin{array}{l}14.41812 \\
14.75045 \\
15.05398 \\
15.33331 \\
15.59203\end{array}$ & $\begin{array}{l}33233 \\
30353 \\
27933 \\
25872 \\
24097\end{array}$ & $\begin{array}{l}17.90427 \\
18.23815 \\
18.54307 \\
18.82375 \\
19.08387\end{array}$ & $\begin{array}{l}33388 \\
30492 \\
28068 \\
26012 \\
24250\end{array}$ \\
\hline $\begin{array}{l}150 \\
160 \\
170 \\
180 \\
190\end{array}$ & $\begin{array}{l}3.51791 \\
3.52542 \\
3.53483 \\
3.54612 \\
3.55926\end{array}$ & $\begin{array}{r}751 \\
941 \\
1129 \\
1314 \\
1484\end{array}$ & $\begin{array}{l}3.49337 \\
3.49513 \\
3.49718 \\
3.49957 \\
3.50236\end{array}$ & $\begin{array}{l}176 \\
205 \\
239 \\
279 \\
321\end{array}$ & $\begin{array}{l}15.83300 \\
16.05851 \\
16.27046 \\
16.47042 \\
16.65970\end{array}$ & $\begin{array}{l}22551 \\
21195 \\
19996 \\
18928 \\
17973\end{array}$ & $\begin{array}{l}19.32637 \\
19.55364 \\
19.76764 \\
19.96999 \\
20.16207\end{array}$ & $\begin{array}{l}22727 \\
21400 \\
20235 \\
19208 \\
18293\end{array}$ \\
\hline $\begin{array}{l}200 \\
210 \\
220 \\
230 \\
240\end{array}$ & $\begin{array}{l}3.57410 \\
3.59049 \\
3.60823 \\
3.62713 \\
3.64697\end{array}$ & $\begin{array}{l}1639 \\
1774 \\
1890 \\
1984 \\
2059\end{array}$ & $\begin{array}{l}3.50557 \\
3.50922 \\
3.51331 \\
3.51784 \\
3.52281\end{array}$ & $\begin{array}{l}365 \\
409 \\
453 \\
497 \\
537\end{array}$ & $\begin{array}{l}16.83943 \\
17.01055 \\
17.17390 \\
17.33017 \\
17.47999\end{array}$ & $\begin{array}{l}1712 \\
16335 \\
15627 \\
14982 \\
14392\end{array}$ & $\begin{array}{l}20.34500 \\
20.51978 \\
20.68721 \\
20.84802 \\
21.00280\end{array}$ & $\begin{array}{l}17478 \\
16743 \\
16081 \\
15478 \\
14929\end{array}$ \\
\hline $\begin{array}{l}250 \\
260 \\
270 \\
280 \\
290\end{array}$ & $\begin{array}{l}3.66756 \\
3.68871 \\
3.71025 \\
3.73201 \\
3.75386\end{array}$ & $\begin{array}{l}2115 \\
2154 \\
2176 \\
2185 \\
2182\end{array}$ & $\begin{array}{l}3.52818 \\
3.53395 \\
3.54008 \\
3.54654 \\
3.55332\end{array}$ & $\begin{array}{l}577 \\
613 \\
646 \\
678 \\
705\end{array}$ & $\begin{array}{l}17.62391 \\
17.76239 \\
17.89588 \\
18.02474 \\
18.14931\end{array}$ & $\begin{array}{l}13848 \\
13349 \\
12886 \\
12457 \\
12058\end{array}$ & $\begin{array}{l}21.15209 \\
21.29635 \\
21.43596 \\
21.57129 \\
21.70263\end{array}$ & $\begin{array}{l}14426 \\
13961 \\
13533 \\
13134 \\
12763\end{array}$ \\
\hline $\begin{array}{l}300 \\
310 \\
320 \\
330 \\
340\end{array}$ & $\begin{array}{l}3.77568 \\
3.79736 \\
3.81881 \\
3.83997 \\
3.86076\end{array}$ & $\begin{array}{l}2168 \\
2145 \\
2116 \\
2079 \\
2039\end{array}$ & $\begin{array}{l}3.56037 \\
3.56766 \\
3.57517 \\
3.58288 \\
3.59075\end{array}$ & $\begin{array}{l}729 \\
751 \\
771 \\
787 \\
800\end{array}$ & $\begin{array}{l}18.26989 \\
18.38676 \\
18.50014 \\
18.61027 \\
18.71735\end{array}$ & $\begin{array}{l}11687 \\
11338 \\
11013 \\
10708 \\
10420\end{array}$ & $\begin{array}{l}21.83026 \\
21.95442 \\
22.07532 \\
22.19316 \\
22.30810\end{array}$ & $\begin{array}{l}12416 \\
12090 \\
11784 \\
11494 \\
11221\end{array}$ \\
\hline $\begin{array}{l}350 \\
360 \\
370 \\
380 \\
390\end{array}$ & $\begin{array}{l}3.88115 \\
3.90109 \\
3.92056 \\
3.93954 \\
3.95800\end{array}$ & $\begin{array}{l}1994 \\
1947 \\
1898 \\
1846 \\
1794\end{array}$ & $\begin{array}{l}3.59875 \\
3.60688 \\
3.61509 \\
3.62338 \\
3.63173\end{array}$ & $\begin{array}{l}813 \\
821 \\
829 \\
835 \\
838\end{array}$ & $\begin{array}{l}18.82155 \\
18.92305 \\
19.02198 \\
19.11850 \\
19.21273\end{array}$ & $\begin{array}{r}10150 \\
9893 \\
9652 \\
9423 \\
9205\end{array}$ & $\begin{array}{l}22.42031 \\
22.52993 \\
22.63708 \\
22.74189 \\
22.84446\end{array}$ & $\begin{array}{l}10962 \\
10715 \\
10481 \\
10257 \\
10043\end{array}$ \\
\hline $\begin{array}{l}400 \\
450 \\
500 \\
550 \\
600\end{array}$ & $\begin{array}{l}3.97594 \\
4.05786 \\
4.12747 \\
4.18632 \\
4.23613\end{array}$ & $\begin{array}{l}8192 \\
6961 \\
5885 \\
4981 \\
4237\end{array}$ & $\begin{array}{l}3.64011 \\
3.68209 \\
3.72325 \\
3.76275 \\
3.80018\end{array}$ & $\begin{array}{l}4198 \\
4116 \\
3950 \\
3743 \\
3520\end{array}$ & $\begin{array}{l}19.30478 \\
19.73595 \\
20.12604 \\
20.48277 \\
20.81179\end{array}$ & $\begin{array}{l}43117 \\
39009 \\
35673 \\
32902 \\
30559\end{array}$ & $\begin{array}{l}22.94489 \\
23.41805 \\
23.84929 \\
24.24552 \\
24.61197\end{array}$ & $\begin{array}{l}47316 \\
43124 \\
39623 \\
36645 \\
34079\end{array}$ \\
\hline $\begin{array}{l}650 \\
700 \\
750 \\
800 \\
850\end{array}$ & $\begin{array}{l}4.27850 \\
4.31480 \\
4.34613 \\
4.37339 \\
4.39732\end{array}$ & $\begin{array}{l}3630 \\
3133 \\
2726 \\
2393 \\
2117\end{array}$ & $\begin{array}{l}3.83538 \\
3.86836 \\
3.89919 \\
3.92800 \\
3.95492\end{array}$ & $\begin{array}{l}3298 \\
3083 \\
2881 \\
2692 \\
2517\end{array}$ & $\begin{array}{l}21.11738 \\
21.40283 \\
21.67078 \\
21.92336 \\
22.16231\end{array}$ & $\begin{array}{l}28545 \\
26795 \\
25258 \\
23895 \\
22678\end{array}$ & $\begin{array}{l}24.95276 \\
25.27120 \\
25.56998 \\
25.85137 \\
26.11723\end{array}$ & $\begin{array}{l}31844 \\
29878 \\
28139 \\
26586 \\
25196\end{array}$ \\
\hline $\begin{array}{r}900 \\
950 \\
1000 \\
1050 \\
1100\end{array}$ & $\begin{array}{l}4.41849 \\
4.43737 \\
4.45434 \\
4.46970 \\
4.48369\end{array}$ & $\begin{array}{l}1888 \\
1697 \\
1536 \\
1399 \\
1284\end{array}$ & $\begin{array}{l}3.98009 \\
4.00367 \\
4.02579 \\
4.04657 \\
4.06613\end{array}$ & $\begin{array}{l}2358 \\
2212 \\
2078 \\
1956 \\
1843\end{array}$ & $\begin{array}{l}22.38909 \\
22.60492 \\
22.81085 \\
23.00778 \\
23.19648\end{array}$ & $\begin{array}{l}21583 \\
20593 \\
19693 \\
18870 \\
18116\end{array}$ & $\begin{array}{l}26.36919 \\
26.60860 \\
26.83665 \\
27.05435 \\
27.26261\end{array}$ & $\begin{array}{l}23941 \\
22805 \\
21770 \\
20826 \\
19960\end{array}$ \\
\hline 1150 & 4.49653 & 1185 & 4.08456 & 1742 & 23.37764 & 17421 & 27.46221 & 19162 \\
\hline
\end{tabular}


Table 2.015. LiT (Cont.)

\begin{tabular}{|c|c|c|c|c|c|c|c|c|}
\hline${ }^{\circ} K$ & $\frac{C_{p}^{0}}{R}$ & & $\frac{\left(H^{0}-E_{0}^{0}\right)}{R T}$ & & $\frac{-1 F^{0}-E}{R T}$ & & $\frac{S^{0}}{R}$ & \\
\hline $\begin{array}{l}1200 \\
1300 \\
1400 \\
1500 \\
1600\end{array}$ & $\begin{array}{l}4.50838 \\
4.52965 \\
4.54835 \\
4.56509 \\
4.58030\end{array}$ & $\begin{array}{l}2127 \\
1870 \\
1674 \\
1521 \\
1401\end{array}$ & $\begin{array}{l}4.10198 \\
4.13408 \\
4.16301 \\
4.18927 \\
4.21324\end{array}$ & $\begin{array}{l}3210 \\
2893 \\
2626 \\
2397 \\
2201\end{array}$ & $\begin{array}{l}23.55185 \\
23.88147 \\
24.18892 \\
24.47705 \\
24.74819\end{array}$ & $\begin{array}{l}32962 \\
30745 \\
28813 \\
27114 \\
25610\end{array}$ & $\begin{array}{l}27.65383 \\
28.01555 \\
28.35194 \\
28.66632 \\
28.96144\end{array}$ & $\begin{array}{l}36172 \\
33639 \\
31438 \\
29512 \\
27810\end{array}$ \\
\hline $\begin{array}{l}1700 \\
1800 \\
1900 \\
2000 \\
2100\end{array}$ & $\begin{array}{l}4.59431 \\
4.60736 \\
4.61962 \\
4.63125 \\
4.64235\end{array}$ & $\begin{array}{l}1305 \\
1226 \\
1163 \\
1110 \\
1065\end{array}$ & $\begin{array}{l}4.23525 \\
4.25556 \\
4.27441 \\
4.29196 \\
4.30838\end{array}$ & $\begin{array}{l}2031 \\
1885 \\
1755 \\
1642 \\
1543\end{array}$ & $\begin{array}{l}25.00429 \\
25.24695 \\
25.47755 \\
25.69725 \\
25.90706\end{array}$ & $\begin{array}{l}24266 \\
23060 \\
21970 \\
20981 \\
20078\end{array}$ & $\begin{array}{l}29.23954 \\
29.50252 \\
29.75196 \\
29.98921 \\
30.21544\end{array}$ & $\begin{array}{l}26298 \\
24944 \\
23725 \\
22623 \\
21621\end{array}$ \\
\hline $\begin{array}{l}2200 \\
2300 \\
2400 \\
2500 \\
2600\end{array}$ & $\begin{array}{l}4.65300 \\
4.66327 \\
4.67322 \\
4.68289 \\
4.69231\end{array}$ & $\begin{array}{r}1027 \\
995 \\
967 \\
942 \\
922\end{array}$ & $\begin{array}{l}4.32381 \\
4.33834 \\
4.35209 \\
4.36513 \\
4.37753\end{array}$ & $\begin{array}{l}1453 \\
1375 \\
1304 \\
1240 \\
1183\end{array}$ & $\begin{array}{l}26.10784 \\
26.30037 \\
26.48530 \\
26.66323 \\
26.83467\end{array}$ & $\begin{array}{l}19253 \\
18493 \\
17793 \\
17144 \\
16544 \\
\end{array}$ & $\begin{array}{l}30.43165 \\
30.63871 \\
30.83739 \\
31.02836 \\
31.21221\end{array}$ & $\begin{array}{l}20706 \\
19868 \\
19097 \\
18385 \\
17726\end{array}$ \\
\hline $\begin{array}{l}2700 \\
2800 \\
2900 \\
3000\end{array}$ & $\begin{array}{l}4.70153 \\
4.71056 \\
4.71942 \\
4.72813\end{array}$ & $\begin{array}{r}903 \\
886 \\
871 \\
1703\end{array}$ & $\begin{array}{l}4.38936 \\
4.40067 \\
4.41151 \\
4.42192\end{array}$ & $\begin{array}{l}1131 \\
1084 \\
1041 \\
1967\end{array}$ & $\begin{array}{l}27.00011 \\
27.15994 \\
27.31456 \\
27.46429\end{array}$ & $\begin{array}{l}15983 \\
15462 \\
14973 \\
28602\end{array}$ & $\begin{array}{l}31.38947 \\
31.56062 \\
31.72608 \\
31.88622\end{array}$ & $\begin{array}{l}17115 \\
16546 \\
16014 \\
30569\end{array}$ \\
\hline $\begin{array}{l}3200 \\
3400 \\
3600 \\
3800 \\
4000\end{array}$ & $\begin{array}{l}4.74516 \\
4.76174 \\
4.77794 \\
4.79382 \\
4.80941\end{array}$ & $\begin{array}{l}1658 \\
1620 \\
1588 \\
1559 \\
1534\end{array}$ & $\begin{array}{l}4.44159 \\
4.45994 \\
4.47716 \\
4.49341 \\
4.50882\end{array}$ & $\begin{array}{l}1835 \\
1722 \\
1625 \\
1541 \\
1468\end{array}$ & $\begin{array}{l}27.75031 \\
28.02014 \\
28.27556 \\
28.51806 \\
28.74894\end{array}$ & $\begin{array}{l}26983 \\
25542 \\
24250 \\
23088 \\
22034\end{array}$ & $\begin{array}{l}32.19191 \\
32.48009 \\
32.75272 \\
33.01148 \\
33.25777\end{array}$ & $\begin{array}{l}28818 \\
27263 \\
25876 \\
24629 \\
23502\end{array}$ \\
\hline $\begin{array}{l}4200 \\
4400 \\
4600 \\
4800 \\
5000\end{array}$ & $\begin{array}{l}4.82475 \\
4.83986 \\
4.85476 \\
4.86946 \\
4.88399\end{array}$ & $\begin{array}{l}1511 \\
1490 \\
1470 \\
1453\end{array}$ & $\begin{array}{l}4.52350 \\
4.53754 \\
4.55101 \\
4.56397 \\
4.57648\end{array}$ & $\begin{array}{l}1404 \\
1347 \\
1296 \\
1251\end{array}$ & $\begin{array}{l}28.96928 \\
29.18004 \\
29.38205 \\
29.57601 \\
29.76258\end{array}$ & $\begin{array}{l}21076 \\
20201 \\
19396 \\
18657\end{array}$ & $\begin{array}{l}33.49279 \\
33.71759 \\
33.93306 \\
34.13999 \\
34.33906\end{array}$ & $\begin{array}{l}22480 \\
21547 \\
20693 \\
19907\end{array}$ \\
\hline
\end{tabular}


Tabie 2.016. $\mathrm{NaH}$

\begin{tabular}{|l|l|l|l|l|}
\hline$K$ & $\frac{C_{p}^{0}}{R}$ & $\frac{\left(H^{0}-E_{0}^{0}\right)}{R T}$ & $\frac{-\left(F^{0}-E_{0}^{0}\right)}{R T}$ & $\frac{S^{0}}{R}$ \\
\hline
\end{tabular}

\begin{tabular}{|c|c|c|c|c|c|c|c|c|}
\hline $\begin{array}{l}50 \\
60 \\
70 \\
80 \\
90\end{array}$ & $\begin{array}{l}3.50242 \\
3.50266 \\
3.50297 \\
3.50330 \\
3.50366\end{array}$ & $\begin{array}{l}24 \\
31 \\
33 \\
36 \\
38\end{array}$ & $\begin{array}{l}3.45417 \\
3.46223 \\
3.46803 \\
3.47242 \\
3.47587\end{array}$ & $\begin{array}{l}836 \\
580 \\
439 \\
345 \\
280\end{array}$ & $\begin{array}{l}12.90333 \\
13.53386 \\
14.06802 \\
14.53141 \\
14.94061\end{array}$ & $\begin{array}{l}63053 \\
53416 \\
46339 \\
40920 \\
36637\end{array}$ & $\begin{array}{l}16.35750 \\
16.99609 \\
17.53605 \\
18.00383 \\
18.41648\end{array}$ & $\begin{array}{l}63859 \\
53996 \\
46778 \\
41265 \\
36917\end{array}$ \\
\hline $\begin{array}{l}100 \\
110 \\
120 \\
130 \\
140\end{array}$ & $\begin{array}{l}3.50404 \\
3.50447 \\
3.50500 \\
3.50572 \\
3.50674\end{array}$ & $\begin{array}{r}43 \\
53 \\
72 \\
102 \\
147\end{array}$ & $\begin{array}{l}3.47867 \\
3.48099 \\
3.48297 \\
3.48469 \\
3.48623\end{array}$ & $\begin{array}{l}232 \\
198 \\
172 \\
154 \\
141\end{array}$ & $\begin{array}{l}15.30698 \\
15.63864 \\
15.94162 \\
16.22047 \\
16.47877\end{array}$ & $\begin{array}{l}33166 \\
30298 \\
27885 \\
25830 \\
24058\end{array}$ & $\begin{array}{l}18.78565 \\
19.11964 \\
19.42459 \\
19.70517 \\
19.96500\end{array}$ & $\begin{array}{l}33399 \\
30495 \\
28058 \\
25983 \\
24199\end{array}$ \\
\hline $\begin{array}{l}150 \\
160 \\
170 \\
180 \\
190\end{array}$ & $\begin{array}{l}3.50821 \\
3.51029 \\
3.51314 \\
3.51688 \\
3.52165\end{array}$ & $\begin{array}{l}208 \\
285 \\
374 \\
477 \\
588\end{array}$ & $\begin{array}{l}3.48764 \\
3.48899 \\
3.49032 \\
3.49169 \\
3.49313\end{array}$ & $\begin{array}{l}135 \\
133 \\
137 \\
144 \\
157\end{array}$ & $\begin{array}{l}16.71935 \\
16.94448 \\
17.15604 \\
17.35558 \\
17.54440\end{array}$ & $\begin{array}{l}22513 \\
21156 \\
19954 \\
18882 \\
17921\end{array}$ & $\begin{array}{l}20.20699 \\
20.43347 \\
20.64636 \\
20.84727 \\
21.03754\end{array}$ & $\begin{array}{l}22648 \\
21289 \\
20091 \\
19027 \\
18078\end{array}$ \\
\hline $\begin{array}{l}200 \\
210 \\
220 \\
230 \\
240\end{array}$ & $\begin{array}{l}3.52753 \\
3.53458 \\
3.54280 \\
3.55221 \\
3.56275\end{array}$ & $\begin{array}{r}705 \\
822 \\
941 \\
1054 \\
1162\end{array}$ & $\begin{array}{l}3.49470 \\
3.49643 \\
3.49834 \\
3.50048 \\
3.50285\end{array}$ & $\begin{array}{l}173 \\
191 \\
214 \\
237 \\
262\end{array}$ & $\begin{array}{l}17.72361 \\
17.89416 \\
18.05686 \\
18.21241 \\
18.36144\end{array}$ & $\begin{array}{l}17055 \\
16270 \\
15555 \\
14703 \\
14305\end{array}$ & $\begin{array}{l}21.21832 \\
21.39060 \\
21.55521 \\
21.71290 \\
21.86430\end{array}$ & $\begin{array}{l}17228 \\
16461 \\
15769 \\
15140 \\
14567\end{array}$ \\
\hline $\begin{array}{l}250 \\
260 \\
270 \\
280 \\
290\end{array}$ & $\begin{array}{l}3.57437 \\
3.58700 \\
3.60054 \\
3.61491 \\
3.63001\end{array}$ & $\begin{array}{l}1263 \\
1354 \\
1437 \\
1510 \\
1572\end{array}$ & $\begin{array}{l}3.50547 \\
3.50836 \\
3.51152 \\
3.51496 \\
3.51866\end{array}$ & $\begin{array}{l}289 \\
316 \\
344 \\
370 \\
397\end{array}$ & $\begin{array}{l}18.50449 \\
18.64203 \\
18.77450 \\
18.90226 \\
19.02567\end{array}$ & $\begin{array}{l}13754 \\
13247 \\
12776 \\
12341 \\
11936\end{array}$ & $\begin{array}{l}22.00997 \\
22.15040 \\
22.28603 \\
22.41723 \\
22.54434\end{array}$ & $\begin{array}{l}14043 \\
13563 \\
13120 \\
12711 \\
12333\end{array}$ \\
\hline $\begin{array}{l}300 \\
310 \\
320 \\
330 \\
340\end{array}$ & $\begin{array}{l}3.64573 \\
3.66197 \\
3.67865 \\
3.69567 \\
3.71295\end{array}$ & $\begin{array}{l}1624 \\
1668 \\
1702 \\
1728 \\
1745\end{array}$ & $\begin{array}{l}3.52263 \\
3.52687 \\
3.53135 \\
3.53607 \\
3.54102\end{array}$ & $\begin{array}{l}424 \\
448 \\
472 \\
495 \\
516\end{array}$ & $\begin{array}{l}19.14503 \\
19.26060 \\
19.37265 \\
19.48138 \\
19.58702\end{array}$ & $\begin{array}{l}11557 \\
11205 \\
10873 \\
10564 \\
10272\end{array}$ & $\begin{array}{l}22.66767 \\
22.78747 \\
22.90400 \\
23.01746 \\
23.12804\end{array}$ & $\begin{array}{l}11980 \\
11653 \\
11346 \\
11058 \\
10788\end{array}$ \\
\hline $\begin{array}{l}350 \\
360 \\
370 \\
380 \\
390\end{array}$ & $\begin{array}{l}3.73040 \\
3.74796 \\
3.76557 \\
3.78316 \\
3.80068\end{array}$ & $\begin{array}{l}1756 \\
1761 \\
1759 \\
1752 \\
1742\end{array}$ & $\begin{array}{l}3.54618 \\
3.55154 \\
3.55709 \\
3.56280 \\
3.56868\end{array}$ & $\begin{array}{l}536 \\
555 \\
571 \\
588 \\
602\end{array}$ & $\begin{array}{l}19.68974 \\
19.78971 \\
19.88710 \\
19.98203 \\
20.07465\end{array}$ & $\begin{array}{l}9997 \\
9739 \\
9493 \\
9262 \\
9043\end{array}$ & $\begin{array}{l}23.23592 \\
23.34125 \\
23.44419 \\
23.54484 \\
23.64334\end{array}$ & $\begin{array}{r}10533 \\
10294 \\
10065 \\
9850 \\
9644\end{array}$ \\
\hline $\begin{array}{l}400 \\
450 \\
500 \\
550 \\
600\end{array}$ & $\begin{array}{l}3.81810 \\
3.90229 \\
3.97974 \\
4.04935 \\
4.11114\end{array}$ & $\begin{array}{l}8419 \\
7745 \\
6961 \\
6179 \\
5458\end{array}$ & $\begin{array}{l}3.57470 \\
3.60647 \\
3.63999 \\
3.67410 \\
3.70800\end{array}$ & $\begin{array}{l}3177 \\
3352 \\
3411 \\
3390 \\
3315\end{array}$ & $\begin{array}{l}20.16508 \\
20.58793 \\
20.96964 \\
21.31816 \\
21.63931\end{array}$ & $\begin{array}{l}42285 \\
38171 \\
34852 \\
32115 \\
29811\end{array}$ & $\begin{array}{l}23.73978 \\
24.19440 \\
24.60963 \\
24.99227 \\
25.34731\end{array}$ & $\begin{array}{l}45462 \\
41523 \\
38264 \\
35504 \\
33127\end{array}$ \\
\hline $\begin{array}{l}650 \\
700 \\
750 \\
800 \\
850\end{array}$ & $\begin{array}{l}4.16572 \\
4.21388 \\
4.25644 \\
4.29419 \\
4.32782\end{array}$ & $\begin{array}{l}4816 \\
4256 \\
3775 \\
3363 \\
3011\end{array}$ & $\begin{array}{l}3.74115 \\
3.77323 \\
3.80406 \\
3.83353 \\
3.86164\end{array}$ & $\begin{array}{l}3208 \\
3083 \\
2947 \\
2811 \\
2675\end{array}$ & $\begin{array}{l}21.93742 \\
22.21585 \\
22.47724 \\
22.72369 \\
22.95695\end{array}$ & $\begin{array}{l}27843 \\
26139 \\
24645 \\
23326 \\
22149\end{array}$ & $\begin{array}{l}25.67858 \\
25.98909 \\
26.28130 \\
26.55723 \\
26.81860\end{array}$ & $\begin{array}{l}31051 \\
29221 \\
27593 \\
26137 \\
24824\end{array}$ \\
\hline $\begin{array}{r}900 \\
950 \\
1000 \\
1050 \\
1100\end{array}$ & $\begin{array}{l}4.35793 \\
4.38502 \\
4.40955 \\
4.43186 \\
4.45227\end{array}$ & $\begin{array}{l}2709 \\
2453 \\
2231 \\
2041 \\
1878\end{array}$ & $\begin{array}{l}3.88839 \\
3.91383 \\
3.93801 \\
3.96100 \\
3.98288\end{array}$ & $\begin{array}{l}2544 \\
2418 \\
2299 \\
2188 \\
2082\end{array}$ & $\begin{array}{l}23.17844 \\
23.38936 \\
23.59074 \\
23.78343 \\
23.96821\end{array}$ & $\begin{array}{l}21092 \\
20138 \\
19269 \\
18478 \\
17751\end{array}$ & $\begin{array}{l}27.06684 \\
27.30320 \\
27.52875 \\
27.74444 \\
27.95109\end{array}$ & $\begin{array}{l}23636 \\
22555 \\
21569 \\
20665 \\
19833\end{array}$ \\
\hline 15 & 4.47105 & 1734 & 4.00370 & 1984 & 24.14572 & 17082 & 28.14942 & 19066 \\
\hline
\end{tabular}


Table 2.016. NaH (Cont.)

\begin{tabular}{|c|c|c|c|c|c|c|c|c|}
\hline${ }^{\circ} \mathrm{K}$ & $\frac{C_{p}^{0}}{R}$ & & $\frac{\left(H^{0}-E_{0}^{0}\right)}{R T}$ & & $\frac{-l F^{\circ}-E}{R T}$ & & $\frac{S^{0}}{R}$ & \\
\hline $\begin{array}{l}1200 \\
1300 \\
1400 \\
1500 \\
1600\end{array}$ & $\begin{array}{l}4.48839 \\
4.51954 \\
4.54687 \\
4.57125 \\
4.59330\end{array}$ & $\begin{array}{l}3115 \\
2733 \\
2438 \\
2205 \\
2020\end{array}$ & $\begin{array}{l}4.02354 \\
4.06052 \\
4.09430 \\
4.12530 \\
4.15387\end{array}$ & $\begin{array}{l}3698 \\
3378 \\
3100 \\
2857 \\
2645\end{array}$ & $\begin{array}{l}24.31654 \\
24.64008 \\
24.94225 \\
25.22580 \\
25.49296\end{array}$ & $\begin{array}{l}32354 \\
30217 \\
28355 \\
26716 \\
25263\end{array}$ & $\begin{array}{l}28.34008 \\
28.70060 \\
29.03656 \\
29.35111 \\
29.64684\end{array}$ & $\begin{array}{l}36052 \\
33596 \\
31455 \\
29573 \\
27908\end{array}$ \\
\hline $\begin{array}{l}1700 \\
1800 \\
1900 \\
2000 \\
2100\end{array}$ & $\begin{array}{l}4.61350 \\
4.63219 \\
4.64966 \\
4.66612 \\
4.68173\end{array}$ & $\begin{array}{l}1869 \\
1747 \\
1646 \\
1561 \\
1490\end{array}$ & $\begin{array}{l}4.18032 \\
4.20492 \\
4.22787 \\
4.24937 \\
4.26959\end{array}$ & $\begin{array}{l}2460 \\
2295 \\
2150 \\
2022 \\
1908\end{array}$ & $\begin{array}{l}25.74559 \\
25.98524 \\
26.21321 \\
26.43062 \\
26.63845\end{array}$ & $\begin{array}{l}23965 \\
22797 \\
21741 \\
20783 \\
19906\end{array}$ & $\begin{array}{l}29.92592 \\
30.19016 \\
30.44108 \\
30.68000 \\
30.90804\end{array}$ & $\begin{array}{l}26424 \\
25092 \\
23892 \\
22804 \\
21814\end{array}$ \\
\hline $\begin{array}{l}2200 \\
2300 \\
2400 \\
2500 \\
2600\end{array}$ & $\begin{array}{l}4.69663 \\
4.71092 \\
4.72469 \\
4.73801 \\
4.75094\end{array}$ & $\begin{array}{l}1429 \\
1377 \\
1332 \\
1293 \\
1258\end{array}$ & $\begin{array}{l}4.28867 \\
4.30672 \\
4.32385 \\
4.34015 \\
4.35570\end{array}$ & $\begin{array}{l}1805 \\
1713 \\
1630 \\
1555 \\
1487\end{array}$ & $\begin{array}{l}26.83751 \\
27.02855 \\
27.21221 \\
27.38905 \\
27.55958\end{array}$ & $\begin{array}{l}19104 \\
18366 \\
17684 \\
17053 \\
16467\end{array}$ & $\begin{array}{l}31.12618 \\
31.33528 \\
31.53606 \\
31.72921 \\
31.91529\end{array}$ & $\begin{array}{l}20910 \\
20078 \\
19315 \\
18608 \\
17954\end{array}$ \\
\hline $\begin{array}{l}2700 \\
2800 \\
2900 \\
3000\end{array}$ & $\begin{array}{l}4.76352 \\
4.77579 \\
4.78779 \\
4.79954\end{array}$ & $\begin{array}{l}1227 \\
1200 \\
1175 \\
2286\end{array}$ & $\begin{array}{l}4.37057 \\
4.38483 \\
4.39852 \\
4.41169\end{array}$ & $\begin{array}{l}1426 \\
1369 \\
1317 \\
2496\end{array}$ & $\begin{array}{l}27.72425 \\
27.88345 \\
28.03756 \\
28.18690\end{array}$ & $\begin{array}{l}15920 \\
15411 \\
14934 \\
28554\end{array}$ & $\begin{array}{l}32.09483 \\
32.26829 \\
32.43609 \\
32.59860\end{array}$ & $\begin{array}{l}17346 \\
16780 \\
16251 \\
31049\end{array}$ \\
\hline $\begin{array}{l}3200 \\
3400 \\
3600 \\
3800 \\
4000\end{array}$ & $\begin{array}{l}4.82240 \\
4.84452 \\
4.86601 \\
4.88696 \\
4.90744\end{array}$ & $\begin{array}{l}2212 \\
2149 \\
2095 \\
2048 \\
2006\end{array}$ & $\begin{array}{l}4.43665 \\
4.45999 \\
4.48196 \\
4.50272 \\
4.52245\end{array}$ & $\begin{array}{l}2334 \\
2197 \\
2076 \\
1973 \\
1881\end{array}$ & $\begin{array}{l}28.47244 \\
28.74211 \\
28.99767 \\
29.24056 \\
29.47202\end{array}$ & $\begin{array}{l}26967 \\
25556 \\
24289 \\
23146 \\
22111\end{array}$ & $\begin{array}{l}32.90909 \\
33.20211 \\
33.47963 \\
33.74328 \\
33.99448\end{array}$ & $\begin{array}{l}29302 \\
27752 \\
26365 \\
25120 \\
23992\end{array}$ \\
\hline $\begin{array}{l}4200 \\
4400 \\
4600 \\
4800 \\
5000\end{array}$ & $\begin{array}{l}4.92750 \\
4.94717 \\
4.96649 \\
4.98549 \\
5.00419\end{array}$ & $\begin{array}{l}1967 \\
1932 \\
1900 \\
1870\end{array}$ & $\begin{array}{l}4.54126 \\
4.55927 \\
4.57655 \\
4.59320 \\
4.60926\end{array}$ & $\begin{array}{l}1801 \\
1728 \\
1665 \\
1606\end{array}$ & $\begin{array}{l}29.69313 \\
29.90481 \\
30.10786 \\
30.30299 \\
30.49082\end{array}$ & $\begin{array}{l}21168 \\
20305 \\
19513 \\
18783\end{array}$ & $\begin{array}{l}34.23440 \\
34.46408 \\
34.68442 \\
34.89619 \\
35.10009\end{array}$ & $\begin{array}{l}22968 \\
22034 \\
21177 \\
20390\end{array}$ \\
\hline
\end{tabular}


Table 2.017. NaD

\begin{tabular}{|l|l|l|l|l|}
\hline${ }^{\circ}$ & $\frac{C_{p}^{0}}{R}$ & $\frac{\left(H^{\circ}-E_{0}^{0}\right)}{R T}$ & $\frac{-\left(F^{\circ}-E_{0}^{0}\right)}{R T}$ & $\frac{S^{\circ}}{R}$ \\
\hline
\end{tabular}

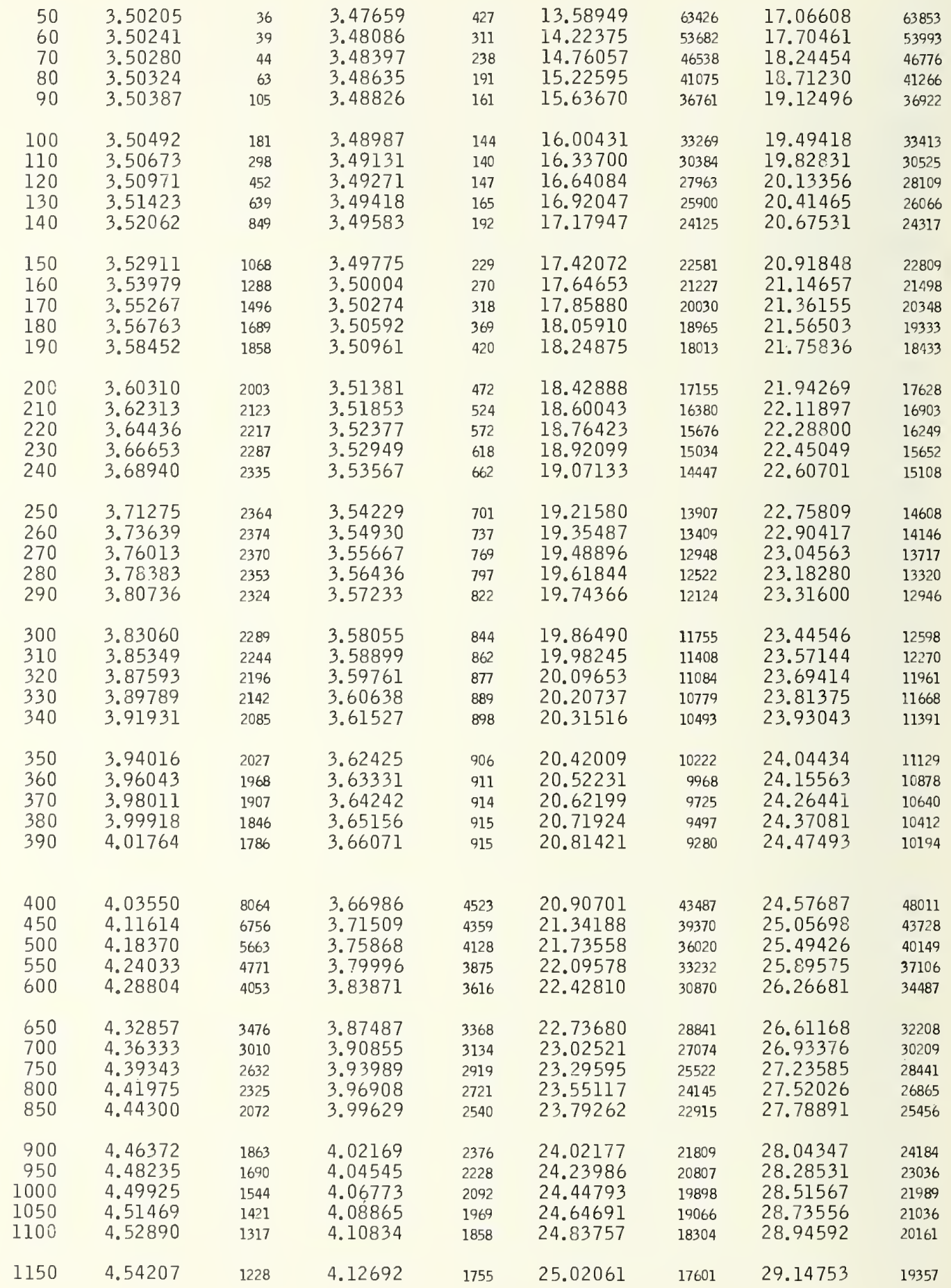


Table 2.017. NaD (Cont.)

\begin{tabular}{|l|l|l|l|l|}
\hline${ }^{\circ}$ & $\frac{C_{p}^{0}}{R}$ & $\frac{\left(H^{\circ}-E_{0}^{0}\right)}{R T}$ & $\frac{-\left(F^{\circ}-E_{0}^{0}\right)}{R T}$ & $\frac{S^{\circ}}{R}$ \\
\hline
\end{tabular}

\begin{tabular}{|c|c|c|c|c|c|c|c|c|}
\hline $\begin{array}{l}1200 \\
1300 \\
1400 \\
1500 \\
1600\end{array}$ & $\begin{array}{l}4.55435 \\
4.57672 \\
4.59678 \\
4.61508 \\
4.63200\end{array}$ & $\begin{array}{l}2237 \\
2006 \\
1830 \\
1692 \\
1584\end{array}$ & $\begin{array}{l}4.14447 \\
4.17688 \\
4.20617 \\
4.23283 \\
4.25725\end{array}$ & $\begin{array}{l}3241 \\
2929 \\
2666 \\
2442 \\
225 ?\end{array}$ & $\begin{array}{l}25.19662 \\
25.52966 \\
25.84029 \\
26.13141 \\
26.40538\end{array}$ & $\begin{array}{l}33304 \\
31063 \\
29112 \\
27397 \\
25878\end{array}$ & $\begin{array}{l}29.34110 \\
29.70655 \\
30.04647 \\
30.36424 \\
30.66264\end{array}$ & $\begin{array}{l}36545 \\
33992 \\
31777 \\
29840 \\
28130\end{array}$ \\
\hline $\begin{array}{l}1700 \\
1800 \\
1900 \\
2000 \\
2100\end{array}$ & $\begin{array}{l}4.64784 \\
4.66280 \\
4.67705 \\
4.69071 \\
4.70387\end{array}$ & $\begin{array}{l}1496 \\
1425 \\
1366 \\
1316 \\
1274\end{array}$ & $\begin{array}{l}4.27977 \\
4.30064 \\
4.32008 \\
4.33827 \\
4.35537\end{array}$ & $\begin{array}{l}2087 \\
1944 \\
1819 \\
1710 \\
1613\end{array}$ & $\begin{array}{l}26.66416 \\
26.90938 \\
27.14244 \\
27.36449 \\
27.57658\end{array}$ & $\begin{array}{l}24522 \\
23306 \\
22205 \\
21209 \\
20298\end{array}$ & $\begin{array}{l}30.94394 \\
31.21003 \\
31.46252 \\
31.70277 \\
31.93195\end{array}$ & $\begin{array}{l}26609 \\
25249 \\
24025 \\
22918 \\
21912\end{array}$ \\
\hline $\begin{array}{l}2200 \\
2300 \\
2400 \\
2500 \\
2600\end{array}$ & $\begin{array}{l}4.71661 \\
4.72900 \\
4.74107 \\
4.75287 \\
4.76444\end{array}$ & $\begin{array}{l}1239 \\
1207 \\
1180 \\
1157 \\
1135\end{array}$ & $\begin{array}{l}4.37150 \\
4.38677 \\
4.40129 \\
4.41511 \\
4.42833\end{array}$ & $\begin{array}{l}1527 \\
1452 \\
1382 \\
1322 \\
1266\end{array}$ & $\begin{array}{l}27.77956 \\
27.97423 \\
28.16123 \\
28.34119 \\
28.51461\end{array}$ & $\begin{array}{l}19467 \\
18700 \\
17996 \\
17342 \\
16737\end{array}$ & $\begin{array}{l}32.15107 \\
32.36100 \\
32.56252 \\
32.75630 \\
32.94294\end{array}$ & $\begin{array}{l}20993 \\
20152 \\
19378 \\
18664 \\
18003\end{array}$ \\
\hline $\begin{array}{l}2700 \\
2800 \\
2900 \\
3000\end{array}$ & $\begin{array}{l}4.77579 \\
4.78695 \\
4.79794 \\
4.80877\end{array}$ & $\begin{array}{l}1116 \\
1099 \\
1083 \\
2124\end{array}$ & $\begin{array}{l}4.44099 \\
4.45314 \\
4.46484 \\
4.47613\end{array}$ & $\begin{array}{l}1215 \\
1170 \\
1129 \\
2146\end{array}$ & $\begin{array}{l}28.68198 \\
28.84371 \\
29.00018 \\
29.15173\end{array}$ & $\begin{array}{l}16173 \\
15647 \\
15155 \\
28958\end{array}$ & $\begin{array}{l}33.12297 \\
33.29685 \\
33.46503 \\
33.62787\end{array}$ & $\begin{array}{l}17388 \\
16818 \\
16284 \\
31103\end{array}$ \\
\hline $\begin{array}{l}3200 \\
3400 \\
3600 \\
3800 \\
4000\end{array}$ & $\begin{array}{l}4.83001 \\
4.85076 \\
4.87108 \\
4.89102 \\
4.91060\end{array}$ & $\begin{array}{l}2075 \\
2032 \\
1994 \\
1958 \\
1927\end{array}$ & $\begin{array}{l}4.49759 \\
4.51775 \\
4.53682 \\
4.55494 \\
4.57223\end{array}$ & $\begin{array}{l}2016 \\
1907 \\
1812 \\
1729 \\
1658\end{array}$ & $\begin{array}{l}29.44131 \\
29.71459 \\
29.97336 \\
30.21914 \\
30.45322\end{array}$ & $\begin{array}{l}27328 \\
25877 \\
24578 \\
23408 \\
22349\end{array}$ & $\begin{array}{l}33.93890 \\
34.23234 \\
34.51018 \\
34.77408 \\
35.02546\end{array}$ & $\begin{array}{l}29344 \\
2784 \\
26390 \\
25138 \\
24006\end{array}$ \\
\hline $\begin{array}{l}4200 \\
4400 \\
4600 \\
4800 \\
5000\end{array}$ & $\begin{array}{l}4.92987 \\
4.94885 \\
4.96755 \\
4.98599 \\
5.00418\end{array}$ & $\begin{array}{l}1898 \\
1870 \\
1844 \\
1819\end{array}$ & $\begin{array}{l}4.58881 \\
4.60474 \\
4.62011 \\
4.63497 \\
4.64938\end{array}$ & $\begin{array}{l}1593 \\
1537 \\
1486 \\
1441\end{array}$ & $\begin{array}{l}30.67671 \\
30.89055 \\
31.09558 \\
31.29252 \\
31.48203\end{array}$ & $\begin{array}{l}21384 \\
20503 \\
19694 \\
18951\end{array}$ & $\begin{array}{l}35.26552 \\
35.49529 \\
35.71569 \\
35.92750 \\
36.13141\end{array}$ & $\begin{array}{l}22977 \\
22040 \\
21181 \\
20391\end{array}$ \\
\hline
\end{tabular}


Table 2.018. NaT

\begin{tabular}{|l|l|l|l|l|}
\hline${ }^{\circ} K$ & $\frac{C_{p}^{0}}{R}$ & $\frac{\left(H^{0}-E_{0}^{0}\right)}{R T}$ & $\frac{-\left(F^{0}-E_{0}^{0}\right)}{R T}$ & $\frac{S^{0}}{R}$ \\
\hline
\end{tabular}

\begin{tabular}{|c|c|c|c|c|c|c|c|c|}
\hline $\begin{array}{l}50 \\
60 \\
70 \\
80 \\
90\end{array}$ & $\begin{array}{l}3.50201 \\
3.50240 \\
3.50290 \\
3.50377 \\
3.50550\end{array}$ & $\begin{array}{r}39 \\
50 \\
87 \\
173 \\
324\end{array}$ & $\begin{array}{l}3.48402 \\
3.48705 \\
3.48928 \\
3.49103 \\
3.49253\end{array}$ & $\begin{array}{l}303 \\
223 \\
175 \\
150 \\
145\end{array}$ & $\begin{array}{l}14.00348 \\
14.63897 \\
15.17668 \\
15.64273 \\
16.05400\end{array}$ & $\begin{array}{l}63549 \\
53771 \\
46605 \\
41127 \\
36805\end{array}$ & $\begin{array}{l}17.48750 \\
18.12603 \\
18.66596 \\
19.13376 \\
19.54654\end{array}$ & $\begin{array}{l}63853 \\
53993 \\
46780 \\
41278 \\
36949\end{array}$ \\
\hline $\begin{array}{l}100 \\
110 \\
120 \\
130 \\
140\end{array}$ & $\begin{array}{l}3.50874 \\
3.51415 \\
3.52228 \\
3.53344 \\
3.54774\end{array}$ & $\begin{array}{r}541 \\
813 \\
1116 \\
1430 \\
1732\end{array}$ & $\begin{array}{l}3.49398 \\
3.49554 \\
3.49741 \\
3.49974 \\
3.50264\end{array}$ & $\begin{array}{l}156 \\
187 \\
233 \\
290 \\
356\end{array}$ & $\begin{array}{l}16.42205 \\
16.75513 \\
17.05936 \\
17.33939 \\
17.59886\end{array}$ & $\begin{array}{l}33308 \\
30423 \\
28003 \\
25947 \\
24177\end{array}$ & $\begin{array}{l}19.91603 \\
20.25068 \\
20.55678 \\
20.83913 \\
21.10150\end{array}$ & $\begin{array}{l}33465 \\
30610 \\
28235 \\
26237 \\
24534\end{array}$ \\
\hline $\begin{array}{l}150 \\
160 \\
170 \\
180 \\
190\end{array}$ & $\begin{array}{l}3.56506 \\
3.58512 \\
3.60755 \\
3.63194 \\
3.65783\end{array}$ & $\begin{array}{l}2006 \\
2243 \\
2439 \\
2589 \\
2698\end{array}$ & $\begin{array}{l}3.50620 \\
3.51050 \\
3.51553 \\
3.52132 \\
3.52781\end{array}$ & $\begin{array}{l}430 \\
503 \\
579 \\
649 \\
718\end{array}$ & $\begin{array}{l}17.84063 \\
18.06705 \\
18.28002 \\
18.48112 \\
18.67168\end{array}$ & $\begin{array}{l}22642 \\
21297 \\
20110 \\
19056 \\
18113\end{array}$ & $\begin{array}{l}21.34684 \\
21.57755 \\
21.79556 \\
22.00244 \\
22.19950\end{array}$ & $\begin{array}{l}23071 \\
21801 \\
20688 \\
19706 \\
18830\end{array}$ \\
\hline $\begin{array}{l}200 \\
210 \\
220 \\
230 \\
240\end{array}$ & $\begin{array}{l}3.68481 \\
3.71251 \\
3.74058 \\
3.76872 \\
3.79671\end{array}$ & $\begin{array}{l}2770 \\
2807 \\
2814 \\
2799 \\
2763\end{array}$ & $\begin{array}{l}3.53499 \\
3.54278 \\
3.55113 \\
3.55998 \\
3.56926\end{array}$ & $\begin{array}{l}779 \\
835 \\
885 \\
928 \\
965\end{array}$ & $\begin{array}{l}18.85281 \\
19.02547 \\
19.19047 \\
19.34852 \\
19.50023\end{array}$ & $\begin{array}{l}17266 \\
16500 \\
15805 \\
15171 \\
14590\end{array}$ & $\begin{array}{l}22.38780 \\
22.56825 \\
22.74161 \\
22.90850 \\
23.06949\end{array}$ & $\begin{array}{l}18045 \\
17336 \\
16689 \\
16099 \\
15555\end{array}$ \\
\hline $\begin{array}{l}250 \\
260 \\
270 \\
280 \\
290\end{array}$ & $\begin{array}{l}3.82434 \\
3.85145 \\
3.87794 \\
3.90370 \\
3.92867\end{array}$ & $\begin{array}{l}2711 \\
2649 \\
2576 \\
2497 \\
2414\end{array}$ & $\begin{array}{l}3.57891 \\
3.58887 \\
3.59909 \\
3.60951 \\
3.62009\end{array}$ & $\begin{array}{r}996 \\
1022 \\
1042 \\
1058 \\
1069\end{array}$ & $\begin{array}{l}19.64613 \\
19.78669 \\
19.92232 \\
20.05340 \\
20.18025\end{array}$ & $\begin{array}{l}14056 \\
13563 \\
13108 \\
12685 \\
12291\end{array}$ & $\begin{array}{l}23.22504 \\
23.37557 \\
23.52142 \\
23.66292 \\
23.80034\end{array}$ & $\begin{array}{l}15053 \\
14585 \\
14150 \\
13742 \\
13360\end{array}$ \\
\hline $\begin{array}{l}300 \\
310 \\
320 \\
330 \\
340\end{array}$ & $\begin{array}{l}3.95281 \\
3.97610 \\
3.99853 \\
4.02009 \\
4.04079\end{array}$ & $\begin{array}{l}2329 \\
2243 \\
2156 \\
2070 \\
1987\end{array}$ & $\begin{array}{l}3.63078 \\
3.64155 \\
3.65235 \\
3.66317 \\
3.67398\end{array}$ & $\begin{array}{l}1077 \\
1080 \\
1082 \\
1081 \\
1076\end{array}$ & $\begin{array}{l}20.30316 \\
20.42238 \\
20.53817 \\
20.65072 \\
20.76024\end{array}$ & $\begin{array}{l}11922 \\
11579 \\
11255 \\
10952 \\
10666\end{array}$ & $\begin{array}{l}23.93394 \\
24.06394 \\
24.19053 \\
24.31390 \\
24.43422\end{array}$ & $\begin{array}{l}13000 \\
12659 \\
12337 \\
12032 \\
11742\end{array}$ \\
\hline $\begin{array}{l}350 \\
360 \\
370 \\
380 \\
390\end{array}$ & $\begin{array}{l}4.06066 \\
4.07970 \\
4.09795 \\
4.11543 \\
4.13218\end{array}$ & $\begin{array}{l}1904 \\
1825 \\
1748 \\
1675 \\
1603\end{array}$ & $\begin{array}{l}3.68474 \\
3.69545 \\
3.70609 \\
3.71663 \\
3.72707\end{array}$ & $\begin{array}{l}1071 \\
1064 \\
1054 \\
1044 \\
1033\end{array}$ & $\begin{array}{l}20.86690 \\
20.97085 \\
21.07225 \\
21.17122 \\
21.26790\end{array}$ & $\begin{array}{r}10395 \\
10140 \\
9897 \\
9668 \\
9447\end{array}$ & $\begin{array}{l}24.55164 \\
24.66631 \\
24.77834 \\
24.88786 \\
24.99497\end{array}$ & $\begin{array}{l}11467 \\
11203 \\
10952 \\
10711 \\
10483\end{array}$ \\
\hline $\begin{array}{l}400 \\
450 \\
500 \\
550 \\
600\end{array}$ & $\begin{array}{l}4.14821 \\
4.21879 \\
4.27599 \\
4.32282 \\
4.36167\end{array}$ & $\begin{array}{l}7058 \\
5720 \\
4683 \\
3885 \\
3268\end{array}$ & $\begin{array}{l}3.73740 \\
3.78711 \\
3.83323 \\
3.87568 \\
3.91461\end{array}$ & $\begin{array}{l}4971 \\
4612 \\
4245 \\
3893 \\
3568\end{array}$ & $\begin{array}{l}21.36239 \\
21.80550 \\
22.20693 \\
22.57429 \\
22.91322\end{array}$ & $\begin{array}{r}44311 \\
40143 \\
36736 \\
33893 \\
31476\end{array}$ & $\begin{array}{l}25.09980 \\
25.59261 \\
26.04017 \\
26.44998 \\
26.82783\end{array}$ & $\begin{array}{l}49281 \\
44756 \\
40981 \\
37785 \\
35045\end{array}$ \\
\hline $\begin{array}{l}650 \\
700 \\
750 \\
800 \\
850\end{array}$ & $\begin{array}{l}4.39435 \\
4.42221 \\
4.44629 \\
4.46736 \\
4.48602\end{array}$ & $\begin{array}{l}2786 \\
2408 \\
2107 \\
1866 \\
1670\end{array}$ & $\begin{array}{l}3.95029 \\
3.98303 \\
4.01313 \\
4.04088 \\
4.06652\end{array}$ & $\begin{array}{l}3274 \\
3010 \\
2775 \\
2564 \\
2378\end{array}$ & $\begin{array}{l}23.22798 \\
23.52195 \\
23.79779 \\
24.05769 \\
24.30344\end{array}$ & $\begin{array}{l}29397 \\
27584 \\
25990 \\
24575 \\
23312\end{array}$ & $\begin{array}{l}27.17828 \\
27.50498 \\
27.81092 \\
28.09857 \\
28.36997\end{array}$ & $\begin{array}{l}32670 \\
30594 \\
28765 \\
27140 \\
25689\end{array}$ \\
\hline $\begin{array}{r}900 \\
950 \\
1000 \\
1050 \\
1100\end{array}$ & $\begin{array}{l}4.50272 \\
4.51782 \\
4.53160 \\
4.54428 \\
4.55605\end{array}$ & $\begin{array}{l}1510 \\
1378 \\
1268 \\
1177 \\
1099\end{array}$ & $\begin{array}{l}4.09030 \\
4.11241 \\
4.13303 \\
4.15231 \\
4.17040\end{array}$ & $\begin{array}{l}2211 \\
2062 \\
1928 \\
1809 \\
1701\end{array}$ & $\begin{array}{l}24.53656 \\
24.75831 \\
24.96978 \\
25.17190 \\
25.36549\end{array}$ & $\begin{array}{l}22175 \\
21147 \\
20212 \\
19359 \\
18576\end{array}$ & $\begin{array}{l}28.62686 \\
28.87073 \\
29.10282 \\
29.32422 \\
29.53590\end{array}$ & $\begin{array}{l}24387 \\
23209 \\
22140 \\
21168 \\
20277\end{array}$ \\
\hline 1150 & 4.56704 & 1033 & 4.18741 & 1604 & 25.55125 & 17856 & 29.73867 & 19459 \\
\hline
\end{tabular}


Table 2.018. NaT (Cont.)

\begin{tabular}{|l|l|l|l|l|}
\hline$K$ & $\frac{C_{p}^{0}}{R}$ & $\frac{\left(H^{0}-E_{0}^{0}\right)}{R T}$ & $\frac{-\left(F^{0}-E_{0}^{0}\right)}{R T}$ & $\frac{S^{0}}{R}$ \\
\hline
\end{tabular}

\begin{tabular}{|c|c|c|c|c|c|c|c|c|}
\hline $\begin{array}{l}1200 \\
1300 \\
1400 \\
1500 \\
1600\end{array}$ & $\begin{array}{l}4.57737 \\
4.59641 \\
4.61375 \\
4.62981 \\
4.64485\end{array}$ & $\begin{array}{l}1904 \\
1734 \\
1606 \\
1504 \\
1425\end{array}$ & $\begin{array}{l}4.20345 \\
4.23295 \\
4.25954 \\
4.28370 \\
4.30581\end{array}$ & $\begin{array}{l}2950 \\
2659 \\
2416 \\
2211 \\
2036\end{array}$ & $\begin{array}{l}25.72981 \\
26.06745 \\
26.38214 \\
26.67685 \\
26.95403\end{array}$ & $\begin{array}{l}33764 \\
31469 \\
29471 \\
27718 \\
26166\end{array}$ & $\begin{array}{l}29.93326 \\
30.30041 \\
30.64169 \\
30.96056 \\
31.25984\end{array}$ & $\begin{array}{l}36715 \\
34128 \\
31887 \\
29928 \\
28203\end{array}$ \\
\hline $\begin{array}{l}1700 \\
1800 \\
1900 \\
2000 \\
2100\end{array}$ & $\begin{array}{l}4.65910 \\
4.67271 \\
4.68579 \\
4.69844 \\
4.71072\end{array}$ & $\begin{array}{l}1361 \\
1308 \\
1265 \\
1228 \\
1197\end{array}$ & $\begin{array}{l}4.32617 \\
4.34505 \\
4.36264 \\
4.37912 \\
4.39462\end{array}$ & $\begin{array}{l}1888 \\
1759 \\
1648 \\
1550 \\
1464\end{array}$ & $\begin{array}{l}27.21569 \\
27.46351 \\
27.69891 \\
27.92311 \\
28.13714\end{array}$ & $\begin{array}{l}24782 \\
23540 \\
22420 \\
21403 \\
20478\end{array}$ & $\begin{array}{l}31.54187 \\
31.80856 \\
32.06155 \\
32.30223 \\
32.53176\end{array}$ & $\begin{array}{l}26669 \\
25299 \\
24068 \\
22953 \\
21943\end{array}$ \\
\hline $\begin{array}{l}2200 \\
2300 \\
2400 \\
2500 \\
2600\end{array}$ & $\begin{array}{l}4.72269 \\
4.73439 \\
4.74585 \\
4.75711 \\
4.76818\end{array}$ & $\begin{array}{l}1170 \\
1146 \\
1126 \\
1107 \\
1090\end{array}$ & $\begin{array}{l}4.40926 \\
4.42314 \\
4.43635 \\
4.44895 \\
4.46102\end{array}$ & $\begin{array}{l}1388 \\
1321 \\
1260 \\
1207 \\
1158\end{array}$ & $\begin{array}{l}28.34192 \\
28.53823 \\
28.72676 \\
28.90812 \\
29.08285\end{array}$ & $\begin{array}{l}19631 \\
18853 \\
18136 \\
17473 \\
16858\end{array}$ & $\begin{array}{l}32.75119 \\
32.96138 \\
33.16311 \\
33.35708 \\
33.54387\end{array}$ & $\begin{array}{l}21019 \\
20173 \\
19397 \\
18679 \\
18016\end{array}$ \\
\hline $\begin{array}{l}2700 \\
2800 \\
2900 \\
3000\end{array}$ & $\begin{array}{l}4.77908 \\
4.78984 \\
4.80046 \\
4.81095\end{array}$ & $\begin{array}{l}1076 \\
1062 \\
1049 \\
2064\end{array}$ & $\begin{array}{l}4.47260 \\
4.48374 \\
4.49448 \\
4.50485\end{array}$ & $\begin{array}{l}1114 \\
1074 \\
1037 \\
1978\end{array}$ & $\begin{array}{l}29.25143 \\
29.41429 \\
29.57181 \\
29.72436\end{array}$ & $\begin{array}{l}16286 \\
15752 \\
15255 \\
29138\end{array}$ & $\begin{array}{l}33.72403 \\
33.89803 \\
34.06630 \\
34.22922\end{array}$ & $\begin{array}{l}17400 \\
16827 \\
16292 \\
31115\end{array}$ \\
\hline $\begin{array}{l}3200 \\
3400 \\
3600 \\
3800 \\
4000\end{array}$ & $\begin{array}{l}4.83159 \\
4.85183 \\
4.87170 \\
4.89124 \\
4.91048\end{array}$ & $\begin{array}{l}2024 \\
1987 \\
1954 \\
1924 \\
1897\end{array}$ & $\begin{array}{l}4.52463 \\
4.54328 \\
4.56098 \\
4.57785 \\
4.59400\end{array}$ & $\begin{array}{l}1865 \\
1770 \\
1687 \\
1615 \\
1552\end{array}$ & $\begin{array}{l}30.01574 \\
30.29060 \\
30.55080 \\
30.79785 \\
31.03308\end{array}$ & $\begin{array}{l}27486 \\
26020 \\
24705 \\
23523 \\
22452\end{array}$ & $\begin{array}{l}34.54037 \\
34.83389 \\
35.11178 \\
35.37570 \\
35.62708\end{array}$ & $\begin{array}{l}29352 \\
27789 \\
26392 \\
25138 \\
24004\end{array}$ \\
\hline $\begin{array}{l}4200 \\
4400 \\
4600 \\
4800 \\
5000\end{array}$ & $\begin{array}{l}4.92945 \\
4.94814 \\
4.96659 \\
4.98480 \\
5.00278\end{array}$ & $\begin{array}{l}1869 \\
1845 \\
1821 \\
1798\end{array}$ & $\begin{array}{l}4.60952 \\
4.62449 \\
4.63896 \\
4.65299 \\
4.66663\end{array}$ & $\begin{array}{l}1497 \\
1447 \\
1403 \\
1364\end{array}$ & $\begin{array}{l}31.25760 \\
31.47238 \\
31.67827 \\
31.87600 \\
32.06622\end{array}$ & $\begin{array}{l}21478 \\
20589 \\
19773 \\
19022\end{array}$ & $\begin{array}{l}35.86712 \\
36.09688 \\
36.31724 \\
36.52900 \\
36.73285\end{array}$ & $\begin{array}{l}22976 \\
22036 \\
21176 \\
20385\end{array}$ \\
\hline
\end{tabular}


Table 2.019. KH

\begin{tabular}{|c|c|c|c|c|c|c|c|c|}
\hline${ }^{\circ} \mathrm{K}$ & $\frac{C_{p}^{0}}{R}$ & & $\frac{\left(H^{0}-E_{0}^{0}\right)}{R T}$ & & $\frac{-\left(F^{0}-E\right.}{R T}$ & & $\frac{S^{0}}{R}$ & \\
\hline $\begin{array}{l}50 \\
60 \\
70 \\
80 \\
90\end{array}$ & $\begin{array}{l}3.50182 \\
3.50207 \\
3.50234 \\
3.50265 \\
3.50299\end{array}$ & $\begin{array}{l}25 \\
27 \\
31 \\
34 \\
46\end{array}$ & $\begin{array}{l}3.46823 \\
3.47385 \\
3.47790 \\
3.48097 \\
3.48340\end{array}$ & $\begin{array}{l}562 \\
405 \\
307 \\
243 \\
198\end{array}$ & $\begin{array}{l}14.01883 \\
14.65169 \\
15.18751 \\
15.65212 \\
16.06227\end{array}$ & $\begin{array}{l}63286 \\
53582 \\
46461 \\
41015 \\
36712\end{array}$ & $\begin{array}{l}17.48706 \\
18.12554 \\
18.66541 \\
19.13310 \\
19.54567\end{array}$ & $\begin{array}{l}63848 \\
53987 \\
46769 \\
41257 \\
36910\end{array}$ \\
\hline $\begin{array}{l}100 \\
110 \\
120 \\
130 \\
140\end{array}$ & $\begin{array}{l}3.50345 \\
3.50414 \\
3.50528 \\
3.50709 \\
3.50984\end{array}$ & $\begin{array}{r}69 \\
114 \\
181 \\
275 \\
393\end{array}$ & $\begin{array}{l}3.48538 \\
3.48705 \\
3.48852 \\
3.48987 \\
3.49120\end{array}$ & $\begin{array}{l}167 \\
147 \\
135 \\
133 \\
136\end{array}$ & $\begin{array}{l}16.42939 \\
16.76166 \\
17.06514 \\
17.34442 \\
17.60310\end{array}$ & $\begin{array}{l}33227 \\
30348 \\
27928 \\
25868 \\
24091\end{array}$ & $\begin{array}{l}19.91477 \\
20.24872 \\
20.55366 \\
20.83430 \\
21.09430\end{array}$ & $\begin{array}{l}33395 \\
30494 \\
28064 \\
26000 \\
24228\end{array}$ \\
\hline $\begin{array}{l}150 \\
160 \\
170 \\
180 \\
190\end{array}$ & $\begin{array}{l}3.51377 \\
3.51910 \\
3.52597 \\
3.53447 \\
3.54461\end{array}$ & $\begin{array}{r}533 \\
687 \\
850 \\
1014 \\
1177\end{array}$ & $\begin{array}{l}3.49256 \\
3.49405 \\
3.49571 \\
3.49762 \\
3.49982\end{array}$ & $\begin{array}{l}149 \\
166 \\
191 \\
220 \\
253\end{array}$ & $\begin{array}{l}17.84401 \\
18.06947 \\
18.28134 \\
18.48120 \\
18.67037\end{array}$ & $\begin{array}{l}22546 \\
21187 \\
19986 \\
18917 \\
17958\end{array}$ & $\begin{array}{l}21.33658 \\
21.56352 \\
21.77706 \\
21.97883 \\
22.17019\end{array}$ & $\begin{array}{l}22694 \\
21354 \\
20177 \\
19136 \\
18211\end{array}$ \\
\hline $\begin{array}{l}200 \\
210 \\
220 \\
230 \\
240\end{array}$ & $\begin{array}{l}3.55638 \\
3.56967 \\
3.58438 \\
3.60036 \\
3.61744\end{array}$ & $\begin{array}{l}1329 \\
1471 \\
1598 \\
1708 \\
1803\end{array}$ & $\begin{array}{l}3.50235 \\
3.50523 \\
3.50849 \\
3.51213 \\
3.51616\end{array}$ & $\begin{array}{l}288 \\
326 \\
364 \\
403 \\
441\end{array}$ & $\begin{array}{l}18.84995 \\
19.02090 \\
19.18403 \\
19.34007 \\
19.48963\end{array}$ & $\begin{array}{l}17095 \\
16313 \\
15604 \\
14956 \\
14363\end{array}$ & $\begin{array}{l}22.35230 \\
22.52613 \\
22.69253 \\
22.85221 \\
23.00580\end{array}$ & $\begin{array}{l}17383 \\
16640 \\
15968 \\
15359 \\
14803\end{array}$ \\
\hline $\begin{array}{l}250 \\
260 \\
270 \\
280 \\
290\end{array}$ & $\begin{array}{l}3.63547 \\
3.65428 \\
3.67371 \\
3.69361 \\
3.71385\end{array}$ & $\begin{array}{l}1881 \\
1943 \\
1990 \\
2024 \\
2043\end{array}$ & $\begin{array}{l}3.52057 \\
3.52535 \\
3.53048 \\
3.53595 \\
3.54174\end{array}$ & $\begin{array}{l}478 \\
513 \\
547 \\
579 \\
607\end{array}$ & $\begin{array}{l}19.63326 \\
19.77143 \\
19.90457 \\
20.03306 \\
20.15725\end{array}$ & $\begin{array}{l}13817 \\
13314 \\
12849 \\
12419 \\
12017\end{array}$ & $\begin{array}{l}23.15383 \\
23.29678 \\
23.43506 \\
23.56902 \\
23.69899\end{array}$ & $\begin{array}{l}14295 \\
13828 \\
13396 \\
12997 \\
12625\end{array}$ \\
\hline $\begin{array}{l}300 \\
310 \\
320 \\
330 \\
340\end{array}$ & $\begin{array}{l}3.73428 \\
3.75481 \\
3.77534 \\
3.79577 \\
3.81603\end{array}$ & $\begin{array}{l}2053 \\
2053 \\
2043 \\
2026 \\
2004\end{array}$ & $\begin{array}{l}3.54781 \\
3.55416 \\
3.56075 \\
3.56756 \\
3.57457\end{array}$ & $\begin{array}{l}635 \\
659 \\
681 \\
701 \\
719\end{array}$ & $\begin{array}{l}20.27742 \\
20.39385 \\
20.50680 \\
20.61647 \\
20.72308\end{array}$ & $\begin{array}{l}11643 \\
11295 \\
10967 \\
10661 \\
10372\end{array}$ & $\begin{array}{l}23.82524 \\
23.94802 \\
24.06755 \\
24.18404 \\
24.29766\end{array}$ & $\begin{array}{l}12278 \\
11953 \\
11649 \\
11362 \\
11090\end{array}$ \\
\hline $\begin{array}{l}350 \\
360 \\
370 \\
380 \\
390\end{array}$ & $\begin{array}{l}3.83607 \\
3.85581 \\
3.87524 \\
3.89429 \\
3.91296\end{array}$ & $\begin{array}{l}1974 \\
1943 \\
1905 \\
1867 \\
1826\end{array}$ & $\begin{array}{l}3.58176 \\
3.58910 \\
3.59657 \\
3.60416 \\
3.61184\end{array}$ & $\begin{array}{l}734 \\
747 \\
759 \\
768 \\
775\end{array}$ & $\begin{array}{l}20.82680 \\
20.92780 \\
21.02624 \\
21.12226 \\
21.21598\end{array}$ & $\begin{array}{r}10100 \\
9844 \\
9602 \\
9372 \\
9154\end{array}$ & $\begin{array}{l}24.40856 \\
24.51691 \\
24.62282 \\
24.72642 \\
24.82782\end{array}$ & $\begin{array}{r}10835 \\
10591 \\
10360 \\
10140 \\
9929\end{array}$ \\
\hline $\begin{array}{l}400 \\
450 \\
500 \\
550 \\
600\end{array}$ & $\begin{array}{l}3.93122 \\
4.01592 \\
4.08953 \\
4.15289 \\
4.20729\end{array}$ & $\begin{array}{l}8470 \\
7361 \\
6336 \\
5440 \\
4682\end{array}$ & $\begin{array}{l}3.61959 \\
3.65903 \\
3.69849 \\
3.73699 \\
3.77397\end{array}$ & $\begin{array}{l}3944 \\
3946 \\
3850 \\
3698 \\
3518\end{array}$ & $\begin{array}{l}21.30752 \\
21.73611 \\
22.12368 \\
22.47800 \\
22.80476\end{array}$ & $\begin{array}{l}42859 \\
38757 \\
35432 \\
32676 \\
30348\end{array}$ & $\begin{array}{l}24.92711 \\
25.39515 \\
25.82217 \\
26.21499 \\
26.57873\end{array}$ & $\begin{array}{l}46804 \\
42702 \\
39282 \\
36374 \\
33866\end{array}$ \\
\hline $\begin{array}{l}650 \\
700 \\
750 \\
800 \\
850\end{array}$ & $\begin{array}{l}4.25411 \\
4.29461 \\
4.32986 \\
4.36076 \\
4.38804\end{array}$ & $\begin{array}{l}4050 \\
3525 \\
3090 \\
2728 \\
2427\end{array}$ & $\begin{array}{l}3.80915 \\
3.84241 \\
3.87376 \\
3.90325 \\
3.93098\end{array}$ & $\begin{array}{l}3326 \\
3135 \\
2949 \\
2773 \\
2608\end{array}$ & $\begin{array}{l}23.10824 \\
23.39176 \\
23.65794 \\
23.90889 \\
24.14637\end{array}$ & $\begin{array}{l}28352 \\
26618 \\
25095 \\
23748 \\
22543\end{array}$ & $\begin{array}{l}26.91739 \\
27.23417 \\
27.53170 \\
27.81215 \\
28.07736\end{array}$ & $\begin{array}{l}31678 \\
29753 \\
28045 \\
26521 \\
25151\end{array}$ \\
\hline $\begin{array}{r}900 \\
950 \\
1000 \\
1050 \\
1100\end{array}$ & $\begin{array}{l}4.41231 \\
4.43406 \\
4.45368 \\
4.47152 \\
4.48783\end{array}$ & $\begin{array}{l}2175 \\
1962 \\
1784 \\
1631 \\
1501\end{array}$ & $\begin{array}{l}3.95706 \\
3.98160 \\
4.00473 \\
4.02654 \\
4.04714\end{array}$ & $\begin{array}{l}2454 \\
2313 \\
2181 \\
2060 \\
1949\end{array}$ & $\begin{array}{l}24.37180 \\
24.58641 \\
24.79124 \\
24.98716 \\
25.17496\end{array}$ & $\begin{array}{l}21461 \\
20483 \\
19592 \\
18780 \\
18033\end{array}$ & $\begin{array}{l}28.32887 \\
28.56802 \\
28.79597 \\
29.01370 \\
29.22210\end{array}$ & $\begin{array}{l}23915 \\
22795 \\
21773 \\
20840 \\
19983\end{array}$ \\
\hline 1150 & 4.50284 & 1389 & 4.06663 & 1847 & 25.35529 & 17347 & 29.42193 & 19193 \\
\hline
\end{tabular}


Table 2.019. KH (Cont.)

\begin{tabular}{|c|c|c|c|c|c|c|c|c|}
\hline${ }^{\circ} \mathrm{K}$ & $\frac{C_{p}^{0}}{R}$ & & $\frac{\left(H^{0}-E_{0}^{0}\right)}{R T}$ & & $\frac{-\left(F^{0}-E\right.}{R T}$ & & $\frac{S^{0}}{R}$ & \\
\hline $\begin{array}{l}1200 \\
1300 \\
1400 \\
1500 \\
1600\end{array}$ & $\begin{array}{l}4.51673 \\
4.54174 \\
4.56383 \\
4.58367 \\
4.60176\end{array}$ & $\begin{array}{l}2501 \\
2209 \\
1984 \\
1809 \\
1669\end{array}$ & $\begin{array}{l}4.08510 \\
4.11928 \\
4.15026 \\
4.17851 \\
4.20440\end{array}$ & $\begin{array}{l}3418 \\
3098 \\
2825 \\
2589 \\
2387\end{array}$ & $\begin{array}{l}25.52876 \\
25.85712 \\
26.16354 \\
26.45086 \\
26.72137\end{array}$ & $\begin{array}{l}32836 \\
30642 \\
28732 \\
27051 \\
25562\end{array}$ & $\begin{array}{l}29.61386 \\
29.97641 \\
30.31381 \\
30.62937 \\
30.92578\end{array}$ & $\begin{array}{l}36255 \\
33740 \\
31556 \\
29641 \\
27949\end{array}$ \\
\hline $\begin{array}{l}1700 \\
1800 \\
1900 \\
2000 \\
2100\end{array}$ & $\begin{array}{l}4.61845 \\
4.63403 \\
4.64869 \\
4.66260 \\
4.67588\end{array}$ & $\begin{array}{l}1558 \\
1466 \\
1391 \\
1328 \\
1276\end{array}$ & $\begin{array}{l}4.22827 \\
4.25039 \\
4.27097 \\
4.29021 \\
4.30826\end{array}$ & $\begin{array}{l}2212 \\
2058 \\
1924 \\
1805 \\
1700\end{array}$ & $\begin{array}{l}26.97699 \\
27.21930 \\
27.44967 \\
27.66923 \\
27.87900\end{array}$ & $\begin{array}{l}24231 \\
23037 \\
21956 \\
20977 \\
20081\end{array}$ & $\begin{array}{l}31.20527 \\
31.46970 \\
31.72064 \\
31.95944 \\
32.18726\end{array}$ & $\begin{array}{l}26443 \\
25094 \\
23880 \\
22782 \\
21782\end{array}$ \\
\hline $\begin{array}{l}2200 \\
2300 \\
2400 \\
2500 \\
2600\end{array}$ & $\begin{array}{l}4.68864 \\
4.70095 \\
4.71287 \\
4.72446 \\
4.73575\end{array}$ & $\begin{array}{l}1231 \\
1192 \\
1159 \\
1129 \\
1104\end{array}$ & $\begin{array}{l}4.32526 \\
4.34133 \\
4.35656 \\
4.37105 \\
4.38486\end{array}$ & $\begin{array}{l}1607 \\
1523 \\
1449 \\
1381 \\
1320\end{array}$ & $\begin{array}{l}28.07981 \\
28.27244 \\
28.45753 \\
28.63567 \\
28.80737\end{array}$ & $\begin{array}{l}19263 \\
18509 \\
17814 \\
17170 \\
16574\end{array}$ & $\begin{array}{l}32.40508 \\
32.61377 \\
32.81409 \\
33.00672 \\
33.19223\end{array}$ & $\begin{array}{l}20869 \\
20032 \\
19263 \\
18551 \\
17894\end{array}$ \\
\hline $\begin{array}{l}2700 \\
2800 \\
2900 \\
3000\end{array}$ & $\begin{array}{l}4.74679 \\
4.75760 \\
4.76820 \\
4.77862\end{array}$ & $\begin{array}{l}1081 \\
1060 \\
1042 \\
2035\end{array}$ & $\begin{array}{r}/ 4.39806 \\
4.41071 \\
4.42285 \\
4.43454\end{array}$ & $\begin{array}{l}1265 \\
1214 \\
1169 \\
2214\end{array}$ & $\begin{array}{l}28.97311 \\
29.13329 \\
29.28828 \\
29.43842\end{array}$ & $\begin{array}{l}16018 \\
15499 \\
15014 \\
28691\end{array}$ & $\begin{array}{l}33.37117 \\
33.54400 \\
33.71113 \\
33.87296\end{array}$ & $\begin{array}{l}17283 \\
16713 \\
16183 \\
30906\end{array}$ \\
\hline $\begin{array}{l}3200 \\
3400 \\
3600 \\
3800 \\
4000\end{array}$ & $\begin{array}{l}4.79897 \\
4.81876 \\
4.83808 \\
4.85697 \\
4.87550\end{array}$ & $\begin{array}{l}1979 \\
1932 \\
1889 \\
1853 \\
1819\end{array}$ & $\begin{array}{l}4.45668 \\
4.47740 \\
4.49690 \\
4.51536 \\
4.53290\end{array}$ & $\begin{array}{l}2072 \\
1950 \\
1846 \\
1754 \\
1675\end{array}$ & $\begin{array}{l}29.72533 \\
29.99614 \\
30.25262 \\
30.49626 \\
30.72831\end{array}$ & $\begin{array}{l}27081 \\
25648 \\
24364 \\
23205 \\
22157\end{array}$ & $\begin{array}{l}34.18202 \\
34.47355 \\
34.74953 \\
35.01162 \\
35.26122\end{array}$ & $\begin{array}{l}29153 \\
27598 \\
26209 \\
24960 \\
23832\end{array}$ \\
\hline $\begin{array}{l}4200 \\
4400 \\
4600 \\
4800 \\
5000\end{array}$ & $\begin{array}{l}4.89369 \\
4.91159 \\
4.92920 \\
4.94655 \\
4.96366\end{array}$ & $\begin{array}{l}1790 \\
1761 \\
1735 \\
1711\end{array}$ & $\begin{array}{l}4.54965 \\
4.56570 \\
4.58112 \\
4.59599 \\
4.61035\end{array}$ & $\begin{array}{l}1605 \\
1542 \\
1487 \\
1436\end{array}$ & $\begin{array}{l}30.94988 \\
31.16191 \\
31.36520 \\
31.56049 \\
31.74840\end{array}$ & $\begin{array}{l}21203 \\
20329 \\
19529 \\
18791\end{array}$ & $\begin{array}{l}35.49954 \\
35.72761 \\
35.94633 \\
36.15648 \\
36.35876\end{array}$ & $\begin{array}{l}22807 \\
21872 \\
21015 \\
20228\end{array}$ \\
\hline
\end{tabular}


Table 2.020. KD

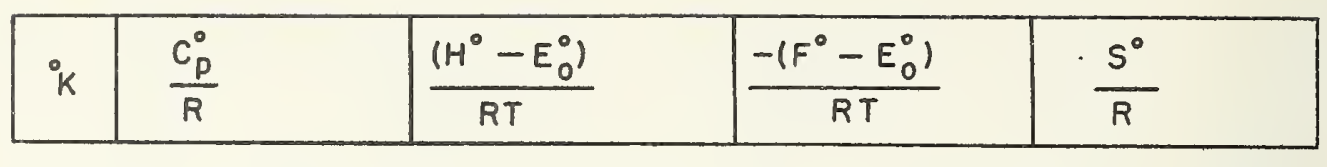

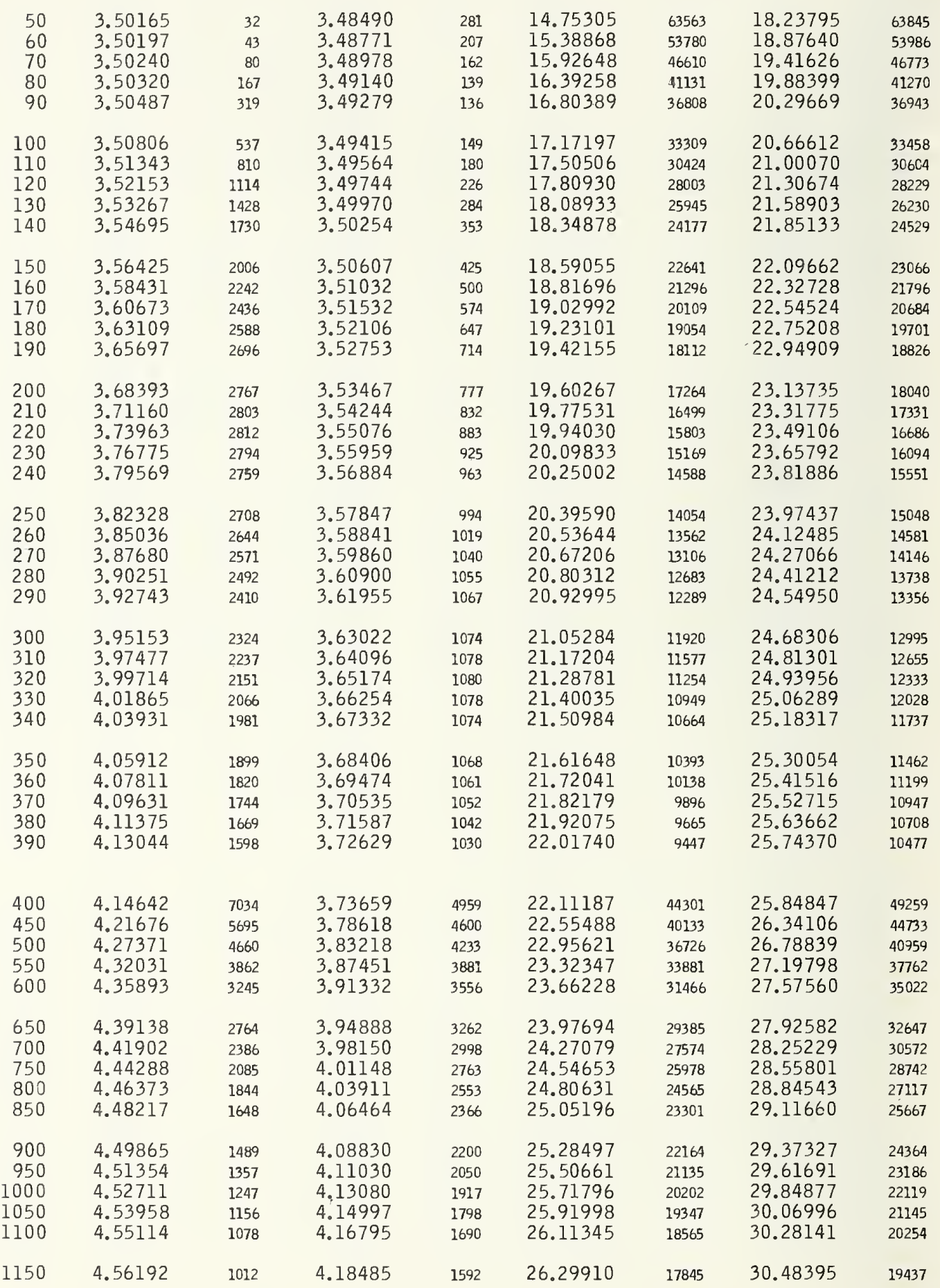


Table 2.020. KD (Cont.)

\begin{tabular}{|l|l|l|l|l|}
\hline${ }^{\circ} K$ & $\frac{C_{p}^{0}}{R}$ & $\frac{\left(H^{\circ}-E_{0}^{0}\right)}{R T}$ & $\frac{-\left(F^{\circ}-E_{0}^{0}\right)}{R T}$ & $\frac{S^{\circ}}{R}$ \\
\hline
\end{tabular}

\begin{tabular}{|c|c|c|c|c|c|c|c|c|}
\hline 1200 & 4.57204 & 1862 & 4.20077 & 2929 & 26.47755 & 33742 & 30.67832 & 36671 \\
\hline 1300 & 4.59066 & 1694 & 4.23006 & 2637 & 26.81497 & 31446 & 31.04503 & 34083 \\
\hline 1400 & 4.60760 & 1564 & 4.25643 & 2394 & 27.12943 & 29449 & 31.38586 & 31844 \\
\hline 1500 & 4.62324 & 1464 & 4.28037 & 2189 & 27.42392 & 27696 & 31.70430 & 29885 \\
\hline 1600 & 4.63788 & 1385 & 4.30226 & 2015 & 27.70088 & 26144 & 32.00315 & 28159 \\
\hline 1700 & 4.65173 & 1322 & 4.32241 & 1867 & 27.96232 & 24759 & 32.28474 & 26626 \\
\hline 1800 & 4.66495 & 1269 & 4.34108 & 1738 & 28.20991 & 23519 & 32.55100 & 25256 \\
\hline 1900 & 4.67764 & 1225 & 4.35846 & 1627 & 28.44510 & 22397 & 32.80356 & 24025 \\
\hline 2000 & 4.68989 & 1190 & 4.37473 & 1529 & 28.66907 & 21382 & 33.04381 & 22911 \\
\hline 2100 & 4.70179 & 1158 & 4.39002 & 1444 & 28.88289 & 20456 & 33.27292 & 21899 \\
\hline 2200 & 4.71337 & 1132 & 4.40446 & 1367 & 29.08745 & 19609 & 33.49191 & 20977 \\
\hline 2300 & 4. 72469 & 1109 & 4.41813 & 1301 & 29.28354 & 18831 & 33.70168 & 20132 \\
\hline 2400 & 4.73578 & 1088 & 4.43114 & 1240 & 29.47185 & 18114 & 33.90300 & 19354 \\
\hline 2500 & 4.74666 & 1070 & 4.44354 & 1187 & 29.65299 & 17452 & 34.09654 & 18638 \\
\hline 2600 & 4.75736 & 1054 & 4.45541 & 1138 & 29.82751 & 16836 & 34.28292 & 17974 \\
\hline 2700 & 4.76790 & 1040 & 4.46679 & 1094 & 29.99587 & 16265 & 34.46266 & 17359 \\
\hline 2800 & 4.77830 & 1025 & 4.47773 & 1054 & 30.15852 & 15731 & 34.63 & 16785 \\
\hline 2900 & 4.78855 & 1014 & 4.48827 & 1018 & 30.31583 & 15233 & 34.80410 & 16251 \\
\hline 3000 & 4.79869 & 1994 & 4.49845 & 1939 & 30.46816 & 29095 & 34.96661 & 31034 \\
\hline 3200 & 4.81863 & 1955 & 4.51784 & 1827 & 30.75911 & 27445 & 35.27695 & 29272 \\
\hline 3400 & 4.83818 & 1920 & 4.53611 & 1731 & 31.03356 & 25977 & 35.56967 & 27709 \\
\hline 3600 & 4.85738 & 1888 & 4.55342 & 1650 & 31.29333 & 24663 & 35.84676 & 26313 \\
\hline 3800 & 4.87626 & 1859 & 4.56992 & 1578 & 31.53996 & 23482 & 36.10989 & 25059 \\
\hline 4000 & 4.89485 & 1832 & 4.58570 & 1516 & 31.77478 & 22410 & 36.36048 & 23927 \\
\hline 4200 & 4.91317 & 1807 & 4.60086 & 1461 & 31.99888 & 21437 & 36.59975 & 22898 \\
\hline 4400 & 4.93124 & 1783 & 4.61547 & 1412 & 32.21325 & 20548 & 36.82873 & 21959 \\
\hline 4600 & 4.94907 & 1760 & 4.62959 & 1368 & 32.41873 & 19733 & 37.04832 & 21101 \\
\hline 4800 & 4.96667 & 1739 & 4.64327 & 1328 & 32.61606 & 18982 & 37.25933 & 20310 \\
\hline 500 & 4.98406 & & 4.65655 & & 32.80588 & & 46243 & \\
\hline
\end{tabular}


Table 2.021. KT

\begin{tabular}{|c|c|c|c|c|c|c|c|c|}
\hline${ }^{\circ} \mathrm{K}$ & $\frac{C_{p}^{0}}{R}$ & & $\frac{\left(H^{0}-E_{0}^{0}\right)}{R T}$ & & $\frac{-\left(F^{0}-E\right.}{R T}$ & & $\frac{S^{0}}{R}$ & \\
\hline $\begin{array}{l}50 \\
60 \\
70 \\
80 \\
90\end{array}$ & $\begin{array}{l}3.50164 \\
3.50213 \\
3.50332 \\
3.50614 \\
3.51178\end{array}$ & $\begin{array}{r}49 \\
119 \\
282 \\
564 \\
946\end{array}$ & $\begin{array}{l}3.48955 \\
3.49160 \\
3.49318 \\
3.49460 \\
3.49616\end{array}$ & $\begin{array}{l}205 \\
158 \\
142 \\
156 \\
200\end{array}$ & $\begin{array}{l}15.16261 \\
15.79902 \\
16.33737 \\
16.80392 \\
17.21561\end{array}$ & $\begin{array}{l}63641 \\
53835 \\
46655 \\
41169 \\
36846\end{array}$ & $\begin{array}{l}18.65216 \\
19.29063 \\
19.83056 \\
20.29852 \\
20.71178\end{array}$ & $\begin{array}{l}63847 \\
53993 \\
46796 \\
41326 \\
37045\end{array}$ \\
\hline $\begin{array}{l}100 \\
110 \\
120 \\
130 \\
140\end{array}$ & $\begin{array}{l}3.52124 \\
3.53511 \\
3.55349 \\
3.57607 \\
3.60227\end{array}$ & $\begin{array}{l}1387 \\
1838 \\
2258 \\
2620 \\
2910\end{array}$ & $\begin{array}{l}3.49816 \\
3.50086 \\
3.50445 \\
3.50906 \\
3.51477\end{array}$ & $\begin{array}{l}270 \\
359 \\
461 \\
571 \\
679\end{array}$ & $\begin{array}{l}17.58407 \\
17.91760 \\
18.22236 \\
18.50304 \\
18.76329\end{array}$ & $\begin{array}{l}33353 \\
30476 \\
28068 \\
26025 \\
24272\end{array}$ & $\begin{array}{l}21.08223 \\
21.41846 \\
21.72681 \\
22.01211 \\
22.27806\end{array}$ & $\begin{array}{l}33623 \\
30835 \\
28530 \\
26595 \\
24951\end{array}$ \\
\hline $\begin{array}{l}150 \\
160 \\
170 \\
180 \\
190\end{array}$ & $\begin{array}{l}3.63137 \\
3.66260 \\
3.69524 \\
3.72865 \\
3.76230\end{array}$ & $\begin{array}{l}3123 \\
3264 \\
3341 \\
3365 \\
3344\end{array}$ & $\begin{array}{l}3.52156 \\
3.52939 \\
3.53818 \\
3.54783 \\
3.55823\end{array}$ & $\begin{array}{r}783 \\
879 \\
965 \\
1040 \\
1104\end{array}$ & $\begin{array}{l}19.00601 \\
19.23353 \\
19.44776 \\
19.65026 \\
19.84236\end{array}$ & $\begin{array}{l}22752 \\
21423 \\
20250 \\
19210 \\
18279\end{array}$ & $\begin{array}{l}22.52757 \\
22.76292 \\
22.98594 \\
23.19810 \\
23.40060\end{array}$ & $\begin{array}{l}23535 \\
22302 \\
21216 \\
20250 \\
19383\end{array}$ \\
\hline $\begin{array}{l}200 \\
210 \\
220 \\
230 \\
240\end{array}$ & $\begin{array}{l}3.79574 \\
3.82863 \\
3.86070 \\
3.89179 \\
3.92175\end{array}$ & $\begin{array}{l}3289 \\
3207 \\
3109 \\
2996 \\
2875\end{array}$ & $\begin{array}{l}3.56927 \\
3.58084 \\
3.59284 \\
3.60516 \\
3.61773\end{array}$ & $\begin{array}{l}1157 \\
1200 \\
1232 \\
1257 \\
1274\end{array}$ & $\begin{array}{l}20.02515 \\
20.19958 \\
20.36643 \\
20.52641 \\
20.68011\end{array}$ & $\begin{array}{l}17443 \\
16685 \\
15998 \\
15370 \\
14794\end{array}$ & $\begin{array}{l}23.59443 \\
23.78042 \\
23.95928 \\
24.13158 \\
24.29785\end{array}$ & $\begin{array}{l}18599 \\
17886 \\
17230 \\
16627 \\
16068\end{array}$ \\
\hline $\begin{array}{l}250 \\
260 \\
270 \\
280 \\
290\end{array}$ & $\begin{array}{l}3.95050 \\
3.97801 \\
4.00426 \\
4.02926 \\
4.05303\end{array}$ & $\begin{array}{l}2751 \\
2625 \\
2500 \\
2377 \\
2258\end{array}$ & $\begin{array}{l}3.63047 \\
3.64332 \\
3.65620 \\
3.66908 \\
3.68192\end{array}$ & $\begin{array}{l}1285 \\
1288 \\
1288 \\
1284 \\
1275\end{array}$ & $\begin{array}{l}20.82805 \\
20.97069 \\
21.10843 \\
21.24163 \\
21.37061\end{array}$ & $\begin{array}{l}14264 \\
13774 \\
13320 \\
12898 \\
12504\end{array}$ & $\begin{array}{l}24.45853 \\
24.61401 \\
24.76464 \\
24.91072 \\
25.05253\end{array}$ & $\begin{array}{l}15548 \\
15063 \\
14608 \\
14181 \\
13779\end{array}$ \\
\hline $\begin{array}{l}300 \\
310 \\
320 \\
330 \\
340\end{array}$ & $\begin{array}{l}4.07561 \\
4.09704 \\
4.11737 \\
4.13666 \\
4.15494\end{array}$ & $\begin{array}{l}2143 \\
2033 \\
1929 \\
1828 \\
1735\end{array}$ & $\begin{array}{l}3.69467 \\
3.70730 \\
3.71980 \\
3.73215 \\
3.74431\end{array}$ & $\begin{array}{l}1263 \\
1250 \\
1235 \\
1216 \\
1199\end{array}$ & $\begin{array}{l}21.49565 \\
21.61700 \\
21.73490 \\
21.84956 \\
21.96115\end{array}$ & $\begin{array}{l}12135 \\
11790 \\
11466 \\
11159 \\
10872\end{array}$ & $\begin{array}{l}25.19032 \\
25.32431 \\
25.45471 \\
25.58171 \\
25.70547\end{array}$ & $\begin{array}{l}13399 \\
13040 \\
12700 \\
12376 \\
12070\end{array}$ \\
\hline $\begin{array}{l}350 \\
360 \\
370 \\
380 \\
390\end{array}$ & $\begin{array}{l}4.17229 \\
4.18874 \\
4.20436 \\
4.21919 \\
4.23327\end{array}$ & $\begin{array}{l}1645 \\
1562 \\
1483 \\
1408 \\
1338\end{array}$ & $\begin{array}{l}3.75630 \\
3.76808 \\
3.77966 \\
3.79104 \\
3.80220\end{array}$ & $\begin{array}{l}1178 \\
1158 \\
1138 \\
1116 \\
1094\end{array}$ & $\begin{array}{l}22.06987 \\
22.17585 \\
22.27925 \\
22.38020 \\
22.47882\end{array}$ & $\begin{array}{r}10598 \\
10340 \\
10095 \\
9862 \\
9640\end{array}$ & $\begin{array}{l}25.82617 \\
25.94394 \\
26.05892 \\
26.17124 \\
26.28102\end{array}$ & $\begin{array}{l}11777 \\
11498 \\
11232 \\
10978 \\
10735\end{array}$ \\
\hline $\begin{array}{l}400 \\
450 \\
500 \\
550 \\
600\end{array}$ & $\begin{array}{l}4.24665 \\
4.30449 \\
4.35027 \\
4.38721 \\
4.41759\end{array}$ & $\begin{array}{l}5784 \\
4578 \\
3694 \\
3038 \\
2545\end{array}$ & $\begin{array}{l}3.81314 \\
3.86465 \\
3.91101 \\
3.95268 \\
3.99020\end{array}$ & $\begin{array}{l}5151 \\
4636 \\
4167 \\
3752 \\
3388\end{array}$ & $\begin{array}{l}22.57522 \\
23.02737 \\
23.43700 \\
23.81174 \\
24.15731\end{array}$ & $\begin{array}{l}45215 \\
40963 \\
37474 \\
34557 \\
32075\end{array}$ & $\begin{array}{l}26.38837 \\
26.89203 \\
27.34801 \\
27.76443 \\
28.14751\end{array}$ & $\begin{array}{l}50366 \\
45598 \\
41642 \\
38308 \\
35463\end{array}$ \\
\hline $\begin{array}{l}650 \\
700 \\
750 \\
800 \\
850\end{array}$ & $\begin{array}{l}4.44304 \\
4.46474 \\
4.48353 \\
4.50005 \\
4.51476\end{array}$ & $\begin{array}{l}2170 \\
1879 \\
1652 \\
1471 \\
1327\end{array}$ & $\begin{array}{l}4.02408 \\
4.05480 \\
4.08277 \\
4.10834 \\
4.13183\end{array}$ & $\begin{array}{l}3072 \\
2797 \\
2557 \\
2349 \\
2164\end{array}$ & $\begin{array}{l}24.47806 \\
24.77742 \\
25.05814 \\
25.32246 \\
25.57224\end{array}$ & $\begin{array}{l}29936 \\
28072 \\
26432 \\
24978 \\
23679\end{array}$ & $\begin{array}{l}28.50214 \\
28.83222 \\
29.14091 \\
29.43081 \\
29.70407\end{array}$ & $\begin{array}{l}33008 \\
30869 \\
28990 \\
27326 \\
25844\end{array}$ \\
\hline $\begin{array}{r}900 \\
950 \\
1000 \\
1050 \\
1100\end{array}$ & $\begin{array}{l}4.52803 \\
4.54012 \\
4.55125 \\
4.56158 \\
4.57124\end{array}$ & $\begin{array}{r}1209 \\
1113 \\
1033 \\
966 \\
911\end{array}$ & $\begin{array}{l}4.15347 \\
4.17351 \\
4.19212 \\
4.20947 \\
4.22570\end{array}$ & $\begin{array}{l}2004 \\
1861 \\
1735 \\
1623 \\
1522\end{array}$ & $\begin{array}{l}25.80903 \\
26.03414 \\
26.24870 \\
26.45366 \\
26.64986\end{array}$ & $\begin{array}{l}22511 \\
21456 \\
20496 \\
19620 \\
18818\end{array}$ & $\begin{array}{l}29.96251 \\
30.20766 \\
30.44082 \\
30.66313 \\
30.87556\end{array}$ & $\begin{array}{l}24515 \\
23316 \\
22231 \\
21243 \\
20341\end{array}$ \\
\hline 1150 & 4.58035 & 862 & 4.24092 & 1433 & 26.83804 & 18080 & 31.07897 & 19512 \\
\hline
\end{tabular}


Table 2.021. KT (Cont.)

\begin{tabular}{|c|c|c|c|c|c|c|c|c|}
\hline${ }^{\circ} \mathrm{K}$ & $\frac{C_{p}^{0}}{R}$ & & $\frac{\left(H^{0}-E_{0}^{0}\right)}{R T}$ & & $\frac{-l F^{\circ}-E}{R T}$ & & $\frac{S^{0}}{R}$ & \\
\hline $\begin{array}{l}200 \\
300 \\
400 \\
500 \\
600\end{array}$ & $\begin{array}{l}4.58897 \\
4.60507 \\
4.61996 \\
4.63392 \\
4.64716\end{array}$ & $\begin{array}{l}1610 \\
1489 \\
1396 \\
1324 \\
1267\end{array}$ & $\begin{array}{l}4.25525 \\
4.28155 \\
4.30519 \\
4.32665 \\
4.34627\end{array}$ & $\begin{array}{l}2630 \\
2364 \\
2146 \\
1962 \\
1807\end{array}$ & $\begin{array}{l}27.01884 \\
27.36050 \\
27.67867 \\
27.97644 \\
28.25632\end{array}$ & $\begin{array}{l}34166 \\
31817 \\
29777 \\
27988 \\
26404\end{array}$ & $\begin{array}{l}31.27409 \\
31.64205 \\
31.98387 \\
32.30310 \\
32.60259\end{array}$ & $\begin{array}{l}36796 \\
34182 \\
31923 \\
29949 \\
28211\end{array}$ \\
\hline $\begin{array}{l}700 \\
800 \\
900 \\
000 \\
100\end{array}$ & $\begin{array}{l}4.65983 \\
4.67203 \\
4.68386 \\
4.69537 \\
4.70661\end{array}$ & $\begin{array}{l}1220 \\
1183 \\
1151 \\
1124 \\
1102\end{array}$ & $\begin{array}{l}4.36434 \\
4.38110 \\
4.39673 \\
4.41137 \\
4.42516\end{array}$ & $\begin{array}{l}1676 \\
1563 \\
1464 \\
1379 \\
1305\end{array}$ & $\begin{array}{l}28.52036 \\
28.77030 \\
29.00759 \\
29.23349 \\
29.44906\end{array}$ & $\begin{array}{l}24994 \\
23729 \\
22590 \\
21557 \\
20616\end{array}$ & $\begin{array}{l}32.88470 \\
33.15140 \\
33.40432 \\
33.64487 \\
33.87423\end{array}$ & $\begin{array}{l}26670 \\
25292 \\
24055 \\
22936 \\
21921\end{array}$ \\
\hline $\begin{array}{l}200 \\
300 \\
400 \\
500 \\
600\end{array}$ & $\begin{array}{l}4.71763 \\
4.72844 \\
4.73907 \\
4.74955 \\
4.75989\end{array}$ & $\begin{array}{l}1081 \\
1063 \\
1048 \\
1034 \\
1021\end{array}$ & $\begin{array}{l}4.43821 \\
4.45059 \\
4.46239 \\
4.47367 \\
4.48448\end{array}$ & $\begin{array}{l}1238 \\
1180 \\
1128 \\
1081 \\
1039\end{array}$ & $\begin{array}{l}29.65522 \\
29.85279 \\
30.04245 \\
30.22485 \\
30.40052\end{array}$ & $\begin{array}{l}19757 \\
18966 \\
18240 \\
17567 \\
16944\end{array}$ & $\begin{array}{l}34.09344 \\
34.30338 \\
34.50485 \\
34.69852 \\
34.88500\end{array}$ & $\begin{array}{l}20994 \\
20147 \\
19367 \\
18648 \\
17983\end{array}$ \\
\hline $\begin{array}{r}2700 \\
2800 \\
2900 \\
3000\end{array}$ & $\begin{array}{l}4.77010 \\
4.78019 \\
4.79017 \\
4.80006\end{array}$ & $\begin{array}{r}1009 \\
998 \\
989 \\
1950\end{array}$ & $\begin{array}{l}4.49487 \\
4.50488 \\
4.51454 \\
4.52390\end{array}$ & $\begin{array}{r}1001 \\
966 \\
936 \\
1787\end{array}$ & $\begin{array}{l}30.56996 \\
30.73361 \\
30.89186 \\
31.04507\end{array}$ & $\begin{array}{l}16365 \\
15825 \\
15321 \\
29254\end{array}$ & $\begin{array}{l}35.06483 \\
35.23849 \\
35.40641 \\
35.56897\end{array}$ & $\begin{array}{l}17366 \\
16792 \\
16256 \\
31042\end{array}$ \\
\hline $\begin{array}{l}200 \\
400 \\
600 \\
800 \\
000\end{array}$ & $\begin{array}{l}4.81956 \\
4.83872 \\
4.85759 \\
4.87617 \\
4.89451\end{array}$ & $\begin{array}{l}1916 \\
1887 \\
1858 \\
1834 \\
1808\end{array}$ & $\begin{array}{l}4.54177 \\
4.55867 \\
4.57476 \\
4.59013 \\
4.60489\end{array}$ & $\begin{array}{l}1690 \\
1609 \\
1537 \\
1476 \\
1423\end{array}$ & $\begin{array}{l}31.33761 \\
31.61347 \\
31.87449 \\
32.12225 \\
32.35808\end{array}$ & $\begin{array}{l}27586 \\
26102 \\
24776 \\
23583 \\
22502\end{array}$ & $\begin{array}{l}35.87939 \\
36.17215 \\
36.44926 \\
36.71239 \\
36.96297\end{array}$ & $\begin{array}{l}29276 \\
27111 \\
26313 \\
25058 \\
23925\end{array}$ \\
\hline $\begin{array}{l}200 \\
400 \\
600 \\
800 \\
000\end{array}$ & $\begin{array}{l}4.91259 \\
4.93045 \\
4.94809 \\
4.96552 \\
4.98275\end{array}$ & $\begin{array}{l}1786 \\
1764 \\
1743 \\
1723\end{array}$ & $\begin{array}{l}4.61912 \\
4.63286 \\
4.64619 \\
4.65913 \\
4.67173\end{array}$ & $\begin{array}{l}1374 \\
1333 \\
1294 \\
1260\end{array}$ & $\begin{array}{l}32.58310 \\
32.79830 \\
33.00453 \\
33.20255 \\
33.39300\end{array}$ & $\begin{array}{l}21520 \\
20623 \\
19802 \\
19045\end{array}$ & $\begin{array}{l}37.20222 \\
37.43116 \\
37.65072 \\
37.86168 \\
38.06473\end{array}$ & $\begin{array}{l}22894 \\
21956 \\
21096 \\
20305\end{array}$ \\
\hline
\end{tabular}


Table 2.022. $\mathrm{RbH}$

\begin{tabular}{|l|l|l|l|l|}
\hline$K^{\circ}$ & $\frac{C_{p}^{0}}{R}$ & $\frac{\left(H^{0}-E_{0}^{0}\right)}{R T}$ & $\frac{-\left(F^{0}-E_{0}^{0}\right)}{R T}$ & $\frac{S^{0}}{R}$ \\
\hline
\end{tabular}

\begin{tabular}{|c|c|c|c|c|c|c|c|c|}
\hline 50 & 3.50207 & 32 & 3.47216 & 501 & 15.29042 & 63352 & 18.76259 & 63853 \\
\hline 60 & 3.50239 & 35 & 3.47717 & 363 & 15.92394 & 53630 & 19.40112 & 53992 \\
\hline 70 & 3.50274 & 38 & 3.48080 & 277 & 16.46024 & 46498 & 19.94104 & 46775 \\
\hline 80 & 3.50312 & 44 & 3.48357 & 219 & 16.92522 & 41044 & 20.40879 & 41263 \\
\hline 90 & 3.50356 & 63 & 3.48576 & 181 & 17.33566 & 36736 & 20.82142 & 36917 \\
\hline 100 & 3.50419 & 100 & 3.48757 & 155 & 17.70302 & 33247 & 21.19059 & 33403 \\
\hline 110 & 3.50519 & 163 & 3.48912 & 141 & 18.03549 & 30366 & 21.52462 & 30506 \\
\hline 120 & 3.50682 & 257 & 3.49053 & 134 & 18.33915 & 27944 & 21.82968 & 28079 \\
\hline 130 & 3.50939 & 380 & 3.49187 & 138 & 18.61859 & 25883 & 22.11047 & 26020 \\
\hline 140 & 3.51319 & 528 & 3.49325 & 150 & 18.87742 & 24106 & 22.37067 & 24256 \\
\hline 150 & 3.51847 & 696 & 3.49475 & 169 & 19.11848 & 22560 & 22.61323 & 22729 \\
\hline 160 & 3.52543 & 876 & 3.49644 & 195 & 19.34408 & 21202 & 22.84052 & 21398 \\
\hline 170 & 3.53419 & 1060 & 3.49839 & 228 & 19.55610 & 20003 & 23.05450 & 20230 \\
\hline 180 & 3.54479 & 1239 & 3.50067 & 264 & 19.75613 & 18934 & 23.25680 & 19198 \\
\hline 190 & 3.55718 & 1410 & 3.50331 & 304 & 19.94547 & 17977 & 23.44878 & 18281 \\
\hline 200 & 3.57128 & 1566 & 3.50635 & 346 & 20.12524 & 17116 & 23.63159 & 17462 \\
\hline 210 & 3.58694 & 1705 & 3.50981 & 388 & 20.29640 & 16336 & 23.80621 & 16725 \\
\hline 220 & 3.60399 & 1826 & 3.51369 & 432 & 20.45976 & 15629 & 23.97346 & 16061 \\
\hline 230 & 3.62225 & 1928 & 3.51801 & 474 & 20.61605 & 14982 & 24.13407 & 15456 \\
\hline 240 & 3.64153 & 2010 & 3.52275 & 516 & 20.76587 & 14391 & 24.28863 & 14906 \\
\hline 250 & 3.66163 & 2074 & 3.52791 & 554 & 20.90978 & 13848 & 24.43769 & 14402 \\
\hline 260 & 3.68237 & 2120 & 3.53345 & 590 & 21.04826 & 13346 & 24.58171 & 13937 \\
\hline 270 & 3.70357 & 2152 & 3.53935 & 625 & 21.18172 & 12883 & 24.72108 & 13507 \\
\hline 280 & 3.72509 & 2170 & 3.54560 & 656 & 21.31055 & 12453 & 24.85615 & 13110 \\
\hline 290 & 3.74679 & 2174 & 3.55216 & 685 & 21.43508 & 12054 & 24.98725 & 12739 \\
\hline 300 & 3.76853 & 2167 & 3.55901 & 711 & 21.55562 & 11682 & 25.11464 & 12392 \\
\hline 310 & 3.79020 & 2153 & 3.56612 & 734 & 21.67244 & 11333 & 25.23856 & 12068 \\
\hline 320 & 3.81173 & 2129 & 3.57346 & 755 & 21.78577 & 11008 & 25.35924 & 11762 \\
\hline 330 & 3.83302 & 2100 & 3.58101 & 772 & 21.89585 & 10702 & 25.47686 & 11474 \\
\hline 340 & 3.85402 & 2064 & 3.58873 & 787 & 22.00287 & 10414 & 25.59160 & 11201 \\
\hline 350 & 3.87466 & 2025 & 3.59660 & 801 & 22.10701 & 10143 & 25.70361 & 10944 \\
\hline 360 & 3.89491 & 1982 & 3.60461 & 811 & 22.20844 & 9887 & 25.81305 & 10699 \\
\hline 370 & 3.91473 & 1937 & 3.61272 & 821 & 22.30731 & 9646 & 25.92004 & 10466 \\
\hline 380 & 3.93410 & 1889 & 3.62093 & 827 & 22.40377 & 9416 & 26.02470 & 10243 \\
\hline 390 & 3.95299 & 1840 & 3.62920 & 833 & 22.49793 & 9199 & 26.12713 & 10032 \\
\hline 400 & 3.97139 & 8460 & 3.63753 & 4191 & 22.58992 & 43085 & 26.22745 & 47277 \\
\hline 450 & 4.05599 & 7262 & 3.67944 & 4138 & 02077 & 38982 & 26.70022 & 43120 \\
\hline 500 & 4.12861 & 6198 & 3.72082 & 3996 & 41059 & 35652 & 27.13142 & 39648 \\
\hline 550 & 4.19059 & 5294 & 3.76078 & 3808 & 23.76711 & 32888 & 27.52790 & 36696 \\
\hline 600 & 4.24353 & 4541 & 3.79886 & 3600 & 24.09599 & 30551 & 27.89486 & 34150 \\
\hline 650 & 4.28894 & 3923 & 3.83486 & 3387 & 24.40150 & 28545 & 28.23636 & 31932 \\
\hline 700 & 4.32817 & 3415 & 3.86873 & 3179 & 24.68695 & 26801 & 28.55568 & 29980 \\
\hline 75 & 4.36232 & 2996 & 3.90052 & 2982 & 24.95496 & 25269 & 28.85548 & 28252 \\
\hline 800 & 4.39228 & 2652 & 3.93034 & 2797 & 25.20765 & 23913 & 29.13800 & 26709 \\
\hline 850 & 4.41880 & 2367 & 3.95831 & 2625 & 25.44678 & 22700 & 29.40509 & 25325 \\
\hline 900 & 4.44247 & 2128 & 3.98456 & 2467 & 25.67378 & 21610 & 29.65834 & 24078 \\
\hline 950 & 4.46375 & 1929 & 4.00923 & $232 ?$ & 25.88988 & 20624 & 29.89912 & 22945 \\
\hline 1000 & 4.48304 & 1761 & 4.03245 & 2188 & 26.09612 & 19728 & 30.12857 & 21917 \\
\hline 1050 & 4.50065 & 1618 & 4.05433 & 2066 & 26.29340 & 18909 & 30.34774 & 20974 \\
\hline & 4.51683 & 1496 & 4.07499 & 1954 & 26.48249 & 18158 & 30.55748 & 20112 \\
\hline 1150 & 4.53179 & 1393 & 4.09453 & 1851 & 26.66407 & 17465 & 30.75860 & 19317 \\
\hline
\end{tabular}


Table 2.022. RbH (Cont.)

\begin{tabular}{|l|l|l|l|l|}
\hline$K$ & $\frac{C_{p}^{0}}{R}$ & $\frac{\left(H^{0}-E_{0}^{0}\right)}{R T}$ & $\frac{-\left(F^{0}-E_{0}^{0}\right)}{R T}$ & $\frac{S^{0}}{R}$ \\
\hline
\end{tabular}

\begin{tabular}{|c|c|c|c|c|c|c|c|c|}
\hline 1200 & 4.54572 & 2527 & 4.11304 & 3428 & 26.83872 & 33060 & 30.95177 & 36487 \\
\hline 1300 & 4.57099 & 2255 & 4.14732 & 3108 & 27.16932 & 30850 & 31.31664 & 33959 \\
\hline 1400 & 4.59354 & 2045 & 4.17840 & 2837 & 27.47782 & 28927 & 31.65623 & 31763 \\
\hline 1500 & 4.61399 & 1883 & 4.20677 & 2604 & 27.76709 & 27234 & 31.97386 & 29839 \\
\hline 1600 & 4.63282 & 1752 & 4.23281 & 2405 & 28.03943 & 25734 & 32.27225 & 28139 \\
\hline 1700 & 4.65034 & 1649 & 4.25686 & 2233 & 28.29677 & 24396 & 32.55364 & 26628 \\
\hline 1800 & 4.66683 & 1562 & 4.27919 & 2081 & 28.54073 & 23193 & 32.81992 & 25274 \\
\hline 1900 & 4.68245 & 1493 & 4.30000 & 1950 & 28.77266 & 22106 & 33.07266 & 24056 \\
\hline 2000 & 4.69738 & 1433 & 4.31950 & 1834 & 28.99372 & 21120 & 22 & 22954 \\
\hline 2100 & 4.71171 & 1383 & 4.33784 & 1731 & 29.20492 & 20219 & 33.54276 & 21951 \\
\hline 2200 & 4.72554 & 1340 & 4.35515 & 1640 & 29.40711 & 19396 & 33.76227 & 21035 \\
\hline 2300 & 4.73894 & 1303 & 4.37155 & 1558 & 29.60107 & 18639 & 33.97262 & 20197 \\
\hline 2400 & 4.75197 & 1270 & 4.38713 & 1484 & 29.78746 & 17939 & 34.17459 & 19424 \\
\hline 2500 & 4.76467 & 1243 & 4.40197 & 1419 & 29.96685 & 17293 & 34.36883 & 18712 \\
\hline 2600 & 4.77710 & 1216 & 4.41616 & 1360 & 30.13978 & 16692 & 34.55595 & 18052 \\
\hline 2700 & 4.78926 & 1195 & 4.42976 & 1305 & 30.30670 & 16134 & 34.73647 & 17439 \\
\hline 2800 & 4.80121 & 1173 & 4.44281 & 1256 & 30.46804 & 15613 & 34.91086 & 16868 \\
\hline 2900 & 4.81294 & 1156 & 4.45537 & 1212 & 30.62417 & 15125 & 35.07954 & 16337 \\
\hline 3000 & 4.82450 & 2260 & 4.46749 & 2302 & 30.77542 & 28906 & 35.24291 & 31209 \\
\hline 3200 & 4.84710 & 2203 & 4.49051 & 2162 & 31.06448 & 27290 & 35.55500 & 29451 \\
\hline 3400 & 4.86913 & 2153 & 4.51213 & 2044 & 31.33738 & 25849 & 35.84951 & 27893 \\
\hline 3600 & 4.89066 & 2108 & 4.53257 & 1940 & 31.59587 & 24558 & 36.12844 & 26499 \\
\hline 3800 & 4.91174 & 2067 & 4.55197 & 1851 & 31.84145 & 23396 & 36.39343 & 25247 \\
\hline 400 & 4.93241 & 2031 & 4.57048 & 1772 & 32.07541 & 22343 & 36.64590 & 24114 \\
\hline 4200 & 4.95272 & 1997 & 4.58820 & 1702 & 32.29884 & 21384 & 36.88704 & 23087 \\
\hline 4400 & 4.97269 & 1966 & 4.60522 & 1641 & 32.51268 & 20507 & 37.11791 & 22148 \\
\hline 4600 & 4.99235 & 1936 & 4.62163 & 1585 & 32.71775 & 19703 & 37.33939 & 21288 \\
\hline 4800 & 5.01171 & 1907 & 4.63748 & 1535 & 32.91478 & 18963 & 37.55227 & 20497 \\
\hline & 5.03078 & & 4.6528 & & 10441 & & 75724 & \\
\hline
\end{tabular}


Table 2.023. RbD

\begin{tabular}{|l|l|l|l|l|}
\hline$K$ & $\frac{C_{p}^{0}}{R}$ & $\frac{\left(H^{\circ}-E_{0}^{0}\right)}{R T}$ & $\frac{-\left(F^{0}-E_{0}^{0}\right)}{R T}$ & $\frac{S^{0}}{R}$ \\
\hline
\end{tabular}

\begin{tabular}{|c|c|c|c|c|c|c|c|c|}
\hline 50 & 3.50194 & 40 & 3.48636 & 263 & 15.97069 & 63588 & 19.45705 & 63852 \\
\hline 60 & 3.50234 & 61 & 3.48899 & 195 & 16.60657 & 53798 & 20.09557 & 53993 \\
\hline 70 & 3.50295 & 122 & 3.49094 & 156 & 17.14455 & 46626 & 20.63550 & 46782 \\
\hline 80 & 3.50417 & 253 & 3.49250 & 143 & 17.61081 & 41144 & 21.10332 & 41286 \\
\hline 90 & 3.50670 & 463 & 3.49393 & 148 & 18.02225 & 36820 & 21.51618 & 36969 \\
\hline 100 & 3.51133 & 747 & 3.49541 & 177 & 18.39045 & 33323 & 21.88587 & 33499 \\
\hline 110 & 3.51880 & 1078 & 3.49718 & 222 & 18.72368 & 30438 & 22.22086 & 30661 \\
\hline 120 & 3.52958 & 1429 & 3.49940 & 285 & 19.02806 & 28021 & 22.52747 & 28306 \\
\hline 130 & 3.54387 & 1774 & 3.50225 & 359 & 19.30827 & 25967 & 22.81053 & 26326 \\
\hline 140 & 3.56161 & 2088 & 3.50584 & 440 & 19.56794 & 24203 & 23.07379 & 24642 \\
\hline 150 & 3.58249 & 2358 & 3.51024 & 524 & 19.80997 & 22671 & 23.32021 & 23195 \\
\hline 160 & 3.60607 & 2580 & 3.51548 & 607 & 20.03668 & 21330 & 23.55216 & 21938 \\
\hline 170 & 3.63187 & 2749 & 3.52155 & 689 & 20.24998 & 20148 & 23.77154 & 20836 \\
\hline 180 & 3.65936 & 2868 & 3.52844 & 764 & 20.45146 & 19097 & 23.97990 & 19862 \\
\hline 190 & 3.68804 & 2943 & 3.53608 & 833 & 20.64243 & 18159 & 24.17852 & 18992 \\
\hline 200 & 3.71747 & 2977 & 3.54441 & 895 & 20.82402 & 17315 & 24.36844 & 18209 \\
\hline 210 & 3.74724 & 2980 & 3.55336 & 949 & 20.99717 & 16552 & 24.55053 & 17501 \\
\hline 220 & 3.77704 & 2954 & 3.56285 & 996 & 21.16269 & 15859 & 24.72554 & 16855 \\
\hline 230 & 3.80658 & 2907 & 3.57281 & 1035 & 21.32128 & 15227 & 24.89409 & 16262 \\
\hline 240 & 3.83565 & 2843 & 3.58316 & 1067 & 21.47355 & 14649 & 25.05671 & 15716 \\
\hline 250 & 3.86408 & 2767 & 3.59383 & 1093 & 21.62004 & 14117 & 25.21387 & 15210 \\
\hline 260 & 3.89175 & 2682 & 3.60476 & 1112 & 21.76121 & 13625 & 25.36597 & 14738 \\
\hline 270 & 3.91857 & 2590 & 3.61588 & 1128 & 21.89746 & 13170 & 25.51335 & 14298 \\
\hline 280 & 3.94447 & 2495 & 3.62716 & 1137 & 22.02916 & 12748 & 25.65633 & 13885 \\
\hline 290 & 3.96942 & 2398 & 3.63853 & 1144 & 22.15664 & 12355 & 25.79518 & 13498 \\
\hline 300 & 3.99340 & 2301 & 3.64997 & 1145 & 22.28019 & 11987 & 25.93016 & 13132 \\
\hline 310 & 4.01641 & 2205 & 3.66142 & 1144 & 22.40006 & 11642 & 26.06148 & 12787 \\
\hline 320 & 4.03846 & 2110 & 3.67286 & 1140 & 22.51648 & 11320 & 26.18935 & 12459 \\
\hline 330 & 4.05956 & 2018 & 3.68426 & 1134 & 22.62968 & 11015 & 26.31394 & 12149 \\
\hline 340 & 4.07974 & 1930 & 3.69560 & 1125 & 22.73983 & 10729 & 26.43543 & 11855 \\
\hline 350 & 4.09904 & 1843 & 3.70685 & 1115 & 22.84712 & 10458 & 26.55398 & 11573 \\
\hline 360 & 4.11747 & 1761 & 3.71800 & 1104 & 22.95170 & 10202 & 26.66971 & 11306 \\
\hline 370 & 4.13508 & 1682 & 3.72904 & 1091 & 23.05372 & 9960 & 26.78277 & 11050 \\
\hline 380 & 4.15190 & 1606 & 3.73995 & 1077 & 23.15332 & 9728 & 26.89327 & 10805 \\
\hline 390 & 4.16796 & 1535 & 3.75072 & 1062 & 23.25060 & 9510 & 27.00132 & 10572 \\
\hline 400 & 4.18331 & 6719 & 3.76134 & 5076 & 23.34570 & 44599 & 27.10704 & 49675 \\
\hline 450 & 4.25050 & 5406 & 3.81210 & 4664 & 23.79169 & 40409 & 27.60379 & 45074 \\
\hline 500 & 4.30456 & 4410 & 3.85874 & 4260 & 24.19578 & 36981 & 28.05453 & 41240 \\
\hline 550 & 4.34866 & 3653 & 3.90134 & 3884 & 24.56559 & 34115 & 28.46693 & 38000 \\
\hline 600 & 4.38519 & 3072 & 3.94018 & 3545 & 24.90674 & 31681 & 28.84693 & 35225 \\
\hline 650 & 4.41591 & 2624 & 3.97563 & 3241 & 25.22355 & 29583 & 29.19918 & 32824 \\
\hline 700 & 4.44215 & 2272 & 4.00804 & 2971 & 25.51938 & 27755 & 29.52742 & 30727 \\
\hline 750 & 4.46487 & 1995 & 4.03775 & 2733 & 25.79693 & 26148 & 29.83469 & 28881 \\
\hline 800 & 4.48482 & 1773 & 4.06508 & 2523 & 26.05841 & 24721 & 30.12350 & 27243 \\
\hline 850 & 4.50255 & 1593 & 4.09031 & 2335 & 26.30562 & 23446 & 30.39593 & 25782 \\
\hline 900 & 4.51848 & 1448 & 4.11366 & 2169 & 26.54008 & 22301 & 30.65375 & 24469 \\
\hline 950 & 4.53296 & 1326 & 4.13535 & 2022 & 26.76309 & 21263 & 30.89844 & 23285 \\
\hline 1000 & 4.54622 & 1227 & $4: 15557$ & 1890 & 26.97572 & 20321 & 31.13129 & 22212 \\
\hline 1050 & 4.55849 & 1143 & 4.17447 & 1771 & 27.17893 & 19461 & 31.35341 & 21232 \\
\hline 1100 & 4.56992 & 1072 & 4.19218 & 1666 & 27.37354 & 18673 & 31.56573 & 20338 \\
\hline 1150 & 4.58064 & 1013 & 4.20884 & 1571 & 27.56027 & 17946 & 31.76911 & 19517 \\
\hline
\end{tabular}


Table 2. 023. RbD (Cont.)

\begin{tabular}{|l|l|l|l|l|}
\hline${ }^{\circ}$ & $\frac{C_{p}^{0}}{R}$ & $\frac{\left(H^{\circ}-E_{0}^{0}\right)}{R T}$ & $\frac{-\left(F^{0}-E_{0}^{0}\right)}{R T}$ & $\frac{S^{\circ}}{R}$ \\
\hline
\end{tabular}

\begin{tabular}{|c|c|c|c|c|c|c|c|c|}
\hline $\begin{array}{l}1200 \\
1300 \\
1400 \\
1500 \\
1600\end{array}$ & $\begin{array}{l}4.59077 \\
4.60954 \\
4.62677 \\
4.64283 \\
4.65796\end{array}$ & $\begin{array}{l}1877 \\
1723 \\
1606 \\
1513 \\
1441\end{array}$ & $\begin{array}{l}4.22455 \\
4.25345 \\
4.27951 \\
4.30320 \\
4.32491\end{array}$ & $\begin{array}{l}2890 \\
2606 \\
2369 \\
2171 \\
2001\end{array}$ & $\begin{array}{l}27.73973 \\
28.07903 \\
28.39522 \\
28.69130 \\
28.96972\end{array}$ & $\begin{array}{l}33930 \\
31619 \\
29608 \\
27842 \\
26281\end{array}$ & $\begin{array}{l}31.96428 \\
32.33249 \\
32.67473 \\
32.99450 \\
33.29463\end{array}$ & $\begin{array}{l}36821 \\
34224 \\
31977 \\
30013 \\
28282\end{array}$ \\
\hline $\begin{array}{l}1700 \\
1800 \\
1900 \\
2000 \\
2100\end{array}$ & $\begin{array}{l}4.67237 \\
4.68619 \\
4.69952 \\
4.71245 \\
4.72504\end{array}$ & $\begin{array}{l}1382 \\
1333 \\
1293 \\
1259 \\
1229\end{array}$ & $\begin{array}{l}4.34492 \\
4.36350 \\
4.38084 \\
4.39710 \\
4.41241\end{array}$ & $\begin{array}{l}1858 \\
1734 \\
1626 \\
1531 \\
1449\end{array}$ & $\begin{array}{l}29.23253 \\
29.48141 \\
29.71780 \\
29.94293 \\
30.15783\end{array}$ & $\begin{array}{l}24888 \\
23639 \\
22513 \\
21490 \\
20561\end{array}$ & $\begin{array}{l}33.57745 \\
33.84491 \\
34.09864 \\
34.34003 \\
34.57025\end{array}$ & $\begin{array}{l}26746 \\
25373 \\
24139 \\
23022 \\
22010\end{array}$ \\
\hline $\begin{array}{l}2200 \\
2300 \\
2400 \\
2500 \\
2600\end{array}$ & $\begin{array}{l}4.73733 \\
4.74937 \\
4.76119 \\
4.77281 \\
4.78425\end{array}$ & $\begin{array}{l}1204 \\
1182 \\
1162 \\
1144 \\
1128\end{array}$ & $\begin{array}{l}4.42690 \\
4.44066 \\
4.45377 \\
4.46630 \\
4.47831\end{array}$ & $\begin{array}{l}1376 \\
1311 \\
1253 \\
1201 \\
1154\end{array}$ & $\begin{array}{l}30.36344 \\
30.56053 \\
30.74980 \\
30.93187 \\
31.10728\end{array}$ & $\begin{array}{l}19709 \\
18927 \\
18207 \\
17541 \\
16923\end{array}$ & $\begin{array}{l}34.79035 \\
35.00120 \\
35.20358 \\
35.39818 \\
35.58559\end{array}$ & $\begin{array}{l}21085 \\
20238 \\
19460 \\
18741 \\
18077\end{array}$ \\
\hline $\begin{array}{l}2700 \\
2800 \\
2900 \\
3000\end{array}$ & $\begin{array}{l}4.79553 \\
4.80667 \\
4.81767 \\
4.82855\end{array}$ & $\begin{array}{l}1114 \\
1100 \\
1088 \\
2141\end{array}$ & $\begin{array}{l}4.48985 \\
4.50097 \\
4.51170 \\
4.52208\end{array}$ & $\begin{array}{l}1112 \\
1073 \\
1038 \\
1983\end{array}$ & $\begin{array}{l}31.27651 \\
31.43999 \\
31.59813 \\
31.75126\end{array}$ & $\begin{array}{l}16348 \\
15814 \\
15313 \\
29249\end{array}$ & $\begin{array}{l}35.76636 \\
35.94097 \\
36.10983 \\
36.27334\end{array}$ & $\begin{array}{l}17461 \\
16886 \\
16351 \\
31232\end{array}$ \\
\hline $\begin{array}{l}3200 \\
3400 \\
3600 \\
3800 \\
4000\end{array}$ & $\begin{array}{l}4.84996 \\
4.87097 \\
4.89160 \\
4.91190 \\
4.93188\end{array}$ & $\begin{array}{l}2101 \\
2063 \\
2030 \\
1998 \\
1970\end{array}$ & $\begin{array}{l}4.54191 \\
4.56065 \\
4.57846 \\
4.59548 \\
4.61180\end{array}$ & $\begin{array}{l}1874 \\
1781 \\
1702 \\
1632 \\
1571\end{array}$ & $\begin{array}{l}32.04375 \\
32.31966 \\
32.58085 \\
32.82886 \\
33.06499\end{array}$ & $\begin{array}{l}27591 \\
26119 \\
24801 \\
23613 \\
22539\end{array}$ & $\begin{array}{l}36.58566 \\
36.88032 \\
37.15932 \\
37.42434 \\
37.67680\end{array}$ & $\begin{array}{l}29466 \\
27900 \\
26502 \\
25246 \\
24110\end{array}$ \\
\hline $\begin{array}{l}4200 \\
4400 \\
4600 \\
4800 \\
5000\end{array}$ & $\begin{array}{l}4.95158 \\
4.97099 \\
4.99014 \\
5.00904 \\
5.02769\end{array}$ & $\begin{array}{l}1941 \\
1915 \\
1890 \\
1865\end{array}$ & $\begin{array}{l}4.62751 \\
4.64269 \\
4.65738 \\
4.67164 \\
4.68551\end{array}$ & $\begin{array}{l}1518 \\
1469 \\
1426 \\
1387\end{array}$ & $\begin{array}{l}33.29038 \\
33.50601 \\
33.71271 \\
33.91123 \\
34.10222\end{array}$ & $\begin{array}{l}21563 \\
20670 \\
19852 \\
19099\end{array}$ & $\begin{array}{l}37.91790 \\
38.14870 \\
38.37009 \\
38.58287 \\
38.78773\end{array}$ & $\begin{array}{l}23080 \\
22139 \\
21278 \\
20486\end{array}$ \\
\hline
\end{tabular}


Table 2.024. RbT

\begin{tabular}{|l|l|l|l|l|}
\hline${ }^{\circ} K$ & $\frac{C_{p}^{0}}{R}$ & $\frac{\left(H^{\circ}-E_{0}^{0}\right)}{R T}$ & $\frac{-\left(F^{\circ}-E_{0}^{0}\right)}{R T}$ & $\frac{S^{\circ}}{R}$ \\
\hline
\end{tabular}

\begin{tabular}{|c|c|c|c|c|c|c|c|c|}
\hline $\begin{array}{l}50 \\
60 \\
70 \\
80 \\
90\end{array}$ & $\begin{array}{l}3.50196 \\
3.50271 \\
3.50461 \\
3.50900 \\
3.51722\end{array}$ & $\begin{array}{r}75 \\
190 \\
439 \\
822 \\
1301\end{array}$ & $\begin{array}{l}3.49110 \\
3.49296 \\
3.49447 \\
3.49598 \\
3.49784\end{array}$ & $\begin{array}{l}186 \\
151 \\
151 \\
186 \\
255\end{array}$ & $\begin{array}{l}16.37456 \\
17.01124 \\
17.54980 \\
18.01651 \\
18.42838\end{array}$ & $\begin{array}{l}63668 \\
53856 \\
46671 \\
41187 \\
36866\end{array}$ & $\begin{array}{l}19.86567 \\
20.50421 \\
21.04427 \\
21.51250 \\
21.92623\end{array}$ & $\begin{array}{l}63854 \\
54006 \\
46823 \\
41373 \\
37121\end{array}$ \\
\hline $\begin{array}{l}100 \\
110 \\
120 \\
130 \\
140\end{array}$ & $\begin{array}{l}3.53023 \\
3.54838 \\
3.57144 \\
3.59876 \\
3.62949\end{array}$ & $\begin{array}{l}1815 \\
2306 \\
2732 \\
3073 \\
3322\end{array}$ & $\begin{array}{l}3.50039 \\
3.50389 \\
3.50852 \\
3.51439 \\
3.52149\end{array}$ & $\begin{array}{l}350 \\
463 \\
587 \\
710 \\
830\end{array}$ & $\begin{array}{l}18.79704 \\
19.13082 \\
19.43589 \\
19.71695 \\
19.97764\end{array}$ & $\begin{array}{l}33378 \\
30507 \\
28106 \\
26069 \\
24324\end{array}$ & $\begin{array}{l}22.29744 \\
22.63471 \\
22.94442 \\
23.23134 \\
23.49914\end{array}$ & $\begin{array}{l}33727 \\
30971 \\
28692 \\
26780 \\
25153\end{array}$ \\
\hline $\begin{array}{l}150 \\
160 \\
170 \\
180 \\
190\end{array}$ & $\begin{array}{l}3.66271 \\
3.69754 \\
3.73322 \\
3.76909 \\
3.80466\end{array}$ & $\begin{array}{l}3483 \\
3568 \\
3587 \\
3557 \\
3485\end{array}$ & $\begin{array}{l}3.52979 \\
3.53918 \\
3.54954 \\
3.56074 \\
3.57265\end{array}$ & $\begin{array}{r}939 \\
1036 \\
1120 \\
1191 \\
1247\end{array}$ & $\begin{array}{l}20.22088 \\
20.44898 \\
20.66385 \\
20.86705 \\
21.05988\end{array}$ & $\begin{array}{l}22810 \\
21487 \\
20320 \\
19283 \\
18357\end{array}$ & $\begin{array}{l}23.75067 \\
23.98817 \\
24.21340 \\
24.42780 \\
24.63254\end{array}$ & $\begin{array}{l}23750 \\
22523 \\
21440 \\
20474 \\
19604\end{array}$ \\
\hline $\begin{array}{l}200 \\
210 \\
220 \\
230 \\
240\end{array}$ & $\begin{array}{l}3.83951 \\
3.87337 \\
3.90604 \\
3.93739 \\
3.96734\end{array}$ & $\begin{array}{l}3386 \\
3267 \\
3135 \\
2995 \\
2852\end{array}$ & $\begin{array}{l}3.58512 \\
3.59805 \\
3.61131 \\
3.62481 \\
3.63846\end{array}$ & $\begin{array}{l}1293 \\
1326 \\
1350 \\
1365 \\
1373\end{array}$ & $\begin{array}{l}21.24345 \\
21.41868 \\
21.58637 \\
21.74719 \\
21.90175\end{array}$ & $\begin{array}{l}17523 \\
16769 \\
16082 \\
15456 \\
14881\end{array}$ & $\begin{array}{l}24.82858 \\
25.01673 \\
25.19768 \\
25.37201 \\
25.54022\end{array}$ & $\begin{array}{l}18815 \\
18095 \\
17433 \\
16821 \\
16254\end{array}$ \\
\hline $\begin{array}{l}250 \\
260 \\
270 \\
280 \\
290\end{array}$ & $\begin{array}{l}3.99586 \\
4.02295 \\
4.04864 \\
4.07297 \\
4.09599\end{array}$ & $\begin{array}{l}2709 \\
2569 \\
2433 \\
2302 \\
2176\end{array}$ & $\begin{array}{l}3.65219 \\
3.66594 \\
3.67964 \\
3.69326 \\
3.70675\end{array}$ & $\begin{array}{l}1375 \\
1370 \\
1362 \\
1349 \\
1334\end{array}$ & $\begin{array}{l}22.05056 \\
22.19407 \\
22.33268 \\
22.46675 \\
22.59659\end{array}$ & $\begin{array}{l}14351 \\
13861 \\
13407 \\
12984 \\
12589\end{array}$ & $\begin{array}{l}25.70276 \\
25.86001 \\
26.01233 \\
26.16001 \\
26.30334\end{array}$ & $\begin{array}{l}15725 \\
15232 \\
14768 \\
14333 \\
13923\end{array}$ \\
\hline $\begin{array}{l}300 \\
310 \\
320 \\
330 \\
340\end{array}$ & $\begin{array}{l}4.11775 \\
4.13832 \\
4.15776 \\
4.17613 \\
4.19351\end{array}$ & $\begin{array}{l}2057 \\
1944 \\
1837 \\
1738 \\
1643\end{array}$ & $\begin{array}{l}3.72009 \\
3.73325 \\
3.74622 \\
3.75897 \\
3.77150\end{array}$ & $\begin{array}{l}1316 \\
1297 \\
1275 \\
1253 \\
1229\end{array}$ & $\begin{array}{l}22.72248 \\
22.84467 \\
22.96340 \\
23.07888 \\
23.19128\end{array}$ & $\begin{array}{l}12219 \\
11873 \\
11548 \\
11240 \\
10950\end{array}$ & $\begin{array}{l}26.44257 \\
26.57793 \\
26.70963 \\
26.83785 \\
26.96278\end{array}$ & $\begin{array}{l}13536 \\
13170 \\
12822 \\
12493 \\
12180\end{array}$ \\
\hline $\begin{array}{l}350 \\
360 \\
370 \\
380 \\
390\end{array}$ & $\begin{array}{l}4.20994 \\
4.22550 \\
4.24023 \\
4.25418 \\
4.26742\end{array}$ & $\begin{array}{l}1556 \\
1473 \\
1395 \\
1324 \\
1256\end{array}$ & $\begin{array}{l}3.78379 \\
3.79585 \\
3.80766 \\
3.81923 \\
3.83055\end{array}$ & $\begin{array}{l}1206 \\
1181 \\
1157 \\
1132 \\
1108\end{array}$ & $\begin{array}{l}23.30078 \\
23.40755 \\
23.51171 \\
23.61341 \\
23.71276\end{array}$ & $\begin{array}{r}10677 \\
10416 \\
10170 \\
9935 \\
9712\end{array}$ & $\begin{array}{l}27.08458 \\
27.20340 \\
27.31938 \\
27.43264 \\
27.54332\end{array}$ & $\begin{array}{l}11882 \\
11598 \\
11326 \\
11068 \\
10820\end{array}$ \\
\hline $\begin{array}{l}400 \\
450 \\
500 \\
550 \\
600\end{array}$ & $\begin{array}{l}4.27998 \\
4.33409 \\
4.37679 \\
4.41125 \\
4.43966\end{array}$ & $\begin{array}{l}5411 \\
4270 \\
3446 \\
2841 \\
2389\end{array}$ & $\begin{array}{l}3.84163 \\
3.89347 \\
3.93975 \\
3.98110 \\
4.01816\end{array}$ & $\begin{array}{l}5184 \\
4628 \\
4135 \\
3705 \\
3337\end{array}$ & $\begin{array}{l}23.80988 \\
24.26541 \\
24.67807 \\
25.05555 \\
25.40357\end{array}$ & $\begin{array}{l}45553 \\
41266 \\
37748 \\
34802 \\
32296\end{array}$ & $\begin{array}{l}27.65152 \\
28.15889 \\
28.61782 \\
29.03665 \\
29.42173\end{array}$ & $\begin{array}{l}50737 \\
45893 \\
41883 \\
38508 \\
35634\end{array}$ \\
\hline $\begin{array}{l}650 \\
700 \\
750 \\
800 \\
850\end{array}$ & $\begin{array}{l}4.46355 \\
4.48402 \\
4.50186 \\
4.51763 \\
4.53178\end{array}$ & $\begin{array}{l}2047 \\
1784 \\
1577 \\
1415 \\
1284\end{array}$ & $\begin{array}{l}4.05153 \\
4.08171 \\
4.10914 \\
4.13418 \\
4.15716\end{array}$ & $\begin{array}{l}3018 \\
2743 \\
2504 \\
2298 \\
2118\end{array}$ & $\begin{array}{l}25.72653 \\
26.02791 \\
26.31046 \\
26.57647 \\
26.82781\end{array}$ & $\begin{array}{l}30138 \\
28255 \\
26601 \\
25134 \\
23822\end{array}$ & $\begin{array}{l}29.77807 \\
30.10962 \\
30.41961 \\
30.71066 \\
30.98497\end{array}$ & $\begin{array}{l}33155 \\
30999 \\
29105 \\
27431 \\
25940\end{array}$ \\
\hline $\begin{array}{r}900 \\
950 \\
1000 \\
1050 \\
1100\end{array}$ & $\begin{array}{l}4.54462 \\
4.55641 \\
4.56733 \\
4.57753 \\
4.58714\end{array}$ & $\begin{array}{r}1179 \\
1092 \\
1020 \\
961 \\
910\end{array}$ & $\begin{array}{l}4.17834 \\
4.19793 \\
4.21613 \\
4.23310 \\
4.24898\end{array}$ & $\begin{array}{l}1959 \\
1820 \\
1697 \\
1588 \\
1490\end{array}$ & $\begin{array}{l}27.06603 \\
27.29247 \\
27.50827 \\
27.71439 \\
27.91168\end{array}$ & $\begin{array}{l}22644 \\
21580 \\
20612 \\
19729 \\
18921\end{array}$ & $\begin{array}{l}31.24437 \\
31.49041 \\
31.72440 \\
31.94749 \\
32.16066\end{array}$ & $\begin{array}{l}24604 \\
23399 \\
22309 \\
21317 \\
20411\end{array}$ \\
\hline 1150 & 4.59624 & 868 & 4.26388 & 1403 & 28.10089 & 18177 & 32.36477 & 19580 \\
\hline
\end{tabular}


Table 2. 024. RbT (Cont.)

\begin{tabular}{|c|c|c|c|c|c|c|c|c|}
\hline${ }^{\circ} \mathrm{K}$ & $\frac{C_{p}^{0}}{R}$ & & $\frac{\left(H^{\circ}-E_{0}^{0}\right)}{R T}$ & & $\frac{-\left(F^{\circ}-E\right.}{R T}$ & & $\frac{S^{0}}{R}$ & \\
\hline $\begin{array}{l}1200 \\
1300 \\
1400 \\
1500 \\
1600\end{array}$ & $\begin{array}{l}4.60492 \\
4.62123 \\
4.63645 \\
4.65083 \\
4.66455\end{array}$ & $\begin{array}{l}1631 \\
1522 \\
1438 \\
1372 \\
1321\end{array}$ & $\begin{array}{l}4.27791 \\
4.30370 \\
4.32693 \\
4.34805 \\
4.36740\end{array}$ & $\begin{array}{l}2579 \\
2323 \\
2112 \\
1935 \\
1787\end{array}$ & $\begin{array}{l}28.28266 \\
28.62611 \\
28.94592 \\
29.24517 \\
29.52642\end{array}$ & $\begin{array}{l}34345 \\
31981 \\
29925 \\
28125 \\
26531\end{array}$ & $\begin{array}{l}32.56057 \\
32.92982 \\
33.27285 \\
33.59323 \\
33.89383\end{array}$ & $\begin{array}{l}36925 \\
34303 \\
32038 \\
30060 \\
28318\end{array}$ \\
\hline $\begin{array}{l}1700 \\
1800 \\
1900 \\
2000 \\
2100\end{array}$ & $\begin{array}{l}4.67776 \\
4.69054 \\
4.70297 \\
4.71510 \\
4.72698\end{array}$ & $\begin{array}{l}1278 \\
1243 \\
1213 \\
1188 \\
1166\end{array}$ & $\begin{array}{l}4.38527 \\
4.40188 \\
4.41740 \\
4.43198 \\
4.44575\end{array}$ & $\begin{array}{l}1661 \\
1552 \\
1458 \\
1377 \\
1305\end{array}$ & $\begin{array}{l}29.79173 \\
30.04286 \\
30.28128 \\
30.50824 \\
30.72481\end{array}$ & $\begin{array}{l}25113 \\
23842 \\
22696 \\
21657 \\
20712\end{array}$ & $\begin{array}{l}34.17701 \\
34.44475 \\
34.69869 \\
34.94023 \\
35.17057\end{array}$ & $\begin{array}{l}26774 \\
25394 \\
24154 \\
23034 \\
22017\end{array}$ \\
\hline $\begin{array}{l}2200 \\
2300 \\
2400 \\
2500 \\
2600\end{array}$ & $\begin{array}{l}4.73864 \\
4.75012 \\
4.76141 \\
4.77256 \\
4.78357\end{array}$ & $\begin{array}{l}1148 \\
1129 \\
1115 \\
1101 \\
1088\end{array}$ & $\begin{array}{l}4.45880 \\
4.47121 \\
4.48307 \\
4.49443 \\
4.50534\end{array}$ & $\begin{array}{l}1241 \\
1186 \\
1136 \\
1091 \\
1051\end{array}$ & $\begin{array}{l}30.93193 \\
31.13041 \\
31.32096 \\
31.50420 \\
31.68069\end{array}$ & $\begin{array}{l}19848 \\
19055 \\
18324 \\
17649 \\
17023\end{array}$ & $\begin{array}{l}35.39074 \\
35.60163 \\
35.80403 \\
35.99863 \\
36.18603\end{array}$ & $\begin{array}{l}21089 \\
20240 \\
19460 \\
18740 \\
18074\end{array}$ \\
\hline $\begin{array}{l}2700 \\
2800 \\
2900 \\
3000\end{array}$ & $\begin{array}{l}4.79445 \\
4.80521 \\
4.81586 \\
4.82642\end{array}$ & $\begin{array}{l}1076 \\
1065 \\
1056 \\
2081\end{array}$ & $\begin{array}{l}4.51585 \\
4.52599 \\
4.53580 \\
4.54531\end{array}$ & $\begin{array}{r}1014 \\
981 \\
951 \\
1822\end{array}$ & $\begin{array}{l}31.85092 \\
32.01533 \\
32.17433 \\
32.32826\end{array}$ & $\begin{array}{l}16441 \\
15900 \\
15393 \\
29393\end{array}$ & $\begin{array}{l}36.36677 \\
36.54133 \\
36.71013 \\
36.87358\end{array}$ & $\begin{array}{l}17456 \\
16880 \\
16345 \\
31215\end{array}$ \\
\hline $\begin{array}{l}3200 \\
3400 \\
3600 \\
3800 \\
4000\end{array}$ & $\begin{array}{l}4.84723 \\
4.86770 \\
4.88786 \\
4.90771 \\
4.92729\end{array}$ & $\begin{array}{l}2047 \\
2016 \\
1985 \\
1958 \\
1931\end{array}$ & $\begin{array}{l}4.56353 \\
4.58083 \\
4.59732 \\
4.61314 \\
4.62836\end{array}$ & $\begin{array}{l}1730 \\
1649 \\
1582 \\
1522 \\
1469\end{array}$ & $\begin{array}{l}32.62219 \\
32.89938 \\
33.16168 \\
33.41068 \\
33.64769\end{array}$ & $\begin{array}{l}27719 \\
26230 \\
24900 \\
23701 \\
22617\end{array}$ & $\begin{array}{l}37.18573 \\
37.48021 \\
37.75901 \\
38.02382 \\
38.27605\end{array}$ & $\begin{array}{l}29448 \\
27880 \\
26481 \\
25223 \\
24087\end{array}$ \\
\hline $\begin{array}{l}4200 \\
4400 \\
4600 \\
4800 \\
5000\end{array}$ & $\begin{array}{l}4.94660 \\
4.96566 \\
4.98447 \\
5.00306 \\
5.02141\end{array}$ & $\begin{array}{l}1906 \\
1881 \\
1859 \\
1835\end{array}$ & $\begin{array}{l}4.64305 \\
4.65729 \\
4.67110 \\
4.68455 \\
4.69766\end{array}$ & $\begin{array}{l}1424 \\
1381 \\
1345 \\
1311\end{array}$ & $\begin{array}{l}33.87386 \\
34.09019 \\
34.29752 \\
34.49661 \\
34.68811\end{array}$ & $\begin{array}{l}21633 \\
20733 \\
19909 \\
19150\end{array}$ & $\begin{array}{l}38.51692 \\
38.74748 \\
38.96863 \\
39.18116 \\
39.38577\end{array}$ & $\begin{array}{l}23056 \\
22115 \\
21253 \\
20461\end{array}$ \\
\hline
\end{tabular}


Table 2.025. CsH

\begin{tabular}{|l|l|l|l|l|}
\hline${ }^{\circ}$ & $\frac{C_{p}^{0}}{R}$ & $\frac{\left(H^{0}-E_{0}^{0}\right)}{R T}$ & $\frac{-\left(F^{0}-E_{0}^{0}\right)}{R T}$ & $\frac{S^{0}}{R}$ \\
\hline
\end{tabular}

\begin{tabular}{|c|c|c|c|c|c|c|c|c|}
\hline $\begin{array}{l}50 \\
60 \\
70 \\
80 \\
90\end{array}$ & $\begin{array}{l}3.50207 \\
3.50241 \\
3.50278 \\
3.50319 \\
3.50371\end{array}$ & $\begin{array}{l}34 \\
37 \\
41 \\
52 \\
81\end{array}$ & $\begin{array}{l}3.47513 \\
3.47965 \\
3.48293 \\
3.48543 \\
3.48743\end{array}$ & $\begin{array}{l}452 \\
328 \\
250 \\
200 \\
167\end{array}$ & $\begin{array}{l}16.05081 \\
16.68483 \\
17.22148 \\
17.68673 \\
18.09737\end{array}$ & $\begin{array}{l}63402 \\
53665 \\
46525 \\
41054 \\
36753\end{array}$ & $\begin{array}{l}19.52594 \\
20.16448 \\
20.70441 \\
21.17216 \\
21.58481\end{array}$ & $\begin{array}{l}63854 \\
53993 \\
46775 \\
41265 \\
36919\end{array}$ \\
\hline $\begin{array}{l}100 \\
110 \\
120 \\
130 \\
140\end{array}$ & $\begin{array}{l}3.50452 \\
3.50586 \\
3.50808 \\
3.51153 \\
3.51652\end{array}$ & $\begin{array}{l}134 \\
222 \\
345 \\
499 \\
678\end{array}$ & $\begin{array}{l}3.48910 \\
3.49056 \\
3.49192 \\
3.49328 \\
3.49476\end{array}$ & $\begin{array}{l}146 \\
136 \\
136 \\
148 \\
166\end{array}$ & $\begin{array}{l}18.46490 \\
18.79751 \\
19.10129 \\
19.38085 \\
19.63978\end{array}$ & $\begin{array}{l}33261 \\
30378 \\
27956 \\
25893 \\
24117\end{array}$ & $\begin{array}{l}21.95400 \\
22.28807 \\
22.59321 \\
22.87414 \\
23.13454\end{array}$ & $\begin{array}{l}33407 \\
30514 \\
28093 \\
26040 \\
24284\end{array}$ \\
\hline $\begin{array}{l}150 \\
160 \\
170 \\
180 \\
190\end{array}$ & $\begin{array}{l}3.52330 \\
3.53203 \\
3.54278 \\
3.55551 \\
3.57012\end{array}$ & $\begin{array}{r}873 \\
1075 \\
1273 \\
1461 \\
1634\end{array}$ & $\begin{array}{l}3.49642 \\
3.49836 \\
3.50065 \\
3.50334 \\
3.50646\end{array}$ & $\begin{array}{l}194 \\
229 \\
269 \\
312 \\
358\end{array}$ & $\begin{array}{l}19.88095 \\
20.10667 \\
20.31882 \\
20.51899 \\
20.70848\end{array}$ & $\begin{array}{l}22572 \\
21215 \\
20017 \\
18949 \\
17995\end{array}$ & $\begin{array}{l}23.37738 \\
23.60503 \\
23.81948 \\
24.02233 \\
24.21495\end{array}$ & $\begin{array}{l}22765 \\
21445 \\
20285 \\
19262 \\
18353\end{array}$ \\
\hline $\begin{array}{l}200 \\
210 \\
220 \\
230 \\
240\end{array}$ & $\begin{array}{l}3.58646 \\
3.60433 \\
3.62352 \\
3.64380 \\
3.66495\end{array}$ & $\begin{array}{l}1787 \\
1919 \\
2028 \\
2115 \\
2182\end{array}$ & $\begin{array}{l}3.51004 \\
3.51410 \\
3.51864 \\
3.52363 \\
3.52908\end{array}$ & $\begin{array}{l}406 \\
454 \\
499 \\
545 \\
587\end{array}$ & $\begin{array}{l}20.88843 \\
21.05978 \\
21.22336 \\
21.37988 \\
21.52996\end{array}$ & $\begin{array}{l}17135 \\
16358 \\
15552 \\
15008 \\
14418\end{array}$ & $\begin{array}{l}24.39848 \\
24.57389 \\
24.74200 \\
24.90352 \\
25.05904\end{array}$ & $\begin{array}{l}17541 \\
16811 \\
16152 \\
15552 \\
15005\end{array}$ \\
\hline $\begin{array}{l}250 \\
260 \\
270 \\
280 \\
290\end{array}$ & $\begin{array}{l}3.68677 \\
3.70905 \\
3.73163 \\
3.75434 \\
3.77706\end{array}$ & $\begin{array}{l}2228 \\
2258 \\
2271 \\
2272 \\
2260\end{array}$ & $\begin{array}{l}3.53495 \\
3.54121 \\
3.54785 \\
3.55482 \\
3.56209\end{array}$ & $\begin{array}{l}626 \\
664 \\
697 \\
727 \\
754\end{array}$ & $\begin{array}{l}21.67414 \\
21.51290 \\
21.94667 \\
22.07583 \\
22.20069\end{array}$ & $\begin{array}{l}13876 \\
13377 \\
12916 \\
12486 \\
12089\end{array}$ & $\begin{array}{l}25.20909 \\
25.35412 \\
25.49453 \\
25.63065 \\
25.76279\end{array}$ & $\begin{array}{l}14503 \\
14041 \\
13612 \\
13214 \\
12843\end{array}$ \\
\hline $\begin{array}{l}300 \\
310 \\
320 \\
330 \\
340\end{array}$ & $\begin{array}{l}3.79966 \\
3.82204 \\
3.84412 \\
3.86584 \\
3.88713\end{array}$ & $\begin{array}{l}2238 \\
2208 \\
2172 \\
2129 \\
2083\end{array}$ & $\begin{array}{l}3.56963 \\
3.57741 \\
3.58540 \\
3.59357 \\
3.60190\end{array}$ & $\begin{array}{l}778 \\
799 \\
817 \\
833 \\
844\end{array}$ & $\begin{array}{l}22.32158 \\
22.43876 \\
22.55246 \\
22.66291 \\
22.77032\end{array}$ & $\begin{array}{l}11718 \\
11370 \\
11045 \\
10741 \\
10453\end{array}$ & $\begin{array}{l}25.89122 \\
26.01617 \\
26.13787 \\
26.25649 \\
26.37222\end{array}$ & $\begin{array}{l}12495 \\
12170 \\
11862 \\
11573 \\
11298\end{array}$ \\
\hline $\begin{array}{l}350 \\
360 \\
370 \\
380 \\
390\end{array}$ & $\begin{array}{l}3.90796 \\
3.92830 \\
3.94812 \\
3.96740 \\
3.98614\end{array}$ & $\begin{array}{l}2034 \\
1982 \\
1928 \\
1874 \\
1819\end{array}$ & $\begin{array}{l}3.61034 \\
3.61890 \\
3.62753 \\
3.63622 \\
3.64495\end{array}$ & $\begin{array}{l}856 \\
863 \\
869 \\
873 \\
876\end{array}$ & $\begin{array}{l}22.87485 \\
22.97667 \\
23.07595 \\
23.17280 \\
23.26737\end{array}$ & $\begin{array}{r}10182 \\
9928 \\
9685 \\
9457 \\
9239\end{array}$ & $\begin{array}{l}26.48520 \\
26.59557 \\
26.70348 \\
26.30902 \\
26.91232\end{array}$ & $\begin{array}{l}11037 \\
10791 \\
10554 \\
10330 \\
10115\end{array}$ \\
\hline $\begin{array}{l}400 \\
450 \\
500 \\
550 \\
600\end{array}$ & $\begin{array}{l}4.00433 \\
4.08715 \\
4.15736 \\
4.21672 \\
4.26706\end{array}$ & $\begin{array}{l}8282 \\
7021 \\
5936 \\
5034 \\
4297\end{array}$ & $\begin{array}{l}3.65371 \\
3.69739 \\
3.73998 \\
3.78069 \\
3.81918\end{array}$ & $\begin{array}{l}4368 \\
4259 \\
4071 \\
3849 \\
3615\end{array}$ & $\begin{array}{l}23.35976 \\
23.79263 \\
24.18441 \\
24.54279 \\
24.87342\end{array}$ & $\begin{array}{l}43287 \\
39178 \\
35838 \\
33063 \\
30715\end{array}$ & $\begin{array}{l}27.01347 \\
27.49003 \\
27.92439 \\
28.32349 \\
28.69261\end{array}$ & $\begin{array}{l}47656 \\
43436 \\
39910 \\
36912 \\
34329\end{array}$ \\
\hline $\begin{array}{l}650 \\
700 \\
750 \\
800 \\
850\end{array}$ & $\begin{array}{l}4.31003 \\
4.34701 \\
4.37912 \\
4.40725 \\
4.43211\end{array}$ & $\begin{array}{l}3698 \\
3211 \\
2813 \\
2486 \\
2218\end{array}$ & $\begin{array}{l}3.85533 \\
3.88916 \\
3.92078 \\
3.95032 \\
3.97795\end{array}$ & $\begin{array}{l}3383 \\
3162 \\
2954 \\
2763 \\
2586\end{array}$ & $\begin{array}{l}25.18057 \\
25.46753 \\
25.73694 \\
25.99094 \\
26.23126\end{array}$ & $\begin{array}{l}28696 \\
26941 \\
25400 \\
24032 \\
22812\end{array}$ & $\begin{array}{l}29.03590 \\
29.35670 \\
29.65773 \\
29.94127 \\
30.20922\end{array}$ & $\begin{array}{l}32080 \\
30103 \\
28354 \\
26795 \\
25397\end{array}$ \\
\hline $\begin{array}{r}900 \\
950 \\
1000 \\
1050 \\
1100\end{array}$ & $\begin{array}{l}4.45429 \\
4.47423 \\
4.49232 \\
4.50883 \\
4.52402\end{array}$ & $\begin{array}{l}1994 \\
1809 \\
1651 \\
1519 \\
1407\end{array}$ & $\begin{array}{l}4.00381 \\
4.02805 \\
4.05082 \\
4.07224 \\
4.09244\end{array}$ & $\begin{array}{l}2424 \\
2277 \\
2142 \\
2020 \\
1907\end{array}$ & $\begin{array}{l}26.45938 \\
26.67651 \\
26.88371 \\
27.08187 \\
27.27178\end{array}$ & $\begin{array}{l}21713 \\
20720 \\
19816 \\
18991 \\
18234\end{array}$ & $\begin{array}{l}30.46319 \\
30.70456 \\
30.93453 \\
31.15412 \\
31.36422\end{array}$ & $\begin{array}{l}24137 \\
22997 \\
21959 \\
21010 \\
20142\end{array}$ \\
\hline 15 & 4.53809 & 1310 & 4.11151 & 1805 & 27.45412 & 17537 & 31.56564 & 19342 \\
\hline
\end{tabular}


Table 2. 025. CsH (Cont.)

\begin{tabular}{|c|c|c|c|c|c|c|c|c|}
\hline ik & $\frac{C_{p}^{0}}{R}$ & & $\frac{\left(H^{\circ}-E_{0}^{0}\right)}{R T}$ & & $\frac{-\left(F^{0}-E\right.}{R T}$ & & $\frac{S^{0}}{R}$ & \\
\hline $\begin{array}{l}1200 \\
1300 \\
1400 \\
1500 \\
1600\end{array}$ & $\begin{array}{l}4.55119 \\
4.57502 \\
4.59633 \\
4.61573 \\
4.63362\end{array}$ & $\begin{array}{l}2383 \\
2131 \\
1940 \\
1789 \\
1671\end{array}$ & $\begin{array}{l}4.12956 \\
4.16293 \\
4.19314 \\
4.22067 \\
4.24593\end{array}$ & $\begin{array}{l}3337 \\
3021 \\
2753 \\
2526 \\
2330\end{array}$ & $\begin{array}{l}27.62949 \\
27.96137 \\
28.27100 \\
28.56125 \\
28.83447\end{array}$ & $\begin{array}{l}33188 \\
30963 \\
29025 \\
27322 \\
25811\end{array}$ & $\begin{array}{l}31.75906 \\
32.12431 \\
32.46415 \\
32.78193 \\
33.08040\end{array}$ & $\begin{array}{l}36525 \\
33984 \\
31778 \\
29847 \\
28142\end{array}$ \\
\hline $\begin{array}{l}1700 \\
1800 \\
1900 \\
2000 \\
2100\end{array}$ & $\begin{array}{l}4.65033 \\
4.66608 \\
4.68105 \\
4.69537 \\
4.70915\end{array}$ & $\begin{array}{l}1575 \\
1497 \\
1432 \\
1378 \\
1332\end{array}$ & $\begin{array}{l}4.26923 \\
4.29085 \\
4.31099 \\
4.32986 \\
4.34759\end{array}$ & $\begin{array}{l}2162 \\
2014 \\
1887 \\
1773 \\
1674\end{array}$ & $\begin{array}{l}29.09258 \\
29.33723 \\
29.56977 \\
29.79138 \\
30.00307\end{array}$ & $\begin{array}{l}24465 \\
23254 \\
22161 \\
21169 \\
20264\end{array}$ & $\begin{array}{l}33.36182 \\
33.62808 \\
33.88076 \\
34.12124 \\
34.35066\end{array}$ & $\begin{array}{l}26626 \\
25268 \\
24048 \\
22942 \\
21938\end{array}$ \\
\hline $\begin{array}{l}2200 \\
2300 \\
2400 \\
2500 \\
2600\end{array}$ & $\begin{array}{l}4.72247 \\
4.73540 \\
4.74798 \\
4.76027 \\
4.77230\end{array}$ & $\begin{array}{l}1293 \\
1258 \\
1229 \\
1203 \\
1180\end{array}$ & $\begin{array}{l}4.36433 \\
4.38018 \\
4.39525 \\
4.40960 \\
4.42332\end{array}$ & $\begin{array}{l}1585 \\
1507 \\
1435 \\
1372 \\
1315\end{array}$ & $\begin{array}{l}30.20571 \\
30.40006 \\
30.58680 \\
30.76652 \\
30.93973\end{array}$ & $\begin{array}{l}19435 \\
18674 \\
17972 \\
17321 \\
16719\end{array}$ & $\begin{array}{l}34.57004 \\
34.78025 \\
34.98205 \\
35.17613 \\
35.36306\end{array}$ & $\begin{array}{l}21021 \\
20180 \\
19408 \\
18693 \\
18033\end{array}$ \\
\hline $\begin{array}{l}2700 \\
2800 \\
2900 \\
3000\end{array}$ & $\begin{array}{l}4.78410 \\
4.79568 \\
4.80708 \\
4.81830\end{array}$ & $\begin{array}{l}1158 \\
1140 \\
1122 \\
2200\end{array}$ & $\begin{array}{l}4.43647 \\
4.44909 \\
4.46124 \\
4.47295\end{array}$ & $\begin{array}{l}1262 \\
1215 \\
1171 \\
2228\end{array}$ & $\begin{array}{l}31.10692 \\
31.26849 \\
31.42483 \\
31.57627\end{array}$ & $\begin{array}{l}16157 \\
15634 \\
15144 \\
28940\end{array}$ & $\begin{array}{l}35.54339 \\
35.71759 \\
35.88607 \\
36.04923\end{array}$ & $\begin{array}{l}17420 \\
16848 \\
16316 \\
31167\end{array}$ \\
\hline $\begin{array}{l}3200 \\
3400 \\
3600 \\
3800 \\
4000\end{array}$ & $\begin{array}{l}4.84030 \\
4.86175 \\
4.38273 \\
4.90330 \\
4.92349\end{array}$ & $\begin{array}{l}2145 \\
2098 \\
2057 \\
2019 \\
1985\end{array}$ & $\begin{array}{l}4.49523 \\
4.51616 \\
4.53594 \\
4.55474 \\
4.57267\end{array}$ & $\begin{array}{l}2093 \\
1978 \\
1880 \\
1793 \\
1718\end{array}$ & $\begin{array}{l}31.86567 \\
32.13883 \\
32.39753 \\
32.64328 \\
32.87737\end{array}$ & $\begin{array}{l}27316 \\
25870 \\
24575 \\
23409 \\
22352\end{array}$ & $\begin{array}{l}36.36090 \\
36.65499 \\
36.93348 \\
37.19803 \\
37.45005\end{array}$ & $\begin{array}{l}29409 \\
27849 \\
26455 \\
25202 \\
24070\end{array}$ \\
\hline $\begin{array}{l}4200 \\
4400 \\
4600 \\
4800 \\
5000\end{array}$ & $\begin{array}{l}4.94334 \\
4.96287 \\
4.98210 \\
5.00105 \\
5.01973\end{array}$ & $\begin{array}{l}1953 \\
1923 \\
1895 \\
1868\end{array}$ & $\begin{array}{l}4.58985 \\
4.60637 \\
4.62228 \\
4.63767 \\
4.65258\end{array}$ & $\begin{array}{l}1652 \\
1591 \\
1539 \\
1491\end{array}$ & $\begin{array}{l}33.10089 \\
33.31479 \\
33.51991 \\
33.71696 \\
33.90658\end{array}$ & $\begin{array}{l}21390 \\
20512 \\
19705 \\
18962\end{array}$ & $\begin{array}{l}37.69075 \\
37.92116 \\
38.14220 \\
38.35464 \\
38.55917\end{array}$ & $\begin{array}{l}23041 \\
22104 \\
21244 \\
20453\end{array}$ \\
\hline
\end{tabular}


Table 2.026. CsD

\begin{tabular}{|l|l|l|l|l|}
\hline$K$ & $\frac{C_{p}^{0}}{R}$ & $\frac{\left(H^{0}-E_{0}^{0}\right)}{R T}$ & $\frac{-\left(F^{0}-E_{0}^{0}\right)}{R T}$ & $\frac{S^{0}}{R}$ \\
\hline
\end{tabular}

\begin{tabular}{|c|c|c|c|c|c|c|c|c|}
\hline 50 & 3.50197 & 45 & 3.48792 & 238 & 16.73047 & 63614 & 20.21840 & 63852 \\
\hline 60 & 3.50242 & 79 & 3.49030 & 178 & 17.36661 & 53818 & 20.85692 & 53995 \\
\hline 70 & 3.50321 & 172 & 3.49208 & 149 & 17.90479 & 46640 & 21.39687 & 46789 \\
\hline 80 & 3.50493 & 353 & 3.49357 & 143 & 18.37119 & 41156 & 21.86476 & 41300 \\
\hline 90 & 3.50846 & 626 & 3.49500 & 164 & 18.78275 & 36832 & 22.27776 & 36995 \\
\hline 100 & 3.51472 & 970 & 3.49664 & 205 & 19.15107 & 33336 & 22.64771 & 33542 \\
\hline 110 & 3.52442 & 1354 & 3.49869 & 268 & 19.48443 & 30454 & 22.98313 & 30721 \\
\hline 120 & 3.53796 & 1739 & 3.50137 & 346 & 19.78897 & 28039 & 23.29034 & 28385 \\
\hline 130 & 3.55535 & 2099 & 3.50483 & 434 & 20.06936 & 25989 & 23.57419 & 26423 \\
\hline 140 & 3.57634 & 2411 & 3.50917 & 527 & 20.32925 & 24228 & 23.83842 & 24755 \\
\hline 150 & 3.60045 & 2668 & 3.51444 & 619 & 20.57153 & 22701 & 24.08597 & 23321 \\
\hline 160 & 3.62713 & 2864 & 3.52063 & 710 & 20.79854 & 21364 & 24.31918 & 22074 \\
\hline 170 & 3.65577 & 3000 & 3.52773 & 794 & 21.01218 & 20186 & 24.53992 & 20980 \\
\hline 180 & 3.68577 & 3085 & 3.53567 & 871 & 21.21404 & 19140 & 24.74972 & 20011 \\
\hline 190 & 3.71662 & 3124 & 3.54438 & 940 & 21.40544 & 18204 & 24.94983 & 19143 \\
\hline 200 & 3.74786 & 3124 & 3.55378 & 998 & 21.58748 & 17363 & 25.14126 & 18361 \\
\hline 210 & 3.77910 & 3092 & 3.56376 & 1049 & 21.76111 & 16602 & 25.32487 & 17652 \\
\hline 220 & 3.81002 & 3037 & 3.57425 & 1092 & 21.92713 & 15913 & 25.50139 & 17004 \\
\hline 230 & 3.84039 & 2963 & 3.58517 & 1125 & 22.08626 & 15282 & 25.67143 & 16407 \\
\hline 240 & 3.87002 & 2875 & 3.59642 & 1152 & 22.23908 & 14704 & 25.83550 & 15857 \\
\hline 250 & 3.89877 & 2778 & 3.60794 & 1173 & 22.38612 & 14174 & 25.99407 & 15346 \\
\hline 260 & 3.92655 & 2675 & 3.61967 & 1186 & 22.52786 & 13683 & 26.14753 & 14869 \\
\hline 270 & 3.95330 & 2569 & 3.63153 & 1196 & 22.66469 & 13228 & 26.29622 & 14424 \\
\hline 280 & 3.97899 & 2461 & 3.64349 & 1199 & 22.79697 & 12807 & 26.44046 & 14006 \\
\hline 290 & 4.00360 & 2353 & 3.65548 & 1200 & 22.92504 & 12412 & 26.58052 & 13613 \\
\hline 300 & 4.02713 & 2248 & 3.66748 & 1197 & 23.04916 & 12046 & 26.71665 & 13242 \\
\hline 310 & 4.04961 & 2145 & 3.67945 & 1190 & 23.16962 & 11700 & 26.84907 & 12891 \\
\hline 320 & 4.07106 & 2045 & 3.69135 & 1182 & 23.28662 & 11377 & 26.97798 & 12559 \\
\hline 330 & 4.09151 & 1950 & 3.70317 & 1171 & 23.40039 & 11073 & 27.10357 & 12244 \\
\hline 340 & 4.11101 & 1857 & 3.71488 & 1159 & 23.51112 & 10785 & 27.22601 & 11943 \\
\hline 350 & 4. 12958 & 1769 & 3.72647 & 1144 & 23.61897 & 10514 & 27.34544 & 11659 \\
\hline 360 & 4.14727 & 1685 & 3.73791 & 1130 & 23.72411 & 10257 & 27.46203 & 11386 \\
\hline 370 & 4.16412 & 1606 & 3.74921 & 1113 & 23.82668 & 10013 & 27.57589 & 11126 \\
\hline 380 & 4.18018 & 1530 & 3.76034 & 1096 & 23.92681 & 9782 & 27.68715 & 10879 \\
\hline 390 & 4.19548 & 1458 & 3.77130 & 1079 & 24.02463 & 9562 & 27.79594 & 10640 \\
\hline 400 & 4.21006 & 6351 & 3.78209 & 5122 & 24.12025 & 44847 & 27.90234 & 49969 \\
\hline 450 & 4.27357 & 5075 & 3.83331 & 4665 & 24.56872 & 40633 & 28.40203 & 45299 \\
\hline 500 & 4.32432 & 4120 & 3.87996 & 4233 & 24.97505 & 37182 & 28.85502 & 41415 \\
\hline 550 & 4.36552 & 3403 & 3.92229 & 3840 & 25.34687 & 34296 & 29.26917 & 38135 \\
\hline 600 & 4.39955 & 2858 & 3.96069 & 3489 & 25.68983 & 31842 & 29.65052 & 35331 \\
\hline 650 & 4.42813 & 2438 & 3.99558 & 3179 & 26.00825 & 29729 & 30.00383 & 32908 \\
\hline 700 & 4.45251 & 2113 & 4.02737 & 2906 & 26.30554 & 27886 & 30.33291 & 30793 \\
\hline 750 & 4.47364 & 1855 & 4.05643 & 2667 & 26.58440 & 26266 & 30.64084 & 28932 \\
\hline 800 & 4.49219 & 1651 & 4.08310 & 2456 & 26.84706 & 24828 & 30.93016 & 27285 \\
\hline 850 & 4.50870 & 1485 & 4.10766 & 2269 & 27.09534 & 23544 & 31.20301 & 25813 \\
\hline 900 & 4.52355 & 1351 & 4.13035 & 2106 & 27.33078 & 22389 & 31.46114 & 24495 \\
\hline 950 & 4.53706 & 1241 & 4.15141 & 1960 & 27.55467 & 21345 & 31.70609 & 23304 \\
\hline 1000 & 4.54947 & 1150 & 4.17101 & 1830 & 27.76812 & 20395 & 31.93913 & 22225 \\
\hline 1050 & 4.56097 & 1073 & 4.18931 & 1714 & 27.97207 & 19528 & 32.16138 & 21243 \\
\hline & 4.57170 & 1008 & 4.20645 & 1610 & 28.16735 & 18735 & 32.37381 & 20344 \\
\hline 1150 & 4.58178 & 954 & 4.22255 & 1517 & 28.35470 & 18003 & 32.57725 & 19520 \\
\hline
\end{tabular}


Table 2.026. CsD (Cont.)

\begin{tabular}{|l|l|l|l|l|}
\hline$K_{K}$ & $\frac{C_{P}^{0}}{R}$ & $\frac{\left(H^{0}-E_{0}^{0}\right)}{R T}$ & $\frac{-\left(F^{0}-E_{0}^{0}\right)}{R T}$ & $\frac{S^{0}}{R}$ \\
\hline
\end{tabular}

\begin{tabular}{|c|c|c|c|c|c|c|c|c|}
\hline $\begin{array}{l}1200 \\
1300 \\
1400 \\
1500 \\
1600\end{array}$ & $\begin{array}{l}4.59132 \\
4.60905 \\
4.62538 \\
4.64064 \\
4.65506\end{array}$ & $\begin{array}{l}1773 \\
1633 \\
1526 \\
1442 \\
1376\end{array}$ & $\begin{array}{l}4.23772 \\
4.26561 \\
4.29073 \\
4.31356 \\
4.33445\end{array}$ & $\begin{array}{l}2789 \\
2512 \\
2283 \\
2089 \\
1927\end{array}$ & $\begin{array}{l}28.53473 \\
28.87505 \\
29.19210 \\
29.48892 \\
29.76799\end{array}$ & $\begin{array}{l}34032 \\
31705 \\
29682 \\
27907 \\
26336\end{array}$ & $\begin{array}{l}32.77245 \\
33.14067 \\
33.48284 \\
33.80248 \\
34.10245\end{array}$ & $\begin{array}{l}36822 \\
34217 \\
31964 \\
29997 \\
28263\end{array}$ \\
\hline $\begin{array}{l}1700 \\
1800 \\
1900 \\
2000 \\
2100\end{array}$ & $\begin{array}{l}4.66882 \\
4.68204 \\
4.69483 \\
4.70724 \\
4.71934\end{array}$ & $\begin{array}{l}1322 \\
1279 \\
1241 \\
1210 \\
1184\end{array}$ & $\begin{array}{l}4.35372 \\
4.37159 \\
4.38827 \\
4.40391 \\
4.41865\end{array}$ & $\begin{array}{l}1787 \\
1668 \\
1564 \\
1474 \\
1393\end{array}$ & $\begin{array}{l}30.03135 \\
30.28071 \\
30.51753 \\
30.74302 \\
30.95825\end{array}$ & $\begin{array}{l}24936 \\
23682 \\
22549 \\
21523 \\
20588\end{array}$ & $\begin{array}{l}34.38508 \\
34.65231 \\
34.90580 \\
35.14693 \\
35.37690\end{array}$ & $\begin{array}{l}26723 \\
25349 \\
24113 \\
22997 \\
21981\end{array}$ \\
\hline $\begin{array}{l}2200 \\
2300 \\
2400 \\
2500 \\
2600\end{array}$ & $\begin{array}{l}4.73118 \\
4.74278 \\
4.75418 \\
4.76540 \\
4.77646\end{array}$ & $\begin{array}{l}1160 \\
1140 \\
1122 \\
1106 \\
1091\end{array}$ & $\begin{array}{l}4.43258 \\
4.44582 \\
4.45843 \\
4.47049 \\
4.48204\end{array}$ & $\begin{array}{l}1324 \\
1261 \\
1206 \\
1155 \\
1111\end{array}$ & $\begin{array}{l}31.16413 \\
31.36146 \\
31.55094 \\
31.73319 \\
31.90875\end{array}$ & $\begin{array}{l}19733 \\
18948 \\
18225 \\
17556 \\
16936\end{array}$ & $\begin{array}{l}35.59671 \\
35.80728 \\
36.00937 \\
36.20368 \\
36.39080\end{array}$ & $\begin{array}{l}21057 \\
20209 \\
19431 \\
18712 \\
18047\end{array}$ \\
\hline $\begin{array}{l}2700 \\
2800 \\
2900 \\
3000\end{array}$ & $\begin{array}{l}4.78737 \\
4.79814 \\
4.80879 \\
4.81933\end{array}$ & $\begin{array}{l}1077 \\
1065 \\
1054 \\
2075\end{array}$ & $\begin{array}{l}4.49315 \\
4.50385 \\
4.51418 \\
4.52418\end{array}$ & $\begin{array}{l}1070 \\
1033 \\
1000 \\
1910\end{array}$ & $\begin{array}{l}32.07811 \\
32.24171 \\
32.39994 \\
32.55315\end{array}$ & $\begin{array}{l}16360 \\
15823 \\
15321 \\
29260\end{array}$ & $\begin{array}{l}36.57127 \\
36.74557 \\
36.91413 \\
37.07733\end{array}$ & $\begin{array}{l}17430 \\
16856 \\
16320 \\
31170\end{array}$ \\
\hline $\begin{array}{l}3200 \\
3400 \\
3600 \\
3800 \\
4000\end{array}$ & $\begin{array}{l}4.84008 \\
4.86046 \\
4.88049 \\
4.90021 \\
4.91963\end{array}$ & $\begin{array}{l}2038 \\
2003 \\
1972 \\
1942 \\
1915\end{array}$ & $\begin{array}{l}4.54328 \\
4.56134 \\
4.57851 \\
4.59492 \\
4.61068\end{array}$ & $\begin{array}{l}1806 \\
1717 \\
1641 \\
1576 \\
1516\end{array}$ & $\begin{array}{l}32.84575 \\
33.12173 \\
33.38294 \\
33.63093 \\
33.86702\end{array}$ & $\begin{array}{l}27598 \\
26121 \\
24799 \\
23609 \\
22532\end{array}$ & $\begin{array}{l}37.38903 \\
37.68307 \\
37.96145 \\
38.22586 \\
38.47770\end{array}$ & $\begin{array}{l}29404 \\
27838 \\
26441 \\
25184 \\
24049\end{array}$ \\
\hline $\begin{array}{l}4200 \\
4400 \\
4600 \\
4800 \\
5000\end{array}$ & $\begin{array}{l}4.93878 \\
4.95767 \\
4.97631 \\
4.99471 \\
5.01288\end{array}$ & $\begin{array}{l}1889 \\
1864 \\
1840 \\
1817\end{array}$ & $\begin{array}{l}4.62584 \\
4.64050 \\
4.65470 \\
4.66848 \\
4.68189\end{array}$ & $\begin{array}{l}1466 \\
1420 \\
1378 \\
1341\end{array}$ & $\begin{array}{l}34.09234 \\
34.30788 \\
34.51447 \\
34.71287 \\
34.90372\end{array}$ & $\begin{array}{l}21554 \\
20659 \\
19840 \\
19085\end{array}$ & $\begin{array}{l}38.71819 \\
38.94838 \\
39.16917 \\
39.38135 \\
39.58562\end{array}$ & $\begin{array}{l}23019 \\
22079 \\
21218 \\
20427\end{array}$ \\
\hline
\end{tabular}


Table 2.027. CsT

\begin{tabular}{|l|l|l|l|l|}
\hline${ }^{\circ} K$ & $\frac{C_{p}^{0}}{R}$ & $\frac{\left(H^{0}-E_{0}^{0}\right)}{R T}$ & $\frac{-\left(F^{0}-E_{0}^{0}\right)}{R T}$ & $\frac{S^{0}}{R}$ \\
\hline
\end{tabular}

\begin{tabular}{|c|c|c|c|c|c|c|c|c|}
\hline 50 & 3.50204 & 103 & 3.49220 & 171 & 17.13301 & 63686 & 20.62521 & 63858 \\
\hline 60 & 3.50307 & 275 & 3.49391 & 148 & 17.76987 & 53870 & 21.26379 & 54017 \\
\hline 70 & 3.50582 & 611 & 3.49539 & 164 & 18.30857 & 46685 & 21.80396 & 46849 \\
\hline 80 & 3.51193 & 1091 & 3.49703 & 221 & 18.77542 & 41201 & 22.27245 & 41423 \\
\hline 90 & 3.52284 & 1650 & 3.49924 & 314 & 19.18743 & 36883 & 22.68668 & 37197 \\
\hline 100 & 3.53934 & 2212 & 3.50238 & 432 & 19.55626 & 33401 & 23.05865 & 33833 \\
\hline 110 & 3.56146 & 2718 & 3.50670 & 567 & 19.89027 & 30536 & 23.39698 & 31102 \\
\hline 120 & 3.58864 & 3130 & 3.51237 & 704 & 20.19563 & 28140 & 23.70800 & 28845 \\
\hline 130 & 3.61994 & 3436 & 3.51941 & 840 & 20.47703 & 26112 & 23.99645 & 26951 \\
\hline 140 & 3.65430 & 3636 & 3.52781 & 963 & 20.73815 & 24372 & 24.26596 & 25335 \\
\hline 150 & 3.69066 & 3745 & 3.53744 & 1075 & 20.98187 & 22863 & 24.51931 & 23939 \\
\hline 160 & 3.72811 & 3774 & 3.54819 & 1169 & 21.21050 & 21546 & 24.75870 & 22714 \\
\hline 170 & 3.76585 & 3743 & 3.55988 & 1249 & 21.42596 & 20383 & 24.98584 & 21632 \\
\hline 180 & 3.80328 & 3664 & 3.57237 & 1312 & 21.62979 & 19349 & 25.20216 & 20662 \\
\hline 190 & 3.83992 & 3553 & 3.58549 & 1361 & 21.82328 & 18426 & 25.40878 & 19787 \\
\hline 200 & 3.87545 & 3418 & 3.59910 & 1398 & 22.00754 & 17594 & 25.60665 & 18991 \\
\hline 210 & 3.90963 & 3269 & 3.61308 & 1423 & 22.18348 & 16841 & 25.79656 & 18264 \\
\hline 220 & 3.94232 & 3113 & 3.62731 & 1438 & 22.35189 & 16155 & 25.97920 & 17594 \\
\hline 230 & 3.97345 & 2954 & 3.64169 & 1444 & 22.51344 & 15530 & 26.15514 & 16974 \\
\hline 240 & 4.00299 & 2795 & 3.65613 & 1444 & 22.66874 & 14954 & 26.32488 & 16398 \\
\hline 250 & 4.03094 & 2641 & 3.67057 & 1437 & 22.81828 & 14424 & 26.48886 & 15861 \\
\hline 260 & 4.05735 & 2492 & 3.68494 & 1426 & 22.96252 & 13934 & 26.64747 & 15360 \\
\hline 270 & 4.08227 & 2348 & 3.69920 & 1411 & 23.10186 & 13479 & 26.80107 & 14889 \\
\hline 280 & 4.10575 & 2213 & 3.71331 & 1392 & 23.23665 & 13055 & 26.94996 & 14447 \\
\hline 290 & 4.12788 & 2084 & 3.72723 & 1370 & 23.36720 & 12659 & 27.09443 & 14030 \\
\hline 300 & 4.14872 & 1963 & 3.74093 & 1348 & 23.49379 & 12288 & 27.23473 & 13635 \\
\hline 310 & 4.16835 & 1850 & 3.75441 & 1322 & 23.61667 & 11941 & 27.37108 & 13264 \\
\hline 320 & 4.18685 & 1744 & 3.76763 & 1297 & 23.73608 & 11614 & 27.50372 & 12911 \\
\hline 330 & 4.20429 & 1644 & 3.78060 & 1271 & 23.85222 & 11305 & 27.63283 & 12575 \\
\hline 340 & 4.22073 & 1552 & 3.79331 & 1243 & 23.96527 & 11014 & 27.75858 & 12258 \\
\hline 350 & 4.23625 & 1466 & 3.80574 & 1217 & 24.07541 & 10738 & 27.88116 & 11954 \\
\hline 360 & 4.25091 & 1385 & 3.81791 & 1189 & 24.18279 & 10477 & 28.00070 & 11666 \\
\hline 370 & 4.26476 & 1310 & 3.82980 & 1162 & 24.28756 & 10229 & 28.11736 & 11391 \\
\hline 380 & 4.27786 & 1240 & 3.84142 & 1135 & 24.38985 & 9993 & 28.23127 & 11129 \\
\hline 390 & 4.29026 & 1176 & 3.85277 & 1109 & 24.48978 & 9768 & 28.34256 & 10877 \\
\hline 400 & 4.30202 & 5047 & 3.86386 & 5160 & 24.58746 & 45814 & 28.45133 & 50974 \\
\hline 450 & 4.35249 & 3969 & 3.91546 & 4577 & 25.04560 & 41495 & 28.96107 & 46071 \\
\hline 500 & 4.39218 & 3195 & 3.96123 & 4068 & 25.46055 & 37949 & 29.42178 & 42017 \\
\hline 550 & 4.42413 & 2633 & 4.00191 & 3631 & 25.84004 & 34980 & 29.84195 & 38612 \\
\hline 600 & 4.45046 & 2215 & 4.03822 & 3259 & 26.18984 & 32454 & 30.22807 & 35712 \\
\hline 650 & 4.47261 & 1901 & 4.07081 & 2939 & 26.51438 & 30278 & 30.58519 & 33217 \\
\hline 700 & 4.49162 & 1653 & 4.10020 & 2666 & 26.81716 & 28380 & 30.9 & 31047 \\
\hline 750 & 4.50820 & 1470 & 4.12686 & 2430 & 27.10096 & 26713 & 31.2 & 29143 \\
\hline 800 & 4.52290 & 1322 & 4.15116 & 2227 & 27.36809 & 25234 & 31.51926 & 27461 \\
\hline 850 & 4.53612 & 1203 & 4.17343 & 2048 & 27.62043 & 23914 & 31.79387 & 25962 \\
\hline 900 & 4.54815 & 1106 & 4.19391 & 1894 & 27.85957 & 22727 & 32.05349 & 24620 \\
\hline 950 & 4.55921 & 1028 & 4.21285 & 1758 & 28.08684 & 21654 & 32.29969 & 23413 \\
\hline 1000 & 4.56949 & 963 & 4.23043 & 1638 & 28.30338 & 20680 & 32.53382 & 22318 \\
\hline 1050 & 4.57912 & 908 & 4.24681 & 1531 & 28.51018 & 19792 & 32.75700 & 21323 \\
\hline 1100 & 4.58820 & 863 & 4.26212 & 1437 & 28.70810 & 18978 & 32.97023 & 20415 \\
\hline 1150 & 4.59683 & 825 & 4.27649 & 1352 & 28.89788 & 18230 & 33.17438 & 19581 \\
\hline
\end{tabular}


Table 2.027. CsT (Cont.)

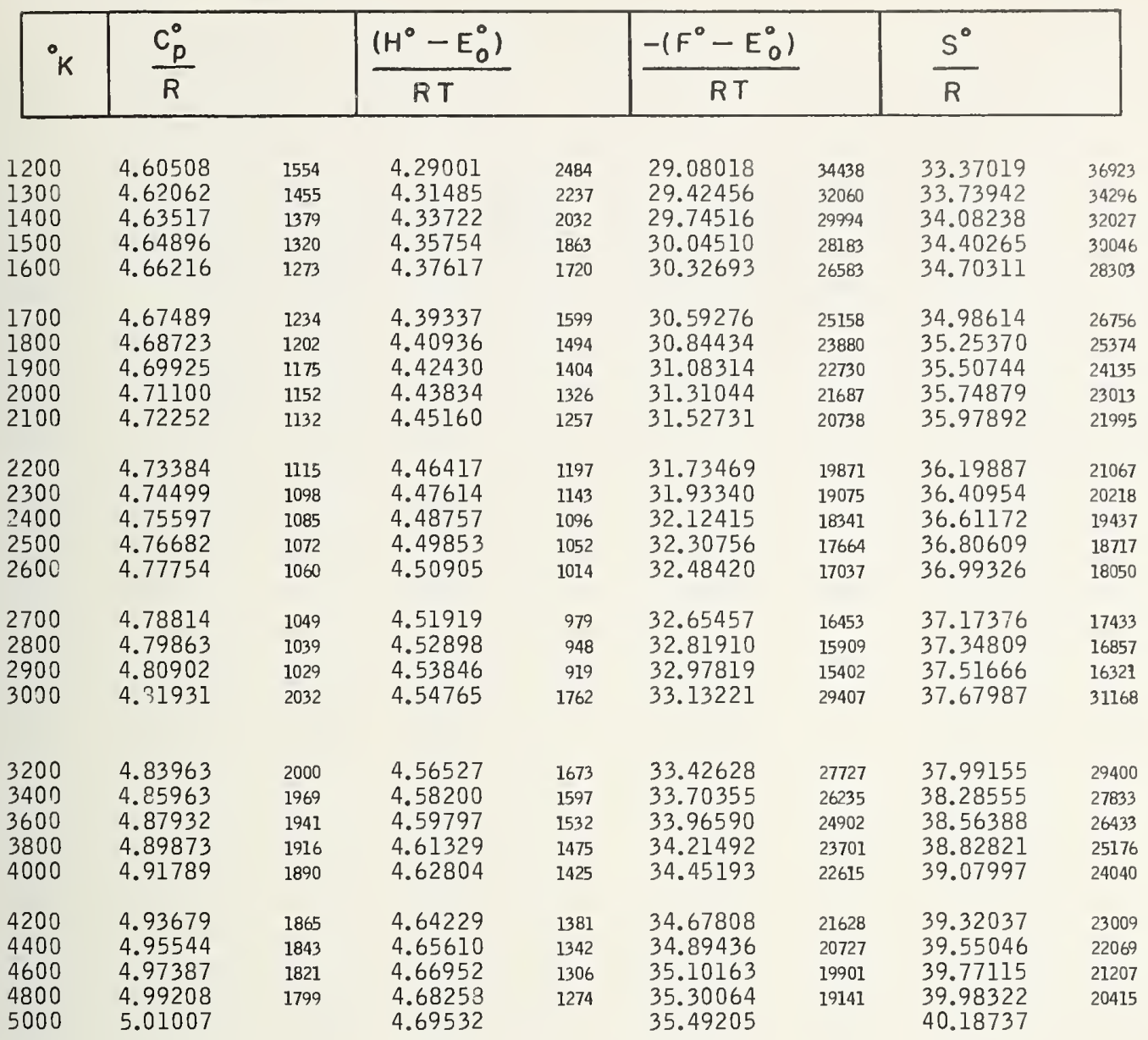


Table 2.028, $\mathrm{BeH}$

\begin{tabular}{|l|l|l|l|l|}
\hline${ }^{\circ}$ & $\frac{C_{p}^{0}}{R}$ & $\frac{\left(H^{0}-E_{0}^{0}\right)}{R T}$ & $\frac{-\left(F^{0}-E_{0}^{0}\right)}{R T}$ & $\frac{S^{0}}{R}$ \\
\hline
\end{tabular}

\begin{tabular}{|c|c|c|c|c|c|c|c|c|}
\hline $\begin{array}{l}50 \\
60 \\
70 \\
80 \\
90\end{array}$ & $\begin{array}{l}3.50346 \\
3.50306 \\
3.50292 \\
3.50292 \\
3.50301\end{array}$ & $\begin{array}{l}-40 \\
-14\end{array}$ & $\begin{array}{l}3.40119 \\
3.41820 \\
3.43031 \\
3.43938 \\
3.44645\end{array}$ & $\begin{array}{r}1701 \\
1211 \\
907 \\
707 \\
566\end{array}$ & $\begin{array}{l}11.59495 \\
12.21666 \\
12.74454 \\
13.20321 \\
13.60874\end{array}$ & $\begin{array}{l}62171 \\
52788 \\
45867 \\
40553 \\
36342\end{array}$ & $\begin{array}{l}14.99615 \\
15.63486 \\
16.17485 \\
16.64260 \\
17.05519\end{array}$ & $\begin{array}{l}63871 \\
53999 \\
46775 \\
41259 \\
36908\end{array}$ \\
\hline $\begin{array}{l}100 \\
110 \\
120 \\
130 \\
140\end{array}$ & $\begin{array}{l}3.50315 \\
3.50332 \\
3.50351 \\
3.50372 \\
3.50394\end{array}$ & $\begin{array}{l}17 \\
19 \\
21 \\
22 \\
23\end{array}$ & $\begin{array}{l}3.45211 \\
3.45676 \\
3.46064 \\
3.46395 \\
3.46680\end{array}$ & $\begin{array}{l}465 \\
388 \\
331 \\
285 \\
248\end{array}$ & $\begin{array}{l}13.97216 \\
14.30141 \\
14.60236 \\
14.87949 \\
15.13630\end{array}$ & $\begin{array}{l}32925 \\
30095 \\
27713 \\
25681 \\
23927\end{array}$ & $\begin{array}{l}17.42427 \\
17.75817 \\
18.06301 \\
18.34344 \\
18.60311\end{array}$ & $\begin{array}{l}33390 \\
30484 \\
28043 \\
25967 \\
24175\end{array}$ \\
\hline $\begin{array}{l}150 \\
160 \\
170 \\
180 \\
190\end{array}$ & $\begin{array}{l}3.50417 \\
3.50441 \\
3.50466 \\
3.50492 \\
3.50520\end{array}$ & $\begin{array}{l}24 \\
25 \\
26 \\
28 \\
31\end{array}$ & $\begin{array}{l}3.46928 \\
3.47147 \\
3.47341 \\
3.47516 \\
3.47673\end{array}$ & $\begin{array}{l}219 \\
194 \\
175 \\
157 \\
143\end{array}$ & $\begin{array}{l}15.37557 \\
15.59955 \\
15.81006 \\
16.00865 \\
16.19658\end{array}$ & $\begin{array}{l}22398 \\
21051 \\
19859 \\
18793 \\
17837\end{array}$ & $\begin{array}{l}18.84486 \\
19.07102 \\
19.28348 \\
19.48381 \\
19.67332\end{array}$ & $\begin{array}{l}22616 \\
21246 \\
20033 \\
18951 \\
17980\end{array}$ \\
\hline $\begin{array}{l}200 \\
210 \\
220 \\
230 \\
240\end{array}$ & $\begin{array}{l}3.50551 \\
3.50586 \\
3.50628 \\
3.50677 \\
3.50737\end{array}$ & $\begin{array}{l}35 \\
42 \\
49 \\
60 \\
74\end{array}$ & $\begin{array}{l}3.47816 \\
3.47947 \\
3.48068 \\
3.48180 \\
3.48286\end{array}$ & $\begin{array}{r}131 \\
121 \\
112 \\
106 \\
99\end{array}$ & $\begin{array}{l}16.37495 \\
16.54469 \\
16.70658 \\
16.86133 \\
17.00954\end{array}$ & $\begin{array}{l}16974 \\
16189 \\
15475 \\
14821 \\
14219\end{array}$ & $\begin{array}{l}19.85312 \\
20.02417 \\
20.18727 \\
20.34314 \\
20.49240\end{array}$ & $\begin{array}{l}17105 \\
16310 \\
15587 \\
14926 \\
14319\end{array}$ \\
\hline $\begin{array}{l}250 \\
260 \\
270 \\
280 \\
290\end{array}$ & $\begin{array}{l}3.50811 \\
3.50900 \\
3.51008 \\
3.51138 \\
3.51293\end{array}$ & $\begin{array}{r}89 \\
108 \\
130 \\
155 \\
183\end{array}$ & $\begin{array}{l}3.48385 \\
3.48480 \\
3.48572 \\
3.48661 \\
3.48749\end{array}$ & $\begin{array}{l}95 \\
92 \\
89 \\
88 \\
88\end{array}$ & $\begin{array}{l}17.15173 \\
17.28839 \\
17.41993 \\
17.54671 \\
17.66908\end{array}$ & $\begin{array}{l}13666 \\
13154 \\
12678 \\
12237 \\
11824\end{array}$ & $\begin{array}{l}20.63559 \\
20.77320 \\
20.90565 \\
21.03332 \\
21.15657\end{array}$ & $\begin{array}{l}13761 \\
13245 \\
12767 \\
12325 \\
11912\end{array}$ \\
\hline $\begin{array}{l}300 \\
310 \\
320 \\
330 \\
340\end{array}$ & $\begin{array}{l}3.51476 \\
3.51688 \\
3.51933 \\
3.52212 \\
3.52527\end{array}$ & $\begin{array}{l}212 \\
245 \\
279 \\
315 \\
352\end{array}$ & $\begin{array}{l}3.48837 \\
3.48925 \\
3.49015 \\
3.49108 \\
3.49204\end{array}$ & $\begin{array}{r}88 \\
90 \\
93 \\
96 \\
100\end{array}$ & $\begin{array}{l}17.78732 \\
17.90172 \\
18.01251 \\
18.11992 \\
18.22416\end{array}$ & $\begin{array}{l}11440 \\
11079 \\
10741 \\
10424 \\
10124\end{array}$ & $\begin{array}{l}21.27569 \\
21.39098 \\
21.50267 \\
21.61101 \\
21.71620\end{array}$ & $\begin{array}{l}11529 \\
11169 \\
10834 \\
10519 \\
10224\end{array}$ \\
\hline $\begin{array}{l}350 \\
360 \\
370 \\
380 \\
390\end{array}$ & $\begin{array}{l}3.52879 \\
3.53269 \\
3.53698 \\
3.54165 \\
3.54671\end{array}$ & $\begin{array}{l}390 \\
429 \\
467 \\
506 \\
543\end{array}$ & $\begin{array}{l}3.49304 \\
3.49408 \\
3.49518 \\
3.49634 \\
3.49757\end{array}$ & $\begin{array}{l}104 \\
110 \\
116 \\
123 \\
129\end{array}$ & $\begin{array}{l}18.32540 \\
18.42381 \\
18.51956 \\
18.61279 \\
18.70362\end{array}$ & $\begin{array}{l}9841 \\
9575 \\
9323 \\
9083 \\
8857\end{array}$ & $\begin{array}{l}21.81844 \\
21.91790 \\
22.01475 \\
22.10914 \\
22.20120\end{array}$ & $\begin{array}{l}9946 \\
9685 \\
9439 \\
9206 \\
8986\end{array}$ \\
\hline $\begin{array}{l}400 \\
450 \\
500 \\
550 \\
600\end{array}$ & $\begin{array}{l}3.55214 \\
3.58474 \\
3.62502 \\
3.67078 \\
3.71980\end{array}$ & $\begin{array}{l}3260 \\
4028 \\
4576 \\
4902 \\
5037\end{array}$ & $\begin{array}{l}3.49886 \\
3.50652 \\
3.51630 \\
3.52823 \\
3.54213\end{array}$ & $\begin{array}{r}766 \\
978 \\
1193 \\
1390 \\
1560\end{array}$ & $\begin{array}{l}18.79219 \\
19.20472 \\
19.57466 \\
19.91034 \\
20.21792\end{array}$ & $\begin{array}{l}41253 \\
36994 \\
33568 \\
30758 \\
28413\end{array}$ & $\begin{array}{l}22.29106 \\
22.71124 \\
23.09096 \\
23.43857 \\
23.76006\end{array}$ & $\begin{array}{l}42018 \\
37972 \\
34761 \\
32149 \\
29973\end{array}$ \\
\hline $\begin{array}{l}650 \\
700 \\
750 \\
800 \\
850\end{array}$ & $\begin{array}{l}3.77017 \\
3.82042 \\
3.86948 \\
3.91666 \\
3.96154\end{array}$ & $\begin{array}{l}5025 \\
4906 \\
4718 \\
4488 \\
4235\end{array}$ & $\begin{array}{l}3.55773 \\
3.57471 \\
3.59273 \\
3.61151 \\
3.63080\end{array}$ & $\begin{array}{l}1698 \\
1802 \\
1878 \\
1929 \\
1956\end{array}$ & $\begin{array}{l}20.50205 \\
20.76632 \\
21.01356 \\
21.24602 \\
21.46555\end{array}$ & $\begin{array}{l}26427 \\
24724 \\
23246 \\
21953 \\
20808\end{array}$ & $\begin{array}{l}24.05979 \\
24.34103 \\
24.60629 \\
24.85754 \\
25.09635\end{array}$ & $\begin{array}{l}28124 \\
26526 \\
25125 \\
23881 \\
22764\end{array}$ \\
\hline $\begin{array}{r}900 \\
950 \\
1000 \\
1050 \\
1100\end{array}$ & $\begin{array}{l}4.00389 \\
4.04365 \\
4.08083 \\
4.11553 \\
4.14787\end{array}$ & \begin{tabular}{l|l}
3976 \\
3718 \\
3470 \\
3234 \\
3013
\end{tabular} & $\begin{array}{l}3.65036 \\
3.67002 \\
3.68964 \\
3.70911 \\
3.72833\end{array}$ & $\begin{array}{l}1966 \\
1962 \\
1947 \\
1922 \\
1890\end{array}$ & $\begin{array}{l}21.67363 \\
21.87152 \\
22.06027 \\
22.24076 \\
22.41375\end{array}$ & $\begin{array}{l}19789 \\
18875 \\
18049 \\
17299 \\
16615\end{array}$ & $\begin{array}{l}25.32399 \\
25.54155 \\
25.74992 \\
25.94987 \\
26.14208\end{array}$ & $\begin{array}{l}21756 \\
20837 \\
19995 \\
19221 \\
18505\end{array}$ \\
\hline 1150 & 4.17800 & 2807 & 3.74723 & 1854 & 22.57990 & 15987 & 26.32713 & 17842 \\
\hline
\end{tabular}


Table 2. 028. BeH (Cont.)

\begin{tabular}{|l|l|l|l|l|}
\hline${ }^{\circ}$ & $\frac{C_{p}^{0}}{R}$ & $\frac{\left(H^{0}-E_{0}^{0}\right)}{R T}$ & $\frac{-\left(F^{\circ}-E_{0}^{0}\right)}{R T}$ & $\frac{S^{0}}{R}$ \\
\hline
\end{tabular}

\begin{tabular}{|c|c|c|c|c|c|c|c|c|}
\hline $\begin{array}{l}1200 \\
1300 \\
1400 \\
1500 \\
1600\end{array}$ & $\begin{array}{l}4.20607 \\
4.25665 \\
4.30079 \\
4.33953 \\
4.37376\end{array}$ & $\begin{array}{l}5058 \\
4414 \\
3874 \\
3423 \\
3046\end{array}$ & $\begin{array}{l}3.76577 \\
3.80163 \\
3.83574 \\
3.86806 \\
3.89862\end{array}$ & $\begin{array}{l}3586 \\
3411 \\
3232 \\
3056 \\
2886\end{array}$ & $\begin{array}{l}22.73977 \\
23.04262 \\
23.32561 \\
23.59137 \\
23.84199\end{array}$ & $\begin{array}{l}30285 \\
28299 \\
26576 \\
25062 \\
23723\end{array}$ & $\begin{array}{l}26.50555 \\
26.84426 \\
27.16136 \\
27.45944 \\
27.74062\end{array}$ & $\begin{array}{l}33871 \\
31710 \\
29808 \\
28118 \\
26609\end{array}$ \\
\hline $\begin{array}{l}1700 \\
1800 \\
1900 \\
2000 \\
2100\end{array}$ & $\begin{array}{l}4.40422 \\
4.43152 \\
4.45616 \\
4.47856 \\
4.49904\end{array}$ & $\begin{array}{l}2730 \\
2464 \\
2240 \\
2048 \\
1886\end{array}$ & $\begin{array}{l}3.92748 \\
3.95474 \\
3.98049 \\
4.00485 \\
4.02790\end{array}$ & $\begin{array}{l}2726 \\
2575 \\
2436 \\
2305 \\
2185\end{array}$ & $\begin{array}{l}24.07922 \\
24.30449 \\
24.51900 \\
24.72380 \\
24.91976\end{array}$ & $\begin{array}{l}22527 \\
21451 \\
20480 \\
19596 \\
18789\end{array}$ & $\begin{array}{l}28.00671 \\
28.25923 \\
28.49950 \\
28.72865 \\
28.94767\end{array}$ & $\begin{array}{l}25252 \\
24027 \\
22915 \\
21902 \\
20973\end{array}$ \\
\hline $\begin{array}{l}2200 \\
2300 \\
2400 \\
2500 \\
2600\end{array}$ & $\begin{array}{l}4.51790 \\
4.53537 \\
4.55164 \\
4.56687 \\
4.58120\end{array}$ & $\begin{array}{l}1747 \\
1627 \\
1523 \\
1433 \\
1353\end{array}$ & $\begin{array}{l}4.04975 \\
4.07049 \\
4.09020 \\
4.10897 \\
4.12686\end{array}$ & $\begin{array}{l}2074 \\
1971 \\
1877 \\
1789 \\
1708\end{array}$ & $\begin{array}{l}25.10765 \\
25.28813 \\
25.46179 \\
25.62914 \\
25.79065\end{array}$ & $\begin{array}{l}18048 \\
17366 \\
16735 \\
16151 \\
15607\end{array}$ & $\begin{array}{l}29.15740 \\
29.35862 \\
29.55199 \\
29.73811 \\
29.91751\end{array}$ & $\begin{array}{l}20122 \\
19337 \\
18612 \\
17940 \\
17315\end{array}$ \\
\hline $\begin{array}{l}2700 \\
2800 \\
2900 \\
3000\end{array}$ & $\begin{array}{l}4.59473 \\
4.60758 \\
4.61982 \\
4.63152\end{array}$ & $\begin{array}{l}1285 \\
1224 \\
1170 \\
2201\end{array}$ & $\begin{array}{l}4.14394 \\
4.16027 \\
4.17590 \\
4.19090\end{array}$ & $\begin{array}{l}1633 \\
1563 \\
1500 \\
2823\end{array}$ & $\begin{array}{l}25.94672 \\
26.09772 \\
26.24399 \\
26.38581\end{array}$ & $\begin{array}{l}15100 \\
14627 \\
14182 \\
27139\end{array}$ & $\begin{array}{l}30.09066 \\
30.25800 \\
30.41990 \\
30.57672\end{array}$ & $\begin{array}{l}16734 \\
16190 \\
15682 \\
29962\end{array}$ \\
\hline $\begin{array}{l}3200 \\
3400 \\
3600 \\
3800 \\
4000\end{array}$ & $\begin{array}{l}4.65353 \\
4.67400 \\
4.69324 \\
4.71145 \\
4.72882\end{array}$ & $\begin{array}{l}2047 \\
1924 \\
1821 \\
1737 \\
1666\end{array}$ & $\begin{array}{l}4.21913 \\
4.24529 \\
4.26965 \\
4.29243 \\
4.31382\end{array}$ & $\begin{array}{l}2616 \\
2436 \\
2278 \\
2139 \\
2016\end{array}$ & $\begin{array}{l}26.65720 \\
26.91378 \\
27.15713 \\
27.38860 \\
27.60932\end{array}$ & $\begin{array}{l}25658 \\
24335 \\
23147 \\
22072 \\
21096\end{array}$ & $\begin{array}{l}30.87634 \\
31.15908 \\
31.42679 \\
31.68103 \\
31.92314\end{array}$ & $\begin{array}{l}28274 \\
26771 \\
25424 \\
24211 \\
23113\end{array}$ \\
\hline $\begin{array}{l}4200 \\
4400 \\
4600 \\
4800 \\
5000\end{array}$ & $\begin{array}{l}4.74548 \\
4.76153 \\
4.77707 \\
4.79215 \\
4.80684\end{array}$ & $\begin{array}{l}1605 \\
1554 \\
1508 \\
1469\end{array}$ & $\begin{array}{l}4.33398 \\
4.35305 \\
4.37115 \\
4.38838 \\
4.40482\end{array}$ & $\begin{array}{l}1907 \\
1810 \\
1723 \\
1644\end{array}$ & $\begin{array}{l}27.82028 \\
28.02235 \\
28.21625 \\
28.40265 \\
28.58213\end{array}$ & $\begin{array}{l}20207 \\
19390 \\
18640 \\
17948\end{array}$ & $\begin{array}{l}32.15427 \\
32.37540 \\
32.58740 \\
32.79103 \\
32.98696\end{array}$ & $\begin{array}{l}22113 \\
21200 \\
20363 \\
19593\end{array}$ \\
\hline
\end{tabular}


Table 2.029. BeD

\begin{tabular}{|l|l|l|l|l|}
\hline \multirow{K}{*}{} & $\frac{C_{P}^{0}}{R}$ & $\frac{\left(H^{\circ}-E_{0}^{0}\right)}{R T}$ & $\frac{-\left(F^{0}-E_{0}^{0}\right)}{R T}$ & $\frac{S^{0}}{R}$ \\
\hline
\end{tabular}

\begin{tabular}{|c|c|c|c|c|c|c|c|c|}
\hline $\begin{array}{l}50 \\
60 \\
70 \\
80 \\
90\end{array}$ & $\begin{array}{l}3.50198 \\
3.50205 \\
3.50220 \\
3.50239 \\
3.50261\end{array}$ & $\begin{array}{r}7 \\
15 \\
19 \\
22 \\
23\end{array}$ & $\begin{array}{l}3.44617 \\
3.45547 \\
3.46214 \\
3.46716 \\
3.47108\end{array}$ & $\begin{array}{l}930 \\
667 \\
502 \\
392 \\
317\end{array}$ & $\begin{array}{l}12.28634 \\
12.91553 \\
13.44872 \\
13.91137 \\
14.31997\end{array}$ & $\begin{array}{l}62919 \\
53319 \\
46265 \\
40860 \\
36589\end{array}$ & $\begin{array}{l}15.73251 \\
16.37101 \\
16.91086 \\
17.37853 \\
17.79106\end{array}$ & $\begin{array}{l}63850 \\
53985 \\
46767 \\
41253 \\
36905\end{array}$ \\
\hline $\begin{array}{l}100 \\
110 \\
120 \\
130 \\
140\end{array}$ & $\begin{array}{l}3.50284 \\
3.50309 \\
3.50334 \\
3.50361 \\
3.50390\end{array}$ & $\begin{array}{l}25 \\
25 \\
27 \\
29 \\
33\end{array}$ & $\begin{array}{l}3.47425 \\
3.47686 \\
3.47905 \\
3.48093 \\
3.48256\end{array}$ & $\begin{array}{l}261 \\
219 \\
188 \\
163 \\
144\end{array}$ & $\begin{array}{l}14.68586 \\
15.01712 \\
15.31974 \\
15.59829 \\
15.85632\end{array}$ & $\begin{array}{l}33126 \\
30262 \\
27855 \\
25803 \\
24032\end{array}$ & $\begin{array}{l}18.16011 \\
18.49398 \\
18.79880 \\
19.07923 \\
19.33888\end{array}$ & $\begin{array}{l}33387 \\
30482 \\
28043 \\
25965 \\
24176\end{array}$ \\
\hline $\begin{array}{l}150 \\
160 \\
170 \\
180 \\
190\end{array}$ & $\begin{array}{l}3.50423 \\
3.50464 \\
3.50517 \\
3.50587 \\
3.50681\end{array}$ & $\begin{array}{r}41 \\
53 \\
70 \\
94 \\
123\end{array}$ & $\begin{array}{l}3.48400 \\
3.48527 \\
3.48643 \\
3.48749 \\
3.48848\end{array}$ & $\begin{array}{r}127 \\
116 \\
106 \\
99 \\
94\end{array}$ & $\begin{array}{l}16.09664 \\
16.32153 \\
16.53286 \\
16.73217 \\
16.92075\end{array}$ & $\begin{array}{l}22489 \\
21133 \\
19931 \\
18858 \\
17896\end{array}$ & $\begin{array}{l}19.58064 \\
19.80681 \\
20.01929 \\
20.21966 \\
20.40924\end{array}$ & $\begin{array}{l}22617 \\
21248 \\
20037 \\
18958 \\
17990\end{array}$ \\
\hline $\begin{array}{l}200 \\
210 \\
220 \\
230 \\
240\end{array}$ & $\begin{array}{l}3.50804 \\
3.50965 \\
3.51170 \\
3.51425 \\
3.51735\end{array}$ & $\begin{array}{l}161 \\
205 \\
255 \\
310 \\
372\end{array}$ & $\begin{array}{l}3.48942 \\
3.49035 \\
3.49127 \\
3.49221 \\
3.49319\end{array}$ & $\begin{array}{r}93 \\
92 \\
94 \\
98 \\
104\end{array}$ & $\begin{array}{l}17.09971 \\
17.26999 \\
17.43238 \\
17.58759 \\
17.73624\end{array}$ & $\begin{array}{l}17028 \\
16239 \\
15521 \\
14865 \\
14262\end{array}$ & $\begin{array}{l}20.58914 \\
20.76034 \\
20.92365 \\
21.07981 \\
21.22944\end{array}$ & $\begin{array}{l}17120 \\
16331 \\
15616 \\
14963 \\
14366\end{array}$ \\
\hline $\begin{array}{l}250 \\
260 \\
270 \\
280 \\
290\end{array}$ & $\begin{array}{l}3.52107 \\
3.52542 \\
3.53045 \\
3.53615 \\
3.54253\end{array}$ & $\begin{array}{l}435 \\
503 \\
570 \\
638 \\
707\end{array}$ & $\begin{array}{l}3.49423 \\
3.49534 \\
3.49655 \\
3.49786 \\
3.49929\end{array}$ & $\begin{array}{l}111 \\
121 \\
131 \\
143 \\
156\end{array}$ & $\begin{array}{l}17.87886 \\
18.01593 \\
18.14787 \\
18.27505 \\
18.39782\end{array}$ & $\begin{array}{l}13707 \\
13194 \\
12718 \\
12277 \\
11866\end{array}$ & $\begin{array}{l}21.37310 \\
21.51128 \\
21.64442 \\
21.77292 \\
21.89711\end{array}$ & $\begin{array}{l}13818 \\
13314 \\
12850 \\
12419 \\
12022\end{array}$ \\
\hline $\begin{array}{l}300 \\
310 \\
320 \\
330 \\
340\end{array}$ & $\begin{array}{l}3.54960 \\
3.55732 \\
3.56567 \\
3.57464 \\
3.58418\end{array}$ & $\begin{array}{r}772 \\
835 \\
897 \\
954 \\
1007\end{array}$ & $\begin{array}{l}3.50085 \\
3.50254 \\
3.50438 \\
3.50637 \\
3.50852\end{array}$ & $\begin{array}{l}169 \\
184 \\
199 \\
215 \\
230\end{array}$ & $\begin{array}{l}18.51648 \\
18.63130 \\
18.74253 \\
18.85039 \\
18.95510\end{array}$ & $\begin{array}{l}11482 \\
11123 \\
10786 \\
10471 \\
10174\end{array}$ & $\begin{array}{l}22.01733 \\
22.13384 \\
22.24691 \\
22.35677 \\
22.46363\end{array}$ & $\begin{array}{l}11651 \\
11307 \\
10986 \\
10686 \\
10404\end{array}$ \\
\hline $\begin{array}{l}350 \\
360 \\
370 \\
380 \\
390\end{array}$ & $\begin{array}{l}3.59425 \\
3.60481 \\
3.61583 \\
3.62725 \\
3.63903\end{array}$ & $\begin{array}{l}1056 \\
1102 \\
1142 \\
1178 \\
1209\end{array}$ & $\begin{array}{l}3.51082 \\
3.51329 \\
3.51591 \\
3.51869 \\
3.52162\end{array}$ & $\begin{array}{l}247 \\
262 \\
278 \\
293 \\
309\end{array}$ & $\begin{array}{l}19.05684 \\
19.15577 \\
19.25207 \\
19.34587 \\
19.43731\end{array}$ & $\begin{array}{l}9893 \\
9630 \\
9380 \\
9144 \\
8920\end{array}$ & $\begin{array}{l}22.56767 \\
22.66907 \\
22.76798 \\
22.86456 \\
22.95893\end{array}$ & $\begin{array}{r}10140 \\
9891 \\
9658 \\
9437 \\
9229\end{array}$ \\
\hline $\begin{array}{l}400 \\
450 \\
500 \\
550 \\
600\end{array}$ & $\begin{array}{l}3.65112 \\
3.71494 \\
3.78107 \\
3.84621 \\
3.90830\end{array}$ & $\begin{array}{l}6382 \\
6613 \\
6514 \\
6209 \\
5795\end{array}$ & $\begin{array}{l}3.52471 \\
3.54226 \\
3.56283 \\
3.58565 \\
3.60997\end{array}$ & $\begin{array}{l}1755 \\
2057 \\
2282 \\
2432 \\
2521\end{array}$ & $\begin{array}{l}19.52651 \\
19.94264 \\
20.31689 \\
20.65752 \\
20.97055\end{array}$ & $\begin{array}{l}41613 \\
37425 \\
34063 \\
31303 \\
28994\end{array}$ & $\begin{array}{l}23.05122 \\
23.48490 \\
23.87973 \\
24.24318 \\
24.58053\end{array}$ & $\begin{array}{l}43368 \\
39483 \\
36345 \\
33735 \\
31515\end{array}$ \\
\hline $\begin{array}{l}650 \\
700 \\
750 \\
800 \\
850\end{array}$ & $\begin{array}{l}3.96625 \\
4.01961 \\
4.06835 \\
4.11267 \\
4.15287\end{array}$ & $\begin{array}{l}5336 \\
4874 \\
4432 \\
4020 \\
3646\end{array}$ & $\begin{array}{l}3.63518 \\
3.66076 \\
3.68633 \\
3.71162 \\
3.73641\end{array}$ & $\begin{array}{l}2558 \\
2557 \\
2529 \\
2479 \\
2417\end{array}$ & $\begin{array}{l}21.26049 \\
21.53083 \\
21.78427 \\
22.02299 \\
22.24875\end{array}$ & $\begin{array}{l}27034 \\
25344 \\
23872 \\
22576 \\
21425\end{array}$ & $\begin{array}{l}24.89568 \\
25.19159 \\
25.47060 \\
25.73461 \\
25.98516\end{array}$ & $\begin{array}{l}29591 \\
27901 \\
26401 \\
25055 \\
23842\end{array}$ \\
\hline $\begin{array}{r}900 \\
950 \\
1000 \\
1050\end{array}$ & $\begin{array}{l}4.18933 \\
4.22241 \\
4.25247 \\
4.27985 \\
4.30484\end{array}$ & $\begin{array}{l}3308 \\
3006 \\
2738 \\
2499 \\
2289\end{array}$ & $\begin{array}{l}3.76058 \\
3.78403 \\
3.80671 \\
3.82860 \\
3.84969\end{array}$ & $\begin{array}{l}2345 \\
2268 \\
2189 \\
2109 \\
2029\end{array}$ & $\begin{array}{l}22.46300 \\
22.66696 \\
22.86163 \\
23.04790 \\
23.22649\end{array}$ & $\begin{array}{l}20396 \\
19467 \\
18627 \\
17859 \\
17158\end{array}$ & $\begin{array}{l}26.22358 \\
26.45099 \\
26.66835 \\
26.87650 \\
27.07618\end{array}$ & $\begin{array}{l}22741 \\
21736 \\
20815 \\
19968 \\
19187\end{array}$ \\
\hline 150 & 4.32773 & 2102 & 3.86998 & 1952 & 23.39807 & 16512 & 27.26805 & 18464 \\
\hline
\end{tabular}


Table 2.029. BeD (Cont.)

\begin{tabular}{|l|l|l|l|l|}
\hline$K$ & $\frac{C_{p}^{0}}{R}$ & $\frac{\left(H^{0}-E_{0}^{0}\right)}{R T}$ & $\frac{-\left(F^{0}-E_{0}^{0}\right)}{R T}$ & $\frac{S^{0}}{R}$ \\
\hline
\end{tabular}

$\begin{array}{lllllllll}1200 & 4.34875 & 3726 & 3.88950 & 3680 & 23.56319 & 31280 & 27.45269 & 34960 \\ 1300 & 4.38601 & 3200 & 3.92630 & 3400 & 23.87599 & 29223 & 27.80229 & 32624 \\ 1400 & 4.41801 & 2783 & 3.96030 & 3146 & 24.16822 & 27432 & 28.12853 & 30578 \\ 1500 & 4.44584 & 2448 & 3.99176 & 2916 & 24.44254 & 25856 & 28.43431 & 28772 \\ 1600 & 4.47032 & 2178 & 4.02092 & 2709 & 24.70110 & 24459 & 28.72203 & 27168 \\ & & & & & & & \\ 1700 & 4.49210 & 1959 & 4.04801 & 2523 & 24.94569 & 23211 & 28.99371 & 25733 \\ 1800 & 4.51169 & 1778 & 4.07324 & 2355 & 25.17780 & 22086 & 29.25104 & 24442 \\ 1900 & 4.52947 & 1627 & 4.09679 & 2204 & 25.39866 & 21071 & 29.49546 & 23275 \\ 2000 & 4.54574 & 1503 & 4.11883 & 2070 & 25.60937 & 20146 & 29.72821 & 22215 \\ 2100 & 4.56077 & 1398 & 4.13953 & 1947 & 25.81083 & 19303 & 29.95036 & 21250 \\ & & & & & & & & \\ 2200 & 4.57475 & 1308 & 4.15900 & 1836 & 26.00386 & 18528 & 30.16286 & 20364 \\ 2300 & 4.58783 & 1232 & 4.17736 & 1736 & 26.18914 & 17816 & 30.36650 & 19552 \\ 2400 & 4.60015 & 1167 & 4.19472 & 1645 & 26.36730 & 17157 & 30.56202 & 18803 \\ 2500 & 4.61182 & 1111 & 4.21117 & 1563 & 26.53887 & 16547 & 30.75005 & 18110 \\ 2600 & 4.62293 & 1062 & 4.22680 & 1487 & 26.70434 & 15981 & 30.93115 & 17467 \\ & & & & & & & & \\ 2700 & 4.63355 & 1019 & 4.24167 & 1418 & 26.86415 & 15451 & 31.10582 & 16870 \\ 2800 & 4.64374 & 981 & 4.25585 & 1354 & 27.01866 & 14959 & 31.27452 & 16312 \\ 2900 & 4.65355 & 948 & 4.26939 & 1297 & 27.16825 & 14495 & 31.43764 & 15793 \\ 3000 & 4.66303 & 1811 & 4.28236 & 2436 & 27.31320 & 27717 & 31.59557 & 30153 \\ & & & & & & & & \\ 3200 & 4.68114 & 1717 & 4.30672 & 2253 & 27.59037 & 26178 & 31.89710 & 28431 \\ 3400 & 4.69831 & 1641 & 4.32925 & 2097 & 27.85215 & 24805 & 32.18141 & 26901 \\ 3600 & 4.71472 & 1577 & 4.35022 & 1960 & 28.10020 & 23574 & 32.45042 & 25534 \\ 3800 & 4.73049 & 1525 & 4.36982 & 1842 & 28.33594 & 22461 & 32.70576 & 24303 \\ 4000 & 4.74574 & 1481 & 4.38824 & 1737 & 28.56055 & 21453 & 32.94879 & 23191 \\ & & & & & & & & \\ 4200 & 4.76055 & 1442 & 4.40561 & 1647 & 28.77508 & 20533 & 33.18070 & 22179 \\ 4400 & 4.77497 & 1409 & 4.42208 & 1565 & 28.98041 & 19692 & 33.40249 & 21257 \\ 4600 & 4.78906 & 1379 & 4.43773 & 1492 & 29.17733 & 18919 & 33.61506 & 20412 \\ 4800 & 4.80285 & 1353 & 4.45265 & 1428 & 29.36652 & 18205 & 33.81918 & 19633 \\ 5000 & 4.81638 & & 4.46693 & & 29.54857 & & 34.01551 & \end{array}$


Table 2.030. BeT

\begin{tabular}{|l|l|l|l|l|}
\hline${ }^{\circ} K$ & $\frac{C_{p}^{0}}{R}$ & $\frac{\left(H^{0}-E_{0}^{0}\right)}{R T}$ & $\frac{-\left(F^{0}-E_{0}^{0}\right)}{R T}$ & $\frac{S^{0}}{R}$ \\
\hline
\end{tabular}

\begin{tabular}{|c|c|c|c|c|c|c|c|c|}
\hline $\begin{array}{l}50 \\
60 \\
70 \\
80 \\
90\end{array}$ & $\begin{array}{l}3.50167 \\
3.50183 \\
3.50203 \\
3.50226 \\
3.50250\end{array}$ & $\begin{array}{l}16 \\
20 \\
23 \\
24 \\
25\end{array}$ & $\begin{array}{l}3.46106 \\
3.46784 \\
3.47271 \\
3.47639 \\
3.47928\end{array}$ & $\begin{array}{l}678 \\
487 \\
368 \\
289 \\
233\end{array}$ & $\begin{array}{l}12.71799 \\
13.34965 \\
13.88461 \\
14.34857 \\
14.75821\end{array}$ & $\begin{array}{l}63166 \\
53496 \\
46396 \\
40964 \\
36670\end{array}$ & $\begin{array}{l}16.17905 \\
16.81749 \\
17.35732 \\
17.82497 \\
18.23749\end{array}$ & $\begin{array}{l}63844 \\
53983 \\
46765 \\
41252 \\
36904\end{array}$ \\
\hline $\begin{array}{l}100 \\
110 \\
120 \\
130 \\
140\end{array}$ & $\begin{array}{l}3.50275 \\
3.50302 \\
3.50331 \\
3.50367 \\
3.50413\end{array}$ & $\begin{array}{l}27 \\
29 \\
36 \\
46 \\
65\end{array}$ & $\begin{array}{l}3.48161 \\
3.48355 \\
3.48518 \\
3.48659 \\
3.48782\end{array}$ & $\begin{array}{l}194 \\
163 \\
141 \\
123 \\
111\end{array}$ & $\begin{array}{l}15.12491 \\
15.45684 \\
15.76002 \\
16.03904 \\
16.29747\end{array}$ & $\begin{array}{l}33193 \\
30318 \\
27902 \\
25843 \\
24067\end{array}$ & $\begin{array}{l}18.60653 \\
18.94039 \\
19.24520 \\
19.52563 \\
19.78530\end{array}$ & $\begin{array}{l}33386 \\
30481 \\
28043 \\
25967 \\
24178\end{array}$ \\
\hline $\begin{array}{l}150 \\
160 \\
170 \\
180 \\
190\end{array}$ & $\begin{array}{l}3.50478 \\
3.50570 \\
3.50700 \\
3.50879 \\
3.51119\end{array}$ & $\begin{array}{r}92 \\
130 \\
179 \\
240 \\
308\end{array}$ & $\begin{array}{l}3.48893 \\
3.48995 \\
3.49091 \\
3.49185 \\
3.49281\end{array}$ & $\begin{array}{r}102 \\
96 \\
94 \\
96 \\
99\end{array}$ & $\begin{array}{l}16.53814 \\
16.76335 \\
16.97495 \\
17.17451 \\
17.36333\end{array}$ & $\begin{array}{l}22521 \\
21160 \\
19956 \\
18882 \\
17919\end{array}$ & $\begin{array}{l}20.02708 \\
20.25330 \\
20.46587 \\
20.66637 \\
20.85615\end{array}$ & $\begin{array}{l}22622 \\
21257 \\
20050 \\
18978 \\
18017\end{array}$ \\
\hline $\begin{array}{l}200 \\
210 \\
220 \\
230 \\
240\end{array}$ & $\begin{array}{l}3.51427 \\
3.51814 \\
3.52285 \\
3.52846 \\
3.53499\end{array}$ & $\begin{array}{l}387 \\
471 \\
561 \\
653 \\
746\end{array}$ & $\begin{array}{l}3.49380 \\
3.49486 \\
3.49602 \\
3.49731 \\
3.49874\end{array}$ & $\begin{array}{l}106 \\
116 \\
129 \\
143 \\
160\end{array}$ & $\begin{array}{l}17.54252 \\
17.71301 \\
17.87561 \\
18.03105 \\
18.17992\end{array}$ & $\begin{array}{l}17049 \\
16260 \\
15544 \\
14887 \\
14286\end{array}$ & $\begin{array}{l}21.03632 \\
21.20787 \\
21.37164 \\
21.52836 \\
21.67867\end{array}$ & $\begin{array}{l}17155 \\
16377 \\
15672 \\
15031 \\
14445\end{array}$ \\
\hline $\begin{array}{l}250 \\
260 \\
270 \\
280 \\
290\end{array}$ & $\begin{array}{l}3.54245 \\
3.55082 \\
3.56008 \\
3.57018 \\
3.58107\end{array}$ & $\begin{array}{r}837 \\
926 \\
1010 \\
1089 \\
1163\end{array}$ & $\begin{array}{l}3.50034 \\
3.50211 \\
3.50409 \\
3.50626 \\
3.50865\end{array}$ & $\begin{array}{l}177 \\
198 \\
217 \\
239 \\
261\end{array}$ & $\begin{array}{l}18.32278 \\
18.46010 \\
18.59231 \\
18.71978 \\
18.84286\end{array}$ & $\begin{array}{l}13732 \\
13221 \\
12747 \\
12308 \\
11899\end{array}$ & $\begin{array}{l}21.82312 \\
21.96222 \\
22.09640 \\
22.22605 \\
22.35152\end{array}$ & $\begin{array}{l}13910 \\
13418 \\
12965 \\
12547 \\
12160\end{array}$ \\
\hline $\begin{array}{l}300 \\
310 \\
320 \\
330 \\
340\end{array}$ & $\begin{array}{l}3.59270 \\
3.60499 \\
3.61788 \\
3.63130 \\
3.64518\end{array}$ & $\begin{array}{l}1229 \\
1289 \\
1342 \\
1388 \\
1427\end{array}$ & $\begin{array}{l}3.51126 \\
3.51408 \\
3.51712 \\
3.52038 \\
3.52385\end{array}$ & $\begin{array}{l}282 \\
304 \\
326 \\
347 \\
366\end{array}$ & $\begin{array}{l}18.96185 \\
19.07703 \\
19.18865 \\
19.29692 \\
19.40207\end{array}$ & $\begin{array}{l}11518 \\
11162 \\
10827 \\
10515 \\
10220\end{array}$ & $\begin{array}{l}22.47312 \\
22.59112 \\
22.70578 \\
22.81731 \\
22.92592\end{array}$ & $\begin{array}{l}11800 \\
11466 \\
11153 \\
10861 \\
10587\end{array}$ \\
\hline $\begin{array}{l}350 \\
360 \\
370 \\
380 \\
390\end{array}$ & $\begin{array}{l}3.65945 \\
3.67404 \\
3.68889 \\
3.70395 \\
3.71915\end{array}$ & $\begin{array}{l}1459 \\
1485 \\
1506 \\
1520 \\
1530\end{array}$ & $\begin{array}{l}3.52751 \\
3.53138 \\
3.53544 \\
3.53967 \\
3.54408\end{array}$ & $\begin{array}{l}387 \\
406 \\
423 \\
441 \\
457\end{array}$ & $\begin{array}{l}19.50427 \\
19.60370 \\
19.70051 \\
19.79485 \\
19.88685\end{array}$ & $\begin{array}{l}9943 \\
9681 \\
9434 \\
9200 \\
8978\end{array}$ & $\begin{array}{l}23.03179 \\
23.13508 \\
23.23595 \\
23.33453 \\
23.43093\end{array}$ & $\begin{array}{r}10329 \\
10087 \\
9858 \\
9640 \\
9436\end{array}$ \\
\hline $\begin{array}{l}400 \\
450 \\
500 \\
550 \\
600\end{array}$ & $\begin{array}{l}3.73445 \\
3.81088 \\
3.88436 \\
3.95257 \\
4.01459\end{array}$ & $\begin{array}{l}7643 \\
7348 \\
6821 \\
6202 \\
5572\end{array}$ & $\begin{array}{l}3.54865 \\
3.57355 \\
3.60099 \\
3.62990 \\
3.65942\end{array}$ & $\begin{array}{l}2490 \\
2744 \\
2891 \\
2952 \\
2950\end{array}$ & $\begin{array}{l}19.97663 \\
20.39601 \\
20.77393 \\
21.11849 \\
21.43559\end{array}$ & $\begin{array}{l}41938 \\
37792 \\
34456 \\
31710 \\
29408\end{array}$ & $\begin{array}{l}23.52529 \\
23.96957 \\
24.37493 \\
24.74839 \\
25.09501\end{array}$ & $\begin{array}{l}44428 \\
40536 \\
37346 \\
34662 \\
32358\end{array}$ \\
\hline $\begin{array}{l}650 \\
700 \\
750 \\
800 \\
850\end{array}$ & $\begin{array}{l}4.07031 \\
4.12007 \\
4.16438 \\
4.20386 \\
4.23907\end{array}$ & $\begin{array}{l}4976 \\
4431 \\
3948 \\
3521 \\
3150\end{array}$ & $\begin{array}{l}3.68892 \\
3.71797 \\
3.74628 \\
3.77367 \\
3.80003\end{array}$ & $\begin{array}{l}2905 \\
2831 \\
2739 \\
2636 \\
2529\end{array}$ & $\begin{array}{l}21.72967 \\
22.00412 \\
22.26160 \\
22.50426 \\
22.73383\end{array}$ & $\begin{array}{l}27445 \\
25748 \\
24266 \\
22957 \\
21793\end{array}$ & $\begin{array}{l}25.41859 \\
25.72209 \\
26.00789 \\
26.27794 \\
26.53387\end{array}$ & $\begin{array}{l}30350 \\
28580 \\
27005 \\
25593 \\
24321\end{array}$ \\
\hline $\begin{array}{r}900 \\
950 \\
1000 \\
1050 \\
1100\end{array}$ & $\begin{array}{l}4.27057 \\
4.29883 \\
4.32429 \\
4.34730 \\
4.36820\end{array}$ & $\begin{array}{l}2826 \\
2546 \\
2301 \\
2090 \\
1906\end{array}$ & $\begin{array}{l}3.82532 \\
3.84951 \\
3.87262 \\
3.89469 \\
3.91574\end{array}$ & $\begin{array}{l}2419 \\
2311 \\
2207 \\
2105 \\
2009\end{array}$ & $\begin{array}{l}22.95176 \\
23.15924 \\
23.35728 \\
23.54677 \\
23.72844\end{array}$ & \begin{tabular}{l|}
20748 \\
19804 \\
18949 \\
18167 \\
17451
\end{tabular} & $\begin{array}{l}26.77708 \\
27.00875 \\
27.22991 \\
27.44146 \\
27.64418\end{array}$ & $\begin{array}{l}23167 \\
22116 \\
21155 \\
20272 \\
19460\end{array}$ \\
\hline 1150 & 4.38726 & 1745 & 3.93583 & 1918 & 23.90295 & 16791 & 27.83878 & 18710 \\
\hline
\end{tabular}


Table 2.030. BeT (Cont.)

\begin{tabular}{|l|l|l|l|l|}
\hline${ }^{\circ} K$ & $\frac{C_{p}^{0}}{R}$ & $\frac{\left(H^{0}-E_{0}^{0}\right)}{R T}$ & $\frac{-\left(F^{0}-E_{0}^{0}\right)}{R T}$ & $\frac{S^{0}}{R}$ \\
\hline
\end{tabular}

\begin{tabular}{|c|c|c|c|c|c|c|c|c|}
\hline 1200 & 4.40471 & 3084 & 3.95501 & 3581 & 24.07086 & 31801 & 28.02588 & 35382 \\
\hline 1300 & 4.43555 & 2648 & 3.99082 & 3274 & 24.38887 & 29697 & 28.37970 & 32970 \\
\hline 1400 & 4.46203 & 2306 & 4.02356 & 3001 & 24.68584 & 27863 & 28.70940 & 30865 \\
\hline 1500 & 4.48509 & 2037 & 4.05357 & 2762 & 24.96447 & 26251 & 29.01805 & 29012 \\
\hline 1600 & 4.50546 & 1822 & 4.08119 & 2550 & 25.22698 & 24819 & 29.30817 & 27370 \\
\hline 1700 & 4.52368 & 1648 & 4.10669 & 2364 & 25.47517 & 23541 & 29.58187 & 25904 \\
\hline 1800 & 4.54016 & 1507 & 4.13033 & 2197 & 25.71058 & 22391 & 29.84091 & 24589 \\
\hline 1900 & 4.55523 & 1389 & 4.15230 & 2050 & 25.93449 & 21352 & 30.08680 & 23401 \\
\hline 2000 & 4.56912 & 1293 & 4.17280 & 1918 & 26.14801 & 20406 & 30.32081 & 22324 \\
\hline 2100 & 4.58205 & 1212 & 4.19198 & 1801 & 26.35207 & 19543 & 30.54405 & 21344 \\
\hline 2200 & 4.59417 & 1143 & 4.20999 & 1695 & 26.54750 & 18752 & 30.75749 & 20448 \\
\hline 2300 & 4.60560 & 1084 & 4.22694 & 1601 & 26.73502 & 18024 & 30.96197 & 19624 \\
\hline 2400 & 4.61644 & 1035 & 4.24295 & 1515 & 26.91526 & 17351 & 31.15821 & 18866 \\
\hline 2500 & 4.62679 & 991 & 4.25810 & 1437 & 27.08877 & 16729 & 31.34687 & 18166 \\
\hline 2600 & 4.63670 & 954 & 4.27247 & 1367 & 27.25606 & 16150 & 31.52853 & 17517 \\
\hline 2700 & 4.64624 & 921 & 4.28614 & 1302 & 27.41756 & 15612 & 31.70370 & 16914 \\
\hline 2800 & 4.65545 & 892 & 4.29916 & 1244 & 27.57368 & 15108 & 31.87284 & 16353 \\
\hline 2900 & 4.66437 & 867 & 4.31160 & 1191 & 27.72476 & 14637 & 32.03637 & 15827 \\
\hline 3000 & 4.67304 & 1669 & 4.32351 & 2237 & 27.87113 & 27976 & 32.19464 & 30213 \\
\hline 3200 & 4.68973 & 1596 & 4.34588 & 2070 & 28.15089 & 26410 & 32.49677 & 28480 \\
\hline 3400 & 4.70569 & 1538 & 4.36658 & 1927 & 28.41499 & 25013 & 32.78157 & 26941 \\
\hline 3600 & 4.72107 & 1488 & 4.38585 & 1803 & 28.66512 & 23762 & 33.05098 & 25565 \\
\hline 3800 & 4.73595 & 1447 & 4.40388 & 1697 & 28.90274 & 22633 & 33.30663 & 24329 \\
\hline 4000 & 4.75042 & 1412 & 4.42085 & 1603 & 29.12907 & 21608 & 33.54992 & 23212 \\
\hline 4200 & 4.76454 & 1382 & 4.43688 & 1521 & 29.34515 & 20676 & 33.78204 & 22197 \\
\hline 4400 & 4.77836 & 1355 & 4.45209 & 1448 & 29.55191 & 19823 & 34.00401 & 21270 \\
\hline 4600 & 4.79191 & 1331 & 4.46657 & 1384 & 29.75014 & 19039 & 34.21671 & 20423 \\
\hline 4800 & 4.80522 & 1310 & 4.48041 & 1325 & 29.94053 & 18317 & 34.42094 & 19642 \\
\hline & 4.81832 & & 4.49366 & & 30.12370 & & 34.61736 & \\
\hline
\end{tabular}


Table 2.031. MgH

\begin{tabular}{|l|l|l|l|l|}
\hline${ }^{\circ}$ & $\frac{C_{p}^{0}}{R}$ & $\frac{\left(H^{0}-E_{0}^{0}\right)}{R T}$ & $\frac{-\left(F^{0}-E_{0}^{0}\right)}{R T}$ & $\frac{S^{0}}{R}$ \\
\hline
\end{tabular}

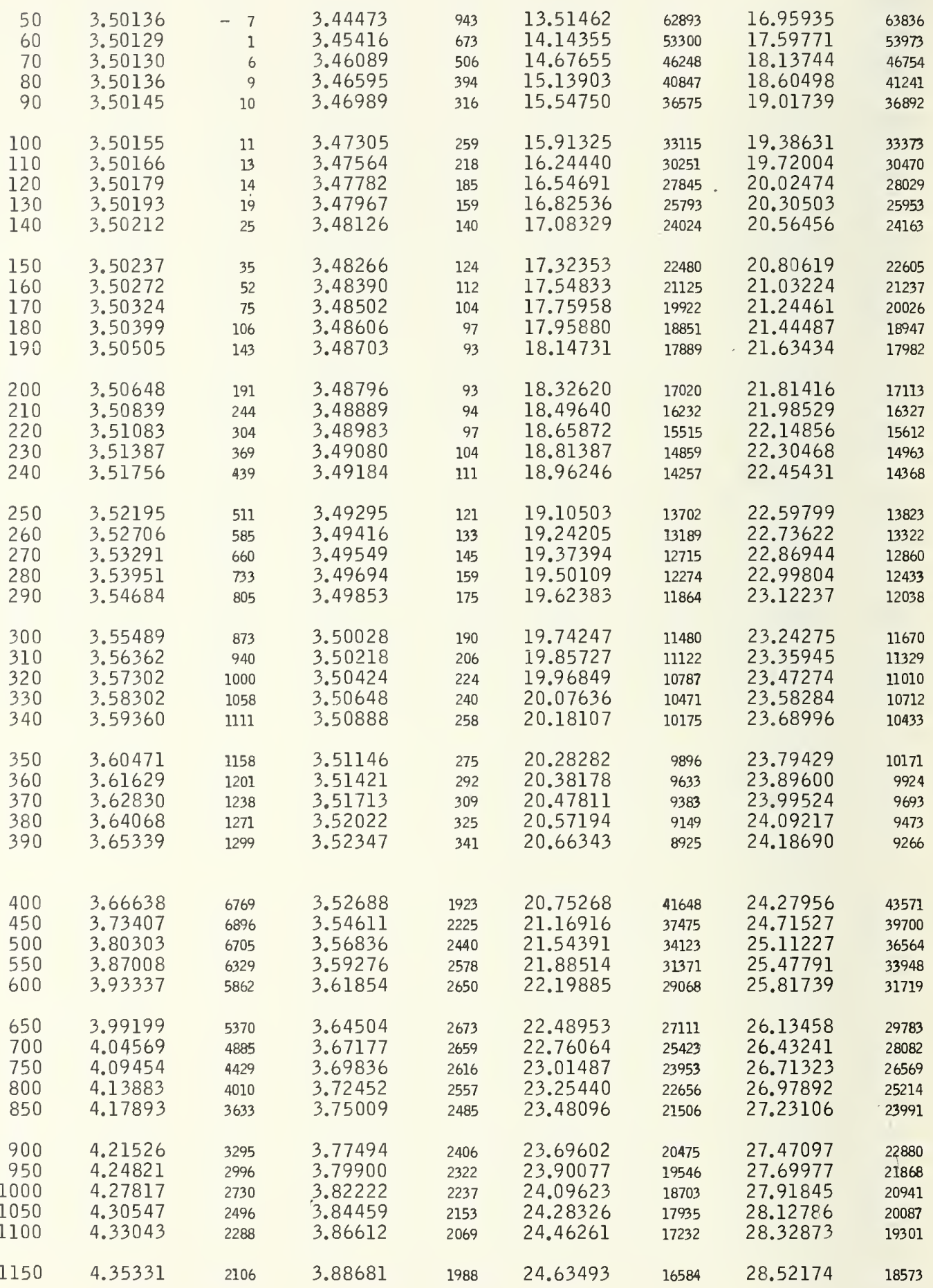


Table 2.031. MgH (Cont.)

\begin{tabular}{|l|l|l|l|l|}
\hline$K$ & $\frac{C_{p}^{0}}{R}$ & $\frac{\left(H^{0}-E_{0}^{0}\right)}{R T}$ & $\frac{-\left(F^{0}-E_{0}^{0}\right)}{R T}$ & $\frac{S^{0}}{R}$ \\
\hline
\end{tabular}

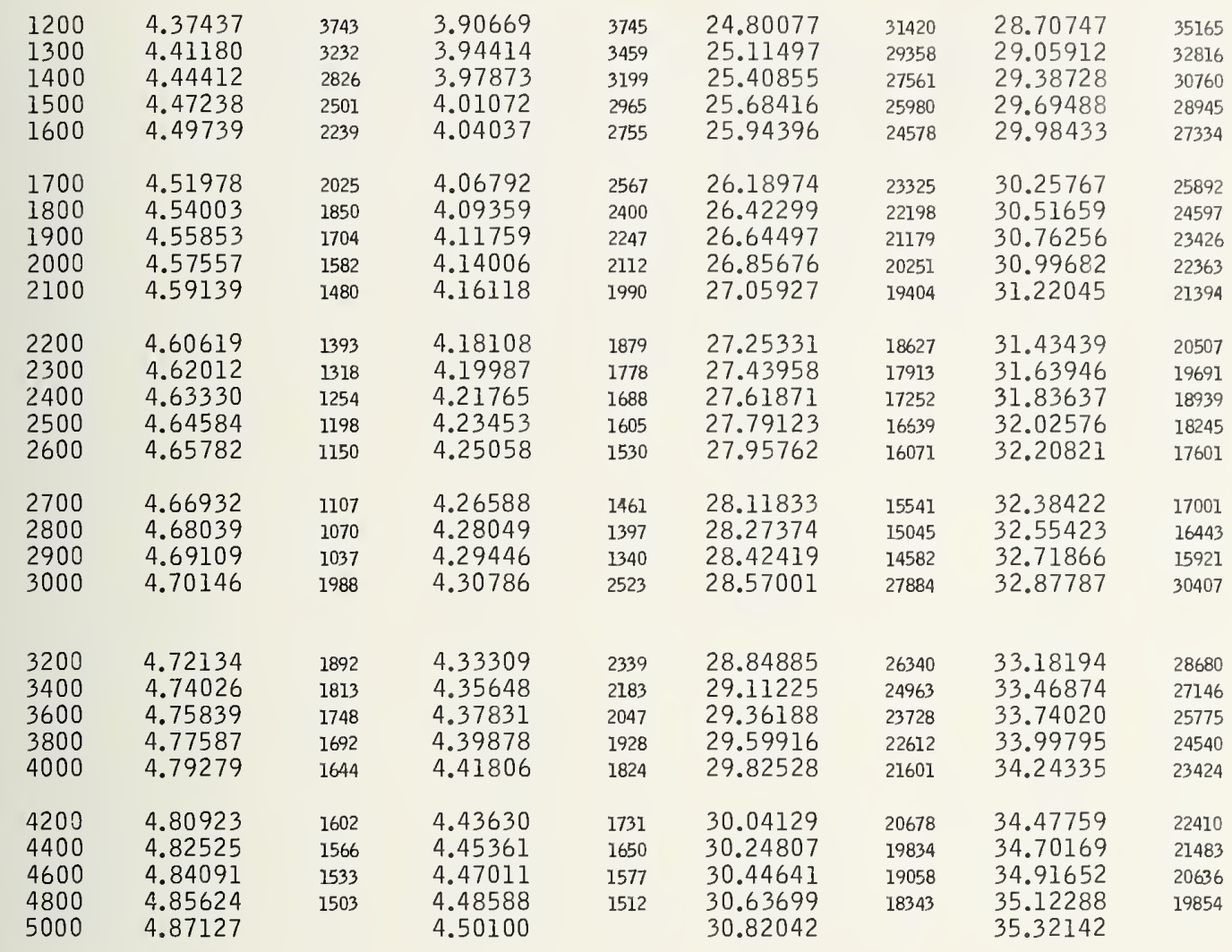


Table 2.032. MgD

\begin{tabular}{|l|l|l|l|l|}
\hline$K^{\circ}$ & $\frac{C_{P}^{0}}{R}$ & $\frac{\left(H^{0}-E_{0}^{0}\right)}{R T}$ & $\frac{-\left(F^{\circ}-E_{0}^{0}\right)}{R T}$ & $\frac{S^{0}}{R}$ \\
\hline
\end{tabular}

\begin{tabular}{|c|c|c|c|c|c|c|c|c|}
\hline $\begin{array}{l}50 \\
60 \\
70 \\
80 \\
90\end{array}$ & $\begin{array}{l}3.50087 \\
3.50095 \\
3.50106 \\
3.50118 \\
3.50132\end{array}$ & $\begin{array}{r}8 \\
11 \\
12 \\
14 \\
18\end{array}$ & $\begin{array}{l}3.47142 \\
3.47634 \\
3.47986 \\
3.48252 \\
3.48460\end{array}$ & $\begin{array}{l}492 \\
352 \\
266 \\
208 \\
168\end{array}$ & $\begin{array}{l}14.19456 \\
14.82793 \\
15.36409 \\
15.82895 \\
16.23925\end{array}$ & $\begin{array}{l}63337 \\
53616 \\
46486 \\
41030 \\
36723\end{array}$ & $\begin{array}{l}17.66599 \\
18.30428 \\
18.84396 \\
19.31147 \\
19.72386\end{array}$ & $\begin{array}{l}63829 \\
53968 \\
46751 \\
41239 \\
36891\end{array}$ \\
\hline $\begin{array}{l}100 \\
110 \\
120 \\
130 \\
140\end{array}$ & $\begin{array}{l}3.50150 \\
3.50178 \\
3.50228 \\
3.50312 \\
3.50451\end{array}$ & $\begin{array}{r}28 \\
50 \\
84 \\
139 \\
213\end{array}$ & $\begin{array}{l}3.48628 \\
3.48768 \\
3.48887 \\
3.48993 \\
3.49092\end{array}$ & $\begin{array}{r}140 \\
119 \\
106 \\
99 \\
97\end{array}$ & $\begin{array}{l}16.60648 \\
16.93883 \\
17.24235 \\
17.52165 \\
17.78032\end{array}$ & $\begin{array}{l}33235 \\
30352 \\
27930 \\
25867 \\
24088\end{array}$ & $\begin{array}{l}20.09277 \\
20.42651 \\
20.73122 \\
21.01159 \\
21.27124\end{array}$ & $\begin{array}{l}33374 \\
30471 \\
28037 \\
25965 \\
24186\end{array}$ \\
\hline $\begin{array}{l}150 \\
160 \\
170 \\
180 \\
190\end{array}$ & $\begin{array}{l}3.50664 \\
3.50971 \\
3.51388 \\
3.51930 \\
3.52604\end{array}$ & $\begin{array}{l}307 \\
417 \\
542 \\
674 \\
812\end{array}$ & $\begin{array}{l}3.49189 \\
3.49290 \\
3.49401 \\
3.49526 \\
3.49669\end{array}$ & $\begin{array}{l}101 \\
111 \\
125 \\
143 \\
167\end{array}$ & $\begin{array}{l}18.02120 \\
18.24659 \\
18.45838 \\
18.65813 \\
18.84715\end{array}$ & $\begin{array}{l}22539 \\
21179 \\
19975 \\
18902 \\
17940\end{array}$ & $\begin{array}{l}21.51310 \\
21.73950 \\
21.95240 \\
22.15339 \\
22.34385\end{array}$ & $\begin{array}{l}22640 \\
21290 \\
20099 \\
19046 \\
18106\end{array}$ \\
\hline $\begin{array}{l}200 \\
210 \\
220 \\
230 \\
240\end{array}$ & $\begin{array}{l}3.53416 \\
3.54366 \\
3.55450 \\
3.56662 \\
3.57991\end{array}$ & $\begin{array}{r}950 \\
1084 \\
1212 \\
1329 \\
1436\end{array}$ & $\begin{array}{l}3.49836 \\
3.50029 \\
3.50250 \\
3.50502 \\
3.50786\end{array}$ & $\begin{array}{l}193 \\
221 \\
252 \\
284 \\
316\end{array}$ & $\begin{array}{l}19.02655 \\
19.19728 \\
19.36016 \\
19.51591 \\
19.66514\end{array}$ & $\begin{array}{l}17073 \\
16288 \\
15575 \\
14923 \\
14326\end{array}$ & $\begin{array}{l}22.52491 \\
22.69757 \\
22.86266 \\
23.02093 \\
23.17300\end{array}$ & $\begin{array}{l}17266 \\
16509 \\
15827 \\
15207 \\
14643\end{array}$ \\
\hline $\begin{array}{l}250 \\
260 \\
270 \\
280 \\
290\end{array}$ & $\begin{array}{l}3.59427 \\
3.60958 \\
3.62572 \\
3.64255 \\
3.65996\end{array}$ & $\begin{array}{l}1531 \\
1614 \\
1683 \\
1741 \\
1786\end{array}$ & $\begin{array}{l}3.51102 \\
3.51452 \\
3.51833 \\
3.52247 \\
3.52691\end{array}$ & $\begin{array}{l}350 \\
381 \\
414 \\
444 \\
473\end{array}$ & $\begin{array}{l}19.80840 \\
19.94617 \\
20.07888 \\
20.20691 \\
20.33059\end{array}$ & $\begin{array}{l}13777 \\
13271 \\
12803 \\
12368 \\
11965\end{array}$ & $\begin{array}{l}23.31943 \\
23.46069 \\
23.59722 \\
23.72938 \\
23.85751\end{array}$ & $\begin{array}{l}14126 \\
13653 \\
13216 \\
12813 \\
12437\end{array}$ \\
\hline $\begin{array}{l}300 \\
310 \\
320 \\
330 \\
340\end{array}$ & $\begin{array}{l}3.67782 \\
3.69602 \\
3.71447 \\
3.73306 \\
3.75172\end{array}$ & $\begin{array}{l}1820 \\
1845 \\
1859 \\
1866 \\
1864\end{array}$ & $\begin{array}{l}3.53164 \\
3.53665 \\
3.54192 \\
3.54743 \\
3.55316\end{array}$ & $\begin{array}{l}501 \\
527 \\
551 \\
573 \\
594\end{array}$ & $\begin{array}{l}20.45024 \\
20.56612 \\
20.67849 \\
20.78756 \\
20.89355\end{array}$ & $\begin{array}{l}11588 \\
11237 \\
10907 \\
10599 \\
10308\end{array}$ & $\begin{array}{l}23.98188 \\
24.10278 \\
24.22041 \\
24.33500 \\
24.44672\end{array}$ & $\begin{array}{l}12090 \\
11763 \\
11459 \\
11172 \\
10902\end{array}$ \\
\hline $\begin{array}{l}350 \\
360 \\
370 \\
380 \\
390\end{array}$ & $\begin{array}{l}3.77036 \\
3.78893 \\
3.80737 \\
3.82562 \\
3.84364\end{array}$ & $\begin{array}{l}1857 \\
1844 \\
1825 \\
1802 \\
1776\end{array}$ & $\begin{array}{l}3.55910 \\
3.56523 \\
3.57152 \\
3.57797 \\
3.58455\end{array}$ & $\begin{array}{l}613 \\
629 \\
645 \\
658 \\
670\end{array}$ & $\begin{array}{l}20.99663 \\
21.09698 \\
21.19475 \\
21.29008 \\
21.38311\end{array}$ & $\begin{array}{r}10035 \\
9777 \\
9533 \\
9303 \\
9083\end{array}$ & $\begin{array}{l}24.55574 \\
24.66221 \\
24.76628 \\
24.86806 \\
24.96766\end{array}$ & \begin{tabular}{r|}
10647 \\
10407 \\
10178 \\
9960 \\
9754
\end{tabular} \\
\hline $\begin{array}{l}400 \\
450 \\
500 \\
550 \\
600\end{array}$ & $\begin{array}{l}3.86140 \\
3.94544 \\
4.02042 \\
4.08617 \\
4.14339\end{array}$ & $\begin{array}{l}8404 \\
7498 \\
6575 \\
5722 \\
4971\end{array}$ & $\begin{array}{l}3.59125 \\
3.62602 \\
3.66178 \\
3.69744 \\
3.73228\end{array}$ & $\begin{array}{l}3477 \\
3576 \\
3566 \\
3484 \\
3358\end{array}$ & $\begin{array}{l}21.47394 \\
21.89892 \\
22.28281 \\
22.63349 \\
22.95671\end{array}$ & $\begin{array}{l}42498 \\
38389 \\
35068 \\
32322 \\
30008\end{array}$ & $\begin{array}{l}25.06520 \\
25.52494 \\
25.94460 \\
26.33094 \\
26.68900\end{array}$ & $\begin{array}{l}45974 \\
41966 \\
38634 \\
35806 \\
33366\end{array}$ \\
\hline $\begin{array}{l}650 \\
700 \\
750 \\
800 \\
850\end{array}$ & $\begin{array}{l}4.19310 \\
4.23634 \\
4.27 .409 \\
4.30721 \\
4.33644\end{array}$ & $\begin{array}{l}4324 \\
3775 \\
3312 \\
2923 \\
2593\end{array}$ & $\begin{array}{l}3.76586 \\
3.79796 \\
3.82847 \\
3.85738 \\
3.88471\end{array}$ & $\begin{array}{l}3210 \\
3051 \\
2891 \\
2733 \\
2583\end{array}$ & $\begin{array}{l}23.25679 \\
23.53706 \\
23.80014 \\
24.04815 \\
24.28283\end{array}$ & $\begin{array}{l}28027 \\
26308 \\
24801 \\
23468 \\
22279\end{array}$ & $\begin{array}{l}27.02266 \\
27.33502 \\
27.62861 \\
27.90554 \\
28.16755\end{array}$ & $\begin{array}{l}31236 \\
29359 \\
27693 \\
26201 \\
24862\end{array}$ \\
\hline $\begin{array}{r}900 \\
950 \\
1000 \\
1050 \\
1100\end{array}$ & $\begin{array}{l}4.36237 \\
4.38553 \\
4.40634 \\
4.42514 \\
4.44222\end{array}$ & $\begin{array}{l}2316 \\
2081 \\
1880 \\
1708 \\
1562\end{array}$ & $\begin{array}{l}3.91054 \\
3.93495 \\
3.95800 \\
3.97981 \\
4.00045\end{array}$ & $\begin{array}{l}2441 \\
2305 \\
2181 \\
2064 \\
1955\end{array}$ & $\begin{array}{l}24.50562 \\
24.71771 \\
24.92014 \\
25.11378 \\
25.29940\end{array}$ & $\begin{array}{l}21209 \\
20243 \\
19364 \\
18562 \\
17827\end{array}$ & $\begin{array}{l}28.41617 \\
28.65266 \\
28.87815 \\
29.09360 \\
29.29985\end{array}$ & $\begin{array}{l}23649 \\
22549 \\
21545 \\
20625 \\
19782\end{array}$ \\
\hline 1150 & 4.45784 & 1435 & 4.02000 & 1854 & 25.47767 & 17148 & 29.49767 & 19003 \\
\hline
\end{tabular}


Table 2.032. MgD (Cont.)

\begin{tabular}{|l|l|l|l|l|}
\hline${ }^{\circ}$ & $\frac{C_{p}^{0}}{R}$ & $\frac{\left(H^{0}-E_{0}^{0}\right)}{R T}$ & $\frac{-\left(F^{0}-E_{0}^{0}\right)}{R T}$ & $\frac{S^{0}}{R}$ \\
\hline
\end{tabular}

$\begin{array}{lllllllll}1200 & 4.47219 & 2556 & 4.03854 & 3437 & 25.64915 & 32464 & 29.68770 & 35900 \\ 1300 & 4.49775 & 2220 & 4.07291 & 3115 & 25.97379 & 30299 & 30.04670 & 33415 \\ 1400 & 4.51995 & 1963 & 4.10406 & 2840 & 26.27678 & 28414 & 30.38085 & 31253 \\ 1500 & 4.53958 & 1762 & 4.13246 & 2600 & 26.56092 & 26754 & 30.69338 & 29355 \\ 1600 & 4.55720 & 1601 & 4.15846 & 2394 & 26.82846 & 25283 & 30.98693 & 27676 \\ & & & & & & & & \\ 1700 & 4.57321 & 1473 & 4.18240 & 2212 & 27.08129 & 23970 & 31.26369 & 26183 \\ 1800 & 4.58794 & 1369 & 4.20452 & 2055 & 27.32099 & 22788 & 31.52552 & 24842 \\ 1900 & 4.60163 & 1282 & 4.22507 & 1915 & 27.54887 & 21721 & 31.77394 & 23637 \\ 2000 & 4.61445 & 1212 & 4.24422 & 1792 & 27.76608 & 20752 & 32.01031 & 22543 \\ 2100 & 4.62657 & 1151 & 4.26214 & 1683 & 27.97360 & 19866 & 32.23574 & 21550 \\ & & & & & & & & \\ 2200 & 4.63808 & 1101 & 4.27897 & 1585 & 28.17226 & 19057 & 32.45124 & 20641 \\ 2300 & 4.64909 & 1057 & 4.29482 & 1499 & 28.36283 & 18310 & 32.65765 & 19809 \\ 2400 & 4.65966 & 1020 & 4.30981 & 1419 & 28.54593 & 17623 & 32.85574 & 19043 \\ 2500 & 4.66986 & 988 & 4.32400 & 1350 & 28.72216 & 16985 & 33.04617 & 18335 \\ 2600 & 4.67974 & 960 & 4.33750 & 1285 & 28.89201 & 16394 & 33.22952 & 17679 \\ 2700 & 4.68934 & 935 & 4.35035 & 1228 & 29.05595 & 15844 & 33.40631 & 17071 \\ 2800 & 4.69869 & 913 & 4.36263 & 1174 & 29.21439 & 15330 & 33.57702 & 16504 \\ 2900 & 4.70782 & 893 & 4.37437 & 1127 & 29.36769 & 14849 & 33.74206 & 15976 \\ 3000 & 4.71675 & 1736 & 4.38564 & 2124 & 29.51618 & 28373 & 33.90182 & 30497 \\ & & & & & & & & \\ 3200 & 4.73411 & 1677 & 4.40688 & 1974 & 29.79991 & 26776 & 34.20679 & 28751 \\ 3400 & 4.75088 & 1630 & 4.42662 & 1847 & 30.06767 & 25355 & 34.49430 & 27201 \\ 3600 & 4.76718 & 1588 & 4.44509 & 1737 & 30.32122 & 24080 & 34.76631 & 25818 \\ 3800 & 4.78306 & 1553 & 4.46246 & 1642 & 30.56202 & 22932 & 35.02449 & 24574 \\ 4000 & 4.79859 & 1522 & 4.47888 & 1559 & 30.79134 & 21890 & 35.27023 & 23449 \\ & & & & & & & \\ 4200 & 4.81381 & 1494 & 4.49447 & 1486 & 31.01024 & 20943 & 35.50472 & 22428 \\ 4400 & 4.82875 & 1469 & 4.50933 & 1420 & 31.21967 & 20077 & 35.72900 & 21498 \\ 4600 & 4.84344 & 1445 & 4.52353 & 1364 & 31.42044 & 19281 & 35.94398 & 20644 \\ 4800 & 4.85789 & 1425 & 4.53717 & 1311 & 31.61325 & 18548 & 36.15042 & 19860 \\ 5000 & 4.87214 & & 4.55028 & & 31.79873 & & 36.34902 & \\ & & & & & & & & \end{array}$


Table 2.033. $\quad \operatorname{MgT}$

\begin{tabular}{|l|l|l|l|l|}
\hline$K$ & $\frac{C_{p}^{0}}{R}$ & $\frac{\left(H^{0}-E_{0}^{0}\right)}{R T}$ & $\frac{-\left(F^{0}-E_{0}^{0}\right)}{R T}$ & $\frac{S^{0}}{R}$ \\
\hline
\end{tabular}

\begin{tabular}{|c|c|c|c|c|c|c|c|c|}
\hline $\begin{array}{l}50 \\
60 \\
70 \\
80 \\
90\end{array}$ & $\begin{array}{l}3.50078 \\
3.50089 \\
3.50102 \\
3.50118 \\
3.50144\end{array}$ & $\begin{array}{l}11 \\
13 \\
16 \\
26 \\
52\end{array}$ & $\begin{array}{l}3.48032 \\
3.48374 \\
3.43619 \\
3.48806 \\
3.43953\end{array}$ & $\begin{array}{l}342 \\
245 \\
187 \\
147 \\
121\end{array}$ & $\begin{array}{l}14.60781 \\
15.24267 \\
15.77983 \\
16.24553 \\
16.65645\end{array}$ & $\begin{array}{l}63486 \\
53721 \\
46565 \\
41092 \\
36772\end{array}$ & $\begin{array}{l}18.98813 \\
18.72641 \\
19.26608 \\
19.73359 \\
20.14598\end{array}$ & $\begin{array}{l}63828 \\
53967 \\
46751 \\
41239 \\
36894\end{array}$ \\
\hline $\begin{array}{l}100 \\
110 \\
120 \\
130 \\
140\end{array}$ & $\begin{array}{l}3.50196 \\
3.50298 \\
3.50481 \\
3.50780 \\
3.51225\end{array}$ & $\begin{array}{l}102 \\
183 \\
299 \\
445 \\
617\end{array}$ & $\begin{array}{l}3.49074 \\
3.49180 \\
3.49281 \\
3.49384 \\
3.49498\end{array}$ & $\begin{array}{l}106 \\
101 \\
103 \\
114 \\
135\end{array}$ & $\begin{array}{l}17.02417 \\
17.35693 \\
17.66080 \\
17.94041 \\
18.19937\end{array}$ & $\begin{array}{l}33276 \\
30387 \\
27961 \\
25896 \\
24118\end{array}$ & $\begin{array}{l}20.51492 \\
20.84874 \\
21.15361 \\
21.43425 \\
21.69436\end{array}$ & $\begin{array}{l}33382 \\
30487 \\
28064 \\
26011 \\
24252\end{array}$ \\
\hline $\begin{array}{l}150 \\
160 \\
170 \\
180 \\
190\end{array}$ & $\begin{array}{l}3.51842 \\
3.52648 \\
3.53650 \\
3.54847 \\
3.56230\end{array}$ & $\begin{array}{r}806 \\
1002 \\
1197 \\
1383 \\
1554\end{array}$ & $\begin{array}{l}3.49633 \\
3.49795 \\
3.49991 \\
3.50227 \\
3.50506\end{array}$ & $\begin{array}{l}162 \\
196 \\
236 \\
279 \\
324\end{array}$ & $\begin{array}{l}18.44055 \\
18.66625 \\
18.87837 \\
19.07848 \\
19.26791\end{array}$ & $\begin{array}{l}22570 \\
21212 \\
20011 \\
18943 \\
17987\end{array}$ & $\begin{array}{l}21.93688 \\
22.16420 \\
22.37829 \\
22.58076 \\
22.77297\end{array}$ & $\begin{array}{l}22732 \\
21409 \\
20247 \\
19221 \\
18312\end{array}$ \\
\hline $\begin{array}{l}200 \\
210 \\
220 \\
230 \\
240\end{array}$ & $\begin{array}{l}3.57784 \\
3.59492 \\
3.61334 \\
3.63286 \\
3.65329\end{array}$ & $\begin{array}{l}1708 \\
1842 \\
1952 \\
2043 \\
2111\end{array}$ & $\begin{array}{l}3.50830 \\
3.51201 \\
3.51620 \\
3.52084 \\
3.52593\end{array}$ & $\begin{array}{l}371 \\
419 \\
464 \\
509 \\
552\end{array}$ & $\begin{array}{l}19.44778 \\
19.61904 \\
19.78251 \\
19.93891 \\
20.08886\end{array}$ & $\begin{array}{l}17126 \\
16347 \\
15640 \\
14995 \\
14405\end{array}$ & $\begin{array}{l}22.95609 \\
23.13106 \\
23.29871 \\
23.45976 \\
23.61480\end{array}$ & $\begin{array}{l}17497 \\
16765 \\
16105 \\
15504 \\
14956\end{array}$ \\
\hline $\begin{array}{l}250 \\
260 \\
270 \\
280 \\
290\end{array}$ & $\begin{array}{l}3.67440 \\
3.69603 \\
3.71797 \\
3.74009 \\
3.76224\end{array}$ & $\begin{array}{l}2163 \\
2194 \\
2212 \\
2215 \\
2207\end{array}$ & $\begin{array}{l}3.53145 \\
3.53736 \\
3.54364 \\
3.55026 \\
3.55719\end{array}$ & $\begin{array}{l}591 \\
628 \\
662 \\
693 \\
720\end{array}$ & $\begin{array}{l}20.23291 \\
20.37153 \\
20.50515 \\
20.63414 \\
20.75885\end{array}$ & $\begin{array}{l}13862 \\
13362 \\
12899 \\
12471 \\
12071\end{array}$ & $\begin{array}{l}23.76436 \\
23.90889 \\
24.04879 \\
24.18441 \\
24.31604\end{array}$ & $\begin{array}{l}14453 \\
13990 \\
13562 \\
13163 \\
12792\end{array}$ \\
\hline $\begin{array}{l}300 \\
310 \\
320 \\
330 \\
340\end{array}$ & $\begin{array}{l}3.78431 \\
3.80618 \\
3.82779 \\
3.84905 \\
3.86992\end{array}$ & $\begin{array}{l}2187 \\
2161 \\
2126 \\
2087 \\
2042\end{array}$ & $\begin{array}{l}3.56439 \\
3.57184 \\
3.57950 \\
3.58735 \\
3.59535\end{array}$ & $\begin{array}{l}745 \\
766 \\
785 \\
800 \\
814\end{array}$ & $\begin{array}{l}20.87956 \\
20.99656 \\
21.11008 \\
21.22035 \\
21.32756\end{array}$ & $\begin{array}{l}11700 \\
11352 \\
11027 \\
10721 \\
10434\end{array}$ & $\begin{array}{l}24.44396 \\
24.56840 \\
24.68959 \\
24.80770 \\
24.92292\end{array}$ & $\begin{array}{l}12444 \\
12119 \\
11811 \\
11522 \\
11247\end{array}$ \\
\hline $\begin{array}{l}350 \\
360 \\
370 \\
380 \\
390\end{array}$ & $\begin{array}{l}3.89034 \\
3.91029 \\
3.92974 \\
3.94866 \\
3.96705\end{array}$ & $\begin{array}{l}1995 \\
1945 \\
1892 \\
1839 \\
1786\end{array}$ & $\begin{array}{l}3.60349 \\
3.61174 \\
3.62007 \\
3.62847 \\
3.63692\end{array}$ & $\begin{array}{l}825 \\
833 \\
840 \\
845 \\
847\end{array}$ & $\begin{array}{l}21.43190 \\
21.53352 \\
21.63260 \\
21.72925 \\
21.82361\end{array}$ & $\begin{array}{r}10162 \\
9908 \\
9665 \\
9436 \\
9218\end{array}$ & $\begin{array}{l}25.03539 \\
25.14527 \\
25.25267 \\
25.35772 \\
25.46053\end{array}$ & $\begin{array}{l}10988 \\
10740 \\
10505 \\
10281 \\
10066\end{array}$ \\
\hline $\begin{array}{l}400 \\
450 \\
500 \\
550 \\
600\end{array}$ & $\begin{array}{l}3.98491 \\
4.06619 \\
4.13497 \\
4.19295 \\
4.24191\end{array}$ & $\begin{array}{l}8128 \\
6878 \\
5798 \\
4896 \\
4157\end{array}$ & $\begin{array}{l}3.64539 \\
3.68776 \\
3.72914 \\
3.76874 \\
3.80619\end{array}$ & $\begin{array}{l}4237 \\
4138 \\
3960 \\
3745 \\
3516\end{array}$ & $\begin{array}{l}21.91579 \\
22.34761 \\
22.73831 \\
23.09561 \\
23.42515\end{array}$ & $\begin{array}{l}43182 \\
39070 \\
35730 \\
32954 \\
30606\end{array}$ & $\begin{array}{l}25.56119 \\
26.03537 \\
26.46745 \\
26.86435 \\
27.23135\end{array}$ & $\begin{array}{l}47418 \\
43208 \\
39690 \\
36700 \\
34122\end{array}$ \\
\hline $\begin{array}{l}650 \\
700 \\
750 \\
800 \\
850\end{array}$ & $\begin{array}{l}4.28348 \\
4.31904 \\
4.34970 \\
4.37635 \\
4.39973\end{array}$ & $\begin{array}{l}3556 \\
3066 \\
2665 \\
2338 \\
2067\end{array}$ & $\begin{array}{l}3.84135 \\
3.87423 \\
3.90493 \\
3.93358 \\
3.96033\end{array}$ & $\begin{array}{l}3288 \\
3070 \\
2865 \\
2675 \\
2499\end{array}$ & $\begin{array}{l}23.73121 \\
24.01711 \\
24.28546 \\
24.53840 \\
24.77769\end{array}$ & $\begin{array}{l}28590 \\
26835 \\
25294 \\
23929 \\
22708\end{array}$ & $\begin{array}{l}27.5725: \\
27.89134 \\
28.19040 \\
28.47199 \\
28.73802\end{array}$ & $\begin{array}{l}31877 \\
29906 \\
28159 \\
26603 \\
25208\end{array}$ \\
\hline $\begin{array}{r}900 \\
950 \\
1000 \\
1050 \\
1100\end{array}$ & $\begin{array}{l}4.42040 \\
4.43882 \\
4.455 .36 \\
4.47033 \\
4.48397\end{array}$ & $\begin{array}{l}1842 \\
1654 \\
1497 \\
1364 \\
1251\end{array}$ & $\begin{array}{l}3.98532 \\
4.00872 \\
4.03064 \\
4.05123 \\
4.07059\end{array}$ & $\begin{array}{l}2340 \\
2192 \\
2059 \\
1936 \\
1825\end{array}$ & $\begin{array}{l}25.00477 \\
25.22088 \\
25.42706 \\
25.62422 \\
25.81314\end{array}$ & $\begin{array}{l}21611 \\
20618 \\
19716 \\
18892 \\
18135\end{array}$ & $\begin{array}{l}28.99010 \\
29.22960 \\
29.45771 \\
29.67546 \\
29.88374\end{array}$ & $\begin{array}{l}23950 \\
22811 \\
21775 \\
20828 \\
19960\end{array}$ \\
\hline 1150 & 4.49648 & 1153 & 4.08884 & 1723 & 25.99449 & 17439 & 30.08334 & 19161 \\
\hline
\end{tabular}


Table 2.033. MgT (Cont.)

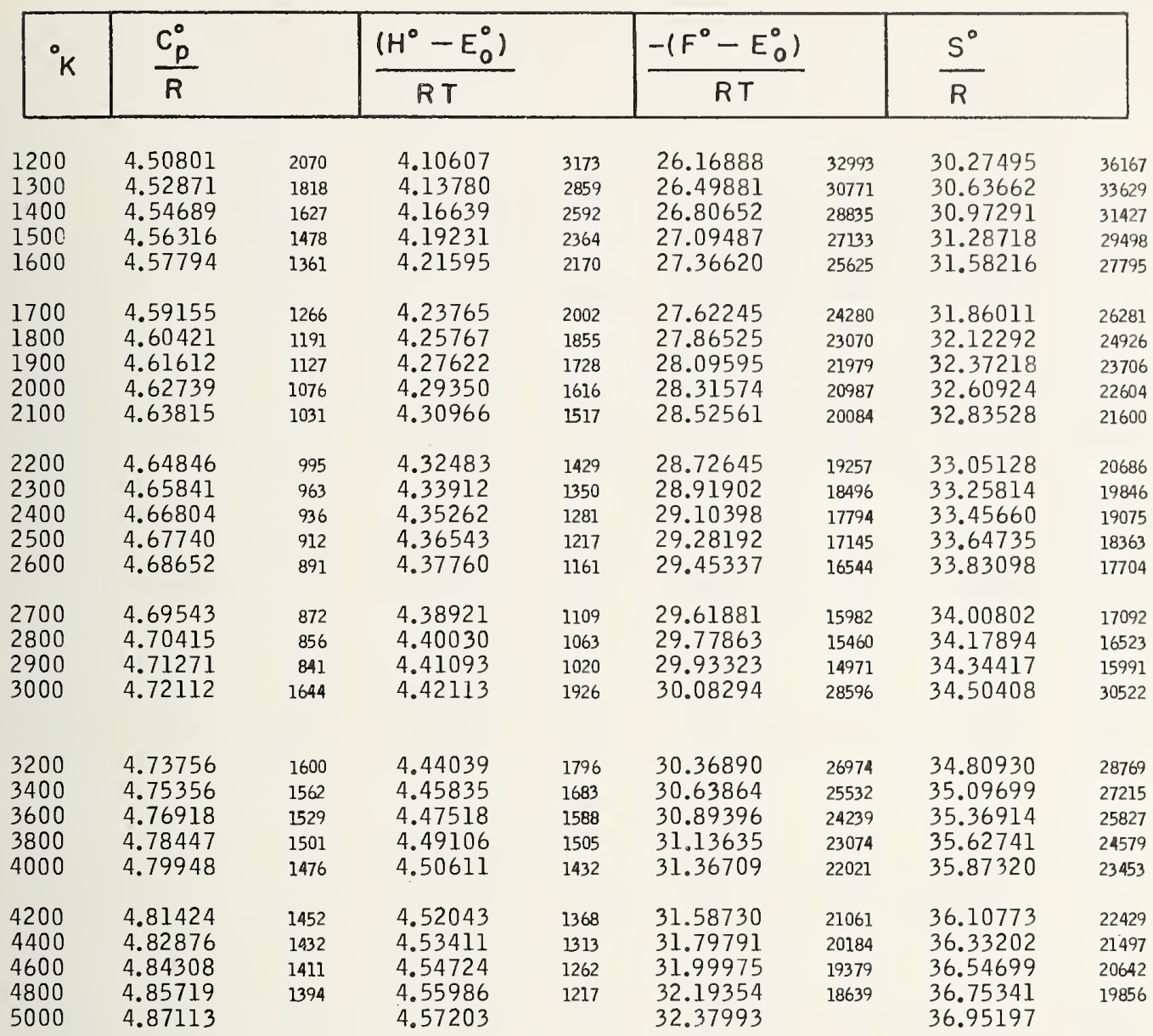


Table 2.034. $\mathrm{CaH}$

\begin{tabular}{|l|l|l|l|l|}
\hline$K$ & $\frac{C_{p}^{0}}{R}$ & $\frac{\left(H^{0}-E_{0}^{0}\right)}{R T}$ & $\frac{-\left(F^{0}-E_{0}^{0}\right)}{R T}$ & $\frac{S^{0}}{R}$ \\
\hline
\end{tabular}

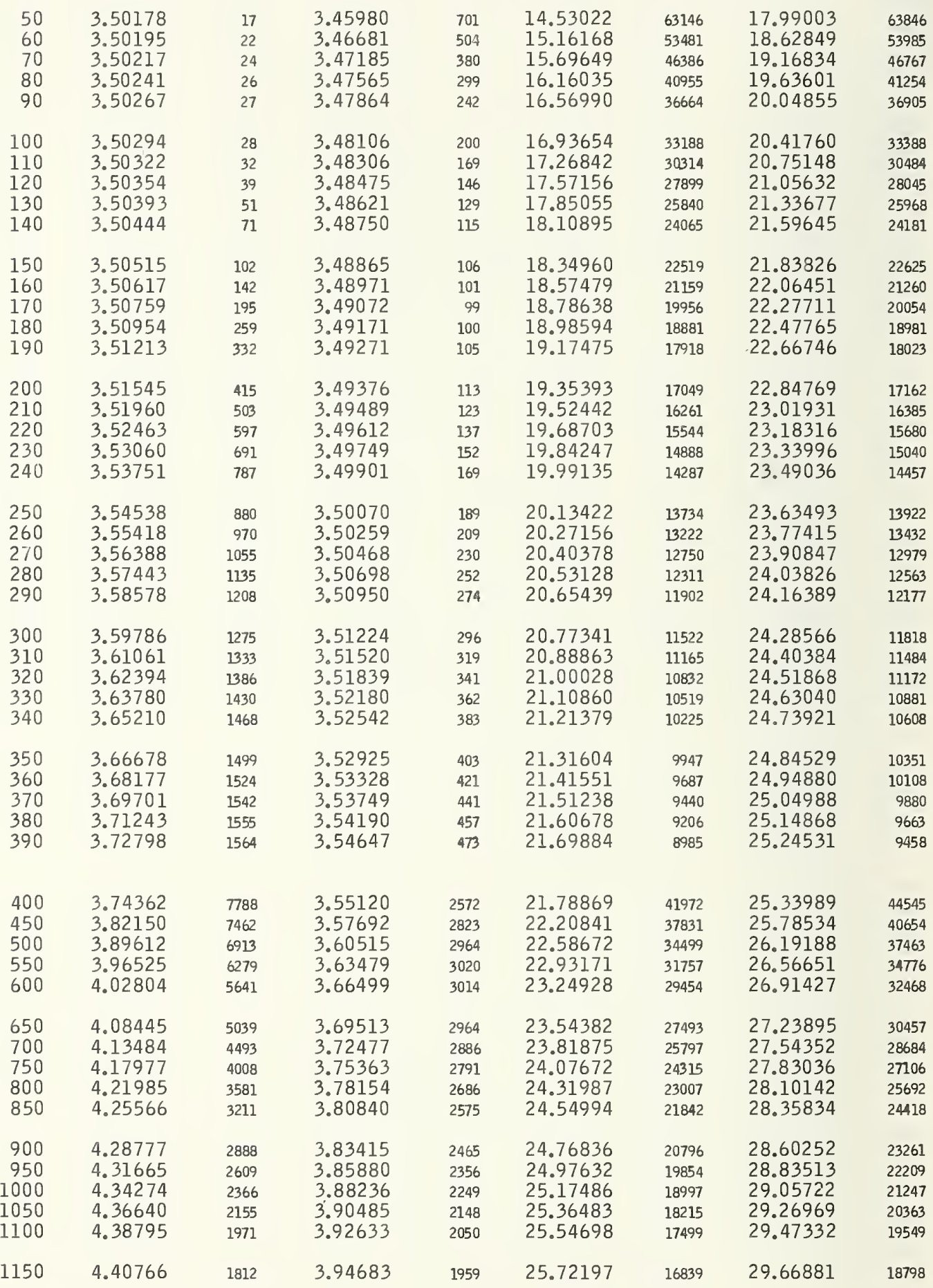


Table 2.034. CaH (Cont.)

\begin{tabular}{|l|l|l|l|l|}
\hline$K$ & $\frac{C_{p}^{0}}{R}$ & $\frac{\left(H^{\circ}-E_{0}^{0}\right)}{R T}$ & $\frac{-\left(F^{\circ}-E_{0}^{0}\right)}{R T}$ & $\frac{S^{0}}{R}$ \\
\hline
\end{tabular}

\begin{tabular}{|c|c|c|c|c|c|c|c|c|}
\hline $\begin{array}{l}1200 \\
1300 \\
1400 \\
1500 \\
1600\end{array}$ & $\begin{array}{l}4.42578 \\
4.45798 \\
4.48583 \\
4.51029 \\
4.53206\end{array}$ & $\begin{array}{l}3220 \\
2785 \\
2446 \\
2177 \\
1962\end{array}$ & $\begin{array}{l}3.96642 \\
4.00302 \\
4.03654 \\
4.06732 \\
4.09570\end{array}$ & $\begin{array}{l}3660 \\
3352 \\
3078 \\
2838 \\
2625\end{array}$ & $\begin{array}{l}25.89036 \\
26.20931 \\
26.50721 \\
26.78677 \\
27.05019\end{array}$ & $\begin{array}{l}31895 \\
29790 \\
27956 \\
26342 \\
24910\end{array}$ & $\begin{array}{l}29.85679 \\
30.21234 \\
30.54376 \\
30.85410 \\
31.14589\end{array}$ & $\begin{array}{l}35555 \\
33142 \\
31034 \\
29179 \\
27536\end{array}$ \\
\hline $\begin{array}{l}1700 \\
1800 \\
1900 \\
2000 \\
2100\end{array}$ & $\begin{array}{l}4.55168 \\
4.56957 \\
4.58605 \\
4.60135 \\
4.61568\end{array}$ & $\begin{array}{l}1789 \\
1648 \\
1530 \\
1433 \\
1351\end{array}$ & $\begin{array}{l}4.12195 \\
4.14633 \\
4.16905 \\
4.19028 \\
4.21020\end{array}$ & $\begin{array}{l}2438 \\
2272 \\
2123 \\
1992 \\
1874\end{array}$ & $\begin{array}{l}27.29929 \\
27.53559 \\
27.76039 \\
27.97478 \\
28.17971\end{array}$ & $\begin{array}{l}23630 \\
22480 \\
21439 \\
20493 \\
19629\end{array}$ & $\begin{array}{l}31.42125 \\
31.68193 \\
31.92944 \\
32.16507 \\
32.38992\end{array}$ & $\begin{array}{l}26068 \\
24751 \\
23563 \\
22485 \\
21503\end{array}$ \\
\hline $\begin{array}{l}2200 \\
2300 \\
2400 \\
2500 \\
2600\end{array}$ & $\begin{array}{l}4.62919 \\
4.64201 \\
4.65424 \\
4.66596 \\
4.67724\end{array}$ & $\begin{array}{l}1282 \\
1223 \\
1172 \\
1128 \\
1089\end{array}$ & $\begin{array}{l}4.22894 \\
4.24663 \\
4.26336 \\
4.27923 \\
4.29432\end{array}$ & $\begin{array}{l}1769 \\
1673 \\
1587 \\
1509 \\
1439\end{array}$ & $\begin{array}{l}28.37600 \\
28.56438 \\
28.74547 \\
28.91984 \\
29.08797\end{array}$ & $\begin{array}{l}18838 \\
18107 \\
17437 \\
16813 \\
16234\end{array}$ & $\begin{array}{l}32.60495 \\
32.81101 \\
33.00884 \\
33.19907 \\
33.38230\end{array}$ & $\begin{array}{l}20606 \\
19783 \\
19023 \\
18323 \\
17672\end{array}$ \\
\hline $\begin{array}{l}2700 \\
2800 \\
2900 \\
3000\end{array}$ & $\begin{array}{l}4.68813 \\
4.69869 \\
4.70896 \\
4.71896\end{array}$ & $\begin{array}{l}1056 \\
1027 \\
1000 \\
1932\end{array}$ & $\begin{array}{l}4.30871 \\
4.32245 \\
4.33560 \\
4.34821\end{array}$ & $\begin{array}{l}1374 \\
1315 \\
1261 \\
2378\end{array}$ & $\begin{array}{l}29.25031 \\
29.40726 \\
29.55917 \\
29.70637\end{array}$ & $\begin{array}{l}15695 \\
15191 \\
14720 \\
28139\end{array}$ & $\begin{array}{l}33.55902 \\
33.72971 \\
33.89477 \\
34.05458\end{array}$ & $\begin{array}{l}17069 \\
16506 \\
15981 \\
30518\end{array}$ \\
\hline $\begin{array}{l}3200 \\
3400 \\
3600 \\
3800 \\
4000\end{array}$ & $\begin{array}{l}4.73828 \\
4.75683 \\
4.77476 \\
4.79216 \\
4.80911\end{array}$ & $\begin{array}{l}1855 \\
1793 \\
1740 \\
1695 \\
1655\end{array}$ & $\begin{array}{l}4.37199 \\
4.39409 \\
4.41474 \\
4.43415 \\
4.45247\end{array}$ & $\begin{array}{l}2210 \\
2065 \\
1941 \\
1832 \\
1738\end{array}$ & $\begin{array}{l}29.98776 \\
30.25349 \\
30.50524 \\
30.74445 \\
30.97237\end{array}$ & $\begin{array}{l}26573 \\
25175 \\
23921 \\
22792 \\
21766\end{array}$ & $\begin{array}{l}34.35976 \\
34.64758 \\
34.91998 \\
35.17861 \\
35.42484\end{array}$ & $\begin{array}{l}28782 \\
27240 \\
25863 \\
24623 \\
23504\end{array}$ \\
\hline $\begin{array}{l}4200 \\
4400 \\
4600 \\
4800 \\
5000\end{array}$ & $\begin{array}{l}4.82566 \\
4.84188 \\
4.85779 \\
4.87342 \\
4.88880\end{array}$ & $\begin{array}{l}1622 \\
1591 \\
1563 \\
1538\end{array}$ & $\begin{array}{l}4.46985 \\
4.48640 \\
4.50220 \\
4.51734 \\
4.53189\end{array}$ & $\begin{array}{l}1655 \\
1580 \\
1514 \\
1455\end{array}$ & $\begin{array}{l}31.19003 \\
31.39835 \\
31.59813 \\
31.79006 \\
31.97477\end{array}$ & $\begin{array}{l}20832 \\
19978 \\
19193 \\
18471\end{array}$ & $\begin{array}{l}35.65988 \\
35.88475 \\
36.10033 \\
36.30741 \\
36.50666\end{array}$ & $\begin{array}{l}22487 \\
21558 \\
20708 \\
19925\end{array}$ \\
\hline
\end{tabular}


Table 2.035. CaD

\begin{tabular}{|l|l|l|l|l|}
\hline$K^{\circ}$ & $\frac{C_{p}^{\circ}}{R}$ & $\frac{\left(H^{\circ}-E_{0}^{\circ}\right)}{R T}$ & $\frac{-\left(F^{\circ}-E_{0}^{\circ}\right)}{R T}$ & $\frac{S^{\circ}}{R}$ \\
\hline
\end{tabular}

\begin{tabular}{|c|c|c|c|c|c|c|c|c|}
\hline $\begin{array}{l}50 \\
60 \\
70 \\
80 \\
90\end{array}$ & $\begin{array}{l}3.50151 \\
3.50177 \\
3.50204 \\
3.50234 \\
3.50270\end{array}$ & $\begin{array}{l}26 \\
27 \\
30 \\
36 \\
53\end{array}$ & $\begin{array}{l}3.47973 \\
3.48339 \\
3.48603 \\
3.48805 \\
3.48966\end{array}$ & $\begin{array}{l}366 \\
264 \\
202 \\
161 \\
133\end{array}$ & $\begin{array}{l}15.21022 \\
15.84499 \\
16.38217 \\
16.84780 \\
17.25873\end{array}$ & $\begin{array}{l}63477 \\
53718 \\
46563 \\
41093 \\
36774\end{array}$ & $\begin{array}{l}18.68996 \\
19.32838 \\
19.86820 \\
20.33586 \\
20.74839\end{array}$ & $\begin{array}{l}63842 \\
53982 \\
46766 \\
41253 \\
36907\end{array}$ \\
\hline $\begin{array}{l}100 \\
110 \\
120 \\
130 \\
140\end{array}$ & $\begin{array}{l}3.50323 \\
3.50413 \\
3.50564 \\
3.50808 \\
3.51172\end{array}$ & $\begin{array}{r}90 \\
151 \\
244 \\
364 \\
512\end{array}$ & $\begin{array}{l}3.49099 \\
3.49214 \\
3.49319 \\
3.49424 \\
3.49535\end{array}$ & $\begin{array}{l}115 \\
105 \\
105 \\
111 \\
125\end{array}$ & $\begin{array}{l}17.62647 \\
17.95926 \\
18.26316 \\
18.54280 \\
18.80180\end{array}$ & $\begin{array}{l}33279 \\
30390 \\
27964 \\
25900 \\
24119\end{array}$ & $\begin{array}{l}21.11746 \\
21.45140 \\
21.75636 \\
22.03705 \\
22.29715\end{array}$ & $\begin{array}{l}33394 \\
30496 \\
28069 \\
26010 \\
24245\end{array}$ \\
\hline $\begin{array}{l}150 \\
160 \\
170 \\
180 \\
190\end{array}$ & $\begin{array}{l}3.51684 \\
3.52361 \\
3.53216 \\
3.54252 \\
3.55466\end{array}$ & $\begin{array}{r}677 \\
855 \\
1036 \\
1214 \\
1382\end{array}$ & $\begin{array}{l}3.49660 \\
3.49807 \\
3.49981 \\
3.50189 \\
3.50434\end{array}$ & $\begin{array}{l}147 \\
174 \\
208 \\
245 \\
286\end{array}$ & $\begin{array}{l}19.04299 \\
19.26870 \\
19.48082 \\
19.68092 \\
19.87033\end{array}$ & $\begin{array}{l}22571 \\
21212 \\
20010 \\
18941 \\
17982\end{array}$ & $\begin{array}{l}22.53960 \\
22.76678 \\
22.98064 \\
23.18282 \\
23.37467\end{array}$ & $\begin{array}{l}22718 \\
21386 \\
20218 \\
19185 \\
18268\end{array}$ \\
\hline $\begin{array}{l}200 \\
210 \\
220 \\
230 \\
240\end{array}$ & $\begin{array}{l}3.56848 \\
3.58385 \\
3.60060 \\
3.61854 \\
3.63749\end{array}$ & $\begin{array}{l}1537 \\
1675 \\
1794 \\
1895 \\
1977\end{array}$ & $\begin{array}{l}3.50720 \\
3.51047 \\
3.51418 \\
3.51833 \\
3.52290\end{array}$ & $\begin{array}{l}327 \\
371 \\
415 \\
457 \\
497\end{array}$ & $\begin{array}{l}20.05015 \\
20.22134 \\
20.38473 \\
20.54103 \\
20.69087\end{array}$ & $\begin{array}{l}17119 \\
16339 \\
15630 \\
14984 \\
14391\end{array}$ & $\begin{array}{l}23.55735 \\
23.73182 \\
23.89892 \\
24.05937 \\
24.21377\end{array}$ & $\begin{array}{l}17447 \\
16710 \\
16045 \\
15440 \\
14889\end{array}$ \\
\hline $\begin{array}{l}250 \\
260 \\
270 \\
280 \\
290\end{array}$ & $\begin{array}{l}3.65726 \\
3.67765 \\
3.69851 \\
3.71967 \\
3.74099\end{array}$ & $\begin{array}{l}2039 \\
2086 \\
2116 \\
2132 \\
2137\end{array}$ & $\begin{array}{l}3.52787 \\
3.53324 \\
3.53897 \\
3.54505 \\
3.55144\end{array}$ & $\begin{array}{l}537 \\
573 \\
608 \\
639 \\
667\end{array}$ & $\begin{array}{l}20.83478 \\
20.97325 \\
21.10670 \\
21.23551 \\
21.36002\end{array}$ & $\begin{array}{l}13847 \\
13345 \\
12881 \\
12451 \\
12052\end{array}$ & $\begin{array}{l}24.36266 \\
24.50649 \\
24.64568 \\
24.78057 \\
24.91147\end{array}$ & $\begin{array}{l}14383 \\
13919 \\
13489 \\
13090 \\
12718\end{array}$ \\
\hline $\begin{array}{l}300 \\
310 \\
320 \\
330 \\
340\end{array}$ & $\begin{array}{l}3.76236 \\
3.78366 \\
3.80481 \\
3.82571 \\
3.84632\end{array}$ & $\begin{array}{l}2130 \\
2115 \\
2090 \\
2061 \\
2025\end{array}$ & $\begin{array}{l}3.55811 \\
3.56504 \\
3.57221 \\
3.57957 \\
3.58712\end{array}$ & $\begin{array}{l}693 \\
717 \\
736 \\
755 \\
769\end{array}$ & $\begin{array}{l}21.48054 \\
21.59732 \\
21.71062 \\
21.82065 \\
21.92762\end{array}$ & $\begin{array}{l}11678 \\
11330 \\
11003 \\
10697 \\
10410\end{array}$ & $\begin{array}{l}25.03865 \\
25.16237 \\
25.28283 \\
25.40023 \\
25.51474\end{array}$ & $\begin{array}{l}12372 \\
12046 \\
11740 \\
11451 \\
11179\end{array}$ \\
\hline $\begin{array}{l}350 \\
360 \\
370 \\
380 \\
390\end{array}$ & $\begin{array}{l}3.86657 \\
3.88643 \\
3.90585 \\
3.92482 \\
3.94331\end{array}$ & $\begin{array}{l}1986 \\
1942 \\
1897 \\
1849 \\
1800\end{array}$ & $\begin{array}{l}3.59481 \\
3.60264 \\
3.61057 \\
3.61859 \\
3.62668\end{array}$ & $\begin{array}{l}783 \\
793 \\
802 \\
809 \\
814\end{array}$ & $\begin{array}{l}22.03172 \\
22.13309 \\
22.23191 \\
22.32831 \\
22.42240\end{array}$ & $\begin{array}{r}10137 \\
9882 \\
9640 \\
9409 \\
9193\end{array}$ & $\begin{array}{l}25.62653 \\
25.73574 \\
25.84249 \\
25.94690 \\
26.04909\end{array}$ & $\begin{array}{l}10921 \\
10675 \\
10441 \\
10219 \\
10007\end{array}$ \\
\hline $\begin{array}{l}400 \\
450 \\
500 \\
550 \\
600\end{array}$ & $\begin{array}{l}3.96131 \\
4.04387 \\
4.11445 \\
4.17439 \\
4.22531\end{array}$ & $\begin{array}{l}8256 \\
7058 \\
5994 \\
5092 \\
4343\end{array}$ & $\begin{array}{l}3.63482 \\
3.67580 \\
3.71623 \\
3.75523 \\
3.79234\end{array}$ & $\begin{array}{l}4098 \\
4043 \\
3900 \\
3711 \\
350 ?\end{array}$ & $\begin{array}{l}22.51433 \\
22.94481 \\
23.33420 \\
23.69024 \\
24.01859\end{array}$ & $\begin{array}{l}43048 \\
38939 \\
35604 \\
32835 \\
30495\end{array}$ & $\begin{array}{l}26.14916 \\
26.62062 \\
27.05044 \\
27.44548 \\
27.81094\end{array}$ & $\begin{array}{l}47146 \\
42982 \\
39504 \\
36546 \\
33996\end{array}$ \\
\hline $\begin{array}{l}650 \\
700 \\
750 \\
800 \\
850\end{array}$ & $\begin{array}{l}4.26874 \\
4.30601 \\
4.33823 \\
4.36630 \\
4.39095\end{array}$ & $\begin{array}{l}3727 \\
3222 \\
2807 \\
2465 \\
2182\end{array}$ & $\begin{array}{l}3.82736 \\
3.86025 \\
3.89107 \\
3.91991 \\
3.94691\end{array}$ & $\begin{array}{l}3289 \\
3082 \\
2884 \\
2700 \\
2529\end{array}$ & $\begin{array}{l}24.32354 \\
24.60839 \\
24.87579 \\
25.12784 \\
25.36630\end{array}$ & $\begin{array}{l}28485 \\
26740 \\
25205 \\
23846 \\
22633\end{array}$ & $\begin{array}{l}28.15090 \\
28.46865 \\
28.76686 \\
29.04776 \\
29.31322\end{array}$ & $\begin{array}{l}31775 \\
29821 \\
28090 \\
26546 \\
25161\end{array}$ \\
\hline $\begin{array}{r}900 \\
950 \\
1000 \\
1050 \\
1100\end{array}$ & $\begin{array}{l}4.41277 \\
4.43224 \\
4.44973 \\
4.46556 \\
4.47999\end{array}$ & $\begin{array}{l}1947 \\
1749 \\
1583 \\
1443 \\
1323\end{array}$ & $\begin{array}{l}3.97220 \\
3.99591 \\
4.01817 \\
4.03910 \\
4.05882\end{array}$ & $\begin{array}{l}2371 \\
2226 \\
2093 \\
1972 \\
1860\end{array}$ & $\begin{array}{l}25.59263 \\
25.80804 \\
26.01357 \\
26.21013 \\
26.39849\end{array}$ & $\begin{array}{l}21541 \\
20553 \\
19656 \\
18836 \\
18084\end{array}$ & $\begin{array}{l}29.56483 \\
29.80395 \\
30.03175 \\
30.24924 \\
30.45731\end{array}$ & $\begin{array}{l}23912 \\
22780 \\
21749 \\
20807 \\
19944\end{array}$ \\
\hline 115 & 4.49322 & 1221 & 4.07742 & 1759 & 26.57933 & 17390 & 30.65675 & 19150 \\
\hline
\end{tabular}


Table 2.035. CaD (Cont.)

\begin{tabular}{|l|l|l|l|l|}
\hline${ }^{\circ} K$ & $\frac{C_{p}^{0}}{R}$ & $\frac{\left(H^{0}-E_{0}^{0}\right)}{R T}$ & $\frac{-\left(F^{0}-E_{0}^{0}\right)}{R T}$ & $\frac{S^{\circ}}{R}$ \\
\hline
\end{tabular}

\begin{tabular}{|c|c|c|c|c|c|c|c|c|}
\hline $\begin{array}{l}1200 \\
1300 \\
1400 \\
1500 \\
1600\end{array}$ & $\begin{array}{l}4.50543 \\
4.52732 \\
4.54655 \\
4.56373 \\
4.57934\end{array}$ & $\begin{array}{l}2189 \\
1923 \\
1718 \\
1561 \\
1436\end{array}$ & $\begin{array}{l}4.09501 \\
4.12744 \\
4.15670 \\
4.18328 \\
4.20755\end{array}$ & $\begin{array}{l}3243 \\
2926 \\
2658 \\
2427 \\
2230\end{array}$ & $\begin{array}{l}26.75323 \\
27.08231 \\
27.38928 \\
27.67698 \\
27.94775\end{array}$ & $\begin{array}{l}32908 \\
30697 \\
28770 \\
27077 \\
25576\end{array}$ & $\begin{array}{l}30.84825 \\
31.20976 \\
31.54599 \\
31.86026 \\
32.15531\end{array}$ & $\begin{array}{l}36151 \\
33623 \\
31427 \\
29505 \\
27805\end{array}$ \\
\hline $\begin{array}{l}1700 \\
1800 \\
1900 \\
2000 \\
2100\end{array}$ & $\begin{array}{l}4.59370 \\
4.60705 \\
4.61960 \\
4.63147 \\
4.64280\end{array}$ & $\begin{array}{l}1335 \\
1255 \\
1187 \\
1133 \\
1086\end{array}$ & $\begin{array}{l}4.22985 \\
4.25044 \\
4.26954 \\
4.28734 \\
4.30400\end{array}$ & $\begin{array}{l}2059 \\
1910 \\
1780 \\
1666 \\
1565\end{array}$ & $\begin{array}{l}28.20351 \\
28.44587 \\
28.67620 \\
28.89566 \\
29.10525\end{array}$ & $\begin{array}{l}24236 \\
23033 \\
21946 \\
20959 \\
20058\end{array}$ & $\begin{array}{l}32.43336 \\
32.69631 \\
32.94574 \\
33.18300 \\
33.40925\end{array}$ & $\begin{array}{l}26295 \\
24943 \\
23726 \\
22625 \\
21624\end{array}$ \\
\hline $\begin{array}{l}2200 \\
2300 \\
2400 \\
2500 \\
2600\end{array}$ & $\begin{array}{l}4.65366 \\
4.66413 \\
4.67426 \\
4.68410 \\
4.69369\end{array}$ & $\begin{array}{r}1047 \\
1013 \\
984 \\
959 \\
937\end{array}$ & $\begin{array}{l}4.31965 \\
4.33440 \\
4.34835 \\
4.36158 \\
4.37417\end{array}$ & $\begin{array}{l}1475 \\
1395 \\
1323 \\
1259 \\
1201\end{array}$ & $\begin{array}{l}29.30583 \\
29.49818 \\
29.68295 \\
29.86072 \\
30.03204\end{array}$ & $\begin{array}{l}19235 \\
18477 \\
17777 \\
17132 \\
16531\end{array}$ & $\begin{array}{l}33.62549 \\
33.83258 \\
34.03130 \\
34.22231 \\
34.40621\end{array}$ & $\begin{array}{l}20709 \\
19872 \\
19101 \\
18390 \\
17732\end{array}$ \\
\hline $\begin{array}{l}2700 \\
2800 \\
2900 \\
3000\end{array}$ & $\begin{array}{l}4.70306 \\
4.71224 \\
4.72124 \\
4.73008\end{array}$ & $\begin{array}{r}918 \\
900 \\
884 \\
1729\end{array}$ & $\begin{array}{l}4.38618 \\
4.39766 \\
4.40867 \\
4.41923\end{array}$ & $\begin{array}{l}1148 \\
1101 \\
1056 \\
1997\end{array}$ & $\begin{array}{l}30.19735 \\
30.35707 \\
30.51158 \\
30.66122\end{array}$ & $\begin{array}{l}15972 \\
15.451 \\
14964 \\
28586\end{array}$ & $\begin{array}{l}34.58353 \\
34.75474 \\
34.92025 \\
35.08046\end{array}$ & $\begin{array}{l}17121 \\
16551 \\
16021 \\
30583\end{array}$ \\
\hline $\begin{array}{l}3200 \\
3400 \\
3600 \\
3800 \\
4000\end{array}$ & $\begin{array}{l}4.74737 \\
4.76420 \\
4.78064 \\
4.79674 \\
4.81254\end{array}$ & $\begin{array}{l}1683 \\
1644 \\
1610 \\
1580 \\
1555\end{array}$ & $\begin{array}{l}4.43920 \\
4.45783 \\
4.47531 \\
4.49180 \\
4.50745\end{array}$ & $\begin{array}{l}1863 \\
1748 \\
1649 \\
1555 \\
1490\end{array}$ & $\begin{array}{l}30.94708 \\
31.21677 \\
31.47207 \\
31.71449 \\
31.94529\end{array}$ & $\begin{array}{l}26969 \\
25530 \\
24242 \\
23080 \\
22028\end{array}$ & $\begin{array}{l}35.38629 \\
35.67460 \\
35.94739 \\
36.20629 \\
36.45274\end{array}$ & $\begin{array}{l}28831 \\
27279 \\
25390 \\
24645 \\
23518\end{array}$ \\
\hline $\begin{array}{l}4200 \\
4400 \\
4600 \\
4800 \\
5000\end{array}$ & $\begin{array}{l}4.82809 \\
4.84340 \\
4.85849 \\
4.87339 \\
4.88810\end{array}$ & $\begin{array}{l}1531 \\
1509 \\
1490 \\
1471\end{array}$ & $\begin{array}{l}4.52235 \\
4.53659 \\
4.55026 \\
4.56341 \\
4.57611\end{array}$ & $\begin{array}{l}1424 \\
1367 \\
1315 \\
1270\end{array}$ & $\begin{array}{l}32.16557 \\
32.37628 \\
32.57824 \\
32.77218 \\
32.95873\end{array}$ & $\begin{array}{l}21071 \\
20196 \\
19394 \\
18655\end{array}$ & $\begin{array}{l}36.68792 \\
36.91288 \\
37.12851 \\
37.33560 \\
37.53484\end{array}$ & $\begin{array}{l}22496 \\
21563 \\
20709 \\
19924\end{array}$ \\
\hline
\end{tabular}


Table 2.036. CaT

\begin{tabular}{|l|l|l|l|l|}
\hline${ }^{\circ} K$ & $\frac{C_{p}^{0}}{R}$ & $\frac{\left(H^{0}-E_{0}^{0}\right)}{R T}$ & $\frac{-\left(F^{0}-E_{0}^{0}\right)}{R T}$ & $\frac{S^{0}}{R}$ \\
\hline
\end{tabular}

\begin{tabular}{|c|c|c|c|c|c|c|c|c|}
\hline $\begin{array}{l}50 \\
60 \\
70 \\
80 \\
90\end{array}$ & $\begin{array}{l}3.50147 \\
3.50174 \\
3.50206 \\
3.50253 \\
3.50342\end{array}$ & $\begin{array}{r}27 \\
32 \\
47 \\
89 \\
173\end{array}$ & $\begin{array}{l}3.48639 \\
3.48892 \\
3.49078 \\
3.49221 \\
3.49340\end{array}$ & $\begin{array}{l}253 \\
186 \\
143 \\
119 \\
108\end{array}$ & $\begin{array}{l}15.61939 \\
16.25527 \\
16.79324 \\
17.25946 \\
17.67086\end{array}$ & $\begin{array}{l}63588 \\
53797 \\
46622 \\
41140 \\
36812\end{array}$ & $\begin{array}{l}19.10578 \\
19.74420 \\
20.28402 \\
20.75168 \\
21.16427\end{array}$ & $\begin{array}{l}63842 \\
53982 \\
46766 \\
41259 \\
36920\end{array}$ \\
\hline $\begin{array}{l}100 \\
110 \\
120 \\
130 \\
140\end{array}$ & $\begin{array}{l}3.50515 \\
3.50823 \\
3.51317 \\
3.52037 \\
3.53009\end{array}$ & $\begin{array}{r}308 \\
494 \\
720 \\
972 \\
1235\end{array}$ & $\begin{array}{l}3.49448 \\
3.49558 \\
3.49683 \\
3.49835 \\
3.50025\end{array}$ & $\begin{array}{l}110 \\
125 \\
152 \\
190 \\
239\end{array}$ & $\begin{array}{l}18.03898 \\
18.37209 \\
18.67630 \\
18.95625 \\
19.21558\end{array}$ & $\begin{array}{l}33311 \\
30421 \\
27995 \\
25933 \\
24157\end{array}$ & $\begin{array}{l}21.53347 \\
21.86768 \\
22.17313 \\
22.45461 \\
22.71583\end{array}$ & $\begin{array}{l}33421 \\
30545 \\
28148 \\
26122 \\
24396\end{array}$ \\
\hline $\begin{array}{l}150 \\
160 \\
170 \\
180 \\
190\end{array}$ & $\begin{array}{l}3.54244 \\
3.55735 \\
3.57464 \\
3.59406 \\
3.61529\end{array}$ & $\begin{array}{l}1491 \\
1729 \\
1942 \\
2123 \\
2271\end{array}$ & $\begin{array}{l}3.50264 \\
3.50558 \\
3.50912 \\
3.51329 \\
3.51809\end{array}$ & $\begin{array}{l}294 \\
354 \\
417 \\
480 \\
543\end{array}$ & $\begin{array}{l}19.45715 \\
19.68329 \\
19.89592 \\
20.09661 \\
20.28669\end{array}$ & $\begin{array}{l}22614 \\
21263 \\
20069 \\
19008 \\
18059\end{array}$ & $\begin{array}{l}22.95979 \\
23.18888 \\
23.40505 \\
23.60991 \\
23.80479\end{array}$ & $\begin{array}{l}22909 \\
21617 \\
20486 \\
19488 \\
18601\end{array}$ \\
\hline $\begin{array}{l}200 \\
210 \\
220 \\
230 \\
240\end{array}$ & $\begin{array}{l}3.63800 \\
3.66185 \\
3.68653 \\
3.71176 \\
3.73728\end{array}$ & $\begin{array}{l}2385 \\
2468 \\
2523 \\
2552 \\
2559\end{array}$ & $\begin{array}{l}3.52352 \\
3.52953 \\
3.53610 \\
3.54319 \\
3.55075\end{array}$ & $\begin{array}{l}601 \\
657 \\
709 \\
756 \\
797\end{array}$ & $\begin{array}{l}20.46728 \\
20.63934 \\
20.80368 \\
20.96102 \\
21.11198\end{array}$ & $\begin{array}{l}17206 \\
16434 \\
15734 \\
15096 \\
14511\end{array}$ & $\begin{array}{l}23.99080 \\
24.16888 \\
24.33979 \\
24.50422 \\
24.66273\end{array}$ & $\begin{array}{l}17808 \\
17091 \\
16443 \\
15851 \\
15308\end{array}$ \\
\hline $\begin{array}{l}250 \\
260 \\
270 \\
280 \\
290\end{array}$ & $\begin{array}{l}3.76287 \\
3.78833 \\
3.81352 \\
3.83832 \\
3.86260\end{array}$ & $\begin{array}{l}2546 \\
2519 \\
2480 \\
2428 \\
2371\end{array}$ & $\begin{array}{l}3.55872 \\
3.56706 \\
3.57572 \\
3.58466 \\
3.59383\end{array}$ & $\begin{array}{l}834 \\
866 \\
894 \\
917 \\
935\end{array}$ & $\begin{array}{l}21.25709 \\
21.39683 \\
21.53161 \\
21.66181 \\
21.78776\end{array}$ & $\begin{array}{l}13974 \\
13478 \\
13020 \\
12595 \\
12199\end{array}$ & $\begin{array}{l}24.81581 \\
24.96389 \\
25.10734 \\
25.24648 \\
25.38159\end{array}$ & $\begin{array}{l}14808 \\
14345 \\
13914 \\
13511 \\
13135\end{array}$ \\
\hline $\begin{array}{l}300 \\
310 \\
320 \\
330 \\
340\end{array}$ & $\begin{array}{l}3.88631 \\
3.90939 \\
3.93179 \\
3.95348 \\
3.97445\end{array}$ & $\begin{array}{l}2308 \\
2240 \\
2169 \\
2097 \\
2024\end{array}$ & $\begin{array}{l}3.60318 \\
3.61269 \\
3.62232 \\
3.63202 \\
3.64179\end{array}$ & $\begin{array}{l}951 \\
963 \\
970 \\
977 \\
979\end{array}$ & $\begin{array}{l}21.90975 \\
22.02806 \\
22.14291 \\
22.25452 \\
22.36309\end{array}$ & $\begin{array}{l}11831 \\
11485 \\
11161 \\
10857 \\
10571\end{array}$ & $\begin{array}{l}25.51294 \\
25.64075 \\
25.76523 \\
25.88655 \\
26.00489\end{array}$ & $\begin{array}{l}12781 \\
12448 \\
12132 \\
11834 \\
11550\end{array}$ \\
\hline $\begin{array}{l}350 \\
360 \\
370 \\
380 \\
390\end{array}$ & $\begin{array}{l}3.99469 \\
4.01421 \\
4.03302 \\
4.05111 \\
4.06851\end{array}$ & $\begin{array}{l}1952 \\
1881 \\
1809 \\
1740 \\
1673\end{array}$ & $\begin{array}{l}3.65158 \\
3.66139 \\
3.67118 \\
3.68094 \\
3.69066\end{array}$ & $\begin{array}{l}981 \\
979 \\
976 \\
972 \\
965\end{array}$ & $\begin{array}{l}22.46880 \\
22.57181 \\
22.67226 \\
22.77029 \\
22.86603\end{array}$ & $\begin{array}{r}10301 \\
10045 \\
9803 \\
9574 \\
9356\end{array}$ & $\begin{array}{l}26.12039 \\
26.23320 \\
26.34344 \\
26.45124 \\
26.55669\end{array}$ & $\begin{array}{l}11281 \\
11024 \\
10780 \\
10545 \\
10322\end{array}$ \\
\hline $\begin{array}{l}400 \\
450 \\
500 \\
550 \\
600\end{array}$ & $\begin{array}{l}4.08524 \\
4.15956 \\
4.22042 \\
4.27050 \\
4.31209\end{array}$ & $\begin{array}{l}7432 \\
6086 \\
5008 \\
4159 \\
3490\end{array}$ & $\begin{array}{l}3.70031 \\
3.74735 \\
3.79172 \\
3.83304 \\
3.87128\end{array}$ & $\begin{array}{l}4704 \\
4437 \\
4132 \\
3824 \\
3529\end{array}$ & $\begin{array}{l}22.95959 \\
23.39817 \\
23.79531 \\
24.15866 \\
24.49384\end{array}$ & $\begin{array}{l}43858 \\
39714 \\
36335 \\
33518 \\
31128 \\
\end{array}$ & $\begin{array}{l}26.65991 \\
27.14552 \\
27.58703 \\
27.99171 \\
28.36513\end{array}$ & $\begin{array}{l}48561 \\
44151 \\
40468 \\
37342 \\
34656\end{array}$ \\
\hline $\begin{array}{l}650 \\
700 \\
750 \\
800 \\
850\end{array}$ & $\begin{array}{l}4.34699 \\
4.37661 \\
4.40203 \\
4.42409 \\
4.44343\end{array}$ & $\begin{array}{l}2962 \\
2542 \\
2206 \\
1934 \\
1714\end{array}$ & $\begin{array}{l}3.90657 \\
3.93911 \\
3.96915 \\
3.99691 \\
4.02262\end{array}$ & $\begin{array}{l}3254 \\
3004 \\
2776 \\
2571 \\
2386\end{array}$ & $\begin{array}{l}24.80512 \\
25.09584 \\
25.36865 \\
25.62571 \\
25.86880\end{array}$ & $\begin{array}{l}29072 \\
27281 \\
25706 \\
24309 \\
23061\end{array}$ & $\begin{array}{l}28.71169 \\
29.03495 \\
29.33780 \\
29.62262 \\
29.89142\end{array}$ & $\begin{array}{l}32326 \\
30285 \\
28482 \\
26880 \\
25448\end{array}$ \\
\hline $\begin{array}{r}900 \\
950 \\
1000 \\
1050 \\
1100\end{array}$ & $\begin{array}{l}4.46057 \\
4.47588 \\
4.48970 \\
4.50227 \\
4.51378\end{array}$ & $\begin{array}{l}1531 \\
1382 \\
1257 \\
1151 \\
1063\end{array}$ & $\begin{array}{l}4.04648 \\
4.06868 \\
4.08939 \\
4.10876 \\
4.12691\end{array}$ & $\begin{array}{l}2220 \\
2071 \\
1937 \\
1815 \\
1706\end{array}$ & $\begin{array}{l}26.09941 \\
26.31880 \\
26.52802 \\
26.72802 \\
26.91958\end{array}$ & $\begin{array}{l}21939 \\
20922 \\
20000 \\
19156 \\
18383\end{array}$ & $\begin{array}{l}30.14590 \\
30.38748 \\
30.61742 \\
30.83679 \\
31.04650\end{array}$ & $\begin{array}{l}24158 \\
22994 \\
21937 \\
20971 \\
20088\end{array}$ \\
\hline 1150 & 4.52441 & 987 & 4.14397 & 1606 & 27.10341 & 17671 & 31.24738 & 19277 \\
\hline
\end{tabular}


Table 2.036. CaT (Cont.)

\begin{tabular}{|l|l|l|l|l|}
\hline${ }^{\circ} K$ & $\frac{C_{p}^{0}}{R}$ & $\frac{\left(H^{0}-E_{0}^{0}\right)}{R T}$ & $\frac{-\left(F^{0}-E_{0}^{0}\right)}{R T}$ & $\frac{S^{0}}{R}$ \\
\hline
\end{tabular}

\begin{tabular}{|c|c|c|c|c|c|c|c|c|}
\hline $\begin{array}{l}1200 \\
1300 \\
1400 \\
1500 \\
1600\end{array}$ & $\begin{array}{l}4.53428 \\
4.55217 \\
4.56813 \\
4.58261 \\
4.59595\end{array}$ & $\begin{array}{l}1789 \\
1596 \\
1448 \\
1334 \\
1244\end{array}$ & $\begin{array}{l}4.16003 \\
4.18952 \\
4.21600 \\
4.23997 \\
4.26180\end{array}$ & $\begin{array}{l}2949 \\
2648 \\
2397 \\
2183 \\
2003\end{array}$ & $\begin{array}{l}27.28012 \\
27.61429 \\
27.92575 \\
28.21746 \\
28.49181\end{array}$ & $\begin{array}{l}33417 \\
31146 \\
29171 \\
27435 \\
25898\end{array}$ & $\begin{array}{l}31.44015 \\
31.80381 \\
32.14176 \\
32.45743 \\
32.75361\end{array}$ & $\begin{array}{l}36366 \\
33795 \\
31567 \\
29618 \\
27901\end{array}$ \\
\hline $\begin{array}{l}1700 \\
1800 \\
1900 \\
2000 \\
2100\end{array}$ & $\begin{array}{l}4.60839 \\
4.62012 \\
4.63126 \\
4.64193 \\
4.65220\end{array}$ & $\begin{array}{r}1173 \\
1114 \\
1067 \\
1027 \\
993\end{array}$ & $\begin{array}{l}4.28183 \\
4.30030 \\
4.31743 \\
4.33339 \\
4.34833\end{array}$ & $\begin{array}{l}1847 \\
1713 \\
1596 \\
1494 \\
1404\end{array}$ & $\begin{array}{l}28.75079 \\
28.99606 \\
29.22903 \\
29.45089 \\
29.66269\end{array}$ & $\begin{array}{l}24527 \\
23297 \\
22186 \\
21180 \\
20261\end{array}$ & $\begin{array}{l}33.03262 \\
33.29636 \\
33.54646 \\
33.78429 \\
34.01102\end{array}$ & $\begin{array}{l}26374 \\
25010 \\
23783 \\
22673 \\
21665\end{array}$ \\
\hline $\begin{array}{l}2200 \\
2300 \\
2400 \\
2500 \\
2600\end{array}$ & $\begin{array}{l}4.66213 \\
4.67178 \\
4.68119 \\
4.69039 \\
4.69940\end{array}$ & $\begin{array}{l}965 \\
941 \\
920 \\
901 \\
884\end{array}$ & $\begin{array}{l}4.36237 \\
4.37561 \\
4.38815 \\
4.40005 \\
4.41139\end{array}$ & $\begin{array}{l}1324 \\
1254 \\
1190 \\
1134 \\
1084\end{array}$ & $\begin{array}{l}29.86530 \\
30.05951 \\
30.24600 \\
30.42538 \\
30.59817\end{array}$ & $\begin{array}{l}19421 \\
18649 \\
17938 \\
17279 \\
16669\end{array}$ & $\begin{array}{l}34.22767 \\
34.43512 \\
34.63415 \\
34.82543 \\
35.00957\end{array}$ & $\begin{array}{l}20745 \\
19903 \\
19128 \\
18414 \\
17752\end{array}$ \\
\hline $\begin{array}{l}2700 \\
2800 \\
2900 \\
3000\end{array}$ & $\begin{array}{l}4.70824 \\
4.71695 \\
4.72552 \\
4.73398\end{array}$ & $\begin{array}{r}871 \\
857 \\
846 \\
1660\end{array}$ & $\begin{array}{l}4.42223 \\
4.43260 \\
4.44255 \\
4.45212\end{array}$ & $\begin{array}{r}1037 \\
995 \\
957 \\
1814\end{array}$ & $\begin{array}{l}30.76486 \\
30.92588 \\
31.08160 \\
31.23237\end{array}$ & $\begin{array}{l}16102 \\
15572 \\
15077 \\
28792\end{array}$ & $\begin{array}{l}35.18709 \\
35.35848 \\
35.52415 \\
35.68450\end{array}$ & $\begin{array}{l}17139 \\
16567 \\
16035 \\
30606\end{array}$ \\
\hline $\begin{array}{l}3200 \\
3400 \\
3600 \\
3800 \\
4000\end{array}$ & $\begin{array}{l}4.75058 \\
4.76683 \\
4.78277 \\
4.79844 \\
4.81387\end{array}$ & $\begin{array}{l}1625 \\
1594 \\
1567 \\
1543 \\
1521\end{array}$ & $\begin{array}{l}4.47026 \\
4.48723 \\
4.50321 \\
4.51833 \\
4.53272\end{array}$ & $\begin{array}{l}1697 \\
1598 \\
1512 \\
1439 \\
1376\end{array}$ & $\begin{array}{l}31.52029 \\
31.79181 \\
32.04875 \\
32.29264 \\
32.52477\end{array}$ & $\begin{array}{l}27152 \\
25694 \\
24389 \\
23213 \\
22149\end{array}$ & $\begin{array}{l}35.99056 \\
36.27905 \\
36.55196 \\
36.81098 \\
37.05750\end{array}$ & $\begin{array}{l}28849 \\
27291 \\
25902 \\
24652 \\
23524\end{array}$ \\
\hline $\begin{array}{l}4200 \\
4400 \\
4600 \\
4800 \\
5000\end{array}$ & $\begin{array}{l}4.82908 \\
4.84409 \\
4.85892 \\
4.87358 \\
4.88807\end{array}$ & $\begin{array}{l}1501 \\
1483 \\
1466 \\
1449\end{array}$ & $\begin{array}{l}4.54648 \\
4.55966 \\
4.57235 \\
4.58460 \\
4.59645\end{array}$ & $\begin{array}{l}1318 \\
1269 \\
1225 \\
1185\end{array}$ & $\begin{array}{l}32.74626 \\
32.95806 \\
33.16103 \\
33.35589 \\
33.54328\end{array}$ & $\begin{array}{l}21180 \\
20297 \\
19486 \\
18739\end{array}$ & $\begin{array}{l}37.29274 \\
37.51773 \\
37.73339 \\
37.94049 \\
38.13974\end{array}$ & $\begin{array}{l}22499 \\
21566 \\
20710 \\
19925\end{array}$ \\
\hline
\end{tabular}


Table 2.037. $\mathrm{SrH}$

\begin{tabular}{|l|l|l|l|l|}
\hline${ }^{\circ} K$ & $\frac{C_{p}^{0}}{R}$ & $\frac{\left(H^{\circ}-E_{0}^{0}\right)}{R T}$ & $\frac{-\left(F^{\circ}-E_{0}^{0}\right)}{R T}$ & $\frac{S^{\circ}}{R}$ \\
\hline
\end{tabular}

\begin{tabular}{|c|c|c|c|c|c|c|c|c|}
\hline $\begin{array}{l}50 \\
60 \\
70 \\
80 \\
90\end{array}$ & $\begin{array}{l}3.50167 \\
3.50188 \\
3.50211 \\
3.50236 \\
3.50263\end{array}$ & $\begin{array}{l}21 \\
23 \\
25 \\
27 \\
28\end{array}$ & $\begin{array}{l}3.46560 \\
3.47163 \\
3.47597 \\
3.47925 \\
3.48183\end{array}$ & $\begin{array}{l}603 \\
434 \\
328 \\
258 \\
210\end{array}$ & $\begin{array}{l}15.82936 \\
16.46178 \\
16.99728 \\
17.46165 \\
17.87161\end{array}$ & $\begin{array}{l}63242 \\
53550 \\
46437 \\
40996 \\
36696\end{array}$ & $\begin{array}{l}19.29497 \\
19.93341 \\
20.47325 \\
20.94091 \\
21.35344\end{array}$ & $\begin{array}{l}63844 \\
53984 \\
46766 \\
41253 \\
36906\end{array}$ \\
\hline $\begin{array}{l}100 \\
110 \\
120 \\
130 \\
140\end{array}$ & $\begin{array}{l}3.50291 \\
3.50322 \\
3.50360 \\
3.50411 \\
3.50486\end{array}$ & $\begin{array}{r}31 \\
38 \\
51 \\
75 \\
110\end{array}$ & $\begin{array}{l}3.48393 \\
3.48566 \\
3.48714 \\
3.48843 \\
3.48957\end{array}$ & $\begin{array}{l}173 \\
148 \\
129 \\
114 \\
106\end{array}$ & $\begin{array}{l}18.23857 \\
18.57070 \\
18.87406 \\
19.15323 \\
19.41180\end{array}$ & $\begin{array}{l}33213 \\
30336 \\
27917 \\
25857 \\
24079\end{array}$ & $\begin{array}{l}21.72250 \\
22.05637 \\
22.36121 \\
22.64167 \\
22.90137\end{array}$ & $\begin{array}{l}33387 \\
30484 \\
28646 \\
25970 \\
24185\end{array}$ \\
\hline $\begin{array}{l}150 \\
160 \\
170 \\
180 \\
190\end{array}$ & $\begin{array}{l}3.50596 \\
3.50755 \\
3.50978 \\
3.51277 \\
3.51665\end{array}$ & $\begin{array}{l}159 \\
223 \\
299 \\
388 \\
487\end{array}$ & $\begin{array}{l}3.49063 \\
3.49163 \\
3.49263 \\
3.49366 \\
3.49477\end{array}$ & $\begin{array}{l}100 \\
100 \\
103 \\
111 \\
121\end{array}$ & $\begin{array}{l}19.65259 \\
19.87790 \\
20.08961 \\
20.28927 \\
20.47820\end{array}$ & $\begin{array}{l}22531 \\
21171 \\
19966 \\
18893 \\
17928\end{array}$ & $\begin{array}{l}23.14322 \\
23.36954 \\
23.58225 \\
23.78294 \\
23.97297\end{array}$ & $\begin{array}{l}22632 \\
21271 \\
20069 \\
19003 \\
18050\end{array}$ \\
\hline $\begin{array}{l}200 \\
210 \\
220 \\
230 \\
240\end{array}$ & $\begin{array}{l}3.52152 \\
3.52744 \\
3.53444 \\
3.54255 \\
3.55174\end{array}$ & $\begin{array}{r}592 \\
700 \\
811 \\
919 \\
1024\end{array}$ & $\begin{array}{l}3.49598 \\
3.49733 \\
3.49885 \\
3.50057 \\
3.50251\end{array}$ & $\begin{array}{l}135 \\
152 \\
172 \\
194 \\
217\end{array}$ & $\begin{array}{l}20.65748 \\
20.82809 \\
20.99082 \\
21.14638 \\
21.29541\end{array}$ & $\begin{array}{l}17061 \\
16273 \\
15556 \\
14903 \\
14302\end{array}$ & $\begin{array}{l}24.15347 \\
24.32542 \\
24.48968 \\
24.64696 \\
24.79792\end{array}$ & $\begin{array}{l}17195 \\
164 ? 6 \\
15728 \\
15396 \\
14520\end{array}$ \\
\hline $\begin{array}{l}250 \\
260 \\
270 \\
280 \\
290\end{array}$ & $\begin{array}{l}3.56198 \\
3.57322 \\
3.58537 \\
3.59837 \\
3.61213\end{array}$ & $\begin{array}{l}1124 \\
1215 \\
1300 \\
1376 \\
1443\end{array}$ & $\begin{array}{l}3.50468 \\
3.50710 \\
3.50977 \\
3.51270 \\
3.51589\end{array}$ & $\begin{array}{l}242 \\
267 \\
293 \\
319 \\
345\end{array}$ & $\begin{array}{l}21.43843 \\
21.57593 \\
21.70834 \\
21.83604 \\
21.95936\end{array}$ & $\begin{array}{l}13750 \\
13241 \\
12770 \\
12332 \\
11925\end{array}$ & $\begin{array}{l}24.94312 \\
25.08304 \\
25.21812 \\
25.34874 \\
25.47525\end{array}$ & $\begin{array}{l}13992 \\
13508 \\
13062 \\
12651 \\
12270\end{array}$ \\
\hline $\begin{array}{l}300 \\
310 \\
320 \\
330 \\
340\end{array}$ & $\begin{array}{l}3.62656 \\
3.64156 \\
3.65705 \\
3.67295 \\
3.68917\end{array}$ & $\begin{array}{l}1500 \\
1549 \\
1590 \\
1622 \\
1646\end{array}$ & $\begin{array}{l}3.51934 \\
3.52303 \\
3.52698 \\
3.53116 \\
3.53557\end{array}$ & $\begin{array}{l}369 \\
395 \\
418 \\
441 \\
462\end{array}$ & $\begin{array}{l}22.07861 \\
22.19407 \\
22.30598 \\
22.41457 \\
22.52005\end{array}$ & $\begin{array}{l}11546 \\
11191 \\
10859 \\
10548 \\
10256\end{array}$ & $\begin{array}{l}25.59795 \\
25.71711 \\
25.83296 \\
25.94574 \\
26.05563\end{array}$ & $\begin{array}{l}11916 \\
11585 \\
11278 \\
10989 \\
10718\end{array}$ \\
\hline $\begin{array}{l}350 \\
360 \\
370 \\
380 \\
390\end{array}$ & $\begin{array}{l}3.70563 \\
3.72227 \\
3.73901 \\
3.75581 \\
3.77260\end{array}$ & $\begin{array}{l}1664 \\
1674 \\
1680 \\
1679 \\
1673\end{array}$ & $\begin{array}{l}3.54019 \\
3.54502 \\
3.55004 \\
3.55523 \\
3.56059\end{array}$ & $\begin{array}{l}483 \\
502 \\
519 \\
536 \\
551\end{array}$ & $\begin{array}{l}22.62261 \\
22.72241 \\
22.81960 \\
22.91435 \\
23.00676\end{array}$ & $\begin{array}{l}9980 \\
9719 \\
9475 \\
9241 \\
9022\end{array}$ & $\begin{array}{l}26.16281 \\
26.26743 \\
26.36964 \\
26.46958 \\
26.56736\end{array}$ & $\begin{array}{r}10462 \\
10221 \\
9994 \\
9778 \\
9572\end{array}$ \\
\hline $\begin{array}{l}400 \\
450 \\
500 \\
550 \\
600\end{array}$ & $\begin{array}{l}3.78933 \\
3.87094 \\
3.94683 \\
4.01553 \\
4.07681\end{array}$ & $\begin{array}{l}8161 \\
7589 \\
6870 \\
6128 \\
5428\end{array}$ & $\begin{array}{l}3.56610 \\
3.59548 \\
3.62687 \\
3.65914 \\
3.69144\end{array}$ & $\begin{array}{l}2938 \\
3139 \\
3227 \\
3230 \\
3178\end{array}$ & $\begin{array}{l}23.09698 \\
23.51867 \\
23.89911 \\
24.24630 \\
24.56607\end{array}$ & $\begin{array}{l}42167 \\
38044 \\
34719 \\
31977 \\
29673\end{array}$ & $\begin{array}{l}26.66308 \\
27.11416 \\
27.52599 \\
27.90544 \\
28.25752\end{array}$ & $\begin{array}{l}45108 \\
41183 \\
37945 \\
35208 \\
32851\end{array}$ \\
\hline $\begin{array}{l}650 \\
700 \\
750 \\
800 \\
850\end{array}$ & $\begin{array}{l}4.13109 \\
4.17903 \\
4.22140 \\
4.25893 \\
4.29229\end{array}$ & $\begin{array}{l}4794 \\
4237 \\
3753 \\
3336 \\
2978\end{array}$ & $\begin{array}{l}3.72322 \\
3.75410 \\
3.78387 \\
3.81241 \\
3.83968\end{array}$ & $\begin{array}{l}3088 \\
2977 \\
2854 \\
2727 \\
2598\end{array}$ & $\begin{array}{l}24.86280 \\
25.13986 \\
25.39989 \\
25.64501 \\
25.87696\end{array}$ & $\begin{array}{l}27706 \\
26003 \\
24512 \\
23195 \\
22022\end{array}$ & $\begin{array}{l}28.58603 \\
28.89396 \\
29.18376 \\
29.45743 \\
29.71665\end{array}$ & $\begin{array}{l}30793 \\
28980 \\
27367 \\
25922 \\
24619\end{array}$ \\
\hline $\begin{array}{l}900 \\
950\end{array}$ & $\begin{array}{l}4.32207 \\
4.34878 \\
4.37285 \\
4.39465 \\
4.41449\end{array}$ & $\begin{array}{l}2671 \\
2407 \\
2180 \\
1984 \\
1815\end{array}$ & $\begin{array}{l}3.86566 \\
3.89040 \\
3.91393 \\
3.93631 \\
3.95760\end{array}$ & $\begin{array}{l}2474 \\
2353 \\
2238 \\
2129 \\
2027\end{array}$ & $\begin{array}{l}26.09718 \\
26.30685 \\
26.50701 \\
26.69851 \\
26.88213\end{array}$ & $\begin{array}{l}20967 \\
20016 \\
19150 \\
18362 \\
17637\end{array}$ & $\begin{array}{l}29.96284 \\
30.19726 \\
30.42094 \\
30.63483 \\
30.83973\end{array}$ & $\begin{array}{l}23442 \\
22368 \\
21389 \\
20490 \\
19664\end{array}$ \\
\hline & 4.43264 & 1668 & 3.97787 & 1930 & 27.05850 & 16971 & 31.03637 & 18901 \\
\hline
\end{tabular}


Table 2.037. SrH (Cont.)

\begin{tabular}{|l|l|l|l|l|}
\hline$K^{\circ}$ & $\frac{C_{p}^{0}}{R}$ & $\frac{\left(H^{0}-E_{0}^{0}\right)}{R T}$ & $\frac{-\left(F^{0}-E_{0}^{0}\right)}{R T}$ & $\frac{S^{0}}{R}$ \\
\hline
\end{tabular}

\begin{tabular}{|c|c|c|c|c|c|c|c|c|}
\hline 1200 & 4.44932 & 2963 & 3.99717 & 3595 & 27.22821 & 32138 & 31.22538 & 35734 \\
\hline 1300 & 4.47900 & 2574 & 4.03312 & 3279 & 27.54959 & 30011 & 31.58272 & 33289 \\
\hline 1400 & 4.50474 & 2269 & 4.06591 & 3003 & 27.84970 & 28156 & 31.91561 & 31159 \\
\hline 1500 & 4.52743 & 2027 & 4.09594 & 2761 & 28.13126 & 26524 & 32.22720 & 29285 \\
\hline 1600 & 4.54770 & 1836 & 4.12355 & 2550 & 28.39650 & 25076 & 32.52005 & 27626 \\
\hline 1700 & 4.56606 & 1682 & 4.14905 & 2364 & 28.64726 & 23783 & 32.79631 & 26147 \\
\hline 1800 & 4.58288 & 1555 & 4.17269 & 2200 & 28.88509 & 22620 & 33.05778 & 24821 \\
\hline 1900 & 4.59843 & 1452 & 4.19469 & 2056 & 29.11129 & 21569 & 33.30599 & 23624 \\
\hline 2000 & 4.61295 & 1365 & 4.21525 & 1926 & 29.32698 & 20614 & 33.54223 & 22540 \\
\hline 2100 & 4.62660 & 1293 & 4.23451 & 1812 & 29.53312 & 19741 & 33.76763 & 21553 \\
\hline 2200 & 4.63953 & 1232 & 4.25263 & 1709 & 29.73053 & 18942 & 33.98316 & 20651 \\
\hline 2300 & 4.65185 & 1180 & 4.26972 & 1617 & 29.91995 & 18206 & 34.18967 & 19823 \\
\hline 2400 & 4.66365 & 1134 & 4.28589 & 1534 & 30.10201 & 17527 & 34.38790 & 19062 \\
\hline 2500 & 4.67499 & 1095 & 4.30123 & 1459 & 30.27728 & 16898 & 34.57852 & 18357 \\
\hline 2600 & 4.68594 & 1062 & 4.31582 & 1390 & 30.44626 & 16315 & 34.76209 & 17705 \\
\hline 2700 & 4.69656 & 1031 & 4.32972 & 1329 & 30.60941 & 15770 & 34.93914 & 17099 \\
\hline 2800 & 4.70687 & 1005 & 4.34301 & 1272 & 30.76711 & 15263 & 35.11013 & 16534 \\
\hline 29 & 4.71692 & 982 & 4.35573 & 1220 & 30.91974 & 14787 & 35.27547 & 16008 \\
\hline 30 & 4.72674 & 1902 & 4.36793 & 2303 & 31.06761 & 28265 & 35.43555 & 30567 \\
\hline 3200 & 4.74576 & 1834 & 4.39096 & 2141 & 31.35026 & 26685 & 35.74122 & 28826 \\
\hline 3. & 4.76410 & 1778 & 4.41237 & 2004 & 31.61711 & 25277 & 36.02948 & 27282 \\
\hline 3600 & 4.78188 & 1729 & 4.43241 & 1885 & 31.86988 & 24016 & 36.30230 & 25901 \\
\hline 3800 & 4.79917 & 1688 & 4.45126 & 1782 & 32.11004 & 22878 & 36.56131 & 24659 \\
\hline 400 & 4.81605 & 1653 & 4.46908 & 1692 & 32.33882 & 21846 & 36.80790 & 23538 \\
\hline 4200 & 4.83258 & 1620 & 4.48600 & 1612 & 32.55728 & 20906 & 37.04328 & 22519 \\
\hline 4400 & 4.84878 & 1592 & 4.50212 & 1542 & 32.76634 & 20047 & 37.26847 & 21599 \\
\hline 4600 & 4.86470 & 1567 & 4.51754 & 1479 & 32.96681 & 19258 & 37.48436 & 20737 \\
\hline 4800 & 4.88037 & 1542 & 4.53233 & 1423 & 33.15939 & 18531 & 37.69173 & 19954 \\
\hline & 4.89579 & & 4.54656 & & 3.3447 & & 9127 & \\
\hline
\end{tabular}


Table 2.038. SrD

\begin{tabular}{|l|l|l|l|l|}
\hline${ }^{\circ}$ & $\frac{C_{P}^{0}}{R}$ & $\frac{\left(H^{0}-E_{0}^{0}\right)}{R T}$ & $\frac{-\left(F^{0}-E_{0}^{0}\right)}{R T}$ & $\frac{S^{0}}{R}$ \\
\hline
\end{tabular}

\begin{tabular}{|c|c|c|c|c|c|c|c|c|}
\hline $\begin{array}{l}50 \\
60 \\
70 \\
80 \\
90\end{array}$ & $\begin{array}{l}3.50148 \\
3.50174 \\
3.50202 \\
3.50235 \\
3.50283\end{array}$ & $\begin{array}{l}26 \\
28 \\
33 \\
48 \\
84\end{array}$ & $\begin{array}{l}3.48293 \\
3.48605 \\
3.48831 \\
3.49004 \\
3.49143\end{array}$ & $\begin{array}{l}312 \\
226 \\
173 \\
139 \\
118\end{array}$ & $\begin{array}{l}16.50612 \\
17.14143 \\
17.67898 \\
18.14490 \\
18.55605\end{array}$ & $\begin{array}{l}63531 \\
53755 \\
46592 \\
41115 \\
36792\end{array}$ & $\begin{array}{l}19.98906 \\
20.62748 \\
21.16730 \\
21.63495 \\
22.04749\end{array}$ & $\begin{array}{l}63842 \\
53982 \\
46765 \\
41254 \\
36910\end{array}$ \\
\hline $\begin{array}{l}100 \\
110 \\
120 \\
130 \\
140\end{array}$ & $\begin{array}{l}3.50367 \\
3.50518 \\
3.50773 \\
3.51169 \\
3.51739\end{array}$ & $\begin{array}{l}151 \\
255 \\
396 \\
570 \\
766\end{array}$ & $\begin{array}{l}3.49261 \\
3.49368 \\
3.49474 \\
3.49588 \\
3.49720\end{array}$ & $\begin{array}{l}107 \\
106 \\
114 \\
132 \\
159\end{array}$ & $\begin{array}{l}18.92397 \\
19.25691 \\
19.56094 \\
19.84072 \\
20.09984\end{array}$ & $\begin{array}{l}33294 \\
30403 \\
27978 \\
25912 \\
24133\end{array}$ & $\begin{array}{l}22.41659 \\
22.75059 \\
23.05568 \\
23.33660 \\
23.59704\end{array}$ & $\begin{array}{l}33400 \\
30509 \\
28092 \\
26044 \\
24292\end{array}$ \\
\hline $\begin{array}{l}150 \\
160 \\
170 \\
180 \\
190\end{array}$ & $\begin{array}{l}3.52505 \\
3.53481 \\
3.54668 \\
3.56060 \\
3.57642\end{array}$ & $\begin{array}{r}976 \\
1187 \\
1392 \\
1582 \\
1752\end{array}$ & $\begin{array}{l}3.49879 \\
3.50072 \\
3.50307 \\
3.50587 \\
3.50916\end{array}$ & $\begin{array}{l}193 \\
235 \\
280 \\
329 \\
379\end{array}$ & $\begin{array}{l}20.34117 \\
20.56704 \\
20.77934 \\
20.97964 \\
21.16928\end{array}$ & $\begin{array}{l}22587 \\
21230 \\
20030 \\
18964 \\
18009\end{array}$ & $\begin{array}{l}23.83996 \\
24.06777 \\
24.28241 \\
24.48552 \\
24.67844\end{array}$ & $\begin{array}{l}22781 \\
21464 \\
20311 \\
19292 \\
18389\end{array}$ \\
\hline $\begin{array}{l}200 \\
210 \\
220 \\
230 \\
240\end{array}$ & $\begin{array}{l}3.59394 \\
3.61294 \\
3.63318 \\
3.65441 \\
3.67640\end{array}$ & $\begin{array}{l}1900 \\
2024 \\
2123 \\
2199 \\
2253\end{array}$ & $\begin{array}{l}3.51295 \\
3.51726 \\
3.52206 \\
3.52735 \\
3.53310\end{array}$ & $\begin{array}{l}431 \\
480 \\
529 \\
575 \\
618\end{array}$ & $\begin{array}{l}21.34937 \\
21.52087 \\
21.68460 \\
21.84128 \\
21.99152\end{array}$ & $\begin{array}{l}17150 \\
16373 \\
15568 \\
15024 \\
14436\end{array}$ & $\begin{array}{l}24.86233 \\
25.03813 \\
25.20667 \\
25.36864 \\
25.52463\end{array}$ & $\begin{array}{l}17580 \\
16854 \\
16197 \\
15599 \\
15053\end{array}$ \\
\hline $\begin{array}{l}250 \\
260 \\
270 \\
280 \\
290\end{array}$ & $\begin{array}{l}3.69893 \\
3.72181 \\
3.74486 \\
3.76793 \\
3.79089\end{array}$ & $\begin{array}{l}2288 \\
2305 \\
2307 \\
2296 \\
2273\end{array}$ & $\begin{array}{l}3.53928 \\
3.54586 \\
3.55280 \\
3.56008 \\
3.56764\end{array}$ & $\begin{array}{l}658 \\
694 \\
728 \\
756 \\
782\end{array}$ & $\begin{array}{l}22.13588 \\
22.27482 \\
22.40877 \\
22.53811 \\
22.66316\end{array}$ & $\begin{array}{l}13894 \\
13395 \\
12934 \\
12505 \\
12108\end{array}$ & $\begin{array}{l}25.67516 \\
25.82068 \\
25.96158 \\
26.09819 \\
26.23081\end{array}$ & $\begin{array}{l}14552 \\
14090 \\
13661 \\
13262 \\
12890\end{array}$ \\
\hline $\begin{array}{l}300 \\
310 \\
320 \\
330 \\
340\end{array}$ & $\begin{array}{l}3.81362 \\
3.83604 \\
3.85807 \\
3.87965 \\
3.90073\end{array}$ & $\begin{array}{l}2242 \\
2203 \\
2158 \\
2108 \\
2055\end{array}$ & $\begin{array}{l}3.57546 \\
3.58351 \\
3.59174 \\
3.60014 \\
3.60867\end{array}$ & $\begin{array}{l}805 \\
823 \\
840 \\
853 \\
864\end{array}$ & $\begin{array}{l}22.78424 \\
22.90161 \\
23.01552 \\
23.12617 \\
23.23377\end{array}$ & $\begin{array}{l}11737 \\
11391 \\
11065 \\
10760 \\
10473\end{array}$ & $\begin{array}{l}26.35971 \\
26.48512 \\
26.60726 \\
26.72631 \\
26.84245\end{array}$ & $\begin{array}{l}12541 \\
12214 \\
11905 \\
11614 \\
11337\end{array}$ \\
\hline $\begin{array}{l}350 \\
360 \\
370 \\
380 \\
390\end{array}$ & $\begin{array}{l}3.92128 \\
3.94128 \\
3.96070 \\
3.97954 \\
3.99780\end{array}$ & $\begin{array}{l}2030 \\
1942 \\
1884 \\
1826 \\
1767\end{array}$ & $\begin{array}{l}3.61731 \\
3.62604 \\
3.63482 \\
3.64364 \\
3.65249\end{array}$ & $\begin{array}{l}873 \\
878 \\
882 \\
885 \\
886\end{array}$ & $\begin{array}{l}23.33850 \\
23.44052 \\
23.53999 \\
23.63705 \\
23.73181\end{array}$ & $\begin{array}{r}10202 \\
9947 \\
9706 \\
9476 \\
9258\end{array}$ & $\begin{array}{l}26.95582 \\
27.06657 \\
27.17482 \\
27.28070 \\
27.38430\end{array}$ & $\begin{array}{l}11075 \\
10825 \\
10588 \\
10360 \\
10144\end{array}$ \\
\hline $\begin{array}{l}400 \\
450 \\
500 \\
550 \\
600\end{array}$ & $\begin{array}{l}4.01547 \\
4.09534 \\
4.16228 \\
4.21831 \\
4.26541\end{array}$ & $\begin{array}{l}7987 \\
6694 \\
5603 \\
4710 \\
3986\end{array}$ & $\begin{array}{l}3.66135 \\
3.70526 \\
3.74771 \\
3.78802 \\
3.82590\end{array}$ & $\begin{array}{l}4391 \\
4245 \\
4031 \\
3788 \\
3538\end{array}$ & $\begin{array}{l}23.82439 \\
24.25818 \\
24.65078 \\
25.00989 \\
25.34113\end{array}$ & $\begin{array}{l}43379 \\
39260 \\
35911 \\
33124 \\
30765\end{array}$ & $\begin{array}{l}27.48574 \\
27.96344 \\
28.39850 \\
28.79792 \\
29.16704\end{array}$ & $\begin{array}{l}47770 \\
43506 \\
39942 \\
36912 \\
34303\end{array}$ \\
\hline $\begin{array}{l}650 \\
700 \\
750 \\
800 \\
850\end{array}$ & $\begin{array}{l}4.30527 \\
4.33930 \\
4.36861 \\
4.39409 \\
4.41645\end{array}$ & $\begin{array}{l}3403 \\
2931 \\
2548 \\
2236 \\
1980\end{array}$ & $\begin{array}{l}3.86128 \\
3.89424 \\
3.92491 \\
3.95346 \\
3.98005\end{array}$ & $\begin{array}{l}3296 \\
3067 \\
2855 \\
2659 \\
2481\end{array}$ & $\begin{array}{l}25.64878 \\
25.93615 \\
26.20589 \\
26.46012 \\
26.70060\end{array}$ & $\begin{array}{l}28737 \\
26974 \\
25423 \\
24048 \\
22821\end{array}$ & $\begin{array}{l}29.51007 \\
29.83040 \\
30.13081 \\
30.41358 \\
30.68066\end{array}$ & $\begin{array}{l}32033 \\
30041 \\
28277 \\
26708 \\
25301\end{array}$ \\
\hline $\begin{array}{r}900 \\
950 \\
1000 \\
1050 \\
1100\end{array}$ & $\begin{array}{l}4.43625 \\
4.45392 \\
4.46983 \\
4.48425 \\
4.49743\end{array}$ & $\begin{array}{l}1767 \\
1591 \\
1442 \\
1318 \\
1212\end{array}$ & $\begin{array}{l}4.00486 \\
4.02803 \\
4.04973 \\
4.07009 \\
4.08922\end{array}$ & $\begin{array}{l}2317 \\
2170 \\
2036 \\
1913 \\
1801\end{array}$ & $\begin{array}{l}26.92881 \\
27.14597 \\
27.35314 \\
27.55122 \\
27.74101\end{array}$ & $\begin{array}{l}21716 \\
20717 \\
19808 \\
18979 \\
18217\end{array}$ & $\begin{array}{l}30.93367 \\
31.17401 \\
31.40287 \\
31.62131 \\
31.83023\end{array}$ & $\begin{array}{l}24034 \\
22886 \\
21844 \\
20892 \\
20019\end{array}$ \\
\hline 1150 & 4.50955 & 1121 & 4.10723 & 1700 & 27.92318 & 17517 & 32.03042 & 19216 \\
\hline
\end{tabular}


Table 2.038. SrD (Cont.)

\begin{tabular}{|l|l|l|l|l|}
\hline${ }^{\circ} K$ & $\frac{C_{p}^{\circ}}{R}$ & $\frac{\left(H^{0}-E_{0}^{0}\right)}{R T}$ & $\frac{-\left(F^{\circ}-E_{o}^{\circ}\right)}{R T}$ & $\frac{S^{\circ}}{R}$ \\
\hline
\end{tabular}

$\begin{array}{lllllllll}1200 & 4.52076 & 2021 & 4.12423 & 3130 & 28.09835 & 33137 & 32.22258 & 36267 \\ 1300 & 4.54097 & 1787 & 4.15553 & 2818 & 28.42972 & 30901 & 32.58525 & 33719 \\ 1400 & 4.55884 & 1609 & 4.18371 & 2555 & 28.73873 & 28953 & 32.92244 & 31509 \\ 1500 & 4.57493 & 1471 & 4.20926 & 2332 & 29.02826 & 27241 & 33.23753 & 29573 \\ 1600 & 4.58964 & 1362 & 4.23258 & 2141 & 29.30067 & 25725 & 33.53326 & 27866 \\ & & & & & & & \\ 1700 & 4.60326 & 1275 & 4.25399 & 1976 & 29.55792 & 24372 & 33.81192 & 26348 \\ 1800 & 4.61601 & 1204 & 4.27375 & 1834 & 29.80164 & 23157 & 34.07540 & 24990 \\ 1900 & 4.62805 & 1146 & 4.29209 & 1708 & 30.03321 & 22060 & 34.32530 & 23768 \\ 2000 & 4.63951 & 1097 & 4.30917 & 1600 & 30.25381 & 21063 & 34.56298 & 22663 \\ 2100 & 4.65048 & 1058 & 4.32517 & 1503 & 30.46444 & 20156 & 34.78961 & 21659 \\ & & & & & & & & \\ 2200 & 4.66106 & 1022 & 4.34020 & 1417 & 30.66600 & 19324 & 35.00620 & 20742 \\ 2300 & 4.67128 & 994 & 4.35437 & 1341 & 30.85924 & 18561 & 35.21362 & 19902 \\ 2400 & 4.68122 & 968 & 4.36778 & 1273 & 31.04485 & 17856 & 35.41264 & 19129 \\ 2500 & 4.69090 & 946 & 4.38051 & 1212 & 31.22341 & 17205 & 35.60393 & 18417 \\ 2600 & 4.70036 & 926 & 4.39263 & 1157 & 31.39546 & 16600 & 35.78810 & 17756 \\ & & & & & & & & \\ 2700 & 4.70962 & 909 & 4.40420 & 1107 & 31.56146 & 16037 & 35.96566 & 17145 \\ 2800 & 4.71871 & 894 & 4.41527 & 1062 & 31.72183 & 15512 & 36.13711 & 16574 \\ 2900 & 4.72765 & 880 & 4.42589 & 1021 & 31.87695 & 15022 & 36.30285 & 16042 \\ 3000 & 4.73645 & 1723 & 4.43610 & 1931 & 32.02717 & 28692 & 36.46327 & 30624 \\ & & & & & & & & \\ 3200 & 4.75368 & 1682 & 4.45541 & 1804 & 32.31409 & 27066 & 36.76951 & 28870 \\ 3400 & 4.77050 & 1646 & 4.47345 & 1696 & 32.58475 & 25618 & 37.05821 & 27314 \\ 3600 & 4.78696 & 1615 & 4.49041 & 1604 & 32.84093 & 24322 & 37.33135 & 25925 \\ 3800 & 4.80311 & 1588 & 4.50645 & 1523 & 33.08415 & 23154 & 37.59060 & 24677 \\ 4000 & 4.81899 & 1563 & 4.52168 & 1453 & 33.31569 & 22097 & 37.83737 & 23550 \\ 4200 & 4.83462 & 1542 & 4.53621 & 1391 & 33.53666 & 21135 & 38.07287 & 22527 \\ 4400 & 4.85004 & 1520 & 4.55012 & 1338 & 33.74801 & 20255 & 38.29814 & 21592 \\ 4600 & 4.86524 & 1502 & 4.56350 & 1288 & 33.95056 & 19450 & 38.51406 & 20739 \\ 4800 & 4.88026 & 1484 & 4.57638 & 1245 & 34.14506 & 18707 & 38.72145 & 19952 \\ 5000 & 4.89510 & & 4.58883 & & 34.33213 & & 38.92097 & \end{array}$


Table 2.039. SrT

\begin{tabular}{|l|l|l|l|l|}
\hline${ }^{\circ} K$ & $\frac{C_{p}^{0}}{R}$ & $\frac{\left(H^{\circ}-E_{0}^{0}\right)}{R T}$ & $\frac{-\left(F^{\circ}-E_{0}^{0}\right)}{R T}$ & $\frac{S^{0}}{R}$ \\
\hline
\end{tabular}

\begin{tabular}{|c|c|c|c|c|c|c|c|c|}
\hline $\begin{array}{l}50 \\
60 \\
70 \\
80 \\
90\end{array}$ & $\begin{array}{l}3.50144 \\
3.50173 \\
3.50213 \\
3.50288 \\
3.50446\end{array}$ & $\begin{array}{r}29 \\
40 \\
75 \\
158 \\
307\end{array}$ & $\begin{array}{l}3.48871 \\
3.49086 \\
3.49244 \\
3.49369 \\
3.49479\end{array}$ & $\begin{array}{l}215 \\
158 \\
125 \\
110 \\
110\end{array}$ & $\begin{array}{l}16.90927 \\
17.54553 \\
18.08378 \\
18.55021 \\
18.96177\end{array}$ & $\begin{array}{l}63626 \\
53825 \\
46643 \\
41156 \\
36827\end{array}$ & $\begin{array}{l}20.39798 \\
21.03639 \\
21.57622 \\
22.04390 \\
22.45656\end{array}$ & $\begin{array}{l}63841 \\
53983 \\
46768 \\
41266 \\
36938\end{array}$ \\
\hline $\begin{array}{l}100 \\
110 \\
120 \\
130 \\
140\end{array}$ & $\begin{array}{l}3.50753 \\
3.51272 \\
3.52058 \\
3.53145 \\
3.54542\end{array}$ & $\begin{array}{r}519 \\
786 \\
1087 \\
1397 \\
1696\end{array}$ & $\begin{array}{l}3.49589 \\
3.49717 \\
3.49877 \\
3.50085 \\
3.50351\end{array}$ & $\begin{array}{l}128 \\
160 \\
208 \\
266 \\
335\end{array}$ & $\begin{array}{l}19.33004 \\
19.66329 \\
19.96765 \\
20.24778 \\
20.50732\end{array}$ & $\begin{array}{l}33325 \\
30436 \\
28013 \\
25954 \\
24183\end{array}$ & $\begin{array}{l}22.82594 \\
23.16047 \\
23.46643 \\
23.74864 \\
24.01084\end{array}$ & $\begin{array}{l}33453 \\
30596 \\
28221 \\
26220 \\
24517\end{array}$ \\
\hline $\begin{array}{l}150 \\
160 \\
170 \\
180 \\
190\end{array}$ & $\begin{array}{l}3.56238 \\
3.58207 \\
3.60413 \\
3.62813 \\
3.65365\end{array}$ & $\begin{array}{l}1969 \\
2206 \\
2400 \\
2552 \\
2662\end{array}$ & $\begin{array}{l}3.50686 \\
3.51093 \\
3.51575 \\
3.52132 \\
3.52761\end{array}$ & $\begin{array}{l}407 \\
482 \\
557 \\
629 \\
696\end{array}$ & $\begin{array}{l}20.74915 \\
20.97560 \\
21.18859 \\
21.38970 \\
21.58025\end{array}$ & $\begin{array}{l}22645 \\
21299 \\
20111 \\
19055 \\
18112\end{array}$ & $\begin{array}{l}24.25601 \\
24.48653 \\
24.70435 \\
24.91102 \\
25.10787\end{array}$ & $\begin{array}{l}23052 \\
21782 \\
20667 \\
19685 \\
18808\end{array}$ \\
\hline $\begin{array}{l}200 \\
210 \\
220 \\
230 \\
240\end{array}$ & $\begin{array}{l}3.68027 \\
3.70760 \\
3.73532 \\
3.76312 \\
3.79077\end{array}$ & $\begin{array}{l}2733 \\
2772 \\
2780 \\
2765 \\
2731\end{array}$ & $\begin{array}{l}3.53457 \\
3.54216 \\
3.55031 \\
3.55896 \\
3.56804\end{array}$ & $\begin{array}{l}759 \\
815 \\
865 \\
908 \\
946\end{array}$ & $\begin{array}{l}21.75137 \\
21.93400 \\
22.09897 \\
22.25698 \\
22.40864\end{array}$ & $\begin{array}{l}17263 \\
16497 \\
15801 \\
15166 \\
14584\end{array}$ & $\begin{array}{l}25.29595 \\
25.47617 \\
25.64928 \\
25.81594 \\
25.97668\end{array}$ & $\begin{array}{l}18022 \\
17311 \\
16666 \\
16074 \\
15530\end{array}$ \\
\hline $\begin{array}{l}250 \\
260 \\
270 \\
280 \\
290\end{array}$ & $\begin{array}{l}3.81808 \\
3.84488 \\
3.87105 \\
3.89651 \\
3.92118\end{array}$ & $\begin{array}{l}2680 \\
2617 \\
2546 \\
2467 \\
2386\end{array}$ & $\begin{array}{l}3.57750 \\
3.58727 \\
3.59730 \\
3.60753 \\
3.61792\end{array}$ & $\begin{array}{r}977 \\
1003 \\
1023 \\
1039 \\
1051\end{array}$ & $\begin{array}{l}22.55448 \\
22.69498 \\
22.83056 \\
22.96156 \\
23.08834\end{array}$ & $\begin{array}{l}14050 \\
13558 \\
13100 \\
12678 \\
12283\end{array}$ & $\begin{array}{l}26.13198 \\
26.28226 \\
26.42786 \\
26.56910 \\
26.70627\end{array}$ & $\begin{array}{l}15028 \\
14560 \\
14124 \\
13717 \\
13333\end{array}$ \\
\hline $\begin{array}{l}300 \\
310 \\
320 \\
330 \\
340\end{array}$ & $\begin{array}{l}3.94504 \\
3.96804 \\
3.99018 \\
4.01146 \\
4.03188\end{array}$ & $\begin{array}{l}2300 \\
2214 \\
2128 \\
2042 \\
1959\end{array}$ & $\begin{array}{l}3.62843 \\
3.63902 \\
3.64965 \\
3.66029 \\
3.67092\end{array}$ & $\begin{array}{l}1059 \\
1063 \\
1064 \\
1063 \\
1060\end{array}$ & $\begin{array}{l}23.21117 \\
23.33032 \\
23.44602 \\
23.55849 \\
23.66792\end{array}$ & $\begin{array}{l}11915 \\
11570 \\
11247 \\
10943 \\
10656\end{array}$ & $\begin{array}{l}26.83960 \\
26.96934 \\
27.09567 \\
27.21878 \\
27.33884\end{array}$ & $\begin{array}{l}12974 \\
12633 \\
12311 \\
12006 \\
11716\end{array}$ \\
\hline $\begin{array}{l}350 \\
360 \\
370 \\
380 \\
390\end{array}$ & $\begin{array}{l}4.05147 \\
4.07024 \\
4.08822 \\
4.10543 \\
4.12191\end{array}$ & $\begin{array}{l}1877 \\
1798 \\
1721 \\
1648 \\
1576\end{array}$ & $\begin{array}{l}3.68152 \\
3.69206 \\
3.70252 \\
3.71290 \\
3.72318\end{array}$ & $\begin{array}{l}1054 \\
1046 \\
1038 \\
1028 \\
1016\end{array}$ & $\begin{array}{l}23.77448 \\
23.87834 \\
23.97964 \\
24.07852 \\
24.17510\end{array}$ & $\begin{array}{r}10386 \\
10130 \\
9888 \\
9658 \\
9439\end{array}$ & $\begin{array}{l}27.45600 \\
27.57040 \\
27.68217 \\
27.79142 \\
27.89828\end{array}$ & $\begin{array}{l}11440 \\
11177 \\
10925 \\
10686 \\
10456\end{array}$ \\
\hline $\begin{array}{l}400 \\
450 \\
500 \\
550 \\
600\end{array}$ & $\begin{array}{l}4.13767 \\
4.20693 \\
4.26281 \\
4.30833 \\
4.34589\end{array}$ & $\begin{array}{l}6926 \\
5588 \\
4552 \\
3756 \\
3138\end{array}$ & $\begin{array}{l}3.73334 \\
3.78226 \\
3.82762 \\
3.86932 \\
3.90751\end{array}$ & $\begin{array}{l}4892 \\
4536 \\
4170 \\
3819 \\
3497\end{array}$ & $\begin{array}{l}24.26949 \\
24.71207 \\
25.11295 \\
25.47975 \\
25.81808\end{array}$ & $\begin{array}{l}44258 \\
40088 \\
36680 \\
33833 \\
31417\end{array}$ & $\begin{array}{l}28.00284 \\
28.49433 \\
28.94057 \\
29.34907 \\
29.72560\end{array}$ & $\begin{array}{l}49149 \\
44624 \\
40850 \\
37653 \\
34914\end{array}$ \\
\hline $\begin{array}{l}650 \\
700 \\
750 \\
800 \\
850\end{array}$ & $\begin{array}{l}4.37727 \\
4.40385 \\
4.42665 \\
4.44645 \\
4.46384\end{array}$ & $\begin{array}{l}2658 \\
2280 \\
1980 \\
1739 \\
1544\end{array}$ & $\begin{array}{l}3.94248 \\
3.97451 \\
4.00391 \\
4.03096 \\
4.05593\end{array}$ & $\begin{array}{l}3203 \\
2940 \\
2705 \\
2497 \\
2310\end{array}$ & $\begin{array}{l}26.13225 \\
26.42561 \\
26.70084 \\
26.96012 \\
27.20526\end{array}$ & $\begin{array}{l}29336 \\
27523 \\
25928 \\
24514 \\
23249\end{array}$ & $\begin{array}{l}30.07474 \\
30.40013 \\
30.70476 \\
30.99109 \\
31.26119\end{array}$ & $\begin{array}{l}32539 \\
30463 \\
28633 \\
27010 \\
25559\end{array}$ \\
\hline $\begin{array}{r}900 \\
950 \\
1000 \\
1050 \\
1100\end{array}$ & $\begin{array}{l}4.47928 \\
4.49313 \\
4.50567 \\
4.51712 \\
4.52765\end{array}$ & $\begin{array}{r}1385 \\
1254 \\
1145 \\
1053 \\
976\end{array}$ & $\begin{array}{l}4.07903 \\
4.10046 \\
4.12041 \\
4.13904 \\
4.15646\end{array}$ & $\begin{array}{l}2143 \\
1995 \\
1863 \\
1742 \\
1636\end{array}$ & $\begin{array}{l}27.43775 \\
27.65888 \\
27.86972 \\
28.07121 \\
28.26416\end{array}$ & $\begin{array}{l}22113 \\
21084 \\
20149 \\
19295 \\
18513\end{array}$ & $\begin{array}{l}31.51678 \\
31.75934 \\
31.99013 \\
32.21025 \\
32.42063\end{array}$ & $\begin{array}{l}24256 \\
23079 \\
22012 \\
21038 \\
20148\end{array}$ \\
\hline 1150 & 4.53741 & 911 & 4.17282 & 1538 & 28.44929 & 17792 & 32.62211 & 19331 \\
\hline
\end{tabular}


Table 2.039. SrT (Cont.)

\begin{tabular}{|l|l|l|l|l|}
\hline${ }^{\circ} K$ & $\frac{C_{p}^{0}}{R}$ & $\frac{\left(H^{0}-E_{0}^{0}\right)}{R T}$ & $\frac{-\left(F^{0}-E_{0}^{0}\right)}{R T}$ & $\frac{S^{0}}{R}$ \\
\hline
\end{tabular}

\begin{tabular}{|c|c|c|c|c|c|c|c|c|}
\hline $\begin{array}{l}1200 \\
1300 \\
1400 \\
1500 \\
1600\end{array}$ & $\begin{array}{l}4.54652 \\
4.56315 \\
4.57810 \\
4.59179 \\
4.60449\end{array}$ & $\begin{array}{l}1663 \\
1495 \\
1369 \\
1270 \\
1194\end{array}$ & $\begin{array}{l}4.18820 \\
4.21642 \\
4.24173 \\
4.26461 \\
4.28546\end{array}$ & $\begin{array}{l}2822 \\
2531 \\
2288 \\
2085 \\
1912\end{array}$ & $\begin{array}{l}28.62721 \\
28.96358 \\
29.27699 \\
29.57044 \\
29.84634\end{array}$ & $\begin{array}{l}33637 \\
31341 \\
29345 \\
27590 \\
26039\end{array}$ & $\begin{array}{l}32.81542 \\
33.18000 \\
33.51872 \\
33.83505 \\
34.13181\end{array}$ & $\begin{array}{l}36458 \\
33872 \\
31633 \\
29676 \\
27951\end{array}$ \\
\hline $\begin{array}{l}1700 \\
1800 \\
1900 \\
2000 \\
2100\end{array}$ & $\begin{array}{l}4.61643 \\
4.62774 \\
4.63856 \\
4.64896 \\
4.65902\end{array}$ & $\begin{array}{r}1131 \\
1082 \\
1040 \\
1006 \\
977\end{array}$ & $\begin{array}{l}4.30458 \\
4.32223 \\
4.33859 \\
4.35385 \\
4.36815\end{array}$ & $\begin{array}{l}1765 \\
1636 \\
1526 \\
1430 \\
1344\end{array}$ & $\begin{array}{l}30.10673 \\
30.35328 \\
30.58742 \\
30.81035 \\
31.02312\end{array}$ & $\begin{array}{l}24655 \\
23414 \\
22293 \\
21277 \\
20352\end{array}$ & $\begin{array}{l}34.41132 \\
34.67551 \\
34.92601 \\
35.16421 \\
35.39127\end{array}$ & $\begin{array}{l}26419 \\
25050 \\
23820 \\
22706 \\
21697\end{array}$ \\
\hline $\begin{array}{l}2200 \\
2300 \\
2400 \\
2500 \\
2600\end{array}$ & $\begin{array}{l}4.66879 \\
4.67831 \\
4.68762 \\
4.69674 \\
4.70570\end{array}$ & $\begin{array}{l}952 \\
931 \\
912 \\
896 \\
882\end{array}$ & $\begin{array}{l}4.38159 \\
4.39428 \\
4.40631 \\
4.41775 \\
4.42865\end{array}$ & $\begin{array}{l}1269 \\
1203 \\
1144 \\
1090 \\
1043\end{array}$ & $\begin{array}{l}31.22664 \\
31.42170 \\
31.60897 \\
31.78908 \\
31.96256\end{array}$ & $\begin{array}{l}19506 \\
18727 \\
18011 \\
17348 \\
16734\end{array}$ & $\begin{array}{l}35.60824 \\
35.81598 \\
36.01529 \\
36.20683 \\
36.39122\end{array}$ & $\begin{array}{l}20774 \\
19931 \\
19154 \\
18439 \\
17776\end{array}$ \\
\hline $\begin{array}{l}2700 \\
2800 \\
2900 \\
3000\end{array}$ & $\begin{array}{l}4.71452 \\
4.72320 \\
4.73178 \\
4.74024\end{array}$ & $\begin{array}{r}868 \\
858 \\
846 \\
1666\end{array}$ & $\begin{array}{l}4.43908 \\
4.44907 \\
4.45867 \\
4.46791\end{array}$ & $\begin{array}{r}999 \\
960 \\
924 \\
1755\end{array}$ & $\begin{array}{l}32.12990 \\
32.29152 \\
32.44781 \\
32.59912\end{array}$ & $\begin{array}{l}16162 \\
15629 \\
15131 \\
28892\end{array}$ & $\begin{array}{l}36.56898 \\
36.74059 \\
36.90649 \\
37.06704\end{array}$ & $\begin{array}{l}17161 \\
16590 \\
16055 \\
30646\end{array}$ \\
\hline $\begin{array}{l}3200 \\
3400 \\
3600 \\
3800 \\
4000\end{array}$ & $\begin{array}{l}4.75690 \\
4.77323 \\
4.78928 \\
4.80507 \\
4.82064\end{array}$ & $\begin{array}{l}1633 \\
1605 \\
1579 \\
1557 \\
1536\end{array}$ & $\begin{array}{l}4.48546 \\
4.50191 \\
4.51743 \\
4.53215 \\
4.54619\end{array}$ & $\begin{array}{l}1645 \\
1552 \\
1472 \\
1404 \\
1343\end{array}$ & $\begin{array}{l}32.88804 \\
33.16047 \\
33.41824 \\
33.66288 \\
33.89571\end{array}$ & $\begin{array}{l}27243 \\
25777 \\
24464 \\
23283 \\
22213\end{array}$ & $\begin{array}{l}37.37350 \\
37.66238 \\
37.93567 \\
38.19504 \\
38.44190\end{array}$ & $\begin{array}{l}28888 \\
27329 \\
25937 \\
24686 \\
23557\end{array}$ \\
\hline $\begin{array}{l}4200 \\
4400 \\
4600 \\
4800 \\
5000\end{array}$ & $\begin{array}{l}4.83600 \\
4.85118 \\
4.86617 \\
4.88100 \\
4.89567\end{array}$ & $\begin{array}{l}1518 \\
1499 \\
1483 \\
1467\end{array}$ & $\begin{array}{l}4.55962 \\
4.57253 \\
4.58497 \\
4.59700 \\
4.60865\end{array}$ & $\begin{array}{l}1291 \\
1244 \\
1203 \\
1165\end{array}$ & $\begin{array}{l}34.11784 \\
34.33026 \\
34.53379 \\
34.72918 \\
34.91708\end{array}$ & $\begin{array}{l}21242 \\
20353 \\
19539 \\
18790\end{array}$ & $\begin{array}{l}38.67747 \\
38.90279 \\
39.11877 \\
39.32619 \\
39.52574\end{array}$ & $\begin{array}{l}22532 \\
21598 \\
20742 \\
19955\end{array}$ \\
\hline
\end{tabular}


Table 2.040. $\mathrm{BaH}$

\begin{tabular}{|l|l|l|l|l|}
\hline${ }^{\circ}$ & $\frac{C_{p}^{0}}{R}$ & $\frac{\left(H^{0}-E_{0}^{0}\right)}{R T}$ & $\frac{-\left(F^{0}-E_{0}^{0}\right)}{R T}$ & $\frac{S^{0}}{R}$ \\
\hline
\end{tabular}

\begin{tabular}{|c|c|c|c|c|c|c|c|c|}
\hline 50 & 3.50159 & 21 & 3.46835 & 556 & 16.57619 & 63288 & 20.04455 & 63843 \\
\hline 60 & 3.50180 & 23 & 3.47391 & 400 & 17.20907 & 53582 & 20.68298 & 53982 \\
\hline 70 & 3.50203 & 25 & 3.47791 & 303 & 17.74489 & 46462 & 21.22280 & 46765 \\
\hline 80 & 3.50228 & 26 & 3.48094 & 239 & 18.20951 & 41014 & 21.69045 & 41253 \\
\hline 90 & 3.50254 & 28 & 3.48333 & 193 & 18.61965 & 36711 & 22.10298 & 36904 \\
\hline 100 & 3.50282 & 32 & 3.48526 & 161 & 18.98676 & 33225 & 22.47202 & 33387 \\
\hline 110 & 3.50314 & 42 & 3.48687 & 137 & 19.31901 & 30346 & 22.80589 & 30483 \\
\hline 120 & 3.50356 & 58 & 3.48824 & 120 & 19.62247 & 27926 & 23.11072 & 28046 \\
\hline 130 & 3.50414 & 88 & 3.48944 & 108 & 19.90173 & 25864 & 23.39118 & 25971 \\
\hline 140 & 3.50502 & 130 & 3.49052 & 101 & 20.16037 & 24085 & 23.65089 & 24187 \\
\hline 150 & 3.50632 & 189 & 3.49153 & 98 & 20.40122 & 22537 & 23.89276 & 22635 \\
\hline 160 & 3.50821 & 262 & 3.49251 & 100 & 20.62659 & 21176 & 24.11911 & 21275 \\
\hline 170 & 3.51083 & 349 & 3.49351 & 105 & 20.83835 & 19972 & 24.33186 & 20077 \\
\hline 180 & 3.51432 & 448 & 3.49456 & 115 & 21.03807 & 18897 & 24.53263 & 19013 \\
\hline 190 & 3.51880 & 556 & 3.49571 & 129 & 21.22704 & 17934 & 24.72276 & 18062 \\
\hline 200 & 3.52436 & 670 & 3.49700 & 146 & 21.40638 & 17065 & 24.90338 & 17212 \\
\hline 210 & 3.53106 & 785 & 3.49846 & 166 & 21.57703 & 16279 & 25.07550 & 16444 \\
\hline 220 & 3.53891 & 901 & 3.50012 & 187 & 21.73982 & 15562 & 25.23994 & 15750 \\
\hline 230 & 3.54792 & 1013 & 3.50199 & 213 & 21.89544 & 14909 & 25.39744 & 15121 \\
\hline 240 & 3.55805 & 1120 & 3.50412 & 237 & 22.04453 & 14309 & 25.54865 & 14547 \\
\hline 250 & 3.56925 & 1219 & 3.50649 & 265 & 22.18762 & 13758 & 25.69412 & 14023 \\
\hline 260 & 3.58144 & 1311 & 3.50914 & 292 & 22.32520 & 13249 & 25.83435 & 13540 \\
\hline 270 & 3.59455 & 1392 & 3.51206 & 319 & 22.45769 & 12778 & 25.96975 & 13098 \\
\hline 280 & 3.60847 & 1465 & 3.51525 & 346 & 22.58547 & 12342 & 26.10073 & 12688 \\
\hline 290 & 3.62312 & 1528 & 3.51871 & 374 & 22.70889 & 11935 & 26.22761 & 12308 \\
\hline 300 & 3.63840 & 1580 & 3.52245 & 399 & 22.82824 & 11557 & 26.35069 & 11956 \\
\hline 310 & 3.65420 & 1624 & 3.52644 & 425 & 22.94381 & 11202 & 26.47025 & 11627 \\
\hline 320 & 3.67044 & 1659 & 3.53069 & 448 & 23.05583 & 10872 & 26.58652 & 11320 \\
\hline 330 & 3.68703 & 1685 & 3.53517 & 472 & 23.16455 & 10560 & 26.69972 & 11032 \\
\hline 340 & 3.70388 & 1704 & 3.53989 & 492 & 23.27015 & 10268 & 26.81004 & 10761 \\
\hline 350 & 3.72092 & 1715 & 3.54481 & 513 & 23.37283 & 9994 & 26.91765 & 10507 \\
\hline 360 & 3.73807 & 1720 & 3.54994 & 532 & 23.47277 & 9733 & 27.02272 & 10265 \\
\hline 370 & 3.75527 & 1719 & 3.55526 & 549 & 23.57010 & 9489 & 27.12537 & 10037 \\
\hline 380 & 3.77246 & 1714 & 3.56075 & 565 & 23.66499 & 9256 & 27.22574 & 9822 \\
\hline 390 & 3.78960 & 1703 & 3.56640 & 579 & 23.75755 & 9037 & 27.32396 & 9616 \\
\hline 400 & 3.80663 & 8237 & 3.57219 & 3068 & 23.84792 & 42249 & 27.42012 & 45316 \\
\hline 450 & 3.88900 & 7576 & 3.60287 & 3246 & 24.27041 & 38127 & 27.87328 & 41374 \\
\hline 500 & 3.96476 & 6800 & 3.6 & 3310 & 24.65168 & 34804 & 28. & 38113 \\
\hline 550 & 4.03276 & 6025 & 3.66843 & 3292 & 24.99972 & 32061 & 28.66815 & 35354 \\
\hline 600 & 4.09301 & 5308 & 3.70135 & 3222 & 25.32033 & 29754 & 29.02169 & 32975 \\
\hline 650 & 4.14609 & 4667 & 3.73357 & 3116 & 25.61787 & 27784 & 29.35144 & 30901 \\
\hline 700 & 4.19276 & 4111 & 3.76473 & 2994 & 25.89571 & 26077 & 29.66045 & 29070 \\
\hline 750 & 4.23387 & 3630 & 3.79467 & 2861 & 26.15648 & 24582 & 29.95115 & 27443 \\
\hline 800 & 4.27017 & 3219 & 3.82 & 2725 & 40230 & 23261 & & 25986 \\
\hline 850 & 4.30236 & 2868 & 3.85 & 2591 & 63491 & 22083 & 30.4 & 24675 \\
\hline 900 & 4.33104 & 2568 & 3.87644 & 2462 & 26.85574 & 21025 & 30.73219 & 23486 \\
\hline 950 & 4.35672 & 2310 & 3.90106 & 2337 & 27.06599 & 20070 & 30.96705 & 22407 \\
\hline 1000 & 4.37982 & 2099 & 3.92443 & 2219 & 27.26669 & 19202 & 31.19112 & 21421 \\
\hline 1050 & 4.40073 & 1901 & 3.94662 & 2108 & 27.45871 & 18408 & 31.40533 & 20517 \\
\hline 1100 & 4.41974 & 1738 & 3.96770 & 2004 & 4279 & 17682 & 31.61050 & 19685 \\
\hline 1150 & 4.43712 & 1596 & 3.98774 & 1906 & 27.81961 & 17012 & 31.80735 & 18919 \\
\hline
\end{tabular}


Table 2. 040. BaH (Cont.)

\begin{tabular}{|l|l|l|l|l|}
\hline$K$ & $\frac{C_{p}^{0}}{R}$ & $\frac{\left(H^{0}-E_{0}^{0}\right)}{R T}$ & $\frac{-\left(F^{0}-E_{0}^{0}\right)}{R T}$ & $\frac{S^{0}}{R}$ \\
\hline
\end{tabular}

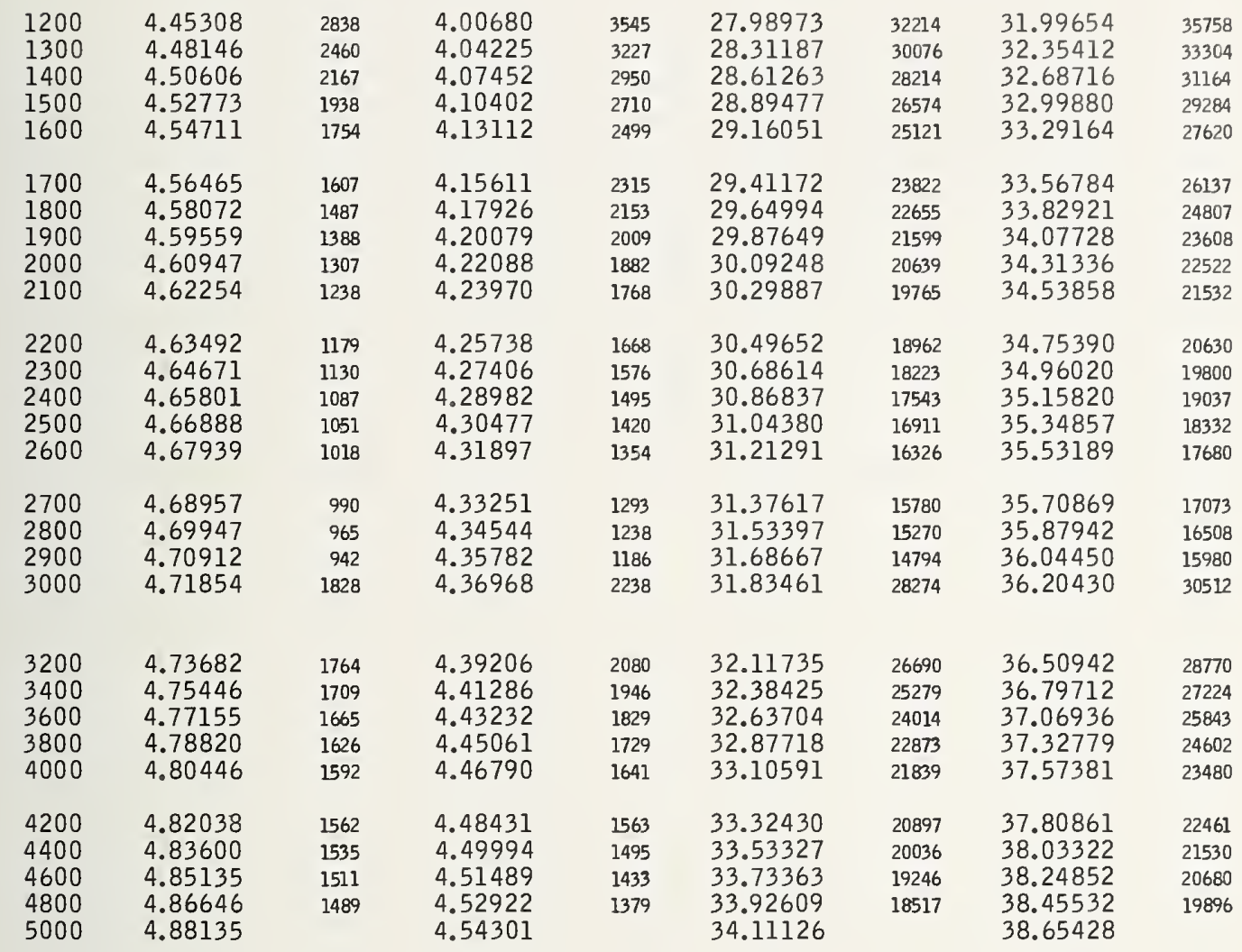


Table 2.041. BaD

\begin{tabular}{|l|l|l|l|l|}
\hline$K$ & $\frac{C_{p}^{0}}{R}$ & $\frac{\left(H^{0}-E_{0}^{0}\right)}{R T}$ & $\frac{-\left(F^{0}-E_{0}^{0}\right)}{R T}$ & $\frac{S^{0}}{R}$ \\
\hline
\end{tabular}

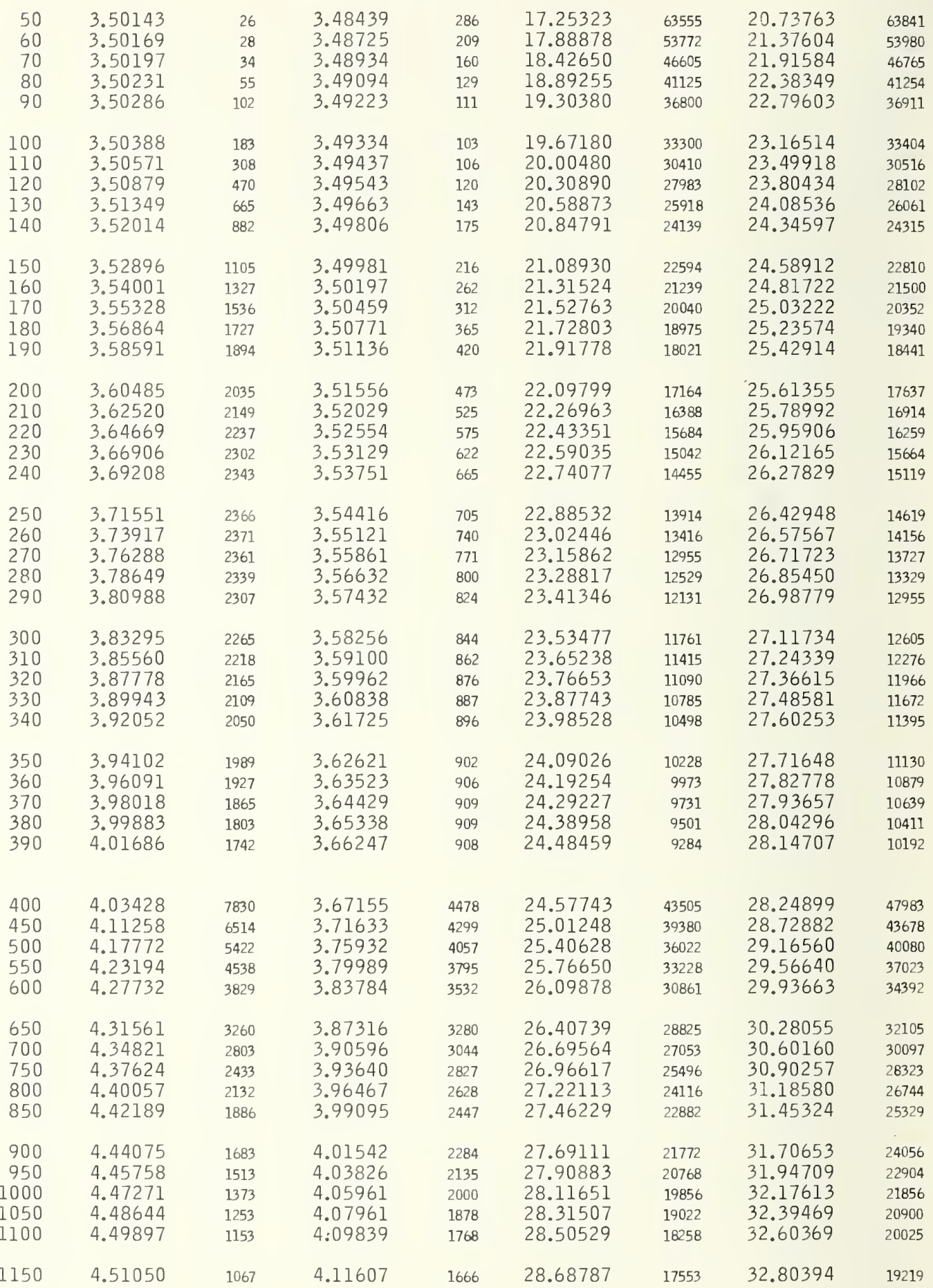


Table 2.041. BaD (Cont.)

\begin{tabular}{|c|c|c|c|c|c|c|c|c|}
\hline${ }^{\circ} \mathrm{K}$ & $\frac{C_{p}^{0}}{R}$ & & $\frac{\left(H^{0}-E_{0}^{0}\right)}{R T}$ & & $\frac{-\left(F^{0}-E_{0}^{0}\right)}{R T}$ & & $\frac{S^{\circ}}{R}$ & \\
\hline 1200 & 4.52117 & 1922 & 4.13273 & 3063 & 28.86340 & 33203 & 32.99613 & 36266 \\
\hline 1300 & 4.54039 & 1701 & 4.16336 & 2755 & 29.19543 & 30956 & 33.35879 & 33712 \\
\hline 1400 & 4.55740 & 1532 & 4.19091 & 2495 & 29.50499 & 29001 & 33.69591 & 31496 \\
\hline 1500 & 4.57272 & 1402 & 4.21586 & 2275 & 29.79500 & 27282 & 34.01087 & 29557 \\
\hline 1600 & 4.58674 & 1298 & 4.23861 & 2086 & 30.06782 & 25760 & 34.30644 & 27846 \\
\hline 1700 & 4.59972 & 1216 & 4.25947 & 1925 & 30.32542 & 24402 & 34.58490 & 26326 \\
\hline 1800 & 4.61188 & 1150 & 4.27872 & 1784 & 30.56944 & 23182 & 34.84816 & 24967 \\
\hline 1900 & 4.62338 & 1094 & 4.29656 & 1662 & 30.80126 & 22081 & 35.09783 & 23742 \\
\hline 2000 & 4.63432 & 1050 & 4.31318 & 1554 & 31.02207 & 21083 & 35.33525 & 22637 \\
\hline 2100 & 4.64482 & 1011 & 4.32872 & 1460 & 31.23290 & 20171 & 35.56162 & 21631 \\
\hline 2200 & 4.65493 & 978 & 4.34332 & 1376 & 31.43461 & 19337 & 35.77793 & 20714 \\
\hline 2300 & 4.66471 & 951 & 4.35708 & 1302 & 31.62798 & 18572 & 35.98507 & 19873 \\
\hline 2400 & 4.67422 & 927 & 4.37010 & 1235 & 31.81370 & 17865 & 36.18380 & 19100 \\
\hline 2500 & 4.68349 & 906 & 4.38245 & 1175 & 31.99235 & 17211 & 36.37480 & 18387 \\
\hline 2600 & 4.69255 & 888 & 4.39420 & 1122 & 32.16446 & 16605 & 36.55867 & 17726 \\
\hline 2700 & 4.70143 & 872 & 4.40542 & 1073 & 32.33051 & 16041 & 36.73593 & 17114 \\
\hline 2800 & 4.71015 & 857 & 4.41615 & 1028 & 32.49092 & 15515 & 36.90707 & 16544 \\
\hline 2900 & 4.71872 & 844 & 4.42643 & 989 & 32.64607 & 15023 & 37.07251 & 16011 \\
\hline 3000 & 4.72716 & 1655 & 4.43632 & 1869 & 32.79630 & 28692 & 37.23262 & 30562 \\
\hline 3200 & 4.74371 & 1615 & 4.45501 & 1746 & 33.08322 & 27061 & 37.53824 & 28807 \\
\hline 3400 & 4.75986 & 1581 & 4.47247 & 1641 & 33.35383 & 25611 & 37.82631 & 27251 \\
\hline 3600 & 4.77567 & 1553 & 4.48888 & 1550 & 33.60994 & 24312 & 38.09882 & 25863 \\
\hline 3800 & 4.79120 & 1528 & 4.50438 & 1473 & 33.85306 & 23142 & 38.35745 & 24614 \\
\hline 4000 & 4.80648 & 1504 & 4.51911 & 1404 & 34.08448 & 22083 & 38.60359 & 23488 \\
\hline 4200 & 4.82152 & 1484 & 4.53315 & 1345 & 34.30531 & 21120 & 38.83847 & 22464 \\
\hline 4400 & 4.83636 & 1464 & 4.54660 & 1291 & 34.51651 & 20239 & 39.06311 & 21531 \\
\hline 4600 & 4.85100 & 1447 & 4.55951 & 1245 & 34.71890 & 19432 & 39.27842 & 20676 \\
\hline 4800 & 4.86547 & 1430 & 4.57196 & 1203 & 34.91322 & 18688 & 39.48518 & 19391 \\
\hline 5000 & 4.87977 & & 4.58399 & & 35.10010 & & 39.68409 & \\
\hline
\end{tabular}


Table 2.042. BaT

\begin{tabular}{|l|l|l|l|l|}
\hline${ }^{\circ} K$ & $\frac{C_{p}^{0}}{R}$ & $\frac{\left(H^{0}-E_{0}^{0}\right)}{R T}$ & $\frac{-\left(F^{0}-E_{0}^{0}\right)}{R T}$ & $\frac{S^{0}}{R}$ \\
\hline
\end{tabular}

\begin{tabular}{|c|c|c|c|c|c|c|c|c|}
\hline $\begin{array}{l}50 \\
60 \\
70 \\
80 \\
90\end{array}$ & $\begin{array}{l}3.50140 \\
3.50169 \\
3.50213 \\
3.50306 \\
3.50504\end{array}$ & $\begin{array}{r}29 \\
44 \\
93 \\
193 \\
377\end{array}$ & $\begin{array}{l}3.48973 \\
3.49170 \\
3.49315 \\
3.49433 \\
3.49539\end{array}$ & $\begin{array}{l}197 \\
145 \\
118 \\
106 \\
114\end{array}$ & $\begin{array}{l}17.65441 \\
18.29085 \\
18.82921 \\
19.29573 \\
19.70737\end{array}$ & $\begin{array}{l}63644 \\
53836 \\
46652 \\
41164 \\
36833\end{array}$ & $\begin{array}{l}21.14414 \\
21.78255 \\
22.32237 \\
22.79006 \\
23.20277\end{array}$ & $\begin{array}{l}63841 \\
53982 \\
46769 \\
41271 \\
36947\end{array}$ \\
\hline $\begin{array}{l}100 \\
110 \\
120 \\
130 \\
140\end{array}$ & $\begin{array}{l}3.50881 \\
3.51507 \\
3.52434 \\
3.53687 \\
3.55269\end{array}$ & $\begin{array}{r}626 \\
927 \\
1253 \\
1582 \\
1889\end{array}$ & $\begin{array}{l}3.49653 \\
3.49791 \\
3.49970 \\
3.50206 \\
3.50509\end{array}$ & $\begin{array}{l}138 \\
179 \\
236 \\
303 \\
379\end{array}$ & $\begin{array}{l}20.07570 \\
20.40902 \\
20.71345 \\
20.99367 \\
21.25330\end{array}$ & $\begin{array}{l}33332 \\
30443 \\
28022 \\
25963 \\
24195\end{array}$ & $\begin{array}{l}23.57224 \\
23.90693 \\
24.21316 \\
24.49573 \\
24.75840\end{array}$ & $\begin{array}{l}33469 \\
30623 \\
28257 \\
26267 \\
24574\end{array}$ \\
\hline $\begin{array}{l}150 \\
160 \\
170 \\
180 \\
190\end{array}$ & $\begin{array}{l}3.57158 \\
3.59320 \\
3.61710 \\
3.64282 \\
3.66987\end{array}$ & $\begin{array}{l}2162 \\
2390 \\
2572 \\
2705 \\
2796\end{array}$ & $\begin{array}{l}3.50888 \\
3.51346 \\
3.51884 \\
3.52501 \\
3.53192\end{array}$ & $\begin{array}{l}458 \\
538 \\
617 \\
691 \\
759\end{array}$ & $\begin{array}{l}21.49525 \\
21.72185 \\
21.93501 \\
22.13632 \\
22.32709\end{array}$ & $\begin{array}{l}22660 \\
21316 \\
20131 \\
19077 \\
18135\end{array}$ & $\begin{array}{l}25.00414 \\
25.23532 \\
25.45386 \\
25.66133 \\
25.85901\end{array}$ & $\begin{array}{l}23118 \\
21854 \\
20747 \\
19768 \\
18895\end{array}$ \\
\hline $\begin{array}{l}200 \\
210 \\
220 \\
230 \\
240\end{array}$ & $\begin{array}{l}3.69783 \\
3.72631 \\
3.75497 \\
3.78353 \\
3.81176\end{array}$ & $\begin{array}{l}2848 \\
2866 \\
2856 \\
2823 \\
2773\end{array}$ & $\begin{array}{l}3.53951 \\
3.54773 \\
3.55649 \\
3.56575 \\
3.57541\end{array}$ & $\begin{array}{r}822 \\
876 \\
926 \\
966 \\
1001\end{array}$ & $\begin{array}{l}22.50844 \\
22.68133 \\
22.84657 \\
23.00487 \\
23.15683\end{array}$ & $\begin{array}{l}17289 \\
16524 \\
15830 \\
15196 \\
14615\end{array}$ & $\begin{array}{l}26.04796 \\
26.22906 \\
26.40307 \\
26.57062 \\
26.73224\end{array}$ & $\begin{array}{l}18110 \\
17401 \\
16755 \\
16162 \\
15617\end{array}$ \\
\hline $\begin{array}{l}250 \\
260 \\
270 \\
280 \\
290\end{array}$ & $\begin{array}{l}3.83949 \\
3.86656 \\
3.89287 \\
3.91836 \\
3.94297\end{array}$ & $\begin{array}{l}2707 \\
2631 \\
2549 \\
2461 \\
2370\end{array}$ & $\begin{array}{l}3.58542 \\
3.59571 \\
3.60624 \\
3.61693 \\
3.62775\end{array}$ & $\begin{array}{l}1029 \\
1053 \\
1069 \\
1082 \\
1091\end{array}$ & $\begin{array}{l}23.30298 \\
23.44381 \\
23.57971 \\
23.71105 \\
23.83816\end{array}$ & $\begin{array}{l}14083 \\
13590 \\
13134 \\
12711 \\
12317\end{array}$ & $\begin{array}{l}26.88841 \\
27.03953 \\
27.18595 \\
27.32799 \\
27.46592\end{array}$ & $\begin{array}{l}15112 \\
14642 \\
14204 \\
13793 \\
13407\end{array}$ \\
\hline $\begin{array}{l}300 \\
310 \\
320 \\
330 \\
340\end{array}$ & $\begin{array}{l}3.96667 \\
3.98945 \\
4.01131 \\
4.03226 \\
4.05232\end{array}$ & $\begin{array}{l}2278 \\
2186 \\
2095 \\
2006 \\
1919\end{array}$ & $\begin{array}{l}3.63866 \\
3.64961 \\
3.66057 \\
3.67152 \\
3.68243\end{array}$ & $\begin{array}{l}1095 \\
1096 \\
1095 \\
1091 \\
1084\end{array}$ & $\begin{array}{l}23.96133 \\
24.08082 \\
24.19686 \\
24.30967 \\
24.41944\end{array}$ & $\begin{array}{l}11949 \\
11604 \\
11281 \\
10977 \\
10690\end{array}$ & $\begin{array}{l}27.59999 \\
27.73043 \\
27.85744 \\
27.98120 \\
28.10187\end{array}$ & $\begin{array}{l}13044 \\
12701 \\
12376 \\
12067 \\
11775\end{array}$ \\
\hline $\begin{array}{l}350 \\
360 \\
370 \\
380 \\
390\end{array}$ & $\begin{array}{l}4.07151 \\
4.08986 \\
4.10740 \\
4.12415 \\
4.14016\end{array}$ & $\begin{array}{l}1835 \\
1754 \\
1675 \\
1601 \\
1530\end{array}$ & $\begin{array}{l}3.69327 \\
3.70403 \\
3.71470 \\
3.72526 \\
3.73569\end{array}$ & $\begin{array}{l}1076 \\
1067 \\
1056 \\
1043 \\
1031\end{array}$ & $\begin{array}{l}24.52634 \\
24.63054 \\
24.73217 \\
24.83138 \\
24.92828\end{array}$ & $\begin{array}{r}10420 \\
10163 \\
9921 \\
9690 \\
9471\end{array}$ & $\begin{array}{l}28.21962 \\
28.33458 \\
28.44688 \\
28.55664 \\
28.66397\end{array}$ & $\begin{array}{l}11496 \\
11230 \\
10976 \\
10733 \\
10502\end{array}$ \\
\hline $\begin{array}{l}400 \\
450 \\
500 \\
550 \\
600\end{array}$ & $\begin{array}{l}4.15546 \\
4.22237 \\
4.27604 \\
4.31959 \\
4.35540\end{array}$ & $\begin{array}{l}6691 \\
5367 \\
4355 \\
3581 \\
2986\end{array}$ & $\begin{array}{l}3.74600 \\
3.79535 \\
3.84083 \\
3.88244 \\
3.92041\end{array}$ & $\begin{array}{l}4935 \\
4548 \\
4161 \\
3797 \\
3464\end{array}$ & $\begin{array}{l}25.02299 \\
25.46709 \\
25.86936 \\
26.23741 \\
26.57688\end{array}$ & $\begin{array}{l}44410 \\
40227 \\
36805 \\
33947 \\
31519\end{array}$ & $\begin{array}{l}28.76899 \\
29.26244 \\
29.71019 \\
30.11986 \\
30.49729\end{array}$ & $\begin{array}{l}49345 \\
44775 \\
40967 \\
37743 \\
34984\end{array}$ \\
\hline $\begin{array}{l}650 \\
700 \\
750 \\
800 \\
850\end{array}$ & $\begin{array}{l}4.38526 \\
4.41051 \\
4.43214 \\
4.45091 \\
4.46739\end{array}$ & $\begin{array}{l}2525 \\
2163 \\
1877 \\
1648 \\
1462\end{array}$ & $\begin{array}{l}3.95505 \\
3.98671 \\
4.01570 \\
4.04233 \\
4.06686\end{array}$ & $\begin{array}{l}3166 \\
2899 \\
2663 \\
2453 \\
2266\end{array}$ & $\begin{array}{l}26.89207 \\
27.18635 \\
27.46240 \\
27.72243 \\
27.96824\end{array}$ & $\begin{array}{l}29428 \\
27605 \\
26003 \\
24581 \\
23311\end{array}$ & $\begin{array}{l}30.84713 \\
31.17306 \\
31.47811 \\
31.76476 \\
32.03510\end{array}$ & $\begin{array}{l}32593 \\
30505 \\
28665 \\
27034 \\
25578\end{array}$ \\
\hline $\begin{array}{l}900 \\
950 \\
1000 \\
1050\end{array}$ & $\begin{array}{l}4.48201 \\
4.49513 \\
4.50701 \\
4.51785 \\
4.52784\end{array}$ & $\begin{array}{r}1312 \\
1188 \\
1084 \\
999 \\
925\end{array}$ & $\begin{array}{l}4.08952 \\
4.11053 \\
4.13006 \\
4.14827 \\
4.16530\end{array}$ & $\begin{array}{l}2101 \\
1953 \\
1821 \\
1703 \\
1597\end{array}$ & $\begin{array}{l}28.20135 \\
28.42303 \\
28.63437 \\
28.83633 \\
29.02970\end{array}$ & $\begin{array}{l}22168 \\
21134 \\
20196 \\
19337 \\
18551\end{array}$ & $\begin{array}{l}32.29088 \\
32.53356 \\
32.76444 \\
32.98460 \\
33.19501\end{array}$ & $\begin{array}{l}24268 \\
23088 \\
22016 \\
21041 \\
20148\end{array}$ \\
\hline 75 & 4.53709 & 864 & 4.18127 & 1501 & 29.21521 & 17828 & 33.39649 & 19328 \\
\hline
\end{tabular}


Table 2.042. BaT (Cont.)

\begin{tabular}{|l|l|l|l|l|}
\hline$K$ & $\frac{C_{p}^{0}}{R}$ & $\frac{\left(H^{0}-E_{0}^{0}\right)}{R T}$ & $\frac{-\left(F^{0}-E_{0}^{0}\right)}{R T}$ & $\frac{S^{0}}{R}$ \\
\hline
\end{tabular}

\begin{tabular}{|c|c|c|c|c|c|c|c|c|}
\hline $\begin{array}{l}1200 \\
1300 \\
1400 \\
1500 \\
1600\end{array}$ & $\begin{array}{l}4.54573 \\
4.56151 \\
4.57571 \\
4.58872 \\
4.60082\end{array}$ & $\begin{array}{l}1578 \\
1420 \\
1301 \\
1210 \\
1136\end{array}$ & $\begin{array}{l}4.19628 \\
4.22378 \\
4.24842 \\
4.27067 \\
4.29093\end{array}$ & $\begin{array}{l}2750 \\
2464 \\
2225 \\
2026 \\
1857\end{array}$ & $\begin{array}{l}29.39349 \\
29.73047 \\
30.04441 \\
30.33829 \\
30.61457\end{array}$ & $\begin{array}{l}33698 \\
31394 \\
29388 \\
27628 \\
26070\end{array}$ & $\begin{array}{l}33.58977 \\
33.95426 \\
34.29283 \\
34.60897 \\
34.90551\end{array}$ & $\begin{array}{l}36449 \\
33857 \\
31614 \\
29654 \\
27927\end{array}$ \\
\hline $\begin{array}{l}1700 \\
1800 \\
1900 \\
2000 \\
2100\end{array}$ & $\begin{array}{l}4.61218 \\
4.62297 \\
4.63329 \\
4.64322 \\
4.65282\end{array}$ & $\begin{array}{r}1079 \\
1032 \\
993 \\
960 \\
934\end{array}$ & $\begin{array}{l}4.30950 \\
4.32662 \\
4.34249 \\
4.35728 \\
4.37112\end{array}$ & $\begin{array}{l}1712 \\
1587 \\
1479 \\
1384 \\
1302\end{array}$ & $\begin{array}{l}30.87527 \\
31.12209 \\
31.35645 \\
31.57957 \\
31.79250\end{array}$ & $\begin{array}{l}24682 \\
23436 \\
22312 \\
21293 \\
20365\end{array}$ & $\begin{array}{l}35.18478 \\
35.44871 \\
35.69894 \\
35.93685 \\
36.16363\end{array}$ & $\begin{array}{l}26393 \\
25023 \\
23791 \\
22678 \\
21666\end{array}$ \\
\hline $\begin{array}{l}2200 \\
2300 \\
2400 \\
2500 \\
2600\end{array}$ & $\begin{array}{l}4.66216 \\
4.67127 \\
4.68017 \\
4.68891 \\
4.69749\end{array}$ & $\begin{array}{l}911 \\
890 \\
874 \\
858 \\
845\end{array}$ & $\begin{array}{l}4.38414 \\
4.39643 \\
4.40807 \\
4.41913 \\
4.42967\end{array}$ & $\begin{array}{l}1229 \\
1164 \\
1106 \\
1054 \\
1007\end{array}$ & $\begin{array}{l}31.99615 \\
32.19130 \\
32.37866 \\
32.55883 \\
32.73236\end{array}$ & $\begin{array}{l}19515 \\
18736 \\
18017 \\
17353 \\
16737\end{array}$ & $\begin{array}{l}36.38029 \\
36.58774 \\
36.78673 \\
36.97797 \\
37.16204\end{array}$ & $\begin{array}{l}20745 \\
19899 \\
19124 \\
18407 \\
17744\end{array}$ \\
\hline $\begin{array}{l}2700 \\
2800 \\
2900 \\
3000\end{array}$ & $\begin{array}{l}4.70594 \\
4.71426 \\
4.72248 \\
4.73060\end{array}$ & $\begin{array}{r}832 \\
822 \\
812 \\
1598\end{array}$ & $\begin{array}{l}4.43974 \\
4.44940 \\
4.45867 \\
4.46760\end{array}$ & $\begin{array}{r}966 \\
927 \\
893 \\
1694\end{array}$ & $\begin{array}{l}32.89973 \\
33.06137 \\
33.21767 \\
33.36898\end{array}$ & $\begin{array}{l}16164 \\
15630 \\
15131 \\
28888\end{array}$ & $\begin{array}{l}37.33948 \\
37.51077 \\
37.67635 \\
37.83658\end{array}$ & $\begin{array}{l}17129 \\
16558 \\
16023 \\
30582\end{array}$ \\
\hline $\begin{array}{l}3200 \\
3400 \\
3600 \\
3800 \\
4000\end{array}$ & $\begin{array}{l}4.74658 \\
4.76226 \\
4.77767 \\
4.79285 \\
4.80781\end{array}$ & $\begin{array}{l}1568 \\
1541 \\
1518 \\
1496 \\
1478\end{array}$ & $\begin{array}{l}4.48454 \\
4.50042 \\
4.51539 \\
4.52960 \\
4.54314\end{array}$ & $\begin{array}{l}1588 \\
1497 \\
1421 \\
1354 \\
1295\end{array}$ & $\begin{array}{l}33.65786 \\
33.93021 \\
34.18788 \\
34.43239 \\
34.66508\end{array}$ & $\begin{array}{l}27235 \\
25767 \\
24451 \\
23269 \\
22198\end{array}$ & $\begin{array}{l}38.14240 \\
38.43063 \\
38.70328 \\
38.96200 \\
39.20822\end{array}$ & $\begin{array}{l}28823 \\
27265 \\
25872 \\
24622 \\
23493\end{array}$ \\
\hline $\begin{array}{l}4200 \\
4400 \\
4600 \\
4800 \\
5000\end{array}$ & $\begin{array}{l}4.82259 \\
4.83718 \\
4.85162 \\
4.86589 \\
4.88002\end{array}$ & $\begin{array}{l}1459 \\
1444 \\
1427 \\
1413\end{array}$ & $\begin{array}{l}4.55609 \\
4.56854 \\
4.58053 \\
4.59213 \\
4.60336\end{array}$ & $\begin{array}{l}1245 \\
1199 \\
1160 \\
1123\end{array}$ & $\begin{array}{l}34.88706 \\
35.09929 \\
35.30264 \\
35.49783 \\
35.68552\end{array}$ & $\begin{array}{l}21223 \\
20335 \\
19519 \\
18769\end{array}$ & $\begin{array}{l}39.44315 \\
39.66784 \\
39.88318 \\
40.08996 \\
40.28889\end{array}$ & $\begin{array}{l}22469 \\
21534 \\
20678 \\
19893\end{array}$ \\
\hline
\end{tabular}


Table 2.043. CuH

\begin{tabular}{|c|c|c|c|c|c|c|c|c|}
\hline${ }^{\circ} \mathrm{K}$ & $\frac{C_{P}^{0}}{R}$ & & $\frac{\left(H^{0}-E_{0}^{0}\right)}{R T}$ & & $\frac{-\left(F^{\circ}-E\right.}{R T}$ & & $\frac{S^{0}}{R}$ & \\
\hline $\begin{array}{l}50 \\
60 \\
70 \\
80 \\
90\end{array}$ & $\begin{array}{l}3.50094 \\
3.50139 \\
3.50171 \\
3.50197 \\
3.50222\end{array}$ & $\begin{array}{l}45 \\
32 \\
26 \\
25 \\
23\end{array}$ & $\begin{array}{l}3.42524 \\
3.43790 \\
3.44699 \\
3.45385 \\
3.45921\end{array}$ & $\begin{array}{r}1266 \\
909 \\
686 \\
536 \\
431\end{array}$ & $\begin{array}{l}13.93521 \\
14.56090 \\
15.09157 \\
15.55232 \\
15.95945\end{array}$ & $\begin{array}{l}62569 \\
53067 \\
46075 \\
40713 \\
36469\end{array}$ & $\begin{array}{l}17.36046 \\
17.99880 \\
18.53857 \\
19.00617 \\
19.41866\end{array}$ & $\begin{array}{l}63834 \\
53977 \\
46760 \\
41249 \\
36901\end{array}$ \\
\hline $\begin{array}{l}100 \\
110 \\
120 \\
130 \\
140\end{array}$ & $\begin{array}{l}3.50245 \\
3.50268 \\
3.50291 \\
3.50314 \\
3.50337\end{array}$ & $\begin{array}{l}23 \\
23 \\
23 \\
23 \\
23\end{array}$ & $\begin{array}{l}3.46352 \\
3.46707 \\
3.47005 \\
3.47259 \\
3.47478\end{array}$ & $\begin{array}{l}355 \\
298 \\
254 \\
219 \\
191\end{array}$ & $\begin{array}{l}16.32414 \\
16.65442 \\
16.95623 \\
17.23408 \\
17.49151\end{array}$ & $\begin{array}{l}33028 \\
30181 \\
27785 \\
25743 \\
23980\end{array}$ & $\begin{array}{l}19.78767 \\
20.12150 \\
20.42628 \\
20.70667 \\
20.96629\end{array}$ & $\begin{array}{l}33383 \\
30478 \\
28039 \\
25962 \\
24172\end{array}$ \\
\hline $\begin{array}{l}150 \\
160 \\
170 \\
180 \\
190\end{array}$ & $\begin{array}{l}3.50360 \\
3.50384 \\
3.50409 \\
3.50436 \\
3.50466\end{array}$ & $\begin{array}{l}24 \\
25 \\
27 \\
30 \\
36\end{array}$ & $\begin{array}{l}3.47669 \\
3.47838 \\
3.47988 \\
3.48124 \\
3.48246\end{array}$ & $\begin{array}{l}169 \\
150 \\
136 \\
122 \\
112\end{array}$ & $\begin{array}{l}17.73131 \\
17.95575 \\
18.16667 \\
18.36562 \\
18.55387\end{array}$ & $\begin{array}{l}22444 \\
21092 \\
19895 \\
18825 \\
17866\end{array}$ & $\begin{array}{l}21.20801 \\
21.43413 \\
21.64656 \\
21.84686 \\
22.03634\end{array}$ & $\begin{array}{l}22612 \\
21243 \\
20030 \\
18948 \\
17977\end{array}$ \\
\hline $\begin{array}{l}200 \\
210 \\
220 \\
230 \\
240\end{array}$ & $\begin{array}{l}3.50502 \\
3.50545 \\
3.50598 \\
3.50664 \\
3.50746\end{array}$ & $\begin{array}{r}43 \\
53 \\
66 \\
82 \\
102\end{array}$ & $\begin{array}{l}3.48358 \\
3.48461 \\
3.48557 \\
3.48647 \\
3.48733\end{array}$ & $\begin{array}{r}103 \\
96 \\
90 \\
86 \\
82\end{array}$ & $\begin{array}{l}18.73253 \\
18.90252 \\
19.06464 \\
19.21960 \\
19.36800\end{array}$ & $\begin{array}{l}16999 \\
16212 \\
15496 \\
14840 \\
14238\end{array}$ & $\begin{array}{l}22.21611 \\
22.38713 \\
22.55022 \\
22.70608 \\
22.85534\end{array}$ & $\begin{array}{l}17102 \\
16309 \\
15586 \\
14926 \\
14320\end{array}$ \\
\hline $\begin{array}{l}250 \\
260 \\
270 \\
280 \\
290\end{array}$ & $\begin{array}{l}3.50848 \\
3.50974 \\
3.51127 \\
3.51310 \\
3.51527\end{array}$ & $\begin{array}{l}126 \\
153 \\
183 \\
217 \\
253\end{array}$ & $\begin{array}{l}3.48815 \\
3.48896 \\
3.48975 \\
3.49055 \\
3.49137\end{array}$ & $\begin{array}{l}81 \\
79 \\
80 \\
82 \\
84\end{array}$ & $\begin{array}{l}19.51038 \\
19.64720 \\
19.77889 \\
19.90582 \\
20.02832\end{array}$ & $\begin{array}{l}13682 \\
13169 \\
12693 \\
12250 \\
11838\end{array}$ & $\begin{array}{l}22.99854 \\
23.13617 \\
23.26865 \\
23.39638 \\
23.51970\end{array}$ & $\begin{array}{l}13763 \\
13248 \\
12773 \\
12332 \\
11921\end{array}$ \\
\hline $\begin{array}{l}300 \\
310 \\
320 \\
330 \\
340\end{array}$ & $\begin{array}{l}3.51780 \\
3.52071 \\
3.52404 \\
3.52778 \\
3.53196\end{array}$ & $\begin{array}{l}291 \\
333 \\
374 \\
418 \\
461\end{array}$ & $\begin{array}{l}3.49221 \\
3.49308 \\
3.49399 \\
3.49496 \\
3.49598\end{array}$ & $\begin{array}{r}87 \\
91 \\
97 \\
102 \\
110\end{array}$ & $\begin{array}{l}20.14670 \\
20.26122 \\
20.37214 \\
20.47967 \\
20.58402\end{array}$ & $\begin{array}{l}11452 \\
11092 \\
10753 \\
10435 \\
10136\end{array}$ & $\begin{array}{l}23.63891 \\
23.75431 \\
23.86614 \\
23.97463 \\
24.08001\end{array}$ & $\begin{array}{l}11540 \\
11183 \\
10849 \\
10538 \\
10245\end{array}$ \\
\hline $\begin{array}{l}350 \\
360 \\
370 \\
380 \\
390\end{array}$ & $\begin{array}{l}3.53657 \\
3.54163 \\
3.54712 \\
3.55304 \\
3.55938\end{array}$ & $\begin{array}{l}506 \\
549 \\
592 \\
634 \\
675\end{array}$ & $\begin{array}{l}3.49708 \\
3.49824 \\
3.49949 \\
3.50082 \\
3.50224\end{array}$ & $\begin{array}{l}116 \\
125 \\
133 \\
142 \\
151\end{array}$ & $\begin{array}{l}20.68538 \\
20.78391 \\
20.87977 \\
20.97312 \\
21.06407\end{array}$ & $\begin{array}{l}9853 \\
9586 \\
9335 \\
9095 \\
8869\end{array}$ & $\begin{array}{l}24.18246 \\
24.28216 \\
24.37927 \\
24.47394 \\
24.56631\end{array}$ & $\begin{array}{l}9970 \\
9711 \\
9467 \\
9237 \\
9020\end{array}$ \\
\hline $\begin{array}{l}400 \\
450 \\
500 \\
550 \\
600\end{array}$ & $\begin{array}{l}3.56613 \\
3.60541 \\
3.65202 \\
3.70322 \\
3.75658\end{array}$ & $\begin{array}{l}3928 \\
4661 \\
5120 \\
5336 \\
5358\end{array}$ & $\begin{array}{l}3.50375 \\
3.51278 \\
3.52433 \\
3.53824 \\
3.55420\end{array}$ & $\begin{array}{r}903 \\
1155 \\
1391 \\
1596 \\
1763\end{array}$ & $\begin{array}{l}21.15276 \\
21.56594 \\
21.93662 \\
22.27316 \\
22.58170\end{array}$ & $\begin{array}{l}41318 \\
37068 \\
33654 \\
30854 \\
28518\end{array}$ & $\begin{array}{l}24.65651 \\
25.07873 \\
25.46096 \\
25.81141 \\
26.13591\end{array}$ & $\begin{array}{l}42222 \\
38223 \\
35045 \\
32450 \\
30280\end{array}$ \\
\hline $\begin{array}{l}650 \\
700 \\
750 \\
800 \\
850\end{array}$ & $\begin{array}{l}3.81016 \\
3.86260 \\
3.91300 \\
3.96082 \\
4.00579\end{array}$ & $\begin{array}{l}5244 \\
5040 \\
4782 \\
4497 \\
4204\end{array}$ & $\begin{array}{l}3.57183 \\
3.59074 \\
3.61056 \\
3.63097 \\
3.65171\end{array}$ & $\begin{array}{l}1891 \\
1982 \\
2041 \\
2074 \\
2085\end{array}$ & $\begin{array}{l}22.86688 \\
23.13226 \\
23.38067 \\
23.61434 \\
23.83509\end{array}$ & $\begin{array}{l}26538 \\
24841 \\
23367 \\
22075 \\
20931\end{array}$ & $\begin{array}{l}26.43871 \\
26.72301 \\
26.99123 \\
27.24531 \\
27.48680\end{array}$ & $\begin{array}{l}28430 \\
26822 \\
25408 \\
24149 \\
23017\end{array}$ \\
\hline $\begin{array}{r}900 \\
950 \\
1000 \\
1050 \\
1100\end{array}$ & $\begin{array}{l}4.04783 \\
4.08698 \\
4.12334 \\
4.15708 \\
4.18837\end{array}$ & $\begin{array}{l}3915 \\
3636 \\
3374 \\
3129 \\
2904\end{array}$ & $\begin{array}{l}3.67256 \\
3.69336 \\
3.71396 \\
3.73426 \\
3.75420\end{array}$ & $\begin{array}{l}2080 \\
2060 \\
2030 \\
1994 \\
1952\end{array}$ & $\begin{array}{l}24.04440 \\
24.24353 \\
24.43350 \\
24.61519 \\
24.78937\end{array}$ & $\begin{array}{l}19913 \\
18997 \\
18169 \\
17418 \\
16732\end{array}$ & $\begin{array}{l}27.71697 \\
27.93689 \\
28.14746 \\
28.34946 \\
28.54358\end{array}$ & $\begin{array}{l}21992 \\
21057 \\
20200 \\
19412 \\
18683\end{array}$ \\
\hline 1150 & 4.21741 & 2697 & 3.77372 & 1906 & 24.95669 & 16101 & 28.73041 & 18007 \\
\hline
\end{tabular}


Table 2.043. CuH (Cont.)

\begin{tabular}{|c|c|c|c|c|c|c|c|c|}
\hline${ }^{\circ} \mathrm{K}$ & $\frac{C_{p}^{0}}{R}$ & & $\frac{\left(H^{0}-E_{0}^{0}\right)}{R T}$ & & $\frac{-\left(F^{\circ}-E\right.}{R T}$ & & $\frac{S^{\circ}}{R}$ & \\
\hline $\begin{array}{l}1200 \\
1300 \\
1400 \\
1500 \\
1600\end{array}$ & $\begin{array}{l}4.24438 \\
4.29278 \\
4.33488 \\
4.37177 \\
4.40434\end{array}$ & $\begin{array}{l}4840 \\
4210 \\
3689 \\
3257 \\
2901\end{array}$ & $\begin{array}{l}3.79278 \\
3.82942 \\
3.86405 \\
3.89670 \\
3.92743\end{array}$ & $\begin{array}{l}3664 \\
3463 \\
3265 \\
3073 \\
2892\end{array}$ & $\begin{array}{l}25.11770 \\
25.42274 \\
25.70781 \\
25.97553 \\
26.22801\end{array}$ & $\begin{array}{l}30504 \\
28507 \\
26772 \\
25248 \\
23898\end{array}$ & $\begin{array}{l}28.91048 \\
29.25217 \\
29.57187 \\
29.87224 \\
30.15544\end{array}$ & $\begin{array}{l}34169 \\
31970 \\
30037 \\
28320 \\
26790\end{array}$ \\
\hline $\begin{array}{l}1700 \\
1800 \\
1900 \\
2000 \\
2100\end{array}$ & $\begin{array}{l}4.43335 \\
4.45937 \\
4.48291 \\
4.50436 \\
4.52403\end{array}$ & $\begin{array}{l}2602 \\
2354 \\
2145 \\
1967 \\
1818\end{array}$ & $\begin{array}{l}3.95635 \\
3.98359 \\
4.00926 \\
4.03348 \\
4.05638\end{array}$ & $\begin{array}{l}2724 \\
2567 \\
2422 \\
2290 \\
2168\end{array}$ & $\begin{array}{l}26.46699 \\
26.69390 \\
26.90998 \\
27.11625 \\
27.31360\end{array}$ & $\begin{array}{l}22691 \\
21608 \\
20627 \\
19735 \\
18921\end{array}$ & $\begin{array}{l}30.42334 \\
30.67750 \\
30.91924 \\
31.14974 \\
31.36999\end{array}$ & $\begin{array}{l}25416 \\
24174 \\
23050 \\
22025 \\
21088\end{array}$ \\
\hline $\begin{array}{l}2200 \\
2300 \\
2400 \\
2500 \\
2600\end{array}$ & $\begin{array}{l}4.54221 \\
4.55909 \\
4.57487 \\
4.58970 \\
4.60370\end{array}$ & $\begin{array}{l}1688 \\
1578 \\
1483 \\
1400 \\
1328\end{array}$ & $\begin{array}{l}4.07806 \\
4.09861 \\
4.11813 \\
4.13670 \\
4.15439\end{array}$ & $\begin{array}{l}2055 \\
1952 \\
1857 \\
1769 \\
1689\end{array}$ & $\begin{array}{l}27.50281 \\
27.68455 \\
27.85940 \\
28.02789 \\
28.19048\end{array}$ & $\begin{array}{l}18174 \\
17485 \\
16849 \\
16259 \\
15711\end{array}$ & $\begin{array}{l}31.58087 \\
31.78316 \\
31.97753 \\
32.16459 \\
32.34488\end{array}$ & $\begin{array}{l}20229 \\
19437 \\
18706 \\
18029 \\
17399\end{array}$ \\
\hline $\begin{array}{l}2700 \\
2800 \\
2900 \\
3000\end{array}$ & $\begin{array}{l}4.61698 \\
4.62962 \\
4.64170 \\
4.65329\end{array}$ & $\begin{array}{l}1264 \\
1208 \\
1159 \\
2190\end{array}$ & $\begin{array}{l}4.17128 \\
4.18743 \\
4.20288 \\
4.21\end{array}$ & $\begin{array}{l}1615 \\
1545 \\
1483 \\
2791\end{array}$ & $\begin{array}{l}28.34759 \\
28.49958 \\
28.64679 \\
28.78953\end{array}$ & $\begin{array}{l}15199 \\
14721 \\
14274 \\
27311\end{array}$ & $\begin{array}{l}32.51887 \\
32.68701 \\
32.84968 \\
33.00724\end{array}$ & $\begin{array}{l}16814 \\
16267 \\
15756 \\
30103\end{array}$ \\
\hline $\begin{array}{l}3200 \\
3400 \\
3600 \\
3800 \\
4000\end{array}$ & $\begin{array}{l}4.67519 \\
4.69569 \\
4.71505 \\
4.73346 \\
4.75110\end{array}$ & $\begin{array}{l}2050 \\
1936 \\
1841 \\
1764 \\
1697\end{array}$ & $\begin{array}{l}4.2456 c \\
4.27150 \\
4.29561 \\
4.31817 \\
4.33938\end{array}$ & $\begin{array}{l}2588 \\
2411 \\
2256 \\
2121 \\
2001\end{array}$ & $\begin{array}{l}29.06264 \\
29.32081 \\
29.56566 \\
29.79852 \\
30.02056\end{array}$ & $\begin{array}{l}25817 \\
24485 \\
23286 \\
22204 \\
21221\end{array}$ & $\begin{array}{l}33.30827 \\
33.59232 \\
33.86127 \\
34.11670 \\
34.35994\end{array}$ & $\begin{array}{l}28405 \\
26895 \\
25543 \\
24324 \\
23222\end{array}$ \\
\hline $\begin{array}{l}4200 \\
4400 \\
4600 \\
4800 \\
5000\end{array}$ & $\begin{array}{l}4.76807 \\
4.78449 \\
4.80041 \\
4.81591 \\
4.83104\end{array}$ & $\begin{array}{l}1642 \\
1592 \\
1550 \\
1513\end{array}$ & $\begin{array}{l}4.35939 \\
4.37834 \\
4.39635 \\
4.41351 \\
4.42991\end{array}$ & $\begin{array}{l}1895 \\
1801 \\
1- \\
i\end{array}$ & $\begin{array}{l}30.23277 \\
30.43601 \\
30.63103 \\
30.81851 \\
30.99901\end{array}$ & $\begin{array}{l}20324 \\
19502 \\
18748 \\
18050\end{array}$ & $\begin{array}{l}34.59216 \\
34.81436 \\
35.02739 \\
35.23202 \\
35.42892\end{array}$ & $\begin{array}{l}22220 \\
21303 \\
20463 \\
19690\end{array}$ \\
\hline
\end{tabular}


Table 2.044. CuD

\begin{tabular}{|c|c|c|c|c|c|c|c|c|}
\hline${ }^{\circ} \mathrm{K}$ & $\frac{C_{p}^{0}}{R}$ & & $\frac{\left(H^{0}-E_{0}^{0}\right)}{R T}$ & & $\frac{-\left(F^{\circ}-E\right.}{R T}$ & & $\frac{S^{0}}{R}$ & \\
\hline $\begin{array}{l}50 \\
60 \\
70 \\
80 \\
90\end{array}$ & $\begin{array}{l}3.50149 \\
3.50163 \\
3.50180 \\
3.50200 \\
3.50221\end{array}$ & $\begin{array}{l}14 \\
17 \\
20 \\
21 \\
22\end{array}$ & $\begin{array}{l}3.46200 \\
3.46859 \\
3.47332 \\
3.47690 \\
3.47970\end{array}$ & $\begin{array}{l}659 \\
473 \\
358 \\
280 \\
226\end{array}$ & $\begin{array}{l}14.59299 \\
15.22481 \\
15.75987 \\
16.22391 \\
16.63360\end{array}$ & $\begin{array}{l}63182 \\
53506 \\
46404 \\
40969 \\
36675\end{array}$ & $\begin{array}{l}18.05500 \\
18.69341 \\
19.23320 \\
19.70081 \\
20.11330\end{array}$ & $\begin{array}{l}63841 \\
53979 \\
46761 \\
41249 \\
36901\end{array}$ \\
\hline $\begin{array}{l}100 \\
110 \\
120 \\
130 \\
140\end{array}$ & $\begin{array}{l}3.50243 \\
3.50266 \\
3.50290 \\
3.50318 \\
3.50352\end{array}$ & $\begin{array}{l}23 \\
24 \\
28 \\
34 \\
46\end{array}$ & $\begin{array}{l}3.48196 \\
3.48383 \\
3.48541 \\
3.48676 \\
3.48795\end{array}$ & $\begin{array}{l}187 \\
158 \\
135 \\
119 \\
105\end{array}$ & $\begin{array}{l}17.00035 \\
17.33230 \\
17.63551 \\
17.91454 \\
18.17298\end{array}$ & $\begin{array}{l}33195 \\
30321 \\
27903 \\
25844 \\
24068\end{array}$ & $\begin{array}{l}20.48231 \\
20.81614 \\
21.12092 \\
21.40131 \\
21.66094\end{array}$ & $\begin{array}{l}33383 \\
30478 \\
28039 \\
25963 \\
24173\end{array}$ \\
\hline $\begin{array}{l}150 \\
160 \\
170 \\
180 \\
190\end{array}$ & $\begin{array}{l}3.50398 \\
3.50462 \\
3.50552 \\
3.50677 \\
3.50846\end{array}$ & $\begin{array}{r}64 \\
90 \\
125 \\
169 \\
224\end{array}$ & $\begin{array}{l}3.48900 \\
3.48996 \\
3.49084 \\
3.49169 \\
3.49253\end{array}$ & $\begin{array}{l}96 \\
88 \\
85 \\
84 \\
85\end{array}$ & $\begin{array}{l}18.41366 \\
18.63887 \\
18.85047 \\
19.05003 \\
19.23884\end{array}$ & $\begin{array}{l}22521 \\
21160 \\
19956 \\
18881 \\
17916\end{array}$ & $\begin{array}{l}21.90267 \\
22.12883 \\
22.34132 \\
22.54173 \\
22.73137\end{array}$ & $\begin{array}{l}22616 \\
21249 \\
20041 \\
18964 \\
18002\end{array}$ \\
\hline $\begin{array}{l}200 \\
210 \\
220 \\
230 \\
240\end{array}$ & $\begin{array}{l}3.51070 \\
3.51356 \\
3.51711 \\
3.52142 \\
3.52652\end{array}$ & $\begin{array}{l}286 \\
355 \\
431 \\
510 \\
594\end{array}$ & $\begin{array}{l}3.49338 \\
3.49427 \\
3.49522 \\
3.49627 \\
3.49742\end{array}$ & $\begin{array}{r}89 \\
95 \\
105 \\
115 \\
128\end{array}$ & $\begin{array}{l}19.41800 \\
19.58847 \\
19.75104 \\
19.90644 \\
20.05526\end{array}$ & $\begin{array}{l}17047 \\
16257 \\
15540 \\
14882 \\
14280\end{array}$ & $\begin{array}{l}22.91139 \\
23.08274 \\
23.24627 \\
23.40271 \\
23.55268\end{array}$ & $\begin{array}{l}17135 \\
16353 \\
15644 \\
14997 \\
14408\end{array}$ \\
\hline $\begin{array}{l}250 \\
260 \\
270 \\
280 \\
290\end{array}$ & $\begin{array}{l}3.53246 \\
3.53923 \\
3.54683 \\
3.55524 \\
3.56443\end{array}$ & $\begin{array}{l}677 \\
760 \\
841 \\
919 \\
993\end{array}$ & $\begin{array}{l}3.49870 \\
3.50012 \\
3.50171 \\
3.50347 \\
3.50541\end{array}$ & $\begin{array}{l}142 \\
159 \\
176 \\
194 \\
213\end{array}$ & $\begin{array}{l}20.19806 \\
20.33531 \\
20.46743 \\
20.59481 \\
20.71779\end{array}$ & $\begin{array}{l}13725 \\
13212 \\
12738 \\
12298 \\
11887\end{array}$ & $\begin{array}{l}23.69676 \\
23.83543 \\
23.96915 \\
24.09828 \\
24.22320\end{array}$ & $\begin{array}{l}13867 \\
13372 \\
12913 \\
12492 \\
12101\end{array}$ \\
\hline $\begin{array}{l}300 \\
310 \\
320 \\
330 \\
340\end{array}$ & $\begin{array}{l}3.57436 \\
3.58498 \\
3.59625 \\
3.60810 \\
3.62047\end{array}$ & $\begin{array}{l}1062 \\
1127 \\
1185 \\
1237 \\
1284\end{array}$ & $\begin{array}{l}3.50754 \\
3.50987 \\
3.51239 \\
3.51511 \\
3.51802\end{array}$ & $\begin{array}{l}233 \\
252 \\
272 \\
291 \\
311\end{array}$ & $\begin{array}{l}20.83666 \\
20.95171 \\
21.06318 \\
21.17131 \\
21.27628\end{array}$ & $\begin{array}{l}11505 \\
11147 \\
10813 \\
10497 \\
10203\end{array}$ & $\begin{array}{l}24.34421 \\
24.46158 \\
24.57558 \\
24.68642 \\
24.79431\end{array}$ & $\begin{array}{l}11737 \\
11400 \\
11084 \\
10789 \\
10514\end{array}$ \\
\hline $\begin{array}{l}350 \\
360 \\
370 \\
380 \\
390\end{array}$ & $\begin{array}{l}3.63331 \\
3.64656 \\
3.66015 \\
3.67404 \\
3.68816\end{array}$ & $\begin{array}{l}1325 \\
1359 \\
1389 \\
1412 \\
1431\end{array}$ & $\begin{array}{l}3.52113 \\
3.52443 \\
3.52792 \\
3.53158 \\
3.53541\end{array}$ & $\begin{array}{l}330 \\
349 \\
366 \\
383 \\
400\end{array}$ & $\begin{array}{l}21.37831 \\
21.47755 \\
21.57416 \\
21.66829 \\
21.76008\end{array}$ & $\begin{array}{l}9924 \\
9661 \\
9413 \\
9179 \\
8956\end{array}$ & $\begin{array}{l}24.89945 \\
25.00198 \\
25.10208 \\
25.19987 \\
25.29549\end{array}$ & $\begin{array}{r}10253 \\
10010 \\
9779 \\
9562 \\
9356\end{array}$ \\
\hline $\begin{array}{l}400 \\
450 \\
500 \\
550 \\
600\end{array}$ & $\begin{array}{l}3.70247 \\
3.77531 \\
3.84716 \\
3.91522 \\
3.97813\end{array}$ & $\begin{array}{l}7284 \\
7185 \\
6906 \\
6291 \\
5728\end{array}$ & $\begin{array}{l}3.53941 \\
3.56156 \\
3.58655 \\
3.61337 \\
3.64119\end{array}$ & $\begin{array}{l}2215 \\
2499 \\
2682 \\
2782 \\
2816\end{array}$ & $\begin{array}{l}21.84964 \\
22.26776 \\
22.64429 \\
22.98737 \\
23.30296\end{array}$ & $\begin{array}{l}41812 \\
37653 \\
34308 \\
31559 \\
29256\end{array}$ & $\begin{array}{l}25.38905 \\
25.82933 \\
26.23084 \\
26.60075 \\
26.94415\end{array}$ & $\begin{array}{l}44028 \\
40151 \\
36991 \\
34340 \\
32072\end{array}$ \\
\hline $\begin{array}{l}650 \\
700 \\
750 \\
800 \\
850\end{array}$ & $\begin{array}{l}4.03541 \\
4.08712 \\
4.13360 \\
4.17531 \\
4.21275\end{array}$ & $\begin{array}{l}5171 \\
4648 \\
4171 \\
3744 \\
3366\end{array}$ & $\begin{array}{l}3.66935 \\
3.69737 \\
3.72493 \\
3.75180 \\
3.77783\end{array}$ & $\begin{array}{l}2802 \\
2756 \\
2687 \\
2603 \\
2512\end{array}$ & $\begin{array}{l}23.59552 \\
23.86848 \\
24.12452 \\
24.36578 \\
24.59402\end{array}$ & $\begin{array}{l}27296 \\
25604 \\
24126 \\
22824 \\
21665\end{array}$ & $\begin{array}{l}27.26487 \\
27.56586 \\
27.84945 \\
28.11759 \\
28.37186\end{array}$ & $\begin{array}{l}30099 \\
28359 \\
26814 \\
25427 \\
24176\end{array}$ \\
\hline $\begin{array}{r}900 \\
950 \\
1000 \\
1050 \\
1100\end{array}$ & $\begin{array}{l}4.24641 \\
4.27676 \\
4.30419 \\
4.32908 \\
4.35174\end{array}$ & $\begin{array}{l}3035 \\
2743 \\
2489 \\
2266 \\
2071\end{array}$ & $\begin{array}{l}3.80295 \\
3.82710 \\
3.85028 \\
3.87250 \\
3.89377\end{array}$ & $\begin{array}{l}2415 \\
2318 \\
2222 \\
2127 \\
2037\end{array}$ & $\begin{array}{l}24.81067 \\
25.01693 \\
25.21383 \\
25.40223 \\
25.58287\end{array}$ & $\begin{array}{l}20626 \\
19690 \\
18840 \\
18064 \\
17354\end{array}$ & $\begin{array}{l}28.61362 \\
28.84404 \\
29.06412 \\
29.27473 \\
29.47665\end{array}$ & $\begin{array}{l}23042 \\
22008 \\
21061 \\
20192 \\
19391\end{array}$ \\
\hline 1150 & 4.37245 & 1900 & 3.91414 & 1950 & 25.75641 & 16700 & 29.67056 & 18650 \\
\hline
\end{tabular}


Table 2.044. CuD (Cont.)

\begin{tabular}{|c|c|c|c|c|c|c|c|c|}
\hline${ }^{\circ} K$ & $\frac{C_{p}^{0}}{R}$ & & $\frac{\left(H^{0}-E_{0}^{0}\right)}{R T}$ & & $\frac{-\left(F^{0}-\right.}{R T}$ & & $\frac{S^{\circ}}{R}$ & \\
\hline $\begin{array}{l}1200 \\
1300 \\
1400 \\
1500 \\
1600\end{array}$ & $\begin{array}{l}4.39145 \\
4.42510 \\
4.45405 \\
4.47930 \\
4.50162\end{array}$ & $\begin{array}{l}3365 \\
2895 \\
2525 \\
2232 \\
1997\end{array}$ & $\begin{array}{l}3.93364 \\
3.97018 \\
4.00374 \\
4.03462 \\
4.06312\end{array}$ & $\begin{array}{l}3654 \\
3356 \\
3088 \\
2850 \\
2639\end{array}$ & $\begin{array}{l}25.92341 \\
26.23973 \\
26.53520 \\
26.81250 \\
27.07381\end{array}$ & $\begin{array}{l}31632 \\
29547 \\
27730 \\
26131 \\
24713\end{array}$ & $\begin{array}{l}29.85706 \\
30.20992 \\
30.53894 \\
30.84712 \\
31.13694\end{array}$ & $\begin{array}{l}35286 \\
32902 \\
30818 \\
28982 \\
27352\end{array}$ \\
\hline $\begin{array}{l}1700 \\
1800 \\
1900 \\
2000 \\
2100\end{array}$ & $\begin{array}{l}4.52159 \\
4.53964 \\
4.55614 \\
4.57135 \\
4.58549\end{array}$ & $\begin{array}{l}1805 \\
1650 \\
1521 \\
1414 \\
1323\end{array}$ & $\begin{array}{l}4.08951 \\
4.11403 \\
4.13687 \\
4.15822 \\
4.17823\end{array}$ & $\begin{array}{l}2452 \\
2284 \\
2135 \\
2001 \\
1882\end{array}$ & $\begin{array}{l}27.32094 \\
27.55539 \\
27.77844 \\
27.99119 \\
28.19456\end{array}$ & $\begin{array}{l}23445 \\
22305 \\
21275 \\
20337 \\
19481\end{array}$ & $\begin{array}{l}31.41046 \\
31.66942 \\
31.91532 \\
32.14941 \\
32.37279\end{array}$ & $\begin{array}{l}25896 \\
24590 \\
23409 \\
22338 \\
21363\end{array}$ \\
\hline $\begin{array}{l}2200 \\
2300 \\
2400 \\
2500 \\
2600\end{array}$ & $\begin{array}{l}4.59872 \\
4.61120 \\
4.62302 \\
4.63429 \\
4.64508\end{array}$ & $\begin{array}{l}1248 \\
1182 \\
1127 \\
1079 \\
1037\end{array}$ & $\begin{array}{l}4.19705 \\
4.21478 \\
4.23155 \\
4.24744 \\
4.26252\end{array}$ & $\begin{array}{l}1773 \\
1677 \\
1589 \\
1508 \\
1437\end{array}$ & $\begin{array}{l}28.38937 \\
28.57633 \\
28.75606 \\
28.92913 \\
29.09601\end{array}$ & $\begin{array}{l}18696 \\
17973 \\
17307 \\
16688 \\
16114\end{array}$ & $\begin{array}{l}32.58642 \\
32.79112 \\
32.98762 \\
33.17657 \\
33.35854\end{array}$ & $\begin{array}{l}20470 \\
19650 \\
18895 \\
18197 \\
17551\end{array}$ \\
\hline $\begin{array}{l}2700 \\
2800 \\
2900 \\
3000\end{array}$ & $\begin{array}{l}4.65545 \\
4.66545 \\
4.67513 \\
4.68452\end{array}$ & $\begin{array}{r}1000 \\
968 \\
939 \\
1806\end{array}$ & $\begin{array}{l}4.27689 \\
4.29059 \\
4.30368 \\
4.31622\end{array}$ & $\begin{array}{l}1370 \\
1309 \\
1254 \\
2359\end{array}$ & $\begin{array}{l}29.25715 \\
29.41294 \\
29.56374 \\
29.70985\end{array}$ & $\begin{array}{l}15579 \\
15080 \\
14611 \\
27933\end{array}$ & $\begin{array}{l}33.53405 \\
33.70353 \\
33.86742 \\
34.02607\end{array}$ & $\begin{array}{l}16948 \\
16389 \\
15865 \\
30292\end{array}$ \\
\hline $\begin{array}{l}3200 \\
3400 \\
3600 \\
3800 \\
4000\end{array}$ & $\begin{array}{l}4.70258 \\
4.71983 \\
4.73641 \\
4.75245 \\
4.76801\end{array}$ & $\begin{array}{l}1725 \\
1658 \\
1604 \\
1556 \\
1518\end{array}$ & $\begin{array}{l}4.33981 \\
4.36166 \\
4.38202 \\
4.40110 \\
4.41905\end{array}$ & $\begin{array}{l}2185 \\
2036 \\
1908 \\
1795 \\
1698\end{array}$ & $\begin{array}{l}29.98918 \\
30.25294 \\
30.50283 \\
30.74027 \\
30.96648\end{array}$ & $\begin{array}{l}26376 \\
24989 \\
23744 \\
22621 \\
21602\end{array}$ & $\begin{array}{l}34.32899 \\
34.61460 \\
34.88485 \\
35.14137 \\
35.38554\end{array}$ & $\begin{array}{l}28561 \\
27025 \\
25652 \\
24417 \\
23300\end{array}$ \\
\hline $\begin{array}{l}4200 \\
4400 \\
4600 \\
4800 \\
5000\end{array}$ & $\begin{array}{l}4.78319 \\
4.79801 \\
4.81254 \\
4.82679 \\
4.84081\end{array}$ & $\begin{array}{l}1482 \\
1453 \\
1425 \\
1402\end{array}$ & $\begin{array}{l}4.43603 \\
4.45215 \\
4.46751 \\
4.48218 \\
4.49625\end{array}$ & $\begin{array}{l}1612 \\
1536 \\
1467 \\
1407\end{array}$ & $\begin{array}{l}31.18250 \\
31.38924 \\
31.58749 \\
31.77793 \\
31.96119\end{array}$ & $\begin{array}{l}20674 \\
19825 \\
19044 \\
18326\end{array}$ & $\begin{array}{l}35.61854 \\
35.84139 \\
36.05500 \\
36.26012 \\
36.45744\end{array}$ & $\begin{array}{l}22285 \\
21361 \\
20512 \\
19732\end{array}$ \\
\hline
\end{tabular}


Table 2.045. CuT

\begin{tabular}{|c|c|c|c|c|c|c|c|c|}
\hline${ }^{\circ} \mathrm{K}$ & $\frac{C_{p}^{0}}{R}$ & & $\frac{\left(H^{0}-E_{0}^{0}\right)}{R T}$ & & $\frac{-\left(F^{0}-E\right.}{R T}$ & & $\frac{S^{0}}{R}$ & \\
\hline $\begin{array}{l}50 \\
60 \\
70 \\
80 \\
90\end{array}$ & $\begin{array}{l}3.50132 \\
3.50151 \\
3.50172 \\
3.50193 \\
3.50216\end{array}$ & $\begin{array}{l}19 \\
21 \\
21 \\
23 \\
25\end{array}$ & $\begin{array}{l}3.47446 \\
3.47895 \\
3.48219 \\
3.48464 \\
3.48658\end{array}$ & $\begin{array}{l}449 \\
324 \\
245 \\
194 \\
157\end{array}$ & $\begin{array}{l}14.99082 \\
15.62471 \\
16.16125 \\
16.62640 \\
17.03695\end{array}$ & $\begin{array}{l}63389 \\
53554 \\
46515 \\
41055 \\
36743\end{array}$ & $\begin{array}{l}18.46528 \\
19.10367 \\
19.64344 \\
20.11105 \\
20.52353\end{array}$ & $\begin{array}{l}63839 \\
53977 \\
46761 \\
41248 \\
36900\end{array}$ \\
\hline $\begin{array}{l}100 \\
110 \\
120 \\
130 \\
140\end{array}$ & $\begin{array}{l}3.50241 \\
3.50272 \\
3.50313 \\
3.50376 \\
3.50473\end{array}$ & $\begin{array}{r}31 \\
41 \\
63 \\
97 \\
148\end{array}$ & $\begin{array}{l}3.48815 \\
3.48946 \\
3.49058 \\
3.49157 \\
3.49247\end{array}$ & $\begin{array}{r}131 \\
112 \\
99 \\
90 \\
86\end{array}$ & $\begin{array}{l}17.40438 \\
17.73690 \\
18.04057 \\
18.32001 \\
18.57879\end{array}$ & $\begin{array}{l}33252 \\
30367 \\
27944 \\
25878 \\
24099\end{array}$ & $\begin{array}{l}20.89253 \\
21.22636 \\
21.53115 \\
21.81158 \\
22.07127\end{array}$ & $\begin{array}{l}33383 \\
30479 \\
28043 \\
25969 \\
24185\end{array}$ \\
\hline $\begin{array}{l}150 \\
160 \\
170 \\
180 \\
190\end{array}$ & $\begin{array}{l}3.50621 \\
3.50834 \\
3.51130 \\
3.51523 \\
3.52024\end{array}$ & $\begin{array}{l}213 \\
296 \\
393 \\
501 \\
616\end{array}$ & $\begin{array}{l}3.49333 \\
3.49420 \\
3.49512 \\
3.49612 \\
3.49725\end{array}$ & $\begin{array}{r}87 \\
92 \\
100 \\
113 \\
130\end{array}$ & $\begin{array}{l}18.81978 \\
19.04526 \\
19.25712 \\
19.45693 \\
19.64598\end{array}$ & $\begin{array}{l}22548 \\
21186 \\
19981 \\
18905 \\
17942\end{array}$ & $\begin{array}{l}22.31312 \\
22.53947 \\
22.75224 \\
22.95305 \\
23.14324\end{array}$ & $\begin{array}{l}22635 \\
21277 \\
20081 \\
19019 \\
18072\end{array}$ \\
\hline $\begin{array}{l}200 \\
210 \\
220 \\
230 \\
240\end{array}$ & $\begin{array}{l}3.52640 \\
3.53377 \\
3.54236 \\
3.55213 \\
3.56305\end{array}$ & $\begin{array}{r}737 \\
859 \\
977 \\
1092 \\
1200\end{array}$ & $\begin{array}{l}3.49855 \\
3.50005 \\
3.50177 \\
3.50374 \\
3.50598\end{array}$ & $\begin{array}{l}150 \\
172 \\
197 \\
224 \\
252\end{array}$ & $\begin{array}{l}19.82540 \\
19.99613 \\
20.15899 \\
20.31469 \\
20.46386\end{array}$ & $\begin{array}{l}17073 \\
16286 \\
15570 \\
14917 \\
14317\end{array}$ & $\begin{array}{l}23.32396 \\
23.49618 \\
23.66077 \\
23.81844 \\
23.96985\end{array}$ & $\begin{array}{l}17222 \\
16459 \\
15767 \\
15141 \\
14569\end{array}$ \\
\hline $\begin{array}{l}250 \\
260 \\
270 \\
280 \\
290\end{array}$ & $\begin{array}{l}3.57505 \\
3.58803 \\
3.60191 \\
3.61659 \\
3.63195\end{array}$ & $\begin{array}{l}1298 \\
1388 \\
1468 \\
1536 \\
1595\end{array}$ & $\begin{array}{l}3.50850 \\
3.51131 \\
3.51441 \\
3.51779 \\
3.52146\end{array}$ & $\begin{array}{l}281 \\
310 \\
338 \\
367 \\
395\end{array}$ & $\begin{array}{l}20.60703 \\
20.74469 \\
20.87727 \\
21.00514 \\
21.12864\end{array}$ & $\begin{array}{l}13766 \\
13258 \\
12787 \\
12350 \\
11945\end{array}$ & $\begin{array}{l}24.11554 \\
24.25600 \\
24.39168 \\
24.52293 \\
24.65011\end{array}$ & $\begin{array}{l}14046 \\
13568 \\
13125 \\
12718 \\
12340\end{array}$ \\
\hline $\begin{array}{l}300 \\
310 \\
320 \\
330 \\
340\end{array}$ & $\begin{array}{l}3.64790 \\
3.66433 \\
3.68115 \\
3.69826 \\
3.71557\end{array}$ & $\begin{array}{l}1643 \\
1682 \\
1711 \\
1731 \\
1745\end{array}$ & $\begin{array}{l}3.52541 \\
3.52962 \\
3.53409 \\
3.53881 \\
3.54375\end{array}$ & $\begin{array}{l}421 \\
447 \\
472 \\
494 \\
516\end{array}$ & $\begin{array}{l}21.24809 \\
21.36376 \\
21.47589 \\
21.58471 \\
21.69043\end{array}$ & $\begin{array}{l}11567 \\
11213 \\
10882 \\
10572 \\
10280\end{array}$ & $\begin{array}{l}24.77351 \\
24.89339 \\
25.00999 \\
25.12352 \\
25.23419\end{array}$ & $\begin{array}{l}11988 \\
11660 \\
11353 \\
11067 \\
10795\end{array}$ \\
\hline $\begin{array}{l}350 \\
360 \\
370 \\
380 \\
390\end{array}$ & $\begin{array}{l}3.73302 \\
3.75054 \\
3.76805 \\
3.78550 \\
3.80284\end{array}$ & $\begin{array}{l}1752 \\
1751 \\
1745 \\
1734 \\
1720\end{array}$ & $\begin{array}{l}3.54891 \\
3.55427 \\
3.55981 \\
3.56552 \\
3.57138\end{array}$ & $\begin{array}{l}536 \\
554 \\
571 \\
586 \\
600\end{array}$ & $\begin{array}{l}21.79323 \\
21.89328 \\
21.99074 \\
22.08575 \\
22.17844\end{array}$ & $\begin{array}{r}10005 \\
9746 \\
9501 \\
9269 \\
9049\end{array}$ & $\begin{array}{l}25.34214 \\
25.44755 \\
25.55055 \\
25.65127 \\
25.74982\end{array}$ & $\begin{array}{r}10541 \\
10300 \\
10072 \\
9855 \\
9650\end{array}$ \\
\hline $\begin{array}{l}400 \\
450 \\
500 \\
550 \\
600\end{array}$ & $\begin{array}{l}3.82004 \\
3.90263 \\
3.97785 \\
4.04483 \\
4.10379\end{array}$ & $\begin{array}{l}8259 \\
7522 \\
6698 \\
5896 \\
5164\end{array}$ & $\begin{array}{l}3.57738 \\
3.60899 \\
3.64219 \\
3.67581 \\
3.70907\end{array}$ & $\begin{array}{l}3161 \\
3320 \\
3362 \\
3326 \\
3239\end{array}$ & $\begin{array}{l}22.26893 \\
22.69209 \\
23.07405 \\
23.42276 \\
23.74403\end{array}$ & $\begin{array}{l}42316 \\
38196 \\
34871 \\
32127 \\
29817\end{array}$ & $\begin{array}{l}25.84632 \\
26.30109 \\
26.71624 \\
27.09857 \\
27.45310\end{array}$ & $\begin{array}{l}45477 \\
41515 \\
38233 \\
35453 \\
33057\end{array}$ \\
\hline $\begin{array}{l}650 \\
700 \\
750 \\
800 \\
850\end{array}$ & $\begin{array}{l}4.15543 \\
4.20064 \\
4.24027 \\
4.27515 \\
4.30597\end{array}$ & $\begin{array}{l}4521 \\
3963 \\
3488 \\
3082 \\
2737\end{array}$ & $\begin{array}{l}3.74146 \\
3.77268 \\
3.80256 \\
3.83103 \\
3.85808\end{array}$ & $\begin{array}{l}3122 \\
2988 \\
2847 \\
2705 \\
2566\end{array}$ & $\begin{array}{l}24.04220 \\
24.32062 \\
24.58194 \\
24.82827 \\
25.06134\end{array}$ & $\begin{array}{l}27842 \\
26132 \\
24633 \\
23307 \\
22126\end{array}$ & $\begin{array}{l}27.78367 \\
28.09331 \\
28.38451 \\
28.65930 \\
28.91943\end{array}$ & $\begin{array}{l}30964 \\
29120 \\
27479 \\
26013 \\
24691\end{array}$ \\
\hline $\begin{array}{r}900 \\
950 \\
1000 \\
1050 \\
1100\end{array}$ & $\begin{array}{l}4.33334 \\
4.35778 \\
4.37972 \\
4.39951 \\
4.41747\end{array}$ & $\begin{array}{l}2444 \\
2194 \\
1979 \\
1796 \\
1638\end{array}$ & $\begin{array}{l}3.88374 \\
3.90806 \\
3.93110 \\
3.95294 \\
3.97366\end{array}$ & $\begin{array}{l}2432 \\
2304 \\
2184 \\
2072 \\
1965\end{array}$ & $\begin{array}{l}25.28260 \\
25.49324 \\
25.69428 \\
25.88662 \\
26.07099\end{array}$ & $\begin{array}{l}21064 \\
20104 \\
19234 \\
18437 \\
17707\end{array}$ & $\begin{array}{l}29.16634 \\
29.40130 \\
29.62539 \\
29.83957 \\
30.04465\end{array}$ & $\begin{array}{l}23496 \\
22409 \\
21418 \\
20508 \\
19673\end{array}$ \\
\hline 1150 & 4.43385 & 1500 & 3.99331 & 1868 & 26.24806 & 17036 & 30.24138 & 18903 \\
\hline
\end{tabular}


Table 2.045. CuT (Cont.)

\begin{tabular}{|c|c|c|c|c|c|c|c|c|}
\hline${ }^{\circ} \mathrm{K}$ & $\frac{C_{p}^{0}}{R}$ & & $\frac{\left(H^{\circ}-E_{0}^{0}\right)}{R T}$ & & $\frac{-\left(F^{0}-\right.}{R T}$ & & $\frac{S^{0}}{R}$ & \\
\hline $\begin{array}{l}1200 \\
1300 \\
1400 \\
1500 \\
1600\end{array}$ & $\begin{array}{l}4.44885 \\
4.47546 \\
4.49844 \\
4.51861 \\
4.53658\end{array}$ & $\begin{array}{l}2661 \\
2298 \\
2017 \\
1797 \\
1623\end{array}$ & $\begin{array}{l}4.01199 \\
4.04664 \\
4.07811 \\
4.10682 \\
4.13313\end{array}$ & $\begin{array}{l}3465 \\
3147 \\
2871 \\
2631 \\
2422\end{array}$ & $\begin{array}{l}26.41842 \\
26.74094 \\
27.04200 \\
27.32435 \\
27.59025\end{array}$ & $\begin{array}{l}32252 \\
30106 \\
28235 \\
26590 \\
25131\end{array}$ & $\begin{array}{l}30.43041 \\
30.78759 \\
31.12011 \\
31.43118 \\
31.72339\end{array}$ & $\begin{array}{l}35718 \\
33252 \\
31107 \\
29221 \\
27552\end{array}$ \\
\hline $\begin{array}{l}1700 \\
1800 \\
1900 \\
2000 \\
2100\end{array}$ & $\begin{array}{l}4.55281 \\
4.56763 \\
4.58132 \\
4.59407 \\
4.60604\end{array}$ & $\begin{array}{l}1482 \\
1369 \\
1275 \\
1197 \\
1133\end{array}$ & $\begin{array}{l}4.15735 \\
4.17973 \\
4.20051 \\
4.21988 \\
4.23798\end{array}$ & $\begin{array}{l}2238 \\
2078 \\
1937 \\
1810 \\
1699\end{array}$ & $\begin{array}{l}27.84156 \\
28.07983 \\
28.30638 \\
28.52233 \\
28.72867\end{array}$ & $\begin{array}{l}23827 \\
22655 \\
21595 \\
20634 \\
19754\end{array}$ & $\begin{array}{l}31.99891 \\
32.25957 \\
32.50690 \\
32.74222 \\
32.96665\end{array}$ & $\begin{array}{l}26066 \\
24733 \\
23532 \\
22443 \\
21454\end{array}$ \\
\hline $\begin{array}{l}2200 \\
2300 \\
2400 \\
2500 \\
2600\end{array}$ & $\begin{array}{l}4.61737 \\
4.62814 \\
4.63845 \\
4.64836 \\
4.65792\end{array}$ & $\begin{array}{r}1077 \\
1031 \\
991 \\
956 \\
927\end{array}$ & $\begin{array}{l}4.25497 \\
4.27097 \\
4.28606 \\
4.30036 \\
4.31393\end{array}$ & $\begin{array}{l}1600 \\
1509 \\
1430 \\
1357 \\
1291\end{array}$ & $\begin{array}{l}28.92621 \\
29.11571 \\
29.29780 \\
29.47306 \\
29.64199\end{array}$ & $\begin{array}{l}18950 \\
18209 \\
17526 \\
16893 \\
16306\end{array}$ & $\begin{array}{l}33.18119 \\
33.38668 \\
33.58387 \\
33.77343 \\
33.95593\end{array}$ & $\begin{array}{l}20549 \\
19719 \\
18956 \\
18250 \\
17596\end{array}$ \\
\hline $\begin{array}{l}2700 \\
2800 \\
2900 \\
3000\end{array}$ & $\begin{array}{l}4.66719 \\
4.67619 \\
4.68495 \\
4.69352\end{array}$ & $\begin{array}{r}900 \\
876 \\
857 \\
1659\end{array}$ & $\begin{array}{l}4.32684 \\
4.33916 \\
4.35093 \\
4.36221\end{array}$ & $\begin{array}{l}1232 \\
1177 \\
1128 \\
2123\end{array}$ & $\begin{array}{l}29.80505 \\
29.96263 \\
30.11510 \\
30.26280\end{array}$ & $\begin{array}{l}15758 \\
15247 \\
14770 \\
28221\end{array}$ & $\begin{array}{l}34.13189 \\
34.30179 \\
34.46604 \\
34.62501\end{array}$ & $\begin{array}{l}16990 \\
16425 \\
15897 \\
30345\end{array}$ \\
\hline $\begin{array}{l}3200 \\
3400 \\
3600 \\
3800 \\
4000\end{array}$ & $\begin{array}{l}4.71011 \\
4.72611 \\
4.74163 \\
4.75673 \\
4.77149\end{array}$ & $\begin{array}{l}1600 \\
1552 \\
1510 \\
1476 \\
1445\end{array}$ & $\begin{array}{l}4.38344 \\
4.40313 \\
4.42150 \\
4.43875 \\
4.45502\end{array}$ & $\begin{array}{l}1969 \\
1837 \\
1725 \\
1627 \\
1542\end{array}$ & $\begin{array}{l}30.54501 \\
30.81136 \\
31.06356 \\
31.30308 \\
31.53118\end{array}$ & $\begin{array}{l}26635 \\
25220 \\
23952 \\
22810 \\
21774\end{array}$ & $\begin{array}{l}34.92846 \\
35.21449 \\
35.48507 \\
35.74184 \\
35.98621\end{array}$ & $\begin{array}{l}28603 \\
27058 \\
25677 \\
24437 \\
23315\end{array}$ \\
\hline $\begin{array}{l}4200 \\
4400 \\
4600 \\
4800 \\
5000\end{array}$ & $\begin{array}{l}4.78594 \\
4.80013 \\
4.81408 \\
4.82782 \\
4.84137\end{array}$ & $\begin{array}{l}1419 \\
1395 \\
1374 \\
1355\end{array}$ & $\begin{array}{l}4.47044 \\
4.48510 \\
4.49910 \\
4.51251 \\
4.52540\end{array}$ & $\begin{array}{l}1466 \\
1400 \\
1341 \\
1289\end{array}$ & $\begin{array}{l}31.74892 \\
31.95722 \\
32.15691 \\
32.34867 \\
32.53314\end{array}$ & $\begin{array}{l}20830 \\
19969 \\
19176 \\
18447\end{array}$ & $\begin{array}{l}36.21936 \\
36.44233 \\
36.65601 \\
36.86119 \\
37.05855\end{array}$ & $\begin{array}{l}22297 \\
21368 \\
20518 \\
19736\end{array}$ \\
\hline
\end{tabular}


Table 2. 046. AgH

\begin{tabular}{|c|c|c|c|c|c|c|c|c|}
\hline${ }^{\circ} K$ & $\frac{C_{p}^{0}}{R}$ & & $\frac{\left(H^{\circ}-E_{0}^{0}\right)}{R T}$ & & $\frac{-\left(F^{0}-E\right.}{R T}$ & & $\frac{S^{0}}{R}$ & \\
\hline $\begin{array}{l}50 \\
60 \\
70 \\
80 \\
90\end{array}$ & $\begin{array}{l}3.50199 \\
3.50198 \\
3.50207 \\
3.50222 \\
3.50239\end{array}$ & $\begin{array}{r}-\quad 1 \\
9 \\
15 \\
17 \\
19\end{array}$ & $\begin{array}{l}3.43962 \\
3.45001 \\
3.45744 \\
3.46303 \\
3.46739\end{array}$ & $\begin{array}{r}1039 \\
743 \\
559 \\
436 \\
351\end{array}$ & $\begin{array}{l}14.92296 \\
15.55106 \\
16.08347 \\
16.54552 \\
16.95367\end{array}$ & $\begin{array}{l}62810 \\
53241 \\
46205 \\
40815 \\
36552\end{array}$ & $\begin{array}{l}18.36258 \\
19.00107 \\
19.54091 \\
20.00856 \\
20.42107\end{array}$ & $\begin{array}{l}63849 \\
53984 \\
46765 \\
41251 \\
36902\end{array}$ \\
\hline $\begin{array}{l}100 \\
110 \\
120 \\
130 \\
140\end{array}$ & $\begin{array}{l}3.50258 \\
3.50279 \\
3.50300 \\
3.50322 \\
3.50345\end{array}$ & $\begin{array}{l}21 \\
21 \\
22 \\
23 \\
24\end{array}$ & $\begin{array}{l}3.47090 \\
3.47379 \\
3.47621 \\
3.47828 \\
3.48007\end{array}$ & $\begin{array}{l}289 \\
242 \\
207 \\
179 \\
157\end{array}$ & $\begin{array}{l}17.31919 \\
17.65014 \\
17.95251 \\
18.23084 \\
18.48867\end{array}$ & $\begin{array}{l}33095 \\
30237 \\
27833 \\
25783 \\
24016\end{array}$ & $\begin{array}{l}20.79009 \\
21.12393 \\
21.42873 \\
21.70913 \\
21.96875\end{array}$ & $\begin{array}{l}33384 \\
30480 \\
28040 \\
25962 \\
24172\end{array}$ \\
\hline $\begin{array}{l}150 \\
160 \\
170 \\
180 \\
190\end{array}$ & $\begin{array}{l}3.50369 \\
3.50396 \\
3.50426 \\
3.50462 \\
3.50506\end{array}$ & $\begin{array}{l}27 \\
30 \\
36 \\
44 \\
57\end{array}$ & $\begin{array}{l}3.48164 \\
3.48303 \\
3.48427 \\
3.48539 \\
3.48641\end{array}$ & $\begin{array}{r}139 \\
124 \\
112 \\
102 \\
95\end{array}$ & $\begin{array}{l}18.72883 \\
18.95357 \\
19.16477 \\
19.36396 \\
19.55243\end{array}$ & $\begin{array}{l}22474 \\
21120 \\
19919 \\
18847 \\
17885\end{array}$ & $\begin{array}{l}22.21047 \\
22.43660 \\
22.64904 \\
22.84935 \\
23.03884\end{array}$ & $\begin{array}{l}22613 \\
21244 \\
20031 \\
18949 \\
17980\end{array}$ \\
\hline $\begin{array}{l}200 \\
210 \\
220 \\
230 \\
240\end{array}$ & $\begin{array}{l}3.50563 \\
3.50637 \\
3.50732 \\
3.50852 \\
3.51004\end{array}$ & $\begin{array}{r}74 \\
95 \\
120 \\
152 \\
188\end{array}$ & $\begin{array}{l}3.48736 \\
3.48824 \\
3.48909 \\
3.48991 \\
3.49071\end{array}$ & $\begin{array}{l}88 \\
85 \\
82 \\
80 \\
81\end{array}$ & $\begin{array}{l}19.73128 \\
19.90145 \\
20.06375 \\
20.21886 \\
20.36741\end{array}$ & $\begin{array}{l}17017 \\
16230 \\
15511 \\
14855 \\
14251\end{array}$ & $\begin{array}{l}23.21864 \\
23.38970 \\
23.55284 \\
23.70877 \\
23.85812\end{array}$ & $\begin{array}{l}17106 \\
16314 \\
15593 \\
14935 \\
14333\end{array}$ \\
\hline $\begin{array}{l}250 \\
260 \\
270 \\
280 \\
290\end{array}$ & $\begin{array}{l}3.51192 \\
3.51419 \\
3.51691 \\
3.52010 \\
3.52380\end{array}$ & $\begin{array}{l}227 \\
272 \\
319 \\
370 \\
422\end{array}$ & $\begin{array}{l}3.49152 \\
3.49235 \\
3.49321 \\
3.49411 \\
3.49507\end{array}$ & $\begin{array}{r}83 \\
86 \\
90 \\
96 \\
102\end{array}$ & $\begin{array}{l}20.50992 \\
20.64688 \\
20.77870 \\
20.90575 \\
21.02838\end{array}$ & $\begin{array}{l}13696 \\
13182 \\
12705 \\
12263 \\
11851\end{array}$ & $\begin{array}{l}24.00145 \\
24.13923 \\
24.27191 \\
24.39987 \\
24.52345\end{array}$ & $\begin{array}{l}13778 \\
13268 \\
12796 \\
12358 \\
11954\end{array}$ \\
\hline $\begin{array}{l}300 \\
310 \\
320 \\
330 \\
340\end{array}$ & $\begin{array}{l}3.52802 \\
3.53278 \\
3.53808 \\
3.54393 \\
3.55032\end{array}$ & $\begin{array}{l}476 \\
530 \\
585 \\
639 \\
692\end{array}$ & $\begin{array}{l}3.49609 \\
3.49720 \\
3.49839 \\
3.49968 \\
3.50108\end{array}$ & $\begin{array}{l}111 \\
119 \\
129 \\
140 \\
150\end{array}$ & $\begin{array}{l}21.14689 \\
21.26154 \\
21.37259 \\
21.48026 \\
21.58476\end{array}$ & $\begin{array}{l}11465 \\
11105 \\
10767 \\
10450 \\
10151\end{array}$ & $\begin{array}{l}24.64299 \\
24.75875 \\
24.87099 \\
24.97995 \\
25.08584\end{array}$ & $\begin{array}{l}11576 \\
11224 \\
10896 \\
10589 \\
10301\end{array}$ \\
\hline $\begin{array}{l}350 \\
360 \\
370 \\
380 \\
390\end{array}$ & $\begin{array}{l}3.55724 \\
3.56468 \\
3.57261 \\
3.58101 \\
3.58987\end{array}$ & $\begin{array}{l}744 \\
793 \\
840 \\
886 \\
926\end{array}$ & $\begin{array}{l}3.50258 \\
3.50420 \\
3.50594 \\
3.50781 \\
3.50980\end{array}$ & $\begin{array}{l}162 \\
174 \\
187 \\
199 \\
211\end{array}$ & $\begin{array}{l}21.68627 \\
21.78496 \\
21.88100 \\
21.97452 \\
22.06566\end{array}$ & $\begin{array}{l}9869 \\
9604 \\
9352 \\
9114 \\
8889\end{array}$ & $\begin{array}{l}25.18885 \\
25.28917 \\
25.38694 \\
25.48233 \\
25.57546\end{array}$ & $\begin{array}{r}10032 \\
9777 \\
9539 \\
9313 \\
9101\end{array}$ \\
\hline $\begin{array}{l}400 \\
450 \\
500 \\
550 \\
600\end{array}$ & $\begin{array}{l}3.59913 \\
3.65073 \\
3.70830 \\
3.76851 \\
3.82878\end{array}$ & $\begin{array}{l}5160 \\
5757 \\
6021 \\
6027 \\
5858\end{array}$ & $\begin{array}{l}3.51191 \\
3.52440 \\
3.53987 \\
3.55791 \\
3.57798\end{array}$ & $\begin{array}{l}1249 \\
1547 \\
1804 \\
2007 \\
2156\end{array}$ & $\begin{array}{l}22.15455 \\
22.56888 \\
22.94099 \\
23.27921 \\
23.58963\end{array}$ & $\begin{array}{l}41433 \\
37211 \\
33822 \\
31042 \\
28724\end{array}$ & $\begin{array}{l}25.66647 \\
26.09329 \\
26.48087 \\
26.83712 \\
27.16762\end{array}$ & $\begin{array}{l}42682 \\
38758 \\
35625 \\
33050 \\
30879\end{array}$ \\
\hline $\begin{array}{l}650 \\
700 \\
750 \\
800 \\
850\end{array}$ & $\begin{array}{l}3.88736 \\
3.94317 \\
3.99563 \\
4.04452 \\
4.08982\end{array}$ & $\begin{array}{l}5581 \\
5246 \\
4889 \\
4530 \\
4184\end{array}$ & $\begin{array}{l}3.59954 \\
3.62211 \\
3.64528 \\
3.66873 \\
3.69218\end{array}$ & $\begin{array}{l}2257 \\
2317 \\
2345 \\
2345 \\
2327\end{array}$ & $\begin{array}{l}23.87687 \\
24.14444 \\
24.39513 \\
24.63114 \\
24.85426\end{array}$ & $\begin{array}{l}26757 \\
25069 \\
23601 \\
22312 \\
21170\end{array}$ & $\begin{array}{l}27.47641 \\
27.76656 \\
28.04042 \\
28.29987 \\
28.54645\end{array}$ & $\begin{array}{l}29015 \\
27386 \\
25945 \\
24658 \\
23497\end{array}$ \\
\hline $\begin{array}{r}900 \\
950 \\
1000 \\
1050 \\
1100\end{array}$ & $\begin{array}{l}4.13166 \\
4.17025 \\
4.20581 \\
4.23861 \\
4.26890\end{array}$ & $\begin{array}{l}3859 \\
3556 \\
3280 \\
3029 \\
2801\end{array}$ & $\begin{array}{l}3.71545 \\
3.73839 \\
3.76088 \\
3.78286 \\
3.80427\end{array}$ & $\begin{array}{l}2294 \\
2249 \\
2198 \\
2141 \\
2082\end{array}$ & $\begin{array}{l}25.06596 \\
25.26746 \\
25.45979 \\
25.64382 \\
25.82029\end{array}$ & $\begin{array}{l}20150 \\
19233 \\
18403 \\
17647 \\
16957\end{array}$ & $\begin{array}{l}28.78142 \\
29.00585 \\
29.22067 \\
29.42668 \\
29.62457\end{array}$ & $\begin{array}{l}22443 \\
21482 \\
20601 \\
19789 \\
19039\end{array}$ \\
\hline 1150 & 4.29691 & 2596 & 3.82509 & 2021 & 25.98986 & 16322 & 29.81496 & 18343 \\
\hline
\end{tabular}


Table 2.046. AgH (Cont.)

\begin{tabular}{|c|c|c|c|c|c|c|c|c|}
\hline${ }^{\circ} \mathrm{K}$ & $\frac{C_{p}^{0}}{R}$ & & $\frac{\left(H^{\circ}-E_{0}^{0}\right)}{R T}$ & & $\frac{-l F^{0}-}{R T}$ & & $\frac{S^{0}}{R}$ & \\
\hline $\begin{array}{l}1200 \\
1300 \\
1400 \\
1500 \\
1600\end{array}$ & $\begin{array}{l}4.32287 \\
4.36941 \\
4.40994 \\
4.44559 \\
4.47726\end{array}$ & $\begin{array}{l}4654 \\
4053 \\
3565 \\
3167 \\
2840\end{array}$ & $\begin{array}{l}3.84530 \\
3.88387 \\
3.92003 \\
3.95390 \\
3.98564\end{array}$ & $\begin{array}{l}3857 \\
3616 \\
3387 \\
3174 \\
2977\end{array}$ & $\begin{array}{l}26.15308 \\
26.46241 \\
26.75158 \\
27.02320 \\
27.27940\end{array}$ & $\begin{array}{l}30933 \\
28917 \\
27162 \\
25620 \\
24253\end{array}$ & $\begin{array}{l}29.99839 \\
30.34628 \\
30.67161 \\
30.97710 \\
31.26505\end{array}$ & $\begin{array}{l}34789 \\
32533 \\
30549 \\
28795 \\
27230\end{array}$ \\
\hline $\begin{array}{l}1700 \\
1800 \\
1900 \\
2000 \\
2100\end{array}$ & $\begin{array}{l}4.50566 \\
4.53136 \\
4.55483 \\
4.57643 \\
4.59645\end{array}$ & $\begin{array}{l}2570 \\
2347 \\
2160 \\
2002 \\
1869\end{array}$ & $\begin{array}{l}4.01541 \\
4.04337 \\
4.06968 \\
4.09449 \\
4.11792\end{array}$ & $\begin{array}{l}2796 \\
2631 \\
2481 \\
2343 \\
2218\end{array}$ & $\begin{array}{l}27.52193 \\
27.75225 \\
27.97157 \\
28.18096 \\
28.38130\end{array}$ & $\begin{array}{l}23032 \\
21932 \\
20939 \\
20034 \\
19208\end{array}$ & $\begin{array}{l}31.53735 \\
31.79562 \\
32.04126 \\
32.27545 \\
32.49922\end{array}$ & $\begin{array}{l}25827 \\
24564 \\
23419 \\
22377 \\
21427\end{array}$ \\
\hline $\begin{array}{l}2200 \\
2300 \\
2400 \\
2500 \\
2600\end{array}$ & $\begin{array}{l}4.61514 \\
4.63268 \\
4.64924 \\
4.66496 \\
4.67994\end{array}$ & $\begin{array}{l}1754 \\
1656 \\
1572 \\
1498 \\
1433\end{array}$ & $\begin{array}{l}4.14010 \\
4.16114 \\
4.18113 \\
4.20017 \\
4.21834\end{array}$ & $\begin{array}{l}2104 \\
1999 \\
1904 \\
1817 \\
1736\end{array}$ & $\begin{array}{l}28.57338 \\
28.75789 \\
28.93541 \\
29.10648 \\
29.27157\end{array}$ & $\begin{array}{l}18451 \\
17752 \\
17107 \\
16509 \\
15953\end{array}$ & $\begin{array}{l}32.71349 \\
32.91903 \\
33.11655 \\
33.30666 \\
33.48992\end{array}$ & $\begin{array}{l}20554 \\
19752 \\
19011 \\
18326 \\
17689\end{array}$ \\
\hline $\begin{array}{l}2700 \\
2800 \\
2900 \\
3000\end{array}$ & $\begin{array}{l}4.69427 \\
4.70804 \\
4.72131 \\
4.73413\end{array}$ & $\begin{array}{l}1377 \\
1327 \\
1282 \\
2450\end{array}$ & $\begin{array}{l}4.23570 \\
4.25233 \\
4.26827 \\
4.28359\end{array}$ & $\begin{array}{l}1663 \\
1594 \\
1532 \\
2893\end{array}$ & $\begin{array}{l}29.43110 \\
29.58545 \\
29.73495 \\
29.87991\end{array}$ & $\begin{array}{l}15435 \\
14950 \\
14496 \\
27739\end{array}$ & $\begin{array}{l}33.66681 \\
33.83778 \\
34.00322 \\
34.16350\end{array}$ & $\begin{array}{l}17097 \\
16544 \\
16028 \\
30633\end{array}$ \\
\hline $\begin{array}{l}3200 \\
3400 \\
3600 \\
3800 \\
4000\end{array}$ & $\begin{array}{l}4.75863 \\
4.78184 \\
4.80399 \\
4.82526 \\
4.84578\end{array}$ & $\begin{array}{l}2321 \\
2215 \\
2127 \\
2052 \\
1987\end{array}$ & $\begin{array}{l}4.31252 \\
4.33945 \\
4.36465 \\
4.38833 \\
4.41070\end{array}$ & $\begin{array}{l}2693 \\
2520 \\
2368 \\
2237 \\
2119\end{array}$ & $\begin{array}{l}30.15730 \\
30.41956 \\
30.66832 \\
30.90494 \\
31.13061\end{array}$ & $\begin{array}{l}26226 \\
24876 \\
23662 \\
22567 \\
21572\end{array}$ & $\begin{array}{l}34.46983 \\
34.75902 \\
35.03297 \\
35.29328 \\
35.54131\end{array}$ & $\begin{array}{l}28919 \\
27395 \\
26031 \\
24803 \\
23691\end{array}$ \\
\hline $\begin{array}{l}4200 \\
4400 \\
4600 \\
4800 \\
5000\end{array}$ & $\begin{array}{l}4.86565 \\
4.88497 \\
4.90379 \\
4.92218 \\
4.94016\end{array}$ & $\begin{array}{l}1932 \\
1882 \\
1839 \\
1798\end{array}$ & $\begin{array}{l}4.43189 \\
4.45205 \\
4.47128 \\
4.48969 \\
4.50735\end{array}$ & $\begin{array}{l}2016 \\
1923 \\
1841 \\
1766\end{array}$ & $\begin{array}{l}31.34633 \\
31.55297 \\
31.75130 \\
31.94198 \\
32.12562\end{array}$ & $\begin{array}{l}20664 \\
19833 \\
19068 \\
18364\end{array}$ & $\begin{array}{l}35.77822 \\
36.00502 \\
36.22258 \\
36.43168 \\
36.63298\end{array}$ & $\begin{array}{l}22680 \\
21756 \\
20910 \\
20130\end{array}$ \\
\hline
\end{tabular}


Table 2.047. AgD

\begin{tabular}{|c|c|c|c|c|c|c|c|c|}
\hline${ }^{\circ} K$ & $\frac{C_{p}^{0}}{R}$ & & $\frac{\left(H^{0}-\varepsilon_{0}^{0}\right)}{R T}$ & & $\frac{-\left(F^{0}-1\right.}{R T}$ & & $\frac{S^{0}}{R}$ & \\
\hline $\begin{array}{l}50 \\
60 \\
70 \\
80 \\
90\end{array}$ & $\begin{array}{l}3.50140 \\
3.50157 \\
3.50177 \\
3.50199 \\
3.50221\end{array}$ & $\begin{array}{l}17 \\
20 \\
22 \\
22 \\
24\end{array}$ & $\begin{array}{l}3.46949 \\
3.47482 \\
3.47866 \\
3.48156 \\
3.48384\end{array}$ & $\begin{array}{l}533 \\
384 \\
290 \\
228 \\
185\end{array}$ & $\begin{array}{l}15.57488 \\
16.20794 \\
16.74389 \\
17.20860 \\
17.61881\end{array}$ & $\begin{array}{l}63306 \\
53595 \\
46471 \\
41021 \\
36716\end{array}$ & $\begin{array}{l}19.04437 \\
19.68277 \\
20.22256 \\
20.69017 \\
21.10266\end{array}$ & $\begin{array}{l}63840 \\
53979 \\
46761 \\
41249 \\
36900\end{array}$ \\
\hline $\begin{array}{l}100 \\
110 \\
120 \\
130 \\
140\end{array}$ & $\begin{array}{l}3.50245 \\
3.50270 \\
3.50300 \\
3.50340 \\
3.50397\end{array}$ & $\begin{array}{l}25 \\
30 \\
40 \\
57 \\
84\end{array}$ & $\begin{array}{l}3.48569 \\
3.48723 \\
3.48853 \\
3.48966 \\
3.49066\end{array}$ & $\begin{array}{r}154 \\
130 \\
113 \\
100 \\
91\end{array}$ & $\begin{array}{l}17.98597 \\
18.31826 \\
18.62175 \\
18.90103 \\
19.15967\end{array}$ & $\begin{array}{l}33229 \\
30349 \\
27928 \\
25864 \\
24087\end{array}$ & $\begin{array}{l}21.47166 \\
21.80549 \\
22.11028 \\
22.39069 \\
22.65034\end{array}$ & $\begin{array}{l}33383 \\
30479 \\
28041 \\
25965 \\
24177\end{array}$ \\
\hline $\begin{array}{l}150 \\
160 \\
170 \\
180 \\
190\end{array}$ & $\begin{array}{l}3.50481 \\
3.50603 \\
3.50776 \\
3.51013 \\
3.51326\end{array}$ & $\begin{array}{l}122 \\
173 \\
237 \\
313 \\
399\end{array}$ & $\begin{array}{l}3.49157 \\
3.49243 \\
3.49328 \\
3.49415 \\
3.49507\end{array}$ & $\begin{array}{r}86 \\
85 \\
87 \\
92 \\
100\end{array}$ & $\begin{array}{l}19.40054 \\
19.62591 \\
19.83766 \\
20.03735 \\
20.22630\end{array}$ & $\begin{array}{l}22537 \\
21175 \\
19969 \\
18895 \\
17930\end{array}$ & $\begin{array}{l}22.89211 \\
23.11834 \\
23.33095 \\
23.53151 \\
23.72137\end{array}$ & $\begin{array}{l}22623 \\
21261 \\
20056 \\
18986 \\
18031\end{array}$ \\
\hline $\begin{array}{l}200 \\
210 \\
220 \\
230 \\
240\end{array}$ & $\begin{array}{l}3.51725 \\
3.52217 \\
3.52807 \\
3.53500 \\
3.54295\end{array}$ & $\begin{array}{l}492 \\
590 \\
693 \\
795 \\
896\end{array}$ & $\begin{array}{l}3.49607 \\
3.49720 \\
3.49846 \\
3.49990 \\
3.50152\end{array}$ & $\begin{array}{l}113 \\
126 \\
144 \\
162 \\
183\end{array}$ & $\begin{array}{l}20.40560 \\
20.57620 \\
20.73892 \\
20.89446 \\
21.04345\end{array}$ & $\begin{array}{l}17060 \\
16272 \\
15554 \\
14899 \\
14297\end{array}$ & $\begin{array}{l}23.90168 \\
24.07340 \\
24.23738 \\
24.39436 \\
24.54497\end{array}$ & $\begin{array}{l}17172 \\
16398 \\
15698 \\
15061 \\
14481\end{array}$ \\
\hline $\begin{array}{l}250 \\
260 \\
270 \\
280 \\
290\end{array}$ & $\begin{array}{l}3.55191 \\
3.56183 \\
3.57268 \\
3.58438 \\
3.59687\end{array}$ & $\begin{array}{r}992 \\
1085 \\
1170 \\
1249 \\
1319\end{array}$ & $\begin{array}{l}3.50335 \\
3.50541 \\
3.50770 \\
3.51022 \\
3.51299\end{array}$ & $\begin{array}{l}206 \\
229 \\
252 \\
277 \\
302\end{array}$ & $\begin{array}{l}21.18642 \\
21.32387 \\
21.45620 \\
21.58382 \\
21.70704\end{array}$ & $\begin{array}{l}13745 \\
13233 \\
12762 \\
12322 \\
11915\end{array}$ & $\begin{array}{l}24.68978 \\
24.82928 \\
24.96391 \\
25.09405 \\
25.22004\end{array}$ & $\begin{array}{l}13950 \\
13463 \\
13014 \\
12599 \\
12216\end{array}$ \\
\hline $\begin{array}{l}300 \\
310 \\
320 \\
330 \\
340\end{array}$ & $\begin{array}{l}3.61006 \\
3.62389 \\
3.63826 \\
3.65309 \\
3.66832\end{array}$ & $\begin{array}{l}1383 \\
1437 \\
1483 \\
1523 \\
1554\end{array}$ & $\begin{array}{l}3.51601 \\
3.51926 \\
3.52276 \\
3.52648 \\
3.53043\end{array}$ & $\begin{array}{l}325 \\
350 \\
372 \\
395 \\
416\end{array}$ & $\begin{array}{l}21.82619 \\
21.94153 \\
22.05332 \\
22.16178 \\
22.26711\end{array}$ & $\begin{array}{l}11534 \\
11179 \\
10846 \\
10533 \\
10240\end{array}$ & $\begin{array}{l}25.34220 \\
25.46080 \\
25.57608 \\
25.68826 \\
25.79754\end{array}$ & $\begin{array}{l}11860 \\
11528 \\
11218 \\
10928 \\
10656\end{array}$ \\
\hline $\begin{array}{l}350 \\
360 \\
370 \\
380 \\
390\end{array}$ & $\begin{array}{l}3.68386 \\
3.69965 \\
3.71562 \\
3.73172 \\
3.74787\end{array}$ & $\begin{array}{l}1579 \\
1597 \\
1610 \\
1615 \\
1617\end{array}$ & $\begin{array}{l}3.53459 \\
3.53895 \\
3.54351 \\
3.54825 \\
3.55316\end{array}$ & $\begin{array}{l}436 \\
456 \\
474 \\
491 \\
507\end{array}$ & $\begin{array}{l}22.36951 \\
22.46914 \\
22.56617 \\
22.66073 \\
22.75296\end{array}$ & $\begin{array}{l}9963 \\
9703 \\
9456 \\
9223 \\
9002\end{array}$ & $\begin{array}{l}25.90410 \\
26.00810 \\
26.10968 \\
26.20899 \\
26.30613\end{array}$ & $\begin{array}{r}10400 \\
10158 \\
9931 \\
9714 \\
9509\end{array}$ \\
\hline $\begin{array}{l}400 \\
450 \\
500 \\
550 \\
600\end{array}$ & $\begin{array}{l}3.76404 \\
3.84373 \\
3.91892 \\
3.98773 \\
4.04964\end{array}$ & $\begin{array}{l}7969 \\
7519 \\
6881 \\
6191 \\
5517\end{array}$ & $\begin{array}{l}3.55823 \\
3.58556 \\
3.61518 \\
3.64597 \\
3.67708\end{array}$ & $\begin{array}{l}2733 \\
2962 \\
3079 \\
3111 \\
3082\end{array}$ & $\begin{array}{l}22.84298 \\
23.26363 \\
23.64292 \\
23.98893 \\
24.30750\end{array}$ & $\begin{array}{l}42065 \\
37929 \\
34601 \\
31857 \\
29555\end{array}$ & $\begin{array}{l}26.40122 \\
26.84919 \\
27.25811 \\
27.63491 \\
27.98459\end{array}$ & $\begin{array}{l}44797 \\
40892 \\
37680 \\
34968 \\
32636\end{array}$ \\
\hline $\begin{array}{l}650 \\
700 \\
750 \\
800 \\
850\end{array}$ & $\begin{array}{l}4.10481 \\
4.15379 \\
4.19724 \\
4.23582 \\
4.27018\end{array}$ & $\begin{array}{l}4898 \\
4345 \\
3858 \\
3436 \\
3071\end{array}$ & $\begin{array}{l}3.70790 \\
3.73804 \\
3.76723 \\
3.79534 \\
3.82228\end{array}$ & $\begin{array}{l}3014 \\
2919 \\
2811 \\
2694 \\
2575\end{array}$ & $\begin{array}{l}24.60305 \\
24.87894 \\
25.13784 \\
25.38188 \\
25.61278\end{array}$ & $\begin{array}{l}27589 \\
25890 \\
24404 \\
23090 \\
21921\end{array}$ & $\begin{array}{l}28.31095 \\
28.61698 \\
28.90508 \\
29.17722 \\
29.43506\end{array}$ & $\begin{array}{l}30603 \\
28810 \\
27214 \\
25784 \\
24497\end{array}$ \\
\hline $\begin{array}{r}900 \\
950 \\
1000 \\
1050 \\
1100\end{array}$ & $\begin{array}{l}4.30089 \\
4.32844 \\
4.35327 \\
4.37575 \\
4.39620\end{array}$ & $\begin{array}{l}2755 \\
2483 \\
2248 \\
2045 \\
1868\end{array}$ & $\begin{array}{l}3.84803 \\
3.87260 \\
3.89602 \\
3.91834 \\
3.93961\end{array}$ & $\begin{array}{l}2457 \\
2342 \\
2232 \\
2127 \\
2026\end{array}$ & $\begin{array}{l}25.83199 \\
26.04071 \\
26.23995 \\
26.43058 \\
26.61335\end{array}$ & $\begin{array}{l}20872 \\
19924 \\
19063 \\
18277 \\
17558\end{array}$ & $\begin{array}{l}29.68003 \\
29.91331 \\
30.13598 \\
30.34893 \\
30.55297\end{array}$ & $\begin{array}{l}23328 \\
22267 \\
21295 \\
20404 \\
19583\end{array}$ \\
\hline 1150 & 4.41488 & 1715 & 3.95987 & 1932 & 26.78893 & 16894 & 30.74880 & 18827 \\
\hline
\end{tabular}


Table 2. 047. AgD (Cont.)

\begin{tabular}{|c|c|c|c|c|c|c|c|c|}
\hline${ }^{\circ} \mathrm{K}$ & $\frac{C_{p}^{0}}{R}$ & & $\frac{\left(H^{\circ}-E_{0}^{0}\right)}{R T}$ & & $\frac{-\left(F^{0}-E\right.}{R T}$ & & $\frac{S^{0}}{R}$ & \\
\hline $\begin{array}{l}1200 \\
1300 \\
1400 \\
1500 \\
1600\end{array}$ & $\begin{array}{l}4.43203 \\
4.46248 \\
4.48879 \\
4.51187 \\
4.53242\end{array}$ & $\begin{array}{l}3045 \\
2631 \\
2308 \\
2055 \\
1852\end{array}$ & $\begin{array}{l}3.97919 \\
4.01523 \\
4.04813 \\
4.07830 \\
4.10605\end{array}$ & $\begin{array}{l}3604 \\
3290 \\
3017 \\
2775 \\
2563\end{array}$ & $\begin{array}{l}26.95787 \\
27.27782 \\
27.57660 \\
27.85694 \\
28.12105\end{array}$ & $\begin{array}{l}31995 \\
29878 \\
28034 \\
26411 \\
24970\end{array}$ & $\begin{array}{l}30.93707 \\
31.29305 \\
31.62474 \\
31.93524 \\
32.22710\end{array}$ & $\begin{array}{l}35598 \\
33169 \\
31050 \\
29186 \\
27534\end{array}$ \\
\hline $\begin{array}{l}1700 \\
1800 \\
1900 \\
2000 \\
2100\end{array}$ & $\begin{array}{l}4.55094 \\
4.56783 \\
4.58338 \\
4.59784 \\
4.61138\end{array}$ & $\begin{array}{l}1689 \\
1555 \\
1446 \\
1354 \\
1278\end{array}$ & $\begin{array}{l}4.13168 \\
4.15545 \\
4.17757 \\
4.19823 \\
4.21758\end{array}$ & $\begin{array}{l}2377 \\
2212 \\
2066 \\
1935 \\
1819\end{array}$ & $\begin{array}{l}28.37075 \\
28.60759 \\
28.83287 \\
29.04768 \\
29.25299\end{array}$ & $\begin{array}{l}23684 \\
22528 \\
21481 \\
20531 \\
19662\end{array}$ & $\begin{array}{l}32.50244 \\
32.76305 \\
33.01044 \\
33.24591 \\
33.47057\end{array}$ & $\begin{array}{l}26061 \\
24739 \\
23547 \\
22466 \\
21482\end{array}$ \\
\hline $\begin{array}{l}2200 \\
2300 \\
2400 \\
2500 \\
2600\end{array}$ & $\begin{array}{l}4.62416 \\
4.63629 \\
4.64787 \\
4.65897 \\
4.66966\end{array}$ & $\begin{array}{l}1213 \\
1158 \\
1110 \\
1069 \\
1034\end{array}$ & $\begin{array}{l}4.23577 \\
4.25293 \\
4.26914 \\
4.28452 \\
4.29912\end{array}$ & $\begin{array}{l}1716 \\
1621 \\
1538 \\
1460 \\
1392\end{array}$ & $\begin{array}{l}29.44961 \\
29.63828 \\
29.81963 \\
29.99422 \\
30.16255\end{array}$ & $\begin{array}{l}18867 \\
18135 \\
17459 \\
16833 \\
16251\end{array}$ & $\begin{array}{l}33.68539 \\
33.89121 \\
34.08878 \\
34.27874 \\
34.46168\end{array}$ & $\begin{array}{l}20582 \\
19757 \\
18996 \\
18294 \\
17643\end{array}$ \\
\hline $\begin{array}{l}2700 \\
2800 \\
2900 \\
3000\end{array}$ & $\begin{array}{l}4.68000 \\
4.69002 \\
4.69976 \\
4.70926\end{array}$ & $\begin{array}{r}1002 \\
974 \\
950 \\
1837\end{array}$ & $\begin{array}{l}4.31304 \\
4.32633 \\
4.33904 \\
4.35122\end{array}$ & $\begin{array}{l}1329 \\
1271 \\
1218 \\
2295\end{array}$ & $\begin{array}{l}30.32506 \\
30.48216 \\
30.63420 \\
30.78151\end{array}$ & $\begin{array}{l}15710 \\
15204 \\
14731 \\
28156\end{array}$ & $\begin{array}{l}34.63811 \\
34.80849 \\
34.97324 \\
35.13273\end{array}$ & $\begin{array}{l}17038 \\
16475 \\
15949 \\
30452\end{array}$ \\
\hline $\begin{array}{l}3200 \\
3400 \\
3600 \\
3800 \\
4000\end{array}$ & $\begin{array}{l}4.72763 \\
4.74529 \\
4.76236 \\
4.77895 \\
4.79512\end{array}$ & $\begin{array}{l}1766 \\
1707 \\
1659 \\
1617 \\
1580\end{array}$ & $\begin{array}{l}4.37417 \\
4.39549 \\
4.41540 \\
4.43410 \\
4.45175\end{array}$ & $\begin{array}{l}2132 \\
1991 \\
1870 \\
1765 \\
1673\end{array}$ & $\begin{array}{l}31.06307 \\
31.32890 \\
31.58071 \\
31.81995 \\
32.04784\end{array}$ & $\begin{array}{l}26583 \\
25181 \\
23924 \\
22789 \\
21761\end{array}$ & $\begin{array}{l}35.43725 \\
35.72440 \\
35.99611 \\
36.25405 \\
36.49959\end{array}$ & $\begin{array}{l}28715 \\
27171 \\
25794 \\
24554 \\
23434\end{array}$ \\
\hline $\begin{array}{l}4200 \\
4400 \\
4600 \\
4800 \\
5000\end{array}$ & $\begin{array}{l}4.81092 \\
4.82641 \\
4.84162 \\
4.85657 \\
4.87129\end{array}$ & $\begin{array}{l}1549 \\
1521 \\
1495 \\
1472\end{array}$ & $\begin{array}{l}4.46848 \\
4.48439 \\
4.49960 \\
4.51416 \\
4.52815\end{array}$ & $\begin{array}{l}1591 \\
1521 \\
1456 \\
1399\end{array}$ & $\begin{array}{l}32.26545 \\
32.47369 \\
32.67337 \\
32.86518 \\
33.04974\end{array}$ & $\begin{array}{l}20824 \\
19968 \\
19181 \\
18456\end{array}$ & $\begin{array}{l}36.73393 \\
36.95809 \\
37.17297 \\
37.37935 \\
37.57790\end{array}$ & $\begin{array}{l}22416 \\
21488 \\
20638 \\
19855\end{array}$ \\
\hline
\end{tabular}


Table 2.048. AgT

\begin{tabular}{|c|c|c|c|c|c|c|c|c|}
\hline${ }^{\circ} \mathrm{K}$ & $\frac{C_{p}^{0}}{R}$ & & $\frac{\left(H^{\circ}-E_{0}^{0}\right)}{R T}$ & & $\frac{-\left(F^{\circ}-E\right.}{R T}$ & & $\frac{S^{0}}{R}$ & \\
\hline $\begin{array}{l}50 \\
60 \\
70 \\
80 \\
90\end{array}$ & $\begin{array}{l}3.50128 \\
3.50150 \\
3.50172 \\
3.50195 \\
3.50221\end{array}$ & $\begin{array}{l}22 \\
22 \\
23 \\
26 \\
32\end{array}$ & $\begin{array}{l}3.47973 \\
3.48334 \\
3.48595 \\
3.48793 \\
3.48950\end{array}$ & $\begin{array}{l}361 \\
261 \\
198 \\
157 \\
129\end{array}$ & $\begin{array}{l}15.97510 \\
16.60987 \\
17.14703 \\
17.61265 \\
18.02356\end{array}$ & $\begin{array}{l}63477 \\
53716 \\
46562 \\
41091 \\
36773\end{array}$ & $\begin{array}{l}19.45483 \\
20.09321 \\
20.63298 \\
21.10059 \\
21.51307\end{array}$ & $\begin{array}{l}63838 \\
53977 \\
46761 \\
41248 \\
36901\end{array}$ \\
\hline $\begin{array}{l}100 \\
110 \\
120 \\
130 \\
140\end{array}$ & $\begin{array}{l}3.50253 \\
3.50300 \\
3.50376 \\
3.50500 \\
3.50694\end{array}$ & $\begin{array}{r}47 \\
76 \\
124 \\
194 \\
288\end{array}$ & $\begin{array}{l}3.49079 \\
3.49188 \\
3.49283 \\
3.49372 \\
3.49459\end{array}$ & $\begin{array}{r}109 \\
95 \\
89 \\
87 \\
91\end{array}$ & $\begin{array}{l}18.39129 \\
18.72405 \\
19.02793 \\
19.30754 \\
19.56648\end{array}$ & $\begin{array}{l}33276 \\
30388 \\
27961 \\
25894 \\
24113\end{array}$ & $\begin{array}{l}21.88208 \\
22.21593 \\
22.52076 \\
22.80126 \\
23.06107\end{array}$ & $\begin{array}{l}33385 \\
30483 \\
28050 \\
25981 \\
24205\end{array}$ \\
\hline $\begin{array}{l}150 \\
160 \\
170 \\
180 \\
190\end{array}$ & $\begin{array}{l}3.50982 \\
3.51381 \\
3.51911 \\
3.52582 \\
3.53401\end{array}$ & $\begin{array}{l}399 \\
530 \\
671 \\
819 \\
969\end{array}$ & $\begin{array}{l}3.49550 \\
3.49651 \\
3.49768 \\
3.49905 \\
3.50067\end{array}$ & $\begin{array}{l}101 \\
117 \\
137 \\
162 \\
190\end{array}$ & $\begin{array}{l}19.80761 \\
20.03324 \\
20.24525 \\
20.44521 \\
20.63444\end{array}$ & $\begin{array}{l}22563 \\
21201 \\
19996 \\
18923 \\
17960\end{array}$ & $\begin{array}{l}23.30312 \\
23.52976 \\
23.74294 \\
23.94427 \\
24.13511\end{array}$ & $\begin{array}{l}22664 \\
21318 \\
20133 \\
19084 \\
18151\end{array}$ \\
\hline $\begin{array}{l}200 \\
210 \\
220 \\
230 \\
240\end{array}$ & $\begin{array}{l}3.54370 \\
3.55484 \\
3.56737 \\
3.58118 \\
3.59616\end{array}$ & $\begin{array}{l}1114 \\
1253 \\
1381 \\
1498 \\
1599\end{array}$ & $\begin{array}{l}3.50257 \\
3.50479 \\
3.50735 \\
3.51025 \\
3.51351\end{array}$ & $\begin{array}{l}222 \\
256 \\
290 \\
326 \\
363\end{array}$ & $\begin{array}{l}20.81404 \\
20.98499 \\
21.14809 \\
21.30406 \\
21.45352\end{array}$ & $\begin{array}{l}17095 \\
16310 \\
15597 \\
14946 \\
14350\end{array}$ & $\begin{array}{l}24.31662 \\
24.48978 \\
24.65544 \\
24.81432 \\
24.96704\end{array}$ & $\begin{array}{l}17316 \\
16566 \\
15888 \\
15272 \\
14713\end{array}$ \\
\hline $\begin{array}{l}250 \\
260 \\
270 \\
280 \\
290\end{array}$ & $\begin{array}{l}3.61215 \\
3.62903 \\
3.64665 \\
3.66487 \\
3.68355\end{array}$ & $\begin{array}{l}1688 \\
1762 \\
1822 \\
1868 \\
1903\end{array}$ & $\begin{array}{l}3.51714 \\
3.52111 \\
3.52543 \\
3.53009 \\
3.53506\end{array}$ & $\begin{array}{l}397 \\
432 \\
466 \\
497 \\
526\end{array}$ & $\begin{array}{l}21.59702 \\
21.73504 \\
21.86801 \\
21.99631 \\
22.12027\end{array}$ & $\begin{array}{l}13802 \\
13297 \\
12830 \\
12396 \\
11993\end{array}$ & $\begin{array}{l}25.11417 \\
25.25616 \\
25.39345 \\
25.52640 \\
25.65533\end{array}$ & $\begin{array}{l}14199 \\
13729 \\
13295 \\
12893 \\
12520\end{array}$ \\
\hline $\begin{array}{l}300 \\
310 \\
320 \\
330 \\
340\end{array}$ & $\begin{array}{l}3.70258 \\
3.72184 \\
3.74123 \\
3.76065 \\
3.78003\end{array}$ & $\begin{array}{l}1926 \\
1939 \\
1942 \\
1938 \\
1926\end{array}$ & $\begin{array}{l}3.54032 \\
3.54587 \\
3.55167 \\
3.55771 \\
3.56396\end{array}$ & $\begin{array}{l}555 \\
580 \\
604 \\
625 \\
645\end{array}$ & $\begin{array}{l}22.24020 \\
22.35638 \\
22.46904 \\
22.57843 \\
22.68473\end{array}$ & $\begin{array}{l}11618 \\
11266 \\
10939 \\
10630 \\
10340\end{array}$ & $\begin{array}{l}25.78053 \\
25.90225 \\
26.02072 \\
26.13614 \\
26.24869\end{array}$ & $\begin{array}{l}12172 \\
11847 \\
11542 \\
11255 \\
10986\end{array}$ \\
\hline $\begin{array}{l}350 \\
360 \\
370 \\
380 \\
390\end{array}$ & $\begin{array}{l}3.79929 \\
3.81837 \\
3.83723 \\
3.85582 \\
3.87410\end{array}$ & $\begin{array}{l}1908 \\
1886 \\
1859 \\
1828 \\
1794\end{array}$ & $\begin{array}{l}3.57041 \\
3.57703 \\
3.58381 \\
3.59073 \\
3.59776\end{array}$ & $\begin{array}{l}662 \\
678 \\
692 \\
703 \\
713\end{array}$ & $\begin{array}{l}22.78813 \\
22.88881 \\
22.98690 \\
23.08257 \\
23.17593\end{array}$ & $\begin{array}{r}10068 \\
9809 \\
9567 \\
9336 \\
9118\end{array}$ & $\begin{array}{l}26.35855 \\
26.46584 \\
26.57072 \\
26.67330 \\
26.77369\end{array}$ & $\begin{array}{r}10729 \\
10488 \\
10258 \\
10039 \\
9832\end{array}$ \\
\hline $\begin{array}{l}400 \\
450 \\
500 \\
550 \\
600\end{array}$ & $\begin{array}{l}3.89204 \\
3.97608 \\
4.05000 \\
4.11413 \\
4.16947\end{array}$ & $\begin{array}{l}8404 \\
7392 \\
6413 \\
5534 \\
4776\end{array}$ & $\begin{array}{l}3.60489 \\
3.64156 \\
3.67879 \\
3.71552 \\
3.75110\end{array}$ & $\begin{array}{l}3667 \\
3723 \\
3673 \\
3558 \\
3407\end{array}$ & $\begin{array}{l}23.26711 \\
23.69381 \\
24.07941 \\
24.43177 \\
24.75660\end{array}$ & $\begin{array}{l}42670 \\
38560 \\
35236 \\
32483 \\
30160\end{array}$ & $\begin{array}{l}26.87201 \\
27.33537 \\
27.75821 \\
28.14730 \\
28.50770\end{array}$ & $\begin{array}{l}46336 \\
42284 \\
38909 \\
36040 \\
33567\end{array}$ \\
\hline $\begin{array}{l}650 \\
700 \\
750 \\
800 \\
850\end{array}$ & $\begin{array}{l}4.21723 \\
4.25857 \\
4.29453 \\
4.32598 \\
4.35367\end{array}$ & $\begin{array}{l}4134 \\
3596 \\
3145 \\
2769 \\
2454\end{array}$ & $\begin{array}{l}3.78517 \\
3.81754 \\
3.84817 \\
3.87707 \\
3.90431\end{array}$ & $\begin{array}{l}3237 \\
3063 \\
2890 \\
2724 \\
2566\end{array}$ & $\begin{array}{l}25.05820 \\
25.33991 \\
25.60435 \\
25.85363 \\
26.08951\end{array}$ & $\begin{array}{l}28171 \\
26444 \\
24928 \\
23588 \\
22389\end{array}$ & $\begin{array}{l}28.84337 \\
29.15745 \\
29.45252 \\
29.73071 \\
29.99382\end{array}$ & $\begin{array}{l}31408 \\
29507 \\
27819 \\
26311 \\
24955\end{array}$ \\
\hline $\begin{array}{r}900 \\
950 \\
1000 \\
1050 \\
1100\end{array}$ & $\begin{array}{l}4.37821 \\
4.40010 \\
4.41976 \\
4.43751 \\
4.45366\end{array}$ & $\begin{array}{l}2189 \\
1966 \\
1775 \\
1615 \\
1476\end{array}$ & $\begin{array}{l}3.92997 \\
3.95414 \\
3.97694 \\
3.99846 \\
4.01879\end{array}$ & $\begin{array}{l}2417 \\
2280 \\
2152 \\
2033 \\
1923\end{array}$ & $\begin{array}{l}26.31340 \\
26.52654 \\
26.72995 \\
26.92451 \\
27.11099\end{array}$ & $\begin{array}{l}21314 \\
20341 \\
19456 \\
18648 \\
17907\end{array}$ & $\begin{array}{l}30.24337 \\
30.48069 \\
30.70689 \\
30.92297 \\
31.12978\end{array}$ & $\begin{array}{l}23732 \\
22620 \\
21608 \\
20681 \\
19831\end{array}$ \\
\hline 1150 & 4.46842 & 1358 & 4.03802 & 1822 & 27.29006 & 17225 & 31.32809 & 19046 \\
\hline
\end{tabular}


Table 2.048. AgT (Cont.)

\begin{tabular}{|c|c|c|c|c|c|c|c|c|}
\hline${ }^{\circ} \mathrm{K}$ & $\frac{C_{p}^{\circ}}{R}$ & & $\frac{\left(H^{0}-E_{0}^{0}\right)}{R T}$ & & $\frac{- \text { IF }^{\circ}-E}{R T}$ & & $\frac{S^{\circ}}{R}$ & \\
\hline $\begin{array}{l}1200 \\
1300 \\
1400 \\
1500 \\
1600\end{array}$ & $\begin{array}{l}4.48200 \\
4.50621 \\
4.52730 \\
4.54599 \\
4.56282\end{array}$ & $\begin{array}{l}2421 \\
2109 \\
1869 \\
1683 \\
1536\end{array}$ & $\begin{array}{l}4.05624 \\
4.08995 \\
4.12045 \\
4.14821 \\
4.17360\end{array}$ & $\begin{array}{l}3371 \\
3050 \\
2776 \\
2539 \\
2336\end{array}$ & $\begin{array}{l}27.46231 \\
27.78833 \\
28.09257 \\
28.37781 \\
28.64635\end{array}$ & $\begin{array}{l}32602 \\
30424 \\
28524 \\
26854 \\
25373\end{array}$ & $\begin{array}{l}31.51855 \\
31.87828 \\
32.21302 \\
32.52602 \\
32.81996\end{array}$ & $\begin{array}{l}35973 \\
33474 \\
31300 \\
29394 \\
27709\end{array}$ \\
\hline $\begin{array}{l}1700 \\
1800 \\
1900 \\
2000 \\
2100\end{array}$ & $\begin{array}{l}4.57818 \\
4.59234 \\
4.60555 \\
4.61796 \\
4.62972\end{array}$ & $\begin{array}{l}1416 \\
1321 \\
1241 \\
1176 \\
1121\end{array}$ & $\begin{array}{l}4.19696 \\
4.21853 \\
4.23856 \\
4.25722 \\
4.27468\end{array}$ & $\begin{array}{l}2157 \\
2003 \\
1866 \\
1746 \\
1640\end{array}$ & $\begin{array}{l}28.90008 \\
29.14060 \\
29.36922 \\
29.58711 \\
29.79525\end{array}$ & $\begin{array}{l}24052 \\
22862 \\
21789 \\
20814 \\
19924\end{array}$ & $\begin{array}{l}33.09705 \\
33.35913 \\
33.60779 \\
33.84434 \\
34.06994\end{array}$ & $\begin{array}{l}26208 \\
24866 \\
23655 \\
22560 \\
21563\end{array}$ \\
\hline $\begin{array}{l}2200 \\
2300 \\
2400 \\
2500 \\
2600\end{array}$ & $\begin{array}{l}4.64093 \\
4.65168 \\
4.66203 \\
4.67204 \\
4.68176\end{array}$ & $\begin{array}{r}1075 \\
1035 \\
1001 \\
972 \\
946\end{array}$ & $\begin{array}{l}4.29108 \\
4.30652 \\
4.32112 \\
4.33496 \\
4.34811\end{array}$ & $\begin{array}{l}1544 \\
1460 \\
1384 \\
1315 \\
1253\end{array}$ & $\begin{array}{l}29.99449 \\
30.18558 \\
30.36918 \\
30.54586 \\
30.71614\end{array}$ & $\begin{array}{l}19109 \\
18360 \\
17668 \\
17028 \\
16433\end{array}$ & $\begin{array}{l}34.28557 \\
34.49211 \\
34.69030 \\
34.88082 \\
35.06425\end{array}$ & $\begin{array}{l}20654 \\
19819 \\
19052 \\
18343 \\
17687\end{array}$ \\
\hline $\begin{array}{l}2700 \\
2800 \\
2900 \\
3000\end{array}$ & $\begin{array}{l}4.69122 \\
4.70045 \\
4.70948 \\
4.71833\end{array}$ & $\begin{array}{r}923 \\
903 \\
885 \\
1724\end{array}$ & $\begin{array}{l}4.36064 \\
4.37262 \\
4.38408 \\
4.39507\end{array}$ & $\begin{array}{l}1198 \\
1146 \\
1099 \\
2075\end{array}$ & $\begin{array}{l}30.88047 \\
31.03928 \\
31.19292 \\
31.34173\end{array}$ & $\begin{array}{l}15881 \\
15364 \\
14881 \\
28432\end{array}$ & $\begin{array}{l}35.24112 \\
35.41190 \\
35.57700 \\
35.73681\end{array}$ & $\begin{array}{l}17078 \\
16510 \\
15981 \\
30507\end{array}$ \\
\hline $\begin{array}{l}3200 \\
3400 \\
3600 \\
3800 \\
4000\end{array}$ & $\begin{array}{l}4.73557 \\
4.75228 \\
4.76855 \\
4.78446 \\
4.80003\end{array}$ & $\begin{array}{l}1671 \\
1627 \\
1591 \\
1557 \\
1530\end{array}$ & $\begin{array}{l}4.41582 \\
4.43512 \\
4.45319 \\
4.47021 \\
4.48631\end{array}$ & $\begin{array}{l}1930 \\
1807 \\
1702 \\
1610 \\
1531\end{array}$ & $\begin{array}{l}31.62605 \\
31.89435 \\
32.14837 \\
32.38960 \\
32.61931\end{array}$ & $\begin{array}{l}26830 \\
25402 \\
24123 \\
22971 \\
21926\end{array}$ & $\begin{array}{l}36.04188 \\
36.32947 \\
36.60157 \\
36.85982 \\
37.10563\end{array}$ & $\begin{array}{l}28759 \\
27210 \\
25825 \\
24581 \\
23456\end{array}$ \\
\hline $\begin{array}{l}4200 \\
4400 \\
4600 \\
4800 \\
5000\end{array}$ & $\begin{array}{l}4.81533 \\
4.83037 \\
4.84518 \\
4.85979 \\
4.87420\end{array}$ & $\begin{array}{l}1504 \\
1481 \\
1461 \\
1441\end{array}$ & $\begin{array}{l}4.50162 \\
4.51622 \\
4.53020 \\
4.54363 \\
4.55657\end{array}$ & $\begin{array}{l}1460 \\
1398 \\
1343 \\
1294\end{array}$ & $\begin{array}{l}32.83857 \\
33.04832 \\
33.24939 \\
33.44248 \\
33.62822\end{array}$ & $\begin{array}{l}20975 \\
20107 \\
19309 \\
18574\end{array}$ & $\begin{array}{l}37.34019 \\
37.56455 \\
37.77960 \\
37.98612 \\
38.18479\end{array}$ & $\begin{array}{l}22436 \\
21505 \\
20652 \\
19867\end{array}$ \\
\hline
\end{tabular}


Table 2.049. AuH

\begin{tabular}{|c|c|c|c|c|c|c|c|c|}
\hline${ }^{\circ} K$ & $\frac{C_{p}^{0}}{R}$ & & $\frac{\left(H^{\circ}-E_{0}^{0}\right)}{R T}$ & & $\frac{-\left(F^{\circ}-E\right.}{R T}$ & & $\frac{S^{0}}{R}$ & \\
\hline $\begin{array}{l}50 \\
60 \\
70 \\
80 \\
90\end{array}$ & $\begin{array}{l}3.50180 \\
3.50162 \\
3.50158 \\
3.50161 \\
3.50168\end{array}$ & $\begin{array}{r}-18 \\
-\quad 4 \\
3 \\
7 \\
9\end{array}$ & $\begin{array}{l}3.43097 \\
3.44276 \\
3.45117 \\
3.45747 \\
3.46238\end{array}$ & $\begin{array}{r}1179 \\
841 \\
630 \\
491 \\
393\end{array}$ & $\begin{array}{l}15.70245 \\
16.32910 \\
16.86047 \\
17.32174 \\
17.72926\end{array}$ & $\begin{array}{l}62665 \\
53137 \\
46127 \\
40752 \\
36501\end{array}$ & $\begin{array}{l}19.13343 \\
19.77187 \\
20.31164 \\
20.77921 \\
21.19165\end{array}$ & $\begin{array}{l}63844 \\
53977 \\
46757 \\
41244 \\
36894\end{array}$ \\
\hline $\begin{array}{l}100 \\
110 \\
120 \\
130 \\
140\end{array}$ & $\begin{array}{l}3.50177 \\
3.50187 \\
3.50199 \\
3.50212 \\
3.50225\end{array}$ & $\begin{array}{l}10 \\
12 \\
13 \\
13 \\
14\end{array}$ & $\begin{array}{l}3.46631 \\
3.46954 \\
3.47224 \\
3.47453 \\
3.47651\end{array}$ & $\begin{array}{l}323 \\
270 \\
229 \\
198 \\
172\end{array}$ & $\begin{array}{l}18.09427 \\
18.42481 \\
18.72681 \\
19.00483 \\
19.26240\end{array}$ & $\begin{array}{l}33054 \\
30200 \\
27802 \\
25757 \\
23991\end{array}$ & $\begin{array}{l}21.56059 \\
21.89435 \\
22.19906 \\
22.47937 \\
22.73891\end{array}$ & $\begin{array}{l}33376 \\
30471 \\
28031 \\
25954 \\
24164\end{array}$ \\
\hline $\begin{array}{l}150 \\
160 \\
170 \\
180 \\
190\end{array}$ & $\begin{array}{l}3.50239 \\
3.50253 \\
3.50267 \\
3.50281 \\
3.50297\end{array}$ & $\begin{array}{l}14 \\
14 \\
14 \\
16 \\
16\end{array}$ & $\begin{array}{l}3.47823 \\
3.47974 \\
3.48109 \\
3.48229 \\
3.48337\end{array}$ & $\begin{array}{r}151 \\
135 \\
120 \\
108 \\
99\end{array}$ & $\begin{array}{l}19.50231 \\
19.72684 \\
19.93784 \\
20.13685 \\
20.32516\end{array}$ & $\begin{array}{l}22453 \\
21100 \\
19901 \\
18831 \\
17870\end{array}$ & $\begin{array}{l}22.98055 \\
23.20659 \\
23.41893 \\
23.61915 \\
23.80854\end{array}$ & $\begin{array}{l}22604 \\
21234 \\
20022 \\
18939 \\
17968\end{array}$ \\
\hline $\begin{array}{l}200 \\
210 \\
220 \\
230 \\
240\end{array}$ & $\begin{array}{l}3.50313 \\
3.50330 \\
3.50350 \\
3.50372 \\
3.50399\end{array}$ & $\begin{array}{l}17 \\
20 \\
22 \\
27 \\
32\end{array}$ & $\begin{array}{l}3.48436 \\
3.48526 \\
3.48608 \\
3.48684 \\
3.48755\end{array}$ & $\begin{array}{l}90 \\
82 \\
76 \\
71 \\
66\end{array}$ & $\begin{array}{l}20.50386 \\
20.67388 \\
20.83604 \\
20.99102 \\
21.13943\end{array}$ & $\begin{array}{l}17002 \\
16216 \\
15498 \\
14841 \\
14238\end{array}$ & $\begin{array}{l}23.98822 \\
24.15914 \\
24.32212 \\
24.47786 \\
24.62699\end{array}$ & $\begin{array}{l}17092 \\
16298 \\
15574 \\
14913 \\
14304\end{array}$ \\
\hline $\begin{array}{l}250 \\
260 \\
270 \\
280 \\
290\end{array}$ & $\begin{array}{l}3.50431 \\
3.50470 \\
3.50519 \\
3.50578 \\
3.50650\end{array}$ & $\begin{array}{l}39 \\
49 \\
59 \\
72 \\
87\end{array}$ & $\begin{array}{l}3.48821 \\
3.48884 \\
3.48944 \\
3.49001 \\
3.49057\end{array}$ & $\begin{array}{l}63 \\
60 \\
57 \\
56 \\
54\end{array}$ & $\begin{array}{l}21.28181 \\
21.41864 \\
21.55032 \\
21.67723 \\
21.79971\end{array}$ & $\begin{array}{l}13683 \\
13168 \\
12691 \\
12248 \\
11834\end{array}$ & $\begin{array}{l}24.77003 \\
24.90748 \\
25.03976 \\
25.16724 \\
25.29028\end{array}$ & $\begin{array}{l}13745 \\
13228 \\
12748 \\
12304 \\
11889\end{array}$ \\
\hline $\begin{array}{l}300 \\
310 \\
320 \\
330 \\
340\end{array}$ & $\begin{array}{l}3.50737 \\
3.50841 \\
3.50965 \\
3.51110 \\
3.51278\end{array}$ & $\begin{array}{l}104 \\
124 \\
145 \\
168 \\
193\end{array}$ & $\begin{array}{l}3.49111 \\
3.49165 \\
3.49219 \\
3.49275 \\
3.49331\end{array}$ & $\begin{array}{l}54 \\
54 \\
56 \\
56 \\
58\end{array}$ & $\begin{array}{l}21.91805 \\
22.03254 \\
22.14340 \\
22.25087 \\
22.35515\end{array}$ & $\begin{array}{l}11449 \\
11086 \\
10747 \\
10428 \\
10127\end{array}$ & $\begin{array}{l}25.40917 \\
25.52419 \\
25.63560 \\
25.74362 \\
25.84846\end{array}$ & $\begin{array}{l}11502 \\
11141 \\
10802 \\
10484 \\
10185\end{array}$ \\
\hline $\begin{array}{l}350 \\
360 \\
370 \\
380 \\
390\end{array}$ & $\begin{array}{l}3.51471 \\
3.51691 \\
3.51938 \\
3.52214 \\
3.52520\end{array}$ & $\begin{array}{l}220 \\
247 \\
276 \\
306 \\
336\end{array}$ & $\begin{array}{l}3.49389 \\
3.49450 \\
3.49514 \\
3.49581 \\
3.49653\end{array}$ & $\begin{array}{l}61 \\
64 \\
67 \\
72 \\
75\end{array}$ & $\begin{array}{l}22.45642 \\
22.55485 \\
22.65061 \\
22.74382 \\
22.83464\end{array}$ & $\begin{array}{l}9843 \\
9576 \\
9321 \\
9082 \\
8853\end{array}$ & $\begin{array}{l}25.95031 \\
26.04936 \\
26.14575 \\
26.23964 \\
26.33117\end{array}$ & $\begin{array}{l}9905 \\
9639 \\
9389 \\
9153 \\
8929\end{array}$ \\
\hline $\begin{array}{l}400 \\
450 \\
500 \\
550 \\
600\end{array}$ & $\begin{array}{l}3.52856 \\
3.55000 \\
3.57883 \\
3.61391 \\
3.65367\end{array}$ & $\begin{array}{l}2144 \\
2883 \\
3508 \\
3976 \\
4282\end{array}$ & $\begin{array}{l}3.49728 \\
3.50188 \\
3.50808 \\
3.51606 \\
3.52584\end{array}$ & $\begin{array}{r}460 \\
620 \\
798 \\
978 \\
1147\end{array}$ & $\begin{array}{l}22.92317 \\
23.33535 \\
23.70461 \\
24.03933 \\
24.34567\end{array}$ & $\begin{array}{l}41218 \\
36926 \\
33472 \\
30634 \\
28266\end{array}$ & $\begin{array}{l}26.42046 \\
26.83723 \\
27.21269 \\
27.55539 \\
27.87152\end{array}$ & $\begin{array}{l}41677 \\
37546 \\
34270 \\
31613 \\
29413\end{array}$ \\
\hline $\begin{array}{l}650 \\
700 \\
750 \\
800 \\
850\end{array}$ & $\begin{array}{l}3.69649 \\
3.74093 \\
3.78578 \\
3.83014 \\
3.87335\end{array}$ & $\begin{array}{l}4444 \\
4485 \\
4436 \\
4321 \\
4162\end{array}$ & $\begin{array}{l}3.53731 \\
3.55026 \\
3.56447 \\
3.57969 \\
3.59570\end{array}$ & $\begin{array}{l}1295 \\
1421 \\
1522 \\
1601 \\
1659\end{array}$ & $\begin{array}{l}24.62833 \\
24.89094 \\
25.13636 \\
25.36689 \\
25.58438\end{array}$ & $\begin{array}{l}26261 \\
24542 \\
23053 \\
21749 \\
20600\end{array}$ & $\begin{array}{l}28.16565 \\
28.44121 \\
28.70083 \\
28.94658 \\
29.18009\end{array}$ & $\begin{array}{l}27556 \\
25962 \\
24575 \\
23351 \\
22258\end{array}$ \\
\hline $\begin{array}{r}900 \\
950 \\
1000 \\
1050 \\
1100\end{array}$ & $\begin{array}{l}3.91497 \\
3.95473 \\
3.99247 \\
4.02814 \\
4.06174\end{array}$ & $\begin{array}{l}3976 \\
3774 \\
3567 \\
3360 \\
3159\end{array}$ & $\begin{array}{l}3.61229 \\
3.62928 \\
3.64650 \\
3.66383 \\
3.68116\end{array}$ & $\begin{array}{l}1699 \\
1722 \\
1733 \\
1733 \\
1724\end{array}$ & $\begin{array}{l}25.79038 \\
25.98614 \\
26.17273 \\
26.35106 \\
26.52191\end{array}$ & $\begin{array}{l}19576 \\
18659 \\
17833 \\
17085 \\
16401\end{array}$ & $\begin{array}{l}29.40267 \\
29.61542 \\
29.81924 \\
30.01490 \\
30.20307\end{array}$ & $\begin{array}{l}21275 \\
20382 \\
19566 \\
18817 \\
18126\end{array}$ \\
\hline 1150 & 4.09333 & 2966 & 3.69840 & 1708 & 26.68592 & 15777 & 30.38433 & 17484 \\
\hline
\end{tabular}


Table 2. 049. AuH (Cont.)

\begin{tabular}{|c|c|c|c|c|c|c|c|c|}
\hline${ }^{\circ} \mathrm{K}$ & $\frac{C_{p}^{0}}{R}$ & & $\frac{\left(H^{0}-E_{0}^{0}\right)}{R T}$ & & $\frac{-\left(F^{0}-\right.}{R T}$ & & $\frac{S^{0}}{R}$ & \\
\hline $\begin{array}{l}1200 \\
1300 \\
1400 \\
1500 \\
1600\end{array}$ & $\begin{array}{l}4.12299 \\
4.17692 \\
4.22440 \\
4.26629 \\
4.30340\end{array}$ & $\begin{array}{l}5393 \\
4748 \\
4189 \\
3711 \\
3303\end{array}$ & $\begin{array}{l}3.71548 \\
3.74895 \\
3.78125 \\
3.81222 \\
3.84178\end{array}$ & $\begin{array}{l}3347 \\
3230 \\
3097 \\
2956 \\
2814\end{array}$ & $\begin{array}{l}26.84369 \\
27.14241 \\
27.42143 \\
27.68337 \\
27.93036\end{array}$ & $\begin{array}{l}29872 \\
27902 \\
26194 \\
24699 \\
23376\end{array}$ & $\begin{array}{l}30.55917 \\
30.89137 \\
31.20268 \\
31.49560 \\
31.77214\end{array}$ & $\begin{array}{l}33220 \\
31131 \\
29292 \\
27654 \\
26191\end{array}$ \\
\hline $\begin{array}{l}1700 \\
1800 \\
1900 \\
2000 \\
2100\end{array}$ & $\begin{array}{l}4.33643 \\
4.36599 \\
4.39258 \\
4.41664 \\
4.43854\end{array}$ & $\begin{array}{l}2956 \\
2659 \\
2406 \\
2190 \\
2004\end{array}$ & $\begin{array}{l}3.86992 \\
3.89668 \\
3.92209 \\
3.94623 \\
3.96916\end{array}$ & $\begin{array}{l}2676 \\
2541 \\
2414 \\
2293 \\
2179\end{array}$ & $\begin{array}{l}28.16412 \\
28.38608 \\
28.59745 \\
28.79925 \\
28.99234\end{array}$ & $\begin{array}{l}22196 \\
21137 \\
20180 \\
19309 \\
18516\end{array}$ & $\begin{array}{l}32.03405 \\
32.28276 \\
32.51954 \\
32.74548 \\
32.96150\end{array}$ & $\begin{array}{l}24871 \\
23678 \\
22594 \\
21602 \\
20695\end{array}$ \\
\hline $\begin{array}{l}2200 \\
2300 \\
2400 \\
2500 \\
2600\end{array}$ & $\begin{array}{l}4.45858 \\
4.47701 \\
4.49405 \\
4.50988 \\
4.52466\end{array}$ & $\begin{array}{l}1843 \\
1704 \\
1583 \\
1478 \\
1385\end{array}$ & $\begin{array}{l}3.99095 \\
4.01169 \\
4.03144 \\
4.05026 \\
4.06823\end{array}$ & $\begin{array}{l}2074 \\
1975 \\
1882 \\
1797 \\
1716\end{array}$ & $\begin{array}{l}29.17750 \\
29.35536 \\
29.52652 \\
29.69147 \\
29.85068\end{array}$ & $\begin{array}{l}17786 \\
17116 \\
16495 \\
15921 \\
15386\end{array}$ & $\begin{array}{l}33.16845 \\
33.36706 \\
33.55796 \\
33.74174 \\
33.91891\end{array}$ & $\begin{array}{l}19861 \\
19090 \\
18378 \\
17717 \\
17103\end{array}$ \\
\hline $\begin{array}{l}2700 \\
2800 \\
2900 \\
3000\end{array}$ & $\begin{array}{l}4.53851 \\
4.55155 \\
4.56388 \\
4.57557\end{array}$ & $\begin{array}{l}1304 \\
1233 \\
1169 \\
2175\end{array}$ & $\begin{array}{l}4.08539 \\
4.10181 \\
4.11753 \\
4.13261\end{array}$ & $\begin{array}{l}1642 \\
1572 \\
1508 \\
2837\end{array}$ & $\begin{array}{l}30.00454 \\
30.15342 \\
30.29763 \\
30.43748\end{array}$ & $\begin{array}{l}14888 \\
14421 \\
13985 \\
26763\end{array}$ & $\begin{array}{l}34.08994 \\
34.25523 \\
34.41517 \\
34.57009\end{array}$ & $\begin{array}{l}16529 \\
15994 \\
15492 \\
29601\end{array}$ \\
\hline $\begin{array}{l}3200 \\
3400 \\
3600 \\
3800 \\
4000\end{array}$ & $\begin{array}{l}4.59732 \\
4.61727 \\
4.63576 \\
4.65305 \\
4.66936\end{array}$ & $\begin{array}{l}1995 \\
1849 \\
1729 \\
1631 \\
1549\end{array}$ & $\begin{array}{l}4.16098 \\
4.18724 \\
4.21165 \\
4.23444 \\
4.25578\end{array}$ & $\begin{array}{l}2626 \\
2441 \\
2279 \\
2134 \\
2006\end{array}$ & $\begin{array}{l}30.70511 \\
30.95817 \\
31.19820 \\
31.42653 \\
31.64428\end{array}$ & $\begin{array}{l}25306 \\
24003 \\
22833 \\
21775 \\
20813\end{array}$ & $\begin{array}{l}34.86610 \\
35.14541 \\
35.40986 \\
35.66097 \\
35.90006\end{array}$ & $\begin{array}{l}27931 \\
26445 \\
25111 \\
23909 \\
22820\end{array}$ \\
\hline $\begin{array}{l}4200 \\
4400 \\
4600 \\
4800 \\
5000\end{array}$ & $\begin{array}{l}4.68485 \\
4.69964 \\
4.71383 \\
4.72752 \\
4.74077\end{array}$ & $\begin{array}{l}1479 \\
1419 \\
1369 \\
1325\end{array}$ & $\begin{array}{l}4.27584 \\
4.29477 \\
4.31269 \\
4.32969 \\
4.34587\end{array}$ & $\begin{array}{l}1893 \\
1792 \\
1700 \\
1618\end{array}$ & $\begin{array}{l}31.85241 \\
32.05176 \\
32.24307 \\
32.42698 \\
32.60406\end{array}$ & $\begin{array}{l}19935 \\
19131 \\
18391 \\
17708\end{array}$ & $\begin{array}{l}36.12826 \\
36.34654 \\
36.55576 \\
36.75667 \\
36.94993\end{array}$ & $\begin{array}{l}21828 \\
20922 \\
20091 \\
19326\end{array}$ \\
\hline
\end{tabular}


Table 2.050. AuD

\begin{tabular}{|c|c|c|c|c|c|c|c|c|}
\hline${ }^{\circ} \mathrm{K}$ & $\frac{C_{p}^{0}}{R}$ & & $\frac{\left(H^{\circ}-E_{0}^{0}\right)}{R T}$ & & $\frac{-\left(F^{0}-1\right.}{R T}$ & & $\frac{S^{0}}{R}$ & \\
\hline $\begin{array}{l}50 \\
60 \\
70 \\
80 \\
90\end{array}$ & $\begin{array}{l}3.50101 \\
3.50108 \\
3.50119 \\
3.50131 \\
3.50144\end{array}$ & $\begin{array}{r}7 \\
11 \\
12 \\
13 \\
14\end{array}$ & $\begin{array}{l}3.46557 \\
3.47148 \\
3.47572 \\
3.47891 \\
3.48141\end{array}$ & $\begin{array}{l}591 \\
424 \\
319 \\
250 \\
201\end{array}$ & $\begin{array}{l}16.35863 \\
16.99104 \\
17.52650 \\
17.99084 \\
18.40075\end{array}$ & $\begin{array}{l}63241 \\
53546 \\
46434 \\
40991 \\
36691\end{array}$ & $\begin{array}{l}19.82421 \\
20.46253 \\
21.00223 \\
21.46976 \\
21.88216\end{array}$ & $\begin{array}{l}63832 \\
53970 \\
46753 \\
41240 \\
36892\end{array}$ \\
\hline $\begin{array}{l}100 \\
110 \\
120 \\
130 \\
140\end{array}$ & $\begin{array}{l}3.50158 \\
3.50172 \\
3.50187 \\
3.50202 \\
3.50218\end{array}$ & $\begin{array}{l}14 \\
15 \\
15 \\
16 \\
18\end{array}$ & $\begin{array}{l}3.48342 \\
3.48508 \\
3.48647 \\
3.48766 \\
3.48869\end{array}$ & $\begin{array}{r}166 \\
139 \\
119 \\
103 \\
90\end{array}$ & $\begin{array}{l}18.76766 \\
19.09974 \\
19.40305 \\
19.68216 \\
19.94066\end{array}$ & $\begin{array}{l}33208 \\
30331 \\
27911 \\
25850 \\
24073\end{array}$ & $\begin{array}{l}22.25108 \\
22.58482 \\
22.88952 \\
23.16983 \\
23.42936\end{array}$ & $\begin{array}{l}33374 \\
30470 \\
28031 \\
25953 \\
24163\end{array}$ \\
\hline $\begin{array}{l}150 \\
160 \\
170 \\
180 \\
190\end{array}$ & $\begin{array}{l}3.50236 \\
3.50258 \\
3.50286 \\
3.50325 \\
3.50377\end{array}$ & $\begin{array}{l}22 \\
28 \\
39 \\
52 \\
71\end{array}$ & $\begin{array}{l}3.48959 \\
3.49040 \\
3.49112 \\
3.49179 \\
3.49240\end{array}$ & $\begin{array}{l}81 \\
72 \\
67 \\
61 \\
59\end{array}$ & $\begin{array}{l}20.18139 \\
20.40663 \\
20.61826 \\
20.81782 \\
21.00663\end{array}$ & $\begin{array}{l}22524 \\
21163 \\
19956 \\
18881 \\
17915\end{array}$ & $\begin{array}{l}23.67099 \\
23.89704 \\
24.10939 \\
24.30962 \\
24.49904\end{array}$ & $\begin{array}{l}22605 \\
21235 \\
20023 \\
18942 \\
17974\end{array}$ \\
\hline $\begin{array}{l}200 \\
210 \\
220 \\
230 \\
240\end{array}$ & $\begin{array}{l}3.50448 \\
3.50543 \\
3.50669 \\
3.50831 \\
3.51034\end{array}$ & $\begin{array}{r}95 \\
126 \\
162 \\
203 \\
249\end{array}$ & $\begin{array}{l}3.49299 \\
3.49356 \\
3.49412 \\
3.49470 \\
3.49531\end{array}$ & $\begin{array}{l}57 \\
56 \\
58 \\
61 \\
65\end{array}$ & $\begin{array}{l}21.18578 \\
21.35622 \\
21.51876 \\
21.67409 \\
21.82283\end{array}$ & $\begin{array}{l}17044 \\
16254 \\
15533 \\
14874 \\
14270\end{array}$ & $\begin{array}{l}24.67878 \\
24.84978 \\
25.01288 \\
25.16880 \\
25.31815\end{array}$ & $\begin{array}{l}17100 \\
16310 \\
15592 \\
14935 \\
14335\end{array}$ \\
\hline $\begin{array}{l}250 \\
260 \\
270 \\
280 \\
290\end{array}$ & $\begin{array}{l}3.51283 \\
3.51583 \\
3.51936 \\
3.52347 \\
3.52815\end{array}$ & $\begin{array}{l}300 \\
353 \\
411 \\
468 \\
529\end{array}$ & $\begin{array}{l}3.49596 \\
3.49667 \\
3.49744 \\
3.49829 \\
3.49924\end{array}$ & $\begin{array}{r}71 \\
77 \\
85 \\
95 \\
105\end{array}$ & $\begin{array}{l}21.96553 \\
22.10266 \\
22.23464 \\
22.36185 \\
22.48463\end{array}$ & $\begin{array}{l}13713 \\
13198 \\
12721 \\
12278 \\
11864\end{array}$ & $\begin{array}{l}25.46150 \\
25.59933 \\
25.73209 \\
25.86015 \\
25.98387\end{array}$ & $\begin{array}{l}13783 \\
13276 \\
12806 \\
12372 \\
11970\end{array}$ \\
\hline $\begin{array}{l}300 \\
310 \\
320 \\
330 \\
340\end{array}$ & $\begin{array}{l}3.53344 \\
3.53931 \\
3.54578 \\
3.55283 \\
3.56044\end{array}$ & $\begin{array}{l}587 \\
647 \\
705 \\
761 \\
814\end{array}$ & $\begin{array}{l}3.50029 \\
3.50145 \\
3.50274 \\
3.50415 \\
3.50569\end{array}$ & $\begin{array}{l}116 \\
129 \\
141 \\
154 \\
168\end{array}$ & $\begin{array}{l}22.60327 \\
22.71807 \\
22.82925 \\
22.93706 \\
23.04169\end{array}$ & $\begin{array}{l}11480 \\
11118 \\
10781 \\
10463 \\
10165\end{array}$ & $\begin{array}{l}26.10357 \\
26.21952 \\
26.33199 \\
26.44121 \\
26.54738\end{array}$ & $\begin{array}{l}11595 \\
11247 \\
10922 \\
10617 \\
10333\end{array}$ \\
\hline $\begin{array}{l}350 \\
360 \\
370 \\
380 \\
390\end{array}$ & $\begin{array}{l}3.56858 \\
3.57724 \\
3.58637 \\
3.59595 \\
3.60593\end{array}$ & $\begin{array}{r}866 \\
913 \\
958 \\
998 \\
1037\end{array}$ & $\begin{array}{l}3.50737 \\
3.50919 \\
3.51115 \\
3.51325 \\
3.51550\end{array}$ & $\begin{array}{l}182 \\
196 \\
210 \\
225 \\
239\end{array}$ & $\begin{array}{l}23.14334 \\
23.24217 \\
23.33834 \\
23.43201 \\
23.52329\end{array}$ & $\begin{array}{l}9883 \\
9617 \\
9367 \\
9128 \\
8904\end{array}$ & $\begin{array}{l}26.65071 \\
26.75136 \\
26.84950 \\
26.94526 \\
27.03880\end{array}$ & $\begin{array}{r}10065 \\
9814 \\
9576 \\
9354 \\
9142\end{array}$ \\
\hline $\begin{array}{l}400 \\
450 \\
500 \\
550 \\
600\end{array}$ & $\begin{array}{l}3.61630 \\
3.67249 \\
3.73295 \\
3.79427 \\
3.85411\end{array}$ & $\begin{array}{l}5619 \\
6046 \\
6132 \\
5984 \\
5691\end{array}$ & $\begin{array}{l}3.51789 \\
3.53189 \\
3.54895 \\
3.56847 \\
3.58980\end{array}$ & $\begin{array}{l}1400 \\
1706 \\
1952 \\
2133 \\
2254\end{array}$ & $\begin{array}{l}23.61233 \\
24.02745 \\
24.40043 \\
24.73958 \\
25.05098\end{array}$ & $\begin{array}{l}41512 \\
37298 \\
33915 \\
31140 \\
28823\end{array}$ & $\begin{array}{l}27.13022 \\
27.55935 \\
27.94939 \\
28.30806 \\
28.64079\end{array}$ & $\begin{array}{l}42913 \\
39004 \\
35867 \\
33273 \\
31076\end{array}$ \\
\hline $\begin{array}{l}650 \\
700 \\
750 \\
800 \\
850\end{array}$ & $\begin{array}{l}3.91102 \\
3.96422 \\
4.01340 \\
4.05855 \\
4.09983\end{array}$ & $\begin{array}{l}5320 \\
4918 \\
4515 \\
4128 \\
3765\end{array}$ & $\begin{array}{l}3.61234 \\
3.63560 \\
3.65917 \\
3.68274 \\
3.70608\end{array}$ & $\begin{array}{l}2326 \\
2357 \\
2357 \\
2334 \\
2293\end{array}$ & $\begin{array}{l}25.33921 \\
25.60776 \\
25.85939 \\
26.09630 \\
26.32027\end{array}$ & $\begin{array}{l}26855 \\
25163 \\
23691 \\
22397 \\
21248\end{array}$ & $\begin{array}{l}28.95155 \\
29.24336 \\
29.51856 \\
29.77904 \\
30.02635\end{array}$ & $\begin{array}{l}29181 \\
27520 \\
26048 \\
24731 \\
23542\end{array}$ \\
\hline $\begin{array}{r}900 \\
950 \\
1000 \\
1050 \\
1100\end{array}$ & $\begin{array}{l}4.13748 \\
4.17180 \\
4.20309 \\
4.23166 \\
4.25778\end{array}$ & $\begin{array}{l}3432 \\
3129 \\
2857 \\
2612 \\
2394\end{array}$ & $\begin{array}{l}3.72901 \\
3.75143 \\
3.77324 \\
3.79440 \\
3.81488\end{array}$ & $\begin{array}{l}2242 \\
2181 \\
2116 \\
2048 \\
1978\end{array}$ & $\begin{array}{l}26.53275 \\
26.73497 \\
26.92795 \\
27.11257 \\
27.28956\end{array}$ & $\begin{array}{l}20222 \\
19298 \\
18462 \\
17699 \\
17002\end{array}$ & $\begin{array}{l}30.26177 \\
30.48641 \\
30.70120 \\
30.90697 \\
31.10444\end{array}$ & $\begin{array}{l}22464 \\
21479 \\
20577 \\
19747 \\
18980\end{array}$ \\
\hline 1150 & 4.28172 & 2197 & 3.83466 & 1910 & 27.45958 & 16360 & 31.29424 & 18270 \\
\hline
\end{tabular}


Table 2.050. AuD (Cont.)

\begin{tabular}{|c|c|c|c|c|c|c|c|c|}
\hline${ }^{\circ} K$ & $\frac{C_{p}^{0}}{R}$ & & $\frac{\left(H^{0}-E_{0}^{0}\right)}{R T}$ & & $\frac{-\left(F^{\circ}-E\right.}{R T}$ & & $\frac{S^{0}}{R}$ & \\
\hline $\begin{array}{l}1200 \\
1300 \\
1400 \\
1500 \\
1600\end{array}$ & $\begin{array}{l}4.30369 \\
4.34259 \\
4.37589 \\
4.40470 \\
4.42989\end{array}$ & $\begin{array}{l}3890 \\
3330 \\
2881 \\
2519 \\
2224\end{array}$ & $\begin{array}{l}3.85376 \\
3.88990 \\
3.92346 \\
3.95460 \\
3.98354\end{array}$ & $\begin{array}{l}3614 \\
3356 \\
3114 \\
2894 \\
2692\end{array}$ & $\begin{array}{l}27.62318 \\
27.93309 \\
28.22261 \\
28.49438 \\
28.75054\end{array}$ & $\begin{array}{l}30991 \\
28952 \\
27177 \\
25616 \\
24231\end{array}$ & $\begin{array}{l}31.47694 \\
31.82300 \\
32.14607 \\
32.44898 \\
32.73408\end{array}$ & $\begin{array}{l}34606 \\
32307 \\
30291 \\
28510 \\
26924\end{array}$ \\
\hline $\begin{array}{l}1700 \\
1800 \\
1900 \\
2000 \\
2100\end{array}$ & $\begin{array}{l}4.45213 \\
4.47196 \\
4.48981 \\
4.50601 \\
4.52083\end{array}$ & $\begin{array}{l}1983 \\
1785 \\
1620 \\
1482 \\
1366\end{array}$ & $\begin{array}{l}4.01046 \\
4.03556 \\
4.05900 \\
4.08096 \\
4.10155\end{array}$ & $\begin{array}{l}2510 \\
2344 \\
2196 \\
2059 \\
1938\end{array}$ & $\begin{array}{l}28.99285 \\
29.22280 \\
29.44163 \\
29.65040 \\
29.85001\end{array}$ & $\begin{array}{l}22995 \\
21883 \\
20877 \\
19961 \\
19126\end{array}$ & $\begin{array}{l}33.00332 \\
33.25837 \\
33.50064 \\
33.73136 \\
33.95157\end{array}$ & $\begin{array}{l}25505 \\
24227 \\
23072 \\
22021 \\
21063\end{array}$ \\
\hline $\begin{array}{l}2200 \\
2300 \\
2400 \\
2500 \\
2600\end{array}$ & $\begin{array}{l}4.53449 \\
4.54716 \\
4.55899 \\
4.57010 \\
4.58058\end{array}$ & $\begin{array}{r}1267 \\
1183 \\
1111 \\
1048 \\
995\end{array}$ & $\begin{array}{l}4.12093 \\
4.13919 \\
4.15643 \\
4.17276 \\
4.18825\end{array}$ & $\begin{array}{l}1826 \\
1724 \\
1633 \\
1549 \\
1471\end{array}$ & $\begin{array}{l}30.04127 \\
30.22486 \\
30.40139 \\
30.57139 \\
30.73536\end{array}$ & $\begin{array}{l}18359 \\
17653 \\
17000 \\
16397 \\
15834\end{array}$ & $\begin{array}{l}34.16220 \\
34.36405 \\
34.55782 \\
34.74416 \\
34.92361\end{array}$ & $\begin{array}{l}20185 \\
19377 \\
18634 \\
17945 \\
17306\end{array}$ \\
\hline $\begin{array}{l}2700 \\
2800 \\
2900 \\
3000\end{array}$ & $\begin{array}{l}4.59053 \\
4.60000 \\
4.60906 \\
4.61775\end{array}$ & $\begin{array}{r}947 \\
906 \\
869 \\
1646\end{array}$ & $\begin{array}{l}4.20296 \\
4.21698 \\
4.23034 \\
4.24311\end{array}$ & $\begin{array}{l}1402 \\
1336 \\
1277 \\
2394\end{array}$ & $\begin{array}{l}30.89370 \\
31.04681 \\
31.19502 \\
31.33865\end{array}$ & $\begin{array}{l}15311 \\
14821 \\
14363 \\
27462\end{array}$ & $\begin{array}{l}35.09667 \\
35.26379 \\
35.42537 \\
35.58177\end{array}$ & $\begin{array}{l}16712 \\
16158 \\
15640 \\
29855\end{array}$ \\
\hline $\begin{array}{l}3200 \\
3400 \\
3600 \\
3800 \\
4000\end{array}$ & $\begin{array}{l}4.63421 \\
4.64965 \\
4.66426 \\
4.67819 \\
4.69157\end{array}$ & $\begin{array}{l}1544 \\
1461 \\
1393 \\
1338 \\
1291\end{array}$ & $\begin{array}{l}4.26705 \\
4.28910 \\
4.30954 \\
4.32858 \\
4.34640\end{array}$ & $\begin{array}{l}2205 \\
2044 \\
1904 \\
1782 \\
1674\end{array}$ & $\begin{array}{l}31.61327 \\
31.87263 \\
32.11838 \\
32.35190 \\
32.57438\end{array}$ & $\begin{array}{l}25936 \\
24575 \\
23352 \\
22248 \\
21247\end{array}$ & $\begin{array}{l}35.88032 \\
36.16174 \\
36.42792 \\
36.68048 \\
36.92079\end{array}$ & $\begin{array}{l}28142 \\
26618 \\
25256 \\
24031 \\
22921\end{array}$ \\
\hline $\begin{array}{l}4200 \\
4400 \\
4600 \\
4800 \\
5000\end{array}$ & $\begin{array}{l}4.70448 \\
4.71700 \\
4.72918 \\
4.74106 \\
4.75268\end{array}$ & $\begin{array}{l}1252 \\
1218 \\
1188 \\
1162\end{array}$ & $\begin{array}{l}4.36314 \\
4.37894 \\
4.39391 \\
4.40813 \\
4.42168\end{array}$ & $\begin{array}{l}1580 \\
1497 \\
1422 \\
1355\end{array}$ & $\begin{array}{l}32.78685 \\
32.99020 \\
33.18518 \\
33.37249 \\
33.55271\end{array}$ & $\begin{array}{l}20335 \\
19498 \\
18731 \\
18022\end{array}$ & $\begin{array}{l}37.15000 \\
37.36915 \\
37.57909 \\
37.78062 \\
37.97440\end{array}$ & $\begin{array}{l}21915 \\
20994 \\
20153 \\
19378\end{array}$ \\
\hline
\end{tabular}


Table 2.051. AuT

\begin{tabular}{|c|c|c|c|c|c|c|c|c|}
\hline${ }^{\circ} \mathrm{K}$ & $\frac{C_{p}^{0}}{R}$ & & $\frac{\left(H^{0}-E_{0}^{0}\right)}{R T}$ & & $\frac{-\left(F^{0}-E\right.}{R T}$ & & $\frac{S^{0}}{R}$ & \\
\hline $\begin{array}{l}50 \\
60 \\
70 \\
80 \\
90\end{array}$ & $\begin{array}{l}3.50087 \\
3.50099 \\
3.50112 \\
3.50126 \\
3.50140\end{array}$ & $\begin{array}{l}12 \\
13 \\
14 \\
14 \\
14 \\
15\end{array}$ & $\begin{array}{l}3.47703 \\
3.48101 \\
3.48388 \\
3.48604 \\
3.48774\end{array}$ & $\begin{array}{l}398 \\
287 \\
216 \\
170 \\
137\end{array}$ & $\begin{array}{l}16.80314 \\
17.43746 \\
17.97428 \\
18.43964 \\
18.85034\end{array}$ & $\begin{array}{l}63432 \\
53682 \\
46536 \\
41070 \\
36754\end{array}$ & $\begin{array}{l}20.28018 \\
20.91848 \\
21.45817 \\
21.92568 \\
22.33808\end{array}$ & $\begin{array}{l}63830 \\
53969 \\
46751 \\
41240 \\
36892\end{array}$ \\
\hline $\begin{array}{l}100 \\
110 \\
120 \\
130 \\
140\end{array}$ & $\begin{array}{l}3.50155 \\
3.50170 \\
3.50188 \\
3.50210 \\
3.50240\end{array}$ & $\begin{array}{l}15 \\
18 \\
22 \\
30 \\
45\end{array}$ & $\begin{array}{l}3.48911 \\
3.49025 \\
3.49121 \\
3.49204 \\
3.49277\end{array}$ & $\begin{array}{r}114 \\
96 \\
83 \\
73 \\
65\end{array}$ & $\begin{array}{l}19.21788 \\
19.55048 \\
19.85422 \\
20.13370 \\
20.39251\end{array}$ & $\begin{array}{l}33260 \\
30374 \\
27948 \\
25881 \\
24100\end{array}$ & $\begin{array}{l}22.70700 \\
23.04074 \\
23.34544 \\
23.62574 \\
23.88529\end{array}$ & $\begin{array}{l}33374 \\
30470 \\
28030 \\
25955 \\
24165\end{array}$ \\
\hline $\begin{array}{l}150 \\
160 \\
170 \\
180 \\
190\end{array}$ & $\begin{array}{l}3.50285 \\
3.50352 \\
3.50451 \\
3.50591 \\
3.50782\end{array}$ & $\begin{array}{r}67 \\
99 \\
140 \\
191 \\
253\end{array}$ & $\begin{array}{l}3.49342 \\
3.49403 \\
3.49462 \\
3.49521 \\
3.49582\end{array}$ & $\begin{array}{l}61 \\
59 \\
59 \\
61 \\
66\end{array}$ & $\begin{array}{l}20.63351 \\
20.85899 \\
21.07084 \\
21.27060 \\
21.45959\end{array}$ & $\begin{array}{l}22548 \\
21185 \\
19976 \\
18899 \\
17933\end{array}$ & $\begin{array}{l}24.12694 \\
24.35303 \\
24.56546 \\
24.76581 \\
24.95541\end{array}$ & $\begin{array}{l}22609 \\
21243 \\
20035 \\
18960 \\
17999\end{array}$ \\
\hline $\begin{array}{l}200 \\
210 \\
220 \\
230 \\
240\end{array}$ & $\begin{array}{l}3.51035 \\
3.51358 \\
3.51757 \\
3.52239 \\
3.52807\end{array}$ & $\begin{array}{l}323 \\
399 \\
482 \\
568 \\
655\end{array}$ & $\begin{array}{l}3.49648 \\
3.49721 \\
3.49804 \\
3.49899 \\
3.50008\end{array}$ & $\begin{array}{r}73 \\
83 \\
95 \\
109 \\
125\end{array}$ & $\begin{array}{l}21.63892 \\
21.80953 \\
21.97224 \\
22.12776 \\
22.27669\end{array}$ & $\begin{array}{l}17061 \\
16271 \\
15552 \\
14893 \\
14291\end{array}$ & $\begin{array}{l}25.13540 \\
25.30675 \\
25.47029 \\
25.62676 \\
25.77678\end{array}$ & $\begin{array}{l}17135 \\
16354 \\
15647 \\
15002 \\
14416\end{array}$ \\
\hline $\begin{array}{l}250 \\
260 \\
270 \\
280 \\
290\end{array}$ & $\begin{array}{l}3.53462 \\
3.54205 \\
3.55033 \\
3.55944 \\
3.56934\end{array}$ & $\begin{array}{r}743 \\
828 \\
911 \\
990 \\
1064\end{array}$ & $\begin{array}{l}3.50133 \\
3.50275 \\
3.50436 \\
3.50616 \\
3.50817\end{array}$ & $\begin{array}{l}142 \\
161 \\
180 \\
201 \\
221\end{array}$ & $\begin{array}{l}22.41960 \\
22.55695 \\
22.68918 \\
22.81665 \\
22.93972\end{array}$ & $\begin{array}{l}13735 \\
13223 \\
12747 \\
12307 \\
11897\end{array}$ & $\begin{array}{l}25.92094 \\
26.05971 \\
26.19354 \\
26.32282 \\
26.44790\end{array}$ & $\begin{array}{l}13877 \\
13383 \\
12928 \\
12508 \\
12118\end{array}$ \\
\hline $\begin{array}{l}300 \\
310 \\
320 \\
330 \\
340\end{array}$ & $\begin{array}{l}3.57998 \\
3.59129 \\
3.60323 \\
3.61572 \\
3.62871\end{array}$ & $\begin{array}{l}1131 \\
1194 \\
1249 \\
1299 \\
1341\end{array}$ & $\begin{array}{l}3.51038 \\
3.51281 \\
3.51544 \\
3.51829 \\
3.52135\end{array}$ & $\begin{array}{l}243 \\
263 \\
285 \\
306 \\
326\end{array}$ & $\begin{array}{l}23.05869 \\
23.17384 \\
23.28541 \\
23.39363 \\
23.49870\end{array}$ & $\begin{array}{l}11515 \\
11157 \\
10822 \\
10507 \\
10212\end{array}$ & $\begin{array}{l}26.56908 \\
26.68665 \\
26.80086 \\
26.91192 \\
27.02005\end{array}$ & $\begin{array}{l}11757 \\
11421 \\
11106 \\
10813 \\
10538\end{array}$ \\
\hline $\begin{array}{l}350 \\
360 \\
370 \\
380 \\
390\end{array}$ & $\begin{array}{l}3.64212 \\
3.65590 \\
3.66998 \\
3.68431 \\
3.69883\end{array}$ & $\begin{array}{l}1378 \\
1408 \\
1433 \\
1452 \\
1466\end{array}$ & $\begin{array}{l}3.52461 \\
3.52806 \\
3.53171 \\
3.53553 \\
3.53953\end{array}$ & $\begin{array}{l}345 \\
365 \\
382 \\
400 \\
417\end{array}$ & $\begin{array}{l}23.60082 \\
23.70016 \\
23.79688 \\
23.89111 \\
23.98300\end{array}$ & $\begin{array}{l}9934 \\
9672 \\
9423 \\
9189 \\
8967\end{array}$ & $\begin{array}{l}27.12543 \\
27.22823 \\
27.32859 \\
27.42665 \\
27.52254\end{array}$ & $\begin{array}{r}10280 \\
10036 \\
9806 \\
9589 \\
9383\end{array}$ \\
\hline $\begin{array}{l}400 \\
450 \\
500 \\
550 \\
600\end{array}$ & $\begin{array}{l}3.71349 \\
3.78737 \\
3.85925 \\
3.92656 \\
3.98817\end{array}$ & $\begin{array}{l}7388 \\
7188 \\
6731 \\
6161 \\
5561\end{array}$ & $\begin{array}{l}3.54370 \\
3.56667 \\
3.59236 \\
3.61973 \\
3.64790\end{array}$ & $\begin{array}{l}2297 \\
2569 \\
2737 \\
2817 \\
2836\end{array}$ & $\begin{array}{l}24.07267 \\
24.49135 \\
24.86845 \\
25.21211 \\
25.52827\end{array}$ & $\begin{array}{l}41868 \\
37710 \\
34366 \\
31616 \\
29311\end{array}$ & $\begin{array}{l}27.61637 \\
28.05802 \\
28.46082 \\
28.83184 \\
29.17618\end{array}$ & $\begin{array}{l}44165 \\
40280 \\
37102 \\
34434 \\
32146\end{array}$ \\
\hline $\begin{array}{l}650 \\
700 \\
750 \\
800 \\
850\end{array}$ & $\begin{array}{l}4.04378 \\
4.09361 \\
4.13809 \\
4.17775 \\
4.21316\end{array}$ & $\begin{array}{l}4983 \\
4448 \\
3966 \\
3541 \\
3166\end{array}$ & $\begin{array}{l}3.67626 \\
3.70432 \\
3.73178 \\
3.75844 \\
3.78417\end{array}$ & $\begin{array}{l}2806 \\
2746 \\
2666 \\
2573 \\
2473\end{array}$ & $\begin{array}{l}25.82138 \\
26.09485 \\
26.35136 \\
26.59306 \\
26.82169\end{array}$ & $\begin{array}{l}27347 \\
25651 \\
24170 \\
22863 \\
21701\end{array}$ & $\begin{array}{l}29.49764 \\
29.79918 \\
30.08315 \\
30.35151 \\
30.60587\end{array}$ & $\begin{array}{l}30154 \\
28397 \\
26836 \\
25436 \\
24173\end{array}$ \\
\hline $\begin{array}{r}900 \\
950 \\
1000 \\
1050 \\
1100\end{array}$ & $\begin{array}{l}4.24482 \\
4.27320 \\
4.29873 \\
4.32178 \\
4.34266\end{array}$ & $\begin{array}{l}2838 \\
2553 \\
2305 \\
2088 \\
1899\end{array}$ & $\begin{array}{l}3.80890 \\
3.83260 \\
3.85528 \\
3.87695 \\
3.89765\end{array}$ & $\begin{array}{l}2370 \\
2268 \\
2167 \\
2070 \\
1977\end{array}$ & $\begin{array}{l}27.03870 \\
27.24527 \\
27.44244 \\
27.63107 \\
27.81191\end{array}$ & $\begin{array}{l}20657 \\
19717 \\
18863 \\
18084 \\
17369\end{array}$ & $\begin{array}{l}30.84760 \\
31.07788 \\
31.29772 \\
31.50803 \\
31.70957\end{array}$ & $\begin{array}{l}23028 \\
21984 \\
21031 \\
20154 \\
19346\end{array}$ \\
\hline 1150 & 4.36165 & 1733 & 3.91742 & 1888 & 27.98560 & 16713 & 31.90303 & 18600 \\
\hline
\end{tabular}


Table 2.051. AuT (Cont.)

\begin{tabular}{|c|c|c|c|c|c|c|c|c|}
\hline${ }^{\circ} \mathrm{K}$ & $\frac{C_{p}^{0}}{R}$ & & $\frac{\left(H^{0}-E_{0}^{0}\right)}{R T}$ & & $\frac{-\left(F^{\circ}-E\right.}{R T}$ & & $\frac{S^{0}}{R}$ & \\
\hline $\begin{array}{l}1200 \\
1300 \\
1400 \\
1500 \\
1600\end{array}$ & $\begin{array}{l}4.37898 \\
4.40947 \\
4.43546 \\
4.45791 \\
4.47758\end{array}$ & $\begin{array}{l}3049 \\
2599 \\
2245 \\
1967 \\
1743\end{array}$ & $\begin{array}{l}3.93630 \\
3.97156 \\
4.00379 \\
4.03333 \\
4.06050\end{array}$ & $\begin{array}{l}3526 \\
3223 \\
2954 \\
2717 \\
2505\end{array}$ & $\begin{array}{l}28.15273 \\
28.46922 \\
28.76474 \\
29.04199 \\
29.30318\end{array}$ & $\begin{array}{l}31649 \\
29552 \\
27725 \\
26119 \\
24692\end{array}$ & $\begin{array}{l}32.08903 \\
32.44078 \\
32.76853 \\
33.07533 \\
33.36368\end{array}$ & $\begin{array}{l}35175 \\
32775 \\
30680 \\
28835 \\
27198\end{array}$ \\
\hline $\begin{array}{l}1700 \\
1800 \\
1900 \\
2000 \\
2100\end{array}$ & $\begin{array}{l}4.49501 \\
4.51064 \\
4.52480 \\
4.53775 \\
4.54969\end{array}$ & $\begin{array}{l}1563 \\
1416 \\
1295 \\
1194 \\
1111\end{array}$ & $\begin{array}{l}4.08555 \\
4.10874 \\
4.13027 \\
4.15033 \\
4.16906\end{array}$ & $\begin{array}{l}2319 \\
2153 \\
2006 \\
1873 \\
1756\end{array}$ & $\begin{array}{l}29.55010 \\
29.78429 \\
30.00703 \\
30.21940 \\
30.42235\end{array}$ & $\begin{array}{l}23419 \\
22274 \\
21237 \\
20295 \\
19436\end{array}$ & $\begin{array}{l}33.63566 \\
33.89304 \\
34.13730 \\
34.36973 \\
34.59142\end{array}$ & $\begin{array}{l}25738 \\
24426 \\
23243 \\
22169 \\
21191\end{array}$ \\
\hline $\begin{array}{l}2200 \\
2300 \\
2400 \\
2500 \\
2600\end{array}$ & $\begin{array}{l}4.56080 \\
4.57119 \\
4.58098 \\
4.59025 \\
4.59908\end{array}$ & $\begin{array}{r}1039 \\
979 \\
927 \\
883 \\
845\end{array}$ & $\begin{array}{l}4.18662 \\
4.20312 \\
4.21866 \\
4.23334 \\
4.24724\end{array}$ & $\begin{array}{l}1650 \\
1554 \\
1468 \\
1390 \\
1319\end{array}$ & $\begin{array}{l}30.61671 \\
30.80318 \\
30.98239 \\
31.15491 \\
31.32121\end{array}$ & $\begin{array}{l}18647 \\
17921 \\
17252 \\
16630 \\
16055\end{array}$ & $\begin{array}{l}34.80333 \\
35.00630 \\
35.20106 \\
35.38825 \\
35.56846\end{array}$ & $\begin{array}{l}20297 \\
19476 \\
18719 \\
18021 \\
17373\end{array}$ \\
\hline $\begin{array}{l}2700 \\
2800 \\
2900 \\
3000\end{array}$ & $\begin{array}{l}4.60753 \\
4.61564 \\
4.62346 \\
4.63102\end{array}$ & $\begin{array}{r}811 \\
782 \\
756 \\
1446\end{array}$ & $\begin{array}{l}4.26043 \\
4.27297 \\
4.28492 \\
4.29633\end{array}$ & $\begin{array}{l}1254 \\
1195 \\
1141 \\
2138\end{array}$ & $\begin{array}{l}31.48176 \\
31.63693 \\
31.78708 \\
31.93254\end{array}$ & $\begin{array}{l}15517 \\
15015 \\
14546 \\
27797\end{array}$ & $\begin{array}{l}35.74219 \\
35.90990 \\
36.07201 \\
36.22888\end{array}$ & $\begin{array}{l}16771 \\
16211 \\
15687 \\
29934\end{array}$ \\
\hline $\begin{array}{l}3200 \\
3400 \\
3600 \\
3800 \\
4000\end{array}$ & $\begin{array}{l}4.64548 \\
4.65921 \\
4.67236 \\
4.68503 \\
4.69729\end{array}$ & $\begin{array}{l}1373 \\
1315 \\
1267 \\
1226 \\
1193\end{array}$ & $\begin{array}{l}4.31771 \\
4.33739 \\
4.35564 \\
4.37264 \\
4.38857\end{array}$ & $\begin{array}{l}1968 \\
1825 \\
1700 \\
1593 \\
1499\end{array}$ & $\begin{array}{l}32.21051 \\
32.47287 \\
32.72131 \\
32.95727 \\
33.18197\end{array}$ & $\begin{array}{l}26236 \\
24844 \\
23596 \\
22470 \\
21448\end{array}$ & $\begin{array}{l}36.52822 \\
36.81027 \\
37.07696 \\
37.32992 \\
37.57054\end{array}$ & $\begin{array}{l}28205 \\
26669 \\
25296 \\
24062 \\
22948\end{array}$ \\
\hline $\begin{array}{l}4200 \\
4400 \\
4600 \\
4800 \\
5000\end{array}$ & $\begin{array}{l}4.70922 \\
4.72087 \\
4.73226 \\
4.74344 \\
4.75442\end{array}$ & $\begin{array}{l}1165 \\
1139 \\
1118 \\
1098\end{array}$ & $\begin{array}{l}4.40356 \\
4.41772 \\
4.43115 \\
4.44393 \\
4.45613\end{array}$ & $\begin{array}{l}1416 \\
1343 \\
1278 \\
1220\end{array}$ & $\begin{array}{l}33.39645 \\
33.60164 \\
33.79831 \\
33.98717 \\
34.16883\end{array}$ & $\begin{array}{l}20519 \\
19667 \\
18886 \\
18166\end{array}$ & $\begin{array}{l}37.80002 \\
38.01936 \\
38.22946 \\
38.43110 \\
38.62496\end{array}$ & $\begin{array}{l}21934 \\
21010 \\
20164 \\
19386\end{array}$ \\
\hline
\end{tabular}


Table 2.052. $\mathrm{ZnH}$

\begin{tabular}{|l|l|l|l|l|}
\hline${ }^{\circ}$ & $\frac{C_{p}^{0}}{R}$ & $\frac{\left(H^{\circ}-E_{0}^{0}\right)}{R T}$ & $\frac{-\left(F^{\circ}-E_{0}^{0}\right)}{R T}$ & $\frac{S^{\circ}}{R}$ \\
\hline
\end{tabular}

\begin{tabular}{|c|c|c|c|c|c|c|c|c|}
\hline $\begin{array}{l}50 \\
60 \\
70 \\
80 \\
90\end{array}$ & $\begin{array}{l}3.50238 \\
3.50241 \\
3.50255 \\
3.50275 \\
3.50299\end{array}$ & $\begin{array}{r}3 \\
14 \\
20 \\
24 \\
25\end{array}$ & $\begin{array}{l}3.43715 \\
3.44802 \\
3.45580 \\
3.46166 \\
3.46624\end{array}$ & $\begin{array}{r}1087 \\
778 \\
586 \\
458 \\
368\end{array}$ & $\begin{array}{l}14.83552 \\
15.46321 \\
15.99534 \\
16.45719 \\
16.86519\end{array}$ & $\begin{array}{l}62769 \\
53213 \\
46185 \\
40800 \\
36541\end{array}$ & $\begin{array}{l}18.27267 \\
18.91123 \\
19.45114 \\
19.91885 \\
20.33143\end{array}$ & $\begin{array}{l}63856 \\
53991 \\
46771 \\
41258 \\
36909\end{array}$ \\
\hline $\begin{array}{l}100 \\
110 \\
120 \\
130 \\
140\end{array}$ & $\begin{array}{l}3.50324 \\
3.50350 \\
3.50378 \\
3.50408 \\
3.50439\end{array}$ & $\begin{array}{l}26 \\
28 \\
30 \\
31 \\
37\end{array}$ & $\begin{array}{l}3.46992 \\
3.47296 \\
3.47552 \\
3.47771 \\
3.47960\end{array}$ & $\begin{array}{l}304 \\
256 \\
219 \\
189 \\
166\end{array}$ & $\begin{array}{l}17.23060 \\
17.56146 \\
17.86376 \\
18.14204 \\
18.39984\end{array}$ & $\begin{array}{l}33086 \\
30230 \\
27828 \\
25780 \\
24012\end{array}$ & $\begin{array}{l}20.70052 \\
21.03443 \\
21.33929 \\
21.61975 \\
21.87944\end{array}$ & $\begin{array}{l}33391 \\
30486 \\
28046 \\
25969 \\
24179\end{array}$ \\
\hline $\begin{array}{l}150 \\
160 \\
170 \\
180 \\
190\end{array}$ & $\begin{array}{l}3.50476 \\
3.50519 \\
3.50575 \\
3.50647 \\
3.50742\end{array}$ & $\begin{array}{r}43 \\
56 \\
72 \\
95 \\
124\end{array}$ & $\begin{array}{l}3.48126 \\
3.48275 \\
3.48408 \\
3.48530 \\
3.48644\end{array}$ & $\begin{array}{l}149 \\
133 \\
122 \\
114 \\
108\end{array}$ & $\begin{array}{l}18.63996 \\
18.86469 \\
19.07587 \\
19.27505 \\
19.46352\end{array}$ & $\begin{array}{l}22473 \\
21118 \\
19918 \\
18847 \\
17886\end{array}$ & $\begin{array}{l}22.12123 \\
22.34744 \\
22.55996 \\
22.76036 \\
22.94997\end{array}$ & $\begin{array}{l}22621 \\
21252 \\
20040 \\
18961 \\
17994\end{array}$ \\
\hline $\begin{array}{l}200 \\
210 \\
220 \\
230 \\
240\end{array}$ & $\begin{array}{l}3.50866 \\
3.51028 \\
3.51234 \\
3.51489 \\
3.51801\end{array}$ & $\begin{array}{l}162 \\
206 \\
255 \\
312 \\
372\end{array}$ & $\begin{array}{l}3.48752 \\
3.48856 \\
3.48960 \\
3.49064 \\
3.49171\end{array}$ & $\begin{array}{l}104 \\
104 \\
104 \\
107 \\
113\end{array}$ & $\begin{array}{l}19.64238 \\
19.81256 \\
19.97487 \\
20.13002 \\
20.27860\end{array}$ & $\begin{array}{l}17018 \\
16231 \\
15515 \\
14858 \\
14256\end{array}$ & $\begin{array}{l}23.12991 \\
23.30113 \\
23.46448 \\
23.62066 \\
23.77032\end{array}$ & $\begin{array}{l}17122 \\
16335 \\
15618 \\
14966 \\
14368\end{array}$ \\
\hline $\begin{array}{l}250 \\
260 \\
270 \\
280 \\
290\end{array}$ & $\begin{array}{l}3.52173 \\
3.52610 \\
3.53115 \\
3.53688 \\
3.54331\end{array}$ & $\begin{array}{l}437 \\
505 \\
573 \\
643 \\
712\end{array}$ & $\begin{array}{l}3.49284 \\
3.49403 \\
3.49531 \\
3.49669 \\
3.49818\end{array}$ & $\begin{array}{l}119 \\
128 \\
138 \\
149 \\
163\end{array}$ & $\begin{array}{l}20.42116 \\
20.55818 \\
20.69006 \\
20.81721 \\
20.93994\end{array}$ & $\begin{array}{l}13702 \\
13188 \\
12715 \\
12273 \\
11862\end{array}$ & $\begin{array}{l}23.91400 \\
24.05221 \\
24.18538 \\
24.31390 \\
24.43812\end{array}$ & $\begin{array}{l}13821 \\
13317 \\
12852 \\
12422 \\
12025\end{array}$ \\
\hline $\begin{array}{l}300 \\
310 \\
320 \\
330 \\
340\end{array}$ & $\begin{array}{l}3.55043 \\
3.55823 \\
3.56668 \\
3.57575 \\
3.58543\end{array}$ & $\begin{array}{r}780 \\
845 \\
907 \\
968 \\
1022\end{array}$ & $\begin{array}{l}3.49981 \\
3.50156 \\
3.50346 \\
3.50551 \\
3.50772\end{array}$ & $\begin{array}{l}175 \\
190 \\
205 \\
221 \\
237\end{array}$ & $\begin{array}{l}21.05856 \\
21.17334 \\
21.28454 \\
21.39238 \\
21.49706\end{array}$ & $\begin{array}{l}11478 \\
11120 \\
10784 \\
10468 \\
10172\end{array}$ & $\begin{array}{l}24.55837 \\
24.67491 \\
24.78801 \\
24.89790 \\
25.00479\end{array}$ & $\begin{array}{l}11654 \\
11310 \\
10989 \\
10689 \\
10408\end{array}$ \\
\hline $\begin{array}{l}350 \\
360 \\
370 \\
380 \\
390\end{array}$ & $\begin{array}{l}3.59565 \\
3.60640 \\
3.61762 \\
3.62928 \\
3.64132\end{array}$ & $\begin{array}{l}1075 \\
1122 \\
1166 \\
1204 \\
1239\end{array}$ & $\begin{array}{l}3.51009 \\
3.51261 \\
3.51530 \\
3.51814 \\
3.52115\end{array}$ & $\begin{array}{l}252 \\
269 \\
284 \\
301 \\
315\end{array}$ & $\begin{array}{l}21.59878 \\
21.69769 \\
21.79397 \\
21.88776 \\
21.97918\end{array}$ & $\begin{array}{l}9891 \\
9628 \\
9379 \\
9142 \\
8919\end{array}$ & $\begin{array}{l}25.10887 \\
25.21031 \\
25.30928 \\
25.40590 \\
25.50033\end{array}$ & $\begin{array}{r}10144 \\
9897 \\
9662 \\
9443 \\
9235\end{array}$ \\
\hline $\begin{array}{l}400 \\
450 \\
500 \\
550 \\
600\end{array}$ & $\begin{array}{l}3.65371 \\
3.71942 \\
3.78815 \\
3.85651 \\
3.92235\end{array}$ & $\begin{array}{l}6571 \\
6873 \\
6836 \\
6584 \\
6211\end{array}$ & $\begin{array}{l}3.52430 \\
3.54229 \\
3.56343 \\
3.58698 \\
3.61220\end{array}$ & $\begin{array}{l}1799 \\
2114 \\
2355 \\
2522 \\
2628\end{array}$ & $\begin{array}{l}22.06837 \\
22.48448 \\
22.85876 \\
23.19948 \\
23.51266\end{array}$ & $\begin{array}{l}41611 \\
37428 \\
34072 \\
31318 \\
29017\end{array}$ & $\begin{array}{l}25.59268 \\
26.02677 \\
26.42219 \\
26.78646 \\
27.12487\end{array}$ & $\begin{array}{l}43409 \\
39542 \\
36427 \\
33841 \\
31644\end{array}$ \\
\hline $\begin{array}{l}650 \\
700 \\
750 \\
800 \\
850\end{array}$ & $\begin{array}{l}3.98446 \\
4.04228 \\
4.09568 \\
4.14478 \\
4.18984\end{array}$ & $\begin{array}{l}5782 \\
5340 \\
4910 \\
4506 \\
4133\end{array}$ & $\begin{array}{l}3.63848 \\
3.66528 \\
3.69222 \\
3.71899 \\
3.74538\end{array}$ & $\begin{array}{l}2680 \\
2694 \\
2677 \\
2639 \\
2586\end{array}$ & $\begin{array}{l}23.80283 \\
24.07345 \\
24.32724 \\
24.56639 \\
24.79265\end{array}$ & $\begin{array}{l}27062 \\
25379 \\
23915 \\
22626 \\
21481\end{array}$ & $\begin{array}{l}27.44131 \\
27.73873 \\
28.01946 \\
28.28538 \\
28.53803\end{array}$ & $\begin{array}{l}29742 \\
28073 \\
26592 \\
25265 \\
24067\end{array}$ \\
\hline $\begin{array}{r}900 \\
950 \\
1000 \\
1050 \\
1100\end{array}$ & $\begin{array}{l}4.23117 \\
4.26911 \\
4.30399 \\
4.33615 \\
4.36585\end{array}$ & $\begin{array}{l}3794 \\
3488 \\
3216 \\
2970 \\
2753\end{array}$ & $\begin{array}{l}3.77124 \\
3.79646 \\
3.82097 \\
3.84475 \\
3.86777\end{array}$ & $\begin{array}{l}2522 \\
2451 \\
2378 \\
2302 \\
2226\end{array}$ & $\begin{array}{l}25.00746 \\
25.21204 \\
25.40740 \\
25.59440 \\
25.77380\end{array}$ & $\begin{array}{l}20458 \\
19536 \\
18700 \\
17940 \\
17242\end{array}$ & $\begin{array}{l}28.77870 \\
29.00850 \\
29.22838 \\
29.43916 \\
29.64157\end{array}$ & $\begin{array}{l}22980 \\
21988 \\
21078 \\
20241 \\
19469\end{array}$ \\
\hline 1150 & 4.39338 & 2559 & 3.89003 & 2151 & 25.94622 & 16602 & 29.83626 & 18753 \\
\hline
\end{tabular}


Table 2. 052. ZnH (Cont.)

\begin{tabular}{|l|l|l|l|l|}
\hline${ }^{\circ} K$ & $\frac{C_{p}^{\circ}}{R}$ & $\frac{\left(H^{0}-E_{0}^{0}\right)}{R T}$ & $\frac{-\left(F^{0}-E_{0}^{0}\right)}{R T}$ & $\frac{S^{\circ}}{R}$ \\
\hline
\end{tabular}

$\begin{array}{lllllllll}1200 & 4.41897 & 4616 & 3.91154 & 4085 & 26.11224 & 31472 & 30.02379 & 35557 \\ 1300 & 4.46513 & 4060 & 3.95239 & 3811 & 26.42696 & 29431 & 30.37936 & 33241 \\ 1400 & 4.50573 & 3615 & 3.99050 & 3557 & 26.72127 & 27655 & 30.71177 & 31212 \\ 1500 & 4.54188 & 3254 & 4.02607 & 3327 & 26.99782 & 26091 & 31.02389 & 29419 \\ 1600 & 4.57442 & 2959 & 4.05934 & 3119 & 27.25873 & 24704 & 31.31808 & 27822 \\ & & & & & & & \\ 1700 & 4.60401 & 2717 & 4.09053 & 2929 & 27.50577 & 23465 & 31.59630 & 26394 \\ 1800 & 4.63118 & 2516 & 4.11982 & 2758 & 27.74042 & 22349 & 31.86024 & 25108 \\ 1900 & 4.65634 & 2346 & 4.14740 & 2604 & 27.96391 & 21340 & 32.11132 & 23944 \\ 2000 & 4.67980 & 2204 & 4.17344 & 2464 & 28.17731 & 20423 & 32.35076 & 22887 \\ 2100 & 4.70184 & 2083 & 4.19808 & 2338 & 28.38154 & 19584 & 32.57963 & 21921 \\ & & & & & & & & \\ 2200 & 4.72267 & 1978 & 4.22146 & 2223 & 28.57738 & 18814 & 32.79884 & 21037 \\ 2300 & 4.74245 & 1888 & 4.24369 & 2117 & 28.76552 & 18106 & 33.00921 & 20224 \\ 2400 & 4.76133 & 1809 & 4.26486 & 2023 & 28.94658 & 17452 & 33.21145 & 19474 \\ 2500 & 4.77942 & 1741 & 4.28509 & 1935 & 29.12110 & 16844 & 33.40619 & 18779 \\ 2600 & 4.79683 & 1679 & 4.30444 & 1855 & 29.28954 & 16280 & 33.59398 & 18135 \\ & & & & & & & & \\ 2700 & 4.81362 & 1626 & 4.32299 & 1781 & 29.45234 & 15754 & 33.77533 & 17536 \\ 2800 & 4.82988 & 1577 & 4.34080 & 1744 & 29.60988 & 15263 & 33.95069 & 16976 \\ 2900 & 4.84565 & 1533 & 4.35794 & 1651 & 29.76251 & 14802 & 34.12045 & 16454 \\ 3000 & 4.86098 & 2953 & 4.37445 & 3134 & 29.91053 & 28333 & 34.28499 & 31467 \\ & & & & & & & & \\ 3200 & 4.89051 & 2822 & 4.40579 & 2935 & 30.19386 & 26799 & 34.59966 & 29734 \\ 3400 & 4.91873 & 2711 & 4.43514 & 2762 & 30.46185 & 25430 & 34.89700 & 28191 \\ 3600 & 4.94584 & 2616 & 4.46276 & 2612 & 30.71615 & 24199 & 35.17891 & 26812 \\ 3800 & 4.97200 & 2532 & 4.48888 & 2479 & 30.95814 & 23089 & 35.44703 & 25567 \\ 4000 & 4.99732 & 2459 & 4.51367 & 2362 & 31.18903 & 22080 & 35.70270 & 24442 \\ & & & & & & & \\ 4200 & 5.02191 & 2392 & 4.53729 & 2258 & 31.40983 & 21160 & 35.94712 & 23418 \\ 4400 & 5.04583 & 2332 & 4.55987 & 2163 & 31.62143 & 20317 & 36.18130 & 22481 \\ 4600 & 5.06915 & 2278 & 4.58150 & 2080 & 31.82460 & 19543 & 36.40611 & 21622 \\ 4800 & 5.09193 & 2227 & 4.60230 & 2003 & 32.02003 & 18828 & 36.62233 & 20832 \\ 5000 & 5.11420 & & 4.62233 & & 32.20831 & & 36.83065 & \end{array}$


Table 2.053. ZnD

\begin{tabular}{|l|l|l|l|l|}
\hline${ }^{\circ} K$ & $\frac{C_{P}^{0}}{R}$ & $\frac{\left(H^{0}-E_{0}^{0}\right)}{R T}$ & $\frac{-\left(F^{0}-E_{0}^{0}\right)}{R T}$ & $\frac{S^{0}}{R}$ \\
\hline
\end{tabular}

\begin{tabular}{|c|c|c|c|c|c|c|c|c|}
\hline $\begin{array}{l}50 \\
60 \\
70 \\
80 \\
90\end{array}$ & $\begin{array}{l}3.50173 \\
3.50197 \\
3.50223 \\
3.50251 \\
3.50280\end{array}$ & $\begin{array}{l}24 \\
26 \\
28 \\
29 \\
32\end{array}$ & $\begin{array}{l}3.46844 \\
3.47401 \\
3.47802 \\
3.48106 \\
3.48346\end{array}$ & $\begin{array}{l}557 \\
401 \\
304 \\
240 \\
195\end{array}$ & $\begin{array}{l}15.49694 \\
16.12983 \\
16.66567 \\
17.13031 \\
17.54046\end{array}$ & $\begin{array}{l}63289 \\
53584 \\
46464 \\
41015 \\
36712 \\
\end{array}$ & $\begin{array}{l}18.96539 \\
19.60385 \\
20.14370 \\
20.61138 \\
21.02393\end{array}$ & $\begin{array}{l}63846 \\
53985 \\
46768 \\
41255 \\
36907\end{array}$ \\
\hline $\begin{array}{l}100 \\
110 \\
120 \\
130 \\
140\end{array}$ & $\begin{array}{l}3.50312 \\
3.50351 \\
3.50404 \\
3.50483 \\
3.50602\end{array}$ & $\begin{array}{r}39 \\
53 \\
79 \\
119 \\
177\end{array}$ & $\begin{array}{l}3.48541 \\
3.48704 \\
3.48843 \\
3.48966 \\
3.49078\end{array}$ & $\begin{array}{l}163 \\
139 \\
123 \\
112 \\
108\end{array}$ & $\begin{array}{l}17.90758 \\
18.23986 \\
18.54333 \\
18.82261 \\
19.08126\end{array}$ & $\begin{array}{l}33228 \\
30347 \\
27928 \\
25865 \\
24088\end{array}$ & $\begin{array}{l}21.39300 \\
21.72690 \\
22.03177 \\
22.31227 \\
22.57205\end{array}$ & $\begin{array}{l}33390 \\
30487 \\
28050 \\
25978 \\
24195\end{array}$ \\
\hline $\begin{array}{l}150 \\
160 \\
170 \\
180 \\
190\end{array}$ & $\begin{array}{l}3.50779 \\
3.51033 \\
3.51378 \\
3.51831 \\
3.52401\end{array}$ & $\begin{array}{l}254 \\
345 \\
453 \\
570 \\
695\end{array}$ & $\begin{array}{l}3.49186 \\
3.49293 \\
3.49405 \\
3.49526 \\
3.49662\end{array}$ & $\begin{array}{l}107 \\
112 \\
121 \\
136 \\
154\end{array}$ & $\begin{array}{l}19.32214 \\
19.54753 \\
19.75932 \\
19.95907 \\
20.14808\end{array}$ & $\begin{array}{l}22539 \\
21179 \\
19975 \\
18901 \\
17939\end{array}$ & $\begin{array}{l}22.81400 \\
23.04046 \\
23.25337 \\
23.45434 \\
23.64471\end{array}$ & $\begin{array}{l}22646 \\
21291 \\
20097 \\
19037 \\
18093\end{array}$ \\
\hline $\begin{array}{l}200 \\
210 \\
220 \\
230 \\
240\end{array}$ & $\begin{array}{l}3.53096 \\
3.53920 \\
3.54872 \\
3.55948 \\
3.57143\end{array}$ & $\begin{array}{r}824 \\
952 \\
1076 \\
1195 \\
1304\end{array}$ & $\begin{array}{l}3.49816 \\
3.49991 \\
3.50191 \\
3.50417 \\
3.50672\end{array}$ & $\begin{array}{l}175 \\
200 \\
226 \\
255 \\
285\end{array}$ & $\begin{array}{l}20.32747 \\
20.49819 \\
20.66105 \\
20.81677 \\
20.96596\end{array}$ & $\begin{array}{l}17072 \\
16286 \\
15572 \\
14919 \\
14321\end{array}$ & $\begin{array}{l}23.92564 \\
23.99811 \\
24.16297 \\
24.32095 \\
24.47269\end{array}$ & $\begin{array}{l}17247 \\
16486 \\
15798 \\
15174 \\
14605\end{array}$ \\
\hline $\begin{array}{l}250 \\
260 \\
270 \\
280 \\
290\end{array}$ & $\begin{array}{l}3.58447 \\
3.59853 \\
3.61348 \\
3.62922 \\
3.64565\end{array}$ & $\begin{array}{l}1406 \\
1495 \\
1574 \\
1643 \\
1699\end{array}$ & $\begin{array}{l}3.50957 \\
3.51272 \\
3.51617 \\
3.51992 \\
3.52397\end{array}$ & $\begin{array}{l}315 \\
345 \\
375 \\
405 \\
434\end{array}$ & $\begin{array}{l}21.10917 \\
21.24687 \\
21.37951 \\
21.50745 \\
21.63104\end{array}$ & $\begin{array}{l}13770 \\
13264 \\
12794 \\
12359 \\
11954\end{array}$ & $\begin{array}{l}24.61874 \\
24.75960 \\
24.89568 \\
25.02738 \\
25.15502\end{array}$ & $\begin{array}{l}14086 \\
13608 \\
13170 \\
12764 \\
12388\end{array}$ \\
\hline $\begin{array}{l}300 \\
310 \\
320 \\
330 \\
340\end{array}$ & $\begin{array}{l}3.66264 \\
3.68009 \\
3.69791 \\
3.71600 \\
3.73427\end{array}$ & $\begin{array}{l}1745 \\
1782 \\
1809 \\
1827 \\
1838\end{array}$ & $\begin{array}{l}3.52831 \\
3.53293 \\
3.53780 \\
3.54293 \\
3.54829\end{array}$ & $\begin{array}{l}462 \\
487 \\
513 \\
536 \\
557\end{array}$ & $\begin{array}{l}21.75058 \\
21.86635 \\
21.97859 \\
22.08753 \\
22.19338\end{array}$ & $\begin{array}{l}11577 \\
11224 \\
10894 \\
10585 \\
10293\end{array}$ & $\begin{array}{l}25.27890 \\
25.39928 \\
25.51640 \\
25.63046 \\
25.74167\end{array}$ & $\begin{array}{l}12038 \\
11712 \\
11406 \\
11121 \\
10851\end{array}$ \\
\hline $\begin{array}{l}350 \\
360 \\
370 \\
380 \\
390\end{array}$ & $\begin{array}{l}3.75265 \\
3.77107 \\
3.78946 \\
3.80777 \\
3.82595\end{array}$ & $\begin{array}{l}1842 \\
1839 \\
1831 \\
1818 \\
1801\end{array}$ & $\begin{array}{l}3.55386 \\
3.55964 \\
3.56560 \\
3.57173 \\
3.57802\end{array}$ & $\begin{array}{l}578 \\
596 \\
613 \\
629 \\
642\end{array}$ & $\begin{array}{l}22.29631 \\
22.39651 \\
22.49412 \\
22.58929 \\
22.68215\end{array}$ & $\begin{array}{r}10020 \\
9761 \\
9517 \\
9286 \\
9067\end{array}$ & $\begin{array}{l}25.85018 \\
25.95616 \\
26.05973 \\
26.16103 \\
26.26018\end{array}$ & $\begin{array}{r}10598 \\
10357 \\
10130 \\
9915 \\
9709\end{array}$ \\
\hline $\begin{array}{l}400 \\
450 \\
500 \\
550 \\
600\end{array}$ & $\begin{array}{l}3.84396 \\
3.93041 \\
4.00920 \\
4.07958 \\
4.14186\end{array}$ & $\begin{array}{l}8645 \\
7879 \\
7038 \\
6228 \\
5492\end{array}$ & $\begin{array}{l}3.58444 \\
3.61814 \\
3.65338 \\
3.68899 \\
3.72419\end{array}$ & $\begin{array}{l}3370 \\
3524 \\
3561 \\
3520 \\
3428\end{array}$ & $\begin{array}{l}22.77282 \\
23.19693 \\
23.57996 \\
23.92983 \\
24.25233\end{array}$ & $\begin{array}{l}42411 \\
38303 \\
34987 \\
32250 \\
29946\end{array}$ & $\begin{array}{l}26.35727 \\
26.81508 \\
27.23334 \\
27.61883 \\
27.97652\end{array}$ & $\begin{array}{l}45781 \\
41826 \\
38549 \\
35769 \\
33374\end{array}$ \\
\hline $\begin{array}{l}650 \\
700 \\
750 \\
800 \\
850\end{array}$ & $\begin{array}{l}4.19678 \\
4.24524 \\
4.28811 \\
4.32622 \\
4.36026\end{array}$ & $\begin{array}{l}4846 \\
4287 \\
3811 \\
3404 \\
3057\end{array}$ & $\begin{array}{l}3.75847 \\
3.79155 \\
3.82325 \\
3.85352 \\
3.88234\end{array}$ & $\begin{array}{l}3308 \\
3170 \\
3027 \\
2882 \\
2742\end{array}$ & $\begin{array}{l}24.55179 \\
24.83154 \\
25.09422 \\
25.34194 \\
25.57643\end{array}$ & $\begin{array}{l}27975 \\
26268 \\
24772 \\
23449 \\
22269\end{array}$ & $\begin{array}{l}28.31026 \\
28.62309 \\
28.91747 \\
29.19546 \\
29.45878\end{array}$ & $\begin{array}{l}31283 \\
29438 \\
27799 \\
26332 \\
25010\end{array}$ \\
\hline $\begin{array}{r}900 \\
950 \\
1000 \\
1050 \\
1100\end{array}$ & $\begin{array}{l}4.39083 \\
4.41846 \\
4.44357 \\
4.46651 \\
4.48761\end{array}$ & $\begin{array}{l}2763 \\
2511 \\
2294 \\
2110 \\
1949\end{array}$ & $\begin{array}{l}3.90976 \\
3.93582 \\
3.96059 \\
3.98414 \\
4.00655\end{array}$ & $\begin{array}{l}2606 \\
2477 \\
2355 \\
2241 \\
2135\end{array}$ & $\begin{array}{l}25.79912 \\
26.01121 \\
26.21373 \\
26.40754 \\
26.59341\end{array}$ & $\begin{array}{l}21209 \\
20252 \\
19381 \\
18587 \\
17857\end{array}$ & $\begin{array}{l}29.70888 \\
29.94704 \\
30.17432 \\
30.39169 \\
30.59996\end{array}$ & $\begin{array}{l}23816 \\
22728 \\
21737 \\
20827 \\
19992\end{array}$ \\
\hline 1150 & 4.50710 & 1810 & 4.02790 & 2034 & 26.77198 & 17186 & 30.79988 & 19221 \\
\hline
\end{tabular}


Table 2.053. ZnD (Cont.)

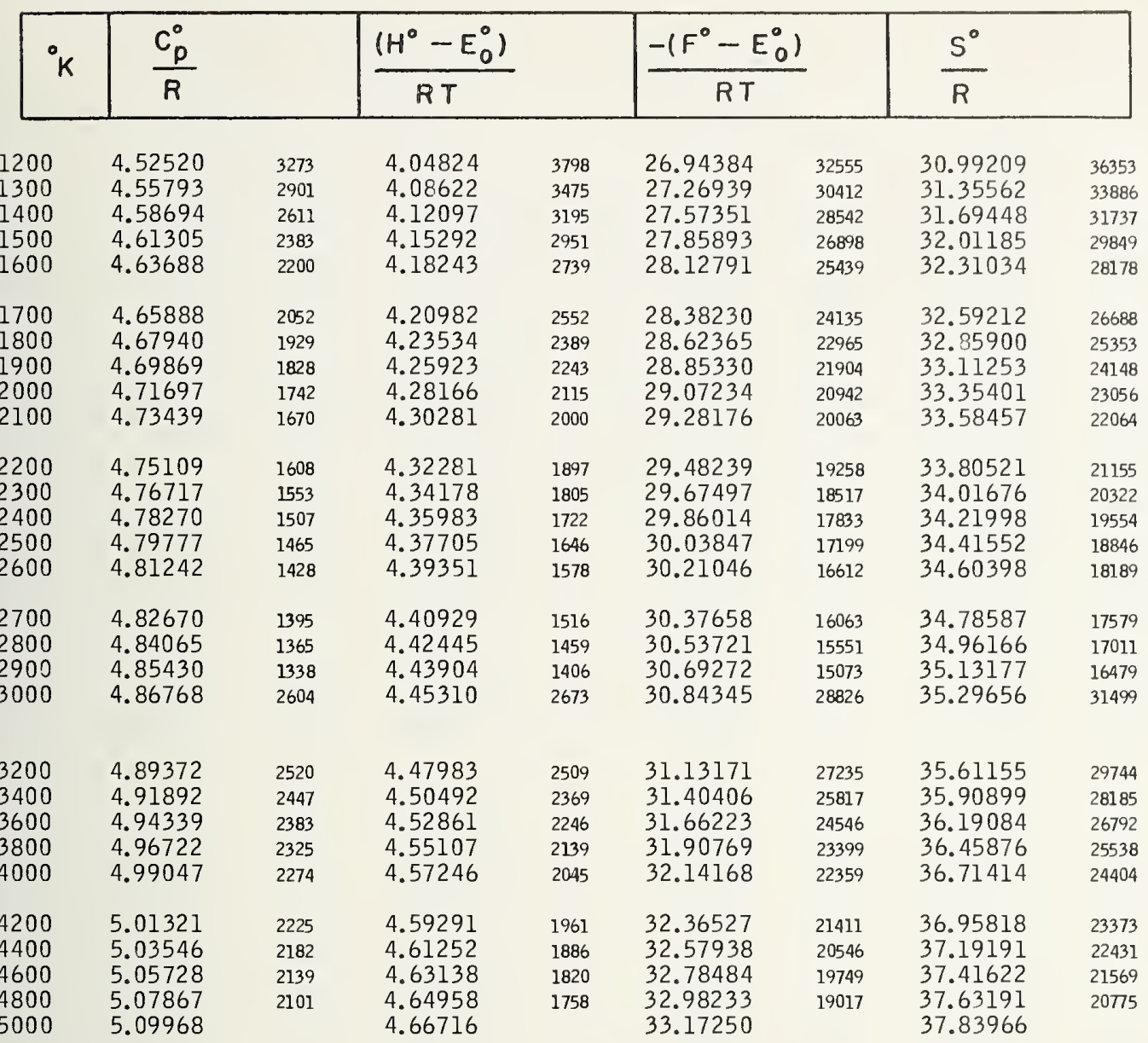


Table 2.054. ZnT

\begin{tabular}{|l|l|l|l|l|}
\hline${ }^{\circ} K$ & $\frac{C_{p}^{0}}{R}$ & $\frac{\left(H^{\circ}-E_{0}^{0}\right)}{R T}$ & $\frac{-\left(F^{\circ}-E_{0}^{0}\right)}{R T}$ & $\frac{S^{0}}{R}$ \\
\hline
\end{tabular}

\begin{tabular}{|c|c|c|c|c|c|c|c|c|}
\hline $\begin{array}{l}50 \\
60 \\
70 \\
80 \\
90\end{array}$ & $\begin{array}{l}3.50161 \\
3.50189 \\
3.50217 \\
3.50248 \\
3.50286\end{array}$ & $\begin{array}{l}28 \\
28 \\
31 \\
38 \\
57\end{array}$ & $\begin{array}{l}3.47882 \\
3.48264 \\
3.48541 \\
3.48753 \\
3.48921\end{array}$ & $\begin{array}{l}382 \\
277 \\
212 \\
168 \\
139\end{array}$ & $\begin{array}{l}15.85352 \\
16.48814 \\
17.02521 \\
17.49077 \\
17.90164\end{array}$ & $\begin{array}{l}63462 \\
53707 \\
46556 \\
41087 \\
36770\end{array}$ & $\begin{array}{l}19.33234 \\
19.97079 \\
20.51063 \\
20.97830 \\
21.39085\end{array}$ & $\begin{array}{l}63845 \\
53984 \\
46767 \\
41255 \\
36909\end{array}$ \\
\hline $\begin{array}{l}100 \\
110 \\
120 \\
130 \\
140\end{array}$ & $\begin{array}{l}3.50343 \\
3.50437 \\
3.50595 \\
3.50847 \\
3.51225\end{array}$ & $\begin{array}{r}94 \\
158 \\
252 \\
378 \\
528\end{array}$ & $\begin{array}{l}3.49060 \\
3.49181 \\
3.49291 \\
3.49401 \\
3.49517\end{array}$ & $\begin{array}{l}121 \\
110 \\
110 \\
116 \\
130\end{array}$ & $\begin{array}{l}18.26934 \\
18.60209 \\
18.90596 \\
19.18559 \\
19.44456\end{array}$ & $\begin{array}{l}33275 \\
30387 \\
27963 \\
25897 \\
24119\end{array}$ & $\begin{array}{l}21.75994 \\
22.09390 \\
22.39888 \\
22.67960 \\
22.93974\end{array}$ & $\begin{array}{l}33396 \\
30498 \\
28072 \\
26014 \\
24249\end{array}$ \\
\hline $\begin{array}{l}150 \\
160 \\
170 \\
180 \\
190\end{array}$ & $\begin{array}{l}3.51753 \\
3.52450 \\
3.53329 \\
3.54392 \\
3.55637\end{array}$ & $\begin{array}{r}697 \\
879 \\
1063 \\
1245 \\
1415\end{array}$ & $\begin{array}{l}3.49647 \\
3.49800 \\
3.49981 \\
3.50195 \\
3.50448\end{array}$ & $\begin{array}{l}153 \\
181 \\
214 \\
253 \\
294\end{array}$ & $\begin{array}{l}19.68575 \\
19.91145 \\
20.12357 \\
20.32367 \\
20.51308\end{array}$ & $\begin{array}{l}22570 \\
21212 \\
20010 \\
18941 \\
17983\end{array}$ & $\begin{array}{l}23.18223 \\
23.40946 \\
23.62338 \\
23.82563 \\
24.01757\end{array}$ & $\begin{array}{l}22723 \\
21392 \\
20225 \\
19194 \\
18277\end{array}$ \\
\hline $\begin{array}{l}200 \\
210 \\
220 \\
230 \\
240\end{array}$ & $\begin{array}{l}3.57052 \\
3.58625 \\
3.60339 \\
3.62173 \\
3.64111\end{array}$ & $\begin{array}{l}1573 \\
1714 \\
1834 \\
1938 \\
2020\end{array}$ & $\begin{array}{l}3.50742 \\
3.51080 \\
3.51461 \\
3.51887 \\
3.52355\end{array}$ & $\begin{array}{l}338 \\
381 \\
426 \\
468 \\
511\end{array}$ & $\begin{array}{l}20.69291 \\
20.86412 \\
21.02753 \\
21.18385 \\
21.33371\end{array}$ & $\begin{array}{l}17121 \\
16341 \\
15632 \\
14986 \\
14394\end{array}$ & $\begin{array}{l}24.20034 \\
24.37492 \\
24.54214 \\
24.70272 \\
24.85727\end{array}$ & $\begin{array}{l}17458 \\
16722 \\
16058 \\
15455 \\
14904\end{array}$ \\
\hline $\begin{array}{l}250 \\
260 \\
270 \\
280 \\
290\end{array}$ & $\begin{array}{l}3.66131 \\
3.68216 \\
3.70348 \\
3.72513 \\
3.74695\end{array}$ & $\begin{array}{l}2085 \\
2132 \\
2165 \\
2182 \\
2188\end{array}$ & $\begin{array}{l}3.52866 \\
3.53416 \\
3.54003 \\
3.54626 \\
3.55280\end{array}$ & $\begin{array}{l}550 \\
587 \\
623 \\
654 \\
684\end{array}$ & $\begin{array}{l}21.47765 \\
21.61615 \\
21.74964 \\
21.87850 \\
22.00305\end{array}$ & $\begin{array}{l}13850 \\
13349 \\
12886 \\
12455 \\
12056\end{array}$ & $\begin{array}{l}25.00631 \\
25.15031 \\
25.28968 \\
25.42476 \\
25.55586\end{array}$ & $\begin{array}{l}14400 \\
13937 \\
13508 \\
13110 \\
12739\end{array}$ \\
\hline $\begin{array}{l}300 \\
310 \\
320 \\
330 \\
340\end{array}$ & $\begin{array}{l}3.76883 \\
3.79066 \\
3.81234 \\
3.83379 \\
3.85496\end{array}$ & $\begin{array}{l}2183 \\
2168 \\
2145 \\
2117 \\
2082\end{array}$ & $\begin{array}{l}3.55964 \\
3.56674 \\
3.57407 \\
3.58162 \\
3.58935\end{array}$ & $\begin{array}{l}710 \\
733 \\
755 \\
773 \\
788\end{array}$ & $\begin{array}{l}22.12361 \\
22.24045 \\
22.35380 \\
22.46390 \\
22.57093\end{array}$ & $\begin{array}{l}11684 \\
11335 \\
11010 \\
10703 \\
10416\end{array}$ & $\begin{array}{l}25.68325 \\
25.80719 \\
25.92788 \\
26.04552 \\
26.16029\end{array}$ & $\begin{array}{l}12394 \\
12069 \\
11764 \\
11477 \\
11204\end{array}$ \\
\hline $\begin{array}{l}350 \\
360 \\
370 \\
380 \\
390\end{array}$ & $\begin{array}{l}3.87578 \\
3.89621 \\
3.91622 \\
3.93578 \\
3.95487\end{array}$ & $\begin{array}{l}2043 \\
2001 \\
1956 \\
1909 \\
1861\end{array}$ & $\begin{array}{l}3.59723 \\
3.60526 \\
3.61339 \\
3.62162 \\
3.62992\end{array}$ & $\begin{array}{l}803 \\
813 \\
823 \\
830 \\
836\end{array}$ & $\begin{array}{l}22.67509 \\
22.77654 \\
22.87543 \\
22.97191 \\
23.06609\end{array}$ & $\begin{array}{r}10145 \\
9889 \\
9648 \\
9418 \\
9200\end{array}$ & $\begin{array}{l}26.27233 \\
26.38181 \\
26.48883 \\
26.59353 \\
26.69601\end{array}$ & $\begin{array}{l}10948 \\
10702 \\
10470 \\
10248 \\
10037\end{array}$ \\
\hline $\begin{array}{l}400 \\
450 \\
500 \\
550 \\
600\end{array}$ & $\begin{array}{l}3.97348 \\
4.05916 \\
4.13295 \\
4.19613 \\
4.25027\end{array}$ & $\begin{array}{l}8568 \\
7379 \\
6318 \\
5414 \\
4662\end{array}$ & $\begin{array}{l}3.63828 \\
3.68040 \\
3.72206 \\
3.76236 \\
3.80082\end{array}$ & $\begin{array}{l}4212 \\
4166 \\
4030 \\
3846 \\
3641\end{array}$ & $\begin{array}{l}23.15809 \\
23.58905 \\
23.97899 \\
24.33564 \\
24.66467\end{array}$ & $\begin{array}{l}43096 \\
38994 \\
35665 \\
32903 \\
30568\end{array}$ & $\begin{array}{l}26.79638 \\
27.26945 \\
27.70105 \\
28.09800 \\
28.46550\end{array}$ & $\begin{array}{l}47307 \\
43160 \\
39695 \\
36750 \\
34209\end{array}$ \\
\hline $\begin{array}{l}650 \\
700 \\
750 \\
800 \\
850\end{array}$ & $\begin{array}{l}4.29689 \\
4.33729 \\
4.37259 \\
4.40369 \\
4.43130\end{array}$ & $\begin{array}{l}4040 \\
3530 \\
3110 \\
2761 \\
2473\end{array}$ & $\begin{array}{l}3.83723 \\
3.87154 \\
3.90379 \\
3.93408 \\
3.96253\end{array}$ & $\begin{array}{l}3431 \\
3225 \\
3029 \\
2845 \\
2675\end{array}$ & $\begin{array}{l}24.97035 \\
25.25599 \\
25.52421 \\
25.77713 \\
26.01650\end{array}$ & $\begin{array}{l}28564 \\
26822 \\
25292 \\
23937 \\
22725\end{array}$ & $\begin{array}{l}28.80759 \\
29.12753 \\
29.42301 \\
29.71122 \\
29.97904\end{array}$ & $\begin{array}{l}31994 \\
30048 \\
28321 \\
26782 \\
25400\end{array}$ \\
\hline $\begin{array}{r}900 \\
950 \\
1000 \\
1050 \\
1100\end{array}$ & $\begin{array}{l}4.45603 \\
4.47836 \\
4.49866 \\
4.51726 \\
4.53440\end{array}$ & $\begin{array}{l}2233 \\
2030 \\
1860 \\
1714 \\
1591\end{array}$ & $\begin{array}{l}3.98928 \\
4.01444 \\
4.03815 \\
4.06053 \\
4.08168\end{array}$ & $\begin{array}{l}2516 \\
2371 \\
2238 \\
2115 \\
2004\end{array}$ & $\begin{array}{l}26.24375 \\
26.46012 \\
26.66665 \\
26.86422 \\
27.05361\end{array}$ & $\begin{array}{l}21637 \\
20653 \\
19757 \\
18939 \\
18188\end{array}$ & $\begin{array}{l}30.23304 \\
30.47457 \\
30.70480 \\
30.92475 \\
31.13529\end{array}$ & $\begin{array}{l}24153 \\
23023 \\
21995 \\
21054 \\
20192\end{array}$ \\
\hline 1150 & 4.55031 & 1485 & 4.10172 & 1900 & 27.23549 & 17497 & 31.33721 & 19398 \\
\hline
\end{tabular}


Table 2.054. ZnT (Cont.)

\begin{tabular}{|l|l|l|l|l|}
\hline \multirow{K}{*}{} & $\frac{C_{p}^{0}}{R}$ & $\frac{\left(H^{0}-E_{0}^{0}\right)}{R T}$ & $\frac{-\left(F^{0}-E_{0}^{0}\right)}{R T}$ & $\frac{S^{0}}{R}$ \\
\hline
\end{tabular}

\begin{tabular}{|c|c|c|c|c|c|c|c|c|}
\hline $\begin{array}{l}1200 \\
1300 \\
1400 \\
1500 \\
1600\end{array}$ & $\begin{array}{l}4.56516 \\
4.59222 \\
4.61649 \\
4.63862 \\
4.65905\end{array}$ & $\begin{array}{l}2706 \\
2427 \\
2213 \\
2043 \\
1909\end{array}$ & $\begin{array}{l}4.12072 \\
4.15597 \\
4.18801 \\
4.21733 \\
4.24430\end{array}$ & $\begin{array}{l}3525 \\
3204 \\
2932 \\
2697 \\
2497\end{array}$ & $\begin{array}{l}27.41046 \\
27.74171 \\
28.05089 \\
28.34085 \\
28.61390\end{array}$ & $\begin{array}{l}33125 \\
30918 \\
28996 \\
27305 \\
25807\end{array}$ & $\begin{array}{l}31.53119 \\
31.89769 \\
32.23891 \\
32.55818 \\
32.85821\end{array}$ & $\begin{array}{l}36650 \\
34122 \\
31927 \\
30003 \\
28303\end{array}$ \\
\hline $\begin{array}{l}1700 \\
1800 \\
1900 \\
2000 \\
2100\end{array}$ & $\begin{array}{l}4.67814 \\
4.69614 \\
4.71324 \\
4.72960 \\
4.74533\end{array}$ & $\begin{array}{l}1800 \\
1710 \\
1636 \\
1573 \\
1519\end{array}$ & $\begin{array}{l}4.26927 \\
4.29249 \\
4.31418 \\
4.33455 \\
4.35374\end{array}$ & $\begin{array}{l}2322 \\
2169 \\
2037 \\
1919 \\
1814\end{array}$ & $\begin{array}{l}28.87197 \\
29.11666 \\
29.34933 \\
29.57114 \\
29.78310\end{array}$ & $\begin{array}{l}24469 \\
23267 \\
22181 \\
21196 \\
20296\end{array}$ & $\begin{array}{l}33.14124 \\
33.40915 \\
33.66352 \\
33.90570 \\
34.13684\end{array}$ & $\begin{array}{l}26791 \\
25437 \\
24218 \\
23114 \\
22110\end{array}$ \\
\hline $\begin{array}{l}2200 \\
2300 \\
2400 \\
2500 \\
2600\end{array}$ & $\begin{array}{l}4.76052 \\
4.77525 \\
4.78958 \\
4.80355 \\
4.81721\end{array}$ & $\begin{array}{l}1473 \\
1433 \\
1397 \\
1366 \\
1338\end{array}$ & $\begin{array}{l}4.37188 \\
4.38910 \\
4.40549 \\
4.42114 \\
4.43611\end{array}$ & $\begin{array}{l}1722 \\
1639 \\
1565 \\
1497 \\
1436\end{array}$ & $\begin{array}{l}29.98606 \\
30.18078 \\
30.36792 \\
30.54809 \\
30.72178\end{array}$ & $\begin{array}{l}19472 \\
18714 \\
18017 \\
17369 \\
16769\end{array}$ & $\begin{array}{l}34.35794 \\
34.56988 \\
34.77342 \\
34.96923 \\
35.15789\end{array}$ & $\begin{array}{l}21194 \\
20354 \\
19581 \\
18866 \\
18206\end{array}$ \\
\hline $\begin{array}{l}2700 \\
2800 \\
2900 \\
3000\end{array}$ & $\begin{array}{l}4.83059 \\
4.84372 \\
4.85661 \\
4.86929\end{array}$ & $\begin{array}{l}1313 \\
1289 \\
1268 \\
2480\end{array}$ & $\begin{array}{l}4.45047 \\
4.46428 \\
4.47759 \\
4.49044\end{array}$ & $\begin{array}{l}1381 \\
1331 \\
1285 \\
2445\end{array}$ & $\begin{array}{l}30.88947 \\
31.05158 \\
31.20847 \\
31.36048\end{array}$ & $\begin{array}{l}16211 \\
15689 \\
15201 \\
29060\end{array}$ & $\begin{array}{l}35.33995 \\
35.51586 \\
35.68606 \\
35.85092\end{array}$ & $\begin{array}{l}17591 \\
17020 \\
16486 \\
31506\end{array}$ \\
\hline $\begin{array}{l}3200 \\
3400 \\
3600 \\
3800 \\
4000\end{array}$ & $\begin{array}{l}4.89409 \\
4.91821 \\
4.94174 \\
4.96474 \\
4.98725\end{array}$ & $\begin{array}{l}2412 \\
2353 \\
2300 \\
2251 \\
2206\end{array}$ & $\begin{array}{l}4.51489 \\
4.53791 \\
4.55970 \\
4.58041 \\
4.60019\end{array}$ & $\begin{array}{l}2302 \\
2179 \\
2071 \\
1978 \\
1896\end{array}$ & $\begin{array}{l}31.65108 \\
31.92549 \\
32.18549 \\
32.43258 \\
32.66803\end{array}$ & $\begin{array}{l}27441 \\
26000 \\
24709 \\
23545 \\
22491\end{array}$ & $\begin{array}{l}36.16598 \\
36.46341 \\
36.74519 \\
37.01300 \\
37.26823\end{array}$ & $\begin{array}{l}29743 \\
28178 \\
26781 \\
25523 \\
24386\end{array}$ \\
\hline $\begin{array}{l}4200 \\
4400 \\
4600 \\
4800 \\
5000\end{array}$ & $\begin{array}{l}5.00931 \\
5.03096 \\
5.05222 \\
5.07312 \\
5.09367\end{array}$ & $\begin{array}{l}2165 \\
2126 \\
2090 \\
2055\end{array}$ & $\begin{array}{l}4.61915 \\
4.63738 \\
4.65495 \\
4.67194 \\
4.68840\end{array}$ & $\begin{array}{l}1823 \\
1757 \\
1699 \\
1646\end{array}$ & $\begin{array}{l}32.89294 \\
33.10824 \\
33.31477 \\
33.51325 \\
33.70430\end{array}$ & $\begin{array}{l}21530 \\
20653 \\
19848 \\
19105\end{array}$ & $\begin{array}{l}37.51209 \\
37.74563 \\
37.96973 \\
38.18520 \\
38.39271\end{array}$ & $\begin{array}{l}23354 \\
22410 \\
21547 \\
20751\end{array}$ \\
\hline
\end{tabular}


Table 2.055. CdH

\begin{tabular}{|c|c|c|c|c|c|c|c|c|}
\hline${ }^{\circ} K$ & $\frac{C_{p}^{0}}{R}$ & & $\frac{\left(H^{\circ}-E_{0}^{0}\right)}{R T}$ & & $\frac{-\left\langle F^{0}-E\right.}{R T}$ & & $\frac{S^{\circ}}{R}$ & \\
\hline $\begin{array}{l}50 \\
60 \\
70 \\
80 \\
90\end{array}$ & $\begin{array}{l}3.50212 \\
3.50225 \\
3.50246 \\
3.50270 \\
3.50297\end{array}$ & $\begin{array}{l}13 \\
21 \\
24 \\
27 \\
28\end{array}$ & $\begin{array}{l}3.44936 \\
3.45817 \\
3.46448 \\
3.46924 \\
3.47297\end{array}$ & $\begin{array}{l}881 \\
631 \\
476 \\
373 \\
302\end{array}$ & $\begin{array}{l}15.83719 \\
16.46691 \\
17.00049 \\
17.46343 \\
17.87227\end{array}$ & $\begin{array}{l}62972 \\
53358 \\
46294 \\
40884 \\
36608\end{array}$ & $\begin{array}{l}19.28656 \\
19.92508 \\
20.46497 \\
20.93267 \\
21.34525\end{array}$ & $\begin{array}{l}63852 \\
53989 \\
46770 \\
41258 \\
36909\end{array}$ \\
\hline $\begin{array}{l}100 \\
110 \\
120 \\
130 \\
140\end{array}$ & $\begin{array}{l}3.50325 \\
3.50354 \\
3.50385 \\
3.50421 \\
3.50463\end{array}$ & $\begin{array}{l}29 \\
31 \\
36 \\
42 \\
56\end{array}$ & $\begin{array}{l}3.47599 \\
3.47848 \\
3.48058 \\
3.48238 \\
3.48396\end{array}$ & $\begin{array}{l}249 \\
210 \\
180 \\
158 \\
139\end{array}$ & $\begin{array}{l}18.23835 \\
18.56976 \\
18.87252 \\
19.15119 \\
19.40932\end{array}$ & $\begin{array}{l}33141 \\
30276 \\
27867 \\
25813 \\
24042\end{array}$ & $\begin{array}{l}21.71434 \\
22.04825 \\
22.35311 \\
22.63358 \\
22.89329\end{array}$ & $\begin{array}{l}33391 \\
30486 \\
28047 \\
25971 \\
24181\end{array}$ \\
\hline $\begin{array}{l}150 \\
160 \\
170 \\
180 \\
190\end{array}$ & $\begin{array}{l}3.50519 \\
3.50595 \\
3.50699 \\
3.50842 \\
3.51033\end{array}$ & $\begin{array}{r}76 \\
104 \\
143 \\
191 \\
251\end{array}$ & $\begin{array}{l}3.48535 \\
3.48661 \\
3.48778 \\
3.48889 \\
3.48996\end{array}$ & $\begin{array}{l}126 \\
117 \\
111 \\
107 \\
108\end{array}$ & $\begin{array}{l}19.64974 \\
19.87472 \\
20.08613 \\
20.28552 \\
20.47418\end{array}$ & $\begin{array}{l}22498 \\
21141 \\
19939 \\
18866 \\
17904\end{array}$ & $\begin{array}{l}23.13510 \\
23.36134 \\
23.57392 \\
23.77441 \\
23.96415\end{array}$ & $\begin{array}{l}22624 \\
21258 \\
20049 \\
18974 \\
18012\end{array}$ \\
\hline $\begin{array}{l}200 \\
210 \\
220 \\
230 \\
240\end{array}$ & $\begin{array}{l}3.51284 \\
3.51601 \\
3.51993 \\
3.52467 \\
3.53027\end{array}$ & $\begin{array}{l}317 \\
392 \\
474 \\
560 \\
648\end{array}$ & $\begin{array}{l}3.49104 \\
3.49215 \\
3.49332 \\
3.49458 \\
3.49595\end{array}$ & $\begin{array}{l}111 \\
117 \\
126 \\
137 \\
150\end{array}$ & $\begin{array}{l}20.65322 \\
20.82358 \\
20.98606 \\
21.14137 \\
21.29013\end{array}$ & $\begin{array}{l}17036 \\
16248 \\
15531 \\
14876 \\
14274\end{array}$ & $\begin{array}{l}24.14427 \\
24.31574 \\
24.47939 \\
24.63596 \\
24.78608\end{array}$ & $\begin{array}{l}17147 \\
16365 \\
15657 \\
15012 \\
14424\end{array}$ \\
\hline $\begin{array}{l}250 \\
260 \\
270 \\
280 \\
290\end{array}$ & $\begin{array}{l}3.53675 \\
3.54412 \\
3.55238 \\
3.56151 \\
3.57147\end{array}$ & $\begin{array}{r}737 \\
826 \\
913 \\
996 \\
1074\end{array}$ & $\begin{array}{l}3.49745 \\
3.49910 \\
3.50091 \\
3.50291 \\
3.50510\end{array}$ & $\begin{array}{l}165 \\
181 \\
200 \\
219 \\
239\end{array}$ & $\begin{array}{l}21.43287 \\
21.57007 \\
21.70217 \\
21.82952 \\
21.95248\end{array}$ & $\begin{array}{l}13720 \\
13210 \\
12735 \\
12296 \\
11887\end{array}$ & $\begin{array}{l}24.93032 \\
25.06918 \\
25.20309 \\
25.33244 \\
25.45759\end{array}$ & $\begin{array}{l}13886 \\
13391 \\
12935 \\
12515 \\
12126\end{array}$ \\
\hline $\begin{array}{l}300 \\
310 \\
320 \\
330 \\
340\end{array}$ & $\begin{array}{l}3.58221 \\
3.59369 \\
3.60585 \\
3.61864 \\
3.63198\end{array}$ & $\begin{array}{l}1148 \\
1216 \\
1279 \\
1334 \\
1384\end{array}$ & $\begin{array}{l}3.50749 \\
3.51009 \\
3.51289 \\
3.51590 \\
3.51911\end{array}$ & $\begin{array}{l}260 \\
280 \\
301 \\
321 \\
342\end{array}$ & $\begin{array}{l}22.07135 \\
22.18640 \\
22.29789 \\
22.40603 \\
22.51104\end{array}$ & $\begin{array}{l}11505 \\
11149 \\
10814 \\
10501 \\
10206\end{array}$ & $\begin{array}{l}25.57885 \\
25.69649 \\
25.81078 \\
25.92193 \\
26.03015\end{array}$ & $\begin{array}{l}11764 \\
11429 \\
11115 \\
10822 \\
10549\end{array}$ \\
\hline $\begin{array}{l}350 \\
360 \\
370 \\
380 \\
390\end{array}$ & $\begin{array}{l}3.64582 \\
3.66010 \\
3.67475 \\
3.68971 \\
3.70494\end{array}$ & $\begin{array}{l}1428 \\
1465 \\
1496 \\
1523 \\
1543\end{array}$ & $\begin{array}{l}3.52253 \\
3.52616 \\
3.52997 \\
3.53398 \\
3.53817\end{array}$ & $\begin{array}{l}363 \\
381 \\
401 \\
419 \\
436\end{array}$ & $\begin{array}{l}22.61310 \\
22.71238 \\
22.80904 \\
22.90323 \\
22.99509\end{array}$ & $\begin{array}{l}9928 \\
9666 \\
9419 \\
9186 \\
8963\end{array}$ & $\begin{array}{l}26.13564 \\
26.23854 \\
26.33902 \\
26.43722 \\
26.53326\end{array}$ & $\begin{array}{r}10290 \\
10048 \\
9820 \\
9604 \\
9399\end{array}$ \\
\hline $\begin{array}{l}400 \\
450 \\
500 \\
550 \\
600\end{array}$ & $\begin{array}{l}3.72037 \\
3.79905 \\
3.87701 \\
3.95133 \\
4.02056\end{array}$ & $\begin{array}{l}7868 \\
7796 \\
7432 \\
6923 \\
6362\end{array}$ & $\begin{array}{l}3.54253 \\
3.56665 \\
3.59381 \\
3.62297 \\
3.65325\end{array}$ & $\begin{array}{l}2412 \\
2716 \\
2916 \\
3028 \\
3074\end{array}$ & $\begin{array}{l}23.08472 \\
23.50333 \\
23.88049 \\
24.22438 \\
24.54091\end{array}$ & $\begin{array}{l}41861 \\
37716 \\
34389 \\
31653 \\
29363\end{array}$ & $\begin{array}{l}26.62725 \\
27.06998 \\
27.47431 \\
27.84735 \\
28.19417\end{array}$ & $\begin{array}{l}44273 \\
40433 \\
37304 \\
34682 \\
32436\end{array}$ \\
\hline $\begin{array}{l}650 \\
700 \\
750 \\
800 \\
850\end{array}$ & $\begin{array}{l}4.08418 \\
4.14219 \\
4.19491 \\
4.24279 \\
4.28631\end{array}$ & $\begin{array}{l}5801 \\
5272 \\
4788 \\
4352 \\
3965\end{array}$ & $\begin{array}{l}3.68399 \\
3.71468 \\
3.74497 \\
3.77461 \\
3.80345\end{array}$ & $\begin{array}{l}3069 \\
3029 \\
2964 \\
2884 \\
2794\end{array}$ & $\begin{array}{l}24.83454 \\
25.10868 \\
25.36600 \\
25.60864 \\
25.83835\end{array}$ & $\begin{array}{l}27414 \\
25732 \\
24264 \\
22971 \\
21819\end{array}$ & $\begin{array}{l}28.51853 \\
28.82336 \\
29.11097 \\
29.38326 \\
29.64180\end{array}$ & $\begin{array}{l}30483 \\
28761 \\
27229 \\
25854 \\
24614\end{array}$ \\
\hline $\begin{array}{r}900 \\
950 \\
1000 \\
1050 \\
1100\end{array}$ & $\begin{array}{l}4.32596 \\
4.36219 \\
4.39541 \\
4.42599 \\
4.45425\end{array}$ & $\begin{array}{l}3623 \\
3322 \\
3058 \\
2826 \\
2621\end{array}$ & $\begin{array}{l}3.83139 \\
3.85839 \\
3.88442 \\
3.90949 \\
3.93362\end{array}$ & $\begin{array}{l}2700 \\
2603 \\
2507 \\
2413 \\
2321\end{array}$ & $\begin{array}{l}26.05654 \\
26.26442 \\
26.46300 \\
26.65313 \\
26.83556\end{array}$ & $\begin{array}{l}20788 \\
19858 \\
19013 \\
18243 \\
17537\end{array}$ & $\begin{array}{l}29.88794 \\
30.12282 \\
30.34743 \\
30.56263 \\
30.76919\end{array}$ & $\begin{array}{l}23488 \\
22461 \\
21520 \\
20656 \\
19858\end{array}$ \\
\hline 1150 & 4.48046 & 2442 & 3.95683 & 2234 & 27.01093 & 16888 & 30.96777 & 19121 \\
\hline
\end{tabular}


Table 2. 055. CdH (Cont.)

\begin{tabular}{|c|c|c|c|c|c|c|c|c|}
\hline${ }^{\circ} K$ & $\frac{C_{p}^{\circ}}{R}$ & & $\frac{\left(H^{0}-E_{0}^{0}\right)}{R T}$ & & $\frac{-\left(F^{\circ}-E\right.}{R T}$ & & $\frac{S^{\circ}}{R}$ & \\
\hline $\begin{array}{l}1200 \\
1300 \\
1400 \\
1500 \\
1600\end{array}$ & $\begin{array}{l}4.50488 \\
4.54912 \\
4.58836 \\
4.62362 \\
4.65569\end{array}$ & $\begin{array}{l}4424 \\
3924 \\
3526 \\
3207 \\
2948\end{array}$ & $\begin{array}{l}3.97917 \\
4.02134 \\
4.06047 \\
4.09686 \\
4.13080\end{array}$ & $\begin{array}{l}4217 \\
3913 \\
3639 \\
3394 \\
3175\end{array}$ & $\begin{array}{l}27.17981 \\
27.50000 \\
27.79946 \\
28.08086 \\
28.34637\end{array}$ & $\begin{array}{l}32019 \\
29946 \\
28140 \\
26551 \\
25139\end{array}$ & $\begin{array}{l}31.15898 \\
31.52135 \\
31.85994 \\
32.17773 \\
32.47717\end{array}$ & $\begin{array}{l}36237 \\
33859 \\
31779 \\
29944 \\
28314\end{array}$ \\
\hline $\begin{array}{l}1700 \\
1800 \\
1900 \\
2000 \\
2100\end{array}$ & $\begin{array}{l}4.68517 \\
4.71252 \\
4.73811 \\
4.76223 \\
4.78509\end{array}$ & $\begin{array}{l}2735 \\
2559 \\
2412 \\
2286 \\
2178\end{array}$ & $\begin{array}{l}4.16255 \\
4.19235 \\
4.22041 \\
4.24690 \\
4.27199\end{array}$ & $\begin{array}{l}2980 \\
2806 \\
2649 \\
2509 \\
2382\end{array}$ & $\begin{array}{l}28.59776 \\
28.83653 \\
29.06396 \\
29.28112 \\
29.48894\end{array}$ & $\begin{array}{l}23877 \\
22743 \\
21716 \\
20782 \\
19929\end{array}$ & $\begin{array}{l}32.76031 \\
33.02889 \\
33.28438 \\
33.52803 \\
33.76094\end{array}$ & $\begin{array}{l}26858 \\
25549 \\
24365 \\
23291 \\
22311\end{array}$ \\
\hline $\begin{array}{l}2200 \\
2300 \\
2400 \\
2500 \\
2600\end{array}$ & $\begin{array}{l}4.80687 \\
4.82774 \\
4.84780 \\
4.86715 \\
4.88588\end{array}$ & $\begin{array}{l}2087 \\
2006 \\
1935 \\
1873 \\
1818\end{array}$ & $\begin{array}{l}4.29581 \\
4.31849 \\
4.34013 \\
4.36083 \\
4.38066\end{array}$ & $\begin{array}{l}2268 \\
2164 \\
2070 \\
1983 \\
1905\end{array}$ & $\begin{array}{l}29.68823 \\
29.87969 \\
30.06394 \\
30.24154 \\
30.41296\end{array}$ & $\begin{array}{l}19146 \\
18425 \\
17760 \\
17142 \\
16569\end{array}$ & $\begin{array}{l}33.98405 \\
34.19819 \\
34.40408 \\
34.60237 \\
34.79363\end{array}$ & $\begin{array}{l}21414 \\
20589 \\
19829 \\
19126 \\
18474\end{array}$ \\
\hline $\begin{array}{l}2700 \\
2800 \\
2900 \\
3000\end{array}$ & $\begin{array}{l}4.90406 \\
4.92173 \\
4.93895 \\
4.95577\end{array}$ & $\begin{array}{l}1767 \\
1722 \\
1682 \\
3253\end{array}$ & $\begin{array}{l}4.39971 \\
4.41804 \\
4.43571 \\
4.45276\end{array}$ & $\begin{array}{l}1833 \\
1767 \\
1705 \\
3247\end{array}$ & $\begin{array}{l}30.57865 \\
30.73899 \\
30.89434 \\
31.04500\end{array}$ & $\begin{array}{l}16034 \\
15535 \\
15066 \\
28843\end{array}$ & $\begin{array}{l}34.97837 \\
35.15704 \\
35.33005 \\
35.49777\end{array}$ & $\begin{array}{l}17867 \\
17301 \\
16772 \\
32089\end{array}$ \\
\hline $\begin{array}{l}3200 \\
3400 \\
3600 \\
3800 \\
4000\end{array}$ & $\begin{array}{l}4.98830 \\
5.01955 \\
5.04969 \\
5.07886 \\
5.10716\end{array}$ & $\begin{array}{l}3125 \\
3014 \\
2917 \\
2830 \\
2751\end{array}$ & $\begin{array}{l}4.48523 \\
4.51574 \\
4.54457 \\
4.57193 \\
4.59799\end{array}$ & $\begin{array}{l}3051 \\
2883 \\
2736 \\
2606 \\
2490\end{array}$ & $\begin{array}{l}31.33343 \\
31.60627 \\
31.86520 \\
32.11166 \\
32.34683\end{array}$ & $\begin{array}{l}27284 \\
25893 \\
24646 \\
23517 \\
22495\end{array}$ & $\begin{array}{l}35.81866 \\
36.12202 \\
36.40978 \\
36.68359 \\
36.94483\end{array}$ & $\begin{array}{l}30336 \\
28776 \\
27381 \\
26124 \\
24984\end{array}$ \\
\hline $\begin{array}{l}4200 \\
4400 \\
4600 \\
4800 \\
5000\end{array}$ & $\begin{array}{l}5.13467 \\
5.16145 \\
5.18757 \\
5.21307 \\
5.23799\end{array}$ & $\begin{array}{l}2678 \\
2612 \\
2550 \\
2492\end{array}$ & $\begin{array}{l}4.62289 \\
4.64677 \\
4.66971 \\
4.69183 \\
4.71318\end{array}$ & $\begin{array}{l}2388 \\
2294 \\
2212 \\
2135\end{array}$ & $\begin{array}{l}32.57178 \\
32.78739 \\
32.99446 \\
33.19367 \\
33.38563\end{array}$ & $\begin{array}{l}21561 \\
20707 \\
19921 \\
19196\end{array}$ & $\begin{array}{l}37.19467 \\
37.43416 \\
37.66417 \\
37.88550 \\
38.09881\end{array}$ & $\begin{array}{l}23949 \\
23001 \\
22133 \\
21331\end{array}$ \\
\hline
\end{tabular}


Table 2.056. CdD

\begin{tabular}{|c|c|c|c|c|c|c|c|c|}
\hline${ }^{\circ} K$ & $\frac{C_{p}^{0}}{R}$ & & $\frac{\left(H^{\circ}-E_{0}^{0}\right)}{R T}$ & & $\frac{-L F^{\circ}-E}{R T}$ & & $\frac{S^{\circ}}{R}$ & \\
\hline $\begin{array}{l}50 \\
60 \\
70 \\
80 \\
90\end{array}$ & $\begin{array}{l}3.50169 \\
3.50196 \\
3.50225 \\
3.50255 \\
3.50289\end{array}$ & $\begin{array}{l}27 \\
29 \\
30 \\
34 \\
43\end{array}$ & $\begin{array}{l}3.47470 \\
3.47922 \\
3.48249 \\
3.48498 \\
3.48695\end{array}$ & $\begin{array}{l}452 \\
327 \\
249 \\
197 \\
161\end{array}$ & $\begin{array}{l}16.49896 \\
17.13289 \\
17.66948 \\
18.13467 \\
18.54526\end{array}$ & $\begin{array}{l}63393 \\
53659 \\
46519 \\
41059 \\
36747\end{array}$ & $\begin{array}{l}19.97366 \\
20.61212 \\
21.15197 \\
21.61965 \\
22.03221\end{array}$ & $\begin{array}{l}63846 \\
53985 \\
46768 \\
41256 \\
36909\end{array}$ \\
\hline $\begin{array}{l}100 \\
110 \\
120 \\
130 \\
140\end{array}$ & $\begin{array}{l}3.50332 \\
3.50398 \\
3.50503 \\
3.50673 \\
3.50933\end{array}$ & $\begin{array}{r}66 \\
105 \\
170 \\
260 \\
374\end{array}$ & $\begin{array}{l}3.48856 \\
3.48993 \\
3.49114 \\
3.49227 \\
3.49339\end{array}$ & $\begin{array}{l}137 \\
121 \\
113 \\
112 \\
118\end{array}$ & $\begin{array}{l}18.91273 \\
19.24529 \\
19.54901 \\
19.82850 \\
20.08734\end{array}$ & $\begin{array}{l}33256 \\
30372 \\
27949 \\
25884 \\
24106\end{array}$ & $\begin{array}{l}22.40130 \\
22.73523 \\
23.04016 \\
23.32078 \\
23.58074\end{array}$ & $\begin{array}{l}33393 \\
30493 \\
28062 \\
25996 \\
24224\end{array}$ \\
\hline $\begin{array}{l}150 \\
160 \\
170 \\
180 \\
190\end{array}$ & $\begin{array}{l}3.51307 \\
3.51819 \\
3.52483 \\
3.53311 \\
3.54306\end{array}$ & $\begin{array}{r}512 \\
664 \\
828 \\
995 \\
1161\end{array}$ & $\begin{array}{l}3.49457 \\
3.49588 \\
3.49738 \\
3.49913 \\
3.50117\end{array}$ & $\begin{array}{l}131 \\
150 \\
175 \\
204 \\
238\end{array}$ & $\begin{array}{l}20.32840 \\
20.55398 \\
20.76596 \\
20.96591 \\
21.15515\end{array}$ & $\begin{array}{l}22558 \\
21198 \\
19995 \\
18924 \\
17965\end{array}$ & $\begin{array}{l}23.82298 \\
24.04987 \\
24.26335 \\
24.46505 \\
24.65633\end{array}$ & $\begin{array}{l}22689 \\
21348 \\
20170 \\
19128 \\
18202\end{array}$ \\
\hline $\begin{array}{l}200 \\
210 \\
220 \\
230 \\
240\end{array}$ & $\begin{array}{l}3.55467 \\
3.56785 \\
3.58251 \\
3.59851 \\
3.61569\end{array}$ & $\begin{array}{l}1318 \\
1466 \\
1600 \\
1718 \\
1821\end{array}$ & $\begin{array}{l}3.50355 \\
3.50629 \\
3.50942 \\
3.51294 \\
3.51686\end{array}$ & $\begin{array}{l}274 \\
313 \\
352 \\
392 \\
431\end{array}$ & $\begin{array}{l}21.33480 \\
21.50580 \\
21.66899 \\
21.82506 \\
21.97465\end{array}$ & $\begin{array}{l}17100 \\
16319 \\
15607 \\
14959 \\
14366\end{array}$ & $\begin{array}{l}24.83835 \\
25.01210 \\
25.17841 \\
25.33801 \\
25.49152\end{array}$ & $\begin{array}{l}17375 \\
16631 \\
15960 \\
15351 \\
14796\end{array}$ \\
\hline $\begin{array}{l}250 \\
260 \\
270 \\
280 \\
290\end{array}$ & $\begin{array}{l}3.63390 \\
3.65297 \\
3.67275 \\
3.69307 \\
3.71380\end{array}$ & $\begin{array}{l}1907 \\
1978 \\
2032 \\
2073 \\
2101\end{array}$ & $\begin{array}{l}3.52117 \\
3.52587 \\
3.53094 \\
3.53637 \\
3.54213\end{array}$ & $\begin{array}{l}470 \\
507 \\
543 \\
576 \\
607\end{array}$ & $\begin{array}{l}22.11831 \\
22.25650 \\
22.38966 \\
22.51817 \\
22.64237\end{array}$ & $\begin{array}{l}13819 \\
13316 \\
12851 \\
12420 \\
12018\end{array}$ & $\begin{array}{l}25.63948 \\
25.78238 \\
25.92061 \\
26.05455 \\
26.18450\end{array}$ & $\begin{array}{l}14290 \\
13823 \\
13394 \\
12995 \\
12626\end{array}$ \\
\hline $\begin{array}{l}300 \\
310 \\
320 \\
330 \\
340\end{array}$ & $\begin{array}{l}3.73481 \\
3.75599 \\
3.77722 \\
3.79841 \\
3.81950\end{array}$ & $\begin{array}{l}2118 \\
2123 \\
2119 \\
2109 \\
2090\end{array}$ & $\begin{array}{l}3.54820 \\
3.55456 \\
3.56119 \\
3.56806 \\
3.57514\end{array}$ & $\begin{array}{l}636 \\
663 \\
687 \\
708 \\
728\end{array}$ & $\begin{array}{l}22.76255 \\
22.87900 \\
22.99196 \\
23.10164 \\
23.20827\end{array}$ & $\begin{array}{l}11645 \\
11296 \\
10968 \\
10663 \\
10374\end{array}$ & $\begin{array}{l}26.31076 \\
26.43357 \\
26.55315 \\
26.66971 \\
26.78341\end{array}$ & $\begin{array}{l}12281 \\
11958 \\
11656 \\
11370 \\
11102\end{array}$ \\
\hline $\begin{array}{l}350 \\
360 \\
370 \\
380 \\
390\end{array}$ & $\begin{array}{l}3.84040 \\
3.86106 \\
3.88143 \\
3.90147 \\
3.92116\end{array}$ & $\begin{array}{l}2066 \\
2037 \\
2004 \\
1969 \\
1930\end{array}$ & $\begin{array}{l}3.58242 \\
3.58988 \\
3.59748 \\
3.60522 \\
3.61307\end{array}$ & $\begin{array}{l}746 \\
760 \\
774 \\
785 \\
794\end{array}$ & $\begin{array}{l}23.31201 \\
23.41303 \\
23.51149 \\
23.60753 \\
23.70128\end{array}$ & $\begin{array}{r}10102 \\
9846 \\
9604 \\
9375 \\
9158\end{array}$ & $\begin{array}{l}26.89443 \\
27.00291 \\
27.10898 \\
27.21276 \\
27.31436\end{array}$ & $\begin{array}{r}10848 \\
10607 \\
10378 \\
10160 \\
9951\end{array}$ \\
\hline $\begin{array}{l}400 \\
450 \\
500 \\
550 \\
600\end{array}$ & $\begin{array}{l}3.94046 \\
4.03065 \\
4.11001 \\
4.17912 \\
4.23920\end{array}$ & $\begin{array}{l}9019 \\
7936 \\
6911 \\
6008 \\
5236\end{array}$ & $\begin{array}{l}3.62101 \\
3.66162 \\
3.70258 \\
3.74283 \\
3.78175\end{array}$ & $\begin{array}{l}4061 \\
4096 \\
4025 \\
3892 \\
3725\end{array}$ & $\begin{array}{l}23.79286 \\
24.22169 \\
24.60960 \\
24.96439 \\
25.29174\end{array}$ & $\begin{array}{l}42883 \\
38791 \\
35479 \\
32735 \\
30418\end{array}$ & $\begin{array}{l}27.41387 \\
27.88331 \\
28.31218 \\
28.70722 \\
29.07349\end{array}$ & $\begin{array}{l}46944 \\
42887 \\
39504 \\
36627 \\
34144\end{array}$ \\
\hline $\begin{array}{l}650 \\
700 \\
750 \\
800 \\
850\end{array}$ & $\begin{array}{l}4.29156 \\
4.33742 \\
4.37785 \\
4.41377 \\
4.44591\end{array}$ & $\begin{array}{l}4586 \\
4043 \\
3592 \\
3214 \\
2898\end{array}$ & $\begin{array}{l}3.81900 \\
3.85443 \\
3.88800 \\
3.91976 \\
3.94978\end{array}$ & $\begin{array}{l}3543 \\
3357 \\
3176 \\
3002 \\
2838\end{array}$ & $\begin{array}{l}25.59592 \\
25.88025 \\
26.14733 \\
26.39928 \\
26.63783\end{array}$ & $\begin{array}{l}28433 \\
26708 \\
25195 \\
23855 \\
22657\end{array}$ & $\begin{array}{l}29.41493 \\
29.73468 \\
30.03534 \\
30.31905 \\
30.58761\end{array}$ & $\begin{array}{l}31975 \\
30066 \\
28371 \\
26856 \\
25496\end{array}$ \\
\hline $\begin{array}{r}900 \\
950 \\
1000 \\
1050 \\
1100\end{array}$ & $\begin{array}{l}4.47489 \\
4.50122 \\
4.52530 \\
4.54748 \\
4.56803\end{array}$ & $\begin{array}{l}2633 \\
2408 \\
2218 \\
2055 \\
1916\end{array}$ & $\begin{array}{l}3.97816 \\
4.00501 \\
4.03043 \\
4.05453 \\
4.07741\end{array}$ & $\begin{array}{l}2685 \\
2542 \\
2410 \\
2288 \\
2175\end{array}$ & $\begin{array}{l}26.86440 \\
27.08021 \\
27.28630 \\
27.48353 \\
27.67268\end{array}$ & $\begin{array}{l}21581 \\
20609 \\
19723 \\
18915 \\
18173\end{array}$ & $\begin{array}{l}30.84257 \\
31.08523 \\
31.31673 \\
31.53807 \\
31.75010\end{array}$ & $\begin{array}{l}24266 \\
23150 \\
22134 \\
21203 \\
20348\end{array}$ \\
\hline 1150 & 4.58719 & 1795 & 4.09916 & 2072 & 27.85441 & 17490 & 31.95358 & 19561 \\
\hline
\end{tabular}


Table 2.056. CdD (Cont.)

\begin{tabular}{|c|c|c|c|c|c|c|c|c|}
\hline${ }^{\circ} K$ & $\frac{C_{p}^{0}}{R}$ & & $\frac{\left(H^{\circ}-E_{0}^{0}\right)}{R T}$ & & $\frac{-\left(F^{0}-\right.}{R T}$ & & $\frac{S^{\circ}}{R}$ & \\
\hline $\begin{array}{l}1200 \\
1300 \\
1400 \\
1500 \\
1600\end{array}$ & $\begin{array}{l}4.60514 \\
4.63805 \\
4.66776 \\
4.69498 \\
4.72022\end{array}$ & $\begin{array}{l}3291 \\
2971 \\
2722 \\
2524 \\
2366\end{array}$ & $\begin{array}{l}4.11988 \\
4.15849 \\
4.19382 \\
4.22634 \\
4.25643\end{array}$ & $\begin{array}{l}3861 \\
3533 \\
3252 \\
3009 \\
2798\end{array}$ & $\begin{array}{l}28.02931 \\
28.36063 \\
28.67012 \\
28.96059 \\
29.23432\end{array}$ & $\begin{array}{l}33132 \\
30949 \\
29047 \\
27373 \\
25889\end{array}$ & $\begin{array}{l}32.14919 \\
32.51913 \\
32.86395 \\
33.18693 \\
33.49075\end{array}$ & $\begin{array}{l}36994 \\
34482 \\
32298 \\
30382 \\
28688\end{array}$ \\
\hline $\begin{array}{l}1700 \\
1800 \\
1900 \\
2000 \\
2100\end{array}$ & $\begin{array}{l}4.74388 \\
4.76625 \\
4.78754 \\
4.80792 \\
4.82754\end{array}$ & $\begin{array}{l}2237 \\
2129 \\
2038 \\
1962 \\
1895\end{array}$ & $\begin{array}{l}4.28441 \\
4.31057 \\
4.33511 \\
4.35825 \\
4.38013\end{array}$ & $\begin{array}{l}2616 \\
2454 \\
2314 \\
2188 \\
2077\end{array}$ & $\begin{array}{l}29.49321 \\
29.73885 \\
29.97258 \\
30.19553 \\
30.40871\end{array}$ & $\begin{array}{l}24564 \\
23373 \\
22295 \\
21318 \\
20425\end{array}$ & $\begin{array}{l}33.77763 \\
34.04942 \\
34.30770 \\
34.55379 \\
34.78884\end{array}$ & $\begin{array}{l}27779 \\
25828 \\
24609 \\
23505 \\
22502\end{array}$ \\
\hline $\begin{array}{l}2200 \\
2300 \\
2400 \\
2500 \\
2600\end{array}$ & $\begin{array}{l}4.84649 \\
4.86486 \\
4.88272 \\
4.90012 \\
4.91712\end{array}$ & $\begin{array}{l}1837 \\
1786 \\
1740 \\
1700 \\
1663\end{array}$ & $\begin{array}{l}4.40090 \\
4.42068 \\
4.43956 \\
4.45763 \\
4.47498\end{array}$ & $\begin{array}{l}1978 \\
1888 \\
1807 \\
1735 \\
1668\end{array}$ & $\begin{array}{l}30.61296 \\
30.80902 \\
30.99757 \\
31.17917 \\
31.35434\end{array}$ & $\begin{array}{l}19606 \\
18855 \\
18160 \\
17517 \\
16920\end{array}$ & $\begin{array}{l}35.01386 \\
35.22970 \\
35.43713 \\
35.63681 \\
35.82933\end{array}$ & $\begin{array}{l}21584 \\
20743 \\
19968 \\
19252 \\
18588\end{array}$ \\
\hline $\begin{array}{l}2700 \\
2800 \\
2900 \\
3000\end{array}$ & $\begin{array}{l}4.93375 \\
4.95004 \\
4.96602 \\
4.98171\end{array}$ & $\begin{array}{l}1629 \\
1598 \\
1569 \\
3061\end{array}$ & $\begin{array}{l}4.49166 \\
4.50774 \\
4.52327 \\
4.53829\end{array}$ & $\begin{array}{l}1608 \\
1553 \\
1502 \\
2868\end{array}$ & $\begin{array}{l}31.52354 \\
31.68719 \\
31.84564 \\
31.99924\end{array}$ & $\begin{array}{l}16365 \\
15845 \\
15360 \\
29382\end{array}$ & $\begin{array}{l}36.01521 \\
36.19494 \\
36.36892 \\
36.53754\end{array}$ & $\begin{array}{l}17973 \\
17398 \\
16862 \\
32250\end{array}$ \\
\hline $\begin{array}{l}3200 \\
3400 \\
3600 \\
3800 \\
4000\end{array}$ & $\begin{array}{l}5.01232 \\
5.04199 \\
5.07082 \\
5.09888 \\
5.12625\end{array}$ & $\begin{array}{l}2967 \\
2883 \\
2806 \\
2737 \\
2671\end{array}$ & $\begin{array}{l}4.56697 \\
4.59404 \\
4.61973 \\
4.64422 \\
4.66764\end{array}$ & $\begin{array}{l}2707 \\
2569 \\
2449 \\
2342 \\
2.247\end{array}$ & $\begin{array}{l}32.29306 \\
32.57075 \\
32.83408 \\
33.08451 \\
33.32333\end{array}$ & $\begin{array}{l}27769 \\
26333 \\
25043 \\
23882 \\
22828\end{array}$ & $\begin{array}{l}36.86004 \\
37.16480 \\
37.45381 \\
37.72873 \\
37.99097\end{array}$ & $\begin{array}{l}30476 \\
28901 \\
27492 \\
26224 \\
25076\end{array}$ \\
\hline $\begin{array}{l}4200 \\
4400 \\
4600 \\
4800 \\
5000\end{array}$ & $\begin{array}{l}5.15296 \\
5.17907 \\
5.20460 \\
5.22959 \\
5.25406\end{array}$ & $\begin{array}{l}2611 \\
2553 \\
2499 \\
2447\end{array}$ & $\begin{array}{l}4.69011 \\
4.71175 \\
4.73262 \\
4.75281 \\
4.77237\end{array}$ & $\begin{array}{l}2164 \\
2087 \\
2019 \\
1956\end{array}$ & $\begin{array}{l}33.55161 \\
33.77030 \\
33.98021 \\
34.18206 \\
34.37647\end{array}$ & $\begin{array}{l}21869 \\
20991 \\
20185 \\
19441\end{array}$ & $\begin{array}{l}38.24173 \\
38.48205 \\
38.71284 \\
38.93487 \\
39.14885\end{array}$ & $\begin{array}{l}24032 \\
23079 \\
22203 \\
21398\end{array}$ \\
\hline
\end{tabular}


Table 2. 057.

CdT

\begin{tabular}{|c|c|c|c|c|c|c|c|c|}
\hline${ }^{\circ} K$ & $\frac{C_{p}^{0}}{R}$ & & $\frac{\left(H^{\circ}-E_{0}^{0}\right)}{R T}$ & & $\frac{-\left(F^{\circ}-\right.}{R T}$ & & $\frac{S^{0}}{R}$ & \\
\hline $\begin{array}{l}50 \\
60 \\
70 \\
80 \\
90\end{array}$ & $\begin{array}{l}3.50162 \\
3.50191 \\
3.50223 \\
3.50263 \\
3.50328\end{array}$ & $\begin{array}{r}29 \\
32 \\
40 \\
65 \\
119\end{array}$ & $\begin{array}{l}3.48314 \\
3.48625 \\
3.48851 \\
3.49025 \\
3.49165\end{array}$ & $\begin{array}{l}311 \\
226 \\
174 \\
140 \\
122\end{array}$ & $\begin{array}{l}16.89305 \\
17.52840 \\
18.06598 \\
18.53193 \\
18.94310\end{array}$ & $\begin{array}{l}63535 \\
53758 \\
46595 \\
41117 \\
36795\end{array}$ & $\begin{array}{l}20.37620 \\
21.01465 \\
21.55449 \\
22.02218 \\
22.43476\end{array}$ & $\begin{array}{l}63845 \\
53984 \\
46769 \\
41258 \\
36916\end{array}$ \\
\hline $\begin{array}{l}100 \\
110 \\
120 \\
130 \\
140\end{array}$ & $\begin{array}{l}3.50447 \\
3.50662 \\
3.51016 \\
3.51553 \\
3.52303\end{array}$ & $\begin{array}{l}215 \\
354 \\
537 \\
750 \\
985\end{array}$ & $\begin{array}{l}3.49287 \\
3.49401 \\
3.49520 \\
3.49654 \\
3.49816\end{array}$ & $\begin{array}{l}114 \\
119 \\
134 \\
162 \\
197\end{array}$ & $\begin{array}{l}19.31105 \\
19.64401 \\
19.94808 \\
20.22790 \\
20.48708\end{array}$ & $\begin{array}{l}33296 \\
30407 \\
27982 \\
25918 \\
24141\end{array}$ & $\begin{array}{l}22.80392 \\
23.13803 \\
23.44328 \\
23.72445 \\
23.98524\end{array}$ & $\begin{array}{l}33411 \\
30525 \\
28117 \\
26079 \\
24338\end{array}$ \\
\hline $\begin{array}{l}150 \\
160 \\
170 \\
180 \\
190\end{array}$ & $\begin{array}{l}3.53288 \\
3.54514 \\
3.55974 \\
3.57655 \\
3.59533\end{array}$ & $\begin{array}{l}1226 \\
1460 \\
1681 \\
1878 \\
2050\end{array}$ & $\begin{array}{l}3.50013 \\
3.50255 \\
3.50547 \\
3.50894 \\
3.51299\end{array}$ & $\begin{array}{l}242 \\
292 \\
347 \\
405 \\
462\end{array}$ & $\begin{array}{l}20.72849 \\
20.95446 \\
21.16688 \\
21.36735 \\
21.55717\end{array}$ & $\begin{array}{l}22597 \\
21242 \\
20047 \\
18982 \\
18031\end{array}$ & $\begin{array}{l}24.22862 \\
24.45701 \\
24.67236 \\
24.87629 \\
25.07016\end{array}$ & $\begin{array}{l}22839 \\
21535 \\
20393 \\
19387 \\
18493\end{array}$ \\
\hline $\begin{array}{l}200 \\
210 \\
220 \\
230 \\
240\end{array}$ & $\begin{array}{l}3.61583 \\
3.63777 \\
3.66085 \\
3.68481 \\
3.70939\end{array}$ & $\begin{array}{l}2194 \\
2308 \\
2396 \\
2458 \\
2498\end{array}$ & $\begin{array}{l}3.51761 \\
3.52280 \\
3.52855 \\
3.53482 \\
3.54158\end{array}$ & $\begin{array}{l}519 \\
575 \\
627 \\
676 \\
721\end{array}$ & $\begin{array}{l}21.73748 \\
21.90923 \\
22.07324 \\
22.23023 \\
22.38081\end{array}$ & $\begin{array}{l}17175 \\
16401 \\
15699 \\
15058 \\
14472\end{array}$ & $\begin{array}{l}25.25509 \\
25.43204 \\
25.60179 \\
25.76505 \\
25.92239\end{array}$ & $\begin{array}{l}17695 \\
16975 \\
16326 \\
15734 \\
15193\end{array}$ \\
\hline $\begin{array}{l}250 \\
260 \\
270 \\
280 \\
290\end{array}$ & $\begin{array}{l}3.73437 \\
3.75953 \\
3.78472 \\
3.80976 \\
3.83455\end{array}$ & $\begin{array}{l}2516 \\
2519 \\
2504 \\
2479 \\
2443\end{array}$ & $\begin{array}{l}3.54879 \\
3.55641 \\
3.56440 \\
3.57272 \\
3.58132\end{array}$ & $\begin{array}{l}762 \\
799 \\
832 \\
860 \\
885\end{array}$ & $\begin{array}{l}22.52553 \\
22.66486 \\
22.79923 \\
22.92901 \\
23.05453\end{array}$ & $\begin{array}{l}13933 \\
13437 \\
12978 \\
12552 \\
12156\end{array}$ & $\begin{array}{l}26.07432 \\
26.22128 \\
26.36364 \\
26.50173 \\
26.63585\end{array}$ & $\begin{array}{l}14696 \\
14236 \\
13809 \\
13412 \\
13041\end{array}$ \\
\hline $\begin{array}{l}300 \\
310 \\
320 \\
330 \\
340\end{array}$ & $\begin{array}{l}3.85898 \\
3.88297 \\
3.90646 \\
3.92938 \\
3.95172\end{array}$ & $\begin{array}{l}2399 \\
2349 \\
2292 \\
2234 \\
2172\end{array}$ & $\begin{array}{l}3.59017 \\
3.59923 \\
3.60847 \\
3.61784 \\
3.62734\end{array}$ & $\begin{array}{l}906 \\
924 \\
937 \\
950 \\
958\end{array}$ & $\begin{array}{l}23.17609 \\
23.29396 \\
23.40837 \\
23.51956 \\
23.62770\end{array}$ & $\begin{array}{l}11787 \\
11441 \\
11119 \\
10814 \\
10529\end{array}$ & $\begin{array}{l}26.76626 \\
26.89319 \\
27.01684 \\
27.13741 \\
27.25504\end{array}$ & $\begin{array}{l}12693 \\
12365 \\
12057 \\
11763 \\
11487\end{array}$ \\
\hline $\begin{array}{l}350 \\
360 \\
370 \\
380 \\
390\end{array}$ & $\begin{array}{l}3.97344 \\
3.99453 \\
4.01498 \\
4.03478 \\
4.05396\end{array}$ & $\begin{array}{l}2109 \\
2045 \\
1980 \\
1918 \\
1854\end{array}$ & $\begin{array}{l}3.63692 \\
3.64656 \\
3.65624 \\
3.66594 \\
3.67565\end{array}$ & $\begin{array}{l}964 \\
968 \\
970 \\
971 \\
969\end{array}$ & $\begin{array}{l}23.73299 \\
23.83558 \\
23.93562 \\
24.03325 \\
24.12861\end{array}$ & $\begin{array}{r}10259 \\
10004 \\
9763 \\
9536 \\
9318\end{array}$ & $\begin{array}{l}27.36991 \\
27.48214 \\
27.59187 \\
27.69920 \\
27.80426\end{array}$ & $\begin{array}{l}11223 \\
10973 \\
10733 \\
10506 \\
10287\end{array}$ \\
\hline $\begin{array}{l}400 \\
450 \\
500 \\
550 \\
600\end{array}$ & $\begin{array}{l}4.07250 \\
4.15623 \\
4.22657 \\
4.28585 \\
4.33618\end{array}$ & $\begin{array}{l}8373 \\
7034 \\
5928 \\
5033 \\
4316\end{array}$ & $\begin{array}{l}3.68534 \\
3.73314 \\
3.77907 \\
3.82252 \\
3.86329\end{array}$ & $\begin{array}{l}4780 \\
4593 \\
4345 \\
4077 \\
3807\end{array}$ & $\begin{array}{l}24.22179 \\
24.65863 \\
25.05435 \\
25.41660 \\
25.75097\end{array}$ & $\begin{array}{l}43684 \\
39572 \\
36225 \\
33437 \\
31075\end{array}$ & $\begin{array}{l}27.90713 \\
28.39178 \\
28.83343 \\
29.23912 \\
29.61426\end{array}$ & $\begin{array}{l}48465 \\
44165 \\
40569 \\
37514 \\
34883\end{array}$ \\
\hline $\begin{array}{l}650 \\
700 \\
750 \\
800 \\
850\end{array}$ & $\begin{array}{l}4.37934 \\
4.41675 \\
4.44953 \\
4.47856 \\
4.50454\end{array}$ & $\begin{array}{l}3741 \\
3278 \\
2903 \\
2598 \\
2347\end{array}$ & $\begin{array}{l}3.90136 \\
3.93687 \\
3.96998 \\
4.00087 \\
4.02975\end{array}$ & $\begin{array}{l}3551 \\
3311 \\
3089 \\
2888 \\
2704\end{array}$ & $\begin{array}{l}26.06172 \\
26.35215 \\
26.62491 \\
26.88213 \\
27.12556\end{array}$ & $\begin{array}{l}29043 \\
27276 \\
25722 \\
24343 \\
23110\end{array}$ & $\begin{array}{l}29.96309 \\
30.28903 \\
30.59490 \\
30.88301 \\
31.15531\end{array}$ & $\begin{array}{l}32594 \\
30587 \\
28811 \\
27230 \\
25815\end{array}$ \\
\hline $\begin{array}{r}900 \\
950 \\
1000 \\
1050 \\
1100\end{array}$ & $\begin{array}{l}4.52801 \\
4.54940 \\
4.56907 \\
4.58728 \\
4.60427\end{array}$ & $\begin{array}{l}2139 \\
1967 \\
1821 \\
1699 \\
1593\end{array}$ & $\begin{array}{l}4.05679 \\
4.08216 \\
4.10602 \\
4.12851 \\
4.14976\end{array}$ & $\begin{array}{l}2537 \\
2386 \\
2249 \\
2125 \\
2011\end{array}$ & $\begin{array}{l}27.35666 \\
27.57669 \\
27.78669 \\
27.98757 \\
28.18013\end{array}$ & $\begin{array}{l}22003 \\
21000 \\
20088 \\
19256 \\
18491\end{array}$ & $\begin{array}{l}31.41346 \\
31.65886 \\
31.89272 \\
32.11609 \\
32.32989\end{array}$ & $\begin{array}{l}24540 \\
23386 \\
22337 \\
21380 \\
20502\end{array}$ \\
\hline 1150 & 4.62020 & 1505 & 4.16987 & 1908 & 28.36504 & 17787 & 32.53491 & 19696 \\
\hline
\end{tabular}


Table 2. 057. CdT (Cont.)

\begin{tabular}{|c|c|c|c|c|c|c|c|c|}
\hline${ }^{\circ} \mathrm{K}$ & $\frac{C_{p}^{0}}{R}$ & & $\frac{\left(H^{0}-E_{0}^{0}\right)}{R T}$ & & $\frac{-\left(F^{0}-\right.}{R T}$ & & $\frac{S^{\circ}}{R}$ & \\
\hline $\begin{array}{l}1200 \\
1300 \\
1400 \\
1500 \\
1600\end{array}$ & $\begin{array}{l}4.63525 \\
4.66311 \\
4.68861 \\
4.71229 \\
4.73452\end{array}$ & $\begin{array}{l}2786 \\
2550 \\
2368 \\
2223 \\
2107\end{array}$ & $\begin{array}{l}4.18895 \\
4.22437 \\
4.25663 \\
4.28623 \\
4.31356\end{array}$ & $\begin{array}{l}3542 \\
3226 \\
2960 \\
2733 \\
2538\end{array}$ & $\begin{array}{l}28.54291 \\
28.87963 \\
29.19389 \\
29.48859 \\
29.76610\end{array}$ & $\begin{array}{l}33672 \\
31426 \\
29470 \\
27751 \\
26228\end{array}$ & $\begin{array}{l}32.73187 \\
33.10400 \\
33.45052 \\
33.77482 \\
34.07966\end{array}$ & $\begin{array}{l}37213 \\
34652 \\
32430 \\
30484 \\
28767\end{array}$ \\
\hline $\begin{array}{l}1700 \\
1800 \\
1900 \\
2000 \\
2100\end{array}$ & $\begin{array}{l}4.75559 \\
4.77571 \\
4.79503 \\
4.81369 \\
4.83176\end{array}$ & $\begin{array}{l}2012 \\
1932 \\
1866 \\
1807 \\
1757\end{array}$ & $\begin{array}{l}4.33894 \\
4.36265 \\
4.38491 \\
4.40588 \\
4.42573\end{array}$ & $\begin{array}{l}2371 \\
2226 \\
2097 \\
1985 \\
1886\end{array}$ & $\begin{array}{l}30.02838 \\
30.27707 \\
30.51354 \\
30.73900 \\
30.95445\end{array}$ & $\begin{array}{l}24869 \\
23647 \\
22546 \\
21545 \\
20632\end{array}$ & $\begin{array}{l}34.36733 \\
34.63972 \\
34.89845 \\
35.14488 \\
35.38019\end{array}$ & $\begin{array}{l}27239 \\
25873 \\
24643 \\
23531 \\
22518\end{array}$ \\
\hline $\begin{array}{l}2200 \\
2300 \\
2400 \\
2500 \\
2600\end{array}$ & $\begin{array}{l}4.84933 \\
4.86646 \\
4.88320 \\
4.89958 \\
4.91564\end{array}$ & $\begin{array}{l}1713 \\
1674 \\
1638 \\
1606 \\
1577\end{array}$ & $\begin{array}{l}4.44459 \\
4.46256 \\
4.47974 \\
4.49621 \\
4.51203\end{array}$ & $\begin{array}{l}1797 \\
1718 \\
1647 \\
1582 \\
1524\end{array}$ & $\begin{array}{l}31.16077 \\
31.35874 \\
31.54903 \\
31.73224 \\
31.90890\end{array}$ & $\begin{array}{l}19797 \\
19029 \\
18321 \\
17666 \\
17057\end{array}$ & $\begin{array}{l}35.60537 \\
35.82131 \\
36.02878 \\
36.22845 \\
36.42093\end{array}$ & $\begin{array}{l}21594 \\
20747 \\
19967 \\
19248 \\
18582\end{array}$ \\
\hline $\begin{array}{l}2700 \\
2800 \\
2900 \\
3000\end{array}$ & $\begin{array}{l}4.93141 \\
4.94691 \\
4.96216 \\
4.97718\end{array}$ & $\begin{array}{l}1550 \\
1525 \\
1502 \\
2938\end{array}$ & $\begin{array}{l}4.52727 \\
4.54198 \\
4.55621 \\
4.56999\end{array}$ & $\begin{array}{l}1471 \\
1423 \\
1378 \\
2637\end{array}$ & $\begin{array}{l}32.07947 \\
32.24438 \\
32.40402 \\
32.55871\end{array}$ & $\begin{array}{l}16491 \\
15964 \\
15469 \\
29579\end{array}$ & $\begin{array}{l}36.60675 \\
36.78637 \\
36.96023 \\
37.12871\end{array}$ & $\begin{array}{l}17962 \\
17386 \\
16848 \\
32216\end{array}$ \\
\hline $\begin{array}{l}3200 \\
3400 \\
3600 \\
3800 \\
4000\end{array}$ & $\begin{array}{l}5.00656 \\
5.03515 \\
5.06302 \\
5.09024 \\
5.11683\end{array}$ & $\begin{array}{l}2859 \\
2787 \\
2722 \\
2659 \\
2602\end{array}$ & $\begin{array}{l}4.59636 \\
4.62134 \\
4.64511 \\
4.66782 \\
4.68961\end{array}$ & $\begin{array}{l}2498 \\
2377 \\
2271 \\
2179 \\
2096\end{array}$ & $\begin{array}{l}32.85450 \\
33.13391 \\
33.39874 \\
33.65050 \\
33.89049\end{array}$ & $\begin{array}{l}27941 \\
26483 \\
25176 \\
23999 \\
22931\end{array}$ & $\begin{array}{l}37.45087 \\
37.75526 \\
38.04385 \\
38.31833 \\
38.58010\end{array}$ & $\begin{array}{l}30439 \\
28859 \\
27448 \\
2617 \\
25028\end{array}$ \\
\hline $\begin{array}{l}4200 \\
4400 \\
4600 \\
4800 \\
5000\end{array}$ & $\begin{array}{l}5.14285 \\
5.16832 \\
5.19327 \\
5.21773 \\
5.24170\end{array}$ & $\begin{array}{l}2547 \\
2495 \\
2446 \\
2397\end{array}$ & $\begin{array}{l}4.71057 \\
4.73080 \\
4.75037 \\
4.76934 \\
4.78775\end{array}$ & $\begin{array}{l}2023 \\
1957 \\
1897 \\
1841\end{array}$ & $\begin{array}{l}34.11980 \\
34.33941 \\
34.55014 \\
34.75271 \\
34.94778\end{array}$ & $\begin{array}{l}21961 \\
21073 \\
20257 \\
19507\end{array}$ & $\begin{array}{l}38.83038 \\
39.07022 \\
39.30051 \\
39.52205 \\
39.73554\end{array}$ & $\begin{array}{l}23984 \\
23029 \\
22154 \\
21349\end{array}$ \\
\hline
\end{tabular}


Table 2.058. $\mathrm{HgH}$

\begin{tabular}{|c|c|c|c|c|c|c|c|c|}
\hline${ }^{\circ} K$ & $\frac{C_{p}^{0}}{R}$ & & $\frac{\left(H^{0}-E_{0}^{0}\right)}{R T}$ & & $\frac{-\left(F^{0}-E\right.}{R T}$ & & $\frac{S^{\circ}}{R}$ & \\
\hline $\begin{array}{l}50 \\
60 \\
70 \\
80 \\
90\end{array}$ & $\begin{array}{l}3.50208 \\
3.50220 \\
3.50240 \\
3.50263 \\
3.50288\end{array}$ & $\begin{array}{l}12 \\
20 \\
23 \\
25 \\
28\end{array}$ & $\begin{array}{l}3.44863 \\
3.45755 \\
3.46394 \\
3.46876 \\
3.47254\end{array}$ & $\begin{array}{l}892 \\
639 \\
482 \\
378 \\
305\end{array}$ & $\begin{array}{l}16.68766 \\
17.31726 \\
17.85075 \\
18.31363 \\
18.72241\end{array}$ & $\begin{array}{l}62960 \\
53349 \\
46288 \\
40878 \\
36604\end{array}$ & $\begin{array}{l}20.13630 \\
20.77482 \\
21.31470 \\
21.78239 \\
22.19496\end{array}$ & $\begin{array}{l}63852 \\
53988 \\
46769 \\
41257 \\
36908\end{array}$ \\
\hline $\begin{array}{l}100 \\
110 \\
120 \\
130 \\
140\end{array}$ & $\begin{array}{l}3.50316 \\
3.50346 \\
3.50383 \\
3.50431 \\
3.50499\end{array}$ & $\begin{array}{l}30 \\
37 \\
48 \\
68 \\
99\end{array}$ & $\begin{array}{l}3.47559 \\
3.47811 \\
3.48023 \\
3.48207 \\
3.48368\end{array}$ & $\begin{array}{l}252 \\
212 \\
184 \\
161 \\
145\end{array}$ & $\begin{array}{l}19.08845 \\
19.41983 \\
19.72256 \\
20.00120 \\
20.25931\end{array}$ & $\begin{array}{l}33138 \\
30273 \\
27864 \\
25811 \\
24040\end{array}$ & $\begin{array}{l}22.56404 \\
22.89794 \\
23.20280 \\
23.48327 \\
23.74299\end{array}$ & $\begin{array}{l}33390 \\
30486 \\
28047 \\
25972 \\
24185\end{array}$ \\
\hline $\begin{array}{l}150 \\
160 \\
170 \\
180 \\
190\end{array}$ & $\begin{array}{l}3.50598 \\
3.50742 \\
3.50944 \\
3.51217 \\
3.51576\end{array}$ & $\begin{array}{l}144 \\
202 \\
273 \\
359 \\
454\end{array}$ & $\begin{array}{l}3.48513 \\
3.48648 \\
3.48776 \\
3.48904 \\
3.49035\end{array}$ & $\begin{array}{l}135 \\
128 \\
128 \\
131 \\
138\end{array}$ & $\begin{array}{l}20.49971 \\
20.72468 \\
20.93608 \\
21.13547 \\
21.32415\end{array}$ & $\begin{array}{l}22497 \\
21140 \\
19939 \\
18868 \\
17907\end{array}$ & $\begin{array}{l}23.98484 \\
24.21116 \\
24.42385 \\
24.62452 \\
24.81450\end{array}$ & $\begin{array}{l}22632 \\
21269 \\
20067 \\
18998 \\
18045\end{array}$ \\
\hline $\begin{array}{l}200 \\
210 \\
220 \\
230 \\
240\end{array}$ & $\begin{array}{l}3.52030 \\
3.52588 \\
3.53255 \\
3.54035 \\
3.54928\end{array}$ & $\begin{array}{r}558 \\
667 \\
780 \\
893 \\
1003\end{array}$ & $\begin{array}{l}3.49173 \\
3.49322 \\
3.49485 \\
3.49665 \\
3.49866\end{array}$ & $\begin{array}{l}149 \\
163 \\
180 \\
201 \\
222\end{array}$ & $\begin{array}{l}21.50322 \\
21.67361 \\
21.83616 \\
21.99155 \\
22.14041\end{array}$ & $\begin{array}{l}17039 \\
16255 \\
15539 \\
14886 \\
14286\end{array}$ & $\begin{array}{l}24.99495 \\
25.16684 \\
25.33101 \\
25.48821 \\
25.63907\end{array}$ & $\begin{array}{l}17189 \\
16417 \\
15720 \\
15086 \\
14509\end{array}$ \\
\hline $\begin{array}{l}250 \\
260 \\
270 \\
280 \\
290\end{array}$ & $\begin{array}{l}3.55931 \\
3.57042 \\
3.58255 \\
3.59563 \\
3.60959\end{array}$ & $\begin{array}{l}1111 \\
1213 \\
1308 \\
1396 \\
1476\end{array}$ & $\begin{array}{l}3.50088 \\
3.50334 \\
3.50604 \\
3.50901 \\
3.51223\end{array}$ & $\begin{array}{l}246 \\
270 \\
297 \\
322 \\
349\end{array}$ & $\begin{array}{l}22.28327 \\
22.42063 \\
22.55289 \\
22.68045 \\
22.80364\end{array}$ & $\begin{array}{l}13736 \\
13226 \\
12756 \\
12319 \\
11913\end{array}$ & $\begin{array}{l}25.78416 \\
25.92397 \\
26.05894 \\
26.18946 \\
26.31588\end{array}$ & $\begin{array}{l}13981 \\
13497 \\
13052 \\
12642 \\
12262\end{array}$ \\
\hline $\begin{array}{l}300 \\
310 \\
320 \\
330 \\
340\end{array}$ & $\begin{array}{l}3.62435 \\
3.63983 \\
3.65595 \\
3.67262 \\
3.68978\end{array}$ & $\begin{array}{l}1548 \\
1612 \\
1667 \\
1716 \\
1755\end{array}$ & $\begin{array}{l}3.51572 \\
3.51947 \\
3.52348 \\
3.52775 \\
3.53226\end{array}$ & $\begin{array}{l}375 \\
401 \\
427 \\
451 \\
475\end{array}$ & $\begin{array}{l}22.92277 \\
23.03811 \\
23.14991 \\
23.25840 \\
23.36378\end{array}$ & $\begin{array}{l}11534 \\
11180 \\
10849 \\
10538 \\
10246\end{array}$ & $\begin{array}{l}26.43850 \\
26.55759 \\
26.67340 \\
26.78616 \\
26.89605\end{array}$ & $\begin{array}{l}11909 \\
11581 \\
11276 \\
10989 \\
10721\end{array}$ \\
\hline $\begin{array}{l}350 \\
360 \\
370 \\
380 \\
390\end{array}$ & $\begin{array}{l}3.70733 \\
3.72521 \\
3.74336 \\
3.76171 \\
3.78020\end{array}$ & $\begin{array}{l}1788 \\
1815 \\
1835 \\
1849 \\
1858\end{array}$ & $\begin{array}{l}3.53701 \\
3.54199 \\
3.54719 \\
3.55259 \\
3.55819\end{array}$ & $\begin{array}{l}498 \\
520 \\
540 \\
560 \\
578\end{array}$ & $\begin{array}{l}23.46624 \\
23.56595 \\
23.66307 \\
23.75774 \\
23.85009\end{array}$ & $\begin{array}{l}9971 \\
9712 \\
9467 \\
9235 \\
9016\end{array}$ & $\begin{array}{l}27.00326 \\
27.10795 \\
27.21026 \\
27.31033 \\
27.40829\end{array}$ & $\begin{array}{r}10469 \\
10231 \\
10007 \\
9796 \\
9594\end{array}$ \\
\hline $\begin{array}{l}400 \\
450 \\
500 \\
550 \\
600\end{array}$ & $\begin{array}{l}3.79878 \\
3.89160 \\
3.98142 \\
4.06598 \\
4.14436\end{array}$ & $\begin{array}{l}9282 \\
8982 \\
8456 \\
7838 \\
7204\end{array}$ & $\begin{array}{l}3.56397 \\
3.59523 \\
3.62940 \\
3.66529 \\
3.70199\end{array}$ & $\begin{array}{l}3126 \\
3417 \\
3589 \\
3670 \\
3684\end{array}$ & $\begin{array}{l}23.94025 \\
24.36179 \\
24.74234 \\
25.08993 \\
25.41042\end{array}$ & $\begin{array}{l}42154 \\
38055 \\
34759 \\
32049 \\
29778\end{array}$ & $\begin{array}{l}27.50423 \\
27.95703 \\
28.37174 \\
28.75522 \\
29.11242\end{array}$ & $\begin{array}{l}45280 \\
41471 \\
38348 \\
35720 \\
33461\end{array}$ \\
\hline $\begin{array}{l}650 \\
700 \\
750 \\
800 \\
850\end{array}$ & $\begin{array}{l}4.21640 \\
4.28240 \\
4.34281 \\
4.39817 \\
4.44903\end{array}$ & $\begin{array}{l}6600 \\
6041 \\
5536 \\
5086 \\
4686\end{array}$ & $\begin{array}{l}3.73883 \\
3.77533 \\
3.81118 \\
3.84616 \\
3.88015\end{array}$ & $\begin{array}{l}3650 \\
3585 \\
3498 \\
3399 \\
3292\end{array}$ & $\begin{array}{l}25.70820 \\
25.98661 \\
26.24831 \\
26.49540 \\
26.72960\end{array}$ & $\begin{array}{l}27841 \\
26170 \\
24709 \\
23420 \\
22272\end{array}$ & $\begin{array}{l}29.44703 \\
29.76195 \\
30.05949 \\
30.34157 \\
30.60975\end{array}$ & $\begin{array}{l}31492 \\
29754 \\
28208 \\
26818 \\
25565\end{array}$ \\
\hline $\begin{array}{r}900 \\
950 \\
1000 \\
1050 \\
1100\end{array}$ & $\begin{array}{l}4.49589 \\
4.53923 \\
4.57946 \\
4.61693 \\
4.65198\end{array}$ & $\begin{array}{l}4334 \\
4023 \\
3747 \\
3505 \\
3288\end{array}$ & $\begin{array}{l}3.91307 \\
3.94490 \\
3.97564 \\
4.00529 \\
4.03390\end{array}$ & $\begin{array}{l}3183 \\
3074 \\
2965 \\
2861 \\
2759\end{array}$ & $\begin{array}{l}26.95232 \\
27.16475 \\
27.36788 \\
27.56257 \\
27.74956\end{array}$ & $\begin{array}{l}21243 \\
20313 \\
19469 \\
18699 \\
17993\end{array}$ & $\begin{array}{l}30.86540 \\
31.10965 \\
31.34352 \\
31.56787 \\
31.78347\end{array}$ & $\begin{array}{l}24425 \\
23387 \\
22435 \\
21560 \\
20752\end{array}$ \\
\hline 1150 & 4.68486 & 3097 & 4.06149 & 2663 & 27.92949 & 17342 & 31.99099 & 20005 \\
\hline
\end{tabular}


Table 2.058. $\mathrm{HgH}$ (Cont.)

\begin{tabular}{|c|c|c|c|c|c|c|c|c|}
\hline${ }^{\circ} \mathrm{K}$ & $\frac{C_{\dot{p}}^{\circ}}{R}$ & & $\frac{\left(H^{\circ}-E_{0}^{0}\right)}{R T}$ & & $\frac{-\left(F^{\circ}-E\right.}{R T}$ & & $\frac{S^{0}}{R}$ & \\
\hline $\begin{array}{l}1200 \\
1300 \\
1400 \\
1500 \\
1600\end{array}$ & $\begin{array}{l}4.71583 \\
4.77283 \\
4.82431 \\
4.87131 \\
4.91461\end{array}$ & $\begin{array}{l}5700 \\
5148 \\
4700 \\
4330 \\
4021\end{array}$ & $\begin{array}{l}4.08812 \\
4.13864 \\
4.18580 \\
4.22996 \\
4.27141\end{array}$ & $\begin{array}{l}5052 \\
4716 \\
4416 \\
4145 \\
3904\end{array}$ & $\begin{array}{l}28.10291 \\
28.43216 \\
28.74061 \\
29.03092 \\
29.30525\end{array}$ & $\begin{array}{l}32925 \\
30845 \\
29031 \\
27433 \\
26014\end{array}$ & $\begin{array}{l}32.19104 \\
32.57080 \\
32.92642 \\
33.26089 \\
33.57667\end{array}$ & $\begin{array}{l}37976 \\
35562 \\
33447 \\
31578 \\
29917\end{array}$ \\
\hline $\begin{array}{l}1700 \\
1800 \\
1900 \\
2000 \\
2100\end{array}$ & $\begin{array}{l}4.95482 \\
4.99241 \\
5.02777 \\
5.06118 \\
5.09290\end{array}$ & $\begin{array}{l}3759 \\
3536 \\
3341 \\
3172 \\
3023\end{array}$ & $\begin{array}{l}4.31045 \\
4.34730 \\
4.38219 \\
4.41531 \\
4.44683\end{array}$ & $\begin{array}{l}3685 \\
3489 \\
3312 \\
3152 \\
3006\end{array}$ & $\begin{array}{l}29.56539 \\
29.81282 \\
30.04881 \\
30.27444 \\
30.49063\end{array}$ & $\begin{array}{l}24743 \\
23599 \\
22563 \\
21619 \\
20757\end{array}$ & $\begin{array}{l}33.87584 \\
34.16013 \\
34.43101 \\
34.68976 \\
34.93747\end{array}$ & $\begin{array}{l}28429 \\
27088 \\
25875 \\
24771 \\
23762\end{array}$ \\
\hline $\begin{array}{l}2200 \\
2300 \\
2400 \\
2500 \\
2600\end{array}$ & $\begin{array}{l}5.12313 \\
5.15202 \\
5.17972 \\
5.20634 \\
5.23198\end{array}$ & $\begin{array}{l}2889 \\
2770 \\
2662 \\
2564 \\
2474\end{array}$ & $\begin{array}{l}4.47689 \\
4.50562 \\
4.53314 \\
4.55953 \\
4.58491\end{array}$ & $\begin{array}{l}2873 \\
2752 \\
2639 \\
2538 \\
2442\end{array}$ & $\begin{array}{l}30.69820 \\
30.89784 \\
31.09018 \\
31.27577 \\
31.45510\end{array}$ & $\begin{array}{l}19964 \\
19234 \\
18559 \\
17933 \\
17350\end{array}$ & $\begin{array}{l}35.17509 \\
35.40347 \\
35.62332 \\
35.83531 \\
36.04001\end{array}$ & $\begin{array}{l}22838 \\
21985 \\
21199 \\
20470 \\
19793\end{array}$ \\
\hline $\begin{array}{l}2700 \\
2800 \\
2900 \\
3000\end{array}$ & $\begin{array}{l}5.25672 \\
5.28064 \\
5.30379 \\
5.32623\end{array}$ & $\begin{array}{l}2392 \\
2315 \\
2244 \\
4295\end{array}$ & $\begin{array}{l}4.60933 \\
4.63288 \\
4.65562 \\
4.67760\end{array}$ & $\begin{array}{l}2355 \\
2274 \\
2198 \\
4190\end{array}$ & $\begin{array}{l}31.62860 \\
31.79666 \\
31.95963 \\
32.11783\end{array}$ & $\begin{array}{l}16806 \\
16297 \\
15820 \\
30324\end{array}$ & $\begin{array}{l}36.23794 \\
36.42955 \\
36.61526 \\
36.79544\end{array}$ & $\begin{array}{l}19161 \\
18571 \\
18018 \\
34513\end{array}$ \\
\hline $\begin{array}{l}3200 \\
3400 \\
3600 \\
3800 \\
4000\end{array}$ & $\begin{array}{l}5.36918 \\
5.40980 \\
5.44836 \\
5.48507 \\
5.52010\end{array}$ & $\begin{array}{l}4062 \\
3856 \\
3671 \\
3503 \\
3351\end{array}$ & $\begin{array}{l}4.71950 \\
4.75892 \\
4.79616 \\
4.83146 \\
4.86502\end{array}$ & $\begin{array}{l}3942 \\
3724 \\
3530 \\
3356 \\
3200\end{array}$ & $\begin{array}{l}32.42107 \\
32.70838 \\
32.98146 \\
33.24173 \\
33.49041\end{array}$ & $\begin{array}{l}28731 \\
27308 \\
26027 \\
24868 \\
23815\end{array}$ & $\begin{array}{l}37.14057 \\
37.46731 \\
37.77763 \\
38.07320 \\
38.35544\end{array}$ & $\begin{array}{l}32674 \\
31032 \\
29557 \\
28224 \\
27014\end{array}$ \\
\hline $\begin{array}{l}4200 \\
4400 \\
4600 \\
4800 \\
5000\end{array}$ & $\begin{array}{l}5.55361 \\
5.58573 \\
5.61655 \\
5.64618 \\
5.67470\end{array}$ & $\begin{array}{l}3212 \\
3082 \\
2963 \\
2852\end{array}$ & $\begin{array}{l}4.89702 \\
4.92760 \\
4.95689 \\
4.98500 \\
5.01202\end{array}$ & $\begin{array}{l}3058 \\
2929 \\
2811 \\
2702\end{array}$ & $\begin{array}{l}33.72856 \\
33.95708 \\
34.17677 \\
34.38833 \\
34.59238\end{array}$ & $\begin{array}{l}22852 \\
21969 \\
21156 \\
20405\end{array}$ & $\begin{array}{l}38.62558 \\
38.88468 \\
39.13366 \\
39.37333 \\
39.60440\end{array}$ & $\begin{array}{l}25910 \\
24898 \\
23967 \\
23107\end{array}$ \\
\hline
\end{tabular}


Table 2.059, HgD

\begin{tabular}{|c|c|c|c|c|c|c|c|c|}
\hline${ }^{\circ} K$ & $\frac{C_{p}^{0}}{R}$ & & $\frac{\left(H^{0}-E_{0}^{0}\right)}{R T}$ & & $\frac{-\left\langle F^{\circ}-E\right.}{R T}$ & & $\frac{S^{0}}{R}$ & \\
\hline $\begin{array}{l}50 \\
60 \\
70 \\
80 \\
90\end{array}$ & $\begin{array}{l}3.50181 \\
3.50210 \\
3.50241 \\
3.50274 \\
3.50317\end{array}$ & $\begin{array}{l}29 \\
31 \\
33 \\
43 \\
63\end{array}$ & $\begin{array}{l}3.47442 \\
3.47901 \\
3.48233 \\
3.48486 \\
3.48687\end{array}$ & $\begin{array}{l}459 \\
332 \\
253 \\
201 \\
166\end{array}$ & $\begin{array}{l}17.34385 \\
17.97775 \\
18.51430 \\
18.97947 \\
19.39005\end{array}$ & $\begin{array}{l}63390 \\
53655 \\
46517 \\
41058 \\
36747\end{array}$ & $\begin{array}{l}20.81828 \\
21.45676 \\
21.99664 \\
22.46434 \\
22.87693\end{array}$ & $\begin{array}{l}63848 \\
53988 \\
46770 \\
41259 \\
36912\end{array}$ \\
\hline $\begin{array}{l}100 \\
110 \\
120 \\
130 \\
140\end{array}$ & $\begin{array}{l}3.50380 \\
3.50487 \\
3.50664 \\
3.50946 \\
3.51363\end{array}$ & $\begin{array}{l}107 \\
177 \\
282 \\
417 \\
580\end{array}$ & $\begin{array}{l}3.48853 \\
3.48996 \\
3.49127 \\
3.49256 \\
3.49390\end{array}$ & $\begin{array}{l}143 \\
131 \\
129 \\
134 \\
150\end{array}$ & $\begin{array}{l}19.75752 \\
20.09008 \\
20.39380 \\
20.67331 \\
20.93218\end{array}$ & $\begin{array}{l}33256 \\
30372 \\
27951 \\
25887 \\
24111\end{array}$ & $\begin{array}{l}23.24605 \\
23.58005 \\
23.88508 \\
24.16587 \\
24.42609\end{array}$ & $\begin{array}{l}33400 \\
30503 \\
28079 \\
26022 \\
24260\end{array}$ \\
\hline $\begin{array}{l}150 \\
160 \\
170 \\
180 \\
190\end{array}$ & $\begin{array}{l}3.51943 \\
3.52704 \\
3.53657 \\
3.54806 \\
3.56144\end{array}$ & $\begin{array}{r}761 \\
953 \\
1149 \\
1338 \\
1518\end{array}$ & $\begin{array}{l}3.49540 \\
3.49713 \\
3.49916 \\
3.50155 \\
3.50434\end{array}$ & $\begin{array}{l}173 \\
203 \\
239 \\
279 \\
323\end{array}$ & $\begin{array}{l}21.17329 \\
21.39893 \\
21.61100 \\
21.81107 \\
22.00046\end{array}$ & $\begin{array}{l}22564 \\
21207 \\
20007 \\
18939 \\
17983\end{array}$ & $\begin{array}{l}24.66869 \\
24.89606 \\
25.11017 \\
25.31263 \\
25.50481\end{array}$ & $\begin{array}{l}22737 \\
21411 \\
20246 \\
19218 \\
18306\end{array}$ \\
\hline $\begin{array}{l}200 \\
210 \\
220 \\
230 \\
240\end{array}$ & $\begin{array}{l}3.57662 \\
3.59344 \\
3.61172 \\
3.63127 \\
3.65189\end{array}$ & $\begin{array}{l}1682 \\
1828 \\
1955 \\
2062 \\
2149\end{array}$ & $\begin{array}{l}3.50757 \\
3.51125 \\
3.51540 \\
3.52001 \\
3.52507\end{array}$ & $\begin{array}{l}368 \\
415 \\
461 \\
506 \\
550\end{array}$ & $\begin{array}{l}22.18029 \\
22.35152 \\
22.51495 \\
22.67132 \\
22.82124\end{array}$ & $\begin{array}{l}17123 \\
16343 \\
15637 \\
14992 \\
14401\end{array}$ & $\begin{array}{l}25.68787 \\
25.86277 \\
26.03036 \\
26.19133 \\
26.34631\end{array}$ & $\begin{array}{l}17490 \\
16759 \\
16097 \\
15498 \\
14951\end{array}$ \\
\hline $\begin{array}{l}250 \\
260 \\
270 \\
280 \\
290\end{array}$ & $\begin{array}{l}3.67338 \\
3.69556 \\
3.71826 \\
3.74131 \\
3.76458\end{array}$ & $\begin{array}{l}2218 \\
2270 \\
2305 \\
2327 \\
2336\end{array}$ & $\begin{array}{l}3.53057 \\
3.53649 \\
3.54280 \\
3.54947 \\
3.55649\end{array}$ & $\begin{array}{l}592 \\
631 \\
667 \\
702 \\
733\end{array}$ & $\begin{array}{l}22.96525 \\
23.10383 \\
23.23742 \\
23.36638 \\
23.49106\end{array}$ & $\begin{array}{l}13858 \\
13359 \\
12896 \\
12468 \\
12069\end{array}$ & $\begin{array}{l}26.49582 \\
26.64032 \\
26.78022 \\
26.91586 \\
27.04755\end{array}$ & $\begin{array}{l}14450 \\
13990 \\
13564 \\
13169 \\
12802\end{array}$ \\
\hline $\begin{array}{l}300 \\
310 \\
320 \\
330 \\
340\end{array}$ & $\begin{array}{l}3.78794 \\
3.81128 \\
3.83450 \\
3.85753 \\
3.88031\end{array}$ & $\begin{array}{l}2334 \\
2322 \\
2303 \\
2278 \\
2246\end{array}$ & $\begin{array}{l}3.56382 \\
3.57142 \\
3.57928 \\
3.58736 \\
3.59565\end{array}$ & $\begin{array}{l}760 \\
786 \\
808 \\
829 \\
845\end{array}$ & $\begin{array}{l}23.61175 \\
23.72873 \\
23.84224 \\
23.95251 \\
24.05972\end{array}$ & $\begin{array}{l}11698 \\
11351 \\
11027 \\
10721 \\
10435\end{array}$ & $\begin{array}{l}27.17557 \\
27.30016 \\
27.42153 \\
27.53988 \\
27.65537\end{array}$ & $\begin{array}{l}12459 \\
12137 \\
11835 \\
11549 \\
11281\end{array}$ \\
\hline $\begin{array}{l}350 \\
360 \\
370 \\
380 \\
390\end{array}$ & $\begin{array}{l}3.90277 \\
3.92486 \\
3.94656 \\
3.96784 \\
3.98867\end{array}$ & $\begin{array}{l}2209 \\
2170 \\
2128 \\
2083 \\
2037\end{array}$ & $\begin{array}{l}3.60410 \\
3.61270 \\
3.62144 \\
3.63027 \\
3.63920\end{array}$ & $\begin{array}{l}860 \\
874 \\
883 \\
893 \\
899\end{array}$ & $\begin{array}{l}24.16407 \\
24.26572 \\
24.36483 \\
24.46152 \\
24.55594\end{array}$ & $\begin{array}{r}10165 \\
9911 \\
9669 \\
9442 \\
9225\end{array}$ & $\begin{array}{l}27.76818 \\
27.87843 \\
27.98627 \\
28.09180 \\
28.19514\end{array}$ & $\begin{array}{l}11025 \\
10784 \\
10553 \\
10334 \\
10124\end{array}$ \\
\hline $\begin{array}{l}400 \\
450 \\
500 \\
550 \\
600\end{array}$ & $\begin{array}{l}4.00904 \\
4.10376 \\
4.18682 \\
4.25933 \\
4.32272\end{array}$ & $\begin{array}{l}9472 \\
8306 \\
7251 \\
6339 \\
5572\end{array}$ & $\begin{array}{l}3.64819 \\
3.69366 \\
3.73891 \\
3.78300 \\
3.82540\end{array}$ & $\begin{array}{l}4547 \\
4525 \\
4409 \\
4240 \\
4044\end{array}$ & $\begin{array}{l}24.64819 \\
25.08050 \\
25.47202 \\
25.83045 \\
26.16145\end{array}$ & $\begin{array}{l}43231 \\
39152 \\
35843 \\
33100 \\
30781\end{array}$ & $\begin{array}{l}28.29638 \\
28.77416 \\
29.21094 \\
29.61346 \\
29.98685\end{array}$ & $\begin{array}{l}47778 \\
43678 \\
40252 \\
37339 \\
34825\end{array}$ \\
\hline $\begin{array}{l}650 \\
700 \\
750 \\
800 \\
850\end{array}$ & $\begin{array}{l}4.37844 \\
4.42775 \\
4.47172 \\
4.51126 \\
4.54708\end{array}$ & $\begin{array}{l}4931 \\
4397 \\
3954 \\
3582 \\
3270\end{array}$ & $\begin{array}{l}3.86584 \\
3.90425 \\
3.94064 \\
3.97509 \\
4.00770\end{array}$ & $\begin{array}{l}3841 \\
3639 \\
3445 \\
3261 \\
3089\end{array}$ & $\begin{array}{l}26.46926 \\
26.75716 \\
27.02778 \\
27.28322 \\
27.52519\end{array}$ & $\begin{array}{l}28790 \\
27062 \\
25544 \\
24197 \\
22976\end{array}$ & $\begin{array}{l}30.33510 \\
30.66142 \\
30.96843 \\
31.25831 \\
31.53290\end{array}$ & $\begin{array}{l}32632 \\
30701 \\
28988 \\
27459 \\
26084\end{array}$ \\
\hline $\begin{array}{r}900 \\
950 \\
1000 \\
1050 \\
1100\end{array}$ & $\begin{array}{l}4.57978 \\
4.60986 \\
4.63770 \\
4.66363 \\
4.68792\end{array}$ & $\begin{array}{l}3008 \\
2784 \\
2593 \\
2429 \\
2287\end{array}$ & $\begin{array}{l}4.03859 \\
4.06787 \\
4.09568 \\
4.12211 \\
4.14729\end{array}$ & $\begin{array}{l}2928 \\
2781 \\
2643 \\
2518 \\
2400\end{array}$ & $\begin{array}{l}27.75515 \\
27.97429 \\
28.18366 \\
28.38414 \\
28.57648\end{array}$ & $\begin{array}{l}21914 \\
20937 \\
20048 \\
19234 \\
18489\end{array}$ & $\begin{array}{l}31.79374 \\
32.04217 \\
32.27935 \\
32.50625 \\
32.72377\end{array}$ & $\begin{array}{l}24843 \\
23718 \\
22690 \\
21752 \\
20890\end{array}$ \\
\hline 1150 & 4.71079 & 2164 & 4.17129 & 2294 & 28.76137 & 17802 & 32.93267 & 20095 \\
\hline
\end{tabular}


Table 2. 059. HgD (Cont.)

\begin{tabular}{|c|c|c|c|c|c|c|c|c|}
\hline${ }^{\circ} \mathrm{K}$ & $\frac{c_{p}^{\circ}}{R}$ & & $\frac{\left(H^{\circ}-E_{0}^{0}\right)}{R T}$ & & $\frac{-\left(F^{0}-E\right.}{R T}$ & & $\frac{S^{0}}{R}$ & \\
\hline $\begin{array}{l}1200 \\
1300 \\
1400 \\
1500 \\
1600\end{array}$ & $\begin{array}{l}4.73243 \\
4.77258 \\
4.80934 \\
4.84340 \\
4.87527\end{array}$ & $\begin{array}{l}4015 \\
3676 \\
3406 \\
3187 \\
3005\end{array}$ & $\begin{array}{l}4.19423 \\
4.23720 \\
4.27677 \\
4.31342 \\
4.34755\end{array}$ & $\begin{array}{l}4297 \\
3957 \\
3665 \\
3413 \\
3194\end{array}$ & $\begin{array}{l}28.93939 \\
29.27683 \\
29.59231 \\
29.88864 \\
30.16812\end{array}$ & $\begin{array}{l}33744 \\
31548 \\
29633 \\
27948 \\
26454\end{array}$ & $\begin{array}{l}33.13362 \\
33.51403 \\
33.86908 \\
34.20206 \\
34.51568\end{array}$ & $\begin{array}{l}38041 \\
35505 \\
33298 \\
31362 \\
29647\end{array}$ \\
\hline $\begin{array}{l}1700 \\
1800 \\
1900 \\
2000 \\
2100\end{array}$ & $\begin{array}{l}4.90532 \\
4.93385 \\
4.96107 \\
4.98717 \\
5.01227\end{array}$ & $\begin{array}{l}2853 \\
2722 \\
2610 \\
2510 \\
2422\end{array}$ & $\begin{array}{l}4.37949 \\
4.40950 \\
4.43782 \\
4.46464 \\
4.49012\end{array}$ & $\begin{array}{l}3001 \\
2832 \\
2682 \\
2548 \\
2429\end{array}$ & $\begin{array}{l}30.43266 \\
30.68384 \\
30.92302 \\
31.15134 \\
31.36979\end{array}$ & $\begin{array}{l}25118 \\
23918 \\
22832 \\
21845 \\
20944\end{array}$ & $\begin{array}{l}34.81215 \\
35.09335 \\
35.36084 \\
35.61598 \\
35.85991\end{array}$ & $\begin{array}{l}28120 \\
26749 \\
25514 \\
24393 \\
23374\end{array}$ \\
\hline $\begin{array}{l}2200 \\
2300 \\
2400 \\
2500 \\
2600\end{array}$ & $\begin{array}{l}5.03649 \\
5.05993 \\
5.08265 \\
5.10473 \\
5.12620\end{array}$ & $\begin{array}{l}2344 \\
2272 \\
2208 \\
2147 \\
2092\end{array}$ & $\begin{array}{l}4.51441 \\
4.53762 \\
4.55986 \\
4.58121 \\
4.60176\end{array}$ & $\begin{array}{l}2321 \\
2224 \\
2135 \\
2055 \\
1982\end{array}$ & $\begin{array}{l}31.57923 \\
31.78042 \\
31.97402 \\
32.16059 \\
32.34068\end{array}$ & $\begin{array}{l}20119 \\
19360 \\
18657 \\
18009 \\
17404\end{array}$ & $\begin{array}{l}36.09365 \\
36.31805 \\
36.53388 \\
36.74181 \\
36.94244\end{array}$ & $\begin{array}{l}22440 \\
21583 \\
20793 \\
20063 \\
19386\end{array}$ \\
\hline $\begin{array}{l}2700 \\
2800 \\
2900 \\
3000\end{array}$ & $\begin{array}{l}5.14712 \\
5.16753 \\
5.18746 \\
5.20693\end{array}$ & $\begin{array}{l}2041 \\
1993 \\
1947 \\
3770\end{array}$ & $\begin{array}{l}4.62158 \\
4.64071 \\
4.65922 \\
4.67716\end{array}$ & $\begin{array}{l}1913 \\
1851 \\
1794 \\
3429\end{array}$ & $\begin{array}{l}32.51472 \\
32.68315 \\
32.84632 \\
33.00458\end{array}$ & $\begin{array}{l}16843 \\
16317 \\
15826 \\
30296\end{array}$ & $\begin{array}{l}37.13630 \\
37.32386 \\
37.50555 \\
37.68174\end{array}$ & $\begin{array}{l}18756 \\
18169 \\
17619 \\
33726\end{array}$ \\
\hline $\begin{array}{l}3200 \\
3400 \\
3600 \\
3800 \\
4000\end{array}$ & $\begin{array}{l}5.24463 \\
5.28080 \\
5.31558 \\
5.34908 \\
5.38141\end{array}$ & $\begin{array}{l}3617 \\
3478 \\
3350 \\
3233 \\
3124\end{array}$ & $\begin{array}{l}4.71145 \\
4.74389 \\
4.77469 \\
4.80404 \\
4.83211\end{array}$ & $\begin{array}{l}3244 \\
3080 \\
2935 \\
2807 \\
2690\end{array}$ & $\begin{array}{l}33.30754 \\
33.59415 \\
33.86619 \\
34.12513 \\
34.37227\end{array}$ & $\begin{array}{l}28661 \\
27204 \\
25894 \\
24714 \\
23641\end{array}$ & $\begin{array}{l}38.01900 \\
38.33805 \\
38.64088 \\
38.92918 \\
39.20438\end{array}$ & $\begin{array}{l}31905 \\
30283 \\
28830 \\
27520 \\
26332\end{array}$ \\
\hline $\begin{array}{l}4200 \\
4400 \\
4600 \\
4800 \\
5000\end{array}$ & $\begin{array}{l}5.41265 \\
5.44286 \\
5.47212 \\
5.50048 \\
5.52800\end{array}$ & $\begin{array}{l}3021 \\
2926 \\
2836 \\
2752\end{array}$ & $\begin{array}{l}4.85901 \\
4.88487 \\
4.90977 \\
4.93380 \\
4.95702\end{array}$ & $\begin{array}{l}2586 \\
2490 \\
2403 \\
2322\end{array}$ & $\begin{array}{l}34.60868 \\
34.83532 \\
35.05302 \\
35.26249 \\
35.46437\end{array}$ & $\begin{array}{l}22664 \\
21770 \\
20947 \\
20188\end{array}$ & $\begin{array}{l}39.46770 \\
39.72020 \\
39.96279 \\
40.19629 \\
40.42139\end{array}$ & $\begin{array}{l}25250 \\
24259 \\
23350 \\
22510\end{array}$ \\
\hline
\end{tabular}


Table 2.060. $\mathrm{HgT}$

\begin{tabular}{|c|c|c|c|c|c|c|c|c|}
\hline${ }^{\circ} K$ & $\frac{C_{\hat{p}}^{0}}{R}$ & & $\frac{\left(H^{0}-E_{0}^{0}\right)}{R T}$ & & $\frac{-\left(F^{0}-E\right.}{R T}$ & & $\frac{S^{0}}{R}$ & \\
\hline $\begin{array}{l}50 \\
60 \\
70 \\
80 \\
90\end{array}$ & $\begin{array}{l}3.50173 \\
3.50205 \\
3.50241 \\
3.50295 \\
3.50395\end{array}$ & $\begin{array}{r}32 \\
36 \\
54 \\
100 \\
190\end{array}$ & $\begin{array}{l}3.48307 \\
3.48620 \\
3.48849 \\
3.49026 \\
3.49172\end{array}$ & $\begin{array}{l}313 \\
229 \\
177 \\
146 \\
131\end{array}$ & $\begin{array}{l}17.73714 \\
18.37247 \\
18.91005 \\
19.37600 \\
19.78718\end{array}$ & $\begin{array}{l}63533 \\
53758 \\
46595 \\
41118 \\
36796\end{array}$ & $\begin{array}{l}21.22021 \\
21.85868 \\
22.39855 \\
22.86626 \\
23.27890\end{array}$ & $\begin{array}{l}63847 \\
53987 \\
46771 \\
41264 \\
36927\end{array}$ \\
\hline $\begin{array}{l}100 \\
110 \\
120 \\
130 \\
140\end{array}$ & $\begin{array}{l}3.50585 \\
3.50919 \\
3.51451 \\
3.52223 \\
3.53260\end{array}$ & $\begin{array}{r}334 \\
532 \\
772 \\
1037 \\
1312\end{array}$ & $\begin{array}{l}3.49303 \\
3.49433 \\
3.49578 \\
3.49750 \\
3.49962\end{array}$ & $\begin{array}{l}130 \\
145 \\
172 \\
212 \\
262\end{array}$ & $\begin{array}{l}20.15514 \\
20.48812 \\
20.79223 \\
21.07210 \\
21.33137\end{array}$ & $\begin{array}{l}33298 \\
30411 \\
27987 \\
25927 \\
24154\end{array}$ & $\begin{array}{l}23.64817 \\
23.98246 \\
24.28801 \\
24.56961 \\
24.83100\end{array}$ & $\begin{array}{l}33429 \\
30555 \\
28160 \\
26139 \\
24415\end{array}$ \\
\hline $\begin{array}{l}150 \\
160 \\
170 \\
180 \\
190\end{array}$ & $\begin{array}{l}3.54572 \\
3.56152 \\
3.57983 \\
3.60036 \\
3.62281\end{array}$ & $\begin{array}{l}1580 \\
1831 \\
2053 \\
2245 \\
2401\end{array}$ & $\begin{array}{l}3.50224 \\
3.50544 \\
3.50926 \\
3.51374 \\
3.51889\end{array}$ & $\begin{array}{l}320 \\
382 \\
448 \\
515 \\
579\end{array}$ & $\begin{array}{l}21.57291 \\
21.79904 \\
22.01166 \\
22.21237 \\
22.40248\end{array}$ & $\begin{array}{l}22613 \\
21262 \\
20071 \\
19011 \\
18064\end{array}$ & $\begin{array}{l}25.07515 \\
25.30448 \\
25.52093 \\
25.72612 \\
25.92138\end{array}$ & $\begin{array}{l}22933 \\
21645 \\
20519 \\
19526 \\
18643\end{array}$ \\
\hline $\begin{array}{l}200 \\
210 \\
220 \\
230 \\
240\end{array}$ & $\begin{array}{l}3.64682 \\
3.67208 \\
3.69825 \\
3.72506 \\
3.75223\end{array}$ & $\begin{array}{l}2526 \\
2617 \\
2681 \\
2717 \\
2733\end{array}$ & $\begin{array}{l}3.52468 \\
3.53109 \\
3.53809 \\
3.54564 \\
3.55368\end{array}$ & $\begin{array}{l}641 \\
700 \\
755 \\
804 \\
849\end{array}$ & $\begin{array}{l}22.58312 \\
22.75525 \\
22.91967 \\
23.07711 \\
23.22818\end{array}$ & $\begin{array}{l}17213 \\
16442 \\
15744 \\
15107 \\
14524\end{array}$ & $\begin{array}{l}26.10781 \\
26.28634 \\
26.45777 \\
26.62275 \\
26.78186\end{array}$ & $\begin{array}{l}17853 \\
17143 \\
16498 \\
15911 \\
15373\end{array}$ \\
\hline $\begin{array}{l}250 \\
260 \\
270 \\
280 \\
290\end{array}$ & $\begin{array}{l}3.77956 \\
3.80684 \\
3.83391 \\
3.86066 \\
3.88696\end{array}$ & $\begin{array}{l}2728 \\
2707 \\
2675 \\
2630 \\
2579\end{array}$ & $\begin{array}{l}3.56217 \\
3.57105 \\
3.58029 \\
3.58982 \\
3.59962\end{array}$ & $\begin{array}{r}888 \\
924 \\
953 \\
980 \\
1001\end{array}$ & $\begin{array}{l}23.37342 \\
23.51330 \\
23.64825 \\
23.77863 \\
23.90477\end{array}$ & $\begin{array}{l}13988 \\
13495 \\
13038 \\
12614 \\
12220\end{array}$ & $\begin{array}{l}26.93559 \\
27.08436 \\
27.22854 \\
27.36845 \\
27.50439\end{array}$ & $\begin{array}{l}14877 \\
14418 \\
13991 \\
13594 \\
13221\end{array}$ \\
\hline $\begin{array}{l}300 \\
310 \\
320 \\
330 \\
340\end{array}$ & $\begin{array}{l}3.91275 \\
3.93795 \\
3.96253 \\
3.98644 \\
4.00968\end{array}$ & $\begin{array}{l}2520 \\
2458 \\
2391 \\
2324 \\
2253\end{array}$ & $\begin{array}{l}3.60963 \\
3.61981 \\
3.63014 \\
3.64058 \\
3.65109\end{array}$ & $\begin{array}{l}1018 \\
1033 \\
1044 \\
1051 \\
1057\end{array}$ & $\begin{array}{l}24.02697 \\
24.14549 \\
24.26058 \\
24.37245 \\
24.48128\end{array}$ & $\begin{array}{l}11852 \\
11509 \\
11187 \\
10883 \\
10599\end{array}$ & $\begin{array}{l}27.63660 \\
27.76531 \\
27.89073 \\
28.01303 \\
28.13238\end{array}$ & $\begin{array}{l}12871 \\
12542 \\
12230 \\
11935 \\
11656\end{array}$ \\
\hline $\begin{array}{l}350 \\
360 \\
370 \\
380 \\
390\end{array}$ & $\begin{array}{l}4.03221 \\
4.05406 \\
4.07521 \\
4.09567 \\
4.11546\end{array}$ & $\begin{array}{l}2185 \\
2115 \\
2046 \\
1979 \\
1913\end{array}$ & $\begin{array}{l}3.66166 \\
3.67226 \\
3.68287 \\
3.69346 \\
3.70403\end{array}$ & $\begin{array}{l}1060 \\
1061 \\
1059 \\
1057 \\
1053\end{array}$ & $\begin{array}{l}24.58727 \\
24.69057 \\
24.79134 \\
24.88969 \\
24.98577\end{array}$ & $\begin{array}{r}10330 \\
10077 \\
9835 \\
9608 \\
9391\end{array}$ & $\begin{array}{l}28.24894 \\
28.36284 \\
28.47421 \\
28.58316 \\
28.68980\end{array}$ & $\begin{array}{l}11390 \\
11137 \\
10895 \\
10664 \\
10444\end{array}$ \\
\hline $\begin{array}{l}400 \\
450 \\
500 \\
550 \\
600\end{array}$ & $\begin{array}{l}4.13459 \\
4.22102 \\
4.29395 \\
4.35591 \\
4.40909\end{array}$ & $\begin{array}{l}8643 \\
7293 \\
6196 \\
5318 \\
4617\end{array}$ & $\begin{array}{l}3.71456 \\
3.76617 \\
3.81540 \\
3.86179 \\
3.90524\end{array}$ & $\begin{array}{l}5161 \\
4923 \\
4639 \\
4345 \\
4057\end{array}$ & $\begin{array}{l}25.07968 \\
25.52019 \\
25.91957 \\
26.28541 \\
26.62332\end{array}$ & $\begin{array}{l}44051 \\
39938 \\
36584 \\
33791 \\
31421\end{array}$ & $\begin{array}{l}28.79424 \\
29.28636 \\
29.73497 \\
30.14721 \\
30.52856\end{array}$ & $\begin{array}{l}49212 \\
44861 \\
41224 \\
38135 \\
35478\end{array}$ \\
\hline $\begin{array}{l}650 \\
700 \\
750 \\
800 \\
850\end{array}$ & $\begin{array}{l}4.45526 \\
4.49582 \\
4.53187 \\
4.56426 \\
4.59365\end{array}$ & $\begin{array}{l}4056 \\
3605 \\
3239 \\
2939 \\
2691\end{array}$ & $\begin{array}{l}3.94581 \\
3.98368 \\
4.01905 \\
4.05213 \\
4.08313\end{array}$ & $\begin{array}{l}3787 \\
3537 \\
3308 \\
3100 \\
2912\end{array}$ & $\begin{array}{l}26.93753 \\
27.23134 \\
27.50741 \\
27.76786 \\
28.01446\end{array}$ & $\begin{array}{l}29381 \\
27607 \\
26045 \\
24660 \\
23422\end{array}$ & $\begin{array}{l}30.88334 \\
31.21503 \\
31.52646 \\
31.81999 \\
32.09760\end{array}$ & $\begin{array}{l}33169 \\
31143 \\
29353 \\
27761 \\
26333\end{array}$ \\
\hline $\begin{array}{r}900 \\
950 \\
1000 \\
1050 \\
1100\end{array}$ & $\begin{array}{l}4.62056 \\
4.64542 \\
4.66856 \\
4.69023 \\
4.71066\end{array}$ & $\begin{array}{l}2486 \\
2314 \\
2167 \\
2043 \\
1935\end{array}$ & $\begin{array}{l}4.11225 \\
4.13967 \\
4.16554 \\
4.19001 \\
4.21322\end{array}$ & $\begin{array}{l}2742 \\
2587 \\
2447 \\
2321 \\
2205\end{array}$ & $\begin{array}{l}28.24868 \\
28.47176 \\
28.68476 \\
28.88859 \\
29.08405\end{array}$ & $\begin{array}{l}22308 \\
21300 \\
20383 \\
19546 \\
18778\end{array}$ & $\begin{array}{l}32.36093 \\
32.61143 \\
32.85030 \\
33.07861 \\
33.29728\end{array}$ & $\begin{array}{l}25050 \\
23887 \\
22831 \\
21867 \\
20983\end{array}$ \\
\hline 1150 & 4.73001 & 1842 & 4.23527 & 2100 & 29.27183 & 18070 & 33.50711 & 20170 \\
\hline
\end{tabular}


Table 2.060. HgT (Cont.)

\begin{tabular}{|c|c|c|c|c|c|c|c|c|}
\hline${ }^{\circ} \mathrm{K}$ & $\frac{C_{p}^{\circ}}{R}$ & & $\frac{\left(H^{\circ}-E_{0}^{0}\right)}{R T}$ & & $\frac{- \text { LF }^{\circ}-E}{R T}$ & & $\frac{S^{\circ}}{R}$ & \\
\hline $\begin{array}{l}1200 \\
1300 \\
1400 \\
1500 \\
1600\end{array}$ & $\begin{array}{l}4.74843 \\
4.78295 \\
4.81494 \\
4.84491 \\
4.87325\end{array}$ & $\begin{array}{l}3452 \\
3199 \\
2997 \\
2834 \\
2697\end{array}$ & $\begin{array}{l}4.25627 \\
4.29548 \\
4.33145 \\
4.36469 \\
4.39560\end{array}$ & $\begin{array}{l}3921 \\
3597 \\
3324 \\
3091 \\
2890\end{array}$ & $\begin{array}{l}29.45253 \\
29.79479 \\
30.11445 \\
30.41444 \\
30.69713\end{array}$ & $\begin{array}{l}34226 \\
31966 \\
29999 \\
28269 \\
26736\end{array}$ & $\begin{array}{l}33.70881 \\
34.09027 \\
34.44591 \\
34.77914 \\
35.09273\end{array}$ & $\begin{array}{l}38146 \\
35564 \\
33323 \\
31359 \\
29626\end{array}$ \\
\hline $\begin{array}{l}1700 \\
1800 \\
1900 \\
2000 \\
2100\end{array}$ & $\begin{array}{l}4.90022 \\
4.92604 \\
4.95088 \\
4.97484 \\
4.99804\end{array}$ & $\begin{array}{l}2582 \\
2484 \\
2396 \\
2320 \\
2251\end{array}$ & $\begin{array}{l}4.42450 \\
4.45165 \\
4.47727 \\
4.50156 \\
4.52465\end{array}$ & $\begin{array}{l}2715 \\
2562 \\
2429 \\
2309 \\
2203\end{array}$ & $\begin{array}{l}30.96449 \\
31.21816 \\
31.45954 \\
31.68982 \\
31.91001\end{array}$ & $\begin{array}{l}25367 \\
24138 \\
23028 \\
22019 \\
21100\end{array}$ & $\begin{array}{l}35.38899 \\
35.66981 \\
35.93682 \\
36.19138 \\
36.43467\end{array}$ & $\begin{array}{l}28082 \\
26701 \\
25456 \\
24329 \\
23303\end{array}$ \\
\hline $\begin{array}{l}2200 \\
2300 \\
2400 \\
2500 \\
2600\end{array}$ & $\begin{array}{l}5.02055 \\
5.04244 \\
5.06376 \\
5.08456 \\
5.10487\end{array}$ & $\begin{array}{l}2189 \\
2132 \\
2080 \\
2031 \\
1986\end{array}$ & $\begin{array}{l}4.54668 \\
4.56776 \\
4.58799 \\
4.60743 \\
4.62618\end{array}$ & $\begin{array}{l}2108 \\
2023 \\
1944 \\
1875 \\
1810\end{array}$ & $\begin{array}{l}32.12101 \\
32.32359 \\
32.51842 \\
32.70611 \\
32.88718\end{array}$ & $\begin{array}{l}20258 \\
19483 \\
18769 \\
18107 \\
17494\end{array}$ & $\begin{array}{l}36.66770 \\
36.89136 \\
37.10641 \\
37.31355 \\
37.51337\end{array}$ & $\begin{array}{l}22366 \\
21505 \\
20714 \\
19982 \\
19303\end{array}$ \\
\hline $\begin{array}{l}2700 \\
2800 \\
2900 \\
3000\end{array}$ & $\begin{array}{l}5.12473 \\
5.14416 \\
5.16319 \\
5.18185\end{array}$ & $\begin{array}{l}1943 \\
1903 \\
1866 \\
3624\end{array}$ & $\begin{array}{l}4.64428 \\
4.66178 \\
4.67875 \\
4.69521\end{array}$ & $\begin{array}{l}1750 \\
1697 \\
1646 \\
3155\end{array}$ & $\begin{array}{l}33.06212 \\
33.23134 \\
33.39522 \\
33.55412\end{array}$ & $\begin{array}{l}16922 \\
16388 \\
15890 \\
30404\end{array}$ & $\begin{array}{l}37.70640 \\
37.89313 \\
38.07397 \\
38.24933\end{array}$ & $\begin{array}{l}18673 \\
18084 \\
17536 \\
33559\end{array}$ \\
\hline $\begin{array}{l}3200 \\
3400 \\
3600 \\
3800 \\
4000\end{array}$ & $\begin{array}{l}5.21809 \\
5.25302 \\
5.28674 \\
5.31935 \\
5.35091\end{array}$ & $\begin{array}{l}3493 \\
3372 \\
3261 \\
3156 \\
3058\end{array}$ & $\begin{array}{l}4.72676 \\
4.75670 \\
4.78521 \\
4.81247 \\
4.83861\end{array}$ & $\begin{array}{l}2994 \\
2851 \\
2726 \\
2614 \\
2513\end{array}$ & $\begin{array}{l}33.85816 \\
34.14562 \\
34.41832 \\
34.67778 \\
34.92530\end{array}$ & $\begin{array}{l}28746 \\
27270 \\
25946 \\
24752 \\
23669\end{array}$ & $\begin{array}{l}38.58492 \\
38.90232 \\
39.20354 \\
39.49026 \\
39.76391\end{array}$ & $\begin{array}{l}31740 \\
30122 \\
28672 \\
27365 \\
26182\end{array}$ \\
\hline $\begin{array}{l}4200 \\
4400 \\
4600 \\
4800 \\
5000\end{array}$ & $\begin{array}{l}5.38149 \\
5.41115 \\
5.43995 \\
5.46792 \\
5.49511\end{array}$ & $\begin{array}{l}2966 \\
2880 \\
2797 \\
2719\end{array}$ & $\begin{array}{l}4.86374 \\
4.88795 \\
4.91132 \\
4.93394 \\
4.95584\end{array}$ & $\begin{array}{l}2421 \\
2337 \\
2262 \\
2190\end{array}$ & $\begin{array}{l}35.16199 \\
35.38881 \\
35.60661 \\
35.81611 \\
36.01797\end{array}$ & $\begin{array}{l}22682 \\
21780 \\
20950 \\
20186\end{array}$ & $\begin{array}{l}40.02573 \\
40.27676 \\
40.51794 \\
40.75005 \\
40.97382\end{array}$ & $\begin{array}{l}25103 \\
24118 \\
23211 \\
22377\end{array}$ \\
\hline
\end{tabular}


Table 2.061. BH

\begin{tabular}{|l|l|l|l|l|}
\hline$K$ & $\frac{C_{p}^{\circ}}{R}$ & $\frac{\left(H^{0}-E_{0}^{0}\right)}{R T}$ & $\frac{-\left(F^{0}-E_{0}^{0}\right)}{R T}$ & $\frac{S^{0}}{R}$ \\
\hline
\end{tabular}

\begin{tabular}{|c|c|c|c|c|c|c|c|c|}
\hline $\begin{array}{l}50 \\
60 \\
70 \\
80 \\
90\end{array}$ & $\begin{array}{l}3.50414 \\
3.50347 \\
3.50317 \\
3.50306 \\
3.50306\end{array}$ & $\begin{array}{l}-67 \\
-30 \\
-11\end{array}$ & $\begin{array}{l}3.38456 \\
3.40443 \\
3.41855 \\
3.42912 \\
3.43734\end{array}$ & $\begin{array}{r}1987 \\
1412 \\
1057 \\
822 \\
657\end{array}$ & $\begin{array}{l}11.01480 \\
11.63375 \\
12.15966 \\
12.61687 \\
13.02126\end{array}$ & $\begin{array}{l}61895 \\
52591 \\
45721 \\
40439 \\
36251\end{array}$ & $\begin{array}{l}14.39937 \\
15.03818 \\
15.57822 \\
16.04599 \\
16.45860\end{array}$ & $\begin{array}{l}63881 \\
54004 \\
46777 \\
41261 \\
36908\end{array}$ \\
\hline $\begin{array}{l}100 \\
110 \\
120 \\
130 \\
140\end{array}$ & $\begin{array}{l}3.50313 \\
3.50325 \\
3.50340 \\
3.50357 \\
3.50375\end{array}$ & $\begin{array}{l}12 \\
15 \\
17 \\
18 \\
20\end{array}$ & $\begin{array}{l}3.44391 \\
3.44930 \\
3.45380 \\
3.45762 \\
3.46091\end{array}$ & $\begin{array}{l}539 \\
450 \\
382 \\
329 \\
286\end{array}$ & $\begin{array}{l}13.38377 \\
13.71227 \\
14.01260 \\
14.28920 \\
14.54556\end{array}$ & $\begin{array}{l}32850 \\
30033 \\
27660 \\
25636 \\
23888\end{array}$ & $\begin{array}{l}16.82768 \\
17.16157 \\
17.46640 \\
17.74683 \\
18.00648\end{array}$ & $\begin{array}{l}33389 \\
30483 \\
28043 \\
25965 \\
24174\end{array}$ \\
\hline $\begin{array}{l}150 \\
160 \\
170 \\
180 \\
190\end{array}$ & $\begin{array}{l}3.50395 \\
3.50416 \\
3.50437 \\
3.50459 \\
3.50481\end{array}$ & $\begin{array}{l}21 \\
21 \\
22 \\
22 \\
24\end{array}$ & $\begin{array}{l}3.46377 \\
3.46629 \\
3.46853 \\
3.47052 \\
3.47232\end{array}$ & $\begin{array}{l}252 \\
224 \\
199 \\
180 \\
163\end{array}$ & $\begin{array}{l}14.78444 \\
15.00807 \\
15.21828 \\
15.41660 \\
15.60429\end{array}$ & $\begin{array}{l}22363 \\
21021 \\
19832 \\
18769 \\
17815\end{array}$ & $\begin{array}{l}18.24822 \\
18.47437 \\
18.68681 \\
18.88712 \\
19.07661\end{array}$ & $\begin{array}{l}22615 \\
21244 \\
20031 \\
18949 \\
17978\end{array}$ \\
\hline $\begin{array}{l}200 \\
210 \\
220 \\
230 \\
240\end{array}$ & $\begin{array}{l}3.50505 \\
3.50530 \\
3.50556 \\
3.50585 \\
3.50618\end{array}$ & $\begin{array}{l}25 \\
26 \\
29 \\
33 \\
37\end{array}$ & $\begin{array}{l}3.47395 \\
3.47544 \\
3.47680 \\
3.47806 \\
3.47922\end{array}$ & $\begin{array}{l}149 \\
136 \\
126 \\
116 \\
109\end{array}$ & $\begin{array}{l}15.78244 \\
15.95197 \\
16.11368 \\
16.26826 \\
16.41631\end{array}$ & $\begin{array}{l}16953 \\
16171 \\
15458 \\
14805 \\
14205\end{array}$ & $\begin{array}{l}19.25639 \\
19.42741 \\
19.59048 \\
19.74632 \\
19.89553\end{array}$ & $\begin{array}{l}17102 \\
16307 \\
15584 \\
14921 \\
14314\end{array}$ \\
\hline $\begin{array}{l}250 \\
260 \\
270 \\
280 \\
290\end{array}$ & $\begin{array}{l}3.50655 \\
3.50699 \\
3.50750 \\
3.50810 \\
3.50881\end{array}$ & $\begin{array}{l}44 \\
51 \\
60 \\
71 \\
85\end{array}$ & $\begin{array}{l}3.48031 \\
3.48133 \\
3.48229 \\
3.48320 \\
3.48407\end{array}$ & $\begin{array}{r}102 \\
96 \\
91 \\
87 \\
84\end{array}$ & $\begin{array}{l}16.55836 \\
16.69488 \\
16.82628 \\
16.95294 \\
17.07519\end{array}$ & $\begin{array}{l}13652 \\
13140 \\
12666 \\
12225 \\
11813\end{array}$ & $\begin{array}{l}20.03867 \\
20.17621 \\
20.30857 \\
20.43614 \\
20.55926\end{array}$ & $\begin{array}{l}13754 \\
13236 \\
12757 \\
12312 \\
11897\end{array}$ \\
\hline $\begin{array}{l}300 \\
310 \\
320 \\
330 \\
340\end{array}$ & $\begin{array}{l}3.50966 \\
3.51066 \\
3.51184 \\
3.51321 \\
3.51478\end{array}$ & $\begin{array}{l}100 \\
118 \\
137 \\
157 \\
180\end{array}$ & $\begin{array}{l}3.48491 \\
3.48572 \\
3.48652 \\
3.48731 \\
3.48809\end{array}$ & $\begin{array}{l}81 \\
80 \\
79 \\
78 \\
79\end{array}$ & $\begin{array}{l}17.19332 \\
17.30760 \\
17.41828 \\
17.52558 \\
17.62970\end{array}$ & $\begin{array}{l}11428 \\
11068 \\
10730 \\
10412 \\
10112\end{array}$ & $\begin{array}{l}20.67823 \\
20.79332 \\
20.90480 \\
21.01289 \\
21.11779\end{array}$ & $\begin{array}{l}11509 \\
11148 \\
10809 \\
10490 \\
10191\end{array}$ \\
\hline $\begin{array}{l}350 \\
360 \\
370 \\
380 \\
390\end{array}$ & $\begin{array}{l}3.51658 \\
3.51863 \\
3.52094 \\
3.52351 \\
3.52636\end{array}$ & $\begin{array}{l}205 \\
231 \\
257 \\
285 \\
314\end{array}$ & $\begin{array}{l}3.48888 \\
3.48968 \\
3.49049 \\
3.49132 \\
3.49218\end{array}$ & $\begin{array}{l}80 \\
81 \\
83 \\
86 \\
90\end{array}$ & $\begin{array}{l}17.73082 \\
17.82911 \\
17.92474 \\
18.01784 \\
18.10854\end{array}$ & $\begin{array}{l}9829 \\
9563 \\
9310 \\
9070 \\
8842\end{array}$ & $\begin{array}{l}21.21970 \\
21.31879 \\
21.41523 \\
21.50916 \\
21.60072\end{array}$ & $\begin{array}{l}9909 \\
9644 \\
9393 \\
9156 \\
8932\end{array}$ \\
\hline $\begin{array}{l}400 \\
450 \\
500 \\
550 \\
600\end{array}$ & $\begin{array}{l}3.52950 \\
3.54964 \\
3.57701 \\
3.61069 \\
3.64926\end{array}$ & $\begin{array}{l}2014 \\
2737 \\
3368 \\
3857 \\
4192\end{array}$ & $\begin{array}{l}3.49308 \\
3.49817 \\
3.50463 \\
3.51270 \\
3.52244\end{array}$ & $\begin{array}{r}509 \\
646 \\
807 \\
974 \\
1135\end{array}$ & $\begin{array}{l}18.19696 \\
18.60867 \\
18.97756 \\
19.31195 \\
19.61800\end{array}$ & $\begin{array}{l}41171 \\
36889 \\
33439 \\
30605 \\
28239\end{array}$ & $\begin{array}{l}21.69004 \\
22.10685 \\
22.48220 \\
22.82466 \\
23.14045\end{array}$ & $\begin{array}{l}41681 \\
37535 \\
34246 \\
31579 \\
29374\end{array}$ \\
\hline $\begin{array}{l}650 \\
700 \\
750 \\
800 \\
850\end{array}$ & $\begin{array}{l}3.69118 \\
3.73504 \\
3.77964 \\
3.82404 \\
3.86754\end{array}$ & $\begin{array}{l}4386 \\
4460 \\
4440 \\
4350 \\
4213\end{array}$ & $\begin{array}{l}3.53379 \\
3.54659 \\
3.56064 \\
3.57572 \\
3.59161\end{array}$ & $\begin{array}{l}1280 \\
1405 \\
1508 \\
1589 \\
1651\end{array}$ & $\begin{array}{l}19.90039 \\
20.16273 \\
20.40789 \\
20.63817 \\
20.85542\end{array}$ & $\begin{array}{l}26234 \\
24516 \\
23028 \\
21725 \\
20575\end{array}$ & $\begin{array}{l}23.43419 \\
23.70933 \\
23.96854 \\
24.21389 \\
24.44704\end{array}$ & $\begin{array}{l}27514 \\
25921 \\
24535 \\
23315 \\
22226\end{array}$ \\
\hline $\begin{array}{r}900 \\
950 \\
1000 \\
1050 \\
1100\end{array}$ & $\begin{array}{l}3.90967 \\
3.95011 \\
3.98867 \\
4.02526 \\
4.05987\end{array}$ & $\begin{array}{l}4044 \\
3856 \\
3659 \\
3461 \\
3266\end{array}$ & $\begin{array}{l}3.60812 \\
3.62506 \\
3.64229 \\
3.65966 \\
3.67707\end{array}$ & $\begin{array}{l}1694 \\
1723 \\
1737 \\
1741 \\
1736\end{array}$ & $\begin{array}{l}21.06117 \\
21.25671 \\
21.44309 \\
21.62122 \\
21.79186\end{array}$ & $\begin{array}{l}19554 \\
18638 \\
17813 \\
17064 \\
16384\end{array}$ & $\begin{array}{l}24.66930 \\
24.88178 \\
25.08538 \\
25.28088 \\
25.46894\end{array}$ & $\begin{array}{l}21248 \\
20360 \\
19550 \\
18806 \\
18120\end{array}$ \\
\hline 1150 & 4.09253 & 3076 & 3.69443 & 1724 & 21.95570 & 15760 & 25.65014 & 17483 \\
\hline
\end{tabular}


Table 2.061. BH (Cont.)

\begin{tabular}{|l|l|l|l|l|}
\hline$K$ & $\frac{C_{p}^{0}}{R}$ & $\frac{\left(H^{\circ}-E_{0}^{0}\right)}{R T}$ & $\frac{-\left(F^{\circ}-E_{0}^{0}\right)}{R T}$ & $\frac{S^{0}}{R}$ \\
\hline
\end{tabular}

\begin{tabular}{|c|c|c|c|c|c|c|c|c|}
\hline $\begin{array}{l}1200 \\
1300 \\
1400 \\
1500 \\
1600\end{array}$ & $\begin{array}{l}4.12329 \\
4.17950 \\
4.22928 \\
4.27346 \\
4.31280\end{array}$ & $\begin{array}{l}5621 \\
4978 \\
4418 \\
3934 \\
3519\end{array}$ & $\begin{array}{l}3.71167 \\
3.74554 \\
3.77835 \\
3.80991 \\
3.84014\end{array}$ & $\begin{array}{l}3387 \\
3281 \\
3156 \\
3023 \\
2885\end{array}$ & $\begin{array}{l}22.11330 \\
22.41174 \\
22.69052 \\
22.95228 \\
23.19914\end{array}$ & $\begin{array}{l}29844 \\
27878 \\
26176 \\
24686 \\
23368\end{array}$ & $\begin{array}{l}25.82497 \\
26.15728 \\
26.46887 \\
26.76220 \\
27.03928\end{array}$ & $\begin{array}{l}33231 \\
31159 \\
29333 \\
27708 \\
26254\end{array}$ \\
\hline $\begin{array}{l}1700 \\
1800 \\
1900 \\
2000 \\
2100\end{array}$ & $\begin{array}{l}4.34799 \\
4.37964 \\
4.40826 \\
4.43427 \\
4.45805\end{array}$ & $\begin{array}{l}3165 \\
2862 \\
2601 \\
2378 \\
2186\end{array}$ & $\begin{array}{l}3.86899 \\
3.89650 \\
3.92269 \\
3.94763 \\
3.97138\end{array}$ & $\begin{array}{l}2751 \\
2619 \\
2494 \\
2375 \\
2262\end{array}$ & $\begin{array}{l}23.43282 \\
23.65475 \\
23.86613 \\
24.06798 \\
24.26116\end{array}$ & $\begin{array}{l}22193 \\
21138 \\
20185 \\
19318 \\
18528\end{array}$ & $\begin{array}{l}27.30182 \\
27.55125 \\
27.78883 \\
28.01562 \\
28.23255\end{array}$ & $\begin{array}{l}24943 \\
23758 \\
22679 \\
21693 \\
20790\end{array}$ \\
\hline $\begin{array}{l}2200 \\
2300 \\
2400 \\
2500 \\
2600\end{array}$ & $\begin{array}{l}4.47991 \\
4.50010 \\
4.51885 \\
4.53634 \\
4.55274\end{array}$ & $\begin{array}{l}2019 \\
1875 \\
1749 \\
1640 \\
1542\end{array}$ & $\begin{array}{l}3.99400 \\
4.01558 \\
4.03616 \\
4.05582 \\
4.07462\end{array}$ & $\begin{array}{l}2158 \\
2058 \\
1966 \\
1880 \\
1800\end{array}$ & $\begin{array}{l}24.44644 \\
24.62446 \\
24.79580 \\
24.96096 \\
25.12041\end{array}$ & $\begin{array}{l}17802 \\
17134 \\
16516 \\
15945 \\
15411\end{array}$ & $\begin{array}{l}28.44045 \\
28.64004 \\
28.83196 \\
29.01679 \\
29.19503\end{array}$ & $\begin{array}{l}19959 \\
19192 \\
18483 \\
17824 \\
17212\end{array}$ \\
\hline $\begin{array}{l}2700 \\
2800 \\
2900 \\
3000\end{array}$ & $\begin{array}{l}4.56816 \\
4.58274 \\
4.59657 \\
4.60973\end{array}$ & $\begin{array}{l}1458 \\
1383 \\
1316 \\
2460\end{array}$ & $\begin{array}{l}4.09262 \\
4.10986 \\
4.12641 \\
4.14230\end{array}$ & $\begin{array}{l}1724 \\
1655 \\
1589 \\
3000\end{array}$ & $\begin{array}{l}25.27452 \\
25.42368 \\
25.56819 \\
25.70835\end{array}$ & $\begin{array}{l}14916 \\
14451 \\
14016 \\
26831\end{array}$ & $\begin{array}{l}29.36715 \\
29.53355 \\
29.69460 \\
29.85066\end{array}$ & $\begin{array}{l}16640 \\
16105 \\
15606 \\
29830\end{array}$ \\
\hline $\begin{array}{l}3200 \\
3400 \\
3600 \\
3800 \\
4000\end{array}$ & $\begin{array}{l}4.63433 \\
4.65702 \\
4.67817 \\
4.69804 \\
4.71686\end{array}$ & $\begin{array}{l}2269 \\
2115 \\
1987 \\
1882 \\
1793\end{array}$ & $\begin{array}{l}4.17230 \\
4.20015 \\
4.22613 \\
4.25045 \\
4.27330\end{array}$ & $\begin{array}{l}2785 \\
2598 \\
2432 \\
2285 \\
2155\end{array}$ & $\begin{array}{l}25.97666 \\
26.23045 \\
26.47127 \\
26.70042 \\
26.91903\end{array}$ & $\begin{array}{l}25379 \\
24082 \\
22915 \\
21861 \\
20902\end{array}$ & $\begin{array}{l}30.14896 \\
30.43060 \\
30.69740 \\
30.95087 \\
31.19233\end{array}$ & $\begin{array}{l}28164 \\
26680 \\
25347 \\
24146 \\
23058\end{array}$ \\
\hline $\begin{array}{l}4200 \\
4400 \\
4600 \\
4800 \\
5000\end{array}$ & $\begin{array}{l}4.73479 \\
4.75196 \\
4.76849 \\
4.78447 \\
4.79996\end{array}$ & $\begin{array}{l}1717 \\
1653 \\
1598 \\
1549\end{array}$ & $\begin{array}{l}4.29485 \\
4.31524 \\
4.33459 \\
4.35301 \\
4.37058\end{array}$ & $\begin{array}{l}2039 \\
1935 \\
1842 \\
1757\end{array}$ & $\begin{array}{l}27.12805 \\
27.32832 \\
27.52057 \\
27.70544 \\
27.88350\end{array}$ & $\begin{array}{l}20027 \\
19225 \\
18487 \\
17806\end{array}$ & $\begin{array}{l}31.42291 \\
31.64357 \\
31.85517 \\
32.05845 \\
32.25408\end{array}$ & $\begin{array}{l}22066 \\
21160 \\
20328 \\
19563\end{array}$ \\
\hline
\end{tabular}


Table 2.062. BD

\begin{tabular}{|l|l|l|l|l|}
\hline$K$ & $\frac{C_{p}^{0}}{R}$ & $\frac{\left(H^{0}-E_{0}^{0}\right)}{R T}$ & $\frac{-\left(F^{\circ}-E_{0}^{0}\right)}{R T}$ & $\frac{S^{\circ}}{R}$ \\
\hline
\end{tabular}

\begin{tabular}{|c|c|c|c|c|c|c|c|c|}
\hline $\begin{array}{l}50 \\
60 \\
70 \\
80 \\
90\end{array}$ & $\begin{array}{l}3.50204 \\
3.50201 \\
3.50209 \\
3.50223 \\
3.50240\end{array}$ & $\begin{array}{r}-3 \\
8 \\
14 \\
17 \\
19\end{array}$ & $\begin{array}{l}3.43803 \\
3.44869 \\
3.45631 \\
3.46204 \\
3.46652\end{array}$ & $\begin{array}{r}1066 \\
762 \\
573 \\
448 \\
360\end{array}$ & $\begin{array}{l}11.69143 \\
12.31926 \\
12.85148 \\
13.31340 \\
13.72144\end{array}$ & $\begin{array}{l}62783 \\
53222 \\
46192 \\
40804 \\
36543\end{array}$ & $\begin{array}{l}15.12946 \\
15.76796 \\
16.30780 \\
16.77545 \\
17.18796\end{array}$ & $\begin{array}{l}63850 \\
53984 \\
46765 \\
41251 \\
36903\end{array}$ \\
\hline $\begin{array}{l}100 \\
110 \\
120 \\
130 \\
140\end{array}$ & $\begin{array}{l}3.50259 \\
3.50280 \\
3.50301 \\
3.50323 \\
3.50345\end{array}$ & $\begin{array}{l}21 \\
21 \\
22 \\
22 \\
24\end{array}$ & $\begin{array}{l}3.47012 \\
3.47308 \\
3.47556 \\
3.47768 \\
3.47952\end{array}$ & $\begin{array}{l}296 \\
248 \\
212 \\
184 \\
160\end{array}$ & $\begin{array}{l}14.08687 \\
14.41775 \\
14.72005 \\
14.99833 \\
15.25613\end{array}$ & $\begin{array}{l}33088 \\
30230 \\
27828 \\
25780 \\
24011\end{array}$ & $\begin{array}{l}17.55699 \\
17.89083 \\
18.19562 \\
18.47602 \\
18.73565\end{array}$ & $\begin{array}{l}33384 \\
30479 \\
28040 \\
25963 \\
24172\end{array}$ \\
\hline $\begin{array}{l}150 \\
160 \\
170 \\
180 \\
190\end{array}$ & $\begin{array}{l}3.50369 \\
3.50395 \\
3.50423 \\
3.50456 \\
3.50496\end{array}$ & $\begin{array}{l}26 \\
28 \\
33 \\
40 \\
51\end{array}$ & $\begin{array}{l}3.48112 \\
3.48254 \\
3.48381 \\
3.48495 \\
3.48599\end{array}$ & $\begin{array}{r}142 \\
127 \\
114 \\
104 \\
96\end{array}$ & $\begin{array}{l}15.49624 \\
15.72096 \\
15.93212 \\
16.13129 \\
16.31974\end{array}$ & $\begin{array}{l}22472 \\
21116 \\
19917 \\
18845 \\
17883\end{array}$ & $\begin{array}{l}18.97737 \\
19.20350 \\
19.41593 \\
19.61624 \\
19.80573\end{array}$ & $\begin{array}{l}22613 \\
21243 \\
20031 \\
18949 \\
17980\end{array}$ \\
\hline $\begin{array}{l}200 \\
210 \\
220 \\
230 \\
240\end{array}$ & $\begin{array}{l}3.50547 \\
3.50611 \\
3.50693 \\
3.50798 \\
3.50929\end{array}$ & $\begin{array}{r}64 \\
82 \\
105 \\
131 \\
163\end{array}$ & $\begin{array}{l}3.48695 \\
3.48785 \\
3.48870 \\
3.48951 \\
3.49031\end{array}$ & $\begin{array}{l}90 \\
85 \\
81 \\
80 \\
79\end{array}$ & $\begin{array}{l}16.49857 \\
16.66872 \\
16.83100 \\
16.98609 \\
17.13462\end{array}$ & $\begin{array}{l}17015 \\
16228 \\
15509 \\
14853 \\
14250\end{array}$ & $\begin{array}{l}19.98553 \\
20.15657 \\
20.31970 \\
20.47561 \\
20.62493\end{array}$ & $\begin{array}{l}17104 \\
16313 \\
15591 \\
14932 \\
14329\end{array}$ \\
\hline $\begin{array}{l}250 \\
260 \\
270 \\
280 \\
290\end{array}$ & $\begin{array}{l}3.51092 \\
3.51290 \\
3.51528 \\
3.51808 \\
3.52134\end{array}$ & $\begin{array}{l}198 \\
238 \\
280 \\
326 \\
374\end{array}$ & $\begin{array}{l}3.49110 \\
3.49190 \\
3.49272 \\
3.49357 \\
3.49447\end{array}$ & $\begin{array}{l}80 \\
82 \\
85 \\
90 \\
96\end{array}$ & $\begin{array}{l}17.27712 \\
17.41406 \\
17.54586 \\
17.67290 \\
17.79551\end{array}$ & $\begin{array}{l}13694 \\
13180 \\
12704 \\
12261 \\
11848\end{array}$ & $\begin{array}{l}20.76822 \\
20.90596 \\
21.03858 \\
21.16647 \\
21.28998\end{array}$ & $\begin{array}{l}13774 \\
13262 \\
12789 \\
12351 \\
11944\end{array}$ \\
\hline $\begin{array}{l}300 \\
310 \\
320 \\
330 \\
340\end{array}$ & $\begin{array}{l}3.52508 \\
3.52931 \\
3.53405 \\
3.53930 \\
3.54506\end{array}$ & $\begin{array}{l}423 \\
474 \\
525 \\
576 \\
626\end{array}$ & $\begin{array}{l}3.49543 \\
3.49645 \\
3.49755 \\
3.49874 \\
3.50001\end{array}$ & $\begin{array}{l}102 \\
110 \\
119 \\
127 \\
138\end{array}$ & $\begin{array}{l}17.91399 \\
18.02862 \\
18.13965 \\
18.24729 \\
18.35176\end{array}$ & $\begin{array}{l}11463 \\
11103 \\
10764 \\
10447 \\
10147\end{array}$ & $\begin{array}{l}21.40942 \\
21.52508 \\
21.63720 \\
21.74603 \\
21.85177\end{array}$ & $\begin{array}{l}11566 \\
11212 \\
10883 \\
10574 \\
10286\end{array}$ \\
\hline $\begin{array}{l}350 \\
360 \\
370 \\
380 \\
390\end{array}$ & $\begin{array}{l}3.55132 \\
3.55807 \\
3.56529 \\
3.57298 \\
3.58109\end{array}$ & $\begin{array}{l}675 \\
722 \\
769 \\
811 \\
853\end{array}$ & $\begin{array}{l}3.50139 \\
3.50287 \\
3.50446 \\
3.50616 \\
3.50797\end{array}$ & $\begin{array}{l}148 \\
159 \\
170 \\
181 \\
194\end{array}$ & $\begin{array}{l}18.45323 \\
18.55189 \\
18.64789 \\
18.74137 \\
18.83247\end{array}$ & $\begin{array}{l}9866 \\
9600 \\
9348 \\
9110 \\
8883\end{array}$ & $\begin{array}{l}21.95463 \\
22.05476 \\
22.15235 \\
22.24753 \\
22.34044\end{array}$ & \begin{tabular}{r|}
10013 \\
9759 \\
9518 \\
9291 \\
9078
\end{tabular} \\
\hline $\begin{array}{l}400 \\
450 \\
500 \\
550 \\
600\end{array}$ & $\begin{array}{l}3.58962 \\
3.63746 \\
3.69145 \\
3.74838 \\
3.80573\end{array}$ & $\begin{array}{l}4784 \\
5399 \\
5693 \\
5735 \\
5601\end{array}$ & $\begin{array}{l}3.50991 \\
3.52135 \\
3.53562 \\
3.55236 \\
3.57109\end{array}$ & $\begin{array}{l}1144 \\
1427 \\
1674 \\
1873 \\
2022\end{array}$ & $\begin{array}{l}18.92130 \\
19.33534 \\
19.70707 \\
20.04482 \\
20.35471\end{array}$ & $\begin{array}{l}41404 \\
37173 \\
33775 \\
30989 \\
28663\end{array}$ & $\begin{array}{l}22.43122 \\
22.85670 \\
23.24270 \\
23.59719 \\
23.92580\end{array}$ & $\begin{array}{l}42548 \\
38600 \\
35449 \\
32861 \\
30685\end{array}$ \\
\hline $\begin{array}{l}650 \\
700 \\
750 \\
800 \\
850\end{array}$ & $\begin{array}{l}3.86174 \\
3.91527 \\
3.96569 \\
4.01273 \\
4.05633\end{array}$ & $\begin{array}{l}5353 \\
5042 \\
4704 \\
4360 \\
4025\end{array}$ & $\begin{array}{l}3.59131 \\
3.61255 \\
3.63443 \\
3.65663 \\
3.67887\end{array}$ & $\begin{array}{l}2124 \\
2188 \\
2220 \\
2224 \\
2211\end{array}$ & $\begin{array}{l}20.64134 \\
20.90825 \\
21.15824 \\
21.39351 \\
21.61586\end{array}$ & $\begin{array}{l}26691 \\
24999 \\
23527 \\
22235 \\
21090\end{array}$ & $\begin{array}{l}24.23265 \\
24.52081 \\
24.79268 \\
25.05014 \\
25.29473\end{array}$ & $\begin{array}{l}28816 \\
27187 \\
25746 \\
24459 \\
23301\end{array}$ \\
\hline $\begin{array}{r}900 \\
950 \\
1000 \\
1050 \\
1100\end{array}$ & $\begin{array}{l}4.09658 \\
4.13365 \\
4.16776 \\
4.19916 \\
4.22807\end{array}$ & $\begin{array}{l}3707 \\
3411 \\
3140 \\
2891 \\
2666\end{array}$ & $\begin{array}{l}3.70098 \\
3.72279 \\
3.74419 \\
3.76512 \\
3.78552\end{array}$ & $\begin{array}{l}2181 \\
2140 \\
2093 \\
2040 \\
1983\end{array}$ & $\begin{array}{l}21.82676 \\
22.02745 \\
22.21895 \\
22.40214 \\
22.57776\end{array}$ & $\begin{array}{l}20069 \\
19150 \\
18319 \\
17562 \\
16872\end{array}$ & $\begin{array}{l}25.52774 \\
25.75024 \\
25.96315 \\
26.16726 \\
26.36329\end{array}$ & $\begin{array}{l}22250 \\
21291 \\
20411 \\
19603 \\
18854\end{array}$ \\
\hline 1150 & 4.25473 & 2462 & 3.80535 & 1924 & 22.74648 & 16236 & 26.55183 & 18160 \\
\hline
\end{tabular}


Table 2.062. BD (Cont.)

\begin{tabular}{|l|l|l|l|l|}
\hline${ }^{\circ} K$ & $\frac{C_{p}^{0}}{R}$ & $\frac{\left(H^{\circ}-E_{0}^{0}\right)}{R T}$ & $\frac{-\left(F^{\circ}-E_{0}^{0}\right)}{R T}$ & $\frac{S^{0}}{R}$ \\
\hline
\end{tabular}

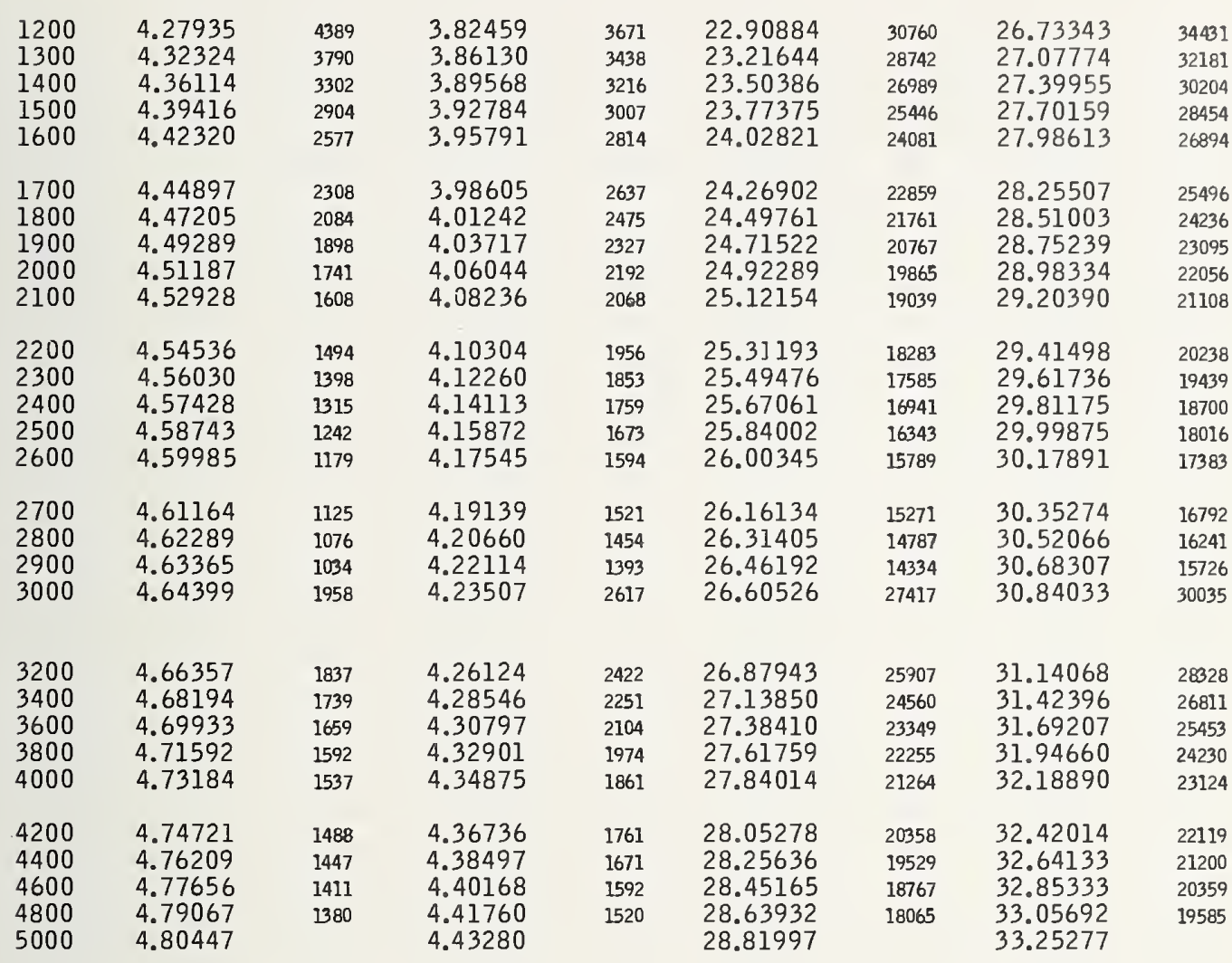


Table 2.063. BT

\begin{tabular}{|l|l|l|l|l|}
\hline${ }^{\circ} K$ & $\frac{C_{p}^{0}}{R}$ & $\frac{\left(H^{0}-E_{0}^{0}\right)}{R T}$ & $\frac{-\left(F^{0}-E_{0}^{0}\right)}{R T}$ & $\frac{S^{0}}{R}$ \\
\hline
\end{tabular}

\begin{tabular}{|c|c|c|c|c|c|c|c|c|}
\hline $\begin{array}{l}50 \\
60 \\
70 \\
80 \\
90\end{array}$ & $\begin{array}{l}3.50163 \\
3.50174 \\
3.50190 \\
3.50209 \\
3.50230\end{array}$ & $\begin{array}{l}11 \\
16 \\
19 \\
21 \\
21\end{array}$ & $\begin{array}{l}3.45559 \\
3.46327 \\
3.46878 \\
3.47293 \\
3.47618\end{array}$ & $\begin{array}{l}768 \\
551 \\
415 \\
325 \\
263\end{array}$ & $\begin{array}{l}12.11258 \\
12.74333 \\
13.27763 \\
13.74110 \\
14.15035\end{array}$ & $\begin{array}{l}63075 \\
53430 \\
46347 \\
40925 \\
36640\end{array}$ & $\begin{array}{l}15.56817 \\
16.20661 \\
16.74641 \\
17.21404 \\
17.62654\end{array}$ & $\begin{array}{l}63844 \\
53980 \\
46763 \\
41250 \\
36902\end{array}$ \\
\hline $\begin{array}{l}100 \\
110 \\
120 \\
130 \\
140\end{array}$ & $\begin{array}{l}3.50251 \\
3.50274 \\
3.50297 \\
3.50323 \\
3.50351\end{array}$ & $\begin{array}{l}23 \\
23 \\
26 \\
28 \\
35\end{array}$ & $\begin{array}{l}3.47881 \\
3.48097 \\
3.48279 \\
3.48436 \\
3.48571\end{array}$ & $\begin{array}{l}216 \\
182 \\
157 \\
135 \\
120\end{array}$ & $\begin{array}{l}14.51675 \\
14.84842 \\
15.15138 \\
15.43022 \\
15.68849\end{array}$ & $\begin{array}{l}33167 \\
30296 \\
27884 \\
25827 \\
24053\end{array}$ & $\begin{array}{l}17.99556 \\
18.32939 \\
18.63418 \\
18.91458 \\
19.17421\end{array}$ & $\begin{array}{l}33383 \\
30479 \\
28040 \\
25963 \\
24172\end{array}$ \\
\hline $\begin{array}{l}150 \\
160 \\
170 \\
180 \\
190\end{array}$ & $\begin{array}{l}3.50386 \\
3.50430 \\
3.50490 \\
3.50572 \\
3.50683\end{array}$ & $\begin{array}{r}44 \\
60 \\
82 \\
111 \\
147\end{array}$ & $\begin{array}{l}3.48691 \\
3.48798 \\
3.48896 \\
3.48987 \\
3.49073\end{array}$ & $\begin{array}{r}107 \\
98 \\
91 \\
86 \\
84\end{array}$ & $\begin{array}{l}15.92902 \\
16.15409 \\
16.36558 \\
16.56503 \\
16.75374\end{array}$ & $\begin{array}{l}22507 \\
21149 \\
19945 \\
18871 \\
17907\end{array}$ & $\begin{array}{l}19.41593 \\
19.64208 \\
19.85455 \\
20.05490 \\
20.24448\end{array}$ & $\begin{array}{l}22615 \\
21247 \\
20035 \\
18958 \\
17991\end{array}$ \\
\hline $\begin{array}{l}200 \\
210 \\
220 \\
230 \\
240\end{array}$ & $\begin{array}{l}3.50830 \\
3.51022 \\
3.51266 \\
3.51567 \\
3.51932\end{array}$ & $\begin{array}{l}192 \\
244 \\
301 \\
365 \\
433\end{array}$ & $\begin{array}{l}3.49157 \\
3.49241 \\
3.49327 \\
3.49418 \\
3.49515\end{array}$ & $\begin{array}{r}84 \\
86 \\
91 \\
97 \\
105\end{array}$ & $\begin{array}{l}16.93281 \\
17.10319 \\
17.26568 \\
17.42098 \\
17.56971\end{array}$ & $\begin{array}{l}17038 \\
16249 \\
15530 \\
14873 \\
14270\end{array}$ & $\begin{array}{l}20.42439 \\
20.59560 \\
20.75895 \\
20.91516 \\
21.06486\end{array}$ & $\begin{array}{l}17121 \\
16335 \\
15621 \\
14970 \\
14375\end{array}$ \\
\hline $\begin{array}{l}250 \\
260 \\
270 \\
280 \\
290\end{array}$ & $\begin{array}{l}3.52365 \\
3.52868 \\
3.53444 \\
3.54092 \\
3.54813\end{array}$ & $\begin{array}{l}503 \\
576 \\
648 \\
721 \\
791\end{array}$ & $\begin{array}{l}3.49620 \\
3.49735 \\
3.49862 \\
3.50001 \\
3.50154\end{array}$ & $\begin{array}{l}115 \\
127 \\
139 \\
153 \\
168\end{array}$ & $\begin{array}{l}17.71241 \\
17.84956 \\
17.98157 \\
18.10883 \\
18.23168\end{array}$ & $\begin{array}{l}13715 \\
13201 \\
12726 \\
12285 \\
11873\end{array}$ & $\begin{array}{l}21.20861 \\
21.34691 \\
21.48019 \\
21.60884 \\
21.73322\end{array}$ & $\begin{array}{l}13830 \\
13328 \\
12865 \\
12438 \\
12042\end{array}$ \\
\hline $\begin{array}{l}300 \\
310 \\
320 \\
330 \\
340\end{array}$ & $\begin{array}{l}3.55604 \\
3.56463 \\
3.57387 \\
3.58371 \\
3.59413\end{array}$ & $\begin{array}{r}859 \\
924 \\
984 \\
1042 \\
1093\end{array}$ & $\begin{array}{l}3.50322 \\
3.50506 \\
3.50707 \\
3.50924 \\
3.51158\end{array}$ & $\begin{array}{l}184 \\
201 \\
217 \\
234 \\
252\end{array}$ & $\begin{array}{l}18.35041 \\
18.46531 \\
18.57663 \\
18.68458 \\
18.78937\end{array}$ & $\begin{array}{l}11490 \\
11132 \\
10795 \\
10479 \\
10183\end{array}$ & $\begin{array}{l}21.85364 \\
21.97038 \\
22.08370 \\
22.19382 \\
22.30096\end{array}$ & $\begin{array}{l}11674 \\
11332 \\
11012 \\
10714 \\
10434\end{array}$ \\
\hline $\begin{array}{l}350 \\
360 \\
370 \\
380 \\
390\end{array}$ & $\begin{array}{l}3.60506 \\
3.61646 \\
3.62829 \\
3.64049 \\
3.65303\end{array}$ & $\begin{array}{l}1140 \\
1183 \\
1220 \\
1254 \\
1281\end{array}$ & $\begin{array}{l}3.51410 \\
3.51678 \\
3.51963 \\
3.52265 \\
3.52583\end{array}$ & $\begin{array}{l}268 \\
285 \\
302 \\
318 \\
334\end{array}$ & $\begin{array}{l}18.89120 \\
18.99023 \\
19.08663 \\
19.18053 \\
19.27207\end{array}$ & $\begin{array}{l}9903 \\
9640 \\
9390 \\
9154 \\
8931\end{array}$ & $\begin{array}{l}22.40530 \\
22.50702 \\
22.60627 \\
22.70319 \\
22.79791\end{array}$ & $\begin{array}{r}10172 \\
9925 \\
9692 \\
9472 \\
9265\end{array}$ \\
\hline $\begin{array}{l}400 \\
450 \\
500 \\
550 \\
600\end{array}$ & $\begin{array}{l}3.66584 \\
3.73263 \\
3.80071 \\
3.86689 \\
3.92930\end{array}$ & $\begin{array}{l}6679 \\
6808 \\
6618 \\
6241 \\
5776\end{array}$ & $\begin{array}{l}3.52917 \\
3.54804 \\
3.56991 \\
3.59392 \\
3.61930\end{array}$ & $\begin{array}{l}1887 \\
2187 \\
2401 \\
2538 \\
2610\end{array}$ & $\begin{array}{l}19.36138 \\
19.77812 \\
20.15305 \\
20.49441 \\
20.80820\end{array}$ & $\begin{array}{l}41674 \\
37493 \\
34136 \\
31379 \\
29073\end{array}$ & $\begin{array}{l}22.89056 \\
23.32616 \\
23.72296 \\
24.08833 \\
24.42750\end{array}$ & $\begin{array}{l}43560 \\
39680 \\
36537 \\
33917 \\
31683\end{array}$ \\
\hline $\begin{array}{l}650 \\
700 \\
750 \\
800 \\
850\end{array}$ & $\begin{array}{l}3.98706 \\
4.03988 \\
4.08784 \\
4.13124 \\
4.17045\end{array}$ & $\begin{array}{l}5282 \\
4796 \\
4340 \\
3921 \\
3544\end{array}$ & $\begin{array}{l}3.64540 \\
3.67172 \\
3.69789 \\
3.72364 \\
3.74879\end{array}$ & $\begin{array}{l}2632 \\
2617 \\
2575 \\
2515 \\
2442\end{array}$ & $\begin{array}{l}21.09893 \\
21.37004 \\
21.62426 \\
21.86374 \\
22.09025\end{array}$ & $\begin{array}{l}27111 \\
25422 \\
23948 \\
22651 \\
21497\end{array}$ & $\begin{array}{l}24.74433 \\
25.04177 \\
25.32215 \\
25.58738 \\
25.83904\end{array}$ & $\begin{array}{l}29744 \\
28038 \\
26523 \\
25166 \\
23939\end{array}$ \\
\hline $\begin{array}{r}900 \\
950 \\
1000 \\
1050 \\
1100\end{array}$ & $\begin{array}{l}4.20589 \\
4.23796 \\
4.26704 \\
4.29346 \\
4.31756\end{array}$ & $\begin{array}{l}3207 \\
2908 \\
2642 \\
2410 \\
2203\end{array}$ & $\begin{array}{l}3.77321 \\
3.79684 \\
3.81964 \\
3.84158 \\
3.86268\end{array}$ & $\begin{array}{l}2363 \\
2280 \\
2194 \\
2110 \\
2026\end{array}$ & $\begin{array}{l}22.30522 \\
22.50986 \\
22.70520 \\
22.89209 \\
23.07129\end{array}$ & $\begin{array}{l}20464 \\
19534 \\
18689 \\
17920 \\
17215\end{array}$ & $\begin{array}{l}26.07843 \\
26.30671 \\
26.52484 \\
26.73368 \\
26.93397\end{array}$ & $\begin{array}{l}22828 \\
21813 \\
20884 \\
20029 \\
19242\end{array}$ \\
\hline 1150 & 4.33959 & 2021 & 3.88294 & 1946 & 23.24344 & 16568 & 27.12639 & 18513 \\
\hline
\end{tabular}


Table 2.063. BT (Cont.)

\begin{tabular}{|l|l|l|l|l|}
\hline$K$ & $\frac{C_{p}^{0}}{R}$ & $\frac{\left(H^{0}-E_{0}^{0}\right)}{R T}$ & $\frac{-\left(F^{\circ}-E_{0}^{0}\right)}{R T}$ & $\frac{S^{\circ}}{R}$ \\
\hline
\end{tabular}

$\begin{array}{lllllllll}1200 & 4.35980 & 3578 & 3.90240 & 3657 & 23.40912 & 31382 & 27.31152 & 35042 \\ 1300 & 4.39558 & 3070 & 3.93899 & 3374 & 23.72294 & 29316 & 27.66194 & 32690 \\ 1400 & 4.42628 & 2669 & 3.97273 & 3115 & 24.01610 & 27517 & 27.98884 & 30631 \\ 1500 & 4.45297 & 2348 & 4.00388 & 2881 & 24.29127 & 25933 & 28.29515 & 28815 \\ 1600 & 4.47645 & 2090 & 4.03269 & 2673 & 24.55060 & 24530 & 28.58330 & 27203 \\ & & & & & & & & \\ 1700 & 4.49735 & 1880 & 4.05942 & 2486 & 24.79590 & 23274 & 28.85533 & 25760 \\ 1800 & 4.51615 & 1708 & 4.08428 & 2319 & 25.02864 & 22145 & 29.11293 & 24464 \\ 1900 & 4.53323 & 1565 & 4.10747 & 2168 & 25.25009 & 21125 & 29.35757 & 23293 \\ 2000 & 4.54888 & 1446 & 4.12915 & 2034 & 25.46134 & 20196 & 29.59050 & 22229 \\ 2100 & 4.56334 & 1346 & 4.14949 & 1912 & 25.66330 & 19348 & 29.81279 & 21260 \\ & & & & & & & & \\ 2200 & 4.57680 & 1262 & 4.16861 & 1802 & 25.85678 & 18570 & 30.02539 & 20373 \\ 2300 & 4.58942 & 1190 & 4.18663 & 1704 & 26.04248 & 17855 & 30.22912 & 19558 \\ 2400 & 4.60132 & 1127 & 4.20367 & 1613 & 26.22103 & 17193 & 30.42470 & 18806 \\ 2500 & 4.61259 & 1075 & 4.21980 & 1532 & 26.39296 & 16580 & 30.61276 & 18113 \\ 2600 & 4.62334 & 1028 & 4.23512 & 1457 & 26.55876 & 16011 & 30.79389 & 17468 \\ & & & & & & & & \\ 2700 & 4.63362 & 988 & 4.24969 & 1389 & 26.71887 & 15481 & 30.96857 & 16869 \\ 2800 & 4.64350 & 952 & 4.26358 & 1326 & 26.87368 & 14985 & 31.13726 & 16311 \\ 2900 & 4.65302 & 921 & 4.27684 & 1270 & 27.02353 & 14520 & 31.30037 & 15790 \\ 3000 & 4.66223 & 1761 & 4.28954 & 2384 & 27.16873 & 27762 & 31.45827 & 30146 \\ & & & & & & & & \\ 3200 & 4.67984 & 1672 & 4.31338 & 2206 & 27.44635 & 26216 & 31.75973 & 28422 \\ 3400 & 4.69656 & 1600 & 4.33544 & 2051 & 27.70851 & 24840 & 32.04395 & 26891 \\ 3600 & 4.71256 & 1540 & 4.35595 & 1917 & 27.95691 & 23603 & 32.31286 & 25521 \\ 3800 & 4.72796 & 1491 & 4.37512 & 1802 & 28.19294 & 22488 & 32.56807 & 24289 \\ 4000 & 4.74287 & 1448 & 4.39314 & 1700 & 28.41782 & 21476 & 32.81096 & 23176 \\ 4200 & 4.75735 & 1413 & 4.41014 & 1610 & 28.63258 & 20553 & 33.04272 & 22164 \\ 4400 & 4.77148 & 1380 & 4.42624 & 1532 & 28.83811 & 19710 & 33.26436 & 21241 \\ 4600 & 4.78528 & 1353 & 4.44156 & 1460 & 29.03521 & 18934 & 33.47677 & 20394 \\ 4800 & 4.79881 & 1328 & 4.45616 & 1397 & 29.22455 & 18219 & 33.68071 & 19617 \\ 5000 & 4.81209 & & 4.47013 & & 29.40674 & & 33.87688 & \end{array}$


Table 2.064. AlH

\begin{tabular}{|l|l|l|l|l|}
\hline$K^{\circ}$ & $\frac{C_{p}^{0}}{R}$ & $\frac{\left(H^{0}-E_{0}^{0}\right)}{R T}$ & $\frac{-\left(F^{\circ}-E_{0}^{0}\right)}{R T}$ & $\frac{S^{0}}{R}$ \\
\hline
\end{tabular}

\begin{tabular}{|c|c|c|c|c|c|c|c|c|}
\hline $\begin{array}{l}50 \\
60 \\
70 \\
80 \\
90\end{array}$ & $\begin{array}{l}3.50210 \\
3.50211 \\
3.50221 \\
3.50238 \\
3.50257\end{array}$ & $\begin{array}{r}1 \\
10 \\
17 \\
19 \\
21\end{array}$ & $\begin{array}{l}3.43946 \\
3.44990 \\
3.45736 \\
3.46298 \\
3.46737\end{array}$ & $\begin{array}{r}1044 \\
746 \\
562 \\
439 \\
353\end{array}$ & $\begin{array}{l}12.88285 \\
13.51092 \\
14.04331 \\
14.50536 \\
14.91350\end{array}$ & $\begin{array}{l}62807 \\
53239 \\
46205 \\
40814 \\
36552\end{array}$ & $\begin{array}{l}16.32231 \\
16.96082 \\
17.50068 \\
17.96835 \\
18.38088\end{array}$ & $\begin{array}{l}63851 \\
53986 \\
46767 \\
41253 \\
36904\end{array}$ \\
\hline $\begin{array}{l}100 \\
110 \\
120 \\
130 \\
140\end{array}$ & $\begin{array}{l}3.50278 \\
3.50301 \\
3.50324 \\
3.50348 \\
3.50374\end{array}$ & $\begin{array}{l}23 \\
23 \\
24 \\
26 \\
27\end{array}$ & $\begin{array}{l}3.47090 \\
3.47381 \\
3.47625 \\
3.47834 \\
3.48014\end{array}$ & $\begin{array}{l}291 \\
244 \\
209 \\
180 \\
158\end{array}$ & $\begin{array}{l}15.27902 \\
15.60997 \\
15.91234 \\
16.19067 \\
16.44851\end{array}$ & $\begin{array}{l}33095 \\
30237 \\
27833 \\
25784 \\
24016\end{array}$ & $\begin{array}{l}18.74992 \\
19.08378 \\
19.38860 \\
19.66901 \\
19.92866\end{array}$ & $\begin{array}{l}33386 \\
30482 \\
28041 \\
25965 \\
24174\end{array}$ \\
\hline $\begin{array}{l}150 \\
160 \\
170 \\
180 \\
190\end{array}$ & $\begin{array}{l}3.50401 \\
3.50432 \\
3.50467 \\
3.50511 \\
3.50567\end{array}$ & $\begin{array}{l}31 \\
35 \\
44 \\
56 \\
73\end{array}$ & $\begin{array}{l}3.48172 \\
3.48313 \\
3.48438 \\
3.48552 \\
3.48657\end{array}$ & $\begin{array}{r}141 \\
125 \\
114 \\
105 \\
97\end{array}$ & $\begin{array}{l}16.68867 \\
16.91343 \\
17.12463 \\
17.32382 \\
17.51230\end{array}$ & $\begin{array}{l}22476 \\
21120 \\
19919 \\
18848 \\
17887\end{array}$ & $\begin{array}{l}20.17040 \\
20.39656 \\
20.60901 \\
20.80935 \\
20.99887\end{array}$ & $\begin{array}{l}22616 \\
21245 \\
20034 \\
18952 \\
17984\end{array}$ \\
\hline $\begin{array}{l}200 \\
210 \\
220 \\
230 \\
240\end{array}$ & $\begin{array}{l}3.50640 \\
3.50734 \\
3.50855 \\
3.51008 \\
3.51199\end{array}$ & $\begin{array}{r}94 \\
121 \\
153 \\
191 \\
233\end{array}$ & $\begin{array}{l}3.48754 \\
3.48846 \\
3.48934 \\
3.49021 \\
3.49108\end{array}$ & $\begin{array}{l}92 \\
88 \\
87 \\
87 \\
88\end{array}$ & $\begin{array}{l}17.69117 \\
17.86135 \\
18.02365 \\
18.17878 \\
18.32734\end{array}$ & $\begin{array}{l}17018 \\
16230 \\
15513 \\
14856 \\
14253\end{array}$ & $\begin{array}{l}21.17871 \\
21.34981 \\
21.51300 \\
21.66899 \\
21.81842\end{array}$ & $\begin{array}{l}17110 \\
16319 \\
15599 \\
14943 \\
14341\end{array}$ \\
\hline $\begin{array}{l}250 \\
260 \\
270 \\
280 \\
290\end{array}$ & $\begin{array}{l}3.51432 \\
3.51712 \\
3.52042 \\
3.52426 \\
3.52866\end{array}$ & $\begin{array}{l}280 \\
330 \\
384 \\
440 \\
496\end{array}$ & $\begin{array}{l}3.49196 \\
3.49287 \\
3.49383 \\
3.49485 \\
3.49593\end{array}$ & $\begin{array}{r}91 \\
96 \\
102 \\
108 \\
118\end{array}$ & $\begin{array}{l}18.46987 \\
18.60684 \\
18.73868 \\
18.86576 \\
18.98842\end{array}$ & $\begin{array}{l}13697 \\
13184 \\
12708 \\
12266 \\
11854\end{array}$ & $\begin{array}{l}21.96183 \\
22.09972 \\
22.23252 \\
22.36061 \\
22.48436\end{array}$ & $\begin{array}{l}13789 \\
13280 \\
12809 \\
12375 \\
11971\end{array}$ \\
\hline $\begin{array}{l}300 \\
310 \\
320 \\
330 \\
340\end{array}$ & $\begin{array}{l}3.53362 \\
3.53917 \\
3.54529 \\
3.55198 \\
3.55922\end{array}$ & $\begin{array}{l}555 \\
612 \\
669 \\
724 \\
779\end{array}$ & $\begin{array}{l}3.49711 \\
3.49837 \\
3.49974 \\
3.50122 \\
3.50282\end{array}$ & $\begin{array}{l}126 \\
137 \\
148 \\
160 \\
172\end{array}$ & $\begin{array}{l}19.10696 \\
19.22165 \\
19.33274 \\
19.44045 \\
19.54500\end{array}$ & $\begin{array}{l}11469 \\
11109 \\
10771 \\
10455 \\
10156\end{array}$ & $\begin{array}{l}22.60407 \\
22.72003 \\
22.83248 \\
22.94168 \\
23.04782\end{array}$ & $\begin{array}{l}11596 \\
11245 \\
10920 \\
10614 \\
10329\end{array}$ \\
\hline $\begin{array}{l}350 \\
360 \\
370 \\
380 \\
390\end{array}$ & $\begin{array}{l}3.56701 \\
3.57530 \\
3.58408 \\
3.59332 \\
3.60298\end{array}$ & $\begin{array}{r}829 \\
878 \\
924 \\
966 \\
1006\end{array}$ & $\begin{array}{l}3.50454 \\
3.50639 \\
3.50837 \\
3.51048 \\
3.51273\end{array}$ & $\begin{array}{l}185 \\
198 \\
211 \\
225 \\
238\end{array}$ & $\begin{array}{l}19.64656 \\
19.74531 \\
19.84141 \\
19.93500 \\
20.02622\end{array}$ & $\begin{array}{l}9875 \\
9610 \\
9359 \\
9122 \\
8896\end{array}$ & $\begin{array}{l}23.15111 \\
23.25171 \\
23.34979 \\
23.44549 \\
23.53895\end{array}$ & $\begin{array}{r}10060 \\
9808 \\
9570 \\
9346 \\
9135\end{array}$ \\
\hline $\begin{array}{l}400 \\
450 \\
500 \\
550 \\
600\end{array}$ & $\begin{array}{l}3.61304 \\
3.66801 \\
3.72789 \\
3.78926 \\
3.84970\end{array}$ & $\begin{array}{l}5497 \\
5988 \\
6137 \\
6044 \\
5794\end{array}$ & $\begin{array}{l}3.51511 \\
3.52898 \\
3.54585 \\
3.56519 \\
3.58639\end{array}$ & $\begin{array}{l}1387 \\
1687 \\
1934 \\
2120 \\
2251\end{array}$ & $\begin{array}{l}20.11518 \\
20.52997 \\
20.90264 \\
21.24148 \\
21.55259\end{array}$ & $\begin{array}{l}41479 \\
37267 \\
33884 \\
31111 \\
28795\end{array}$ & $\begin{array}{l}23.63030 \\
24.05896 \\
24.44850 \\
24.80668 \\
25.13899\end{array}$ & $\begin{array}{l}42866 \\
38954 \\
35818 \\
33231 \\
31045\end{array}$ \\
\hline $\begin{array}{l}650 \\
700 \\
750 \\
800 \\
850\end{array}$ & $\begin{array}{l}3.90764 \\
3.96221 \\
4.01299 \\
4.05989 \\
4.10302\end{array}$ & $\begin{array}{l}5457 \\
5078 \\
4690 \\
4313 \\
3957\end{array}$ & $\begin{array}{l}3.60890 \\
3.63221 \\
3.65592 \\
3.67972 \\
3.70337\end{array}$ & $\begin{array}{l}2331 \\
2371 \\
2380 \\
2365 \\
2332\end{array}$ & $\begin{array}{l}21.84054 \\
22.10884 \\
22.36024 \\
22.59695 \\
22.82074\end{array}$ & $\begin{array}{l}26830 \\
25140 \\
23671 \\
22379 \\
21239\end{array}$ & $\begin{array}{l}25.44944 \\
25.74105 \\
26.01617 \\
26.27668 \\
26.52412\end{array}$ & $\begin{array}{l}29161 \\
27512 \\
26051 \\
24744 \\
23566\end{array}$ \\
\hline $\begin{array}{r}900 \\
950 \\
1000 \\
1050 \\
1100\end{array}$ & $\begin{array}{l}4.14259 \\
4.17884 \\
4.21207 \\
4.24256 \\
4.27058\end{array}$ & $\begin{array}{l}3625 \\
3323 \\
3049 \\
2802 \\
2580\end{array}$ & $\begin{array}{l}3.72669 \\
3.74955 \\
3.77186 \\
3.79355 \\
3.81461\end{array}$ & $\begin{array}{l}2286 \\
2231 \\
2169 \\
2106 \\
2039\end{array}$ & $\begin{array}{l}23.03308 \\
23.23519 \\
23.42809 \\
23.61265 \\
23.78961\end{array}$ & $\begin{array}{l}20211 \\
19290 \\
18456 \\
17696 \\
17002\end{array}$ & $\begin{array}{l}26.75978 \\
26.98474 \\
27.19995 \\
27.40620 \\
27.60422\end{array}$ & $\begin{array}{l}22496 \\
21521 \\
20625 \\
19802 \\
19042\end{array}$ \\
\hline 1150 & 4.29638 & 2380 & 3.83500 & 1973 & 23.95963 & 16363 & 27.79464 & 18336 \\
\hline
\end{tabular}


Table 2.064. AlH (Cont.)

\begin{tabular}{|c|c|c|c|c|c|c|c|c|}
\hline${ }^{\circ} \mathrm{K}$ & $\frac{C_{p}^{0}}{R}$ & & $\frac{\left(H^{0}-E_{0}^{0}\right)}{R T}$ & & $\frac{-l F^{0}-}{R T}$ & & $\frac{S^{0}}{R}$ & \\
\hline $\begin{array}{l}200 \\
300 \\
400 \\
500 \\
600\end{array}$ & $\begin{array}{l}4.32018 \\
4.36262 \\
4.39931 \\
4.43137 \\
4.45967\end{array}$ & $\begin{array}{l}4244 \\
3669 \\
3206 \\
2830 \\
2524\end{array}$ & $\begin{array}{l}3.85473 \\
3.89221 \\
3.92715 \\
3.95972 \\
3.99010\end{array}$ & $\begin{array}{l}3748 \\
3494 \\
3257 \\
3038 \\
2838\end{array}$ & $\begin{array}{l}24.12326 \\
24.43330 \\
24.72304 \\
24.99511 \\
25.25165\end{array}$ & $\begin{array}{l}31004 \\
28974 \\
27207 \\
25654 \\
24276\end{array}$ & $\begin{array}{l}27.97800 \\
28.32552 \\
28.65020 \\
28.95483 \\
29.24175\end{array}$ & $\begin{array}{l}34752 \\
32468 \\
30463 \\
28692 \\
2714\end{array}$ \\
\hline $\begin{array}{l}700 \\
800 \\
1900 \\
1000 \\
100\end{array}$ & $\begin{array}{l}4.48491 \\
4.50764 \\
4.52829 \\
4.54721 \\
4.56467\end{array}$ & $\begin{array}{l}2273 \\
2065 \\
1892 \\
1746 \\
1624\end{array}$ & $\begin{array}{l}4.01848 \\
4.04503 \\
4.06993 \\
4.09333 \\
4.11536\end{array}$ & $\begin{array}{l}2655 \\
2490 \\
2340 \\
2203 \\
2080\end{array}$ & $\begin{array}{l}25.49441 \\
25.72486 \\
25.94424 \\
26.15360 \\
26.35385\end{array}$ & $\begin{array}{l}23045 \\
21938 \\
20936 \\
20025 \\
19193\end{array}$ & $\begin{array}{l}29.51289 \\
29.76989 \\
30.01417 \\
30.24693 \\
30.46922\end{array}$ & $\begin{array}{l}25700 \\
24428 \\
23276 \\
22229 \\
21273\end{array}$ \\
\hline $\begin{array}{l}2 \\
200 \\
300 \\
2400 \\
500 \\
2600\end{array}$ & $\begin{array}{l}4.58091 \\
4.59610 \\
4.61040 \\
4.62393 \\
4.63679\end{array}$ & $\begin{array}{l}1519 \\
1430 \\
1353 \\
1286 \\
1228\end{array}$ & $\begin{array}{l}4.13616 \\
4.15583 \\
4.17448 \\
4.19218 \\
4.20904\end{array}$ & $\begin{array}{l}1967 \\
1865 \\
1770 \\
1686 \\
1607\end{array}$ & $\begin{array}{l}26.54578 \\
26.73008 \\
26.90735 \\
27.07812 \\
27.24287\end{array}$ & $\begin{array}{l}18430 \\
17727 \\
17077 \\
16475 \\
15916\end{array}$ & $\begin{array}{l}30.68195 \\
30.88591 \\
31.08183 \\
31.27031 \\
31.45192\end{array}$ & $\begin{array}{l}20396 \\
19592 \\
18848 \\
18161 \\
17522\end{array}$ \\
\hline $\begin{array}{l}700 \\
300 \\
900 \\
000\end{array}$ & $\begin{array}{l}4.64907 \\
4.66084 \\
4.67216 \\
4.68309\end{array}$ & $\begin{array}{l}1177 \\
1132 \\
1093 \\
2084\end{array}$ & $\begin{array}{l}4.22511 \\
4.24046 \\
4.25516 \\
4.26924\end{array}$ & $\begin{array}{l}1535 \\
1470 \\
1408 \\
2652\end{array}$ & $\begin{array}{l}27.40203 \\
27.55596 \\
27.70502 \\
27.84952\end{array}$ & $\begin{array}{l}15393 \\
14906 \\
14450 \\
27639\end{array}$ & $\begin{array}{l}31.62714 \\
31.79643 \\
31.96019 \\
32.11876\end{array}$ & $\begin{array}{l}16929 \\
16376 \\
15857 \\
30292\end{array}$ \\
\hline $\begin{array}{l}3200 \\
3400 \\
3600 \\
800 \\
1000\end{array}$ & $\begin{array}{l}4.70393 \\
4.72364 \\
4.74242 \\
4.76045 \\
4.77784\end{array}$ & $\begin{array}{l}1971 \\
1878 \\
1803 \\
1739 \\
1685\end{array}$ & $\begin{array}{l}4.29576 \\
4.32036 \\
4.34329 \\
4.36477 \\
4.38499\end{array}$ & $\begin{array}{l}2460 \\
2293 \\
2148 \\
2022 \\
1911\end{array}$ & $\begin{array}{l}28.12591 \\
28.38709 \\
28.63469 \\
28.87010 \\
29.09450\end{array}$ & $\begin{array}{l}26118 \\
24760 \\
23541 \\
22440 \\
21441\end{array}$ & $\begin{array}{l}32.42168 \\
32.70745 \\
32.97798 \\
33.23488 \\
33.47950\end{array}$ & $\begin{array}{l}28577 \\
27053 \\
25690 \\
24462 \\
23352\end{array}$ \\
\hline $\begin{array}{l}200 \\
400 \\
600 \\
800 \\
000\end{array}$ & $\begin{array}{l}4.79469 \\
4.81107 \\
4.82704 \\
4.84266 \\
4.85796\end{array}$ & $\begin{array}{l}1638 \\
1597 \\
1562 \\
1530\end{array}$ & $\begin{array}{l}4.40410 \\
4.42223 \\
4.43949 \\
4.45596 \\
4.47174\end{array}$ & $\begin{array}{l}1813 \\
1726 \\
1647 \\
1578\end{array}$ & $\begin{array}{l}29.30891 \\
29.51421 \\
29.71117 \\
29.90047 \\
30.08269\end{array}$ & $\begin{array}{l}20530 \\
19696 \\
18930 \\
18222\end{array}$ & $\begin{array}{l}33.71302 \\
33.93645 \\
34.15066 \\
34.35643 \\
34.55443\end{array}$ & $\begin{array}{l}22343 \\
21421 \\
20577 \\
19800\end{array}$ \\
\hline
\end{tabular}


Table 2.065. AlD

\begin{tabular}{|l|l|l|l|l|}
\hline${ }^{\circ}$ & $\frac{C_{p}^{0}}{R}$ & $\frac{\left(H^{0}-E_{0}^{0}\right)}{R T}$ & $\frac{-\left(F^{0}-E_{0}^{0}\right)}{R T}$ & $\frac{S^{0}}{R}$ \\
\hline
\end{tabular}

\begin{tabular}{|c|c|c|c|c|c|c|c|c|}
\hline $\begin{array}{l}50 \\
60 \\
70 \\
80 \\
90\end{array}$ & $\begin{array}{l}3.50145 \\
3.50164 \\
3.50185 \\
3.50207 \\
3.50231\end{array}$ & $\begin{array}{l}19 \\
21 \\
22 \\
24 \\
24\end{array}$ & $\begin{array}{l}3.46896 \\
3.47439 \\
3.47830 \\
3.48126 \\
3.48358\end{array}$ & $\begin{array}{l}543 \\
391 \\
296 \\
232 \\
189\end{array}$ & $\begin{array}{l}13.55931 \\
14.19229 \\
14.72818 \\
15.19284 \\
15.60302\end{array}$ & $\begin{array}{l}63298 \\
53589 \\
46466 \\
41018 \\
36713\end{array}$ & $\begin{array}{l}17.02828 \\
17.66669 \\
18.20648 \\
18.67411 \\
19.08660\end{array}$ & $\begin{array}{l}63841 \\
53979 \\
46763 \\
41249 \\
36902\end{array}$ \\
\hline $\begin{array}{l}100 \\
110 \\
120 \\
130 \\
140\end{array}$ & $\begin{array}{l}3.50255 \\
3.50283 \\
3.50317 \\
3.50363 \\
3.50431\end{array}$ & $\begin{array}{r}28 \\
34 \\
46 \\
68 \\
101\end{array}$ & $\begin{array}{l}3.48547 \\
3.48703 \\
3.48836 \\
3.48952 \\
3.49055\end{array}$ & $\begin{array}{r}156 \\
133 \\
116 \\
103 \\
95\end{array}$ & $\begin{array}{l}15.97015 \\
16.30243 \\
16.60590 \\
16.88516 \\
17.14380\end{array}$ & $\begin{array}{l}33228 \\
30347 \\
27926 \\
25864 \\
24086\end{array}$ & $\begin{array}{l}19.45562 \\
19.78946 \\
20.09426 \\
20.37469 \\
20.63436\end{array}$ & $\begin{array}{l}33384 \\
30480 \\
28043 \\
25967 \\
24180\end{array}$ \\
\hline $\begin{array}{l}150 \\
160 \\
170 \\
180 \\
190\end{array}$ & $\begin{array}{l}3.50532 \\
3.50680 \\
3.50888 \\
3.51170 \\
3.51538\end{array}$ & $\begin{array}{l}148 \\
208 \\
282 \\
368 \\
462\end{array}$ & $\begin{array}{l}3.49150 \\
3.49241 \\
3.49331 \\
3.49425 \\
3.49526\end{array}$ & $\begin{array}{r}91 \\
90 \\
94 \\
101 \\
112\end{array}$ & $\begin{array}{l}17.38466 \\
17.61002 \\
17.82178 \\
18.02148 \\
18.21043\end{array}$ & $\begin{array}{l}22536 \\
21176 \\
19970 \\
18895 \\
17931\end{array}$ & $\begin{array}{l}20.87616 \\
21.10243 \\
21.31509 \\
21.51573 \\
21.70569\end{array}$ & $\begin{array}{l}22627 \\
21266 \\
20064 \\
18996 \\
18043\end{array}$ \\
\hline $\begin{array}{l}200 \\
210 \\
220 \\
230 \\
240\end{array}$ & $\begin{array}{l}3.52000 \\
3.52566 \\
3.53237 \\
3.54018 \\
3.54905\end{array}$ & $\begin{array}{l}566 \\
671 \\
781 \\
887 \\
991\end{array}$ & $\begin{array}{l}3.49638 \\
3.49763 \\
3.49906 \\
3.50067 \\
3.50250\end{array}$ & $\begin{array}{l}125 \\
143 \\
161 \\
183 \\
205\end{array}$ & $\begin{array}{l}18.38974 \\
18.56036 \\
18.72310 \\
18.87867 \\
19.02770\end{array}$ & $\begin{array}{l}17062 \\
16274 \\
15557 \\
14903 \\
14302\end{array}$ & $\begin{array}{l}21.88612 \\
22.05800 \\
22.22216 \\
22.37935 \\
22.53020\end{array}$ & $\begin{array}{l}17188 \\
16416 \\
15719 \\
15085 \\
14508\end{array}$ \\
\hline $\begin{array}{l}250 \\
260 \\
270 \\
280 \\
290\end{array}$ & $\begin{array}{l}3.55896 \\
3.56985 \\
3.58167 \\
3.59434 \\
3.60776\end{array}$ & $\begin{array}{l}1089 \\
1182 \\
1267 \\
1342 \\
1411\end{array}$ & $\begin{array}{l}3.50455 \\
3.50685 \\
3.50940 \\
3.51221 \\
3.51527\end{array}$ & $\begin{array}{l}230 \\
255 \\
281 \\
306 \\
332\end{array}$ & $\begin{array}{l}19.17072 \\
19.30821 \\
19.44061 \\
19.56829 \\
19.69159\end{array}$ & $\begin{array}{l}13749 \\
13240 \\
12768 \\
12330 \\
11923\end{array}$ & $\begin{array}{l}22.67528 \\
22.81507 \\
22.95002 \\
23.08050 \\
23.20687\end{array}$ & $\begin{array}{l}13979 \\
13495 \\
13048 \\
12637 \\
12254\end{array}$ \\
\hline $\begin{array}{l}300 \\
310 \\
320 \\
330 \\
340\end{array}$ & $\begin{array}{l}3.62187 \\
3.63656 \\
3.65175 \\
3.66736 \\
3.68331\end{array}$ & $\begin{array}{l}1469 \\
1519 \\
1561 \\
1595 \\
1620\end{array}$ & $\begin{array}{l}3.51859 \\
3.52215 \\
3.52596 \\
3.53001 \\
3.53429\end{array}$ & $\begin{array}{l}356 \\
381 \\
405 \\
428 \\
448\end{array}$ & $\begin{array}{l}19.81082 \\
19.92625 \\
20.03813 \\
20.14670 \\
20.25214\end{array}$ & $\begin{array}{l}11543 \\
11188 \\
10857 \\
10544 \\
10251\end{array}$ & $\begin{array}{l}23.32941 \\
23.44841 \\
23.56410 \\
23.67671 \\
23.78643\end{array}$ & $\begin{array}{l}11900 \\
11569 \\
11261 \\
10972 \\
10700\end{array}$ \\
\hline $\begin{array}{l}350 \\
360 \\
370 \\
380 \\
390\end{array}$ & $\begin{array}{l}3.69951 \\
3.71591 \\
3.73242 \\
3.74900 \\
3.76559\end{array}$ & $\begin{array}{l}1640 \\
1651 \\
1658 \\
1659 \\
1655\end{array}$ & $\begin{array}{l}3.53877 \\
3.54347 \\
3.54835 \\
3.55341 \\
3.55864\end{array}$ & $\begin{array}{l}470 \\
488 \\
506 \\
523 \\
538\end{array}$ & $\begin{array}{l}20.35465 \\
20.45441 \\
20.55156 \\
20.64626 \\
20.73863\end{array}$ & $\begin{array}{l}9976 \\
9715 \\
9470 \\
9237 \\
9016\end{array}$ & $\begin{array}{l}23.89343 \\
23.99788 \\
24.09992 \\
24.19968 \\
24.29727\end{array}$ & $\begin{array}{r}10445 \\
10204 \\
9976 \\
9759 \\
9555\end{array}$ \\
\hline $\begin{array}{l}400 \\
450 \\
500 \\
550 \\
600\end{array}$ & $\begin{array}{l}3.78214 \\
3.86298 \\
3.93834 \\
4.00665 \\
4.06763\end{array}$ & $\begin{array}{l}8084 \\
7536 \\
6831 \\
6098 \\
5403\end{array}$ & $\begin{array}{l}3.56402 \\
3.59279 \\
3.62363 \\
3.65540 \\
3.68726\end{array}$ & $\begin{array}{l}2877 \\
3084 \\
3177 \\
3186 \\
3138\end{array}$ & $\begin{array}{l}20.82879 \\
21.25021 \\
21.63033 \\
21.97719 \\
22.29661\end{array}$ & $\begin{array}{l}42142 \\
38012 \\
34686 \\
31942 \\
29639\end{array}$ & $\begin{array}{l}24.39282 \\
24.84300 \\
25.25396 \\
25.63259 \\
25.98388\end{array}$ & $\begin{array}{l}45018 \\
41096 \\
37863 \\
35129 \\
32776\end{array}$ \\
\hline $\begin{array}{l}650 \\
700 \\
750 \\
800 \\
850\end{array}$ & $\begin{array}{l}4.12166 \\
4.16937 \\
4.21151 \\
4.24881 \\
4.28193\end{array}$ & $\begin{array}{l}4771 \\
4214 \\
3730 \\
3312 \\
2952\end{array}$ & $\begin{array}{l}3.71864 \\
3.74917 \\
3.77861 \\
3.80686 \\
3.83385\end{array}$ & $\begin{array}{l}3053 \\
2944 \\
2825 \\
2699 \\
2573\end{array}$ & $\begin{array}{l}22.59300 \\
22.86970 \\
23.12938 \\
23.37415 \\
23.60576\end{array}$ & $\begin{array}{l}27670 \\
25968 \\
24477 \\
23161 \\
21987\end{array}$ & $\begin{array}{l}26.31164 \\
26.61887 \\
26.90800 \\
27.18102 \\
27.43961\end{array}$ & $\begin{array}{l}30723 \\
28913 \\
27302 \\
25859 \\
24560\end{array}$ \\
\hline $\begin{array}{r}900 \\
950 \\
1000 \\
1050 \\
1100\end{array}$ & $\begin{array}{l}4.31145 \\
4.33789 \\
4.36168 \\
4.38318 \\
4.40272\end{array}$ & $\begin{array}{l}2644 \\
2379 \\
2150 \\
1954 \\
1783\end{array}$ & $\begin{array}{l}3.85958 \\
3.88407 \\
3.90736 \\
3: 92952 \\
3.95059\end{array}$ & $\begin{array}{l}2449 \\
2329 \\
2216 \\
2107 \\
2005\end{array}$ & $\begin{array}{l}23.82563 \\
24.03497 \\
24.23479 \\
24.42598 \\
24.60927\end{array}$ & $\begin{array}{l}20934 \\
19982 \\
19119 \\
18329 \\
17605\end{array}$ & $\begin{array}{l}27.68521 \\
27.91904 \\
28.14216 \\
28.35550 \\
28.55986\end{array}$ & $\begin{array}{l}23383 \\
22312 \\
21334 \\
20436 \\
19611\end{array}$ \\
\hline 1150 & 4.42055 & 1636 & 3.97064 & 1909 & 24.78532 & 16940 & 28.75597 & 18849 \\
\hline
\end{tabular}


Table 2.065. AlD (Cont.)

\begin{tabular}{|l|l|l|l|l|}
\hline${ }^{\circ} K$ & $\frac{C_{p}^{0}}{R}$ & $\frac{\left(H^{0}-E_{0}^{0}\right)}{R T}$ & $\frac{-\left(F^{0}-E_{0}^{0}\right)}{R T}$ & $\frac{S^{0}}{R}$ \\
\hline
\end{tabular}

$\begin{array}{lllllllll}1200 & 4.43691 & 2901 & 3.98973 & 3555 & 24.95472 & 32078 & 28.94446 & 35632 \\ 1300 & 4.46592 & 2505 & 4.02528 & 3239 & 25.27550 & 29950 & 29.30078 & 33190 \\ 1400 & 4.49097 & 2197 & 4.05767 & 2963 & 25.57500 & 28098 & 29.63268 & 31061 \\ 1500 & 4.51294 & 1955 & 4.08730 & 2723 & 25.85598 & 26467 & 29.94329 & 29189 \\ 1600 & 4.53249 & 1762 & 4.11453 & 2511 & 26.12065 & 25021 & 30.23518 & 27532 \\ & & & & & & & \\ 1700 & 4.55011 & 1608 & 4.13964 & 2326 & 26.37086 & 23728 & 30.51050 & 26054 \\ 1800 & 4.56619 & 1480 & 4.16290 & 2162 & 26.60814 & 22566 & 30.77104 & 24728 \\ 1900 & 4.58099 & 1377 & 4.18452 & 2017 & 26.83380 & 21516 & 31.01832 & 23533 \\ 2000 & 4.59476 & 1291 & 4.20469 & 1888 & 27.04896 & 20561 & 31.25365 & 22449 \\ 2100 & 4.60767 & 1218 & 4.22357 & 1774 & 27.25457 & 19689 & 31.47814 & 21464 \\ & & & & & & & \\ 2200 & 4.61985 & 1157 & 4.24131 & 1671 & 27.45146 & 18891 & 31.69278 & 20562 \\ 2300 & 4.63142 & 1105 & 4.25802 & 1579 & 27.64037 & 18155 & 31.89840 & 19734 \\ 2400 & 4.64247 & 1060 & 4.27381 & 1496 & 27.82192 & 17478 & 32.09574 & 18973 \\ 2500 & 4.65307 & 1022 & 4.28877 & 1421 & 27.99670 & 16848 & 32.28547 & 18270 \\ 2600 & 4.66329 & 987 & 4.30298 & 1353 & 28.16518 & 16266 & 32.46817 & 17618 \\ & & & & & & & & \\ 2700 & 4.67316 & 959 & 4.31651 & 1291 & 28.32784 & 15721 & 32.64435 & 17013 \\ 2800 & 4.68275 & 932 & 4.32942 & 1235 & 28.48505 & 15214 & 32.81448 & 16448 \\ 2900 & 4.69207 & 910 & 4.34177 & 1182 & 28.63719 & 14740 & 32.97896 & 15923 \\ 3000 & 4.70117 & 1760 & 4.35359 & 2228 & 28.78459 & 28169 & 33.13819 & 30397 \\ & & & & & & & & \\ 3200 & 4.71877 & 1693 & 4.37587 & 2067 & 29.06628 & 26592 & 33.44216 & 28658 \\ 3400 & 4.73570 & 1639 & 4.39654 & 1930 & 29.33220 & 25185 & 33.72874 & 27116 \\ 3600 & 4.75209 & 1593 & 4.41584 & 1812 & 29.58405 & 23924 & 33.99990 & 25736 \\ 3800 & 4.76802 & 1554 & 4.43396 & 1709 & 29.82329 & 22787 & 34.25726 & 24496 \\ 4000 & 4.78356 & 1520 & 4.45105 & 1620 & 30.05116 & 21757 & 34.50222 & 23376 \\ & & & & & & & \\ 4200 & 4.79876 & 1491 & 4.46725 & 1541 & 30.26873 & 20817 & 34.73598 & 22359 \\ 4400 & 4.81367 & 1464 & 4.48266 & 1471 & 30.47690 & 19959 & 34.95957 & 21430 \\ 4600 & 4.82831 & 1441 & 4.49737 & 1409 & 30.67649 & 19171 & 35.17387 & 20579 \\ 4800 & 4.84272 & 1419 & 4.51146 & 1354 & 30.86820 & 18444 & 35.37966 & 19798 \\ 5000 & 4.85691 & & 4.52500 & & 31.05264 & & 35.57764 & \end{array}$


Table 2.066. AlT

\begin{tabular}{|c|c|c|c|c|c|c|c|c|}
\hline${ }^{\circ} \mathrm{K}$ & $\frac{C_{p}^{0}}{R}$ & & $\frac{\left(H^{0}-E_{0}^{0}\right)}{R T}$ & & $\frac{-\left(F^{\circ}-E\right.}{R T}$ & & $\frac{S^{0}}{R}$ & \\
\hline $\begin{array}{l}50 \\
60 \\
70 \\
80 \\
90\end{array}$ & $\begin{array}{l}3.50138 \\
3.50161 \\
3.50185 \\
3.50210 \\
3.50238\end{array}$ & $\begin{array}{l}23 \\
24 \\
25 \\
28 \\
36\end{array}$ & $\begin{array}{l}3.47878 \\
3.48256 \\
3.48530 \\
3.48738 \\
3.48903\end{array}$ & $\begin{array}{l}378 \\
274 \\
208 \\
165 \\
136\end{array}$ & $\begin{array}{l}13.96859 \\
14.60321 \\
15.14026 \\
15.60580 \\
16.01666\end{array}$ & $\begin{array}{l}63462 \\
53705 \\
46554 \\
41086 \\
36767\end{array}$ & $\begin{array}{l}17.44737 \\
18.08577 \\
18.62557 \\
19.09319 \\
19.50569\end{array}$ & $\begin{array}{l}63840 \\
53980 \\
46762 \\
41250 \\
36904\end{array}$ \\
\hline $\begin{array}{l}100 \\
110 \\
120 \\
130 \\
140\end{array}$ & $\begin{array}{l}3.50274 \\
3.50327 \\
3.50414 \\
3.50556 \\
3.50776\end{array}$ & $\begin{array}{r}53 \\
87 \\
142 \\
220 \\
320\end{array}$ & $\begin{array}{l}3.49039 \\
3.49153 \\
3.49254 \\
3.49349 \\
3.49442\end{array}$ & $\begin{array}{r}114 \\
101 \\
95 \\
93 \\
99\end{array}$ & $\begin{array}{l}16.38433 \\
16.71706 \\
17.02091 \\
17.30050 \\
17.55943\end{array}$ & $\begin{array}{l}33273 \\
30385 \\
27959 \\
25893 \\
24112\end{array}$ & $\begin{array}{l}19.87473 \\
20.20860 \\
20.51345 \\
20.79399 \\
21.05385\end{array}$ & $\begin{array}{l}33387 \\
30485 \\
28054 \\
25986 \\
24212\end{array}$ \\
\hline $\begin{array}{l}150 \\
160 \\
170 \\
180 \\
190\end{array}$ & $\begin{array}{l}3.51096 \\
3.51539 \\
3.52120 \\
3.52850 \\
3.53734\end{array}$ & $\begin{array}{r}443 \\
581 \\
730 \\
884 \\
1038\end{array}$ & $\begin{array}{l}3.49541 \\
3.49651 \\
3.49779 \\
3.49929 \\
3.50105\end{array}$ & $\begin{array}{l}110 \\
128 \\
150 \\
176 \\
207\end{array}$ & $\begin{array}{l}17.80055 \\
18.02617 \\
18.23819 \\
18.43816 \\
18.62740\end{array}$ & $\begin{array}{l}22562 \\
21202 \\
19997 \\
18924 \\
17963\end{array}$ & $\begin{array}{l}21.29597 \\
21.52269 \\
21.73598 \\
21.93745 \\
22.12845\end{array}$ & $\begin{array}{l}22672 \\
21329 \\
20147 \\
19100 \\
18170\end{array}$ \\
\hline $\begin{array}{l}200 \\
210 \\
220 \\
230 \\
240\end{array}$ & $\begin{array}{l}3.54772 \\
3.55959 \\
3.57285 \\
3.58738 \\
3.60306\end{array}$ & $\begin{array}{l}1187 \\
1326 \\
1453 \\
1568 \\
1668\end{array}$ & $\begin{array}{l}3.50312 \\
3.50552 \\
3.50827 \\
3.51139 \\
3.51488\end{array}$ & $\begin{array}{l}240 \\
275 \\
312 \\
349 \\
386\end{array}$ & $\begin{array}{l}18.80703 \\
18.97800 \\
19.14114 \\
19.29716 \\
19.44668\end{array}$ & $\begin{array}{l}17097 \\
16314 \\
15602 \\
14952 \\
14356\end{array}$ & $\begin{array}{l}22.31015 \\
22.48353 \\
22.64942 \\
22.80856 \\
22.96156\end{array}$ & $\begin{array}{l}17338 \\
16589 \\
15914 \\
15300 \\
14742\end{array}$ \\
\hline $\begin{array}{l}250 \\
260 \\
270 \\
280 \\
290\end{array}$ & $\begin{array}{l}3.61974 \\
3.63726 \\
3.65548 \\
3.67424 \\
3.69343\end{array}$ & $\begin{array}{l}1752 \\
1822 \\
1876 \\
1919 \\
1947\end{array}$ & $\begin{array}{l}3.51874 \\
3.52296 \\
3.52753 \\
3.53243 \\
3.53765\end{array}$ & $\begin{array}{l}422 \\
457 \\
490 \\
522 \\
552\end{array}$ & $\begin{array}{l}19.59024 \\
19.72833 \\
19.86137 \\
19.98974 \\
20.11379\end{array}$ & $\begin{array}{l}13809 \\
13304 \\
12837 \\
12405 \\
12003\end{array}$ & $\begin{array}{l}23.10898 \\
23.25129 \\
23.38890 \\
23.52218 \\
23.65145\end{array}$ & $\begin{array}{l}14231 \\
13761 \\
13328 \\
12927 \\
12554\end{array}$ \\
\hline $\begin{array}{l}300 \\
310 \\
320 \\
330 \\
340\end{array}$ & $\begin{array}{l}3.71290 \\
3.73255 \\
3.75227 \\
3.77199 \\
3.79160\end{array}$ & $\begin{array}{l}1965 \\
1972 \\
1972 \\
1961 \\
1946\end{array}$ & $\begin{array}{l}3.54317 \\
3.54896 \\
3.55500 \\
3.56128 \\
3.56777\end{array}$ & $\begin{array}{l}579 \\
604 \\
628 \\
649 \\
661\end{array}$ & $\begin{array}{l}20.23382 \\
20.35009 \\
20.46286 \\
20.57235 \\
20.67876\end{array}$ & $\begin{array}{l}11627 \\
11277 \\
10949 \\
10641 \\
10352\end{array}$ & $\begin{array}{l}23.77699 \\
23.89905 \\
24.01787 \\
24.13363 \\
24.24653\end{array}$ & $\begin{array}{l}12206 \\
11882 \\
11576 \\
11290 \\
11019\end{array}$ \\
\hline $\begin{array}{l}350 \\
360 \\
370 \\
380 \\
390\end{array}$ & $\begin{array}{l}3.81106 \\
3.83030 \\
3.84926 \\
3.86792 \\
3.88624\end{array}$ & $\begin{array}{l}1924 \\
1896 \\
1866 \\
1832 \\
1795\end{array}$ & $\begin{array}{l}3.57444 \\
3.58128 \\
3.58827 \\
3.59538 \\
3.60261\end{array}$ & $\begin{array}{l}684 \\
699 \\
711 \\
723 \\
731\end{array}$ & $\begin{array}{l}20.78228 \\
20.88307 \\
20.98129 \\
21.07707 \\
21.17056\end{array}$ & $\begin{array}{r}10079 \\
9822 \\
9578 \\
9349 \\
9130\end{array}$ & $\begin{array}{l}24.35672 \\
24.46435 \\
24.56956 \\
24.67246 \\
24.77317\end{array}$ & $\begin{array}{r}10763 \\
10521 \\
10290 \\
10071 \\
9862\end{array}$ \\
\hline $\begin{array}{l}400 \\
450 \\
500 \\
550 \\
600\end{array}$ & $\begin{array}{l}3.90419 \\
3.98789 \\
4.06105 \\
4.12421 \\
4.17850\end{array}$ & $\begin{array}{l}8370 \\
7316 \\
6316 \\
5429 \\
4670\end{array}$ & $\begin{array}{l}3.60992 \\
3.64736 \\
3.68516 \\
3.72228 \\
3.75809\end{array}$ & $\begin{array}{l}3744 \\
3780 \\
3712 \\
3581 \\
3418\end{array}$ & $\begin{array}{l}21.26186 \\
21.68920 \\
22.07545 \\
22.42843 \\
22.75386\end{array}$ & $\begin{array}{l}42734 \\
38625 \\
35298 \\
32543 \\
30217\end{array}$ & $\begin{array}{l}24.87179 \\
25.33657 \\
25.76061 \\
26.15071 \\
26.51195\end{array}$ & $\begin{array}{l}46478 \\
42404 \\
39010 \\
36124 \\
33635\end{array}$ \\
\hline $\begin{array}{l}650 \\
700 \\
750 \\
800 \\
850\end{array}$ & $\begin{array}{l}4.22520 \\
4.26551 \\
4.30050 \\
4.33105 \\
4.35790\end{array}$ & $\begin{array}{l}4031 \\
3499 \\
3055 \\
2685 \\
2376\end{array}$ & $\begin{array}{l}3.79227 \\
3.82467 \\
3.85525 \\
3.88406 \\
3.91116\end{array}$ & $\begin{array}{l}3240 \\
3058 \\
2881 \\
2710 \\
2549\end{array}$ & $\begin{array}{l}23.05603 \\
23.33826 \\
23.60319 \\
23.85293 \\
24.08922\end{array}$ & $\begin{array}{l}28223 \\
26493 \\
24974 \\
23629 \\
22429\end{array}$ & $\begin{array}{l}26.84830 \\
27.16293 \\
27.45845 \\
27.73699 \\
28.00038\end{array}$ & $\begin{array}{l}31463 \\
29552 \\
27854 \\
26339 \\
24978\end{array}$ \\
\hline $\begin{array}{l}900 \\
950 \\
1000 \\
1050 \\
100\end{array}$ & $\begin{array}{l}4.38166 \\
4.40283 \\
4.42181 \\
4.43895 \\
4.45452\end{array}$ & $\begin{array}{l}2117 \\
1898 \\
1714 \\
1557 \\
1423\end{array}$ & $\begin{array}{l}3.93665 \\
3.96064 \\
3.98323 \\
4.00453 \\
4.02463\end{array}$ & $\begin{array}{l}2399 \\
2259 \\
2130 \\
2010 \\
1901\end{array}$ & $\begin{array}{l}24.31351 \\
24.52700 \\
24.73073 \\
24.92560 \\
25.11236\end{array}$ & $\begin{array}{l}21349 \\
20373 \\
19487 \\
18676 \\
17932\end{array}$ & $\begin{array}{l}28.25016 \\
28.48764 \\
28.71397 \\
28.93013 \\
29.13700\end{array}$ & $\begin{array}{l}23748 \\
22633 \\
21616 \\
20687 \\
19833\end{array}$ \\
\hline 150 & 4.46875 & 1307 & 4.04364 & 1799 & 25.29168 & 17248 & 29.33533 & 19046 \\
\hline
\end{tabular}


Table 2.066. AlT (Cont.)

\begin{tabular}{|l|l|l|l|l|}
\hline$K^{\circ}$ & $\frac{C_{p}^{0}}{R}$ & $\frac{\left(H^{0}-E_{0}^{0}\right)}{R T}$ & $\frac{-\left(F^{0}-E_{0}^{0}\right)}{R T}$ & $\frac{S^{0}}{R}$ \\
\hline
\end{tabular}

$\begin{array}{lllllllll}1200 & 4.48182 & 2329 & 4.06163 & 3324 & 25.46416 & 32644 & 29.52579 & 35968 \\ 1300 & 4.50511 & 2028 & 4.09487 & 3004 & 25.79060 & 30458 & 29.88547 & 33463 \\ 1400 & 4.52539 & 1796 & 4.12491 & 2731 & 26.09518 & 28554 & 30.22010 & 31284 \\ 1500 & 4.54335 & 1616 & 4.15222 & 2496 & 26.38072 & 26878 & 30.53294 & 29375 \\ 1600 & 4.55951 & 1473 & 4.17718 & 2293 & 26.64950 & 25394 & 30.82669 & 27686 \\ & & & & & & & & \\ 1700 & 4.57424 & 1359 & 4.20011 & 2117 & 26.90344 & 24068 & 31.10355 & 26185 \\ 1800 & 4.58783 & 1267 & 4.22128 & 1963 & 27.14412 & 22877 & 31.36540 & 24840 \\ 1900 & 4.60050 & 1191 & 4.24091 & 1828 & 27.37289 & 21800 & 31.61380 & 23628 \\ 2000 & 4.61241 & 1127 & 4.25919 & 1709 & 27.59089 & 20822 & 31.85008 & 22531 \\ 2100 & 4.62368 & 1075 & 4.27628 & 1603 & 27.79911 & 19931 & 32.07539 & 21535 \\ & & & & & & & & \\ 2200 & 4.63443 & 1031 & 4.29231 & 1510 & 27.99842 & 19113 & 32.29074 & 20623 \\ 2300 & 4.64474 & 993 & 4.30741 & 1427 & 28.18955 & 18363 & 32.49697 & 19789 \\ 2400 & 4.65467 & 960 & 4.32168 & 1351 & 28.37318 & 17670 & 32.69486 & 19021 \\ 2500 & 4.66427 & 932 & 4.33519 & 1284 & 28.54988 & 17028 & 32.88507 & 18312 \\ 2600 & 4.67359 & 907 & 4.34803 & 1222 & 28.72016 & 16433 & 33.06819 & 17655 \\ & & & & & & & \\ 2700 & 4.68266 & 885 & 4.36025 & 1168 & 28.88449 & 15878 & 33.24474 & 17046 \\ 2800 & 4.69151 & 867 & 4.37193 & 1117 & 29.04327 & 15361 & 33.41520 & 16478 \\ 2900 & 4.70018 & 850 & 4.38310 & 1071 & 29.19688 & 14878 & 33.57998 & 15949 \\ 3000 & 4.70868 & 1655 & 4.39381 & 2020 & 29.34566 & 28422 & 33.73947 & 30442 \\ & & & & & & & & \\ 3200 & 4.72523 & 1605 & 4.41401 & 1878 & 29.62988 & 26817 & 34.04389 & 28695 \\ 3400 & 4.74128 & 1563 & 4.43279 & 1757 & 29.89805 & 25387 & 34.33084 & 27145 \\ 3600 & 4.75691 & 1529 & 4.45036 & 1654 & 30.15192 & 24107 & 34.60229 & 25761 \\ 3800 & 4.77220 & 1498 & 4.46690 & 1564 & 30.39299 & 22952 & 34.85990 & 24516 \\ 4000 & 4.78718 & 1472 & 4.48254 & 1486 & 30.62251 & 21907 & 35.10506 & 23393 \\ 4200 & 4.80190 & 1448 & 4.49740 & 1417 & 30.84158 & 20955 & 35.33899 & 22372 \\ 4400 & 4.81638 & 1426 & 4.51157 & 1356 & 31.05113 & 20085 & 35.56271 & 21441 \\ 4600 & 4.83064 & 1407 & 4.52513 & 1303 & 31.25198 & 19286 & 35.77712 & 20589 \\ 4800 & 4.84471 & 1389 & 4.53816 & 1254 & 31.44484 & 18552 & 35.98301 & 19805 \\ 5000 & 4.85860 & & 4.55070 & & 31.63036 & & 36.18106 & \end{array}$


Table 2.067. InH

\begin{tabular}{|c|c|c|c|c|c|c|c|c|}
\hline$\stackrel{\circ}{K}$ & $\frac{C_{p}^{0}}{R}$ & & $\frac{\left(H^{0}-E_{0}^{0}\right.}{R T}$ & & $\frac{-\left(F^{0}-E\right.}{R T}$ & & $\frac{S^{\circ}}{R}$ & \\
\hline $\begin{array}{l}50 \\
60 \\
70 \\
80 \\
90\end{array}$ & $\begin{array}{l}3.50158 \\
3.50176 \\
3.50196 \\
3.50218 \\
3.50241\end{array}$ & $\begin{array}{l}18 \\
20 \\
22 \\
23 \\
23\end{array}$ & $\begin{array}{l}3.45304 \\
3.46114 \\
3.46696 \\
3.47135 \\
3.47479\end{array}$ & $\begin{array}{l}810 \\
582 \\
439 \\
344 \\
277\end{array}$ & $\begin{array}{l}15.24193 \\
15.87226 \\
16.40626 \\
16.86950 \\
17.27858\end{array}$ & $\begin{array}{l}63033 \\
53400 \\
46324 \\
40908 \\
36625\end{array}$ & $\begin{array}{l}18.69498 \\
19.33341 \\
19.87322 \\
20.34086 \\
20.75337\end{array}$ & $\begin{array}{l}63843 \\
53981 \\
46764 \\
41251 \\
36903\end{array}$ \\
\hline $\begin{array}{l}100 \\
110 \\
120 \\
130 \\
140\end{array}$ & $\begin{array}{l}3.50264 \\
3.50289 \\
3.50314 \\
3.50341 \\
3.50372\end{array}$ & $\begin{array}{l}25 \\
25 \\
27 \\
31 \\
37\end{array}$ & $\begin{array}{l}3.47756 \\
3.47985 \\
3.48178 \\
3.48344 \\
3.48487\end{array}$ & $\begin{array}{l}229 \\
193 \\
166 \\
143 \\
127\end{array}$ & $\begin{array}{l}17.64483 \\
17.97639 \\
18.27926 \\
18.55802 \\
18.81622\end{array}$ & $\begin{array}{l}33156 \\
30287 \\
27876 \\
25820 \\
24048\end{array}$ & $\begin{array}{l}21.12240 \\
21.45625 \\
21.76105 \\
22.04146 \\
22.30110\end{array}$ & $\begin{array}{l}33385 \\
30480 \\
28041 \\
25964 \\
24175\end{array}$ \\
\hline $\begin{array}{l}150 \\
160 \\
170 \\
180 \\
190\end{array}$ & $\begin{array}{l}3.50409 \\
3.50458 \\
3.50523 \\
3.50612 \\
3.50733\end{array}$ & $\begin{array}{r}49 \\
65 \\
89 \\
121 \\
160\end{array}$ & $\begin{array}{l}3.48614 \\
3.48728 \\
3.48831 \\
3.48928 \\
3.49019\end{array}$ & $\begin{array}{r}114 \\
103 \\
97 \\
91 \\
90\end{array}$ & $\begin{array}{l}19.05670 \\
19.28173 \\
19.49317 \\
19.69259 \\
19.88127\end{array}$ & $\begin{array}{l}22503 \\
21144 \\
19942 \\
18868 \\
17905\end{array}$ & $\begin{array}{l}22.54285 \\
22.76901 \\
22.98149 \\
23.18187 \\
23.37147\end{array}$ & $\begin{array}{l}22616 \\
21248 \\
20038 \\
18960 \\
17994\end{array}$ \\
\hline $\begin{array}{l}200 \\
210 \\
220 \\
230 \\
240\end{array}$ & $\begin{array}{l}3.50893 \\
3.51100 \\
3.51362 \\
3.51685 \\
3.52074\end{array}$ & $\begin{array}{l}207 \\
262 \\
323 \\
389 \\
460\end{array}$ & $\begin{array}{l}3.49109 \\
3.49198 \\
3.49291 \\
3.49387 \\
3.49491\end{array}$ & $\begin{array}{r}89 \\
93 \\
96 \\
104 \\
112\end{array}$ & $\begin{array}{l}20.06032 \\
20.23067 \\
20.39314 \\
20.54842 \\
20.69714\end{array}$ & $\begin{array}{l}17035 \\
16247 \\
15528 \\
14872 \\
14270\end{array}$ & $\begin{array}{l}23.55141 \\
23.72266 \\
23.88605 \\
24.04230 \\
24.19206\end{array}$ & $\begin{array}{l}17125 \\
16339 \\
15625 \\
14976 \\
14381\end{array}$ \\
\hline $\begin{array}{l}250 \\
260 \\
270 \\
280 \\
290\end{array}$ & $\begin{array}{l}3.52534 \\
3.53067 \\
3.53675 \\
3.54358 \\
3.55114\end{array}$ & $\begin{array}{l}533 \\
608 \\
683 \\
756 \\
829\end{array}$ & $\begin{array}{l}3.49603 \\
3.49726 \\
3.49861 \\
3.50009 \\
3.50172\end{array}$ & $\begin{array}{l}123 \\
135 \\
148 \\
163 \\
178\end{array}$ & $\begin{array}{l}20.83984 \\
20.97698 \\
21.10899 \\
21.23625 \\
21.35910\end{array}$ & $\begin{array}{l}13714 \\
13201 \\
12726 \\
12285 \\
11875\end{array}$ & $\begin{array}{l}24.33587 \\
24.47424 \\
24.60760 \\
24.73635 \\
24.86083\end{array}$ & $\begin{array}{l}13837 \\
13336 \\
12875 \\
12448 \\
12052\end{array}$ \\
\hline $\begin{array}{l}300 \\
310 \\
320 \\
330 \\
340\end{array}$ & $\begin{array}{l}3.55943 \\
3.56840 \\
3.57803 \\
3.58827 \\
3.59908\end{array}$ & $\begin{array}{r}897 \\
963 \\
1024 \\
1081 \\
1133\end{array}$ & $\begin{array}{l}3.50350 \\
3.50545 \\
3.50756 \\
3.50985 \\
3.51232\end{array}$ & $\begin{array}{l}195 \\
211 \\
229 \\
247 \\
264\end{array}$ & $\begin{array}{l}21.47785 \\
21.59276 \\
21.70408 \\
21.81205 \\
21.91687\end{array}$ & $\begin{array}{l}11491 \\
11132 \\
10797 \\
10482 \\
10185\end{array}$ & $\begin{array}{l}24.98135 \\
25.09821 \\
25.21165 \\
25.32191 \\
25.42919\end{array}$ & $\begin{array}{l}11686 \\
11344 \\
11026 \\
10728 \\
10449\end{array}$ \\
\hline $\begin{array}{l}350 \\
360 \\
370 \\
380 \\
390\end{array}$ & $\begin{array}{l}3.61041 \\
3.62221 \\
3.63442 \\
3.64701 \\
3.65991\end{array}$ & $\begin{array}{l}1180 \\
1221 \\
1259 \\
1290 \\
1318\end{array}$ & $\begin{array}{l}3.51496 \\
3.51777 \\
3.52076 \\
3.52391 \\
3.52724\end{array}$ & $\begin{array}{l}281 \\
299 \\
315 \\
333 \\
348\end{array}$ & $\begin{array}{l}22.01872 \\
22.11778 \\
22.21420 \\
22.30813 \\
22.39971\end{array}$ & $\begin{array}{l}9906 \\
9642 \\
9393 \\
9158 \\
8935\end{array}$ & $\begin{array}{l}25.53368 \\
25.63555 \\
25.73496 \\
25.83205 \\
25.92695\end{array}$ & $\begin{array}{r}10187 \\
9941 \\
9709 \\
9490 \\
9283\end{array}$ \\
\hline $\begin{array}{l}400 \\
450 \\
500 \\
550 \\
600\end{array}$ & $\begin{array}{l}3.67309 \\
3.74156 \\
3.81105 \\
3.87840 \\
3.94182\end{array}$ & $\begin{array}{l}6847 \\
6949 \\
6735 \\
6342 \\
5862\end{array}$ & $\begin{array}{l}3.53072 \\
3.55031 \\
3.57292 \\
3.59765 \\
3.62372\end{array}$ & $\begin{array}{l}1959 \\
2261 \\
2473 \\
2607 \\
2676\end{array}$ & $\begin{array}{l}22.48906 \\
22.90601 \\
23.28123 \\
23.62291 \\
23.93706\end{array}$ & $\begin{array}{l}41695 \\
37522 \\
34168 \\
31415 \\
29110\end{array}$ & $\begin{array}{l}26.01978 \\
26.45633 \\
26.85415 \\
27.22056 \\
27.56078\end{array}$ & $\begin{array}{l}43655 \\
39782 \\
36641 \\
34022 \\
31786\end{array}$ \\
\hline $\begin{array}{l}650 \\
700 \\
750 \\
800 \\
850\end{array}$ & $\begin{array}{l}4.00044 \\
4.05403 \\
4.10270 \\
4.14678 \\
4.18664\end{array}$ & $\begin{array}{l}5359 \\
4867 \\
4408 \\
3986 \\
3608\end{array}$ & $\begin{array}{l}3.65048 \\
3.67742 \\
3.70417 \\
3.73048 \\
3.75616\end{array}$ & $\begin{array}{l}2694 \\
2675 \\
2631 \\
2568 \\
2494\end{array}$ & $\begin{array}{l}24.22816 \\
24.49968 \\
24.75431 \\
24.99421 \\
25.22115\end{array}$ & $\begin{array}{l}27152 \\
25463 \\
23990 \\
22694 \\
21540\end{array}$ & $\begin{array}{l}27.87864 \\
28.17710 \\
28.45849 \\
28.72470 \\
28.97731\end{array}$ & $\begin{array}{l}29846 \\
28139 \\
26621 \\
25261 \\
24034\end{array}$ \\
\hline $\begin{array}{l}900 \\
950 \\
000 \\
050 \\
100\end{array}$ & $\begin{array}{l}4.22272 \\
4.25542 \\
4.28513 \\
4.31220 \\
4.33693\end{array}$ & $\begin{array}{l}3270 \\
2971 \\
2707 \\
2473 \\
2268\end{array}$ & $\begin{array}{l}3.78110 \\
3.80521 \\
3.82848 \\
3.85088 \\
3.87242\end{array}$ & $\begin{array}{l}2411 \\
2327 \\
2240 \\
2154 \\
2069\end{array}$ & $\begin{array}{l}25.43655 \\
25.64164 \\
25.83741 \\
26.02475 \\
26.20439\end{array}$ & $\begin{array}{l}20509 \\
19577 \\
18734 \\
17964 \\
17260\end{array}$ & $\begin{array}{l}29.21765 \\
29.44686 \\
29.66590 \\
29.87563 \\
30.07682\end{array}$ & $\begin{array}{l}22921 \\
21904 \\
20973 \\
20119 \\
19329\end{array}$ \\
\hline 50 & 4.35961 & 2086 & 3.89311 & 1988 & 26.37699 & 16611 & 30.27011 & 18599 \\
\hline
\end{tabular}


Table 2.067. InH (Cont.)

\begin{tabular}{|c|c|c|c|c|c|c|c|c|}
\hline${ }^{\circ} \mathrm{K}$ & $\frac{C_{p}^{0}}{R}$ & & $\frac{\left(H^{0}-E_{0}^{0}\right)}{R T}$ & & $\frac{-\left(F^{\circ}-E\right.}{R T}$ & & $\frac{S^{\circ}}{R}$ & \\
\hline $\begin{array}{l}1200 \\
1300 \\
1400 \\
1500 \\
1600\end{array}$ & $\begin{array}{l}4.38047 \\
4.41756 \\
4.44959 \\
4.47761 \\
4.50244\end{array}$ & $\begin{array}{l}3709 \\
3203 \\
2802 \\
2483 \\
2226\end{array}$ & $\begin{array}{l}3.91299 \\
3.95041 \\
3.98495 \\
4.01688 \\
4.04647\end{array}$ & $\begin{array}{l}3742 \\
3454 \\
3193 \\
2959 \\
2749\end{array}$ & $\begin{array}{l}26.54310 \\
26.85781 \\
27.15185 \\
27.42788 \\
27.68808\end{array}$ & $\begin{array}{l}31471 \\
29404 \\
27603 \\
26020 \\
24615\end{array}$ & $\begin{array}{l}30.45610 \\
30.80823 \\
31.13680 \\
31.44477 \\
31.73456\end{array}$ & $\begin{array}{l}35213 \\
32857 \\
30797 \\
28979 \\
27363\end{array}$ \\
\hline $\begin{array}{l}1700 \\
1800 \\
1900 \\
2000 \\
2100\end{array}$ & $\begin{array}{l}4.52470 \\
4.54485 \\
4.56328 \\
4.58029 \\
4.59610\end{array}$ & $\begin{array}{l}2015 \\
1843 \\
1701 \\
1581 \\
1481\end{array}$ & $\begin{array}{l}4.07396 \\
4.09957 \\
4.12349 \\
4.14591 \\
4.16698\end{array}$ & $\begin{array}{l}2561 \\
2392 \\
2242 \\
2107 \\
1984\end{array}$ & $\begin{array}{l}27.93423 \\
28.16783 \\
28.39013 \\
28.60221 \\
28.80501\end{array}$ & $\begin{array}{l}23360 \\
22230 \\
21208 \\
20280 \\
19431\end{array}$ & $\begin{array}{l}32.00819 \\
32.26740 \\
32.51363 \\
32.74813 \\
32.97199\end{array}$ & $\begin{array}{l}25921 \\
24623 \\
23450 \\
22386 \\
21416\end{array}$ \\
\hline $\begin{array}{l}2200 \\
2300 \\
2400 \\
2500 \\
2600\end{array}$ & $\begin{array}{l}4.61091 \\
4.62487 \\
4.63811 \\
4.65072 \\
4.66278\end{array}$ & $\begin{array}{l}1396 \\
1324 \\
1261 \\
1206 \\
1160\end{array}$ & $\begin{array}{l}4.18682 \\
4.20557 \\
4.22332 \\
4.24016 \\
4.25619\end{array}$ & $\begin{array}{l}1875 \\
1775 \\
1684 \\
1603 \\
1527\end{array}$ & $\begin{array}{l}28.99932 \\
29.18585 \\
29.36521 \\
29.53796 \\
29.70458\end{array}$ & $\begin{array}{l}18653 \\
17936 \\
17275 \\
16662 \\
16092\end{array}$ & $\begin{array}{l}33.18615 \\
33.39142 \\
33.58854 \\
33.77813 \\
33.96077\end{array}$ & $\begin{array}{l}20527 \\
19712 \\
18959 \\
18264 \\
17620\end{array}$ \\
\hline $\begin{array}{l}2700 \\
2800 \\
2900 \\
3000\end{array}$ & $\begin{array}{l}4.67438 \\
4.68557 \\
4.69639 \\
4.70688\end{array}$ & $\begin{array}{l}1119 \\
1082 \\
1049 \\
2017\end{array}$ & $\begin{array}{l}4.27146 \\
4.28605 \\
4.30002 \\
4.31341\end{array}$ & $\begin{array}{l}1459 \\
1397 \\
1339 \\
2522\end{array}$ & $\begin{array}{l}29.86550 \\
30.02111 \\
30.17176 \\
30.31776\end{array}$ & $\begin{array}{l}15561 \\
15065 \\
14600 \\
27920\end{array}$ & $\begin{array}{l}34.13697 \\
34.30717 \\
34.47178 \\
34.63117\end{array}$ & $\begin{array}{l}17020 \\
16461 \\
15939 \\
30443\end{array}$ \\
\hline $\begin{array}{l}3200 \\
3400 \\
3600 \\
3800 \\
4000\end{array}$ & $\begin{array}{l}4.72705 \\
4.74629 \\
4.76477 \\
4.78262 \\
4.79994\end{array}$ & $\begin{array}{l}1924 \\
1848 \\
1785 \\
1732 \\
1685\end{array}$ & $\begin{array}{l}4.33863 \\
4.36205 \\
4.38392 \\
4.40443 \\
4.42378\end{array}$ & $\begin{array}{l}2342 \\
2187 \\
2051 \\
1935 \\
1831\end{array}$ & $\begin{array}{l}30.59696 \\
30.86070 \\
31.11065 \\
31.34823 \\
31.57465\end{array}$ & $\begin{array}{l}26374 \\
24995 \\
23758 \\
22642 \\
21628 \\
\end{array}$ & $\begin{array}{l}34.93560 \\
35.22275 \\
35.49457 \\
35.75267 \\
35.99843\end{array}$ & $\begin{array}{l}28715 \\
27182 \\
25810 \\
24576 \\
23460\end{array}$ \\
\hline $\begin{array}{l}4200 \\
4400 \\
4600 \\
4800 \\
5000\end{array}$ & $\begin{array}{l}4.81679 \\
4.83325 \\
4.84935 \\
4.86514 \\
4.88065\end{array}$ & $\begin{array}{l}1646 \\
1610 \\
1579 \\
1551\end{array}$ & $\begin{array}{l}4.44209 \\
4.45950 \\
4.47610 \\
4.49198 \\
4.50722\end{array}$ & $\begin{array}{l}1741 \\
1660 \\
1588 \\
1524\end{array}$ & $\begin{array}{l}31.79093 \\
31.99798 \\
32.19659 \\
32.38742 \\
32.57111\end{array}$ & $\begin{array}{l}20705 \\
19861 \\
19083 \\
18369\end{array}$ & $\begin{array}{l}36.23303 \\
36.45749 \\
36.67269 \\
36.87941 \\
37.07833\end{array}$ & $\begin{array}{l}22446 \\
21520 \\
20672 \\
19892\end{array}$ \\
\hline
\end{tabular}


Table 2. 068. InD

\begin{tabular}{|c|c|c|c|c|c|c|c|c|}
\hline$\stackrel{\circ}{K}$ & $\frac{C_{p}^{0}}{R}$ & & $\frac{\left(H^{\circ}-E\right.}{R T}$ & & $\frac{-l F^{\circ}-}{R T}$ & & $\frac{S^{0}}{R}$ & \\
\hline $\begin{array}{l}50 \\
60 \\
70 \\
80 \\
90\end{array}$ & $\begin{array}{l}3.50137 \\
3.50160 \\
3.50184 \\
3.50208 \\
3.50235\end{array}$ & $\begin{array}{l}23 \\
24 \\
24 \\
27 \\
32\end{array}$ & $\begin{array}{l}3.47660 \\
3.48074 \\
3.48374 \\
3.48602 \\
3.48782\end{array}$ & $\begin{array}{l}414 \\
300 \\
228 \\
180 \\
147\end{array}$ & $\begin{array}{l}15.91570 \\
16.54995 \\
17.08675 \\
17.55209 \\
17.96279\end{array}$ & $\begin{array}{l}63425 \\
53680 \\
46534 \\
41070 \\
36756\end{array}$ & $\begin{array}{l}19.39230 \\
20.03070 \\
20.57049 \\
21.03811 \\
21.45061\end{array}$ & $\begin{array}{l}63840 \\
53979 \\
46762 \\
41250 \\
36903\end{array}$ \\
\hline $\begin{array}{l}100 \\
110 \\
120 \\
130 \\
140\end{array}$ & $\begin{array}{l}3.50267 \\
3.50311 \\
3.50380 \\
3.50491 \\
3.50665\end{array}$ & $\begin{array}{r}44 \\
69 \\
111 \\
174 \\
257\end{array}$ & $\begin{array}{l}3.48929 \\
3.49052 \\
3.49160 \\
3.49257 \\
3.49351\end{array}$ & $\begin{array}{r}123 \\
108 \\
97 \\
94 \\
96\end{array}$ & $\begin{array}{l}18.33035 \\
18.66297 \\
18.96674 \\
19.24625 \\
19.50511\end{array}$ & $\begin{array}{l}33262 \\
30377 \\
27951 \\
25886 \\
24106\end{array}$ & $\begin{array}{l}21.81964 \\
22.15350 \\
22.45834 \\
22.73883 \\
22.99863\end{array}$ & $\begin{array}{l}33386 \\
30484 \\
28049 \\
25980 \\
24202\end{array}$ \\
\hline $\begin{array}{l}150 \\
160 \\
170 \\
180 \\
190\end{array}$ & $\begin{array}{l}3.50922 \\
3.51283 \\
3.51765 \\
3.52380 \\
3.53137\end{array}$ & $\begin{array}{l}361 \\
482 \\
615 \\
757 \\
900\end{array}$ & $\begin{array}{l}3.49447 \\
3.49550 \\
3.49665 \\
3.49799 \\
3.49954\end{array}$ & $\begin{array}{l}103 \\
115 \\
134 \\
155 \\
181\end{array}$ & $\begin{array}{l}19.74617 \\
19.97174 \\
20.18368 \\
20.38358 \\
20.57275\end{array}$ & $\begin{array}{l}22557 \\
21194 \\
19990 \\
18917 \\
17955\end{array}$ & $\begin{array}{l}23.24065 \\
23.46724 \\
23.68034 \\
23.88157 \\
24.07229\end{array}$ & $\begin{array}{l}22659 \\
21310 \\
20123 \\
19072 \\
18136\end{array}$ \\
\hline $\begin{array}{l}200 \\
210 \\
220 \\
230 \\
240\end{array}$ & $\begin{array}{l}3.54037 \\
3.55080 \\
3.56259 \\
3.57567 \\
3.58992\end{array}$ & $\begin{array}{l}1043 \\
1179 \\
1308 \\
1425 \\
1529\end{array}$ & $\begin{array}{l}3.50135 \\
3.50345 \\
3.50586 \\
3.50861 \\
3.51170\end{array}$ & $\begin{array}{l}210 \\
241 \\
275 \\
309 \\
343\end{array}$ & $\begin{array}{l}20.75230 \\
20.92318 \\
21.08621 \\
21.24212 \\
21.39151\end{array}$ & $\begin{array}{l}17088 \\
16303 \\
15591 \\
14939 \\
14342\end{array}$ & $\begin{array}{l}24.25365 \\
24.42663 \\
24.59208 \\
24.75073 \\
24.90321\end{array}$ & $\begin{array}{l}17298 \\
16545 \\
15865 \\
15248 \\
14685\end{array}$ \\
\hline $\begin{array}{l}250 \\
260 \\
270 \\
280 \\
290\end{array}$ & $\begin{array}{l}3.60521 \\
3.62142 \\
3.63841 \\
3.65604 \\
3.67419\end{array}$ & $\begin{array}{l}1621 \\
1699 \\
1763 \\
1815 \\
1854\end{array}$ & $\begin{array}{l}3.51513 \\
3.51890 \\
3.52301 \\
3.52745 \\
3.53219\end{array}$ & $\begin{array}{l}377 \\
411 \\
444 \\
474 \\
504\end{array}$ & $\begin{array}{l}21.53493 \\
21.67287 \\
21.80575 \\
21.93395 \\
22.05782\end{array}$ & $\begin{array}{l}13794 \\
13288 \\
12820 \\
12387 \\
11983\end{array}$ & $\begin{array}{l}25.05006 \\
25.19177 \\
25.32876 \\
25.46140 \\
25.59001\end{array}$ & $\begin{array}{l}14171 \\
13699 \\
13264 \\
12861 \\
12487\end{array}$ \\
\hline $\begin{array}{l}300 \\
310 \\
320 \\
330 \\
340\end{array}$ & $\begin{array}{l}3.69273 \\
3.71156 \\
3.73056 \\
3.74965 \\
3.76875\end{array}$ & $\begin{array}{l}1883 \\
1900 \\
1909 \\
1910 \\
1902\end{array}$ & $\begin{array}{l}3.53723 \\
3.54255 \\
3.54813 \\
3.55395 \\
3.55998\end{array}$ & $\begin{array}{l}532 \\
558 \\
582 \\
603 \\
624\end{array}$ & $\begin{array}{l}22.17765 \\
22.29372 \\
22.40628 \\
22.51555 \\
22.62173\end{array}$ & $\begin{array}{l}11607 \\
11256 \\
10927 \\
10618 \\
10329\end{array}$ & $\begin{array}{l}25.71488 \\
25.83628 \\
25.95441 \\
26.06950 \\
26.18172\end{array}$ & $\begin{array}{l}12140 \\
11813 \\
11509 \\
11222 \\
10952\end{array}$ \\
\hline $\begin{array}{l}350 \\
360 \\
370 \\
380 \\
390\end{array}$ & $\begin{array}{l}3.78777 \\
3.80666 \\
3.82537 \\
3.84384 \\
3.86204\end{array}$ & $\begin{array}{l}1889 \\
1871 \\
1847 \\
1820 \\
1789\end{array}$ & $\begin{array}{l}3.56622 \\
3.57264 \\
3.57922 \\
3.58594 \\
3.59278\end{array}$ & $\begin{array}{l}642 \\
658 \\
672 \\
684 \\
696\end{array}$ & $\begin{array}{l}22.72502 \\
22.82557 \\
22.92355 \\
23.01909 \\
23.11232\end{array}$ & $\begin{array}{r}10055 \\
9798 \\
9554 \\
9323 \\
9105\end{array}$ & $\begin{array}{l}26.29124 \\
26.39821 \\
26.50277 \\
26.60503 \\
26.70511\end{array}$ & $\begin{array}{r}10697 \\
10456 \\
10226 \\
10008 \\
9801\end{array}$ \\
\hline $\begin{array}{l}400 \\
450 \\
500 \\
550 \\
600\end{array}$ & $\begin{array}{l}3.87993 \\
3.96410 \\
4.03860 \\
4.10353 \\
4.15977\end{array}$ & $\begin{array}{l}8417 \\
7450 \\
6493 \\
5624 \\
4867\end{array}$ & $\begin{array}{l}3.59974 \\
3.63563 \\
3.67229 \\
3.70861 \\
3.74392\end{array}$ & $\begin{array}{l}3589 \\
3666 \\
3632 \\
3531 \\
3391\end{array}$ & $\begin{array}{l}23.20337 \\
23.62942 \\
24.01437 \\
24.36608 \\
24.69030\end{array}$ & $\begin{array}{l}42605 \\
38495 \\
35171 \\
32422 \\
30102\end{array}$ & $\begin{array}{l}26.80312 \\
27.26506 \\
27.68666 \\
28.07470 \\
28.43422\end{array}$ & $\begin{array}{l}46194 \\
42160 \\
38804 \\
35952 \\
33493\end{array}$ \\
\hline $\begin{array}{l}650 \\
700 \\
750 \\
800 \\
850\end{array}$ & $\begin{array}{l}4.20844 \\
4.25066 \\
4.28745 \\
4.31968 \\
4.34808\end{array}$ & $\begin{array}{l}4222 \\
3679 \\
3223 \\
2840 \\
2520\end{array}$ & $\begin{array}{l}3.77783 \\
3.81013 \\
3.84075 \\
3.86970 \\
3.89702\end{array}$ & $\begin{array}{l}3230 \\
3062 \\
2895 \\
2732 \\
2577\end{array}$ & $\begin{array}{l}24.99132 \\
25.27248 \\
25.53641 \\
25.78522 \\
26.02064\end{array}$ & $\begin{array}{l}28116 \\
26393 \\
24881 \\
23542 \\
22349\end{array}$ & $\begin{array}{l}28.76915 \\
29.08261 \\
29.37716 \\
29.65492 \\
29.91767\end{array}$ & $\begin{array}{l}31346 \\
29455 \\
27776 \\
26275 \\
24925\end{array}$ \\
\hline $\begin{array}{l}900 \\
950 \\
1000 \\
1050 \\
100\end{array}$ & $\begin{array}{l}4.37328 \\
4.39577 \\
4.41597 \\
4.43424 \\
4.45084\end{array}$ & $\begin{array}{l}2249 \\
2020 \\
1827 \\
1660 \\
1519\end{array}$ & $\begin{array}{l}3.92279 \\
3.94710 \\
3.97005 \\
3.99173 \\
4.01223\end{array}$ & $\begin{array}{l}2431 \\
2295 \\
2168 \\
2050 \\
1940\end{array}$ & $\begin{array}{l}26.24413 \\
26.45688 \\
26.65993 \\
26.85416 \\
27.04033\end{array}$ & $\begin{array}{l}21275 \\
20305 \\
19423 \\
18617 \\
17878\end{array}$ & $\begin{array}{l}30.16692 \\
30.40399 \\
30.62999 \\
30.84589 \\
31.05256\end{array}$ & $\begin{array}{l}23707 \\
22600 \\
21590 \\
20667 \\
19819\end{array}$ \\
\hline 150 & 4.46603 & 1397 & 4.03163 & 1840 & 27.21911 & 17198 & 31.25075 & 19037 \\
\hline
\end{tabular}


Table 2. 068. InD (Cont.)

\begin{tabular}{|c|c|c|c|c|c|c|c|c|}
\hline${ }^{\circ} \mathrm{K}$ & $\frac{C_{p}^{0}}{R}$ & & $\frac{\left(H^{0}-E_{0}^{0}\right)}{R T}$ & & $\frac{-\left(F^{0}-E_{0}^{0}\right)}{R T}$ & & $\frac{S^{0}}{R}$ & \\
\hline $\begin{array}{l}1200 \\
1300 \\
1400 \\
1500 \\
1600\end{array}$ & $\begin{array}{l}4.48000 \\
4.50491 \\
4.52662 \\
4.54585 \\
4.56316\end{array}$ & $\begin{array}{l}2491 \\
2171 \\
1923 \\
1731 \\
1578\end{array}$ & $\begin{array}{l}4.05003 \\
4.08408 \\
4.11493 \\
4.14303 \\
4.16876\end{array}$ & $\begin{array}{l}3405 \\
3085 \\
2810 \\
2573 \\
2367\end{array}$ & $\begin{array}{l}27.39109 \\
27.71663 \\
28.02044 \\
28.30532 \\
28.57354\end{array}$ & $\begin{array}{l}32554 \\
30381 \\
28488 \\
26822 \\
25345\end{array}$ & $\begin{array}{l}31.44112 \\
31.80072 \\
32.13538 \\
32.44836 \\
32.74230\end{array}$ & $\begin{array}{l}35960 \\
33466 \\
31298 \\
29394 \\
27712\end{array}$ \\
\hline $\begin{array}{l}1700 \\
1800 \\
1900 \\
2000 \\
2100\end{array}$ & $\begin{array}{l}4.57894 \\
4.59349 \\
4.60705 \\
4.61980 \\
4.63186\end{array}$ & $\begin{array}{l}1455 \\
1356 \\
1275 \\
1206 \\
1150\end{array}$ & $\begin{array}{l}4.19243 \\
4.21431 \\
4.23463 \\
4.25357 \\
4.27130\end{array}$ & $\begin{array}{l}2188 \\
2032 \\
1894 \\
1773 \\
1665\end{array}$ & $\begin{array}{l}28.82699 \\
29.06725 \\
29.29565 \\
29.51335 \\
29.72132\end{array}$ & $\begin{array}{l}24026 \\
22840 \\
21770 \\
20797 \\
19909\end{array}$ & $\begin{array}{l}33.01942 \\
33.28156 \\
33.53029 \\
33.76693 \\
33.99262\end{array}$ & $\begin{array}{l}26214 \\
24873 \\
23664 \\
22569 \\
21574\end{array}$ \\
\hline $\begin{array}{l}2200 \\
2300 \\
2400 \\
2500 \\
2600\end{array}$ & $\begin{array}{l}4.64336 \\
4.65438 \\
4.66499 \\
4.67524 \\
4.68519\end{array}$ & $\begin{array}{r}1102 \\
1061 \\
1025 \\
995 \\
968\end{array}$ & $\begin{array}{l}4.28795 \\
4.30365 \\
4.31848 \\
4.33255 \\
4.34592\end{array}$ & $\begin{array}{l}1570 \\
1483 \\
1407 \\
1337 \\
1275\end{array}$ & $\begin{array}{l}29.92041 \\
30.11136 \\
30.29484 \\
30.47142 \\
30.64161\end{array}$ & $\begin{array}{l}19095 \\
18348 \\
17658 \\
17019 \\
16425\end{array}$ & $\begin{array}{l}34.20836 \\
34.41501 \\
34.61333 \\
34.80397 \\
34.98753\end{array}$ & $\begin{array}{l}20665 \\
19832 \\
19064 \\
18356 \\
17701\end{array}$ \\
\hline $\begin{array}{l}2700 \\
2800 \\
2900 \\
3000\end{array}$ & $\begin{array}{l}4.69487 \\
4.70432 \\
4.71356 \\
4.72262\end{array}$ & $\begin{array}{r}945 \\
924 \\
906 \\
1763\end{array}$ & $\begin{array}{l}4.35867 \\
4.37084 \\
4.38250 \\
4.39369\end{array}$ & $\begin{array}{l}1217 \\
1166 \\
1119 \\
2111\end{array}$ & $\begin{array}{l}30.80586 \\
30.96460 \\
31.11818 \\
31.26695\end{array}$ & $\begin{array}{l}15874 \\
15358 \\
14877 \\
28424\end{array}$ & $\begin{array}{l}35.16454 \\
35.33545 \\
35.50069 \\
35.66064\end{array}$ & $\begin{array}{l}17091 \\
16524 \\
15995 \\
30536\end{array}$ \\
\hline $\begin{array}{l}3200 \\
3400 \\
3600 \\
3800 \\
4000\end{array}$ & $\begin{array}{l}4.74025 \\
4.75733 \\
4.77396 \\
4.79020 \\
4.80611\end{array}$ & $\begin{array}{l}1708 \\
1663 \\
1624 \\
1591 \\
1561\end{array}$ & $\begin{array}{l}4.41480 \\
4.43445 \\
4.45285 \\
4.47018 \\
4.48658\end{array}$ & $\begin{array}{l}1965 \\
1840 \\
1733 \\
1640 \\
1559\end{array}$ & $\begin{array}{l}31.55119 \\
31.81943 \\
32.07343 \\
32.31465 \\
32.54436\end{array}$ & $\begin{array}{l}26824 \\
25400 \\
24122 \\
22971 \\
21928\end{array}$ & $\begin{array}{l}35.96600 \\
36.25389 \\
36.52628 \\
36.78484 \\
37.03095\end{array}$ & $\begin{array}{l}28789 \\
27239 \\
25856 \\
24611 \\
23487\end{array}$ \\
\hline $\begin{array}{l}4200 \\
4400 \\
4600 \\
4800 \\
5000\end{array}$ & $\begin{array}{l}4.82172 \\
4.83708 \\
4.85219 \\
4.86709 \\
4.88179\end{array}$ & $\begin{array}{l}1536 \\
1511 \\
1490 \\
1470\end{array}$ & $\begin{array}{l}4.50217 \\
4.51704 \\
4.53129 \\
4.54497 \\
4.55815\end{array}$ & $\begin{array}{l}1487 \\
1425 \\
1368 \\
1318\end{array}$ & $\begin{array}{l}32.76364 \\
32.97343 \\
33.17454 \\
33.36768 \\
33.55348\end{array}$ & $\begin{array}{l}20979 \\
20111 \\
19314 \\
18580\end{array}$ & $\begin{array}{l}37.26582 \\
37.49048 \\
37.70583 \\
37.91265 \\
38.11164\end{array}$ & $\begin{array}{l}22466 \\
21535 \\
20682 \\
19899\end{array}$ \\
\hline
\end{tabular}


Table 2.069. InT

\begin{tabular}{|c|c|c|c|c|c|c|c|c|}
\hline${ }^{\circ} \mathrm{K}$ & $\frac{C_{p}^{0}}{R}$ & & $\frac{\left(H^{0}-E_{0}^{0}\right)}{R T}$ & & $\frac{-l F^{0}-E}{R T}$ & & $\frac{S^{0}}{R}$ & \\
\hline $\begin{array}{l}50 \\
60 \\
70 \\
80 \\
90\end{array}$ & $\begin{array}{l}3.50132 \\
3.50156 \\
3.50182 \\
3.50212 \\
3.50257\end{array}$ & $\begin{array}{l}24 \\
26 \\
30 \\
45 \\
80\end{array}$ & $\begin{array}{l}3.48444 \\
3.48727 \\
3.48933 \\
3.49091 \\
3.49218\end{array}$ & $\begin{array}{l}283 \\
206 \\
158 \\
127 \\
107\end{array}$ & $\begin{array}{l}16.31289 \\
16.94844 \\
17.48617 \\
17.95221 \\
18.36346\end{array}$ & $\begin{array}{l}63555 \\
53773 \\
46604 \\
41125 \\
36799\end{array}$ & $\begin{array}{l}19.79733 \\
20.43572 \\
20.97551 \\
21.44313 \\
21.85564\end{array}$ & $\begin{array}{l}63839 \\
53979 \\
46762 \\
41251 \\
36907\end{array}$ \\
\hline $\begin{array}{l}100 \\
110 \\
120 \\
130 \\
140\end{array}$ & $\begin{array}{l}3.50337 \\
3.50483 \\
3.50731 \\
3.51119 \\
3.51679\end{array}$ & $\begin{array}{l}146 \\
248 \\
388 \\
560 \\
755\end{array}$ & $\begin{array}{l}3.49325 \\
3.49423 \\
3.49521 \\
3.49628 \\
3.49754\end{array}$ & $\begin{array}{r}98 \\
98 \\
107 \\
126 \\
152\end{array}$ & $\begin{array}{l}18.73145 \\
19.06444 \\
19.36852 \\
19.64833 \\
19.90748\end{array}$ & $\begin{array}{l}33299 \\
30408 \\
27981 \\
25915 \\
24135\end{array}$ & $\begin{array}{l}22.22471 \\
22.55868 \\
22.86374 \\
23.14462 \\
23.40502\end{array}$ & $\begin{array}{l}33397 \\
30506 \\
28088 \\
26040 \\
24288\end{array}$ \\
\hline $\begin{array}{l}150 \\
160 \\
170 \\
180 \\
190\end{array}$ & $\begin{array}{l}3.52434 \\
3.53398 \\
3.54572 \\
3.55951 \\
3.57520\end{array}$ & $\begin{array}{r}964 \\
1174 \\
1379 \\
1569 \\
1739\end{array}$ & $\begin{array}{l}3.49906 \\
3.50093 \\
3.50321 \\
3.50595 \\
3.50917\end{array}$ & $\begin{array}{l}187 \\
228 \\
274 \\
322 \\
373\end{array}$ & $\begin{array}{l}20.14883 \\
20.37472 \\
20.58702 \\
20.78734 \\
20.97698\end{array}$ & $\begin{array}{l}22589 \\
21230 \\
20032 \\
18964 \\
18009\end{array}$ & $\begin{array}{l}23.64790 \\
23.87565 \\
24.09024 \\
24.29329 \\
24.48615\end{array}$ & $\begin{array}{l}22775 \\
21459 \\
20305 \\
19286 \\
18382\end{array}$ \\
\hline $\begin{array}{l}200 \\
210 \\
220 \\
230 \\
240\end{array}$ & $\begin{array}{l}3.59259 \\
3.61147 \\
3.63158 \\
3.65270 \\
3.67459\end{array}$ & $\begin{array}{l}1888 \\
2011 \\
2112 \\
2189 \\
2244\end{array}$ & $\begin{array}{l}3.51290 \\
3.51714 \\
3.52188 \\
3.52710 \\
3.53279\end{array}$ & $\begin{array}{l}424 \\
474 \\
522 \\
569 \\
612\end{array}$ & $\begin{array}{l}21.15707 \\
21.32856 \\
21.49229 \\
21.64896 \\
21.79919\end{array}$ & $\begin{array}{l}17149 \\
16373 \\
15667 \\
15023 \\
14434\end{array}$ & $\begin{array}{l}24.66997 \\
24.84571 \\
25.01417 \\
25.17607 \\
25.33198\end{array}$ & $\begin{array}{l}17574 \\
16846 \\
16190 \\
15591 \\
15046\end{array}$ \\
\hline $\begin{array}{l}250 \\
260 \\
270 \\
280 \\
290\end{array}$ & $\begin{array}{l}3.69703 \\
3.71983 \\
3.74281 \\
3.76582 \\
3.78872\end{array}$ & $\begin{array}{l}2280 \\
2298 \\
2301 \\
2290 \\
2268\end{array}$ & $\begin{array}{l}3.53891 \\
3.54543 \\
3.55231 \\
3.55953 \\
3.56704\end{array}$ & $\begin{array}{l}652 \\
688 \\
722 \\
751 \\
777\end{array}$ & $\begin{array}{l}21.94353 \\
22.08245 \\
22.21638 \\
22.34570 \\
22.47074\end{array}$ & $\begin{array}{l}13892 \\
13393 \\
12932 \\
12504 \\
12106\end{array}$ & $\begin{array}{l}25.48244 \\
25.62789 \\
25.76870 \\
25.90524 \\
26.03778\end{array}$ & $\begin{array}{l}14545 \\
14081 \\
13654 \\
13254 \\
12883\end{array}$ \\
\hline $\begin{array}{l}300 \\
310 \\
320 \\
330 \\
340\end{array}$ & $\begin{array}{l}3.81140 \\
3.83377 \\
3.85576 \\
3.87731 \\
3.89836\end{array}$ & $\begin{array}{l}2237 \\
2199 \\
2155 \\
2105 \\
2053\end{array}$ & $\begin{array}{l}3.57481 \\
3.58280 \\
3.59099 \\
3.59934 \\
3.60782\end{array}$ & $\begin{array}{l}799 \\
819 \\
835 \\
848 \\
860\end{array}$ & $\begin{array}{l}22.59180 \\
22.70915 \\
22.82303 \\
22.93365 \\
23.04123\end{array}$ & $\begin{array}{l}11735 \\
11388 \\
11062 \\
10758 \\
10471\end{array}$ & $\begin{array}{l}26.16661 \\
26.29195 \\
26.41402 \\
26.53300 \\
26.64906\end{array}$ & $\begin{array}{l}12534 \\
12207 \\
11898 \\
11606 \\
11330\end{array}$ \\
\hline $\begin{array}{l}350 \\
360 \\
370 \\
380 \\
390\end{array}$ & $\begin{array}{l}3.91889 \\
3.93886 \\
3.95827 \\
3.97709 \\
3.99533\end{array}$ & $\begin{array}{l}1997 \\
1941 \\
1882 \\
1824 \\
1766\end{array}$ & $\begin{array}{l}3.61642 \\
3.62510 \\
3.63384 \\
3.64263 \\
3.65144\end{array}$ & $\begin{array}{l}868 \\
874 \\
879 \\
881 \\
882\end{array}$ & $\begin{array}{l}23.14594 \\
23.24794 \\
23.34738 \\
23.44440 \\
23.53914\end{array}$ & $\begin{array}{r}10200 \\
9944 \\
9702 \\
9474 \\
9256\end{array}$ & $\begin{array}{l}26.76236 \\
26.27304 \\
26.98123 \\
27.08704 \\
27.19058\end{array}$ & $\begin{array}{l}11068 \\
10819 \\
10581 \\
10354 \\
10138\end{array}$ \\
\hline $\begin{array}{l}400 \\
450 \\
500 \\
550 \\
600\end{array}$ & $\begin{array}{l}4.01299 \\
4.09281 \\
4.15971 \\
4.21569 \\
4.26272\end{array}$ & $\begin{array}{l}7982 \\
6690 \\
5598 \\
4703 \\
3979\end{array}$ & $\begin{array}{l}3.66026 \\
3.70402 \\
3.74634 \\
3.78654 \\
3.82432\end{array}$ & $\begin{array}{l}4376 \\
4232 \\
4020 \\
3778 \\
3529\end{array}$ & $\begin{array}{l}23.63170 \\
24.06535 \\
24.45781 \\
24.81678 \\
25.14789\end{array}$ & $\begin{array}{l}43365 \\
39246 \\
35897 \\
33111 \\
30752\end{array}$ & $\begin{array}{l}27.29196 \\
27.76937 \\
28.20416 \\
28.60332 \\
28.97221\end{array}$ & $\begin{array}{l}47741 \\
43479 \\
39916 \\
36889 \\
34282\end{array}$ \\
\hline $\begin{array}{l}650 \\
700 \\
750 \\
800 \\
850\end{array}$ & $\begin{array}{l}4.30251 \\
4.33646 \\
4.36568 \\
4.39106 \\
4.41331\end{array}$ & $\begin{array}{l}3395 \\
2922 \\
2538 \\
2225 \\
1969\end{array}$ & $\begin{array}{l}3.85961 \\
3.89249 \\
3.92309 \\
3.95156 \\
3.97808\end{array}$ & $\begin{array}{l}3288 \\
3060 \\
2847 \\
2652 \\
2474\end{array}$ & $\begin{array}{l}25.45541 \\
25.74266 \\
26.01227 \\
26.26638 \\
26.50674\end{array}$ & $\begin{array}{l}28725 \\
26961 \\
25411 \\
24036 \\
22809\end{array}$ & $\begin{array}{l}29.31503 \\
29.63515 \\
29.93536 \\
30.21794 \\
30.48483\end{array}$ & $\begin{array}{l}32012 \\
30021 \\
28258 \\
26689 \\
25283\end{array}$ \\
\hline $\begin{array}{l}900 \\
950 \\
000 \\
.050 \\
100\end{array}$ & $\begin{array}{l}4.43300 \\
4.45055 \\
4.46634 \\
4.48064 \\
4.49369\end{array}$ & $\begin{array}{l}1755 \\
1579 \\
1430 \\
1305 \\
1199\end{array}$ & $\begin{array}{l}4.00282 \\
4.02593 \\
4.04756 \\
4.06785 \\
4.08691\end{array}$ & $\begin{array}{l}2311 \\
2163 \\
2029 \\
1906 \\
1795\end{array}$ & $\begin{array}{l}26.73483 \\
26.95188 \\
27.15894 \\
27.35692 \\
27.54660\end{array}$ & $\begin{array}{l}21705 \\
20706 \\
19798 \\
18968 \\
18207\end{array}$ & $\begin{array}{l}30.73766 \\
30.97781 \\
31.20651 \\
31.42477 \\
31.63352\end{array}$ & $\begin{array}{l}24015 \\
22870 \\
21826 \\
20875 \\
20002\end{array}$ \\
\hline 50 & 4.50568 & 1108 & 4.10486 & 1694 & 27.72867 & 17506 & 31.83354 & 19199 \\
\hline
\end{tabular}


Table 2.069. In T (Cont.)

\begin{tabular}{|c|c|c|c|c|c|c|c|c|}
\hline${ }^{\circ} \mathrm{K}$ & $\frac{C_{p}^{0}}{R}$ & & $\frac{\left(H^{\circ}-E_{0}^{0}\right)}{R T}$ & & $\frac{-\left(F^{0}-E_{0}^{0}\right)}{R T}$ & & $\frac{S^{0}}{R}$ & \\
\hline $\begin{array}{l}1200 \\
1300 \\
1400 \\
1500 \\
1600\end{array}$ & $\begin{array}{l}4.51676 \\
4.53670 \\
4.55430 \\
4.57012 \\
4.58456\end{array}$ & $\begin{array}{l}1994 \\
1760 \\
1582 \\
1444 \\
1334\end{array}$ & $\begin{array}{l}4.12180 \\
4.15296 \\
4.18101 \\
4.20644 \\
4.22962\end{array}$ & $\begin{array}{l}3116 \\
2805 \\
2543 \\
2318 \\
2128\end{array}$ & $\begin{array}{l}27.90373 \\
28.23491 \\
28.54372 \\
28.83306 \\
29.10529\end{array}$ & $\begin{array}{l}33118 \\
30881 \\
28934 \\
27223 \\
25706\end{array}$ & $\begin{array}{l}32.02553 \\
32.38787 \\
32.72474 \\
33.03950 \\
33.33492\end{array}$ & $\begin{array}{l}36234 \\
33687 \\
31476 \\
29542 \\
27834\end{array}$ \\
\hline $\begin{array}{l}1700 \\
1800 \\
1900 \\
2000 \\
2100\end{array}$ & $\begin{array}{l}4.59790 \\
4.61038 \\
4.62215 \\
4.63334 \\
4.64405\end{array}$ & $\begin{array}{l}1248 \\
1177 \\
1119 \\
1071 \\
1030\end{array}$ & $\begin{array}{l}4.25090 \\
4.27053 \\
4.28873 \\
4.30568 \\
4.32154\end{array}$ & $\begin{array}{l}1963 \\
1820 \\
1695 \\
1586 \\
1489\end{array}$ & $\begin{array}{l}29.36235 \\
29.60589 \\
29.83728 \\
30.05770 \\
30.26816\end{array}$ & $\begin{array}{l}24354 \\
23139 \\
22042 \\
21046 \\
20139\end{array}$ & $\begin{array}{l}33.61326 \\
33.87642 \\
34.12601 \\
34.36339 \\
34.58971\end{array}$ & $\begin{array}{l}26316 \\
24959 \\
23738 \\
22632 \\
21628\end{array}$ \\
\hline $\begin{array}{l}2200 \\
2300 \\
2400 \\
2500 \\
2600\end{array}$ & $\begin{array}{l}4.65435 \\
4.66432 \\
4.67399 \\
4.68341 \\
4.69261\end{array}$ & $\begin{array}{l}997 \\
967 \\
942 \\
920 \\
900\end{array}$ & $\begin{array}{l}4.33643 \\
4.35047 \\
4.36375 \\
4.37635 \\
4.38834\end{array}$ & $\begin{array}{l}1404 \\
1328 \\
1260 \\
1199 \\
1144\end{array}$ & $\begin{array}{l}30.46955 \\
30.66262 \\
30.84806 \\
31.02646 \\
31.19834\end{array}$ & $\begin{array}{l}19307 \\
18544 \\
17840 \\
17188 \\
16583\end{array}$ & $\begin{array}{l}34.80599 \\
35.01310 \\
35.21182 \\
35.40281 \\
35.58668\end{array}$ & $\begin{array}{l}20711 \\
19872 \\
19099 \\
18387 \\
17727\end{array}$ \\
\hline $\begin{array}{l}2700 \\
2800 \\
2900 \\
3000\end{array}$ & $\begin{array}{l}4.70161 \\
4.71045 \\
4.71913 \\
4.72768\end{array}$ & $\begin{array}{r}884 \\
868 \\
855 \\
1674\end{array}$ & $\begin{array}{l}4.39978 \\
4.41071 \\
4.42120 \\
4.43127\end{array}$ & $\begin{array}{l}1093 \\
1049 \\
1007 \\
1905\end{array}$ & $\begin{array}{l}31.36417 \\
31.52438 \\
31.67934 \\
31.82940\end{array}$ & $\begin{array}{l}16021 \\
15496 \\
15006 \\
28660\end{array}$ & $\begin{array}{l}35.76395 \\
35.93510 \\
36.10055 \\
36.26068\end{array}$ & $\begin{array}{l}17115 \\
16545 \\
16013 \\
30565\end{array}$ \\
\hline $\begin{array}{l}3200 \\
3400 \\
3600 \\
3800 \\
4000\end{array}$ & $\begin{array}{l}4.74442 \\
4.76075 \\
4.77673 \\
4.79241 \\
4.80783\end{array}$ & $\begin{array}{l}1633 \\
1598 \\
1568 \\
1542 \\
1518\end{array}$ & $\begin{array}{l}4.45032 \\
4.46811 \\
4.48481 \\
4.50059 \\
4.51557\end{array}$ & $\begin{array}{l}1779 \\
1670 \\
1578 \\
1498 \\
1428\end{array}$ & $\begin{array}{l}32.11600 \\
32.38634 \\
32.64221 \\
32.88512 \\
33.11635\end{array}$ & $\begin{array}{l}27034 \\
25587 \\
24291 \\
23123 \\
22066\end{array}$ & $\begin{array}{l}36.56633 \\
36.85445 \\
37.12702 \\
37.38571 \\
37.63192\end{array}$ & $\begin{array}{l}28812 \\
27257 \\
25869 \\
24621 \\
23494\end{array}$ \\
\hline $\begin{array}{l}4200 \\
4400 \\
4600 \\
4800 \\
5000\end{array}$ & $\begin{array}{l}4.82301 \\
4.83798 \\
4.85275 \\
4.86734 \\
4.88176\end{array}$ & $\begin{array}{l}1497 \\
1477 \\
1459 \\
1442\end{array}$ & $\begin{array}{l}4.52985 \\
4.54351 \\
4.55664 \\
4.56928 \\
4.58149\end{array}$ & $\begin{array}{l}1366 \\
1313 \\
1264 \\
1221\end{array}$ & $\begin{array}{l}33.33701 \\
33.54806 \\
33.75032 \\
33.94452 \\
34.13129\end{array}$ & $\begin{array}{l}21105 \\
20226 \\
19420 \\
18677\end{array}$ & $\begin{array}{l}37.86686 \\
38.09158 \\
38.30696 \\
38.51380 \\
38.71279\end{array}$ & $\begin{array}{l}22472 \\
21538 \\
20684 \\
19899\end{array}$ \\
\hline
\end{tabular}


Table 2.070. TlH

\begin{tabular}{|c|c|c|c|c|c|c|c|c|}
\hline$\stackrel{\circ}{K}$ & $\frac{C_{p}^{0}}{R}$ & & $\frac{\left(H^{0}-E_{0}^{0}\right.}{R T}$ & & $\frac{-\left(F^{0}-t\right.}{R T}$ & & $\frac{S^{0}}{R}$ & \\
\hline $\begin{array}{l}50 \\
60 \\
70 \\
80 \\
90\end{array}$ & $\begin{array}{l}3.50246 \\
3.50272 \\
3.50304 \\
3.50339 \\
3.50375\end{array}$ & $\begin{array}{l}26 \\
32 \\
35 \\
36 \\
38\end{array}$ & $\begin{array}{l}3.45521 \\
3.46311 \\
3.46879 \\
3.47309 \\
3.47648\end{array}$ & $\begin{array}{l}790 \\
568 \\
430 \\
339 \\
275\end{array}$ & $\begin{array}{l}16.14416 \\
16.77486 \\
17.30915 \\
17.77263 \\
18.18191\end{array}$ & $\begin{array}{l}63070 \\
53429 \\
46348 \\
40928 \\
36643\end{array}$ & $\begin{array}{l}19.59937 \\
20.23797 \\
20.77794 \\
21.24573 \\
21.65839\end{array}$ & $\begin{array}{l}63860 \\
53997 \\
46779 \\
41266 \\
36918\end{array}$ \\
\hline $\begin{array}{l}100 \\
110 \\
120 \\
130 \\
140\end{array}$ & $\begin{array}{l}3.50413 \\
3.50452 \\
3.50493 \\
3.5053 \vec{i} \\
3.50588\end{array}$ & $\begin{array}{l}39 \\
41 \\
44 \\
51 \\
62\end{array}$ & $\begin{array}{l}3.47923 \\
3.48151 \\
3.48344 \\
3.48511 \\
3.48658\end{array}$ & $\begin{array}{l}228 \\
193 \\
167 \\
147 \\
130\end{array}$ & $\begin{array}{l}18.54834 \\
18.88006 \\
19.18307 \\
19.46196 \\
19.72029\end{array}$ & $\begin{array}{l}33172 \\
30301 \\
27889 \\
25833 \\
24060\end{array}$ & $\begin{array}{l}22.02757 \\
22.36157 \\
22.66652 \\
22.94708 \\
23.20687\end{array}$ & $\begin{array}{l}33400 \\
30495 \\
28056 \\
25979 \\
24191\end{array}$ \\
\hline $\begin{array}{l}150 \\
160 \\
170 \\
180 \\
190\end{array}$ & $\begin{array}{l}3.50650 \\
3.50731 \\
3.50838 \\
3.50981 \\
3.51169\end{array}$ & $\begin{array}{r}81 \\
107 \\
143 \\
188 \\
242\end{array}$ & $\begin{array}{l}3.48788 \\
3.48907 \\
3.49017 \\
3.49122 \\
3.49225\end{array}$ & $\begin{array}{l}119 \\
110 \\
105 \\
103 \\
103\end{array}$ & $\begin{array}{l}19.96089 \\
20.18603 \\
20.39759 \\
20.59711 \\
20.78590\end{array}$ & $\begin{array}{l}22514 \\
21156 \\
19952 \\
18879 \\
17915\end{array}$ & $\begin{array}{l}23.44878 \\
23.67510 \\
23.88777 \\
24.08834 \\
24.27815\end{array}$ & $\begin{array}{l}22632 \\
21267 \\
20057 \\
18981 \\
18019\end{array}$ \\
\hline $\begin{array}{l}200 \\
210 \\
220 \\
230 \\
240\end{array}$ & $\begin{array}{l}3.51411 \\
3.51716 \\
3.52092 \\
3.52543 \\
3.53076\end{array}$ & $\begin{array}{l}305 \\
376 \\
451 \\
533 \\
616\end{array}$ & $\begin{array}{l}3.49323 \\
3.49434 \\
3.49546 \\
3.49666 \\
3.49797\end{array}$ & $\begin{array}{l}106 \\
112 \\
120 \\
131 \\
143\end{array}$ & $\begin{array}{l}20.96505 \\
21.13552 \\
21.29810 \\
21.45351 \\
21.60235\end{array}$ & $\begin{array}{l}17047 \\
16258 \\
15541 \\
14884 \\
14282\end{array}$ & $\begin{array}{l}24.45834 \\
24.62986 \\
24.79357 \\
24.95017 \\
25.10032\end{array}$ & $\begin{array}{l}17152 \\
16371 \\
15660 \\
15015 \\
14426\end{array}$ \\
\hline $\begin{array}{l}250 \\
260 \\
270 \\
280 \\
290\end{array}$ & $\begin{array}{l}3.53692 \\
3.54392 \\
3.55176 \\
3.56042 \\
3.56986\end{array}$ & $\begin{array}{r}703 \\
784 \\
866 \\
944 \\
1019\end{array}$ & $\begin{array}{l}3.49940 \\
3.50098 \\
3.50271 \\
3.50461 \\
3.50670\end{array}$ & $\begin{array}{l}158 \\
173 \\
190 \\
209 \\
227\end{array}$ & $\begin{array}{l}21.74517 \\
21.88245 \\
22.01461 \\
22.14203 \\
22.26505\end{array}$ & $\begin{array}{l}13728 \\
13216 \\
12742 \\
12302 \\
11892\end{array}$ & $\begin{array}{l}25.24458 \\
25.38343 \\
25.51733 \\
25.64665 \\
25.77175\end{array}$ & $\begin{array}{l}13885 \\
13390 \\
12932 \\
12510 \\
12120\end{array}$ \\
\hline $\begin{array}{l}300 \\
310 \\
320 \\
330 \\
340\end{array}$ & $\begin{array}{l}3.58005 \\
3.59095 \\
3.60248 \\
3.61461 \\
3.62727\end{array}$ & $\begin{array}{l}1090 \\
1153 \\
1213 \\
1266 \\
1312\end{array}$ & $\begin{array}{l}3.50897 \\
3.51144 \\
3.51410 \\
3.51696 \\
3.52002\end{array}$ & $\begin{array}{l}247 \\
266 \\
286 \\
306 \\
325\end{array}$ & $\begin{array}{l}22.38397 \\
22.49907 \\
22.61059 \\
22.71877 \\
22.82381\end{array}$ & $\begin{array}{l}11510 \\
11152 \\
10818 \\
10504 \\
10208\end{array}$ & $\begin{array}{l}25.89295 \\
26.01051 \\
26.12470 \\
26.23574 \\
26.34383\end{array}$ & $\begin{array}{l}11756 \\
11419 \\
11104 \\
10809 \\
10534\end{array}$ \\
\hline $\begin{array}{l}350 \\
360 \\
370 \\
380 \\
390\end{array}$ & $\begin{array}{l}3.64039 \\
3.65393 \\
3.66782 \\
3.68200 \\
3.69642\end{array}$ & $\begin{array}{l}1354 \\
1389 \\
1418 \\
1442 \\
1462\end{array}$ & $\begin{array}{l}3.52327 \\
3.52671 \\
3.53034 \\
3.53414 \\
3.53812\end{array}$ & $\begin{array}{l}344 \\
363 \\
380 \\
398 \\
414\end{array}$ & $\begin{array}{l}22.92589 \\
23.02519 \\
23.12187 \\
23.21607 \\
23.30792\end{array}$ & $\begin{array}{l}9930 \\
9668 \\
9420 \\
9185 \\
8963\end{array}$ & $\begin{array}{l}26.44917 \\
26.55191 \\
26.65221 \\
26.75021 \\
26.84604\end{array}$ & $\begin{array}{r}10274 \\
10030 \\
9800 \\
9583 \\
9377\end{array}$ \\
\hline $\begin{array}{l}400 \\
450 \\
500 \\
550 \\
600\end{array}$ & $\begin{array}{l}3.71104 \\
3.78543 \\
3.85886 \\
3.92854 \\
3.99306\end{array}$ & $\begin{array}{l}7439 \\
7343 \\
6968 \\
6452 \\
5889\end{array}$ & $\begin{array}{l}3.54226 \\
3.56513 \\
3.59086 \\
3.61842 \\
3.64699\end{array}$ & $\begin{array}{l}2287 \\
2573 \\
2756 \\
2857 \\
2892\end{array}$ & $\begin{array}{l}23.39755 \\
23.81606 \\
24.19299 \\
24.53652 \\
24.85258\end{array}$ & $\begin{array}{l}41851 \\
37693 \\
34353 \\
31606 \\
29306\end{array}$ & $\begin{array}{l}26.93981 \\
27.38119 \\
27.78385 \\
28.15495 \\
28.49958\end{array}$ & $\begin{array}{l}44138 \\
40266 \\
37110 \\
34463 \\
32198\end{array}$ \\
\hline $\begin{array}{l}650 \\
700 \\
750 \\
800 \\
850\end{array}$ & $\begin{array}{l}4.05195 \\
4.10527 \\
4.15334 \\
4.19663 \\
4.23564\end{array}$ & $\begin{array}{l}5332 \\
4807 \\
4329 \\
3901 \\
3522\end{array}$ & $\begin{array}{l}3.67591 \\
3.70471 \\
3.73304 \\
3.76069 \\
3.78750\end{array}$ & $\begin{array}{l}2880 \\
2833 \\
2765 \\
2681 \\
2589\end{array}$ & $\begin{array}{l}25.14564 \\
25.41911 \\
25.67568 \\
25.91749 \\
26.14629\end{array}$ & $\begin{array}{l}27347 \\
25657 \\
24181 \\
22880 \\
21723\end{array}$ & $\begin{array}{l}28.82156 \\
29.12383 \\
29.40873 \\
29.67819 \\
29.93380\end{array}$ & $\begin{array}{l}30227 \\
28490 \\
26946 \\
25561 \\
24311\end{array}$ \\
\hline $\begin{array}{l}900 \\
950 \\
000 \\
050 \\
100\end{array}$ & $\begin{array}{l}4.27086 \\
4.30274 \\
4.33170 \\
4.35810 \\
4.38225\end{array}$ & $\begin{array}{l}3188 \\
2896 \\
2640 \\
2415 \\
2219\end{array}$ & $\begin{array}{l}3.81339 \\
3.83832 \\
3.86228 \\
3.88527 \\
3.90732\end{array}$ & $\begin{array}{l}2493 \\
2396 \\
2299 \\
2205 \\
2114\end{array}$ & $\begin{array}{l}26.36352 \\
26.57037 \\
26.76786 \\
26.95686 \\
27.13812\end{array}$ & $\begin{array}{l}20685 \\
19749 \\
18900 \\
18126 \\
17416\end{array}$ & $\begin{array}{l}30.17691 \\
30.40869 \\
30.63014 \\
30.84214 \\
31.04544\end{array}$ & $\begin{array}{l}23178 \\
22145 \\
21200 \\
20330 \\
19530\end{array}$ \\
\hline 50 & 4.40444 & 2046 & 3.92846 & 2026 & 27.31228 & 16762 & 31.24074 & 18789 \\
\hline
\end{tabular}


Table 2.070. TlH (Cont.)

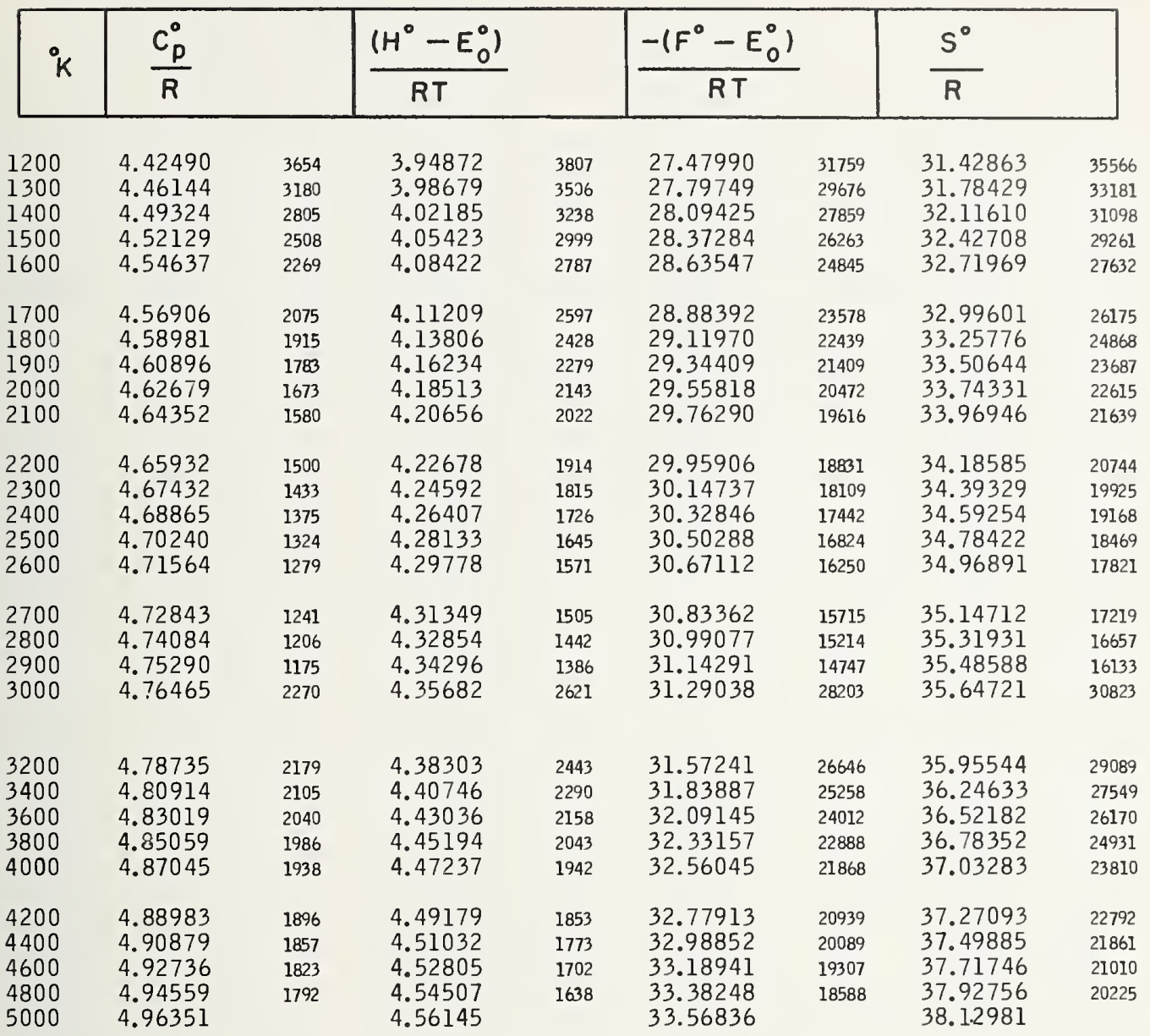


Table 2.071.

TID

\begin{tabular}{|c|c|c|c|c|c|c|c|c|}
\hline${ }^{\circ} \mathrm{K}$ & $\frac{C_{p}^{0}}{R}$ & & $\frac{\left(H^{0}-E_{0}^{0}\right)}{R T}$ & & $\frac{-l F^{\circ}-E}{R T}$ & & $\frac{S^{0}}{R}$ & \\
\hline $\begin{array}{l}50 \\
60 \\
70 \\
80 \\
90\end{array}$ & $\begin{array}{l}3.50212 \\
3.50249 \\
3.50287 \\
3.50327 \\
3.50370\end{array}$ & $\begin{array}{l}37 \\
38 \\
40 \\
43 \\
54\end{array}$ & $\begin{array}{l}3.47798 \\
3.48204 \\
3.48499 \\
3.48725 \\
3.48905\end{array}$ & $\begin{array}{l}406 \\
295 \\
226 \\
180 \\
149\end{array}$ & $\begin{array}{l}16.81185 \\
17.44634 \\
17.98333 \\
18.44884 \\
18.85969\end{array}$ & $\begin{array}{l}63449 \\
53699 \\
46551 \\
41085 \\
36769\end{array}$ & $\begin{array}{l}20.28984 \\
20.92838 \\
21.46832 \\
21.93609 \\
22.34875\end{array}$ & $\begin{array}{l}63854 \\
53994 \\
46777 \\
41266 \\
36917\end{array}$ \\
\hline $\begin{array}{l}100 \\
110 \\
120 \\
130 \\
140\end{array}$ & $\begin{array}{l}3.50424 \\
3.50500 \\
3.50616 \\
3.50797 \\
3.51069\end{array}$ & $\begin{array}{r}76 \\
116 \\
181 \\
272 \\
386\end{array}$ & $\begin{array}{l}3.49054 \\
3.49182 \\
3.49296 \\
3.49404 \\
3.49513\end{array}$ & $\begin{array}{l}128 \\
114 \\
108 \\
109 \\
116\end{array}$ & $\begin{array}{l}19.22738 \\
19.56012 \\
19.86400 \\
20.14363 \\
20.40261\end{array}$ & $\begin{array}{l}33274 \\
30388 \\
27963 \\
25898 \\
24118\end{array}$ & $\begin{array}{l}22.71792 \\
23.05195 \\
23.35697 \\
23.63768 \\
23.89774\end{array}$ & $\begin{array}{l}33403 \\
30502 \\
28071 \\
26006 \\
24234\end{array}$ \\
\hline $\begin{array}{l}150 \\
160 \\
170 \\
180 \\
190\end{array}$ & $\begin{array}{l}3.51455 \\
3.51976 \\
3.52647 \\
3.53479 \\
3.54474\end{array}$ & $\begin{array}{r}521 \\
671 \\
832 \\
995 \\
1155\end{array}$ & $\begin{array}{l}3.49629 \\
3.49758 \\
3.49908 \\
3.50082 \\
3.50287\end{array}$ & $\begin{array}{l}129 \\
150 \\
174 \\
205 \\
237\end{array}$ & $\begin{array}{l}20.64379 \\
20.86947 \\
21.08156 \\
21.28161 \\
21.47094\end{array}$ & $\begin{array}{l}22568 \\
21209 \\
20005 \\
18933 \\
17973\end{array}$ & $\begin{array}{l}24.14008 \\
24.36706 \\
24.58064 \\
24.78243 \\
24.97381\end{array}$ & $\begin{array}{l}22698 \\
21358 \\
20179 \\
19138 \\
18211\end{array}$ \\
\hline $\begin{array}{l}200 \\
210 \\
220 \\
230 \\
240\end{array}$ & $\begin{array}{l}3.55629 \\
3.56936 \\
3.58384 \\
3.59960 \\
3.61648\end{array}$ & $\begin{array}{l}1307 \\
1448 \\
1576 \\
1688 \\
1783\end{array}$ & $\begin{array}{l}3.50524 \\
3.50798 \\
3.51109 \\
3.51459 \\
3.51848\end{array}$ & $\begin{array}{l}274 \\
311 \\
350 \\
389 \\
428\end{array}$ & $\begin{array}{l}21.65067 \\
21.82176 \\
21.98502 \\
22.14117 \\
22.29083\end{array}$ & $\begin{array}{l}17109 \\
16326 \\
15615 \\
14966 \\
14372\end{array}$ & $\begin{array}{l}25.15592 \\
25.32974 \\
25.49612 \\
25.65577 \\
25.80932\end{array}$ & $\begin{array}{l}17382 \\
16638 \\
15965 \\
15355 \\
14799\end{array}$ \\
\hline $\begin{array}{l}250 \\
260 \\
270 \\
280 \\
290\end{array}$ & $\begin{array}{l}3.63431 \\
3.65294 \\
3.67221 \\
3.69197 \\
3.71208\end{array}$ & $\begin{array}{l}1863 \\
1927 \\
1976 \\
2011 \\
2033\end{array}$ & $\begin{array}{l}3.52276 \\
3.52740 \\
3.53241 \\
3.53775 \\
3.54342\end{array}$ & $\begin{array}{l}464 \\
501 \\
534 \\
567 \\
596\end{array}$ & $\begin{array}{l}22.43455 \\
22.57280 \\
22.70602 \\
22.83458 \\
22.95882\end{array}$ & $\begin{array}{l}13825 \\
13322 \\
12856 \\
12424 \\
12023\end{array}$ & $\begin{array}{l}25.95731 \\
26.10021 \\
26.23843 \\
26.37234 \\
26.50224\end{array}$ & $\begin{array}{l}14290 \\
13822 \\
13391 \\
12990 \\
12619\end{array}$ \\
\hline $\begin{array}{l}300 \\
310 \\
320 \\
330 \\
340\end{array}$ & $\begin{array}{l}3.73241 \\
3.75285 \\
3.77331 \\
3.79368 \\
3.81390\end{array}$ & $\begin{array}{l}2044 \\
2046 \\
2037 \\
2022 \\
2001\end{array}$ & $\begin{array}{l}3.54938 \\
3.55561 \\
3.56209 \\
3.56880 \\
3.57572\end{array}$ & $\begin{array}{l}623 \\
648 \\
671 \\
692 \\
709\end{array}$ & $\begin{array}{l}23.07905 \\
23.19554 \\
23.30852 \\
23.41824 \\
23.52488\end{array}$ & $\begin{array}{l}11649 \\
11298 \\
10972 \\
10664 \\
10375\end{array}$ & $\begin{array}{l}26.62843 \\
26.75115 \\
26.87062 \\
26.98705 \\
27.10060\end{array}$ & $\begin{array}{l}12272 \\
11947 \\
11643 \\
11355 \\
11084\end{array}$ \\
\hline $\begin{array}{l}350 \\
360 \\
370 \\
380 \\
390\end{array}$ & $\begin{array}{l}3.83391 \\
3.85364 \\
3.87306 \\
3.89212 \\
3.91080\end{array}$ & $\begin{array}{l}1973 \\
1942 \\
1906 \\
1868 \\
1827\end{array}$ & $\begin{array}{l}3.58281 \\
3.59006 \\
3.59744 \\
3.60495 \\
3.61255\end{array}$ & $\begin{array}{l}725 \\
738 \\
751 \\
760 \\
769\end{array}$ & $\begin{array}{l}23.62863 \\
23.72966 \\
23.82813 \\
23.92417 \\
24.01790\end{array}$ & $\begin{array}{r}10103 \\
9847 \\
9604 \\
9373 \\
9156\end{array}$ & $\begin{array}{l}27.21144 \\
27.31973 \\
27.42558 \\
27.52912 \\
27.63046\end{array}$ & $\begin{array}{r}10829 \\
10585 \\
10354 \\
10134 \\
9925\end{array}$ \\
\hline $\begin{array}{l}400 \\
450 \\
500 \\
550 \\
600\end{array}$ & $\begin{array}{l}3.92907 \\
4.01394 \\
4.08780 \\
4.15142 \\
4.20607\end{array}$ & $\begin{array}{l}8487 \\
7386 \\
6362 \\
5465 \\
4706\end{array}$ & $\begin{array}{l}3.62024 \\
3.65937 \\
3.69861 \\
3.73696 \\
3.77383\end{array}$ & $\begin{array}{l}3913 \\
3924 \\
3835 \\
3687 \\
3510\end{array}$ & $\begin{array}{l}24.10946 \\
24.53812 \\
24.92571 \\
25.28003 \\
25.60678\end{array}$ & $\begin{array}{l}42866 \\
38759 \\
35432 \\
32675 \\
30347\end{array}$ & $\begin{array}{l}27.72971 \\
28.19749 \\
28.62432 \\
29.01699 \\
29.38062\end{array}$ & $\begin{array}{l}46778 \\
42683 \\
39267 \\
36363 \\
33857\end{array}$ \\
\hline $\begin{array}{l}650 \\
700 \\
750 \\
800 \\
850\end{array}$ & $\begin{array}{l}4.25313 \\
4.29385 \\
4.32929 \\
4.36035 \\
4.38779\end{array}$ & $\begin{array}{l}4072 \\
3544 \\
3106 \\
2744 \\
2440\end{array}$ & $\begin{array}{l}3.80893 \\
3.84215 \\
3.87347 \\
3.90295 \\
3.93068\end{array}$ & $\begin{array}{l}3322 \\
3132 \\
2948 \\
2773 \\
2609\end{array}$ & $\begin{array}{l}25.91025 \\
26.19375 \\
26.45991 \\
26.71085 \\
26.94830\end{array}$ & $\begin{array}{l}28350 \\
26616 \\
25394 \\
23745 \\
22542\end{array}$ & $\begin{array}{l}29.71919 \\
30.03590 \\
30.33338 \\
30.61380 \\
30.87899\end{array}$ & $\begin{array}{l}31671 \\
29748 \\
28042 \\
26519 \\
25150\end{array}$ \\
\hline $\begin{array}{l}900 \\
950 \\
000 \\
050 \\
100\end{array}$ & $\begin{array}{l}4.41219 \\
4.43406 \\
4.45380 \\
4.47174 \\
4.48814\end{array}$ & $\begin{array}{l}2187 \\
1974 \\
1794 \\
1640 \\
1510\end{array}$ & $\begin{array}{l}3.95677 \\
3.98132 \\
4.00446 \\
4.02629 \\
4.04692\end{array}$ & $\begin{array}{l}2455 \\
2314 \\
2183 \\
2063 \\
1951\end{array}$ & $\begin{array}{l}27.17372 \\
27.38832 \\
27.59313 \\
27.78904 \\
27.97682\end{array}$ & $\begin{array}{l}21460 \\
20481 \\
19591 \\
18778 \\
18033\end{array}$ & $\begin{array}{l}31.13049 \\
31.36964 \\
31.59759 \\
31.81533 \\
32.02374\end{array}$ & $\begin{array}{l}23915 \\
22795 \\
21774 \\
20841 \\
19985\end{array}$ \\
\hline 50 & 4.50324 & 1397 & 4.06643 & 1850 & 28.15715 & 17346 & 32.22359 & 19195 \\
\hline
\end{tabular}


Table 2.071. TID (Cont.)

\begin{tabular}{|c|c|c|c|c|c|c|c|c|}
\hline${ }^{\circ} \mathrm{K}$ & $\frac{C_{p}^{0}}{R}$ & & $\frac{\left(H^{\circ}-E_{0}^{0}\right)}{R T}$ & & $\frac{-\left(F^{0}-E_{0}^{0}\right)}{R T}$ & & $\frac{S^{0}}{R}$ & \\
\hline $\begin{array}{l}1200 \\
1300 \\
1400 \\
1500 \\
1600\end{array}$ & $\begin{array}{l}4.51721 \\
4.54238 \\
4.56460 \\
4.58457 \\
4.60277\end{array}$ & $\begin{array}{l}2517 \\
2222 \\
1997 \\
1820 \\
1680\end{array}$ & $\begin{array}{l}4.08493 \\
4.11917 \\
4.15021 \\
4.17851 \\
4.20447\end{array}$ & $\begin{array}{l}3424 \\
3104 \\
2830 \\
2596 \\
2393\end{array}$ & $\begin{array}{l}28.33061 \\
28.65895 \\
28.96537 \\
29.25269 \\
29.52320\end{array}$ & $\begin{array}{l}32834 \\
30642 \\
28732 \\
27051 \\
25562\end{array}$ & $\begin{array}{l}32.41554 \\
32.77813 \\
33.11559 \\
33.43120 \\
33.72767\end{array}$ & $\begin{array}{l}36259 \\
33746 \\
31561 \\
29647 \\
27956\end{array}$ \\
\hline $\begin{array}{l}1700 \\
1800 \\
1900 \\
2000 \\
2100\end{array}$ & $\begin{array}{l}4.61957 \\
4.63525 \\
4.65002 \\
4.66403 \\
4.67741\end{array}$ & $\begin{array}{l}1568 \\
1477 \\
1401 \\
1338 \\
1286\end{array}$ & $\begin{array}{l}4.22840 \\
4.25057 \\
4.27121 \\
4.29050 \\
4.30861\end{array}$ & $\begin{array}{l}2217 \\
2064 \\
1929 \\
1811 \\
1706\end{array}$ & $\begin{array}{l}29.77882 \\
30.02115 \\
30.25152 \\
30.47111 \\
30.68088\end{array}$ & $\begin{array}{l}24233 \\
23037 \\
21959 \\
20977 \\
20084\end{array}$ & $\begin{array}{l}34.00723 \\
34.27172 \\
34.52274 \\
34.76161 \\
34.98950\end{array}$ & $\begin{array}{l}26449 \\
25102 \\
23887 \\
22789 \\
21789\end{array}$ \\
\hline $\begin{array}{l}2200 \\
2300 \\
2400 \\
2500 \\
2600\end{array}$ & $\begin{array}{l}4.69027 \\
4.70267 \\
4.71469 \\
4.72637 \\
4.73776\end{array}$ & $\begin{array}{l}1240 \\
1202 \\
1169 \\
1139 \\
1113\end{array}$ & $\begin{array}{l}4.32567 \\
4.34179 \\
4.35708 \\
4.37162 \\
4.38548\end{array}$ & $\begin{array}{l}1612 \\
1529 \\
1454 \\
1386 \\
1326\end{array}$ & $\begin{array}{l}30.88172 \\
31.07436 \\
31.25947 \\
31.43763 \\
31.60936\end{array}$ & $\begin{array}{l}19264 \\
18511 \\
17816 \\
17173 \\
16576\end{array}$ & $\begin{array}{l}35.20739 \\
35.41615 \\
35.61655 \\
35.80926 \\
35.99485\end{array}$ & $\begin{array}{l}20876 \\
20040 \\
19271 \\
18559 \\
17901\end{array}$ \\
\hline $\begin{array}{l}2700 \\
2800 \\
2900 \\
3000\end{array}$ & $\begin{array}{l}4.74889 \\
4.75979 \\
4.77049 \\
4.78100\end{array}$ & $\begin{array}{l}1090 \\
1070 \\
1051 \\
2054\end{array}$ & $\begin{array}{l}4.39874 \\
4.41144 \\
4.42363 \\
4.43537\end{array}$ & $\begin{array}{l}1270 \\
1219 \\
1174 \\
2225\end{array}$ & $\begin{array}{l}31.77512 \\
31.93533 \\
32.09034 \\
32.24051\end{array}$ & $\begin{array}{l}16021 \\
15501 \\
15017 \\
28697\end{array}$ & $\begin{array}{l}36.17386 \\
36.34677 \\
36.51398 \\
36.67589\end{array}$ & $\begin{array}{l}17291 \\
16721 \\
16191 \\
30921\end{array}$ \\
\hline $\begin{array}{l}3200 \\
3400 \\
3600 \\
3800 \\
4000\end{array}$ & $\begin{array}{l}4.80154 \\
4.82152 \\
4.84102 \\
4.86010 \\
4.87882\end{array}$ & $\begin{array}{l}1998 \\
1950 \\
1908 \\
1872 \\
1838\end{array}$ & $\begin{array}{l}4.45762 \\
4.47844 \\
4.49804 \\
4.51660 \\
4.53424\end{array}$ & $\begin{array}{l}2082 \\
1960 \\
1856 \\
1764 \\
1685\end{array}$ & $\begin{array}{l}32.52748 \\
32.79835 \\
33.05490 \\
33.29859 \\
33.53072\end{array}$ & $\begin{array}{l}27087 \\
25655 \\
24369 \\
23213 \\
22164\end{array}$ & $\begin{array}{l}36.98510 \\
37.27680 \\
37.55294 \\
37.81520 \\
38.06497\end{array}$ & $\begin{array}{l}29170 \\
27614 \\
26226 \\
24977 \\
23848\end{array}$ \\
\hline $\begin{array}{l}4200 \\
4400 \\
4600 \\
4800 \\
5000\end{array}$ & $\begin{array}{l}4.89720 \\
4.91528 \\
4.93308 \\
4.95063 \\
4.96793\end{array}$ & $\begin{array}{l}1808 \\
1780 \\
1755 \\
1730\end{array}$ & $\begin{array}{l}4.55109 \\
4.56723 \\
4.58275 \\
4.59772 \\
4.61218\end{array}$ & $\begin{array}{l}1614 \\
1552 \\
1497 \\
1446\end{array}$ & $\begin{array}{l}33.75236 \\
33.96445 \\
34.16781 \\
34.36317 \\
34.55116\end{array}$ & $\begin{array}{l}21209 \\
20336 \\
19536 \\
18799\end{array}$ & $\begin{array}{l}38.30345 \\
38.53169 \\
38.75057 \\
38.96090 \\
39.16334\end{array}$ & $\begin{array}{l}22824 \\
21888 \\
21033 \\
20244\end{array}$ \\
\hline
\end{tabular}


Table 2.072. TlT

\begin{tabular}{|c|c|c|c|c|c|c|c|c|}
\hline${ }^{\circ} \mathrm{K}$ & $\frac{C_{p}^{0}}{R}$ & & $\frac{\left(H^{0}-E_{0}^{0}\right)}{R T}$ & & $\frac{-l F^{\circ}-E}{R T}$ & & $\frac{S^{0}}{R}$ & \\
\hline $\begin{array}{l}50 \\
60 \\
70 \\
80 \\
90\end{array}$ & $\begin{array}{l}3.50206 \\
3.50245 \\
3.50286 \\
3.50337 \\
3.50415\end{array}$ & $\begin{array}{r}39 \\
41 \\
51 \\
78 \\
137\end{array}$ & $\begin{array}{l}3.48556 \\
3.48834 \\
3.49038 \\
3.49197 \\
3.49328\end{array}$ & $\begin{array}{l}278 \\
204 \\
159 \\
131 \\
115\end{array}$ & $\begin{array}{l}17.20871 \\
17.84446 \\
18.38235 \\
18.84854 \\
19.25991\end{array}$ & $\begin{array}{l}63575 \\
53789 \\
46619 \\
41137 \\
36811\end{array}$ & $\begin{array}{l}20.69427 \\
21.33280 \\
21.87274 \\
22.34052 \\
22.75320\end{array}$ & $\begin{array}{l}63853 \\
53994 \\
46778 \\
41268 \\
36726\end{array}$ \\
\hline $\begin{array}{l}100 \\
110 \\
120 \\
130 \\
140\end{array}$ & $\begin{array}{l}3.50552 \\
3.50790 \\
3.51174 \\
3.51745 \\
3.52534\end{array}$ & $\begin{array}{r}238 \\
384 \\
571 \\
789 \\
1023\end{array}$ & $\begin{array}{l}3.49443 \\
3.49554 \\
3.49672 \\
3.49808 \\
3.49973\end{array}$ & $\begin{array}{l}111 \\
118 \\
136 \\
165 \\
203\end{array}$ & $\begin{array}{l}19.62802 \\
19.96113 \\
20.26533 \\
20.54527 \\
20.80457\end{array}$ & $\begin{array}{l}33311 \\
30420 \\
27994 \\
25930 \\
24152\end{array}$ & $\begin{array}{l}23.12246 \\
23.45667 \\
23.76205 \\
24.04335 \\
24.30430\end{array}$ & $\begin{array}{l}33421 \\
30538 \\
28130 \\
26095 \\
24356\end{array}$ \\
\hline $\begin{array}{l}150 \\
160 \\
170 \\
180 \\
190\end{array}$ & $\begin{array}{l}3.53557 \\
3.54820 \\
3.56313 \\
3.58020 \\
3.59916\end{array}$ & $\begin{array}{l}1263 \\
1493 \\
1707 \\
1896 \\
2058\end{array}$ & $\begin{array}{l}3.50176 \\
3.50426 \\
3.50727 \\
3.51084 \\
3.51498\end{array}$ & $\begin{array}{l}250 \\
301 \\
357 \\
414 \\
472\end{array}$ & $\begin{array}{l}21.04609 \\
21.27217 \\
21.48470 \\
21.68527 \\
21.87520\end{array}$ & $\begin{array}{l}22608 \\
21253 \\
20057 \\
18993 \\
18041\end{array}$ & $\begin{array}{l}24.54786 \\
24.77643 \\
24.99198 \\
25.19611 \\
25.39018\end{array}$ & $\begin{array}{l}22857 \\
21555 \\
20413 \\
19407 \\
18513\end{array}$ \\
\hline $\begin{array}{l}200 \\
210 \\
220 \\
230 \\
240\end{array}$ & $\begin{array}{l}3.61974 \\
3.64165 \\
3.66460 \\
3.68832 \\
3.71256\end{array}$ & $\begin{array}{l}2191 \\
2295 \\
2372 \\
2424 \\
2452\end{array}$ & $\begin{array}{l}3.51970 \\
3.52498 \\
3.53080 \\
3.53713 \\
3.54394\end{array}$ & $\begin{array}{l}528 \\
582 \\
633 \\
681 \\
723\end{array}$ & $\begin{array}{l}22.05561 \\
22.22746 \\
22.39158 \\
22.54867 \\
22.69935\end{array}$ & $\begin{array}{l}17185 \\
16412 \\
15709 \\
15068 \\
14481\end{array}$ & $\begin{array}{l}25.57531 \\
25.75245 \\
25.92238 \\
26.08580 \\
26.24329\end{array}$ & $\begin{array}{l}17714 \\
16993 \\
16342 \\
15749 \\
15205\end{array}$ \\
\hline $\begin{array}{l}250 \\
260 \\
270 \\
280 \\
290\end{array}$ & $\begin{array}{l}3.73708 \\
3.76170 \\
3.78625 \\
3.81058 \\
3.83459\end{array}$ & $\begin{array}{l}2462 \\
2455 \\
2433 \\
2401 \\
2357\end{array}$ & $\begin{array}{l}3.55117 \\
3.55879 \\
3.56676 \\
3.57504 \\
3.58358\end{array}$ & $\begin{array}{l}762 \\
797 \\
828 \\
854 \\
876\end{array}$ & $\begin{array}{l}22.84416 \\
22.98359 \\
23.11805 \\
23.24791 \\
23.37351\end{array}$ & $\begin{array}{l}13943 \\
13446 \\
12986 \\
12560 \\
12164\end{array}$ & $\begin{array}{l}26.39534 \\
26.54239 \\
26.68482 \\
26.82296 \\
26.95709\end{array}$ & $\begin{array}{l}14705 \\
14243 \\
13814 \\
13413 \\
13040\end{array}$ \\
\hline $\begin{array}{l}300 \\
310 \\
320 \\
330 \\
340\end{array}$ & $\begin{array}{l}3.35816 \\
3.88124 \\
3.90376 \\
3.92568 \\
3.94698\end{array}$ & $\begin{array}{l}2308 \\
2252 \\
2192 \\
2130 \\
2064\end{array}$ & $\begin{array}{l}3.59234 \\
3.60129 \\
3.61039 \\
3.61961 \\
3.62893\end{array}$ & $\begin{array}{l}895 \\
910 \\
922 \\
932 \\
938\end{array}$ & $\begin{array}{l}23.49515 \\
23.61309 \\
23.72757 \\
23.83881 \\
23.94700\end{array}$ & $\begin{array}{l}11794 \\
11448 \\
11124 \\
10819 \\
10533\end{array}$ & $\begin{array}{l}27.08749 \\
27.21438 \\
27.33796 \\
27.45842 \\
27.57593\end{array}$ & $\begin{array}{l}12689 \\
12358 \\
12046 \\
11751 \\
11472\end{array}$ \\
\hline $\begin{array}{l}350 \\
360 \\
370 \\
380 \\
390\end{array}$ & $\begin{array}{l}3.96762 \\
3.98761 \\
4.00693 \\
4.02560 \\
4.04362\end{array}$ & $\begin{array}{l}1999 \\
1932 \\
1867 \\
1802 \\
1737\end{array}$ & $\begin{array}{l}3.63831 \\
3.64774 \\
3.65719 \\
3.66664 \\
3.67607\end{array}$ & $\begin{array}{l}943 \\
945 \\
945 \\
943 \\
941\end{array}$ & $\begin{array}{l}24.05233 \\
24.15496 \\
24.25503 \\
24.35269 \\
24.44805\end{array}$ & $\begin{array}{r}10263 \\
10007 \\
9766 \\
9536 \\
9319\end{array}$ & $\begin{array}{l}27.69065 \\
27.80270 \\
27.91222 \\
28.01933 \\
28.12413\end{array}$ & $\begin{array}{l}11205 \\
10952 \\
10711 \\
10480 \\
10260\end{array}$ \\
\hline $\begin{array}{l}400 \\
450 \\
500 \\
550 \\
600\end{array}$ & $\begin{array}{l}4.06099 \\
4.13881 \\
4.20329 \\
4.25686 \\
4.30168\end{array}$ & $\begin{array}{l}7782 \\
6448 \\
5357 \\
4482 \\
3787\end{array}$ & $\begin{array}{l}3.68548 \\
3.73166 \\
3.77570 \\
3.81708 \\
3.85565\end{array}$ & $\begin{array}{l}4618 \\
4404 \\
4138 \\
3857 \\
3581\end{array}$ & $\begin{array}{l}24.54124 \\
24.97801 \\
25.37348 \\
25.73531 \\
26.06911\end{array}$ & $\begin{array}{l}43677 \\
39547 \\
36183 \\
33380 \\
31005\end{array}$ & $\begin{array}{l}28.22673 \\
28.70968 \\
29.14919 \\
29.55240 \\
29.92477\end{array}$ & $\begin{array}{l}48295 \\
43951 \\
40321 \\
37237 \\
34585\end{array}$ \\
\hline $\begin{array}{l}650 \\
700 \\
750 \\
800 \\
850\end{array}$ & $\begin{array}{l}4.33955 \\
4.37187 \\
4.39975 \\
4.42405 \\
4.44545\end{array}$ & $\begin{array}{l}3232 \\
2788 \\
2430 \\
2140 \\
1903\end{array}$ & $\begin{array}{l}3.89146 \\
3.92465 \\
3.95542 \\
3.98396 \\
4.01049\end{array}$ & $\begin{array}{l}3319 \\
3077 \\
2854 \\
2653 \\
2471\end{array}$ & $\begin{array}{l}26.37916 \\
26.66878 \\
26.94062 \\
27.19681 \\
27.43915\end{array}$ & $\begin{array}{l}28962 \\
27184 \\
25619 \\
24234 \\
22994\end{array}$ & $\begin{array}{l}30.27062 \\
30.59343 \\
30.89604 \\
31.18078 \\
31.44964\end{array}$ & $\begin{array}{l}32281 \\
30261 \\
28474 \\
26886 \\
25465\end{array}$ \\
\hline $\begin{array}{l}900 \\
950 \\
000 \\
050 \\
100\end{array}$ & $\begin{array}{l}4.46448 \\
4.48156 \\
4.49702 \\
4.51112 \\
4.52408\end{array}$ & \begin{tabular}{l|}
1708 \\
1546 \\
1410 \\
1296 \\
1200
\end{tabular} & $\begin{array}{l}4.03520 \\
4.05825 \\
4.07980 \\
4.10001 \\
4.11900\end{array}$ & $\begin{array}{l}2305 \\
2155 \\
2021 \\
1899 \\
1787\end{array}$ & $\begin{array}{l}27.66909 \\
27.88788 \\
28.09660 \\
28.29615 \\
28.48732\end{array}$ & $\begin{array}{l}21879 \\
20872 \\
19955 \\
19117 \\
18350\end{array}$ & $\begin{array}{l}31.70429 \\
31.94614 \\
32.17641 \\
32.39617 \\
32.60633\end{array}$ & $\begin{array}{l}24185 \\
23027 \\
21976 \\
21016 \\
20137\end{array}$ \\
\hline 150 & 4.53608 & 1118 & 4.13687 & 1687 & 28.67082 & 17642 & 32.80770 & 19329 \\
\hline
\end{tabular}


Table 2.072. TIT (Cont.)

\begin{tabular}{|l|l|l|l|l|}
\hline$K$ & $\frac{C_{p}^{0}}{R}$ & $\frac{\left(H^{0}-E_{0}^{0}\right)}{R T}$ & $\frac{-\left(F^{0}-E_{0}^{0}\right)}{R T}$ & $\frac{S^{0}}{R}$ \\
\hline
\end{tabular}

\begin{tabular}{|c|c|c|c|c|c|c|c|c|}
\hline 1200 & 4.54726 & 2033 & 4.15374 & 3107 & 28.84724 & 33373 & 33.00099 & 36480 \\
\hline 1300 & 4.56759 & 1820 & 4.18481 & 2800 & 29.18097 & 31117 & 33.36579 & 33917 \\
\hline 1400 & 4.58579 & 1659 & 4.21281 & 2543 & 29.49214 & 29154 & 33.70496 & 31696 \\
\hline 1500 & 4.60238 & 1534 & 4.23824 & 2324 & 29.78368 & 27428 & 34.02192 & 29753 \\
\hline 1600 & 4.61772 & 1434 & 4.26148 & 2138 & 30.05796 & 25900 & 34.31945 & 28038 \\
\hline 1700 & 4.63206 & 1354 & 4.28286 & 1978 & 30.31696 & 24537 & 34.59983 & 26515 \\
\hline 1800 & 4.64560 & 1290 & 4.30264 & 1840 & 30.562 .33 & 23313 & 34.86498 & 25152 \\
\hline 1900 & 4.65850 & 1236 & 4.32104 & 1718 & 30.79546 & 22208 & 35.11650 & 23927 \\
\hline 2000 & 4.67086 & 1191 & 4.33822 & 1613 & 31.01754 & 21206 & 35.35577 & 22818 \\
\hline 2100 & 4.68277 & 1154 & 4.35435 & 1519 & 31.22960 & 20292 & 35.58395 & 21811 \\
\hline 2200 & 4.69431 & 1122 & 4.36954 & 1436 & 31.43252 & 19455 & 35.80206 & 20892 \\
\hline 2300 & 4.70553 & 1094 & 4.38390 & 1363 & 31.62707 & 18687 & 36.01098 & 20050 \\
\hline 2400 & 4.71647 & 1069 & 4.39753 & 1298 & 31.1394 & 17978 & 36.21148 & 19275 \\
\hline 2500 & 4.72716 & 1048 & 4.41051 & 1238 & 31.99372 & 17323 & 36.40423 & 18561 \\
\hline 2600 & 4.73764 & 1030 & 4.42289 & 1185 & 32.16695 & 16714 & 36.58984 & 17899 \\
\hline 2700 & 4.74794 & 1012 & 4.43474 & 1136 & 32.33409 & 16149 & 36.76883 & 17286 \\
\hline 2800 & 4.75806 & 998 & 4.44610 & 1093 & 32.49558 & 15621 & 36.94169 & 16714 \\
\hline 2900 & 4.76804 & 983 & 4.45703 & 1053 & 32.65179 & 15128 & 37.10883 & 16181 \\
\hline 3000 & 4.77787 & 1930 & 4.46756 & 2000 & 32.80307 & 28898 & 37.27064 & 30897 \\
\hline 3200 & 4.79717 & 1888 & 4.48756 & 1877 & 33.09205 & 27262 & 37.57961 & 29140 \\
\hline 3400 & 4.81605 & 1850 & 4.50633 & 1772 & 33.36467 & 25808 & 37.87101 & 27580 \\
\hline 3600 & 4.83455 & 1816 & 4.52405 & 1683 & 33.62275 & 24506 & 38.14681 & 26188 \\
\hline 3800 & 4.85271 & 1787 & 4.54088 & 1604 & 33.86781 & 23333 & 38.40869 & 24937 \\
\hline 4000 & 4.87058 & 1760 & 4.55692 & 1535 & 34.10114 & 22271 & 38.65806 & 23806 \\
\hline 4200 & 4.88818 & 1735 & 4.57227 & 1476 & 34.32385 & 21304 & 38.89612 & 22780 \\
\hline 4400 & 4.90553 & 1711 & 4.58703 & 1422 & 34.53689 & 20422 & 39.12392 & 21844 \\
\hline 4600 & 4.92264 & 1689 & 4.60125 & 1374 & 34.74111 & 19612 & 39.34236 & 20986 \\
\hline $\begin{array}{l}4800 \\
5000\end{array}$ & $\begin{array}{l}4.93953 \\
4.95621\end{array}$ & 1668 & $\begin{array}{l}4.61499 \\
4.62831\end{array}$ & 1332 & $\begin{array}{l}34.93723 \\
35.12589\end{array}$ & 18866 & $\begin{array}{l}39.55222 \\
39.75420\end{array}$ & 20198 \\
\hline
\end{tabular}


Table 2.073. $\mathrm{CH}$

\begin{tabular}{|l|l|l|l|l|}
\hline${ }^{\circ} K$ & $\frac{C_{\dot{p}}^{\circ}}{R}$ & $\frac{\left(H^{\circ}-E_{0}^{0}\right)}{R T}$ & $\frac{-\left(F^{0}-E_{0}^{0}\right)}{R T}$ & $\frac{S^{0}}{R}$ \\
\hline
\end{tabular}

\begin{tabular}{|c|c|c|c|c|c|c|c|c|}
\hline $\begin{array}{l}50 \\
60 \\
70 \\
80 \\
90\end{array}$ & $\begin{array}{l}3.52178 \\
3.52536 \\
3.52451 \\
3.52244 \\
3.52023\end{array}$ & $\begin{array}{r}358 \\
-\quad 85 \\
-207 \\
-221 \\
-202\end{array}$ & $\begin{array}{l}3.29318 \\
3.33169 \\
3.35932 \\
3.37985 \\
3.39557\end{array}$ & $\begin{array}{l}3851 \\
2763 \\
2053 \\
1572 \\
1236\end{array}$ & $\begin{array}{l}12.42968 \\
13.03371 \\
13.54949 \\
13.99947 \\
14.39850\end{array}$ & $\begin{array}{l}60403 \\
51578 \\
44998 \\
39903 \\
35843\end{array}$ & $\begin{array}{l}15.72286 \\
16.36540 \\
16.90881 \\
17.37932 \\
17.79407\end{array}$ & $\begin{array}{l}64254 \\
54341 \\
47051 \\
41475 \\
37079\end{array}$ \\
\hline $\begin{array}{l}100 \\
110 \\
120 \\
130 \\
140\end{array}$ & $\begin{array}{l}3.51821 \\
3.51646 \\
3.51499 \\
3.51377 \\
3.51275\end{array}$ & $\begin{array}{l}-175 \\
-147 \\
-122 \\
-102 \\
-\quad 84\end{array}$ & $\begin{array}{l}3.40793 \\
3.41788 \\
3.42603 \\
3.43282 \\
3.43856\end{array}$ & $\begin{array}{l}995 \\
815 \\
679 \\
574 \\
493\end{array}$ & $\begin{array}{l}14.75693 \\
15.08221 \\
15.37997 \\
15.65448 \\
15.90909\end{array}$ & $\begin{array}{l}32528 \\
29776 \\
27451 \\
25461 \\
23741\end{array}$ & $\begin{array}{l}18.16486 \\
18.50009 \\
18.80600 \\
19.08730 \\
19.34765\end{array}$ & $\begin{array}{l}33523 \\
30591 \\
28130 \\
26035 \\
24234\end{array}$ \\
\hline $\begin{array}{l}150 \\
160 \\
170 \\
180 \\
190\end{array}$ & $\begin{array}{l}3.51191 \\
3.51122 \\
3.51065 \\
3.51018 \\
3.50979\end{array}$ & $\begin{array}{l}-\quad 69 \\
=\quad 57 \\
-\quad 47 \\
-\quad 39 \\
-\quad 31\end{array}$ & $\begin{array}{l}3.44349 \\
3.44773 \\
3.45146 \\
3.45473 \\
3.45764\end{array}$ & $\begin{array}{l}424 \\
373 \\
327 \\
291 \\
259\end{array}$ & $\begin{array}{l}16.14650 \\
16.36888 \\
16.57801 \\
16.77539 \\
16.96226\end{array}$ & $\begin{array}{l}22238 \\
20913 \\
19738 \\
18687 \\
17742\end{array}$ & $\begin{array}{l}19.58999 \\
19.81661 \\
20.02947 \\
20.23012 \\
20.41990\end{array}$ & $\begin{array}{l}22662 \\
21286 \\
20065 \\
18978 \\
18001\end{array}$ \\
\hline $\begin{array}{l}200 \\
210 \\
220 \\
230 \\
240\end{array}$ & $\begin{array}{l}3.50948 \\
3.50923 \\
3.50904 \\
3.50889 \\
3.50878\end{array}$ & $\begin{array}{l}-\quad 25 \\
-\quad 19 \\
-\quad 15 \\
-\quad 11 \\
-\quad 7\end{array}$ & $\begin{array}{l}3.46023 \\
3.46257 \\
3.46469 \\
3.46662 \\
3.46838\end{array}$ & $\begin{array}{l}234 \\
212 \\
193 \\
176 \\
161\end{array}$ & $\begin{array}{l}17.13968 \\
17.30857 \\
17.46969 \\
17.62374 \\
17.77132\end{array}$ & $\begin{array}{l}16889 \\
16112 \\
15405 \\
14758 \\
14162\end{array}$ & $\begin{array}{l}20.59991 \\
20.77114 \\
20.93438 \\
21.09036 \\
21.23970\end{array}$ & $\begin{array}{l}17123 \\
16324 \\
15598 \\
14934 \\
14323\end{array}$ \\
\hline $\begin{array}{l}250 \\
260 \\
270 \\
280 \\
290\end{array}$ & $\begin{array}{l}3.50871 \\
3.50868 \\
3.50870 \\
3.50874 \\
3.50884\end{array}$ & $\begin{array}{r}-\quad 3 \\
2 \\
4 \\
10 \\
14\end{array}$ & $\begin{array}{l}3.46999 \\
3.47147 \\
3.47285 \\
3.47413 \\
3.47533\end{array}$ & $\begin{array}{l}148 \\
138 \\
128 \\
120 \\
111\end{array}$ & $\begin{array}{l}17.91294 \\
18.04907 \\
18.18011 \\
18.30643 \\
18.42835\end{array}$ & $\begin{array}{l}13613 \\
13104 \\
12632 \\
12192 \\
11785\end{array}$ & $\begin{array}{l}21.38293 \\
21.52054 \\
21.65296 \\
21.78056 \\
21.90368\end{array}$ & $\begin{array}{l}13761 \\
13242 \\
12760 \\
12312 \\
11896\end{array}$ \\
\hline $\begin{array}{l}300 \\
310 \\
320 \\
330 \\
340\end{array}$ & $\begin{array}{l}3.50898 \\
3.50918 \\
3.50944 \\
3.50977 \\
3.51018\end{array}$ & $\begin{array}{l}20 \\
26 \\
33 \\
41 \\
50\end{array}$ & $\begin{array}{l}3.47644 \\
3.47750 \\
3.47850 \\
3.47945 \\
3.48034\end{array}$ & $\begin{array}{r}106 \\
100 \\
95 \\
89 \\
86\end{array}$ & $\begin{array}{l}18.54620 \\
18.66021 \\
18.77063 \\
18.87768 \\
18.98157\end{array}$ & $\begin{array}{l}11401 \\
11042 \\
10705 \\
10389 \\
10090\end{array}$ & $\begin{array}{l}22.02264 \\
22.13771 \\
22.24913 \\
22.35713 \\
22.46191\end{array}$ & $\begin{array}{l}11507 \\
11142 \\
10800 \\
10478 \\
10176\end{array}$ \\
\hline $\begin{array}{l}350 \\
360 \\
370 \\
380 \\
390\end{array}$ & $\begin{array}{l}3.51068 \\
3.51128 \\
3.51199 \\
3.51283 \\
3.51378\end{array}$ & $\begin{array}{r}60 \\
71 \\
84 \\
95 \\
112\end{array}$ & $\begin{array}{l}3.48120 \\
3.48203 \\
3.48282 \\
3.48360 \\
3.48437\end{array}$ & $\begin{array}{l}83 \\
79 \\
78 \\
77 \\
75\end{array}$ & $\begin{array}{l}19.08247 \\
19.18055 \\
19.27596 \\
19.36886 \\
19.45935\end{array}$ & $\begin{array}{l}9808 \\
9541 \\
9290 \\
9049 \\
8823\end{array}$ & $\begin{array}{l}22.56367 \\
22.66258 \\
22.75878 \\
22.85246 \\
22.94372\end{array}$ & $\begin{array}{l}9891 \\
9620 \\
9368 \\
9126 \\
8898\end{array}$ \\
\hline $\begin{array}{l}400 \\
450 \\
500 \\
550 \\
600\end{array}$ & $\begin{array}{l}3.51490 \\
3.52290 \\
3.53564 \\
3.55354 \\
3.57643\end{array}$ & $\begin{array}{r}800 \\
1274 \\
1790 \\
2289 \\
2732\end{array}$ & $\begin{array}{l}3.48512 \\
3.48883 \\
3.49283 \\
3.49750 \\
3.50309\end{array}$ & $\begin{array}{l}371 \\
400 \\
467 \\
559 \\
666\end{array}$ & $\begin{array}{l}19.54758 \\
19.95828 \\
20.32607 \\
20.65918 \\
20.96374\end{array}$ & $\begin{array}{l}41070 \\
36779 \\
33311 \\
30456 \\
28065\end{array}$ & $\begin{array}{l}23.03270 \\
23.44711 \\
23.81890 \\
24.15668 \\
24.46683\end{array}$ & $\begin{array}{l}41441 \\
37179 \\
33778 \\
31015 \\
28731\end{array}$ \\
\hline $\begin{array}{l}650 \\
700 \\
750 \\
800 \\
850\end{array}$ & $\begin{array}{l}3.60375 \\
3.63471 \\
3.66839 \\
3.70397 \\
3.74063\end{array}$ & $\begin{array}{l}3096 \\
3368 \\
3558 \\
3666 \\
3711\end{array}$ & $\begin{array}{l}3.50975 \\
3.51755 \\
3.52647 \\
3.53645 \\
3.54738\end{array}$ & $\begin{array}{r}780 \\
892 \\
998 \\
1093 \\
1177\end{array}$ & $\begin{array}{l}21.24439 \\
21.50476 \\
21.74775 \\
21.97566 \\
22.19039\end{array}$ & $\begin{array}{l}26037 \\
24299 \\
22791 \\
21473 \\
20308\end{array}$ & $\begin{array}{l}24.75414 \\
25.02231 \\
25.27422 \\
25.51211 \\
25.73777\end{array}$ & $\begin{array}{l}26817 \\
25191 \\
23789 \\
22566 \\
21485\end{array}$ \\
\hline $\begin{array}{r}900 \\
950 \\
1000 \\
1050 \\
1100\end{array}$ & $\begin{array}{l}3.77774 \\
3.81477 \\
3.85129 \\
3.88699 \\
3.92164\end{array}$ & $\begin{array}{l}3703 \\
3652 \\
3570 \\
3465 \\
3345\end{array}$ & $\begin{array}{l}3.55915 \\
3.57162 \\
3.58469 \\
3.59825 \\
3.61216\end{array}$ & $\begin{array}{l}1247 \\
1307 \\
1356 \\
1391 \\
1419\end{array}$ & $\begin{array}{l}22.39347 \\
22.58624 \\
22.76977 \\
22.94499 \\
23.11271\end{array}$ & $\begin{array}{l}19277 \\
18353 \\
17522 \\
16772 \\
16088\end{array}$ & $\begin{array}{l}25.95262 \\
26.15786 \\
26.35446 \\
26.54324 \\
26.72487\end{array}$ & $\begin{array}{l}20524 \\
19660 \\
18878 \\
18163 \\
17507\end{array}$ \\
\hline 150 & 3.95509 & 3216 & 3.62635 & 1438 & 23.27359 & 15463 & 26.89994 & 16901 \\
\hline
\end{tabular}


Table 2.073. CH (Cont.)

\begin{tabular}{|l|l|l|l|l|}
\hline${ }^{\circ} K$ & $\frac{C_{p}^{0}}{R}$ & $\frac{\left(H^{0}-E_{0}^{0}\right)}{R T}$ & $\frac{-\left(F^{0}-E_{0}^{0}\right)}{R T}$ & $\frac{S^{\circ}}{R}$ \\
\hline
\end{tabular}

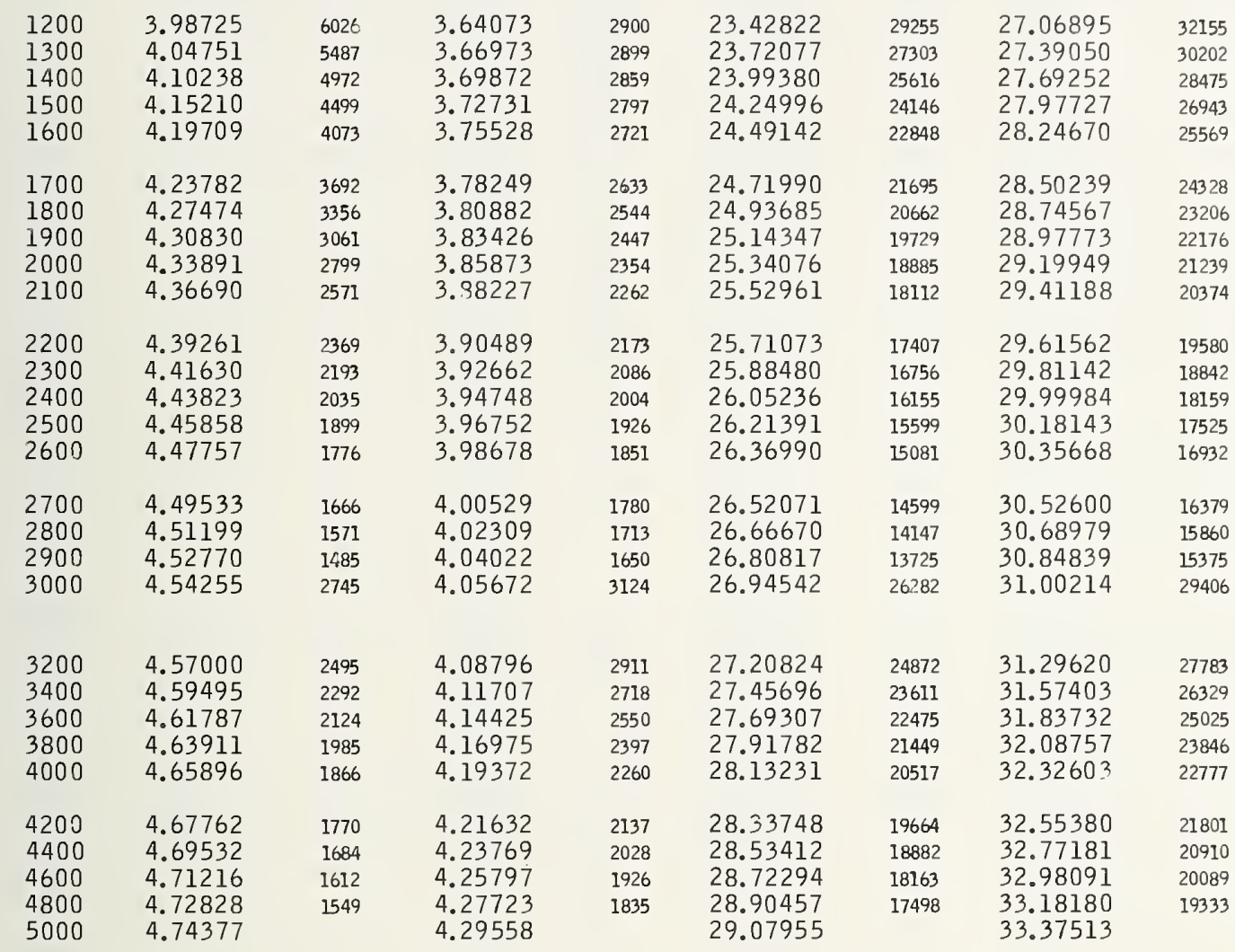


Table 2.074. CD

\begin{tabular}{|c|c|c|c|c|c|c|c|c|}
\hline${ }^{\circ} \mathrm{K}$ & $\frac{C_{p}^{0}}{R}$ & & $\frac{\left(H^{0}-E_{0}^{0}\right)}{R T}$ & & $\frac{-l F^{\circ}-}{R T}$ & & $\frac{S^{\circ}}{R}$ & \\
\hline $\begin{array}{l}50 \\
60 \\
70 \\
80 \\
90\end{array}$ & $\begin{array}{l}3.59427 \\
3.57280 \\
3.55758 \\
3.54664 \\
3.53861\end{array}$ & $\begin{array}{l}-2147 \\
-1522 \\
-1094 \\
-803 \\
-601\end{array}$ & $\begin{array}{l}3.48637 \\
3.50247 \\
3.51136 \\
3.51641 \\
3.51931\end{array}$ & $\begin{array}{r}1610 \\
889 \\
505 \\
290 \\
162\end{array}$ & $\begin{array}{l}12.92815 \\
13.56537 \\
14.10601 \\
14.57526 \\
14.98961\end{array}$ & $\begin{array}{l}63722 \\
54064 \\
46925 \\
41435 \\
37089\end{array}$ & $\begin{array}{l}16.41452 \\
17.06784 \\
17.61737 \\
18.09167 \\
18.50892\end{array}$ & $\begin{array}{l}65332 \\
54953 \\
47430 \\
41725 \\
37251\end{array}$ \\
\hline $\begin{array}{l}100 \\
110 \\
120 \\
130 \\
140\end{array}$ & $\begin{array}{l}3.53260 \\
3.52800 \\
3.52444 \\
3.52163 \\
3.51939\end{array}$ & $\begin{array}{l}-460 \\
-356 \\
-281 \\
-224 \\
-180\end{array}$ & $\begin{array}{l}3.52093 \\
3.52177 \\
3.52213 \\
3.52220 \\
3.52208\end{array}$ & $\begin{array}{r}84 \\
36 \\
-\quad 7 \\
-\quad 12 \\
-\quad 25\end{array}$ & $\begin{array}{l}15.36050 \\
15.69611 \\
16.00257 \\
16.28449 \\
16.54552\end{array}$ & $\begin{array}{l}33561 \\
30646 \\
28192 \\
26103 \\
24300\end{array}$ & $\begin{array}{l}18.88143 \\
19.21788 \\
19.52470 \\
19.80669 \\
20.06760\end{array}$ & $\begin{array}{l}33645 \\
30682 \\
28199 \\
26091 \\
24275\end{array}$ \\
\hline $\begin{array}{l}150 \\
160 \\
170 \\
180 \\
190\end{array}$ & $\begin{array}{l}3.51759 \\
3.51612 \\
3.51494 \\
3.51396 \\
3.51317\end{array}$ & $\begin{array}{l}-147 \\
-\quad 118 \\
-\quad 98 \\
-\quad 79 \\
-\quad 62\end{array}$ & $\begin{array}{l}3.52183 \\
3.52152 \\
3.52117 \\
3.52080 \\
3.52041\end{array}$ & $\begin{array}{l}-31 \\
-\quad 35 \\
-\quad 37 \\
-\quad 39 \\
-\quad 37\end{array}$ & $\begin{array}{l}16.78852 \\
17.01580 \\
17.22928 \\
17.43052 \\
17.62089\end{array}$ & $\begin{array}{l}22728 \\
21348 \\
20124 \\
19037 \\
18056\end{array}$ & $\begin{array}{l}20.31035 \\
20.53732 \\
20.75045 \\
20.95132 \\
21.14130\end{array}$ & $\begin{array}{l}22697 \\
21313 \\
20087 \\
18998 \\
18019\end{array}$ \\
\hline $\begin{array}{l}200 \\
210 \\
220 \\
230 \\
240\end{array}$ & $\begin{array}{l}3.51255 \\
3.51208 \\
3.51175 \\
3.51156 \\
3.51153\end{array}$ & $\begin{array}{l}-\quad 47 \\
-\quad 33 \\
-\quad 19 \\
-\quad 3 \\
14\end{array}$ & $\begin{array}{l}3.52004 \\
3.51966 \\
3.51931 \\
3.51899 \\
3.51867\end{array}$ & $\begin{array}{l}-\quad 38 \\
-\quad 35 \\
-\quad 32 \\
-\quad 32 \\
-\quad 28\end{array}$ & $\begin{array}{l}17.80145 \\
17.97319 \\
18.13692 \\
18.29333 \\
18.44309\end{array}$ & $\begin{array}{l}17174 \\
16373 \\
15641 \\
14976 \\
14364\end{array}$ & $\begin{array}{l}21.32149 \\
21.49285 \\
21.65623 \\
21.81232 \\
21.96176\end{array}$ & $\begin{array}{l}17136 \\
16338 \\
15609 \\
14944 \\
14336\end{array}$ \\
\hline $\begin{array}{l}250 \\
260 \\
270 \\
280 \\
290\end{array}$ & $\begin{array}{l}3.51167 \\
3.51200 \\
3.51252 \\
3.51328 \\
3.51428\end{array}$ & $\begin{array}{r}33 \\
52 \\
76 \\
100 \\
130\end{array}$ & $\begin{array}{l}3.51839 \\
3.51814 \\
3.51792 \\
3.51774 \\
3.51759\end{array}$ & $\begin{array}{l}-\quad 25 \\
-\quad 22 \\
-\quad 18 \\
-\quad 15 \\
-\quad 8\end{array}$ & $\begin{array}{l}18.58673 \\
18.72473 \\
18.85750 \\
18.98542 \\
19.10888\end{array}$ & $\begin{array}{l}13800 \\
1327 \\
12792 \\
12346 \\
11924\end{array}$ & $\begin{array}{l}22.10512 \\
22.24287 \\
22.37542 \\
22.50316 \\
22.62647\end{array}$ & $\begin{array}{l}13775 \\
13255 \\
12774 \\
12331 \\
11916\end{array}$ \\
\hline $\begin{array}{l}300 \\
310 \\
320 \\
330 \\
340\end{array}$ & $\begin{array}{l}3.51558 \\
3.51716 \\
3.51905 \\
3.52127 \\
3.52385\end{array}$ & $\begin{array}{l}158 \\
189 \\
222 \\
258 \\
293\end{array}$ & $\begin{array}{l}3.51751 \\
3.51748 \\
3.51750 \\
3.51757 \\
3.51772\end{array}$ & $\begin{array}{r}-\quad 3 \\
2 \\
7 \\
15 \\
21\end{array}$ & $\begin{array}{l}19.22812 \\
19.34346 \\
19.45512 \\
19.56337 \\
19.66839\end{array}$ & $\begin{array}{l}11534 \\
11166 \\
10825 \\
10502 \\
10197\end{array}$ & $\begin{array}{l}22.74563 \\
22.86094 \\
22.97262 \\
23.08094 \\
23.18611\end{array}$ & $\begin{array}{l}11531 \\
11168 \\
10832 \\
10517 \\
10218\end{array}$ \\
\hline $\begin{array}{l}350 \\
360 \\
370 \\
380 \\
390\end{array}$ & $\begin{array}{l}3.52678 \\
3.53008 \\
3.53375 \\
3.53781 \\
3.54224\end{array}$ & $\begin{array}{l}330 \\
367 \\
406 \\
443 \\
481\end{array}$ & $\begin{array}{l}3.51793 \\
3.51823 \\
3.51860 \\
3.51904 \\
3.51958\end{array}$ & $\begin{array}{l}30 \\
37 \\
44 \\
54 \\
62\end{array}$ & $\begin{array}{l}19.77036 \\
19.86947 \\
19.96587 \\
20.05970 \\
20.15112\end{array}$ & $\begin{array}{l}9911 \\
9640 \\
9383 \\
9142 \\
8913\end{array}$ & $\begin{array}{l}23.28829 \\
23.38770 \\
23.48447 \\
23.57874 \\
23.67070\end{array}$ & $\begin{array}{l}9941 \\
9677 \\
9427 \\
9196 \\
8975\end{array}$ \\
\hline $\begin{array}{l}400 \\
450 \\
500 \\
550 \\
600\end{array}$ & $\begin{array}{l}3.54705 \\
3.57648 \\
3.61374 \\
3.65679 \\
3.70351\end{array}$ & $\begin{array}{l}2943 \\
3726 \\
4305 \\
4672 \\
4849\end{array}$ & $\begin{array}{l}3.52020 \\
3.52475 \\
3.53172 \\
3.54110 \\
3.55267\end{array}$ & $\begin{array}{r}455 \\
697 \\
938 \\
1157 \\
1346\end{array}$ & $\begin{array}{l}20.24025 \\
20.65510 \\
21.02681 \\
21.36384 \\
21.67244\end{array}$ & $\begin{array}{l}41485 \\
37171 \\
33703 \\
30860 \\
28489\end{array}$ & $\begin{array}{l}23.76045 \\
24.17985 \\
24.55853 \\
24.90494 \\
25.22511\end{array}$ & $\begin{array}{l}41940 \\
37868 \\
34641 \\
32017 \\
29835\end{array}$ \\
\hline $\begin{array}{l}650 \\
700 \\
750 \\
800 \\
850\end{array}$ & $\begin{array}{l}3.75200 \\
3.80076 \\
3.84867 \\
3.89498 \\
3.93923\end{array}$ & $\begin{array}{l}4876 \\
4791 \\
4631 \\
4425 \\
4190\end{array}$ & $\begin{array}{l}3.56613 \\
3.58116 \\
3.59740 \\
3.61456 \\
3.63237\end{array}$ & $\begin{array}{l}1503 \\
1624 \\
1716 \\
1781 \\
1823\end{array}$ & $\begin{array}{l}21.95733 \\
22.22214 \\
22.46976 \\
22.70248 \\
22.92214\end{array}$ & $\begin{array}{l}26481 \\
24762 \\
23272 \\
21966 \\
20813\end{array}$ & $\begin{array}{l}25.52346 \\
25.80330 \\
26.06716 \\
26.31704 \\
26.55451\end{array}$ & $\begin{array}{l}27984 \\
26386 \\
24988 \\
23747 \\
22636\end{array}$ \\
\hline $\begin{array}{l}900 \\
950 \\
000 \\
050 \\
100\end{array}$ & $\begin{array}{l}3.98113 \\
4.02057 \\
4.05754 \\
4.09209 \\
4.12434\end{array}$ & $\begin{array}{l}3944 \\
3697 \\
3455 \\
3225 \\
3008\end{array}$ & $\begin{array}{l}3.65060 \\
3.66903 \\
3.68755 \\
3.70599 \\
3.72428\end{array}$ & $\begin{array}{l}1843 \\
1852 \\
1844 \\
1829 \\
1807\end{array}$ & $\begin{array}{l}23.13027 \\
23.32815 \\
23.51682 \\
23.69718 \\
23.87000\end{array}$ & $\begin{array}{l}19788 \\
18867 \\
18036 \\
17282 \\
16595\end{array}$ & $\begin{array}{l}26.78087 \\
26.99718 \\
27.20437 \\
27.40317 \\
27.59428\end{array}$ & $\begin{array}{l}21631 \\
20719 \\
19880 \\
19111 \\
18402\end{array}$ \\
\hline 5 & 4.15442 & 2803 & 3.74235 & 1775 & 24.03595 & 15966 & 27.77830 & 17741 \\
\hline
\end{tabular}


Table 2.074. CD (Cont.)

\begin{tabular}{|c|c|c|c|c|c|c|c|c|}
\hline${ }^{\circ} \mathrm{K}$ & $\frac{C_{p}^{0}}{R}$ & & $\frac{\left(H^{\circ}-E_{0}^{0}\right)}{R T}$ & & $\frac{-\left(F^{\circ}-E\right.}{R T}$ & & $\frac{S^{\circ}}{R}$ & \\
\hline $\begin{array}{l}1200 \\
1300 \\
1400 \\
1500 \\
1600\end{array}$ & $\begin{array}{l}4.18245 \\
4.23298 \\
4.27704 \\
4.31568 \\
4.34975\end{array}$ & $\begin{array}{l}5053 \\
4406 \\
3864 \\
3407 \\
3023\end{array}$ & $\begin{array}{l}3.76010 \\
3.79458 \\
3.82750 \\
3.85879 \\
3.88844\end{array}$ & $\begin{array}{l}3448 \\
3292 \\
3129 \\
2965 \\
2803\end{array}$ & $\begin{array}{l}24.19561 \\
24.49794 \\
24.78036 \\
25.04553 \\
25.29552\end{array}$ & $\begin{array}{l}30233 \\
28242 \\
26517 \\
24999 \\
23658\end{array}$ & $\begin{array}{l}27.95571 \\
28.29252 \\
28.60786 \\
28.90432 \\
29.18396\end{array}$ & $\begin{array}{l}33681 \\
31534 \\
29646 \\
27964 \\
26461\end{array}$ \\
\hline $\begin{array}{l}1700 \\
1800 \\
1900 \\
2000 \\
2100\end{array}$ & $\begin{array}{l}4.37998 \\
4.40701 \\
4.43132 \\
4.45334 \\
4.47340\end{array}$ & $\begin{array}{l}2703 \\
2431 \\
2202 \\
2006 \\
1840\end{array}$ & $\begin{array}{l}3.91647 \\
3.94300 \\
3.96806 \\
3.99178 \\
4.01425\end{array}$ & $\begin{array}{l}2653 \\
2506 \\
2372 \\
2247 \\
2128\end{array}$ & $\begin{array}{l}25.53210 \\
25.75671 \\
25.97058 \\
26.17473 \\
26.37003\end{array}$ & $\begin{array}{l}22461 \\
21387 \\
20415 \\
19530 \\
18724\end{array}$ & $\begin{array}{l}29.44857 \\
29.69971 \\
29.93864 \\
30.16651 \\
30.38428\end{array}$ & $\begin{array}{l}25114 \\
23893 \\
22787 \\
21777 \\
20852\end{array}$ \\
\hline $\begin{array}{l}2200 \\
2300 \\
2400 \\
2500 \\
2600\end{array}$ & $\begin{array}{l}4.49180 \\
4.50877 \\
4.52452 \\
4.53919 \\
4.55295\end{array}$ & $\begin{array}{l}1697 \\
1575 \\
1467 \\
1376 \\
1294\end{array}$ & $\begin{array}{l}4.03553 \\
4.05575 \\
4.07495 \\
4.09324 \\
4.11067\end{array}$ & $\begin{array}{l}2022 \\
1920 \\
1829 \\
1743 \\
166 ?\end{array}$ & $\begin{array}{l}26.55727 \\
26.73711 \\
26.91013 \\
27.07685 \\
27.23773\end{array}$ & $\begin{array}{l}17984 \\
17302 \\
16672 \\
16088 \\
15545\end{array}$ & $\begin{array}{l}30.59280 \\
30.79286 \\
30.98508 \\
31.17009 \\
31.34840\end{array}$ & $\begin{array}{l}20006 \\
19222 \\
18501 \\
17831 \\
17207\end{array}$ \\
\hline $\begin{array}{l}2700 \\
2800 \\
2900 \\
3000\end{array}$ & $\begin{array}{l}4.55589 \\
4.57813 \\
4.58974 \\
4.60080\end{array}$ & $\begin{array}{l}1224 \\
1161 \\
1106 \\
2071\end{array}$ & $\begin{array}{l}4.12729 \\
4.14316 \\
4.15836 \\
4.17293\end{array}$ & $\begin{array}{l}1587 \\
1520 \\
1457 \\
2740\end{array}$ & $\begin{array}{l}27.39318 \\
27.54358 \\
27.68924 \\
27.83045\end{array}$ & $\begin{array}{l}15040 \\
14566 \\
14121 \\
27020\end{array}$ & $\begin{array}{l}31.52047 \\
31.68674 \\
31.84760 \\
32.00338\end{array}$ & $\begin{array}{l}16627 \\
16086 \\
15578 \\
29760\end{array}$ \\
\hline $\begin{array}{l}3200 \\
3400 \\
3600 \\
3800 \\
4000\end{array}$ & $\begin{array}{l}4.62151 \\
4.64065 \\
4.65853 \\
4.67539 \\
4.69138\end{array}$ & $\begin{array}{l}1914 \\
1788 \\
1686 \\
1599 \\
1528\end{array}$ & $\begin{array}{l}4.20033 \\
4.22568 \\
4.24923 \\
4.27122 \\
4.29183\end{array}$ & $\begin{array}{l}2535 \\
2355 \\
2199 \\
2061 \\
1940\end{array}$ & $\begin{array}{l}28.10065 \\
28.35606 \\
28.59828 \\
28.82862 \\
29.04823\end{array}$ & $\begin{array}{l}25541 \\
24222 \\
23034 \\
21961 \\
20987\end{array}$ & $\begin{array}{l}32.30098 \\
32.58174 \\
32.84751 \\
33.09984 \\
33.34006\end{array}$ & $\begin{array}{l}28076 \\
26577 \\
25233 \\
24022 \\
22927\end{array}$ \\
\hline $\begin{array}{l}4200 \\
4400 \\
4600 \\
4800 \\
5000\end{array}$ & $\begin{array}{l}4.70666 \\
4.72134 \\
4.73551 \\
4.74923 \\
4.76256\end{array}$ & $\begin{array}{l}1468 \\
1417 \\
1372 \\
1333\end{array}$ & $\begin{array}{l}4.31123 \\
4.32953 \\
4.34688 \\
4.36336 \\
4.37906\end{array}$ & $\begin{array}{l}1830 \\
1735 \\
1648 \\
1570\end{array}$ & $\begin{array}{l}29.25810 \\
29.45909 \\
29.65193 \\
29.83728 \\
30.01573\end{array}$ & $\begin{array}{l}20099 \\
19284 \\
18535 \\
17845\end{array}$ & $\begin{array}{l}33.56933 \\
33.78862 \\
33.99881 \\
34.20064 \\
34.39479\end{array}$ & $\begin{array}{l}21929 \\
21019 \\
20183 \\
19415\end{array}$ \\
\hline
\end{tabular}


Table 2.075. CT

\begin{tabular}{|c|c|c|c|c|c|c|c|c|}
\hline${ }^{\circ} \mathrm{K}$ & $\frac{C_{p}^{0}}{R}$ & & $\frac{\left(H^{\circ}-E_{0}^{0}\right)}{R T}$ & & $\frac{-\left(F^{0}-E\right.}{R T}$ & & $\frac{S^{0}}{R}$ & \\
\hline $\begin{array}{l}50 \\
60 \\
70 \\
80 \\
90\end{array}$ & $\begin{array}{l}3.61066 \\
3.58353 \\
3.56504 \\
3.55208 \\
3.54273\end{array}$ & $\begin{array}{l}-2713 \\
-1849 \\
-1296 \\
-935 \\
-693\end{array}$ & $\begin{array}{l}3.47237 \\
3.49301 \\
3.50454 \\
3.51125 \\
3.51523\end{array}$ & $\begin{array}{r}2064 \\
1153 \\
671 \\
398 \\
239\end{array}$ & $\begin{array}{l}13.37350 \\
14.00861 \\
14.54801 \\
15.01645 \\
15.43026\end{array}$ & $\begin{array}{l}63511 \\
53940 \\
46844 \\
41381 \\
37052\end{array}$ & $\begin{array}{l}16.84587 \\
17.50162 \\
18.05255 \\
18.52770 \\
18.94549\end{array}$ & $\begin{array}{l}65575 \\
55093 \\
47515 \\
41779 \\
37291\end{array}$ \\
\hline $\begin{array}{l}100 \\
110 \\
120 \\
130 \\
140\end{array}$ & $\begin{array}{l}3.53580 \\
3.53056 \\
3.52653 \\
3.52336 \\
3.52086\end{array}$ & $\begin{array}{l}-524 \\
-403 \\
-317 \\
-250 \\
-201\end{array}$ & $\begin{array}{l}3.51762 \\
3.51902 \\
3.51981 \\
3.52020 \\
3.52033\end{array}$ & $\begin{array}{r}140 \\
79 \\
39 \\
13 \\
-\quad 3\end{array}$ & $\begin{array}{l}15.80078 \\
16.13610 \\
16.44234 \\
16.72409 \\
16.98497\end{array}$ & $\begin{array}{l}33532 \\
30624 \\
28175 \\
26088 \\
24289\end{array}$ & $\begin{array}{l}19.31840 \\
19.65512 \\
19.96215 \\
20.24429 \\
20.50530\end{array}$ & $\begin{array}{l}33672 \\
30703 \\
28214 \\
26101 \\
24286\end{array}$ \\
\hline $\begin{array}{l}150 \\
160 \\
170 \\
180 \\
190\end{array}$ & $\begin{array}{l}3.51885 \\
3.51723 \\
3.51595 \\
3.51495 \\
3.51422\end{array}$ & $\begin{array}{l}-162 \\
-128 \\
-\quad 100 \\
-\quad 73 \\
-\quad 49\end{array}$ & $\begin{array}{l}3.52030 \\
3.52016 \\
3.51995 \\
3.51970 \\
3.51943\end{array}$ & $\begin{array}{l}-\quad 14 \\
-\quad 21 \\
-\quad 25 \\
-\quad 27 \\
-\quad 28\end{array}$ & $\begin{array}{l}17.22786 \\
17.45504 \\
17.66845 \\
17.86964 \\
18.05993\end{array}$ & $\begin{array}{l}22718 \\
21341 \\
20119 \\
19029 \\
18051\end{array}$ & $\begin{array}{l}20.74816 \\
20.97520 \\
21.18840 \\
21.38934 \\
21.57936\end{array}$ & $\begin{array}{l}22704 \\
21320 \\
20094 \\
19002 \\
18023\end{array}$ \\
\hline $\begin{array}{l}200 \\
210 \\
220 \\
230 \\
240\end{array}$ & $\begin{array}{l}3.51373 \\
3.51352 \\
3.51357 \\
3.51394 \\
3.51465\end{array}$ & $\begin{array}{r}-\quad 21 \\
5 \\
37 \\
71 \\
109\end{array}$ & $\begin{array}{l}3.51915 \\
3.51889 \\
3.51864 \\
3.51843 \\
3.51825\end{array}$ & $\begin{array}{l}-26 \\
-\quad 25 \\
-\quad 21 \\
-\quad 18 \\
-\quad 11\end{array}$ & $\begin{array}{l}18.24044 \\
18.41214 \\
18.57583 \\
18.73224 \\
18.88199\end{array}$ & $\begin{array}{l}17170 \\
16369 \\
15641 \\
14975 \\
14360\end{array}$ & $\begin{array}{l}21.75959 \\
21.93103 \\
22.09447 \\
22.25067 \\
22.40024\end{array}$ & $\begin{array}{l}17144 \\
16344 \\
15620 \\
14957 \\
14349\end{array}$ \\
\hline $\begin{array}{l}250 \\
260 \\
270 \\
280 \\
290\end{array}$ & $\begin{array}{l}3.51574 \\
3.51722 \\
3.51915 \\
3.52154 \\
3.52442\end{array}$ & $\begin{array}{l}148 \\
193 \\
239 \\
288 \\
338\end{array}$ & $\begin{array}{l}3.51814 \\
3.51807 \\
3.51807 \\
3.51815 \\
3.51832\end{array}$ & $\begin{array}{r}-7 \\
8 \\
17 \\
25\end{array}$ & $\begin{array}{l}19.02559 \\
19.16359 \\
19.29636 \\
19.42430 \\
19.54776\end{array}$ & $\begin{array}{l}13800 \\
13277 \\
12794 \\
12346 \\
11927\end{array}$ & $\begin{array}{l}22.54373 \\
22.68166 \\
22.81443 \\
22.94245 \\
23.06608\end{array}$ & $\begin{array}{l}13793 \\
13277 \\
12802 \\
12363 \\
11952\end{array}$ \\
\hline $\begin{array}{l}300 \\
310 \\
320 \\
330 \\
340\end{array}$ & $\begin{array}{l}3.52780 \\
3.53170 \\
3.53614 \\
3.54109 \\
3.54658\end{array}$ & $\begin{array}{l}390 \\
444 \\
495 \\
549 \\
599\end{array}$ & $\begin{array}{l}3.51857 \\
3.51893 \\
3.51940 \\
3.51998 \\
3.52068\end{array}$ & $\begin{array}{l}36 \\
47 \\
58 \\
70 \\
83\end{array}$ & $\begin{array}{l}19.66703 \\
19.78241 \\
19.89414 \\
20.00245 \\
20.10754\end{array}$ & $\begin{array}{l}11538 \\
11173 \\
10831 \\
10509 \\
10207\end{array}$ & $\begin{array}{l}23.18560 \\
23.30134 \\
23.41354 \\
23.52243 \\
23.62822\end{array}$ & $\begin{array}{l}11574 \\
11220 \\
10889 \\
10579 \\
10290\end{array}$ \\
\hline $\begin{array}{l}350 \\
360 \\
370 \\
380 \\
390\end{array}$ & $\begin{array}{l}3.55257 \\
3.55907 \\
3.56605 \\
3.57350 \\
3.58139\end{array}$ & $\begin{array}{l}650 \\
698 \\
745 \\
789 \\
833\end{array}$ & $\begin{array}{l}3.52151 \\
3.52246 \\
3.52354 \\
3.52475 \\
3.52611\end{array}$ & $\begin{array}{r}95 \\
108 \\
121 \\
136 \\
148\end{array}$ & $\begin{array}{l}20.20961 \\
20.30883 \\
20.40535 \\
20.49933 \\
20.59090\end{array}$ & $\begin{array}{l}9922 \\
9652 \\
9398 \\
9157 \\
8931\end{array}$ & $\begin{array}{l}23.73112 \\
23.83129 \\
23.92889 \\
24.02408 \\
24.11701\end{array}$ & $\begin{array}{r}10017 \\
9760 \\
9519 \\
9293 \\
9079\end{array}$ \\
\hline $\begin{array}{l}400 \\
450 \\
500 \\
550 \\
600\end{array}$ & $\begin{array}{l}3.58972 \\
3.63662 \\
3.68980 \\
3.74603 \\
3.80276\end{array}$ & $\begin{array}{l}4690 \\
5318 \\
5623 \\
5673 \\
5545\end{array}$ & $\begin{array}{l}3.52759 \\
3.53702 \\
3.54960 \\
3.56489 \\
3.58236\end{array}$ & $\begin{array}{r}943 \\
1258 \\
1529 \\
1747 \\
1910\end{array}$ & $\begin{array}{l}20.68021 \\
21.09621 \\
21.46949 \\
21.80851 \\
22.11943\end{array}$ & $\begin{array}{l}41600 \\
37328 \\
33902 \\
31092 \\
28749\end{array}$ & $\begin{array}{l}24.20780 \\
24.63323 \\
25.01909 \\
25.37340 \\
25.70179\end{array}$ & $\begin{array}{l}42543 \\
38586 \\
35431 \\
32839 \\
30659\end{array}$ \\
\hline $\begin{array}{l}650 \\
700 \\
750 \\
800 \\
850\end{array}$ & $\begin{array}{l}3.85821 \\
3.91121 \\
3.96117 \\
4.00775 \\
4.05091\end{array}$ & $\begin{array}{l}5300 \\
4996 \\
4658 \\
4316 \\
3982\end{array}$ & $\begin{array}{l}3.60146 \\
3.62171 \\
3.64269 \\
3.66407 \\
3.68557\end{array}$ & $\begin{array}{l}2025 \\
2098 \\
2138 \\
2150 \\
2142\end{array}$ & $\begin{array}{l}22.40692 \\
22.67457 \\
22.92513 \\
23.16091 \\
23.38369\end{array}$ & $\begin{array}{l}26765 \\
25056 \\
23578 \\
22278 \\
21127\end{array}$ & $\begin{array}{l}26.00838 \\
26.29628 \\
26.56782 \\
26.82498 \\
27.06926\end{array}$ & $\begin{array}{l}28790 \\
27154 \\
25716 \\
24428 \\
23269\end{array}$ \\
\hline $\begin{array}{r}900 \\
950 \\
1000 \\
1050 \\
1100\end{array}$ & $\begin{array}{l}4.09073 \\
4.12739 \\
4.16110 \\
4.1 .9210 \\
4.22062\end{array}$ & $\begin{array}{l}3666 \\
337 ? \\
3100 \\
2852 \\
2627\end{array}$ & $\begin{array}{l}3.70699 \\
3.72816 \\
3.74898 \\
3.76935 \\
3.78922\end{array}$ & $\begin{array}{l}2117 \\
2082 \\
2037 \\
1987 \\
1934\end{array}$ & $\begin{array}{l}23.59496 \\
23.79596 \\
23.98771 \\
24.17112 \\
24.34694\end{array}$ & $\begin{array}{l}20100 \\
19175 \\
18341 \\
17582 \\
16886\end{array}$ & $\begin{array}{l}27.30195 \\
27.52412 \\
27.73669 \\
27.94047 \\
28.13616\end{array}$ & $\begin{array}{l}22217 \\
21257 \\
20378 \\
19569 \\
18820\end{array}$ \\
\hline 1150 & 4.24689 & 2425 & 3.80856 & 1878 & 24.51580 & 16249 & 28.32436 & 18127 \\
\hline
\end{tabular}


Table 2.075. CT (Cont.)

\begin{tabular}{|l|l|l|l|l|}
\hline$K$ & $\frac{C_{p}^{0}}{R}$ & $\frac{\left(H^{0}-E_{0}^{0}\right)}{R T}$ & $\frac{-\left(F^{0}-E_{0}^{0}\right)}{R T}$ & $\frac{S^{0}}{R}$ \\
\hline
\end{tabular}

$\begin{array}{lllllllll}1200 & 4.27114 & 4315 & 3.82734 & 3584 & 24.67829 & 30777 & 28.50563 & 34361 \\ 1300 & 4.31429 & 3718 & 3.86318 & 3358 & 24.98606 & 28754 & 28.84924 & 32112 \\ 1400 & 4.35147 & 3230 & 3.89676 & 3141 & 25.27360 & 26993 & 29.17036 & 30134 \\ 1500 & 4.38377 & 2833 & 3.92817 & 2938 & 25.54353 & 25447 & 29.47170 & 28385 \\ 1600 & 4.41210 & 2508 & 3.95755 & 2750 & 25.79800 & 24076 & 29.75555 & 26826 \\ & & & & & & & & \\ 1700 & 4.43718 & 2238 & 3.98505 & 2575 & 26.03876 & 22852 & 30.02381 & 25427 \\ 1800 & 4.45956 & 2016 & 4.01080 & 2415 & 26.26728 & 21751 & 30.27808 & 24166 \\ 1900 & 4.47972 & 1830 & 4.03495 & 2270 & 26.48479 & 20755 & 30.51974 & 23025 \\ 2000 & 4.49802 & 1674 & 4.05765 & 2138 & 26.69234 & 19849 & 30.74999 & 21987 \\ 2100 & 4.51476 & 1541 & 4.07903 & 2016 & 26.89083 & 19023 & 30.96986 & 21039 \\ 2200 & 4.53017 & 1428 & 4.09919 & 1905 & 27.08106 & 18264 & 31.18025 & 20169 \\ 2300 & 4.54445 & 1333 & 4.11824 & 1804 & 27.26370 & 17566 & 31.38194 & 19370 \\ 2400 & 4.55778 & 1249 & 4.13628 & 1712 & 27.43936 & 16920 & 31.57564 & 18632 \\ 2500 & 4.57027 & 1179 & 4.15340 & 1626 & 27.60856 & 16321 & 31.76196 & 17947 \\ 2600 & 4.58206 & 1115 & 4.16966 & 1548 & 27.77177 & 15766 & 31.94143 & 17314 \\ 2700 & 4.59321 & 1061 & 4.18514 & 1476 & 27.92943 & 15248 & 32.11457 & 16724 \\ 2800 & 4.60382 & 1014 & 4.19990 & 1411 & 28.08191 & 14762 & 32.28181 & 16173 \\ 2900 & 4.61396 & 970 & 4.21401 & 1349 & 28.22953 & 14309 & 32.44354 & 15658 \\ 3000 & 4.62366 & 1836 & 4.22750 & 2535 & 28.37262 & 27367 & 32.60012 & 29902 \\ & & & & & & & & \\ 3200 & 4.64202 & 1714 & 4.25285 & 2340 & 28.64629 & 25852 & 32.89914 & 28192 \\ 3400 & 4.65916 & 1620 & 4.27625 & 2172 & 28.90481 & 24506 & 33.18106 & 26678 \\ 3600 & 4.67536 & 1542 & 4.29797 & 2028 & 29.14987 & 23293 & 33.44784 & 25321 \\ 3800 & 4.69078 & 1475 & 4.31825 & 1899 & 29.38280 & 22198 & 33.70105 & 24097 \\ 4000 & 4.70553 & 1422 & 4.33724 & 1788 & 29.60478 & 21205 & 33.94202 & 22993 \\ & & & & & & & \\ 4200 & 4.71975 & 1375 & 4.35512 & 1688 & 29.81683 & 20300 & 34.17195 & 21988 \\ 4400 & 4.73350 & 1336 & 4.37200 & 1601 & 30.01983 & 19470 & 34.39183 & 21071 \\ 4600 & 4.74686 & 1301 & 4.38801 & 1523 & 30.21453 & 18707 & 34.60254 & 20230 \\ 4800 & 4.75987 & 1271 & 4.40324 & 1452 & 30.40160 & 18005 & 34.80484 & 19457 \\ 5000 & 4.77258 & & 4.41776 & & 30.58165 & & 34.99941 & \end{array}$


Table 2.076. SiH

\begin{tabular}{|l|l|l|l|l|}
\hline$K$ & $\frac{C_{p}^{0}}{R}$ & $\frac{\left(H^{0}-E_{0}^{0}\right)}{R T}$ & $\frac{-\left(F^{0}-E_{0}^{0}\right)}{R T}$ & $\frac{S^{0}}{R}$ \\
\hline
\end{tabular}

\begin{tabular}{|c|c|c|c|c|c|c|c|c|}
\hline $\begin{array}{l}50 \\
60 \\
70 \\
80 \\
90\end{array}$ & $\begin{array}{l}3.73696 \\
3.82052 \\
3.87201 \\
3.89566 \\
3.89914\end{array}$ & $\begin{array}{r}8356 \\
5149 \\
2365 \\
-348 \\
-\quad 955\end{array}$ & $\begin{array}{l}3.39401 \\
3.45857 \\
3.51433 \\
3.56077 \\
3.59833\end{array}$ & $\begin{array}{l}6456 \\
5576 \\
4644 \\
3756 \\
2967\end{array}$ & $\begin{array}{l}13.65530 \\
14.27993 \\
14.81738 \\
15.28979 \\
15.71144\end{array}$ & $\begin{array}{l}62463 \\
53745 \\
47241 \\
42165 \\
38072\end{array}$ & $\begin{array}{l}17.04931 \\
17.73850 \\
18.33171 \\
18.85056 \\
19.30977\end{array}$ & $\begin{array}{l}68919 \\
59321 \\
51885 \\
45921 \\
41039\end{array}$ \\
\hline $\begin{array}{l}100 \\
110 \\
120 \\
130 \\
140\end{array}$ & $\begin{array}{l}3.88959 \\
3.87246 \\
3.85143 \\
3.82887 \\
3.80627\end{array}$ & $\begin{array}{l}-1713 \\
-2103 \\
-2256 \\
-2260 \\
-2179\end{array}$ & $\begin{array}{l}3.62800 \\
3.65105 \\
3.66864 \\
3.68184 \\
3.69153\end{array}$ & $\begin{array}{r}2305 \\
1759 \\
1320 \\
969 \\
692\end{array}$ & $\begin{array}{l}16.09216 \\
16.43907 \\
16.75755 \\
17.05175 \\
17.32498\end{array}$ & $\begin{array}{l}34691 \\
31848 \\
29420 \\
27323 \\
25493\end{array}$ & $\begin{array}{l}19.72016 \\
20.09012 \\
20.42619 \\
20.73359 \\
21.01651\end{array}$ & $\begin{array}{l}36996 \\
33607 \\
30740 \\
28292 \\
26185\end{array}$ \\
\hline $\begin{array}{l}150 \\
160 \\
170 \\
180 \\
190\end{array}$ & $\begin{array}{l}3.78448 \\
3.76395 \\
3.74486 \\
3.72731 \\
3.71125\end{array}$ & $\begin{array}{l}-2053 \\
-1909 \\
-1755 \\
-1606 \\
-1460\end{array}$ & $\begin{array}{l}3.69845 \\
3.70319 \\
3.70619 \\
3.70783 \\
3.70842\end{array}$ & $\begin{array}{r}474 \\
300 \\
164 \\
-\quad 59 \\
-\quad 22\end{array}$ & $\begin{array}{l}17.57991 \\
17.81876 \\
18.04337 \\
18.25526 \\
18.45576\end{array}$ & $\begin{array}{l}23885 \\
22461 \\
21189 \\
20050 \\
19021\end{array}$ & $\begin{array}{l}21.27836 \\
21.52195 \\
21.74956 \\
21.96309 \\
22.16418\end{array}$ & $\begin{array}{l}24359 \\
22761 \\
21353 \\
20109 \\
18999\end{array}$ \\
\hline $\begin{array}{l}200 \\
210 \\
220 \\
230 \\
240\end{array}$ & $\begin{array}{l}3.69665 \\
3.68340 \\
3.67142 \\
3.66062 \\
3.65094\end{array}$ & $\begin{array}{l}-1325 \\
-1198 \\
-1080 \\
-968 \\
-864\end{array}$ & $\begin{array}{l}3.70820 \\
3.70733 \\
3.70598 \\
3.70423 \\
3.70220\end{array}$ & $\begin{array}{l}-\quad 87 \\
-135 \\
-175 \\
-203 \\
-222\end{array}$ & $\begin{array}{l}18.64597 \\
18.82688 \\
18.99931 \\
19.16401 \\
19.32162\end{array}$ & $\begin{array}{l}18091 \\
17243 \\
16470 \\
15761 \\
15107\end{array}$ & $\begin{array}{l}22.35417 \\
22.53421 \\
22.70529 \\
22.86824 \\
23.02382\end{array}$ & $\begin{array}{l}18004 \\
17108 \\
16295 \\
15558 \\
14885\end{array}$ \\
\hline $\begin{array}{l}250 \\
260 \\
270 \\
280 \\
290\end{array}$ & $\begin{array}{l}3.64230 \\
3.63462 \\
3.62787 \\
3.62200 \\
3.61699\end{array}$ & $\begin{array}{l}-768 \\
-675 \\
-587 \\
-501 \\
-420\end{array}$ & $\begin{array}{l}3.69998 \\
3.69760 \\
3.69516 \\
3.69264 \\
3.69012\end{array}$ & $\begin{array}{l}-238 \\
-244 \\
-252 \\
-252 \\
-252\end{array}$ & $\begin{array}{l}19.47269 \\
19.61778 \\
19.75728 \\
19.89162 \\
20.02115\end{array}$ & $\begin{array}{l}14509 \\
13950 \\
13434 \\
12953 \\
12506\end{array}$ & $\begin{array}{l}23.17267 \\
23.31538 \\
23.45244 \\
23.58425 \\
23.71127\end{array}$ & $\begin{array}{l}14271 \\
13706 \\
13181 \\
12702 \\
12253\end{array}$ \\
\hline $\begin{array}{l}300 \\
310 \\
320 \\
330 \\
340\end{array}$ & $\begin{array}{l}3.61279 \\
3.60938 \\
3.60674 \\
3.60483 \\
3.60365\end{array}$ & $\begin{array}{l}-341 \\
-264 \\
-191 \\
-118 \\
-\quad 48\end{array}$ & $\begin{array}{l}3.68760 \\
3.68512 \\
3.68273 \\
3.68040 \\
3.67815\end{array}$ & $\begin{array}{l}-248 \\
-239 \\
-233 \\
-225 \\
-214\end{array}$ & $\begin{array}{l}20.14621 \\
20.26709 \\
20.38405 \\
20.49734 \\
20.60718\end{array}$ & $\begin{array}{l}12088 \\
11696 \\
11329 \\
10984 \\
10658\end{array}$ & $\begin{array}{l}23.83380 \\
23.95222 \\
24.06677 \\
24.17774 \\
24.28532\end{array}$ & $\begin{array}{l}11842 \\
11455 \\
11097 \\
10758 \\
10445\end{array}$ \\
\hline $\begin{array}{l}350 \\
360 \\
370 \\
380 \\
390\end{array}$ & $\begin{array}{l}3.60317 \\
3.60336 \\
3.60421 \\
3.60570 \\
3.60779\end{array}$ & $\begin{array}{r}19 \\
85 \\
149 \\
209 \\
269\end{array}$ & $\begin{array}{l}3.67601 \\
3.67400 \\
3.67209 \\
3.67033 \\
3.66869\end{array}$ & $\begin{array}{l}-201 \\
-191 \\
-176 \\
-164 \\
-149\end{array}$ & $\begin{array}{l}20.71376 \\
20.81729 \\
20.91794 \\
21.01583 \\
21.11115\end{array}$ & $\begin{array}{r}10353 \\
10065 \\
9789 \\
9532 \\
9286\end{array}$ & $\begin{array}{l}24.38977 \\
24.49128 \\
24.59002 \\
24.68616 \\
24.77987\end{array}$ & $\begin{array}{r}10151 \\
9874 \\
9614 \\
9371 \\
9134\end{array}$ \\
\hline $\begin{array}{l}400 \\
450 \\
500 \\
550 \\
600\end{array}$ & $\begin{array}{l}3.61048 \\
3.63182 \\
3.66389 \\
3.70346 \\
3.74763\end{array}$ & $\begin{array}{l}2134 \\
3207 \\
3957 \\
4417 \\
4646\end{array}$ & $\begin{array}{l}3.66720 \\
3.66197 \\
3.66048 \\
3.66254 \\
3.66777\end{array}$ & $\begin{array}{r}-523 \\
-149 \\
206 \\
523 \\
792\end{array}$ & $\begin{array}{l}21.20401 \\
21.63561 \\
22.02133 \\
22.37028 \\
22.68916\end{array}$ & $\begin{array}{l}43160 \\
38572 \\
34895 \\
31888 \\
29388\end{array}$ & $\begin{array}{l}24.87121 \\
25.29758 \\
25.68181 \\
26.03282 \\
26.35693\end{array}$ & $\begin{array}{l}42637 \\
38423 \\
35101 \\
32411 \\
30180\end{array}$ \\
\hline $\begin{array}{l}650 \\
700 \\
750 \\
800 \\
850\end{array}$ & $\begin{array}{l}3.79409 \\
3.84107 \\
3.88737 \\
3.93214 \\
3.97490\end{array}$ & $\begin{array}{l}4698 \\
4630 \\
4477 \\
4276 \\
4047\end{array}$ & $\begin{array}{l}3.67569 \\
3.68582 \\
3.69773 \\
3.71099 \\
3.72527\end{array}$ & $\begin{array}{l}1013 \\
1191 \\
1326 \\
1428 \\
1501\end{array}$ & $\begin{array}{l}22.98304 \\
23.25580 \\
23.51049 \\
23.74955 \\
23.97495\end{array}$ & $\begin{array}{l}27276 \\
25469 \\
23906 \\
22540 \\
21335\end{array}$ & $\begin{array}{l}26.65873 \\
26.94163 \\
27.20822 \\
27.46053 \\
27.70022\end{array}$ & $\begin{array}{l}28290 \\
26659 \\
25231 \\
23969 \\
22836\end{array}$ \\
\hline $\begin{array}{r}900 \\
950 \\
1000 \\
1050 \\
1100\end{array}$ & $\begin{array}{l}4.01537 \\
4.05342 \\
4.08908 \\
4.12235 \\
4.15341\end{array}$ & $\begin{array}{l}3805 \\
3566 \\
3327 \\
3106 \\
2892\end{array}$ & $\begin{array}{l}3.74028 \\
3.75575 \\
3.77155 \\
3.78746 \\
3.80341\end{array}$ & $\begin{array}{l}1547 \\
1580 \\
1591 \\
1595 \\
1585\end{array}$ & $\begin{array}{l}24.18830 \\
24.39094 \\
24.58399 \\
24.76839 \\
24.94494\end{array}$ & $\begin{array}{l}20264 \\
19305 \\
18440 \\
17655 \\
16943\end{array}$ & $\begin{array}{l}27.92858 \\
28.14670 \\
28.35554 \\
28.55585 \\
28.74835\end{array}$ & $\begin{array}{l}21812 \\
20884 \\
20031 \\
19250 \\
18528\end{array}$ \\
\hline 1150 & 4.18233 & 2696 & 3.81926 & 1569 & 25.11437 & 16288 & 28.93363 & 17857 \\
\hline
\end{tabular}


Table 2.076. SiH (Cont.)

\begin{tabular}{|l|l|l|l|l|}
\hline${ }^{\circ}$ & $\frac{C_{p}^{0}}{R}$ & $\frac{\left(H^{0}-E_{0}^{0}\right)}{R T}$ & $\frac{-\left(F^{0}-E_{0}^{0}\right)}{R T}$ & $\frac{S^{0}}{R}$ \\
\hline
\end{tabular}

$\begin{array}{lllllllll}1200 & 4.20929 & 4856 & 3.83495 & 3070 & 25.27725 & 30818 & 29.11220 & 33888 \\ 1300 & 4.25785 & 4237 & 3.86565 & 2957 & 25.58543 & 28757 & 29.45108 & 31714 \\ 1400 & 4.30022 & 3716 & 3.89522 & 2827 & 25.87300 & 26971 & 29.76822 & 29798 \\ 1500 & 4.33738 & 3280 & 3.92349 & 2691 & 26.14271 & 25408 & 30.06620 & 28099 \\ 1600 & 4.37018 & 2914 & 3.95040 & 2557 & 26.39679 & 24026 & 30.34719 & 26583 \\ & & & & & & & & \\ 1700 & 4.39932 & 2610 & 3.97597 & 2426 & 26.63705 & 22796 & 30.61302 & 25222 \\ 1800 & 4.42542 & 2352 & 4.00023 & 2300 & 26.86501 & 21690 & 30.86524 & 23990 \\ 1.900 & 4.44894 & 2135 & 4.02323 & 2183 & 27.08191 & 20694 & 31.10514 & 22876 \\ 2000 & 4.47029 & 1949 & 4.04506 & 2072 & 27.28885 & 19785 & 31.33390 & 21858 \\ 2100 & 4.48978 & 1792 & 4.06578 & 1968 & 27.48670 & 18961 & 31.55248 & 20929 \\ & & & & & & & & \\ 2200 & 4.50770 & 1658 & 4.08546 & 1873 & 27.67631 & 18202 & 31.76177 & 20075 \\ 2300 & 4.52428 & 1542 & 4.10419 & 1783 & 27.85833 & 17505 & 31.96252 & 19287 \\ 2400 & 4.53970 & 1442 & 4.12202 & 1700 & 28.03338 & 16861 & 32.15539 & 18562 \\ 2500 & 4.55412 & 1354 & 4.13902 & 1624 & 28.20199 & 16266 & 32.34101 & 17889 \\ 2600 & 4.56766 & 1278 & 4.15526 & 1549 & 28.36465 & 15711 & 32.51990 & 17261 \\ & & & & & & & & \\ 2700 & 4.58044 & 1211 & 4.17075 & 1486 & 28.52176 & 15195 & 32.69251 & 16681 \\ 2800 & 4.59255 & 1153 & 4.18561 & 1423 & 28.67371 & 14713 & 32.85932 & 16137 \\ 2900 & 4.60408 & 1100 & 4.19984 & 1366 & 28.82084 & 14262 & 33.02069 & 15627 \\ 3000 & 4.61508 & 2067 & 4.21350 & 2575 & 28.96346 & 27275 & 33.17696 & 29851 \\ & & & & & & & & \\ 3200 & 4.63575 & 1920 & 4.23925 & 2390 & 29.23621 & 25774 & 33.47547 & 28163 \\ 3400 & 4.65495 & 1801 & 4.26315 & 2228 & 29.49395 & 24431 & 33.75710 & 26659 \\ 3600 & 4.67296 & 1704 & 4.28543 & 2083 & 29.73826 & 23228 & 34.02369 & 25310 \\ 3800 & 4.69000 & 1621 & 4.30626 & 1962 & 29.97054 & 22137 & 34.27679 & 24099 \\ 4000 & 4.70621 & 1556 & 4.32588 & 1846 & 30.19191 & 21151 & 34.51778 & 22999 \\ & & & & & & & \\ 4200 & 4.72177 & 1497 & 4.34434 & 1751 & 30.40342 & 20251 & 34.74777 & 22001 \\ 4400 & 4.73674 & 1447 & 4.36185 & 1661 & 30.60593 & 19426 & 34.96778 & 21087 \\ 4600 & 4.75121 & 1406 & 4.37846 & 1582 & 30.80019 & 18668 & 35.17865 & 20250 \\ 4800 & 4.76527 & 1369 & 4.39428 & 1513 & 30.98687 & 17969 & 35.38115 & 19482 \\ 5000 & 4.77896 & & 4.40941 & & 31.16656 & & 35.57597 & \end{array}$


Table 2.077. SiD

\begin{tabular}{|l|l|l|l|l|}
\hline$K$ & $\frac{C_{p}^{0}}{R}$ & $\frac{\left(H^{0}-E_{0}^{0}\right)}{R T}$ & $\frac{-\left(F^{0}-E_{0}^{0}\right)}{R T}$ & $\frac{S^{0}}{R}$ \\
\hline
\end{tabular}

\begin{tabular}{|c|c|c|c|c|c|c|c|c|}
\hline $\begin{array}{l}50 \\
60 \\
70 \\
80 \\
90\end{array}$ & $\begin{array}{l}3.75653 \\
3.84246 \\
3.89444 \\
3.91722 \\
3.91902\end{array}$ & $\begin{array}{r}8593 \\
5198 \\
2278 \\
180 \\
-1152\end{array}$ & $\begin{array}{l}3.47695 \\
3.53117 \\
3.57974 \\
3.62077 \\
3.65396\end{array}$ & $\begin{array}{l}5422 \\
4857 \\
4103 \\
3319 \\
2601\end{array}$ & $\begin{array}{l}14.25275 \\
14.89152 \\
15.43959 \\
15.92037 \\
16.34883\end{array}$ & $\begin{array}{l}63877 \\
54807 \\
48078 \\
42846 \\
38638\end{array}$ & $\begin{array}{l}17.72970 \\
18.42269 \\
19.01933 \\
19.54114 \\
20.00279\end{array}$ & $\begin{array}{l}69299 \\
59664 \\
52181 \\
46165 \\
41239\end{array}$ \\
\hline $\begin{array}{l}100 \\
110 \\
120 \\
130 \\
140\end{array}$ & $\begin{array}{l}3.90750 \\
3.88832 \\
3.86536 \\
3.84109 \\
3.81696\end{array}$ & $\begin{array}{l}-1918 \\
-2296 \\
-2427 \\
-2413 \\
-2298\end{array}$ & $\begin{array}{l}3.67997 \\
3.69983 \\
3.71459 \\
3.72526 \\
3.73266\end{array}$ & $\begin{array}{r}1986 \\
1476 \\
1067 \\
740 \\
486\end{array}$ & $\begin{array}{l}16.73521 \\
17.08692 \\
17.40952 \\
17.70730 \\
17.98365\end{array}$ & $\begin{array}{l}35171 \\
32260 \\
29778 \\
27635 \\
25771\end{array}$ & $\begin{array}{l}20.41518 \\
20.78675 \\
21.12411 \\
21.43256 \\
21.71631\end{array}$ & $\begin{array}{l}37157 \\
33736 \\
30845 \\
28375 \\
26257\end{array}$ \\
\hline $\begin{array}{l}150 \\
160 \\
170 \\
180 \\
190\end{array}$ & $\begin{array}{l}3.79398 \\
3.77251 \\
3.75281 \\
3.73501 \\
3.71913\end{array}$ & $\begin{array}{l}-2147 \\
-1970 \\
-1780 \\
-1588 \\
-1397\end{array}$ & $\begin{array}{l}3.73752 \\
3.74038 \\
3.74167 \\
3.74176 \\
3.74100\end{array}$ & $\begin{array}{r}286 \\
129 \\
-\quad 9 \\
-\quad 76 \\
-145\end{array}$ & $\begin{array}{l}18.24136 \\
18.48266 \\
18.70947 \\
18.92335 \\
19.12565\end{array}$ & $\begin{array}{l}24130 \\
22681 \\
21388 \\
20230 \\
19184\end{array}$ & $\begin{array}{l}21.97888 \\
22.22304 \\
22.45114 \\
22.66514 \\
22.86665\end{array}$ & $\begin{array}{l}24416 \\
22810 \\
21400 \\
20151 \\
19040\end{array}$ \\
\hline $\begin{array}{l}200 \\
210 \\
220 \\
230 \\
240\end{array}$ & $\begin{array}{l}3.70516 \\
3.69309 \\
3.68283 \\
3.67435 \\
3.66759\end{array}$ & $\begin{array}{l}-1207 \\
-1026 \\
-848 \\
-676 \\
-512\end{array}$ & $\begin{array}{l}3.73955 \\
3.73762 \\
3.73536 \\
3.73287 \\
3.73030\end{array}$ & $\begin{array}{l}-193 \\
-226 \\
-249 \\
-257 \\
-262\end{array}$ & $\begin{array}{l}19.31749 \\
19.49990 \\
19.67372 \\
19.83971 \\
19.99853\end{array}$ & $\begin{array}{l}18241 \\
17382 \\
16599 \\
15882 \\
15223\end{array}$ & $\begin{array}{l}23.05705 \\
23.23753 \\
23.40908 \\
23.57258 \\
23.72883\end{array}$ & $\begin{array}{l}18048 \\
17155 \\
16350 \\
15625 \\
14961\end{array}$ \\
\hline $\begin{array}{l}250 \\
260 \\
270 \\
280 \\
290\end{array}$ & $\begin{array}{l}3.66247 \\
3.65893 \\
3.65691 \\
3.65632 \\
3.65709\end{array}$ & $\begin{array}{r}-354 \\
-\quad 202 \\
-\quad 59 \\
77 \\
203\end{array}$ & $\begin{array}{l}3.72768 \\
3.72510 \\
3.72260 \\
3.72024 \\
3.71806\end{array}$ & $\begin{array}{l}-258 \\
-250 \\
-236 \\
-218 \\
-202\end{array}$ & $\begin{array}{l}20.15076 \\
20.29690 \\
20.43745 \\
20.57279 \\
20.70329\end{array}$ & $\begin{array}{l}14614 \\
14055 \\
13534 \\
13050 \\
12603\end{array}$ & $\begin{array}{l}23.87844 \\
24.02200 \\
24.16005 \\
24.29303 \\
24.42135\end{array}$ & $\begin{array}{l}14356 \\
13805 \\
13298 \\
12832 \\
12401\end{array}$ \\
\hline $\begin{array}{l}300 \\
310 \\
320 \\
330 \\
340\end{array}$ & $\begin{array}{l}3.65912 \\
3.66234 \\
3.66665 \\
3.67198 \\
3.67824\end{array}$ & $\begin{array}{l}322 \\
431 \\
533 \\
626 \\
712\end{array}$ & $\begin{array}{l}3.71604 \\
3.71426 \\
3.71271 \\
3.71138 \\
3.71032\end{array}$ & $\begin{array}{l}-178 \\
-155 \\
-133 \\
-106 \\
-\quad 82\end{array}$ & $\begin{array}{l}20.82932 \\
20.95113 \\
21.06902 \\
21.18326 \\
21.29403\end{array}$ & $\begin{array}{l}12181 \\
11789 \\
11424 \\
11077 \\
10755\end{array}$ & $\begin{array}{l}24.54536 \\
24.66539 \\
24.78173 \\
24.89465 \\
25.00436\end{array}$ & $\begin{array}{l}12003 \\
11634 \\
11292 \\
10971 \\
10672\end{array}$ \\
\hline $\begin{array}{l}350 \\
360 \\
370 \\
330 \\
390\end{array}$ & $\begin{array}{l}3.68536 \\
3.69323 \\
3.70178 \\
3.71096 \\
3.72068\end{array}$ & $\begin{array}{r}787 \\
855 \\
918 \\
972 \\
1017\end{array}$ & $\begin{array}{l}3.70950 \\
3.70893 \\
3.70862 \\
3.70856 \\
3.70875\end{array}$ & $\begin{array}{r}-\quad 57 \\
-\quad 31 \\
-\quad 6 \\
19 \\
42\end{array}$ & $\begin{array}{l}21.40158 \\
21.50607 \\
21.60769 \\
21.70659 \\
21.80292\end{array}$ & $\begin{array}{r}10449 \\
10162 \\
9890 \\
9633 \\
9390\end{array}$ & $\begin{array}{l}25.11108 \\
25.21501 \\
25.31631 \\
25.41515 \\
25.51167\end{array}$ & $\begin{array}{r}10393 \\
10130 \\
9884 \\
9652 \\
9432\end{array}$ \\
\hline $\begin{array}{l}400 \\
450 \\
500 \\
550 \\
600\end{array}$ & $\begin{array}{l}3.73085 \\
3.78681 \\
3.84676 \\
3.90655 \\
3.96378\end{array}$ & $\begin{array}{l}5596 \\
5995 \\
5979 \\
5723 \\
5342\end{array}$ & $\begin{array}{l}3.70917 \\
3.71463 \\
3.72483 \\
3.73865 \\
3.75505\end{array}$ & $\begin{array}{r}546 \\
1020 \\
1382 \\
1640 \\
1814\end{array}$ & $\begin{array}{l}21.89682 \\
22.33395 \\
22.72583 \\
23.08146 \\
23.40745\end{array}$ & $\begin{array}{l}43713 \\
39188 \\
35563 \\
32599 \\
30128\end{array}$ & $\begin{array}{l}25.60599 \\
26.04858 \\
26.45066 \\
26.82011 \\
27.16250\end{array}$ & $\begin{array}{l}44259 \\
40208 \\
36945 \\
34239 \\
31942\end{array}$ \\
\hline $\begin{array}{l}650 \\
700 \\
750 \\
800 \\
850\end{array}$ & $\begin{array}{l}4.01720 \\
4.06632 \\
4.11107 \\
4.15164 \\
4.18839\end{array}$ & $\begin{array}{l}4912 \\
4475 \\
4057 \\
3675 \\
3322\end{array}$ & $\begin{array}{l}3.77319 \\
3.79240 \\
3.81216 \\
3.83215 \\
3.85203\end{array}$ & $\begin{array}{l}1921 \\
1976 \\
1999 \\
1988 \\
1962\end{array}$ & $\begin{array}{l}23.70873 \\
23.98905 \\
24.25137 \\
24.49804 \\
24.73096\end{array}$ & $\begin{array}{l}28032 \\
26232 \\
24667 \\
23292 \\
22074\end{array}$ & $\begin{array}{l}27.48192 \\
27.78145 \\
28.06354 \\
28.33019 \\
28.58299\end{array}$ & $\begin{array}{l}29953 \\
28209 \\
26665 \\
25280 \\
24037\end{array}$ \\
\hline $\begin{array}{l}900 \\
950 \\
000 \\
050\end{array}$ & $\begin{array}{l}4.22161 \\
4.25169 \\
4.27897 \\
4.30378 \\
4.32640\end{array}$ & $\begin{array}{l}3008 \\
2728 \\
2481 \\
2262 \\
2068\end{array}$ & $\begin{array}{l}3.87165 \\
3.89088 \\
3.90961 \\
3.92780 \\
3.9 .4541\end{array}$ & $\begin{array}{l}1923 \\
1873 \\
1819 \\
1761 \\
1702\end{array}$ & $\begin{array}{l}24.95170 \\
25.16154 \\
25.36161 \\
25.55279 \\
25.73594\end{array}$ & $\begin{array}{l}20984 \\
20007 \\
19118 \\
18315 \\
17573\end{array}$ & $\begin{array}{l}28.82336 \\
29.05242 \\
29.27122 \\
29.48060 \\
29.68135\end{array}$ & $\begin{array}{l}22906 \\
21880 \\
20938 \\
20075 \\
19275\end{array}$ \\
\hline & 4.34708 & 1896 & 3.96243 & 1643 & 25.91167 & 16900 & 29.87410 & 18543 \\
\hline
\end{tabular}


Table 2.077. SiD (Cont.)

\begin{tabular}{|c|c|c|c|c|c|c|c|c|}
\hline${ }^{\circ} \mathrm{K}$ & $\frac{C_{p}^{0}}{R}$ & & $\frac{\left(H^{\circ}-E_{0}^{0}\right)}{R T}$ & & $\frac{-\left(F^{0}-E_{0}^{0}\right)}{R T}$ & & $\frac{S^{0}}{R}$ & \\
\hline $\begin{array}{l}1200 \\
1300 \\
1400 \\
1500 \\
1600\end{array}$ & $\begin{array}{l}4.36604 \\
4.39961 \\
4.42842 \\
4.45343 \\
4.47543\end{array}$ & $\begin{array}{l}3357 \\
2881 \\
2501 \\
2200 \\
1957\end{array}$ & $\begin{array}{l}3.97886 \\
4.00996 \\
4.03885 \\
4.06567 \\
4.09062\end{array}$ & $\begin{array}{l}3110 \\
2889 \\
2682 \\
2495 \\
2322\end{array}$ & $\begin{array}{l}26.08067 \\
26.40040 \\
26.69864 \\
26.97821 \\
27.24141\end{array}$ & $\begin{array}{l}31973 \\
29824 \\
27957 \\
26320 \\
24869\end{array}$ & $\begin{array}{l}30.05953 \\
30.41036 \\
30.73748 \\
31.04388 \\
31.33203\end{array}$ & $\begin{array}{l}35083 \\
32712 \\
30640 \\
28815 \\
27191\end{array}$ \\
\hline $\begin{array}{l}1700 \\
1800 \\
1900 \\
2000 \\
2100\end{array}$ & $\begin{array}{l}4.49500 \\
4.51260 \\
4.52857 \\
4.54320 \\
4.55671\end{array}$ & $\begin{array}{l}1760 \\
1597 \\
1463 \\
1351 \\
1257\end{array}$ & $\begin{array}{l}4.11384 \\
4.13550 \\
4.15579 \\
4.17479 \\
4.19267\end{array}$ & $\begin{array}{l}2166 \\
2029 \\
1900 \\
1788 \\
1684\end{array}$ & $\begin{array}{l}27.49010 \\
27.72587 \\
27.95001 \\
28.16367 \\
28.36779\end{array}$ & $\begin{array}{l}23577 \\
22414 \\
21366 \\
20412 \\
19544\end{array}$ & $\begin{array}{l}31.60394 \\
31.86138 \\
32.10580 \\
32.33846 \\
32.56046\end{array}$ & $\begin{array}{l}25744 \\
24442 \\
23266 \\
22200 \\
21228\end{array}$ \\
\hline $\begin{array}{l}2200 \\
2300 \\
2400 \\
2500 \\
2600\end{array}$ & $\begin{array}{l}4.56928 \\
4.58107 \\
4.59216 \\
4.60270 \\
4.61271\end{array}$ & $\begin{array}{r}1179 \\
1109 \\
1054 \\
1001 \\
959\end{array}$ & $\begin{array}{l}4.20951 \\
4.22540 \\
4.24046 \\
4.25473 \\
4.26832\end{array}$ & $\begin{array}{l}1589 \\
1506 \\
1427 \\
1359 \\
1293\end{array}$ & $\begin{array}{l}28.56323 \\
28.75070 \\
28.93085 \\
29.10425 \\
29.27139\end{array}$ & $\begin{array}{l}18747 \\
18015 \\
17340 \\
16714 \\
16134\end{array}$ & $\begin{array}{l}32.77274 \\
32.97610 \\
33.17131 \\
33.35898 \\
33.53971\end{array}$ & $\begin{array}{l}20336 \\
19521 \\
18767 \\
18073 \\
17427\end{array}$ \\
\hline $\begin{array}{l}2700 \\
2800 \\
2900 \\
3000\end{array}$ & $\begin{array}{l}4.62230 \\
4.63151 \\
4.64039 \\
4.64896\end{array}$ & $\begin{array}{r}921 \\
888 \\
857 \\
1644\end{array}$ & $\begin{array}{l}4.28125 \\
4.29360 \\
4.30540 \\
4.31671\end{array}$ & $\begin{array}{l}1235 \\
1180 \\
1131 \\
2128\end{array}$ & $\begin{array}{l}29.43273 \\
29.58865 \\
29.73951 \\
29.88567\end{array}$ & $\begin{array}{l}15592 \\
15086 \\
14616 \\
27929\end{array}$ & $\begin{array}{l}33.71398 \\
33.88225 \\
34.04491 \\
34.20238\end{array}$ & $\begin{array}{l}16827 \\
16266 \\
15747 \\
30058\end{array}$ \\
\hline $\begin{array}{l}3200 \\
3400 \\
3600 \\
3800 \\
4000\end{array}$ & $\begin{array}{l}4.66540 \\
4.68099 \\
4.69591 \\
4.71028 \\
4.72419\end{array}$ & $\begin{array}{l}1559 \\
1492 \\
1437 \\
1391 \\
1352\end{array}$ & $\begin{array}{l}4.33799 \\
4.35771 \\
4.37609 \\
4.39330 \\
4.40952\end{array}$ & $\begin{array}{l}1972 \\
1838 \\
1721 \\
1622 \\
1529\end{array}$ & $\begin{array}{l}30.16496 \\
30.42854 \\
30.67816 \\
30.91522 \\
31.14098\end{array}$ & $\begin{array}{l}26358 \\
24962 \\
23706 \\
22576 \\
21553\end{array}$ & $\begin{array}{l}34.50296 \\
34.78626 \\
35.05425 \\
35.30852 \\
35.55050\end{array}$ & $\begin{array}{l}28330 \\
26799 \\
25427 \\
24198 \\
23082\end{array}$ \\
\hline $\begin{array}{l}4200 \\
4400 \\
4600 \\
4800 \\
5000\end{array}$ & $\begin{array}{l}4.73771 \\
4.75090 \\
4.76380 \\
4.77645 \\
4.78888\end{array}$ & $\begin{array}{l}1319 \\
1290 \\
1265 \\
1243\end{array}$ & $\begin{array}{l}4.42481 \\
4.43933 \\
4.45316 \\
4.46637 \\
4.47902\end{array}$ & $\begin{array}{l}1452 \\
1383 \\
1321 \\
1265\end{array}$ & $\begin{array}{l}31.35651 \\
31.56269 \\
31.76033 \\
31.95014 \\
32.13272\end{array}$ & $\begin{array}{l}20618 \\
19764 \\
18981 \\
18258\end{array}$ & $\begin{array}{l}35.78132 \\
36.00202 \\
36.21349 \\
36.41650 \\
36.61173\end{array}$ & $\begin{array}{l}22070 \\
21147 \\
20301 \\
19523\end{array}$ \\
\hline
\end{tabular}


Table 2.078. SiT

\begin{tabular}{|l|l|l|l|l|}
\hline${ }^{\circ} K$ & $\frac{C_{p}^{0}}{R}$ & $\frac{\left(H^{0}-E_{0}^{0}\right)}{R T}$ & $\frac{-\left(F^{0}-E_{0}^{0}\right)}{R T}$ & $\frac{S^{0}}{R}$ \\
\hline
\end{tabular}

\begin{tabular}{|c|c|c|c|c|c|c|c|c|}
\hline $\begin{array}{l}50 \\
60 \\
70 \\
80 \\
90\end{array}$ & $\begin{array}{l}3.76154 \\
3.84862 \\
3.90114 \\
3.92394 \\
3.92545\end{array}$ & $\begin{array}{r}8708 \\
5252 \\
2280 \\
151 \\
-1198\end{array}$ & $\begin{array}{l}3.50325 \\
3.55404 \\
3.60027 \\
3.63957 \\
3.67140\end{array}$ & $\begin{array}{l}5079 \\
4623 \\
3930 \\
3183 \\
2489\end{array}$ & $\begin{array}{l}14.63851 \\
15.28174 \\
15.83316 \\
16.31658 \\
16.74717\end{array}$ & $\begin{array}{l}64323 \\
55142 \\
48342 \\
43059 \\
38815\end{array}$ & $\begin{array}{l}18.14176 \\
18.83578 \\
19.43343 \\
19.95615 \\
20.41857\end{array}$ & $\begin{array}{l}69402 \\
59765 \\
52272 \\
46242 \\
41304\end{array}$ \\
\hline $\begin{array}{l}100 \\
110 \\
120 \\
130 \\
140\end{array}$ & $\begin{array}{l}3.91347 \\
3.89377 \\
3.87034 \\
3.84574 \\
3.82155\end{array}$ & $\begin{array}{l}-1970 \\
-2343 \\
-2460 \\
-2419 \\
-2281\end{array}$ & $\begin{array}{l}3.69629 \\
3.71518 \\
3.72910 \\
3.73902 \\
3.74578\end{array}$ & $\begin{array}{r}1889 \\
1392 \\
992 \\
676 \\
428\end{array}$ & $\begin{array}{l}17.13532 \\
17.48853 \\
17.81244 \\
18.11133 \\
18.38869\end{array}$ & $\begin{array}{l}35321 \\
32391 \\
29889 \\
27736 \\
25858\end{array}$ & $\begin{array}{l}20.83161 \\
21.20371 \\
21.54154 \\
21.85035 \\
22.13447\end{array}$ & $\begin{array}{l}37210 \\
33783 \\
30881 \\
28412 \\
26287\end{array}$ \\
\hline $\begin{array}{l}150 \\
160 \\
170 \\
180 \\
190\end{array}$ & $\begin{array}{l}3.79874 \\
3.77786 \\
3.75927 \\
3.74314 \\
3.72951\end{array}$ & $\begin{array}{l}-2088 \\
-1859 \\
-1613 \\
-1363 \\
-1110\end{array}$ & $\begin{array}{l}3.75006 \\
3.75244 \\
3.75337 \\
3.75324 \\
3.75234\end{array}$ & $\begin{array}{r}238 \\
-\quad 93 \\
-\quad 13 \\
-\quad 90 \\
-\quad 143\end{array}$ & $\begin{array}{l}18.64727 \\
18.88939 \\
19.11692 \\
19.33145 \\
19.53436\end{array}$ & $\begin{array}{l}24212 \\
22753 \\
21453 \\
20291 \\
19243\end{array}$ & $\begin{array}{l}22.39734 \\
22.64183 \\
22.87029 \\
23.08469 \\
23.28670\end{array}$ & $\begin{array}{l}24449 \\
22846 \\
214.40 \\
20201 \\
19100\end{array}$ \\
\hline $\begin{array}{l}200 \\
210 \\
220 \\
230 \\
240\end{array}$ & $\begin{array}{l}3.71841 \\
3.70976 \\
3.70351 \\
3.69954 \\
3.69772\end{array}$ & $\begin{array}{r}-865 \\
-625 \\
-397 \\
-\quad 182 \\
20\end{array}$ & $\begin{array}{l}3.75091 \\
3.74915 \\
3.74721 \\
3.74522 \\
3.74327\end{array}$ & $\begin{array}{l}-176 \\
-194 \\
-199 \\
-195 \\
-183\end{array}$ & $\begin{array}{l}19.72679 \\
19.90974 \\
20.08411 \\
20.25064 \\
20.41000\end{array}$ & $\begin{array}{l}18295 \\
17437 \\
16653 \\
15936 \\
15276\end{array}$ & $\begin{array}{l}23.47770 \\
23.65889 \\
23.83132 \\
23.99586 \\
24.15326\end{array}$ & $\begin{array}{l}18119 \\
17243 \\
16454 \\
15740 \\
15094\end{array}$ \\
\hline $\begin{array}{l}250 \\
260 \\
270 \\
280 \\
290\end{array}$ & $\begin{array}{l}3.69792 \\
3.69996 \\
3.70372 \\
3.70903 \\
3.71571\end{array}$ & $\begin{array}{l}204 \\
376 \\
531 \\
668 \\
792\end{array}$ & $\begin{array}{l}3.74144 \\
3.73981 \\
3.73839 \\
3.73724 \\
3.73638\end{array}$ & $\begin{array}{l}-163 \\
-142 \\
-115 \\
-\quad 86 \\
-\quad 57\end{array}$ & $\begin{array}{l}20.56276 \\
20.70948 \\
20.85062 \\
20.98653 \\
21.11766\end{array}$ & $\begin{array}{l}14672 \\
14114 \\
13591 \\
13113 \\
12666\end{array}$ & $\begin{array}{l}24.30420 \\
24.44929 \\
24.58901 \\
24.72378 \\
24.85404\end{array}$ & $\begin{array}{l}14509 \\
13972 \\
13477 \\
13026 \\
12610\end{array}$ \\
\hline $\begin{array}{l}300 \\
310 \\
320 \\
330 \\
340\end{array}$ & $\begin{array}{l}3.72363 \\
3.73264 \\
3.74261 \\
3.75339 \\
3.76488\end{array}$ & $\begin{array}{r}901 \\
997 \\
1078 \\
1149 \\
1207\end{array}$ & $\begin{array}{l}3.73581 \\
3.73557 \\
3.73563 \\
3.73600 \\
3.73669\end{array}$ & $\begin{array}{r}-\quad 24 \\
6 \\
37 \\
69 \\
97\end{array}$ & $\begin{array}{l}21.24432 \\
21.36681 \\
21.48541 \\
21.60037 \\
21.71190\end{array}$ & $\begin{array}{l}12249 \\
11860 \\
11496 \\
11153 \\
10834\end{array}$ & $\begin{array}{l}24.98014 \\
25.10238 \\
25.22104 \\
25.33637 \\
25.44859\end{array}$ & $\begin{array}{l}12224 \\
11866 \\
11533 \\
11222 \\
10930\end{array}$ \\
\hline $\begin{array}{l}350 \\
360 \\
370 \\
380 \\
390\end{array}$ & $\begin{array}{l}3.77695 \\
3.78950 \\
3.80245 \\
3.81570 \\
3.82917\end{array}$ & $\begin{array}{l}1255 \\
1295 \\
1325 \\
1347 \\
1363\end{array}$ & $\begin{array}{l}3.73766 \\
3.73893 \\
3.74047 \\
3.74227 \\
3.74433\end{array}$ & $\begin{array}{l}127 \\
154 \\
180 \\
206 \\
228\end{array}$ & $\begin{array}{l}21.82024 \\
21.92555 \\
22.02802 \\
22.12779 \\
22.22502\end{array}$ & $\begin{array}{r}10531 \\
10247 \\
9977 \\
9723 \\
9482\end{array}$ & $\begin{array}{l}25.55789 \\
25.66448 \\
25.76848 \\
25.87006 \\
25.96935\end{array}$ & $\begin{array}{r}10659 \\
10400 \\
10158 \\
9929 \\
9711\end{array}$ \\
\hline $\begin{array}{l}400 \\
450 \\
500 \\
550 \\
600\end{array}$ & $\begin{array}{l}3.84280 \\
3.91145 \\
3.97754 \\
4.03856 \\
4.09362\end{array}$ & $\begin{array}{l}6865 \\
6609 \\
6102 \\
5506 \\
4908\end{array}$ & $\begin{array}{l}3.74661 \\
3.76113 \\
3.77950 \\
3.80032 \\
3.82251\end{array}$ & $\begin{array}{l}1452 \\
1837 \\
2082 \\
2219 \\
2277\end{array}$ & $\begin{array}{l}22.31984 \\
22.761 .92 \\
23.15913 \\
23.52031 \\
23.85193\end{array}$ & $\begin{array}{l}44208 \\
39721 \\
36118 \\
33162 \\
30687\end{array}$ & $\begin{array}{l}26.06646 \\
26.52305 \\
26.93863 \\
27.32063 \\
27.67444\end{array}$ & $\begin{array}{l}45659 \\
41558 \\
38200 \\
35381 \\
32963\end{array}$ \\
\hline $\begin{array}{l}650 \\
700 \\
750 \\
800 \\
850\end{array}$ & $\begin{array}{l}4.14270 \\
4.18624 \\
4.22478 \\
4.25893 \\
4.28926\end{array}$ & $\begin{array}{l}4354 \\
3854 \\
3415 \\
3033 \\
2705\end{array}$ & $\begin{array}{l}3.84528 \\
3.86811 \\
3.89063 \\
3.91260 \\
3.93389\end{array}$ & $\begin{array}{l}2283 \\
2252 \\
2197 \\
2129 \\
2051\end{array}$ & $\begin{array}{l}24.15880 \\
24.44460 \\
24.71224 \\
24.96404 \\
25.20189\end{array}$ & $\begin{array}{l}28580 \\
26764 \\
25180 \\
23785 \\
22543\end{array}$ & $\begin{array}{l}28.00407 \\
28.31271 \\
28.60287 \\
28.87664 \\
29.13578\end{array}$ & $\begin{array}{l}30864 \\
29016 \\
27377 \\
25914 \\
24594\end{array}$ \\
\hline $\begin{array}{r}900 \\
950 \\
1000 \\
1050 \\
1100\end{array}$ & $\begin{array}{l}4.31631 \\
4.34051 \\
4.36225 \\
4.38190 \\
4.39972\end{array}$ & $\begin{array}{l}2420 \\
2174 \\
1965 \\
1782 \\
1625\end{array}$ & $\begin{array}{l}3.95440 \\
3.97409 \\
3.99296 \\
4.01103 \\
4.02829\end{array}$ & $\begin{array}{l}1969 \\
1887 \\
1807 \\
1726 \\
1651\end{array}$ & $\begin{array}{l}25.42732 \\
25.64167 \\
25.84597 \\
26.04122 \\
26.22824\end{array}$ & $\begin{array}{l}21435 \\
20430 \\
19525 \\
18702 \\
17944\end{array}$ & $\begin{array}{l}29.38172 \\
29.61576 \\
29.83894 \\
30.05225 \\
30.25654\end{array}$ & $\begin{array}{l}23404 \\
22318 \\
21331 \\
20429 \\
19593\end{array}$ \\
\hline 1150 & 4.41597 & 1486 & 4.04480 & 1578 & 26.40768 & 17248 & 30.45247 & 18827 \\
\hline
\end{tabular}


Table 2.078. SiT (Cont.)

\begin{tabular}{|c|c|c|c|c|c|c|c|c|}
\hline${ }^{\circ} \mathrm{K}$ & $\frac{C_{p}^{0}}{R}$ & & $\frac{\left(H^{0}-E_{0}^{0}\right)}{R T}$ & & $\frac{-\left(F^{0}-E_{0}^{0}\right)}{R T}$ & & $\frac{S^{0}}{R}$ & \\
\hline $\begin{array}{l}1200 \\
1300 \\
1400 \\
1500 \\
1600\end{array}$ & $\begin{array}{l}4.43083 \\
4.45713 \\
4.47973 \\
4.49948 \\
4.51698\end{array}$ & $\begin{array}{l}2630 \\
2260 \\
1975 \\
1750 \\
1569\end{array}$ & $\begin{array}{l}4.06058 \\
4.09010 \\
4.11714 \\
4.14200 \\
4.16488\end{array}$ & $\begin{array}{l}2952 \\
2704 \\
2486 \\
2288 \\
2118\end{array}$ & $\begin{array}{l}26.58016 \\
26.90635 \\
27.21046 \\
27.49537 \\
27.76344\end{array}$ & $\begin{array}{l}32619 \\
30411 \\
28491 \\
26807 \\
25313\end{array}$ & $\begin{array}{l}30.64074 \\
30.99645 \\
31.32760 \\
31.63736 \\
31.92833\end{array}$ & $\begin{array}{l}35571 \\
33115 \\
30976 \\
29097 \\
27431\end{array}$ \\
\hline $\begin{array}{l}1700 \\
1800 \\
1900 \\
2000 \\
2100\end{array}$ & $\begin{array}{l}4.53267 \\
4.54694 \\
4.56005 \\
4.57218 \\
4.58350\end{array}$ & $\begin{array}{l}1427 \\
1311 \\
1213 \\
1132 \\
1066\end{array}$ & $\begin{array}{l}4.18606 \\
4.20572 \\
4.22403 \\
4.24114 \\
4.25719\end{array}$ & $\begin{array}{l}1966 \\
1831 \\
1711 \\
1605 \\
1507\end{array}$ & $\begin{array}{l}28.01657 \\
28.25641 \\
28.48430 \\
28.70140 \\
28.90871\end{array}$ & $\begin{array}{l}23984 \\
22789 \\
21710 \\
20731 \\
19841\end{array}$ & $\begin{array}{l}32.20264 \\
32.46213 \\
32.70833 \\
32.94254 \\
33.16589\end{array}$ & $\begin{array}{l}25949 \\
24620 \\
23421 \\
22335 \\
21349\end{array}$ \\
\hline $\begin{array}{l}2200 \\
2300 \\
2400 \\
2500 \\
2600\end{array}$ & $\begin{array}{l}4.59416 \\
4.60425 \\
4.61387 \\
4.62308 \\
4.63194\end{array}$ & $\begin{array}{r}1009 \\
962 \\
921 \\
886 \\
855\end{array}$ & $\begin{array}{l}4.27226 \\
4.28647 \\
4.29992 \\
4.31267 \\
4.32477\end{array}$ & $\begin{array}{l}1421 \\
1345 \\
1275 \\
1210 \\
1154\end{array}$ & $\begin{array}{l}29.10712 \\
29.29734 \\
29.48005 \\
29.65584 \\
29.82523\end{array}$ & $\begin{array}{l}19022 \\
18271 \\
17579 \\
16939 \\
16343\end{array}$ & $\begin{array}{l}33.37938 \\
33.58381 \\
33.77996 \\
33.96851 \\
34.15000\end{array}$ & $\begin{array}{l}20443 \\
19615 \\
18855 \\
18149 \\
17497\end{array}$ \\
\hline $\begin{array}{l}2700 \\
2800 \\
2900 \\
3000\end{array}$ & $\begin{array}{l}4.64049 \\
4.64876 \\
4.65681 \\
4.66465\end{array}$ & $\begin{array}{r}827 \\
805 \\
784 \\
1513\end{array}$ & $\begin{array}{l}4.33631 \\
4.34732 \\
4.35785 \\
4.36795\end{array}$ & $\begin{array}{l}1101 \\
1053 \\
1010 \\
1902\end{array}$ & $\begin{array}{l}29.98866 \\
30.14657 \\
30.29932 \\
30.44723\end{array}$ & $\begin{array}{l}15791 \\
15275 \\
14791 \\
28251\end{array}$ & $\begin{array}{l}34.32497 \\
34.49389 \\
34.65717 \\
34.81517\end{array}$ & $\begin{array}{l}16892 \\
16328 \\
15800 \\
30154\end{array}$ \\
\hline $\begin{array}{l}3200 \\
3400 \\
3600 \\
3800 \\
4000\end{array}$ & $\begin{array}{l}4.67978 \\
4.69433 \\
4.70838 \\
4.72204 \\
4.73537\end{array}$ & $\begin{array}{l}1455 \\
1405 \\
1366 \\
1333 \\
1303\end{array}$ & $\begin{array}{l}4.38697 \\
4.40462 \\
4.42111 \\
4.43660 \\
4.45120\end{array}$ & $\begin{array}{l}1765 \\
1649 \\
1549 \\
1460 \\
1384\end{array}$ & $\begin{array}{l}30.72974 \\
30.99623 \\
31.24847 \\
31.48792 \\
31.71587\end{array}$ & $\begin{array}{l}26649 \\
25224 \\
23945 \\
22795 \\
21751\end{array}$ & $\begin{array}{l}35.11671 \\
35.40085 \\
35.66958 \\
35.92452 \\
36.16707\end{array}$ & $\begin{array}{l}28414 \\
26873 \\
25494 \\
24255 \\
23135\end{array}$ \\
\hline $\begin{array}{l}4200 \\
4400 \\
4600 \\
4800 \\
5000\end{array}$ & $\begin{array}{l}4.74840 \\
4.76120 \\
4.77376 \\
4.78613 \\
4.79833\end{array}$ & $\begin{array}{l}1280 \\
1256 \\
1237 \\
1220\end{array}$ & $\begin{array}{l}4.46504 \\
4.47821 \\
4.49079 \\
4.50284 \\
4.51441\end{array}$ & $\begin{array}{l}1317 \\
1258 \\
1205 \\
1157\end{array}$ & $\begin{array}{l}31.93338 \\
32.14140 \\
32.34074 \\
32.53213 \\
32.71618\end{array}$ & $\begin{array}{l}20802 \\
19934 \\
19139 \\
18405\end{array}$ & $\begin{array}{l}36.39842 \\
36.61961 \\
36.83153 \\
37.03497 \\
37.23059\end{array}$ & $\begin{array}{l}22119 \\
21192 \\
20344 \\
19562\end{array}$ \\
\hline
\end{tabular}


Table 2.079. SnH

\begin{tabular}{|c|c|c|c|c|c|c|c|c|}
\hline${ }^{\circ} K$ & $\frac{C_{p}^{\circ}}{R}$ & & $\frac{\left(H^{\circ}-E_{0}^{0}\right)}{R T}$ & & $\frac{-\left(F^{0}-\right.}{R T}$ & & $\frac{S^{\circ}}{R}$ & \\
\hline $\begin{array}{l}50 \\
60 \\
70 \\
80 \\
90\end{array}$ & $\begin{array}{l}3.50152 \\
3.50153 \\
3.50162 \\
3.50174 \\
3.50189\end{array}$ & $\begin{array}{r}1 \\
9 \\
12 \\
15 \\
16\end{array}$ & $\begin{array}{l}3.44917 \\
3.45790 \\
3.46414 \\
3.46883 \\
3.47249\end{array}$ & $\begin{array}{l}873 \\
624 \\
469 \\
366 \\
295\end{array}$ & $\begin{array}{l}15.91988 \\
16.54956 \\
17.08309 \\
17.54598 \\
17.95477\end{array}$ & $\begin{array}{l}62968 \\
53353 \\
46289 \\
40879 \\
36602\end{array}$ & $\begin{array}{l}19.36905 \\
20.00746 \\
20.54723 \\
21.01481 \\
21.42726\end{array}$ & $\begin{array}{l}63841 \\
53977 \\
46758 \\
41245 \\
36897\end{array}$ \\
\hline $\begin{array}{l}100 \\
110 \\
120 \\
130 \\
140\end{array}$ & $\begin{array}{l}3.50205 \\
3.50222 \\
3.50239 \\
3.50257 \\
3.50277\end{array}$ & $\begin{array}{l}17 \\
17 \\
18 \\
20 \\
22\end{array}$ & $\begin{array}{l}3.47544 \\
3.47787 \\
3.47990 \\
3.48164 \\
3.48314\end{array}$ & $\begin{array}{l}243 \\
203 \\
174 \\
150 \\
132\end{array}$ & $\begin{array}{l}18.32079 \\
18.65216 \\
18.95486 \\
19.23347 \\
19.49155\end{array}$ & $\begin{array}{l}33137 \\
30270 \\
27861 \\
25808 \\
24035\end{array}$ & $\begin{array}{l}21.79623 \\
22.13003 \\
22.43476 \\
22.71511 \\
22.97469\end{array}$ & $\begin{array}{l}33380 \\
30473 \\
28035 \\
25958 \\
24167\end{array}$ \\
\hline $\begin{array}{l}150 \\
160 \\
170 \\
180 \\
190\end{array}$ & $\begin{array}{l}3.50299 \\
3.50325 \\
3.50358 \\
3.50403 \\
3.50463\end{array}$ & $\begin{array}{l}26 \\
33 \\
45 \\
60 \\
81\end{array}$ & $\begin{array}{l}3.48446 \\
3.48562 \\
3.48667 \\
3.48762 \\
3.48850\end{array}$ & $\begin{array}{r}116 \\
105 \\
95 \\
88 \\
82\end{array}$ & $\begin{array}{l}19.73190 \\
19.95683 \\
20.16817 \\
20.36749 \\
20.55608\end{array}$ & $\begin{array}{l}22493 \\
21134 \\
19932 \\
18859 \\
17896\end{array}$ & $\begin{array}{l}23.21636 \\
23.44245 \\
23.65484 \\
23.85511 \\
24.04458\end{array}$ & $\begin{array}{l}22609 \\
21239 \\
20027 \\
18947 \\
17978\end{array}$ \\
\hline $\begin{array}{l}200 \\
210 \\
220 \\
230 \\
240\end{array}$ & $\begin{array}{l}3.50544 \\
3.50653 \\
3.50795 \\
3.50979 \\
3.51211\end{array}$ & $\begin{array}{l}109 \\
142 \\
184 \\
232 \\
285\end{array}$ & $\begin{array}{l}3.48932 \\
3.49012 \\
3.49090 \\
3.49168 \\
3.49248\end{array}$ & $\begin{array}{l}80 \\
78 \\
78 \\
80 \\
84\end{array}$ & $\begin{array}{l}20.73504 \\
20.90530 \\
21.06768 \\
21.22288 \\
21.37150\end{array}$ & $\begin{array}{l}17026 \\
16238 \\
15520 \\
14862 \\
14258\end{array}$ & $\begin{array}{l}24.22436 \\
24.39542 \\
24.55858 \\
24.71456 \\
24.86398\end{array}$ & $\begin{array}{l}17106 \\
16316 \\
15598 \\
14942 \\
14342\end{array}$ \\
\hline $\begin{array}{l}250 \\
260 \\
270 \\
280 \\
290\end{array}$ & $\begin{array}{l}3.51496 \\
3.51843 \\
3.52256 \\
3.52738 \\
3.53296\end{array}$ & $\begin{array}{l}347 \\
413 \\
482 \\
558 \\
636\end{array}$ & $\begin{array}{l}3.49332 \\
3.49421 \\
3.49519 \\
3.49625 \\
3.49742\end{array}$ & $\begin{array}{r}89 \\
98 \\
106 \\
117 \\
128\end{array}$ & $\begin{array}{l}21.51408 \\
21.65112 \\
21.78300 \\
21.91013 \\
22.03284\end{array}$ & $\begin{array}{l}13704 \\
13188 \\
12713 \\
12271 \\
11859\end{array}$ & $\begin{array}{l}25.00740 \\
25.14533 \\
25.27819 \\
25.40638 \\
25.53026\end{array}$ & $\begin{array}{l}13793 \\
13286 \\
12819 \\
12388 \\
11987\end{array}$ \\
\hline $\begin{array}{l}300 \\
310 \\
320 \\
330 \\
340\end{array}$ & $\begin{array}{l}3.53932 \\
3.54646 \\
3.55443 \\
3.56321 \\
3.57281\end{array}$ & $\begin{array}{r}714 \\
797 \\
878 \\
960 \\
1040\end{array}$ & $\begin{array}{l}3.49870 \\
3.50012 \\
3.50170 \\
3.50343 \\
3.50533\end{array}$ & $\begin{array}{l}142 \\
158 \\
173 \\
190 \\
207\end{array}$ & $\begin{array}{l}22.15143 \\
22.26618 \\
22.37733 \\
22.48511 \\
22.58972\end{array}$ & $\begin{array}{l}11475 \\
11115 \\
10778 \\
10461 \\
10164\end{array}$ & $\begin{array}{l}25.65013 \\
25.76630 \\
25.87903 \\
25.98854 \\
26.09505\end{array}$ & $\begin{array}{l}11617 \\
11273 \\
10951 \\
10651 \\
10371\end{array}$ \\
\hline $\begin{array}{l}350 \\
360 \\
370 \\
380 \\
390\end{array}$ & $\begin{array}{l}3.58321 \\
3.59439 \\
3.60635 \\
3.61906 \\
3.63248\end{array}$ & $\begin{array}{l}1118 \\
1196 \\
1271 \\
1342 \\
1411\end{array}$ & $\begin{array}{l}3.50740 \\
3.50966 \\
3.51211 \\
3.51476 \\
3.51760\end{array}$ & $\begin{array}{l}226 \\
245 \\
265 \\
284 \\
304\end{array}$ & $\begin{array}{l}22.69136 \\
22.79020 \\
22.88640 \\
22.98009 \\
23.07142\end{array}$ & $\begin{array}{l}9884 \\
9620 \\
9369 \\
9133 \\
8910\end{array}$ & $\begin{array}{l}26.19876 \\
26.29986 \\
26.39851 \\
26.49485 \\
26.58902\end{array}$ & $\begin{array}{r}10110 \\
9865 \\
9634 \\
9417 \\
9214\end{array}$ \\
\hline $\begin{array}{l}400 \\
450 \\
500 \\
550 \\
600\end{array}$ & $\begin{array}{l}3.64659 \\
3.72609 \\
3.81676 \\
3.91364 \\
4.01235\end{array}$ & $\begin{array}{l}7950 \\
9067 \\
9688 \\
9871 \\
9713\end{array}$ & $\begin{array}{l}3.52064 \\
3.53893 \\
3.56211 \\
3.58964 \\
3.62074\end{array}$ & $\begin{array}{l}1829 \\
2318 \\
2753 \\
3110 \\
3389\end{array}$ & $\begin{array}{l}23.16052 \\
23.57620 \\
23.95022 \\
24.29098 \\
24.60464\end{array}$ & $\begin{array}{l}41568 \\
37402 \\
34076 \\
31366 \\
29113\end{array}$ & $\begin{array}{l}26.68116 \\
27.11513 \\
27.51233 \\
27.88062 \\
28.22538\end{array}$ & $\begin{array}{l}43397 \\
39720 \\
36829 \\
34476 \\
32502\end{array}$ \\
\hline $\begin{array}{l}650 \\
700 \\
750 \\
800 \\
850\end{array}$ & $\begin{array}{l}4.10948 \\
4.20245 \\
4.28958 \\
4.36986 \\
4.44279\end{array}$ & $\begin{array}{l}9297 \\
8713 \\
8028 \\
7293 \\
6548\end{array}$ & $\begin{array}{l}3.65463 \\
3.69047 \\
3.72754 \\
3.76522 \\
3.80296\end{array}$ & $\begin{array}{l}3584 \\
3707 \\
3768 \\
3774 \\
3740\end{array}$ & $\begin{array}{l}24.89577 \\
25.16791 \\
25.42378 \\
25.66556 \\
25.89496\end{array}$ & $\begin{array}{l}27214 \\
25587 \\
24178 \\
22940 \\
21843\end{array}$ & $\begin{array}{l}28.55040 \\
28.85838 \\
29.15132 \\
29.43078 \\
29.69792\end{array}$ & $\begin{array}{l}30798 \\
29294 \\
27946 \\
26714 \\
25583\end{array}$ \\
\hline $\begin{array}{r}900 \\
950 \\
1000 \\
1050 \\
1100\end{array}$ & $\begin{array}{l}4.50827 \\
4.56647 \\
4.61776 \\
4.66261 \\
4.70157\end{array}$ & $\begin{array}{l}5820 \\
5129 \\
4485 \\
3896 \\
3362\end{array}$ & $\begin{array}{l}3.84036 \\
3.87708 \\
3.91286 \\
3.94752 \\
3.98093\end{array}$ & $\begin{array}{l}3672 \\
3578 \\
3466 \\
3341 \\
3208\end{array}$ & $\begin{array}{l}26.11339 \\
26.32201 \\
26.52179 \\
26.71355 \\
26.89796\end{array}$ & $\begin{array}{l}20862 \\
19978 \\
19176 \\
18441 \\
17768\end{array}$ & $\begin{array}{l}29.95375 \\
30.19909 \\
30.43465 \\
30.66107 \\
30.87889\end{array}$ & $\begin{array}{l}24534 \\
23556 \\
22642 \\
21782 \\
20976\end{array}$ \\
\hline 1150 & 4.73519 & 2884 & 4.01301 & 3070 & 27.07564 & 17144 & 31.08865 & 20214 \\
\hline
\end{tabular}


Table 2.079. SnH (Cont.)

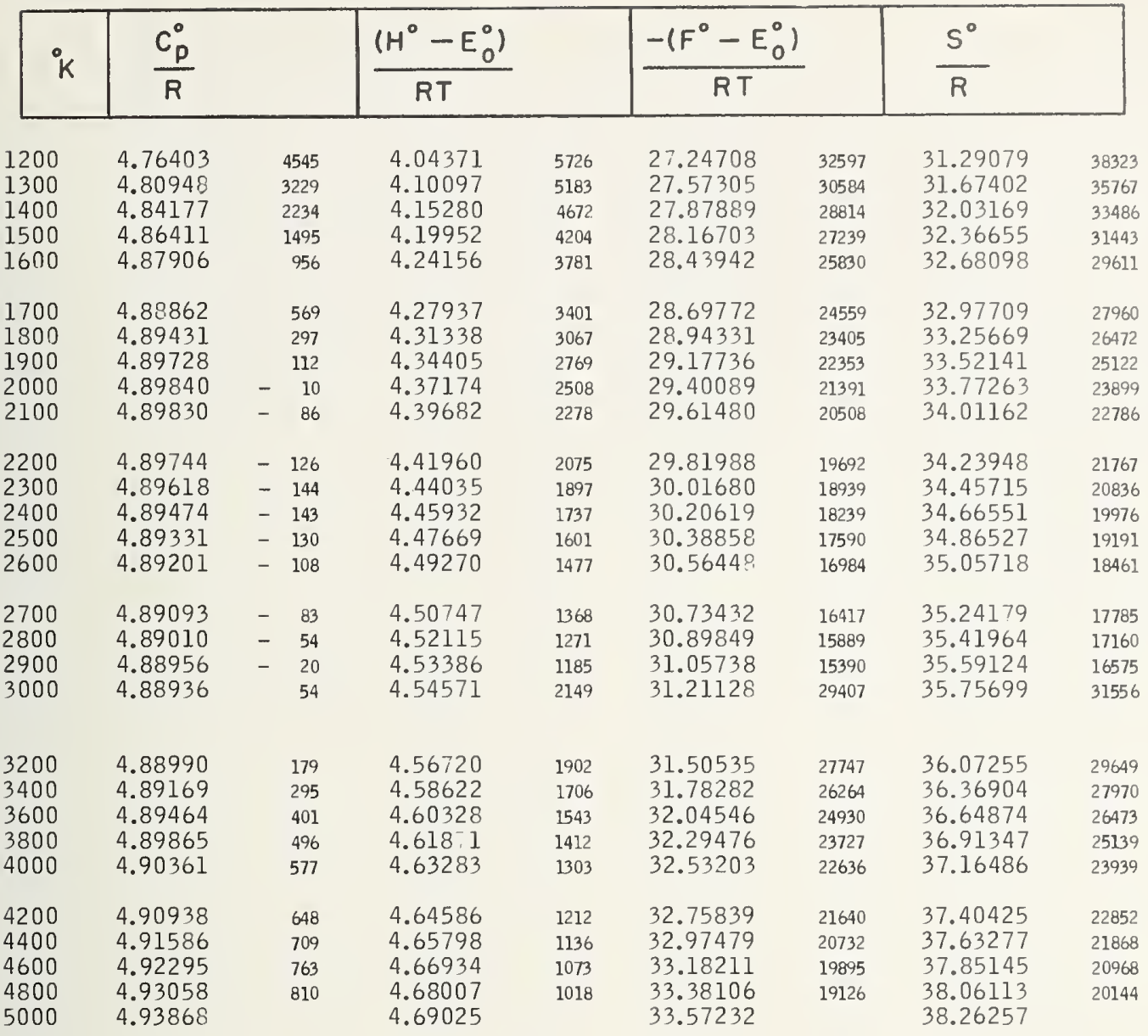


Table 2.080. SnD

\begin{tabular}{|c|c|c|c|c|c|c|c|c|}
\hline${ }^{\circ} \mathrm{K}$ & $\frac{C_{p}^{\circ}}{R}$ & & $\frac{\left(H^{0}-\sigma_{0}^{0}\right)}{R T}$ & & $\frac{-\left(F^{0}-\right.}{R T}$ & & $\frac{S^{0}}{R}$ & \\
\hline $\begin{array}{l}50 \\
60 \\
70 \\
80 \\
90\end{array}$ & $\begin{array}{l}3.50109 \\
3.50124 \\
3.50141 \\
3.50158 \\
3.50177\end{array}$ & $\begin{array}{l}15 \\
17 \\
17 \\
19 \\
20\end{array}$ & $\begin{array}{l}3.47463 \\
3.47905 \\
3.48223 \\
3.48464 \\
3.48653\end{array}$ & $\begin{array}{l}442 \\
318 \\
241 \\
189 \\
154\end{array}$ & $\begin{array}{l}16.58723 \\
17.22115 \\
17.75770 \\
18.22285 \\
18.63340\end{array}$ & $\begin{array}{l}63392 \\
53655 \\
46515 \\
41055 \\
36742\end{array}$ & $\begin{array}{l}20.06186 \\
20.70020 \\
21.23993 \\
21.70749 \\
22.11993\end{array}$ & $\begin{array}{l}63834 \\
53973 \\
46756 \\
41244 \\
36896\end{array}$ \\
\hline $\begin{array}{l}100 \\
110 \\
120 \\
130 \\
140\end{array}$ & $\begin{array}{l}3.50197 \\
3.50222 \\
3.50258 \\
3.50314 \\
3.50403\end{array}$ & $\begin{array}{r}25 \\
36 \\
56 \\
89 \\
136\end{array}$ & $\begin{array}{l}3.48807 \\
3.48934 \\
3.49043 \\
3.49138 \\
3.49225\end{array}$ & $\begin{array}{r}127 \\
109 \\
95 \\
87 \\
83\end{array}$ & $\begin{array}{l}19.00082 \\
19.33333 \\
19.63699 \\
19.91641 \\
20.17519\end{array}$ & $\begin{array}{l}33251 \\
30366 \\
27942 \\
25887 \\
24097\end{array}$ & $\begin{array}{l}22.48889 \\
22.82267 \\
23.12742 \\
23.40779 \\
23.66744\end{array}$ & $\begin{array}{l}33378 \\
30475 \\
28037 \\
25965 \\
24180\end{array}$ \\
\hline $\begin{array}{l}150 \\
160 \\
170 \\
180 \\
190\end{array}$ & $\begin{array}{l}3.50539 \\
3.50739 \\
3.51019 \\
3.51394 \\
3.51874\end{array}$ & $\begin{array}{l}200 \\
280 \\
375 \\
480 \\
595\end{array}$ & $\begin{array}{l}3.49308 \\
3.49391 \\
3.49478 \\
3.49574 \\
3.49681\end{array}$ & $\begin{array}{r}83 \\
87 \\
96 \\
107 \\
124\end{array}$ & $\begin{array}{l}20.41616 \\
20.64162 \\
20.85346 \\
21.05325 \\
21.24228\end{array}$ & $\begin{array}{l}22546 \\
21184 \\
19979 \\
18903 \\
17939\end{array}$ & $\begin{array}{l}23.90924 \\
24.13553 \\
24.34824 \\
24.54899 \\
24.73909\end{array}$ & $\begin{array}{l}22629 \\
21271 \\
20075 \\
19010 \\
18063\end{array}$ \\
\hline $\begin{array}{l}200 \\
210 \\
220 \\
230 \\
240\end{array}$ & $\begin{array}{l}3.52469 \\
3.53184 \\
3.54022 \\
3.54980 \\
3.56059\end{array}$ & $\begin{array}{r}715 \\
838 \\
958 \\
1079 \\
1192\end{array}$ & $\begin{array}{l}3.49805 \\
3.49949 \\
3.50115 \\
3.50305 \\
3.50522\end{array}$ & $\begin{array}{l}144 \\
166 \\
190 \\
217 \\
245\end{array}$ & $\begin{array}{l}21.42167 \\
21.59238 \\
21.75521 \\
21.91088 \\
22.06002\end{array}$ & $\begin{array}{l}17071 \\
16283 \\
15567 \\
14914 \\
14313\end{array}$ & $\begin{array}{l}24.91972 \\
25.09187 \\
25.25636 \\
25.41393 \\
25.56524\end{array}$ & $\begin{array}{l}17215 \\
16449 \\
15757 \\
15131 \\
14558\end{array}$ \\
\hline $\begin{array}{l}250 \\
260 \\
270 \\
280 \\
290\end{array}$ & $\begin{array}{l}3.57251 \\
3.58551 \\
3.59951 \\
3.61443 \\
3.63021\end{array}$ & $\begin{array}{l}1300 \\
1400 \\
1492 \\
1578 \\
1655\end{array}$ & $\begin{array}{l}3.50767 \\
3.51041 \\
3.51345 \\
3.51678 \\
3.52042\end{array}$ & $\begin{array}{l}274 \\
304 \\
333 \\
364 \\
393\end{array}$ & $\begin{array}{l}22.20315 \\
22.34079 \\
22.47333 \\
22.60116 \\
22.72463\end{array}$ & $\begin{array}{l}13764 \\
13254 \\
12783 \\
12347 \\
11941\end{array}$ & $\begin{array}{l}25.71082 \\
25.85120 \\
25.98678 \\
26.11794 \\
26.24505\end{array}$ & $\begin{array}{l}14038 \\
13558 \\
13116 \\
12711 \\
12334\end{array}$ \\
\hline $\begin{array}{l}300 \\
310 \\
320 \\
330 \\
340\end{array}$ & $\begin{array}{l}3.64676 \\
3.66399 \\
3.68184 \\
3.70023 \\
3.71909\end{array}$ & $\begin{array}{l}1723 \\
1785 \\
1839 \\
1886 \\
1929\end{array}$ & $\begin{array}{l}3.52435 \\
3.52858 \\
3.53309 \\
3.53787 \\
3.54293\end{array}$ & $\begin{array}{l}423 \\
451 \\
478 \\
506 \\
531\end{array}$ & $\begin{array}{l}22.84404 \\
22.95967 \\
23.07178 \\
23.18057 \\
23.28625\end{array}$ & $\begin{array}{l}11563 \\
11211 \\
10879 \\
10568 \\
10278\end{array}$ & $\begin{array}{l}26.36839 \\
26.48825 \\
26.60487 \\
26.71844 \\
26.82918\end{array}$ & $\begin{array}{l}11986 \\
11662 \\
11357 \\
11074 \\
10809\end{array}$ \\
\hline $\begin{array}{l}350 \\
360 \\
370 \\
380 \\
390\end{array}$ & $\begin{array}{l}3.73838 \\
3.75800 \\
3.77793 \\
3.79813 \\
3.81854\end{array}$ & $\begin{array}{l}1962 \\
1993 \\
2020 \\
2041 \\
2058\end{array}$ & $\begin{array}{l}3.54824 \\
3.55379 \\
3.55958 \\
3.56559 \\
3.57181\end{array}$ & $\begin{array}{l}555 \\
579 \\
601 \\
622 \\
642\end{array}$ & $\begin{array}{l}23.38903 \\
23.48907 \\
23.58652 \\
23.68152 \\
23.77422\end{array}$ & $\begin{array}{r}10004 \\
9745 \\
9500 \\
9270 \\
9052\end{array}$ & $\begin{array}{l}26.93727 \\
27.04286 \\
27.14610 \\
27.24711 \\
27.34603\end{array}$ & $\begin{array}{r}10559 \\
10324 \\
10101 \\
9892 \\
9694\end{array}$ \\
\hline $\begin{array}{l}400 \\
450 \\
500 \\
550 \\
600\end{array}$ & $\begin{array}{l}3.83912 \\
3.94348 \\
4.04770 \\
4.14928 \\
4.24631\end{array}$ & $\begin{array}{r}10436 \\
10422 \\
10158 \\
9703 \\
9119\end{array}$ & $\begin{array}{l}3.57823 \\
3.61300 \\
3.65128 \\
3.69196 \\
3.73415\end{array}$ & $\begin{array}{l}3477 \\
3828 \\
4068 \\
4219 \\
4295\end{array}$ & $\begin{array}{l}23.86474 \\
24.28816 \\
24.67078 \\
25.02067 \\
25.34372\end{array}$ & $\begin{array}{l}42342 \\
38262 \\
34989 \\
32305 \\
30059\end{array}$ & $\begin{array}{l}27.44297 \\
27.90116 \\
28.32206 \\
28.71263 \\
29.07787\end{array}$ & $\begin{array}{l}45819 \\
42090 \\
39057 \\
36524 \\
34354\end{array}$ \\
\hline $\begin{array}{l}650 \\
700 \\
750 \\
800 \\
850\end{array}$ & $\begin{array}{l}4.33750 \\
4.42184 \\
4.49882 \\
4.56823 \\
4.63009\end{array}$ & $\begin{array}{l}8434 \\
7698 \\
6941 \\
6186 \\
5458\end{array}$ & $\begin{array}{l}3.77710 \\
3.82019 \\
3.86290 \\
3.90486 \\
3.94573\end{array}$ & $\begin{array}{l}4309 \\
4271 \\
4196 \\
4087 \\
3957\end{array}$ & $\begin{array}{l}25.64431 \\
25.92579 \\
26.19082 \\
26.44148 \\
26.67944\end{array}$ & $\begin{array}{l}28148 \\
26503 \\
25066 \\
23796 \\
22665\end{array}$ & $\begin{array}{l}29.42141 \\
29.74598 \\
30.05372 \\
30.34634 \\
30.62517\end{array}$ & $\begin{array}{l}32457 \\
30774 \\
29262 \\
27883 \\
26622\end{array}$ \\
\hline $\begin{array}{r}900 \\
950 \\
1000 \\
1050 \\
1100\end{array}$ & $\begin{array}{l}4.68467 \\
4.73234 \\
4.77361 \\
4.80899 \\
4.83909\end{array}$ & $\begin{array}{l}4767 \\
4127 \\
3538 \\
3010 \\
2534\end{array}$ & $\begin{array}{l}3.98530 \\
4.02339 \\
4.05990 \\
4.09475 \\
4.12792\end{array}$ & $\begin{array}{l}3809 \\
3651 \\
3485 \\
3317 \\
3148\end{array}$ & $\begin{array}{l}26.90609 \\
27.12259 \\
27.32990 \\
27.52884 \\
27.72009\end{array}$ & $\begin{array}{l}21650 \\
20731 \\
19894 \\
19125 \\
18420\end{array}$ & $\begin{array}{l}30.89139 \\
31.14598 \\
31.38980 \\
31.62359 \\
31.84801\end{array}$ & $\begin{array}{l}25459 \\
24382 \\
23379 \\
22442 \\
21568\end{array}$ \\
\hline 1150 & 4.86443 & 2114 & 4.15940 & 2983 & 27.90429 & 17766 & 32.06369 & 20749 \\
\hline
\end{tabular}


Table 2.080. SnD (Cont.)

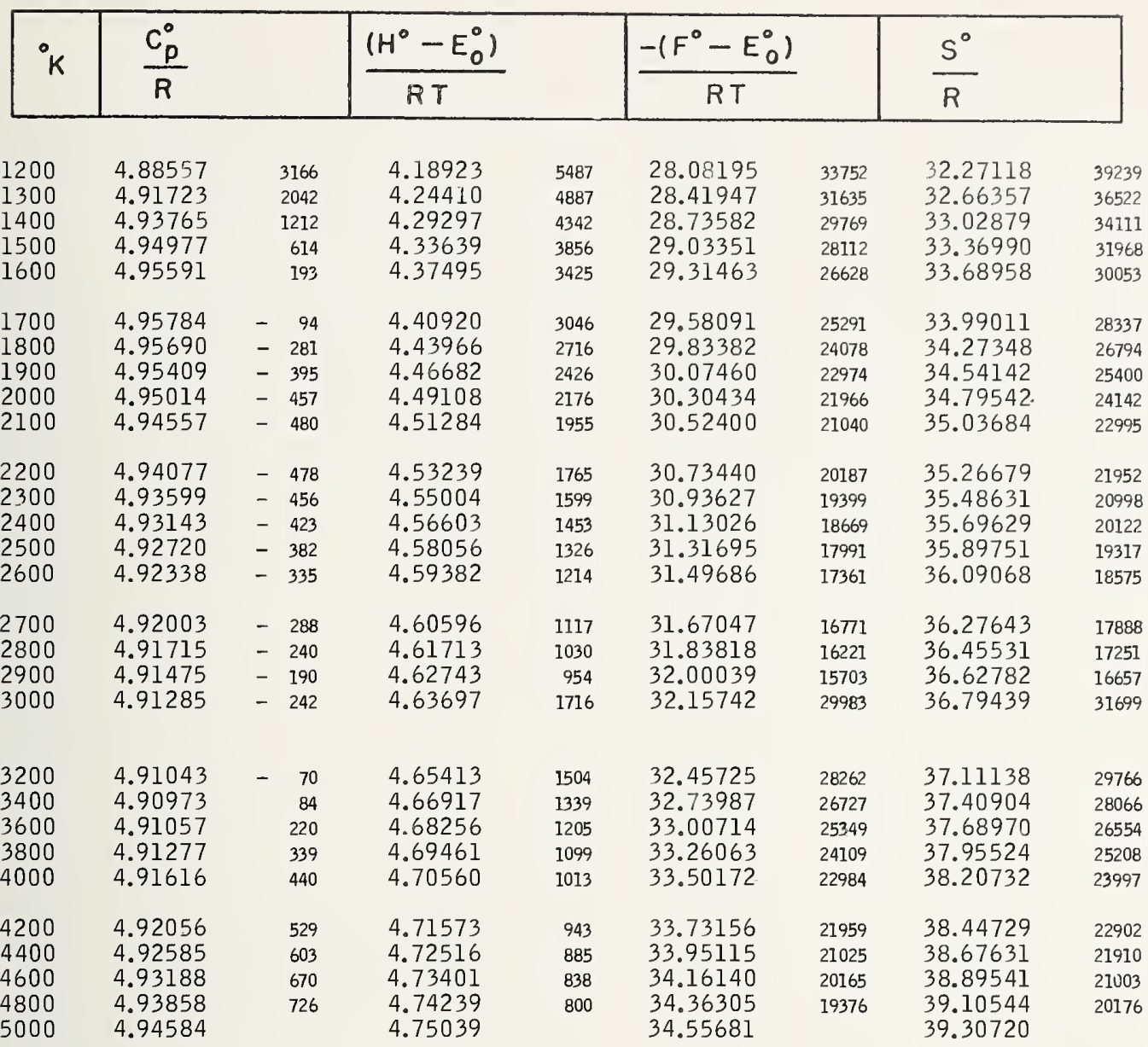


Table 2.081. SnT

\begin{tabular}{|c|c|c|c|c|c|c|c|c|}
\hline${ }^{\circ} \mathrm{K}$ & $\frac{C_{p}^{0}}{R}$ & & $\frac{\left(H^{0}-5_{0}^{0}\right)}{R T}$ & & $\frac{-\left(F^{0}-E\right.}{R T}$ & & $\frac{S^{0}}{R}$ & \\
\hline $\begin{array}{l}50 \\
60 \\
70 \\
80 \\
90\end{array}$ & $\begin{array}{l}3.50102 \\
3.50119 \\
3.50137 \\
3.50157 \\
3.50183\end{array}$ & $\begin{array}{l}17 \\
18 \\
20 \\
26 \\
41\end{array}$ & $\begin{array}{l}3.48307 \\
3.48607 \\
3.48825 \\
3.48990 \\
3.49121\end{array}$ & $\begin{array}{l}300 \\
218 \\
165 \\
131 \\
108\end{array}$ & $\begin{array}{l}16.98473 \\
17.62005 \\
18.15760 \\
18.62351 \\
19.03464\end{array}$ & $\begin{array}{l}63532 \\
53755 \\
46591 \\
41113 \\
36789\end{array}$ & $\begin{array}{l}20.46780 \\
21.10612 \\
21.64585 \\
22.11341 \\
22.52585\end{array}$ & $\begin{array}{l}63832 \\
53973 \\
46756 \\
41244 \\
36897\end{array}$ \\
\hline $\begin{array}{l}100 \\
110 \\
120 \\
130 \\
140\end{array}$ & $\begin{array}{l}3.50224 \\
3.50298 \\
3.50429 \\
3.50645 \\
3.50976\end{array}$ & $\begin{array}{r}74 \\
131 \\
216 \\
331 \\
470\end{array}$ & $\begin{array}{l}3.49229 \\
3.49322 \\
3.49409 \\
3.49495 \\
3.49588\end{array}$ & $\begin{array}{c}93 \\
87 \\
86 \\
93 \\
108\end{array}$ & $\begin{array}{l}19.40253 \\
19.73543 \\
20.03942 \\
20.31913 \\
20.57816\end{array}$ & $\begin{array}{l}33290 \\
30399 \\
27971 \\
25903 \\
24123\end{array}$ & $\begin{array}{l}22.89482 \\
23.22865 \\
23.53351 \\
23.81408 \\
24.07404\end{array}$ & $\begin{array}{l}33383 \\
30486 \\
28057 \\
25996 \\
24231\end{array}$ \\
\hline $\begin{array}{l}150 \\
160 \\
170 \\
180 \\
190\end{array}$ & $\begin{array}{l}3.51446 \\
3.52075 \\
3.52877 \\
3.53856 \\
3.55010\end{array}$ & $\begin{array}{r}629 \\
802 \\
979 \\
1154 \\
1323\end{array}$ & $\begin{array}{l}3.49696 \\
3.49824 \\
3.49979 \\
3.50166 \\
3.50390\end{array}$ & $\begin{array}{l}128 \\
155 \\
187 \\
224 \\
263\end{array}$ & $\begin{array}{l}20.81939 \\
21.04512 \\
21.25724 \\
21.45734 \\
21.64672\end{array}$ & $\begin{array}{l}22573 \\
21212 \\
20010 \\
18938 \\
17979\end{array}$ & $\begin{array}{l}24.31635 \\
24.54336 \\
24.75703 \\
24.95900 \\
25.15062\end{array}$ & $\begin{array}{l}22701 \\
21367 \\
20197 \\
19162 \\
18242\end{array}$ \\
\hline $\begin{array}{l}200 \\
210 \\
220 \\
230 \\
240\end{array}$ & $\begin{array}{l}3.56333 \\
3.57812 \\
3.59432 \\
3.61177 \\
3.63032\end{array}$ & $\begin{array}{l}1479 \\
1620 \\
1745 \\
1855 \\
1944\end{array}$ & $\begin{array}{l}3.50653 \\
3.50959 \\
3.51306 \\
3.51697 \\
3.52131\end{array}$ & $\begin{array}{l}306 \\
347 \\
391 \\
434 \\
474\end{array}$ & $\begin{array}{l}21.82651 \\
21.99767 \\
22.16101 \\
22.31726 \\
22.46703\end{array}$ & $\begin{array}{l}17116 \\
16334 \\
15625 \\
14977 \\
14384\end{array}$ & $\begin{array}{l}25.33304 \\
25.50726 \\
25.67407 \\
25.83423 \\
25.98834\end{array}$ & $\begin{array}{l}17422 \\
16681 \\
16016 \\
15411 \\
14858\end{array}$ \\
\hline $\begin{array}{l}250 \\
260 \\
270 \\
280 \\
290\end{array}$ & $\begin{array}{l}3.64976 \\
3.66996 \\
3.69077 \\
3.71203 \\
3.73366\end{array}$ & $\begin{array}{l}2020 \\
2081 \\
2126 \\
2163 \\
2188\end{array}$ & $\begin{array}{l}3.52605 \\
3.53119 \\
3.53672 \\
3.54260 \\
3.54882\end{array}$ & $\begin{array}{l}514 \\
553 \\
588 \\
622 \\
652\end{array}$ & $\begin{array}{l}22.61087 \\
22.74927 \\
22.88264 \\
23.01136 \\
23.13579\end{array}$ & $\begin{array}{l}13840 \\
13337 \\
12872 \\
12443 \\
12042\end{array}$ & $\begin{array}{l}26.13692 \\
26.28046 \\
26.41936 \\
26.55396 \\
26.68461\end{array}$ & $\begin{array}{l}14354 \\
13890 \\
13460 \\
13065 \\
12694\end{array}$ \\
\hline $\begin{array}{l}300 \\
310 \\
320 \\
330 \\
340\end{array}$ & $\begin{array}{l}3.75554 \\
3.77756 \\
3.79969 \\
3.32183 \\
3.84396\end{array}$ & $\begin{array}{l}2202 \\
2213 \\
2214 \\
2213 \\
2207\end{array}$ & $\begin{array}{l}3.55534 \\
3.56215 \\
3.56923 \\
3.57655 \\
3.58409\end{array}$ & $\begin{array}{l}681 \\
708 \\
732 \\
754 \\
775\end{array}$ & $\begin{array}{l}23.25621 \\
23.37289 \\
23.48610 \\
23.59605 \\
23.70292\end{array}$ & $\begin{array}{l}11668 \\
11321 \\
10995 \\
10687 \\
10401\end{array}$ & $\begin{array}{l}26.81155 \\
26.93504 \\
27.05533 \\
27.17260 \\
27.28701\end{array}$ & $\begin{array}{l}12349 \\
12029 \\
11727 \\
11441 \\
11176\end{array}$ \\
\hline $\begin{array}{l}350 \\
360 \\
370 \\
380 \\
390\end{array}$ & $\begin{array}{l}3.86603 \\
3.88798 \\
3.90982 \\
3.93154 \\
3.95309\end{array}$ & $\begin{array}{l}2195 \\
2184 \\
2172 \\
2155 \\
2139\end{array}$ & $\begin{array}{l}3.59184 \\
3.59976 \\
3.60784 \\
3.61607 \\
3.62444\end{array}$ & $\begin{array}{l}792 \\
808 \\
823 \\
837 \\
848\end{array}$ & $\begin{array}{l}23.80693 \\
23.90823 \\
24.00697 \\
24.10329 \\
24.19733\end{array}$ & $\begin{array}{r}10130 \\
9874 \\
9632 \\
9404 \\
9187\end{array}$ & $\begin{array}{l}27.39877 \\
27.50799 \\
27.61481 \\
27.71936 \\
27.82177\end{array}$ & $\begin{array}{l}10922 \\
10682 \\
10455 \\
10241 \\
10035\end{array}$ \\
\hline $\begin{array}{l}400 \\
450 \\
500 \\
550 \\
600\end{array}$ & $\begin{array}{l}3.97448 \\
4.07879 \\
4.17828 \\
4.27264 \\
4.36136\end{array}$ & $\begin{array}{r}10431 \\
9949 \\
9436 \\
8872 \\
8262\end{array}$ & $\begin{array}{l}3.63292 \\
3.67671 \\
3.72193 \\
3.76775 \\
3.91356\end{array}$ & $\begin{array}{l}4379 \\
4522 \\
4582 \\
4581 \\
4536\end{array}$ & $\begin{array}{l}24.28920 \\
24.71961 \\
25.10932 \\
25.46620 \\
25.79600\end{array}$ & $\begin{array}{l}43041 \\
38971 \\
35688 \\
32980 \\
30705\end{array}$ & $\begin{array}{l}27.92212 \\
28.39632 \\
28.83125 \\
29.23395 \\
29.60956\end{array}$ & $\begin{array}{l}47420 \\
43493 \\
40270 \\
37561 \\
35241\end{array}$ \\
\hline $\begin{array}{l}650 \\
700 \\
750 \\
800 \\
850\end{array}$ & $\begin{array}{l}4.44398 \\
4.51999 \\
4.58910 \\
4.65122 \\
4.70643\end{array}$ & $\begin{array}{l}7601 \\
6911 \\
6212 \\
5521 \\
4851\end{array}$ & $\begin{array}{l}3.85892 \\
3.90347 \\
3.94691 \\
3.98903 \\
4.02963\end{array}$ & $\begin{array}{l}4455 \\
4344 \\
4212 \\
4060 \\
3898\end{array}$ & $\begin{array}{l}26.10305 \\
26.39066 \\
26.66146 \\
26.91754 \\
27.16060\end{array}$ & $\begin{array}{l}28761 \\
27080 \\
25608 \\
24306 \\
23144\end{array}$ & $\begin{array}{l}29.96197 \\
30.29413 \\
30.60837 \\
30.90657 \\
31.19023\end{array}$ & $\begin{array}{l}33216 \\
31424 \\
29820 \\
28366 \\
27042\end{array}$ \\
\hline $\begin{array}{r}900 \\
950 \\
1000 \\
1050 \\
1100\end{array}$ & $\begin{array}{l}4.75494 \\
4.79711 \\
4.83341 \\
4.86430 \\
4.89033\end{array}$ & $\begin{array}{l}4217 \\
3630 \\
3089 \\
2603 \\
2167\end{array}$ & $\begin{array}{l}4.06861 \\
4.10587 \\
4.14136 \\
4.17507 \\
4.20701\end{array}$ & $\begin{array}{l}3726 \\
3549 \\
3371 \\
3194 \\
3020\end{array}$ & $\begin{array}{l}27.39204 \\
27.61302 \\
27.82453 \\
28.02742 \\
28.22238\end{array}$ & $\begin{array}{l}22098 \\
21151 \\
20289 \\
19496 \\
18769\end{array}$ & $\begin{array}{l}31.46065 \\
31.71889 \\
31.96589 \\
32.20249 \\
32.42939\end{array}$ & $\begin{array}{l}25824 \\
24700 \\
23660 \\
22690 \\
21789\end{array}$ \\
\hline 1150 & 4.91200 & 1780 & 4.23721 & 2849 & 28.41007 & 18094 & 32.64728 & 20943 \\
\hline
\end{tabular}


Table 2.081. SnT (Cont.)

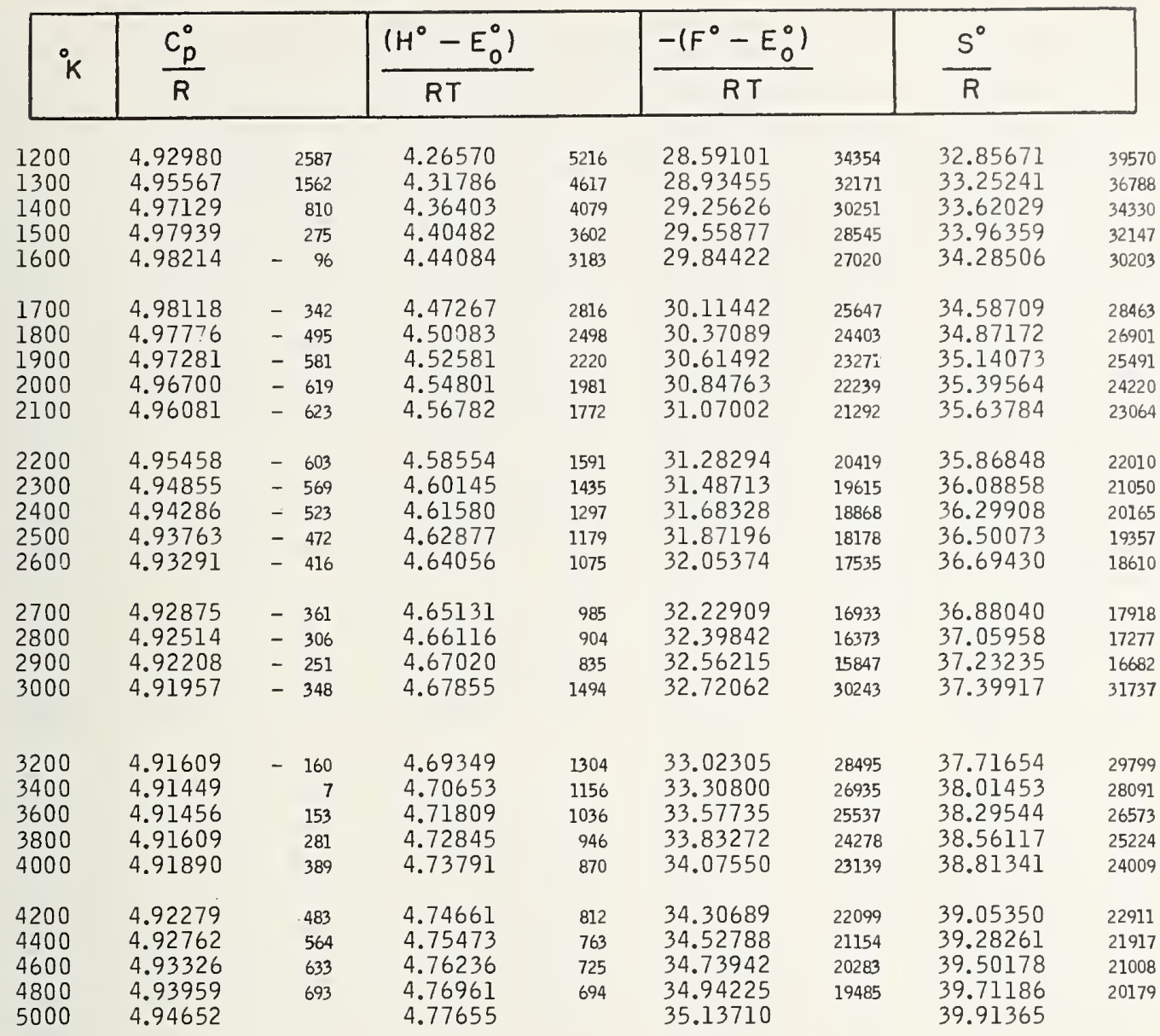


Table 2.082. $\mathrm{PbH}$

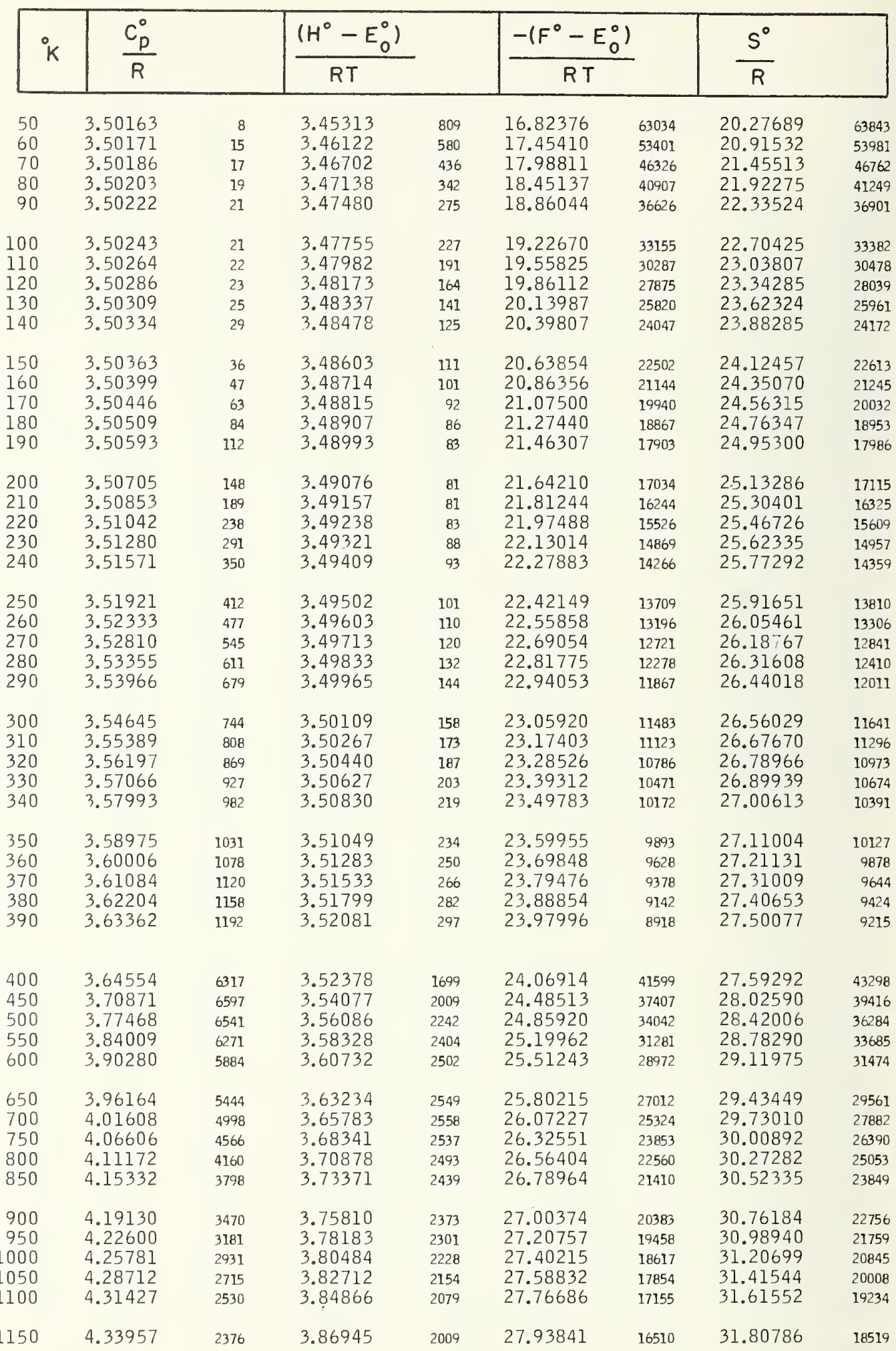


Table 2.082. $\mathrm{PbH}$ (Cont.)

\begin{tabular}{|c|c|c|c|c|c|c|c|c|}
\hline${ }^{\circ} \mathrm{K}$ & $\frac{C_{p}^{0}}{R}$ & & $\frac{\left(H^{0}-E_{0}^{0}\right)}{R T}$ & & $\frac{-\left(F^{\circ}-E\right.}{R T}$ & & $\frac{S^{\circ}}{R}$ & \\
\hline $\begin{array}{l}1200 \\
1300 \\
1400 \\
1500 \\
1600\end{array}$ & $\begin{array}{l}4.36333 \\
4.40717 \\
4.44752 \\
4.48567 \\
4.52254\end{array}$ & $\begin{array}{l}4384 \\
4035 \\
3815 \\
3687 \\
3620\end{array}$ & $\begin{array}{l}3.88954 \\
3.92770 \\
3.96341 \\
3.99696 \\
4.02866\end{array}$ & $\begin{array}{l}3816 \\
3571 \\
3355 \\
3170 \\
3012\end{array}$ & $\begin{array}{l}28.10351 \\
28.41636 \\
28.70876 \\
28.98336 \\
29.24235\end{array}$ & $\begin{array}{l}31285 \\
29240 \\
27460 \\
25897 \\
24514\end{array}$ & $\begin{array}{l}31.99305 \\
32.34406 \\
32.67217 \\
32.98032 \\
33.27101\end{array}$ & $\begin{array}{l}35101 \\
32811 \\
30815 \\
29069 \\
27526\end{array}$ \\
\hline $\begin{array}{l}1700 \\
1800 \\
1900 \\
2000 \\
2100\end{array}$ & $\begin{array}{l}4.55874 \\
4.59464 \\
4.63043 \\
4.66614 \\
4.70175\end{array}$ & $\begin{array}{l}3590 \\
3579 \\
3571 \\
3561 \\
3539\end{array}$ & $\begin{array}{l}4.05878 \\
4.08756 \\
4.11519 \\
4.14185 \\
4.16765\end{array}$ & $\begin{array}{l}2878 \\
2763 \\
2666 \\
2580 \\
2509\end{array}$ & $\begin{array}{l}29.48749 \\
29.72030 \\
29.94205 \\
30.15382 \\
30.35653\end{array}$ & $\begin{array}{l}23281 \\
22175 \\
21177 \\
20271 \\
19446\end{array}$ & $\begin{array}{l}33.54627 \\
33.80786 \\
34.05724 \\
34.29567 \\
34.52418\end{array}$ & $\begin{array}{l}26159 \\
24938 \\
23843 \\
22851 \\
21955\end{array}$ \\
\hline $\begin{array}{l}2200 \\
2300 \\
2400 \\
2500 \\
2600\end{array}$ & $\begin{array}{l}4.73714 \\
4.77217 \\
4.80668 \\
4.84052 \\
4.87354\end{array}$ & $\begin{array}{l}3503 \\
3451 \\
3334 \\
3302 \\
3207\end{array}$ & $\begin{array}{l}4.19274 \\
4.21718 \\
4.24102 \\
4.26432 \\
4.28712\end{array}$ & $\begin{array}{l}2444 \\
2384 \\
2330 \\
2280 \\
2232\end{array}$ & $\begin{array}{l}30.55099 \\
30.73791 \\
30.91789 \\
31.09149 \\
31.25919\end{array}$ & $\begin{array}{l}18692 \\
17998 \\
17360 \\
16770 \\
16222\end{array}$ & $\begin{array}{l}34.74373 \\
34.95509 \\
35.15891 \\
35.35581 \\
35.54631\end{array}$ & $\begin{array}{l}21136 \\
20382 \\
19690 \\
19050 \\
18454\end{array}$ \\
\hline $\begin{array}{l}2700 \\
2800 \\
2900 \\
3000\end{array}$ & $\begin{array}{l}4.90561 \\
4.93662 \\
4.96648 \\
4.99511\end{array}$ & $\begin{array}{l}3101 \\
2936 \\
2863 \\
5339\end{array}$ & $\begin{array}{l}4.30944 \\
4.33129 \\
4.35268 \\
4.37362\end{array}$ & $\begin{array}{l}2185 \\
2139 \\
2094 \\
4054\end{array}$ & $\begin{array}{l}31.42141 \\
31.57853 \\
31.73089 \\
31.87880\end{array}$ & $\begin{array}{l}15712 \\
15236 \\
14791 \\
28358\end{array}$ & $\begin{array}{l}35.73085 \\
35.90982 \\
36.08357 \\
36.25242\end{array}$ & $\begin{array}{l}17897 \\
17375 \\
16885 \\
32412\end{array}$ \\
\hline $\begin{array}{l}3200 \\
3400 \\
3600 \\
3800 \\
4000\end{array}$ & $\begin{array}{l}5.04850 \\
5.09660 \\
5.13946 \\
5.17728 \\
5.21035\end{array}$ & $\begin{array}{l}4810 \\
4286 \\
3782 \\
3307 \\
2879\end{array}$ & $\begin{array}{l}4.41416 \\
4.45291 \\
4.48989 \\
4.52510 \\
4.55855\end{array}$ & $\begin{array}{l}3875 \\
3698 \\
3521 \\
3345 \\
3174\end{array}$ & $\begin{array}{l}32.16238 \\
32.43116 \\
32.68674 \\
32.93044 \\
33.16340\end{array}$ & $\begin{array}{l}26878 \\
25558 \\
24370 \\
23296 \\
22319\end{array}$ & $\begin{array}{l}36.57654 \\
36.88407 \\
37.17663 \\
37.45554 \\
37.72195\end{array}$ & $\begin{array}{l}30753 \\
29256 \\
27891 \\
26641 \\
25493\end{array}$ \\
\hline $\begin{array}{l}4200 \\
4400 \\
4600 \\
4800 \\
5000\end{array}$ & $\begin{array}{l}5.23914 \\
5.26407 \\
5.28555 \\
5.30403 \\
5.31993\end{array}$ & $\begin{array}{l}2493 \\
2148 \\
1848 \\
1590\end{array}$ & $\begin{array}{l}4.59029 \\
4.62036 \\
4.64883 \\
4.67576 \\
4.70122\end{array}$ & $\begin{array}{l}3007 \\
2847 \\
2693 \\
2546\end{array}$ & $\begin{array}{l}33.38659 \\
33.60084 \\
33.80685 \\
34.00528 \\
34.19668\end{array}$ & $\begin{array}{l}21425 \\
20601 \\
19843 \\
19140\end{array}$ & $\begin{array}{l}37.97688 \\
38.22120 \\
38.45568 \\
38.68104 \\
38.89790\end{array}$ & $\begin{array}{l}24432 \\
23448 \\
22536 \\
21696\end{array}$ \\
\hline
\end{tabular}


Table 2.083. PbD

\begin{tabular}{|c|c|c|c|c|c|c|c|c|}
\hline${ }^{\circ} \mathrm{K}$ & $\frac{C_{p}^{0}}{R}$ & & $\frac{\left(H^{\circ}-E_{0}^{0}\right)}{R T}$ & & $\frac{-\left(F^{\circ}-E\right.}{R T}$ & & $\frac{S^{0}}{R}$ & \\
\hline $\begin{array}{l}50 \\
60 \\
70 \\
80 \\
90\end{array}$ & $\begin{array}{l}3.50127 \\
3.50147 \\
3.50168 \\
3.50190 \\
3.50213\end{array}$ & $\begin{array}{l}20 \\
21 \\
22 \\
23 \\
25\end{array}$ & $\begin{array}{l}3.47675 \\
3.48085 \\
3.48381 \\
3.48606 \\
3.48783\end{array}$ & $\begin{array}{l}410 \\
296 \\
225 \\
177 \\
144\end{array}$ & $\begin{array}{l}17.49112 \\
18.12540 \\
18.66221 \\
19.12756 \\
19.53826\end{array}$ & $\begin{array}{l}63428 \\
53681 \\
46535 \\
41070 \\
36756\end{array}$ & $\begin{array}{l}20.96787 \\
21.60625 \\
22.14602 \\
22.61362 \\
23.02609\end{array}$ & $\begin{array}{l}63838 \\
53977 \\
46760 \\
41247 \\
36900\end{array}$ \\
\hline $\begin{array}{l}100 \\
110 \\
120 \\
130 \\
140\end{array}$ & $\begin{array}{l}3.50238 \\
3.50272 \\
3.50321 \\
3.50397 \\
3.50517\end{array}$ & $\begin{array}{r}34 \\
49 \\
76 \\
120 \\
181\end{array}$ & $\begin{array}{l}3.48927 \\
3.49048 \\
3.49152 \\
3.49244 \\
3.49331\end{array}$ & $\begin{array}{r}121 \\
104 \\
92 \\
87 \\
84\end{array}$ & $\begin{array}{l}19.90582 \\
20.23844 \\
20.54220 \\
20.82171 \\
21.08056\end{array}$ & $\begin{array}{l}33262 \\
30376 \\
27951 \\
25885 \\
24104\end{array}$ & $\begin{array}{l}23.39509 \\
23.72892 \\
24.03372 \\
24.31415 \\
24.57387\end{array}$ & $\begin{array}{l}33383 \\
30480 \\
28043 \\
25972 \\
24188\end{array}$ \\
\hline $\begin{array}{l}150 \\
160 \\
170 \\
180 \\
190\end{array}$ & $\begin{array}{l}3.50698 \\
3.50959 \\
3.51316 \\
3.51783 \\
3.52371\end{array}$ & $\begin{array}{l}261 \\
357 \\
467 \\
588 \\
716\end{array}$ & $\begin{array}{l}3.49415 \\
3.49503 \\
3.49599 \\
3.49707 \\
3.49831\end{array}$ & $\begin{array}{r}88 \\
96 \\
108 \\
124 \\
144\end{array}$ & $\begin{array}{l}21.32160 \\
21.54713 \\
21.75905 \\
21.95890 \\
22.14801\end{array}$ & $\begin{array}{l}22553 \\
21192 \\
19985 \\
18911 \\
17948\end{array}$ & $\begin{array}{l}24.81575 \\
25.04216 \\
25.25504 \\
25.45597 \\
25.64632\end{array}$ & $\begin{array}{l}22641 \\
21288 \\
20093 \\
19035 \\
18092\end{array}$ \\
\hline $\begin{array}{l}200 \\
210 \\
220 \\
230 \\
240\end{array}$ & $\begin{array}{l}3.53087 \\
3.53931 \\
3.54905 \\
3.56002 \\
3.57216\end{array}$ & $\begin{array}{r}844 \\
974 \\
1097 \\
1214 \\
1323\end{array}$ & $\begin{array}{l}3.49975 \\
3.50143 \\
3.50337 \\
3.50559 \\
3.50811\end{array}$ & $\begin{array}{l}168 \\
194 \\
222 \\
252 \\
282\end{array}$ & $\begin{array}{l}22.32749 \\
22.49828 \\
22.66121 \\
22.81699 \\
22.96624\end{array}$ & $\begin{array}{l}17079 \\
16293 \\
15578 \\
14925 \\
14326\end{array}$ & $\begin{array}{l}25.82724 \\
25.99971 \\
26.16458 \\
26.32258 \\
26.47435\end{array}$ & $\begin{array}{l}17247 \\
16487 \\
15800 \\
15177 \\
14608\end{array}$ \\
\hline $\begin{array}{l}250 \\
260 \\
270 \\
280 \\
290\end{array}$ & $\begin{array}{l}3.58539 \\
3.59959 \\
3.61466 \\
3.63048 \\
3.64694\end{array}$ & $\begin{array}{l}1420 \\
1507 \\
1582 \\
1646 \\
1698\end{array}$ & $\begin{array}{l}3.51093 \\
3.51406 \\
3.51751 \\
3.52126 \\
3.52531\end{array}$ & $\begin{array}{l}313 \\
345 \\
375 \\
405 \\
433\end{array}$ & $\begin{array}{l}23.10950 \\
23.24727 \\
23.37995 \\
23.50794 \\
23.63158\end{array}$ & $\begin{array}{l}13777 \\
13268 \\
12799 \\
12364 \\
11958\end{array}$ & $\begin{array}{l}26.62043 \\
26.76133 \\
26.89746 \\
27.02920 \\
27.15689\end{array}$ & $\begin{array}{l}14090 \\
13613 \\
13174 \\
12769 \\
12391\end{array}$ \\
\hline $\begin{array}{l}300 \\
310 \\
320 \\
330 \\
340\end{array}$ & $\begin{array}{l}3.66392 \\
3.68132 \\
3.69904 \\
3.71698 \\
3.73505\end{array}$ & $\begin{array}{l}1740 \\
1772 \\
1794 \\
1807 \\
1814\end{array}$ & $\begin{array}{l}3.52964 \\
3.53425 \\
3.53913 \\
3.54424 \\
3.54959\end{array}$ & $\begin{array}{l}461 \\
488 \\
511 \\
535 \\
556\end{array}$ & $\begin{array}{l}23.75116 \\
23.86697 \\
23.97926 \\
24.08824 \\
24.19413\end{array}$ & $\begin{array}{l}11581 \\
11229 \\
10898 \\
10589 \\
10297\end{array}$ & $\begin{array}{l}27.28080 \\
27.40122 \\
27.51839 \\
27.63248 \\
27.74372\end{array}$ & $\begin{array}{l}12042 \\
11717 \\
11409 \\
11124 \\
10853\end{array}$ \\
\hline $\begin{array}{l}350 \\
360 \\
370 \\
380 \\
390\end{array}$ & $\begin{array}{l}3.75319 \\
3.77133 \\
3.78939 \\
3.80734 \\
3.82511\end{array}$ & $\begin{array}{l}1814 \\
1806 \\
1795 \\
1777 \\
1758\end{array}$ & $\begin{array}{l}3.55515 \\
3.56090 \\
3.56683 \\
3.57293 \\
3.57916\end{array}$ & $\begin{array}{l}575 \\
593 \\
610 \\
623 \\
637\end{array}$ & $\begin{array}{l}24.29710 \\
24.39733 \\
24.49498 \\
24.59018 \\
24.68307\end{array}$ & $\begin{array}{r}10023 \\
9765 \\
9520 \\
9289 \\
9070\end{array}$ & $\begin{array}{l}27.85225 \\
27.95823 \\
28.06181 \\
28.16311 \\
28.26223\end{array}$ & $\begin{array}{r}10598 \\
10358 \\
10130 \\
9912 \\
9707\end{array}$ \\
\hline $\begin{array}{l}400 \\
450 \\
500 \\
550 \\
600\end{array}$ & $\begin{array}{l}3.84269 \\
3.92645 \\
4.00198 \\
4.06875 \\
4.12721\end{array}$ & $\begin{array}{l}8376 \\
7553 \\
6677 \\
5846 \\
5103\end{array}$ & $\begin{array}{l}3.58553 \\
3.61883 \\
3.65344 \\
3.68823 \\
3.72243\end{array}$ & $\begin{array}{l}3330 \\
3461 \\
3479 \\
3420 \\
3314\end{array}$ & $\begin{array}{l}24.77377 \\
25.19798 \\
25.58105 \\
25.93089 \\
26.25329\end{array}$ & $\begin{array}{l}42421 \\
38307 \\
34984 \\
32240 \\
29927\end{array}$ & $\begin{array}{l}28.35930 \\
28.81681 \\
29.23449 \\
29.61912 \\
29.97572\end{array}$ & $\begin{array}{l}45751 \\
41768 \\
38463 \\
35660 \\
33241\end{array}$ \\
\hline $\begin{array}{l}650 \\
700 \\
750 \\
800 \\
850\end{array}$ & $\begin{array}{l}4.17824 \\
4.22281 \\
4.26187 \\
4.29626 \\
4.32667\end{array}$ & $\begin{array}{l}4457 \\
3906 \\
3439 \\
3041 \\
2715\end{array}$ & $\begin{array}{l}3.75557 \\
3.78739 \\
3.81775 \\
3.84661 \\
3.87396\end{array}$ & $\begin{array}{l}3182 \\
3036 \\
2886 \\
2735 \\
2593\end{array}$ & $\begin{array}{l}26.55256 \\
26.83205 \\
27.09439 \\
27.34172 \\
27.57574\end{array}$ & $\begin{array}{l}27949 \\
26234 \\
24733 \\
23402 \\
22217\end{array}$ & $\begin{array}{l}30.30813 \\
30.61944 \\
30.91214 \\
31.18833 \\
31.44970\end{array}$ & $\begin{array}{l}31131 \\
29270 \\
27619 \\
26137 \\
24810\end{array}$ \\
\hline $\begin{array}{r}900 \\
950 \\
1000 \\
1050 \\
1100\end{array}$ & $\begin{array}{l}4.35382 \\
4.37822 \\
4.40032 \\
4.42054 \\
4.43924\end{array}$ & $\begin{array}{l}2440 \\
2210 \\
2022 \\
1870 \\
1746\end{array}$ & $\begin{array}{l}3.89989 \\
3.92443 \\
3.94768 \\
3.96973 \\
3.99065\end{array}$ & $\begin{array}{l}2454 \\
2325 \\
2205 \\
2092 \\
1988\end{array}$ & $\begin{array}{l}27.79791 \\
28.00943 \\
28.21133 \\
28.40447 \\
28.58963\end{array}$ & $\begin{array}{l}21152 \\
20190 \\
19314 \\
18516 \\
17784\end{array}$ & $\begin{array}{l}31.69780 \\
31.93386 \\
32.15901 \\
32.37420 \\
32.58028\end{array}$ & $\begin{array}{l}23606 \\
22515 \\
21519 \\
20608 \\
19772\end{array}$ \\
\hline 1150 & 4.45670 & 1649 & 4.01053 & 1894 & 28.76747 & 17108 & 32.77800 & 19002 \\
\hline
\end{tabular}


Table 2.083. PbD (Cont.)

\begin{tabular}{|c|c|c|c|c|c|c|c|c|}
\hline${ }^{\circ} K$ & $\frac{C_{p}^{0}}{R}$ & & $\frac{\left(H^{0}-E_{0}^{0}\right)}{R T}$ & & $\frac{-\left(F^{\circ}-E\right.}{R T}$ & & $\frac{S^{0}}{R}$ & \\
\hline $\begin{array}{l}1200 \\
1300 \\
1400 \\
1500 \\
1600\end{array}$ & $\begin{array}{l}4.47319 \\
4.50412 \\
4.53342 \\
4.56212 \\
4.59086\end{array}$ & $\begin{array}{l}3093 \\
2930 \\
2870 \\
2874 \\
2918\end{array}$ & $\begin{array}{l}4.02947 \\
4.06481 \\
4.09724 \\
4.12727 \\
4.15535\end{array}$ & $\begin{array}{l}3534 \\
3243 \\
3003 \\
2808 \\
2647\end{array}$ & $\begin{array}{l}28.93855 \\
29.26250 \\
29.56494 \\
29.84865 \\
30.11594\end{array}$ & $\begin{array}{l}32395 \\
30244 \\
28371 \\
26729 \\
25271\end{array}$ & $\begin{array}{l}32.96802 \\
33.32731 \\
33.66218 \\
33.97592 \\
34.27129\end{array}$ & $\begin{array}{l}35929 \\
33487 \\
31374 \\
29537 \\
27918\end{array}$ \\
\hline $\begin{array}{l}1700 \\
1800 \\
1900 \\
2000 \\
2100\end{array}$ & $\begin{array}{l}4.62004 \\
4.64986 \\
4.68034 \\
4.71141 \\
4.74292\end{array}$ & $\begin{array}{l}2982 \\
3048 \\
3107 \\
3151 \\
3177\end{array}$ & $\begin{array}{l}4.18182 \\
4.20700 \\
4.23110 \\
4.25434 \\
4.27685\end{array}$ & $\begin{array}{l}2518 \\
2410 \\
2324 \\
2251 \\
2191\end{array}$ & $\begin{array}{l}30.36865 \\
30.60839 \\
30.83651 \\
31.05413 \\
31.26225\end{array}$ & $\begin{array}{l}23974 \\
22812 \\
21762 \\
20812 \\
19946\end{array}$ & $\begin{array}{l}34.55047 \\
34.81539 \\
35.06761 \\
35.30847 \\
35.53910\end{array}$ & $\begin{array}{l}26492 \\
25222 \\
24086 \\
23063 \\
22137\end{array}$ \\
\hline $\begin{array}{l}2200 \\
2300 \\
2400 \\
2500 \\
2600\end{array}$ & $\begin{array}{l}4.77469 \\
4.80651 \\
4.83816 \\
4.86943 \\
4.90014\end{array}$ & $\begin{array}{l}3182 \\
3165 \\
3127 \\
3071 \\
2999\end{array}$ & $\begin{array}{l}4.29876 \\
4.32015 \\
4.34107 \\
4.36158 \\
4.38171\end{array}$ & $\begin{array}{l}2139 \\
2072 \\
2051 \\
2013 \\
1975\end{array}$ & $\begin{array}{l}31.46171 \\
31.65328 \\
31.83758 \\
32.01521 \\
32.18667\end{array}$ & $\begin{array}{l}19157 \\
18430 \\
17763 \\
17146 \\
16574\end{array}$ & $\begin{array}{l}35.76047 \\
35.97343 \\
36.17865 \\
36.37679 \\
36.56838\end{array}$ & $\begin{array}{l}21296 \\
20522 \\
19814 \\
19159 \\
18549\end{array}$ \\
\hline $\begin{array}{l}2700 \\
2800 \\
2900 \\
3000\end{array}$ & $\begin{array}{l}4.93013 \\
4.95925 \\
4.98739 \\
5.01446\end{array}$ & $\begin{array}{l}2912 \\
2814 \\
2707 \\
5065\end{array}$ & $\begin{array}{l}4.40146 \\
4.42087 \\
4.43992 \\
4.45862\end{array}$ & $\begin{array}{l}1941 \\
1905 \\
1870 \\
3635\end{array}$ & $\begin{array}{l}32.35241 \\
32.51283 \\
32.66829 \\
32.81913\end{array}$ & $\begin{array}{l}16042 \\
15546 \\
15084 \\
28893\end{array}$ & $\begin{array}{l}36.75387 \\
36.93370 \\
37.10821 \\
37.27775\end{array}$ & $\begin{array}{l}17983 \\
17451 \\
16954 \\
32528\end{array}$ \\
\hline $\begin{array}{l}3200 \\
3400 \\
3600 \\
3800 \\
4000\end{array}$ & $\begin{array}{l}5.06511 \\
5.11089 \\
5.15177 \\
5.18788 \\
5.21947\end{array}$ & $\begin{array}{l}4578 \\
4088 \\
3611 \\
3159 \\
274 \overline{8}\end{array}$ & $\begin{array}{l}4.49497 \\
4.52988 \\
4.56332 \\
4.59526 \\
4.62569\end{array}$ & $\begin{array}{l}3491 \\
3344 \\
3194 \\
3043 \\
2895\end{array}$ & $\begin{array}{l}33.10806 \\
33.38162 \\
33.64149 \\
33.88908 \\
34.12556\end{array}$ & $\begin{array}{l}27356 \\
25987 \\
24759 \\
23648 \\
22640\end{array}$ & $\begin{array}{l}37.60303 \\
37.91150 \\
38.20481 \\
38.48434 \\
38.75125\end{array}$ & $\begin{array}{l}30847 \\
29331 \\
27953 \\
26691 \\
25535\end{array}$ \\
\hline $\begin{array}{l}4200 \\
4400 \\
4600 \\
4800 \\
5000\end{array}$ & $\begin{array}{l}5.24695 \\
5.27073 \\
5.29119 \\
5.30876 \\
5.32384\end{array}$ & $\begin{array}{l}2378 \\
2046 \\
1757 \\
1508\end{array}$ & $\begin{array}{l}4.65464 \\
4.68212 \\
4.70817 \\
4.73284 \\
4.75619\end{array}$ & $\begin{array}{l}2748 \\
2605 \\
2467 \\
2335\end{array}$ & $\begin{array}{l}34.35196 \\
34.56914 \\
34.77784 \\
34.97875 \\
35.17244\end{array}$ & $\begin{array}{l}21718 \\
20870 \\
20091 \\
19369\end{array}$ & $\begin{array}{l}39.00660 \\
39.25126 \\
39.48601 \\
39.71159 \\
39.92863\end{array}$ & $\begin{array}{l}24466 \\
23475 \\
22558 \\
21704\end{array}$ \\
\hline
\end{tabular}


Table 2.084. PbT

\begin{tabular}{|c|c|c|c|c|c|c|c|c|}
\hline${ }^{\circ} \mathrm{K}$ & $\frac{C_{p}^{0}}{R}$ & & $\frac{\left(H^{\circ}-E_{0}^{0}\right)}{R T}$ & & $\frac{-\left(F^{0}-E\right.}{R T}$ & & $\frac{S^{0}}{R}$ & \\
\hline $\begin{array}{l}50 \\
60 \\
70 \\
80 \\
90\end{array}$ & $\begin{array}{l}3.50121 \\
3.50142 \\
3.50165 \\
3.50190 \\
3.50224\end{array}$ & $\begin{array}{l}21 \\
23 \\
25 \\
34 \\
55\end{array}$ & $\begin{array}{l}3.48458 \\
3.48737 \\
3.48939 \\
3.49094 \\
3.49217\end{array}$ & $\begin{array}{l}279 \\
202 \\
155 \\
123 \\
103\end{array}$ & $\begin{array}{l}17.88743 \\
18.52300 \\
19.06074 \\
19.52679 \\
19.93804\end{array}$ & $\begin{array}{l}63557 \\
53774 \\
46605 \\
41125 \\
36799\end{array}$ & $\begin{array}{l}21.37201 \\
22.01037 \\
22.55013 \\
23.01773 \\
23.43021\end{array}$ & $\begin{array}{l}63836 \\
53976 \\
46760 \\
41248 \\
36902\end{array}$ \\
\hline $\begin{array}{l}100 \\
110 \\
120 \\
130 \\
140\end{array}$ & $\begin{array}{l}3.50279 \\
3.50379 \\
3.50553 \\
3.50832 \\
3.51247\end{array}$ & $\begin{array}{l}100 \\
174 \\
279 \\
415 \\
578\end{array}$ & $\begin{array}{l}3.49320 \\
3.49412 \\
3.49499 \\
3.49590 \\
3.49693\end{array}$ & $\begin{array}{r}92 \\
87 \\
91 \\
103 \\
122\end{array}$ & $\begin{array}{l}20.30603 \\
20.63901 \\
20.94308 \\
21.22286 \\
21.48197\end{array}$ & $\begin{array}{l}33298 \\
30407 \\
27978 \\
25911 \\
24131\end{array}$ & $\begin{array}{l}23.79923 \\
24.13313 \\
24.43807 \\
24.71876 \\
24.97890\end{array}$ & $\begin{array}{l}33390 \\
30494 \\
28069 \\
26014 \\
24253\end{array}$ \\
\hline $\begin{array}{l}150 \\
160 \\
170 \\
180 \\
190\end{array}$ & $\begin{array}{l}3.51825 \\
3.52583 \\
3.53530 \\
3.54666 \\
3.55986\end{array}$ & $\begin{array}{r}758 \\
947 \\
1136 \\
1320 \\
1490\end{array}$ & $\begin{array}{l}3.49815 \\
3.49963 \\
3.50144 \\
3.50363 \\
3.50623\end{array}$ & $\begin{array}{l}148 \\
181 \\
219 \\
260 \\
305\end{array}$ & $\begin{array}{l}21.72328 \\
21.94909 \\
22.16131 \\
22.36150 \\
22.55100\end{array}$ & $\begin{array}{l}22581 \\
21222 \\
20019 \\
18950 \\
17992\end{array}$ & $\begin{array}{l}25.22143 \\
25.44872 \\
25.66275 \\
25.86513 \\
26.05723\end{array}$ & $\begin{array}{l}22729 \\
21403 \\
20238 \\
19210 \\
18297\end{array}$ \\
\hline $\begin{array}{l}200 \\
210 \\
220 \\
230 \\
240\end{array}$ & $\begin{array}{l}3.57476 \\
3.59121 \\
3.60901 \\
3.62796 \\
3.64785\end{array}$ & $\begin{array}{l}1645 \\
1780 \\
1895 \\
1989 \\
2063\end{array}$ & $\begin{array}{l}3.50928 \\
3.51278 \\
3.51675 \\
3.52117 \\
3.52603\end{array}$ & $\begin{array}{l}350 \\
397 \\
442 \\
486 \\
528\end{array}$ & $\begin{array}{l}22.73092 \\
22.90223 \\
23.06573 \\
23.22215 \\
23.37211\end{array}$ & $\begin{array}{l}17131 \\
16350 \\
15642 \\
14996 \\
14405\end{array}$ & $\begin{array}{l}26.24020 \\
26.41501 \\
26.58248 \\
26.74332 \\
26.89814\end{array}$ & $\begin{array}{l}17481 \\
16747 \\
16084 \\
15482 \\
14933\end{array}$ \\
\hline $\begin{array}{l}250 \\
260 \\
270 \\
280 \\
290\end{array}$ & $\begin{array}{l}3.66848 \\
3.68966 \\
3.71122 \\
3.73300 \\
3.75487\end{array}$ & $\begin{array}{l}2118 \\
2156 \\
2178 \\
2187 \\
2183\end{array}$ & $\begin{array}{l}3.53131 \\
3.53699 \\
3.54305 \\
3.54944 \\
3.55615\end{array}$ & $\begin{array}{l}568 \\
606 \\
639 \\
671 \\
699\end{array}$ & $\begin{array}{l}23.51616 \\
23.65477 \\
23.78837 \\
23.91733 \\
24.04201\end{array}$ & $\begin{array}{l}13861 \\
13360 \\
12896 \\
12468 \\
12067\end{array}$ & $\begin{array}{l}27.04747 \\
27.19176 \\
27.33142 \\
27.46677 \\
27.59816\end{array}$ & $\begin{array}{l}14429 \\
13966 \\
13535 \\
13139 \\
12766\end{array}$ \\
\hline $\begin{array}{l}300 \\
310 \\
320 \\
330 \\
340\end{array}$ & $\begin{array}{l}3.77670 \\
3.79838 \\
3.81984 \\
3.84100 \\
3.86179\end{array}$ & $\begin{array}{l}2168 \\
2146 \\
2116 \\
2079 \\
2038\end{array}$ & $\begin{array}{l}3.56314 \\
3.57038 \\
3.57784 \\
3.58549 \\
3.59331\end{array}$ & $\begin{array}{l}724 \\
746 \\
765 \\
782 \\
797\end{array}$ & $\begin{array}{l}24.16268 \\
24.27963 \\
24.39311 \\
24.50332 \\
24.61047\end{array}$ & $\begin{array}{l}11695 \\
11348 \\
11021 \\
10715 \\
10428\end{array}$ & $\begin{array}{l}27.72582 \\
27.85001 \\
27.97095 \\
28.08881 \\
28.20378\end{array}$ & $\begin{array}{l}12419 \\
12094 \\
11786 \\
11497 \\
11225\end{array}$ \\
\hline $\begin{array}{l}350 \\
360 \\
370 \\
380 \\
390\end{array}$ & $\begin{array}{l}3.88217 \\
3.90211 \\
3.92156 \\
3.94052 \\
3.95897\end{array}$ & $\begin{array}{l}1994 \\
1945 \\
1896 \\
1845 \\
1793\end{array}$ & $\begin{array}{l}3.60128 \\
3.60936 \\
3.61753 \\
3.62578 \\
3.63409\end{array}$ & $\begin{array}{l}808 \\
817 \\
825 \\
831 \\
835\end{array}$ & $\begin{array}{l}24.71475 \\
24.81631 \\
24.91532 \\
25.01190 \\
25.10619\end{array}$ & $\begin{array}{r}10156 \\
9901 \\
9658 \\
9429 \\
9211\end{array}$ & $\begin{array}{l}28.31603 \\
28.42567 \\
28.53285 \\
28.63768 \\
28.74028\end{array}$ & $\begin{array}{l}10964 \\
10718 \\
10483 \\
10260 \\
10046\end{array}$ \\
\hline $\begin{array}{l}400 \\
450 \\
500 \\
550 \\
600\end{array}$ & $\begin{array}{l}3.97690 \\
4.05874 \\
4.12827 \\
4.18703 \\
4.23677\end{array}$ & $\begin{array}{l}8184 \\
6953 \\
5876 \\
4974 \\
4231\end{array}$ & $\begin{array}{l}3.64244 \\
3.68427 \\
3.72529 \\
3.76467 \\
3.80199\end{array}$ & $\begin{array}{l}4183 \\
4102 \\
3938 \\
3732 \\
3512\end{array}$ & $\begin{array}{l}25.19830 \\
25.62974 \\
26.02005 \\
26.37697 \\
26.70615\end{array}$ & $\begin{array}{l}43144 \\
39031 \\
35692 \\
32918 \\
30573\end{array}$ & $\begin{array}{l}28.84074 \\
29.31401 \\
29.74534 \\
30.14164 \\
30.50814\end{array}$ & $\begin{array}{l}47327 \\
43133 \\
39630 \\
36650 \\
34085\end{array}$ \\
\hline $\begin{array}{l}650 \\
700 \\
750 \\
800 \\
850\end{array}$ & $\begin{array}{l}4.27908 \\
4.31532 \\
4.34664 \\
4.37393 \\
4.39789\end{array}$ & $\begin{array}{l}3624 \\
3132 \\
2729 \\
2396 \\
2133\end{array}$ & $\begin{array}{l}3.83711 \\
3.87001 \\
3.90076 \\
3.92951 \\
3.95636\end{array}$ & $\begin{array}{l}3290 \\
3075 \\
2875 \\
2685 \\
2514\end{array}$ & $\begin{array}{l}27.01188 \\
27.29746 \\
27.56552 \\
27.81820 \\
28.05724\end{array}$ & $\begin{array}{l}28558 \\
26806 \\
25268 \\
23904 \\
22685\end{array}$ & $\begin{array}{l}30.84899 \\
31.16747 \\
31.46628 \\
31.74771 \\
32.01360\end{array}$ & $\begin{array}{l}31848 \\
29881 \\
28143 \\
26589 \\
25199\end{array}$ \\
\hline $\begin{array}{r}900 \\
950 \\
1000 \\
1050 \\
1100\end{array}$ & $\begin{array}{l}4.41922 \\
4.43836 \\
4.45572 \\
4.47169 \\
4.48654\end{array}$ & $\begin{array}{l}1914 \\
1736 \\
1597 \\
1485 \\
1399\end{array}$ & $\begin{array}{l}3.98150 \\
4.00505 \\
4.02716 \\
4.04795 \\
4.06756\end{array}$ & $\begin{array}{l}2355 \\
2211 \\
2079 \\
1961 \\
1852\end{array}$ & $\begin{array}{l}28.28409 \\
28.50000 \\
28.70601 \\
28.90299 \\
29.09176\end{array}$ & $\begin{array}{l}21591 \\
20601 \\
19698 \\
18877 \\
18123\end{array}$ & $\begin{array}{l}32.26559 \\
32.50505 \\
32.73317 \\
32.95094 \\
33.15932\end{array}$ & $\begin{array}{l}23946 \\
22812 \\
21777 \\
20838 \\
19975\end{array}$ \\
\hline 1150 & 4.50053 & 1335 & 4.08608 & 1755 & 29.27299 & 17427 & 33.35907 & 19182 \\
\hline
\end{tabular}


Table 2. 084 .

PbT (Cont.)

\begin{tabular}{|c|c|c|c|c|c|c|c|c|}
\hline${ }^{\circ} \mathrm{K}$ & $\frac{C_{p}^{0}}{R}$ & & $\frac{\left(H^{\circ}-E_{0}^{0}\right)}{R T}$ & & $\frac{-\left(F^{0}-E\right.}{R T}$ & & $\frac{S^{\circ}}{R}$ & \\
\hline $\begin{array}{l}1200 \\
1300 \\
1400 \\
1500 \\
1600\end{array}$ & $\begin{array}{l}4.51388 \\
4.53938 \\
4.56418 \\
4.58911 \\
4.61468\end{array}$ & $\begin{array}{l}2550 \\
2480 \\
2493 \\
2557 \\
2648\end{array}$ & $\begin{array}{l}4.10363 \\
4.13617 \\
4.16586 \\
4.19324 \\
4.21878\end{array}$ & $\begin{array}{l}3254 \\
2969 \\
2738 \\
2554 \\
2406\end{array}$ & $\begin{array}{l}29.44726 \\
29.77703 \\
30.08466 \\
30.37302 \\
30.64447\end{array}$ & $\begin{array}{l}32977 \\
30763 \\
28836 \\
27145 \\
25649\end{array}$ & $\begin{array}{l}33.55089 \\
33.91320 \\
34.25052 \\
34.56626 \\
34.86325\end{array}$ & $\begin{array}{l}36231 \\
33732 \\
31574 \\
29899 \\
28055\end{array}$ \\
\hline $\begin{array}{l}1700 \\
1800 \\
1900 \\
2000 \\
2100\end{array}$ & $\begin{array}{l}4.64116 \\
4.66867 \\
4.69716 \\
4.72649 \\
4.75649\end{array}$ & $\begin{array}{l}2751 \\
2849 \\
2933 \\
3000 \\
3043\end{array}$ & $\begin{array}{l}4.24284 \\
4.26573 \\
4.28768 \\
4.30889 \\
4.32948\end{array}$ & $\begin{array}{l}2289 \\
2195 \\
2121 \\
2059 \\
2010\end{array}$ & $\begin{array}{l}30.90096 \\
31.14412 \\
31.37536 \\
31.59583 \\
31.80656\end{array}$ & $\begin{array}{l}24316 \\
23124 \\
22047 \\
21073 \\
20187\end{array}$ & $\begin{array}{l}35.14380 \\
35.40985 \\
35.66304 \\
35.90472 \\
36.13604\end{array}$ & $\begin{array}{l}26605 \\
25319 \\
24168 \\
23132 \\
22197\end{array}$ \\
\hline $\begin{array}{l}2200 \\
2300 \\
2400 \\
2500 \\
2600\end{array}$ & $\begin{array}{l}4.78692 \\
4.81756 \\
4.84815 \\
4.87849 \\
4.90835\end{array}$ & $\begin{array}{l}3064 \\
3059 \\
3034 \\
2986 \\
2922\end{array}$ & $\begin{array}{l}4.34958 \\
4.36927 \\
4.38858 \\
4.40757 \\
4.42626\end{array}$ & $\begin{array}{l}1969 \\
1931 \\
1899 \\
1869 \\
1840\end{array}$ & $\begin{array}{l}32.00843 \\
32.20222 \\
32.38858 \\
32.56812 \\
32.74135\end{array}$ & $\begin{array}{l}19379 \\
18636 \\
17954 \\
17323 \\
16740\end{array}$ & $\begin{array}{l}36.35801 \\
36.57149 \\
36.77716 \\
36.97569 \\
37.16761\end{array}$ & $\begin{array}{l}21348 \\
20567 \\
19853 \\
19192 \\
18580\end{array}$ \\
\hline $\begin{array}{l}2700 \\
2800 \\
2900 \\
3000\end{array}$ & $\begin{array}{l}4.93757 \\
4.96601 \\
4.99352 \\
5.02001\end{array}$ & $\begin{array}{l}2844 \\
2751 \\
2649 \\
4964\end{array}$ & $\begin{array}{l}4.44466 \\
4.46277 \\
4.48060 \\
4.49814\end{array}$ & $\begin{array}{l}1811 \\
1783 \\
1754 \\
3420\end{array}$ & $\begin{array}{l}32.90875 \\
33.07072 \\
33.22763 \\
33.37982\end{array}$ & $\begin{array}{l}16197 \\
15691 \\
15219 \\
29141\end{array}$ & $\begin{array}{l}37.35341 \\
37.53349 \\
37.70823 \\
37.87796\end{array}$ & $\begin{array}{l}18008 \\
17474 \\
16973 \\
32561\end{array}$ \\
\hline $\begin{array}{l}3200 \\
3400 \\
3600 \\
3800 \\
4000\end{array}$ & $\begin{array}{l}5.06965 \\
5.11458 \\
5.15472 \\
5.19018 \\
5.22121\end{array}$ & $\begin{array}{l}4493 \\
4014 \\
3546 \\
3103 \\
2698\end{array}$ & $\begin{array}{l}4.53234 \\
4.56529 \\
4.59694 \\
4.62725 \\
4.65619\end{array}$ & $\begin{array}{l}3295 \\
3165 \\
3031 \\
2894 \\
2756\end{array}$ & $\begin{array}{l}33.67123 \\
33.94700 \\
34.20884 \\
34.45820 \\
34.69629\end{array}$ & $\begin{array}{l}27577 \\
26184 \\
24936 \\
23809 \\
22785\end{array}$ & $\begin{array}{l}38.20357 \\
38.51229 \\
38.80578 \\
39.08545 \\
39.35248\end{array}$ & $\begin{array}{l}30872 \\
29349 \\
27967 \\
26703 \\
25541\end{array}$ \\
\hline $\begin{array}{l}4200 \\
4400 \\
4600 \\
4800 \\
5000\end{array}$ & $\begin{array}{l}5.24819 \\
5.27152 \\
5.29158 \\
5.30879 \\
5.32353\end{array}$ & $\begin{array}{l}2333 \\
2006 \\
1721 \\
1474\end{array}$ & $\begin{array}{l}4.68375 \\
4.70995 \\
4.73482 \\
4.75839 \\
4.78071\end{array}$ & $\begin{array}{l}2620 \\
2487 \\
2357 \\
2232\end{array}$ & $\begin{array}{l}34.92414 \\
35.14265 \\
35.35256 \\
35.55458 \\
35.74929\end{array}$ & $\begin{array}{l}21851 \\
20991 \\
20202 \\
19471\end{array}$ & $\begin{array}{l}39.60789 \\
39.85260 \\
40.08738 \\
40.31297 \\
40.53000\end{array}$ & $\begin{array}{l}24471 \\
23478 \\
22559 \\
21703\end{array}$ \\
\hline
\end{tabular}


Table 2.085. NH

\begin{tabular}{|l|l|l|l|l|}
\hline$K$ & $\frac{C_{p}^{0}}{R}$ & $\frac{\left(H^{\circ}-E_{0}^{0}\right)}{R T}$ & $\frac{-\left(F^{0}-E_{0}^{0}\right)}{R T}$ & $\frac{S^{0}}{R}$ \\
\hline
\end{tabular}

\begin{tabular}{|c|c|c|c|c|c|c|c|c|}
\hline $\begin{array}{l}50 \\
60 \\
70 \\
80 \\
90\end{array}$ & $\begin{array}{l}3.50715 \\
3.50543 \\
3.50444 \\
3.50385 \\
3.50350\end{array}$ & $\begin{array}{l}-\quad 172 \\
-\quad 99 \\
-\quad 59 \\
-\quad 35 \\
-\quad 20\end{array}$ & $\begin{array}{l}3.31820 \\
3.34954 \\
3.37173 \\
3.38828 \\
3.40110\end{array}$ & $\begin{array}{l}3134 \\
2219 \\
1655 \\
1282 \\
1023\end{array}$ & $\begin{array}{l}12.19325 \\
12.80444 \\
13.32486 \\
13.77797 \\
14.17918\end{array}$ & $\begin{array}{l}61119 \\
52042 \\
45311 \\
40121 \\
35998\end{array}$ & $\begin{array}{l}15.51146 \\
16.15399 \\
16.69660 \\
17.16625 \\
17.58028\end{array}$ & $\begin{array}{l}64253 \\
5426 ! \\
46965 \\
41403 \\
37021\end{array}$ \\
\hline $\begin{array}{l}100 \\
110 \\
120 \\
130 \\
140\end{array}$ & $\begin{array}{l}3.50330 \\
3.50320 \\
3.50316 \\
3.50317 \\
3.50322\end{array}$ & $\begin{array}{r}-\quad 10 \\
-\quad 4 \\
1 \\
5 \\
7\end{array}$ & $\begin{array}{l}3.41133 \\
3.41968 \\
3.42664 \\
3.43253 \\
3.43758\end{array}$ & $\begin{array}{l}835 \\
696 \\
589 \\
505 \\
438\end{array}$ & $\begin{array}{l}14.53916 \\
14.86558 \\
15.16418 \\
15.43933 \\
15.69444\end{array}$ & $\begin{array}{l}32642 \\
29860 \\
27515 \\
25511 \\
23778\end{array}$ & $\begin{array}{l}17.95049 \\
18.28528 \\
18.59084 \\
18.87187 \\
19.13201\end{array}$ & $\begin{array}{l}33479 \\
30556 \\
28103 \\
26014 \\
24217\end{array}$ \\
\hline $\begin{array}{l}150 \\
160 \\
170 \\
180 \\
190\end{array}$ & $\begin{array}{l}3.50329 \\
3.50338 \\
3.50349 \\
3.50360 \\
3.50373\end{array}$ & $\begin{array}{r}9 \\
11 \\
11 \\
13 \\
13\end{array}$ & $\begin{array}{l}3.44196 \\
3.44579 \\
3.44918 \\
3.45220 \\
3.45491\end{array}$ & $\begin{array}{l}383 \\
339 \\
302 \\
271 \\
245\end{array}$ & $\begin{array}{l}15.93222 \\
16.15489 \\
16.36425 \\
16.56181 \\
16.74883\end{array}$ & $\begin{array}{l}22267 \\
20936 \\
19756 \\
18702 \\
17753\end{array}$ & $\begin{array}{l}19.37418 \\
19.60069 \\
19.81344 \\
20.01403 \\
20.20374\end{array}$ & $\begin{array}{l}22651 \\
21275 \\
20059 \\
18971 \\
17998\end{array}$ \\
\hline $\begin{array}{l}200 \\
210 \\
220 \\
230 \\
240\end{array}$ & $\begin{array}{l}3.50386 \\
3.50400 \\
3.50414 \\
3.50429 \\
3.50445\end{array}$ & $\begin{array}{l}14 \\
14 \\
15 \\
16 \\
15\end{array}$ & $\begin{array}{l}3.45736 \\
3.45957 \\
3.46160 \\
3.46345 \\
3.46515\end{array}$ & $\begin{array}{l}221 \\
203 \\
185 \\
170 \\
157\end{array}$ & $\begin{array}{l}16.92636 \\
17.09533 \\
17.25653 \\
17.41064 \\
17.55826\end{array}$ & $\begin{array}{l}16897 \\
16120 \\
15411 \\
14762 \\
14164\end{array}$ & $\begin{array}{l}20.38372 \\
20.55491 \\
20.71813 \\
20.87410 \\
21.02341\end{array}$ & $\begin{array}{l}17117 \\
16322 \\
15597 \\
14931 \\
14323\end{array}$ \\
\hline $\begin{array}{l}250 \\
260 \\
270 \\
280 \\
290\end{array}$ & $\begin{array}{l}3.50460 \\
3.50476 \\
3.50493 \\
3.50510 \\
3.50528\end{array}$ & $\begin{array}{l}16 \\
17 \\
17 \\
18 \\
19\end{array}$ & $\begin{array}{l}3.46672 \\
3.46819 \\
3.46955 \\
3.47081 \\
3.47199\end{array}$ & $\begin{array}{l}147 \\
136 \\
126 \\
118 \\
112\end{array}$ & $\begin{array}{l}17.69990 \\
17.83606 \\
17.96711 \\
18.09344 \\
18.21538\end{array}$ & $\begin{array}{l}13616 \\
13105 \\
12633 \\
12194 \\
11784\end{array}$ & $\begin{array}{l}21.16664 \\
21.30425 \\
21.43666 \\
21.56426 \\
21.68739\end{array}$ & $\begin{array}{l}13761 \\
13241 \\
12760 \\
12313 \\
11894\end{array}$ \\
\hline $\begin{array}{l}300 \\
310 \\
320 \\
330 \\
340\end{array}$ & $\begin{array}{l}3.50547 \\
3.50567 \\
3.50589 \\
3.50613 \\
3.50640\end{array}$ & $\begin{array}{l}20 \\
22 \\
24 \\
27 \\
30\end{array}$ & $\begin{array}{l}3.47311 \\
3.47416 \\
3.47514 \\
3.47608 \\
3.47697\end{array}$ & $\begin{array}{r}105 \\
98 \\
94 \\
89 \\
84\end{array}$ & $\begin{array}{l}18.33322 \\
18.44722 \\
18.55764 \\
18.66468 \\
18.76855\end{array}$ & $\begin{array}{l}11400 \\
11042 \\
10704 \\
10387 \\
10038\end{array}$ & $\begin{array}{l}21.80633 \\
21.92138 \\
22.03278 \\
22.14077 \\
22.24552\end{array}$ & $\begin{array}{l}11505 \\
11140 \\
10797 \\
10475 \\
10174\end{array}$ \\
\hline $\begin{array}{l}350 \\
360 \\
370 \\
380 \\
390\end{array}$ & $\begin{array}{l}3.50670 \\
3.50703 \\
3.50741 \\
3.50783 \\
3.50832\end{array}$ & $\begin{array}{l}33 \\
38 \\
42 \\
49 \\
55\end{array}$ & $\begin{array}{l}3.47781 \\
3.47862 \\
3.47940 \\
3.48013 \\
3.48086\end{array}$ & $\begin{array}{l}81 \\
78 \\
73 \\
73 \\
69\end{array}$ & $\begin{array}{l}18.86943 \\
18.96750 \\
19.06289 \\
19.15576 \\
19.24624\end{array}$ & $\begin{array}{l}9807 \\
9539 \\
9287 \\
9048 \\
8820\end{array}$ & $\begin{array}{l}22.34726 \\
22.44612 \\
22.54229 \\
22.63591 \\
22.72709\end{array}$ & $\begin{array}{l}9886 \\
9617 \\
9362 \\
9118 \\
8889\end{array}$ \\
\hline $\begin{array}{l}400 \\
450 \\
500 \\
550 \\
600\end{array}$ & $\begin{array}{l}3.50887 \\
3.51286 \\
3.51961 \\
3.52984 \\
3.54398\end{array}$ & $\begin{array}{r}399 \\
675 \\
1023 \\
1414 \\
1807\end{array}$ & $\begin{array}{l}3.48155 \\
3.48478 \\
3.48790 \\
3.49122 \\
3.49500\end{array}$ & $\begin{array}{l}323 \\
312 \\
332 \\
378 \\
444\end{array}$ & $\begin{array}{l}19.33444 \\
19.74496 \\
20.11250 \\
20.44526 \\
20.74934\end{array}$ & $\begin{array}{l}41052 \\
36754 \\
33276 \\
30408 \\
28005\end{array}$ & $\begin{array}{l}22.81598 \\
23.22975 \\
23.60040 \\
23.93649 \\
24.24435\end{array}$ & $\begin{array}{l}41377 \\
3706 ! \\
33009 \\
30786 \\
28449\end{array}$ \\
\hline $\begin{array}{l}650 \\
700 \\
750 \\
800 \\
850\end{array}$ & $\begin{array}{l}3.56205 \\
3.58382 \\
3.60883 \\
3.63651 \\
3.66626\end{array}$ & $\begin{array}{l}2177 \\
2501 \\
2768 \\
2975 \\
3124\end{array}$ & $\begin{array}{l}3.49944 \\
3.50467 \\
3.51076 \\
3.51774 \\
3.52560\end{array}$ & $\begin{array}{l}523 \\
609 \\
698 \\
786 \\
868\end{array}$ & $\begin{array}{l}21.02939 \\
21.28902 \\
21.53112 \\
21.75800 \\
21.97156\end{array}$ & $\begin{array}{l}25963 \\
24210 \\
22688 \\
21355 \\
20183\end{array}$ & $\begin{array}{l}24.52884 \\
24.79369 \\
25.04189 \\
25.27574 \\
25.49716\end{array}$ & $\begin{array}{l}26485 \\
24820 \\
23385 \\
22142 \\
21050\end{array}$ \\
\hline $\begin{array}{r}900 \\
950 \\
1000 \\
1050 \\
1100\end{array}$ & $\begin{array}{l}3.69750 \\
3.72969 \\
3.76236 \\
3.79513 \\
3.82767\end{array}$ & $\begin{array}{l}3219 \\
3267 \\
3277 \\
3254 \\
3206\end{array}$ & $\begin{array}{l}3.53428 \\
3.54371 \\
3.55382 \\
3.56453 \\
3.57576\end{array}$ & $\begin{array}{r}943 \\
1011 \\
1071 \\
1123 \\
1164\end{array}$ & $\begin{array}{l}22.17339 \\
22.36478 \\
22.54685 \\
22.72054 \\
22.88668\end{array}$ & $\begin{array}{l}19139 \\
18207 \\
17369 \\
16614 \\
15923\end{array}$ & $\begin{array}{l}25.70766 \\
25.90849 \\
26.10068 \\
26.28509 \\
26.46243\end{array}$ & $\begin{array}{l}20083 \\
19219 \\
18441 \\
17734 \\
17090\end{array}$ \\
\hline 1150 & 3.85973 & 3138 & 3.58740 & 1201 & 23.04591 & 15297 & 26.63333 & 16497 \\
\hline
\end{tabular}


Table 2.085. NH (Cont.)

\begin{tabular}{|l|l|l|l|l|}
\hline${ }^{\circ} K$ & $\frac{C_{p}^{0}}{R}$ & $\frac{\left(H^{0}-E_{0}^{0}\right)}{R T}$ & $\frac{-\left(F^{0}-E_{0}^{0}\right)}{R T}$ & $\frac{S^{0}}{R}$ \\
\hline
\end{tabular}

\begin{tabular}{|c|c|c|c|c|c|c|c|c|}
\hline $\begin{array}{l}1200 \\
1300 \\
1400 \\
1500 \\
1600\end{array}$ & $\begin{array}{l}3.89111 \\
3.95132 \\
4.00759 \\
4.05968 \\
4.10762\end{array}$ & $\begin{array}{l}6021 \\
5627 \\
5209 \\
4794 \\
4398\end{array}$ & $\begin{array}{l}3.59941 \\
3.62418 \\
3.64959 \\
3.67521 \\
3.70076\end{array}$ & $\begin{array}{l}2477 \\
2541 \\
2562 \\
2555 \\
2525\end{array}$ & $\begin{array}{l}23.19888 \\
23.48802 \\
23.75758 \\
24.01030 \\
24.24835\end{array}$ & $\begin{array}{l}28914 \\
26956 \\
25272 \\
23805 \\
22515\end{array}$ & $\begin{array}{l}26.79830 \\
27.11222 \\
27.40718 \\
27.68552 \\
27.94912\end{array}$ & $\begin{array}{l}31392 \\
29496 \\
27834 \\
26360 \\
25043\end{array}$ \\
\hline $\begin{array}{l}1700 \\
1800 \\
1900 \\
2000 \\
2100\end{array}$ & $\begin{array}{l}4.15160 \\
4.19191 \\
4.22886 \\
4.26276 \\
4.29392\end{array}$ & $\begin{array}{l}4031 \\
3695 \\
3390 \\
3116 \\
2870\end{array}$ & $\begin{array}{l}3.72601 \\
3.75079 \\
3.77499 \\
3.79855 \\
3.82141\end{array}$ & $\begin{array}{l}2478 \\
2420 \\
2356 \\
2286 \\
2213\end{array}$ & $\begin{array}{l}24.47350 \\
24.68722 \\
24.89069 \\
25.08495 \\
25.27086\end{array}$ & $\begin{array}{l}21372 \\
20347 \\
19426 \\
18591 \\
17831\end{array}$ & $\begin{array}{l}28.19952 \\
28.43801 \\
28.66569 \\
28.88350 \\
29.09226\end{array}$ & $\begin{array}{l}23849 \\
22768 \\
21781 \\
20876 \\
20046\end{array}$ \\
\hline $\begin{array}{l}2200 \\
2300 \\
2400 \\
2500 \\
2600\end{array}$ & $\begin{array}{l}4.32262 \\
4.34913 \\
4.37368 \\
4.39648 \\
4.41771\end{array}$ & $\begin{array}{l}2651 \\
2455 \\
2280 \\
2123 \\
1984\end{array}$ & $\begin{array}{l}3.84354 \\
3.86496 \\
3.88565 \\
3.90563 \\
3.92492\end{array}$ & $\begin{array}{l}2142 \\
2069 \\
1998 \\
1929 \\
1863\end{array}$ & $\begin{array}{l}25.44917 \\
25.62052 \\
25.78547 \\
25.94450 \\
26.09808\end{array}$ & $\begin{array}{l}17135 \\
16495 \\
15903 \\
15358 \\
14849\end{array}$ & $\begin{array}{l}29.29272 \\
29.48547 \\
29.67111 \\
29.85014 \\
30.02300\end{array}$ & $\begin{array}{l}19275 \\
18554 \\
17903 \\
17286 \\
16713\end{array}$ \\
\hline $\begin{array}{l}2700 \\
2800 \\
2900 \\
3000\end{array}$ & $\begin{array}{l}4.43755 \\
4.45613 \\
4.47359 \\
4.49005\end{array}$ & $\begin{array}{l}1858 \\
1746 \\
1646 \\
3027\end{array}$ & $\begin{array}{l}3.94355 \\
3.96153 \\
3.97888 \\
3.99565\end{array}$ & $\begin{array}{l}1798 \\
1735 \\
1677 \\
3188\end{array}$ & $\begin{array}{l}26.24657 \\
26.39034 \\
26.52966 \\
26.66485\end{array}$ & $\begin{array}{l}14377 \\
13932 \\
13519 \\
25892\end{array}$ & $\begin{array}{l}30.19013 \\
30.35186 \\
30.50855 \\
30.66050\end{array}$ & $\begin{array}{l}16173 \\
15669 \\
15195 \\
29079\end{array}$ \\
\hline $\begin{array}{l}3200 \\
3400 \\
3600 \\
3800 \\
4000\end{array}$ & $\begin{array}{l}4.52032 \\
4.54763 \\
4.57251 \\
4.59537 \\
4.61655\end{array}$ & $\begin{array}{l}2731 \\
2488 \\
2286 \\
2118 \\
1977\end{array}$ & $\begin{array}{l}4.02753 \\
4.05732 \\
4.08525 \\
4.11151 \\
4.13624\end{array}$ & $\begin{array}{l}2979 \\
2793 \\
2626 \\
2473 \\
2335\end{array}$ & $\begin{array}{l}26.92377 \\
27.16886 \\
27.40158 \\
27.62319 \\
27.83473\end{array}$ & $\begin{array}{l}24509 \\
23272 \\
22161 \\
21154 \\
20239\end{array}$ & $\begin{array}{l}30.95129 \\
31.22618 \\
31.48685 \\
31.73471 \\
31.97098\end{array}$ & $\begin{array}{l}27489 \\
26067 \\
24786 \\
23627 \\
22574\end{array}$ \\
\hline $\begin{array}{l}4200 \\
4400 \\
4500 \\
4800 \\
5000\end{array}$ & $\begin{array}{l}4.63632 \\
4.65488 \\
4.67243 \\
4.68910 \\
4.70501\end{array}$ & $\begin{array}{l}1856 \\
1755 \\
1667 \\
1591\end{array}$ & $\begin{array}{l}4.15959 \\
4.18169 \\
4.20264 \\
4.22257 \\
4.24155\end{array}$ & $\begin{array}{l}2210 \\
2095 \\
1993 \\
1898\end{array}$ & $\begin{array}{l}28.03712 \\
28.23115 \\
28.41751 \\
28.59681 \\
28.76958\end{array}$ & $\begin{array}{l}19403 \\
18636 \\
17930 \\
17277\end{array}$ & $\begin{array}{l}32.19672 \\
32.41284 \\
32.62016 \\
32.81938 \\
33.01113\end{array}$ & $\begin{array}{l}21612 \\
20732 \\
19922 \\
19175\end{array}$ \\
\hline
\end{tabular}


Table 2.086. ND

\begin{tabular}{|l|l|l|l|l|}
\hline${ }^{\circ}$ & $\frac{C_{p}^{0}}{R}$ & $\frac{\left(H^{0}-E_{0}^{0}\right)}{R T}$ & $\frac{-\left(F^{0}-E_{0}^{0}\right)}{R T}$ & $\frac{S^{0}}{R}$ \\
\hline
\end{tabular}

\begin{tabular}{|c|c|c|c|c|c|c|c|c|}
\hline $\begin{array}{l}50 \\
60 \\
70 \\
80 \\
90\end{array}$ & $\begin{array}{l}3.50283 \\
3.50240 \\
3.50220 \\
3.50214 \\
3.50216\end{array}$ & $\begin{array}{r}-\quad 43 \\
-\quad 20 \\
-\quad 6 \\
2 \\
6\end{array}$ & $\begin{array}{l}3.39615 \\
3.41389 \\
3.42652 \\
3.43598 \\
3.44333\end{array}$ & $\begin{array}{r}1774 \\
1263 \\
946 \\
735 \\
589\end{array}$ & $\begin{array}{l}12.83882 \\
13.46275 \\
13.99219 \\
14.45204 \\
14.85846\end{array}$ & $\begin{array}{l}62393 \\
52944 \\
45985 \\
40642 \\
36413\end{array}$ & $\begin{array}{l}16.23498 \\
16.87665 \\
17.41872 \\
17.88801 \\
18.30179\end{array}$ & $\begin{array}{l}64157 \\
54207 \\
46929 \\
41378 \\
37001\end{array}$ \\
\hline $\begin{array}{l}100 \\
110 \\
120 \\
130 \\
140\end{array}$ & $\begin{array}{l}3.50222 \\
3.50231 \\
3.50242 \\
3.50254 \\
3.50268\end{array}$ & $\begin{array}{r}9 \\
11 \\
12 \\
14 \\
14\end{array}$ & $\begin{array}{l}3.44922 \\
3.45404 \\
3.45806 \\
3.46148 \\
3.46442\end{array}$ & $\begin{array}{l}482 \\
402 \\
342 \\
294 \\
255\end{array}$ & $\begin{array}{l}15.22259 \\
15.55241 \\
15.85381 \\
16.13134 \\
16.38848\end{array}$ & $\begin{array}{l}32982 \\
30140 \\
27753 \\
25714 \\
23955\end{array}$ & $\begin{array}{l}18.67180 \\
19.00644 \\
19.31189 \\
19.59282 \\
19.85290\end{array}$ & $\begin{array}{l}33464 \\
30545 \\
28093 \\
26008 \\
24210\end{array}$ \\
\hline $\begin{array}{l}150 \\
160 \\
170 \\
180 \\
190\end{array}$ & $\begin{array}{l}3.50282 \\
3.50297 \\
3.50313 \\
3.50329 \\
3.50345\end{array}$ & $\begin{array}{l}15 \\
16 \\
16 \\
16 \\
17\end{array}$ & $\begin{array}{l}3.46697 \\
3.46921 \\
3.47121 \\
3.47298 \\
3.47459\end{array}$ & $\begin{array}{l}224 \\
200 \\
177 \\
161 \\
144\end{array}$ & $\begin{array}{l}16.62803 \\
16.85223 \\
17.06295 \\
17.26171 \\
17.44981\end{array}$ & $\begin{array}{l}22420 \\
21072 \\
19876 \\
18810 \\
17850\end{array}$ & $\begin{array}{l}20.09500 \\
20.32146 \\
20.53416 \\
20.73471 \\
20.92440\end{array}$ & $\begin{array}{l}22646 \\
21270 \\
20055 \\
18969 \\
17994\end{array}$ \\
\hline $\begin{array}{l}200 \\
210 \\
220 \\
230 \\
240\end{array}$ & $\begin{array}{l}3.50362 \\
3.50380 \\
3.50400 \\
3.50422 \\
3.50446\end{array}$ & $\begin{array}{l}18 \\
20 \\
22 \\
24 \\
29\end{array}$ & $\begin{array}{l}3.47603 \\
3.47735 \\
3.47856 \\
3.47967 \\
3.48069\end{array}$ & $\begin{array}{r}132 \\
121 \\
111 \\
102 \\
96\end{array}$ & $\begin{array}{l}17.62831 \\
17.79816 \\
17.96015 \\
18.11499 \\
18.26327\end{array}$ & $\begin{array}{l}16985 \\
16199 \\
15484 \\
14828 \\
14226\end{array}$ & $\begin{array}{l}21.10434 \\
21.27552 \\
21.43872 \\
21.59466 \\
21.74396\end{array}$ & $\begin{array}{l}17118 \\
16320 \\
15594 \\
14930 \\
14323\end{array}$ \\
\hline $\begin{array}{l}250 \\
260 \\
270 \\
280 \\
290\end{array}$ & $\begin{array}{l}3.50475 \\
3.50509 \\
3.50550 \\
3.50599 \\
3.50658\end{array}$ & $\begin{array}{l}34 \\
41 \\
49 \\
59 \\
71\end{array}$ & $\begin{array}{l}3.48165 \\
3.48255 \\
3.48338 \\
3.48419 \\
3.48495\end{array}$ & $\begin{array}{l}90 \\
83 \\
81 \\
76 \\
74\end{array}$ & $\begin{array}{l}18.40553 \\
18.54224 \\
18.67382 \\
18.80064 \\
18.92303\end{array}$ & $\begin{array}{l}13671 \\
13158 \\
12682 \\
12239 \\
11827\end{array}$ & $\begin{array}{l}21.88719 \\
22.02479 \\
22.15722 \\
22.28483 \\
22.40798\end{array}$ & $\begin{array}{l}13760 \\
13243 \\
12761 \\
12315 \\
11900\end{array}$ \\
\hline $\begin{array}{l}300 \\
310 \\
320 \\
330 \\
340\end{array}$ & $\begin{array}{l}3.50729 \\
3.50814 \\
3.50915 \\
3.51033 \\
3.51171\end{array}$ & $\begin{array}{r}85 \\
101 \\
118 \\
138 \\
159\end{array}$ & $\begin{array}{l}3.48569 \\
3.48640 \\
3.48709 \\
3.48778 \\
3.48846\end{array}$ & $\begin{array}{l}71 \\
69 \\
69 \\
68 \\
68\end{array}$ & $\begin{array}{l}19.04130 \\
19.15570 \\
19.26649 \\
19.37390 \\
19.47811\end{array}$ & $\begin{array}{l}11440 \\
11079 \\
10741 \\
10421 \\
10121\end{array}$ & $\begin{array}{l}22.52698 \\
22.64210 \\
22.75358 \\
22.86167 \\
22.96657\end{array}$ & $\begin{array}{l}11512 \\
11148 \\
10809 \\
10490 \\
10190\end{array}$ \\
\hline $\begin{array}{l}350 \\
360 \\
370 \\
380 \\
390\end{array}$ & $\begin{array}{l}3.51330 \\
3.51511 \\
3.51717 \\
3.51948 \\
3.52205\end{array}$ & $\begin{array}{l}181 \\
206 \\
231 \\
257 \\
285\end{array}$ & $\begin{array}{l}3.48914 \\
3.48984 \\
3.49055 \\
3.49128 \\
3.49204\end{array}$ & $\begin{array}{l}70 \\
71 \\
73 \\
76 \\
78\end{array}$ & $\begin{array}{l}19.57932 \\
19.67769 \\
19.77339 \\
19.86655 \\
19.95731\end{array}$ & $\begin{array}{l}9837 \\
9570 \\
9316 \\
9076 \\
8848\end{array}$ & $\begin{array}{l}23.06847 \\
23.16753 \\
23.26394 \\
23.35784 \\
23.44935\end{array}$ & $\begin{array}{l}9906 \\
9641 \\
9390 \\
9151 \\
8927\end{array}$ \\
\hline $\begin{array}{l}400 \\
450 \\
500 \\
550 \\
500\end{array}$ & $\begin{array}{l}3.52490 \\
3.54339 \\
3.56891 \\
3.60068 \\
3.63739\end{array}$ & $\begin{array}{l}1849 \\
2552 \\
3177 \\
3671 \\
4019\end{array}$ & $\begin{array}{l}3.49282 \\
3.49735 \\
3.50317 \\
3.51054 \\
3.51956\end{array}$ & $\begin{array}{r}453 \\
582 \\
737 \\
902 \\
1059\end{array}$ & $\begin{array}{l}20.04579 \\
20.45770 \\
20.82667 \\
21.16105 \\
21.46703\end{array}$ & $\begin{array}{l}41191 \\
36897 \\
33438 \\
30598 \\
28224\end{array}$ & $\begin{array}{l}23.53862 \\
23.95504 \\
24.32984 \\
24.67161 \\
24.98660\end{array}$ & $\begin{array}{l}41642 \\
37480 \\
34177 \\
31499 \\
29282\end{array}$ \\
\hline $\begin{array}{l}650 \\
700 \\
750 \\
800 \\
850\end{array}$ & $\begin{array}{l}3.67758 \\
3.71986 \\
3.76305 \\
3.80619 \\
3.84859\end{array}$ & $\begin{array}{l}4228 \\
4319 \\
4314 \\
4240 \\
4114\end{array}$ & $\begin{array}{l}3.53015 \\
3.54218 \\
3.55547 \\
3.56980 \\
3.58495\end{array}$ & $\begin{array}{l}1203 \\
1329 \\
1433 \\
1515 \\
1580\end{array}$ & $\begin{array}{l}21.74927 \\
22.01141 \\
22.25634 \\
22.48633 \\
22.70327\end{array}$ & $\begin{array}{l}26214 \\
24493 \\
22999 \\
21694 \\
20541\end{array}$ & $\begin{array}{l}25.27942 \\
25.55361 \\
25.81180 \\
26.05612 \\
26.28822\end{array}$ & $\begin{array}{l}27419 \\
25819 \\
24432 \\
23210 \\
22121\end{array}$ \\
\hline $\begin{array}{r}900 \\
950 \\
1000 \\
1050 \\
1100\end{array}$ & $\begin{array}{l}3.88973 \\
3.92928 \\
3.96703 \\
4.00289 \\
4.03682\end{array}$ & $\begin{array}{l}3955 \\
3775 \\
3586 \\
3393 \\
3201\end{array}$ & $\begin{array}{l}3.60075 \\
3.61701 \\
3.63357 \\
3.65031 \\
3.66711\end{array}$ & $\begin{array}{l}1626 \\
1656 \\
1674 \\
1680 \\
1678\end{array}$ & $\begin{array}{l}22.90868 \\
23.10386 \\
23.28985 \\
23.46758 \\
23.63782\end{array}$ & $\begin{array}{l}19518 \\
18597 \\
17773 \\
17024 \\
16342\end{array}$ & $\begin{array}{l}26.50943 \\
26.72086 \\
26.92342 \\
27.11789 \\
27.30493\end{array}$ & $\begin{array}{l}21143 \\
20256 \\
19447 \\
18704 \\
18020\end{array}$ \\
\hline 1150 & 4.06883 & 3015 & 3.68389 & 1668 & 23.80124 & 15717 & 27.48513 & 17384 \\
\hline
\end{tabular}


Table 2.086. ND (Cont.)

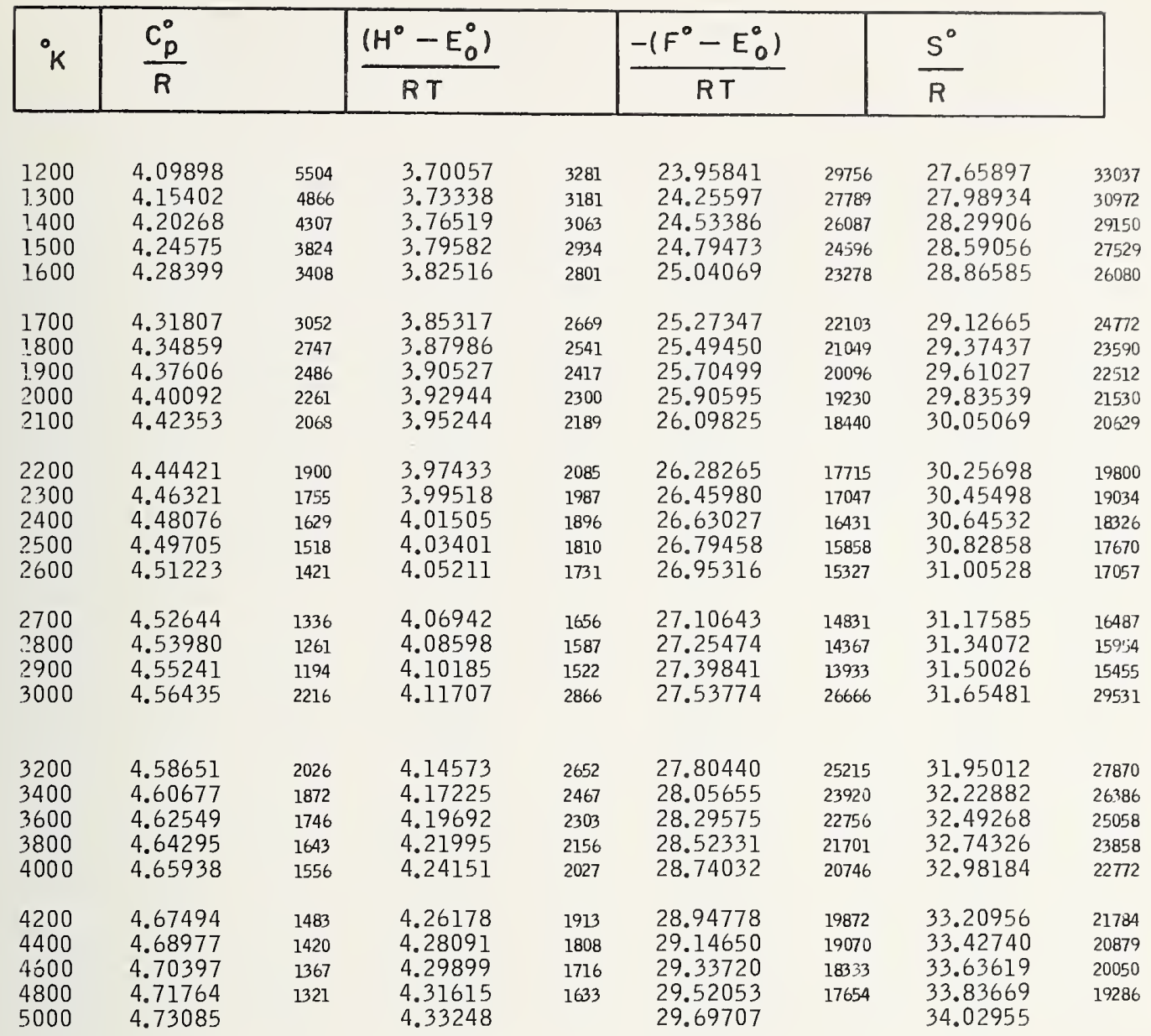


Table 2.087. NT

\begin{tabular}{|l|l|l|l|l|}
\hline${ }^{\circ} K$ & $\frac{C_{D}^{0}}{R}$ & $\frac{\left(H^{\circ}-E_{0}^{0}\right)}{R T}$ & $\frac{-\left(F^{\circ}-E_{0}^{0}\right)}{R T}$ & $\frac{S^{0}}{R}$ \\
\hline
\end{tabular}

\begin{tabular}{|c|c|c|c|c|c|c|c|c|}
\hline $\begin{array}{l}50 \\
60 \\
70 \\
80 \\
90\end{array}$ & $\begin{array}{l}3.50192 \\
3.50176 \\
3.50174 \\
3.50179 \\
3.50188\end{array}$ & $\begin{array}{r}-\quad 16 \\
-\quad 2 \\
5 \\
9 \\
12\end{array}$ & $\begin{array}{l}3.42155 \\
3.43494 \\
3.44448 \\
3.45164 \\
3.45722\end{array}$ & $\begin{array}{r}1339 \\
954 \\
716 \\
558 \\
447\end{array}$ & $\begin{array}{l}13.24727 \\
13.87543 \\
14.40787 \\
14.86995 \\
15.27811\end{array}$ & $\begin{array}{l}62816 \\
53244 \\
46208 \\
40816 \\
36552\end{array}$ & $\begin{array}{l}16.66884 \\
17.31037 \\
17.85236 \\
18.32159 \\
18.73533\end{array}$ & $\begin{array}{l}64153 \\
54199 \\
46923 \\
41374 \\
36999\end{array}$ \\
\hline $\begin{array}{l}100 \\
110 \\
120 \\
130 \\
140\end{array}$ & $\begin{array}{l}3.50200 \\
3.50213 \\
3.50227 \\
3.50242 \\
3.50257\end{array}$ & $\begin{array}{l}13 \\
14 \\
15 \\
15 \\
16\end{array}$ & $\begin{array}{l}3.46169 \\
3.46536 \\
3.46843 \\
3.47104 \\
3.47328\end{array}$ & $\begin{array}{l}367 \\
307 \\
261 \\
224 \\
196\end{array}$ & $\begin{array}{l}15.64363 \\
15.97458 \\
16.27693 \\
16.55525 \\
16.81307\end{array}$ & $\begin{array}{l}33095 \\
30235 \\
27832 \\
25782 \\
24014\end{array}$ & $\begin{array}{l}19.10532 \\
19.43994 \\
19.74537 \\
20.02630 \\
20.28636\end{array}$ & $\begin{array}{l}33462 \\
30543 \\
28093 \\
26006 \\
24210\end{array}$ \\
\hline $\begin{array}{l}150 \\
160 \\
170 \\
180 \\
190\end{array}$ & $\begin{array}{l}3.50273 \\
3.50290 \\
3.50307 \\
3.50326 \\
3.50347\end{array}$ & $\begin{array}{l}17 \\
17 \\
19 \\
21 \\
24\end{array}$ & $\begin{array}{l}3.47524 \\
3.47696 \\
3.47849 \\
3.47986 \\
3.48111\end{array}$ & $\begin{array}{l}172 \\
153 \\
137 \\
125 \\
112\end{array}$ & $\begin{array}{l}17.05321 \\
17.27794 \\
17.48911 \\
17.68828 \\
17.87673\end{array}$ & $\begin{array}{l}22473 \\
21117 \\
19917 \\
18845 \\
17883\end{array}$ & $\begin{array}{l}20.52846 \\
20.75491 \\
20.96761 \\
21.16816 \\
21.35784\end{array}$ & $\begin{array}{l}22645 \\
21270 \\
20055 \\
18968 \\
17996\end{array}$ \\
\hline $\begin{array}{l}200 \\
210 \\
220 \\
230 \\
240\end{array}$ & $\begin{array}{l}3.50371 \\
3.50401 \\
3.50437 \\
3.50482 \\
3.50540\end{array}$ & $\begin{array}{l}30 \\
36 \\
45 \\
58 \\
72\end{array}$ & $\begin{array}{l}3.48223 \\
3.48326 \\
3.48421 \\
3.48510 \\
3.48593\end{array}$ & $\begin{array}{r}103 \\
95 \\
89 \\
83 \\
79\end{array}$ & $\begin{array}{l}18.05556 \\
18.22571 \\
18.38797 \\
18.54305 \\
18.69155\end{array}$ & $\begin{array}{l}17015 \\
16226 \\
15508 \\
14850 \\
14248\end{array}$ & $\begin{array}{l}21.53780 \\
21.70897 \\
21.87218 \\
22.02815 \\
22.17748\end{array}$ & $\begin{array}{l}17117 \\
16321 \\
15597 \\
14933 \\
14328\end{array}$ \\
\hline $\begin{array}{l}250 \\
260 \\
270 \\
280 \\
290\end{array}$ & $\begin{array}{l}3.50612 \\
3.50702 \\
3.50812 \\
3.50947 \\
3.51110\end{array}$ & $\begin{array}{r}90 \\
110 \\
135 \\
163 \\
191\end{array}$ & $\begin{array}{l}3.48672 \\
3.48748 \\
3.48822 \\
3.48896 \\
3.48969\end{array}$ & $\begin{array}{l}76 \\
74 \\
74 \\
73 \\
75\end{array}$ & $\begin{array}{l}18.83403 \\
18.97094 \\
19.10270 \\
19.22970 \\
19.35225\end{array}$ & $\begin{array}{l}13691 \\
13176 \\
12700 \\
12255 \\
11843\end{array}$ & $\begin{array}{l}22.32076 \\
22.45843 \\
22.59093 \\
22.71866 \\
22.84196\end{array}$ & $\begin{array}{l}13767 \\
13250 \\
12773 \\
12330 \\
11916\end{array}$ \\
\hline $\begin{array}{l}300 \\
310 \\
320 \\
330 \\
340\end{array}$ & $\begin{array}{l}3.51301 \\
3.51525 \\
3.51784 \\
3.52078 \\
3.52411\end{array}$ & $\begin{array}{l}224 \\
259 \\
294 \\
333 \\
371\end{array}$ & $\begin{array}{l}3.49044 \\
3.49120 \\
3.49199 \\
3.49282 \\
3.49370\end{array}$ & $\begin{array}{l}76 \\
79 \\
83 \\
88 \\
92\end{array}$ & $\begin{array}{l}19.47068 \\
19.58524 \\
19.69619 \\
19.80374 \\
19.90811\end{array}$ & $\begin{array}{l}11456 \\
11095 \\
10755 \\
10437 \\
10137\end{array}$ & $\begin{array}{l}22.96112 \\
23.07645 \\
23.18818 \\
23.29657 \\
23.40180\end{array}$ & $\begin{array}{l}11533 \\
11173 \\
10839 \\
10523 \\
10229\end{array}$ \\
\hline $\begin{array}{l}350 \\
360 \\
370 \\
380 \\
390\end{array}$ & $\begin{array}{l}3.52782 \\
3.53192 \\
3.53643 \\
3.54133 \\
3.54663\end{array}$ & $\begin{array}{l}410 \\
451 \\
490 \\
530 \\
569\end{array}$ & $\begin{array}{l}3.49462 \\
3.49559 \\
3.49664 \\
3.49774 \\
3.49893\end{array}$ & $\begin{array}{r}97 \\
105 \\
110 \\
119 \\
126\end{array}$ & $\begin{array}{l}20.00948 \\
20.10800 \\
20.20387 \\
20.29719 \\
20.38813\end{array}$ & $\begin{array}{l}9852 \\
9587 \\
9332 \\
9094 \\
8866\end{array}$ & $\begin{array}{l}23.50409 \\
23.60360 \\
23.70050 \\
23.79494 \\
23.88706\end{array}$ & $\begin{array}{l}9951 \\
9690 \\
9444 \\
9212 \\
8992\end{array}$ \\
\hline $\begin{array}{l}400 \\
450 \\
500 \\
550 \\
600\end{array}$ & $\begin{array}{l}3.55232 \\
3.58617 \\
3.62759 \\
3.67421 \\
3.72375\end{array}$ & $\begin{array}{l}3385 \\
4142 \\
4662 \\
4954 \\
5054\end{array}$ & $\begin{array}{l}3.50019 \\
3.50779 \\
3.51764 \\
3.52972 \\
3.54381\end{array}$ & $\begin{array}{r}760 \\
985 \\
1208 \\
1409 \\
1579\end{array}$ & $\begin{array}{l}20.47679 \\
20.88972 \\
21.26000 \\
21.59598 \\
21.90384\end{array}$ & $\begin{array}{l}41293 \\
37028 \\
33598 \\
30785 \\
28439\end{array}$ & $\begin{array}{l}23.97698 \\
24.39751 \\
24.77764 \\
25.12572 \\
25.44766\end{array}$ & $\begin{array}{l}42053 \\
38013 \\
34808 \\
32194 \\
30017\end{array}$ \\
\hline $\begin{array}{l}650 \\
700 \\
750 \\
800 \\
850\end{array}$ & $\begin{array}{l}3.77429 \\
3.82438 \\
3.87301 \\
3.91953 \\
3.96356\end{array}$ & $\begin{array}{l}5009 \\
4863 \\
4652 \\
4403 \\
4138\end{array}$ & $\begin{array}{l}3.55960 \\
3.57673 \\
3.59487 \\
3.61372 \\
3.63302\end{array}$ & $\begin{array}{l}1713 \\
1814 \\
1885 \\
1930 \\
1952\end{array}$ & $\begin{array}{l}22.18823 \\
22.45274 \\
22.70022 \\
22.93290 \\
23.15263\end{array}$ & $\begin{array}{l}26451 \\
24748 \\
23268 \\
21973 \\
20826\end{array}$ & $\begin{array}{l}25.74783 \\
26.02948 \\
26.29509 \\
26.54662 \\
26.78565\end{array}$ & $\begin{array}{l}28165 \\
26561 \\
25153 \\
23903 \\
22779\end{array}$ \\
\hline $\begin{array}{r}900 \\
950 \\
1000 \\
1050 \\
1100\end{array}$ & $\begin{array}{l}4.00494 \\
4.04362 \\
4.07966 \\
4.11317 \\
4.14429\end{array}$ & $\begin{array}{l}3868 \\
3604 \\
3351 \\
3112 \\
2889\end{array}$ & $\begin{array}{l}3.65254 \\
3.67212 \\
3.69161 \\
3.71089 \\
3.72989\end{array}$ & $\begin{array}{l}1958 \\
1949 \\
1928 \\
1900 \\
1866\end{array}$ & $\begin{array}{l}23.36089 \\
23.55896 \\
23.74786 \\
23.92848 \\
24.10159\end{array}$ & $\begin{array}{l}19807 \\
18890 \\
18062 \\
17311 \\
16625\end{array}$ & $\begin{array}{l}27.01344 \\
27.23108 \\
27.43947 \\
27.63937 \\
27.83149\end{array}$ & $\begin{array}{l}21764 \\
20839 \\
19990 \\
19212 \\
18490\end{array}$ \\
\hline 1150 & 4.17318 & 2683 & 3.74855 & 1826 & 24.26784 & 15996 & 28.01639 & 17822 \\
\hline
\end{tabular}


Table 2.087. NT (Cont.)

\begin{tabular}{|l|l|l|l|l|}
\hline$K$ & $\frac{C_{p}^{0}}{R}$ & $\frac{\left(H^{0}-E_{0}^{0}\right)}{R T}$ & $\frac{-\left(F^{0}-E_{0}^{0}\right)}{R T}$ & $\frac{S^{0}}{R}$ \\
\hline
\end{tabular}

\begin{tabular}{|c|c|c|c|c|c|c|c|}
\hline $\begin{array}{l}1200 \\
1300 \\
1400 \\
1500 \\
1600\end{array}$ & $\begin{array}{l}4.20001 \\
4.24813 \\
4.28986 \\
4.32627 \\
4.35825\end{array}$ & $\begin{array}{l}4812 \\
4173 \\
3641 \\
3198 \\
2829\end{array}$ & $\begin{array}{l}3.76681 \\
3.80203 \\
3.83541 \\
3.86695 \\
3.89668\end{array}$ & $\begin{array}{l}3522 \\
3338 \\
3154 \\
2973 \\
2800\end{array}$ & $\begin{array}{l}24.42780 \\
24.73077 \\
25.01381 \\
25.27955 \\
25.53012\end{array}$ & $\begin{array}{l}30297 \\
28304 \\
26574 \\
25057 \\
23712\end{array}$ & $\begin{array}{l}28.19461 \\
28.53279 \\
28.84923 \\
29.14652 \\
29.42680\end{array}$ \\
\hline $\begin{array}{l}1700 \\
1800 \\
1900 \\
2000 \\
2100\end{array}$ & $\begin{array}{l}4.38654 \\
4.41175 \\
4.43437 \\
4.45482 \\
4.47342\end{array}$ & $\begin{array}{l}2521 \\
2262 \\
2045 \\
1860 \\
1702\end{array}$ & $\begin{array}{l}3.92468 \\
3.95105 \\
3.97591 \\
3.99935 \\
4.02148\end{array}$ & $\begin{array}{l}2637 \\
2486 \\
2344 \\
2213 \\
2094\end{array}$ & $\begin{array}{l}25.76724 \\
25.99235 \\
26.20668 \\
26.41124 \\
26.60693\end{array}$ & $\begin{array}{l}22511 \\
21433 \\
20456 \\
19569 \\
18758\end{array}$ & $\begin{array}{l}29.69192 \\
29.94341 \\
30.18259 \\
30.41059 \\
30.62842\end{array}$ \\
\hline $\begin{array}{l}2200 \\
2300 \\
2400 \\
2500 \\
2600\end{array}$ & $\begin{array}{l}4.49044 \\
4.50613 \\
4.52066 \\
4.53420 \\
4.54687\end{array}$ & $\begin{array}{l}1569 \\
1453 \\
1354 \\
1267 \\
1192\end{array}$ & $\begin{array}{l}4.04242 \\
4.06225 \\
4.08105 \\
4.09891 \\
4.11589\end{array}$ & $\begin{array}{l}1983 \\
1880 \\
1786 \\
1698 \\
1619\end{array}$ & $\begin{array}{l}26.79451 \\
26.97467 \\
27.14798 \\
27.31495 \\
27.47606\end{array}$ & $\begin{array}{l}18016 \\
17331 \\
16697 \\
16111 \\
15566\end{array}$ & $\begin{array}{l}30.83694 \\
31.03692 \\
31.22903 \\
31.41386 \\
31.59196\end{array}$ \\
\hline $\begin{array}{l}2700 \\
2800 \\
2900 \\
3000\end{array}$ & $\begin{array}{l}4.55879 \\
4.57005 \\
4.58072 \\
4.59089\end{array}$ & $\begin{array}{l}1126 \\
1067 \\
1017 \\
1902\end{array}$ & $\begin{array}{l}4.13208 \\
4.14753 \\
4.16228 \\
4.17640\end{array}$ & $\begin{array}{l}1545 \\
1475 \\
1412 \\
2651\end{array}$ & $\begin{array}{l}27.63172 \\
27.78229 \\
27.92809 \\
28.06946\end{array}$ & $\begin{array}{l}15057 \\
14580 \\
14137 \\
27041\end{array}$ & $\begin{array}{l}31.76380 \\
31.92981 \\
32.09037 \\
32.24585\end{array}$ \\
\hline $\begin{array}{l}3200 \\
3400 \\
3600 \\
3800 \\
4000\end{array}$ & $\begin{array}{l}4.60991 \\
4.62749 \\
4.64390 \\
4.65936 \\
4.67404\end{array}$ & $\begin{array}{l}1758 \\
1641 \\
1546 \\
1468 \\
1403\end{array}$ & $\begin{array}{l}4.20291 \\
4.22736 \\
4.25006 \\
4.27120 \\
4.29097\end{array}$ & $\begin{array}{l}2445 \\
2270 \\
2114 \\
1977 \\
1858\end{array}$ & $\begin{array}{l}28.33987 \\
28.59542 \\
28.83772 \\
29.06810 \\
29.28770\end{array}$ & $\begin{array}{l}25555 \\
24230 \\
23038 \\
21960 \\
20983\end{array}$ & $\begin{array}{l}32.54278 \\
32.82280 \\
33.08778 \\
33.33931 \\
33.57869\end{array}$ \\
\hline $\begin{array}{l}4200 \\
4400 \\
4600 \\
4800 \\
5000\end{array}$ & $\begin{array}{l}4.68807 \\
4.70154 \\
4.71454 \\
4.72714 \\
4.73939\end{array}$ & $\begin{array}{l}1347 \\
1300 \\
1260 \\
1225\end{array}$ & $\begin{array}{l}4.30955 \\
4.32707 \\
4.34363 \\
4.35935 \\
4.37431\end{array}$ & $\begin{array}{l}1752 \\
1656 \\
1572 \\
1496\end{array}$ & $\begin{array}{l}29.49753 \\
29.69843 \\
29.89115 \\
30.07635 \\
30.25463\end{array}$ & $\begin{array}{l}20090 \\
19272 \\
18520 \\
17828\end{array}$ & $\begin{array}{l}33.80709 \\
34.02550 \\
34.23479 \\
34.43571 \\
34.62894\end{array}$ \\
\hline
\end{tabular}


Table 2.088. PH

\begin{tabular}{|l|l|l|l|l|}
\hline$K$ & $\frac{C_{p}^{0}}{R}$ & $\frac{\left(H^{0}-E_{0}^{0}\right)}{R T}$ & $\frac{-\left(F^{0}-E_{0}^{0}\right)}{R T}$ & $\frac{S^{0}}{R}$ \\
\hline
\end{tabular}

\begin{tabular}{|c|c|c|c|c|c|c|c|c|}
\hline $\begin{array}{l}50 \\
60 \\
70 \\
80 \\
90\end{array}$ & $\begin{array}{l}3.50276 \\
3.50232 \\
3.50212 \\
3.50206 \\
3.50206\end{array}$ & $\begin{array}{r}-\quad 44 \\
-\quad 20 \\
-\quad 6\end{array}$ & $\begin{array}{l}3.28980 \\
3.40858 \\
3.42196 \\
3.43197 \\
3.43976\end{array}$ & $\begin{array}{r}1878 \\
1338 \\
1001 \\
779 \\
623\end{array}$ & $\begin{array}{l}13.90836 \\
14.53359 \\
15.06396 \\
15.52450 \\
15.93146\end{array}$ & $\begin{array}{l}62523 \\
53037 \\
46054 \\
40593 \\
36456\end{array}$ & $\begin{array}{l}17.29816 \\
17.94217 \\
18.48592 \\
18.95647 \\
19.37122\end{array}$ & $\begin{array}{l}64401 \\
54375 \\
47055 \\
41475 \\
37079\end{array}$ \\
\hline $\begin{array}{l}100 \\
110 \\
120 \\
130 \\
140\end{array}$ & $\begin{array}{l}3.50212 \\
3.50220 \\
3.50230 \\
3.50242 \\
3.50255\end{array}$ & $\begin{array}{r}8 \\
10 \\
12 \\
13 \\
13\end{array}$ & $\begin{array}{l}3.44599 \\
3.45110 \\
3.45536 \\
3.45898 \\
3.46209\end{array}$ & $\begin{array}{l}511 \\
426 \\
362 \\
311 \\
270\end{array}$ & $\begin{array}{l}16.29602 \\
16.62619 \\
16.92790 \\
17.20567 \\
17.46302\end{array}$ & $\begin{array}{l}33017 \\
30171 \\
27777 \\
25735 \\
23973\end{array}$ & $\begin{array}{l}19.74201 \\
20.07729 \\
20.38326 \\
20.66465 \\
20.92511\end{array}$ & $\begin{array}{l}33528 \\
30597 \\
28139 \\
26046 \\
24243\end{array}$ \\
\hline $\begin{array}{l}150 \\
160 \\
170 \\
180 \\
190\end{array}$ & $\begin{array}{l}3.50268 \\
3.50282 \\
3.50297 \\
3.50312 \\
3.50327\end{array}$ & $\begin{array}{l}14 \\
15 \\
15 \\
15 \\
17\end{array}$ & $\begin{array}{l}3.46479 \\
3.46716 \\
3.46927 \\
3.47114 \\
3.47283\end{array}$ & $\begin{array}{l}237 \\
211 \\
187 \\
169 \\
152\end{array}$ & $\begin{array}{l}17.70275 \\
17.92712 \\
18.13798 \\
18.33686 \\
18.52506\end{array}$ & $\begin{array}{l}22437 \\
21086 \\
19888 \\
18820 \\
17859\end{array}$ & $\begin{array}{l}21.16754 \\
21.39428 \\
21.60725 \\
21.80800 \\
21.99789\end{array}$ & $\begin{array}{l}22674 \\
21297 \\
20075 \\
18989 \\
18011\end{array}$ \\
\hline $\begin{array}{l}200 \\
210 \\
220 \\
230 \\
240\end{array}$ & $\begin{array}{l}3.50344 \\
3.50361 \\
3.50379 \\
3.50400 \\
3.50424\end{array}$ & $\begin{array}{l}17 \\
18 \\
21 \\
24 \\
27\end{array}$ & $\begin{array}{l}3.47435 \\
3.47574 \\
3.47701 \\
3.47818 \\
3.47926\end{array}$ & $\begin{array}{l}139 \\
127 \\
117 \\
108 \\
101\end{array}$ & $\begin{array}{l}18.70365 \\
18.87360 \\
19.03566 \\
19.19058 \\
19.33892\end{array}$ & $\begin{array}{l}16995 \\
16206 \\
15492 \\
14834 \\
14233\end{array}$ & $\begin{array}{l}22.17800 \\
22.34934 \\
22.51267 \\
22.66876 \\
22.81818\end{array}$ & $\begin{array}{l}17134 \\
16333 \\
15609 \\
14942 \\
14334\end{array}$ \\
\hline $\begin{array}{l}250 \\
260 \\
270 \\
280 \\
290\end{array}$ & $\begin{array}{l}3.50451 \\
3.50484 \\
3.50523 \\
3.50570 \\
3.50627\end{array}$ & $\begin{array}{l}33 \\
39 \\
47 \\
57 \\
69\end{array}$ & $\begin{array}{l}3.48027 \\
3.48120 \\
3.48209 \\
3.48291 \\
3.48372\end{array}$ & $\begin{array}{l}93 \\
89 \\
82 \\
81 \\
76\end{array}$ & $\begin{array}{l}19.48125 \\
19.61802 \\
19.74965 \\
19.87651 \\
19.99894\end{array}$ & $\begin{array}{l}13677 \\
13163 \\
12686 \\
12243 \\
11831\end{array}$ & $\begin{array}{l}22.96152 \\
23.09922 \\
23.23174 \\
23.35942 \\
23.48266\end{array}$ & $\begin{array}{l}13770 \\
13252 \\
12768 \\
12324 \\
11907\end{array}$ \\
\hline $\begin{array}{l}300 \\
310 \\
320 \\
330 \\
340\end{array}$ & $\begin{array}{l}3.50696 \\
3.50778 \\
3.50876 \\
3.50991 \\
3.51126\end{array}$ & $\begin{array}{r}82 \\
98 \\
115 \\
135 \\
155\end{array}$ & $\begin{array}{l}3.48448 \\
3.48522 \\
3.48593 \\
3.48665 \\
3.48735\end{array}$ & $\begin{array}{l}74 \\
71 \\
72 \\
70 \\
70\end{array}$ & $\begin{array}{l}20.11725 \\
20.23169 \\
20.34252 \\
20.44996 \\
20.55420\end{array}$ & $\begin{array}{l}11144 \\
110^{\circ} 3 \\
10744 \\
10424 \\
10123\end{array}$ & $\begin{array}{l}23.60173 \\
23.71691 \\
23.82845 \\
23.93661 \\
24.04155\end{array}$ & $\begin{array}{l}11518 \\
11154 \\
10816 \\
10494 \\
10193\end{array}$ \\
\hline $\begin{array}{l}350 \\
360 \\
370 \\
380 \\
390\end{array}$ & $\begin{array}{l}3.51281 \\
3.51458 \\
3.51660 \\
3.51886 \\
3.52139\end{array}$ & $\begin{array}{l}177 \\
202 \\
226 \\
253 \\
279\end{array}$ & $\begin{array}{l}3.48805 \\
3.48877 \\
3.48949 \\
3.49024 \\
3.49100\end{array}$ & $\begin{array}{l}72 \\
72 \\
75 \\
76 \\
79\end{array}$ & $\begin{array}{l}20.65543 \\
20.75384 \\
20.84956 \\
20.94274 \\
21.03352\end{array}$ & $\begin{array}{l}9841 \\
9572 \\
9318 \\
9078 \\
8850\end{array}$ & $\begin{array}{l}24.14348 \\
24.24261 \\
24.33905 \\
24.43298 \\
24.52452\end{array}$ & $\begin{array}{l}9913 \\
9644 \\
9393 \\
9154 \\
8929\end{array}$ \\
\hline $\begin{array}{l}400 \\
450 \\
500 \\
550 \\
600\end{array}$ & $\begin{array}{l}3.52418 \\
3.54239 \\
3.56761 \\
3.59908 \\
3.63553\end{array}$ & $\begin{array}{l}1821 \\
2522 \\
3147 \\
3645 \\
3998\end{array}$ & $\begin{array}{l}3.49179 \\
3.49634 \\
3.50215 \\
3.50949 \\
3.51844\end{array}$ & $\begin{array}{r}455 \\
581 \\
734 \\
895 \\
1053\end{array}$ & $\begin{array}{l}21.12202 \\
21.53400 \\
21.90303 \\
22.23745 \\
22.54343\end{array}$ & $\begin{array}{l}41198 \\
36903 \\
33442 \\
30598 \\
28225\end{array}$ & $\begin{array}{l}24.61381 \\
25.03034 \\
25.40518 \\
25.74694 \\
26.06187\end{array}$ & $\begin{array}{l}41653 \\
37484 \\
34176 \\
31493 \\
29278\end{array}$ \\
\hline $\begin{array}{l}650 \\
700 \\
750 \\
800 \\
850\end{array}$ & $\begin{array}{l}3.67551 \\
3.71764 \\
3.76074 \\
3.80384 \\
3.84625\end{array}$ & $\begin{array}{l}4213 \\
4310 \\
4310 \\
4241 \\
4120\end{array}$ & $\begin{array}{l}3.52897 \\
3.54094 \\
3.55414 \\
3.56841 \\
3.58351\end{array}$ & $\begin{array}{l}1197 \\
1320 \\
1427 \\
1510 \\
1574\end{array}$ & $\begin{array}{l}22.82568 \\
23.08781 \\
23.33270 \\
23.56267 \\
23.77957\end{array}$ & $\begin{array}{l}26213 \\
24489 \\
22997 \\
21690 \\
20538\end{array}$ & $\begin{array}{l}26.35465 \\
26.62875 \\
26.88684 \\
27.13108 \\
27.36308\end{array}$ & $\begin{array}{l}27+10 \\
25809 \\
24424 \\
23200 \\
22113\end{array}$ \\
\hline $\begin{array}{r}900 \\
950 \\
1000 \\
1050 \\
1100\end{array}$ & $\begin{array}{l}3.88745 \\
3.92709 \\
3.96497 \\
4.00098 \\
4.03507\end{array}$ & $\begin{array}{l}3964 \\
3788 \\
3601 \\
3409 \\
3219\end{array}$ & $\begin{array}{l}3.59925 \\
3.61547 \\
3.63200 \\
3.64873 \\
3.66552\end{array}$ & $\begin{array}{l}1622 \\
1653 \\
1673 \\
1679 \\
1678\end{array}$ & $\begin{array}{l}23.98495 \\
24.18008 \\
24.36604 \\
24.54372 \\
24.71392\end{array}$ & $\begin{array}{l}19513 \\
18596 \\
17768 \\
17020 \\
16337\end{array}$ & $\begin{array}{l}27.58421 \\
27.79555 \\
27.99805 \\
28.19246 \\
28.37945\end{array}$ & $\begin{array}{l}21134 \\
20250 \\
19441 \\
18699 \\
18014\end{array}$ \\
\hline 1150 & 4.06726 & 3034 & 3.68230 & 1667 & 24.87729 & 15713 & 28.55959 & 17381 \\
\hline
\end{tabular}


Table 2.088. PH (Cont.)

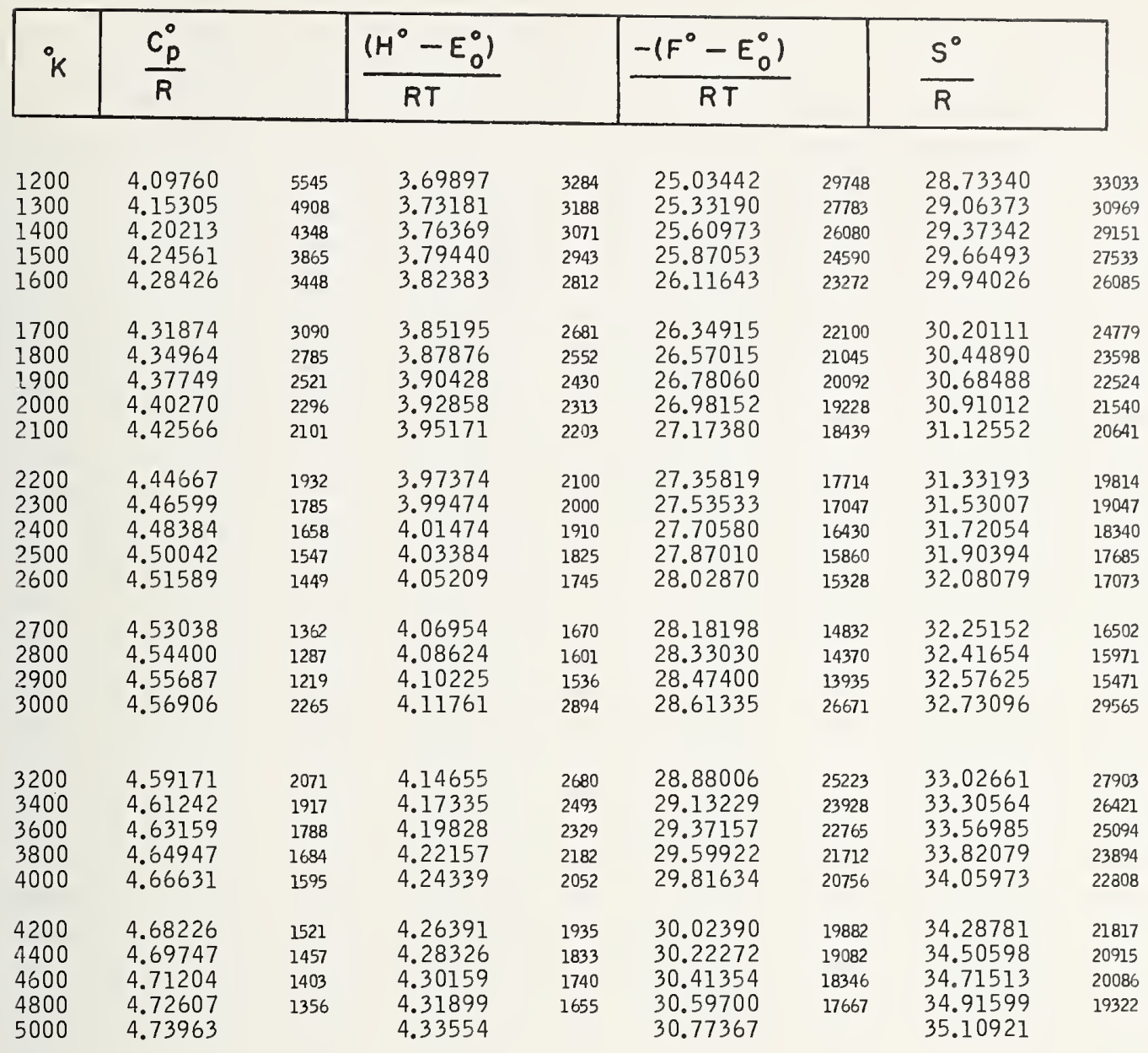


Table 2.089. PD

\begin{tabular}{|l|l|l|l|l|}
\hline$K_{K}$ & $\frac{C_{p}^{0}}{R}$ & $\frac{\left(H^{0}-E_{0}^{0}\right)}{R T}$ & $\frac{-\left(F^{0}-E_{0}^{0}\right)}{R T}$ & $\frac{S^{0}}{R}$ \\
\hline
\end{tabular}

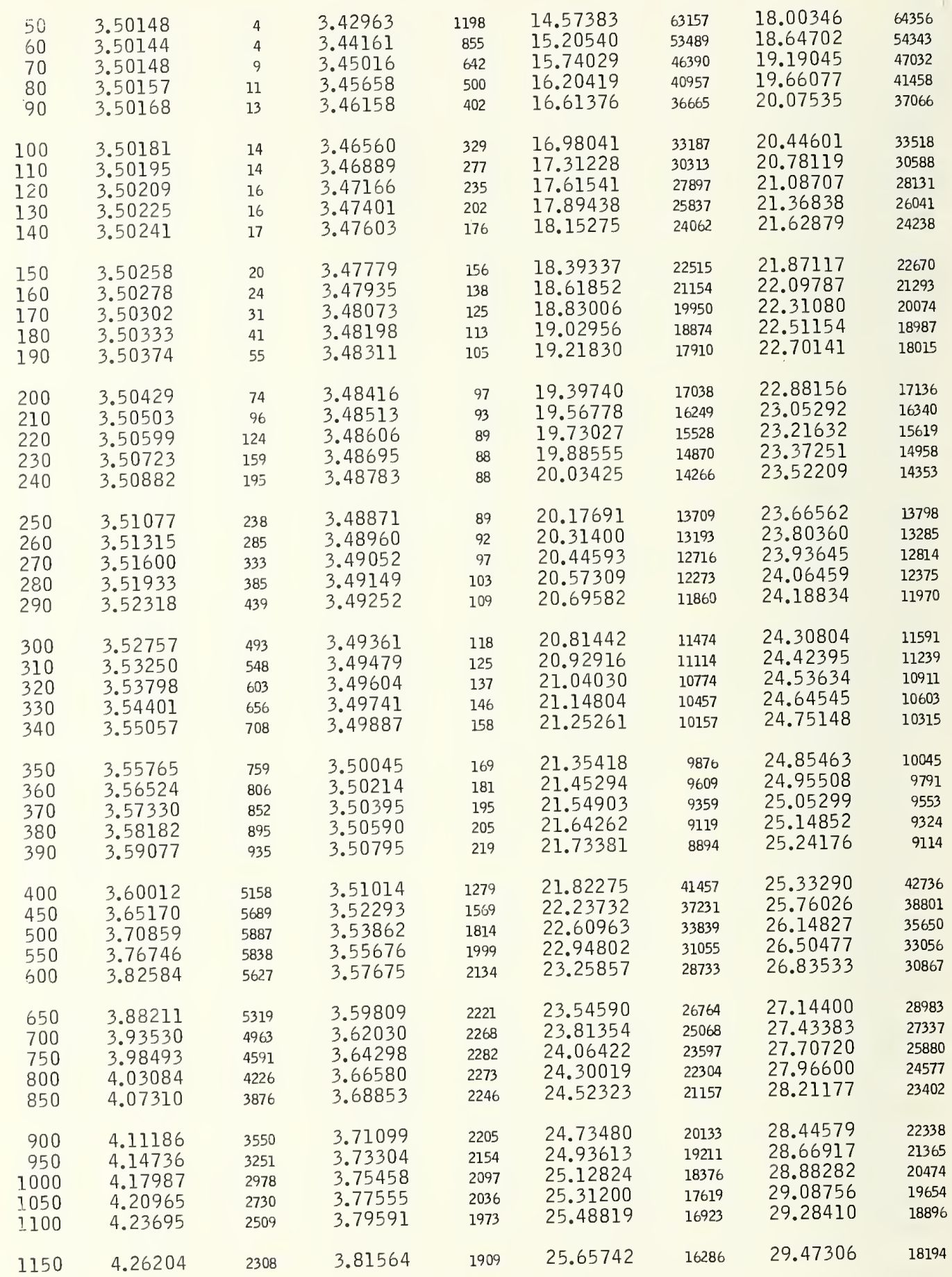


Table 2.089. PD (Cont.)

\begin{tabular}{|c|c|c|c|c|c|c|c|c|}
\hline${ }^{\circ} \mathrm{K}$ & $\frac{C_{p}^{0}}{R}$ & & $\frac{\left(H^{0}-E_{0}^{0}\right)}{R T}$ & & $\frac{-\left(F^{0}-E_{0}^{0}\right)}{R T}$ & & $\frac{S^{0}}{R}$ & \\
\hline $\begin{array}{l}1200 \\
1300 \\
1400 \\
1500 \\
1600\end{array}$ & $\begin{array}{l}4.28512 \\
4.32608 \\
4.36123 \\
4.39169 \\
4.41834\end{array}$ & $\begin{array}{l}4096 \\
3515 \\
3046 \\
2665 \\
2355\end{array}$ & $\begin{array}{l}3.83473 \\
3.87099 \\
3.90478 \\
3.93626 \\
3.96557\end{array}$ & $\begin{array}{l}3626 \\
3379 \\
3148 \\
2931 \\
2734\end{array}$ & $\begin{array}{l}25.82028 \\
26.12877 \\
26.41697 \\
26.68754 \\
26.94259\end{array}$ & $\begin{array}{l}30849 \\
28820 \\
27057 \\
25505 \\
24130\end{array}$ & $\begin{array}{l}29.65500 \\
29.99975 \\
30.32176 \\
30.62379 \\
30.90815\end{array}$ & $\begin{array}{l}34475 \\
32201 \\
30203 \\
28436 \\
26864\end{array}$ \\
\hline $\begin{array}{l}1700 \\
1800 \\
1900 \\
? 000 \\
2100\end{array}$ & $\begin{array}{l}4.44189 \\
4.46289 \\
4.48177 \\
4.49890 \\
4.51456\end{array}$ & $\begin{array}{l}2100 \\
1888 \\
1713 \\
1566 \\
1441\end{array}$ & $\begin{array}{l}3.99291 \\
4.01844 \\
4.04234 \\
4.06474 \\
4.08580\end{array}$ & $\begin{array}{l}2553 \\
2390 \\
2240 \\
2106 \\
1982\end{array}$ & $\begin{array}{l}27.18389 \\
27.41289 \\
27.63085 \\
27.83881 \\
28.03768\end{array}$ & $\begin{array}{l}22900 \\
21796 \\
20796 \\
19887 \\
19057\end{array}$ & $\begin{array}{l}31.17679 \\
31.43134 \\
31.67320 \\
31.90357 \\
32.12348\end{array}$ & $\begin{array}{l}25455 \\
24186 \\
23037 \\
21991 \\
21040\end{array}$ \\
\hline $\begin{array}{l}2200 \\
2300 \\
2400 \\
2500 \\
2600\end{array}$ & $\begin{array}{l}4.52897 \\
4.54232 \\
4.55477 \\
4.56645 \\
4.57745\end{array}$ & $\begin{array}{l}1335 \\
1245 \\
1168 \\
1100 \\
1043\end{array}$ & $\begin{array}{l}4.10562 \\
4.12432 \\
4.14200 \\
4.15875 \\
4.17464\end{array}$ & $\begin{array}{l}1870 \\
1768 \\
1675 \\
1589 \\
1511\end{array}$ & $\begin{array}{l}28.22825 \\
28.41120 \\
28.58714 \\
28.75659 \\
28.92003\end{array}$ & $\begin{array}{l}18295 \\
17594 \\
16945 \\
16344 \\
15786\end{array}$ & $\begin{array}{l}32.33388 \\
32.53553 \\
32.72914 \\
32.91534 \\
33.09468\end{array}$ & $\begin{array}{l}20165 \\
19361 \\
18620 \\
17934 \\
17298\end{array}$ \\
\hline $\begin{array}{l}2700 \\
2800 \\
2900 \\
3000\end{array}$ & $\begin{array}{l}4.58788 \\
4.59779 \\
4.60726 \\
4.61633\end{array}$ & $\begin{array}{r}991 \\
947 \\
907 \\
1715\end{array}$ & $\begin{array}{l}4.18975 \\
4.20415 \\
4.21789 \\
4.23102\end{array}$ & $\begin{array}{l}1440 \\
1374 \\
1313 \\
2463\end{array}$ & $\begin{array}{l}29.07789 \\
29.23054 \\
29.37833 \\
29.52157\end{array}$ & $\begin{array}{l}15265 \\
14779 \\
14324 \\
27390\end{array}$ & $\begin{array}{l}33.26766 \\
33.43471 \\
33.59624 \\
33.75259\end{array}$ & $\begin{array}{l}16705 \\
16153 \\
15635 \\
29853\end{array}$ \\
\hline $\begin{array}{l}3200 \\
3400 \\
3600 \\
3800 \\
4000\end{array}$ & $\begin{array}{l}4.63348 \\
4.64952 \\
4.66466 \\
4.67908 \\
4.69289\end{array}$ & $\begin{array}{l}1604 \\
1514 \\
1442 \\
1381 \\
1331\end{array}$ & $\begin{array}{l}4.25565 \\
4.27834 \\
4.29940 \\
4.31900 \\
4.33735\end{array}$ & $\begin{array}{l}2269 \\
2106 \\
1960 \\
1835 \\
1725\end{array}$ & $\begin{array}{l}29.79547 \\
30.05418 \\
30.29936 \\
30.53237 \\
30.75439\end{array}$ & $\begin{array}{l}25871 \\
24518 \\
23301 \\
22202 \\
21206\end{array}$ & $\begin{array}{l}34.05112 \\
34.33253 \\
34.59875 \\
34.85136 \\
35.09174\end{array}$ & $\begin{array}{l}28141 \\
26622 \\
25261 \\
24038 \\
22931\end{array}$ \\
\hline $\begin{array}{l}4200 \\
4400 \\
4600 \\
4800 \\
5000\end{array}$ & $\begin{array}{l}4.70620 \\
4.71908 \\
4.73160 \\
4.74379 \\
4.75571\end{array}$ & $\begin{array}{l}1288 \\
1252 \\
1219 \\
1192\end{array}$ & $\begin{array}{l}4.35460 \\
4.37087 \\
4.38629 \\
4.40093 \\
4.41489\end{array}$ & $\begin{array}{l}1627 \\
1542 \\
1464 \\
1396\end{array}$ & $\begin{array}{l}30.96645 \\
31.16942 \\
31.36407 \\
31.55108 \\
31.73104\end{array}$ & $\begin{array}{l}20297 \\
19465 \\
18701 \\
17996\end{array}$ & $\begin{array}{l}35.32105 \\
35.54031 \\
35.75036 \\
35.95202 \\
36.14592\end{array}$ & $\begin{array}{l}21926 \\
21005 \\
20166 \\
19390\end{array}$ \\
\hline
\end{tabular}


Table 2.090. PT

\begin{tabular}{|l|l|l|l|l|}
\hline${ }^{\circ} K$ & $\frac{C_{p}^{0}}{R}$ & $\frac{\left(H^{0}-E_{0}^{0}\right)}{R T}$ & $\frac{-\left(F^{0}-E_{0}^{0}\right)}{R T}$ & $\frac{S^{0}}{R}$ \\
\hline
\end{tabular}

\begin{tabular}{|c|c|c|c|c|c|c|c|c|}
\hline $\begin{array}{l}50 \\
60 \\
70 \\
80 \\
90\end{array}$ & $\begin{array}{l}3.50121 \\
3.50125 \\
3.50135 \\
3.50147 \\
3.50160\end{array}$ & $\begin{array}{c}4 \\
10 \\
12 \\
13 \\
15\end{array}$ & $\begin{array}{l}3.44311 \\
3.45279 \\
3.45972 \\
3.46493 \\
3.46900\end{array}$ & $\begin{array}{l}968 \\
693 \\
521 \\
407 \\
327\end{array}$ & $\begin{array}{l}14.98448 \\
15.61809 \\
16.15443 \\
16.61942 \\
17.02984\end{array}$ & $\begin{array}{l}63361 \\
53634 \\
46499 \\
41042 \\
36733\end{array}$ & $\begin{array}{l}18.42759 \\
19.07089 \\
19.61416 \\
20.08436 \\
20.49884\end{array}$ & $\begin{array}{l}64330 \\
54327 \\
47020 \\
41448 \\
37060\end{array}$ \\
\hline $\begin{array}{l}100 \\
110 \\
120 \\
130 \\
140\end{array}$ & $\begin{array}{l}3.50175 \\
3.50190 \\
3.50207 \\
3.50226 \\
3.50250\end{array}$ & $\begin{array}{l}15 \\
17 \\
19 \\
24 \\
34\end{array}$ & $\begin{array}{l}3.47227 \\
3.47495 \\
3.47721 \\
3.47912 \\
3.48079\end{array}$ & $\begin{array}{l}268 \\
226 \\
191 \\
167 \\
146\end{array}$ & $\begin{array}{l}17.39717 \\
17.72959 \\
18.03318 \\
18.31253 \\
18.57124\end{array}$ & $\begin{array}{l}33242 \\
30359 \\
27935 \\
25871 \\
24091\end{array}$ & $\begin{array}{l}20.86944 \\
21.20454 \\
21.51038 \\
21.79167 \\
22.05203\end{array}$ & $\begin{array}{l}33510 \\
30584 \\
28129 \\
26036 \\
24237\end{array}$ \\
\hline $\begin{array}{l}150 \\
160 \\
170 \\
180 \\
190\end{array}$ & $\begin{array}{l}3.50284 \\
3.50332 \\
3.50402 \\
3.50502 \\
3.50639\end{array}$ & $\begin{array}{r}48 \\
70 \\
100 \\
137 \\
186\end{array}$ & $\begin{array}{l}3.48225 \\
3.48354 \\
3.48473 \\
3.48583 \\
3.48687\end{array}$ & $\begin{array}{l}129 \\
119 \\
110 \\
104 \\
102\end{array}$ & $\begin{array}{l}18.81215 \\
19.03756 \\
19.24932 \\
19.44902 \\
19.63796\end{array}$ & $\begin{array}{l}22541 \\
21176 \\
19970 \\
18894 \\
17926\end{array}$ & $\begin{array}{l}22.29440 \\
22.52110 \\
22.73406 \\
22.93485 \\
23.12483\end{array}$ & $\begin{array}{l}22670 \\
21296 \\
20079 \\
18998 \\
18029\end{array}$ \\
\hline $\begin{array}{l}200 \\
210 \\
220 \\
230 \\
240\end{array}$ & $\begin{array}{l}3.50825 \\
3.51065 \\
3.51367 \\
3.51739 \\
3.52184\end{array}$ & $\begin{array}{l}240 \\
302 \\
372 \\
445 \\
523\end{array}$ & $\begin{array}{l}3.48789 \\
3.48892 \\
3.48997 \\
3.49108 \\
3.49226\end{array}$ & $\begin{array}{l}103 \\
105 \\
111 \\
118 \\
129\end{array}$ & $\begin{array}{l}19.81722 \\
19.98778 \\
20.15043 \\
20.30588 \\
20.45475\end{array}$ & $\begin{array}{l}17056 \\
16265 \\
15545 \\
14887 \\
14284\end{array}$ & $\begin{array}{l}23.30512 \\
23.47670 \\
23.64040 \\
23.79697 \\
23.94703\end{array}$ & $\begin{array}{l}17158 \\
16370 \\
15657 \\
15006 \\
14411\end{array}$ \\
\hline $\begin{array}{l}250 \\
260 \\
270 \\
280 \\
290\end{array}$ & $\begin{array}{l}3.52707 \\
3.53308 \\
3.53988 \\
3.54747 \\
3.55582\end{array}$ & $\begin{array}{l}601 \\
680 \\
759 \\
835 \\
908\end{array}$ & $\begin{array}{l}3.49355 \\
3.49495 \\
3.49649 \\
3.49818 \\
3.50002\end{array}$ & $\begin{array}{l}140 \\
154 \\
169 \\
184 \\
200\end{array}$ & $\begin{array}{l}20.59759 \\
20.73486 \\
20.86701 \\
20.99440 \\
21.11737\end{array}$ & $\begin{array}{l}13727 \\
13215 \\
12739 \\
12297 \\
11885\end{array}$ & $\begin{array}{l}24.09114 \\
24.22982 \\
24.36350 \\
24.49257 \\
24.61738\end{array}$ & $\begin{array}{l}13868 \\
133 i 58 \\
12907 \\
12481 \\
12088\end{array}$ \\
\hline $\begin{array}{l}300 \\
310 \\
320 \\
330 \\
340\end{array}$ & $\begin{array}{l}3.56490 \\
3.57467 \\
3.58508 \\
3.59608 \\
3.60763\end{array}$ & $\begin{array}{r}977 \\
1041 \\
1100 \\
1155 \\
1204\end{array}$ & $\begin{array}{l}3.50202 \\
3.50420 \\
3.50657 \\
3.50912 \\
3.51184\end{array}$ & $\begin{array}{l}218 \\
237 \\
255 \\
272 \\
291\end{array}$ & $\begin{array}{l}21.23622 \\
21.35125 \\
21.46269 \\
21.57077 \\
21.67570\end{array}$ & $\begin{array}{l}11503 \\
11144 \\
10808 \\
10493 \\
10197\end{array}$ & $\begin{array}{l}24.73826 \\
24.85546 \\
24.96926 \\
25.07989 \\
25.18755\end{array}$ & $\begin{array}{l}11720 \\
11380 \\
11063 \\
10766 \\
10487\end{array}$ \\
\hline $\begin{array}{l}350 \\
360 \\
370 \\
380 \\
390\end{array}$ & $\begin{array}{l}3.61967 \\
3.63213 \\
3.64497 \\
3.65814 \\
3.67157\end{array}$ & $\begin{array}{l}1245 \\
1284 \\
1317 \\
1343 \\
1366\end{array}$ & $\begin{array}{l}3.51475 \\
3.51784 \\
3.52110 \\
3.52453 \\
3.52812\end{array}$ & $\begin{array}{l}309 \\
326 \\
343 \\
359 \\
376\end{array}$ & $\begin{array}{l}21.77767 \\
21.87684 \\
21.97338 \\
22.06743 \\
22.15913\end{array}$ & $\begin{array}{l}9917 \\
9654 \\
9405 \\
9170 \\
8947\end{array}$ & $\begin{array}{l}25.29242 \\
25.39468 \\
25.49448 \\
25.59197 \\
25.68727\end{array}$ & $\begin{array}{r}10226 \\
9980 \\
9749 \\
9530 \\
9322\end{array}$ \\
\hline $\begin{array}{l}400 \\
450 \\
500 \\
550 \\
600\end{array}$ & $\begin{array}{l}3.68523 \\
3.75528 \\
3.82511 \\
3.89177 \\
3.95370\end{array}$ & $\begin{array}{l}7005 \\
6983 \\
6666 \\
6193 \\
5660\end{array}$ & $\begin{array}{l}3.53188 \\
3.55280 \\
3.57655 \\
3.60221 \\
3.62896\end{array}$ & $\begin{array}{l}2092 \\
2375 \\
2556 \\
2675 \\
2719\end{array}$ & $\begin{array}{l}22.24860 \\
22.66619 \\
23.04205 \\
23.38439 \\
23.69919\end{array}$ & $\begin{array}{l}41759 \\
37586 \\
34234 \\
31480 \\
29174\end{array}$ & $\begin{array}{l}25.78049 \\
26.21899 \\
26.61861 \\
26.98661 \\
27.32815\end{array}$ & $\begin{array}{l}43850 \\
39962 \\
36800 \\
34154 \\
31893\end{array}$ \\
\hline $\begin{array}{l}650 \\
700 \\
750 \\
800 \\
850\end{array}$ & $\begin{array}{l}4.01030 \\
4.06150 \\
4.10758 \\
4.14893 \\
4.18604\end{array}$ & $\begin{array}{l}5120 \\
4608 \\
4135 \\
3711 \\
3332\end{array}$ & $\begin{array}{l}3.65615 \\
3.68331 \\
3.71008 \\
3.73624 \\
3.76163\end{array}$ & $\begin{array}{l}2716 \\
2677 \\
2616 \\
2539 \\
2452\end{array}$ & $\begin{array}{l}23.99093 \\
24.26304 \\
24.51822 \\
24.75862 \\
24.98601\end{array}$ & $\begin{array}{l}27211 \\
25518 \\
24040 \\
22739 \\
21580\end{array}$ & $\begin{array}{l}27.64708 \\
27.94635 \\
28.22831 \\
28.49487 \\
28.74764\end{array}$ & $\begin{array}{l}29927 \\
28196 \\
26556 \\
25277 \\
24033\end{array}$ \\
\hline $\begin{array}{r}900 \\
950 \\
1000 \\
7050 \\
1100\end{array}$ & $\begin{array}{l}4.21936 \\
4.24935 \\
4.27639 \\
4.30086 \\
4.32307\end{array}$ & $\begin{array}{l}2999 \\
2704 \\
2447 \\
2221 \\
2022\end{array}$ & $\begin{array}{l}3.78615 \\
3.80976 \\
3.83242 \\
3.85415 \\
3.87497\end{array}$ & $\begin{array}{l}2361 \\
2266 \\
2173 \\
2082 \\
1993\end{array}$ & $\begin{array}{l}25.20181 \\
25.40724 \\
25.60332 \\
25.79090 \\
25.97074\end{array}$ & $\begin{array}{l}20543 \\
19608 \\
18758 \\
17984 \\
17275\end{array}$ & $\begin{array}{l}28.98797 \\
29.21700 \\
29.43574 \\
29.64506 \\
29.84572\end{array}$ & $\begin{array}{l}22903 \\
21874 \\
20932 \\
20066 \\
19268\end{array}$ \\
\hline 1150 & 4.34329 & 1848 & 3.89490 & 1907 & 26.14349 & 16623 & 30.03840 & 18530 \\
\hline
\end{tabular}


Table 2.090. PT (Cont.)

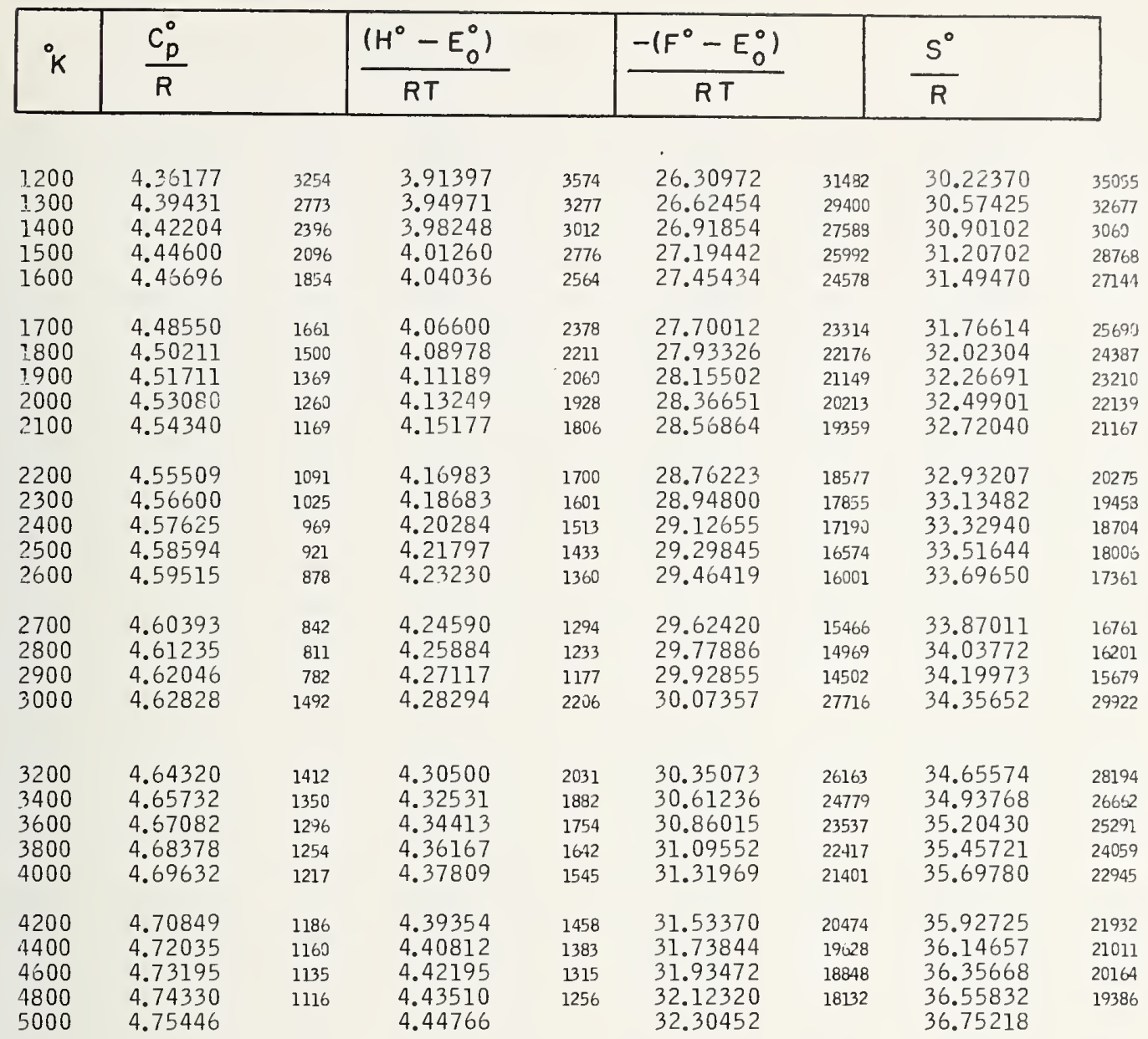


Table 2.091 $\mathrm{BiH}$

\begin{tabular}{|c|c|c|c|c|c|c|c|c|}
\hline${ }^{\circ} K$ & $\frac{C_{p}^{0}}{R}$ & & $\frac{\left(H^{\circ}-\Sigma_{0}^{0}\right)}{R T}$ & & $\frac{-\left(F^{0}-E_{0}^{0}\right.}{R T}$ & & $\frac{S^{0}}{R}$ & \\
\hline $\begin{array}{l}50 \\
60 \\
70 \\
80 \\
90\end{array}$ & $\begin{array}{l}3.50153 \\
3.50157 \\
3.50168 \\
3.50182 \\
3.50198\end{array}$ & $\begin{array}{r}4 \\
11 \\
14 \\
16 \\
18\end{array}$ & $\begin{array}{l}3.45146 \\
3.45980 \\
3.46578 \\
3.47027 \\
3.47379\end{array}$ & $\begin{array}{l}834 \\
598 \\
449 \\
352 \\
283\end{array}$ & $\begin{array}{l}16.11208 \\
16.74214 \\
17.27594 \\
17.73904 \\
18.14799\end{array}$ & $\begin{array}{l}63006 \\
53380 \\
46310 \\
40895 \\
36616\end{array}$ & $\begin{array}{l}19.56354 \\
20.20194 \\
20.74172 \\
21.20931 \\
21.62178\end{array}$ & $\begin{array}{l}63840 \\
53978 \\
46759 \\
41247 \\
36899\end{array}$ \\
\hline $\begin{array}{l}100 \\
110 \\
120 \\
130 \\
140\end{array}$ & $\begin{array}{l}3.50216 \\
3.50234 \\
3.50253 \\
3.50272 \\
3.50292\end{array}$ & $\begin{array}{l}18 \\
19 \\
19 \\
20 \\
22\end{array}$ & $\begin{array}{l}3.47662 \\
3.47895 \\
3.48090 \\
3.48257 \\
3.48402\end{array}$ & $\begin{array}{l}233 \\
195 \\
167 \\
145 \\
127\end{array}$ & $\begin{array}{l}18.51415 \\
18.84562 \\
19.14841 \\
19.42710 \\
19.68524\end{array}$ & $\begin{array}{l}33147 \\
30279 \\
27869 \\
25814 \\
24042\end{array}$ & $\begin{array}{l}21.99077 \\
22.32457 \\
22.62931 \\
22.90967 \\
23.16926\end{array}$ & $\begin{array}{l}33380 \\
30474 \\
28036 \\
25959 \\
24169\end{array}$ \\
\hline $\begin{array}{l}150 \\
160 \\
170 \\
180 \\
190\end{array}$ & $\begin{array}{l}3.50314 \\
3.50339 \\
3.50369 \\
3.50406 \\
3.50455\end{array}$ & $\begin{array}{l}25 \\
30 \\
37 \\
49 \\
65\end{array}$ & $\begin{array}{l}3.48529 \\
3.48641 \\
3.48742 \\
3.48833 \\
3.48917\end{array}$ & $\begin{array}{r}112 \\
101 \\
91 \\
84 \\
79\end{array}$ & $\begin{array}{l}19.92566 \\
20.15063 \\
20.36202 \\
20.56138 \\
20.75001\end{array}$ & $\begin{array}{l}22497 \\
21139 \\
19936 \\
18863 \\
17899\end{array}$ & $\begin{array}{l}23.41095 \\
23.63704 \\
23.84944 \\
24.04971 \\
24.23918\end{array}$ & $\begin{array}{l}22609 \\
21240 \\
20027 \\
18947 \\
17978\end{array}$ \\
\hline $\begin{array}{l}200 \\
210 \\
220 \\
230 \\
240\end{array}$ & $\begin{array}{l}3.50520 \\
3.50605 \\
3.50716 \\
3.50857 \\
3.51036\end{array}$ & $\begin{array}{r}85 \\
111 \\
141 \\
179 \\
219\end{array}$ & $\begin{array}{l}3.48996 \\
3.49070 \\
3.49142 \\
3.49214 \\
3.49286\end{array}$ & $\begin{array}{l}74 \\
72 \\
72 \\
72 \\
74\end{array}$ & $\begin{array}{l}20.92900 \\
21.09930 \\
21.26170 \\
21.41692 \\
21.56556\end{array}$ & $\begin{array}{l}17030 \\
16240 \\
15522 \\
14864 \\
14260\end{array}$ & $\begin{array}{l}24.41896 \\
24.59000 \\
24.75312 \\
24.90906 \\
25.05842\end{array}$ & $\begin{array}{l}17104 \\
16312 \\
15594 \\
14936 \\
14334\end{array}$ \\
\hline $\begin{array}{l}250 \\
260 \\
270 \\
280 \\
290\end{array}$ & $\begin{array}{l}3.51255 \\
3.51520 \\
3.51834 \\
3.52200 \\
3.52621\end{array}$ & $\begin{array}{l}265 \\
314 \\
366 \\
421 \\
477\end{array}$ & $\begin{array}{l}3.49360 \\
3.49438 \\
3.49521 \\
3.49610 \\
3.49706\end{array}$ & $\begin{array}{r}78 \\
83 \\
89 \\
96 \\
105\end{array}$ & $\begin{array}{l}21.70816 \\
21.84519 \\
21.97709 \\
22.10422 \\
22.22692\end{array}$ & $\begin{array}{l}13703 \\
13190 \\
12713 \\
12270 \\
11857\end{array}$ & $\begin{array}{l}25.20176 \\
25.33957 \\
25.47230 \\
25.60032 \\
25.72398\end{array}$ & $\begin{array}{l}13781 \\
13273 \\
12802 \\
12366 \\
11962\end{array}$ \\
\hline $\begin{array}{l}300 \\
310 \\
320 \\
330 \\
340\end{array}$ & $\begin{array}{l}3.53098 \\
3.53632 \\
3.54223 \\
3.54870 \\
3.55572\end{array}$ & $\begin{array}{l}534 \\
591 \\
647 \\
702 \\
756\end{array}$ & $\begin{array}{l}3.49811 \\
3.49926 \\
3.50050 \\
3.50187 \\
3.50334\end{array}$ & $\begin{array}{l}115 \\
124 \\
137 \\
147 \\
161\end{array}$ & $\begin{array}{l}22.34549 \\
22.46021 \\
22.57133 \\
22.67906 \\
22.78363\end{array}$ & $\begin{array}{l}11472 \\
11112 \\
10773 \\
10457 \\
10157\end{array}$ & $\begin{array}{l}25.84360 \\
25.95947 \\
26.07183 \\
26.18093 \\
26.28697\end{array}$ & $\begin{array}{l}11587 \\
11236 \\
10910 \\
10604 \\
10318\end{array}$ \\
\hline $\begin{array}{l}350 \\
360 \\
370 \\
380 \\
390\end{array}$ & $\begin{array}{l}3.56328 \\
3.57135 \\
3.57990 \\
3.58891 \\
3.59835\end{array}$ & $\begin{array}{l}807 \\
855 \\
901 \\
944 \\
986\end{array}$ & $\begin{array}{l}3.50495 \\
3.50668 \\
3.50854 \\
3.51054 \\
3.51267\end{array}$ & $\begin{array}{l}173 \\
186 \\
200 \\
213 \\
226\end{array}$ & $\begin{array}{l}22.88520 \\
22.98396 \\
23.08007 \\
23.17366 \\
23.26488\end{array}$ & $\begin{array}{l}9876 \\
9611 \\
9359 \\
9122 \\
8896\end{array}$ & $\begin{array}{l}26.39015 \\
26.49064 \\
26.58861 \\
26.68420 \\
26.77755\end{array}$ & $\begin{array}{r}10049 \\
9797 \\
9559 \\
9335 \\
9122\end{array}$ \\
\hline $\begin{array}{l}400 \\
450 \\
500 \\
550 \\
600\end{array}$ & $\begin{array}{l}3.60821 \\
3.66223 \\
3.72146 \\
3.78275 \\
3.84394\end{array}$ & $\begin{array}{l}5402 \\
5923 \\
6129 \\
6119 \\
5993\end{array}$ & $\begin{array}{l}3.51493 \\
3.52824 \\
3.54456 \\
3.56343 \\
3.58426\end{array}$ & $\begin{array}{l}1331 \\
1632 \\
1887 \\
2083 \\
2228\end{array}$ & $\begin{array}{l}23.35384 \\
23.76857 \\
24.14113 \\
24.47984 \\
24.79077\end{array}$ & $\begin{array}{l}41473 \\
37256 \\
33871 \\
31093 \\
28777\end{array}$ & $\begin{array}{l}26.86877 \\
27.29681 \\
27.68569 \\
28.04327 \\
28.37503\end{array}$ & $\begin{array}{l}42804 \\
38888 \\
35758 \\
33176 \\
31005\end{array}$ \\
\hline $\begin{array}{l}650 \\
700 \\
750 \\
800 \\
850\end{array}$ & $\begin{array}{l}3.90387 \\
3.96208 \\
4.01860 \\
4.07371 \\
4.12780\end{array}$ & $\begin{array}{l}5821 \\
5652 \\
5511 \\
5409 \\
5347\end{array}$ & $\begin{array}{l}3.60654 \\
3.62988 \\
3.65392 \\
3.67843 \\
3.70328\end{array}$ & $\begin{array}{l}2334 \\
2404 \\
2451 \\
2485 \\
2507\end{array}$ & $\begin{array}{l}25.07854 \\
25.34666 \\
25.59791 \\
25.83452 \\
26.05826\end{array}$ & $\begin{array}{l}26812 \\
25125 \\
23661 \\
22374 \\
21238\end{array}$ & $\begin{array}{l}28.68508 \\
28.97654 \\
29.25183 \\
29.51295 \\
29.76154\end{array}$ & $\begin{array}{l}29146 \\
27529 \\
26112 \\
24859 \\
23745\end{array}$ \\
\hline $\begin{array}{r}900 \\
950 \\
1000 \\
1050 \\
1100\end{array}$ & $\begin{array}{l}4.18127 \\
4.23443 \\
4.28751 \\
4.34064 \\
4.39384\end{array}$ & $\begin{array}{l}5316 \\
5308 \\
5313 \\
5320 \\
5323\end{array}$ & $\begin{array}{l}3.72835 \\
3.75359 \\
3.77896 \\
3.80444 \\
3.83003\end{array}$ & $\begin{array}{l}2524 \\
2537 \\
2548 \\
2559 \\
2566\end{array}$ & $\begin{array}{l}26.27064 \\
26.47290 \\
26.66608 \\
26.85107 \\
27.02864\end{array}$ & $\begin{array}{l}20226 \\
19318 \\
18499 \\
17757 \\
17082\end{array}$ & $\begin{array}{l}29.99899 \\
30.22649 \\
30.44504 \\
30.65551 \\
30.85867\end{array}$ & $\begin{array}{l}22750 \\
21855 \\
21047 \\
20316 \\
19648\end{array}$ \\
\hline 1150 & 4.44707 & 5313 & 3.35569 & 2576 & 27.19946 & 16464 & 31.05515 & 19040 \\
\hline
\end{tabular}


Table 2.091. BiH (Cont.)

\begin{tabular}{|c|c|c|c|c|c|c|c|c|}
\hline${ }^{\circ} \mathrm{K}$ & $\frac{C_{p}^{0}}{R}$ & & $\frac{\left(H^{0}-E_{0}^{0}\right)}{R T}$ & & $\frac{-l F^{\circ}-E}{R T}$ & & $\frac{S^{0}}{R}$ & \\
\hline $\begin{array}{l}1200 \\
1300 \\
1400 \\
1500 \\
1600\end{array}$ & $\begin{array}{l}4.50020 \\
4.60548 \\
4.70802 \\
4.80609 \\
4.89813\end{array}$ & $\begin{array}{r}10528 \\
10254 \\
9807 \\
9204 \\
8479\end{array}$ & $\begin{array}{l}3.88145 \\
3.93310 \\
3.98481 \\
4.03632 \\
4.08735\end{array}$ & $\begin{array}{l}5165 \\
5171 \\
5151 \\
5103 \\
5022\end{array}$ & $\begin{array}{l}27.36410 \\
27.67683 \\
27.97019 \\
28.24687 \\
28.50901\end{array}$ & $\begin{array}{l}31273 \\
29336 \\
27668 \\
26214 \\
24930\end{array}$ & $\begin{array}{l}31.24555 \\
31.60993 \\
31.95500 \\
32.28319 \\
32.59636\end{array}$ & $\begin{array}{l}36438 \\
34507 \\
32819 \\
31317 \\
29952\end{array}$ \\
\hline $\begin{array}{l}1700 \\
1800 \\
1900 \\
2000 \\
2100\end{array}$ & $\begin{array}{l}4.98292 \\
5.05964 \\
5.12787 \\
5.18753 \\
5.23880\end{array}$ & $\begin{array}{l}7672 \\
6823 \\
5966 \\
5127 \\
4328\end{array}$ & $\begin{array}{l}4.13757 \\
4.18671 \\
4.23448 \\
4.28068 \\
4.32511\end{array}$ & $\begin{array}{l}4914 \\
4777 \\
4620 \\
4443 \\
4255\end{array}$ & $\begin{array}{l}28.75831 \\
28.99621 \\
29.22385 \\
29.44224 \\
29.65217\end{array}$ & $\begin{array}{l}23790 \\
22764 \\
21839 \\
20993 \\
20220\end{array}$ & $\begin{array}{l}32.89588 \\
33.18292 \\
33.45833 \\
33.72292 \\
33.97728\end{array}$ & $\begin{array}{l}2870 \mathrm{c} \\
27541 \\
26459 \\
25436 \\
24475\end{array}$ \\
\hline $\begin{array}{l}2200 \\
2300 \\
2400 \\
2500 \\
2600\end{array}$ & $\begin{array}{l}5.28208 \\
5.31791 \\
5.34695 \\
5.36987 \\
5.38736\end{array}$ & $\begin{array}{l}3583 \\
2904 \\
2292 \\
1749 \\
1274\end{array}$ & $\begin{array}{l}4.36766 \\
4.40822 \\
4.44675 \\
4.48324 \\
4.51769\end{array}$ & $\begin{array}{l}4056 \\
3853 \\
3649 \\
3445 \\
3247\end{array}$ & $\begin{array}{l}29.85437 \\
30.04942 \\
30.23786 \\
30.42013 \\
30.59664\end{array}$ & $\begin{array}{l}19505 \\
18844 \\
18227 \\
17651 \\
17112\end{array}$ & $\begin{array}{l}34.22203 \\
34.45764 \\
34.68461 \\
34.90337 \\
35.11433\end{array}$ & $\begin{array}{l}23561 \\
22697 \\
21876 \\
21096 \\
20359\end{array}$ \\
\hline $\begin{array}{l}2700 \\
2800 \\
2900 \\
3000\end{array}$ & $\begin{array}{l}5.40010 \\
5.40873 \\
5.41385 \\
5.41600\end{array}$ & $\begin{array}{r}863 \\
512 \\
215 \\
-\quad 269\end{array}$ & $\begin{array}{l}4.55016 \\
4.58067 \\
4.60932 \\
4.63619\end{array}$ & $\begin{array}{l}3051 \\
2865 \\
2687 \\
4869\end{array}$ & $\begin{array}{l}30.76776 \\
30.93379 \\
31.09504 \\
31.25176\end{array}$ & $\begin{array}{l}16603 \\
16125 \\
15672 \\
30080\end{array}$ & $\begin{array}{l}35.31792 \\
35.51446 \\
35.70436 \\
35.88795\end{array}$ & $\begin{array}{l}19654 \\
18990 \\
18359 \\
34949\end{array}$ \\
\hline $\begin{array}{l}3200 \\
3400 \\
3600 \\
3800 \\
4000\end{array}$ & $\begin{array}{l}5.41331 \\
5.40395 \\
5.39043 \\
5.37459 \\
5.35777\end{array}$ & $\begin{array}{l}-\quad 936 \\
-1352 \\
-1584 \\
-1682 \\
-1688\end{array}$ & $\begin{array}{l}4.68488 \\
4.72748 \\
4.76470 \\
4.79722 \\
4.82567\end{array}$ & $\begin{array}{l}4260 \\
3722 \\
3252 \\
2845 \\
2494\end{array}$ & $\begin{array}{l}31.55256 \\
31.83788 \\
32.10918 \\
32.36769 \\
32.61449\end{array}$ & $\begin{array}{l}28532 \\
27130 \\
25851 \\
24680 \\
23607\end{array}$ & $\begin{array}{l}36.23744 \\
36.56536 \\
36.87388 \\
37.16491 \\
37.44016\end{array}$ & $\begin{array}{l}32792 \\
30852 \\
29103 \\
27525 \\
26101\end{array}$ \\
\hline $\begin{array}{l}4200 \\
4400 \\
4600 \\
4800 \\
5000\end{array}$ & $\begin{array}{l}5.34089 \\
5.32459 \\
5.30926 \\
5.29516 \\
5.28242\end{array}$ & $\begin{array}{l}-1630 \\
-1533 \\
-1410 \\
-1274\end{array}$ & $\begin{array}{l}4.85061 \\
4.87253 \\
4.89183 \\
4.90893 \\
4.92413\end{array}$ & $\begin{array}{l}2192 \\
1930 \\
1710 \\
1520\end{array}$ & $\begin{array}{l}32.85056 \\
33.07672 \\
33.29375 \\
33.5023 .1 \\
33.70302\end{array}$ & $\begin{array}{l}22616 \\
21703 \\
20856 \\
20071\end{array}$ & $\begin{array}{l}37.70117 \\
37.94925 \\
38.18558 \\
38.41124 \\
38.62715\end{array}$ & $\begin{array}{l}24808 \\
23633 \\
22566 \\
21591\end{array}$ \\
\hline
\end{tabular}


Table 2.092. BiD

\begin{tabular}{|c|c|c|c|c|c|c|c|c|}
\hline${ }^{\circ} \mathrm{K}$ & $\frac{C_{p}^{0}}{R}$ & & $\frac{\left(H^{0}-E_{0}^{0}\right)}{R T}$ & & $\frac{-\angle F^{\circ}-E}{R T}$ & & $\frac{S^{0}}{R}$ & \\
\hline $\begin{array}{l}50 \\
60 \\
70 \\
80 \\
90\end{array}$ & $\begin{array}{l}3.50114 \\
3.50131 \\
3.50149 \\
3.50167 \\
3.50187\end{array}$ & $\begin{array}{l}17 \\
18 \\
18 \\
20 \\
21\end{array}$ & $\begin{array}{l}3.47578 \\
3.48002 \\
3.48308 \\
3.48539 \\
3.48721\end{array}$ & $\begin{array}{l}424 \\
306 \\
231 \\
182 \\
148\end{array}$ & $\begin{array}{l}16.77504 \\
17.40915 \\
17.94584 \\
18.41110 \\
18.82173\end{array}$ & $\begin{array}{l}63411 \\
53669 \\
46526 \\
41063 \\
36749\end{array}$ & $\begin{array}{l}20.25082 \\
20.88917 \\
21.42892 \\
21.89649 \\
22.30894\end{array}$ & $\begin{array}{l}63835 \\
53975 \\
46757 \\
41245 \\
36397\end{array}$ \\
\hline $\begin{array}{l}100 \\
110 \\
120 \\
130 \\
140\end{array}$ & $\begin{array}{l}3.50208 \\
3.50231 \\
3.50262 \\
3.50306 \\
3.50372\end{array}$ & $\begin{array}{r}23 \\
31 \\
44 \\
66 \\
102\end{array}$ & $\begin{array}{l}3.48869 \\
3.48991 \\
3.49096 \\
3.49187 \\
3.49269\end{array}$ & $\begin{array}{r}122 \\
105 \\
91 \\
82 \\
77\end{array}$ & $\begin{array}{l}19.18922 \\
19.52179 \\
19.82550 \\
20.10496 \\
20.36377\end{array}$ & $\begin{array}{l}33257 \\
30371 \\
27946 \\
25881 \\
24099\end{array}$ & $\begin{array}{l}22.67791 \\
23.01170 \\
23.31646 \\
23.59683 \\
23.85646\end{array}$ & $\begin{array}{l}33379 \\
30476 \\
28037 \\
25963 \\
24176\end{array}$ \\
\hline $\begin{array}{l}150 \\
160 \\
170 \\
180 \\
190\end{array}$ & $\begin{array}{l}3.50474 \\
3.50624 \\
3.50838 \\
3.51127 \\
3.51505\end{array}$ & $\begin{array}{l}150 \\
214 \\
289 \\
378 \\
476\end{array}$ & $\begin{array}{l}3.49346 \\
3.49421 \\
3.49498 \\
3.49580 \\
3.49671\end{array}$ & $\begin{array}{r}75 \\
77 \\
82 \\
91 \\
103\end{array}$ & $\begin{array}{l}20.60476 \\
20.83025 \\
21.04211 \\
21.24190 \\
21.43093\end{array}$ & $\begin{array}{l}22549 \\
21186 \\
19979 \\
18903 \\
17939\end{array}$ & $\begin{array}{l}24.09822 \\
24.32446 \\
24.53709 \\
24.73770 \\
24.92764\end{array}$ & $\begin{array}{l}22624 \\
21263 \\
20061 \\
18994 \\
18042\end{array}$ \\
\hline $\begin{array}{l}200 \\
210 \\
220 \\
230 \\
240\end{array}$ & $\begin{array}{l}3.51981 \\
3.52561 \\
3.53250 \\
3.54049 \\
3.54956\end{array}$ & $\begin{array}{r}580 \\
689 \\
799 \\
907 \\
1012\end{array}$ & $\begin{array}{l}3.49774 \\
3.49892 \\
3.50029 \\
3.50186 \\
3.50365\end{array}$ & $\begin{array}{l}118 \\
137 \\
157 \\
179 \\
204\end{array}$ & $\begin{array}{l}21.61032 \\
21.78100 \\
21.94380 \\
22.09943 \\
22.24850\end{array}$ & $\begin{array}{l}17068 \\
16280 \\
15563 \\
14907 \\
14307\end{array}$ & $\begin{array}{l}25.10806 \\
25.27992 \\
25.44409 \\
25.60129 \\
25.75215\end{array}$ & $\begin{array}{l}17186 \\
16417 \\
15720 \\
15086 \\
14511\end{array}$ \\
\hline $\begin{array}{l}250 \\
260 \\
270 \\
280 \\
290\end{array}$ & $\begin{array}{l}3.55968 \\
3.57079 \\
3.58281 \\
3.59568 \\
3.60931\end{array}$ & $\begin{array}{l}1111 \\
1202 \\
1287 \\
1363 \\
1430\end{array}$ & $\begin{array}{l}3.50569 \\
3.50798 \\
3.51052 \\
3.51333 \\
3.51640\end{array}$ & $\begin{array}{l}229 \\
254 \\
281 \\
307 \\
334\end{array}$ & $\begin{array}{l}22.39157 \\
22.52911 \\
22.66155 \\
22.78927 \\
22.91261\end{array}$ & $\begin{array}{l}13754 \\
13244 \\
12772 \\
12334 \\
11927\end{array}$ & $\begin{array}{l}25.89726 \\
26.03709 \\
26.17207 \\
26.30260 \\
26.42901\end{array}$ & $\begin{array}{l}13983 \\
13498 \\
13053 \\
12641 \\
12261\end{array}$ \\
\hline $\begin{array}{l}300 \\
310 \\
320 \\
330 \\
340\end{array}$ & $\begin{array}{l}3.62361 \\
3.63848 \\
3.65384 \\
3.66961 \\
3.68570\end{array}$ & $\begin{array}{l}1487 \\
1536 \\
1577 \\
1609 \\
1633\end{array}$ & $\begin{array}{l}3.51974 \\
3.52333 \\
3.52716 \\
3.53124 \\
3.53555\end{array}$ & $\begin{array}{l}359 \\
383 \\
408 \\
431 \\
452\end{array}$ & $\begin{array}{l}23.03188 \\
23.14735 \\
23.25927 \\
23.36787 \\
23.47335\end{array}$ & $\begin{array}{l}11547 \\
11192 \\
10860 \\
10548 \\
10255\end{array}$ & $\begin{array}{l}26.55162 \\
26.67068 \\
26.78643 \\
26.89911 \\
27.00890\end{array}$ & $\begin{array}{l}11906 \\
11575 \\
11268 \\
10979 \\
10707\end{array}$ \\
\hline $\begin{array}{l}350 \\
360 \\
370 \\
380 \\
390\end{array}$ & $\begin{array}{l}3.70203 \\
3.71854 \\
3.73515 \\
3.75182 \\
3.76848\end{array}$ & $\begin{array}{l}1651 \\
1661 \\
1667 \\
1666 \\
1664\end{array}$ & $\begin{array}{l}3.54007 \\
3.54480 \\
3.54972 \\
3.55482 \\
3.56008\end{array}$ & $\begin{array}{l}473 \\
492 \\
510 \\
526 \\
542\end{array}$ & $\begin{array}{l}23.57590 \\
23.67569 \\
23.77288 \\
23.86761 \\
23.96002\end{array}$ & $\begin{array}{l}9979 \\
9719 \\
9473 \\
9241 \\
9020\end{array}$ & $\begin{array}{l}27.11597 \\
27.22049 \\
27.32260 \\
27.42243 \\
27.52010\end{array}$ & $\begin{array}{r}10452 \\
10211 \\
9983 \\
9767 \\
9562\end{array}$ \\
\hline $\begin{array}{l}400 \\
450 \\
500 \\
550 \\
600\end{array}$ & $\begin{array}{l}3.78512 \\
3.86616 \\
3.94163 \\
4.01029 \\
4.07221\end{array}$ & $\begin{array}{l}8104 \\
7547 \\
6866 \\
6192 \\
5599\end{array}$ & $\begin{array}{l}3.56550 \\
3.59445 \\
3.62544 \\
3.65736 \\
3.68940\end{array}$ & $\begin{array}{l}2895 \\
3099 \\
3192 \\
3204 \\
3163\end{array}$ & $\begin{array}{l}24.05022 \\
24.47182 \\
24.85213 \\
25.19917 \\
25.51876\end{array}$ & $\begin{array}{l}42160 \\
38031 \\
34704 \\
31959 \\
29657\end{array}$ & $\begin{array}{l}27.61572 \\
28.06627 \\
28.47757 \\
28.85653 \\
29.20816\end{array}$ & $\begin{array}{l}45055 \\
41130 \\
37896 \\
35163 \\
32820\end{array}$ \\
\hline $\begin{array}{l}650 \\
700 \\
750 \\
800 \\
850\end{array}$ & $\begin{array}{l}4.12820 \\
4.17942 \\
4.22706 \\
4.27227 \\
4.31603\end{array}$ & $\begin{array}{l}5122 \\
4764 \\
4521 \\
4376 \\
4310\end{array}$ & $\begin{array}{l}3.72103 \\
3.75197 \\
3.78207 \\
3.81130 \\
3.83971\end{array}$ & $\begin{array}{l}3094 \\
3010 \\
2923 \\
2841 \\
2766\end{array}$ & $\begin{array}{l}25.81533 \\
26.09223 \\
26.35212 \\
26.59715 \\
26.82906\end{array}$ & $\begin{array}{l}27670 \\
25989 \\
24503 \\
23191 \\
22026\end{array}$ & $\begin{array}{l}29.53636 \\
29.84420 \\
30.13419 \\
30.40845 \\
30.66877\end{array}$ & $\begin{array}{l}30784 \\
28999 \\
27426 \\
26032 \\
24792\end{array}$ \\
\hline $\begin{array}{r}900 \\
950 \\
1000 \\
1050 \\
1100\end{array}$ & $\begin{array}{l}4.35913 \\
4.40214 \\
4.44545 \\
4.48927 \\
4.53369\end{array}$ & $\begin{array}{l}4301 \\
4331 \\
4382 \\
4442 \\
4498\end{array}$ & $\begin{array}{l}3.86737 \\
3.89439 \\
3.92086 \\
3.94688 \\
3.97254\end{array}$ & $\begin{array}{l}2702 \\
2647 \\
2602 \\
2566 \\
2537\end{array}$ & $\begin{array}{l}27.04932 \\
27.25915 \\
27.45958 \\
27.65151 \\
27.83571\end{array}$ & $\begin{array}{l}20983 \\
20043 \\
19193 \\
18420 \\
17714\end{array}$ & $\begin{array}{l}30.91669 \\
31.15354 \\
31.38044 \\
31.59839 \\
31.80825\end{array}$ & $\begin{array}{l}23685 \\
22690 \\
21795 \\
20986 \\
20251\end{array}$ \\
\hline 1150 & 4.57867 & 4542 & 3.99791 & 2515 & 28.01285 & 17068 & 32.01076 & 19583 \\
\hline
\end{tabular}


Table 2. 092. BiD (Cont.)

\begin{tabular}{|c|c|c|c|c|c|c|c|c|}
\hline${ }^{\circ} K$ & $\frac{C_{p}^{0}}{R}$ & & $\frac{\left(H^{\circ}-E_{0}^{0}\right)}{R T}$ & & $\frac{-\angle F^{0}-}{R T}$ & & $\frac{S^{0}}{R}$ & \\
\hline $\begin{array}{l}1200 \\
1300 \\
1400 \\
1500 \\
1600\end{array}$ & $\begin{array}{l}4.62409 \\
4.71546 \\
4.80595 \\
4.89359 \\
4.97659\end{array}$ & $\begin{array}{l}9137 \\
9049 \\
8764 \\
8300 \\
7693\end{array}$ & $\begin{array}{l}4.02306 \\
4.07281 \\
4.12195 \\
4.17049 \\
4.21831\end{array}$ & $\begin{array}{l}4975 \\
4914 \\
4854 \\
4782 \\
4690\end{array}$ & $\begin{array}{l}28.18353 \\
28.50753 \\
28.81115 \\
29.09720 \\
29.36789\end{array}$ & $\begin{array}{l}32400 \\
30362 \\
28605 \\
27069 \\
25714\end{array}$ & $\begin{array}{l}32.20659 \\
32.58034 \\
32.93310 \\
33.26769 \\
33.58620\end{array}$ & $\begin{array}{l}37375 \\
35276 \\
33459 \\
31851 \\
30404\end{array}$ \\
\hline $\begin{array}{l}1700 \\
1800 \\
1900 \\
2000 \\
2100\end{array}$ & $\begin{array}{l}5.05352 \\
5.12339 \\
5.18561 \\
5.23999 \\
5.28660\end{array}$ & $\begin{array}{l}6987 \\
6222 \\
5438 \\
4661 \\
3914\end{array}$ & $\begin{array}{l}4.26521 \\
4.31098 \\
4.35541 \\
4.39831 \\
4.43953\end{array}$ & $\begin{array}{l}4577 \\
4443 \\
4290 \\
4122 \\
3942\end{array}$ & $\begin{array}{l}29.62503 \\
29.87013 \\
30.10441 \\
30.32891 \\
30.54450\end{array}$ & $\begin{array}{l}24510 \\
23428 \\
22450 \\
21559 \\
20746\end{array}$ & $\begin{array}{l}33.89024 \\
34.18111 \\
34.45982 \\
34.72722 \\
34.98403\end{array}$ & $\begin{array}{l}29087 \\
27871 \\
26740 \\
25681 \\
24688\end{array}$ \\
\hline $\begin{array}{l}2200 \\
2300 \\
2400 \\
2500 \\
2600\end{array}$ & $\begin{array}{l}5.32574 \\
5.35788 \\
5.38362 \\
5.40358 \\
5.41841\end{array}$ & $\begin{array}{l}3214 \\
2574 \\
1996 \\
1483 \\
1033\end{array}$ & $\begin{array}{l}4.47895 \\
4.51649 \\
4.55211 \\
4.58578 \\
4.61754\end{array}$ & $\begin{array}{l}3754 \\
3562 \\
3367 \\
3176 \\
2987\end{array}$ & $\begin{array}{l}30.75196 \\
30.95188 \\
31.14487 \\
31.33138 \\
31.51186\end{array}$ & $\begin{array}{l}19992 \\
19299 \\
18651 \\
18048 \\
17484\end{array}$ & $\begin{array}{l}35.23091 \\
35.46837 \\
35.69698 \\
35.91716 \\
36.12940\end{array}$ & $\begin{array}{l}23746 \\
22861 \\
22018 \\
21224 \\
20471\end{array}$ \\
\hline $\begin{array}{l}2700 \\
2800 \\
2900 \\
3000\end{array}$ & $\begin{array}{l}5.42874 \\
5.43518 \\
5.43831 \\
5.43865\end{array}$ & $\begin{array}{r}644 \\
313 \\
-\quad 34 \\
-\quad 588\end{array}$ & $\begin{array}{l}4.64741 \\
4.67544 \\
4.70169 \\
4.72626\end{array}$ & $\begin{array}{l}2803 \\
2625 \\
2457 \\
4438\end{array}$ & $\begin{array}{l}31.68670 \\
31.85622 \\
32.02075 \\
32.18056\end{array}$ & $\begin{array}{l}16952 \\
16453 \\
15981 \\
30648\end{array}$ & $\begin{array}{l}36.33411 \\
36.53166 \\
36.72244 \\
36.90682\end{array}$ & $\begin{array}{l}19755 \\
19078 \\
18438 \\
35086\end{array}$ \\
\hline $\begin{array}{l}3200 \\
3400 \\
3600 \\
3800 \\
4000\end{array}$ & $\begin{array}{l}5.43277 \\
5.42071 \\
5.40488 \\
5.38704 \\
5.36848\end{array}$ & $\begin{array}{l}-1206 \\
-1583 \\
-1784 \\
-1856 \\
-1840\end{array}$ & $\begin{array}{l}4.77064 \\
4.80926 \\
4.84281 \\
4.87192 \\
4.89721\end{array}$ & $\begin{array}{l}3862 \\
3355 \\
2911 \\
2529 \\
2201\end{array}$ & $\begin{array}{l}32.48704 \\
32.77744 \\
33.05331 \\
33.31594 \\
33.56650\end{array}$ & $\begin{array}{l}29040 \\
27587 \\
26263 \\
25056 \\
23948\end{array}$ & $\begin{array}{l}37.25768 \\
37.58670 \\
37.89612 \\
38.18786 \\
38.46371\end{array}$ & $\begin{array}{l}32902 \\
30942 \\
29174 \\
27585 \\
26149\end{array}$ \\
\hline $\begin{array}{l}4200 \\
4400 \\
4600 \\
4800 \\
5000\end{array}$ & $\begin{array}{l}5.35008 \\
5.33243 \\
5.31590 \\
5.30073 \\
5.28703\end{array}$ & $\begin{array}{l}-1765 \\
-1653 \\
-1517 \\
-1370\end{array}$ & $\begin{array}{l}4.91922 \\
4.93840 \\
4.95516 \\
4.96987 \\
4.98283\end{array}$ & $\begin{array}{l}1918 \\
1676 \\
1471 \\
1296\end{array}$ & $\begin{array}{l}33.80598 \\
34.03527 \\
34.25518 \\
34.46638 \\
34.66953\end{array}$ & $\begin{array}{l}22929 \\
21991 \\
21120 \\
20315\end{array}$ & $\begin{array}{l}38.72520 \\
38.97367 \\
39.21034 \\
39.43625 \\
39.65236\end{array}$ & $\begin{array}{l}24847 \\
23667 \\
22591 \\
21611\end{array}$ \\
\hline
\end{tabular}


Table 2.093. BiT

\begin{tabular}{|c|c|c|c|c|c|c|c|c|}
\hline${ }^{\circ} K$ & $\frac{C_{p}^{0}}{R}$ & & $\frac{\left(H^{\circ}-E_{0}^{0}\right)}{R T}$ & & $\frac{-l F^{\circ}-}{R T}$ & & $\frac{S^{0}}{R}$ & \\
\hline $\begin{array}{l}50 \\
60 \\
70 \\
80 \\
90\end{array}$ & $\begin{array}{l}3.50107 \\
3.50126 \\
3.50145 \\
3.50166 \\
3.50189\end{array}$ & $\begin{array}{l}19 \\
19 \\
21 \\
23 \\
34\end{array}$ & $\begin{array}{l}3.48396 \\
3.48683 \\
3.48891 \\
3.49049 \\
3.49174\end{array}$ & $\begin{array}{l}287 \\
208 \\
158 \\
125 \\
103\end{array}$ & $\begin{array}{l}17.17420 \\
17.80967 \\
18.34733 \\
18.81332 \\
19.22451\end{array}$ & $\begin{array}{l}635.47 \\
53766 \\
46599 \\
41119 \\
36795\end{array}$ & $\begin{array}{l}20.65816 \\
21.29650 \\
21.83624 \\
22.30381 \\
22.71625\end{array}$ & $\begin{array}{l}63834 \\
53974 \\
46757 \\
41244 \\
36898\end{array}$ \\
\hline $\begin{array}{l}100 \\
110 \\
120 \\
130 \\
140\end{array}$ & $\begin{array}{l}3.50223 \\
3.50278 \\
3.50373 \\
3.50531 \\
3.50778\end{array}$ & $\begin{array}{r}55 \\
95 \\
158 \\
247 \\
359\end{array}$ & $\begin{array}{l}3.49277 \\
3.49365 \\
3.49445 \\
3.49522 \\
3.49602\end{array}$ & $\begin{array}{l}88 \\
80 \\
77 \\
80 \\
90\end{array}$ & $\begin{array}{l}19.59246 \\
19.92540 \\
20.22942 \\
20.50916 \\
20.76821\end{array}$ & $\begin{array}{l}33294 \\
30402 \\
27974 \\
25705 \\
24123\end{array}$ & $\begin{array}{l}23.08523 \\
23.41905 \\
23.72387 \\
24.00438 \\
24.26423\end{array}$ & $\begin{array}{l}33382 \\
30482 \\
28051 \\
25985 \\
24213\end{array}$ \\
\hline $\begin{array}{l}150 \\
160 \\
170 \\
180 \\
190\end{array}$ & $\begin{array}{l}3.51137 \\
3.51629 \\
3.52270 \\
3.53070 \\
3.54030\end{array}$ & $\begin{array}{r}492 \\
641 \\
800 \\
960 \\
1119\end{array}$ & $\begin{array}{l}3.49692 \\
3.49797 \\
3.49923 \\
3.50075 \\
3.50257\end{array}$ & $\begin{array}{l}105 \\
126 \\
152 \\
182 \\
216\end{array}$ & $\begin{array}{l}21.00944 \\
21.23516 \\
21.44726 \\
21.64731 \\
21.83663\end{array}$ & $\begin{array}{l}22572 \\
21210 \\
20305 \\
18932 \\
17972\end{array}$ & $\begin{array}{l}24.50636 \\
24.73313 \\
24.94649 \\
25.14806 \\
25.33920\end{array}$ & $\begin{array}{l}22677 \\
21336 \\
20157 \\
19114 \\
18188\end{array}$ \\
\hline $\begin{array}{l}200 \\
210 \\
220 \\
230 \\
240\end{array}$ & $\begin{array}{l}3.55149 \\
3.56420 \\
3.57831 \\
3.59369 \\
3.61018\end{array}$ & $\begin{array}{l}1271 \\
1411 \\
1538 \\
1649 \\
1745\end{array}$ & $\begin{array}{l}3.50473 \\
3.50725 \\
3.51016 \\
3.51345 \\
3.51713\end{array}$ & $\begin{array}{l}252 \\
291 \\
329 \\
368 \\
407\end{array}$ & $\begin{array}{l}22.01635 \\
22.18740 \\
22.35063 \\
22.50673 \\
22.65634\end{array}$ & $\begin{array}{l}17105 \\
16323 \\
15610 \\
14961 \\
14366\end{array}$ & $\begin{array}{l}25.52108 \\
25.69465 \\
25.86079 \\
26.02018 \\
26.17347\end{array}$ & $\begin{array}{l}17357 \\
16614 \\
15939 \\
15329 \\
14773\end{array}$ \\
\hline $\begin{array}{l}250 \\
260 \\
270 \\
280 \\
290\end{array}$ & $\begin{array}{l}3.62763 \\
3.64588 \\
3.66477 \\
3.68415 \\
3.70389\end{array}$ & $\begin{array}{l}1825 \\
1889 \\
1938 \\
1974 \\
1996\end{array}$ & $\begin{array}{l}3.52120 \\
3.52564 \\
3.53044 \\
3.53559 \\
3.54105\end{array}$ & $\begin{array}{l}444 \\
480 \\
515 \\
546 \\
576\end{array}$ & $\begin{array}{l}22.80000 \\
22.93818 \\
23.07133 \\
23.19982 \\
23.32398\end{array}$ & $\begin{array}{l}13818 \\
13315 \\
12849 \\
12416 \\
12015\end{array}$ & $\begin{array}{l}26.32120 \\
26.46382 \\
26.60177 \\
26.73541 \\
26.86503\end{array}$ & $\begin{array}{l}14262 \\
13795 \\
13364 \\
12962 \\
12591\end{array}$ \\
\hline $\begin{array}{l}300 \\
310 \\
320 \\
330 \\
340\end{array}$ & $\begin{array}{l}3.72385 \\
3.74393 \\
3.76402 \\
3.78404 \\
3.80391\end{array}$ & $\begin{array}{l}2008 \\
2009 \\
2002 \\
1987 \\
1966\end{array}$ & $\begin{array}{l}3.54681 \\
3.55284 \\
3.55913 \\
3.56564 \\
3.57236\end{array}$ & $\begin{array}{l}603 \\
629 \\
651 \\
672 \\
689\end{array}$ & $\begin{array}{l}23.44413 \\
23.56052 \\
23.67342 \\
23.78304 \\
23.88958\end{array}$ & $\begin{array}{l}11639 \\
11290 \\
10962 \\
10654 \\
10366\end{array}$ & $\begin{array}{l}26.99094 \\
27.11336 \\
27.23255 \\
27.34868 \\
27.46194\end{array}$ & $\begin{array}{l}12242 \\
11919 \\
11613 \\
11326 \\
11055\end{array}$ \\
\hline $\begin{array}{l}350 \\
360 \\
370 \\
380 \\
390\end{array}$ & $\begin{array}{l}3.82357 \\
3.84296 \\
3.86204 \\
3.88077 \\
3.89912\end{array}$ & $\begin{array}{l}1939 \\
1908 \\
1873 \\
1835 \\
1797\end{array}$ & $\begin{array}{l}3.57925 \\
3.58631 \\
3.59351 \\
3.60082 \\
3.60823\end{array}$ & $\begin{array}{l}706 \\
720 \\
731 \\
741 \\
750\end{array}$ & $\begin{array}{l}23.99324 \\
24.09417 \\
24.19252 \\
24.28845 \\
24.38208\end{array}$ & $\begin{array}{r}10093 \\
9835 \\
9593 \\
9363 \\
9145\end{array}$ & $\begin{array}{l}27.57249 \\
27.68048 \\
27.78603 \\
27.88927 \\
27.99031\end{array}$ & $\begin{array}{r}10799 \\
10555 \\
10324 \\
10104 \\
9895\end{array}$ \\
\hline $\begin{array}{l}400 \\
450 \\
500 \\
550 \\
600\end{array}$ & $\begin{array}{l}3.91709 \\
4.00044 \\
4.07298 \\
4.13568 \\
4.19011\end{array}$ & $\begin{array}{l}8335 \\
7254 \\
6270 \\
5443 \\
4794\end{array}$ & $\begin{array}{l}3.61573 \\
3.65395 \\
3.69231 \\
3.72984 \\
3.76598\end{array}$ & $\begin{array}{l}3822 \\
3836 \\
3753 \\
3614 \\
3450\end{array}$ & $\begin{array}{l}24.47353 \\
24.90160 \\
25.28857 \\
25.64226 \\
25.96835\end{array}$ & $\begin{array}{l}42807 \\
38697 \\
35369 \\
32609 \\
30282\end{array}$ & $\begin{array}{l}28.08926 \\
28.55555 \\
28.98088 \\
29.37210 \\
29.73433\end{array}$ & $\begin{array}{l}46629 \\
42533 \\
39122 \\
36223 \\
33732\end{array}$ \\
\hline $\begin{array}{l}650 \\
700 \\
750 \\
800 \\
850\end{array}$ & $\begin{array}{l}4.23805 \\
4.28121 \\
4.32109 \\
4.35901 \\
4.39603\end{array}$ & $\begin{array}{l}4316 \\
3988 \\
3792 \\
3702 \\
3691\end{array}$ & $\begin{array}{l}3.80048 \\
3.83331 \\
3.86451 \\
3.89423 \\
3.92267\end{array}$ & $\begin{array}{l}3283 \\
3120 \\
2972 \\
2844 \\
2731\end{array}$ & $\begin{array}{l}26.27117 \\
26.55403 \\
26.81957 \\
27.06994 \\
27.30688\end{array}$ & $\begin{array}{l}28286 \\
26554 \\
25037 \\
23694 \\
22499\end{array}$ & $\begin{array}{l}30.07165 \\
30.38734 \\
30.68408 \\
30.96417 \\
31.22955\end{array}$ & $\begin{array}{l}31569 \\
29674 \\
28009 \\
26538 \\
25230\end{array}$ \\
\hline $\begin{array}{r}900 \\
950 \\
1000 \\
1050 \\
1100\end{array}$ & $\begin{array}{l}4.43294 \\
4.47030 \\
4.50847 \\
4.54763 \\
4.58781\end{array}$ & $\begin{array}{l}3736 \\
3817 \\
3916 \\
4018 \\
4112\end{array}$ & $\begin{array}{l}3.94998 \\
3.97639 \\
4.00203 \\
4.02708 \\
4.05165\end{array}$ & $\begin{array}{l}2641 \\
2564 \\
2505 \\
2457 \\
2420\end{array}$ & $\begin{array}{l}27.53187 \\
27.74616 \\
27.95077 \\
28.14664 \\
28.33454\end{array}$ & $\begin{array}{l}21429 \\
20461 \\
19587 \\
18790 \\
18064\end{array}$ & $\begin{array}{l}31.48185 \\
31.72255 \\
31.95280 \\
32.17372 \\
32.38619\end{array}$ & $\begin{array}{l}24070 \\
23025 \\
22092 \\
21247 \\
20484\end{array}$ \\
\hline 1150 & 4.62893 & 4191 & 4.07585 & 2392 & 28.51518 & 17397 & 32.59103 & 19789 \\
\hline
\end{tabular}


Table 2.093. BiT (Cont.)

\begin{tabular}{|c|c|c|c|c|c|c|c|c|}
\hline${ }^{\circ} K$ & $\frac{C_{p}^{0}}{R}$ & & $\frac{\left(H^{0}-E_{0}^{0}\right)}{R T}$ & & $\frac{-\left\langle F^{\circ}-E\right.}{R T}$ & & $\frac{S^{0}}{R}$ & \\
\hline $\begin{array}{l}1200 \\
1300 \\
1400 \\
1500 \\
1600\end{array}$ & $\begin{array}{l}4.67084 \\
4.75611 \\
4.84150 \\
4.92485 \\
5.00421\end{array}$ & $\begin{array}{l}8527 \\
8539 \\
8335 \\
7936 \\
7383\end{array}$ & $\begin{array}{l}4.09977 \\
4.14697 \\
4.19353 \\
4.23953 \\
4.28487\end{array}$ & $\begin{array}{l}4720 \\
4656 \\
4600 \\
4534 \\
4451\end{array}$ & $\begin{array}{l}28.68915 \\
29.01919 \\
29.32822 \\
29.61911 \\
29.89418\end{array}$ & $\begin{array}{l}33004 \\
30903 \\
29089 \\
27507 \\
26110\end{array}$ & $\begin{array}{l}32.78892 \\
33.16616 \\
33.52175 \\
33.85864 \\
34.17905\end{array}$ & $\begin{array}{l}37724 \\
35559 \\
33599 \\
32041 \\
30561\end{array}$ \\
\hline $\begin{array}{l}1700 \\
1800 \\
1900 \\
2000 \\
2100\end{array}$ & $\begin{array}{l}5.07804 \\
5.14524 \\
5.20516 \\
5.25753 \\
5.30237\end{array}$ & $\begin{array}{l}6720 \\
5992 \\
5237 \\
4484 \\
3758\end{array}$ & $\begin{array}{l}4.32938 \\
4.37288 \\
4.41513 \\
4.45597 \\
4.49524\end{array}$ & $\begin{array}{l}4350 \\
4225 \\
4084 \\
3927 \\
3757\end{array}$ & $\begin{array}{l}30.15528 \\
30.40398 \\
30.64155 \\
30.86906 \\
31.08742\end{array}$ & $\begin{array}{l}24870 \\
23757 \\
22751 \\
21836 \\
21000\end{array}$ & $\begin{array}{l}34.48466 \\
34.77686 \\
35.05668 \\
35.32503 \\
35.58266\end{array}$ & $\begin{array}{l}29220 \\
27982 \\
26835 \\
25763 \\
24757\end{array}$ \\
\hline $\begin{array}{l}2200 \\
2300 \\
2400 \\
2500 \\
2600\end{array}$ & $\begin{array}{l}5.33995 \\
5.37072 \\
5.39523 \\
5.41409 \\
5.42793\end{array}$ & $\begin{array}{r}3077 \\
2451 \\
1886 \\
1384 \\
943\end{array}$ & $\begin{array}{l}4.53281 \\
4.56860 \\
4.60255 \\
4.63465 \\
4.66491\end{array}$ & $\begin{array}{l}3579 \\
3395 \\
3210 \\
3026 \\
2845\end{array}$ & $\begin{array}{l}31.29742 \\
31.49971 \\
31.69487 \\
31.88341 \\
32.06578\end{array}$ & $\begin{array}{l}20229 \\
19516 \\
18854 \\
18237 \\
17660\end{array}$ & $\begin{array}{l}35.83023 \\
36.06831 \\
36.29742 \\
36.51806 \\
36.73069\end{array}$ & $\begin{array}{l}23808 \\
22911 \\
22064 \\
21263 \\
20505\end{array}$ \\
\hline $\begin{array}{l}2700 \\
2800 \\
2900 \\
3000\end{array}$ & $\begin{array}{l}5.43736 \\
5.44299 \\
5.44538 \\
5.44504\end{array}$ & $\begin{array}{r}563 \\
239 \\
-\quad 34 \\
-\quad 707\end{array}$ & $\begin{array}{l}4.69336 \\
4.72004 \\
4.74502 \\
4.76837\end{array}$ & $\begin{array}{l}2668 \\
2478 \\
2335 \\
4211\end{array}$ & $\begin{array}{l}32.24238 \\
32.41355 \\
32.57962 \\
32.74088\end{array}$ & $\begin{array}{l}17117 \\
16607 \\
16126 \\
30912\end{array}$ & $\begin{array}{l}36.93574 \\
37.13359 \\
37.32464 \\
37.50925\end{array}$ & $\begin{array}{l}19785 \\
19105 \\
18461 \\
35123\end{array}$ \\
\hline $\begin{array}{l}3200 \\
3400 \\
3600 \\
3800 \\
4000\end{array}$ & $\begin{array}{l}5.43797 \\
5.42489 \\
5.40819 \\
5.38959 \\
5.37035\end{array}$ & $\begin{array}{l}-1308 \\
-1670 \\
-1860 \\
-1924 \\
-1899\end{array}$ & $\begin{array}{l}4.81048 \\
4.84702 \\
4.87868 \\
4.90606 \\
4.92976\end{array}$ & $\begin{array}{l}3654 \\
3166 \\
2738 \\
2370 \\
2053\end{array}$ & $\begin{array}{l}33.05000 \\
33.34275 \\
33.62073 \\
33.88525 \\
34.13752\end{array}$ & $\begin{array}{l}29275 \\
27798 \\
26452 \\
25227 \\
24103\end{array}$ & $\begin{array}{l}37.86048 \\
38.18977 \\
38.49941 \\
38.79131 \\
39.06728\end{array}$ & $\begin{array}{l}32929 \\
30964 \\
29190 \\
27597 \\
26156\end{array}$ \\
\hline $\begin{array}{l}4200 \\
4400 \\
4600 \\
4800 \\
5000\end{array}$ & $\begin{array}{l}5.35136 \\
5.33317 \\
5.31616 \\
5.30056 \\
5.28646\end{array}$ & $\begin{array}{l}-1819 \\
-1701 \\
-1560 \\
-1410\end{array}$ & $\begin{array}{l}4.95029 \\
4.96810 \\
4.98359 \\
4.99712 \\
5.00898\end{array}$ & $\begin{array}{l}1781 \\
1549 \\
1353 \\
1186\end{array}$ & $\begin{array}{l}34.37855 \\
34.60926 \\
34.83045 \\
35.04284 \\
35.24708\end{array}$ & $\begin{array}{l}23071 \\
22119 \\
21239 \\
20424\end{array}$ & $\begin{array}{l}39.32884 \\
39.57736 \\
39.81404 \\
40.03996 \\
40.25606\end{array}$ & $\begin{array}{l}24852 \\
23668 \\
22592 \\
21610\end{array}$ \\
\hline
\end{tabular}


Table 2.094. $\mathrm{OH}$

\begin{tabular}{|c|c|c|c|c|c|c|c|c|}
\hline${ }^{\circ} \mathrm{K}$ & $\frac{C_{p}^{0}}{R}$ & & $\frac{\left(H^{0}-E_{0}^{0}\right)}{R T}$ & & $\frac{-\left(F^{\circ}-\right.}{R T}$ & & $\frac{S^{0}}{R}$ & \\
\hline $\begin{array}{l}50 \\
60 \\
70 \\
80 \\
90\end{array}$ & $\begin{array}{l}3.50050 \\
3.64553 \\
3.73245 \\
3.77962 \\
3.80047\end{array}$ & $\begin{array}{r}14503 \\
8692 \\
4717 \\
2085 \\
420\end{array}$ & $\begin{array}{l}2.87580 \\
2.99297 \\
3.09296 \\
3.17619 \\
3.24458\end{array}$ & $\begin{array}{r}11717 \\
9999 \\
8323 \\
6839 \\
5591\end{array}$ & $\begin{array}{l}12.59278 \\
13.12770 \\
13.59680 \\
14.01543 \\
14.39361\end{array}$ & $\begin{array}{l}53492 \\
46910 \\
41863 \\
37818 \\
34483\end{array}$ & $\begin{array}{l}15.46858 \\
16.12067 \\
16.68976 \\
17.19162 \\
17.63819\end{array}$ & $\begin{array}{l}65209 \\
56909 \\
50186 \\
44657 \\
40074\end{array}$ \\
\hline $\begin{array}{l}100 \\
110 \\
120 \\
130 \\
140\end{array}$ & $\begin{array}{l}3.80467 \\
3.79879 \\
3.78713 \\
3.77249 \\
3.75660\end{array}$ & $\begin{array}{l}-\quad 588 \\
-1166 \\
-1454 \\
-1589 \\
-1609\end{array}$ & $\begin{array}{l}3.30049 \\
3.34611 \\
3.38338 \\
3.41389 \\
3.43893\end{array}$ & $\begin{array}{l}4562 \\
3727 \\
3051 \\
2504 \\
2065\end{array}$ & $\begin{array}{l}14.73844 \\
15.05523 \\
15.34803 \\
15.62010 \\
15.87404\end{array}$ & $\begin{array}{l}31679 \\
29280 \\
27207 \\
25394 \\
23798\end{array}$ & $\begin{array}{l}18.03893 \\
18.40134 \\
18.73141 \\
19.03399 \\
19.31297\end{array}$ & $\begin{array}{l}36241 \\
33007 \\
30258 \\
27898 \\
25863\end{array}$ \\
\hline $\begin{array}{l}150 \\
160 \\
170 \\
180 \\
190\end{array}$ & $\begin{array}{l}3.74051 \\
3.72484 \\
3.70993 \\
3.69595 \\
3.68295\end{array}$ & $\begin{array}{l}-1567 \\
-1491 \\
-1398 \\
-1300 \\
-1200\end{array}$ & $\begin{array}{l}3.45958 \\
3.47664 \\
3.49080 \\
3.50258 \\
3.51241\end{array}$ & $\begin{array}{r}1706 \\
1416 \\
1178 \\
983 \\
822\end{array}$ & $\begin{array}{l}16.11202 \\
16.33586 \\
16.54706 \\
16.74694 \\
16.93658\end{array}$ & $\begin{array}{l}22384 \\
21120 \\
19988 \\
18964 \\
18039\end{array}$ & $\begin{array}{l}19.57160 \\
19.81250 \\
20.03786 \\
20.24952 \\
20.44899\end{array}$ & $\begin{array}{l}24090 \\
22536 \\
21166 \\
19947 \\
18861\end{array}$ \\
\hline $\begin{array}{l}200 \\
210 \\
220 \\
230 \\
240\end{array}$ & $\begin{array}{l}3.67095 \\
3.65993 \\
3.64981 \\
3.64053 \\
3.63202\end{array}$ & $\begin{array}{l}-1102 \\
-\quad 1012 \\
-\quad 928 \\
-\quad 851 \\
-\quad 778\end{array}$ & $\begin{array}{l}3.52063 \\
3.52753 \\
3.53331 \\
3.53817 \\
3.54226\end{array}$ & $\begin{array}{l}690 \\
578 \\
486 \\
409 \\
342\end{array}$ & $\begin{array}{l}17.11697 \\
17.28891 \\
17.45315 \\
17.61033 \\
17.76098\end{array}$ & $\begin{array}{l}17194 \\
16424 \\
15718 \\
15065 \\
14469\end{array}$ & $\begin{array}{l}20.63760 \\
20.81644 \\
20.98646 \\
21.14850 \\
21.30324\end{array}$ & $\begin{array}{l}17884 \\
17002 \\
16204 \\
15474 \\
14811\end{array}$ \\
\hline $\begin{array}{l}250 \\
260 \\
270 \\
280 \\
290\end{array}$ & $\begin{array}{l}3.62424 \\
3.61711 \\
3.61057 \\
3.60455 \\
3.59903\end{array}$ & $\begin{array}{l}-\quad 713 \\
-\quad 654 \\
-\quad 602 \\
-\quad 552 \\
-\quad 503\end{array}$ & $\begin{array}{l}3.54568 \\
3.54857 \\
3.55099 \\
3.55300 \\
3.55468\end{array}$ & $\begin{array}{l}289 \\
242 \\
201 \\
168 \\
140\end{array}$ & $\begin{array}{l}17.90567 \\
18.04479 \\
18.17875 \\
18.30794 \\
18.43265\end{array}$ & $\begin{array}{l}13912 \\
13396 \\
12919 \\
12471 \\
12053\end{array}$ & $\begin{array}{l}21.45135 \\
21.59336 \\
21.72974 \\
21.86094 \\
21.98733\end{array}$ & $\begin{array}{l}14201 \\
13638 \\
13120 \\
12639 \\
12193\end{array}$ \\
\hline $\begin{array}{l}300 \\
310 \\
320 \\
330 \\
340\end{array}$ & $\begin{array}{l}3.59395 \\
3.58928 \\
3.58497 \\
3.58098 \\
3.57732\end{array}$ & $\begin{array}{l}-\quad 467 \\
-\quad 431 \\
-\quad 399 \\
-\quad 366 \\
-\quad 339\end{array}$ & $\begin{array}{l}3.55608 \\
3.55723 \\
3.55815 \\
3.55891 \\
3.55951\end{array}$ & $\begin{array}{r}115 \\
92 \\
76 \\
60 \\
46\end{array}$ & $\begin{array}{l}18.55318 \\
18.66979 \\
18.78276 \\
18.89226 \\
18.99851\end{array}$ & $\begin{array}{l}11661 \\
11297 \\
10950 \\
10625 \\
10319\end{array}$ & $\begin{array}{l}22.10926 \\
22.22702 \\
22.34091 \\
22.45117 \\
22.55802\end{array}$ & $\begin{array}{l}11776 \\
11389 \\
11026 \\
10685 \\
10365\end{array}$ \\
\hline $\begin{array}{l}350 \\
360 \\
370 \\
380 \\
390\end{array}$ & $\begin{array}{l}3.57393 \\
3.57080 \\
3.56791 \\
3.56524 \\
3.56279\end{array}$ & $\begin{array}{l}-\quad 313 \\
-\quad 289 \\
-\quad 267 \\
-\quad 245 \\
-\quad 226\end{array}$ & $\begin{array}{l}3.55997 \\
3.56030 \\
3.56054 \\
3.56071 \\
3.56079\end{array}$ & $\begin{array}{r}33 \\
24 \\
17 \\
8 \\
2\end{array}$ & $\begin{array}{l}19.10170 \\
19.20199 \\
19.29955 \\
19.39450 \\
19.48700\end{array}$ & $\begin{array}{r}10029 \\
9756 \\
9495 \\
9250 \\
9014\end{array}$ & $\begin{array}{l}22.66167 \\
22.76229 \\
22.86009 \\
22.95521 \\
23.04779\end{array}$ & $\begin{array}{r}10062 \\
9780 \\
9512 \\
9258 \\
9016\end{array}$ \\
\hline $\begin{array}{l}400 \\
450 \\
500 \\
550 \\
600\end{array}$ & $\begin{array}{l}3.56053 \\
3.55189 \\
3.54721 \\
3.54629 \\
3.54912\end{array}$ & $\begin{array}{r}-\quad 864 \\
-\quad 468 \\
-\quad 92 \\
283 \\
660\end{array}$ & $\begin{array}{l}3.56081 \\
3.56027 \\
3.55916 \\
3.55800 \\
3.55712\end{array}$ & $\begin{array}{r}-\quad 54 \\
-\quad 111 \\
-\quad 116 \\
-\quad 88 \\
-\quad 39\end{array}$ & $\begin{array}{l}19.57714 \\
19.99653 \\
20.37158 \\
20.71075 \\
21.02029\end{array}$ & $\begin{array}{l}41939 \\
37505 \\
33917 \\
30954 \\
28471\end{array}$ & $\begin{array}{l}23.13795 \\
23.55680 \\
23.93074 \\
24.26875 \\
24.57741\end{array}$ & $\begin{array}{l}41885 \\
37394 \\
33801 \\
30866 \\
28432\end{array}$ \\
\hline $\begin{array}{l}650 \\
700 \\
750 \\
800 \\
850\end{array}$ & $\begin{array}{l}3.55572 \\
3.56602 \\
3.57984 \\
3.59683 \\
3.61664\end{array}$ & $\begin{array}{l}1030 \\
1382 \\
1699 \\
1981 \\
2216\end{array}$ & $\begin{array}{l}3.55673 \\
3.55700 \\
3.55805 \\
3.55992 \\
3.56267\end{array}$ & $\begin{array}{r}27 \\
105 \\
187 \\
275 \\
360\end{array}$ & $\begin{array}{l}21.30500 \\
21.56859 \\
21.81403 \\
22.04372 \\
22.25961\end{array}$ & $\begin{array}{l}26359 \\
24544 \\
22969 \\
21589 \\
20373\end{array}$ & $\begin{array}{l}24.86173 \\
25.12559 \\
25.37208 \\
25.60364 \\
25.82228\end{array}$ & $\begin{array}{l}26386 \\
24649 \\
23156 \\
21864 \\
20733\end{array}$ \\
\hline $\begin{array}{r}900 \\
950 \\
1000 \\
1050 \\
1100\end{array}$ & $\begin{array}{l}3.63880 \\
3.66288 \\
3.68844 \\
3.71509 \\
3.74245\end{array}$ & $\begin{array}{l}2408 \\
2556 \\
2665 \\
2736 \\
2776\end{array}$ & $\begin{array}{l}3.56627 \\
3.57072 \\
3.57596 \\
3.58195 \\
3.58861\end{array}$ & $\begin{array}{l}445 \\
524 \\
599 \\
666 \\
729\end{array}$ & $\begin{array}{l}22.46334 \\
22.65628 \\
22.83956 \\
23.01417 \\
23.18096\end{array}$ & $\begin{array}{l}19294 \\
18328 \\
17461 \\
16679 \\
15968\end{array}$ & $\begin{array}{l}26.02961 \\
26.22700 \\
26.41552 \\
26.59612 \\
26.76957\end{array}$ & $\begin{array}{l}19739 \\
18852 \\
18060 \\
17345 \\
16697\end{array}$ \\
\hline 1150 & 3.77021 & 2790 & 3.59590 & 785 & 23.34064 & 15320 & 26.93654 & 16105 \\
\hline
\end{tabular}


Table 2.094. OH (Cont.)

\begin{tabular}{|c|c|c|c|c|c|c|c|c|}
\hline${ }^{\circ} K$ & $\frac{C_{P}^{0}}{R}$ & & $\frac{\left(H^{0}-E_{0}^{0}\right)}{R T}$ & & $\frac{-L F^{\circ}-E}{R T}$ & & $\frac{S^{0}}{R}$ & \\
\hline $\begin{array}{l}1200 \\
1300 \\
1400 \\
1500 \\
1600\end{array}$ & $\begin{array}{l}3.79811 \\
3.85345 \\
3.90711 \\
3.95832 \\
4.00660\end{array}$ & $\begin{array}{l}5534 \\
5366 \\
5121 \\
4828 \\
4519\end{array}$ & $\begin{array}{l}3.60375 \\
3.62083 \\
3.63938 \\
3.65895 \\
3.67918\end{array}$ & $\begin{array}{l}1708 \\
1855 \\
1957 \\
2023 \\
2061\end{array}$ & $\begin{array}{l}23.49384 \\
23.78296 \\
24.05197 \\
24.30372 \\
24.54050\end{array}$ & $\begin{array}{l}28912 \\
26901 \\
25175 \\
23678 \\
22367\end{array}$ & $\begin{array}{l}27.09759 \\
27.40379 \\
27.69135 \\
27.96267 \\
28.21968\end{array}$ & $\begin{array}{l}30620 \\
28756 \\
27132 \\
25701 \\
24428\end{array}$ \\
\hline $\begin{array}{l}1700 \\
1800 \\
1900 \\
2000 \\
2100\end{array}$ & $\begin{array}{l}4.05179 \\
4.09386 \\
4.13294 \\
4.16916 \\
4.20272\end{array}$ & $\begin{array}{l}4207 \\
3908 \\
3622 \\
3356 \\
3110\end{array}$ & $\begin{array}{l}3.69979 \\
3.72052 \\
3.74121 \\
3.76172 \\
3.78193\end{array}$ & $\begin{array}{l}2073 \\
2069 \\
2051 \\
2021 \\
1984\end{array}$ & $\begin{array}{l}24.76417 \\
24.97623 \\
25.17794 \\
25.37037 \\
25.55439\end{array}$ & $\begin{array}{l}21206 \\
20171 \\
19243 \\
18402 \\
17640\end{array}$ & $\begin{array}{l}28.46396 \\
28.69675 \\
28.91915 \\
29.13209 \\
29.33632\end{array}$ & $\begin{array}{l}23279 \\
22240 \\
21294 \\
20423 \\
19624\end{array}$ \\
\hline $\begin{array}{l}2200 \\
2300 \\
2400 \\
2500 \\
2600\end{array}$ & $\begin{array}{l}4.23382 \\
4.26268 \\
4.28949 \\
4.31444 \\
4.33769\end{array}$ & $\begin{array}{l}2886 \\
2681 \\
2495 \\
2325 \\
2173\end{array}$ & $\begin{array}{l}3.80177 \\
3.82119 \\
3.84015 \\
3.85863 \\
3.87661\end{array}$ & $\begin{array}{l}1942 \\
1896 \\
1848 \\
1798 \\
1748\end{array}$ & $\begin{array}{l}25.73079 \\
25.90021 \\
26.06324 \\
26.22038 \\
26.37207\end{array}$ & $\begin{array}{l}16942 \\
16303 \\
15714 \\
15169 \\
14664\end{array}$ & $\begin{array}{l}29.53256 \\
29.72140 \\
29.90339 \\
30.07901 \\
30.24868\end{array}$ & $\begin{array}{l}18884 \\
18199 \\
17562 \\
16967 \\
16412\end{array}$ \\
\hline $\begin{array}{l}2700 \\
2800 \\
2900 \\
3000\end{array}$ & $\begin{array}{l}4.35942 \\
4.37974 \\
4.39882 \\
4.41674\end{array}$ & $\begin{array}{l}2032 \\
1908 \\
1792 \\
3288\end{array}$ & $\begin{array}{l}3.89409 \\
3.91108 \\
3.92757 \\
3.94359\end{array}$ & $\begin{array}{l}1699 \\
1649 \\
1602 \\
3061\end{array}$ & $\begin{array}{l}26.51871 \\
26.66063 \\
26.79817 \\
26.93159\end{array}$ & $\begin{array}{l}14192 \\
13754 \\
13342 \\
25550\end{array}$ & $\begin{array}{l}30.41280 \\
30.57171 \\
30.72574 \\
30.87518\end{array}$ & $\begin{array}{l}15891 \\
15403 \\
14944 \\
28611\end{array}$ \\
\hline $\begin{array}{l}3200 \\
3400 \\
3600 \\
3800 \\
4000\end{array}$ & $\begin{array}{l}4.44962 \\
4.47905 \\
4.50564 \\
4.52985 \\
4.55208\end{array}$ & $\begin{array}{l}2943 \\
2659 \\
2421 \\
2223 \\
2054\end{array}$ & $\begin{array}{l}3.97420 \\
4.00305 \\
4.03025 \\
4.05592 \\
4.08017\end{array}$ & $\begin{array}{l}2885 \\
2720 \\
2567 \\
2425 \\
2297\end{array}$ & $\begin{array}{l}27.18709 \\
27.42890 \\
27.65849 \\
27.87708 \\
28.08574\end{array}$ & $\begin{array}{l}24181 \\
22959 \\
21859 \\
20866 \\
19964\end{array}$ & $\begin{array}{l}31.16129 \\
31.43195 \\
31.68874 \\
31.93300 \\
32.16591\end{array}$ & $\begin{array}{l}27066 \\
25679 \\
24426 \\
23291 \\
22261\end{array}$ \\
\hline $\begin{array}{l}4200 \\
4400 \\
4600 \\
4800 \\
5000\end{array}$ & $\begin{array}{l}4.57262 \\
4.59173 \\
4.60962 \\
4.62645 \\
4.64238\end{array}$ & $\begin{array}{l}1911 \\
1789 \\
1683 \\
1593\end{array}$ & $\begin{array}{l}4.10314 \\
4.12492 \\
4.14561 \\
4.16529 \\
4.18406\end{array}$ & $\begin{array}{l}2178 \\
2069 \\
1968 \\
1877\end{array}$ & $\begin{array}{l}28.28538 \\
28.47676 \\
28.66058 \\
28.83744 \\
29.00786\end{array}$ & $\begin{array}{l}19138 \\
18382 \\
17686 \\
17042\end{array}$ & $\begin{array}{l}32.38852 \\
32.60168 \\
32.80619 \\
33.00273 \\
33.19192\end{array}$ & $\begin{array}{l}21316 \\
20451 \\
19654 \\
18919\end{array}$ \\
\hline
\end{tabular}


Table 2.095. OD

\begin{tabular}{|c|c|c|c|c|c|c|c|c|}
\hline${ }^{\circ} \mathrm{K}$ & $\frac{C_{P}^{0}}{R}$ & & $\frac{\left(H^{0}-E_{0}^{0}\right.}{R T}$ & & $\frac{-\left(F^{0}-\right.}{R T}$ & & $\frac{S^{0}}{R}$ & \\
\hline $\begin{array}{l}50 \\
60 \\
70 \\
80 \\
90\end{array}$ & $\begin{array}{l}3.68420 \\
3.78363 \\
3.84240 \\
3.86980 \\
3.87567\end{array}$ & $\begin{array}{r}9943 \\
5877 \\
2740 \\
587 \\
-\quad 769\end{array}$ & $\begin{array}{l}3.13107 \\
3.23215 \\
3.31558 \\
3.38340 \\
3.43794\end{array}$ & $\begin{array}{r}10108 \\
8343 \\
6782 \\
5454 \\
4347\end{array}$ & $\begin{array}{l}12.94356 \\
13.52364 \\
14.02836 \\
14.47569 \\
14.87746\end{array}$ & $\begin{array}{l}58008 \\
50472 \\
44733 \\
40177 \\
36456\end{array}$ & $\begin{array}{l}16.07463 \\
16.75579 \\
17.34394 \\
17.85909 \\
18.31540\end{array}$ & $\begin{array}{l}68116 \\
58815 \\
51515 \\
45631 \\
40803\end{array}$ \\
\hline $\begin{array}{l}100 \\
110 \\
120 \\
130 \\
140\end{array}$ & $\begin{array}{l}3.86798 \\
3.85244 \\
3.83287 \\
3.81171 \\
3.79040\end{array}$ & $\begin{array}{l}-1554 \\
-1957 \\
-2116 \\
-2131 \\
-2062\end{array}$ & $\begin{array}{l}3.48141 \\
3.51589 \\
3.54314 \\
3.56462 \\
3.58151\end{array}$ & $\begin{array}{l}3448 \\
2725 \\
2148 \\
1689 \\
1323\end{array}$ & $\begin{array}{l}15.24202 \\
15.57553 \\
15.88265 \\
16.16713 \\
16.43194\end{array}$ & $\begin{array}{l}33351 \\
30712 \\
28448 \\
26481 \\
24757\end{array}$ & $\begin{array}{l}18.72343 \\
19.09142 \\
19.42579 \\
19.73175 \\
20.01345\end{array}$ & $\begin{array}{l}36799 \\
33437 \\
30596 \\
28170 \\
26080\end{array}$ \\
\hline $\begin{array}{l}150 \\
160 \\
170 \\
180 \\
190\end{array}$ & $\begin{array}{l}3.76978 \\
3.75032 \\
3.73221 \\
3.71553 \\
3.70024\end{array}$ & $\begin{array}{l}-1946 \\
-1811 \\
-1668 \\
-1529 \\
-1393\end{array}$ & $\begin{array}{l}3.59474 \\
3.60506 \\
3.61306 \\
3.61922 \\
3.62388\end{array}$ & $\begin{array}{r}1032 \\
800 \\
616 \\
466 \\
346\end{array}$ & $\begin{array}{l}16.67951 \\
16.91187 \\
17.13067 \\
17.33737 \\
17.53319\end{array}$ & $\begin{array}{l}23236 \\
21880 \\
20670 \\
19582 \\
18596\end{array}$ & $\begin{array}{l}20.27425 \\
20.51693 \\
20.74373 \\
20.95659 \\
21.15707\end{array}$ & $\begin{array}{l}24268 \\
22680 \\
21286 \\
20048 \\
18942\end{array}$ \\
\hline $\begin{array}{l}200 \\
210 \\
220 \\
230 \\
240\end{array}$ & $\begin{array}{l}3.68631 \\
3.67360 \\
3.66204 \\
3.65153 \\
3.64195\end{array}$ & $\begin{array}{l}-1271 \\
-1156 \\
-1051 \\
-958 \\
-870\end{array}$ & $\begin{array}{l}3.62734 \\
3.62985 \\
3.63156 \\
3.63266 \\
3.63324\end{array}$ & $\begin{array}{r}251 \\
171 \\
110 \\
58 \\
17\end{array}$ & $\begin{array}{l}17.71915 \\
17.89619 \\
18.06510 \\
18.22656 \\
18.38118\end{array}$ & $\begin{array}{l}17704 \\
16391 \\
16146 \\
15462 \\
14831\end{array}$ & $\begin{array}{l}21.34649 \\
21.52604 \\
21.69666 \\
21.85922 \\
22.01442\end{array}$ & $\begin{array}{l}17955 \\
17062 \\
16256 \\
15520 \\
14848\end{array}$ \\
\hline $\begin{array}{l}250 \\
260 \\
270 \\
280 \\
290\end{array}$ & $\begin{array}{l}3.63325 \\
3.62533 \\
3.61812 \\
3.61154 \\
3.60558\end{array}$ & $\begin{array}{l}-792 \\
-721 \\
-658 \\
-596 \\
-544\end{array}$ & $\begin{array}{l}3.63341 \\
3.63325 \\
3.63282 \\
3.63217 \\
3.63137\end{array}$ & $\begin{array}{l}-\quad 16 \\
-\quad 43 \\
-\quad 65 \\
-\quad 80 \\
-\quad 96\end{array}$ & $\begin{array}{l}18.52949 \\
18.67179 \\
18.80912 \\
18.94122 \\
19.06866\end{array}$ & $\begin{array}{l}14250 \\
13713 \\
13210 \\
12744 \\
12309\end{array}$ & $\begin{array}{l}22.16290 \\
22.30524 \\
22.44194 \\
22.57339 \\
22.70003\end{array}$ & $\begin{array}{l}14234 \\
13670 \\
13145 \\
12664 \\
12213\end{array}$ \\
\hline $\begin{array}{l}300 \\
310 \\
320 \\
330 \\
340\end{array}$ & $\begin{array}{l}3.60014 \\
3.59523 \\
3.59080 \\
3.58680 \\
3.58324\end{array}$ & $\begin{array}{l}-491 \\
-443 \\
-400 \\
-356 \\
-317\end{array}$ & $\begin{array}{l}3.63041 \\
3.62934 \\
3.62823 \\
3.62703 \\
3.62579\end{array}$ & $\begin{array}{l}-\quad 107 \\
-\quad 111 \\
-\quad 120 \\
-\quad 124 \\
-\quad 126\end{array}$ & $\begin{array}{l}19.19175 \\
19.31078 \\
19.42599 \\
19.53762 \\
19.64587\end{array}$ & $\begin{array}{l}11903 \\
11521 \\
11163 \\
10825 \\
10508\end{array}$ & $\begin{array}{l}22.82216 \\
22.94012 \\
23.05422 \\
23.16465 \\
23.27166\end{array}$ & $\begin{array}{l}11796 \\
11410 \\
11043 \\
10701 \\
10382\end{array}$ \\
\hline $\begin{array}{l}350 \\
360 \\
370 \\
380 \\
390\end{array}$ & $\begin{array}{l}3.58007 \\
3.57731 \\
3.57491 \\
3.57290 \\
3.57124\end{array}$ & $\begin{array}{l}-276 \\
-240 \\
-201 \\
-166 \\
-130\end{array}$ & $\begin{array}{l}3.62453 \\
3.62324 \\
3.62197 \\
3.62070 \\
3.61945\end{array}$ & $\begin{array}{l}-\quad 129 \\
-\quad 127 \\
-\quad 127 \\
-\quad 125 \\
-\quad 121\end{array}$ & $\begin{array}{l}19.75095 \\
19.85305 \\
19.95230 \\
20.04888 \\
20.14292\end{array}$ & $\begin{array}{r}10210 \\
9925 \\
9658 \\
9404 \\
9162\end{array}$ & $\begin{array}{l}23.37548 \\
23.47629 \\
23.57427 \\
23.66958 \\
23.76237\end{array}$ & $\begin{array}{r}10081 \\
9798 \\
9531 \\
9279 \\
9041\end{array}$ \\
\hline
\end{tabular}

$\begin{array}{rrrrrrrrr}400 & 3.56994 & -138 & 3.61824 & -552 & 20.23454 & 42584 & 23.85278 & 42032 \\ 450 & 3.56856 & 680 & 3.51272 & -414 & 20.66038 & 38041 & 24.27310 & 37627 \\ 500 & 3.57536 & 1424 & 3.60858 & -243 & 21.04079 & 34380 & 24.64937 & 34137 \\ 550 & 3.58960 & 2076 & 3.60615 & -55 & 21.38459 & 31373 & 24.99074 & 31318 \\ 600 & 3.61036 & 2608 & 3.60560 & 134 & 21.69832 & 28865 & 25.30392 & 28999 \\ & & & & & & & & \\ 650 & 3.63644 & 3020 & 3.60694 & 317 & 21.98697 & 26741 & 25.59391 & 27058 \\ 700 & 3.66664 & 3316 & 3.61011 & 485 & 22.25438 & 24923 & 25.86449 & 25408 \\ 750 & 3.69980 & 3507 & 3.61496 & 639 & 22.50361 & 23350 & 26.11857 & 23989 \\ 800 & 3.73487 & 3608 & 3.62135 & 774 & 22.73711 & 21977 & 26.35846 & 22751 \\ 850 & 3.77095 & 3641 & 3.62909 & 889 & 22.95688 & 20768 & 26.58597 & 21657 \\ 900 & 3.80736 & 3615 & 3.63798 & 987 & 23.16456 & 19695 & 26.80254 & 20682 \\ 950 & 3.84351 & 3548 & 3.64785 & 1057 & 23.36151 & 18738 & 27.00936 & 19805 \\ 1000 & 3.87899 & 3453 & 3.65852 & 1133 & 23.54889 & 17878 & 27.20741 & 19011 \\ 1050 & 3.91352 & 3334 & 3.66985 & 1184 & 23.72767 & 17099 & 27.39752 & 18283 \\ 1100 & 3.94686 & 3204 & 3.68169 & 1223 & 23.89866 & 16392 & 27.58035 & 17615 \\ 1150 & 3.97890 & 3067 & 3.69392 & 1252 & 24.06258 & 15748 & 27.75650 & 17000\end{array}$


Table 2.095. OD (Cont.)

\begin{tabular}{|c|c|c|c|c|c|c|c|c|}
\hline${ }^{\circ} \mathrm{K}$ & $\frac{C_{p}^{0}}{R}$ & & $\frac{\left(H^{0}-E_{0}^{0}\right)}{R T}$ & & $\frac{-\left\langle F^{\circ}-E\right.}{R T}$ & & $\frac{S^{0}}{R}$ & \\
\hline $\begin{array}{l}1200 \\
1300 \\
1400 \\
1500 \\
1600\end{array}$ & $\begin{array}{l}4.00957 \\
4.06663 \\
411816 \\
4.16451 \\
4.20615\end{array}$ & $\begin{array}{l}5706 \\
5153 \\
4635 \\
4164 \\
3745\end{array}$ & $\begin{array}{l}3.70644 \\
3.73199 \\
3.75776 \\
3.78336 \\
3.80851\end{array}$ & $\begin{array}{l}2555 \\
2577 \\
2560 \\
2515 \\
2451\end{array}$ & $\begin{array}{l}24.22006 \\
24.51774 \\
24.79525 \\
25.05539 \\
25.30036\end{array}$ & $\begin{array}{l}29768 \\
27751 \\
26014 \\
24497 \\
23163\end{array}$ & $\begin{array}{l}27.92650 \\
28.24973 \\
28.55301 \\
28.83875 \\
29.10887\end{array}$ & $\begin{array}{l}32323 \\
30328 \\
28574 \\
27012 \\
25614\end{array}$ \\
\hline $\begin{array}{l}1700 \\
1800 \\
1900 \\
2000 \\
2100\end{array}$ & $\begin{array}{l}4.24360 \\
4.27734 \\
4.30784 \\
4.33549 \\
4.36066\end{array}$ & $\begin{array}{l}3374 \\
3050 \\
2765 \\
2517 \\
2299\end{array}$ & $\begin{array}{l}3.83302 \\
3.85678 \\
3.87973 \\
3.90184 \\
3.92310\end{array}$ & $\begin{array}{l}2376 \\
2295 \\
2211 \\
2126 \\
2041\end{array}$ & $\begin{array}{l}25.53199 \\
25.75176 \\
25.96090 \\
26.16048 \\
26.35137\end{array}$ & $\begin{array}{l}21977 \\
20914 \\
19958 \\
19089 \\
18297\end{array}$ & $\begin{array}{l}29.36501 \\
29.60854 \\
29.84063 \\
30.06232 \\
30.27447\end{array}$ & $\begin{array}{l}24353 \\
23209 \\
22169 \\
21215 \\
20338\end{array}$ \\
\hline $\begin{array}{l}2200 \\
2300 \\
2400 \\
2500 \\
2600\end{array}$ & $\begin{array}{l}4.38365 \\
4.40475 \\
4.42419 \\
4.44215 \\
4.45883\end{array}$ & $\begin{array}{l}2110 \\
1944 \\
1796 \\
1668 \\
1556\end{array}$ & $\begin{array}{l}3.94351 \\
3.96312 \\
3.98193 \\
3.99999 \\
4.01731\end{array}$ & $\begin{array}{l}1961 \\
1881 \\
1806 \\
1732 \\
1665\end{array}$ & $\begin{array}{l}26.53434 \\
26.71007 \\
26.87914 \\
27.04206 \\
27.19929\end{array}$ & $\begin{array}{l}17573 \\
16907 \\
16292 \\
15723 \\
15193\end{array}$ & $\begin{array}{l}30.47785 \\
30.67319 \\
30.86107 \\
31.04205 \\
31.21660\end{array}$ & $\begin{array}{l}19534 \\
18788 \\
18098 \\
17455 \\
16858\end{array}$ \\
\hline $\begin{array}{l}2700 \\
2800 \\
2900 \\
3000\end{array}$ & $\begin{array}{l}4.47439 \\
4.43892 \\
4.50256 \\
4.51542\end{array}$ & $\begin{array}{l}1453 \\
1364 \\
1286 \\
2365\end{array}$ & $\begin{array}{l}4.03396 \\
4.04995 \\
4.06533 \\
4.08011\end{array}$ & $\begin{array}{l}1599 \\
1538 \\
1478 \\
2796\end{array}$ & $\begin{array}{l}27.35122 \\
27.49821 \\
27.64060 \\
27.77867\end{array}$ & $\begin{array}{l}14699 \\
14239 \\
13807 \\
26423\end{array}$ & $\begin{array}{l}31.38518 \\
31.54816 \\
31.70593 \\
31.8587 ?\end{array}$ & $\begin{array}{l}16298 \\
15777 \\
15285 \\
29219\end{array}$ \\
\hline $\begin{array}{l}3200 \\
3400 \\
3600 \\
3800 \\
4000\end{array}$ & $\begin{array}{l}4.53907 \\
4.56043 \\
4.57994 \\
4.59793 \\
4.61466\end{array}$ & $\begin{array}{l}2136 \\
1951 \\
1799 \\
1673 \\
1569\end{array}$ & $\begin{array}{l}4.10807 \\
4.13407 \\
4.15830 \\
4.18098 \\
4.20225\end{array}$ & $\begin{array}{l}2600 \\
2423 \\
2268 \\
2127 \\
2001\end{array}$ & $\begin{array}{l}28.04290 \\
28.29274 \\
28.52972 \\
28.75518 \\
28.97017\end{array}$ & $\begin{array}{l}24984 \\
23698 \\
22546 \\
21499 \\
20553\end{array}$ & $\begin{array}{l}32.15097 \\
32.42681 \\
32.68802 \\
32.93616 \\
33.17242\end{array}$ & $\begin{array}{l}27584 \\
26121 \\
24814 \\
23626 \\
22554\end{array}$ \\
\hline $\begin{array}{l}4200 \\
4400 \\
4600 \\
4800 \\
5000\end{array}$ & $\begin{array}{l}4.63035 \\
4.64516 \\
4.65920 \\
4.67261 \\
4.68548\end{array}$ & $\begin{array}{l}1481 \\
1404 \\
1341 \\
1287\end{array}$ & $\begin{array}{l}4.22226 \\
4.24115 \\
4.25902 \\
4.27597 \\
4.29211\end{array}$ & $\begin{array}{l}1889 \\
1787 \\
1695 \\
1614\end{array}$ & $\begin{array}{l}29.17570 \\
29.37254 \\
29.56147 \\
29.74310 \\
29.91799\end{array}$ & $\begin{array}{l}19684 \\
18893 \\
18163 \\
17489\end{array}$ & $\begin{array}{l}33.39796 \\
33.61369 \\
33.82049 \\
34.01907 \\
34.21010\end{array}$ & $\begin{array}{l}21573 \\
20580 \\
19858 \\
19103\end{array}$ \\
\hline
\end{tabular}


Table 2.096. OT

\begin{tabular}{|c|c|c|c|c|c|c|c|c|}
\hline${ }^{\circ} \mathrm{K}$ & $\frac{C_{p}^{0}}{R}$ & & $\frac{\left(H^{\circ}-E\right.}{R T}$ & & $\frac{-\left(F^{\circ}-E\right.}{R T}$ & & $\frac{S^{0}}{R}$ & \\
\hline $\begin{array}{l}50 \\
60 \\
70 \\
80 \\
90\end{array}$ & $\begin{array}{l}3.72686 \\
3.81750 \\
3.87087 \\
3.89422 \\
3.89672\end{array}$ & $\begin{array}{r}9064 \\
5337 \\
2335 \\
250 \\
-1061\end{array}$ & $\begin{array}{l}3.24159 \\
3.33057 \\
3.40436 \\
3.46439 \\
3.51244\end{array}$ & $\begin{array}{l}8898 \\
7379 \\
6003 \\
4805 \\
3797\end{array}$ & $\begin{array}{l}13.23305 \\
13.83217 \\
14.35131 \\
14.80995 \\
15.22089\end{array}$ & $\begin{array}{l}59912 \\
51914 \\
45864 \\
41094 \\
37212\end{array}$ & $\begin{array}{l}16.47464 \\
17.16274 \\
17.75567 \\
18.27434 \\
18.73333\end{array}$ & $\begin{array}{l}68810 \\
59293 \\
51867 \\
45899 \\
41009\end{array}$ \\
\hline $\begin{array}{l}20 \\
30 \\
40\end{array}$ & $\begin{array}{l}3.88611 \\
3.86809 \\
3.84639 \\
3.82340 \\
3.80054\end{array}$ & $\begin{array}{l}-1802 \\
-2170 \\
-2299 \\
-2286 \\
-2193\end{array}$ & $\begin{array}{l}3.55041 \\
3.58015 \\
3.60327 \\
3.62108 \\
3.63471\end{array}$ & $\begin{array}{l}2974 \\
2312 \\
1781 \\
1363 \\
1032\end{array}$ & $\begin{array}{l}15.59301 \\
15.93286 \\
16.24540 \\
16.53455 \\
16.80343\end{array}$ & $\begin{array}{l}33985 \\
31254 \\
28915 \\
26988 \\
25113\end{array}$ & $\begin{array}{l}19.14342 \\
19.51301 \\
19.84867 \\
20.15563 \\
20.43814\end{array}$ & $\begin{array}{l}36959 \\
33566 \\
30696 \\
28251 \\
26145\end{array}$ \\
\hline 90 & $\begin{array}{l}3.77861 \\
3.75802 \\
3.73897 \\
3.72149 \\
3.70553\end{array}$ & $\begin{array}{l}-2059 \\
-1905 \\
-1748 \\
-1596 \\
-1454\end{array}$ & $\begin{array}{l}3.64503 \\
3.65273 \\
3.65835 \\
3.66234 \\
3.66503\end{array}$ & $\begin{array}{l}770 \\
562 \\
399 \\
269 \\
165\end{array}$ & $\begin{array}{l}17.05456 \\
17.29007 \\
17.51170 \\
17.72093 \\
17.91902\end{array}$ & $\begin{array}{l}23551 \\
22163 \\
20323 \\
19809 \\
18803\end{array}$ & $\begin{array}{l}20.69959 \\
20.94280 \\
21.17005 \\
21.38327 \\
21.58405\end{array}$ & $\begin{array}{l}24321 \\
22725 \\
21322 \\
20078 \\
18968\end{array}$ \\
\hline 0 & $\begin{array}{l}3.69099 \\
3.67781 \\
3.66586 \\
3.65504 \\
3.64524\end{array}$ & $\begin{array}{l}-1318 \\
-1195 \\
-1082 \\
-980 \\
-885\end{array}$ & $\begin{array}{l}3.66668 \\
3.66753 \\
3.66771 \\
3.66739 \\
3.66667\end{array}$ & $\begin{array}{r}85 \\
18 \\
-\quad 32 \\
-\quad 72 \\
-\quad 103\end{array}$ & $\begin{array}{l}18.10705 \\
18.28597 \\
18.45660 \\
18.61963 \\
18.77570\end{array}$ & $\begin{array}{l}17892 \\
17063 \\
16303 \\
15607 \\
14965\end{array}$ & $\begin{array}{l}21.77373 \\
21.95350 \\
22.12431 \\
22.28702 \\
22.44237\end{array}$ & $\begin{array}{l}17977 \\
17081 \\
16271 \\
15535 \\
14862\end{array}$ \\
\hline 90 & $\begin{array}{l}3.63639 \\
3.62841 \\
3.62123 \\
3.61482 \\
3.60911\end{array}$ & $\begin{array}{l}-798 \\
-718 \\
-641 \\
-571 \\
-504\end{array}$ & $\begin{array}{l}3.66564 \\
3.66435 \\
3.66289 \\
3.66127 \\
3.65959\end{array}$ & $\begin{array}{l}-129 \\
-146 \\
-162 \\
-168 \\
-178\end{array}$ & $\begin{array}{l}18.92535 \\
19.06910 \\
19.20736 \\
19.34055 \\
19.46899\end{array}$ & $\begin{array}{l}14375 \\
13826 \\
13319 \\
12844 \\
12404\end{array}$ & $\begin{array}{l}22.59099 \\
22.73345 \\
22.87025 \\
23.00182 \\
23.12858\end{array}$ & $\begin{array}{l}14246 \\
13680 \\
13157 \\
12676 \\
12226\end{array}$ \\
\hline $\begin{array}{l}320 \\
330 \\
340\end{array}$ & $\begin{array}{l}3.60407 \\
3.59967 \\
3.59590 \\
3.59272 \\
3.59011\end{array}$ & $\begin{array}{l}-440 \\
-377 \\
-318 \\
-261 \\
-204\end{array}$ & $\begin{array}{l}3.65781 \\
3.65600 \\
3.65418 \\
3.65237 \\
3.65058\end{array}$ & $\begin{array}{l}-181 \\
-182 \\
-181 \\
-179 \\
-176\end{array}$ & $\begin{array}{l}19.59303 \\
19.71294 \\
19.82900 \\
19.94140 \\
20.05040\end{array}$ & $\begin{array}{l}11991 \\
11606 \\
11240 \\
10900 \\
10581\end{array}$ & $\begin{array}{l}23.25084 \\
23.36894 \\
23.48318 \\
23.59377 \\
23.70098\end{array}$ & $\begin{array}{l}11810 \\
11424 \\
11059 \\
10721 \\
10405\end{array}$ \\
\hline $\begin{array}{l}350 \\
360 \\
370 \\
380 \\
390\end{array}$ & $\begin{array}{l}3.58807 \\
3.58656 \\
3.58560 \\
3.58516 \\
3.58522\end{array}$ & $\begin{array}{r}-151 \\
-\quad 96 \\
-\quad 44 \\
6 \\
57\end{array}$ & $\begin{array}{l}3.64882 \\
3.64710 \\
3.64546 \\
3.64388 \\
3.64237\end{array}$ & $\begin{array}{l}-172 \\
-164 \\
-158 \\
-151 \\
-142\end{array}$ & $\begin{array}{l}20.15621 \\
20.25898 \\
20.35889 \\
20.45607 \\
20.55071\end{array}$ & $\begin{array}{r}10277 \\
9991 \\
9718 \\
9464 \\
9219\end{array}$ & $\begin{array}{l}23.80503 \\
23.90608 \\
24.00435 \\
24.09995 \\
24.19308\end{array}$ & $\begin{array}{r}10105 \\
9827 \\
9560 \\
9313 \\
9077\end{array}$ \\
\hline
\end{tabular}

$\begin{array}{rrrrrrrrr}400 & 3.58579 & 984 & 3.64095 & -569 & 20.64290 & 42850 & 24.28385 & 42281 \\ 450 & 3.59563 & 2016 & 3.63526 & -303 & 21.07140 & 38283 & 24.70666 & 37980 \\ 500 & 3.61579 & 2842 & 3.63223 & -26 & 21.45423 & 34615 & 25.08646 & 34589 \\ 550 & 3.64421 & 3448 & 3.63197 & 242 & 21.80038 & 31612 & 25.43235 & 31854 \\ 600 & 3.67869 & 3853 & 3.63439 & 488 & 22.11650 & 29107 & 25.75089 & 29595 \\ & & & & & & & & \\ 650 & 3.71722 & 4086 & 3.63927 & 701 & 22.40757 & 26994 & 26.04684 & 27695 \\ 700 & 3.75808 & 4181 & 3.64628 & 885 & 22.67751 & 25186 & 26.32379 & 26071 \\ 750 & 3.79989 & 4173 & 3.65513 & 1036 & 22.92937 & 23623 & 26.58450 & 24659 \\ 800 & 3.84162 & 4091 & 3.66549 & 1156 & 23.16560 & 22256 & 26.83109 & 23412 \\ 850 & 3.88253 & 3956 & 3.67705 & 1252 & 23.38816 & 21053 & 27.06521 & 22305 \\ & & & & & & & & \\ 900 & 3.92209 & 3789 & 3.68957 & 1324 & 23.59869 & 19983 & 27.28826 & 21307 \\ 950 & 3.95998 & 3603 & 3.70281 & 1377 & 23.79852 & 19028 & 27.50133 & 20405 \\ 1000 & 3.99601 & 3409 & 3.71658 & 1413 & 23.98880 & 18167 & 27.70538 & 19580 \\ 1050 & 4.03010 & 3214 & 3.73071 & 1435 & 24.17047 & 17388 & 27.90118 & 18823 \\ 1100 & 4.06224 & 3022 & 3.74506 & 1445 & 24.34435 & 16680 & 28.08941 & 18125 \\ 1150 & 4.09246 & 2836 & 3.75951 & 1447 & 24.51115 & 16031 & 28.27066 & 17478\end{array}$


Table 2.096. OT (Cont.)

\begin{tabular}{|l|l|l|l|l|}
\hline$K$ & $\frac{C_{P}^{0}}{R}$ & $\frac{\left(H^{0}-E_{0}^{0}\right)}{R T}$ & $\frac{-\left(F^{0}-E_{0}^{0}\right)}{R T}$ & $\frac{S^{0}}{R}$ \\
\hline
\end{tabular}

\begin{tabular}{|c|c|c|c|c|c|c|c|c|}
\hline 1200 & 4.12082 & 5154 & 3.77398 & 2870 & 24.67146 & 30321 & 28.44544 & 33191 \\
\hline 1300 & 4.17236 & 4531 & 3.80268 & 2806 & 24.97467 & 28285 & 28.77735 & 31091 \\
\hline 1400 & 4.21767 & 3987 & 3.83074 & 2716 & 25.25752 & 26522 & 29.08826 & 29238 \\
\hline 1500 & 4.25754 & 3523 & 3.85790 & 2609 & 25.52274 & 24982 & 29.38064 & 27591 \\
\hline 1600 & 4.29277 & 3126 & 3.88399 & 2498 & 25.77256 & 23623 & 29.65655 & 26121 \\
\hline 1700 & 4.32403 & 2788 & 3.90897 & 2386 & 26.00879 & 22410 & 29.91776 & 24796 \\
\hline 1800 & 4.35191 & 2498 & 3.93283 & 2272 & 26.23289 & 21325 & 30.16572 & 23597 \\
\hline 1900 & 4.37689 & 2253 & 3.95555 & 2164 & 26.44614 & 20346 & 30.40169 & 22510 \\
\hline 2000 & 4.39942 & 2043 & 3.9 & 2061 & 26.64960 & 19455 & 30.62679 & 21516 \\
\hline 2100 & 4.41985 & 1861 & 3.99780 & 1960 & 26.84415 & 18644 & 30.84195 & 20604 \\
\hline 2200 & 4.43846 & 1706 & 4.01740 & 1869 & 27.03059 & 17898 & 31.04799 & 19767 \\
\hline 2300 & 4.45552 & 1569 & 4.03609 & 1780 & 27.20957 & 17216 & 31.24566 & 18996 \\
\hline 2400 & 4.47121 & 1453 & 4.05389 & 1700 & 27.38173 & 16583 & 31.43562 & 18283 \\
\hline 2500 & 4.48574 & 1351 & 4.07089 & 1620 & 27.54756 & 16000 & 31.61845 & 17620 \\
\hline 2600 & 4.49925 & 1261 & 4.08709 & 1551 & 27.70756 & 15453 & 31.79465 & 17004 \\
\hline 2700 & 4.51186 & 1183 & 4.10260 & 1482 & 27.86209 & 14948 & 31.96469 & 16430 \\
\hline 2800 & 4.52369 & 1112 & 4.11742 & 1421 & 28.01157 & 14473 & 32.12899 & 15894 \\
\hline 2900 & 4.53481 & 1052 & 4.13163 & 1362 & 28.15630 & 14030 & 32.28793 & 15392 \\
\hline 3000 & 4.54533 & 1947 & 4.14525 & 2562 & 28.29660 & 26836 & 32.44185 & 29398 \\
\hline 3200 & 4.56480 & 1773 & 4.17087 & 2370 & 28.56496 & 25358 & 32.73583 & 27728 \\
\hline 3400 & 4.58253 & 1631 & 4.19457 & 2202 & 28.81854 & 24038 & 33.01311 & 26240 \\
\hline 3600 & 4.59884 & 1519 & 4.21659 & 2051 & 29.05892 & 22854 & 33.27551 & 24905 \\
\hline 3800 & 4.61403 & 1425 & 4.23710 & 1922 & 29.28746 & 21782 & 33.52456 & 23704 \\
\hline 4000 & 4.62828 & 1346 & 4.25632 & 1803 & 29.50528 & 20812 & 33.76160 & 22615 \\
\hline 4200 & 4.64174 & 1282 & 4.27435 & 1699 & 29.71340 & 19923 & $33.987^{\prime} 5$ & 21622 \\
\hline 4400 & 4.65456 & 1225 & 4.29134 & 1626 & 29.91263 & 19111 & 34.20397 & 20717 \\
\hline 4600 & 4.66681 & 1178 & 4.30740 & 1523 & 30.10374 & 18365 & 34.41114 & 19888 \\
\hline 480 & 4.67859 & 1137 & 4.32263 & 1446 & 30.28739 & 17676 & 34.61002 & 19122 \\
\hline & 4.68996 & & 4.33709 & & 30.46415 & & 34.80124 & \\
\hline
\end{tabular}


Table 2.097. SH

\begin{tabular}{|c|c|c|c|c|c|c|c|c|}
\hline${ }^{\circ} K$ & $\frac{C_{p}^{\sigma}}{R}$ & & $\frac{\left(H^{0}-E_{0}^{0}\right)}{R T}$ & & $\frac{-\left(F^{0}-E\right.}{R T}$ & & $\frac{S^{0}}{R}$ & \\
\hline $\begin{array}{l}50 \\
60 \\
70 \\
80 \\
90\end{array}$ & $\begin{array}{l}3.42596 \\
3.46203 \\
3.49225 \\
3.52587 \\
3.56468\end{array}$ & $\begin{array}{l}3607 \\
3022 \\
3362 \\
3881 \\
4303\end{array}$ & $\begin{array}{l}3.07447 \\
3.13623 \\
3.18495 \\
3.22539 \\
3.26090\end{array}$ & $\begin{array}{l}6176 \\
4872 \\
4044 \\
3551 \\
3248\end{array}$ & $\begin{array}{l}13.84212 \\
14.40837 \\
14.89561 \\
15.32361 \\
15.70560\end{array}$ & $\begin{array}{l}56625 \\
48724 \\
42800 \\
38199 \\
34529\end{array}$ & $\begin{array}{l}16.91659 \\
17.54460 \\
18.08056 \\
18.54900 \\
18.96650\end{array}$ & $\begin{array}{l}62801 \\
53596 \\
46844 \\
41750 \\
37777\end{array}$ \\
\hline $\begin{array}{l}100 \\
110 \\
120 \\
130 \\
140\end{array}$ & $\begin{array}{l}3.60771 \\
3.65267 \\
3.69727 \\
3.73955 \\
3.77814\end{array}$ & $\begin{array}{l}4496 \\
4460 \\
4228 \\
3859 \\
3402\end{array}$ & $\begin{array}{l}3.29338 \\
3.32402 \\
3.35327 \\
3.38137 \\
3.40835\end{array}$ & $\begin{array}{l}3064 \\
2925 \\
2810 \\
2698 \\
2582\end{array}$ & $\begin{array}{l}16.05089 \\
16.36621 \\
16.65670 \\
16.92620 \\
17.17782\end{array}$ & $\begin{array}{l}31532 \\
29049 \\
26950 \\
25162 \\
23603\end{array}$ & $\begin{array}{l}19.34427 \\
19.69023 \\
20.00997 \\
20.30757 \\
20.58617\end{array}$ & $\begin{array}{l}34596 \\
31974 \\
29760 \\
27860 \\
26185\end{array}$ \\
\hline $\begin{array}{l}150 \\
160 \\
170 \\
180 \\
190\end{array}$ & $\begin{array}{l}3.81216 \\
3.84122 \\
3.86524 \\
3.88443 \\
3.89915\end{array}$ & $\begin{array}{l}2906 \\
2402 \\
1919 \\
1472 \\
1062\end{array}$ & $\begin{array}{l}3.43417 \\
3.45873 \\
3.48197 \\
3.50382 \\
3.52425\end{array}$ & $\begin{array}{l}2456 \\
2324 \\
2185 \\
2043 \\
1902\end{array}$ & $\begin{array}{l}17.41385 \\
17.63628 \\
17.84667 \\
18.04631 \\
18.23631\end{array}$ & $\begin{array}{l}22243 \\
21039 \\
19964 \\
19000 \\
18126\end{array}$ & $\begin{array}{l}20.84802 \\
21.09501 \\
21.32864 \\
21.55013 \\
21.76056\end{array}$ & $\begin{array}{l}24699 \\
23363 \\
22149 \\
21043 \\
20028\end{array}$ \\
\hline $\begin{array}{l}200 \\
210 \\
220 \\
230 \\
240\end{array}$ & $\begin{array}{l}3.90977 \\
3.91700 \\
3.92134 \\
3.92266 \\
3.92166\end{array}$ & $\begin{array}{r}723 \\
434 \\
132 \\
-\quad 100 \\
-\quad 290\end{array}$ & $\begin{array}{l}3.54327 \\
3.56094 \\
3.57724 \\
3.59224 \\
3.60600\end{array}$ & $\begin{array}{l}1767 \\
1630 \\
1500 \\
1376 \\
1257\end{array}$ & $\begin{array}{l}18.41757 \\
18.59015 \\
18.75559 \\
18.91493 \\
19.06811\end{array}$ & $\begin{array}{l}17258 \\
16544 \\
15934 \\
15318 \\
14747\end{array}$ & $\begin{array}{l}21.96084 \\
22.15109 \\
22.33283 \\
22.50717 \\
22.67411\end{array}$ & $\begin{array}{l}19025 \\
18174 \\
17434 \\
16694 \\
16004\end{array}$ \\
\hline $\begin{array}{l}250 \\
260 \\
270 \\
280 \\
290\end{array}$ & $\begin{array}{l}3.91876 \\
3.91435 \\
3.90869 \\
3.90209 \\
3.89472\end{array}$ & $\begin{array}{l}-441 \\
-566 \\
-660 \\
-737 \\
-787\end{array}$ & $\begin{array}{l}3.61857 \\
3.63004 \\
3.64046 \\
3.64993 \\
3.65850\end{array}$ & $\begin{array}{r}1147 \\
1042 \\
947 \\
857 \\
774\end{array}$ & $\begin{array}{l}19.21558 \\
19.35773 \\
19.49492 \\
19.62749 \\
19.75572\end{array}$ & $\begin{array}{l}14215 \\
13719 \\
13257 \\
12823 \\
12417\end{array}$ & $\begin{array}{l}22.83415 \\
22.98777 \\
23.13538 \\
23.27742 \\
23.41422\end{array}$ & $\begin{array}{l}15362 \\
14761 \\
14204 \\
13680 \\
13191\end{array}$ \\
\hline $\begin{array}{l}300 \\
310 \\
320 \\
330 \\
340\end{array}$ & $\begin{array}{l}3.88685 \\
3.87862 \\
3.87014 \\
3.86159 \\
3.85303\end{array}$ & $\begin{array}{l}-823 \\
-848 \\
-855 \\
-856 \\
-846\end{array}$ & $\begin{array}{l}3.66624 \\
3.67323 \\
3.67952 \\
3.68516 \\
3.69022\end{array}$ & $\begin{array}{l}699 \\
629 \\
564 \\
506 \\
453\end{array}$ & $\begin{array}{l}19.87989 \\
20.00021 \\
20.11693 \\
20.23025 \\
20.34035\end{array}$ & $\begin{array}{l}12032 \\
11672 \\
11332 \\
11010 \\
10703\end{array}$ & $\begin{array}{l}23.54613 \\
23.67344 \\
23.79645 \\
23.91541 \\
24.03057\end{array}$ & $\begin{array}{l}12731 \\
12301 \\
11896 \\
11516 \\
11156\end{array}$ \\
\hline $\begin{array}{l}350 \\
360 \\
370 \\
380 \\
390\end{array}$ & $\begin{array}{l}3.84457 \\
3.83629 \\
3.82825 \\
3.82049 \\
3.81306\end{array}$ & $\begin{array}{l}-828 \\
-804 \\
-776 \\
-743 \\
-707\end{array}$ & $\begin{array}{l}3.69475 \\
3.69880 \\
3.70241 \\
3.70562 \\
3.70846\end{array}$ & $\begin{array}{l}405 \\
361 \\
321 \\
284 \\
253\end{array}$ & $\begin{array}{l}20.44738 \\
20.55152 \\
20.65291 \\
20.75170 \\
20.84799\end{array}$ & $\begin{array}{r}10414 \\
10139 \\
9879 \\
9629 \\
9392\end{array}$ & $\begin{array}{l}24.14213 \\
24.25032 \\
24.35532 \\
24.45732 \\
24.55645\end{array}$ & $\begin{array}{r}10819 \\
10500 \\
10200 \\
9913 \\
9645\end{array}$ \\
\hline $\begin{array}{l}400 \\
450 \\
500 \\
550 \\
600\end{array}$ & $\begin{array}{l}3.80599 \\
3.77690 \\
3.75911 \\
3.75239 \\
3.75551\end{array}$ & $\begin{array}{r}-2909 \\
-1779 \\
-\quad 672 \\
312 \\
1133\end{array}$ & $\begin{array}{l}3.71099 \\
3.71983 \\
3.72455 \\
3.72731 \\
3.72947\end{array}$ & $\begin{array}{l}884 \\
472 \\
276 \\
216 \\
238\end{array}$ & $\begin{array}{l}20.94191 \\
21.37957 \\
21.77177 \\
22.12691 \\
22.45129\end{array}$ & $\begin{array}{l}43766 \\
39220 \\
35514 \\
32438 \\
29862\end{array}$ & $\begin{array}{l}24.65290 \\
25.09940 \\
25.49632 \\
25.85422 \\
26.18076\end{array}$ & $\begin{array}{l}44650 \\
39692 \\
35790 \\
32654 \\
30100\end{array}$ \\
\hline $\begin{array}{l}650 \\
700 \\
750 \\
800 \\
850\end{array}$ & $\begin{array}{l}3.76684 \\
3.78465 \\
3.80733 \\
3.83346 \\
3.86190\end{array}$ & $\begin{array}{l}1781 \\
2268 \\
2613 \\
2844 \\
2978\end{array}$ & $\begin{array}{l}3.73185 \\
3.73496 \\
3.73900 \\
3.74408 \\
3.75016\end{array}$ & $\begin{array}{l}311 \\
404 \\
508 \\
608 \\
704\end{array}$ & $\begin{array}{l}22.74991 \\
23.02658 \\
23.28440 \\
23.52586 \\
23.75302\end{array}$ & $\begin{array}{l}27667 \\
25782 \\
24146 \\
22716 \\
21456 \\
\end{array}$ & $\begin{array}{l}26.48176 \\
26.76154 \\
27.02340 \\
27.26994 \\
27.50318\end{array}$ & $\begin{array}{l}27978 \\
26186 \\
24654 \\
23324 \\
22160\end{array}$ \\
\hline $\begin{array}{r}900 \\
950 \\
1000 \\
1050 \\
1100\end{array}$ & $\begin{array}{l}3.89168 \\
3.92206 \\
3.95250 \\
3.98257 \\
4.01198\end{array}$ & $\begin{array}{l}3038 \\
3044 \\
3007 \\
2941 \\
2854\end{array}$ & $\begin{array}{l}3.75720 \\
3.76507 \\
3.77369 \\
3.78292 \\
3.79266\end{array}$ & $\begin{array}{r}787 \\
862 \\
923 \\
974 \\
1016\end{array}$ & $\begin{array}{l}23.96758 \\
24.17092 \\
24.36426 \\
24.54860 \\
24.72481\end{array}$ & $\begin{array}{l}20334 \\
19334 \\
18434 \\
17621 \\
16881\end{array}$ & $\begin{array}{l}27.72478 \\
27.93599 \\
28.13795 \\
28.33152 \\
28.51747\end{array}$ & $\begin{array}{l}21121 \\
20196 \\
19357 \\
18595 \\
17897\end{array}$ \\
\hline 1150 & 4.04052 & 2754 & 3.80282 & 1047 & 24.89362 & 16206 & 28.69644 & 17253 \\
\hline
\end{tabular}


Table 2.097. SH (Cont.)

\begin{tabular}{|c|c|c|c|c|c|c|c|c|}
\hline${ }^{\circ} \mathrm{K}$ & $\frac{C_{p}^{0}}{R}$ & & $\frac{\left(H^{\circ}-E_{0}^{0}\right)}{R T}$ & & $\frac{-\left(F^{0}-\right.}{R T}$ & & $\frac{S^{\circ}}{R}$ & \\
\hline $\begin{array}{l}1200 \\
1300 \\
1400 \\
1500 \\
1600\end{array}$ & $\begin{array}{l}4.06806 \\
4.11986 \\
4.16714 \\
4.21007 \\
4.24893\end{array}$ & $\begin{array}{l}5180 \\
4728 \\
4293 \\
3886 \\
3519\end{array}$ & $\begin{array}{l}3.81329 \\
3.83492 \\
3.85699 \\
3.87912 \\
3.90104\end{array}$ & $\begin{array}{l}2163 \\
2207 \\
2213 \\
2192 \\
2151\end{array}$ & $\begin{array}{l}25.05568 \\
25.36176 \\
25.64677 \\
25.91362 \\
26.16468\end{array}$ & $\begin{array}{l}30608 \\
28501 \\
26635 \\
25106 \\
23715\end{array}$ & $\begin{array}{l}28.86897 \\
29.19668 \\
29.50376 \\
29.79274 \\
30.06572\end{array}$ & $\begin{array}{l}32771 \\
30708 \\
28898 \\
27298 \\
25866\end{array}$ \\
\hline $\begin{array}{l}1700 \\
1800 \\
1900 \\
2000 \\
2100\end{array}$ & $\begin{array}{l}4.28412 \\
4.31603 \\
4.34504 \\
4.37151 \\
4.39573\end{array}$ & $\begin{array}{l}3191 \\
2901 \\
2647 \\
2422 \\
2225\end{array}$ & $\begin{array}{l}3.92255 \\
3.94354 \\
3.96393 \\
3.98365 \\
4.00270\end{array}$ & $\begin{array}{l}2099 \\
2039 \\
1972 \\
1905 \\
1839\end{array}$ & $\begin{array}{l}26.40183 \\
26.62663 \\
26.84039 \\
27.04423 \\
27.23905\end{array}$ & $\begin{array}{l}22480 \\
21376 \\
20384 \\
19482 \\
18663\end{array}$ & $\begin{array}{l}30.32438 \\
30.57017 \\
30.80432 \\
31.02788 \\
31.24175\end{array}$ & $\begin{array}{l}24579 \\
23415 \\
22356 \\
21387 \\
20502\end{array}$ \\
\hline $\begin{array}{l}2200 \\
2300 \\
2400 \\
2500 \\
2600\end{array}$ & $\begin{array}{l}4.41798 \\
4.43851 \\
4.45751 \\
4.47519 \\
4.49166\end{array}$ & $\begin{array}{l}2053 \\
1900 \\
1768 \\
1647 \\
1546\end{array}$ & $\begin{array}{l}4.02109 \\
4.03879 \\
4.05585 \\
4.07227 \\
4.08809\end{array}$ & $\begin{array}{l}1770 \\
1706 \\
1642 \\
1582 \\
1523\end{array}$ & $\begin{array}{l}27.42568 \\
27.60482 \\
27.77707 \\
27.94298 \\
28.10301\end{array}$ & $\begin{array}{l}17914 \\
17225 \\
16591 \\
16003 \\
15457\end{array}$ & $\begin{array}{l}31.44677 \\
31.64361 \\
31.83292 \\
32.01525 \\
32.19110\end{array}$ & $\begin{array}{l}19684 \\
18931 \\
18233 \\
17585 \\
16980\end{array}$ \\
\hline $\begin{array}{l}2700 \\
2800 \\
2900 \\
3000\end{array}$ & $\begin{array}{l}4.50712 \\
4.52163 \\
4.53532 \\
4.54828\end{array}$ & $\begin{array}{l}1451 \\
1369 \\
1296 \\
2401\end{array}$ & $\begin{array}{l}4.10332 \\
4.11800 \\
4.13216 \\
4.14582\end{array}$ & $\begin{array}{l}1468 \\
1416 \\
1366 \\
2591\end{array}$ & $\begin{array}{l}28.25758 \\
28.40708 \\
28.55184 \\
28.69216\end{array}$ & $\begin{array}{l}14950 \\
14476 \\
14032 \\
26840\end{array}$ & $\begin{array}{l}32.36090 \\
32.52508 \\
32.68400 \\
32.83798\end{array}$ & $\begin{array}{l}16418 \\
15892 \\
15398 \\
29431\end{array}$ \\
\hline $\begin{array}{l}3200 \\
3400 \\
3600 \\
3800 \\
4000\end{array}$ & $\begin{array}{l}4.57229 \\
4.59418 \\
4.61433 \\
4.63306 \\
4.65061\end{array}$ & $\begin{array}{l}2189 \\
2015 \\
1873 \\
1755 \\
1655\end{array}$ & $\begin{array}{l}4.17173 \\
4.19595 \\
4.21864 \\
4.23996 \\
4.26006\end{array}$ & $\begin{array}{l}2422 \\
2269 \\
2132 \\
2010 \\
1899\end{array}$ & $\begin{array}{l}28.96056 \\
29.21420 \\
29.45469 \\
29.68336 \\
29.90135\end{array}$ & $\begin{array}{l}25364 \\
24049 \\
22867 \\
21799 \\
20832\end{array}$ & $\begin{array}{l}33.13229 \\
33.41015 \\
33.67333 \\
33.92332 \\
34.16141\end{array}$ & $\begin{array}{l}27786 \\
26318 \\
24999 \\
23809 \\
22731\end{array}$ \\
\hline $\begin{array}{l}4200 \\
4400 \\
4600 \\
4800 \\
5000\end{array}$ & $\begin{array}{l}4.66716 \\
4.68288 \\
4.69789 \\
4.71228 \\
4.72613\end{array}$ & $\begin{array}{l}1572 \\
1501 \\
1439 \\
1385\end{array}$ & $\begin{array}{l}4.27905 \\
4.29706 \\
4.31416 \\
4.33045 \\
4.34601\end{array}$ & $\begin{array}{l}1801 \\
1710 \\
1629 \\
1556\end{array}$ & $\begin{array}{l}30.10967 \\
30.30914 \\
30.50054 \\
30.68449 \\
30.86159\end{array}$ & $\begin{array}{l}19947 \\
19140 \\
18395 \\
17710\end{array}$ & $\begin{array}{l}34.38872 \\
34.60620 \\
34.81470 \\
35.01494 \\
35.20760\end{array}$ & $\begin{array}{l}21748 \\
20850 \\
20024 \\
19266\end{array}$ \\
\hline
\end{tabular}


Table 2.098. SD

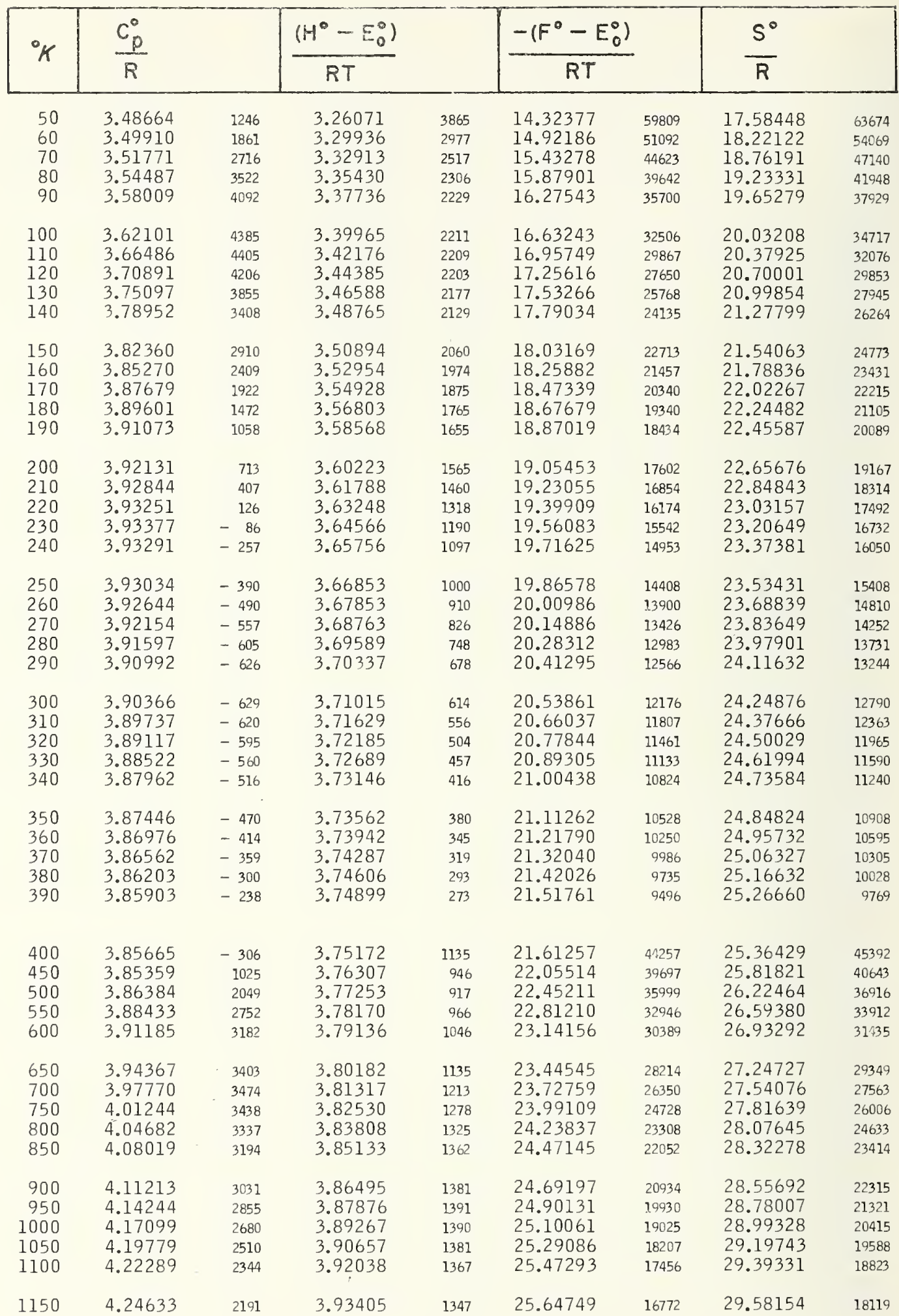


Table 2.098. SD (Cont.)

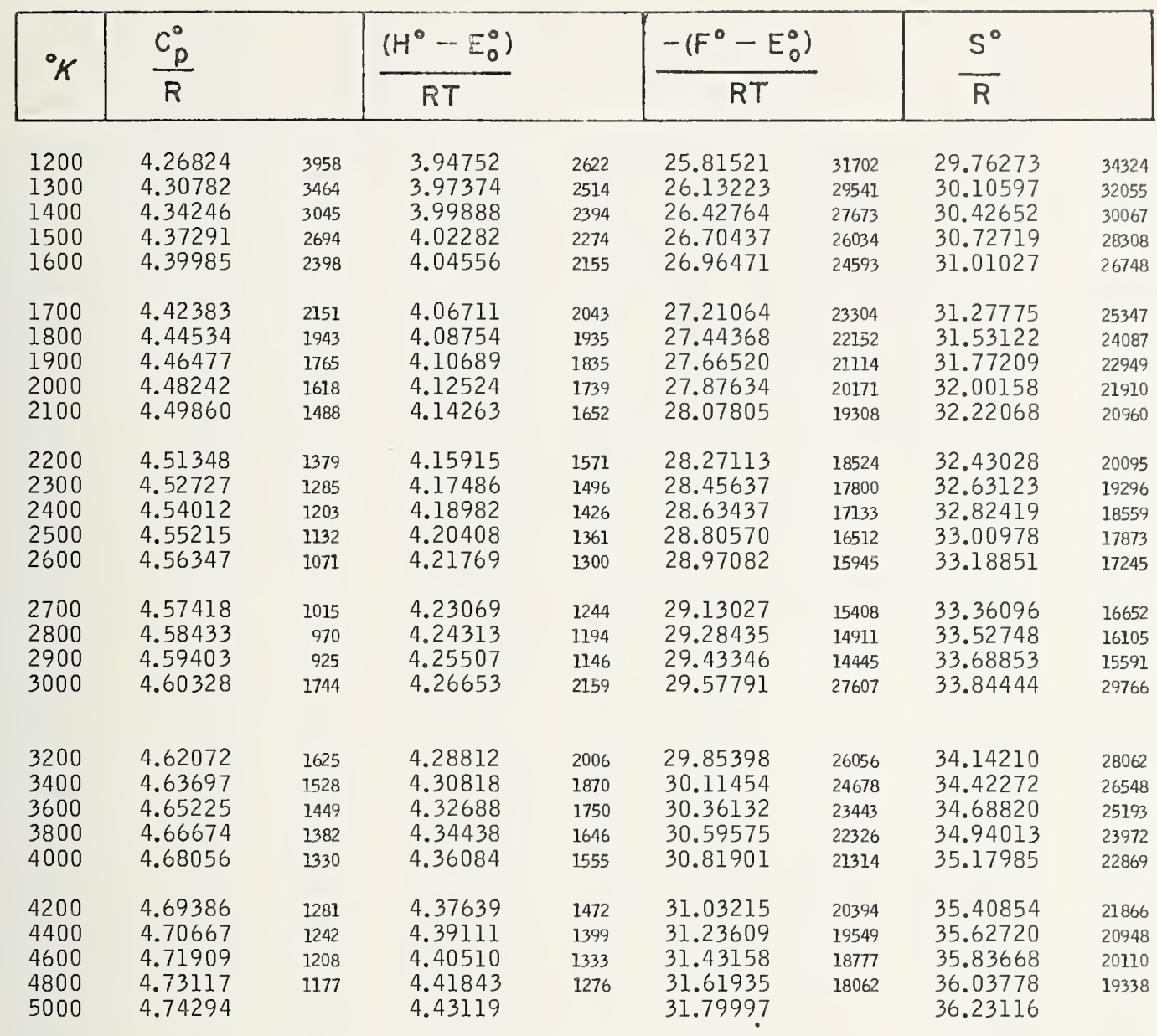


Table 2.099 ST

\begin{tabular}{|c|c|c|c|c|c|c|c|c|}
\hline${ }^{\circ} K$ & $\frac{C_{p}^{0}}{R}$ & & $\frac{\left(H^{0}-E_{0}^{0}\right)}{R T}$ & & $\frac{-F^{\circ}-E}{R T}$ & & $\frac{S^{0}}{R}$ & \\
\hline $\begin{array}{l}50 \\
60 \\
70 \\
80 \\
90\end{array}$ & $\begin{array}{l}3.49544 \\
3.50506 \\
3.52224 \\
3.54870 \\
3.58359\end{array}$ & $\begin{array}{r}962 \\
1718 \\
2646 \\
3489 \\
4091\end{array}$ & $\begin{array}{l}3.32989 \\
3.35821 \\
3.38031 \\
3.39960 \\
3.41804\end{array}$ & $\begin{array}{l}2832 \\
2210 \\
1929 \\
1844 \\
1856\end{array}$ & $\begin{array}{l}14.65352 \\
15.26327 \\
15.78265 \\
16.23532 \\
16.63681\end{array}$ & $\begin{array}{l}60975 \\
51938 \\
45267 \\
40149 \\
36110\end{array}$ & $\begin{array}{l}17.98341 \\
18.62148 \\
19.16296 \\
19.63492 \\
20.05485\end{array}$ & $\begin{array}{l}63807 \\
54148 \\
47196 \\
41993 \\
37966\end{array}$ \\
\hline $\begin{array}{l}100 \\
110 \\
120 \\
130 \\
140\end{array}$ & $\begin{array}{l}3.62450 \\
3.66840 \\
3.71257 \\
3.75473 \\
3.79338\end{array}$ & $\begin{array}{l}4390 \\
4417 \\
4216 \\
3865 \\
3414\end{array}$ & $\begin{array}{l}3.43660 \\
3.45567 \\
3.47524 \\
3.49512 \\
3.51508\end{array}$ & $\begin{array}{l}1907 \\
1957 \\
1988 \\
1996 \\
1970\end{array}$ & $\begin{array}{l}16.99791 \\
17.32637 \\
17.62792 \\
17.90691 \\
18.16671\end{array}$ & $\begin{array}{l}32846 \\
30155 \\
27899 \\
25980 \\
24323\end{array}$ & $\begin{array}{l}20.43451 \\
20.78204 \\
21.10316 \\
21.40203 \\
21.68179\end{array}$ & $\begin{array}{l}34753 \\
32112 \\
29887 \\
27976 \\
26293\end{array}$ \\
\hline $\begin{array}{l}150 \\
160 \\
170 \\
180 \\
190\end{array}$ & $\begin{array}{l}3.82752 \\
3.85670 \\
3.88086 \\
3.90019 \\
3.91513\end{array}$ & $\begin{array}{l}2918 \\
2416 \\
1933 \\
1494 \\
1099\end{array}$ & $\begin{array}{l}3.53478 \\
3.55400 \\
3.57254 \\
3.59022 \\
3.60692\end{array}$ & $\begin{array}{l}1922 \\
1854 \\
1768 \\
1670 \\
1569\end{array}$ & $\begin{array}{l}18.40994 \\
18.63876 \\
18.85483 \\
19.05961 \\
19.25426\end{array}$ & $\begin{array}{l}22882 \\
21607 \\
20478 \\
19465 \\
18548\end{array}$ & $\begin{array}{l}21.94472 \\
22.19276 \\
22.42737 \\
22.64983 \\
22.86118\end{array}$ & $\begin{array}{l}24804 \\
23461 \\
22246 \\
21135 \\
20117\end{array}$ \\
\hline $\begin{array}{l}200 \\
210 \\
220 \\
230 \\
240\end{array}$ & $\begin{array}{l}3.92612 \\
3.93388 \\
3.93871 \\
3.94115 \\
3.94176\end{array}$ & $\begin{array}{r}776 \\
483 \\
244 \\
-\quad 61 \\
-\quad 82\end{array}$ & $\begin{array}{l}3.62261 \\
3.63743 \\
3.65122 \\
3.66377 \\
3.67535\end{array}$ & $\begin{array}{l}1482 \\
1379 \\
1255 \\
1158 \\
1064\end{array}$ & $\begin{array}{l}19.43974 \\
19.61629 \\
19.78500 \\
19.94742 \\
20.10399\end{array}$ & $\begin{array}{l}17655 \\
16871 \\
16242 \\
15657 \\
15045\end{array}$ & $\begin{array}{l}23.06235 \\
23.25372 \\
23.43622 \\
23.61119 \\
23.77934\end{array}$ & $\begin{array}{l}19137 \\
18250 \\
17497 \\
16815 \\
16109\end{array}$ \\
\hline $\begin{array}{l}250 \\
260 \\
270 \\
280 \\
290\end{array}$ & $\begin{array}{l}3.94094 \\
3.93908 \\
3.93655 \\
3.93362 \\
3.93051\end{array}$ & $\begin{array}{l}-186 \\
-253 \\
-293 \\
-311 \\
-306\end{array}$ & $\begin{array}{l}3.68599 \\
3.69577 \\
3.70472 \\
3.71297 \\
3.72052\end{array}$ & $\begin{array}{l}978 \\
895 \\
825 \\
755 \\
695\end{array}$ & $\begin{array}{l}20.25444 \\
20.39900 \\
20.53866 \\
20.67354 \\
20.80397\end{array}$ & $\begin{array}{l}14456 \\
13966 \\
13488 \\
13043 \\
12624\end{array}$ & $\begin{array}{l}23.94043 \\
24.09477 \\
24.24338 \\
24.38651 \\
24.52449\end{array}$ & $\begin{array}{l}15434 \\
14861 \\
14313 \\
13798 \\
13319\end{array}$ \\
\hline $\begin{array}{l}300 \\
310 \\
320 \\
330 \\
340\end{array}$ & $\begin{array}{l}3.92745 \\
3.92459 \\
3.92204 \\
3.91991 \\
3.91828\end{array}$ & $\begin{array}{l}-286 \\
-255 \\
-213 \\
-163 \\
-110\end{array}$ & $\begin{array}{l}3.72747 \\
3.73387 \\
3.73979 \\
3.74527 \\
3.75040\end{array}$ & $\begin{array}{l}640 \\
592 \\
548 \\
513 \\
477\end{array}$ & $\begin{array}{l}20.93021 \\
21.05254 \\
21.17114 \\
21.28635 \\
21.39823\end{array}$ & $\begin{array}{l}12233 \\
11860 \\
11521 \\
11188 \\
10879\end{array}$ & $\begin{array}{l}24.65768 \\
24.78641 \\
24.91093 \\
25.03162 \\
25.14863\end{array}$ & $\begin{array}{l}12873 \\
12452 \\
12069 \\
11701 \\
11356\end{array}$ \\
\hline $\begin{array}{l}350 \\
360 \\
370 \\
380 \\
390\end{array}$ & $\begin{array}{l}3.91718 \\
3.91665 \\
3.91671 \\
3.91735 \\
3.91857\end{array}$ & $\begin{array}{r}-53 \\
6 \\
64 \\
122 \\
178\end{array}$ & $\begin{array}{l}3.75517 \\
3.75966 \\
3.76391 \\
3.76793 \\
3.77178\end{array}$ & $\begin{array}{l}449 \\
425 \\
402 \\
385 \\
369\end{array}$ & $\begin{array}{l}21.50702 \\
21.61287 \\
21.71594 \\
21.81637 \\
21.91429\end{array}$ & $\begin{array}{r}10585 \\
10307 \\
10043 \\
9792 \\
9554\end{array}$ & $\begin{array}{l}25.26219 \\
25.37253 \\
25.47985 \\
25.58430 \\
25.68607\end{array}$ & $\begin{array}{r}11034 \\
10732 \\
10445 \\
10177 \\
9923\end{array}$ \\
\hline $\begin{array}{l}400 \\
450 \\
500 \\
550 \\
600\end{array}$ & $\begin{array}{l}3.92035 \\
3.93692 \\
3.96340 \\
3.99599 \\
4.03161\end{array}$ & $\begin{array}{l}1657 \\
2648 \\
3259 \\
3562 \\
3643\end{array}$ & $\begin{array}{l}3.77547 \\
3.79238 \\
3.80809 \\
3.82365 \\
3.83949\end{array}$ & $\begin{array}{l}1691 \\
1571 \\
1556 \\
1584 \\
1619\end{array}$ & $\begin{array}{l}22.00983 \\
22.45551 \\
22.85590 \\
23.21958 \\
23.55296\end{array}$ & $\begin{array}{l}44568 \\
40039 \\
36368 \\
33338 \\
30795\end{array}$ & $\begin{array}{l}25.78530 \\
26.24789 \\
26.66399 \\
27.04323 \\
27.39245\end{array}$ & $\begin{array}{l}46259 \\
41610 \\
37924 \\
34922 \\
32414\end{array}$ \\
\hline $\begin{array}{l}650 \\
700 \\
750 \\
800 \\
850\end{array}$ & $\begin{array}{l}4.06804 \\
4.10387 \\
4.13823 \\
4.17067 \\
4.20098\end{array}$ & $\begin{array}{l}3583 \\
3436 \\
3244 \\
3031 \\
2814\end{array}$ & $\begin{array}{l}3.85568 \\
3.87213 \\
3.88873 \\
3.90535 \\
3.92185\end{array}$ & $\begin{array}{l}1645 \\
1660 \\
1662 \\
1650 \\
1631\end{array}$ & $\begin{array}{l}23.86091 \\
24.14727 \\
24.41497 \\
24.66647 \\
24.90373\end{array}$ & $\begin{array}{l}28636 \\
26770 \\
25150 \\
23726 \\
22463\end{array}$ & $\begin{array}{l}27.71659 \\
28.01940 \\
28.30370 \\
28.57182 \\
28.82558\end{array}$ & $\begin{array}{l}30281 \\
28430 \\
26812 \\
25376 \\
24094\end{array}$ \\
\hline $\begin{array}{r}900 \\
950 \\
1000 \\
1050 \\
1100\end{array}$ & $\begin{array}{l}4.22912 \\
4.25514 \\
4.27916 \\
4.30133 \\
4.32179\end{array}$ & $\begin{array}{l}2602 \\
2402 \\
2217 \\
2046 \\
1889\end{array}$ & $\begin{array}{l}3.93816 \\
3.95417 \\
3.96982 \\
3.98509 \\
3.99994\end{array}$ & $\begin{array}{l}1601 \\
1565 \\
1527 \\
1485 \\
1440\end{array}$ & $\begin{array}{l}25.12836 \\
25.34171 \\
25.54494 \\
25.73899 \\
25.92472\end{array}$ & $\begin{array}{l}21335 \\
20323 \\
19405 \\
18573 \\
17813\end{array}$ & $\begin{array}{l}29.06652 \\
29.29588 \\
29.51476 \\
29.72408 \\
29.92466\end{array}$ & $\begin{array}{l}22936 \\
21888 \\
20932 \\
20058 \\
19253\end{array}$ \\
\hline 1150 & 4.34068 & 1748 & 4.01434 & 1397 & 26.10285 & 17114 & 30.11719 & 18511 \\
\hline
\end{tabular}


Table 2.099. ST (Cont.)

\begin{tabular}{|c|c|c|c|c|c|c|c|c|}
\hline${ }^{\circ} K$ & $\frac{C_{p}^{0}}{R}$ & & $\frac{\left(H^{0}-E_{0}^{0}\right)}{R T}$ & & $\frac{-\left(F^{0}-E\right.}{R T}$ & & $\frac{S^{\circ}}{R}$ & \\
\hline $\begin{array}{l}1200 \\
1300 \\
1400 \\
1500 \\
1600\end{array}$ & $\begin{array}{l}4.35816 \\
4.38943 \\
4.41650 \\
4.44020 \\
4.46112\end{array}$ & $\begin{array}{l}3127 \\
2707 \\
2370 \\
2092 \\
1865\end{array}$ & $\begin{array}{l}4.02831 \\
4.05492 \\
4.07980 \\
4.10305 \\
4.12479\end{array}$ & $\begin{array}{l}2661 \\
2488 \\
2325 \\
2174 \\
2034\end{array}$ & $\begin{array}{l}26.27399 \\
26.59750 \\
26.89893 \\
27.18121 \\
27.44671\end{array}$ & $\begin{array}{l}32351 \\
30143 \\
28228 \\
26550 \\
25067\end{array}$ & $\begin{array}{l}30.30230 \\
30.65242 \\
30.97873 \\
31.28426 \\
31.57150\end{array}$ & $\begin{array}{l}35012 \\
32631 \\
30553 \\
28724 \\
27101\end{array}$ \\
\hline $\begin{array}{l}1700 \\
1800 \\
1900 \\
2000 \\
2100\end{array}$ & $\begin{array}{l}4.47977 \\
4.49656 \\
4.51179 \\
4.52572 \\
4.53857\end{array}$ & $\begin{array}{l}1679 \\
1523 \\
1393 \\
1285 \\
1193\end{array}$ & $\begin{array}{l}4.14513 \\
4.16419 \\
4.18210 \\
4.19893 \\
4.21480\end{array}$ & $\begin{array}{l}1906 \\
1791 \\
1683 \\
1587 \\
1500\end{array}$ & $\begin{array}{l}27.69738 \\
27.93487 \\
28.16050 \\
28.37545 \\
28.58070\end{array}$ & $\begin{array}{l}23749 \\
22563 \\
21495 \\
20525 \\
19642\end{array}$ & $\begin{array}{l}31.84251 \\
32.09906 \\
32.34260 \\
32.57438 \\
32.79550\end{array}$ & $\begin{array}{l}25655 \\
24354 \\
23178 \\
22112 \\
21142\end{array}$ \\
\hline $\begin{array}{l}2200 \\
2300 \\
2400 \\
2500 \\
2600\end{array}$ & $\begin{array}{l}4.55050 \\
4.56165 \\
4.57213 \\
4.58204 \\
4.59144\end{array}$ & $\begin{array}{r}1115 \\
1048 \\
991 \\
940 \\
897\end{array}$ & $\begin{array}{l}4.22980 \\
4.24398 \\
4.25744 \\
4.27023 \\
4.28240\end{array}$ & $\begin{array}{l}1418 \\
1346 \\
1279 \\
1217 \\
1161\end{array}$ & $\begin{array}{l}28.77712 \\
28.96546 \\
29.14636 \\
29.32043 \\
29.48815\end{array}$ & $\begin{array}{l}18834 \\
18090 \\
17407 \\
16772 \\
16184\end{array}$ & $\begin{array}{l}33.00692 \\
33.20944 \\
33.40380 \\
33.59066 \\
33.77055\end{array}$ & $\begin{array}{l}20252 \\
19436 \\
18686 \\
17989 \\
17345\end{array}$ \\
\hline $\begin{array}{l}2700 \\
2800 \\
2900 \\
3000\end{array}$ & $\begin{array}{l}4.60041 \\
4.60900 \\
4.61726 \\
4.62524\end{array}$ & $\begin{array}{r}859 \\
826 \\
798 \\
1518\end{array}$ & $\begin{array}{l}4.29401 \\
4.30511 \\
4.31574 \\
4.32592\end{array}$ & $\begin{array}{l}1110 \\
1063 \\
1018 \\
1918\end{array}$ & $\begin{array}{l}29.64999 \\
29.80635 \\
29.95761 \\
30.10410\end{array}$ & $\begin{array}{l}15636 \\
15126 \\
14649 \\
27981\end{array}$ & $\begin{array}{l}33.94400 \\
34.11146 \\
34.27335 \\
34.43002\end{array}$ & $\begin{array}{l}16746 \\
16189 \\
15667 \\
29899\end{array}$ \\
\hline $\begin{array}{l}3200 \\
3400 \\
3600 \\
3800 \\
4000\end{array}$ & $\begin{array}{l}4.64042 \\
4.65477 \\
4.66845 \\
4.68157 \\
4.69424\end{array}$ & $\begin{array}{l}1435 \\
1368 \\
1312 \\
1267 \\
1229\end{array}$ & $\begin{array}{l}4.34510 \\
4.36290 \\
4.37950 \\
4.39505 \\
4.40968\end{array}$ & $\begin{array}{l}1780 \\
1660 \\
1555 \\
1463 \\
1387\end{array}$ & $\begin{array}{l}30.38391 \\
30.64788 \\
30.89772 \\
31.13493 \\
31.36075\end{array}$ & $\begin{array}{l}26397 \\
24984 \\
23721 \\
22582 \\
21548\end{array}$ & $\begin{array}{l}34.72901 \\
35.01078 \\
35.27722 \\
35.52998 \\
35.77043\end{array}$ & $\begin{array}{l}28177 \\
26644 \\
25276 \\
24045 \\
22935\end{array}$ \\
\hline $\begin{array}{l}4200 \\
4400 \\
4600 \\
4800 \\
5000\end{array}$ & $\begin{array}{l}4.70653 \\
4.71847 \\
4.73014 \\
4.74156 \\
4.75275\end{array}$ & $\begin{array}{l}1194 \\
1167 \\
1142 \\
1119\end{array}$ & $\begin{array}{l}4.42355 \\
4.43668 \\
4.44918 \\
4.46112 \\
4.47257\end{array}$ & $\begin{array}{l}1313 \\
1250 \\
1194 \\
1145\end{array}$ & $\begin{array}{l}31.57623 \\
31.78232 \\
31.97982 \\
32.16943 \\
32.35176\end{array}$ & $\begin{array}{l}20609 \\
19750 \\
18961 \\
18233\end{array}$ & $\begin{array}{l}35.99978 \\
36.21900 \\
36.42900 \\
36.63055 \\
36.82433\end{array}$ & $\begin{array}{l}21922 \\
21000 \\
20155 \\
19378\end{array}$ \\
\hline
\end{tabular}


Table 2.100. HF

\begin{tabular}{|c|c|c|c|c|c|c|c|c|}
\hline ik & $\frac{C_{p}^{0}}{R}$ & & $\frac{\left(H^{\circ}-E\right.}{R T}$ & & $\frac{-\left(F^{0}-E\right.}{R T}$ & & $\frac{S^{0}}{R}$ & \\
\hline $\begin{array}{l}50 \\
60 \\
70 \\
80 \\
90\end{array}$ & $\begin{array}{l}3.50941 \\
3.50701 \\
3.50555 \\
3.50463 \\
3.50404\end{array}$ & $\begin{array}{l}-240 \\
-146 \\
-92 \\
-59 \\
-38\end{array}$ & $\begin{array}{l}3.29454 \\
3.33013 \\
3.35529 \\
3.37401 \\
3.38849\end{array}$ & $\begin{array}{l}3559 \\
2516 \\
1872 \\
1448 \\
1153\end{array}$ & $\begin{array}{l}11.33668 \\
11.94069 \\
12.45603 \\
12.90535 \\
13.30362\end{array}$ & $\begin{array}{l}60401 \\
51534 \\
44932 \\
39827 \\
35763\end{array}$ & $\begin{array}{l}14.63122 \\
15.27083 \\
15.81132 \\
16.27936 \\
16.69211\end{array}$ & $\begin{array}{l}63961 \\
54049 \\
46804 \\
41275 \\
36917\end{array}$ \\
\hline $\begin{array}{r}20 \\
10 \\
20 \\
30 \\
40\end{array}$ & $\begin{array}{l}3.50366 \\
3.50341 \\
3.50326 \\
3.50318 \\
3.50314\end{array}$ & $\begin{array}{l}-25 \\
-\quad 15 \\
-\quad 8 \\
-\quad 4\end{array}$ & $\begin{array}{l}3.40002 \\
3.40943 \\
3.41726 \\
3.42387 \\
3.42953\end{array}$ & $\begin{array}{l}941 \\
783 \\
661 \\
566 \\
491\end{array}$ & $\begin{array}{l}13.66125 \\
13.98576 \\
14.28277 \\
14.55656 \\
14.81051\end{array}$ & $\begin{array}{l}32451 \\
29701 \\
27379 \\
25395 \\
23679\end{array}$ & $\begin{array}{l}17.06128 \\
17.39520 \\
17.70003 \\
17.98043 \\
18.24005\end{array}$ & $\begin{array}{l}33392 \\
30483 \\
28040 \\
25962 \\
24169\end{array}$ \\
\hline $\begin{array}{l}50 \\
60 \\
70 \\
30 \\
90\end{array}$ & $\begin{array}{l}3.50314 \\
3.50317 \\
3.50321 \\
3.50328 \\
3.50335\end{array}$ & $\begin{array}{l}3 \\
4 \\
7 \\
7 \\
9\end{array}$ & $\begin{array}{l}3.43444 \\
3.43873 \\
3.44252 \\
3.44590 \\
3.44892\end{array}$ & $\begin{array}{l}429 \\
379 \\
338 \\
302 \\
272\end{array}$ & $\begin{array}{l}15.04730 \\
15.26909 \\
15.47768 \\
15.67454 \\
15.86094\end{array}$ & $\begin{array}{l}22179 \\
20859 \\
19686 \\
18640 \\
17697\end{array}$ & $\begin{array}{l}18.48174 \\
18.70783 \\
18.92021 \\
19.12045 \\
19.30986\end{array}$ & $\begin{array}{l}22609 \\
21238 \\
20024 \\
18941 \\
17970\end{array}$ \\
\hline $\begin{array}{l}50 \\
30 \\
40\end{array}$ & $\begin{array}{l}3.50344 \\
3.50353 \\
3.50363 \\
3.50374 \\
3.50385\end{array}$ & $\begin{array}{r}9 \\
10 \\
11 \\
11 \\
12\end{array}$ & $\begin{array}{l}3.45164 \\
3.45411 \\
3.45636 \\
3.45842 \\
3.46031\end{array}$ & $\begin{array}{l}247 \\
225 \\
206 \\
189 \\
174\end{array}$ & $\begin{array}{l}16.03791 \\
16.20638 \\
16.36712 \\
16.52081 \\
16.66304\end{array}$ & $\begin{array}{l}16847 \\
16074 \\
15369 \\
14723 \\
14129\end{array}$ & $\begin{array}{l}19.48956 \\
19.66050 \\
19.82349 \\
19.97923 \\
20.12835\end{array}$ & $\begin{array}{l}17094 \\
16299 \\
15574 \\
14912 \\
14304\end{array}$ \\
\hline $\begin{array}{l}50 \\
60 \\
70 \\
30 \\
90\end{array}$ & $\begin{array}{l}3.50397 \\
3.50409 \\
3.50421 \\
3.50434 \\
3.50446\end{array}$ & $\begin{array}{l}12 \\
12 \\
13 \\
12 \\
13\end{array}$ & $\begin{array}{l}3.46205 \\
3.46367 \\
3.46517 \\
3.46656 \\
3.46787\end{array}$ & $\begin{array}{l}162 \\
150 \\
139 \\
131 \\
122\end{array}$ & $\begin{array}{l}16.80933 \\
16.94515 \\
17.07589 \\
17.20194 \\
17.32361\end{array}$ & $\begin{array}{l}13582 \\
13074 \\
12605 \\
12167 \\
11759\end{array}$ & $\begin{array}{l}20.27139 \\
20.40882 \\
20.54107 \\
20.66851 \\
20.79148\end{array}$ & $\begin{array}{l}13743 \\
13225 \\
12744 \\
12297 \\
11881\end{array}$ \\
\hline $\begin{array}{l}20 \\
30 \\
40\end{array}$ & $\begin{array}{l}3.50459 \\
3.50472 \\
3.50486 \\
3.50499 \\
3.50513\end{array}$ & $\begin{array}{l}13 \\
14 \\
13 \\
14 \\
15\end{array}$ & $\begin{array}{l}3.46909 \\
3.47024 \\
3.47132 \\
3.47234 \\
3.47330\end{array}$ & $\begin{array}{r}115 \\
108 \\
102 \\
96 \\
91\end{array}$ & $\begin{array}{l}17.44120 \\
17.55497 \\
17.66516 \\
17.77199 \\
17.87567\end{array}$ & $\begin{array}{l}11377 \\
11019 \\
10683 \\
10368 \\
10069\end{array}$ & $\begin{array}{l}20.91029 \\
21.02521 \\
21.13648 \\
21.24433 \\
21.34897\end{array}$ & $\begin{array}{l}11492 \\
11127 \\
10785 \\
10464 \\
10161\end{array}$ \\
\hline $\begin{array}{l}70 \\
30 \\
0\end{array}$ & $\begin{array}{l}3.50528 \\
3.50542 \\
3.50558 \\
3.50573 \\
3.50590\end{array}$ & $\begin{array}{l}14 \\
16 \\
15 \\
17 \\
17\end{array}$ & $\begin{array}{l}3.47421 \\
3.47508 \\
3.47590 \\
3.47668 \\
3.47743\end{array}$ & $\begin{array}{l}87 \\
82 \\
78 \\
75 \\
71\end{array}$ & $\begin{array}{l}17.97636 \\
18.07425 \\
18.16947 \\
18.26218 \\
18.35250\end{array}$ & $\begin{array}{l}9789 \\
9522 \\
9271 \\
9032 \\
8805\end{array}$ & $\begin{array}{l}21.45058 \\
21.54933 \\
21.64537 \\
21.73886 \\
21.82993\end{array}$ & $\begin{array}{l}9875 \\
9604 \\
9349 \\
9107 \\
8876\end{array}$ \\
\hline $\begin{array}{l}50 \\
00\end{array}$ & $\begin{array}{l}3.50607 \\
3.50716 \\
3.50885 \\
3.51156 \\
3.51577\end{array}$ & $\begin{array}{l}109 \\
169 \\
271 \\
421 \\
612\end{array}$ & $\begin{array}{l}3.47814 \\
3.48130 \\
3.48396 \\
3.48634 \\
3.48861\end{array}$ & $\begin{array}{l}316 \\
266 \\
238 \\
227 \\
231\end{array}$ & $\begin{array}{l}18.44055 \\
18.85040 \\
19.21734 \\
19.54951 \\
19.85296\end{array}$ & $\begin{array}{l}40985 \\
36694 \\
33217 \\
30345 \\
27932\end{array}$ & $\begin{array}{l}21.91869 \\
22.33171 \\
22.70131 \\
23.03585 \\
23.34157\end{array}$ & $\begin{array}{l}41302 \\
36960 \\
33454 \\
30572 \\
28164\end{array}$ \\
\hline $\begin{array}{l}50 \\
00\end{array}$ & $\begin{array}{l}3.52189 \\
3.53022 \\
3.54096 \\
3.55413 \\
3.56966\end{array}$ & $\begin{array}{r}833 \\
1074 \\
1317 \\
1553 \\
1772\end{array}$ & $\begin{array}{l}3.49092 \\
3.49341 \\
3.49621 \\
3.49941 \\
3.50307\end{array}$ & $\begin{array}{l}249 \\
280 \\
320 \\
366 \\
418\end{array}$ & $\begin{array}{l}20.13228 \\
20.39108 \\
20.63219 \\
20.85793 \\
21.07019\end{array}$ & $\begin{array}{l}25880 \\
24111 \\
22574 \\
21226 \\
20035\end{array}$ & $\begin{array}{l}23.62321 \\
23.88450 \\
24.12841 \\
24.35735 \\
24.57327\end{array}$ & $\begin{array}{l}26129 \\
24391 \\
22894 \\
21592 \\
20453\end{array}$ \\
\hline In & $\begin{array}{l}3.58738 \\
3.60704 \\
3.62838 \\
3.65109 \\
3.67489\end{array}$ & $\begin{array}{l}1966 \\
2134 \\
2271 \\
2380 \\
2462\end{array}$ & $\begin{array}{l}3.50725 \\
3.51198 \\
3.51726 \\
3.52309 \\
3.52944\end{array}$ & $\begin{array}{l}473 \\
528 \\
583 \\
635 \\
686\end{array}$ & $\begin{array}{l}21.27054 \\
21.46029 \\
21.64056 \\
21.81231 \\
21.97635\end{array}$ & $\begin{array}{l}18975 \\
18027 \\
17175 \\
16404 \\
15704\end{array}$ & $\begin{array}{l}24.77780 \\
24.97228 \\
25.15783 \\
25.33540 \\
25.50580\end{array}$ & $\begin{array}{l}19448 \\
18555 \\
17757 \\
17040 \\
16389\end{array}$ \\
\hline 50 & 3.69951 & 2517 & 3.53630 & 732 & 22.13339 & 15066 & 25.66969 & 15798 \\
\hline
\end{tabular}


Table 2.100. HF (Cont.)

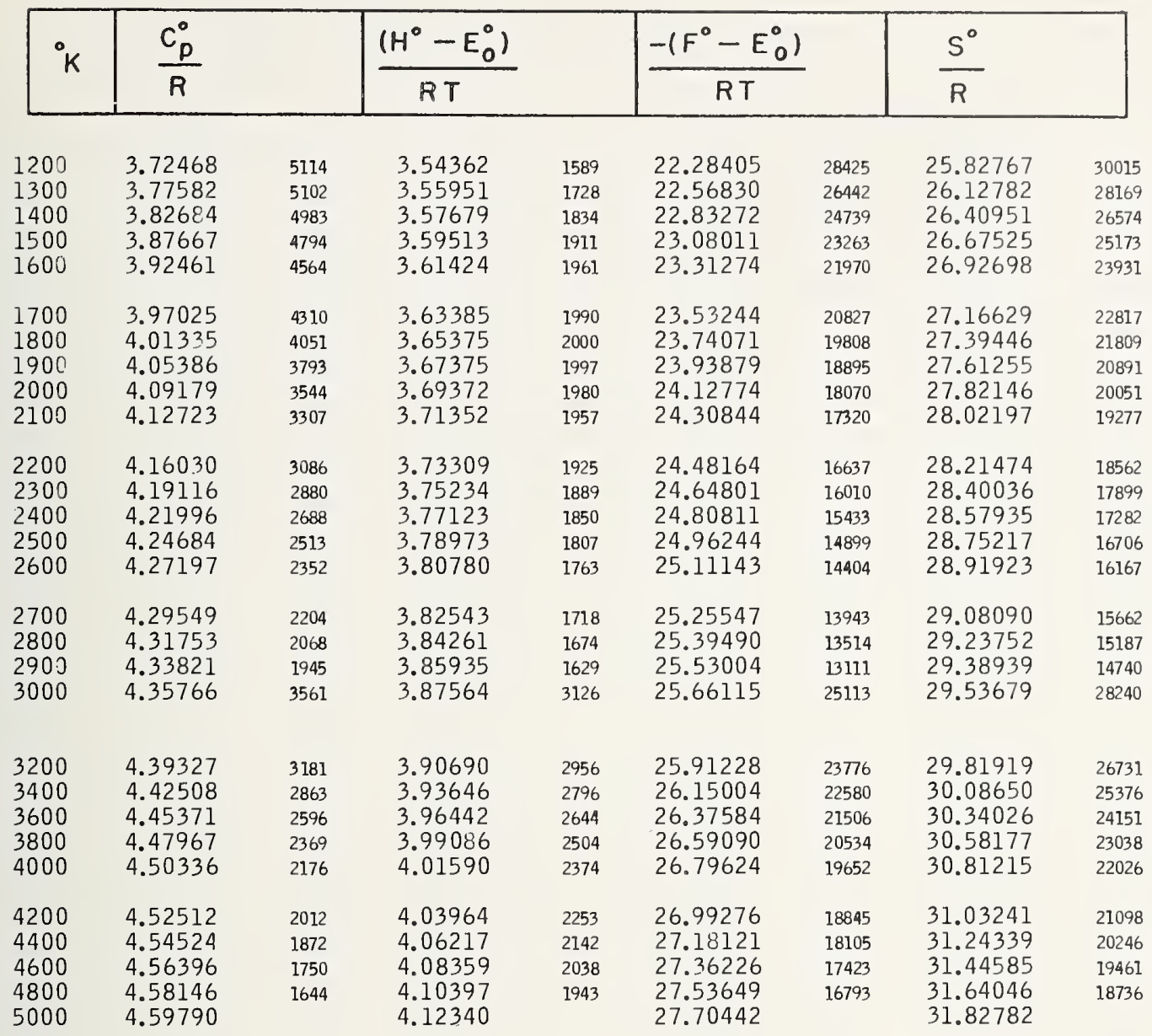


Table 2.101. DF

\begin{tabular}{|c|c|c|c|c|c|c|c|c|}
\hline${ }^{\circ} \mathrm{K}$ & $\frac{C_{p}^{0}}{R}$ & & $\frac{\left(H^{0}-E_{0}^{0}\right)}{R T}$ & & $\frac{-l F^{0}-}{R T}$ & & $\frac{S^{0}}{R}$ & \\
\hline $\begin{array}{l}50 \\
60 \\
70 \\
80 \\
90\end{array}$ & $\begin{array}{l}3.50319 \\
3.50257 \\
3.50225 \\
3.50210 \\
3.50204\end{array}$ & $\begin{array}{r}-62 \\
-\quad 32 \\
-\quad 15 \\
-\quad 6 \\
1\end{array}$ & $\begin{array}{l}3.39388 \\
3.41204 \\
3.42494 \\
3.43460 \\
3.44209\end{array}$ & $\begin{array}{r}1816 \\
1290 \\
965 \\
747 \\
600\end{array}$ & $\begin{array}{l}11.95202 \\
12.57251 \\
13.09950 \\
13.55750 \\
13.96248\end{array}$ & $\begin{array}{l}62049 \\
52699 \\
45830 \\
40498 \\
36299\end{array}$ & $\begin{array}{l}15.34590 \\
15.98455 \\
16.52445 \\
16.99210 \\
17.40458\end{array}$ & $\begin{array}{l}63865 \\
53990 \\
46765 \\
41248 \\
36998\end{array}$ \\
\hline $\begin{array}{l}100 \\
110 \\
120 \\
130 \\
140\end{array}$ & $\begin{array}{l}3.50205 \\
3.50209 \\
3.50215 \\
3.50224 \\
3.50233\end{array}$ & $\begin{array}{r}4 \\
6 \\
9 \\
9 \\
11\end{array}$ & $\begin{array}{l}3.44809 \\
3.45300 \\
3.45709 \\
3.46056 \\
3.46354\end{array}$ & $\begin{array}{l}491 \\
409 \\
347 \\
298 \\
259\end{array}$ & $\begin{array}{l}14.32547 \\
14.65434 \\
14.95497 \\
15.23183 \\
15.48840\end{array}$ & $\begin{array}{l}32887 \\
30063 \\
27686 \\
25657 \\
239 J 5\end{array}$ & $\begin{array}{l}17.77356 \\
18.10734 \\
18.41207 \\
18.69239 \\
18.95194\end{array}$ & $\begin{array}{l}33378 \\
30473 \\
28032 \\
25755 \\
24164\end{array}$ \\
\hline $\begin{array}{l}150 \\
160 \\
170 \\
180 \\
190\end{array}$ & $\begin{array}{l}3.50244 \\
3.50255 \\
3.50267 \\
3.50279 \\
3.50292\end{array}$ & $\begin{array}{l}11 \\
12 \\
12 \\
13 \\
13\end{array}$ & $\begin{array}{l}3.46613 \\
3.46840 \\
3.47041 \\
3.47221 \\
3.47382\end{array}$ & $\begin{array}{l}227 \\
201 \\
180 \\
161 \\
146\end{array}$ & $\begin{array}{l}15.72745 \\
15.95122 \\
16.16155 \\
16.35997 \\
16.54774\end{array}$ & $\begin{array}{l}22377 \\
21033 \\
19842 \\
18777 \\
17823\end{array}$ & $\begin{array}{l}19.19358 \\
19.41962 \\
19.63197 \\
19.83218 \\
20.02157\end{array}$ & $\begin{array}{l}22604 \\
21235 \\
20021 \\
18939 \\
17968\end{array}$ \\
\hline $\begin{array}{l}200 \\
210 \\
220 \\
230 \\
240\end{array}$ & $\begin{array}{l}3.50305 \\
3.50318 \\
3.50332 \\
3.50346 \\
3.50360\end{array}$ & $\begin{array}{l}13 \\
14 \\
14 \\
14 \\
14\end{array}$ & $\begin{array}{l}3.47528 \\
3.47661 \\
3.47782 \\
3.47893 \\
3.47995\end{array}$ & $\begin{array}{r}133 \\
121 \\
111 \\
102 \\
95\end{array}$ & $\begin{array}{l}16.72597 \\
16.89556 \\
17.05732 \\
17.21194 \\
17.36002\end{array}$ & $\begin{array}{l}16959 \\
16176 \\
15462 \\
14808 \\
14208\end{array}$ & $\begin{array}{l}20.20125 \\
20.37217 \\
20.53514 \\
20.69087 \\
20.83998\end{array}$ & $\begin{array}{l}17092 \\
16297 \\
15573 \\
14911 \\
14303\end{array}$ \\
\hline $\begin{array}{l}250 \\
260 \\
270 \\
280 \\
290\end{array}$ & $\begin{array}{l}3.50374 \\
3.50389 \\
3.50405 \\
3.50422 \\
3.50440\end{array}$ & $\begin{array}{l}15 \\
16 \\
17 \\
18 \\
20\end{array}$ & $\begin{array}{l}3.48090 \\
3.48178 \\
3.48261 \\
3.48337 \\
3.48410\end{array}$ & $\begin{array}{l}88 \\
83 \\
76 \\
73 \\
68\end{array}$ & $\begin{array}{l}17.50210 \\
17.63864 \\
17.77006 \\
17.89673 \\
18.01898\end{array}$ & $\begin{array}{l}13654 \\
13142 \\
12667 \\
12225 \\
11813\end{array}$ & $\begin{array}{l}20.98301 \\
21.12043 \\
21.25267 \\
21.38011 \\
21.50308\end{array}$ & $\begin{array}{l}13742 \\
13224 \\
12744 \\
12297 \\
11881\end{array}$ \\
\hline $\begin{array}{l}300 \\
310 \\
320 \\
330 \\
340\end{array}$ & $\begin{array}{l}3.50460 \\
3.50482 \\
3.50508 \\
3.50536 \\
3.50570\end{array}$ & $\begin{array}{l}22 \\
26 \\
28 \\
34 \\
38\end{array}$ & $\begin{array}{l}3.48478 \\
3.48542 \\
3.48603 \\
3.48661 \\
3.48717\end{array}$ & $\begin{array}{l}64 \\
61 \\
58 \\
56 \\
53\end{array}$ & $\begin{array}{l}18.13711 \\
18.25138 \\
18.36205 \\
18.46933 \\
18.57342\end{array}$ & $\begin{array}{l}11427 \\
11067 \\
10728 \\
10409 \\
10110\end{array}$ & $\begin{array}{l}21.62189 \\
21.73681 \\
21.84808 \\
21.95595 \\
22.06060\end{array}$ & $\begin{array}{l}11492 \\
11127 \\
10787 \\
10465 \\
10162\end{array}$ \\
\hline $\begin{array}{l}350 \\
360 \\
370 \\
380 \\
390\end{array}$ & $\begin{array}{l}3.50608 \\
3.50652 \\
3.50704 \\
3.50763 \\
3.50830\end{array}$ & $\begin{array}{l}44 \\
52 \\
59 \\
67 \\
78\end{array}$ & $\begin{array}{l}3.48770 \\
3.48822 \\
3.48872 \\
3.48921 \\
3.48969\end{array}$ & $\begin{array}{l}52 \\
50 \\
49 \\
48 \\
47\end{array}$ & $\begin{array}{l}18.67452 \\
18.77277 \\
18.86836 \\
18.96140 \\
19.05204\end{array}$ & $\begin{array}{l}9825 \\
9559 \\
9304 \\
9064 \\
8836\end{array}$ & $\begin{array}{l}22.16222 \\
22.26100 \\
22.35708 \\
22.45061 \\
22.54174\end{array}$ & $\begin{array}{l}9878 \\
9638 \\
9353 \\
9113 \\
8883\end{array}$ \\
\hline $\begin{array}{l}400 \\
450 \\
500 \\
550 \\
600\end{array}$ & $\begin{array}{l}3.50908 \\
3.51473 \\
3.52404 \\
3.53765 \\
3.55572\end{array}$ & $\begin{array}{r}565 \\
931 \\
1361 \\
1807 \\
2229\end{array}$ & $\begin{array}{l}3.49016 \\
3.49255 \\
3.49520 \\
3.49841 \\
3.50240\end{array}$ & $\begin{array}{l}239 \\
265 \\
321 \\
399 \\
493\end{array}$ & $\begin{array}{l}19.14040 \\
19.55162 \\
19.91973 \\
20.25300 \\
20.55757\end{array}$ & $\begin{array}{l}41122 \\
36811 \\
33327 \\
30457 \\
28053\end{array}$ & $\begin{array}{l}22.63057 \\
23.04417 \\
23.41493 \\
23.75141 \\
24.05997\end{array}$ & $\begin{array}{l}41360 \\
37076 \\
33648 \\
30856 \\
28547\end{array}$ \\
\hline $\begin{array}{l}650 \\
700 \\
750 \\
800 \\
850\end{array}$ & $\begin{array}{l}3.57801 \\
3.60400 \\
3.63299 \\
3.66426 \\
3.69710\end{array}$ & $\begin{array}{l}2599 \\
2899 \\
3127 \\
3284 \\
3376\end{array}$ & $\begin{array}{l}3.50733 \\
3.51329 \\
3.52029 \\
3.52830 \\
3.53726\end{array}$ & $\begin{array}{l}596 \\
700 \\
801 \\
896 \\
981\end{array}$ & $\begin{array}{l}20.83810 \\
21.09823 \\
21.34086 \\
21.56830 \\
21.78247\end{array}$ & $\begin{array}{l}26013 \\
24263 \\
22744 \\
21417 \\
20246\end{array}$ & $\begin{array}{l}24.34544 \\
24.61153 \\
24.86115 \\
25.09661 \\
25.31973\end{array}$ & $\begin{array}{l}26509 \\
24962 \\
23546 \\
22312 \\
21227\end{array}$ \\
\hline $\begin{array}{l}900 \\
950 \\
.000 \\
050 \\
100\end{array}$ & $\begin{array}{l}3.73086 \\
3.76501 \\
3.79909 \\
3.83274 \\
3.86569\end{array}$ & $\begin{array}{l}3415 \\
3408 \\
3365 \\
3295 \\
3205\end{array}$ & $\begin{array}{l}3.54707 \\
3.55764 \\
3.56886 \\
3.58063 \\
3.59284\end{array}$ & $\begin{array}{l}1057 \\
1122 \\
1177 \\
1221 \\
1257\end{array}$ & $\begin{array}{l}21.98493 \\
22.17699 \\
22.35975 \\
22.53416 \\
22.70101\end{array}$ & $\begin{array}{l}19206 \\
18276 \\
17441 \\
16685 \\
15999\end{array}$ & $\begin{array}{l}25.53200 \\
25.73463 \\
25.92862 \\
26.11480 \\
26.29386\end{array}$ & $\begin{array}{l}20263 \\
19399 \\
18618 \\
17906 \\
17255\end{array}$ \\
\hline 50 & 3.89774 & 3101 & 3.60541 & 1283 & 22.86100 & 15371 & 26.46641 & 16654 \\
\hline
\end{tabular}


Table 2.101. DF (Cont.)

\begin{tabular}{|l|l|l|l|l|}
\hline$k$ & $\frac{C_{p}^{0}}{R}$ & $\frac{\left(H^{\circ}-E_{0}^{0}\right)}{R T}$ & $\frac{-\left(F^{0}-E_{0}^{0}\right)}{R T}$ & $\frac{S^{0}}{R}$ \\
\hline
\end{tabular}

\begin{tabular}{|c|c|c|c|c|c|c|c|c|}
\hline $\begin{array}{l}1200 \\
1300 \\
1400 \\
1500 \\
1600\end{array}$ & $\begin{array}{l}3.92875 \\
3.98730 \\
4.04101 \\
4.08993 \\
4.13432\end{array}$ & $\begin{array}{l}5855 \\
5371 \\
4892 \\
4439 \\
4022\end{array}$ & $\begin{array}{l}3.61824 \\
3.64440 \\
3.67084 \\
3.69718 \\
3.72313\end{array}$ & $\begin{array}{l}2616 \\
2644 \\
2634 \\
2595 \\
2539\end{array}$ & $\begin{array}{l}23.01471 \\
23.30536 \\
23.57640 \\
23.83056 \\
24.07001\end{array}$ & $\begin{array}{l}29065 \\
27104 \\
25416 \\
23945 \\
22648\end{array}$ & $\begin{array}{l}26.63295 \\
26.94977 \\
27.24725 \\
27.52775 \\
27.79315\end{array}$ & $\begin{array}{l}31682 \\
29748 \\
28050 \\
26540 \\
25187\end{array}$ \\
\hline $\begin{array}{l}1700 \\
1800 \\
1900 \\
2000 \\
2100\end{array}$ & $\begin{array}{l}4.17454 \\
4.21098 \\
4.24404 \\
4.27410 \\
4.30149\end{array}$ & $\begin{array}{l}3644 \\
3306 \\
3006 \\
2739 \\
2504\end{array}$ & $\begin{array}{l}3.74852 \\
3.77322 \\
3.79714 \\
3.82025 \\
3.84253\end{array}$ & $\begin{array}{l}2470 \\
2392 \\
2311 \\
2228 \\
2144\end{array}$ & $\begin{array}{l}24.29649 \\
24.51145 \\
24.71610 \\
24.91146 \\
25.09839\end{array}$ & $\begin{array}{l}21496 \\
20465 \\
19536 \\
18693 \\
17926\end{array}$ & $\begin{array}{l}28.04502 \\
28.28467 \\
28.51325 \\
28.73172 \\
28.94093\end{array}$ & $\begin{array}{l}23965 \\
22858 \\
21847 \\
20921 \\
20069\end{array}$ \\
\hline $\begin{array}{l}2200 \\
2300 \\
2400 \\
2500 \\
2600\end{array}$ & $\begin{array}{l}4.32653 \\
4.34948 \\
4.37,059 \\
4.39007 \\
4.40811\end{array}$ & $\begin{array}{l}2295 \\
2111 \\
1948 \\
1804 \\
1676\end{array}$ & $\begin{array}{l}3.86397 \\
3.88458 \\
3.90440 \\
3.92344 \\
3.94174\end{array}$ & $\begin{array}{l}2061 \\
1982 \\
1904 \\
1830 \\
1759\end{array}$ & $\begin{array}{l}25.27765 \\
25.44986 \\
25.61561 \\
25.77539 \\
25.92963\end{array}$ & $\begin{array}{l}17221 \\
16575 \\
15978 \\
15424 \\
14909\end{array}$ & $\begin{array}{l}29.14162 \\
29.33445 \\
29.52002 \\
29.69883 \\
29.87137\end{array}$ & $\begin{array}{l}19283 \\
18557 \\
17881 \\
17254 \\
16668\end{array}$ \\
\hline $\begin{array}{l}2700 \\
2800 \\
2900 \\
3000\end{array}$ & $\begin{array}{l}4.42487 \\
4.44048 \\
4.45507 \\
4.46876\end{array}$ & $\begin{array}{l}1561 \\
1459 \\
1369 \\
2502\end{array}$ & $\begin{array}{l}3.95933 \\
3.97624 \\
3.99250 \\
4.00815\end{array}$ & $\begin{array}{l}1691 \\
1626 \\
1565 \\
2958\end{array}$ & $\begin{array}{l}26.07872 \\
26.22302 \\
26.36284 \\
26.49845\end{array}$ & $\begin{array}{l}14430 \\
13982 \\
13561 \\
25964\end{array}$ & $\begin{array}{l}30.03805 \\
30.19926 \\
30.35534 \\
30.50661\end{array}$ & $\begin{array}{l}16121 \\
15608 \\
15127 \\
28922\end{array}$ \\
\hline $\begin{array}{l}3200 \\
3400 \\
3600 \\
3800 \\
4000\end{array}$ & $\begin{array}{l}4.49378 \\
4.51616 \\
4.53640 \\
4.55487 \\
4.57188\end{array}$ & $\begin{array}{l}2238 \\
2024 \\
1847 \\
1701 \\
1579\end{array}$ & $\begin{array}{l}4.03773 \\
4.06523 \\
4.09085 \\
4.11480 \\
4.13723\end{array}$ & $\begin{array}{l}2750 \\
2562 \\
2395 \\
2243 \\
2108\end{array}$ & $\begin{array}{l}26.75809 \\
27.00371 \\
27.23681 \\
27.45864 \\
27.67028\end{array}$ & $\begin{array}{l}24562 \\
23310 \\
22183 \\
21164 \\
20237\end{array}$ & $\begin{array}{l}30.79583 \\
31.06895 \\
31.32767 \\
31.57344 \\
31.80751\end{array}$ & $\begin{array}{l}27312 \\
25872 \\
24577 \\
23407 \\
22345\end{array}$ \\
\hline $\begin{array}{l}4200 \\
4400 \\
4600 \\
4800 \\
5000\end{array}$ & $\begin{array}{l}4.58767 \\
4.60243 \\
4.61632 \\
4.62947 \\
4.64198\end{array}$ & $\begin{array}{l}1476 \\
1389 \\
1315 \\
1251\end{array}$ & $\begin{array}{l}4.15831 \\
4.17816 \\
4.19692 \\
4.21467 \\
4.23151\end{array}$ & $\begin{array}{l}1985 \\
1876 \\
1775 \\
1684\end{array}$ & $\begin{array}{l}27.87265 \\
28.06656 \\
28.25270 \\
28.43170 \\
28.60409\end{array}$ & $\begin{array}{l}19391 \\
18614 \\
17900 \\
17239\end{array}$ & $\begin{array}{l}32.03096 \\
32.24472 \\
32.44962 \\
32.64637 \\
32.83561\end{array}$ & $\begin{array}{l}21376 \\
20490 \\
19675 \\
18924\end{array}$ \\
\hline
\end{tabular}


Table 2.102. TF

\begin{tabular}{|c|c|c|c|c|c|c|c|c|}
\hline${ }^{\circ} \mathrm{K}$ & $\frac{C_{p}^{0}}{R}$ & & $\frac{\left(H^{\circ}-E_{0}^{0}\right)}{R T}$ & & $\frac{-\left(F^{\circ}-E\right.}{R T}$ & & $\frac{S^{0}}{R}$ & \\
\hline $\begin{array}{l}50 \\
60 \\
70 \\
80 \\
90\end{array}$ & $\begin{array}{l}3.50191 \\
3.50169 \\
3.50161 \\
3.50161 \\
3.50166\end{array}$ & $\begin{array}{r}-22 \\
-\quad 8 \\
5 \\
8\end{array}$ & $\begin{array}{l}3.42622 \\
3.43882 \\
3.44779 \\
3.45452 \\
3.45975\end{array}$ & $\begin{array}{r}1260 \\
897 \\
673 \\
523 \\
420\end{array}$ & $\begin{array}{l}12.34640 \\
12.97226 \\
13.50306 \\
13.96391 \\
14.37111\end{array}$ & $\begin{array}{l}62586 \\
53080 \\
46085 \\
40720 \\
36475\end{array}$ & $\begin{array}{l}15.77263 \\
16.41108 \\
16.95086 \\
17.41843 \\
17.83087\end{array}$ & $\begin{array}{l}63845 \\
53978 \\
46757 \\
41244 \\
36894\end{array}$ \\
\hline $\begin{array}{l}100 \\
110 \\
120 \\
130 \\
140\end{array}$ & $\begin{array}{l}3.50174 \\
3.50183 \\
3.50194 \\
3.50206 \\
3.50218\end{array}$ & $\begin{array}{r}9 \\
11 \\
12 \\
12 \\
13\end{array}$ & $\begin{array}{l}3.46395 \\
3.46739 \\
3.47026 \\
3.47270 \\
3.47480\end{array}$ & $\begin{array}{l}344 \\
287 \\
244 \\
210 \\
183\end{array}$ & $\begin{array}{l}14.73586 \\
15.06617 \\
15.36800 \\
15.64587 \\
15.90330\end{array}$ & $\begin{array}{l}33031 \\
30183 \\
27787 \\
25743 \\
23980\end{array}$ & $\begin{array}{l}18.19981 \\
18.53356 \\
18.83827 \\
19.11858 \\
19.37811\end{array}$ & $\begin{array}{l}33375 \\
30471 \\
28031 \\
25953 \\
24163\end{array}$ \\
\hline $\begin{array}{l}150 \\
160 \\
170 \\
180 \\
190\end{array}$ & $\begin{array}{l}3.50231 \\
3.50244 \\
3.50257 \\
3.50270 \\
3.50284\end{array}$ & $\begin{array}{l}13 \\
13 \\
13 \\
14 \\
15\end{array}$ & $\begin{array}{l}3.47663 \\
3.47824 \\
3.47967 \\
3.48095 \\
3.48209\end{array}$ & $\begin{array}{l}161 \\
143 \\
128 \\
114 \\
105\end{array}$ & $\begin{array}{l}16.14310 \\
16.36753 \\
16.57845 \\
16.77737 \\
16.96561\end{array}$ & $\begin{array}{l}22443 \\
21092 \\
19892 \\
18824 \\
17864\end{array}$ & $\begin{array}{l}19.61974 \\
19.84578 \\
20.05812 \\
20.25833 \\
20.44771\end{array}$ & $\begin{array}{l}22604 \\
21234 \\
20021 \\
18938 \\
17968\end{array}$ \\
\hline $\begin{array}{l}200 \\
210 \\
220 \\
230 \\
240\end{array}$ & $\begin{array}{l}3.50299 \\
3.50313 \\
3.50329 \\
3.50345 \\
3.50363\end{array}$ & $\begin{array}{l}14 \\
16 \\
16 \\
18 \\
21\end{array}$ & $\begin{array}{l}3.48314 \\
3.48408 \\
3.48495 \\
3.48575 \\
3.48650\end{array}$ & $\begin{array}{l}94 \\
87 \\
80 \\
75 \\
68\end{array}$ & $\begin{array}{l}17.14425 \\
17.31421 \\
17.47631 \\
17.63124 \\
17.77961\end{array}$ & $\begin{array}{l}16996 \\
16210 \\
15493 \\
14837 \\
14234\end{array}$ & $\begin{array}{l}20.62739 \\
20.79830 \\
20.96127 \\
21.11700 \\
21.26611\end{array}$ & $\begin{array}{l}17091 \\
16297 \\
15573 \\
14911 \\
14303\end{array}$ \\
\hline $\begin{array}{l}250 \\
260 \\
270 \\
280 \\
290\end{array}$ & $\begin{array}{l}3.50384 \\
3.50407 \\
3.50435 \\
3.50467 \\
3.50506\end{array}$ & $\begin{array}{l}23 \\
28 \\
32 \\
39 \\
47\end{array}$ & $\begin{array}{l}3.48718 \\
3.48783 \\
3.48844 \\
3.48901 \\
3.48956\end{array}$ & $\begin{array}{l}65 \\
61 \\
57 \\
55 \\
52\end{array}$ & $\begin{array}{l}17.92195 \\
18.05873 \\
18.19038 \\
18.31726 \\
18.43970\end{array}$ & $\begin{array}{l}13678 \\
13165 \\
12688 \\
12244 \\
11831\end{array}$ & $\begin{array}{l}21.40914 \\
21.54657 \\
21.67882 \\
21.80627 \\
21.92926\end{array}$ & $\begin{array}{l}13743 \\
13225 \\
12745 \\
12299 \\
11884\end{array}$ \\
\hline $\begin{array}{l}300 \\
310 \\
320 \\
330 \\
340\end{array}$ & $\begin{array}{l}3.50553 \\
3.50609 \\
3.50676 \\
3.50756 \\
3.50849\end{array}$ & $\begin{array}{r}56 \\
67 \\
80 \\
93 \\
109\end{array}$ & $\begin{array}{l}3.49008 \\
3.49059 \\
3.49108 \\
3.49157 \\
3.49205\end{array}$ & $\begin{array}{l}51 \\
49 \\
49 \\
48 \\
49\end{array}$ & $\begin{array}{l}18.55801 \\
18.67246 \\
18.78329 \\
18.89072 \\
18.99496\end{array}$ & $\begin{array}{l}11445 \\
11083 \\
10743 \\
10424 \\
10123\end{array}$ & $\begin{array}{l}22.04810 \\
22.16305 \\
22.27437 \\
22.38230 \\
22.48702\end{array}$ & $\begin{array}{l}11495 \\
11132 \\
10793 \\
10472 \\
10172\end{array}$ \\
\hline $\begin{array}{l}350 \\
360 \\
370 \\
380 \\
390\end{array}$ & $\begin{array}{l}3.50958 \\
3.51085 \\
3.51230 \\
3.51394 \\
3.51580\end{array}$ & $\begin{array}{l}127 \\
145 \\
164 \\
186 \\
207\end{array}$ & $\begin{array}{l}3.49254 \\
3.49303 \\
3.49353 \\
3.49405 \\
3.49458\end{array}$ & $\begin{array}{l}49 \\
50 \\
52 \\
53 \\
56\end{array}$ & $\begin{array}{l}19.09619 \\
19.19459 \\
19.29030 \\
19.38348 \\
19.47424\end{array}$ & $\begin{array}{l}9840 \\
9571 \\
9318 \\
9076 \\
8848\end{array}$ & $\begin{array}{l}22.58874 \\
22.68762 \\
22.78384 \\
22.87753 \\
22.96883\end{array}$ & $\begin{array}{l}9888 \\
9622 \\
9369 \\
9130 \\
8903\end{array}$ \\
\hline $\begin{array}{l}400 \\
450 \\
500 \\
550 \\
600\end{array}$ & $\begin{array}{l}3.51787 \\
3.53183 \\
3.55206 \\
3.57827 \\
3.60960\end{array}$ & $\begin{array}{l}1396 \\
2023 \\
2621 \\
3133 \\
3528\end{array}$ & $\begin{array}{l}3.49514 \\
3.49838 \\
3.50268 \\
3.50832 \\
3.51543\end{array}$ & $\begin{array}{l}324 \\
430 \\
564 \\
711 \\
857\end{array}$ & $\begin{array}{l}19.56272 \\
19.97457 \\
20.34337 \\
20.67747 \\
20.98303\end{array}$ & $\begin{array}{l}41135 \\
36880 \\
33410 \\
30556 \\
28171\end{array}$ & $\begin{array}{l}23.05786 \\
23.47296 \\
23.84606 \\
24.18580 \\
24.49846\end{array}$ & $\begin{array}{l}41510 \\
37310 \\
33974 \\
31266 \\
29029\end{array}$ \\
\hline $\begin{array}{l}650 \\
700 \\
750 \\
800 \\
850\end{array}$ & $\begin{array}{l}3.64488 \\
3.68287 \\
3.72247 \\
3.76270 \\
3.80280\end{array}$ & $\begin{array}{l}3799 \\
3960 \\
4023 \\
4010 \\
3939\end{array}$ & $\begin{array}{l}3.52400 \\
3.53398 \\
3.54522 \\
3.55756 \\
3.57081\end{array}$ & $\begin{array}{r}998 \\
1124 \\
1234 \\
1325 \\
1398\end{array}$ & $\begin{array}{l}21.26474 \\
21.52626 \\
21.77045 \\
21.99964 \\
22.21571\end{array}$ & $\begin{array}{l}26152 \\
24419 \\
22919 \\
21607 \\
20450\end{array}$ & $\begin{array}{l}24.78875 \\
25.06024 \\
25.31568 \\
25.55721 \\
25.78652\end{array}$ & $\begin{array}{l}27149 \\
25544 \\
24153 \\
22931 \\
21848\end{array}$ \\
\hline $\begin{array}{l}900 \\
950 \\
000 \\
050 \\
100\end{array}$ & $\begin{array}{l}3.84219 \\
3.88045 \\
3.91730 \\
3.95256 \\
3.98613\end{array}$ & $\begin{array}{l}3826 \\
3685 \\
3526 \\
3357 \\
3185\end{array}$ & $\begin{array}{l}3.58479 \\
3.59935 \\
3.61433 \\
3.62961 \\
3.64506\end{array}$ & $\begin{array}{l}1456 \\
1498 \\
1528 \\
1545 \\
1553\end{array}$ & $\begin{array}{l}22.42021 \\
22.61442 \\
22.79942 \\
22.97613 \\
23.14534\end{array}$ & $\begin{array}{l}19421 \\
18500 \\
17671 \\
16921 \\
16237\end{array}$ & $\begin{array}{l}26.00500 \\
26.21377 \\
26.41376 \\
26.60574 \\
26.79040\end{array}$ & $\begin{array}{l}20877 \\
19999 \\
19198 \\
18466 \\
17790\end{array}$ \\
\hline 50 & 4.01798 & 3012 & 3.66059 & 1552 & 23.30771 & 15612 & 26.96830 & 17164 \\
\hline
\end{tabular}


Table 2. 102. TF (Cont.)

\begin{tabular}{|c|c|c|c|c|c|c|c|c|}
\hline${ }^{\circ} \mathrm{K}$ & $\frac{C_{p}^{0}}{R}$ & & $\frac{\left(H^{0}-E_{0}^{0}\right)}{R T}$ & & $\frac{-\left(F^{0}-E\right.}{R T}$ & & $\frac{S^{0}}{R}$ & \\
\hline $\begin{array}{l}1200 \\
1300 \\
1400 \\
1500 \\
1600\end{array}$ & $\begin{array}{l}4.04810 \\
4.10337 \\
4.15245 \\
4.19600 \\
4.23468\end{array}$ & $\begin{array}{l}5527 \\
4908 \\
4355 \\
3868 \\
3446\end{array}$ & $\begin{array}{l}3.67611 \\
3.70689 \\
3.73700 \\
3.76618 \\
3.79427\end{array}$ & $\begin{array}{l}3078 \\
3011 \\
2918 \\
2809 \\
2694\end{array}$ & $\begin{array}{l}23.46383 \\
23.75929 \\
24.03511 \\
24.29394 \\
24.53791\end{array}$ & $\begin{array}{l}29546 \\
27582 \\
25883 \\
24397 \\
23084\end{array}$ & $\begin{array}{l}27.13994 \\
27.46619 \\
27.77212 \\
28.06012 \\
28.33218\end{array}$ & $\begin{array}{l}32625 \\
30593 \\
28800 \\
27206 \\
25778\end{array}$ \\
\hline $\begin{array}{l}1700 \\
1800 \\
1900 \\
2000 \\
2100\end{array}$ & $\begin{array}{l}4.26914 \\
4.29993 \\
4.32757 \\
4.35247 \\
4.37502\end{array}$ & $\begin{array}{l}3079 \\
2764 \\
2490 \\
2255 \\
2051\end{array}$ & $\begin{array}{l}3.82121 \\
3.84697 \\
3.87155 \\
3.89498 \\
3.91731\end{array}$ & $\begin{array}{l}2576 \\
2458 \\
2343 \\
2233 \\
2128\end{array}$ & $\begin{array}{l}24.76875 \\
24.98790 \\
25.19656 \\
25.39574 \\
25.58632\end{array}$ & $\begin{array}{l}21915 \\
20866 \\
19918 \\
19058 \\
18273\end{array}$ & $\begin{array}{l}28.58996 \\
28.83487 \\
29.06811 \\
29.29073 \\
29.50364\end{array}$ & $\begin{array}{l}24491 \\
23324 \\
22262 \\
21291 \\
20401\end{array}$ \\
\hline $\begin{array}{l}2200 \\
2300 \\
2400 \\
2500 \\
2600\end{array}$ & $\begin{array}{l}4.39553 \\
4.41427 \\
4.43148 \\
4.44733 \\
4.46201\end{array}$ & $\begin{array}{l}1874 \\
1721 \\
1585 \\
1468 \\
1365\end{array}$ & $\begin{array}{l}3.93859 \\
3.95887 \\
3.97821 \\
3.99666 \\
4.01428\end{array}$ & $\begin{array}{l}2028 \\
1934 \\
1845 \\
1762 \\
1684\end{array}$ & $\begin{array}{l}25.76905 \\
25.94458 \\
26.11348 \\
26.27626 \\
26.43335\end{array}$ & $\begin{array}{l}17553 \\
16890 \\
16278 \\
15709 \\
15182\end{array}$ & $\begin{array}{l}29.70765 \\
29.90346 \\
30.09169 \\
30.27292 \\
30.44764\end{array}$ & $\begin{array}{l}19581 \\
18823 \\
18123 \\
17472 \\
16866\end{array}$ \\
\hline $\begin{array}{l}2700 \\
2800 \\
2900 \\
3000\end{array}$ & $\begin{array}{l}4.47566 \\
4.48840 \\
4.50033 \\
4.51155\end{array}$ & $\begin{array}{l}1274 \\
1193 \\
1122 \\
2061\end{array}$ & $\begin{array}{l}4.03112 \\
4.04723 \\
4.06265 \\
4.07743\end{array}$ & $\begin{array}{l}1611 \\
1542 \\
1478 \\
2778\end{array}$ & $\begin{array}{l}26.58517 \\
26.73207 \\
26.87436 \\
27.01234\end{array}$ & $\begin{array}{l}14690 \\
14229 \\
13798 \\
26405\end{array}$ & $\begin{array}{l}30.61630 \\
30.77930 \\
30.93701 \\
31.08977\end{array}$ & $\begin{array}{l}16300 \\
15771 \\
15276 \\
29184\end{array}$ \\
\hline $\begin{array}{l}3200 \\
3400 \\
3600 \\
3800 \\
4000\end{array}$ & $\begin{array}{l}4.53216 \\
4.55073 \\
4.56766 \\
4.58325 \\
4.59774\end{array}$ & $\begin{array}{l}1857 \\
1693 \\
1559 \\
1449 \\
1358\end{array}$ & $\begin{array}{l}4.10521 \\
4.13088 \\
4.15469 \\
4.17684 \\
4.19752\end{array}$ & $\begin{array}{l}2567 \\
2381 \\
2215 \\
2068 \\
1939\end{array}$ & $\begin{array}{l}27.27639 \\
27.52605 \\
27.76285 \\
27.98808 \\
28.20286\end{array}$ & $\begin{array}{l}24966 \\
23680 \\
22523 \\
21478 \\
20527\end{array}$ & $\begin{array}{l}31.38161 \\
31.65694 \\
31.91754 \\
32.16492 \\
32.40039\end{array}$ & $\begin{array}{l}27533 \\
26063 \\
24738 \\
23547 \\
22465\end{array}$ \\
\hline $\begin{array}{l}4200 \\
4400 \\
4600 \\
4800 \\
5000\end{array}$ & $\begin{array}{l}4.61132 \\
4.62413 \\
4.63628 \\
4.64789 \\
4.65902\end{array}$ & $\begin{array}{l}1281 \\
1215 \\
1161 \\
1113\end{array}$ & $\begin{array}{l}4.21691 \\
4.23513 \\
4.25231 \\
4.26855 \\
4.28395\end{array}$ & $\begin{array}{l}1822 \\
1718 \\
1624 \\
1540\end{array}$ & $\begin{array}{l}28.40813 \\
28.60472 \\
28.79337 \\
28.97469 \\
29.14926\end{array}$ & $\begin{array}{l}19659 \\
18865 \\
18132 \\
17457\end{array}$ & $\begin{array}{l}32.62504 \\
32.83986 \\
33.04568 \\
33.24325 \\
33.43321\end{array}$ & $\begin{array}{l}21482 \\
20582 \\
19757 \\
18996\end{array}$ \\
\hline
\end{tabular}


Table 2.103. $\mathrm{ClH}$

\begin{tabular}{|c|c|c|c|c|c|c|c|c|}
\hline K & $\frac{C_{p}^{0}}{R}$ & & $\frac{\left(H^{\circ}-E_{0}^{\circ}\right.}{R T}$ & & $\frac{-\left(F^{0}-E\right.}{R T}$ & & $\frac{S^{0}}{R}$ & \\
\hline $\begin{array}{l}50 \\
60 \\
70 \\
80 \\
90\end{array}$ & $\begin{array}{l}3.50294 \\
3.50237 \\
3.50208 \\
3.50194 \\
3.50189\end{array}$ & $\begin{array}{l}-57 \\
-\quad 29 \\
-\quad 14 \\
-\quad 5\end{array}$ & $\begin{array}{l}3.39804 \\
3.41547 \\
3.42786 \\
3.43713 \\
3.44433\end{array}$ & $\begin{array}{r}1743 \\
1239 \\
927 \\
720 \\
575\end{array}$ & $\begin{array}{l}12.81449 \\
13.43567 \\
13.96315 \\
14.42151 \\
14.82678\end{array}$ & $\begin{array}{l}62118 \\
52748 \\
45836 \\
40527 \\
36320\end{array}$ & $\begin{array}{l}16.21254 \\
16.85115 \\
17.39102 \\
17.85865 \\
18.27111\end{array}$ & $\begin{array}{l}63861 \\
53987 \\
46763 \\
41246 \\
36896\end{array}$ \\
\hline $\begin{array}{l}100 \\
110 \\
120 \\
130 \\
140\end{array}$ & $\begin{array}{l}3.50189 \\
3.50193 \\
3.50199 \\
3.50207 \\
3.50216\end{array}$ & $\begin{array}{r}4 \\
6 \\
8 \\
9 \\
10\end{array}$ & $\begin{array}{l}3.45008 \\
3.45480 \\
3.45873 \\
3.46206 \\
3.46492\end{array}$ & $\begin{array}{l}472 \\
393 \\
333 \\
286 \\
248\end{array}$ & $\begin{array}{l}15.18998 \\
15.51904 \\
15.81982 \\
16.09680 \\
16.35348\end{array}$ & $\begin{array}{l}32906 \\
30078 \\
27698 \\
25668 \\
23914\end{array}$ & $\begin{array}{l}18.64007 \\
18.97384 \\
19.27855 \\
19.55886 \\
19.81840\end{array}$ & $\begin{array}{l}33377 \\
30471 \\
28031 \\
25954 \\
24163\end{array}$ \\
\hline $\begin{array}{l}150 \\
160 \\
170 \\
180 \\
190\end{array}$ & $\begin{array}{l}3.50226 \\
3.50236 \\
3.50247 \\
3.50259 \\
3.50271\end{array}$ & $\begin{array}{l}10 \\
11 \\
12 \\
12 \\
12\end{array}$ & $\begin{array}{l}3.46740 \\
3.46959 \\
3.47152 \\
3.47324 \\
3.47479\end{array}$ & $\begin{array}{l}219 \\
193 \\
172 \\
155 \\
140\end{array}$ & $\begin{array}{l}16.59262 \\
16.81647 \\
17.02687 \\
17.22535 \\
17.41318\end{array}$ & $\begin{array}{l}22385 \\
21040 \\
19848 \\
18783 \\
17827\end{array}$ & $\begin{array}{l}20.06003 \\
20.28606 \\
20.49839 \\
20.69859 \\
20.88797\end{array}$ & $\begin{array}{l}22603 \\
21233 \\
20020 \\
18938 \\
17967\end{array}$ \\
\hline $\begin{array}{l}200 \\
210 \\
220 \\
230 \\
240\end{array}$ & $\begin{array}{l}3.50283 \\
3.50295 \\
3.50307 \\
3.50320 \\
3.50333\end{array}$ & $\begin{array}{l}12 \\
12 \\
13 \\
13 \\
14\end{array}$ & $\begin{array}{l}3.47619 \\
3.47746 \\
3.47862 \\
3.47969 \\
3.48067\end{array}$ & $\begin{array}{r}127 \\
116 \\
107 \\
98 \\
91\end{array}$ & $\begin{array}{l}17.59145 \\
17.76108 \\
17.92288 \\
18.07754 \\
18.22565\end{array}$ & $\begin{array}{l}16963 \\
16180 \\
15466 \\
14811 \\
14211\end{array}$ & $\begin{array}{l}21.06764 \\
21.23855 \\
21.40151 \\
21.55723 \\
21.70633\end{array}$ & $\begin{array}{l}17091 \\
16296 \\
15572 \\
14910 \\
14301\end{array}$ \\
\hline $\begin{array}{l}250 \\
260 \\
270 \\
280 \\
290\end{array}$ & $\begin{array}{l}3.50347 \\
3.50361 \\
3.50376 \\
3.50392 \\
3.50409\end{array}$ & $\begin{array}{l}14 \\
15 \\
16 \\
17 \\
20\end{array}$ & $\begin{array}{l}3.48158 \\
3.48242 \\
3.48321 \\
3.48395 \\
3.48464\end{array}$ & $\begin{array}{l}84 \\
79 \\
74 \\
69 \\
65\end{array}$ & $\begin{array}{l}18.36776 \\
18.50433 \\
18.63577 \\
18.76246 \\
18.88473\end{array}$ & $\begin{array}{l}13657 \\
13144 \\
12669 \\
12227 \\
11814\end{array}$ & $\begin{array}{l}21.84934 \\
21.98675 \\
22.11898 \\
22.24641 \\
22.36937\end{array}$ & $\begin{array}{l}13741 \\
13223 \\
12743 \\
12296 \\
11880\end{array}$ \\
\hline $\begin{array}{l}300 \\
310 \\
320 \\
330 \\
340\end{array}$ & $\begin{array}{l}3.50429 \\
3.50451 \\
3.50476 \\
3.50505 \\
3.50538\end{array}$ & $\begin{array}{l}22 \\
25 \\
29 \\
33 \\
39\end{array}$ & $\begin{array}{l}3.48529 \\
3.48591 \\
3.48649 \\
3.48705 \\
3.48758\end{array}$ & $\begin{array}{l}62 \\
58 \\
56 \\
53 \\
52\end{array}$ & $\begin{array}{l}19.00287 \\
19.11717 \\
19.22785 \\
19.33514 \\
19.43925\end{array}$ & $\begin{array}{l}11430 \\
11068 \\
10729 \\
10411 \\
10110\end{array}$ & $\begin{array}{l}22.48817 \\
22.60308 \\
22.71434 \\
22.82220 \\
22.92684\end{array}$ & $\begin{array}{l}11491 \\
11126 \\
10786 \\
10464 \\
10162\end{array}$ \\
\hline $\begin{array}{l}350 \\
360 \\
370 \\
380 \\
390\end{array}$ & $\begin{array}{l}3.50577 \\
3.50623 \\
3.50675 \\
3.50736 \\
3.50806\end{array}$ & $\begin{array}{l}46 \\
52 \\
61 \\
70 \\
80\end{array}$ & $\begin{array}{l}3.48810 \\
3.48859 \\
3.48908 \\
3.48955 \\
3.49002\end{array}$ & $\begin{array}{l}49 \\
49 \\
47 \\
47 \\
46\end{array}$ & $\begin{array}{l}19.54035 \\
19.63862 \\
19.73421 \\
19.82727 \\
19.91792\end{array}$ & $\begin{array}{l}9827 \\
9559 \\
9306 \\
9065 \\
8836\end{array}$ & $\begin{array}{l}23.02846 \\
23.12722 \\
23.22330 \\
23.31682 \\
23.40794\end{array}$ & $\begin{array}{l}9876 \\
9608 \\
9352 \\
9112 \\
8882\end{array}$ \\
\hline $\begin{array}{l}400 \\
450 \\
500 \\
550 \\
600\end{array}$ & $\begin{array}{l}3.50886 \\
3.51471 \\
3.52434 \\
3.53837 \\
3.55693\end{array}$ & $\begin{array}{r}585 \\
963 \\
1403 \\
1856 \\
2282 \\
\end{array}$ & $\begin{array}{l}3.49048 \\
3.49281 \\
3.49545 \\
3.49868 \\
3.50273\end{array}$ & $\begin{array}{l}233 \\
264 \\
323 \\
405 \\
502\end{array}$ & $\begin{array}{l}20.00628 \\
20.41754 \\
20.78567 \\
21.11897 \\
21.42356\end{array}$ & $\begin{array}{l}41126 \\
36813 \\
33330 \\
30459 \\
28056\end{array}$ & $\begin{array}{l}23.49676 \\
23.91035 \\
24.28113 \\
24.61766 \\
24.92630\end{array}$ & $\begin{array}{l}41359 \\
37078 \\
33653 \\
30864 \\
28558\end{array}$ \\
\hline $\begin{array}{l}650 \\
700 \\
750 \\
800 \\
850\end{array}$ & $\begin{array}{l}3.57975 \\
3.60628 \\
3.63580 \\
3.66756 \\
3.70085\end{array}$ & $\begin{array}{l}2653 \\
2952 \\
3176 \\
3329 \\
3417\end{array}$ & $\begin{array}{l}3.50775 \\
3.51382 \\
3.52095 \\
3.52912 \\
3.53823\end{array}$ & $\begin{array}{l}607 \\
713 \\
817 \\
911 \\
998\end{array}$ & $\begin{array}{l}21.70412 \\
21.96429 \\
22.20696 \\
22.43445 \\
22.64867\end{array}$ & $\begin{array}{l}26017 \\
24267 \\
22749 \\
21422 \\
20252\end{array}$ & $\begin{array}{l}25.21188 \\
25.47812 \\
25.72792 \\
25.96357 \\
26.18691\end{array}$ & $\begin{array}{l}26624 \\
24980 \\
23565 \\
22334 \\
21250\end{array}$ \\
\hline $\begin{array}{l}900 \\
950 \\
000 \\
050 \\
100\end{array}$ & $\begin{array}{l}3.73502 \\
3.76952 \\
3.80392 \\
3.83784 \\
3.87104\end{array}$ & $\begin{array}{l}3450 \\
3440 \\
3392 \\
3320 \\
3226\end{array}$ & $\begin{array}{l}3.54821 \\
3.55895 \\
3.57034 \\
3.58227 \\
3.59465\end{array}$ & $\begin{array}{l}1074 \\
1139 \\
1193 \\
1238 \\
1272\end{array}$ & $\begin{array}{l}22.85119 \\
23.04332 \\
23.22615 \\
23.40064 \\
23.56757\end{array}$ & $\begin{array}{l}19213 \\
18283 \\
17449 \\
16693 \\
16007\end{array}$ & $\begin{array}{l}26.39941 \\
26.60227 \\
26.79650 \\
26.98292 \\
27.16222\end{array}$ & $\begin{array}{l}20286 \\
19423 \\
18642 \\
17930 \\
17279\end{array}$ \\
\hline 50 & 3.90330 & 3119 & 3.60737 & 1299 & 23.72764 & 15380 & 27.33501 & 16679 \\
\hline
\end{tabular}


Table 2.103. ClH (Cont.)

\begin{tabular}{|c|c|c|c|c|c|c|c|c|}
\hline${ }^{\circ} \mathrm{K}$ & $\frac{C_{p}^{0}}{R}$ & & $\frac{\left(H^{0}-E_{0}^{0}\right)}{R T}$ & & $\frac{-\left(F^{0}-E_{0}^{0}\right)}{R T}$ & & $\frac{S^{0}}{R}$ & \\
\hline $\begin{array}{l}1200 \\
1300 \\
1400 \\
1500 \\
1600\end{array}$ & $\begin{array}{l}3.93449 \\
3.99336 \\
4.04732 \\
4.09647 \\
4.14106\end{array}$ & $\begin{array}{l}5887 \\
5396 \\
4915 \\
4459 \\
4040\end{array}$ & $\begin{array}{l}3.62036 \\
3.64681 \\
3.67352 \\
3.70011 \\
3.72630\end{array}$ & $\begin{array}{l}2645 \\
2671 \\
2659 \\
2619 \\
2560\end{array}$ & $\begin{array}{l}23.88144 \\
24.17227 \\
24.44350 \\
24.69786 \\
24.93749\end{array}$ & $\begin{array}{l}29083 \\
27123 \\
25436 \\
23963 \\
22668\end{array}$ & $\begin{array}{l}27.50180 \\
27.81908 \\
28.11703 \\
28.39797 \\
28.66380\end{array}$ & $\begin{array}{l}31728 \\
29795 \\
28094 \\
26583 \\
25228\end{array}$ \\
\hline $\begin{array}{l}1700 \\
1800 \\
1900 \\
2000 \\
2100\end{array}$ & $\begin{array}{l}4.18146 \\
4.21809 \\
4.25134 \\
4.28159 \\
4.30917\end{array}$ & $\begin{array}{l}3663 \\
3325 \\
3025 \\
2758 \\
2523\end{array}$ & $\begin{array}{l}3.75190 \\
3.77680 \\
3.80092 \\
3.82421 \\
3.84665\end{array}$ & $\begin{array}{l}2490 \\
2412 \\
2329 \\
2244 \\
2161\end{array}$ & $\begin{array}{l}25.16417 \\
25.37933 \\
25.58418 \\
25.77974 \\
25.96687\end{array}$ & $\begin{array}{l}21516 \\
20485 \\
19556 \\
18713 \\
17945\end{array}$ & $\begin{array}{l}28.91608 \\
29.15614 \\
29.38511 \\
29.60395 \\
29.81353\end{array}$ & $\begin{array}{l}24006 \\
22897 \\
21884 \\
20958 \\
20105\end{array}$ \\
\hline $\begin{array}{l}2200 \\
2300 \\
2400 \\
2500 \\
2600\end{array}$ & $\begin{array}{l}4.33440 \\
4.35756 \\
4.37887 \\
4.39857 \\
4.41682\end{array}$ & $\begin{array}{l}2316 \\
2131 \\
1970 \\
1825 \\
1697\end{array}$ & $\begin{array}{l}3.86826 \\
3.88903 \\
3.90901 \\
3.92820 \\
3.94665\end{array}$ & $\begin{array}{l}2077 \\
1998 \\
1919 \\
1845 \\
1773\end{array}$ & $\begin{array}{l}26.14632 \\
26.31873 \\
26.48467 \\
26.64464 \\
26.79907\end{array}$ & $\begin{array}{l}17241 \\
16594 \\
15997 \\
15443 \\
14928\end{array}$ & $\begin{array}{l}30.01458 \\
30.20777 \\
30.39369 \\
30.57284 \\
30.74572\end{array}$ & $\begin{array}{l}19319 \\
18592 \\
17915 \\
17288 \\
16701\end{array}$ \\
\hline $\begin{array}{l}2700 \\
2800 \\
2900 \\
3000\end{array}$ & $\begin{array}{l}4.43379 \\
4.44963 \\
4.46445 \\
4.47837\end{array}$ & $\begin{array}{l}1584 \\
1482 \\
1392 \\
2548\end{array}$ & $\begin{array}{l}3.96438 \\
3.98143 \\
3.99783 \\
4.01362\end{array}$ & $\begin{array}{l}1705 \\
1640 \\
1579 \\
2986\end{array}$ & $\begin{array}{l}26.94835 \\
27.09284 \\
27.23284 \\
27.36864\end{array}$ & $\begin{array}{l}14449 \\
14000 \\
13580 \\
26000\end{array}$ & $\begin{array}{l}30.91273 \\
31.07427 \\
31.23068 \\
31.38226\end{array}$ & $\begin{array}{l}16154 \\
15641 \\
15158 \\
28986\end{array}$ \\
\hline $\begin{array}{l}3200 \\
3400 \\
3600 \\
3800 \\
4000\end{array}$ & $\begin{array}{l}4.50385 \\
4.52670 \\
4.54742 \\
4.56637 \\
4.58386\end{array}$ & $\begin{array}{l}2285 \\
2072 \\
1895 \\
1749 \\
1628\end{array}$ & $\begin{array}{l}4.04348 \\
4.07124 \\
4.09713 \\
4.12134 \\
4.14403\end{array}$ & $\begin{array}{l}2776 \\
2589 \\
2421 \\
2269 \\
2134\end{array}$ & $\begin{array}{l}27.62864 \\
27.87461 \\
28.10806 \\
28.33024 \\
28.54222\end{array}$ & $\begin{array}{l}24597 \\
23345 \\
22218 \\
21198 \\
20271\end{array}$ & $\begin{array}{l}31.67212 \\
31.94586 \\
32.20520 \\
32.45158 \\
32.68625\end{array}$ & $\begin{array}{l}27374 \\
25934 \\
24638 \\
23467 \\
22405\end{array}$ \\
\hline $\begin{array}{l}4200 \\
4400 \\
4600 \\
4800 \\
5000\end{array}$ & $\begin{array}{l}4.60014 \\
4.61539 \\
4.62976 \\
4.64339 \\
4.65638\end{array}$ & $\begin{array}{l}1525 \\
1437 \\
1363 \\
1299\end{array}$ & $\begin{array}{l}4.16537 \\
4.18548 \\
4.20449 \\
4.22249 \\
4.23959\end{array}$ & $\begin{array}{l}2011 \\
1901 \\
1800 \\
1710\end{array}$ & $\begin{array}{l}28.74493 \\
28.93917 \\
29.12565 \\
29.30497 \\
29.47769\end{array}$ & $\begin{array}{l}19424 \\
18648 \\
17932 \\
17272\end{array}$ & $\begin{array}{l}32.91030 \\
33.12465 \\
33.33014 \\
33.52747 \\
33.71729\end{array}$ & $\begin{array}{l}21435 \\
20549 \\
19733 \\
18982\end{array}$ \\
\hline
\end{tabular}




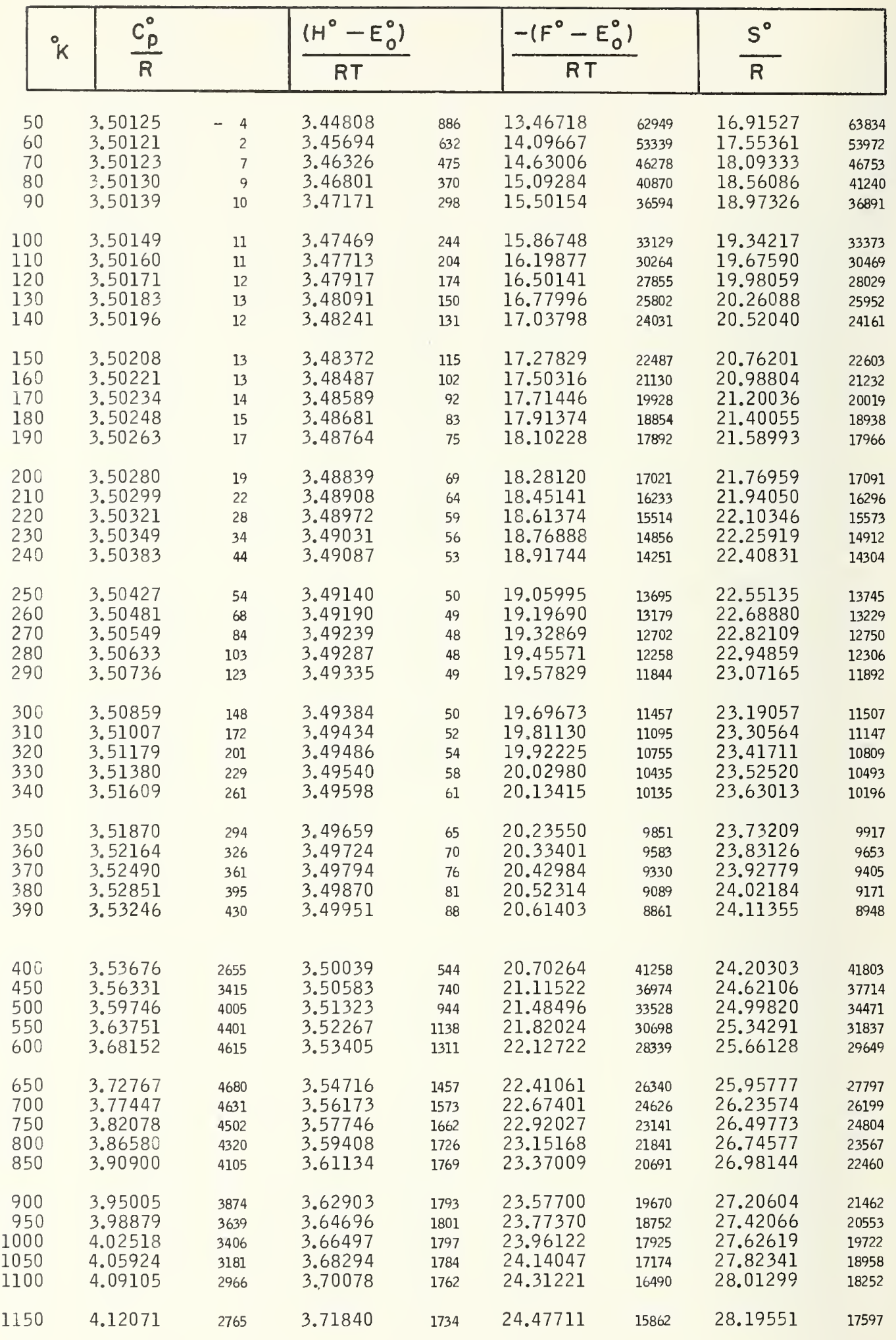


Table 2.104. ClD (Cont.)

\begin{tabular}{|c|c|c|c|c|c|c|c|c|}
\hline${ }^{\circ} \mathrm{K}$ & $\frac{C_{p}^{0}}{R}$ & & $\frac{\left(H^{0}-E_{0}^{0}\right)}{R T}$ & & $\frac{-l F^{0}-E_{0}^{0}}{R T}$ & & $\frac{S^{\circ}}{R}$ & \\
\hline $\begin{array}{l}1200 \\
1300 \\
1400 \\
1500 \\
1600\end{array}$ & $\begin{array}{l}4.14836 \\
4.19814 \\
4.24145 \\
4.27929 \\
4.31250\end{array}$ & $\begin{array}{l}4978 \\
4331 \\
3784 \\
3321 \\
2931\end{array}$ & $\begin{array}{l}3.73574 \\
3.76944 \\
3.80165 \\
3.83226 \\
3.86125\end{array}$ & $\begin{array}{l}3370 \\
3221 \\
3061 \\
2899 \\
2743\end{array}$ & $\begin{array}{l}24.63573 \\
24.93609 \\
25.21662 \\
25.47996 \\
25.72822\end{array}$ & $\begin{array}{l}30036 \\
28053 \\
26334 \\
24826 \\
23492\end{array}$ & $\begin{array}{l}28.37148 \\
28.70554 \\
29.01827 \\
29.31222 \\
29.58948\end{array}$ & $\begin{array}{l}33406 \\
31273 \\
29395 \\
2726 \\
26235\end{array}$ \\
\hline $\begin{array}{l}1700 \\
1800 \\
1900 \\
2000 \\
2100\end{array}$ & $\begin{array}{l}4.34181 \\
4.36785 \\
4.39112 \\
4.41204 \\
4.43097\end{array}$ & $\begin{array}{l}2604 \\
2327 \\
2092 \\
1893 \\
1722\end{array}$ & $\begin{array}{l}3.88868 \\
3.91459 \\
3.93907 \\
3.96220 \\
3.98408\end{array}$ & $\begin{array}{l}2591 \\
2448 \\
2313 \\
2188 \\
2071\end{array}$ & $\begin{array}{l}25.96314 \\
26.18615 \\
26.39847 \\
26.60111 \\
26.79496\end{array}$ & $\begin{array}{l}22301 \\
21232 \\
20264 \\
19385 \\
18582\end{array}$ & $\begin{array}{l}29.85183 \\
30.10075 \\
30.33754 \\
30.56332 \\
30.77905\end{array}$ & $\begin{array}{l}24892 \\
23679 \\
22578 \\
21573 \\
20653\end{array}$ \\
\hline $\begin{array}{l}2200 \\
2300 \\
2400 \\
2500 \\
2600\end{array}$ & $\begin{array}{l}4.44819 \\
4.46395 \\
4.47845 \\
4.49186 \\
4.50432\end{array}$ & $\begin{array}{l}1576 \\
1450 \\
1341 \\
1246 \\
1163\end{array}$ & $\begin{array}{l}4.00479 \\
4.02442 \\
4.04304 \\
4.06073 \\
4.07755\end{array}$ & $\begin{array}{l}1963 \\
1862 \\
1769 \\
1682 \\
1603\end{array}$ & $\begin{array}{l}26.98078 \\
27.15924 \\
27.33091 \\
27.49632 \\
27.65592\end{array}$ & $\begin{array}{l}17846 \\
17167 \\
16541 \\
15960 \\
15419\end{array}$ & $\begin{array}{l}30.98558 \\
31.18366 \\
31.37396 \\
31.55705 \\
31.73347\end{array}$ & $\begin{array}{l}19808 \\
19030 \\
18309 \\
17642 \\
17022\end{array}$ \\
\hline $\begin{array}{l}2700 \\
2800 \\
2900 \\
3000\end{array}$ & $\begin{array}{l}4.51595 \\
4.52686 \\
4.53713 \\
4.54684\end{array}$ & $\begin{array}{r}1091 \\
1027 \\
971 \\
1798\end{array}$ & $\begin{array}{l}4.09358 \\
4.10886 \\
4.12345 \\
4.13740\end{array}$ & $\begin{array}{l}1528 \\
1459 \\
1395 \\
2616\end{array}$ & $\begin{array}{l}27.81011 \\
27.95926 \\
28.10370 \\
28.24373\end{array}$ & $\begin{array}{l}14915 \\
14444 \\
14003 \\
26787\end{array}$ & $\begin{array}{l}31.90369 \\
32.06812 \\
32.22716 \\
32.38114\end{array}$ & $\begin{array}{l}16443 \\
15904 \\
15398 \\
29403\end{array}$ \\
\hline $\begin{array}{l}3200 \\
3400 \\
3600 \\
3800 \\
4000\end{array}$ & $\begin{array}{l}4.56482 \\
4.58121 \\
4.59632 \\
4.61040 \\
4.62363\end{array}$ & $\begin{array}{l}1639 \\
1511 \\
1408 \\
1323 \\
1252\end{array}$ & $\begin{array}{l}4.16356 \\
4.18766 \\
4.20994 \\
4.23065 \\
4.24998\end{array}$ & $\begin{array}{l}2410 \\
2228 \\
2071 \\
1933 \\
1809\end{array}$ & $\begin{array}{l}28.51160 \\
28.76475 \\
29.00475 \\
29.23293 \\
29.45043\end{array}$ & $\begin{array}{l}25315 \\
24000 \\
22818 \\
21750 \\
20780\end{array}$ & $\begin{array}{l}32.67517 \\
32.95241 \\
33.21470 \\
33.46359 \\
33.70041\end{array}$ & $\begin{array}{l}27724 \\
26229 \\
24889 \\
23682 \\
22589\end{array}$ \\
\hline $\begin{array}{l}4200 \\
4400 \\
4600 \\
4800 \\
5000\end{array}$ & $\begin{array}{l}4.63615 \\
4.64808 \\
4.65950 \\
4.67050 \\
4.68112\end{array}$ & $\begin{array}{l}1193 \\
1142 \\
1100 \\
1062\end{array}$ & $\begin{array}{l}4.26807 \\
4.28507 \\
4.30111 \\
4.31627 \\
4.33065\end{array}$ & $\begin{array}{l}1700 \\
1604 \\
1516 \\
1438\end{array}$ & $\begin{array}{l}29.65823 \\
29.85718 \\
30.04801 \\
30.23139 \\
30.40788\end{array}$ & $\begin{array}{l}19895 \\
19083 \\
18338 \\
17649\end{array}$ & $\begin{array}{l}33.92630 \\
34.14225 \\
34.34912 \\
34.54766 \\
34.73854\end{array}$ & $\begin{array}{l}21595 \\
20687 \\
19854 \\
19088\end{array}$ \\
\hline
\end{tabular}


Table 2.105. C1T

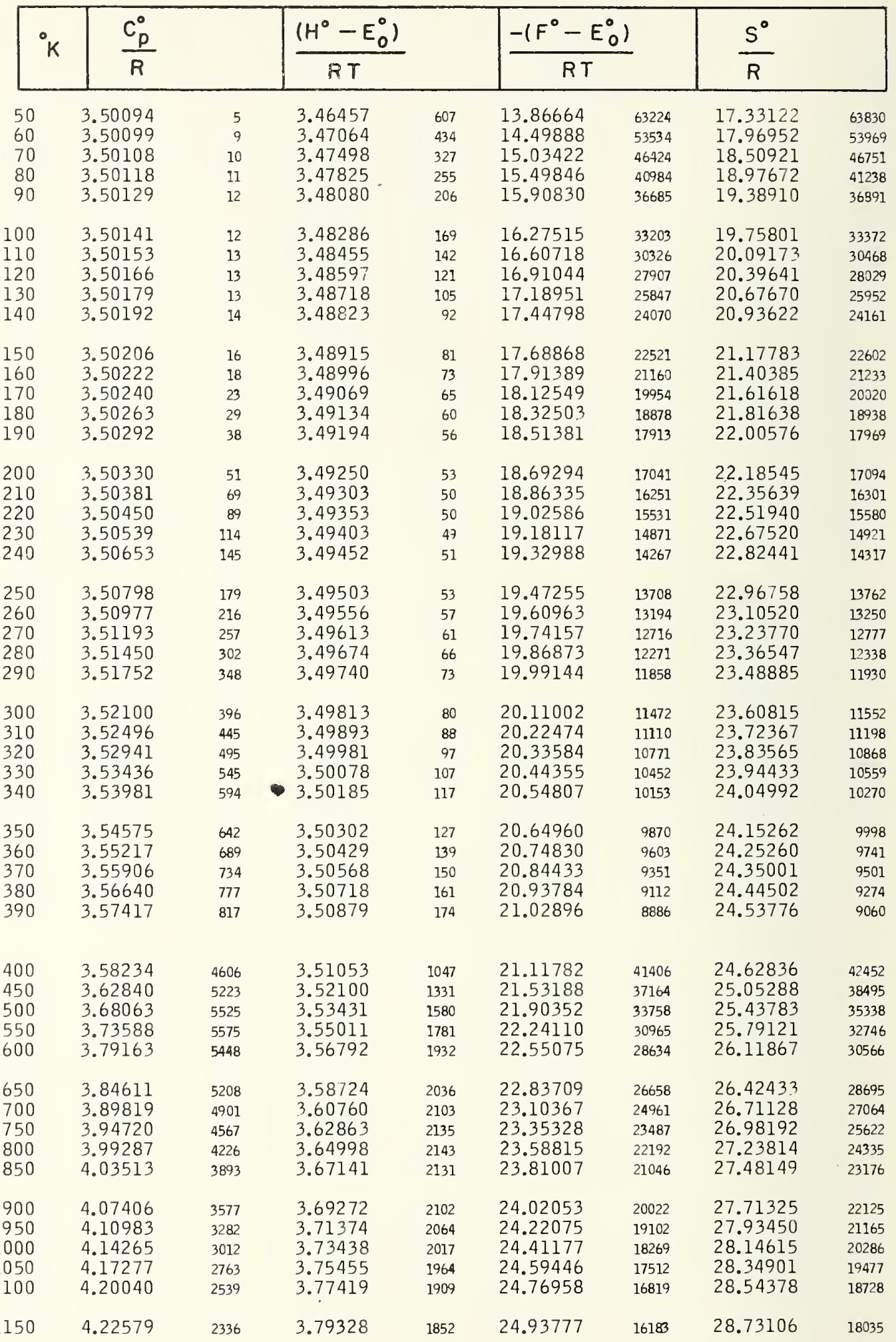


Table 2.105. CIT (Cont.)

\begin{tabular}{|c|c|c|c|c|c|c|c|c|}
\hline k & $\frac{C_{p}^{0}}{R}$ & & $\frac{\left(H^{\circ}-E_{0}^{0}\right)}{R T}$ & & $\frac{-\left(F^{\circ}-E\right.}{R T}$ & & $\frac{S^{0}}{R}$ & \\
\hline 1200 & 4.24915 & 4138 & 3.81180 & 3528 & 25.09960 & 30652 & 22.91141 & 34179 \\
\hline 1300 & 4.29053 & 3540 & 3.84708 & 3297 & 25.40612 & 28632 & 29.25320 & 31929 \\
\hline 1400 & 4.32593 & 3054 & 3.88005 & 3076 & 25.69244 & 26876 & 29.57249 & 29953 \\
\hline 1500 & 4.35647 & 2657 & 3.91081 & 2871 & 25.96120 & 25332 & 29.87202 & 28202 \\
\hline 1600 & 4.38304 & 2332 & 3.93952 & 2679 & 26.21452 & 23965 & 30.15404 & 26644 \\
\hline 1700 & 4.40636 & 2064 & 3.96631 & 2503 & 26.45417 & 22742 & 30.42048 & 25245 \\
\hline 1800 & 4.42700 & 1842 & 3.99134 & 2342 & 26.68159 & 21644 & 30.67293 & 23986 \\
\hline 1900 & 4.44542 & 1657 & 4.01476 & 2196 & 26.89803 & 20649 & 30.91279 & 22845 \\
\hline 2000 & 4.46199 & 1501 & 4.03672 & 2061 & 27.10452 & 19746 & 31.14124 & 21807 \\
\hline 2100 & 4.47700 & 1370 & 4.05733 & 1939 & 27.30198 & 18920 & 31.35931 & 20859 \\
\hline 2200 & 4.49070 & 1259 & 4.07672 & 1828 & 27.49118 & 18162 & 31.56790 & 19991 \\
\hline 2300 & 4.50329 & 1163 & 4.09500 & 1726 & 27.67280 & 17465 & 31.76781 & 19190 \\
\hline 2400 & 4.51492 & 1031 & 4.11226 & 1632 & 27.84745 & 16821 & 31.95971 & 18453 \\
\hline 2500 & 4.52573 & 1011 & 4.12858 & 1547 & 28.01566 & 16223 & 32.14424 & 17770 \\
\hline 2600 & 4.53584 & 949 & 4.14405 & 1469 & 28.17789 & 15667 & 32.32194 & 17137 \\
\hline 2700 & 4.54533 & 895 & 4.15874 & 1397 & 28.33456 & 15150 & 32.49331 & 16546 \\
\hline 2800 & 4.55428 & 849 & 4.17271 & 1330 & 28.48606 & 14666 & 32.65877 & 15997 \\
\hline 2900 & 4.56277 & 808 & 4.18601 & 1270 & 28.63272 & 14213 & 32.81874 & 15482 \\
\hline 3000 & 4.57085 & 1509 & 4.19871 & 2374 & 28.77485 & 27175 & 32.97356 & 29549 \\
\hline 3200 & 4.58594 & 1395 & 4.22245 & 2179 & 29.04660 & 25654 & 33.26905 & 27844 \\
\hline 3400 & 4.59989 & 1302 & 4.24424 & 2013 & 29.30324 & 24318 & 33.54749 & 26330 \\
\hline 3600 & 4.61291 & 1227 & 4.26437 & 1867 & 29.54642 & 23106 & 33.81079 & 24974 \\
\hline 3800 & 4.62518 & 1165 & 4.28304 & 1740 & 29.77748 & 22014 & 34.06053 & 23754 \\
\hline 4000 & 4.63683 & 1114 & 4.30044 & 1628 & 29.99762 & 21022 & 34.29807 & 22650 \\
\hline 4200 & 4.64797 & 1072 & 4.31672 & 1530 & 30.20784 & 20117 & 34.52457 & 21647 \\
\hline 4400 & 4.65869 & 1035 & 4.33202 & 1443 & 30.40901 & 19289 & 34.74104 & 20732 \\
\hline 4600 & 4.66904 & 1004 & 4.34645 & 1366 & 30.60190 & 18527 & 34.94836 & 19892 \\
\hline $\begin{array}{l}4800 \\
5000\end{array}$ & $\begin{array}{l}4.67908 \\
4.68885\end{array}$ & 977 & $\begin{array}{l}4.36011 \\
4.37306\end{array}$ & 1295 & $\begin{array}{l}30.78717 \\
30.96543\end{array}$ & 17826 & $\begin{array}{l}35.14728 \\
35.33849\end{array}$ & 19121 \\
\hline
\end{tabular}


Table 2.106. BrH

\begin{tabular}{|c|c|c|c|c|c|c|c|c|}
\hline$\stackrel{\circ}{K}$ & $\frac{C_{p}^{0}}{R}$ & & $\frac{\left(H^{0}-E_{0}^{0}\right)}{R T}$ & & $\frac{-1 F^{\circ}-E}{R T}$ & & $\frac{S^{0}}{R}$ & \\
\hline $\begin{array}{l}50 \\
60 \\
70 \\
80 \\
90\end{array}$ & $\begin{array}{l}3.50219 \\
3.50189 \\
3.50178 \\
3.50176 \\
3.50179\end{array}$ & $\begin{array}{r}-30 \\
-11 \\
-\quad 2 \\
3 \\
7\end{array}$ & $\begin{array}{l}3.41894 \\
3.43278 \\
3.44265 \\
3.45004 \\
3.45578\end{array}$ & $\begin{array}{r}1384 \\
987 \\
739 \\
574 \\
461\end{array}$ & $\begin{array}{l}14.21309 \\
14.83774 \\
15.36769 \\
15.82790 \\
16.23460\end{array}$ & $\begin{array}{l}62465 \\
52995 \\
46021 \\
40670 \\
36435\end{array}$ & $\begin{array}{l}17.63203 \\
18.27053 \\
18.81034 \\
19.27794 \\
19.69039\end{array}$ & $\begin{array}{l}63850 \\
53981 \\
46760 \\
41245 \\
36895\end{array}$ \\
\hline $\begin{array}{l}100 \\
110 \\
120 \\
130 \\
140\end{array}$ & $\begin{array}{l}3.50186 \\
3.50195 \\
3.50205 \\
3.50217 \\
3.50229\end{array}$ & $\begin{array}{r}9 \\
10 \\
12 \\
12 \\
13\end{array}$ & $\begin{array}{l}3.46039 \\
3.46416 \\
3.46731 \\
3.46999 \\
3.47229\end{array}$ & $\begin{array}{l}377 \\
315 \\
268 \\
230 \\
201\end{array}$ & $\begin{array}{l}16.59895 \\
16.92894 \\
17.23050 \\
17.50814 \\
17.76538\end{array}$ & $\begin{array}{l}32999 \\
30156 \\
27764 \\
25724 \\
23964\end{array}$ & $\begin{array}{l}20.05934 \\
20.39311 \\
20.69782 \\
20.97814 \\
21.23768\end{array}$ & $\begin{array}{l}33377 \\
30471 \\
28032 \\
25954 \\
24164\end{array}$ \\
\hline $\begin{array}{l}150 \\
160 \\
170 \\
180 \\
190\end{array}$ & $\begin{array}{l}3.50242 \\
3.50255 \\
3.50269 \\
3.50283 \\
3.50297\end{array}$ & $\begin{array}{l}13 \\
14 \\
14 \\
14 \\
15\end{array}$ & $\begin{array}{l}3.47430 \\
3.47606 \\
3.47762 \\
3.47902 \\
3.48028\end{array}$ & $\begin{array}{l}176 \\
156 \\
140 \\
126 \\
113\end{array}$ & $\begin{array}{l}18.00502 \\
18.22930 \\
18.44008 \\
18.63890 \\
18.82704\end{array}$ & $\begin{array}{l}22428 \\
21078 \\
19882 \\
18814 \\
17854\end{array}$ & $\begin{array}{l}21.47932 \\
21.70537 \\
21.91771 \\
22.11792 \\
22.30732\end{array}$ & $\begin{array}{l}22605 \\
21234 \\
20021 \\
18940 \\
17968\end{array}$ \\
\hline $\begin{array}{l}200 \\
210 \\
220 \\
230 \\
240\end{array}$ & $\begin{array}{l}3.50312 \\
3.50326 \\
3.50341 \\
3.50357 \\
3.50374\end{array}$ & $\begin{array}{l}14 \\
15 \\
16 \\
17 \\
19\end{array}$ & $\begin{array}{l}3.48141 \\
3.48245 \\
3.48340 \\
3.48427 \\
3.48508\end{array}$ & $\begin{array}{r}104 \\
95 \\
87 \\
81 \\
75\end{array}$ & $\begin{array}{l}19.00558 \\
19.17546 \\
19.33749 \\
19.49235 \\
19.64066\end{array}$ & $\begin{array}{l}16988 \\
16203 \\
15486 \\
14831 \\
14228\end{array}$ & $\begin{array}{l}22.48700 \\
22.65792 \\
22.82090 \\
22.97663 \\
23.12575\end{array}$ & $\begin{array}{l}17092 \\
16298 \\
15573 \\
14912 \\
14303\end{array}$ \\
\hline $\begin{array}{l}250 \\
260 \\
270 \\
280 \\
290\end{array}$ & $\begin{array}{l}3.50393 \\
3.50413 \\
3.50436 \\
3.50463 \\
3.50493\end{array}$ & $\begin{array}{l}20 \\
23 \\
27 \\
30 \\
37\end{array}$ & $\begin{array}{l}3.48583 \\
3.48653 \\
3.48719 \\
3.48781 \\
3.48839\end{array}$ & $\begin{array}{l}70 \\
66 \\
6 ? \\
58 \\
56\end{array}$ & $\begin{array}{l}19.78294 \\
19.91967 \\
20.05127 \\
20.17810 \\
20.30050\end{array}$ & $\begin{array}{l}13673 \\
13160 \\
12683 \\
12240 \\
11828\end{array}$ & $\begin{array}{l}23.26878 \\
23.40621 \\
23.53846 \\
23.66591 \\
23.78890\end{array}$ & $\begin{array}{l}13743 \\
13225 \\
12745 \\
12299 \\
11883\end{array}$ \\
\hline $\begin{array}{l}300 \\
310 \\
320 \\
330 \\
340\end{array}$ & $\begin{array}{l}3.50530 \\
3.50573 \\
3.50624 \\
3.50685 \\
3.50756\end{array}$ & $\begin{array}{l}43 \\
51 \\
61 \\
71 \\
83\end{array}$ & $\begin{array}{l}3.48895 \\
3.48948 \\
3.49000 \\
3.49050 \\
3.49099\end{array}$ & $\begin{array}{l}53 \\
52 \\
50 \\
49 \\
49\end{array}$ & $\begin{array}{l}20.41878 \\
20.53319 \\
20.64398 \\
20.75138 \\
20.85559\end{array}$ & $\begin{array}{l}11441 \\
11079 \\
10740 \\
10421 \\
10120\end{array}$ & $\begin{array}{l}23.90773 \\
24.02267 \\
24.13399 \\
24.24189 \\
24.34659\end{array}$ & $\begin{array}{l}11494 \\
11132 \\
10790 \\
10470 \\
10168\end{array}$ \\
\hline $\begin{array}{l}350 \\
360 \\
370 \\
380 \\
390\end{array}$ & $\begin{array}{l}3.50839 \\
3.50935 \\
3.51047 \\
3.51175 \\
3.51320\end{array}$ & $\begin{array}{r}96 \\
112 \\
128 \\
145 \\
163\end{array}$ & $\begin{array}{l}3.49148 \\
3.49196 \\
3.49244 \\
3.49293 \\
3.49343\end{array}$ & $\begin{array}{l}48 \\
48 \\
49 \\
50 \\
52\end{array}$ & $\begin{array}{l}20.95679 \\
21.05516 \\
21.15084 \\
21.24399 \\
21.33472\end{array}$ & $\begin{array}{l}9837 \\
9568 \\
9315 \\
9073 \\
8846\end{array}$ & $\begin{array}{l}24.44827 \\
24.54712 \\
24.64329 \\
24.73693 \\
24.82816\end{array}$ & $\begin{array}{l}9885 \\
9617 \\
9364 \\
9123 \\
8897\end{array}$ \\
\hline $\begin{array}{l}400 \\
450 \\
500 \\
550 \\
600\end{array}$ & $\begin{array}{l}3.51483 \\
3.52607 \\
3.54295 \\
3.56552 \\
3.59324\end{array}$ & $\begin{array}{l}1124 \\
1688 \\
2257 \\
2772 \\
3195\end{array}$ & $\begin{array}{l}3.49395 \\
3.49684 \\
3.50056 \\
3.50540 \\
3.51153\end{array}$ & $\begin{array}{l}289 \\
372 \\
484 \\
613 \\
749\end{array}$ & $\begin{array}{l}21.42318 \\
21.83487 \\
22.20348 \\
22.53734 \\
22.84260\end{array}$ & $\begin{array}{l}41169 \\
36861 \\
33386 \\
30526 \\
28136\end{array}$ & $\begin{array}{l}24.91713 \\
25.33171 \\
25.70405 \\
26.04274 \\
26.35414\end{array}$ & $\begin{array}{l}41458 \\
37234 \\
33869 \\
31140 \\
28885\end{array}$ \\
\hline $\begin{array}{l}650 \\
700 \\
750 \\
800 \\
850\end{array}$ & $\begin{array}{l}3.62519 \\
3.66031 \\
3.69756 \\
3.73598 \\
3.77481\end{array}$ & $\begin{array}{l}3512 \\
3725 \\
3842 \\
3883 \\
3861\end{array}$ & $\begin{array}{l}3.51902 \\
3.52785 \\
3.53791 \\
3.54908 \\
3.56122\end{array}$ & $\begin{array}{r}883 \\
1006 \\
1117 \\
1214 \\
1294\end{array}$ & $\begin{array}{l}23.12396 \\
23.38506 \\
23.62879 \\
23.85748 \\
24.07300\end{array}$ & $\begin{array}{l}26110 \\
24373 \\
22869 \\
21552 \\
20392\end{array}$ & $\begin{array}{l}26.64299 \\
26.91291 \\
27.16671 \\
27.40657 \\
27.63422\end{array}$ & $\begin{array}{l}26992 \\
25380 \\
23986 \\
22765 \\
21686\end{array}$ \\
\hline $\begin{array}{l}900 \\
950 \\
1000 \\
1050 \\
100\end{array}$ & $\begin{array}{l}3.81342 \\
3.85132 \\
3.88817 \\
3.92373 \\
3.95785\end{array}$ & $\begin{array}{l}3790 \\
3685 \\
3556 \\
3412 \\
3260\end{array}$ & $\begin{array}{l}3.57416 \\
3.58775 \\
3.60186 \\
3.61634 \\
3.63110\end{array}$ & $\begin{array}{l}1359 \\
1411 \\
1448 \\
1476 \\
1492\end{array}$ & $\begin{array}{l}24.27692 \\
24.47052 \\
24.65491 \\
24.83099 \\
24.99956\end{array}$ & $\begin{array}{l}19360 \\
18439 \\
17608 \\
16857 \\
16174\end{array}$ & $\begin{array}{l}27.85108 \\
28.05828 \\
28.25677 \\
28.44734 \\
28.63067\end{array}$ & $\begin{array}{l}20720 \\
19849 \\
19057 \\
18333 \\
17666\end{array}$ \\
\hline 150 & 3.99045 & 3103 & 3.64602 & 1500 & 25.16130 & 15549 & 28.80733 & 17049 \\
\hline
\end{tabular}


Table 2.106. BrH (Cont.)

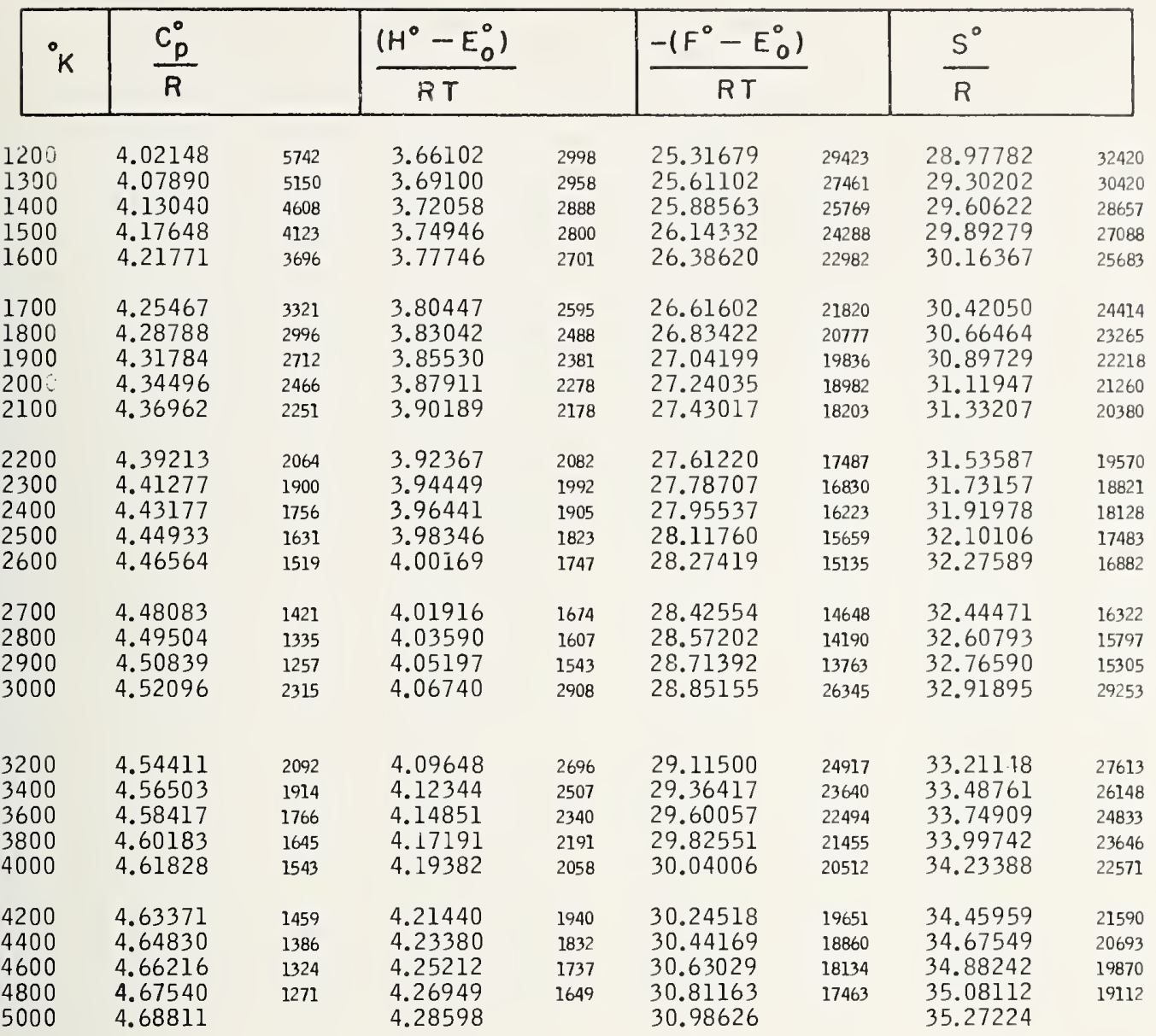


Table 2.107. BrD

\begin{tabular}{|c|c|c|c|c|c|c|c|c|}
\hline${ }^{\circ} \mathrm{K}$ & $\frac{C_{p}^{0}}{R}$ & & $\frac{\left(H^{0}-E_{0}^{0}\right)}{R T}$ & & $\frac{-\left(F^{0}-\right.}{R T}$ & & $\frac{S^{0}}{R}$ & \\
\hline $\begin{array}{l}50 \\
60 \\
70 \\
80 \\
90\end{array}$ & $\begin{array}{l}3.50109 \\
3.50113 \\
3.50121 \\
3.50131 \\
3.50143\end{array}$ & $\begin{array}{r}4 \\
8 \\
10 \\
12 \\
13\end{array}$ & $\begin{array}{l}3.45929 \\
3.46626 \\
3.47124 \\
3.47500 \\
3.47793\end{array}$ & $\begin{array}{l}697 \\
498 \\
376 \\
293 \\
235\end{array}$ & $\begin{array}{l}14.86715 \\
15.49851 \\
16.03323 \\
16.49700 \\
16.90647\end{array}$ & $\begin{array}{l}63136 \\
53472 \\
46377 \\
40947 \\
36557\end{array}$ & $\begin{array}{l}18.32644 \\
18.96477 \\
19.50447 \\
19.97200 \\
20.38441\end{array}$ & $\begin{array}{l}63833 \\
53970 \\
46753 \\
41241 \\
36892\end{array}$ \\
\hline $\begin{array}{l}100 \\
110 \\
120 \\
130 \\
140\end{array}$ & $\begin{array}{l}3.50156 \\
3.50169 \\
3.50183 \\
3.50197 \\
3.50211\end{array}$ & $\begin{array}{l}13 \\
14 \\
14 \\
14 \\
15\end{array}$ & $\begin{array}{l}3.48028 \\
3.48222 \\
3.48385 \\
3.48524 \\
3.48644\end{array}$ & $\begin{array}{l}194 \\
163 \\
139 \\
120 \\
105\end{array}$ & $\begin{array}{l}17.27304 \\
17.60484 \\
17.90790 \\
18.18682 \\
18.44515\end{array}$ & $\begin{array}{l}33180 \\
30306 \\
27892 \\
25833 \\
24057\end{array}$ & $\begin{array}{l}20.75333 \\
21.08707 \\
21.39176 \\
21.67206 \\
21.93159\end{array}$ & $\begin{array}{l}33374 \\
30459 \\
28030 \\
25953 \\
24163\end{array}$ \\
\hline $\begin{array}{l}150 \\
160 \\
170 \\
180 \\
190\end{array}$ & $\begin{array}{l}3.50226 \\
3.50241 \\
3.50258 \\
3.50277 \\
3.50300\end{array}$ & $\begin{array}{l}15 \\
17 \\
19 \\
23 \\
29\end{array}$ & $\begin{array}{l}3.48749 \\
3.48842 \\
3.48924 \\
3.48999 \\
3.49067\end{array}$ & $\begin{array}{l}93 \\
82 \\
75 \\
68 \\
62\end{array}$ & $\begin{array}{l}18.68572 \\
18.91083 \\
19.12234 \\
19.32180 \\
19.51051\end{array}$ & $\begin{array}{l}22511 \\
21151 \\
19946 \\
18871 \\
17907\end{array}$ & $\begin{array}{l}22.17322 \\
22.39925 \\
22.61159 \\
22.81180 \\
23.00119\end{array}$ & $\begin{array}{l}22603 \\
21234 \\
20021 \\
18939 \\
17969\end{array}$ \\
\hline $\begin{array}{l}200 \\
210 \\
220 \\
230 \\
240\end{array}$ & $\begin{array}{l}3.50329 \\
3.50366 \\
3.50414 \\
3.50476 \\
3.50556\end{array}$ & $\begin{array}{r}37 \\
48 \\
62 \\
80 \\
101\end{array}$ & $\begin{array}{l}3.49129 \\
3.49187 \\
3.49242 \\
3.49294 \\
3.49345\end{array}$ & $\begin{array}{l}58 \\
55 \\
52 \\
51 \\
50\end{array}$ & $\begin{array}{l}19.68958 \\
19.85993 \\
20.02239 \\
20.17764 \\
20.32631\end{array}$ & $\begin{array}{l}17035 \\
16246 \\
15525 \\
14867 \\
14262\end{array}$ & $\begin{array}{l}23.18088 \\
23.35181 \\
23.51481 \\
23.67059 \\
23.81977\end{array}$ & $\begin{array}{l}17093 \\
16300 \\
15578 \\
14918 \\
14312\end{array}$ \\
\hline $\begin{array}{l}250 \\
260 \\
270 \\
280 \\
290\end{array}$ & $\begin{array}{l}3.50657 \\
3.50784 \\
3.50940 \\
3.51128 \\
3.51350\end{array}$ & $\begin{array}{l}127 \\
156 \\
188 \\
222 \\
261\end{array}$ & $\begin{array}{l}3.49395 \\
3.49446 \\
3.49499 \\
3.49553 \\
3.49611\end{array}$ & $\begin{array}{l}51 \\
53 \\
54 \\
58 \\
63\end{array}$ & $\begin{array}{l}20.46893 \\
20.60598 \\
20.73787 \\
20.86499 \\
20.98766\end{array}$ & $\begin{array}{l}13705 \\
13189 \\
12712 \\
12267 \\
11853\end{array}$ & $\begin{array}{l}23.96289 \\
24.10045 \\
24.23286 \\
24.36052 \\
24.48378\end{array}$ & $\begin{array}{l}13756 \\
13241 \\
12766 \\
12326 \\
11915\end{array}$ \\
\hline $\begin{array}{l}300 \\
310 \\
320 \\
330 \\
340\end{array}$ & $\begin{array}{l}3.51611 \\
3.51912 \\
3.52255 \\
3.52641 \\
3.53072\end{array}$ & $\begin{array}{l}301 \\
343 \\
386 \\
431 \\
475\end{array}$ & $\begin{array}{l}3.49674 \\
3.49741 \\
3.49814 \\
3.49894 \\
3.49981\end{array}$ & $\begin{array}{l}67 \\
73 \\
80 \\
87 \\
95\end{array}$ & $\begin{array}{l}21.10619 \\
21.22086 \\
21.33191 \\
21.43957 \\
21.54403\end{array}$ & $\begin{array}{l}11467 \\
11105 \\
10766 \\
10446 \\
10147\end{array}$ & $\begin{array}{l}24.60293 \\
24.71827 \\
24.83005 \\
24.93851 \\
25.04384\end{array}$ & $\begin{array}{l}11534 \\
11178 \\
10846 \\
10533 \\
10242\end{array}$ \\
\hline $\begin{array}{l}350 \\
360 \\
370 \\
380 \\
390\end{array}$ & $\begin{array}{l}3.53547 \\
3.54066 \\
3.54629 \\
3.55236 \\
3.55884\end{array}$ & $\begin{array}{l}519 \\
563 \\
607 \\
648 \\
690\end{array}$ & $\begin{array}{l}3.50076 \\
3.50179 \\
3.50292 \\
3.50414 \\
3.50546\end{array}$ & $\begin{array}{l}103 \\
113 \\
122 \\
132 \\
142\end{array}$ & $\begin{array}{l}21.64550 \\
21.74413 \\
21.84009 \\
21.93352 \\
22.02456\end{array}$ & $\begin{array}{l}9863 \\
9596 \\
9343 \\
9104 \\
8877\end{array}$ & $\begin{array}{l}25.14626 \\
25.24593 \\
25.34301 \\
25.43767 \\
25.53002\end{array}$ & $\begin{array}{l}9967 \\
9708 \\
9466 \\
9235 \\
9019\end{array}$ \\
\hline $\begin{array}{l}400 \\
450 \\
500 \\
550 \\
600\end{array}$ & $\begin{array}{l}3.56574 \\
3.60562 \\
3.65253 \\
3.70366 \\
3.75655\end{array}$ & $\begin{array}{l}3988 \\
4691 \\
5113 \\
5289 \\
5276\end{array}$ & $\begin{array}{l}3.50688 \\
3.51555 \\
3.52686 \\
3.54059 \\
3.55637\end{array}$ & $\begin{array}{r}867 \\
1131 \\
1373 \\
1578 \\
1744\end{array}$ & $\begin{array}{l}22.11333 \\
22.52686 \\
22.89782 \\
23.23459 \\
23.54333\end{array}$ & $\begin{array}{l}41353 \\
37096 \\
33677 \\
30874 \\
28534\end{array}$ & $\begin{array}{l}25.62021 \\
26.04242 \\
26.42469 \\
26.77518 \\
27.09971\end{array}$ & $\begin{array}{l}42221 \\
38227 \\
35049 \\
32453 \\
30277\end{array}$ \\
\hline $\begin{array}{l}650 \\
700 \\
750 \\
800 \\
850\end{array}$ & $\begin{array}{l}3.80931 \\
3.86060 \\
3.90960 \\
3.95582 \\
3.99905\end{array}$ & $\begin{array}{l}5129 \\
4900 \\
4622 \\
4323 \\
4019\end{array}$ & $\begin{array}{l}3.57381 \\
3.59247 \\
3.61200 \\
3.63206 \\
3.65239\end{array}$ & $\begin{array}{l}1866 \\
1953 \\
2006 \\
2033 \\
2039\end{array}$ & $\begin{array}{l}23.82867 \\
24.09419 \\
24.34271 \\
24.57646 \\
24.79726\end{array}$ & $\begin{array}{l}26552 \\
24852 \\
23375 \\
22080 \\
20935\end{array}$ & $\begin{array}{l}27.40248 \\
27.68667 \\
27.95471 \\
28.20852 \\
28.44966\end{array}$ & $\begin{array}{l}28419 \\
26804 \\
25381 \\
24114 \\
22973\end{array}$ \\
\hline $\begin{array}{l}900 \\
950 \\
1000 \\
1050 \\
1100\end{array}$ & $\begin{array}{l}4.03924 \\
4.07646 \\
4.11085 \\
4.14260 \\
4.17190\end{array}$ & $\begin{array}{l}3722 \\
3439 \\
3175 \\
2930 \\
2709\end{array}$ & $\begin{array}{l}3.67278 \\
3.69306 \\
3.71310 \\
3.73280 \\
3.75211\end{array}$ & $\begin{array}{l}2028 \\
2004 \\
1970 \\
1931 \\
1884\end{array}$ & $\begin{array}{l}25.00661 \\
25.20573 \\
25.39567 \\
25.57731 \\
25.75141\end{array}$ & $\begin{array}{l}19912 \\
18994 \\
18164 \\
17410 \\
16720\end{array}$ & $\begin{array}{l}28.67939 \\
28.89879 \\
29.10877 \\
29.31012 \\
29.50352\end{array}$ & $\begin{array}{l}21940 \\
20998 \\
20135 \\
19340 \\
18605\end{array}$ \\
\hline 50 & 4.19894 & 2498 & 3.77095 & 1836 & 25.91861 & 16088 & 29.68957 & 17924 \\
\hline
\end{tabular}


Table 2.107. BrD (Cont.)

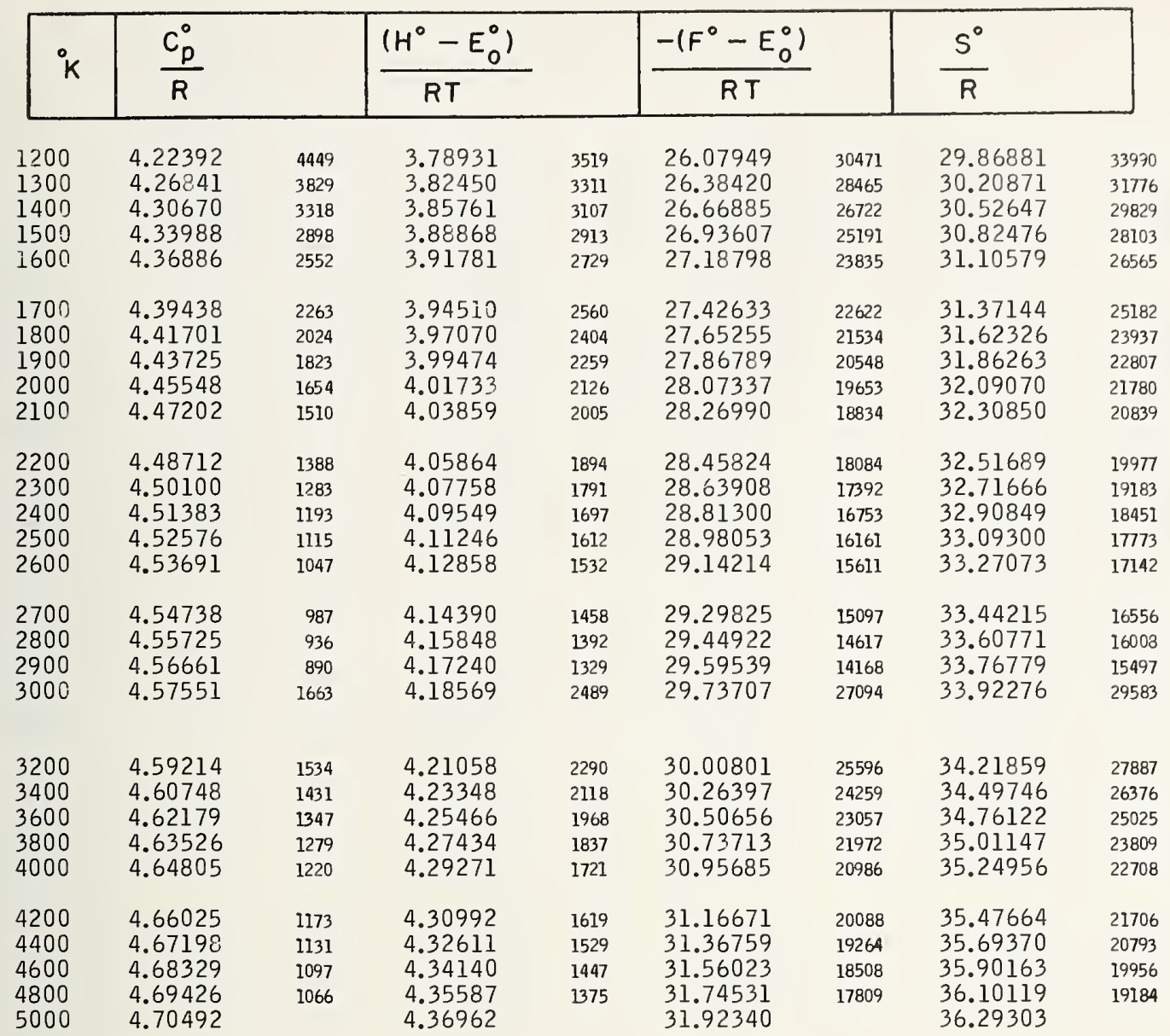


Table 2.108. BrT

\begin{tabular}{|c|c|c|c|c|c|c|c|c|}
\hline${ }^{\circ} \mathrm{K}$ & $\frac{C_{p}^{0}}{R}$ & & $\frac{\left(H^{0}-E_{0}^{0}\right)}{R T}$ & & $\frac{-\left(F^{\circ}-E\right.}{R T}$ & & $\frac{S^{0}}{R}$ & \\
\hline $\begin{array}{l}50 \\
60 \\
70 \\
80 \\
90\end{array}$ & $\begin{array}{l}3.50089 \\
3.50099 \\
3.50111 \\
3.50124 \\
3.50137\end{array}$ & $\begin{array}{l}10 \\
12 \\
13 \\
13 \\
14\end{array}$ & $\begin{array}{l}3.47268 \\
3.47739 \\
3.48077 \\
3.48332 \\
3.48532\end{array}$ & $\begin{array}{l}471 \\
338 \\
255 \\
200 \\
161\end{array}$ & $\begin{array}{l}15.26475 \\
15.89834 \\
16.43465 \\
16.89962 \\
17.31001\end{array}$ & $\begin{array}{l}63359 \\
53631 \\
46497 \\
41039 \\
36730\end{array}$ & $\begin{array}{l}18.73744 \\
19.37573 \\
19.91542 \\
20.38294 \\
20.79533\end{array}$ & $\begin{array}{l}63829 \\
53969 \\
46752 \\
41239 \\
36892\end{array}$ \\
\hline $\begin{array}{l}100 \\
110 \\
120 \\
130 \\
140\end{array}$ & $\begin{array}{l}3.50151 \\
3.50165 \\
3.50180 \\
3.50195 \\
3.50212\end{array}$ & $\begin{array}{l}14 \\
15 \\
15 \\
17 \\
21\end{array}$ & $\begin{array}{l}3.48693 \\
3.48826 \\
3.48938 \\
3.49034 \\
3.49118\end{array}$ & $\begin{array}{r}133 \\
112 \\
96 \\
84 \\
74\end{array}$ & $\begin{array}{l}17.67731 \\
18.00972 \\
18.31329 \\
18.59263 \\
18.85132\end{array}$ & $\begin{array}{l}33241 \\
30357 \\
27934 \\
25869 \\
24089\end{array}$ & $\begin{array}{l}21.16425 \\
21.49799 \\
21.80268 \\
22.08298 \\
22.34251\end{array}$ & $\begin{array}{l}33374 \\
30469 \\
28030 \\
25953 \\
24162\end{array}$ \\
\hline $\begin{array}{l}150 \\
160 \\
170 \\
180 \\
190\end{array}$ & $\begin{array}{l}3.50233 \\
3.50259 \\
3.50296 \\
3.50346 \\
3.50417\end{array}$ & $\begin{array}{l}26 \\
37 \\
50 \\
71 \\
96\end{array}$ & $\begin{array}{l}3.49192 \\
3.49257 \\
3.49317 \\
3.49373 \\
3.49426\end{array}$ & $\begin{array}{l}65 \\
60 \\
56 \\
53 \\
52\end{array}$ & $\begin{array}{l}19.09221 \\
19.31760 \\
19.52935 \\
19.72903 \\
19.91794\end{array}$ & $\begin{array}{l}22539 \\
21175 \\
19968 \\
18891 \\
17925\end{array}$ & $\begin{array}{l}22.58413 \\
22.81018 \\
23.02253 \\
23.22277 \\
23.41221\end{array}$ & $\begin{array}{l}22605 \\
21235 \\
20024 \\
18744 \\
17976\end{array}$ \\
\hline $\begin{array}{l}200 \\
210 \\
220 \\
230 \\
240\end{array}$ & $\begin{array}{l}3.50513 \\
3.50642 \\
3.50810 \\
3.51024 \\
3.51288\end{array}$ & $\begin{array}{l}129 \\
168 \\
214 \\
264 \\
319\end{array}$ & $\begin{array}{l}3.49478 \\
3.49530 \\
3.49584 \\
3.49642 \\
3.49705\end{array}$ & $\begin{array}{l}52 \\
54 \\
58 \\
63 \\
69\end{array}$ & $\begin{array}{l}20.09719 \\
20.26771 \\
20.43033 \\
20.58574 \\
20.73456\end{array}$ & $\begin{array}{l}17052 \\
16262 \\
15541 \\
14882 \\
14277\end{array}$ & $\begin{array}{l}23.59197 \\
23.76302 \\
23.92617 \\
24.08216 \\
24.23161\end{array}$ & $\begin{array}{l}17105 \\
16315 \\
15599 \\
14945 \\
14347\end{array}$ \\
\hline $\begin{array}{l}250 \\
260 \\
270 \\
280 \\
290\end{array}$ & $\begin{array}{l}3.51607 \\
3.51987 \\
3.52428 \\
3.52934 \\
3.53505\end{array}$ & $\begin{array}{l}380 \\
441 \\
506 \\
571 \\
636\end{array}$ & $\begin{array}{l}3.49774 \\
3.49852 \\
3.49939 \\
3.50037 \\
3.50146\end{array}$ & $\begin{array}{r}78 \\
87 \\
98 \\
109 \\
123\end{array}$ & $\begin{array}{l}20.87733 \\
21.01453 \\
21.14658 \\
21.27386 \\
21.39671\end{array}$ & $\begin{array}{l}13720 \\
13205 \\
12728 \\
12285 \\
11873\end{array}$ & $\begin{array}{l}24.37508 \\
24.51305 \\
24.64597 \\
24.77423 \\
24.89818\end{array}$ & $\begin{array}{l}13797 \\
13292 \\
12826 \\
12395 \\
11995\end{array}$ \\
\hline $\begin{array}{l}300 \\
310 \\
320 \\
330 \\
340\end{array}$ & $\begin{array}{l}3.54141 \\
3.54840 \\
3.55602 \\
3.56423 \\
3.57302\end{array}$ & $\begin{array}{l}699 \\
762 \\
821 \\
879 \\
931\end{array}$ & $\begin{array}{l}3.50269 \\
3.50405 \\
3.50555 \\
3.50720 \\
3.50901\end{array}$ & $\begin{array}{l}136 \\
150 \\
165 \\
181 \\
196\end{array}$ & $\begin{array}{l}21.51544 \\
21.63031 \\
21.74158 \\
21.84948 \\
21.95421\end{array}$ & $\begin{array}{l}11487 \\
11127 \\
10790 \\
10473 \\
10174\end{array}$ & $\begin{array}{l}25.01813 \\
25.13436 \\
25.24714 \\
25.35669 \\
25.46322\end{array}$ & $\begin{array}{l}11623 \\
11278 \\
10955 \\
10653 \\
10371\end{array}$ \\
\hline $\begin{array}{l}350 \\
360 \\
370 \\
380 \\
390\end{array}$ & $\begin{array}{l}3.58233 \\
3.59215 \\
3.60242 \\
3.61311 \\
3.62418\end{array}$ & $\begin{array}{r}982 \\
1027 \\
1069 \\
1107 \\
1140\end{array}$ & $\begin{array}{l}3.51097 \\
3.51309 \\
3.51536 \\
3.51779 \\
3.52038\end{array}$ & $\begin{array}{l}212 \\
227 \\
243 \\
259 \\
273\end{array}$ & $\begin{array}{l}22.05595 \\
22.15489 \\
22.25117 \\
22.34495 \\
22.43636\end{array}$ & $\begin{array}{l}9894 \\
9628 \\
9378 \\
9141 \\
8917\end{array}$ & $\begin{array}{l}25.56693 \\
25.66798 \\
25.76654 \\
25.86275 \\
25.95675\end{array}$ & $\begin{array}{r}10105 \\
9856 \\
9621 \\
9400 \\
9190\end{array}$ \\
\hline $\begin{array}{l}400 \\
450 \\
500 \\
550 \\
600\end{array}$ & $\begin{array}{l}3.63558 \\
3.69623 \\
3.75977 \\
3.82285 \\
3.88332\end{array}$ & $\begin{array}{l}6065 \\
6354 \\
6308 \\
6047 \\
5667\end{array}$ & $\begin{array}{l}3.52311 \\
3.53893 \\
3.55783 \\
3.57907 \\
3.60193\end{array}$ & $\begin{array}{l}1582 \\
1890 \\
2124 \\
2286 \\
2385\end{array}$ & $\begin{array}{l}22.52553 \\
22.94137 \\
23.31519 \\
23.65527 \\
23.96766\end{array}$ & $\begin{array}{l}41584 \\
37382 \\
34008 \\
31239 \\
28924\end{array}$ & $\begin{array}{l}26.04865 \\
26.48031 \\
26.87303 \\
27.23434 \\
27.56959\end{array}$ & $\begin{array}{l}43166 \\
39272 \\
36131 \\
33525 \\
31310\end{array}$ \\
\hline $\begin{array}{l}650 \\
700 \\
750 \\
800 \\
850\end{array}$ & $\begin{array}{l}3.93999 \\
3.99231 \\
4.04016 \\
4.08370 \\
4.12318\end{array}$ & $\begin{array}{l}5232 \\
4785 \\
4354 \\
3948 \\
3577\end{array}$ & $\begin{array}{l}3.62578 \\
3.65012 \\
3.67455 \\
3.69878 \\
3.72261\end{array}$ & $\begin{array}{l}2434 \\
2443 \\
2423 \\
2383 \\
2326\end{array}$ & $\begin{array}{l}24.25690 \\
24.52649 \\
24.77916 \\
25.01708 \\
25.24204\end{array}$ & $\begin{array}{l}26959 \\
25267 \\
23792 \\
22496 \\
21344\end{array}$ & $\begin{array}{l}27.88269 \\
28.17662 \\
28.45371 \\
28.71587 \\
28.96465\end{array}$ & $\begin{array}{l}29393 \\
27709 \\
26216 \\
24878 \\
23671\end{array}$ \\
\hline $\begin{array}{l}900 \\
950 \\
000 \\
050 \\
100\end{array}$ & $\begin{array}{l}4.15895 \\
4.19135 \\
4.22073 \\
4.24742 \\
4.27172\end{array}$ & $\begin{array}{l}3240 \\
2938 \\
2669 \\
2430 \\
2216\end{array}$ & $\begin{array}{l}3.74587 \\
3.76848 \\
3.79037 \\
3.81151 \\
3.83188\end{array}$ & $\begin{array}{l}2261 \\
2189 \\
2114 \\
2037 \\
1961\end{array}$ & $\begin{array}{l}25.45548 \\
25.65862 \\
25.85247 \\
26.03792 \\
26.21571\end{array}$ & $\begin{array}{l}20314 \\
19385 \\
18545 \\
17779 \\
17077\end{array}$ & $\begin{array}{l}29.20136 \\
29.42710 \\
29.64285 \\
29.84943 \\
30.04759\end{array}$ & $\begin{array}{l}22574 \\
21575 \\
20658 \\
19816 \\
19039\end{array}$ \\
\hline 50 & 4.29388 & 2028 & 3.85149 & 1887 & 26.38648 & 16432 & 30.23798 & 18318 \\
\hline
\end{tabular}


Table 2. 108. BrT (Cont.)

\begin{tabular}{|c|c|c|c|c|c|c|c|c|}
\hline${ }^{\circ} \mathrm{K}$ & $\frac{C_{p}^{0}}{R}$ & & $\frac{\left(H^{0}-E_{0}^{0}\right)}{R T}$ & & $\frac{-\left(F^{\circ}-E\right.}{R T}$ & & $\frac{S^{\circ}}{R}$ & \\
\hline $\begin{array}{l}1200 \\
1300 \\
1400 \\
1500 \\
1600\end{array}$ & $\begin{array}{l}4.31416 \\
4.34987 \\
4.38025 \\
4.40639 \\
4.42913\end{array}$ & $\begin{array}{l}3571 \\
3038 \\
2614 \\
2274 \\
1999\end{array}$ & $\begin{array}{l}3.87036 \\
3.90591 \\
3.93873 \\
3.96906 \\
3.99712\end{array}$ & $\begin{array}{l}3555 \\
3282 \\
3033 \\
2806 \\
2601\end{array}$ & $\begin{array}{l}26.55080 \\
26.86201 \\
27.15269 \\
27.42548 \\
27.68255\end{array}$ & $\begin{array}{l}31121 \\
29068 \\
27279 \\
25707 \\
24311\end{array}$ & $\begin{array}{l}30.42116 \\
30.76792 \\
31.09143 \\
31.39455 \\
31.67967\end{array}$ & $\begin{array}{l}34676 \\
32351 \\
30312 \\
28512 \\
26913\end{array}$ \\
\hline $\begin{array}{l}1700 \\
1800 \\
1900 \\
2000 \\
2100\end{array}$ & $\begin{array}{l}4.44912 \\
4.46687 \\
4.48279 \\
4.49718 \\
4.51030\end{array}$ & $\begin{array}{l}1775 \\
1592 \\
1439 \\
1312 \\
1205\end{array}$ & $\begin{array}{l}4.02313 \\
4.04730 \\
4.06981 \\
4.09082 \\
4.11049\end{array}$ & $\begin{array}{l}2417 \\
2251 \\
2101 \\
1967 \\
1845\end{array}$ & $\begin{array}{l}27.92566 \\
28.15631 \\
28.37575 \\
28.58504 \\
28.78511\end{array}$ & $\begin{array}{l}23065 \\
21944 \\
20929 \\
20007 \\
19165\end{array}$ & $\begin{array}{l}31.94880 \\
32.20361 \\
32.44556 \\
32.67587 \\
32.89561\end{array}$ & $\begin{array}{l}25181 \\
24195 \\
23031 \\
21974 \\
21010\end{array}$ \\
\hline $\begin{array}{l}2200 \\
2300 \\
2400 \\
2500 \\
2600\end{array}$ & $\begin{array}{l}4.52235 \\
4.53351 \\
4.54389 \\
4.55362 \\
4.56278\end{array}$ & $\begin{array}{r}1116 \\
1038 \\
973 \\
916 \\
867\end{array}$ & $\begin{array}{l}4.12894 \\
4.14629 \\
4.16264 \\
4.17809 \\
4.19271\end{array}$ & $\begin{array}{l}1735 \\
1635 \\
1545 \\
1462 \\
1387\end{array}$ & $\begin{array}{l}28.97676 \\
29.16069 \\
29.33750 \\
29.50775 \\
29.67190\end{array}$ & $\begin{array}{l}18393 \\
17681 \\
17025 \\
16415 \\
15850\end{array}$ & $\begin{array}{l}33.10571 \\
33.30699 \\
33.50015 \\
33.68584 \\
33.86462\end{array}$ & $\begin{array}{l}20128 \\
19316 \\
18569 \\
17878 \\
17236\end{array}$ \\
\hline $\begin{array}{l}2700 \\
2800 \\
2900 \\
3000\end{array}$ & $\begin{array}{l}4.57145 \\
4.57969 \\
4.58756 \\
4.59510\end{array}$ & $\begin{array}{r}824 \\
787 \\
754 \\
1425\end{array}$ & $\begin{array}{l}4.20658 \\
4.21976 \\
4.23231 \\
4.24428\end{array}$ & $\begin{array}{l}1318 \\
1255 \\
1197 \\
2237\end{array}$ & $\begin{array}{l}29.83040 \\
29.98362 \\
30.13192 \\
30.27561\end{array}$ & $\begin{array}{l}15322 \\
14830 \\
14369 \\
27464\end{array}$ & $\begin{array}{l}34.03698 \\
34.20339 \\
34.36423 \\
34.51989\end{array}$ & $\begin{array}{l}16541 \\
16089 \\
15566 \\
29702\end{array}$ \\
\hline $\begin{array}{l}3200 \\
3400 \\
3600 \\
3800 \\
4000\end{array}$ & $\begin{array}{l}4.60935 \\
4.62267 \\
4.63526 \\
4.64725 \\
4.65875\end{array}$ & $\begin{array}{l}1332 \\
1259 \\
1199 \\
1150 \\
1109\end{array}$ & $\begin{array}{l}4.26665 \\
4.28721 \\
4.30620 \\
4.32383 \\
4.34029\end{array}$ & $\begin{array}{l}2056 \\
1899 \\
1763 \\
1646 \\
1543\end{array}$ & $\begin{array}{l}30.55025 \\
30.80954 \\
31.05514 \\
31.28844 \\
31.51065\end{array}$ & $\begin{array}{l}25929 \\
24560 \\
23330 \\
22221 \\
21214\end{array}$ & $\begin{array}{l}34.81691 \\
35.09675 \\
35.36134 \\
35.61228 \\
35.85094\end{array}$ & $\begin{array}{l}27984 \\
26459 \\
25094 \\
23866 \\
22758\end{array}$ \\
\hline $\begin{array}{l}4200 \\
4400 \\
4600 \\
4800 \\
5000\end{array}$ & $\begin{array}{l}4.66984 \\
4.68058 \\
4.69103 \\
4.70122 \\
4.71119\end{array}$ & $\begin{array}{r}1074 \\
1045 \\
1019 \\
997\end{array}$ & $\begin{array}{l}4.35572 \\
4.37025 \\
4.38397 \\
4.39698 \\
4.40935\end{array}$ & $\begin{array}{l}1453 \\
1372 \\
1301 \\
1237\end{array}$ & $\begin{array}{l}31.72279 \\
31.92575 \\
32.12032 \\
32.30718 \\
32.48693\end{array}$ & $\begin{array}{l}20296 \\
19457 \\
18686 \\
17975\end{array}$ & $\begin{array}{l}36.07852 \\
36.29601 \\
36.50430 \\
36.70416 \\
36.89628\end{array}$ & $\begin{array}{l}21749 \\
20829 \\
19986 \\
19212\end{array}$ \\
\hline
\end{tabular}


Table 2. 109.

HI

\begin{tabular}{|c|c|c|c|c|c|c|c|c|}
\hline${ }^{\circ} \mathrm{K}$ & $\frac{C_{p}^{0}}{R}$ & & $\frac{\left(H^{\circ}-E_{0}^{0}\right.}{R T}$ & & $\frac{-l F^{\circ}-E}{R T}$ & & $\frac{S^{0}}{R}$ & \\
\hline $\begin{array}{l}50 \\
60 \\
70 \\
80 \\
90\end{array}$ & $\begin{array}{l}3.50152 \\
3.50140 \\
3.50139 \\
3.50143 \\
3.50150\end{array}$ & $\begin{array}{r}-12 \\
-\quad 1 \\
4 \\
7 \\
9\end{array}$ & $\begin{array}{l}3.43857 \\
3.44905 \\
3.45652 \\
3.46213 \\
3.46650\end{array}$ & $\begin{array}{r}1048 \\
747 \\
561 \\
437 \\
351\end{array}$ & $\begin{array}{l}15.13731 \\
15.76522 \\
16.29748 \\
16.75942 \\
17.16746\end{array}$ & $\begin{array}{l}62791 \\
53226 \\
46194 \\
40804 \\
36542\end{array}$ & $\begin{array}{l}18.57588 \\
19.21427 \\
19.75401 \\
20.22156 \\
20.63397\end{array}$ & $\begin{array}{l}63839 \\
53974 \\
46755 \\
41241 \\
36993\end{array}$ \\
\hline $\begin{array}{l}100 \\
110 \\
120 \\
130 \\
140\end{array}$ & $\begin{array}{l}3.50159 \\
3.50170 \\
3.50181 \\
3.50193 \\
3.50206\end{array}$ & $\begin{array}{l}11 \\
11 \\
12 \\
13 \\
12\end{array}$ & $\begin{array}{l}3.47001 \\
3.47288 \\
3.47529 \\
3.47734 \\
3.47910\end{array}$ & $\begin{array}{l}287 \\
241 \\
205 \\
176 \\
153\end{array}$ & $\begin{array}{l}17.53288 \\
17.86375 \\
18.16604 \\
18.44429 \\
18.70206\end{array}$ & $\begin{array}{l}33087 \\
30229 \\
27825 \\
25777 \\
24008\end{array}$ & $\begin{array}{l}21.00290 \\
21.33664 \\
21.64133 \\
21.92163 \\
22.18116\end{array}$ & $\begin{array}{l}33374 \\
30469 \\
28030 \\
25953 \\
24162\end{array}$ \\
\hline $\begin{array}{l}150 \\
160 \\
170 \\
180 \\
190\end{array}$ & $\begin{array}{l}3.50218 \\
3.50231 \\
3.50245 \\
3.50258 \\
3.50272\end{array}$ & $\begin{array}{l}13 \\
14 \\
13 \\
14 \\
15\end{array}$ & $\begin{array}{l}3.48063 \\
3.48198 \\
3.48318 \\
3.48426 \\
3.48522\end{array}$ & $\begin{array}{r}135 \\
120 \\
108 \\
96 \\
88\end{array}$ & $\begin{array}{l}18.94214 \\
19.16682 \\
19.37795 \\
19.57708 \\
19.76549\end{array}$ & $\begin{array}{l}22468 \\
21113 \\
19913 \\
18841 \\
17879\end{array}$ & $\begin{array}{l}22.42278 \\
22.64881 \\
22.86114 \\
23.06134 \\
23.25072\end{array}$ & $\begin{array}{l}22603 \\
21233 \\
20020 \\
18938 \\
17967\end{array}$ \\
\hline $\begin{array}{l}200 \\
210 \\
220 \\
230 \\
240\end{array}$ & $\begin{array}{l}3.50287 \\
3.50304 \\
3.50322 \\
3.50343 \\
3.50368\end{array}$ & $\begin{array}{l}17 \\
18 \\
21 \\
25 \\
30\end{array}$ & $\begin{array}{l}3.48610 \\
3.48691 \\
3.48764 \\
3.48832 \\
3.48896\end{array}$ & $\begin{array}{l}81 \\
73 \\
68 \\
64 \\
59\end{array}$ & $\begin{array}{l}19.94428 \\
20.11439 \\
20.27661 \\
20.43166 \\
20.58014\end{array}$ & $\begin{array}{l}17011 \\
16222 \\
15505 \\
14848 \\
14244\end{array}$ & $\begin{array}{l}23.43039 \\
23.60130 \\
23.76426 \\
23.91999 \\
24.06910\end{array}$ & $\begin{array}{l}17091 \\
16296 \\
15573 \\
14911 \\
14303\end{array}$ \\
\hline $\begin{array}{l}250 \\
260 \\
270 \\
280 \\
290\end{array}$ & $\begin{array}{l}3.50398 \\
3.50435 \\
3.50480 \\
3.50536 \\
3.50605\end{array}$ & $\begin{array}{l}37 \\
45 \\
56 \\
69 \\
83\end{array}$ & $\begin{array}{l}3.48955 \\
3.49012 \\
3.49065 \\
3.49117 \\
3.49167\end{array}$ & $\begin{array}{l}57 \\
53 \\
52 \\
50 \\
49\end{array}$ & $\begin{array}{l}20.72258 \\
20.85945 \\
20.99118 \\
21.11813 \\
21.24065\end{array}$ & $\begin{array}{l}13687 \\
13173 \\
12695 \\
12252 \\
11838\end{array}$ & $\begin{array}{l}24.21213 \\
24.34957 \\
24.48183 \\
24.60931 \\
24.73232\end{array}$ & $\begin{array}{l}13744 \\
13226 \\
12748 \\
12301 \\
11888\end{array}$ \\
\hline $\begin{array}{l}300 \\
310 \\
320 \\
330 \\
340\end{array}$ & $\begin{array}{l}3.50688 \\
3.50788 \\
3.50906 \\
3.51046 \\
3.51208\end{array}$ & $\begin{array}{l}100 \\
118 \\
140 \\
162 \\
186\end{array}$ & $\begin{array}{l}3.49216 \\
3.49265 \\
3.49314 \\
3.49365 \\
3.49417\end{array}$ & $\begin{array}{l}49 \\
49 \\
51 \\
52 \\
53\end{array}$ & $\begin{array}{l}21.35903 \\
21.47355 \\
21.58445 \\
21.69194 \\
21.79625\end{array}$ & $\begin{array}{l}11452 \\
11070 \\
10749 \\
10431 \\
10129\end{array}$ & $\begin{array}{l}24.85120 \\
24.96621 \\
25.07759 \\
25.18560 \\
25.29042\end{array}$ & $\begin{array}{l}11501 \\
11138 \\
10801 \\
10482 \\
10183\end{array}$ \\
\hline $\begin{array}{l}350 \\
360 \\
370 \\
380 \\
390\end{array}$ & $\begin{array}{l}3.51394 \\
3.51606 \\
3.51845 \\
3.52113 \\
3.52410\end{array}$ & $\begin{array}{l}212 \\
239 \\
268 \\
297 \\
326\end{array}$ & $\begin{array}{l}3.49470 \\
3.49527 \\
3.49586 \\
3.49649 \\
3.49716\end{array}$ & $\begin{array}{l}57 \\
59 \\
63 \\
67 \\
71\end{array}$ & $\begin{array}{l}21.89754 \\
21.99600 \\
22.09177 \\
22.18501 \\
22.27584\end{array}$ & $\begin{array}{l}9846 \\
9577 \\
9324 \\
9083 \\
8855\end{array}$ & $\begin{array}{l}25.39225 \\
25.49127 \\
25.58764 \\
25.68150 \\
25.77300\end{array}$ & $\begin{array}{l}9902 \\
9637 \\
9386 \\
9150 \\
8927\end{array}$ \\
\hline $\begin{array}{l}400 \\
450 \\
500 \\
550 \\
600\end{array}$ & $\begin{array}{l}3.52736 \\
3.54827 \\
3.57650 \\
3.61097 \\
3.65016\end{array}$ & $\begin{array}{l}2091 \\
2823 \\
3447 \\
3919 \\
4229\end{array}$ & $\begin{array}{l}3.49787 \\
3.50224 \\
3.50820 \\
3.51593 \\
3.52546\end{array}$ & $\begin{array}{r}437 \\
596 \\
773 \\
953 \\
1120\end{array}$ & $\begin{array}{l}22.36439 \\
22.77662 \\
23.14591 \\
23.48063 \\
23.78695\end{array}$ & $\begin{array}{l}41223 \\
36929 \\
33472 \\
30632 \\
28262\end{array}$ & $\begin{array}{l}25.86227 \\
26.27886 \\
26.65411 \\
26.99656 \\
27.31241\end{array}$ & $\begin{array}{l}41659 \\
37525 \\
34245 \\
31585 \\
29382\end{array}$ \\
\hline $\begin{array}{l}650 \\
700 \\
750 \\
800 \\
850\end{array}$ & $\begin{array}{l}3.69245 \\
3.73641 \\
3.78084 \\
3.82483 \\
3.86772\end{array}$ & $\begin{array}{l}4396 \\
4443 \\
4399 \\
4289 \\
4133\end{array}$ & $\begin{array}{l}3.53666 \\
3.54935 \\
3.56330 \\
3.57828 \\
3.59405\end{array}$ & $\begin{array}{l}1269 \\
1395 \\
1498 \\
1577 \\
1636\end{array}$ & $\begin{array}{l}24.06957 \\
24.33212 \\
24.57747 \\
24.80791 \\
25.02531\end{array}$ & $\begin{array}{l}26255 \\
24535 \\
23044 \\
21740 \\
20590\end{array}$ & $\begin{array}{l}27.60623 \\
27.88148 \\
28.14078 \\
28.38620 \\
28.61937\end{array}$ & $\begin{array}{l}27525 \\
25930 \\
24542 \\
23317 \\
22225\end{array}$ \\
\hline $\begin{array}{l}900 \\
950 \\
000 \\
050 \\
100\end{array}$ & $\begin{array}{l}3.90905 \\
3.94854 \\
3.98605 \\
4.02150 \\
4.05490\end{array}$ & $\begin{array}{l}3949 \\
3751 \\
3545 \\
3340 \\
3139\end{array}$ & $\begin{array}{l}3.61041 \\
3.62718 \\
3.64419 \\
3.66132 \\
3.67846\end{array}$ & $\begin{array}{l}1677 \\
1701 \\
1713 \\
1714 \\
1706\end{array}$ & $\begin{array}{l}25.23121 \\
25.42686 \\
25.61334 \\
25.79156 \\
25.96228\end{array}$ & $\begin{array}{l}19565 \\
18648 \\
17822 \\
17072 \\
16389\end{array}$ & $\begin{array}{l}28.84162 \\
29.05404 \\
29.25754 \\
29.45288 \\
29.64074\end{array}$ & $\begin{array}{l}21242 \\
20350 \\
19534 \\
18786 \\
18095\end{array}$ \\
\hline 150 & 4.08629 & 2948 & 3.69552 & 1690 & 26.12617 & 15764 & 29.82169 & 17454 \\
\hline
\end{tabular}


Table 2.109. HI (Cont.)

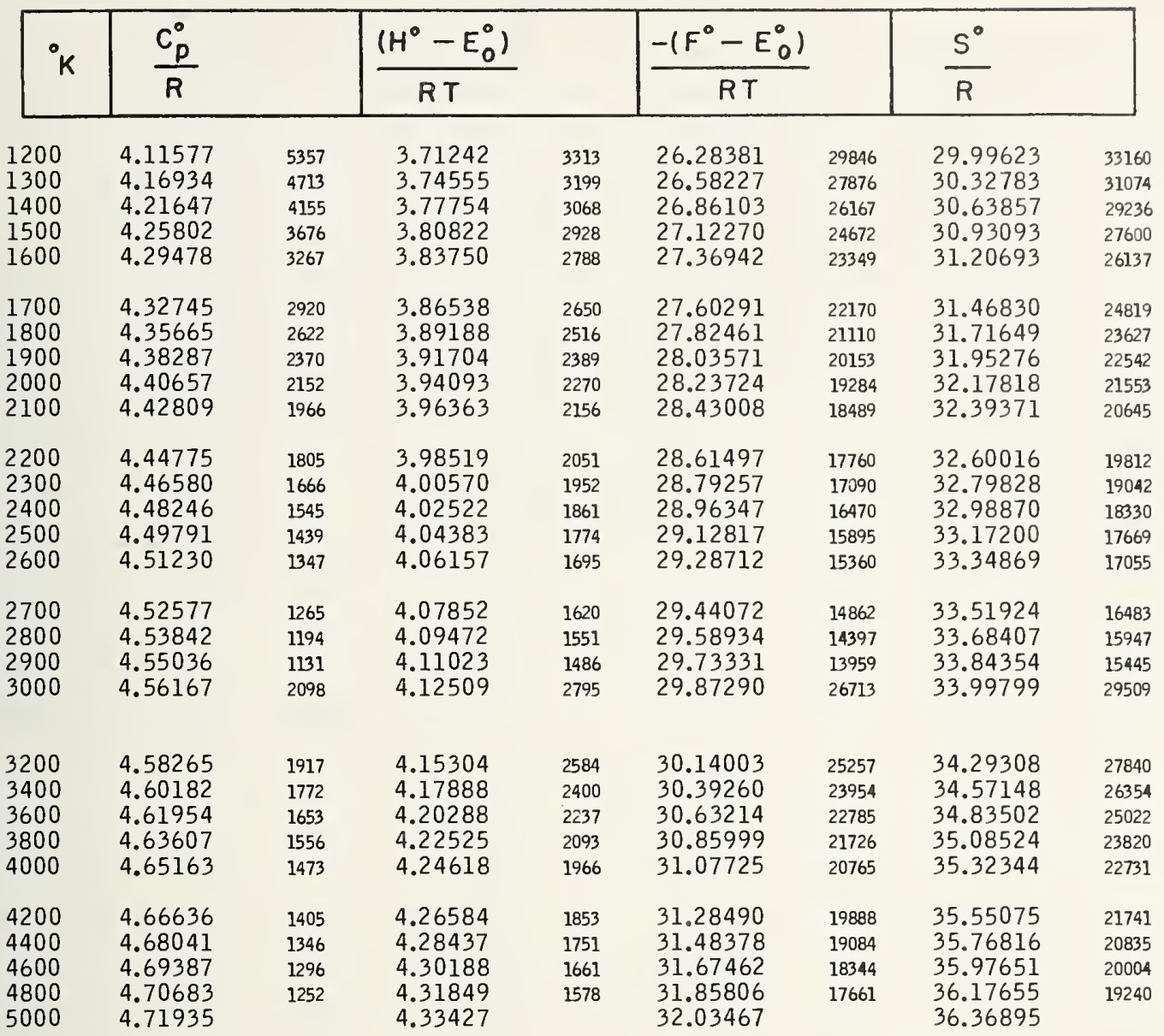


Table 2.110. DI

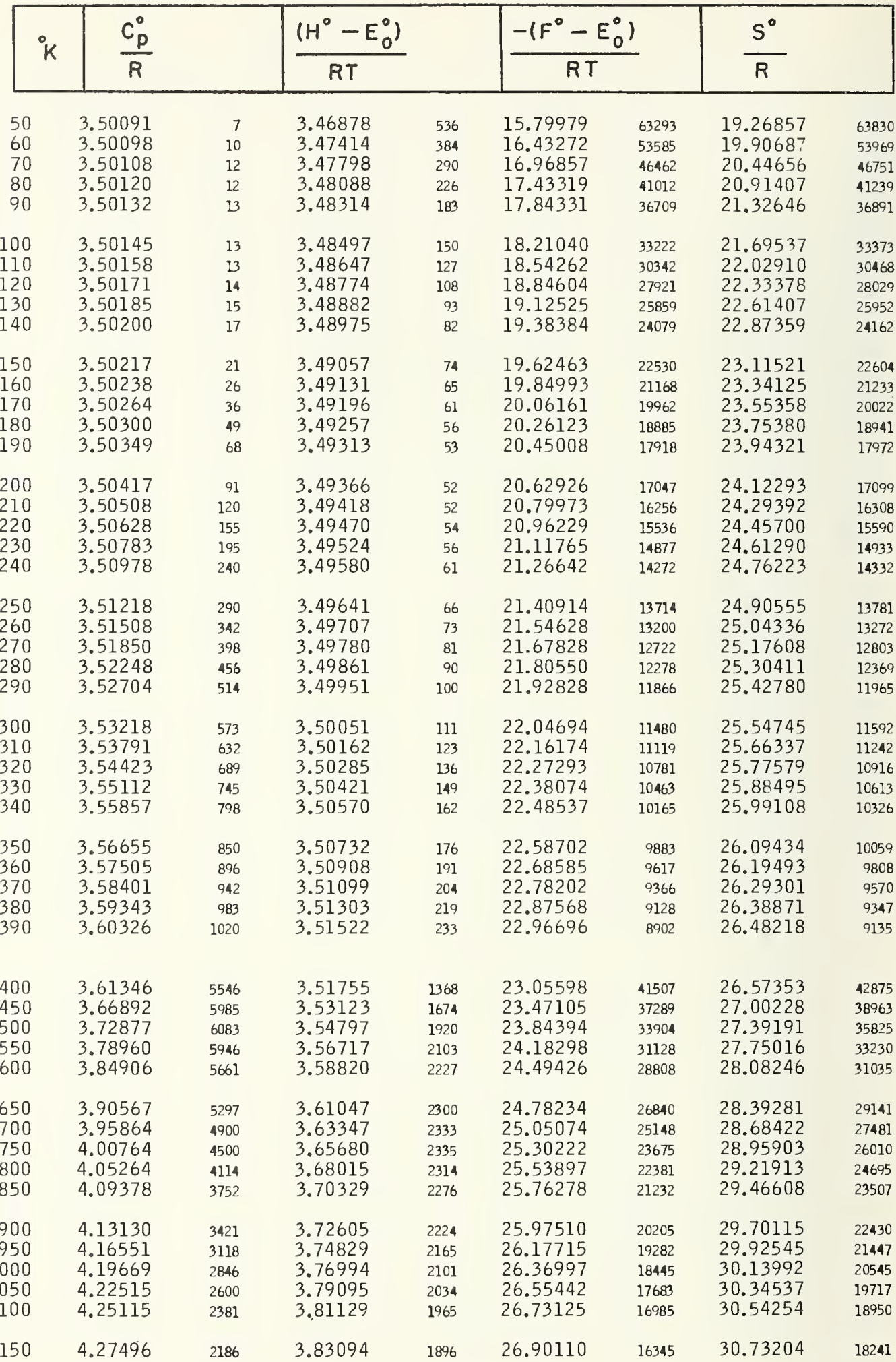


Table 2.110. DI (Cont.)

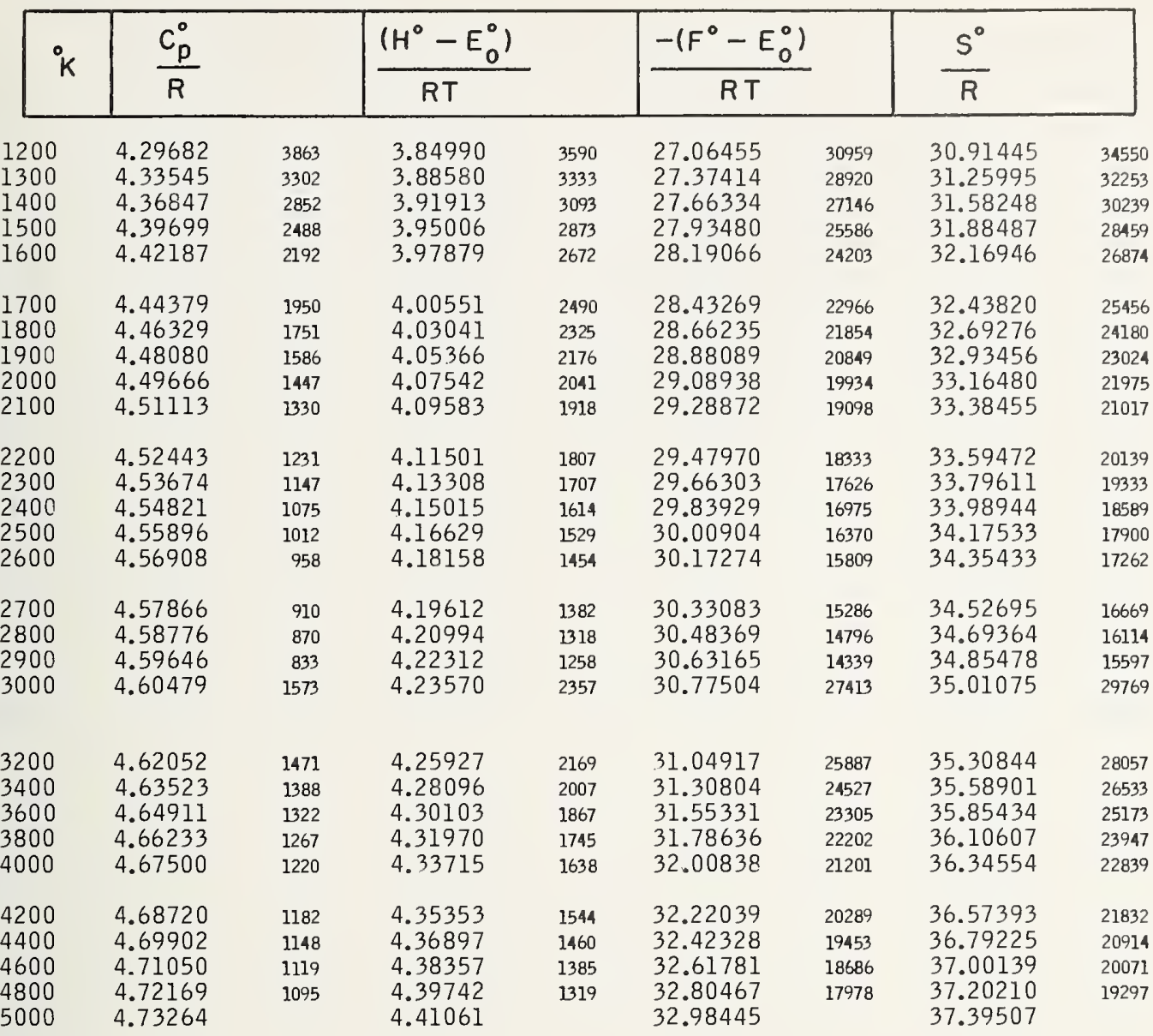


Table 2.111. TI

\begin{tabular}{|c|c|c|c|c|c|c|c|c|}
\hline${ }^{\circ} \mathrm{K}$ & $\frac{C_{p}^{0}}{R}$ & & $\frac{\left(H^{0}-E_{0}^{0}\right)}{R T}$ & & $\frac{-l F^{\circ}-E}{R T}$ & & $\frac{S^{0}}{R}$ & \\
\hline $\begin{array}{l}50 \\
60 \\
70 \\
80 \\
90\end{array}$ & $\begin{array}{l}3.50079 \\
3.50090 \\
3.50102 \\
3.50115 \\
3.50129\end{array}$ & $\begin{array}{l}11 \\
12 \\
13 \\
14 \\
13\end{array}$ & $\begin{array}{l}3.47915 \\
3.48277 \\
3.48537 \\
3.48733 \\
3.48888\end{array}$ & $\begin{array}{l}362 \\
260 \\
196 \\
155 \\
124\end{array}$ & $\begin{array}{l}16.19747 \\
16.83214 \\
17.36921 \\
17.83475 \\
18.24559\end{array}$ & $\begin{array}{l}63467 \\
53707 \\
46554 \\
41084 \\
36766\end{array}$ & $\begin{array}{l}19.67663 \\
20.31491 \\
20.85459 \\
21.32209 \\
21.73448\end{array}$ & $\begin{array}{l}63828 \\
53968 \\
46750 \\
41239 \\
36890\end{array}$ \\
\hline $\begin{array}{l}100 \\
110 \\
120 \\
130 \\
140\end{array}$ & $\begin{array}{l}3.50142 \\
3.50157 \\
3.50173 \\
3.50193 \\
3.50221\end{array}$ & $\begin{array}{l}15 \\
16 \\
20 \\
28 \\
42\end{array}$ & $\begin{array}{l}3.49012 \\
3.49116 \\
3.49203 \\
3.49278 \\
3.49345\end{array}$ & $\begin{array}{r}104 \\
87 \\
75 \\
67 \\
60\end{array}$ & $\begin{array}{l}18.61325 \\
18.94595 \\
19.24976 \\
19.52930 \\
19.78817\end{array}$ & $\begin{array}{l}33270 \\
30381 \\
27954 \\
25887 \\
24104\end{array}$ & $\begin{array}{l}22.10338 \\
22.43711 \\
22.74179 \\
23.02209 \\
23.28162\end{array}$ & $\begin{array}{l}33373 \\
30468 \\
28030 \\
25953 \\
24164\end{array}$ \\
\hline $\begin{array}{l}150 \\
160 \\
170 \\
180 \\
190\end{array}$ & $\begin{array}{l}3.50263 \\
3.50326 \\
3.50419 \\
3.50552 \\
3.50735\end{array}$ & $\begin{array}{r}63 \\
93 \\
133 \\
183 \\
242\end{array}$ & $\begin{array}{l}3.49405 \\
3.49460 \\
3.49514 \\
3.49567 \\
3.49624\end{array}$ & $\begin{array}{l}55 \\
54 \\
53 \\
57 \\
61\end{array}$ & $\begin{array}{l}20.02921 \\
20.25473 \\
20.46660 \\
20.66638 \\
20.85541\end{array}$ & $\begin{array}{l}22552 \\
21187 \\
19978 \\
18903 \\
17935\end{array}$ & $\begin{array}{l}23.52326 \\
23.74933 \\
23.96174 \\
24.16206 \\
24.35166\end{array}$ & $\begin{array}{l}22607 \\
21241 \\
20032 \\
18960 \\
17996\end{array}$ \\
\hline $\begin{array}{l}200 \\
210 \\
220 \\
230 \\
240\end{array}$ & $\begin{array}{l}3.50977 \\
3.51286 \\
3.51671 \\
3.52137 \\
3.52686\end{array}$ & $\begin{array}{l}309 \\
385 \\
466 \\
549 \\
636\end{array}$ & $\begin{array}{l}3.49685 \\
3.49754 \\
3.49832 \\
3.49922 \\
3.50025\end{array}$ & $\begin{array}{r}69 \\
78 \\
90 \\
103 \\
119\end{array}$ & $\begin{array}{l}21.03476 \\
21.20539 \\
21.36811 \\
21.52364 \\
21.67259\end{array}$ & $\begin{array}{l}17063 \\
16272 \\
15553 \\
14895 \\
14291\end{array}$ & $\begin{array}{l}24.53162 \\
24.70293 \\
24.86644 \\
25.02286 \\
25.17284\end{array}$ & $\begin{array}{l}17131 \\
16351 \\
15642 \\
14998 \\
14410\end{array}$ \\
\hline $\begin{array}{l}250 \\
260 \\
270 \\
280 \\
290\end{array}$ & $\begin{array}{l}3.53322 \\
3.54044 \\
3.54852 \\
3.55741 \\
3.56709\end{array}$ & $\begin{array}{r}722 \\
808 \\
889 \\
968 \\
1042\end{array}$ & $\begin{array}{l}3.50144 \\
3.50280 \\
3.50434 \\
3.50607 \\
3.50801\end{array}$ & $\begin{array}{l}136 \\
154 \\
173 \\
194 \\
214\end{array}$ & $\begin{array}{l}21.81550 \\
21.95285 \\
22.08508 \\
22.21255 \\
22.33562\end{array}$ & $\begin{array}{l}13735 \\
13223 \\
12747 \\
12307 \\
11896\end{array}$ & $\begin{array}{l}25.31694 \\
25.45566 \\
25.58942 \\
25.71863 \\
25.84363\end{array}$ & $\begin{array}{l}13872 \\
13376 \\
12921 \\
12500 \\
12111\end{array}$ \\
\hline $\begin{array}{l}300 \\
310 \\
320 \\
330 \\
340\end{array}$ & $\begin{array}{l}3.57751 \\
3.58860 \\
3.60033 \\
3.61261 \\
3.62539\end{array}$ & $\begin{array}{l}1109 \\
1173 \\
1228 \\
1278 \\
1321\end{array}$ & $\begin{array}{l}3.51015 \\
3.51250 \\
3.51506 \\
3.51783 \\
3.52080\end{array}$ & $\begin{array}{l}235 \\
256 \\
277 \\
297 \\
318\end{array}$ & $\begin{array}{l}22.45458 \\
22.56972 \\
22.68127 \\
22.78948 \\
22.89454\end{array}$ & $\begin{array}{l}11514 \\
11155 \\
10821 \\
10506 \\
10211\end{array}$ & $\begin{array}{l}25.96474 \\
26.08222 \\
26.19634 \\
26.30731 \\
26.41535\end{array}$ & $\begin{array}{l}11748 \\
11412 \\
11097 \\
10804 \\
10528\end{array}$ \\
\hline $\begin{array}{l}350 \\
360 \\
370 \\
380 \\
390\end{array}$ & $\begin{array}{l}3.63860 \\
3.65219 \\
3.66609 \\
3.68025 \\
3.69461\end{array}$ & $\begin{array}{l}1359 \\
1390 \\
1416 \\
1436 \\
1451\end{array}$ & $\begin{array}{l}3.52398 \\
3.52735 \\
3.53091 \\
3.53465 \\
3.53857\end{array}$ & $\begin{array}{l}337 \\
356 \\
374 \\
392 \\
408\end{array}$ & $\begin{array}{l}22.99665 \\
23.09597 \\
23.19266 \\
23.28687 \\
23.37874\end{array}$ & $\begin{array}{l}9932 \\
9669 \\
9421 \\
9187 \\
8964\end{array}$ & $\begin{array}{l}26.52063 \\
26.62332 \\
26.72358 \\
26.82153 \\
26.91732\end{array}$ & $\begin{array}{r}10269 \\
10026 \\
9795 \\
9579 \\
9372\end{array}$ \\
\hline $\begin{array}{l}400 \\
450 \\
500 \\
550 \\
600\end{array}$ & $\begin{array}{l}3.70912 \\
3.78239 \\
3.85386 \\
3.92092 \\
3.98237\end{array}$ & $\begin{array}{l}7327 \\
7147 \\
6706 \\
6145 \\
5554\end{array}$ & $\begin{array}{l}3.54265 \\
3.56522 \\
3.59054 \\
3.61756 \\
3.64545\end{array}$ & $\begin{array}{l}2257 \\
2532 \\
2702 \\
2789 \\
2809\end{array}$ & $\begin{array}{l}23.46838 \\
23.88691 \\
24.26384 \\
24.60731 \\
24.92327\end{array}$ & $\begin{array}{l}41853 \\
37693 \\
34347 \\
31596 \\
29290\end{array}$ & $\begin{array}{l}27.01104 \\
27.45214 \\
27.85438 \\
28.22488 \\
28.56872\end{array}$ & $\begin{array}{l}44110 \\
40224 \\
37050 \\
34384 \\
32100\end{array}$ \\
\hline $\begin{array}{l}650 \\
700 \\
750 \\
800 \\
850\end{array}$ & $\begin{array}{l}4.03791 \\
4.08770 \\
4.13216 \\
4.17183 \\
4.20722\end{array}$ & $\begin{array}{l}4979 \\
4446 \\
3967 \\
3539 \\
3165\end{array}$ & $\begin{array}{l}3.67354 \\
3.70137 \\
3.72864 \\
3.75512 \\
3.78070\end{array}$ & $\begin{array}{l}2783 \\
2727 \\
2648 \\
2558 \\
2459\end{array}$ & $\begin{array}{l}25.21617 \\
25.48943 \\
25.74574 \\
25.98723 \\
26.21565\end{array}$ & $\begin{array}{l}27326 \\
25631 \\
24149 \\
22842 \\
21680\end{array}$ & $\begin{array}{l}28.88972 \\
29.19081 \\
29.47438 \\
29.74236 \\
29.99635\end{array}$ & $\begin{array}{l}30109 \\
28357 \\
26798 \\
25399 \\
24140\end{array}$ \\
\hline $\begin{array}{l}900 \\
950 \\
1000 \\
1050 \\
100\end{array}$ & $\begin{array}{l}4.23887 \\
4.26724 \\
4.29274 \\
4.31575 \\
4.33658\end{array}$ & $\begin{array}{l}2837 \\
2550 \\
2301 \\
2083 \\
1893\end{array}$ & $\begin{array}{l}3.80529 \\
3.82887 \\
3.85143 \\
3.87301 \\
3.89361\end{array}$ & $\begin{array}{l}2358 \\
2256 \\
2158 \\
2060 \\
1968\end{array}$ & $\begin{array}{l}26.43245 \\
26.63883 \\
26.83581 \\
27.02424 \\
27.20490\end{array}$ & $\begin{array}{l}20638 \\
19698 \\
18843 \\
18066 \\
17351\end{array}$ & $\begin{array}{l}30.23775 \\
30.46770 \\
30.68724 \\
30.89725 \\
31.09851\end{array}$ & $\begin{array}{l}22995 \\
21954 \\
21001 \\
20126 \\
19320\end{array}$ \\
\hline 150 & 4.35551 & 1727 & 3.91329 & 1879 & 27.37841 & 16695 & 31.29171 & 18574 \\
\hline
\end{tabular}


Table 2.111. TI (Cont.)

\begin{tabular}{|c|c|c|c|c|c|c|c|c|}
\hline${ }^{\circ} \mathrm{K}$ & $\frac{C_{p}^{0}}{R}$ & & $\frac{\left(H^{0}-E_{0}^{0}\right)}{R T}$ & & $\frac{-\left(F^{0}-E_{0}^{0}\right)}{R T}$ & & $\frac{S^{0}}{R}$ & \\
\hline $\begin{array}{l}1200 \\
1300 \\
1400 \\
1500 \\
1600\end{array}$ & $\begin{array}{l}4.37278 \\
4.40312 \\
4.42892 \\
4.45117 \\
4.47060\end{array}$ & $\begin{array}{l}3034 \\
2580 \\
2225 \\
1943 \\
1719\end{array}$ & $\begin{array}{l}3.93208 \\
3.96718 \\
3.99926 \\
4.02867 \\
4.05569\end{array}$ & $\begin{array}{l}3510 \\
3208 \\
2941 \\
2702 \\
2493\end{array}$ & $\begin{array}{l}27.54536 \\
27.86150 \\
28.15669 \\
28.43363 \\
28.69451\end{array}$ & $\begin{array}{l}31614 \\
29519 \\
27694 \\
26088 \\
24663\end{array}$ & $\begin{array}{l}31.47745 \\
31.82869 \\
32.15596 \\
32.46230 \\
32.75021\end{array}$ & $\begin{array}{l}35124 \\
32727 \\
30634 \\
28791 \\
27155\end{array}$ \\
\hline $\begin{array}{l}1700 \\
1800 \\
1900 \\
2000 \\
2100\end{array}$ & $\begin{array}{l}4.48779 \\
4.50316 \\
4.51706 \\
4.52973 \\
4.54139\end{array}$ & $\begin{array}{l}1537 \\
1390 \\
1267 \\
1166 \\
1081\end{array}$ & $\begin{array}{l}4.08062 \\
4.10367 \\
4.12507 \\
4.14499 \\
4.16359\end{array}$ & $\begin{array}{l}2305 \\
2140 \\
1992 \\
1860 \\
1742\end{array}$ & $\begin{array}{l}28.94114 \\
29.17505 \\
29.39750 \\
29.60960 \\
29.81229\end{array}$ & $\begin{array}{l}23391 \\
22245 \\
21210 \\
20269 \\
19410\end{array}$ & $\begin{array}{l}33.02176 \\
33.27872 \\
33.52257 \\
33.75460 \\
33.97589\end{array}$ & $\begin{array}{l}25696 \\
24385 \\
23203 \\
22129 \\
21152\end{array}$ \\
\hline $\begin{array}{l}2200 \\
2300 \\
2400 \\
2500 \\
2600\end{array}$ & $\begin{array}{l}4.55220 \\
4.56230 \\
4.57180 \\
4.58077 \\
4.58930\end{array}$ & $\begin{array}{r}1010 \\
950 \\
897 \\
853 \\
815\end{array}$ & $\begin{array}{l}4.18101 \\
4.19737 \\
4.21278 \\
4.22732 \\
4.24108\end{array}$ & $\begin{array}{l}1636 \\
1541 \\
1454 \\
1376 \\
1305\end{array}$ & $\begin{array}{l}30.00639 \\
30.19261 \\
30.37157 \\
30.54385 \\
30.70992\end{array}$ & $\begin{array}{l}18622 \\
17896 \\
17228 \\
16607 \\
16030\end{array}$ & $\begin{array}{l}34.18741 \\
34.38999 \\
34.58436 \\
34.77117 \\
34.95100\end{array}$ & $\begin{array}{l}20258 \\
19437 \\
18681 \\
17983 \\
17336\end{array}$ \\
\hline $\begin{array}{l}2700 \\
2800 \\
2900 \\
3000\end{array}$ & $\begin{array}{l}4.59745 \\
4.60525 \\
4.61277 \\
4.62002\end{array}$ & $\begin{array}{r}780 \\
752 \\
725 \\
1385\end{array}$ & $\begin{array}{l}4.25413 \\
4.26653 \\
4.27834 \\
4.28961\end{array}$ & $\begin{array}{l}1240 \\
1181 \\
1127 \\
2109\end{array}$ & $\begin{array}{l}30.87022 \\
31.02516 \\
31.17509 \\
31.32032\end{array}$ & $\begin{array}{l}15494 \\
14993 \\
14523 \\
27753\end{array}$ & $\begin{array}{l}35.12436 \\
35.29170 \\
35.45343 \\
35.60994\end{array}$ & $\begin{array}{l}16734 \\
16173 \\
15651 \\
29861\end{array}$ \\
\hline $\begin{array}{l}3200 \\
3400 \\
3600 \\
3800 \\
4000\end{array}$ & $\begin{array}{l}4.63387 \\
4.64700 \\
4.65954 \\
4.67161 \\
4.68328\end{array}$ & $\begin{array}{l}1313 \\
1254 \\
1207 \\
1167 \\
1133\end{array}$ & $\begin{array}{l}4.31070 \\
4.33010 \\
4.34805 \\
4.36477 \\
4.38040\end{array}$ & $\begin{array}{l}1940 \\
1795 \\
1672 \\
1563 \\
1470\end{array}$ & $\begin{array}{l}31.59785 \\
31.85977 \\
32.10779 \\
32.34333 \\
32.56762\end{array}$ & $\begin{array}{l}26192 \\
24802 \\
23554 \\
22429 \\
21408\end{array}$ & $\begin{array}{l}35.90855 \\
36.18988 \\
36.45585 \\
36.70810 \\
36.94802\end{array}$ & $\begin{array}{l}28133 \\
26597 \\
25225 \\
23992 \\
22878\end{array}$ \\
\hline $\begin{array}{l}4200 \\
4400 \\
4600 \\
4800 \\
5000\end{array}$ & $\begin{array}{l}4.69461 \\
4.70567 \\
4.71647 \\
4.72707 \\
4.73748\end{array}$ & $\begin{array}{l}1106 \\
1080 \\
1060 \\
1041\end{array}$ & $\begin{array}{l}4.39510 \\
4.40896 \\
4.42210 \\
4.43459 \\
4.44649\end{array}$ & $\begin{array}{l}1386 \\
1314 \\
1249 \\
1190\end{array}$ & $\begin{array}{l}32.78170 \\
32.98648 \\
33.18276 \\
33.37123 \\
33.55250\end{array}$ & $\begin{array}{l}20478 \\
19628 \\
18847 \\
18127\end{array}$ & $\begin{array}{l}37.17680 \\
37.39545 \\
37.60486 \\
37.80582 \\
37.99900\end{array}$ & $\begin{array}{l}21865 \\
20941 \\
20096 \\
19318\end{array}$ \\
\hline
\end{tabular}




\begin{tabular}{|c|c|c|c|c|c|c|}
\hline $\mathrm{T}^{\circ} \mathrm{K}$ & $\mathrm{F}_{\mathrm{H}_{2}}^{\circ}-\mathrm{F}_{\mathrm{HD}}^{\circ}$ & $\mathrm{F}_{\mathrm{H}_{2}}^{\circ}-\mathrm{F}_{\mathrm{HT}}^{\circ}$ & $\mathrm{F}_{\mathrm{H}_{2}}^{\circ}-\mathrm{F}_{\mathrm{D}_{2}}^{\circ}$ & $\mathrm{H}_{\mathrm{H}_{2}}^{\circ}{ }^{-\mathrm{H}_{\mathrm{HD}}^{\circ}}$ & $\mathrm{H}_{\mathrm{H}_{2}}^{-}{ }^{-\mathrm{H}_{\mathrm{HT}}^{\circ}}$ & $\mathrm{H}_{\mathrm{H}_{2}}^{\circ}{ }^{-\mathrm{H}_{\mathrm{D}_{2}}^{\circ}}$ \\
\hline & $\mathrm{RT}$ & $\mathrm{RT}$ & $\mathrm{RT}$ & $\mathrm{RT}$ & $\mathrm{RT}$ & $\mathrm{RT}$ \\
\hline $\begin{array}{l}100 \\
200 \\
300 \\
400 \\
500\end{array}$ & $\begin{array}{l}5.79042 \\
3.59845 \\
2.91965 \\
2.58535 \\
2.38515\end{array}$ & $\begin{array}{l}7.78968 \\
4.87156 \\
3.95061 \\
3.49521 \\
3.22237\end{array}$ & $\begin{array}{r}10.77864 \\
6.15853 \\
4.67288 \\
3.93536 \\
3.49319\end{array}$ & $\begin{array}{r}4.61516 \\
2.06881 \\
1.34104 \\
1.00130 \\
.80055\end{array}$ & $\begin{array}{l}6.06693 \\
2.79521 \\
1.82541 \\
1.36456 \\
1.09097\end{array}$ & $\begin{array}{l}9.49540 \\
4.48956 \\
2.95474 \\
2.21160 \\
1.76770\end{array}$ \\
\hline $\begin{array}{r}600 \\
700 \\
800 \\
900 \\
1000\end{array}$ & $\begin{array}{l}2.25142 \\
2.15638 \\
2.08542 \\
2.03068 \\
1.98739\end{array}$ & $\begin{array}{l}3.04029 \\
2.91084 \\
2.81429 \\
2.73989 \\
2.68115\end{array}$ & $\begin{array}{l}3.19851 \\
2.98916 \\
2.83337 \\
2.71366 \\
2.61946\end{array}$ & $\begin{array}{l}.66611 \\
.56914 \\
.49492 \\
.43561 \\
.38673\end{array}$ & $\begin{array}{l}.90750 \\
.77484 \\
.67305 \\
.59155 \\
.52433\end{array}$ & $\begin{array}{r}1.46900 \\
1.25182 \\
1.08445 \\
.95017 \\
.83941\end{array}$ \\
\hline $\begin{array}{l}1100 \\
1200 \\
1300 \\
1400 \\
1500\end{array}$ & $\begin{array}{l}1.95252 \\
1.92399 \\
1.90038 \\
1.88061 \\
1.86395\end{array}$ & $\begin{array}{l}2.63391 \\
2.59533 \\
2.56347 \\
2.53684 \\
2.51442\end{array}$ & $\begin{array}{l}2.54395 \\
2.48249 \\
2.43187 \\
2.38969 \\
2.35423\end{array}$ & $\begin{array}{l}.34558 \\
.31038 \\
.27998 \\
.25349 \\
.23030\end{array}$ & $\begin{array}{l}.46771 \\
.41931 \\
.37755 \\
.34122 \\
.30946\end{array}$ & $\begin{array}{l}.74631 \\
.66703 \\
.59890 \\
.53994 \\
.48861\end{array}$ \\
\hline $\begin{array}{l}2000 \\
2500 \\
3000 \\
4000 \\
5000\end{array}$ & $\begin{array}{l}1.80999 \\
1.78222 \\
1.76628 \\
1.74995 \\
1.74234\end{array}$ & $\begin{array}{l}2.44218 \\
2.40532 \\
2.38436 \\
2.36319 \\
2.35363\end{array}$ & $\begin{array}{l}2.24056 \\
2.18270 \\
2.14969 \\
2.11582 \\
2.09986\end{array}$ & $\begin{array}{l}.14892 \\
.10228 \\
.07367 \\
.04241 \\
.02682\end{array}$ & $\begin{array}{l}.19842 \\
.13515 \\
.09643 \\
.05414 \\
.03294\end{array}$ & $\begin{array}{l}.31144 \\
.21233 \\
.15253 \\
.08828 \\
.05688\end{array}$ \\
\hline
\end{tabular}

\begin{tabular}{|c|c|c|c|c|c|c|}
\hline \multirow{2}{*}{$\mathrm{T}^{\circ} \mathrm{K}$} & $\mathrm{C}_{\mathrm{HD}}^{\circ}{ }^{-\mathrm{C}^{\circ}} \mathrm{H}_{2}$ & $\mathrm{C}_{\mathrm{HT}}^{\circ}{ }^{-\mathrm{C}_{\mathrm{H}_{2}}^{\circ}}$ & $\mathrm{C}_{\mathrm{D}_{2}}^{\circ}-\mathrm{C}_{\mathrm{H}_{2}}^{\circ}$ & $\mathrm{S}_{\mathrm{HD}}^{\circ}-\mathrm{S}_{\mathrm{H}_{2}}^{\circ}$ & $\mathrm{S}_{\mathrm{HT}}^{\circ}{ }^{-\mathrm{S}^{\circ}} \mathrm{H}_{2}$ & $\mathrm{~S}_{\mathrm{D}_{2}^{\circ}}^{\circ}-\mathrm{S}^{\circ} \mathrm{H}_{2}$ \\
\hline & $\mathrm{R}$ & $\mathrm{R}$ & $\mathrm{R}$ & $\mathrm{R}$ & $\mathrm{R}$ & $\mathrm{R}$ \\
\hline $\begin{array}{l}100 \\
200 \\
300 \\
400 \\
500\end{array}$ & $\begin{array}{l}.79980 \\
.22975 \\
.04201 \\
.00500 \\
.00221\end{array}$ & $\begin{array}{l}.79786 \\
.22924 \\
.04184 \\
.00527 \\
.00415\end{array}$ & $\begin{array}{l}.90627 \\
.23279 \\
.04200 \\
.00681 \\
.01270\end{array}$ & $\begin{array}{l}1.17527 \\
1.52964 \\
1.57862 \\
1.58406 \\
1.58461\end{array}$ & $\begin{array}{l}1.72275 \\
2.07636 \\
2.12521 \\
2.13065 \\
2.13140\end{array}$ & $\begin{array}{l}1.28324 \\
1.66897 \\
1.71814 \\
1.72377 \\
1.72550\end{array}$ \\
\hline $\begin{array}{r}600 \\
700 \\
800 \\
900 \\
1000\end{array}$ & $\begin{array}{l}.00778 \\
.01811 \\
.03159 \\
.04614 \\
.05992\end{array}$ & $\begin{array}{l}.01361 \\
.02968 \\
.04983 \\
.07083 \\
.09016\end{array}$ & $\begin{array}{l}.03537 \\
.06851 \\
.10586 \\
.14165 \\
.17222\end{array}$ & $\begin{array}{l}1.58530 \\
1.58723 \\
1.59051 \\
1.59507 \\
1.60066\end{array}$ & $\begin{array}{l}2.13278 \\
2.13599 \\
2.14125 \\
2.14834 \\
2.15683\end{array}$ & $\begin{array}{l}1.72950 \\
1.73734 \\
1.74892 \\
1.76350 \\
1.78006\end{array}$ \\
\hline $\begin{array}{l}1100 \\
1200 \\
1300 \\
1400 \\
1500\end{array}$ & $\begin{array}{l}.07180 \\
.08125 \\
.08820 \\
.09288 \\
.09559\end{array}$ & $\begin{array}{l}.10637 \\
.11891 \\
.12784 \\
.13356 \\
.13659\end{array}$ & $\begin{array}{l}.19600 \\
.21286 \\
.22349 \\
.22893 \\
.23024\end{array}$ & $\begin{array}{l}1.60695 \\
1.61361 \\
1.62041 \\
1.62713 \\
1.63364\end{array}$ & $\begin{array}{l}2.16621 \\
2.17602 \\
2.18592 \\
2.19562 \\
2.20495\end{array}$ & $\begin{array}{l}1.79765 \\
1.81547 \\
1.83297 \\
1.84976 \\
1.86561\end{array}$ \\
\hline $\begin{array}{l}2000 \\
2500 \\
3000 \\
4000 \\
5000\end{array}$ & $\begin{array}{l}.09134 \\
.07669 \\
.06249 \\
.04207 \\
.02996\end{array}$ & $\begin{array}{l}.12825 \\
.10724 \\
.08768 \\
.06036 \\
.04465\end{array}$ & $\begin{array}{l}.20408 \\
.16426 \\
.12995 \\
.08335 \\
.05637\end{array}$ & $\begin{array}{l}1.66108 \\
1.67993 \\
1.69262 \\
1.70755 \\
1.71552\end{array}$ & $\begin{array}{l}2.24377 \\
2.27017 \\
2.28794 \\
2.30906 \\
2.32068\end{array}$ & $\begin{array}{l}1.92913 \\
1.97037 \\
1.99717 \\
2.02754 \\
2.04298\end{array}$ \\
\hline
\end{tabular}


Table 3.02

\begin{tabular}{|c|c|c|c|c|c|c|}
\hline \multirow{2}{*}{$\mathrm{T}^{\circ} \mathrm{K}$} & $\mathrm{F}_{\mathrm{H}_{2}}^{\circ}-\mathrm{F}_{\mathrm{DT}}^{\circ}$ & $\mathrm{F}_{\mathrm{H}_{2}}^{\circ}-\mathrm{F}_{\mathrm{T}_{2}}^{\circ}$ & $\mathrm{F}_{\mathrm{HD}}^{\circ}-\mathrm{F}_{\mathrm{HT}}^{\circ}$ & $\mathrm{H}_{\mathrm{H}_{2}}^{\mathrm{o}}-\mathrm{H}_{\mathrm{DT}}^{\circ}$ & $\mathrm{H}_{\mathrm{H}_{2}}^{\circ}-\mathrm{H}_{\mathrm{T}}^{\circ}$ & $\mathrm{H}_{\mathrm{HD}}^{\circ}-\mathrm{H}_{\mathrm{HT}}^{\circ}$ \\
\hline & $\mathrm{RT}$ & $\mathrm{RT}$ & $\mathrm{RT}$ & $\mathrm{RT}$ & $\mathrm{RT}$ & $\mathrm{RT}$ \\
\hline $\begin{array}{l}100 \\
200 \\
300 \\
400 \\
500\end{array}$ & $\begin{array}{r}13.83299 \\
8.29362 \\
6.49864 \\
5.60620 \\
5.07138\end{array}$ & $\begin{array}{r}15.71828 \\
9.13666 \\
6.99405 \\
5.92778 \\
5.28898\end{array}$ & $\begin{array}{r}1.99876 \\
1.27286 \\
1.03079 \\
.90974 \\
.83712\end{array}$ & $\begin{array}{r}11.30817 \\
5.41710 \\
3.57362 \\
2.67538 \\
2.13756\end{array}$ & $\begin{array}{r}13.38651 \\
6.46041 \\
4.26921 \\
3.19639 \\
2.55197\end{array}$ & $\begin{array}{r}1.45127 \\
.72615 \\
.48420 \\
.36314 \\
.29032\end{array}$ \\
\hline $\begin{array}{r}600 \\
700 \\
800 \\
900 \\
1000\end{array}$ & $\begin{array}{r}4.71526 \\
4.46254 \\
4.27483 \\
4.13091 \\
4.01793\end{array}$ & $\begin{array}{l}4.86418 \\
4.56323 \\
4.34026 \\
4.16978 \\
4.03633\end{array}$ & $\begin{array}{l}.78879 \\
.75439 \\
.72881 \\
.70915 \\
.69371\end{array}$ & $\begin{array}{l}1.77463 \\
1.50981 \\
1.30523 \\
1.14095 \\
1.00551\end{array}$ & $\begin{array}{l}2.11544 \\
1.79568 \\
1.54819 \\
1.34946 \\
1.18593\end{array}$ & $\begin{array}{l}.24131 \\
.20563 \\
.17807 \\
.15588 \\
.13755\end{array}$ \\
\hline $\begin{array}{l}1100 \\
1200 \\
1300 \\
1400 \\
1500\end{array}$ & $\begin{array}{l}3.92758 \\
3.85422 \\
3.79391 \\
3.74377 \\
3.70171\end{array}$ & $\begin{array}{l}3.92991 \\
3.84372 \\
3.77303 \\
3.71439 \\
3.66528\end{array}$ & $\begin{array}{l}.68134 \\
.67130 \\
.66306 \\
.65619 \\
.65044\end{array}$ & $\begin{array}{l}.89187 \\
.79533 \\
.71257 \\
.64114 \\
.57915\end{array}$ & $\begin{array}{r}1.04910 \\
.93326 \\
.83431 \\
.74922 \\
.67560\end{array}$ & $\begin{array}{l}.12208 \\
.10889 \\
.09754 \\
.08769 \\
.07913\end{array}$ \\
\hline $\begin{array}{l}2000 \\
2500 \\
3000 \\
4000 \\
5000\end{array}$ & $\begin{array}{l}3.56743 \\
3.49953 \\
3.46100 \\
3.42175 \\
3.40350\end{array}$ & $\begin{array}{l}3.50910 \\
3.43048 \\
3.38596 \\
3.34063 \\
3.31948\end{array}$ & $\begin{array}{l}.63217 \\
.62308 \\
.61806 \\
.61323 \\
.61128\end{array}$ & $\begin{array}{l}.36645 \\
.24845 \\
.17758 \\
.10163 \\
.06449\end{array}$ & $\begin{array}{l}.42491 \\
.28723 \\
.20507 \\
.11753 \\
.07499\end{array}$ & $\begin{array}{l}.04948 \\
.03285 \\
.02274 \\
.01172 \\
.00611\end{array}$ \\
\hline
\end{tabular}

\begin{tabular}{|c|c|c|c|c|c|c|}
\hline \multirow{2}{*}{$\mathrm{T}^{\circ} \mathrm{K}$} & $\mathrm{C}_{\mathrm{DT}}^{\circ}{ }^{-\mathrm{C}_{\mathrm{H}_{2}}^{\circ}}$ & $\mathrm{C}_{\mathrm{T}_{2}}^{\circ}-\mathrm{C}_{\mathrm{H}_{2}}^{\circ}$ & $\mathrm{C}_{\mathrm{HT}}^{\circ}-\mathrm{C}_{\mathrm{HD}}^{\circ}$ & $\mathrm{S}_{\mathrm{DT}}^{\circ}-\mathrm{S}_{\mathrm{H}_{2}}^{\circ}$ & $\mathrm{S}_{\mathrm{T}_{2}}^{\circ}-\mathrm{S}_{\mathrm{H}_{2}}^{\circ}$ & $\mathrm{S}_{\mathrm{HT}}^{\circ}{ }^{-\mathrm{S}_{\mathrm{HD}}^{\circ}}$ \\
\hline & $\mathrm{R}$ & $\mathrm{R}$ & $\mathrm{R}$ & $\mathrm{R}$ & $\mathrm{R}$ & $\mathrm{R}$ \\
\hline $\begin{array}{l}100 \\
200 \\
300 \\
400 \\
500\end{array}$ & $\begin{array}{l}.79296 \\
.22793 \\
.04157 \\
.00939 \\
.02265\end{array}$ & $\begin{array}{l}.74531 \\
.22781 \\
.04214 \\
.01552 \\
.04164\end{array}$ & $\begin{array}{r}-.00194 \\
-.00051 \\
-.00017 \\
.00027 \\
.00194\end{array}$ & $\begin{array}{l}2.52483 \\
2.87653 \\
2.92503 \\
2.93082 \\
2.93382\end{array}$ & $\begin{array}{l}2.33177 \\
2.67625 \\
2.72485 \\
2.73139 \\
2.73701\end{array}$ & $\begin{array}{l}.54748 \\
.54672 \\
.54659 \\
.54659 \\
.54679\end{array}$ \\
\hline $\begin{array}{r}600 \\
700 \\
800 \\
900 \\
1000\end{array}$ & $\begin{array}{l}.05689 \\
.10254 \\
.15081 \\
.19475 \\
.23056\end{array}$ & $\begin{array}{l}.09255 \\
.15376 \\
.21377 \\
.26511 \\
.30457\end{array}$ & $\begin{array}{l}.00583 \\
.01157 \\
.01824 \\
.02469 \\
.03024\end{array}$ & $\begin{array}{l}2.94063 \\
2.95273 \\
2.96960 \\
2.98997 \\
3.01242\end{array}$ & $\begin{array}{l}2.74874 \\
2.76755 \\
2.79207 \\
2.82032 \\
2.85040\end{array}$ & $\begin{array}{l}.54748 \\
.54876 \\
.55074 \\
.55327 \\
.55617\end{array}$ \\
\hline $\begin{array}{l}1100 \\
1200 \\
1300 \\
1400 \\
1500\end{array}$ & $\begin{array}{l}.25709 \\
.27480 \\
.28490 \\
.28887 \\
.28811\end{array}$ & $\begin{array}{l}.33191 \\
.34847 \\
.35615 \\
.35696 \\
.35265\end{array}$ & $\begin{array}{l}.03457 \\
.03766 \\
.03964 \\
.04068 \\
.04100\end{array}$ & $\begin{array}{l}3.03571 \\
3.05890 \\
3.08134 \\
3.10263 \\
3.12256\end{array}$ & $\begin{array}{l}2.88081 \\
2.91047 \\
2.93872 \\
2.96518 \\
2.98968\end{array}$ & $\begin{array}{l}.55926 \\
.56241 \\
.56551 \\
.56849 \\
.57131\end{array}$ \\
\hline $\begin{array}{l}2000 \\
2500 \\
3000 \\
4000 \\
5000\end{array}$ & $\begin{array}{l}.24920 \\
.19863 \\
.15670 \\
.10118 \\
.06970\end{array}$ & $\begin{array}{l}.29611 \\
.23238 \\
.18147 \\
.11536 \\
.07825\end{array}$ & $\begin{array}{l}.03691 \\
.03055 \\
.02519 \\
.01829 \\
.01469\end{array}$ & $\begin{array}{l}3.20099 \\
3.25108 \\
3.28343 \\
3.32013 \\
3.33900\end{array}$ & $\begin{array}{l}3.08420 \\
3.14325 \\
3.18090 \\
3.22310 \\
3.24448\end{array}$ & $\begin{array}{l}.58269 \\
.59024 \\
.59532 \\
.60151 \\
.60516\end{array}$ \\
\hline
\end{tabular}


Table 3.03

\begin{tabular}{|c|c|c|c|c|c|c|}
\hline $\mathrm{T}^{\circ} \mathrm{K}$ & $\frac{\mathrm{F}_{\mathrm{HD}}^{\circ}-\mathrm{F}_{\mathrm{D}_{2}}^{\circ}}{\mathrm{RT}}$ & $\frac{\mathrm{F}_{\mathrm{HD}}^{\circ}-\mathrm{F}_{\mathrm{DT}}^{\circ}}{\mathrm{RT}}$ & $\frac{\mathrm{F}_{\mathrm{HD}}^{\circ}-\mathrm{F}_{\mathrm{T}_{2}}^{\circ}}{\mathrm{RT}}$ & $\frac{\mathrm{H}_{\mathrm{HD}}^{\circ}-\mathrm{H}_{\mathrm{D}_{2}}^{\circ}}{\mathrm{RT}}$ & $\frac{\mathrm{H}_{\mathrm{HD}}^{\circ}{ }^{-\mathrm{H}_{\mathrm{DT}}^{\circ}}}{\mathrm{RT}}$ & $\frac{\mathrm{H}_{\mathrm{HD}}^{\circ}-\mathrm{H}_{\mathrm{T}_{2}}^{\circ}}{\mathrm{RT}}$ \\
\hline $\begin{array}{l}100 \\
200 \\
300 \\
400 \\
500\end{array}$ & $\begin{array}{l}4.98822 \\
2.56008 \\
1.75323 \\
1.35001 \\
1.10804\end{array}$ & $\begin{array}{l}8.04207 \\
4.69492 \\
3.57882 \\
3.02073 \\
2.68613\end{array}$ & $\begin{array}{l}9.92836 \\
5.53846 \\
4.07457 \\
3.34256 \\
2.90393\end{array}$ & $\begin{array}{r}4.88024 \\
2.42075 \\
1.61370 \\
1.21030 \\
.96715\end{array}$ & $\begin{array}{l}6.69251 \\
3.34804 \\
2.23241 \\
1.67396 \\
1.33691\end{array}$ & $\begin{array}{l}8.77185 \\
4.39185 \\
2.92834 \\
2.19522 \\
1.75152\end{array}$ \\
\hline $\begin{array}{r}600 \\
700 \\
800 \\
900 \\
1000\end{array}$ & $\begin{array}{l}.94709 \\
.83278 \\
.74796 \\
.68298 \\
.63207\end{array}$ & $\begin{array}{l}2.46376 \\
2.30609 \\
2.18935 \\
2.10017 \\
2.03049\end{array}$ & $\begin{array}{l}2.61284 \\
2.40693 \\
2.25490 \\
2.13916 \\
2.04899\end{array}$ & $\begin{array}{l}.80289 \\
.68268 \\
.58954 \\
.51456 \\
.45268\end{array}$ & $\begin{array}{r}1.10844 \\
.94060 \\
.81025 \\
.70528 \\
.61873\end{array}$ & $\begin{array}{r}1.44941 \\
1.22662 \\
1.05333 \\
.91391 \\
.79925\end{array}$ \\
\hline $\begin{array}{l}1100 \\
1200 \\
1300 \\
1400 \\
1500\end{array}$ & $\begin{array}{l}.59144 \\
.55851 \\
.53150 \\
.50907 \\
.49028\end{array}$ & $\begin{array}{l}1.97502 \\
1.93019 \\
1.89349 \\
1.86313 \\
1.83773\end{array}$ & $\begin{array}{l}1.97744 \\
1.91978 \\
1.87270 \\
1.83381 \\
1.80136\end{array}$ & $\begin{array}{l}.40074 \\
.35666 \\
.31893 \\
.28644 \\
.25831\end{array}$ & $\begin{array}{l}.54625 \\
.48491 \\
.43255 \\
.38762 \\
.34882\end{array}$ & $\begin{array}{l}.70357 \\
.62293 \\
.55438 \\
.49576 \\
.44533\end{array}$ \\
\hline $\begin{array}{l}2000 \\
2500 \\
3000 \\
4000 \\
5000\end{array}$ & $\begin{array}{l}.43057 \\
.40048 \\
.38341 \\
.36588 \\
.35752\end{array}$ & $\begin{array}{l}1.75742 \\
1.71729 \\
1.69470 \\
1.67179 \\
1.66115\end{array}$ & $\begin{array}{l}1.69914 \\
1.64828 \\
1.61970 \\
1.59069 \\
1.57715\end{array}$ & $\begin{array}{l}.16252 \\
.11005 \\
.07886 \\
.04588 \\
.03006\end{array}$ & $\begin{array}{l}.21751 \\
.14615 \\
.10389 \\
.05921 \\
.03766\end{array}$ & $\begin{array}{l}.27602 \\
.18497 \\
.13142 \\
.07513 \\
.04818\end{array}$ \\
\hline
\end{tabular}

\begin{tabular}{|c|c|c|c|c|c|c|}
\hline \multirow{2}{*}{$\mathrm{T}^{\circ} \mathrm{K}$} & $\mathrm{C}_{\mathrm{D}_{2}}^{-\mathrm{C}_{\mathrm{HD}}^{\circ}}$ & $\mathrm{C}_{\mathrm{DT}}^{\circ}-\mathrm{C}_{\mathrm{HD}}^{\circ}$ & $\mathrm{C}_{\mathrm{T}_{2}}^{\circ}-\mathrm{C}_{\mathrm{HD}}^{\circ}$ & $\mathrm{S}_{\mathrm{D}_{2}}-\mathrm{S}_{\mathrm{HD}}^{\circ}$ & $\mathrm{S}_{\mathrm{DT}}^{\circ}-\mathrm{S}_{\mathrm{HD}}^{\circ}$ & $\mathrm{S}_{\mathrm{T}_{2}}^{\circ}-\mathrm{S}_{\mathrm{HD}}^{\circ}$ \\
\hline & $\mathrm{R}$ & $\mathrm{R}$ & $\mathrm{R}$ & $\mathrm{R}$ & $\mathrm{R}$ & $R$ \\
\hline $\begin{array}{l}100 \\
200 \\
300 \\
400 \\
500\end{array}$ & $\begin{array}{r}.10647 \\
.00304 \\
-.00001 \\
.00181 \\
.01049\end{array}$ & $\begin{array}{r}-.00684 \\
-.00182 \\
-.00044 \\
.00439 \\
.02044\end{array}$ & $\begin{array}{r}-.05449 \\
-.00194 \\
.00013 \\
.01052 \\
.03943\end{array}$ & $\begin{array}{l}.10797 \\
.13933 \\
.13952 \\
.13971 \\
.14089\end{array}$ & $\begin{array}{l}1.34956 \\
1.34689 \\
1.34641 \\
1.34676 \\
1.34921\end{array}$ & $\begin{array}{l}1.15650 \\
1.14661 \\
1.14623 \\
1.14733 \\
1.15240\end{array}$ \\
\hline $\begin{array}{r}600 \\
700 \\
800 \\
900 \\
1000\end{array}$ & $\begin{array}{l}.02759 \\
.05040 \\
.07427 \\
.09551 \\
.11230\end{array}$ & $\begin{array}{l}.04911 \\
.08443 \\
.11922 \\
.14861 \\
.17064\end{array}$ & $\begin{array}{l}.08477 \\
.13565 \\
.18218 \\
.21897 \\
.24465\end{array}$ & $\begin{array}{l}.14420 \\
.15011 \\
.15841 \\
.16843 \\
.17940\end{array}$ & $\begin{array}{l}1.35533 \\
1.36550 \\
1.37909 \\
1.39490 \\
1.41176\end{array}$ & $\begin{array}{l}1.16344 \\
1.18032 \\
1.20156 \\
1.22525 \\
1.24974\end{array}$ \\
\hline $\begin{array}{l}1100 \\
1200 \\
1300 \\
1400 \\
1500\end{array}$ & $\begin{array}{l}.12420 \\
.13161 \\
.13529 \\
.13605 \\
.13465\end{array}$ & $\begin{array}{l}.18529 \\
.19355 \\
.19670 \\
.19599 \\
.19252\end{array}$ & $\begin{array}{l}.26011 \\
.26722 \\
.26795 \\
.26408 \\
.25706\end{array}$ & $\begin{array}{l}.19070 \\
.20186 \\
.21256 \\
.22263 \\
.23197\end{array}$ & $\begin{array}{l}1.42876 \\
1.44529 \\
1.46093 \\
1.47550 \\
1.48892\end{array}$ & $\begin{array}{l}1.27386 \\
1.29686 \\
1.31831 \\
1.33805 \\
1.35604\end{array}$ \\
\hline $\begin{array}{l}2000 \\
2500 \\
3000 \\
4000 \\
5000\end{array}$ & $\begin{array}{l}.11274 \\
.08757 \\
.06746 \\
.04128 \\
.02641\end{array}$ & $\begin{array}{l}.15786 \\
.12194 \\
.09421 \\
.05911 \\
.03974\end{array}$ & $\begin{array}{l}.20477 \\
.15569 \\
.11898 \\
.07329 \\
.04829\end{array}$ & $\begin{array}{l}.26805 \\
.29044 \\
.30455 \\
.31999 \\
.32746\end{array}$ & $\begin{array}{l}1.53991 \\
1.57115 \\
1.59081 \\
1.61258 \\
1.62348\end{array}$ & $\begin{array}{l}1.42312 \\
1.46332 \\
1.48828 \\
1.51555 \\
1.52896\end{array}$ \\
\hline
\end{tabular}


Table. 3.04

\begin{tabular}{|c|c|c|c|c|c|c|}
\hline \multirow{2}{*}{$\mathrm{T}^{\circ} \mathrm{K}$} & $\mathrm{F}_{\mathrm{HT}}^{\circ}-\mathrm{F}_{\mathrm{D}_{2}}^{\circ}$ & $\mathrm{F}_{\mathrm{HT}}^{\circ}-\mathrm{F}_{\mathrm{DT}}^{\circ}$ & $\mathrm{F}_{\mathrm{HT}}^{\circ}{ }^{\circ} \mathrm{F}_{2}^{\circ}$ & $\mathrm{H}_{\mathrm{HT}}^{\circ}-\mathrm{H}^{\circ} \mathrm{D}_{2}$ & $\mathrm{H}_{\mathrm{HT}}^{\circ}-\mathrm{H}_{\mathrm{DT}}^{\circ}$ & $\mathrm{H}_{\mathrm{HT}}^{\circ}-\mathrm{H}_{\mathrm{T}_{2}}^{\circ}$ \\
\hline & $\mathrm{RT}$ & $\mathrm{RT}$ & $\mathrm{RT}$ & $\mathrm{RT}$ & $\mathrm{RT}$ & $\mathrm{RT}$ \\
\hline $\begin{array}{l}100 \\
200 \\
300 \\
400 \\
500\end{array}$ & $\begin{array}{r}2.98926 \\
1.28712 \\
.72237 \\
.44022 \\
.27088\end{array}$ & $\begin{array}{l}6.04331 \\
3.42206 \\
2.54802 \\
2.11099 \\
1.84901\end{array}$ & $\begin{array}{l}7.92860 \\
4.26510 \\
3.04344 \\
2.43257 \\
2.06661\end{array}$ & $\begin{array}{r}3.42877 \\
1.69450 \\
1.12943 \\
.84711 \\
.67679\end{array}$ & $\begin{array}{l}5.24124 \\
2.62189 \\
1.74820 \\
1.31082 \\
1.04659\end{array}$ & $\begin{array}{l}7.31958 \\
3.66520 \\
2.44380 \\
1.83183 \\
1.46100\end{array}$ \\
\hline $\begin{array}{r}600 \\
700 \\
800 \\
900 \\
1000\end{array}$ & $\begin{array}{r}.15827 \\
.07836 \\
.01913 \\
-.02619 \\
-.06166\end{array}$ & $\begin{array}{l}1.67498 \\
1.55170 \\
1.46055 \\
1.39102 \\
1.33678\end{array}$ & $\begin{array}{l}1.82389 \\
1.65239 \\
1.52597 \\
1.42989 \\
1.35518\end{array}$ & $\begin{array}{l}.56155 \\
.47702 \\
.41145 \\
.35866 \\
.31511\end{array}$ & $\begin{array}{l}.86714 \\
.73497 \\
.63219 \\
.54940 \\
.48118\end{array}$ & $\begin{array}{r}1.20794 \\
1.02084 \\
.87514 \\
.75791 \\
.66160\end{array}$ \\
\hline $\begin{array}{l}1100 \\
1200 \\
1300 \\
1400 \\
1500\end{array}$ & $\begin{array}{l}-.08993 \\
-.11281 \\
-.13158 \\
-.14713 \\
-.16017\end{array}$ & $\begin{array}{l}1.29367 \\
1.25888 \\
1.23044 \\
1.20694 \\
1.18730\end{array}$ & $\begin{array}{l}1.29600 \\
1.24839 \\
1.20956 \\
1.17755 \\
1.15086\end{array}$ & $\begin{array}{l}.27863 \\
.24775 \\
.22137 \\
.19874 \\
.17917\end{array}$ & $\begin{array}{l}.42416 \\
.37601 \\
.33502 \\
.29993 \\
.26970\end{array}$ & $\begin{array}{l}.58139 \\
.51395 \\
.45676 \\
.40800 \\
.36614\end{array}$ \\
\hline $\begin{array}{l}2000 \\
2500 \\
3000 \\
4000 \\
5000\end{array}$ & $\begin{array}{l}-.20161 \\
-.22261 \\
-.23466 \\
-.24735 \\
-.25376\end{array}$ & $\begin{array}{l}1.12525 \\
1.09421 \\
1.07663 \\
1.05857 \\
1.04987\end{array}$ & $\begin{array}{r}1.06692 \\
1.02516 \\
1.00160 \\
.97744 \\
.96585\end{array}$ & $\begin{array}{l}.11303 \\
.07719 \\
.05611 \\
.03416 \\
.02395\end{array}$ & $\begin{array}{l}.16803 \\
.11330 \\
.08114 \\
.04750 \\
.03155\end{array}$ & $\begin{array}{l}.22649 \\
.15208 \\
.10864 \\
.06339 \\
.04205\end{array}$ \\
\hline
\end{tabular}

$\mathrm{T}^{\circ} \mathrm{K}$

$\frac{\mathrm{C}_{\mathrm{D}_{2}}^{\circ}-\mathrm{C}_{\mathrm{HT}}^{\circ}}{\mathrm{R}}$

$\frac{\mathrm{C}_{\mathrm{DT}}^{\circ}-\mathrm{C}_{\mathrm{HT}}^{\circ}}{\mathrm{R}}$

$\frac{\mathrm{C}_{\mathrm{T}_{2}}^{\circ}-\mathrm{C}_{\mathrm{HT}}^{\circ}}{\mathrm{R}}$

$$
\begin{array}{rr}
-.00490 & -.05255 \\
-.00131 & -.00143 \\
-.00027 & .00030 \\
.00412 & .01025 \\
.01850 & .03749
\end{array}
$$

.04328

.07286

.10098

.12392

.14040

.15072

.15589

.15706

.15531

.15152

.12095

.09139

.06902

.04082

.02505
.07894

.12408

.16394

.19428

.22554

.22956

.22831

.22340

.21606

.16786

.12514

.09379

.05500

.03360
$\frac{\mathrm{S}_{\mathrm{D}_{2}}^{\circ}-\mathrm{S}_{\mathrm{HT}}^{\circ}}{\mathrm{R}}$

$-.43951$

$-.40739$

$-.40707$

-.40688

-. 40590

$-.40328$

$-.39865$

$-.39233$

-.38484
-.37677

$-.37677$

- .36856

$-.36055$

$-.35295$

$-.34586$

$-.33934$

$-.31464$

$-.29980$

$-.29077$

$-.28152$

$-.27770$
$\frac{\mathrm{S}_{\mathrm{DT}}^{\circ}-\mathrm{S}_{\mathrm{HT}}^{\circ}}{\mathrm{R}} \frac{\mathrm{S}_{\mathrm{T}_{2}}^{\circ}-\mathrm{S}_{\mathrm{HT}}^{\circ}}{\mathrm{R}}$

.80208

.80017

.79982

.80017

.80242

.80785

.81674

.82835

.84163
.85559

.86950

.88288

.89542

.90701

.91761

.95722

.98091

.99549

1.01107

1.01832
.60902

.59989

.59964

.60074

.60561

.61596

.63156

.65082

.67198

.69357

.71460

.73445

.75280

.76956

.78473

.84043

.87308

.89296

.91404

.92380 
Table 3.05

\begin{tabular}{|c|c|c|c|c|c|c|}
\hline \multirow{2}{*}{$\mathrm{T}^{\circ} \mathrm{K}$} & $\mathrm{F}_{2}^{\circ}-\mathrm{F}_{\mathrm{DT}}^{\circ}$ & $\mathrm{F}_{2}^{\circ}-\mathrm{F}^{\circ} \mathrm{T}_{2}$ & $\mathrm{~F}_{\mathrm{DT}}^{\circ}{ }^{-\mathrm{F}_{2}^{\circ}}$ & $\mathrm{H}_{\mathrm{D}_{2}}^{\circ}{ }^{-\mathrm{H}_{\mathrm{DT}}^{\circ}}$ & $\mathrm{H}_{2}^{\circ}-\mathrm{H}_{\mathrm{T}_{2}}^{\circ}$ & ${ }_{\mathrm{DT}}^{\circ}{ }^{-\mathrm{H}_{2}^{\circ}}$ \\
\hline & $\mathrm{RT}$ & $\mathrm{RT}$ & $\mathrm{RT}$ & $\mathrm{RT}^{\mathrm{T}}$ & $\mathrm{RT}$ & RT \\
\hline $\begin{array}{l}100 \\
200 \\
300 \\
400 \\
500\end{array}$ & $\begin{array}{l}3.05405 \\
2.13494 \\
1.82566 \\
1.67077 \\
1.57813\end{array}$ & $\begin{array}{l}4.94034 \\
2.97848 \\
2.32141 \\
1.99260 \\
1.79593\end{array}$ & $\begin{array}{r}1.88629 \\
.84354 \\
.49575 \\
.32183 \\
.21780\end{array}$ & $\begin{array}{r}1.81247 \\
.92739 \\
.61878 \\
.46371 \\
.36980\end{array}$ & $\begin{array}{r}3.89181 \\
1.97120 \\
1.31471 \\
.98497 \\
.78441\end{array}$ & $\begin{array}{r}2.07934 \\
1.04381 \\
.69593 \\
.52126 \\
.41461\end{array}$ \\
\hline $\begin{array}{r}600 \\
700 \\
800 \\
900 \\
1000\end{array}$ & $\begin{array}{l}1.51670 \\
1.47334 \\
1.44142 \\
1.41721 \\
1.39844\end{array}$ & $\begin{array}{l}1.66578 \\
1.57418 \\
1.50697 \\
1.45620 \\
1.41694\end{array}$ & $\begin{array}{l}.14908 \\
.10083 \\
.06555 \\
.03899 \\
.01850\end{array}$ & $\begin{array}{l}.30558 \\
.25795 \\
.22074 \\
.19074 \\
.16607\end{array}$ & $\begin{array}{l}.64655 \\
.54397 \\
.46382 \\
.39937 \\
.34659\end{array}$ & $\begin{array}{l}.34097 \\
.28601 \\
.24308 \\
.20863 \\
.18052\end{array}$ \\
\hline $\begin{array}{l}1100 \\
1200 \\
1300 \\
1400 \\
1500\end{array}$ & $\begin{array}{l}1.38360 \\
1.37170 \\
1.36202 \\
1.35407 \\
1.34746\end{array}$ & $\begin{array}{l}1.38602 \\
1.36129 \\
1.34122 \\
1.32475 \\
1.31109\end{array}$ & $\begin{array}{r}.00242 \\
-.01041 \\
-.02080 \\
-.02931 \\
-.03637\end{array}$ & $\begin{array}{l}.14553 \\
.12827 \\
.11365 \\
.10119 \\
.09052\end{array}$ & $\begin{array}{l}.30285 \\
.26629 \\
.23547 \\
.20933 \\
.18703\end{array}$ & $\begin{array}{l}.15732 \\
.13802 \\
.12182 \\
.10815 \\
.09651\end{array}$ \\
\hline $\begin{array}{l}2000 \\
2500 \\
3000 \\
4000 \\
5000\end{array}$ & $\begin{array}{l}1.32686 \\
1.31682 \\
1.31130 \\
1.30592 \\
1.30363\end{array}$ & $\begin{array}{l}1.26858 \\
1.24781 \\
1.23630 \\
1.22482 \\
1.21963\end{array}$ & $\begin{array}{l}-.05828 \\
-.06901 \\
-.07500 \\
-.088110 \\
-.08400\end{array}$ & $\begin{array}{l}.05500 \\
.03611 \\
.02504 \\
.01334 \\
.00760\end{array}$ & $\begin{array}{l}.11351 \\
.07493 \\
.05257 \\
.02926 \\
.01812\end{array}$ & $\begin{array}{l}.05851 \\
.03882 \\
.02753 \\
.01592 \\
.01052\end{array}$ \\
\hline
\end{tabular}

\begin{tabular}{|c|c|c|c|c|c|c|}
\hline \multirow{2}{*}{$\mathrm{T}^{0} \mathrm{~K}$} & $\mathrm{C}_{\mathrm{DT}}^{\circ}-\mathrm{C}_{\mathrm{D}_{2}}^{\circ}$ & $\mathrm{C}_{\mathrm{T}_{2}}^{\circ}-\mathrm{C}_{\mathrm{D}_{2}}^{\circ}$ & $\mathrm{C}_{\mathrm{T}_{2}}^{\circ}-\mathrm{C}_{\mathrm{DT}}^{\circ}$ & $\mathrm{S}_{\mathrm{DT}}^{\circ}-\mathrm{S}_{\mathrm{D}_{2}}^{\circ}$ & $\mathrm{S}_{\mathrm{T}_{2}}^{\circ}-\mathrm{S}_{\mathrm{D}_{2}}^{\circ}$ & $\mathrm{S}_{\mathrm{T}_{2}}^{\circ}-\mathrm{S}_{\mathrm{DT}}^{\circ}$ \\
\hline & $\mathrm{R}$ & $\mathrm{R}$ & $\mathrm{R}$ & $\mathrm{R}$ & $\mathrm{R}$ & $\mathrm{R}$ \\
\hline $\begin{array}{l}100 \\
200 \\
300 \\
400 \\
500\end{array}$ & $\begin{array}{r}-.11331 \\
-.00486 \\
-.00043 \\
.00258 \\
.00995\end{array}$ & $\begin{array}{r}-.16096 \\
-.00498 \\
.00014 \\
.00871 \\
.02894\end{array}$ & $\begin{array}{r}-.04765 \\
-.00012 \\
.00057 \\
.00613 \\
.01899\end{array}$ & $\begin{array}{l}1.24159 \\
1.20756 \\
1.20689 \\
1.20705 \\
1.20832\end{array}$ & $\begin{array}{l}1.04853 \\
1.00728 \\
1.00671 \\
1.00762 \\
1.01151\end{array}$ & $\begin{array}{l}-.19306 \\
-.20028 \\
-.20018 \\
-.19943 \\
-.19681\end{array}$ \\
\hline $\begin{array}{r}600 \\
700 \\
800 \\
900 \\
1000\end{array}$ & $\begin{array}{l}.02152 \\
.03403 \\
.04495 \\
.05310 \\
.05834\end{array}$ & $\begin{array}{l}.05718 \\
.08525 \\
.10791 \\
.12346 \\
.13235\end{array}$ & $\begin{array}{l}.03566 \\
.05122 \\
.06296 \\
.07036 \\
.07401\end{array}$ & $\begin{array}{l}1.21113 \\
1.21539 \\
1.22068 \\
1.22647 \\
1.23236\end{array}$ & $\begin{array}{l}1.01924 \\
1.03021 \\
1.04315 \\
1.05682 \\
1.07034\end{array}$ & $\begin{array}{l}-.19189 \\
-.18518 \\
-.17753 \\
-.16965 \\
-.16202\end{array}$ \\
\hline $\begin{array}{l}1100 \\
1200 \\
1300 \\
1400 \\
1500\end{array}$ & $\begin{array}{l}.06109 \\
.06194 \\
.06141 \\
.05994 \\
.05787\end{array}$ & $\begin{array}{l}.13591 \\
.13561 \\
.13266 \\
.12803 \\
.12241\end{array}$ & $\begin{array}{l}.07482 \\
.07367 \\
.07125 \\
.06809 \\
.06454\end{array}$ & $\begin{array}{l}1.23806 \\
1.24343 \\
1.24837 \\
1.25287 \\
1.25695\end{array}$ & $\begin{array}{l}1.08316 \\
1.09500 \\
1.10575 \\
1.11542 \\
1.12407\end{array}$ & $\begin{array}{l}-.15490 \\
-. .14843 \\
-. .14262 \\
-. .13745 \\
-.13288\end{array}$ \\
\hline $\begin{array}{l}2000 \\
2500 \\
3000 \\
4000 \\
5000\end{array}$ & $\begin{array}{l}.04512 \\
.03437 \\
.02675 \\
.01783 \\
.01333\end{array}$ & $\begin{array}{l}.09203 \\
.06812 \\
.05152 \\
.03201 \\
.02188\end{array}$ & $\begin{array}{l}.04691 \\
.03375 \\
.02477 \\
.01418 \\
.00855\end{array}$ & $\begin{array}{l}1.27186 \\
1.28071 \\
1.28626 \\
1.29259 \\
1.29602\end{array}$ & $\begin{array}{l}1.15507 \\
1.17288 \\
1.18373 \\
1.19556 \\
1.20150\end{array}$ & $\begin{array}{l}-.11679 \\
-.10783 \\
-.10253 \\
-.09703 \\
-.09452\end{array}$ \\
\hline
\end{tabular}


Table 3. 06

\begin{tabular}{|c|c|c|c|c|c|c|}
\hline \multirow{2}{*}{$\mathrm{T}^{\circ} \mathrm{K}$} & $\mathrm{F}_{\mathrm{LiH}}^{\circ}{ }^{-\mathrm{F}_{\mathrm{iD}}^{\circ}}$ & $\mathrm{F}_{\mathrm{LiH}}^{\circ}{ }^{-\mathrm{F}_{\mathrm{LiT}}^{\circ}}$ & $\mathrm{F}_{\mathrm{LiD}}^{\circ}-\mathrm{F}_{\mathrm{LiT}}^{\circ}$ & $\mathrm{H}_{\mathrm{LiH}}^{\circ}{ }^{-\mathrm{H}_{\mathrm{LiD}}^{\circ}}$ & $\mathrm{H}_{\mathrm{LiH}}^{\circ}{ }^{-\mathrm{H}^{\circ}} \mathrm{LiT}$ & $\mathrm{H}_{\mathrm{LiD}}^{\circ}{ }^{-\mathrm{H}_{\mathrm{T}}^{\circ}}$ \\
\hline & $\mathrm{RT}$ & $\mathrm{RT}$ & $\mathrm{RT}$ & $\mathrm{RT}$ & $\mathrm{RT}$ & $\mathrm{RT}$ \\
\hline $\begin{array}{l}100 \\
200 \\
300 \\
400 \\
500\end{array}$ & $\begin{array}{l}3.21888 \\
1.98453 \\
1.57819 \\
1.38435 \\
1.27675\end{array}$ & $\begin{array}{l}4.69901 \\
2.95372 \\
2.38268 \\
2.11343 \\
1.96559\end{array}$ & $\begin{array}{r}1.48013 \\
.96919 \\
.80450 \\
.72908 \\
.68884\end{array}$ & $\begin{array}{r}2.46978 \\
1.23089 \\
.79660 \\
.55944 \\
.40992\end{array}$ & $\begin{array}{r}3.49375 \\
1.73663 \\
1.11243 \\
.77217 \\
.56050\end{array}$ & $\begin{array}{r}1.02397 \\
.50574 \\
.31584 \\
.21273 \\
.15058\end{array}$ \\
\hline $\begin{array}{r}600 \\
700 \\
800 \\
900 \\
1000\end{array}$ & $\begin{array}{l}1.21141 \\
1.16910 \\
1.14030 \\
1.11990 \\
1.10495\end{array}$ & $\begin{array}{l}1.87655 \\
1.81924 \\
1.78040 \\
1.75297 \\
1.73294\end{array}$ & $\begin{array}{l}.66513 \\
.65014 \\
.64010 \\
.63308 \\
.62799\end{array}$ & $\begin{array}{l}.31016 \\
.24112 \\
.19185 \\
.15573 \\
.12858\end{array}$ & $\begin{array}{l}.42117 \\
.32576 \\
.25821 \\
.20899 \\
.17218\end{array}$ & $\begin{array}{l}.11100 \\
.08464 \\
.06636 \\
.05327 \\
.04360\end{array}$ \\
\hline $\begin{array}{l}1100 \\
1200 \\
1300 \\
1400 \\
1500\end{array}$ & $\begin{array}{l}1.09372 \\
1.08506 \\
107827 \\
1.07285 \\
1.06845\end{array}$ & $\begin{array}{l}1.71791 \\
1.70635 \\
1.69729 \\
1.69006 \\
1.68422\end{array}$ & $\begin{array}{l}.62419 \\
.62130 \\
.61902 \\
.61721 \\
.61576\end{array}$ & $\begin{array}{l}.10776 \\
.09148 \\
.07852 \\
.06809 \\
.05955\end{array}$ & $\begin{array}{l}.14404 \\
.12211 \\
.10471 \\
.09072 \\
.07930\end{array}$ & $\begin{array}{l}.03628 \\
.03064 \\
.02619 \\
.02263 \\
.01974\end{array}$ \\
\hline $\begin{array}{l}2000 \\
2500 \\
3000 \\
4000 \\
5000\end{array}$ & $\begin{array}{l}1.05536 \\
1.04929 \\
1.04600 \\
1.04273 \\
1.04119\end{array}$ & $\begin{array}{l}1.66679 \\
1.65869 \\
1.65427 \\
1.64983 \\
1.64766\end{array}$ & $\begin{array}{l}.61143 \\
.60941 \\
.60828 \\
.60710 \\
.60647\end{array}$ & $\begin{array}{l}.03375 \\
.02159 \\
.01496 \\
.00846 \\
.00553\end{array}$ & $\begin{array}{l}.04493 \\
.02883 \\
.02014 \\
.01171 \\
.00801\end{array}$ & $\begin{array}{l}.01118 \\
.00725 \\
.00519 \\
.00325 \\
.00248\end{array}$ \\
\hline
\end{tabular}

\begin{tabular}{|c|c|c|c|c|c|c|}
\hline \multirow{2}{*}{$\mathrm{T}^{\circ} \mathrm{K}$} & $\mathrm{C}_{\mathrm{LiD}}^{\circ}{ }^{-\mathrm{C}_{\mathrm{LiH}}^{\circ}}$ & $\mathrm{C}_{\mathrm{LiT}}^{\circ}{ }^{-\mathrm{C}_{\mathrm{LiH}}^{\circ}}$ & $\mathrm{C}_{\mathrm{LiT}}^{\circ}-\mathrm{C}_{\mathrm{LiD}}^{\circ}$ & $\mathrm{S}_{\mathrm{LiD}}^{\circ}{ }^{-\mathrm{S}_{\mathrm{LiH}}^{\circ}}$ & $\mathrm{S}_{\mathrm{LiH}}^{\circ}$ & \multirow{2}{*}{$\frac{\mathrm{LiT}^{\circ} \mathrm{S}_{\mathrm{LiD}}^{\circ}}{\mathrm{R}}$} \\
\hline & $\mathrm{R}$ & $\mathrm{R}$ & $\mathrm{R}$ & $\mathrm{R}$ & $\mathrm{R}$ & \\
\hline $\begin{array}{l}100 \\
200 \\
300 \\
400 \\
500\end{array}$ & $\begin{array}{r}-.00011 \\
.02862 \\
.11750 \\
.17788 \\
.19246\end{array}$ & $\begin{array}{l}.00023 \\
.06416 \\
.20448 \\
.27866 \\
.28580\end{array}$ & $\begin{array}{l}.00034 \\
.03554 \\
.08698 \\
.10078 \\
.09334\end{array}$ & $\begin{array}{l}.74910 \\
.75364 \\
.78159 \\
.82491 \\
.86683\end{array}$ & $\begin{array}{l}1.20525 \\
1.21708 \\
1.27024 \\
1.34126 \\
1.40508\end{array}$ & $\begin{array}{l}.45615 \\
.46344 \\
.48865 \\
.51635 \\
.53825\end{array}$ \\
\hline $\begin{array}{r}600 \\
700 \\
800 \\
900 \\
1000\end{array}$ & $\begin{array}{l}.18231 \\
.16328 \\
.14290 \\
.12408 \\
.10766\end{array}$ & $\begin{array}{l}.26249 \\
.23054 \\
.19911 \\
.17125 \\
.14754\end{array}$ & $\begin{array}{l}.08018 \\
.06726 \\
.05621 \\
.04717 \\
.03988\end{array}$ & $\begin{array}{l}.90126 \\
.92798 \\
.94845 \\
.96417 \\
.97637\end{array}$ & $\begin{array}{l}1.45538 \\
1.49348 \\
1.52219 \\
1.54399 \\
1.56077\end{array}$ & $\begin{array}{l}.55412 \\
.56550 \\
.57374 \\
.57982 \\
.58440\end{array}$ \\
\hline $\begin{array}{l}1100 \\
1200 \\
1300 \\
1400 \\
1500\end{array}$ & $\begin{array}{l}.09370 \\
.08191 \\
.07199 \\
.0636 ? \\
.05651\end{array}$ & $\begin{array}{l}.12768 \\
.11110 \\
.09726 \\
.08565 \\
.07584\end{array}$ & $\begin{array}{l}.03398 \\
.02919 \\
.02527 \\
.02203 \\
.01933\end{array}$ & $\begin{array}{r}.98596 \\
.99359 \\
.99974 \\
1.00476 \\
1.00890\end{array}$ & $\begin{array}{l}1.57387 \\
1.58424 \\
1.59257 \\
1.59935 \\
1.60491\end{array}$ & $\begin{array}{l}.58791 \\
.59065 \\
.59283 \\
.59459 \\
.59601\end{array}$ \\
\hline $\begin{array}{l}2000 \\
2500 \\
3000 \\
4000 \\
5000\end{array}$ & $\begin{array}{l}.03355 \\
.02180 \\
.01507 \\
.00804 \\
.00462\end{array}$ & $\begin{array}{l}.04435 \\
.02832 \\
.01911 \\
.00939 \\
.00456\end{array}$ & $\begin{array}{r}.01080 \\
.00652 \\
.00404 \\
.00135 \\
-.00006\end{array}$ & $\begin{array}{l}1.02162 \\
1.02770 \\
1.03103 \\
1.03427 \\
1.03566\end{array}$ & $\begin{array}{l}1.62186 \\
1.62985 \\
1.63413 \\
1.63812 \\
1.63964\end{array}$ & $\begin{array}{l}.60024 \\
.60215 \\
.60310 \\
.60385 \\
.60398\end{array}$ \\
\hline
\end{tabular}


Table 3.07

\begin{tabular}{|c|c|c|c|c|c|c|}
\hline \multirow{2}{*}{$\mathrm{T}^{\circ} \mathrm{K}$} & $\mathrm{F}_{\mathrm{NaH}}^{\circ}-\mathrm{F}_{\mathrm{NaD}}^{\circ}$ & $\mathrm{F}_{\mathrm{NaH}}^{\circ}-\mathrm{F}_{\mathrm{NaT}}^{\circ}$ & $\mathrm{F}_{\mathrm{NaD}}^{\circ}-\mathrm{F}_{\mathrm{NaT}}^{\circ}$ & $\mathrm{H}_{\mathrm{NaH}}^{\circ}{ }^{-\mathrm{H}_{\mathrm{NaD}}^{\circ}}$ & $\mathrm{H}_{\mathrm{NaH}}^{\circ}{ }^{-\mathrm{H}^{\circ}} \mathrm{NaT}$ & $\mathrm{H}_{\mathrm{NaD}}^{\circ}-\mathrm{H}_{\mathrm{NaT}}^{\circ}$ \\
\hline & $\mathrm{RT}$ & $\mathrm{RT}$ & $\mathrm{RT}$ & $\mathrm{RT}$ & $\mathrm{RT}$ & $\mathrm{RT}$ \\
\hline $\begin{array}{l}100 \\
200 \\
300 \\
400 \\
500\end{array}$ & $\begin{array}{l}3.00463 \\
1.85892 \\
1.48897 \\
1.31876 \\
1.22740\end{array}$ & $\begin{array}{l}4.42497 \\
2.78415 \\
2.26143 \\
2.02479 \\
1.89927\end{array}$ & $\begin{array}{r}1.42034 \\
.92523 \\
.77246 \\
.70603 \\
.67187\end{array}$ & $\begin{array}{r}2.29610 \\
1.13454 \\
.71118 \\
.48167 \\
.34277\end{array}$ & $\begin{array}{r}3.29459 \\
1.61466 \\
.99515 \\
.66478 \\
.46874\end{array}$ & $\begin{array}{l}.99849 \\
.48012 \\
.28397 \\
.18311 \\
.12597\end{array}$ \\
\hline $\begin{array}{r}600 \\
700 \\
800 \\
900 \\
1000\end{array}$ & $\begin{array}{l}1.17334 \\
1.13897 \\
1.11589 \\
1.09970 \\
1.08792\end{array}$ & $\begin{array}{l}1.82556 \\
1.77894 \\
1.74774 \\
1.72589 \\
1.71003\end{array}$ & $\begin{array}{l}.65222 \\
.63997 \\
.63185 \\
.62619 \\
.62211\end{array}$ & $\begin{array}{l}.25384 \\
.19429 \\
.15286 \\
.12307 \\
.10101\end{array}$ & $\begin{array}{l}.34504 \\
.26304 \\
.20639 \\
.16586 \\
.13597\end{array}$ & $\begin{array}{l}.09120 \\
.06875 \\
.05353 \\
.04279 \\
.03496\end{array}$ \\
\hline $\begin{array}{l}1100 \\
1200 \\
1300 \\
1400 \\
1500\end{array}$ & $\begin{array}{l}1.07911 \\
1.07235 \\
1.06706 \\
1.06285 \\
1.05943\end{array}$ & $\begin{array}{l}1.69818 \\
1.68909 \\
1.68198 \\
1.67631 \\
1.67171\end{array}$ & $\begin{array}{l}.61907 \\
.61674 \\
.61491 \\
.61346 \\
.61228\end{array}$ & $\begin{array}{l}.08429 \\
.07134 \\
.06112 \\
.05294 \\
.04629\end{array}$ & $\begin{array}{l}.11338 \\
.09591 \\
.08218 \\
.07118 \\
.06226\end{array}$ & $\begin{array}{l}.02909 \\
.02457 \\
.02105 \\
.01824 \\
.01597\end{array}$ \\
\hline $\begin{array}{l}2000 \\
2500 \\
3000 \\
4000 \\
5000\end{array}$ & $\begin{array}{l}1.04924 \\
1.04443 \\
1.04174 \\
1.03888 \\
1.03736\end{array}$ & $\begin{array}{l}1.65799 \\
1.65147 \\
1.64779 \\
1.64381 \\
1.64160\end{array}$ & $\begin{array}{l}.60875 \\
.60703 \\
.60605 \\
.60493 \\
.60424\end{array}$ & $\begin{array}{l}.02647 \\
.01733 \\
.01247 \\
.00790 \\
.00603\end{array}$ & $\begin{array}{l}.03575 \\
.02360 \\
.01717 \\
.01120 \\
.00883\end{array}$ & $\begin{array}{l}.00928 \\
.00626 \\
.00470 \\
.00330 \\
.00280\end{array}$ \\
\hline
\end{tabular}

\begin{tabular}{|c|c|c|c|c|c|c|}
\hline \multirow{2}{*}{$\mathrm{T}^{\circ} \mathrm{K}$} & $\mathrm{C}_{\mathrm{NaD}}^{\circ}-\mathrm{C}_{\mathrm{NaH}}^{\circ}$ & $\mathrm{C}_{\mathrm{NaT}}^{\circ}{ }^{-\mathrm{C}_{\mathrm{NaH}}^{\circ}}$ & $\mathrm{C}_{\mathrm{NaT}}^{\circ}-\mathrm{C}_{\mathrm{NaD}}^{\circ}$ & $\mathrm{S}_{\mathrm{NaD}}^{\circ}{ }^{-} \mathrm{S}_{\mathrm{NaH}}$ & $\mathrm{S}_{\mathrm{NaT}}^{\circ}-\mathrm{S}_{\mathrm{NaH}}^{\circ}$ & $\mathrm{S}_{\mathrm{NaT}}^{\circ}-\mathrm{S}_{\mathrm{NaD}}^{\circ}$ \\
\hline & $\mathrm{R}$ & $\mathrm{R}$ & $\mathrm{R}$ & $\mathrm{R}$ & $\mathrm{R}$ & $\mathrm{R}$ \\
\hline $\begin{array}{l}100 \\
200 \\
300 \\
400 \\
500\end{array}$ & $\begin{array}{l}.00088 \\
.07557 \\
.18487 \\
.21740 \\
.20396\end{array}$ & $\begin{array}{l}.00470 \\
.15728 \\
.30708 \\
.33011 \\
.29625\end{array}$ & $\begin{array}{l}.00382 \\
.08171 \\
.12221 \\
.11271 \\
.09229\end{array}$ & $\begin{array}{l}.70853 \\
.72437 \\
.77779 \\
.83709 \\
.88463\end{array}$ & $\begin{array}{l}1.13038 \\
1.16948 \\
1.26627 \\
1.36002 \\
1.43054\end{array}$ & $\begin{array}{l}.42185 \\
.44511 \\
.48848 \\
.52293 \\
.54591\end{array}$ \\
\hline $\begin{array}{r}600 \\
700 \\
800 \\
900 \\
1000\end{array}$ & $\begin{array}{l}.17690 \\
.14945 \\
.12556 \\
.10579 \\
.08970\end{array}$ & $\begin{array}{l}.25053 \\
.20833 \\
.17317 \\
.14479 \\
.12205\end{array}$ & $\begin{array}{l}.07363 \\
.05888 \\
.04761 \\
.03900 \\
.03235\end{array}$ & $\begin{array}{l}.91950 \\
.94467 \\
.96303 \\
.97663 \\
.98692\end{array}$ & $\begin{array}{l}1.48052 \\
1.51589 \\
1.54134 \\
1.56002 \\
1.57407\end{array}$ & $\begin{array}{l}.56102 \\
.57122 \\
.57831 \\
.58339 \\
.58715\end{array}$ \\
\hline $\begin{array}{l}1100 \\
1200 \\
1300 \\
1400 \\
1500\end{array}$ & $\begin{array}{l}.07663 \\
.06596 \\
.05718 \\
.04991 \\
.04383\end{array}$ & $\begin{array}{l}.10378 \\
.08898 \\
.07687 \\
.06688 \\
.05856\end{array}$ & $\begin{array}{l}.02715 \\
.02302 \\
.01969 \\
.01697 \\
.01473\end{array}$ & $\begin{array}{l}.99483 \\
1.00102 \\
1.00595 \\
1.00991 \\
1.01313\end{array}$ & $\begin{array}{l}1.58481 \\
1.59318 \\
1.59981 \\
1.60513 \\
1.60945\end{array}$ & $\begin{array}{l}.58998 \\
.59216 \\
.59386 \\
.59522 \\
.59632\end{array}$ \\
\hline $\begin{array}{l}2000 \\
2500 \\
3000 \\
4000 \\
5000\end{array}$ & $\begin{array}{r}.02459 \\
.01486 \\
.00923 \\
.00316 \\
-.00001\end{array}$ & $\begin{array}{r}.03232 \\
.01910 \\
.01141 \\
.00304 \\
-.00141\end{array}$ & $\begin{array}{r}.00773 \\
.00424 \\
.00218 \\
-.00012 \\
-.00140\end{array}$ & $\begin{array}{l}1.02277 \\
1.02709 \\
1.02927 \\
1.03098 \\
1.03132\end{array}$ & $\begin{array}{l}1.62223 \\
1.62787 \\
1.63062 \\
1.63260 \\
1.63276\end{array}$ & $\begin{array}{l}.59946 \\
.60078 \\
.60135 \\
.60162 \\
.60144\end{array}$ \\
\hline
\end{tabular}


Table 3.08

\begin{tabular}{|c|c|c|c|c|c|c|}
\hline & $\mathrm{F}_{\mathrm{KH}}^{\circ}-\mathrm{F}_{\mathrm{KD}}^{\circ}$ & $\mathrm{F}_{\mathrm{KH}}^{\circ}-\mathrm{F}_{\mathrm{KT}}^{\circ}$ & $\mathrm{F}_{\mathrm{KD}}^{\circ}-\mathrm{F}_{\mathrm{KT}}^{\circ}$ & $\mathrm{H}_{\mathrm{KH}}^{\circ}-\mathrm{H}_{\mathrm{KD}}^{\circ}$ & $\mathrm{H}_{\mathrm{KH}}^{\circ}-\mathrm{H}_{\mathrm{KT}}^{\circ}$ & $\mathrm{H}_{\mathrm{KD}}^{\circ}-\mathrm{H}_{\mathrm{KT}}^{\circ}$ \\
\hline $1 \mathrm{~N}$ & $\mathrm{RT}$ & $\mathrm{RT}$ & $\mathrm{RT}$ & $\mathrm{RT}$ & $\mathrm{RT}$ & $\mathrm{RT}$ \\
\hline $\begin{array}{l}100 \\
200 \\
300 \\
400 \\
500\end{array}$ & $\begin{array}{l}2.72408 \\
1.74347 \\
1.43592 \\
1.29973 \\
1.22883\end{array}$ & $\begin{array}{l}4.00548 \\
2.60060 \\
2.16850 \\
1.98040 \\
1.88348\end{array}$ & $\begin{array}{r}1.28140 \\
.85713 \\
.73258 \\
.68068 \\
.65465\end{array}$ & $\begin{array}{r}1.97273 \\
.95843 \\
.57809 \\
.37838 \\
.26261\end{array}$ & $\begin{array}{r}2.83802 \\
1.35848 \\
.80341 \\
.51915 \\
.35764\end{array}$ & $\begin{array}{l}.86529 \\
.40005 \\
.22532 \\
.14078 \\
.09503\end{array}$ \\
\hline $\begin{array}{r}600 \\
700 \\
800 \\
900 \\
1000\end{array}$ & $\begin{array}{l}1.18777 \\
1.16210 \\
1.14511 \\
1.13334 \\
1.12487\end{array}$ & $\begin{array}{l}1.82768 \\
1.79292 \\
1.76992 \\
1.75399 \\
1.74254\end{array}$ & $\begin{array}{l}.63991 \\
.63082 \\
.62481 \\
.62065 \\
.61767\end{array}$ & $\begin{array}{l}.19090 \\
.14398 \\
.11183 \\
.08893 \\
.07208\end{array}$ & $\begin{array}{l}.25890 \\
.19487 \\
.15126 \\
.12035 \\
.09769\end{array}$ & $\begin{array}{l}.06800 \\
.05089 \\
.03943 \\
.03142 \\
.02561\end{array}$ \\
\hline $\begin{array}{l}1100 \\
1200 \\
1300 \\
1400 \\
1500\end{array}$ & $\begin{array}{l}1.11863 \\
1.11391 \\
1.11027 \\
1.10743 \\
1.10516\end{array}$ & $\begin{array}{l}1.73406 \\
1.72765 \\
1.72267 \\
1.71876 \\
1.71563\end{array}$ & $\begin{array}{l}.61544 \\
.61373 \\
.61240 \\
.61133 \\
.61047\end{array}$ & $\begin{array}{l}.05933 \\
.04945 \\
.04164 \\
.03537 \\
.03024\end{array}$ & $\begin{array}{l}.08060 \\
.06742 \\
.05702 \\
.04870 \\
.04191\end{array}$ & $\begin{array}{l}.02128 \\
.01796 \\
.01538 \\
.01333 \\
.01167\end{array}$ \\
\hline $\begin{array}{l}2000 \\
2500 \\
3000 \\
4000 \\
5000\end{array}$ & $\begin{array}{l}1.09892 \\
1.09658 \\
1.09579 \\
1.09601 \\
1.09711\end{array}$ & $\begin{array}{l}1.70680 \\
1.70321 \\
1.70168 \\
1.70104 \\
1.70162\end{array}$ & $\begin{array}{l}.60789 \\
.60663 \\
.60589 \\
.60503 \\
.60451\end{array}$ & $\begin{array}{r}.01456 \\
.00677 \\
.00214 \\
-.00326 \\
-.00657\end{array}$ & $\begin{array}{r}.02138 \\
.01141 \\
.00567 \\
-.00072 \\
-.00436\end{array}$ & $\begin{array}{l}.00683 \\
.00464 \\
.00353 \\
.00254 \\
.00221\end{array}$ \\
\hline
\end{tabular}

\begin{tabular}{|c|c|c|c|c|c|c|}
\hline \multirow{2}{*}{$\mathrm{T}^{\circ} \mathrm{K}$} & $\mathrm{C}_{\mathrm{KD}}^{\circ}-\mathrm{C}_{\mathrm{KH}}^{\circ}$ & $\mathrm{C}_{\mathrm{KT}}^{\circ}-\mathrm{C}_{\mathrm{KH}}^{\circ}$ & $\mathrm{C}_{\mathrm{KT}}^{\circ}-\mathrm{C}_{\mathrm{KD}}^{\circ}$ & $\mathrm{S}_{\mathrm{KD}}^{\circ}-\mathrm{S}_{\mathrm{KH}}^{\circ}$ & $\mathrm{S}_{\mathrm{KT}}^{\circ}-\mathrm{S}_{\mathrm{KH}}^{\circ}$ & $\mathrm{S}_{\mathrm{KT}}^{\circ}-\mathrm{S}_{\mathrm{KD}}^{\circ}$ \\
\hline & $\mathrm{R}$ & $\mathrm{R}$ & $\mathrm{R}$ & $\mathrm{R}$ & $\mathrm{R}$ & $\mathrm{R}$ \\
\hline $\begin{array}{l}100 \\
200 \\
300 \\
400 \\
500\end{array}$ & $\begin{array}{l}.00461 \\
.12755 \\
.21725 \\
.21520 \\
.18418\end{array}$ & $\begin{array}{l}.01779 \\
.23936 \\
.34133 \\
.31543 \\
.26074\end{array}$ & $\begin{array}{l}.01318 \\
.11181 \\
.12408 \\
.10023 \\
.07656\end{array}$ & $\begin{array}{l}.75135 \\
.78505 \\
.85782 \\
.92136 \\
.96622\end{array}$ & $\begin{array}{l}1.16746 \\
1.24213 \\
1.36508 \\
1.46126 \\
1.52584\end{array}$ & $\begin{array}{l}.41611 \\
.45708 \\
.50726 \\
.53990 \\
.55962\end{array}$ \\
\hline $\begin{array}{r}600 \\
700 \\
800 \\
900 \\
1000\end{array}$ & $\begin{array}{l}.15164 \\
.12441 \\
.10297 \\
.08634 \\
.07343\end{array}$ & $\begin{array}{l}.21030 \\
.17013 \\
.13929 \\
.11572 \\
.09757\end{array}$ & $\begin{array}{l}.05866 \\
.04572 \\
.03632 \\
.02938 \\
.02414\end{array}$ & $\begin{array}{r}.99687 \\
1.01812 \\
1.03328 \\
1.04440 \\
1.05280\end{array}$ & $\begin{array}{l}1.56878 \\
1.59805 \\
1.61866 \\
1.63364 \\
1.64485\end{array}$ & $\begin{array}{l}.57191 \\
.57993 \\
.58538 \\
.58924 \\
.59205\end{array}$ \\
\hline $\begin{array}{l}1100 \\
1200 \\
1300 \\
1400 \\
1500\end{array}$ & $\begin{array}{l}.06331 \\
.05531 \\
.04892 \\
.04377 \\
.03957\end{array}$ & $\begin{array}{l}.08341 \\
.07224 \\
.06333 \\
.05613 \\
.05025\end{array}$ & $\begin{array}{l}.02010 \\
.01693 \\
.01441 \\
.01236 \\
.01068\end{array}$ & $\begin{array}{l}1.05931 \\
1.06446 \\
1.06862 \\
1.07205 \\
1.07493\end{array}$ & $\begin{array}{l}1.65346 \\
1.66023 \\
1.66564 \\
1.67006 \\
1.67373\end{array}$ & $\begin{array}{l}.59415 \\
.59577 \\
.59702 \\
.59801 \\
.59880\end{array}$ \\
\hline $\begin{array}{l}2000 \\
2500 \\
3000 \\
4000 \\
5000\end{array}$ & $\begin{array}{l}.02729 \\
.02220 \\
.02007 \\
.01935 \\
.02040\end{array}$ & $\begin{array}{l}.03277 \\
.02509 \\
.02144 \\
.01901 \\
.01909\end{array}$ & $\begin{array}{r}.00548 \\
.00289 \\
.00137 \\
-.00034 \\
-.00131\end{array}$ & $\begin{array}{l}1.08437 \\
1.08982 \\
1.09365 \\
1.09926 \\
1.10367\end{array}$ & $\begin{array}{l}1.68543 \\
1.69180 \\
1.69601 \\
1.70175 \\
1.70597\end{array}$ & $\begin{array}{l}.60106 \\
.60198 \\
.60236 \\
.60249 \\
.60230\end{array}$ \\
\hline
\end{tabular}




\begin{tabular}{|c|c|c|c|c|c|c|}
\hline \multirow{2}{*}{$\mathrm{T}^{\circ} \mathrm{K}$} & $\mathrm{F}_{\mathrm{RbH}}^{\circ}-\mathrm{F}^{\circ} \mathrm{RbD}$ & $\begin{array}{c}\mathrm{F}^{\circ}-\mathrm{F}^{\circ} \\
\mathrm{RbH}\end{array}$ & $\mathrm{F}_{\mathrm{RbD}}^{\circ}{ }^{-\mathrm{F}^{\circ}} \mathrm{RbT}$ & $\begin{array}{c}\mathrm{H}^{\circ}-\mathrm{H}^{\circ} \\
\mathrm{RbH}\end{array}$ & $\begin{array}{l}\mathrm{H}_{\mathrm{RbH}}^{\circ} \\
\end{array} \mathrm{H}^{\circ} \mathrm{RbT}$ & \multirow{2}{*}{$\frac{\mathrm{RbD}^{\circ}{ }^{-\mathrm{H}^{\circ}} \mathrm{Rb}}{\mathrm{RT}}$} \\
\hline & $\mathrm{RT}$ & $\mathrm{RT}$ & $\mathrm{RT}$ & RT & RT & \\
\hline $\begin{array}{l}100 \\
200 \\
300 \\
400 \\
500\end{array}$ & $\begin{array}{l}2.60633 \\
1.65823 \\
1.36420 \\
1.23551 \\
1.16897\end{array}$ & $\begin{array}{l}3.85872 \\
2.50056 \\
2.08843 \\
1.91114 \\
1.82042\end{array}$ & $\begin{array}{r}1.25239 \\
.84233 \\
.72422 \\
.67563 \\
.65145\end{array}$ & $\begin{array}{r}1.91106 \\
.92139 \\
.54867 \\
.35592 \\
.24586\end{array}$ & $\begin{array}{r}2.75188 \\
1.30358 \\
.76049 \\
.48708 \\
.33401\end{array}$ & $\begin{array}{r}.84082 \\
.38219 \\
.21181 \\
.13116 \\
.08815\end{array}$ \\
\hline $\begin{array}{r}600 \\
700 \\
800 \\
900 \\
1000\end{array}$ & $\begin{array}{l}1.13057 \\
1.10656 \\
1.09062 \\
1.07951 \\
1.07149\end{array}$ & $\begin{array}{l}1.76836 \\
1.73592 \\
1.71441 \\
1.69944 \\
1.68862\end{array}$ & $\begin{array}{l}.63780 \\
.62936 \\
.62379 \\
.61993 \\
.61713\end{array}$ & $\begin{array}{l}.17850 \\
.13482 \\
.10512 \\
.08411 \\
.06877\end{array}$ & $\begin{array}{r}.24148 \\
.18198 \\
.14175 \\
.11341 \\
.09279\end{array}$ & $\begin{array}{l}.06299 \\
.04716 \\
.03663 \\
.02930 \\
.02402\end{array}$ \\
\hline $\begin{array}{l}1100 \\
1200 \\
1300 \\
1400 \\
1500\end{array}$ & $\begin{array}{l}1.06550 \\
1.06092 \\
1.05732 \\
1.05446 \\
1.05214\end{array}$ & $\begin{array}{l}1.68053 \\
1.67433 \\
1.66946 \\
1.66558 \\
1.66239\end{array}$ & $\begin{array}{l}.61503 \\
.61341 \\
.61214 \\
.61111 \\
.61026\end{array}$ & $\begin{array}{l}.05726 \\
.04840 \\
.04148 \\
.03595 \\
.03150\end{array}$ & $\begin{array}{l}.07735 \\
.06552 \\
.05629 \\
.04895 \\
.04303\end{array}$ & $\begin{array}{l}.02009 \\
.01712 \\
.01481 \\
.01299 \\
.01154\end{array}$ \\
\hline $\begin{array}{l}2000 \\
2500 \\
3000 \\
4000 \\
5000\end{array}$ & $\begin{array}{l}1.04516 \\
1.04178 \\
1.03980 \\
1.03755 \\
1.03619\end{array}$ & $\begin{array}{l}1.65276 \\
1.64794 \\
1.64500 \\
1.64140 \\
1.63899\end{array}$ & $\begin{array}{l}.60760 \\
.60616 \\
.60519 \\
.60385 \\
.60281\end{array}$ & $\begin{array}{l}.01835 \\
.01243 \\
.00937 \\
.00665 \\
.00570\end{array}$ & $\begin{array}{l}.02576 \\
.01813 \\
.01434 \\
.01124 \\
.01046\end{array}$ & $\begin{array}{l}.00741 \\
.00570 \\
.00496 \\
.00459 \\
.00477\end{array}$ \\
\hline
\end{tabular}

\begin{tabular}{|c|c|c|c|c|c|c|}
\hline \multirow{2}{*}{$\mathrm{T}^{\circ} \mathrm{K}$} & $\mathrm{C}_{\mathrm{RbD}}^{\circ}{ }^{-\mathrm{C}_{\mathrm{RbH}}^{\circ}}$ & $\mathrm{C}_{\mathrm{RbT}}^{\circ}{ }^{-\mathrm{C}_{\mathrm{RbH}}^{\circ}}$ & $\mathrm{C}_{\mathrm{RbT}}^{\circ}{ }^{-\mathrm{C}_{\mathrm{RbD}}^{\circ}}$ & $\mathrm{S}_{\mathrm{RbD}}^{\circ}-\mathrm{S}_{\mathrm{RbH}}^{\circ}$ & $\mathrm{S}_{\mathrm{RbT}}^{\circ}{ }^{-\mathrm{S}_{\mathrm{RbH}}^{\circ}}$ & $\mathrm{S}_{\mathrm{RbT}}^{\circ}{ }^{-\mathrm{S}_{\mathrm{RbD}}^{\circ}}$ \\
\hline & $\mathrm{R}$ & $\mathrm{R}$ & $\mathrm{R}$ & $\mathrm{R}$ & $\mathrm{R}$ & $\mathrm{R}$ \\
\hline $\begin{array}{l}100 \\
200 \\
300 \\
400 \\
500\end{array}$ & $\begin{array}{l}.00714 \\
.14619 \\
.22487 \\
.21192 \\
.17595\end{array}$ & $\begin{array}{l}.02604 \\
.26823 \\
.34922 \\
.30859 \\
.24818\end{array}$ & $\begin{array}{l}.01890 \\
.12204 \\
.12435 \\
.09667 \\
.07223\end{array}$ & $\begin{array}{l}.69528 \\
.73685 \\
.81552 \\
.87959 \\
.92311\end{array}$ & $\begin{array}{l}1.10685 \\
1.19699 \\
1.32793 \\
1.42407 \\
1.48640\end{array}$ & $\begin{array}{l}.41157 \\
.46014 \\
.51241 \\
.54448 \\
.56329\end{array}$ \\
\hline $\begin{array}{r}600 \\
700 \\
800 \\
900 \\
1000\end{array}$ & $\begin{array}{l}.14166 \\
.11398 \\
.09254 \\
.07601 \\
.06318\end{array}$ & $\begin{array}{l}.19613 \\
.15585 \\
.12535 \\
.10215 \\
.08429\end{array}$ & $\begin{array}{l}.05447 \\
.04187 \\
.03281 \\
.02614 \\
.02111\end{array}$ & $\begin{array}{r}.95207 \\
.97174 \\
.98550 \\
.99541 \\
1.00272\end{array}$ & $\begin{array}{l}1.52687 \\
1.55394 \\
1.57266 \\
1.58603 \\
1.59583\end{array}$ & $\begin{array}{l}.57480 \\
.58220 \\
.58716 \\
.59062 \\
.59311\end{array}$ \\
\hline $\begin{array}{l}1100 \\
1200 \\
1300 \\
1400 \\
1500\end{array}$ & $\begin{array}{l}.05309 \\
.04505 \\
.03855 \\
.03323 \\
.02884\end{array}$ & $\begin{array}{l}.07031 \\
.05920 \\
.05024 \\
.04291 \\
.03684\end{array}$ & $\begin{array}{l}.01722 \\
.01415 \\
.01169 \\
.00968 \\
.00800\end{array}$ & $\begin{array}{l}1.00825 \\
1.01251 \\
1.01585 \\
1.01850 \\
1.02064\end{array}$ & $\begin{array}{l}1.60318 \\
1.60880 \\
1.61318 \\
1.61662 \\
1.61937\end{array}$ & $\begin{array}{l}.59493 \\
.59629 \\
.59733 \\
.59812 \\
.59873\end{array}$ \\
\hline $\begin{array}{l}2000 \\
2500 \\
3000 \\
4000 \\
5000\end{array}$ & $\begin{array}{r}.01507 \\
.00814 \\
.00405 \\
-.00053 \\
-.00309\end{array}$ & $\begin{array}{r}.01772 \\
.00789 \\
.00192 \\
-.00512 \\
-.00937\end{array}$ & $\begin{array}{r}.00265 \\
-.00025 \\
-.00213 \\
-.00459 \\
-.00628\end{array}$ & $\begin{array}{l}1.02681 \\
1.02935 \\
1.03043 \\
1.03090 \\
1.03049\end{array}$ & $\begin{array}{l}1.62701 \\
1.62980 \\
1.63067 \\
1.63015 \\
1.62853\end{array}$ & $\begin{array}{l}.60020 \\
.60045 \\
.60024 \\
.59925 \\
.59804\end{array}$ \\
\hline
\end{tabular}


Table 3.10

\begin{tabular}{|c|c|c|c|c|c|c|}
\hline \multirow{2}{*}{$\mathrm{T}^{\circ} \mathrm{K}$} & $\mathrm{F}^{\circ} \mathrm{CsH}^{-\mathrm{F}_{\mathrm{CDD}}^{\circ}}$ & $\mathrm{F}_{\mathrm{CsH}}^{\circ}-\mathrm{F}_{\mathrm{CsT}}^{\circ}$ & $\mathrm{F}_{\mathrm{CsD}}^{\circ}{ }^{-\mathrm{F}_{\mathrm{CsT}}^{\circ}}$ & $\mathrm{H}^{\circ} \mathrm{CsH}{ }^{-\mathrm{H}^{\circ}} \mathrm{CsD}$ & $\mathrm{H}_{\mathrm{CsH}}^{\circ}{ }^{-\mathrm{H}^{\circ} \mathrm{CsT}}$ & $\mathrm{H}_{\mathrm{CSD}}^{\circ}{ }^{-\mathrm{H}^{\circ} \mathrm{CsT}}$ \\
\hline & $\mathrm{RT}$ & $\mathrm{RT}$ & $\mathrm{RT}$ & $\mathrm{RT}$ & $\mathrm{RT}$ & $\mathrm{RT}$ \\
\hline 100 & 2.52167 & 3.73666 & 1.39109 & 1.82796 & 2.63202 & .98016 \\
\hline 200 & 1.61680 & 2.44176 & .91301 & .87401 & 1.23359 & .44763 \\
\hline 300 & 1.33941 & 2.05398 & .77326 & .51398 & .71047 & .25518 \\
\hline 400 & 1.21937 & 1.88903 & .71369 & .33050 & .45118 & .16471 \\
\hline 500 & 1.15774 & 1.80520 & .68268 & .22712 & .30781 & .11591 \\
\hline 600 & 1.12233 & 1.75730 & .66433 & .16441 & .22184 & .08679 \\
\hline 700 & 1.10022 & 1.72753 & .65246 & .12400 & .16686 & .06801 \\
\hline 800 & 1.08556 & 1.70781 & .64427 & .09666 & .12982 & .05518 \\
\hline 900 & 1.07534 & 1.69411 & .63833 & .07740 & .10382 & .04598 \\
\hline 1000 & 1.06796 & 1.68420 & .63385 & .06336 & .08492 & .03917 \\
\hline 1100 & 1.06243 & 1.67680 & .63038 & .05285 & .07080 & .03396 \\
\hline 1200 & 1.05820 & 1.67113 & .62761 & .04480 & .05999 & .02987 \\
\hline 1300 & 1.05487 & 1.66667 & .62535 & .03851 & .05156 & .02660 \\
\hline 1400 & 1.05221 & 1.66311 & .62348 & .03352 & .04487 & .02393 \\
\hline 1500 & 1.05004 & 1.66020 & .62191 & .02948 & .03948 & .02175 \\
\hline 2000 & 1.04342 & 1.65133 & .61672 & .01773 & .02379 & .01487 \\
\hline 2500 & 1.04009 & 1.64685 & .61381 & .01253 & .01688 & .01140 \\
\hline 3000 & 1.03806 & 1.64412 & .61192 & .00995 & .01348 & .00939 \\
\hline 4000 & 1.03554 & 1.64069 & .60956 & .00788 & .01076 & .00729 \\
\hline 5000 & 1.03385 & 1.63838 & .60805 & .00740 & .01017 & .00629 \\
\hline
\end{tabular}

$\mathrm{T}^{\circ} \mathrm{K} \frac{\mathrm{C}_{\mathrm{CsD}}^{\circ}-\mathrm{C}_{\mathrm{CsH}}^{\circ}}{\mathrm{R}} \frac{{ }_{\mathrm{CsT}}^{\circ}-\mathrm{C}_{\mathrm{CsH}}^{\circ}}{\mathrm{R}} \frac{{ }_{\mathrm{CST}}^{\circ}{ }_{\mathrm{CSD}}^{\circ}}{\mathrm{R}} \frac{\mathrm{S}_{\mathrm{CsD}}^{\circ}{ }^{-S_{\mathrm{CsH}}^{\circ}}}{\mathrm{R}} \frac{\mathrm{S}_{\mathrm{CST}}^{\circ}-\mathrm{S}_{\mathrm{CsH}}^{\circ}}{\mathrm{R}} \frac{\mathrm{S}_{\mathrm{CST}}^{\circ}-\mathrm{S}_{\mathrm{CsD}}^{\circ}}{\mathrm{R}}$

$\begin{array}{lrrrrrr}100 & .01020 & .03482 & .02462 & .69371 & 1.10465 & .41094 \\ 200 & .16140 & .28899 & .12759 & .74278 & 1.20817 & .46539 \\ 300 & .22747 & .34906 & .12159 & .82543 & 1.34351 & .51808 \\ 400 & .20573 & .29769 & .09196 & .88887 & 1.43786 & .54899 \\ 500 & .16696 & .23482 & .06786 & .93063 & 1.49739 & .56676 \\ 600 & .13249 & .18340 & .05091 & .95791 & 1.53546 & .57755 \\ 700 & .10550 & .14461 & .03911 & .97621 & 1.56066 & .58445 \\ 800 & .08494 & .11565 & .03071 & .98889 & 1.57799 & .58910 \\ 900 & .06926 & .09386 & .02460 & .99795 & 1.59030 & .59235 \\ 1000 & .05715 & .07717 & .02002 & 1.00460 & 1.59929 & .59469 \\ 1100 & .04768 & .06418 & .01650 & 1.00959 & 1.60601 & .59642 \\ 1200 & .04013 & .05389 & .01376 & 1.01339 & 1.61113 & .59774 \\ 1300 & .03403 & .04560 & .01157 & 1.01636 & 1.61511 & .59875 \\ 1400 & .02905 & .03884 & .00979 & 1.01869 & 1.61823 & .59954 \\ 1500 & .02491 & .03323 & .00832 & 1.02055 & 1.62072 & .60017 \\ 2000 & .01187 & .01563 & .00376 & 1.02569 & 1.62755 & .60186 \\ 2500 & .00513 & .00655 & .00142 & 1.02755 & 1.62996 & .60241 \\ 3000 & .00103 & .00101 & -.00002 & 1.02810 & 1.63064 & .60254 \\ 4000 & -.00386 & -.00560 & -.00174 & 1.02765 & 1.62992 & .60227 \\ 5000 & -.00685 & -.00966 & -.00281 & 1.02645 & 1.62820 & .60175\end{array}$




\begin{tabular}{|c|c|c|c|c|c|c|}
\hline \multirow{2}{*}{$\mathrm{T}^{\circ} \mathrm{K}$} & $\mathrm{F}_{\mathrm{BeH}}^{\circ}-\mathrm{F}_{\mathrm{BeD}}^{\circ}$ & $\mathrm{F}_{\mathrm{BeH}}^{\circ}{ }^{-\mathrm{F}_{\mathrm{BeT}}^{\circ}}$ & $\mathrm{F}_{\mathrm{BeD}}^{\circ}{ }^{-\mathrm{F}_{\mathrm{BeT}}^{\circ}}$ & \multirow{2}{*}{$\frac{\mathrm{H}_{\mathrm{BeH}}^{\circ}{ }^{-\mathrm{H}^{\circ}} \mathrm{BeD}}{\mathrm{RT}}$} & $\mathrm{H}_{\mathrm{BeH}}^{\circ}{ }^{-\mathrm{H}^{\circ}} \mathrm{BeT}$ & \multirow{2}{*}{$\frac{\mathrm{HeD}^{\circ} \mathrm{HeT}^{\circ}}{\mathrm{RT}}$} \\
\hline & $\mathrm{RT}$ & $\mathrm{RT}$ & $\mathrm{RT}$ & & $\mathrm{RT}$ & \\
\hline $\begin{array}{l}100 \\
200 \\
300 \\
400 \\
500\end{array}$ & $\begin{array}{l}4.47560 \\
2.60571 \\
1.98313 \\
1.67480 \\
1.49461\end{array}$ & $\begin{array}{l}6.50505 \\
3.84372 \\
2.95863 \\
2.52252 \\
2.26973\end{array}$ & $\begin{array}{r}2.02945 \\
1.23801 \\
.97550 \\
.84772 \\
.77512\end{array}$ & $\begin{array}{r}3.73976 \\
1.86969 \\
1.24149 \\
.91463 \\
.70585\end{array}$ & $\begin{array}{r}5.32280 \\
2.66051 \\
1.76121 \\
1.28829 \\
.98577\end{array}$ & $\begin{array}{r}1.58304 \\
.79082 \\
.51972 \\
.37366 \\
.27992\end{array}$ \\
\hline $\begin{array}{r}600 \\
700 \\
800 \\
900 \\
1000\end{array}$ & $\begin{array}{l}1.37961 \\
1.30192 \\
1.24721 \\
1.20736 \\
1.17755\end{array}$ & $\begin{array}{l}2.10972 \\
2.00241 \\
1.92728 \\
1.87283 \\
1.83224\end{array}$ & $\begin{array}{l}.73011 \\
.70049 \\
.68007 \\
.66547 \\
.65469\end{array}$ & $\begin{array}{l}.55914 \\
.45136 \\
.37013 \\
.30777 \\
.25912\end{array}$ & $\begin{array}{l}.77476 \\
.62135 \\
.50688 \\
.41974 \\
.35225\end{array}$ & $\begin{array}{l}.21562 \\
.16999 \\
.13675 \\
.11197 \\
.09313\end{array}$ \\
\hline $\begin{array}{l}1100 \\
1200 \\
1300 \\
1400 \\
1500\end{array}$ & $\begin{array}{l}1.15473 \\
1.13691 \\
1.12275 \\
1.11132 \\
1.10196\end{array}$ & $\begin{array}{l}1.80126 \\
1.77711 \\
1.75796 \\
1.74254 \\
1.72992\end{array}$ & $\begin{array}{l}.64653 \\
.64020 \\
.63522 \\
.63122 \\
.62796\end{array}$ & $\begin{array}{l}.22063 \\
.18976 \\
.16471 \\
.14415 \\
.12709\end{array}$ & $\begin{array}{l}.29916 \\
.25678 \\
.22252 \\
.19449 \\
.17131\end{array}$ & $\begin{array}{l}.07853 \\
.06702 \\
.05782 \\
.05034 \\
.04422\end{array}$ \\
\hline $\begin{array}{l}2000 \\
2500 \\
3000 \\
4000 \\
5000\end{array}$ & $\begin{array}{l}1.07367 \\
1.06021 \\
1.05279 \\
1.04528 \\
1.04168\end{array}$ & $\begin{array}{l}1.69183 \\
1.67372 \\
1.66373 \\
1.65356 \\
1.64862\end{array}$ & $\begin{array}{l}.61816 \\
.61352 \\
.61094 \\
.60828 \\
.60694\end{array}$ & $\begin{array}{l}.07412 \\
.04828 \\
.03394 \\
.01963 \\
.01313\end{array}$ & $\begin{array}{l}.09967 \\
.06496 \\
.04580 \\
.02678 \\
.01821\end{array}$ & $\begin{array}{l}.02555 \\
.01669 \\
.01186 \\
.00715 \\
.00508\end{array}$ \\
\hline
\end{tabular}

\begin{tabular}{|c|c|c|c|c|c|c|}
\hline \multirow{2}{*}{$\mathrm{T}^{\circ} \mathrm{K}$} & $\mathrm{C}_{\mathrm{BeD}}^{\circ}{ }^{-\mathrm{C}_{\mathrm{BeH}}^{\circ}}$ & $\mathrm{C}_{\mathrm{BeT}}^{\circ}-\mathrm{C}_{\mathrm{BeH}}^{\circ}$ & $\mathrm{C}_{\mathrm{BeT}}^{\circ}-\mathrm{C}_{\mathrm{BeD}}^{\circ}$ & $\mathrm{S}_{\mathrm{BeD}}^{-\mathrm{S}_{\mathrm{BeH}}^{\circ}}$ & $\mathrm{S}_{\mathrm{BeT}}^{\circ}{ }^{-\mathrm{S}_{\mathrm{BeH}}^{\circ}}$ & $\mathrm{S}_{\mathrm{BeT}^{\circ}}^{-\mathrm{S}_{\mathrm{BeD}}^{\circ}}$ \\
\hline & $\mathrm{R}$ & $\mathrm{R}$ & $\mathrm{R}$ & $\mathrm{R}$ & $\mathrm{R}$ & $\mathrm{R}$ \\
\hline $\begin{array}{l}100 \\
200 \\
300 \\
400 \\
500\end{array}$ & $\begin{array}{r}-.00031 \\
.00253 \\
.03484 \\
.09898 \\
.15605\end{array}$ & $\begin{array}{r}-.00040 \\
.00876 \\
.07794 \\
.18231 \\
.25934\end{array}$ & $\begin{array}{r}-.00009 \\
.00623 \\
.04310 \\
.08333 \\
.10329\end{array}$ & $\begin{array}{l}.73584 \\
.73602 \\
.74164 \\
.76016 \\
.78877\end{array}$ & $\begin{array}{l}1.18226 \\
1.18320 \\
1.19743 \\
1.23423 \\
1.28397\end{array}$ & $\begin{array}{l}.44642 \\
.44718 \\
.45579 \\
.47407 \\
.49520\end{array}$ \\
\hline $\begin{array}{r}600 \\
700 \\
800 \\
900 \\
1000\end{array}$ & $\begin{array}{l}.18850 \\
.19919 \\
.19601 \\
.18544 \\
.17164\end{array}$ & $\begin{array}{l}.29479 \\
.29965 \\
.28720 \\
.26668 \\
.24346\end{array}$ & $\begin{array}{l}.10629 \\
.10046 \\
.09119 \\
.08124 \\
.07182\end{array}$ & $\begin{array}{l}.82047 \\
.85056 \\
.87707 \\
.89959 \\
.91843\end{array}$ & $\begin{array}{l}1.33495 \\
1.38106 \\
1.42040 \\
1.45309 \\
1.47999\end{array}$ & $\begin{array}{l}.51448 \\
.53050 \\
.54333 \\
.55350 \\
.56156\end{array}$ \\
\hline $\begin{array}{l}1100 \\
1200 \\
1300 \\
1400 \\
1500\end{array}$ & $\begin{array}{l}.15697 \\
.14268 \\
.12936 \\
.11722 \\
.10631\end{array}$ & $\begin{array}{l}.22033 \\
.19864 \\
.17890 \\
.16124 \\
.14556\end{array}$ & $\begin{array}{l}.06336 \\
.05596 \\
.04954 \\
.04402 \\
.03925\end{array}$ & $\begin{array}{l}.93410 \\
.94714 \\
.95803 \\
.96717 \\
.97487\end{array}$ & $\begin{array}{l}1.50210 \\
1.52033 \\
1.53544 \\
1.54804 \\
1.55861\end{array}$ & $\begin{array}{l}.56800 \\
.57319 \\
.57741 \\
.58087 \\
.58374\end{array}$ \\
\hline $\begin{array}{l}2000 \\
2500 \\
3000 \\
4000 \\
5000\end{array}$ & $\begin{array}{l}.06718 \\
.04495 \\
.03151 \\
.01692 \\
.00954\end{array}$ & $\begin{array}{l}.09056 \\
.05992 \\
.04152 \\
.02160 \\
.01148\end{array}$ & $\begin{array}{l}.02338 \\
.01497 \\
.01001 \\
.00468 \\
.00194\end{array}$ & $\begin{array}{l}.99956 \\
1.01194 \\
1.01885 \\
1.02565 \\
1.02855\end{array}$ & $\begin{array}{l}1.59216 \\
1.60876 \\
1.61792 \\
1.62678 \\
1.63040\end{array}$ & $\begin{array}{l}.59260 \\
.59682 \\
.59907 \\
.60113 \\
.60185\end{array}$ \\
\hline
\end{tabular}


Table 3.12

\begin{tabular}{|c|c|c|c|c|c|c|}
\hline \multirow{2}{*}{$\mathrm{T}^{\circ} \mathrm{K}$} & $\mathrm{F}_{\mathrm{M} \sigma \mathrm{H}}^{\circ}-\mathrm{F}_{\mathrm{MgD}}^{\circ}$ & $\mathrm{F}_{\mathrm{MgH}}^{\circ}-\mathrm{F}_{\mathrm{MgT}}^{\circ}$ & $\mathrm{F}_{\mathrm{MgD}}^{\circ}-\mathrm{F}_{\mathrm{MgT}}^{\circ}$ & $\mathrm{H}_{\mathrm{MgH}}^{\circ}{ }^{-\mathrm{H}_{\mathrm{MgD}}^{\circ}}$ & $\mathrm{H}_{\mathrm{MgH}}^{\circ}-\mathrm{H}_{\mathrm{MgT}}^{\circ}$ & $\mathrm{H}_{\mathrm{MgD}}^{\circ}{ }^{-\mathrm{H}^{\circ}} \mathrm{MgT}$ \\
\hline & $\mathrm{RT}$ & $\mathrm{RT}$ & $\mathrm{RT}$ & RT & $\mathrm{RT}$ & $\mathrm{RT}$ \\
\hline $\begin{array}{l}100 \\
200 \\
300 \\
400 \\
500\end{array}$ & $\begin{array}{l}3.64353 \\
2.17550 \\
1.69120 \\
1.45884 \\
1.32896\end{array}$ & $\begin{array}{l}5.34462 \\
3.23843 \\
2.54832 \\
2.22154 \\
2.04114\end{array}$ & $\begin{array}{r}1.70109 \\
1.06293 \\
.85712 \\
.76270 \\
.71218\end{array}$ & $\begin{array}{r}2.93707 \\
1.46475 \\
.95207 \\
.67321 \\
.49664\end{array}$ & $\begin{array}{r}4.21601 \\
2.09651 \\
1.34712 \\
.93992 \\
.68596\end{array}$ & $\begin{array}{r}1.27894 \\
.63176 \\
.39505 \\
.26671 \\
.18932\end{array}$ \\
\hline $\begin{array}{r}600 \\
700 \\
800 \\
900 \\
1000\end{array}$ & $\begin{array}{l}1.24958 \\
1.19789 \\
1.16254 \\
1.13741 \\
1.11894\end{array}$ & $\begin{array}{l}1.93192 \\
1.86128 \\
1.81321 \\
1.77916 \\
1.75420\end{array}$ & $\begin{array}{l}.68234 \\
.66339 \\
.65068 \\
.64175 \\
.63526\end{array}$ & $\begin{array}{l}.37798 \\
.29528 \\
.23593 \\
.19221 \\
.15925\end{array}$ & $\begin{array}{l}.51797 \\
.40235 \\
.32015 \\
.26003 \\
.21495\end{array}$ & $\begin{array}{l}.13999 \\
.10707 \\
.08423 \\
.06782 \\
.05570\end{array}$ \\
\hline $\begin{array}{l}1100 \\
1200 \\
1300 \\
1400 \\
1500\end{array}$ & $\begin{array}{l}1.10500 \\
1.09424 \\
1.08577 \\
1.07897 \\
1.07345\end{array}$ & $\begin{array}{l}1.73541 \\
1.72092 \\
1.70951 \\
1.70038 \\
1.69296\end{array}$ & $\begin{array}{l}.63041 \\
.62668 \\
.62374 \\
.62141 \\
.61951\end{array}$ & $\begin{array}{l}.13388 \\
.11401 \\
.09818 \\
.08541 \\
.07495\end{array}$ & $\begin{array}{l}.18041 \\
.15343 \\
.13201 \\
.11475 \\
.10066\end{array}$ & $\begin{array}{l}.04653 \\
.03942 \\
.03383 \\
.02934 \\
.02571\end{array}$ \\
\hline $\begin{array}{l}2000 \\
2500 \\
3000 \\
4000 \\
5000\end{array}$ & $\begin{array}{l}1.05684 \\
1.04894 \\
1.04451 \\
1.03982 \\
1.03732\end{array}$ & $\begin{array}{l}1.67067 \\
1.66004 \\
1.65405 \\
1.64765 \\
1.64418\end{array}$ & $\begin{array}{l}.61383 \\
.61110 \\
.60954 \\
.60784 \\
.60687\end{array}$ & $\begin{array}{l}.04336 \\
.02854 \\
.02056 \\
.01294 \\
.00973\end{array}$ & $\begin{array}{l}.05825 \\
.03845 \\
.02785 \\
.01779 \\
.01364\end{array}$ & $\begin{array}{l}.01489 \\
.00991 \\
.00729 \\
.00486 \\
.00392\end{array}$ \\
\hline
\end{tabular}

$\mathrm{T}^{\circ} \mathrm{K}$

$\frac{\mathrm{C}_{\mathrm{MgD}}^{\circ} \mathrm{C}_{\mathrm{MgH}}^{\circ}}{\mathrm{R}} \frac{\mathrm{C}_{\mathrm{MgT}}^{\circ}{ }^{-\mathrm{C}^{\circ}} \mathrm{MgH}}{\mathrm{R}} \frac{\mathrm{C}_{\mathrm{MgT}}^{\circ} \mathrm{C}_{\mathrm{MgD}}^{\circ}}{\mathrm{R}} \frac{\mathrm{S}_{\mathrm{MgD}}^{\circ} \mathrm{S}^{\circ}}{\mathrm{RgH}} \frac{\mathrm{S}_{\mathrm{MgT}}^{\circ} \mathrm{S}_{\mathrm{MgH}}^{\circ}}{\mathrm{R}} \frac{\mathrm{S}_{\mathrm{MgT}}^{\circ} \mathrm{S}^{\circ} \mathrm{MgD}}{\mathrm{R}}$

\begin{tabular}{|c|c|c|c|c|c|c|}
\hline $\begin{array}{l}100 \\
200 \\
300 \\
400 \\
500\end{array}$ & $\begin{array}{r}-.00005 \\
.02768 \\
.12293 \\
.19502 \\
.21739\end{array}$ & $\begin{array}{l}.00041 \\
.07136 \\
.22942 \\
.31853 \\
.33194\end{array}$ & $\begin{array}{l}.00046 \\
.04368 \\
.10649 \\
.12351 \\
.11455\end{array}$ & $\begin{array}{l}.70646 \\
.71075 \\
.73913 \\
.78564 \\
.83233\end{array}$ & $\begin{array}{l}1.12861 \\
1.14193 \\
1.20121 \\
1.28163 \\
1.35518\end{array}$ & $\begin{array}{l}.42215 \\
.43118 \\
.46208 \\
.49599 \\
.52285\end{array}$ \\
\hline $\begin{array}{r}600 \\
700 \\
800 \\
900 \\
1000\end{array}$ & $\begin{array}{l}.21002 \\
.19065 \\
.16838 \\
.14711 \\
.12817\end{array}$ & $\begin{array}{l}.30854 \\
.27335 \\
.23752 \\
.20514 \\
.17719\end{array}$ & $\begin{array}{l}.09852 \\
.08270 \\
.06914 \\
.05803 \\
.04902\end{array}$ & $\begin{array}{l}.87161 \\
.90261 \\
.92662 \\
.94520 \\
.95970\end{array}$ & $\begin{array}{l}1.41396 \\
1.45893 \\
1.49307 \\
1.51913 \\
1.53926\end{array}$ & $\begin{array}{l}.54235 \\
.55632 \\
.56645 \\
.57393 \\
.57956\end{array}$ \\
\hline $\begin{array}{l}1100 \\
1200 \\
1300 \\
1400 \\
1500\end{array}$ & $\begin{array}{l}.11179 \\
.09782 \\
.08595 \\
.07583 \\
.06720\end{array}$ & $\begin{array}{l}.15354 \\
.13364 \\
.11691 \\
.10277 \\
.09078\end{array}$ & $\begin{array}{l}.04175 \\
.03582 \\
.03096 \\
.02694 \\
.02358\end{array}$ & $\begin{array}{l}.97112 \\
.98023 \\
.98758 \\
.99357 \\
.99850\end{array}$ & $\begin{array}{l}1.55501 \\
1.56748 \\
1.57750 \\
1.58563 \\
1.59230\end{array}$ & $\begin{array}{l}.58389 \\
.58725 \\
.58992 \\
.59206 \\
.59380\end{array}$ \\
\hline $\begin{array}{l}2000 \\
2500 \\
3000 \\
4000 \\
5000\end{array}$ & $\begin{array}{l}.03888 \\
.02402 \\
.01529 \\
.00580 \\
.00087\end{array}$ & $\begin{array}{r}.05182 \\
.03156 \\
.01966 \\
.00669 \\
-.00014\end{array}$ & $\begin{array}{r}.01294 \\
.00754 \\
.00437 \\
.00089 \\
-.00101\end{array}$ & $\begin{array}{l}1.01349 \\
1.02041 \\
1.02395 \\
1.02688 \\
1.02760\end{array}$ & $\begin{array}{l}1.61242 \\
1.62159 \\
1.62621 \\
1.62985 \\
1.63055\end{array}$ & $\begin{array}{l}.59893 \\
.60118 \\
.60226 \\
.60297 \\
.60295\end{array}$ \\
\hline
\end{tabular}




\begin{tabular}{|c|c|c|c|c|c|c|}
\hline \multirow{2}{*}{$\mathrm{T}^{\circ} \mathrm{K}$} & $\mathrm{F}_{\mathrm{CaH}}^{\circ}-\mathrm{F}_{\mathrm{CaD}}^{\circ}$ & $\mathrm{F}_{\mathrm{CaH}}^{\circ}{ }^{-\mathrm{F}_{\mathrm{CaT}}^{\circ}}$ & $\mathrm{F}_{\mathrm{CaD}}^{\circ}{ }^{-\mathrm{F}_{\mathrm{CaT}}^{\circ}}$ & $\mathrm{H}_{\mathrm{CaH}}^{\circ}{ }^{-} \mathrm{H}_{\mathrm{CaD}}^{\circ}$ & $\mathrm{H}_{\mathrm{CaH}}^{\circ}{ }^{-\mathrm{H}^{\circ}} \mathrm{CaT}$ & $\mathrm{H}_{\mathrm{CaD}}^{\circ}{ }^{-\mathrm{H}^{\circ}} \mathrm{CaT}$ \\
\hline & $\mathrm{RT}$ & $\mathrm{RT}$ & $\mathrm{RT}$ & RT & $\mathrm{RT}$ & $\mathrm{RT}$ \\
\hline $\begin{array}{l}100 \\
200 \\
300 \\
400 \\
500\end{array}$ & $\begin{array}{l}3.30893 \\
2.00572 \\
1.58013 \\
1.38039 \\
1.27128\end{array}$ & $\begin{array}{l}4.86844 \\
2.99635 \\
2.39167 \\
2.11240 \\
1.96179\end{array}$ & $\begin{array}{r}1.55851 \\
.99013 \\
.81121 \\
.73176 \\
.69031\end{array}$ & $\begin{array}{r}2.60907 \\
1.29606 \\
.82713 \\
.57113 \\
.41272\end{array}$ & $\begin{array}{r}3.75258 \\
1.85324 \\
1.16439 \\
.79239 \\
.56663\end{array}$ & $\begin{array}{r}1.14251 \\
.55668 \\
.33693 \\
.22101 \\
.15371\end{array}$ \\
\hline $\begin{array}{r}600 \\
700 \\
800 \\
900 \\
1000\end{array}$ & $\begin{array}{l}1.20581 \\
1.16378 \\
1.13535 \\
1.11527 \\
1.10061\end{array}$ & $\begin{array}{l}1.87223 \\
1.81509 \\
1.77659 \\
1.74949 \\
1.72976\end{array}$ & $\begin{array}{l}.66625 \\
.65116 \\
.64112 \\
.63411 \\
.62905\end{array}$ & $\begin{array}{l}.30915 \\
.23866 \\
.18901 \\
.15295 \\
.12609\end{array}$ & $\begin{array}{l}.42138 \\
.32366 \\
.25538 \\
.20611 \\
.16957\end{array}$ & $\begin{array}{l}.11206 \\
.08485 \\
.06625 \\
.05305 \\
.04338\end{array}$ \\
\hline $\begin{array}{l}1100 \\
1200 \\
1300 \\
1400 \\
1500\end{array}$ & $\begin{array}{l}1.08960 \\
1.08112 \\
1.07446 \\
1.06914 \\
1.06481\end{array}$ & $\begin{array}{l}1.71496 \\
1.70359 \\
1.69467 \\
1.68754 \\
1.68176\end{array}$ & $\begin{array}{l}.62527 \\
.62239 \\
.62013 \\
.61833 \\
.61688\end{array}$ & $\begin{array}{l}.10560 \\
.08966 \\
.07704 \\
.06691 \\
.05864\end{array}$ & $\begin{array}{l}.14178 \\
.12022 \\
.10319 \\
.08954 \\
.07842\end{array}$ & $\begin{array}{l}.03609 \\
.03048 \\
.02607 \\
.02256 \\
.01971\end{array}$ \\
\hline $\begin{array}{l}2000 \\
2500 \\
3000 \\
4000 \\
5000\end{array}$ & $\begin{array}{l}1.05183 \\
1.04564 \\
1.04215 \\
1.03840 \\
1.03634\end{array}$ & $\begin{array}{l}1.66441 \\
1.65618 \\
1.65153 \\
1.64655 \\
1.64383\end{array}$ & $\begin{array}{l}.61253 \\
.61050 \\
.60935 \\
.60813 \\
.60747\end{array}$ & $\begin{array}{l}.03389 \\
.02241 \\
.01628 \\
.01050 \\
.00816\end{array}$ & $\begin{array}{l}.04519 \\
.02982 \\
.02162 \\
.01390 \\
.01076\end{array}$ & $\begin{array}{l}.01125 \\
.00737 \\
.00531 \\
.00338 \\
.00258\end{array}$ \\
\hline
\end{tabular}

\begin{tabular}{|c|c|c|c|c|c|c|}
\hline \multirow{2}{*}{$\mathrm{T}^{\circ} \mathrm{K}$} & $\mathrm{C}_{\mathrm{CaD}}^{\circ}{ }^{-\mathrm{C}_{\mathrm{CaH}}^{\circ}}$ & $\mathrm{C}_{\mathrm{CaT}}^{\circ}{ }^{-\mathrm{C}_{\mathrm{CaH}}^{\circ}}$ & $\mathrm{C}_{\mathrm{CaT}}^{\circ}{ }^{-} \mathrm{C}_{\mathrm{CaD}}^{\circ}$ & $\mathrm{S}_{\mathrm{CaD}}^{\circ}{ }^{-} \mathrm{S}_{\mathrm{CaH}}^{\circ}$ & $\mathrm{S}_{\mathrm{CaT}}^{\circ}{ }^{-\mathrm{S}_{\mathrm{CaH}}^{\circ}}$ & $\mathrm{S}_{\mathrm{CaT}}^{\circ}{ }^{-\mathrm{S}_{\mathrm{CaD}}^{\circ}}$ \\
\hline & $R$ & $\mathrm{R}$ & $\mathrm{R}$ & $R$ & $\mathrm{R}$ & $R$ \\
\hline $\begin{array}{l}100 \\
200 \\
300 \\
400 \\
500\end{array}$ & $\begin{array}{l}.00029 \\
.05303 \\
.16450 \\
.21769 \\
.21833\end{array}$ & $\begin{array}{l}.00221 \\
.12255 \\
.28845 \\
.34162 \\
.32430\end{array}$ & $\begin{array}{l}.00192 \\
.06952 \\
.12395 \\
.12393 \\
.10597\end{array}$ & $\begin{array}{l}.69986 \\
.70966 \\
.75299 \\
.80927 \\
.85856\end{array}$ & $\begin{array}{l}1.11587 \\
1.14311 \\
1.22728 \\
1.32002 \\
1.39515\end{array}$ & $\begin{array}{l}.41601 \\
.43345 \\
.47429 \\
.51075 \\
.53659\end{array}$ \\
\hline $\begin{array}{r}600 \\
700 \\
800 \\
900 \\
1000\end{array}$ & $\begin{array}{l}.19727 \\
.17117 \\
.14645 \\
.12500 \\
.10699\end{array}$ & $\begin{array}{l}.28405 \\
.24177 \\
.20424 \\
.17280 \\
.14696\end{array}$ & $\begin{array}{l}.08678 \\
.07060 \\
.05779 \\
.04780 \\
.03997\end{array}$ & $\begin{array}{l}.89667 \\
.92513 \\
.94634 \\
.96231 \\
.97453\end{array}$ & $\begin{array}{l}1.45086 \\
1.49143 \\
1.52120 \\
1.54338 \\
1.56020\end{array}$ & $\begin{array}{l}.55419 \\
.56630 \\
.57486 \\
.58107 \\
.58567\end{array}$ \\
\hline $\begin{array}{l}1100 \\
1200 \\
1300 \\
1400 \\
1500\end{array}$ & $\begin{array}{l}.09204 \\
.07965 \\
.06934 \\
.06072 \\
.05344\end{array}$ & $\begin{array}{l}.12583 \\
.10850 \\
.09419 \\
.08230 \\
.07232\end{array}$ & $\begin{array}{l}.03379 \\
.02885 \\
.02485 \\
.02158 \\
.01888\end{array}$ & $\begin{array}{r}.98399 \\
.99146 \\
.99742 \\
1.00223 \\
1.00616\end{array}$ & $\begin{array}{l}1.57318 \\
1.58336 \\
1.59147 \\
1.59800 \\
1.60333\end{array}$ & $\begin{array}{l}.58919 \\
.59190 \\
.59405 \\
.59577 \\
.59717\end{array}$ \\
\hline $\begin{array}{l}2000 \\
2500 \\
3000 \\
4000 \\
5000\end{array}$ & $\begin{array}{r}.03012 \\
.01814 \\
.01112 \\
.00343 \\
-.00070\end{array}$ & $\begin{array}{r}.04058 \\
.02443 \\
.01502 \\
.00476 \\
-.00073\end{array}$ & $\begin{array}{r}.01046 \\
.00629 \\
.00390 \\
.00133 \\
-.00003\end{array}$ & $\begin{array}{l}1.01793 \\
1.02324 \\
1.02588 \\
1.02790 \\
1.02818\end{array}$ & $\begin{array}{l}1.61922 \\
1.62636 \\
1.62992 \\
1.63266 \\
1.63308\end{array}$ & $\begin{array}{l}.60129 \\
.60312 \\
.60404 \\
.60476 \\
.60490\end{array}$ \\
\hline
\end{tabular}


Table 3.14

\begin{tabular}{|c|c|c|c|c|c|c|}
\hline \multirow{2}{*}{$\mathrm{T}^{\circ} \mathrm{K}$} & $\mathrm{F}_{\mathrm{SrH}}^{\circ}-\mathrm{F}_{\mathrm{SrD}}^{\circ}$ & $\mathrm{F}_{\mathrm{SrH}}^{\circ}{ }^{-\mathrm{F}^{\circ} \mathrm{SrT}}$ & $\mathrm{F}_{\mathrm{SrD}}^{\circ}-\mathrm{F}_{\mathrm{Sr} T}^{\circ}$ & $\mathrm{H}_{\mathrm{SrH}}^{\circ}{ }^{-\mathrm{H}^{\circ}} \mathrm{SrD}$ & $\mathrm{H}_{\mathrm{SrH}}^{\circ}{ }^{-\mathrm{H}_{\mathrm{SrT}}^{\circ}}$ & $\mathrm{H}_{\mathrm{SrD}}^{\circ}{ }^{-\mathrm{H}_{\mathrm{SrT}}^{\circ}}$ \\
\hline & $\mathrm{RT}$ & $\mathrm{RT}$ & $\mathrm{RT}$ & $\mathrm{RT}$ & $\mathrm{RT}$ & $\mathrm{RT}$ \\
\hline 100 & 3.15940 & 4.65647 & 1.49607 & 2.46532 & 3.55304 & 1.08672 \\
\hline 200 & 1.92889 & 2.88639 & .95700 & 1.22003 & 1.74391 & .52338 \\
\hline 300 & 1.53030 & 2.32089 & .79026 & .76855 & 1.07924 & .31036 \\
\hline 400 & 1.34591 & 2.06376 & .71760 & .52325 & .72401 & .20051 \\
\hline 500 & 1.24647 & 1.92684 & .68017 & .37396 & .51225 & .13809 \\
\hline 600 & 1.18739 & 1.84618 & .65862 & .27787 & .37810 & .10006 \\
\hline 700 & 1.14972 & 1.79504 & .64517 & .21329 & .28888 & .07544 \\
\hline 800 & 1.12436 & 1.76074 & .63625 & .16820 & .22708 & .05875 \\
\hline 900 & 1.10652 & 1.73668 & .63005 & .13569 & .18274 & .04694 \\
\hline 1000 & 1.09353 & 1.71921 & .62558 & .11160 & .15002 & .03832 \\
\hline 1100 & 1.08379 & 1.70612 & .62224 & .09329 & .12523 & .03185 \\
\hline 1200 & 1.07631 & 1.69608 & .61969 & .07911 & .10605 & .02686 \\
\hline 1300 & 1.07044 & 1.68822 & .61771 & .06790 & .09093 & .02296 \\
\hline 1400 & 1.06574 & 1.68193 & .61612 & .05891 & .07882 & .01984 \\
\hline 1500 & 1.06193 & 1.67685 & .61485 & .05161 & .06900 & .01732 \\
\hline 2000 & 1.05053 & 1.66162 & .61104 & .02978 & .03965 & .00982 \\
\hline 2500 & 1.04509 & 1.65440 & .60927 & .01968 & .02608 & .00636 \\
\hline 3000 & 1.04203 & 1.65034 & .60828 & .01430 & .01885 & .00452 \\
\hline 4000 & 1.03872 & 1.64602 & .60727 & .00925 & .01202 & .00274 \\
\hline 5000 & 1.03691 & 1.64368 & .60675 & .00721 & .00921 & .00198 \\
\hline
\end{tabular}

\begin{tabular}{|c|c|c|c|c|c|c|}
\hline$T^{\circ} \mathrm{K}$ & $\mathrm{C}_{\mathrm{SrD}}^{\circ}-\mathrm{C}_{\mathrm{SrH}}^{\circ}$ & $\mathrm{C}_{\mathrm{SrT}}^{\circ}{ }^{-\mathrm{C}_{\mathrm{SrH}}^{\circ}}$ & $\mathrm{C}_{\mathrm{SrD}}^{\circ}-\mathrm{C}_{\mathrm{SrT}}^{\circ}$ & $\mathrm{S}_{\mathrm{SrD}}^{\circ}-\mathrm{S}_{\mathrm{SrH}}^{\circ}$ & $\mathrm{S}_{\mathrm{SrT}}^{\circ}{ }^{-\mathrm{S}_{\mathrm{SrH}}^{\circ}}$ & $\mathrm{S}_{\mathrm{SrT}}^{\circ}{ }^{-\mathrm{S}_{\mathrm{SrD}}^{\circ}}$ \\
\hline $1 \mathrm{~K}$ & $\mathrm{R}$ & $\mathrm{R}$ & $\mathrm{R}$ & $\mathrm{R}$ & $\mathrm{R}$ & $\mathrm{R}$ \\
\hline $\begin{array}{l}100 \\
200 \\
300 \\
400 \\
500\end{array}$ & $\begin{array}{l}.00076 \\
.07242 \\
.18706 \\
.22614 \\
.21545\end{array}$ & $\begin{array}{l}.00462 \\
.15875 \\
.31848 \\
.34834 \\
.31598\end{array}$ & $\begin{array}{l}.00386 \\
.08633 \\
.13142 \\
.12220 \\
.10053\end{array}$ & $\begin{array}{l}.69409 \\
.70886 \\
.76176 \\
.82266 \\
.87251\end{array}$ & $\begin{array}{l}1.10344 \\
1.14248 \\
1.24165 \\
1.33976 \\
1.41458\end{array}$ & $\begin{array}{l}.40935 \\
.43362 \\
.47989 \\
.51710 \\
.54207\end{array}$ \\
\hline $\begin{array}{r}600 \\
700 \\
800 \\
900 \\
1000\end{array}$ & $\begin{array}{l}.18860 \\
.16027 \\
.13516 \\
.11418 \\
.09698\end{array}$ & $\begin{array}{l}.26908 \\
.22482 \\
.18752 \\
.15721 \\
.13282\end{array}$ & $\begin{array}{l}.08048 \\
.06455 \\
.05236 \\
.04303 \\
.03584\end{array}$ & $\begin{array}{l}.90952 \\
.93644 \\
.95615 \\
.97083 \\
.98193\end{array}$ & $\begin{array}{l}1.46808 \\
1.50617 \\
1.53366 \\
1.55394 \\
1.56919\end{array}$ & $\begin{array}{l}.55856 \\
.56973 \\
.57751 \\
.58311 \\
.58726\end{array}$ \\
\hline $\begin{array}{l}1100 \\
1200 \\
1300 \\
1400 \\
1500\end{array}$ & $\begin{array}{l}.08294 \\
.07144 \\
.06197 \\
.05410 \\
.04750\end{array}$ & $\begin{array}{l}.11316 \\
.09720 \\
.08415 \\
.07336 \\
.06436\end{array}$ & $\begin{array}{l}.03022 \\
.02576 \\
.02218 \\
.01926 \\
.01686\end{array}$ & $\begin{array}{r}.99050 \\
.99720 \\
1.00253 \\
1.00683 \\
1.01033\end{array}$ & $\begin{array}{l}1.58090 \\
1.59004 \\
1.59728 \\
1.60311 \\
1.60785\end{array}$ & $\begin{array}{l}.59040 \\
.59284 \\
.59475 \\
.59628 \\
.59752\end{array}$ \\
\hline $\begin{array}{l}2000 \\
2500 \\
3000 \\
4000 \\
5000\end{array}$ & $\begin{array}{r}.02656 \\
.01591 \\
.00971 \\
.00294 \\
-.00069\end{array}$ & $\begin{array}{r}.03601 \\
.02175 \\
.01350 \\
.00459 \\
-.00012\end{array}$ & $\begin{array}{l}.00945 \\
.00584 \\
.00379 \\
.00165 \\
.00057\end{array}$ & $\begin{array}{l}1.02075 \\
1.02541 \\
1.02772 \\
1.02947 \\
1.02970\end{array}$ & $\begin{array}{l}1.62198 \\
1.62831 \\
1.63149 \\
1.63400 \\
1.63447\end{array}$ & $\begin{array}{l}.60123 \\
.60290 \\
.60377 \\
.60453 \\
.60477\end{array}$ \\
\hline
\end{tabular}


Table 3.15

\begin{tabular}{|c|c|c|c|c|c|c|}
\hline \multirow{2}{*}{$\mathrm{T}^{\circ} \mathrm{K}$} & $\mathrm{F}_{\mathrm{BaH}}^{\circ}-\mathrm{F}_{\mathrm{BaD}}^{\circ}$ & $\mathrm{F}_{\mathrm{BaH}}^{\circ}-\mathrm{F}_{\mathrm{BaT}}^{\circ}$ & $\mathrm{F}_{\mathrm{BaD}}^{\circ}-\mathrm{F}_{\mathrm{BaT}}^{\circ}$ & $\mathrm{H}_{\mathrm{BaH}}^{\circ}-\mathrm{H}_{\mathrm{BaD}}^{\circ}$ & $\mathrm{H}_{\mathrm{BaH}}^{\circ}-\mathrm{H}_{\mathrm{BaT}}^{\circ}$ & $\mathrm{H}_{\mathrm{BaD}}^{\circ}{ }^{-\mathrm{H}_{\mathrm{BaT}}^{\circ}}$ \\
\hline & $\mathrm{RT}$ & $\mathrm{RT}$ & $\mathrm{RT}$ & $\mathrm{RT}$ & $\mathrm{RT}$ & $\mathrm{RT}$ \\
\hline $\begin{array}{l}100 \\
200 \\
300 \\
400 \\
500\end{array}$ & $\begin{array}{l}3.10204 \\
1.90011 \\
1.51220 \\
1.33376 \\
1.23800\end{array}$ & $\begin{array}{l}4.57394 \\
2.84456 \\
2.29476 \\
2.04632 \\
1.91468\end{array}$ & $\begin{array}{r}1.47290 \\
.94495 \\
.78289 \\
.71281 \\
.67688\end{array}$ & $\begin{array}{r}2.40892 \\
1.18994 \\
.74556 \\
.50489 \\
.35941\end{array}$ & $\begin{array}{r}3.47373 \\
1.69999 \\
1.04546 \\
.69744 \\
.49150\end{array}$ & $\begin{array}{r}1.06581 \\
.51055 \\
.30023 \\
.19280 \\
.13229\end{array}$ \\
\hline $\begin{array}{r}600 \\
700 \\
800 \\
900 \\
1000\end{array}$ & $\begin{array}{l}1.18128 \\
1.14522 \\
1.12096 \\
1.10393 \\
1.09152\end{array}$ & $\begin{array}{l}1.83738 \\
1.78850 \\
1.75576 \\
1.73283 \\
1.71618\end{array}$ & $\begin{array}{l}.65627 \\
.64342 \\
.63493 \\
.62902 \\
.62476\end{array}$ & $\begin{array}{l}.26634 \\
.20406 \\
.16074 \\
.12958 \\
.10652\end{array}$ & $\begin{array}{l}.36177 \\
.27588 \\
.21658 \\
.17414 \\
.14287\end{array}$ & $\begin{array}{l}.09560 \\
.07196 \\
.05597 \\
.04468 \\
.03645\end{array}$ \\
\hline $\begin{array}{l}1100 \\
1200 \\
1300 \\
1400 \\
1500\end{array}$ & $\begin{array}{l}1.08223 \\
1.07509 \\
1.06948 \\
1.06500 \\
1.06136\end{array}$ & $\begin{array}{l}1.70373 \\
1.69418 \\
1.68668 \\
1.68071 \\
1.67585\end{array}$ & $\begin{array}{l}.62159 \\
.61917 \\
.61727 \\
.61578 \\
.61456\end{array}$ & $\begin{array}{l}.08904 \\
.07549 \\
.06481 \\
.05625 \\
.04929\end{array}$ & $\begin{array}{l}.11922 \\
.10094 \\
.08655 \\
.07503 \\
.06568\end{array}$ & $\begin{array}{l}.03027 \\
.02553 \\
.02181 \\
.01885 \\
.01646\end{array}$ \\
\hline $\begin{array}{l}2000 \\
2500 \\
3000 \\
4000 \\
5000\end{array}$ & $\begin{array}{l}1.05044 \\
1.04523 \\
1.04226 \\
1.03900 \\
1.03718\end{array}$ & $\begin{array}{l}1.66134 \\
1.65443 \\
1.65054 \\
1.64630 \\
1.64396\end{array}$ & $\begin{array}{l}.61095 \\
.60924 \\
.60831 \\
.60733 \\
.60680\end{array}$ & $\begin{array}{l}.02855 \\
.01900 \\
.01393 \\
.00922 \\
.00736\end{array}$ & $\begin{array}{l}.03785 \\
.02504 \\
.01825 \\
.01189 \\
.00935\end{array}$ & $\begin{array}{l}.00935 \\
.00608 \\
.00435 \\
.00270 \\
.00201\end{array}$ \\
\hline
\end{tabular}

\begin{tabular}{|c|c|c|c|c|c|c|}
\hline \multirow{2}{*}{$\mathrm{T}^{\circ} \mathrm{K}$} & $\mathrm{C}_{\mathrm{BaD}}^{\circ}-\mathrm{C}_{\mathrm{BaH}}^{\circ}$ & $\mathrm{C}_{\mathrm{BaT}}^{\circ}-\mathrm{C}_{\mathrm{BaH}}^{\circ}$ & $\mathrm{C}_{\mathrm{BaT}}^{\circ}-\mathrm{C}_{\mathrm{BaD}}^{\circ}$ & $\mathrm{S}_{\mathrm{BaD}}^{\circ}{ }^{-\mathrm{S}^{\circ} \mathrm{BaH}}$ & $\mathrm{S}_{\mathrm{BaT}}^{\circ}{ }^{-\mathrm{S}_{\mathrm{BaH}}^{\circ}}$ & $\mathrm{S}_{\mathrm{BaT}}^{\circ}-\mathrm{S}_{\mathrm{BaD}}^{\circ}$ \\
\hline & $\mathrm{R}$ & $\mathrm{R}$ & $\mathrm{R}$ & $\mathrm{R}$ & $\mathrm{R}$ & $\mathrm{R}$ \\
\hline $\begin{array}{l}100 \\
200 \\
300 \\
400 \\
500\end{array}$ & $\begin{array}{l}.00106 \\
.08049 \\
.19455 \\
.22765 \\
.21296\end{array}$ & $\begin{array}{l}.00599 \\
.17347 \\
.32827 \\
.34883 \\
.31128\end{array}$ & $\begin{array}{l}.00493 \\
.09298 \\
.13372 \\
.12118 \\
.09832\end{array}$ & $\begin{array}{l}.69312 \\
.71017 \\
.76665 \\
.82887 \\
.87858\end{array}$ & $\begin{array}{l}1.10022 \\
1.14458 \\
1.24930 \\
1.34887 \\
1.42317\end{array}$ & $\begin{array}{l}.40710 \\
.43441 \\
.48265 \\
.52000 \\
.54459\end{array}$ \\
\hline $\begin{array}{r}600 \\
700 \\
800 \\
900 \\
1000\end{array}$ & $\begin{array}{l}.18431 \\
.15545 \\
.13040 \\
.10971 \\
.09289\end{array}$ & $\begin{array}{l}.26239 \\
.21775 \\
.18074 \\
.15097 \\
.12719\end{array}$ & $\begin{array}{l}.07808 \\
.06230 \\
.05034 \\
.04126 \\
.03430\end{array}$ & $\begin{array}{l}.91494 \\
.94115 \\
.96022 \\
.97434 \\
.98501\end{array}$ & $\begin{array}{l}1.47560 \\
1.51261 \\
1.53918 \\
1.55869 \\
1.57332\end{array}$ & $\begin{array}{l}.56066 \\
.57146 \\
.57896 \\
.58435 \\
.58831\end{array}$ \\
\hline $\begin{array}{l}1100 \\
1200 \\
1300 \\
1400 \\
1500\end{array}$ & $\begin{array}{l}.07923 \\
.06809 \\
.05893 \\
.05134 \\
.04499\end{array}$ & $\begin{array}{l}.10810 \\
.09265 \\
.08005 \\
.06965 \\
.06099\end{array}$ & $\begin{array}{l}.02887 \\
.02456 \\
.02112 \\
.01831 \\
.01600\end{array}$ & $\begin{array}{r}.99319 \\
.99959 \\
1.00467 \\
1.00875 \\
1.01207\end{array}$ & $\begin{array}{l}1.58451 \\
1.59323 \\
1.60014 \\
1.60567 \\
1.61017\end{array}$ & $\begin{array}{l}.59132 \\
.59364 \\
.59547 \\
.59692 \\
.59810\end{array}$ \\
\hline $\begin{array}{l}2000 \\
2500 \\
3000 \\
4000 \\
5000\end{array}$ & $\begin{array}{r}.02485 \\
.01461 \\
.00862 \\
.00202 \\
-.00158\end{array}$ & $\begin{array}{r}.03375 \\
.02003 \\
.01206 \\
.00335 \\
-.00133\end{array}$ & $\begin{array}{l}.00890 \\
.00542 \\
.00344 \\
.00133 \\
.00025\end{array}$ & $\begin{array}{l}1.02189 \\
1.02623 \\
1.02832 \\
1.02978 \\
1.02981\end{array}$ & $\begin{array}{l}1.62349 \\
1.62940 \\
1.63228 \\
1.63441 \\
1.63461\end{array}$ & $\begin{array}{l}.60160 \\
.60317 \\
.60396 \\
.60463 \\
.60480\end{array}$ \\
\hline
\end{tabular}


Table 3.16

\begin{tabular}{|c|c|c|c|c|c|c|}
\hline \multirow{2}{*}{$\mathrm{T}^{\circ} \mathrm{K}$} & $\mathrm{F}_{\mathrm{CuH}}^{\circ}{ }^{-\mathrm{F}^{\circ} \mathrm{CuD}}$ & $\mathrm{F}^{\circ}{ }^{-\mathrm{F}^{\circ}} \mathrm{CuT}$ & $\mathrm{F}_{\mathrm{CuD}}^{\circ}{ }^{-\mathrm{F}_{\mathrm{C} T}^{\circ}}$ & $\mathrm{H}_{\mathrm{CuH}}^{\circ}{ }^{-\mathrm{H}^{\circ}} \mathrm{CuD}$ & $\mathrm{H}_{\mathrm{CuH}}^{\circ}{ }^{-\mathrm{H}^{\circ}} \mathrm{CuT}$ & $\mathrm{H}_{\mathrm{CuD}}^{\circ}{ }^{-\mathrm{H}^{\circ}} \mathrm{CuT}$ \\
\hline & $\mathrm{RT}$ & $\mathrm{RT}$ & $\mathrm{RT}^{\prime}$ & RT & $\mathrm{RT}$ & $\mathrm{RT}$ \\
\hline $\begin{array}{l}100 \\
200 \\
300 \\
400 \\
500\end{array}$ & $\begin{array}{l}4.61101 \\
2.65287 \\
2.00156 \\
1.68058 \\
1.49463\end{array}$ & $\begin{array}{l}6.75604 \\
3.93077 \\
2.99332 \\
2.53512 \\
2.27259\end{array}$ & $\begin{array}{r}2.14503 \\
1.27790 \\
.99176 \\
.85454 \\
.77796\end{array}$ & $\begin{array}{r}3.91636 \\
1.95760 \\
1.29627 \\
.94804 \\
.72474\end{array}$ & $\begin{array}{l}5.65117 \\
2.82293 \\
1.85873 \\
1.34532 \\
1.01730\end{array}$ & $\begin{array}{r}1.73481 \\
.86533 \\
.56246 \\
.39728 \\
.29256\end{array}$ \\
\hline $\begin{array}{r}600 \\
700 \\
800 \\
900 \\
1000\end{array}$ & $\begin{array}{l}1.37706 \\
1.29833 \\
1.24329 \\
1.20347 \\
1.17381\end{array}$ & $\begin{array}{l}2.10830 \\
1.99919 \\
1.92341 \\
1.86884 \\
1.82836\end{array}$ & $\begin{array}{l}.73124 \\
.70085 \\
.68012 \\
.66537 \\
.65455\end{array}$ & $\begin{array}{l}.56881 \\
.45548 \\
.37102 \\
.30681 \\
.25716\end{array}$ & $\begin{array}{l}.79110 \\
.62889 \\
.50942 \\
.41946 \\
.35044\end{array}$ & $\begin{array}{l}.22229 \\
.17340 \\
.13840 \\
.11265 \\
.09328\end{array}$ \\
\hline $\begin{array}{l}1100 \\
1200 \\
1300 \\
1400 \\
1500\end{array}$ & $\begin{array}{l}1.15121 \\
1.13361 \\
1.11967 \\
1.10845 \\
1.09929\end{array}$ & $\begin{array}{l}1.79760 \\
1.77370 \\
1.75480 \\
1.73960 \\
1.72721\end{array}$ & $\begin{array}{l}.64639 \\
.64009 \\
.63513 \\
.63116 \\
.62792\end{array}$ & $\begin{array}{r}.21814 \\
.18704 \\
.16192 \\
.14137 \\
.12440\end{array}$ & $\begin{array}{l}.29652 \\
.25377 \\
.21938 \\
.19135 \\
.16827\end{array}$ & $\begin{array}{l}.07838 \\
.06673 \\
.05746 \\
.04999 \\
.04387\end{array}$ \\
\hline $\begin{array}{l}2000 \\
2500 \\
3000 \\
4000 \\
5000\end{array}$ & $\begin{array}{l}1.07168 \\
1.05863 \\
1.05148 \\
1.04429 \\
1.04088\end{array}$ & $\begin{array}{l}1.68987 \\
1.67220 \\
1.66246 \\
1.65252 \\
1.64765\end{array}$ & $\begin{array}{l}.61819 \\
.61357 \\
.61098 \\
.60823 \\
.60677\end{array}$ & $\begin{array}{l}.07200 \\
.04665 \\
.03265 \\
.01870 \\
.01236\end{array}$ & $\begin{array}{l}.09739 \\
.06337 \\
.04469 \\
.02626 \\
.01803\end{array}$ & $\begin{array}{l}.02539 \\
.01672 \\
.01204 \\
.00756 \\
.00567\end{array}$ \\
\hline
\end{tabular}

\begin{tabular}{|c|c|c|c|c|c|c|}
\hline \multirow{2}{*}{$\mathrm{T}^{\circ} \mathrm{K}$} & $\mathrm{C}_{\mathrm{CuD}}^{\circ}{ }^{-\mathrm{C}_{\mathrm{CuH}}^{\circ}}$ & $\mathrm{C}_{\mathrm{CuT}}^{\circ}-\mathrm{C}_{\mathrm{CuH}}^{\circ}$ & $\mathrm{C}_{\mathrm{CuT}}^{\circ}{ }^{-\mathrm{C}_{\mathrm{CuD}}^{\circ}}$ & \multirow{2}{*}{$\frac{\mathrm{SuD}^{\circ}{ }^{-\mathrm{S}^{\circ} \mathrm{CuH}}}{\mathrm{R}}$} & $\mathrm{S}_{\mathrm{CuT}}{ }^{-\mathrm{S}^{\circ} \mathrm{CuH}}$ & $\mathrm{S}_{\mathrm{CuT}}^{\circ}{ }^{-\mathrm{S}_{\mathrm{CuD}}^{\circ}}$ \\
\hline & $\mathrm{R}$ & $\mathrm{R}$ & $\bar{R}$ & & $\mathrm{R}$ & $\mathrm{R}$ \\
\hline $\begin{array}{l}100 \\
200 \\
300 \\
400 \\
500\end{array}$ & $\begin{array}{r}-.00002 \\
.00568 \\
.05656 \\
.13634 \\
.19514\end{array}$ & $\begin{array}{r}-.00004 \\
.02138 \\
.13010 \\
.25391 \\
.32583\end{array}$ & $\begin{array}{r}-.00002 \\
.01570 \\
.07354 \\
.11757 \\
.13069\end{array}$ & $\begin{array}{l}.69464 \\
.69528 \\
.70530 \\
.73254 \\
.76988\end{array}$ & $\begin{array}{l}1.10486 \\
1.10785 \\
1.13460 \\
1.18981 \\
1.25528\end{array}$ & $\begin{array}{l}.41022 \\
.41257 \\
.42930 \\
.45727 \\
.48540\end{array}$ \\
\hline $\begin{array}{r}600 \\
700 \\
800 \\
900 \\
1000\end{array}$ & $\begin{array}{l}.22155 \\
.22452 \\
.21449 \\
.19858 \\
.18085\end{array}$ & $\begin{array}{l}.34721 \\
.33804 \\
.31433 \\
.28551 \\
.25638\end{array}$ & $\begin{array}{l}.12566 \\
.11352 \\
.09984 \\
.08693 \\
.07553\end{array}$ & $\begin{array}{l}.80824 \\
.84285 \\
.87228 \\
.89665 \\
.91666\end{array}$ & $\begin{array}{l}1.31719 \\
1.37030 \\
1.41399 \\
1.44937 \\
1.47793\end{array}$ & $\begin{array}{l}.50895 \\
.52745 \\
.54171 \\
.55272 \\
.56127\end{array}$ \\
\hline $\begin{array}{l}1100 \\
1200 \\
1300 \\
1400 \\
1500\end{array}$ & $\begin{array}{l}.16337 \\
.14707 \\
.13232 \\
.11917 \\
.10753\end{array}$ & $\begin{array}{l}.22910 \\
.20447 \\
.18268 \\
.16356 \\
.14684\end{array}$ & $\begin{array}{l}.06573 \\
.05740 \\
.05036 \\
.04439 \\
.03931\end{array}$ & $\begin{array}{l}.93307 \\
.94658 \\
.95775 \\
.96707 \\
.97488\end{array}$ & $\begin{array}{l}1.50107 \\
1.51993 \\
1.53542 \\
1.54824 \\
1.55894\end{array}$ & $\begin{array}{l}.56800 \\
.57335 \\
.57767 \\
.58117 \\
.58406\end{array}$ \\
\hline $\begin{array}{l}2000 \\
2500 \\
3000 \\
4000 \\
5000\end{array}$ & $\begin{array}{l}.06699 \\
.04459 \\
.03123 \\
.01691 \\
.00977\end{array}$ & $\begin{array}{l}.08971 \\
.05866 \\
.04023 \\
.02039 \\
.01033\end{array}$ & $\begin{array}{l}.02272 \\
.01407 \\
.00900 \\
.00348 \\
.00056\end{array}$ & $\begin{array}{l}.99967 \\
1.01198 \\
1.01883 \\
1.02560 \\
1.02852\end{array}$ & $\begin{array}{l}1.59248 \\
1.60884 \\
1.61777 \\
1.62627 \\
1.62963\end{array}$ & $\begin{array}{l}.59281 \\
.59686 \\
.59894 \\
.60067 \\
.60111\end{array}$ \\
\hline
\end{tabular}


Table 3.17

\begin{tabular}{|c|c|c|c|c|c|c|}
\hline \multirow[b]{2}{*}{$\mathrm{T}^{\circ} \mathrm{K}$} & \multirow{2}{*}{$\frac{\mathrm{F}^{\circ}-\mathrm{F}^{\circ}}{\mathrm{Ag} \mathrm{AgD}}$} & $\mathrm{F}^{\circ} \mathrm{AgH}^{-\mathrm{F}^{\circ}} \mathrm{AgT}$ & $\mathrm{F}_{\mathrm{AgD}}^{\circ}-\mathrm{F}^{\circ} \mathrm{AgT}$ & \multirow{2}{*}{$\frac{\mathrm{HgH}^{\circ}{ }^{-\mathrm{H}^{\circ}} \mathrm{AgD}}{\mathrm{RT}}$} & $\mathrm{H}_{\mathrm{AgH}}^{\circ}{ }^{\circ}{ }^{\circ} \mathrm{AgT}^{\circ}$ & \multirow{2}{*}{$\frac{\mathrm{HgD}^{\circ}{ }^{-\mathrm{H}^{\circ}} \mathrm{AgT}}{\mathrm{RT}}$} \\
\hline & & $\mathrm{RT}$ & $\mathrm{RT}$ & & $\mathrm{RT}$ & \\
\hline 100 & 4.26928 & 6.26470 & 1.99542 & 3.58771 & 5.17271 & 1.58500 \\
\hline 200 & 2.47557 & 3.67906 & 1.20349 & 1.79254 & 2.58109 & .78855 \\
\hline 300 & 1.88013 & 2.82418 & .94404 & 1.18091 & 1.68664 & .50572 \\
\hline 400 & 1.58906 & 2.41071 & .82166 & .85431 & 1.20517 & .35087 \\
\hline 500 & 1.42243 & 2.17694 & .05451 & .64519 & .89960 & .25441 \\
\hline 600 & 1.31829 & 2.03240 & .71412 & .50132 & .69231 & 19100 \\
\hline 700 & 1.24914 & 1.93727 & .68813 & .39871 & .54637 & .14766 \\
\hline 800 & 1.20105 & 1.87157 & .67051 & .32370 & .44074 & .11703 \\
\hline 900 & 1.16631 & 1.82440 & .65809 & .26770 & .36244 & .09474 \\
\hline 1000 & 1.14041 & 1.78942 & .64901 & .22511 & .30320 & .07809 \\
\hline 1100 & 1.12056 & 1.76275 & .64219 & .19216 & .25753 & .06537 \\
\hline 10 & 1.10500 & 1.74195 & .63695 & .16632 & .22178 & .05546 \\
\hline 0 & 1.09253 & 1.72535 & .63283 & .14576 & .19335 & .04760 \\
\hline 1400 & 1.08234 & 1.71189 & .62955 & .12922 & .17048 & .04126 \\
\hline & 1.07391 & 1.70078 & .62688 & .11577 & .15186 & .03610 \\
\hline 200 & 1.04685 & 1.66578 & .61894 & .07639 & .09690 & .02052 \\
\hline 2500 & 1.03184 & 1.64708 & .61524 & .05975 & .07291 & .01316 \\
\hline & 1.02168 & 1.63491 & .61322 & .05245 & .06161 & .00915 \\
\hline 4000 & 1.00729 & 1.61852 & .61122 & .04901 & .05421 & .00519 \\
\hline 5000 & .99617 & 1.60645 & .61028 & .05125 & .05463 & .00338 \\
\hline
\end{tabular}

\begin{tabular}{|c|c|c|c|c|c|c|}
\hline \multirow{2}{*}{$\mathrm{T}^{\circ} \mathrm{K}$} & $\mathrm{C}_{\mathrm{AgD}}^{\circ}{ }^{-\mathrm{C}_{\mathrm{AgH}}^{\circ}}$ & $\mathrm{C}_{\mathrm{AgT}}^{\circ}{ }^{-\mathrm{C}_{\mathrm{AgH}}^{\circ}}$ & $\mathrm{C}_{\mathrm{AgT}}^{\circ}-\mathrm{C}_{\mathrm{AgD}}^{\circ}$ & $\mathrm{S}_{\mathrm{AgD}}^{\circ}-\mathrm{S}_{\mathrm{AgH}}^{\circ}$ & $\mathrm{S}_{\mathrm{AgT}}^{\circ}-\mathrm{S}_{\mathrm{AgH}}^{\circ}$ & $\mathrm{S}_{\mathrm{Ag} \mathrm{T}}{ }^{-\mathrm{S}^{\circ}} \mathrm{AgD}$ \\
\hline & $\mathrm{R}$ & $\mathrm{R}$ & $\bar{R}$ & $\mathrm{R}$ & $\mathrm{R}$ & $\mathrm{R}$ \\
\hline $\begin{array}{l}100 \\
200 \\
300 \\
400 \\
500\end{array}$ & $\begin{array}{r}-.00013 \\
.01162 \\
.08204 \\
.16491 \\
.21062\end{array}$ & $\begin{array}{r}-.00005 \\
.03807 \\
.17456 \\
.29291 \\
.34170\end{array}$ & $\begin{array}{l}.00008 \\
.02645 \\
.09252 \\
.12800 \\
.13108\end{array}$ & $\begin{array}{l}.68157 \\
.68304 \\
.69921 \\
.73475 \\
.77724\end{array}$ & $\begin{array}{l}1.09199 \\
1.09798 \\
1.13754 \\
1.20554 \\
1.27734\end{array}$ & $\begin{array}{l}.41042 \\
.41494 \\
.43833 \\
.47079 \\
.50010\end{array}$ \\
\hline $\begin{array}{r}600 \\
700 \\
800 \\
900 \\
1000\end{array}$ & $\begin{array}{l}.22086 \\
.21062 \\
.19130 \\
.16923 \\
.14746\end{array}$ & $\begin{array}{l}.34069 \\
.31540 \\
.28146 \\
.24655 \\
.21395\end{array}$ & $\begin{array}{l}.11983 \\
.10478 \\
.09016 \\
.07732 \\
.06649\end{array}$ & $\begin{array}{l}.81697 \\
.85042 \\
.87735 \\
.89861 \\
.91531\end{array}$ & $\begin{array}{l}1.34008 \\
1.39089 \\
1.43084 \\
1.46195 \\
1.48622\end{array}$ & $\begin{array}{l}.52311 \\
.54047 \\
.55349 \\
.56334 \\
.57091\end{array}$ \\
\hline $\begin{array}{l}1100 \\
1200 \\
1300 \\
1400 \\
1500\end{array}$ & $\begin{array}{l}.12730 \\
.10916 \\
.09307 \\
.07885 \\
.06628\end{array}$ & $\begin{array}{l}.18476 \\
.15913 \\
.13680 \\
.11736 \\
.10040\end{array}$ & $\begin{array}{l}.05746 \\
.04997 \\
.04373 \\
.03851 \\
.03412\end{array}$ & $\begin{array}{l}.92840 \\
.93868 \\
.94677 \\
.95313 \\
.95814\end{array}$ & $\begin{array}{l}1.50521 \\
1.52016 \\
1.53200 \\
1.54141 \\
1.54892\end{array}$ & $\begin{array}{l}.57681 \\
.58148 \\
.58523 \\
.58828 \\
.59078\end{array}$ \\
\hline $\begin{array}{l}2000 \\
2500 \\
3000 \\
4000 \\
5000\end{array}$ & $\begin{array}{r}.02141 \\
-.00599 \\
-.02487 \\
-.05066 \\
-.06887\end{array}$ & $\begin{array}{r}.04153 \\
-.00708 \\
-.01580 \\
-.04575 \\
-.06596\end{array}$ & $\begin{array}{l}.02012 \\
.01307 \\
.00907 \\
.00491 \\
.00291\end{array}$ & $\begin{array}{l}.97046 \\
.97208 \\
.96923 \\
.95828 \\
.94492\end{array}$ & $\begin{array}{l}1.56889 \\
1.57416 \\
1.57331 \\
1.56432 \\
1.55181\end{array}$ & $\begin{array}{l}.59843 \\
.60208 \\
.60408 \\
.60604 \\
.60689\end{array}$ \\
\hline
\end{tabular}


Table 3.18

\begin{tabular}{|c|c|c|c|c|c|c|}
\hline \multirow{2}{*}{$\mathrm{T}^{\circ} \mathrm{K}$} & $\mathrm{F}_{\mathrm{AuH}}^{\circ}-\mathrm{F}_{\mathrm{AuD}}^{\circ}$ & $\mathrm{F}_{\mathrm{AuH}}^{\circ}-\mathrm{F}_{\mathrm{AuT}}^{\circ}$ & $\mathrm{F}_{\mathrm{AuD}}^{\circ}-\mathrm{F}_{\mathrm{AuT}}^{\circ}$ & $\mathrm{H}_{\mathrm{AuH}}^{\circ}-\mathrm{H}_{\mathrm{AuD}}^{\circ}$ & $\mathrm{H}_{\mathrm{AuH}}^{\circ}{ }^{-\mathrm{H}_{\mathrm{AuT}}^{\circ}}$ & $\mathrm{H}_{\mathrm{AuD}}^{\circ}-\mathrm{H}_{\mathrm{AuT}}^{\circ}$ \\
\hline & $\mathrm{RT}$ & $\mathrm{RT}$ & $\mathrm{RT}$ & $\mathrm{RT}$ & $\mathrm{RT}$ & $\mathrm{RT}$ \\
\hline $\begin{array}{l}100 \\
200 \\
300 \\
400 \\
500\end{array}$ & $\begin{array}{l}5.41549 \\
3.05297 \\
2.26592 \\
1.87469 \\
1.64424\end{array}$ & $\begin{array}{l}7.96811 \\
4.55731 \\
3.42214 \\
2.86063 \\
2.53274\end{array}$ & $\begin{array}{r}2.55272 \\
1.50439 \\
1.15625 \\
.98597 \\
.88852\end{array}$ & $\begin{array}{r}4.72499 \\
2.36242 \\
1.57152 \\
1.16492 \\
.90755\end{array}$ & $\begin{array}{l}6.82170 \\
3.41013 \\
2.26223 \\
1.66471 \\
1.28462\end{array}$ & $\begin{array}{r}2.09681 \\
1.04776 \\
.69074 \\
.49982 \\
.37709\end{array}$ \\
\hline $\begin{array}{r}600 \\
700 \\
800 \\
900 \\
1000\end{array}$ & $\begin{array}{l}1.49566 \\
1.39426 \\
1.32217 \\
1.26927 \\
1.22943\end{array}$ & $\begin{array}{l}2.32335 \\
2.18170 \\
2.08173 \\
2.00882 \\
1.95416\end{array}$ & $\begin{array}{l}.82771 \\
.78745 \\
.75957 \\
.73956 \\
.72474\end{array}$ & $\begin{array}{l}.72639 \\
.59210 \\
.48971 \\
.41018 \\
.34747\end{array}$ & $\begin{array}{r}1.01869 \\
.82373 \\
.67681 \\
.56389 \\
.47567\end{array}$ & $\begin{array}{l}.29232 \\
.23164 \\
.18711 \\
.15372 \\
.12821\end{array}$ \\
\hline $\begin{array}{l}1100 \\
1200 \\
1300 \\
1400 \\
1500\end{array}$ & $\begin{array}{l}1.19875 \\
1.17466 \\
1.15546 \\
1.13990 \\
1.12715\end{array}$ & $\begin{array}{l}1.91223 \\
1.87941 \\
1.85331 \\
1.83220 \\
1.81492\end{array}$ & $\begin{array}{l}.71349 \\
.70476 \\
.69786 \\
.69231 \\
.68778\end{array}$ & $\begin{array}{l}.29738 \\
.25689 \\
.22383 \\
.19651 \\
.17376\end{array}$ & $\begin{array}{l}.40574 \\
.34955 \\
.30389 \\
.26635 \\
.23519\end{array}$ & $\begin{array}{l}.10837 \\
.09267 \\
.08007 \\
.06985 \\
.06144\end{array}$ \\
\hline $\begin{array}{l}2000 \\
2500 \\
3000 \\
4000 \\
5000\end{array}$ & $\begin{array}{l}1.08826 \\
1.06960 \\
1.05924 \\
1.04865 \\
1.04349\end{array}$ & $\begin{array}{l}1.76238 \\
1.73722 \\
1.72321 \\
1.70880 \\
1.70166\end{array}$ & $\begin{array}{l}.67413 \\
.66762 \\
.66397 \\
.66015 \\
.65817\end{array}$ & $\begin{array}{l}.10238 \\
.06718 \\
.04757 \\
.02793 \\
.01903\end{array}$ & $\begin{array}{l}.13813 \\
.09070 \\
.06443 \\
.03832 \\
.02663\end{array}$ & $\begin{array}{l}.03576 \\
.02352 \\
.01686 \\
.01039 \\
.00760\end{array}$ \\
\hline
\end{tabular}

\begin{tabular}{|c|c|c|c|c|c|c|}
\hline \multirow{2}{*}{$\mathrm{T}^{\circ} \mathrm{K}$} & $\mathrm{C}_{\mathrm{AuD}}^{\circ}{ }^{-\mathrm{C}_{\mathrm{AuH}}^{\circ}}$ & $\mathrm{C}_{\mathrm{AuT} T}^{\circ}-\mathrm{C}_{\mathrm{AuH}}^{\circ}$ & $\mathrm{C}_{\mathrm{AuT} T}^{\circ}-\mathrm{C}_{\mathrm{AuD}}^{\circ}$ & $\mathrm{S}_{\mathrm{AuD}}^{\circ}{ }^{-\mathrm{S}_{\mathrm{AuH}}^{\circ}}$ & $\mathrm{S}_{\mathrm{AuTT}}^{\circ}{ }^{-\mathrm{S}_{\mathrm{AuH}}^{\circ}}$ & $\mathrm{S}_{\mathrm{Au} \mathrm{T}}^{\circ}{ }^{-\mathrm{S}_{\mathrm{AuD}}^{\circ}}$ \\
\hline & $\mathrm{R}$ & $\mathrm{R}$ & $\mathrm{R}$ & $\mathrm{R}$ & $\mathrm{R}$ & $\mathrm{R}$ \\
\hline $\begin{array}{l}100 \\
200 \\
300 \\
400 \\
500\end{array}$ & $\begin{array}{r}-.00019 \\
.00135 \\
.02607 \\
.08774 \\
.15412\end{array}$ & $\begin{array}{r}-.00022 \\
.00722 \\
.07261 \\
.18493 \\
.28042\end{array}$ & $\begin{array}{r}-.00003 \\
.00587 \\
.04654 \\
.09719 \\
.12630\end{array}$ & $\begin{array}{l}.69049 \\
.69056 \\
.69440 \\
.70976 \\
.73670\end{array}$ & $\begin{array}{l}1.14641 \\
1.14718 \\
1.15991 \\
1.19591 \\
1.24813\end{array}$ & $\begin{array}{r}.45592 \\
.45662 \\
.46551 \\
.48615 \\
.51143\end{array}$ \\
\hline $\begin{array}{r}600 \\
700 \\
800 \\
900 \\
1000\end{array}$ & $\begin{array}{l}.20044 \\
.22329 \\
.22841 \\
.22251 \\
.21062\end{array}$ & $\begin{array}{l}.33450 \\
.35268 \\
.34761 \\
.32985 \\
.30626\end{array}$ & $\begin{array}{r}.13406 \\
.12939 \\
.11920 \\
.10734 \\
.09564\end{array}$ & $\begin{array}{l}.76927 \\
.80215 \\
.83246 \\
.85910 \\
.88196\end{array}$ & $\begin{array}{l}1.30466 \\
1.35797 \\
1.40493 \\
1.44493 \\
1.47848\end{array}$ & $\begin{array}{l}.53539 \\
.55582 \\
.57247 \\
.58583 \\
.59652\end{array}$ \\
\hline $\begin{array}{l}1100 \\
1200 \\
1300 \\
1400 \\
1500\end{array}$ & $\begin{array}{r}.19604 \\
.18070 \\
.16567 \\
.15149 \\
.13841\end{array}$ & $\begin{array}{l}.28092 \\
.25599 \\
.23255 \\
.21106 \\
.19162\end{array}$ & $\begin{array}{l}.08488 \\
.07529 \\
.06688 \\
.05957 \\
.05321\end{array}$ & $\begin{array}{l}.90137 \\
.91777 \\
.93163 \\
.94339 \\
.95338\end{array}$ & $\begin{array}{l}1.50650 \\
1.52986 \\
1.54941 \\
1.56585 \\
1.57973\end{array}$ & $\begin{array}{l}.60513 \\
.61209 \\
.61778 \\
.62246 \\
.62635\end{array}$ \\
\hline $\begin{array}{l}2000 \\
2500 \\
3000 \\
4000 \\
5000\end{array}$ & $\begin{array}{l}.08937 \\
.06022 \\
.04218 \\
.02221 \\
.01191\end{array}$ & $\begin{array}{l}.12111 \\
.08037 \\
.05545 \\
.02793 \\
.01365\end{array}$ & $\begin{array}{l}.03174 \\
.02015 \\
.01327 \\
.00572 \\
.00174\end{array}$ & $\begin{array}{l}.98588 \\
1.00242 \\
1.01168 \\
1.02073 \\
1.02447\end{array}$ & $\begin{array}{l}1.62425 \\
1.64651 \\
1.65879 \\
1.67048 \\
1.67503\end{array}$ & $\begin{array}{l}.63837 \\
.64409 \\
.64711 \\
.64975 \\
.65056\end{array}$ \\
\hline
\end{tabular}


Table 3.19

\begin{tabular}{|c|c|c|c|c|c|c|}
\hline \multirow{2}{*}{$\mathrm{T}^{\circ} \mathrm{K}$} & $\mathrm{F}_{\mathrm{ZnH}}^{\circ}-\mathrm{F}_{\mathrm{ZnD}}^{\circ}$ & $\mathrm{F}_{\mathrm{ZnH}}^{\circ}{ }^{-\mathrm{F}_{\mathrm{ZnT}}^{\circ}}$ & $\mathrm{F}_{\mathrm{ZnD}}^{\circ}-\mathrm{F}_{\mathrm{ZnT}}^{\circ}$ & $\mathrm{H}_{\mathrm{ZnH}}^{\circ} \mathrm{H}_{\mathrm{ZnD}}^{\circ}$ & $\mathrm{H}_{\mathrm{ZnH}}^{\circ}{ }^{-\mathrm{H}_{\mathrm{ZnT}}^{\circ}}$ & $\mathrm{H}_{\mathrm{ZnD}}^{\circ} \mathrm{H}_{\mathrm{ZnT}}^{\circ}$ \\
\hline & $\mathrm{RT}$ & $\mathrm{RT}$ & $\mathrm{RT}$ & $\mathrm{RT}$ & $\mathrm{RT}$ & $\mathrm{RT}$ \\
\hline $\begin{array}{l}100 \\
200 \\
300 \\
400 \\
500\end{array}$ & $\begin{array}{l}3.90948 \\
2.30134 \\
1.76952 \\
1.51258 \\
1.36770\end{array}$ & $\begin{array}{l}5.69694 \\
3.37963 \\
2.61778 \\
2.25427 \\
2.05187\end{array}$ & $\begin{array}{r}1.78746 \\
1.07829 \\
.84826 \\
.74170 \\
.68417\end{array}$ & $\begin{array}{r}3.21701 \\
1.60561 \\
1.04900 \\
.74799 \\
.55655\end{array}$ & $\begin{array}{r}4.63752 \\
2.30920 \\
1.49290 \\
1.05057 \\
.77301\end{array}$ & $\begin{array}{r}1.42051 \\
.70359 \\
.44390 \\
.30259 \\
.21646\end{array}$ \\
\hline $\begin{array}{r}600 \\
700 \\
800 \\
900 \\
1000\end{array}$ & $\begin{array}{l}1.27842 \\
1.21988 \\
1.17961 \\
1.15083 \\
1.12958\end{array}$ & $\begin{array}{l}1.92838 \\
1.84800 \\
1.79302 \\
1.75387 \\
1.72507\end{array}$ & $\begin{array}{l}.64996 \\
.62812 \\
.61340 \\
.60304 \\
.59549\end{array}$ & $\begin{array}{l}.42676 \\
.33552 \\
.26953 \\
.22065 \\
.18363\end{array}$ & $\begin{array}{l}.58775 \\
.45920 \\
.36719 \\
.29954 \\
.24864\end{array}$ & $\begin{array}{l}.16099 \\
.12368 \\
.09765 \\
.07889 \\
.06501\end{array}$ \\
\hline $\begin{array}{l}1100 \\
1200 \\
1300 \\
1400 \\
1500\end{array}$ & $\begin{array}{l}1.11347 \\
1.10097 \\
1.09108 \\
1.08313 \\
1.07661\end{array}$ & $\begin{array}{l}1.70328 \\
1.68640 \\
1.67307 \\
1.66235 \\
1.65358\end{array}$ & $\begin{array}{r}.58981 \\
.58543 \\
.58199 \\
.57922 \\
.57697\end{array}$ & $\begin{array}{l}.15508 \\
.13267 \\
.11482 \\
.10042 \\
.08865\end{array}$ & $\begin{array}{l}.20956 \\
.17900 \\
.15474 \\
.13522 \\
.11929\end{array}$ & $\begin{array}{l}.05448 \\
.04633 \\
.03992 \\
.03480 \\
.03064\end{array}$ \\
\hline $\begin{array}{l}2000 \\
2500 \\
3000 \\
4000 \\
5000\end{array}$ & $\begin{array}{l}1.05666 \\
1.04667 \\
1.04067 \\
1.03346 \\
1.02884\end{array}$ & $\begin{array}{l}1.62674 \\
1.61332 \\
1.60522 \\
1.59546 \\
1.58915\end{array}$ & $\begin{array}{l}.57009 \\
.56665 \\
.56455 \\
.56199 \\
.56031\end{array}$ & $\begin{array}{l}.05341 \\
.03734 \\
.02910 \\
.02202 \\
.01982\end{array}$ & $\begin{array}{l}.07180 \\
.05028 \\
.03928 \\
.02994 \\
.02709\end{array}$ & $\begin{array}{l}.01840 \\
.01294 \\
.01018 \\
.00791 \\
.00727\end{array}$ \\
\hline
\end{tabular}

\begin{tabular}{|c|c|c|c|c|c|c|}
\hline \multirow{2}{*}{$\mathrm{T}^{\circ} \mathrm{K}$} & $\mathrm{C}_{\mathrm{ZnD}}^{\circ}{ }^{-\mathrm{C}_{\mathrm{ZnH}}^{\circ}}$ & $\mathrm{C}_{\mathrm{ZnT}}^{\circ}-\mathrm{C}_{\mathrm{ZnH}}^{\circ}$ & $\mathrm{C}_{\mathrm{ZnT}}^{\circ}-\mathrm{C}_{\mathrm{ZnD}}^{\circ}$ & $\mathrm{S}_{\mathrm{ZnD}}^{\circ}{ }^{-\mathrm{S}_{\mathrm{ZnH}}^{\circ}}$ & $\mathrm{S}_{\mathrm{ZnT}}^{\circ}{ }^{-\mathrm{S}_{\mathrm{ZnH}}^{\circ}}$ & $\mathrm{S}_{\mathrm{ZnT}}^{\circ}{ }^{-\mathrm{S}_{\mathrm{ZnD}}^{\circ}}$ \\
\hline & $\mathrm{R}$ & $\mathrm{R}$ & $\mathrm{R}$ & $\mathrm{R}$ & $\mathrm{R}$ & $\mathrm{R}$ \\
\hline $\begin{array}{l}100 \\
200 \\
300 \\
400 \\
500\end{array}$ & $\begin{array}{r}-.00012 \\
.02230 \\
.11221 \\
.19025 \\
.22105\end{array}$ & $\begin{array}{l}.00019 \\
.06186 \\
.21840 \\
.31977 \\
.34480\end{array}$ & $\begin{array}{l}.00031 \\
.03956 \\
.10619 \\
.12952 \\
.12375\end{array}$ & $\begin{array}{l}.69248 \\
.69573 \\
.72053 \\
.76459 \\
.81115\end{array}$ & $\begin{array}{l}1.05942 \\
1.07043 \\
1.12488 \\
1.20370 \\
1.27886\end{array}$ & $\begin{array}{l}.36694 \\
.37470 \\
.40435 \\
.43911 \\
.46771\end{array}$ \\
\hline $\begin{array}{r}600 \\
700 \\
800 \\
900 \\
1000\end{array}$ & $\begin{array}{l}.21951 \\
.20296 \\
.18144 \\
.15966 \\
.13958\end{array}$ & $\begin{array}{l}.32792 \\
.29501 \\
.25891 \\
.22486 \\
.19467\end{array}$ & $\begin{array}{l}.10841 \\
.09205 \\
.07747 \\
.06520 \\
.05509\end{array}$ & $\begin{array}{l}.85165 \\
.88436 \\
.91008 \\
.93018 \\
.94594\end{array}$ & $\begin{array}{l}1.34063 \\
1.38880 \\
1.42584 \\
1.45434 \\
1.47642\end{array}$ & $\begin{array}{l}.48898 \\
.50444 \\
.51576 \\
.52416 \\
.53048\end{array}$ \\
\hline $\begin{array}{l}1100 \\
1200 \\
1300 \\
1400 \\
1500\end{array}$ & $\begin{array}{l}.12176 \\
.10623 \\
.09280 \\
.08121 \\
.07117\end{array}$ & $\begin{array}{l}.16855 \\
.14619 \\
.12709 \\
.11076 \\
.09674\end{array}$ & $\begin{array}{l}.04679 \\
.03996 \\
.03429 \\
.02955 \\
.02557\end{array}$ & $\begin{array}{l}.95839 \\
.96830 \\
.97626 \\
.98271 \\
.98796\end{array}$ & $\begin{array}{l}1.49372 \\
1.50740 \\
1.51833 \\
1.52714 \\
1.53429\end{array}$ & $\begin{array}{l}.53533 \\
.53910 \\
.54207 \\
.54443 \\
.54633\end{array}$ \\
\hline $\begin{array}{l}2000 \\
2500 \\
3000 \\
4000 \\
5000\end{array}$ & $\begin{array}{r}.03717 \\
.01835 \\
.00670 \\
-.00685 \\
-.01452\end{array}$ & $\begin{array}{r}.04980 \\
.02413 \\
.00831 \\
-.01007 \\
-.02053\end{array}$ & $\begin{array}{r}.01263 \\
.00578 \\
.00161 \\
-.00322 \\
-.00601\end{array}$ & $\begin{array}{l}1.00325 \\
1.00933 \\
1.01157 \\
1.01144 \\
1.00901\end{array}$ & $\begin{array}{l}1.55494 \\
1.56304 \\
1.56593 \\
1.56553 \\
1.56206\end{array}$ & $\begin{array}{l}.55169 \\
.55371 \\
.55436 \\
.55409 \\
.55305\end{array}$ \\
\hline
\end{tabular}


Table 3.20

\begin{tabular}{|c|c|c|c|c|c|c|}
\hline \multirow{2}{*}{$\mathrm{T}^{\circ} \mathrm{K}$} & $\mathrm{F}_{\mathrm{CdH}}^{\circ}{ }^{-\mathrm{F}_{\mathrm{CdD}}^{\circ}}$ & $\mathrm{F}_{\mathrm{CdH}}^{\circ}{ }^{-\mathrm{F}_{\mathrm{CdT}}^{\circ}}$ & $\mathrm{F}_{\mathrm{CdD}}^{\circ}{ }^{-\mathrm{F}_{\mathrm{Cd}}^{\circ} \mathrm{T}}$ & $\mathrm{H}_{\mathrm{CdH}}^{\circ}{ }^{-\mathrm{H}_{\mathrm{CdD}}^{\circ}}$ & $\mathrm{H}_{\mathrm{CdH}}^{\circ}{ }^{-\mathrm{H}^{\circ} \mathrm{Cd} T}$ & $\mathrm{H}_{\mathrm{CdD}}^{\circ}{ }^{-\mathrm{H}^{\circ} \mathrm{CdT}}$ \\
\hline & $\mathrm{RT}$ & RT & $\mathrm{RT}$ & RT & $\mathrm{RT}$ & $\mathrm{RT}$ \\
\hline $\begin{array}{l}100 \\
200 \\
300 \\
400 \\
500\end{array}$ & $\begin{array}{l}3.57098 \\
2.12988 \\
1.65673 \\
1.43229 \\
1.30843\end{array}$ & $\begin{array}{l}5.25250 \\
3.17416 \\
2.49801 \\
2.18202 \\
2.00982\end{array}$ & $\begin{array}{r}1.68142 \\
1.04423 \\
.84124 \\
.74971 \\
.70137\end{array}$ & $\begin{array}{r}2.88403 \\
1.43579 \\
.92482 \\
.64567 \\
.47055\end{array}$ & $\begin{array}{r}4.16292 \\
2.06333 \\
1.31059 \\
.90214 \\
.65070\end{array}$ & $\begin{array}{r}1.27879 \\
.62749 \\
.38573 \\
.25645 \\
.18013\end{array}$ \\
\hline $\begin{array}{r}600 \\
700 \\
800 \\
900 \\
1000\end{array}$ & $\begin{array}{l}1.23360 \\
1.18537 \\
1.15272 \\
1.12970 \\
1.11296\end{array}$ & $\begin{array}{l}1.90669 \\
1.84058 \\
1.79597 \\
1.76454 \\
1.74167\end{array}$ & $\begin{array}{l}.67308 \\
.65520 \\
.64324 \\
.63483 \\
.62870\end{array}$ & $\begin{array}{l}.35427 \\
.27405 \\
.21693 \\
.17507 \\
.14365\end{array}$ & $\begin{array}{l}.48659 \\
.37492 \\
.29622 \\
.23902 \\
.19638\end{array}$ & $\begin{array}{l}.13231 \\
.10086 \\
.07928 \\
.06394 \\
.05272\end{array}$ \\
\hline $\begin{array}{l}1100 \\
1200 \\
1300 \\
1400 \\
1500\end{array}$ & $\begin{array}{l}1.10045 \\
1.09088 \\
1.08345 \\
1.07756 \\
1.07284\end{array}$ & $\begin{array}{l}1.72455 \\
1.71142 \\
1.70115 \\
1.69299 \\
1.68638\end{array}$ & $\begin{array}{l}.62410 \\
.62052 \\
.61770 \\
.61542 \\
.61354\end{array}$ & $\begin{array}{l}.11954 \\
.10067 \\
.08567 \\
.07355 \\
.06363\end{array}$ & $\begin{array}{l}.16384 \\
.13854 \\
.11849 \\
.10240 \\
.08928\end{array}$ & $\begin{array}{l}.04430 \\
.03785 \\
.03282 \\
.02884 \\
.02565\end{array}$ \\
\hline $\begin{array}{l}2000 \\
2500 \\
3000 \\
4000 \\
5000\end{array}$ & $\begin{array}{l}1.05924 \\
1.05349 \\
1.05079 \\
1.04892 \\
1.04877\end{array}$ & $\begin{array}{l}1.66687 \\
1.65789 \\
1.65304 \\
1.64816 \\
1.64575\end{array}$ & $\begin{array}{l}.60763 \\
.60439 \\
.60224 \\
.59924 \\
.59697\end{array}$ & $\begin{array}{r}.03348 \\
.01906 \\
.01102 \\
.00277 \\
-.00126\end{array}$ & $\begin{array}{l}.05001 \\
.03181 \\
.02210 \\
.01288 \\
.00903\end{array}$ & $\begin{array}{l}.01653 \\
.01274 \\
.01107 \\
.01011 \\
.01028\end{array}$ \\
\hline
\end{tabular}

\begin{tabular}{|c|c|c|c|c|c|c|}
\hline \multirow{2}{*}{$\mathrm{T}^{\circ} \mathrm{K}$} & $\mathrm{C}_{\mathrm{CdD}}^{\circ}{ }^{-\mathrm{C}_{\mathrm{CdH}}^{\circ}}$ & $\mathrm{C}_{\mathrm{CdT}}^{\circ}-\mathrm{C}_{\mathrm{CdH}}^{\circ}$ & $\mathrm{C}_{\mathrm{CdT}}^{\circ}{ }^{-\mathrm{C}_{\mathrm{CdD}}^{\circ}}$ & $\mathrm{S}_{\mathrm{CdD}}^{\circ}{ }^{-\mathrm{S}_{\mathrm{C}}^{\circ} \mathrm{H}}$ & $\mathrm{S}_{\mathrm{Cd} \mathrm{T}}^{\circ}{ }^{-\mathrm{S}_{\mathrm{CdH}}^{\circ}}$ & $\mathrm{S}_{\mathrm{Cd} \mathrm{T}}^{-\mathrm{S}_{\mathrm{CdD}}^{\circ}}$ \\
\hline & $\mathrm{R}$ & $\mathrm{R}$ & $\mathrm{R}$ & $\mathrm{R}$ & $\mathrm{R}$ & $\mathrm{R}$ \\
\hline $\begin{array}{l}100 \\
200 \\
300 \\
400 \\
500\end{array}$ & $\begin{array}{l}.00007 \\
.04183 \\
.15260 \\
.22009 \\
.23300\end{array}$ & $\begin{array}{l}.00122 \\
.10299 \\
.27677 \\
.35213 \\
.34956\end{array}$ & $\begin{array}{l}.00115 \\
.06116 \\
.12417 \\
.13204 \\
.11656\end{array}$ & $\begin{array}{l}.68696 \\
.69408 \\
.73191 \\
.78662 \\
.83787\end{array}$ & $\begin{array}{l}1.08958 \\
1.11082 \\
1.18741 \\
1.27988 \\
1.35912\end{array}$ & $\begin{array}{l}.40262 \\
.41674 \\
.45550 \\
.49326 \\
.52125\end{array}$ \\
\hline $\begin{array}{r}600 \\
700 \\
800 \\
900 \\
1000\end{array}$ & $\begin{array}{r}.21864 \\
.19523 \\
.17098 \\
.14893 \\
.12989\end{array}$ & $\begin{array}{l}.31562 \\
.27456 \\
.23577 \\
.20205 \\
.17366\end{array}$ & $\begin{array}{l}.09698 \\
.07933 \\
.06479 \\
.05312 \\
.04377\end{array}$ & $\begin{array}{l}.87932 \\
.91132 \\
.93579 \\
.95463 \\
.96930\end{array}$ & $\begin{array}{l}1.42009 \\
1.46567 \\
1.49975 \\
1.52552 \\
1.54529\end{array}$ & $\begin{array}{r}.54077 \\
.55435 \\
.56396 \\
.57089 \\
.57599\end{array}$ \\
\hline $\begin{array}{l}1100 \\
1200 \\
1300 \\
1400 \\
1500\end{array}$ & $\begin{array}{l}.11378 \\
.10026 \\
.08893 \\
.07940 \\
.07136\end{array}$ & $\begin{array}{l}.15002 \\
.13037 \\
.11399 \\
.10025 \\
.08867\end{array}$ & $\begin{array}{l}.03624 \\
.03011 \\
.02506 \\
.02085 \\
.01731\end{array}$ & $\begin{array}{r}.98091 \\
.99021 \\
.99778 \\
1.00401 \\
1.00920\end{array}$ & $\begin{array}{l}1.56070 \\
1.57289 \\
1.58265 \\
1.59058 \\
1.59709\end{array}$ & $\begin{array}{r}.57979 \\
.58268 \\
.58487 \\
.58657 \\
.58789\end{array}$ \\
\hline $\begin{array}{l}2000 \\
2500 \\
3000 \\
4000 \\
5000\end{array}$ & $\begin{array}{l}.04569 \\
.03297 \\
.02594 \\
.01909 \\
.01607\end{array}$ & $\begin{array}{l}.05146 \\
.03243 \\
.02141 \\
.00967 \\
.00371\end{array}$ & $\begin{array}{r}.00577 \\
-.00054 \\
-.00453 \\
-.00942 \\
-.01236\end{array}$ & $\begin{array}{l}1.02576 \\
1.03444 \\
1.03977 \\
1.04614 \\
1.05004\end{array}$ & $\begin{array}{l}1.61685 \\
1.62608 \\
1.63094 \\
1.63527 \\
1.63673\end{array}$ & $\begin{array}{l}.59109 \\
.59164 \\
.59117 \\
.58913 \\
.58669\end{array}$ \\
\hline
\end{tabular}




\begin{tabular}{|c|c|c|c|c|c|c|}
\hline \multirow{2}{*}{$\mathrm{T}^{\circ} \mathrm{K}$} & $F_{\mathrm{HgH}}^{\circ}{ }^{-F^{\circ}} \mathrm{HgD}$ & $\mathrm{F}_{\mathrm{HgH}}^{\circ}-\mathrm{F}_{\mathrm{HgT}}^{\circ}$ & $\mathrm{F}_{\mathrm{HgD}}^{\circ}-\mathrm{F}_{\mathrm{HgT}}^{\circ}$ & $\mathrm{H}_{\mathrm{HgH}}^{\circ}{ }^{-H^{\circ}}$ & $\mathrm{H}_{\mathrm{HgH}}^{\circ}{ }^{-\mathrm{H}^{\circ}} \mathrm{HgT}$ & $\mathrm{H}_{\mathrm{HgD}}^{\mathrm{o}}{ }^{-\mathrm{H}^{\mathrm{o}}} \mathrm{Hg \textrm {T }}$ \\
\hline & $\mathrm{R}^{\prime} \mathrm{T}$ & $\mathrm{RT}$ & $\mathrm{RT}^{\prime}$ & $\mathrm{RT}$ & $\mathrm{RT}^{\mathrm{T}}$ & $\mathrm{RT}$ \\
\hline $\begin{array}{l}100 \\
200 \\
300 \\
400 \\
500\end{array}$ & $\begin{array}{l}3.36119 \\
2.02313 \\
1.58636 \\
1.38097 \\
1.26810\end{array}$ & $\begin{array}{l}4.94389 \\
3.01850 \\
2.39660 \\
2.10874 \\
1.95267\end{array}$ & $\begin{array}{r}1.58108 \\
.99456 \\
.80971 \\
.72736 \\
.68424\end{array}$ & $\begin{array}{r}2.67918 \\
1.33022 \\
.84928 \\
.58881 \\
.42891\end{array}$ & $\begin{array}{r}3.85976 \\
1.90565 \\
1.19849 \\
.81872 \\
.58944\end{array}$ & $\begin{array}{r}1.17896 \\
.57462 \\
.34868 \\
.22950 \\
.16020\end{array}$ \\
\hline $\begin{array}{l}600 \\
700 \\
800 \\
900\end{array}$ & $\begin{array}{l}1.19971 \\
1.15513 \\
1.12434 \\
1.10196 \\
1.08499\end{array}$ & $\begin{array}{l}1.85910 \\
1.79862 \\
1.75711 \\
1.72716 \\
1.70460\end{array}$ & $\begin{array}{l}.65911 \\
.64325 \\
.63258 \\
.62503 \\
.61945\end{array}$ & $\begin{array}{l}.32527 \\
.25566 \\
.20759 \\
.17360 \\
.14917\end{array}$ & $\begin{array}{l}.44295 \\
.34554 \\
.27868 \\
.23162 \\
.19782\end{array}$ & $\begin{array}{l}.11740 \\
.08964 \\
.07090 \\
.05784 \\
.04849\end{array}$ \\
\hline 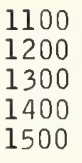 & $\begin{array}{l}1.07166 \\
1.06083 \\
1.05175 \\
1.04400 \\
1.03719\end{array}$ & $\begin{array}{l}1.68697 \\
1.67272 \\
1.66088 \\
1.65078 \\
1.64200\end{array}$ & $\begin{array}{l}.61516 \\
.61177 \\
.60899 \\
.60667 \\
.60469\end{array}$ & $\begin{array}{l}.13135 \\
.11824 \\
.10852 \\
.10133 \\
.09601\end{array}$ & $\begin{array}{l}.17316 \\
.15495 \\
.14141 \\
.13129 \\
.12375\end{array}$ & $\begin{array}{l}.04166 \\
.03659 \\
.03275 \\
.02985 \\
.02762\end{array}$ \\
\hline 00 & $\begin{array}{r}1.01151 \\
.99250 \\
.97649 \\
.94916 \\
.92583\end{array}$ & $\begin{array}{l}1.60925 \\
1.58543 \\
1.56553 \\
1.53182 \\
1.50314\end{array}$ & $\begin{array}{l}.59765 \\
.59286 \\
.58899 \\
.58262 \\
.57727\end{array}$ & $\begin{array}{l}.08528 \\
.08600 \\
.09018 \\
.10021 \\
.10884\end{array}$ & $\begin{array}{l}.10762 \\
.10719 \\
.11163 \\
.12334 \\
.13373\end{array}$ & $\begin{array}{l}.02225 \\
.02112 \\
.02140 \\
.02309 \\
.02485\end{array}$ \\
\hline
\end{tabular}

\begin{tabular}{|c|c|c|c|c|c|c|}
\hline \multirow{2}{*}{$\mathrm{T}^{\circ} \mathrm{K}$} & $\mathrm{C}_{\mathrm{HgD}}^{\circ}{ }^{-\mathrm{C}_{\mathrm{HgH}}^{\circ}}$ & $\mathrm{C}_{\mathrm{HgT}}^{\circ}-\mathrm{C}_{\mathrm{HgH}}^{\circ}$ & $\mathrm{C}_{\mathrm{HgT}}^{\circ}{ }^{-\mathrm{C}^{\circ}}{ }_{\mathrm{HgD}}$ & $\mathrm{S}_{\mathrm{HgD}}^{\circ}{ }^{-\mathrm{S}_{\mathrm{HgH}}^{\circ}}$ & $\mathrm{S}_{\mathrm{HgT}}^{\circ}{ }^{-S_{\mathrm{HgH}}^{\circ}}$ & $\mathrm{S}_{\mathrm{Hg} \mathrm{T}}^{\circ}{ }^{-\mathrm{S}_{\mathrm{HgD}}^{\circ}}$ \\
\hline & $\mathrm{R}$ & $\mathrm{R}$ & $\mathrm{R}$ & $\mathrm{R}$ & $\mathrm{R}$ & $\mathrm{R}$ \\
\hline $\begin{array}{l}100 \\
200 \\
300 \\
400 \\
500\end{array}$ & $\begin{array}{l}.00064 \\
.05632 \\
.16359 \\
.21026 \\
.20540\end{array}$ & $\begin{array}{l}.00269 \\
.12652 \\
.28840 \\
.33581 \\
.31253\end{array}$ & $\begin{array}{l}.00205 \\
.07020 \\
.12481 \\
.12555 \\
.10713\end{array}$ & $\begin{array}{l}.68201 \\
.69292 \\
.73707 \\
.79215 \\
.83920\end{array}$ & $\begin{array}{l}1.08413 \\
1.11286 \\
1.19810 \\
1.29001 \\
1.36323\end{array}$ & $\begin{array}{l}.40212 \\
.41994 \\
.46103 \\
.49786 \\
.52403\end{array}$ \\
\hline $\begin{array}{r}600 \\
700 \\
800 \\
900 \\
1000\end{array}$ & $\begin{array}{l}.17836 \\
.14535 \\
.11309 \\
.08389 \\
.05824\end{array}$ & $\begin{array}{l}.26473 \\
.21342 \\
.16609 \\
.12467 \\
.08910\end{array}$ & $\begin{array}{l}.08637 \\
.06807 \\
.05300 \\
.04078 \\
.03086\end{array}$ & $\begin{array}{l}.87443 \\
.89947 \\
.91674 \\
.92834 \\
.93583\end{array}$ & $\begin{array}{l}1.41614 \\
1.45308 \\
1.47842 \\
1.49553 \\
1.50678\end{array}$ & $\begin{array}{l}.54171 \\
.55361 \\
.56168 \\
.56719 \\
.57095\end{array}$ \\
\hline $\begin{array}{l}1100 \\
1200 \\
1300 \\
1400 \\
1500\end{array}$ & $\begin{array}{r}.03594 \\
.01660 \\
-.00025 \\
-.01497 \\
-.02791\end{array}$ & $\begin{array}{r}.05868 \\
.03260 \\
. .01012 \\
-.00937 \\
-.02640\end{array}$ & $\begin{array}{l}.02274 \\
.01600 \\
.01037 \\
.00560 \\
.00151\end{array}$ & $\begin{array}{l}.94030 \\
.94258 \\
.94323 \\
.94266 \\
.94117\end{array}$ & $\begin{array}{l}1.51381 \\
1.51777 \\
1.51947 \\
1.51949 \\
1.51825\end{array}$ & $\begin{array}{l}.57351 \\
.57519 \\
.57624 \\
.57683 \\
.57708\end{array}$ \\
\hline $\begin{array}{l}2000 \\
2500 \\
3000 \\
4000 \\
5000\end{array}$ & $\begin{array}{l}-.07401 \\
-.10161 \\
-.11930 \\
-.13869 \\
-.14670\end{array}$ & $\begin{array}{l}-.08634 \\
-.12178 \\
-.14438 \\
-.16919 \\
-.17959\end{array}$ & $\begin{array}{l}-.01233 \\
-.02017 \\
-.02508 \\
-.03050 \\
-.03289\end{array}$ & $\begin{array}{l}.92622 \\
.90650 \\
.88630 \\
.84894 \\
.81699\end{array}$ & $\begin{array}{l}1.50162 \\
1.47824 \\
1.45389 \\
1.40847 \\
1.36942\end{array}$ & $\begin{array}{l}.57540 \\
.57174 \\
.56759 \\
.55953 \\
.55243\end{array}$ \\
\hline
\end{tabular}


Table 3.22

\begin{tabular}{|c|c|c|c|c|c|c|}
\hline \multirow{2}{*}{$\mathrm{T}^{\circ} \mathrm{K}$} & $\mathrm{F}_{\mathrm{BH}}^{\circ}{ }^{-\mathrm{F}_{\mathrm{BD}}^{\circ}}$ & $\mathrm{F}_{\mathrm{BH}}^{\circ}-\mathrm{F}_{\mathrm{BT}}^{\circ}$ & $\mathrm{F}_{\mathrm{BD}}^{\circ}-\mathrm{F}_{\mathrm{BT}}^{\circ}$ & $\mathrm{H}_{\mathrm{BH}}^{\circ}{ }^{-\mathrm{H}_{\mathrm{BD}}^{\circ}}$ & $\mathrm{H}_{\mathrm{BH}}^{\circ}{ }^{-\mathrm{H}_{\mathrm{BT}}^{\circ}}$ & $\mathrm{H}_{\mathrm{BD}}^{\circ}{ }^{-\mathrm{H}_{\mathrm{BT}}^{\circ}}$ \\
\hline & $\mathrm{RT}$ & $\mathrm{RT}$ & $\mathrm{RT}$ & $\mathrm{RT}$ & $\mathrm{RT}$ & $\mathrm{RT}$ \\
\hline 100 & 4.83870 & 7.40468 & 2.56598 & 4.10939 & 6.23680 & 2.12741 \\
\hline 200 & 2.78393 & 4.28622 & 1.50229 & 2.05480 & 3.11823 & 1.06343 \\
\hline 300 & 2.09920 & 3.24766 & 1.14845 & 1.36801 & 2.07226 & .70424 \\
\hline 400 & 1.75824 & 2.73235 & .97411 & 1.01707 & 1.53184 & .51477 \\
\hline 500 & 1.55663 & 2.42983 & .87320 & .79613 & 1.18906 & .39293 \\
\hline 600 & 1.42598 & 2.23548 & .80951 & .64062 & .94842 & .30781 \\
\hline 700 & 1.33632 & 2.10327 & .76695 & .52484 & .77083 & .24599 \\
\hline 800 & 1.27229 & 2.00953 & .73724 & .43604 & .63604 & 20000 \\
\hline 900 & 1.22510 & 1.94091 & .71580 & .36665 & .53177 & .16511 \\
\hline 1000 & 1.18942 & 1.88928 & .69986 & .31166 & .44982 & .13816 \\
\hline 1100 & 1.16186 & 1.84958 & .68772 & .26751 & .38454 & .11703 \\
\hline 1200 & 1.14017 & 1.81846 & .67829 & .23171 & .33191 & .10020 \\
\hline 1300 & 1.12282 & 1.79364 & .67082 & .20236 & .28899 & .08663 \\
\hline 1400 & 1.10874 & 1.77356 & .66482 & .17807 & .25360 & .07553 \\
\hline 1500 & 1.09718 & 1.75710 & .65993 & .15778 & .22414 & .06637 \\
\hline 2000 & 1.06169 & 1.70695 & .64526 & .09397 & .13207 & .03810 \\
\hline 2500 & 1.04448 & 1.68287 & .63838 & .06252 & .08689 & .02436 \\
\hline 3000 & 1.03476 & 1.66944 & .63467 & .04508 & .06182 & .01673 \\
\hline 4000 & 1.02450 & 1.65558 & .63108 & .02794 & .03695 & .00901 \\
\hline 5000 & 1.01918 & 1.64867 & .62949 & .02049 & .02588 & .00539 \\
\hline
\end{tabular}

\begin{tabular}{|c|c|c|c|c|c|c|}
\hline $\mathrm{T}^{\circ} \mathrm{K}$ & $\frac{\mathrm{C}_{\mathrm{BD}}^{\circ}-\mathrm{C}_{\mathrm{BH}}^{\circ}}{\mathrm{R}}$ & $\frac{\mathrm{C}_{\mathrm{BT}}^{\circ}-\mathrm{C}_{\mathrm{BH}}^{\circ}}{\mathrm{R}}$ & $\frac{\mathrm{C}_{\mathrm{BT}}^{\circ}-\mathrm{C}_{\mathrm{BD}}^{\circ}}{\mathrm{R}}$ & $\frac{\mathrm{S}_{\mathrm{BD}}^{\circ}{ }^{-\mathrm{S}^{\circ}} \mathrm{BH}}{\mathrm{R}}$ & $\underbrace{\mathrm{S}^{\circ} \mathrm{BH}}_{\mathrm{BT}}$ & $\frac{\mathrm{S}_{\mathrm{BT}}^{\circ}{ }^{-\mathrm{S}^{\circ} \mathrm{BD}}}{\mathrm{R}}$ \\
\hline $\begin{array}{l}100 \\
200 \\
300 \\
400 \\
500\end{array}$ & $\begin{array}{r}-.00054 \\
.00042 \\
.01542 \\
.06012 \\
.11444\end{array}$ & $\begin{array}{r}-.00062 \\
.00325 \\
.04638 \\
.13634 \\
.22370\end{array}$ & $\begin{array}{r}-.00008 \\
.00283 \\
.03096 \\
.07622 \\
.10926\end{array}$ & $\begin{array}{l}.72931 \\
.72914 \\
.73119 \\
.74118 \\
.76050\end{array}$ & $\begin{array}{l}1.16788 \\
1.16800 \\
1.17541 \\
1.20052 \\
1.24076\end{array}$ & $\begin{array}{l}.43857 \\
.43886 \\
.44422 \\
.45934 \\
.48026\end{array}$ \\
\hline $\begin{array}{r}600 \\
700 \\
800 \\
900 \\
1000\end{array}$ & $\begin{array}{l}.15647 \\
.18023 \\
.18869 \\
.18691 \\
.17909\end{array}$ & $\begin{array}{l}.28004 \\
.30484 \\
.30720 \\
.29622 \\
.27837\end{array}$ & $\begin{array}{l}.12357 \\
.12461 \\
.11851 \\
.10931 \\
.09928\end{array}$ & $\begin{array}{l}.78535 \\
.81148 \\
.83625 \\
.85844 \\
.87777\end{array}$ & $\begin{array}{l}1.28705 \\
1.33244 \\
1.37349 \\
1.40913 \\
1.43946\end{array}$ & $\begin{array}{l}.50170 \\
.52096 \\
.53724 \\
.55069 \\
.56169\end{array}$ \\
\hline $\begin{array}{l}1100 \\
1200 \\
1300 \\
1400 \\
1500\end{array}$ & $\begin{array}{l}.16820 \\
.15606 \\
.14374 \\
.13186 \\
.12070\end{array}$ & $\begin{array}{l}.25769 \\
.23651 \\
.21608 \\
.19700 \\
.17951\end{array}$ & $\begin{array}{l}.08949 \\
.08045 \\
.07234 \\
.06514 \\
.05881\end{array}$ & $\begin{array}{l}.89435 \\
.90846 \\
.92046 \\
.93068 \\
.93939\end{array}$ & $\begin{array}{l}1.46503 \\
1.48655 \\
1.50466 \\
1.51997 \\
1.53295\end{array}$ & $\begin{array}{l}.57068 \\
.57809 \\
.58420 \\
.58929 \\
.59356\end{array}$ \\
\hline $\begin{array}{l}2000 \\
2500 \\
3000 \\
4000 \\
5000\end{array}$ & $\begin{array}{l}.07760 \\
.05109 \\
.03426 \\
.01498 \\
.00451\end{array}$ & $\begin{array}{l}.11461 \\
.07625 \\
.05250 \\
.02601 \\
.01213\end{array}$ & $\begin{array}{l}.03701 \\
.02516 \\
.01824 \\
.01103 \\
.00762\end{array}$ & $\begin{array}{l}.96772 \\
.98196 \\
.98967 \\
.99657 \\
.99869\end{array}$ & $\begin{array}{l}1.57488 \\
1.59597 \\
1.60761 \\
1.61863 \\
1.62280\end{array}$ & $\begin{array}{l}.60716 \\
.61401 \\
.61794 \\
.62206 \\
.62411\end{array}$ \\
\hline
\end{tabular}




\begin{tabular}{|c|c|c|c|c|c|c|}
\hline \multirow{2}{*}{$\mathrm{T}^{\circ} \mathrm{K}$} & $\mathrm{F}_{\mathrm{AlH}}^{\circ}-\mathrm{F}_{\mathrm{AlD}}^{\circ}$ & $\mathrm{F}_{\mathrm{AlH}}^{\circ}{ }^{-\mathrm{F}_{\mathrm{ATT}}^{\circ}}$ & $\mathrm{F}_{\mathrm{AlD}}^{\circ}{ }^{-\mathrm{F}^{\circ} \mathrm{AlT}}$ & $\mathrm{H}_{\mathrm{AlH}}^{\circ}-\mathrm{H}^{\circ} \mathrm{AlD}$ & $\mathrm{H}_{\mathrm{AlH}}^{\circ}{ }^{-\mathrm{H}^{\circ}} \mathrm{AlT}$ & $\mathrm{H}_{\mathrm{AlD}}^{\circ}{ }^{-\mathrm{H}_{\mathrm{AIT}}^{\circ}}$ \\
\hline & $\mathrm{RT}$ & $\mathrm{RT}$ & $\mathrm{RT}$ & $\mathrm{RT}$ & $\mathrm{RT}$ & $\mathrm{RT}$ \\
\hline $\begin{array}{l}100 \\
200 \\
300 \\
400 \\
500\end{array}$ & $\begin{array}{l}4.02623 \\
2.36612 \\
1.81556 \\
1.54739 \\
1.39471\end{array}$ & $\begin{array}{l}5.89831 \\
3.51236 \\
2.72453 \\
2.34493 \\
2.13141\end{array}$ & $\begin{array}{r}1.87208 \\
1.14624 \\
.90897 \\
.79755 \\
.73670\end{array}$ & $\begin{array}{r}3.32053 \\
1.65871 \\
1.09022 \\
.78487 \\
.58924\end{array}$ & $\begin{array}{r}4.77351 \\
2.38092 \\
1.55161 \\
1.10344 \\
.81929\end{array}$ & $\begin{array}{c}1.45298 \\
.72221 \\
.46139 \\
.31858 \\
.23005\end{array}$ \\
\hline $\begin{array}{r}600 \\
700 \\
800 \\
900 \\
1000\end{array}$ & $\begin{array}{l}1.29987 \\
1.23730 \\
1.19409 \\
1.16312 \\
1.14021\end{array}$ & $\begin{array}{l}2.00010 \\
1.91413 \\
1.85511 \\
1.81299 \\
1.78194\end{array}$ & $\begin{array}{l}.70023 \\
.67683 \\
.66102 \\
.64987 \\
.64173\end{array}$ & $\begin{array}{l}.45498 \\
.35948 \\
.28975 \\
.23768 \\
.19801\end{array}$ & $\begin{array}{l}.62713 \\
.49225 \\
.39479 \\
.32260 \\
.26793\end{array}$ & $\begin{array}{l}.17215 \\
.13277 \\
.10504 \\
.08492 \\
.06992\end{array}$ \\
\hline $\begin{array}{l}1100 \\
1200 \\
1300 \\
1400 \\
1500\end{array}$ & $\begin{array}{l}1.12285 \\
1.10938 \\
1.09875 \\
1.09018 \\
1.08321\end{array}$ & $\begin{array}{l}1.75848 \\
1.74032 \\
1.72599 \\
1.71450 \\
1.70514\end{array}$ & $\begin{array}{l}.63563 \\
.63093 \\
.62725 \\
.62432 \\
.62193\end{array}$ & $\begin{array}{l}.16721 \\
.14292 \\
.12348 \\
.10770 \\
.09476\end{array}$ & $\begin{array}{l}.22571 \\
.19252 \\
.16603 \\
.14460 \\
.12703\end{array}$ & $\begin{array}{l}.05850 \\
.04959 \\
.04256 \\
.03690 \\
.03227\end{array}$ \\
\hline $\begin{array}{l}2000 \\
2500 \\
3000 \\
4000 \\
5000\end{array}$ & $\begin{array}{l}1.06212 \\
1.05198 \\
1.04624 \\
1.04004 \\
1.03665\end{array}$ & $\begin{array}{l}1.67694 \\
1.66348 \\
1.65591 \\
1.64784 \\
1.64353\end{array}$ & $\begin{array}{l}.61483 \\
.61150 \\
.60967 \\
.60780 \\
.60688\end{array}$ & $\begin{array}{l}.05540 \\
.03681 \\
.02682 \\
.01732 \\
.01344\end{array}$ & $\begin{array}{l}.07379 \\
.04871 \\
.03520 \\
.02228 \\
.01690\end{array}$ & $\begin{array}{l}.01840 \\
.01190 \\
.00838 \\
.00496 \\
.00346\end{array}$ \\
\hline
\end{tabular}

\begin{tabular}{|c|c|c|c|c|c|c|}
\hline $\mathrm{T}^{\circ} \mathrm{K}$ & $\frac{\mathrm{C}_{\mathrm{AlD}}^{\circ}-\mathrm{C}_{\mathrm{AlH}}^{\circ}}{\mathrm{R}}$ & $\frac{\mathrm{C}_{\mathrm{AlT}}^{\circ}-\mathrm{C}_{\mathrm{AIH}}^{\circ}}{\mathrm{R}}$ & $\frac{\mathrm{C}_{\mathrm{AlT}}^{\circ}-\mathrm{C}_{\mathrm{AID}}^{\circ}}{\mathrm{R}}$ & $\frac{\mathrm{S}_{\mathrm{AlD}}^{\circ}{ }^{-\mathrm{S}_{\mathrm{AlH}}^{\circ}}}{\mathrm{R}}$ & $\frac{\mathrm{S}_{\mathrm{AlT}}^{\circ}-\mathrm{S}_{\mathrm{AlH}}^{\circ}}{\mathrm{R}}$ & $\frac{\mathrm{S}_{\mathrm{A} 1 \mathrm{~T}}{ }^{-\mathrm{S}_{\mathrm{A}}^{\circ} \mathrm{D}}}{\mathrm{R}}$ \\
\hline $\begin{array}{l}100 \\
200 \\
300 \\
400 \\
500\end{array}$ & $\begin{array}{r}-.00023 \\
.01360 \\
.08825 \\
.16910 \\
.21045\end{array}$ & $\begin{array}{r}-.00004 \\
.04132 \\
.17928 \\
.29115 \\
.33316\end{array}$ & $\begin{array}{l}.00019 \\
.02772 \\
.09103 \\
.12205 \\
.12271\end{array}$ & $\begin{array}{l}.70570 \\
.70741 \\
.72534 \\
.76252 \\
.80546\end{array}$ & $\begin{array}{l}1.12481 \\
1.13144 \\
1.17292 \\
1.24149 \\
1.31211\end{array}$ & $\begin{array}{l}.41911 \\
.42403 \\
.44758 \\
.47897 \\
.50665\end{array}$ \\
\hline $\begin{array}{r}600 \\
700 \\
800 \\
900 \\
1000\end{array}$ & $\begin{array}{l}.21793 \\
.20716 \\
.18892 \\
.16886 \\
.14961\end{array}$ & $\begin{array}{l}.32880 \\
.30330 \\
.27116 \\
.23907 \\
.20974\end{array}$ & $\begin{array}{l}.11087 \\
.09614 \\
.08224 \\
.07021 \\
.06013\end{array}$ & $\begin{array}{l}.84489 \\
.87782 \\
.90434 \\
.92543 \\
.94221\end{array}$ & $\begin{array}{l}1.37296 \\
1.42188 \\
1.46031 \\
1.49038 \\
1.51402\end{array}$ & $\begin{array}{l}.52807 \\
.54406 \\
.55597 \\
.56495 \\
.57181\end{array}$ \\
\hline $\begin{array}{l}1100 \\
1200 \\
1300 \\
1400 \\
1500\end{array}$ & $\begin{array}{l}.13214 \\
.11673 \\
.10330 \\
.09166 \\
.08157\end{array}$ & $\begin{array}{l}.18394 \\
.16164 \\
.14249 \\
.12608 \\
.11198\end{array}$ & $\begin{array}{l}.05180 \\
.04491 \\
.03919 \\
.03442 \\
.03041\end{array}$ & $\begin{array}{l}.95564 \\
.96646 \\
.97526 \\
.98248 \\
.98846\end{array}$ & $\begin{array}{l}1.53278 \\
1.54779 \\
1.55995 \\
1.56990 \\
1.57811\end{array}$ & $\begin{array}{l}.57714 \\
.58133 \\
.58469 \\
.58742 \\
.58965\end{array}$ \\
\hline $\begin{array}{l}2000 \\
2500 \\
3000 \\
4000 \\
5000\end{array}$ & $\begin{array}{l}.04755 \\
.02914 \\
.01808 \\
.00572 \\
.00105\end{array}$ & $\begin{array}{l}.06520 \\
.04034 \\
.02559 \\
.00934 \\
.00064\end{array}$ & $\begin{array}{l}.01765 \\
.01120 \\
.00751 \\
.00362 \\
.00169\end{array}$ & $\begin{array}{l}1.00672 \\
1.01516 \\
1.01943 \\
1.02272 \\
1.02321\end{array}$ & $\begin{array}{l}1.60315 \\
1.61476 \\
1.62071 \\
1.62556 \\
1.62663\end{array}$ & $\begin{array}{l}.59643 \\
.59960 \\
.60128 \\
.60284 \\
.60342\end{array}$ \\
\hline
\end{tabular}


Table 3.24

\begin{tabular}{|c|c|c|c|c|c|c|}
\hline \multirow{2}{*}{$\mathrm{T}^{\circ} \mathrm{K}$} & $\mathrm{F}_{\mathrm{InH}}^{\circ}-\mathrm{F}_{\mathrm{InD}}^{\circ}$ & $\mathrm{F}_{\text {InH }}^{\circ}-\mathrm{F}_{\text {In } \mathrm{T}}^{\circ}$ & $\mathrm{F}_{\mathrm{InD}}^{\circ}-\mathrm{F}_{\mathrm{InT}}^{\circ}$ & $\mathrm{H}_{\mathrm{InH}}^{\circ}{ }^{-\mathrm{H}_{\mathrm{InD}}^{\circ}}$ & $\mathrm{H}_{\mathrm{InH}}^{\circ}{ }^{-\mathrm{H}^{\circ}} \mathrm{InT}$ & $\mathrm{H}_{\mathrm{InD}}^{\circ}-\mathrm{H}_{\mathrm{InT}}^{\circ}$ \\
\hline & $\mathrm{RT}$ & $\mathrm{RT}$ & $\mathrm{RT}$ & $\mathrm{RT}$ & $\mathrm{RT}$ & RT \\
\hline $\begin{array}{l}100 \\
200 \\
300 \\
400 \\
500\end{array}$ & $\begin{array}{l}3.71162 \\
2.20503 \\
1.70850 \\
1.47084 \\
1.33836\end{array}$ & $\begin{array}{l}5.44932 \\
3.27810 \\
2.56818 \\
2.23332 \\
2.04912\end{array}$ & $\begin{array}{r}1.73770 \\
1.07307 \\
.85968 \\
.76248 \\
.71076\end{array}$ & $\begin{array}{r}3.01437 \\
1.50279 \\
.97497 \\
.68751 \\
.50585\end{array}$ & $\begin{array}{r}4.34701 \\
2.15954 \\
1.38292 \\
.96114 \\
.69912\end{array}$ & $\begin{array}{r}1.33264 \\
.65675 \\
.40795 \\
.27363 \\
.19327\end{array}$ \\
\hline $\begin{array}{r}600 \\
700 \\
800 \\
900 \\
1000\end{array}$ & $\begin{array}{l}1.25759 \\
1.20510 \\
1.16927 \\
1.14381 \\
1.12513\end{array}$ & $\begin{array}{l}1.93795 \\
1.86622 \\
1.81751 \\
1.78302 \\
1.75780\end{array}$ & $\begin{array}{l}.68036 \\
.66112 \\
.64824 \\
.63921 \\
.63267\end{array}$ & $\begin{array}{l}.38415 \\
.29959 \\
.23904 \\
.19454 \\
.16104\end{array}$ & $\begin{array}{l}.52652 \\
.40817 \\
.32426 \\
.26302 \\
.21719\end{array}$ & $\begin{array}{l}.14237 \\
.10858 \\
.08522 \\
.06848 \\
.05615\end{array}$ \\
\hline $\begin{array}{l}1100 \\
1200 \\
1300 \\
1400 \\
1500\end{array}$ & $\begin{array}{l}1.11104 \\
1.10016 \\
1.09160 \\
1.08474 \\
1.07918\end{array}$ & $\begin{array}{l}1.73882 \\
1.72419 \\
1.71269 \\
1.70349 \\
1.69603\end{array}$ & $\begin{array}{l}.62778 \\
.62402 \\
.62110 \\
.61875 \\
.61685\end{array}$ & $\begin{array}{l}.13529 \\
.11513 \\
.09911 \\
.08617 \\
.07559\end{array}$ & $\begin{array}{l}.18212 \\
.15475 \\
.13304 \\
.11556 \\
.10129\end{array}$ & $\begin{array}{l}.04683 \\
.03961 \\
.03394 \\
.02939 \\
.02570\end{array}$ \\
\hline $\begin{array}{l}2000 \\
2500 \\
3000 \\
4000 \\
5000\end{array}$ & $\begin{array}{l}1.06245 \\
1.05450 \\
1.05006 \\
1.04536 \\
1.04289\end{array}$ & $\begin{array}{l}1.67363 \\
1.66301 \\
1.65706 \\
1.65077 \\
1.64743\end{array}$ & $\begin{array}{l}.61118 \\
.60850 \\
.60700 \\
.60541 \\
.60454\end{array}$ & $\begin{array}{l}.04365 \\
.02865 \\
.02059 \\
.01285 \\
.00959\end{array}$ & $\begin{array}{l}.05837 \\
.03832 \\
.02756 \\
.01728 \\
.01298\end{array}$ & $\begin{array}{l}.01472 \\
.00966 \\
.00697 \\
.00443 \\
.00339\end{array}$ \\
\hline
\end{tabular}

\begin{tabular}{|c|c|c|c|c|c|c|}
\hline & $\mathrm{C}_{\text {InD }}^{\circ}-\mathrm{C}_{\mathrm{InH}}^{\circ}$ & $\mathrm{C}_{\mathrm{InT}}^{\circ}{ }^{-\mathrm{C}_{\mathrm{InH}}^{\circ}}$ & $\mathrm{C}_{\operatorname{InT}}^{\circ}-\mathrm{C}_{\operatorname{InD}}^{\circ}$ & $\mathrm{S}_{\mathrm{InD}}^{\circ}{ }^{-\mathrm{S}_{\mathrm{InH}}^{\circ}}$ & $\mathrm{S}_{\mathrm{InT}}^{\circ}{ }^{-\mathrm{S}_{\mathrm{InH}}^{\circ}}$ & $\mathrm{S}_{\text {In } \mathrm{T}^{\circ}}^{-\mathrm{S}_{\mathrm{InD}}^{\circ}}$ \\
\hline$T^{K}$ & $\bar{R}$ & $\mathrm{R}$ & $\mathrm{R}$ & $\mathrm{R}$ & $\mathrm{R}$ & $\mathrm{R}$ \\
\hline $\begin{array}{l}100 \\
200 \\
300 \\
400 \\
500\end{array}$ & $\begin{array}{l}.00003 \\
.03144 \\
.13330 \\
.20684 \\
.22755\end{array}$ & $\begin{array}{l}.00073 \\
.08366 \\
.25197 \\
.33990 \\
.34866\end{array}$ & $\begin{array}{l}.00070 \\
.05222 \\
.11867 \\
.13306 \\
.12111\end{array}$ & $\begin{array}{l}.69724 \\
.70224 \\
.73353 \\
.78334 \\
.83251\end{array}$ & $\begin{array}{l}1.10231 \\
1.11856 \\
1.18526 \\
1.27218 \\
1.35001\end{array}$ & $\begin{array}{l}.40507 \\
.41632 \\
.45173 \\
.48884 \\
.51750\end{array}$ \\
\hline $\begin{array}{r}600 \\
700 \\
800 \\
900 \\
1000\end{array}$ & $\begin{array}{l}.21795 \\
.19663 \\
.17290 \\
.15056 \\
.13084\end{array}$ & $\begin{array}{l}.32090 \\
.28243 \\
.24428 \\
.21028 \\
.18121\end{array}$ & $\begin{array}{l}.10295 \\
.08580 \\
.07138 \\
.05972 \\
.05037\end{array}$ & $\begin{array}{l}.87344 \\
.90551 \\
.93022 \\
.94927 \\
.96409\end{array}$ & $\begin{array}{l}1.41143 \\
1.45805 \\
1.49324 \\
1.52001 \\
1.54061\end{array}$ & $\begin{array}{l}.53799 \\
.55254 \\
.56302 \\
.57074 \\
.57652\end{array}$ \\
\hline $\begin{array}{l}1100 \\
1200 \\
1300 \\
1400 \\
1500\end{array}$ & $\begin{array}{l}.11391 \\
.09953 \\
.08735 \\
.07703 \\
.06824\end{array}$ & $\begin{array}{l}.15676 \\
.13629 \\
.11914 \\
.10471 \\
.09251\end{array}$ & $\begin{array}{l}.04285 \\
.03676 \\
.03179 \\
.02768 \\
.02427\end{array}$ & $\begin{array}{r}.97574 \\
.98502 \\
.99249 \\
.99858 \\
1.00359\end{array}$ & $\begin{array}{l}1.55670 \\
1.56943 \\
1.57964 \\
1.58794 \\
1.59473\end{array}$ & $\begin{array}{l}.58096 \\
.58441 \\
.58715 \\
.58936 \\
.59114\end{array}$ \\
\hline $\begin{array}{l}2000 \\
2500 \\
3000 \\
4000 \\
5000\end{array}$ & $\begin{array}{l}.03951 \\
.02452 \\
.01574 \\
.00617 \\
.00114\end{array}$ & $\begin{array}{l}.05305 \\
.03269 \\
.02080 \\
.00789 \\
.00111\end{array}$ & $\begin{array}{r}.01354 \\
.00817 \\
.00506 \\
.00172 \\
-.00003\end{array}$ & $\begin{array}{l}1.01880 \\
1.02584 \\
1.02947 \\
1.03252 \\
1.03331\end{array}$ & $\begin{array}{l}1.61526 \\
1.62468 \\
1.62951 \\
1.63349 \\
1.63446\end{array}$ & $\begin{array}{l}.59646 \\
.59884 \\
.60004 \\
.60097 \\
.60115\end{array}$ \\
\hline
\end{tabular}


Table 3.25

\begin{tabular}{|c|c|c|c|c|c|c|}
\hline $\mathrm{T}^{\circ} \mathrm{K}$ & $\frac{\mathrm{F}_{\mathrm{TlH}}^{\circ}-\mathrm{F}_{\mathrm{TlD}}^{\circ}}{\mathrm{RT}}$ & $\frac{\mathrm{F}_{\mathrm{T} 1 \mathrm{H}}^{\circ}-\mathrm{F}_{\mathrm{T} 1 \mathrm{~T}}^{\circ}}{\mathrm{RT}}$ & $\frac{\mathrm{F}_{\mathrm{TlD}}^{\circ}-\mathrm{F}_{\mathrm{TlT}}^{\circ}}{\mathrm{RT}}$ & $\frac{\mathrm{H}_{\mathrm{TlH}}^{\circ}-\mathrm{H}_{\mathrm{TlD}}^{\circ}}{\mathrm{RT}}$ & $\frac{\mathrm{H}_{\mathrm{TlH}}^{\circ}-\mathrm{H}_{\mathrm{TlT}}^{\circ}}{\mathrm{RT}}$ & $\frac{\mathrm{H}_{\mathrm{TlD}}^{\circ}-\mathrm{H}_{\mathrm{TlT}}^{\circ}}{\mathrm{RT}}$ \\
\hline $\begin{array}{l}100 \\
200 \\
300 \\
400 \\
500\end{array}$ & $\begin{array}{l}3.54854 \\
2.12037 \\
1.65158 \\
1.42929 \\
1.30662\end{array}$ & $\begin{array}{l}5.21798 \\
3.15971 \\
2.49061 \\
2.17827 \\
2.00815\end{array}$ & $\begin{array}{r}1.66954 \\
1.03939 \\
.83907 \\
.74901 \\
.70155\end{array}$ & $\begin{array}{r}2.85819 \\
1.42279 \\
.91609 \\
.63940 \\
.46615\end{array}$ & $\begin{array}{r}4.12310 \\
2.04273 \\
1.29606 \\
.89136 \\
.64282\end{array}$ & $\begin{array}{r}1.26501 \\
.61999 \\
.38001 \\
.25199 \\
.17669\end{array}$ \\
\hline $\begin{array}{r}600 \\
700 \\
800 \\
900 \\
1000\end{array}$ & $\begin{array}{l}1.23245 \\
1.18457 \\
1.15205 \\
1.12903 \\
1.11222\end{array}$ & $\begin{array}{l}1.90625 \\
1.84086 \\
1.79661 \\
1.76538 \\
1.74257\end{array}$ & $\begin{array}{l}.67381 \\
.65630 \\
.64457 \\
.63636 \\
.63036\end{array}$ & $\begin{array}{l}.35141 \\
.27249 \\
.21643 \\
.17545 \\
.14477\end{array}$ & $\begin{array}{l}.48106 \\
.37125 \\
.29402 \\
.23800 \\
.19631\end{array}$ & $\begin{array}{l}.12966 \\
.09877 \\
.07760 \\
.06256 \\
.05155\end{array}$ \\
\hline $\begin{array}{l}1100 \\
1200 \\
1300 \\
1400 \\
1500\end{array}$ & $\begin{array}{l}1.09956 \\
1.08983 \\
1.08219 \\
1.07608 \\
1.07115\end{array}$ & $\begin{array}{l}1.72541 \\
1.71220 \\
1.70181 \\
1.69348 \\
1.68673\end{array}$ & $\begin{array}{l}.62585 \\
.62237 \\
.61963 \\
.61741 \\
.61558\end{array}$ & $\begin{array}{l}.12126 \\
.10291 \\
.08835 \\
.07660 \\
.06702\end{array}$ & $\begin{array}{l}.16453 \\
.13984 \\
.12031 \\
.10463 \\
.09188\end{array}$ & $\begin{array}{l}.04327 \\
.03693 \\
.03197 \\
.02804 \\
.02486\end{array}$ \\
\hline $\begin{array}{l}2000 \\
2500 \\
3000 \\
4000 \\
5000\end{array}$ & $\begin{array}{l}1.05641 \\
1.04953 \\
1.04578 \\
1.04201 \\
1.04019\end{array}$ & $\begin{array}{l}1.66628 \\
1.65637 \\
1.65063 \\
1.64415 \\
1.64030\end{array}$ & $\begin{array}{l}.60988 \\
.60685 \\
.60486 \\
.60214 \\
.60011\end{array}$ & $\begin{array}{l}.03811 \\
.02449 \\
.01710 \\
.00987 \\
.00666\end{array}$ & $\begin{array}{l}.05383 \\
.03635 \\
.02720 \\
.01891 \\
.01591\end{array}$ & $\begin{array}{l}.01573 \\
.01187 \\
.01011 \\
.00904 \\
.00925\end{array}$ \\
\hline
\end{tabular}

\begin{tabular}{|c|c|c|c|c|c|c|}
\hline \multirow{2}{*}{$\mathrm{T}^{\circ} \mathrm{K}$} & $\mathrm{C}_{\mathrm{TlD}}^{\circ}{ }^{-\mathrm{C}_{\mathrm{TlH}}^{\circ}}$ & $\mathrm{C}_{\mathrm{TlT}}^{\circ}-\mathrm{C}_{\mathrm{TlH}}^{\circ}$ & $\mathrm{C}_{\mathrm{T} 1 \mathrm{~T}}^{\circ}{ }^{-\mathrm{C}_{\mathrm{T}}^{\circ} \mathrm{D}}$ & $\mathrm{S}_{\mathrm{T} 1 \mathrm{D}}^{\circ}{ }^{-\mathrm{S}_{\mathrm{T} 1 \mathrm{H}}^{\circ}}$ & $\mathrm{S}_{\mathrm{TlT}}^{\circ}{ }^{-\mathrm{S}_{\mathrm{T} 1 \mathrm{H}}^{\circ}}$ & $\mathrm{S}_{\mathrm{TlT}}^{\circ}-\mathrm{S}_{\mathrm{TlD}}^{\circ}$ \\
\hline & $\mathrm{R}$ & $\mathrm{R}$ & $\mathrm{R}$ & $\mathrm{R}$ & $\mathrm{R}$ & $\mathrm{R}$ \\
\hline $\begin{array}{l}100 \\
200 \\
300 \\
400 \\
500\end{array}$ & $\begin{array}{l}.00011 \\
.04218 \\
.15236 \\
.21803 \\
.22894\end{array}$ & $\begin{array}{l}.00139 \\
.10563 \\
.27811 \\
.34995 \\
.34443\end{array}$ & $\begin{array}{l}.00128 \\
.06345 \\
.12575 \\
.13192 \\
.11549\end{array}$ & $\begin{array}{l}.69035 \\
.69758 \\
.73548 \\
.78990 \\
.84047\end{array}$ & $\begin{array}{l}1.09489 \\
1.11697 \\
1.19454 \\
1.28692 \\
1.36534\end{array}$ & $\begin{array}{l}.40454 \\
.41939 \\
.45906 \\
.49702 \\
.52487\end{array}$ \\
\hline $\begin{array}{r}600 \\
700 \\
800 \\
900 \\
1000\end{array}$ & $\begin{array}{l}.21301 \\
.18858 \\
.16372 \\
.14133 \\
.12210\end{array}$ & $\begin{array}{l}.30862 \\
.26660 \\
.22742 \\
.19362 \\
.16532\end{array}$ & $\begin{array}{l}.09561 \\
.07802 \\
.06370 \\
.05229 \\
.04322\end{array}$ & $\begin{array}{l}.88104 \\
.91207 \\
.93561 \\
.95358 \\
.96745\end{array}$ & $\begin{array}{l}1.42519 \\
1.46960 \\
1.50259 \\
1.52738 \\
1.54627\end{array}$ & $\begin{array}{l}.54415 \\
.55753 \\
.56698 \\
.57380 \\
.57882\end{array}$ \\
\hline $\begin{array}{l}1100 \\
1200 \\
1300 \\
1400 \\
1500\end{array}$ & $\begin{array}{l}.10589 \\
.09231 \\
.08094 \\
.07136 \\
.06328\end{array}$ & $\begin{array}{l}.14183 \\
.12236 \\
.10615 \\
.09255 \\
.08109\end{array}$ & $\begin{array}{l}.03594 \\
.03005 \\
.02521 \\
.02119 \\
.01781\end{array}$ & $\begin{array}{r}.97830 \\
.98691 \\
.99384 \\
.99949 \\
1.00412\end{array}$ & $\begin{array}{l}1.56089 \\
1.57236 \\
1.58150 \\
1.58886 \\
1.59484\end{array}$ & $\begin{array}{l}.58259 \\
.58545 \\
.58766 \\
.58937 \\
.59072\end{array}$ \\
\hline $\begin{array}{l}2000 \\
2500 \\
3000 \\
4000 \\
5000\end{array}$ & $\begin{array}{l}.03724 \\
.02397 \\
.01635 \\
.00837 \\
.00442\end{array}$ & $\begin{array}{r}.04407 \\
.02476 \\
.01322 \\
.00013 \\
-.00730\end{array}$ & $\begin{array}{r}.00683 \\
.00079 \\
-.00313 \\
-.00824 \\
-.01172\end{array}$ & $\begin{array}{l}1.01830 \\
1.02504 \\
1.02868 \\
1.03214 \\
1.03353\end{array}$ & $\begin{array}{l}1.61246 \\
1.62001 \\
1.62343 \\
1.62523 \\
1.62439\end{array}$ & $\begin{array}{l}.59416 \\
.59497 \\
.59475 \\
.59309 \\
.59086\end{array}$ \\
\hline
\end{tabular}


Table 3.26

\begin{tabular}{|c|c|c|c|c|c|c|}
\hline $\mathrm{T}^{\circ} \mathrm{K}$ & $\frac{\mathrm{F}_{\mathrm{CH}}^{\circ}-\mathrm{F}_{\mathrm{CD}}^{\circ}}{\mathrm{RT}}$ & $\frac{\mathrm{F}_{\mathrm{CH}}^{\circ}-\mathrm{F}_{\mathrm{CT}}^{\circ}}{\mathrm{RT}}$ & $\frac{\mathrm{F}_{\mathrm{CD}}^{\circ}-\mathrm{F}_{\mathrm{C} T}^{\circ}}{\mathrm{RT}}$ & $\frac{\mathrm{H}_{\mathrm{CH}}^{\circ}-\mathrm{H}_{\mathrm{CD}}^{\circ}}{\mathrm{RT}}$ & $\frac{{ }_{\mathrm{CH}}^{\circ}-\mathrm{H}_{\mathrm{C}}^{\circ}}{\mathrm{RT}}$ & $\frac{\mathrm{H}_{\mathrm{CD}}^{\circ}-\mathrm{H}_{\mathrm{C} T}^{\circ}}{\mathrm{RT}}$ \\
\hline $\begin{array}{l}100 \\
200 \\
300 \\
400 \\
500\end{array}$ & $\begin{array}{l}6.06121 \\
3.39059 \\
2.50117 \\
2.05708 \\
1.79227\end{array}$ & $\begin{array}{l}8.82611 \\
4.99189 \\
3.71492 \\
3.07820 \\
2.69987\end{array}$ & $\begin{array}{r}2.76480 \\
1.60125 \\
1.21374 \\
1.02109 \\
.90758\end{array}$ & $\begin{array}{l}5.34464 \\
2.66901 \\
1.77818 \\
1.32933 \\
1.05264\end{array}$ & $\begin{array}{l}7.67257 \\
3.83221 \\
2.55196 \\
1.90310 \\
1.49968\end{array}$ & $\begin{array}{r}2.32783 \\
1.16315 \\
.77377 \\
.57374 \\
.44702\end{array}$ \\
\hline $\begin{array}{r}600 \\
700 \\
800 \\
900 \\
1000\end{array}$ & $\begin{array}{l}1.61831 \\
1.49705 \\
1.40903 \\
1.34320 \\
1.29281\end{array}$ & $\begin{array}{l}2.45273 \\
2.28156 \\
2.15804 \\
2.06618 \\
1.99617\end{array}$ & $\begin{array}{l}.83441 \\
.78450 \\
.74899 \\
.72297 \\
.70334\end{array}$ & $\begin{array}{l}.86003 \\
.71606 \\
.60410 \\
.51495 \\
.44290\end{array}$ & $\begin{array}{r}1.21777 \\
1.00759 \\
.84517 \\
.71685 \\
.61394\end{array}$ & $\begin{array}{l}.35773 \\
.29152 \\
.24105 \\
.20189 \\
.17102\end{array}$ \\
\hline $\begin{array}{l}1100 \\
1200 \\
1300 \\
1400 \\
1500\end{array}$ & $\begin{array}{l}1.25344 \\
1.22219 \\
1.19698 \\
1.17639 \\
1.15941\end{array}$ & $\begin{array}{l}1.94171 \\
1.89859 \\
1.86392 \\
1.83568 \\
1.81238\end{array}$ & $\begin{array}{l}.68826 \\
.67639 \\
.66693 \\
.65928 \\
.65297\end{array}$ & $\begin{array}{l}.38403 \\
.33543 \\
.29496 \\
.26105 \\
.23236\end{array}$ & $\begin{array}{l}.53042 \\
.46191 \\
.40518 \\
.35784 \\
.31795\end{array}$ & $\begin{array}{l}.14638 \\
.12647 \\
.11021 \\
.09678 \\
.08559\end{array}$ \\
\hline $\begin{array}{l}2000 \\
2500 \\
3000 \\
4000 \\
5000\end{array}$ & $\begin{array}{l}1.10685 \\
1.08125 \\
1.06695 \\
1.05236 \\
1.04533\end{array}$ & $\begin{array}{l}1.74069 \\
1.70594 \\
1.68661 \\
1.66703 \\
1.65775\end{array}$ & $\begin{array}{l}.63384 \\
.62469 \\
.61965 \\
.61466 \\
.61241\end{array}$ & $\begin{array}{l}.13983 \\
.09259 \\
.06571 \\
.03833 \\
.02567\end{array}$ & $\begin{array}{l}.19019 \\
.12541 \\
.08863 \\
.05104 \\
.03347\end{array}$ & $\begin{array}{l}.05036 \\
.03282 \\
.02291 \\
.01270 \\
.00779\end{array}$ \\
\hline
\end{tabular}

$\mathrm{T}^{\circ} \mathrm{K} \quad \frac{\mathrm{C}_{\mathrm{CD}}^{\circ}-\mathrm{C}_{\mathrm{CH}}^{\circ}}{\mathrm{R}} \quad \frac{\mathrm{C}_{\mathrm{CT}}^{\circ}-\mathrm{C}_{\mathrm{CH}}^{\circ}}{\mathrm{R}} \quad \frac{\mathrm{C}_{\mathrm{CT}}^{\circ}-\mathrm{C}_{\mathrm{CD}}^{\circ}}{\mathrm{R}}$

$\frac{\mathrm{S}_{\mathrm{CD}}^{\circ}-\mathrm{S}_{\mathrm{CH}}^{\circ}}{R} \quad \frac{\mathrm{S}_{\mathrm{C} T}^{\circ}-\mathrm{S}_{\mathrm{CH}}^{\circ}}{R} \quad \frac{\mathrm{S}_{\mathrm{C} T}^{\circ}-\mathrm{S}_{\mathrm{CD}}^{\circ}}{\mathrm{R}}$

$\begin{array}{rllllll}100 & .01439 & .01759 & .00320 & .71657 & 1.15354 & .43697 \\ 200 & .00307 & .00425 & .00118 & .72158 & 1.15968 & .43810 \\ 300 & .00660 & .01882 & .01222 & .72299 & 1.16296 & .43997 \\ 400 & .03215 & .07482 & .04267 & .72775 & 1.17510 & .44735 \\ 500 & .07810 & .15416 & .07606 & .73963 & 1.20019 & .46056 \\ & & & & & \\ 600 & .12708 & .22633 & .09925 & .75828 & 1.23496 & .47668 \\ 700 & .16605 & .27650 & .11045 & .78099 & 1.27397 & .49298 \\ 800 & .19101 & .30378 & .11277 & .80493 & 1.31287 & .50794 \\ 900 & .20339 & .31299 & .10960 & .82825 & 1.34933 & .52108 \\ 1000 & .20625 & .30981 & .10356 & .84991 & 1.38223 & .53232 \\ 1100 & .20270 & .29898 & .09628 & .86941 & 1.41129 & .54188 \\ 1200 & .19520 & .28389 & .08869 & .88676 & 1.43668 & .54992 \\ 1300 & .18547 & .26678 & .08131 & .90202 & 1.45874 & .55672 \\ 1400 & .17466 & .24909 & .07443 & .91534 & 1.47784 & .56250 \\ 1500 & .16358 & .23167 & .06809 & .92705 & 1.49443 & .56738 \\ 2000 & .11443 & .15911 & .04468 & .96702 & 1.55050 & .58348 \\ 2500 & .08061 & .11169 & .03108 & .98866 & 1.58053 & .59187 \\ 3000 & .05825 & .08111 & .02286 & 1.00124 & 1.59798 & .59674 \\ 4000 & .03242 & .04657 & .01415 & 1.01403 & 1.61599 & .60196 \\ 5000 & .01879 & .02881 & .01002 & 1.01966 & 1.62428 & .60462\end{array}$




\begin{tabular}{|c|c|c|c|c|c|c|}
\hline & $\mathrm{F}_{\mathrm{SiH}}^{\circ} \mathrm{F}_{\mathrm{SiD}}^{\circ}$ & $\mathrm{F}_{\mathrm{SiH}}^{\circ} \quad \mathrm{F}_{\mathrm{SiT}}^{\circ}$ & $\mathrm{F}_{\mathrm{SiD}}^{\circ} \mathrm{F}_{\mathrm{SiT}}^{\circ}$ & $\mathrm{H}_{\mathrm{SiH}}^{\circ} \mathrm{H}_{\mathrm{SiD}}^{\circ}$ & $\mathrm{H}_{\mathrm{SiH}}^{\circ} \quad \mathrm{H}_{\mathrm{SiT}}^{\circ}$ & $\mathrm{H}_{\mathrm{SiD}}^{\circ} \quad \mathrm{H}_{\mathrm{SiT}}^{\circ}$ \\
\hline$T^{2} \mathrm{~K}$ & $\mathrm{RT}^{\prime}$ & $\mathrm{RT}$ & $\mathrm{RT}^{\mathrm{T}}$ & $\mathrm{RT}^{\mathrm{T}}$ & $\mathrm{RT}$ & $\mathrm{RT}$ \\
\hline $\begin{array}{l}100 \\
200 \\
300 \\
400 \\
500\end{array}$ & $\begin{array}{l}4.75492 \\
2.72745 \\
2.05373 \\
1.72078 \\
1.52687\end{array}$ & $\begin{array}{l}6.91888 \\
4.01868 \\
3.05668 \\
2.58476 \\
2.31294\end{array}$ & $\begin{array}{r}2.16396 \\
1.29122 \\
1.00295 \\
.86398 \\
.78607\end{array}$ & $\begin{array}{r}4.05990 \\
2.02458 \\
1.34218 \\
.98600 \\
.75802\end{array}$ & $\begin{array}{l}5.80743 \\
2.89515 \\
1.91036 \\
1.38952 \\
1.05612\end{array}$ & $\begin{array}{r}1.74753 \\
.87056 \\
.56818 \\
.40352 \\
.29810\end{array}$ \\
\hline $\begin{array}{r}600 \\
700 \\
800 \\
900 \\
1000\end{array}$ & $\begin{array}{l}1.40360 \\
1.32066 \\
1.26247 \\
1.22027 \\
1.18881\end{array}$ & $\begin{array}{l}2.14206 \\
2.02819 \\
1.94895 \\
1.89188 \\
1.84955\end{array}$ & $\begin{array}{l}.73845 \\
.70753 \\
.68648 \\
.67160 \\
.66074\end{array}$ & $\begin{array}{l}.59803 \\
.48083 \\
.39282 \\
.32550 \\
.27313\end{array}$ & $\begin{array}{l}.82455 \\
.65710 \\
.53285 \\
.43874 \\
.36616\end{array}$ & $\begin{array}{l}.22651 \\
.17627 \\
.14003 \\
.11323 \\
.09303\end{array}$ \\
\hline $\begin{array}{l}1100 \\
1200 \\
1300 \\
1400 \\
1500\end{array}$ & $\begin{array}{l}1.16481 \\
1.14608 \\
1.13127 \\
1.11934 \\
1.10962\end{array}$ & $\begin{array}{l}1.81746 \\
1.79255 \\
1.77290 \\
1.75715 \\
1.74437\end{array}$ & $\begin{array}{l}.65265 \\
.64648 \\
.64163 \\
.63781 \\
.63475\end{array}$ & $\begin{array}{l}.23181 \\
.19875 \\
.17199 \\
.15007 \\
.13194\end{array}$ & $\begin{array}{l}.30928 \\
.26401 \\
.22753 \\
.19777 \\
.17320\end{array}$ & $\begin{array}{l}.07747 \\
.06527 \\
.05554 \\
.04770 \\
.04126\end{array}$ \\
\hline $\begin{array}{l}2000 \\
2500 \\
3000 \\
4000 \\
5000\end{array}$ & $\begin{array}{l}1.08041 \\
1.06673 \\
1.05927 \\
1.05187 \\
1.04840\end{array}$ & $\begin{array}{l}1.70633 \\
1.68888 \\
1.67963 \\
1.67085 \\
1.66713\end{array}$ & $\begin{array}{l}.62592 \\
.62214 \\
.62036 \\
.61899 \\
.61874\end{array}$ & $\begin{array}{l}.07586 \\
.04876 \\
.03385 \\
.01916 \\
.01263\end{array}$ & $\begin{array}{l}.09770 \\
.06138 \\
.04141 \\
.02157 \\
.01251\end{array}$ & $\begin{array}{l}.02184 \\
.01261 \\
.00756 \\
.00242 \\
.00011\end{array}$ \\
\hline
\end{tabular}

\begin{tabular}{|c|c|c|c|c|c|c|}
\hline \multirow{2}{*}{$\mathrm{T}^{\circ} \mathrm{K}$} & $\mathrm{C}_{\mathrm{SiD}}^{\circ}-\mathrm{C}_{\mathrm{SiH}}^{\circ}$ & $\mathrm{C}_{\mathrm{SiT}}^{\circ}{ }^{-\mathrm{C}_{\mathrm{SiH}}^{\circ}}$ & $\mathrm{C}_{\mathrm{SiT}}^{\circ}-\mathrm{C}_{\mathrm{SiD}}^{\circ}$ & $\mathrm{S}_{\mathrm{SiD}}^{\circ}-\mathrm{S}_{\mathrm{SiH}}^{\circ}$ & $\mathrm{S}_{\mathrm{SiT}}^{\circ}{ }^{-\mathrm{S}_{\mathrm{SiH}}^{\circ}}$ & $\mathrm{S}_{\mathrm{SiT}}^{\circ}-\mathrm{S}_{\mathrm{SiD}}^{\circ}$ \\
\hline & $\mathrm{R}$ & $\mathrm{R}$ & $\mathrm{R}$ & $\mathrm{R}$ & $\mathrm{R}$ & $\mathrm{R}$ \\
\hline $\begin{array}{l}100 \\
200 \\
300 \\
400 \\
500\end{array}$ & $\begin{array}{l}.01791 \\
.00851 \\
.04633 \\
.12037 \\
.18287\end{array}$ & $\begin{array}{l}.02388 \\
.02176 \\
.11034 \\
.23232 \\
.31365\end{array}$ & $\begin{array}{l}.00597 \\
.01325 \\
.06451 \\
.11195 \\
.13078\end{array}$ & $\begin{array}{l}.69502 \\
.70288 \\
.71155 \\
.73478 \\
.76885\end{array}$ & $\begin{array}{l}1.11145 \\
1.12353 \\
1.14633 \\
1.19525 \\
1.25682\end{array}$ & $\begin{array}{l}.41643 \\
.42065 \\
.43478 \\
.46047 \\
.48797\end{array}$ \\
\hline $\begin{array}{r}600 \\
700 \\
800 \\
900 \\
1000\end{array}$ & $\begin{array}{l}.21615 \\
.22525 \\
.21950 \\
.20624 \\
.18989\end{array}$ & $\begin{array}{l}.34599 \\
.34517 \\
.32679 \\
.30094 \\
.27317\end{array}$ & $\begin{array}{l}.12984 \\
.11992 \\
.10729 \\
.09470 \\
.08328\end{array}$ & $\begin{array}{l}.80557 \\
.83982 \\
.86965 \\
.89478 \\
.91568\end{array}$ & $\begin{array}{l}1.31751 \\
1.37108 \\
1.41610 \\
1.45314 \\
1.48339\end{array}$ & $\begin{array}{l}.51194 \\
.53126 \\
.54645 \\
.55836 \\
.56771\end{array}$ \\
\hline $\begin{array}{l}1100 \\
1200 \\
1300 \\
1400 \\
1500\end{array}$ & $\begin{array}{l}.17299 \\
.15675 \\
.14176 \\
.12820 \\
.11605\end{array}$ & $\begin{array}{l}.24631 \\
.22154 \\
.19928 \\
.17951 \\
.16210\end{array}$ & $\begin{array}{l}.07332 \\
.06479 \\
.05752 \\
.05131 \\
.04605\end{array}$ & $\begin{array}{l}.93300 \\
.94733 \\
.95928 \\
.96926 \\
.97768\end{array}$ & $\begin{array}{l}1.50819 \\
1.52854 \\
1.54537 \\
1.55938 \\
1.57117\end{array}$ & $\begin{array}{l}.57519 \\
.58121 \\
.58609 \\
.59012 \\
.59349\end{array}$ \\
\hline $\begin{array}{l}2000 \\
2500 \\
3000 \\
4000 \\
5000\end{array}$ & $\begin{array}{l}.07291 \\
.04858 \\
.03388 \\
.01798 \\
.00992\end{array}$ & $\begin{array}{l}.10189 \\
.06896 \\
.04957 \\
.02916 \\
.01937\end{array}$ & $\begin{array}{l}.02898 \\
.02038 \\
.01569 \\
.01118 \\
.00945\end{array}$ & $\begin{array}{l}1.00455 \\
1.01797 \\
1.02542 \\
1.03272 \\
1.03576\end{array}$ & $\begin{array}{l}1.60863 \\
1.62750 \\
1.63821 \\
1.64929 \\
1.65462\end{array}$ & $\begin{array}{l}.60408 \\
.60953 \\
.61279 \\
.61657 \\
.61886\end{array}$ \\
\hline
\end{tabular}


Table 3.28

\begin{tabular}{|c|c|c|c|c|c|c|}
\hline \multirow{2}{*}{$\mathrm{T}^{\circ} \mathrm{K}$} & $\mathrm{F}_{\mathrm{SnH}}^{\circ}-\mathrm{F}_{\mathrm{SnD}}^{\circ}$ & $\mathrm{F}_{\mathrm{SnH}}^{\circ}-\mathrm{F}^{\circ} \mathrm{SnT}$ & $\mathrm{F}_{\mathrm{SnD}}^{\circ}-\mathrm{F}^{\circ} \mathrm{SnT}$ & $\mathrm{H}_{\mathrm{SnH}}^{\circ}{ }^{-\mathrm{H}^{\circ}} \mathrm{SnD}$ & $\mathrm{H}_{\mathrm{SnH}}^{\circ}{ }^{-\mathrm{H}^{\circ}} \mathrm{SnT}$ & $\mathrm{H}_{\mathrm{SnD}}^{\circ}{ }^{-\mathrm{H}^{\circ}} \mathrm{SnT}$ \\
\hline & $\mathrm{RT}$ & $\mathrm{RT}$ & $\mathrm{RT}$ & $\mathrm{RT}$ & $\mathrm{RT}$ & $\mathrm{RT}$ \\
\hline $\begin{array}{l}100 \\
200 \\
300 \\
400 \\
500\end{array}$ & $\begin{array}{l}3.97413 \\
2.33368 \\
1.79064 \\
1.52775 \\
1.37938\end{array}$ & $\begin{array}{l}5.82864 \\
3.46492 \\
2.68708 \\
2.31541 \\
2.10848\end{array}$ & $\begin{array}{r}1.84741 \\
1.12769 \\
.89407 \\
.78589 \\
.72768\end{array}$ & $\begin{array}{r}3.28147 \\
1.63832 \\
1.07238 \\
.76594 \\
.56965\end{array}$ & $\begin{array}{r}4.73005 \\
2.35624 \\
1.52566 \\
1.07445 \\
.78956\end{array}$ & $\begin{array}{r}1.44148 \\
.71437 \\
.45091 \\
.30674 \\
.21849\end{array}$ \\
\hline $\begin{array}{r}600 \\
700 \\
800 \\
900 \\
1000\end{array}$ & $\begin{array}{l}1.28810 \\
1.22847 \\
1.18768 \\
1.15871 \\
1.13752\end{array}$ & $\begin{array}{l}1.98251 \\
1.90088 \\
1.84534 \\
1.80608 \\
1.77743\end{array}$ & $\begin{array}{l}.69323 \\
.67140 \\
.65677 \\
.64658 \\
.63920\end{array}$ & $\begin{array}{l}.43561 \\
.34087 \\
.27212 \\
.22107 \\
.18237\end{array}$ & $\begin{array}{l}.59833 \\
.46513 \\
.36955 \\
.29918 \\
.24619\end{array}$ & $\begin{array}{l}.16154 \\
.12325 \\
.09654 \\
.07732 \\
.06311\end{array}$ \\
\hline $\begin{array}{l}1100 \\
1200 \\
1300 \\
1400 \\
1500\end{array}$ & $\begin{array}{l}1.12159 \\
1.10938 \\
1.09981 \\
1.09222 \\
1.08609\end{array}$ & $\begin{array}{l}1.75596 \\
1.73950 \\
1.72665 \\
1.71643 \\
1.70820\end{array}$ & $\begin{array}{l}.63372 \\
.62953 \\
.62629 \\
.62370 \\
.62164\end{array}$ & $\begin{array}{l}.15247 \\
.12899 \\
.11026 \\
.09512 \\
.08274\end{array}$ & $\begin{array}{l}.20546 \\
.17358 \\
.14826 \\
.12783 \\
.11116\end{array}$ & $\begin{array}{l}.05234 \\
.04400 \\
.03745 \\
.03220 \\
.02795\end{array}$ \\
\hline $\begin{array}{l}2000 \\
2500 \\
3000 \\
4000 \\
5000\end{array}$ & $\begin{array}{l}1.06816 \\
1.06013 \\
1.05594 \\
1.05204 \\
1.05037\end{array}$ & $\begin{array}{l}1.68409 \\
1.67326 \\
1.66757 \\
1.66214 \\
1.65972\end{array}$ & $\begin{array}{l}.61558 \\
.61284 \\
.61139 \\
.60992 \\
.60920\end{array}$ & $\begin{array}{l}.04537 \\
.02789 \\
.01854 \\
.00958 \\
.00574\end{array}$ & $\begin{array}{l}.06108 \\
.03780 \\
.02539 \\
.01359 \\
.00864\end{array}$ & $\begin{array}{l}.01536 \\
.00962 \\
.00661 \\
.00383 \\
.00275\end{array}$ \\
\hline
\end{tabular}

\begin{tabular}{|c|c|c|c|c|c|c|}
\hline \multirow{2}{*}{$\mathrm{T}^{\circ} \mathrm{K}$} & $\mathrm{C}_{\mathrm{SnD}}^{\circ}{ }^{-\mathrm{C}_{\mathrm{SnH}}^{\circ}}$ & $\mathrm{C}_{\mathrm{SnT}}^{\circ}{ }^{-\mathrm{C}_{\mathrm{SnH}}^{\circ}}$ & $\mathrm{C}_{\mathrm{SnT}}^{\circ}{ }^{-\mathrm{C}_{\mathrm{SnD}}^{\circ}}$ & $\mathrm{S}_{\mathrm{SnD}}^{\circ}{ }^{-\mathrm{S}_{\mathrm{SnH}}^{\circ}}$ & $\mathrm{S}_{\mathrm{Sn}}^{\circ} \mathrm{T}^{-\mathrm{S}_{\mathrm{SnH}}^{\circ}}$ & $\mathrm{S}_{\mathrm{Sn} \mathrm{T}^{\circ}}^{-\mathrm{S}_{\mathrm{SnD}}^{\circ}}$ \\
\hline & $\mathrm{R}$ & $\mathrm{R}$ & $\mathrm{R}$ & $\mathrm{R}$ & $\mathrm{R}$ & $\mathrm{R}$ \\
\hline $\begin{array}{l}100 \\
200 \\
300 \\
400 \\
500\end{array}$ & $\begin{array}{r}-.00008 \\
.01925 \\
.10744 \\
.19253 \\
.23094\end{array}$ & $\begin{array}{l}.00019 \\
.05789 \\
.21622 \\
.32789 \\
.36152\end{array}$ & $\begin{array}{l}.00027 \\
.03864 \\
.10878 \\
.13536 \\
.13058\end{array}$ & $\begin{array}{l}.69266 \\
.69536 \\
.71826 \\
.76181 \\
.80973\end{array}$ & $\begin{array}{l}1.09859 \\
1.10868 \\
1.16142 \\
1.24096 \\
1.31892\end{array}$ & $\begin{array}{l}.40593 \\
.41332 \\
.44316 \\
.47915 \\
.50919\end{array}$ \\
\hline $\begin{array}{r}600 \\
700 \\
800 \\
900 \\
1000\end{array}$ & $\begin{array}{l}.23396 \\
.21939 \\
.19837 \\
.17640 \\
.15585\end{array}$ & $\begin{array}{l}.34901 \\
.31754 \\
.28136 \\
.24667 \\
.21565\end{array}$ & $\begin{array}{l}.11505 \\
.09815 \\
.08299 \\
.07027 \\
.05980\end{array}$ & $\begin{array}{l}.85249 \\
.88760 \\
.91556 \\
.93764 \\
.95515\end{array}$ & $\begin{array}{l}1.38418 \\
1.43575 \\
1.47579 \\
1.50690 \\
1.53124\end{array}$ & $\begin{array}{l}.53169 \\
.54815 \\
.56023 \\
.56926 \\
.57609\end{array}$ \\
\hline $\begin{array}{l}1100 \\
1200 \\
1300 \\
1400 \\
1500\end{array}$ & $\begin{array}{l}.13752 \\
.12154 \\
.10775 \\
.09588 \\
.08566\end{array}$ & $\begin{array}{l}.18876 \\
.16577 \\
.14619 \\
.12952 \\
.11528\end{array}$ & $\begin{array}{l}.05124 \\
.04423 \\
.03844 \\
.03364 \\
.02962\end{array}$ & $\begin{array}{r}.96912 \\
.98039 \\
.98955 \\
.99710 \\
1.00335\end{array}$ & $\begin{array}{l}1.55050 \\
1.56592 \\
1.57839 \\
1.58860 \\
1.59704\end{array}$ & $\begin{array}{l}.58138 \\
.58553 \\
.58884 \\
.59150 \\
.59369\end{array}$ \\
\hline $\begin{array}{l}2000 \\
2500 \\
3000 \\
4000 \\
5000\end{array}$ & $\begin{array}{l}.05174 \\
.03389 \\
.02349 \\
.01255 \\
.00716\end{array}$ & $\begin{array}{l}.06860 \\
.04432 \\
.03021 \\
.01529 \\
.00784\end{array}$ & $\begin{array}{l}.01686 \\
.01043 \\
.00672 \\
.00274 \\
.00068\end{array}$ & $\begin{array}{l}1.02279 \\
1.03224 \\
1.03740 \\
1.04246 \\
1.04463\end{array}$ & $\begin{array}{l}1.62301 \\
1.63546 \\
1.64218 \\
1.64855 \\
1.65108\end{array}$ & $\begin{array}{l}.60022 \\
.60322 \\
.60478 \\
.60609 \\
.60645\end{array}$ \\
\hline
\end{tabular}




\begin{tabular}{|c|c|c|c|c|c|c|}
\hline \multirow{2}{*}{$\mathrm{T}^{\circ} \mathrm{K}$} & $\mathrm{F}_{\mathrm{PbH}}^{\circ}-\mathrm{F}_{\mathrm{PbD}}^{\circ}$ & $\mathrm{F}_{\mathrm{PbH}}^{\circ}-\mathrm{F}_{\mathrm{PbT}}^{\circ}$ & $\mathrm{F}_{\mathrm{PbD}}^{\circ}-\mathrm{F}_{\mathrm{PbT}}^{\circ}$ & \multirow{2}{*}{$\frac{\mathrm{H}_{\mathrm{PbH}}^{\circ}{ }^{-\mathrm{H}_{\mathrm{PbD}}^{\circ}}}{\mathrm{RT}^{\prime}}$} & $\mathrm{H}_{\mathrm{PbH}}^{\circ}{ }^{-\mathrm{H}_{\mathrm{PbT}}^{\circ}}$ & \multirow{2}{*}{$\frac{\mathrm{HbD}^{\circ} \mathrm{HbT}^{\circ}}{\mathrm{RT}}$} \\
\hline & $\mathrm{RT}$ & $\mathrm{RT}$ & $\mathrm{RT}^{\prime}$ & & $\mathrm{RT}$ & \\
\hline $\begin{array}{l}100 \\
200 \\
300 \\
400 \\
500\end{array}$ & $\begin{array}{l}3.89842 \\
2.29504 \\
1.76506 \\
1.50946 \\
1.36571\end{array}$ & $\begin{array}{l}5.72313 \\
3.41072 \\
2.65141 \\
2.29011 \\
2.08961\end{array}$ & $\begin{array}{r}1.82461 \\
1.11563 \\
.88632 \\
.78063 \\
.72388\end{array}$ & $\begin{array}{r}3.20758 \\
1.60066 \\
1.04455 \\
.74308 \\
.55128\end{array}$ & $\begin{array}{r}4.62815 \\
2.30338 \\
1.48588 \\
1.04229 \\
.76433\end{array}$ & $\begin{array}{r}1.42047 \\
.70267 \\
.44130 \\
.29919 \\
.21303\end{array}$ \\
\hline $\begin{array}{r}600 \\
700 \\
800 \\
900 \\
1000\end{array}$ & $\begin{array}{l}1.27741 \\
1.21968 \\
1.18009 \\
1.15187 \\
1.13111\end{array}$ & $\begin{array}{l}1.96769 \\
1.88859 \\
1.83464 \\
1.79633 \\
1.76824\end{array}$ & $\begin{array}{l}.69026 \\
.66890 \\
.65453 \\
.64445 \\
.63712\end{array}$ & $\begin{array}{r}.42144 \\
.33034 \\
.26458 \\
.21591 \\
.17909\end{array}$ & $\begin{array}{r}.57930 \\
.45122 \\
.35975 \\
.29258 \\
.24206\end{array}$ & $\begin{array}{r}.15784 \\
.12087 \\
.09515 \\
.07666 \\
.06296\end{array}$ \\
\hline $\begin{array}{l}1100 \\
1200 \\
1300 \\
1400 \\
1500\end{array}$ & $\begin{array}{l}1.11543 \\
1.10331 \\
1.09378 \\
1.08613 \\
1.07991\end{array}$ & $\begin{array}{l}1.74706 \\
1.73073 \\
1.71789 \\
1.70760 \\
1.69925\end{array}$ & $\begin{array}{l}.63162 \\
.62741 \\
.62410 \\
.62146 \\
.61933\end{array}$ & $\begin{array}{l}.15067 \\
.12834 \\
.11053 \\
.09612 \\
.08431\end{array}$ & $\begin{array}{l}.20326 \\
.17289 \\
.14875 \\
.12925 \\
.11331\end{array}$ & $\begin{array}{l}.05258 \\
.04454 \\
.03821 \\
.03312 \\
.02899\end{array}$ \\
\hline $\begin{array}{l}2000 \\
2500 \\
3000 \\
4000 \\
5000\end{array}$ & $\begin{array}{l}1.06128 \\
1.05249 \\
1.04764 \\
1.04264 \\
1.04015\end{array}$ & $\begin{array}{l}1.67420 \\
1.66238 \\
1.65581 \\
1.64899 \\
1.64549\end{array}$ & $\begin{array}{l}.61292 \\
.60989 \\
.60817 \\
.60634 \\
.60534\end{array}$ & $\begin{array}{r}.04848 \\
.03151 \\
.02231 \\
.01334 \\
.00942\end{array}$ & $\begin{array}{l}.06515 \\
.04250 \\
.03027 \\
.01846 \\
.01339\end{array}$ & $\begin{array}{l}.01667 \\
.01099 \\
.00796 \\
.00511 \\
.00397\end{array}$ \\
\hline
\end{tabular}

\begin{tabular}{|c|c|c|c|c|c|c|}
\hline \multirow{2}{*}{$\mathrm{T}^{\circ} \mathrm{K}$} & $\mathrm{C}_{\mathrm{PbD}}^{\circ}-\mathrm{C}_{\mathrm{PbH}}^{\circ}$ & $\mathrm{C}_{\mathrm{PbT}}^{\circ}-\mathrm{C}_{\mathrm{PbH}}^{\circ}$ & $\mathrm{C}_{\mathrm{PbT}}^{\circ}{ }^{-\mathrm{C}_{\mathrm{PbD}}^{\circ}}$ & $\mathrm{S}_{\mathrm{PbD}}^{\circ}-\mathrm{S}_{\mathrm{PbH}}^{\circ}$ & $\mathrm{S}_{\mathrm{PbT}}^{\circ}{ }^{-\mathrm{S}_{\mathrm{PbH}}^{\circ}}$ & $\mathrm{S}_{\mathrm{PbT}}^{\circ}{ }^{-\mathrm{S}_{\mathrm{PbD}}^{\circ}}$ \\
\hline & $\mathrm{R}$ & $R$ & $\mathrm{R}$ & $\mathrm{R}$ & $\mathrm{R}$ & $\mathrm{R}$ \\
\hline $\begin{array}{l}100 \\
200 \\
300 \\
400 \\
500\end{array}$ & $\begin{array}{r}-.00005 \\
.02382 \\
.11747 \\
.19715 \\
.22730\end{array}$ & $\begin{array}{l}.00036 \\
.06771 \\
.23025 \\
.33136 \\
.35359\end{array}$ & $\begin{array}{l}.00041 \\
.04389 \\
.11278 \\
.13421 \\
.12629\end{array}$ & $\begin{array}{l}.69084 \\
.69438 \\
.72051 \\
.76638 \\
.81443\end{array}$ & $\begin{array}{l}1.09498 \\
1.10734 \\
1.16553 \\
1.24782 \\
1.32528\end{array}$ & $\begin{array}{l}.40414 \\
.41296 \\
.44502 \\
.48144 \\
.51085\end{array}$ \\
\hline $\begin{array}{r}600 \\
700 \\
800 \\
900 \\
1000\end{array}$ & $\begin{array}{l}.22441 \\
.20673 \\
.18454 \\
.16252 \\
.14251\end{array}$ & $\begin{array}{l}.33397 \\
.29924 \\
.26221 \\
.22792 \\
.19791\end{array}$ & $\begin{array}{l}.10956 \\
.09251 \\
.07767 \\
.06540 \\
.05540\end{array}$ & $\begin{array}{l}.85597 \\
.88934 \\
.91551 \\
.93596 \\
.95202\end{array}$ & $\begin{array}{l}1.38839 \\
1.43737 \\
1.47489 \\
1.50375 \\
1.52618\end{array}$ & $\begin{array}{l}.53242 \\
.54803 \\
.55938 \\
.56779 \\
.57416\end{array}$ \\
\hline $\begin{array}{l}1100 \\
1200 \\
1300 \\
1400 \\
1500\end{array}$ & $\begin{array}{l}.12497 \\
.10986 \\
.09695 \\
.08590 \\
.07645\end{array}$ & $\begin{array}{l}.17227 \\
.15055 \\
.13221 \\
.11666 \\
.10344\end{array}$ & $\begin{array}{l}.04730 \\
.04069 \\
.03526 \\
.03076 \\
.02699\end{array}$ & $\begin{array}{l}.96476 \\
.97497 \\
.98325 \\
.99001 \\
.99560\end{array}$ & $\begin{array}{l}1.54380 \\
1.55784 \\
1.56914 \\
1.57835 \\
1.58594\end{array}$ & $\begin{array}{l}.57904 \\
.58287 \\
.58589 \\
.58834 \\
.59034\end{array}$ \\
\hline $\begin{array}{l}2000 \\
2500 \\
3000 \\
4000 \\
5000\end{array}$ & $\begin{array}{l}.04527 \\
.02891 \\
.01935 \\
.00912 \\
.00391\end{array}$ & $\begin{array}{l}.06035 \\
.03797 \\
.02490 \\
.01086 \\
.00360\end{array}$ & $\begin{array}{r}.01508 \\
.00906 \\
.00555 \\
.00174 \\
-.00031\end{array}$ & $\begin{array}{l}1.01280 \\
1.02098 \\
1.02533 \\
1.02930 \\
1.03073\end{array}$ & $\begin{array}{l}1.60905 \\
1.61988 \\
1.62554 \\
1.63053 \\
1.63210\end{array}$ & $\begin{array}{l}.59625 \\
.59890 \\
.60021 \\
.60123 \\
.60137\end{array}$ \\
\hline
\end{tabular}


Table 3.30

\begin{tabular}{|c|c|c|c|c|c|c|}
\hline \multirow{2}{*}{$\mathrm{T}^{\circ} \mathrm{K}$} & $\mathrm{F}_{\mathrm{NH}}^{\circ}-\mathrm{F}_{\mathrm{ND}}^{\circ}$ & $\mathrm{F}_{\mathrm{NH}}^{\circ}-\mathrm{F}_{\mathrm{NT}}^{\circ}$ & $\mathrm{F}_{\mathrm{ND}}^{\circ}-\mathrm{F}^{\circ} \mathrm{NT}$ & $\mathrm{H}_{\mathrm{NH}}^{\circ}{ }^{-\mathrm{H}^{\circ}} \mathrm{ND}$ & $\mathrm{H}_{\mathrm{NH}}^{\circ}{ }^{-\mathrm{H}^{\circ}} \mathrm{NT}$ & $\mathrm{H}_{\mathrm{ND}}^{\circ}-\mathrm{H}_{\mathrm{NT}}^{\circ}$ \\
\hline & $\mathrm{RT}$ & $\mathrm{RT}$ & $\mathrm{RT}$ & RT & RT & $\mathrm{RT}$ \\
\hline 100 & 6.88074 & 9.96228 & 3.08144 & 6.16000 & 8.80803 & 2.64793 \\
\hline 200 & 3.80060 & 5.55810 & 1.75745 & 3.08027 & 4.40432 & 1.32400 \\
\hline 300 & 2.77385 & 4.09006 & 1.31618 & 2.05339 & 2.93547 & .88205 \\
\hline 400 & 2.26068 & 3.35681 & 1.09610 & 1.53820 & 2.19596 & .65773 \\
\hline 500 & 1.95363 & 2.91906 & .96541 & 1.22431 & 1.74194 & .51761 \\
\hline 600 & 1.75057 & 2.63080 & .88021 & 1.00842 & 1.42759 & .41915 \\
\hline 700 & 1.60772 & 2.42912 & .82139 & .84790 & 1.19342 & .34551 \\
\hline 800 & 1.50299 & 2.28212 & .77912 & .72268 & 1.01131 & .28862 \\
\hline 900 & 1.42388 & 2.17170 & .74781 & .62218 & .86600 & .24381 \\
\hline 1000 & 1.36273 & 2.08679 & .72405 & .54004 & .74805 & .20800 \\
\hline 1100 & 1.31454 & 2.02017 & .70562 & .47209 & .65117 & .17907 \\
\hline 1200 & 1.27597 & 1.96706 & .69109 & .41534 & .57080 & .15546 \\
\hline 1300 & 1.24466 & 1.92411 & .67945 & .36757 & .50357 & .13600 \\
\hline 1400 & 1.21895 & 1.88893 & .66998 & .32711 & .44692 & .11981 \\
\hline 1500 & 1.19758 & 1.85977 & .66218 & .29258 & .39882 & .10623 \\
\hline 2000 & 1.13087 & 1.76919 & .63831 & .17900 & .24212 & .06311 \\
\hline 2500 & 1.09796 & 1.72475 & .62679 & .11954 & .16106 & .04152 \\
\hline 3000 & 1.07947 & 1.69987 & .62040 & .08518 & .11453 & .02935 \\
\hline 4000 & 1.06053 & 1.67442 & .61389 & .04967 & .06672 & .01705 \\
\hline 5000 & 1.05144 & 1.66221 & .61077 & .03303 & .04441 & .01138 \\
\hline
\end{tabular}

\begin{tabular}{|c|c|c|c|c|c|c|}
\hline \multirow{2}{*}{$\mathrm{T}^{\circ} \mathrm{K}$} & $\mathrm{C}_{\mathrm{ND}}^{\circ}-\mathrm{C}_{\mathrm{NH}}^{\circ}$ & $\mathrm{C}_{\mathrm{NT}}^{\circ}-\mathrm{C}_{\mathrm{NH}}^{\circ}$ & $\mathrm{C}_{\mathrm{NT}}^{\circ}{ }^{-\mathrm{C}_{\mathrm{ND}}^{\circ}}$ & $\mathrm{S}_{\mathrm{ND}}^{\circ}{ }^{-\mathrm{S}^{\circ}} \mathrm{NH}$ & $\mathrm{S}_{\mathrm{NT}}^{\circ}{ }^{-\mathrm{S}^{\circ}} \mathrm{NH}$ & $\mathrm{S}_{\mathrm{NT}}^{\circ}{ }^{-\mathrm{S}^{\circ}} \mathrm{ND}$ \\
\hline & $\mathrm{R}$ & $\mathrm{R}$ & $\mathrm{R}$ & $\mathrm{R}$ & $\mathrm{R}$ & $\mathrm{R}$ \\
\hline 100 & -.00108 & -.00130 & -.00022 & .72074 & 1.15426 & .43352 \\
\hline 200 & -.00024 & -.00015 & .00009 & .72034 & 1.15379 & .43345 \\
\hline 300 & .00182 & .00754 & .00572 & .72046 & 1.15460 & .43414 \\
\hline 400 & .01603 & .04345 & .02742 & .72249 & 1.16085 & .43836 \\
\hline 500 & .04930 & .10798 & .05868 & .72932 & 1.17712 & .44780 \\
\hline 600 & .09341 & .17977 & .08636 & .74215 & 1.20321 & .46106 \\
\hline 700 & .13604 & .24056 & .10452 & .75983 & 1.23570 & .47587 \\
\hline 800 & .16968 & .28302 & .11334 & .78031 & 1.27081 & .49050 \\
\hline 900 & .19223 & .30744 & .11521 & .80170 & 1.30571 & .50401 \\
\hline 1000 & .20467 & .31730 & .11263 & .82268 & 1.33873 & .51605 \\
\hline 1100 & .20915 & .31662 & .10747 & .84245 & 1.36901 & .52656 \\
\hline 1200 & .20787 & .30890 & .10103 & .86063 & 1.39627 & .53564 \\
\hline 1300 & .20270 & .29681 & .09411 & .87708 & 1.42053 & .54345 \\
\hline 1400 & .19509 & .28227 & .08718 & .89184 & 1.44201 & .55017 \\
\hline & .18607 & .26659 & .08052 & .90500 & 1.46096 & .55596 \\
\hline 2000 & .13816 & .19206 & .05390 & .95186 & 1.52706 & .57520 \\
\hline 2500 & .10057 & .13772 & .03715 & .97842 & 1.56370 & .58528 \\
\hline & .07430 & .10084 & .02654 & .99429 & 1.58533 & .59104 \\
\hline 4000 & .04283 & .05749 & .01466 & 1.01085 & 1.60770 & .59685 \\
\hline 5000 & .02584 & .03438 & .00854 & 1.01840 & 1.61780 & .59940 \\
\hline
\end{tabular}


$\mathrm{T}^{\circ} \mathrm{K} \quad \frac{\mathrm{F}_{\mathrm{PH}}^{\circ}-\mathrm{F}_{\mathrm{PD}}^{\circ}}{\mathrm{RT}} \quad \frac{\mathrm{F}_{\mathrm{PH}}^{\circ}-\mathrm{F}_{\mathrm{PT}}^{\circ}}{\mathrm{RT}} \quad \frac{\mathrm{F}_{\mathrm{PD}}^{\circ}-\mathrm{F}_{\mathrm{PT}}^{\circ}}{\mathrm{RT}}$

$\begin{array}{rlll}100 & 5.50287 & 8.00503 & 2.50226 \\ 200 & 3.10298 & 4.56551 & 1.46258 \\ 300 & 2.30333 & 3.42026 & 1.11697 \\ 400 & 1.90535 & 2.85255 & .94723 \\ 500 & 1.67030 & 2.51980 & .84952 \\ & & & \\ 600 & 1.51822 & 2.30641 & .78820 \\ 700 & 1.41407 & 2.16150 & .74744 \\ 800 & 1.33983 & 2.05894 & .71912 \\ 900 & 1.28523 & 1.98396 & .69874 \\ 1000 & 1.24405 & 1.92767 & .68363 \\ 1100 & 1.21231 & 1.88445 & .67215 \\ 1200 & 1.18739 & 1.85062 & .66324 \\ 1300 & 1.16751 & 1.82370 & .65620 \\ 1400 & 1.15142 & 1.80195 & .65054 \\ 1500 & 1.13824 & 1.78415 & .64591 \\ & & & \\ 2000 & 1.09822 & 1.73018 & .63197 \\ 2500 & 1.07923 & 1.70450 & .62528 \\ 3000 & 1.06884 & 1.69035 & .62152 \\ 4000 & 1.05851 & 1.67596 & .61745 \\ 5000 & 1.05374 & 1.66893 & .61520\end{array}$

$\frac{\mathrm{H}_{\mathrm{PH}}^{\circ}-\mathrm{H}_{\mathrm{PD}}^{\circ}}{\mathrm{RT}} \quad \frac{\mathrm{H}_{\mathrm{PH}}^{\circ}-\mathrm{H}_{\mathrm{PT}}^{\circ}}{\mathrm{RT}} \frac{\mathrm{H}_{\mathrm{PD}}^{\circ}-\mathrm{H}_{\mathrm{PT}}^{\circ}}{\mathrm{RT}}$

$\begin{array}{lll}4.79971 & 6.87904 & 2.07943 \\ 2.39986 & 3.43912 & 1.03931 \\ 1.59731 & 2.28423 & .68696 \\ 1.18649 & 1.68624 & .49978 \\ .92739 & 1.30666 & .37929 \\ & & \\ .74491 & 1.04037 & .29547 \\ .60911 & .84410 & .23500 \\ .50502 & .69534 & .19033 \\ .42374 & .58036 & .15663 \\ .35936 & .49012 & .13077 \\ .30773 & .41830 & .11058 \\ .26585 & .36044 & .09460 \\ .23154 & .31328 & .08175 \\ .20315 & .27445 & .07131 \\ .17943 & .24215 & .06272 \\ & & \\ .10481 & .14136 & .03656 \\ .06787 & .09208 & .02422 \\ .04723 & .06484 & .01762 \\ .02652 & .03793 & .01141 \\ .01705 & .02599 & .00895\end{array}$

\begin{tabular}{|c|c|c|c|c|c|c|}
\hline \multirow{2}{*}{$\mathrm{T}^{\circ} \mathrm{K}$} & $\mathrm{C}_{\mathrm{PD}}^{\circ}-\mathrm{C}_{\mathrm{PH}}^{\circ}$ & $\mathrm{C}_{\mathrm{PT}}^{\circ}-\mathrm{C}_{\mathrm{PH}}^{\circ}$ & $\mathrm{C}_{\mathrm{PT}}^{\circ}{ }^{-\mathrm{C}_{\mathrm{PD}}^{\circ}}$ & $\mathrm{S}_{\mathrm{PD}}^{\circ}{ }^{-\mathrm{S}_{\mathrm{PH}}^{\circ}}$ & $\mathrm{S}_{\mathrm{PT}}^{\circ}{ }^{-\mathrm{S}_{\mathrm{PH}}^{\circ}}$ & $\mathrm{S}_{\mathrm{PD}}^{\circ}{ }^{-\mathrm{S}_{\mathrm{PT}}^{\circ}}$ \\
\hline & $\mathrm{R}$ & $\mathrm{R}$ & $\mathrm{R}$ & $\mathrm{R}$ & $\mathrm{R}$ & $\mathrm{R}$ \\
\hline $\begin{array}{l}100 \\
200 \\
300 \\
400 \\
500\end{array}$ & $\begin{array}{r}-.00031 \\
.00085 \\
.02061 \\
.07594 \\
.14098\end{array}$ & $\begin{array}{r}-.00037 \\
.00481 \\
.05794 \\
.016105 \\
.25750\end{array}$ & $\begin{array}{r}-.00006 \\
.00396 \\
.03733 \\
.08511 \\
.11652\end{array}$ & $\begin{array}{l}.70315 \\
.70312 \\
.70602 \\
.71886 \\
.74292\end{array}$ & $\begin{array}{l}1.12598 \\
1.12638 \\
1.13604 \\
1.16631 \\
1.21314\end{array}$ & $\begin{array}{l}.42283 \\
.42326 \\
.43002 \\
.44745 \\
.47022\end{array}$ \\
\hline $\begin{array}{r}600 \\
700 \\
800 \\
900 \\
1000\end{array}$ & $\begin{array}{l}.19031 \\
.21766 \\
.22700 \\
.22441 \\
.21490\end{array}$ & $\begin{array}{l}.31817 \\
.34386 \\
.34509 \\
.33191 \\
.31142\end{array}$ & $\begin{array}{l}.12786 \\
.12620 \\
.11809 \\
.10750 \\
.09652\end{array}$ & $\begin{array}{l}.77332 \\
.80497 \\
.83481 \\
.86149 \\
.88468\end{array}$ & $\begin{array}{l}1.26604 \\
1.31740 \\
1.36361 \\
1.40360 \\
1.43755\end{array}$ & $\begin{array}{l}.49272 \\
.51243 \\
.52880 \\
.54211 \\
.55287\end{array}$ \\
\hline $\begin{array}{l}1100 \\
1200 \\
1300 \\
1400 \\
1500\end{array}$ & $\begin{array}{l}.20188 \\
.18752 \\
.17303 \\
.15910 \\
.14608\end{array}$ & $\begin{array}{l}.28800 \\
.26417 \\
.24126 \\
.21991 \\
.20039\end{array}$ & $\begin{array}{l}.08612 \\
.07665 \\
.06823 \\
.06081 \\
.05431\end{array}$ & $\begin{array}{l}.90457 \\
.92153 \\
.93596 \\
.94827 \\
.95880\end{array}$ & $\begin{array}{l}1.46614 \\
1.49018 \\
1.51041 \\
1.52749 \\
1.54199\end{array}$ & $\begin{array}{l}.56157 \\
.56865 \\
.57445 \\
.57922 \\
.58319\end{array}$ \\
\hline $\begin{array}{l}2000 \\
2500 \\
3000 \\
4000 \\
5000\end{array}$ & $\begin{array}{l}.09620 \\
.06603 \\
.04727 \\
.02658 \\
.01608\end{array}$ & $\begin{array}{l}.12810 \\
.08552 \\
.05922 \\
.03001 \\
.01483\end{array}$ & $\begin{array}{r}.03190 \\
.01949 \\
.01195 \\
.00343 \\
-.00125\end{array}$ & $\begin{array}{r}.99341 \\
1.01136 \\
1.02160 \\
1.03200 \\
1.03669\end{array}$ & $\begin{array}{l}1.58882 \\
1.61243 \\
1.62551 \\
1.63804 \\
1.64294\end{array}$ & $\begin{array}{l}.59541 \\
.60107 \\
.60391 \\
.60604 \\
.60625\end{array}$ \\
\hline
\end{tabular}


Table 3.32

\begin{tabular}{|c|c|c|c|c|c|c|}
\hline \multirow{2}{*}{$\mathrm{T}^{\circ} \mathrm{K}$} & $\mathrm{F}_{\mathrm{BiH}}^{\circ}-\mathrm{F}_{\mathrm{BiD}}^{\circ}$ & $\mathrm{F}_{\mathrm{BiH}}^{\circ}-\mathrm{F}_{\mathrm{BiT}}^{\circ}$ & $\mathrm{F}_{\mathrm{BiD}}^{\circ}{ }^{-\mathrm{F}_{\mathrm{BiT}}^{\circ}}$ & $\mathrm{H}_{\mathrm{BiH}}^{\circ}{ }^{-\mathrm{H}_{\mathrm{BD}}^{\circ}}$ & $\mathrm{H}_{\mathrm{BiH}}^{\circ}{ }^{-\mathrm{H}^{\circ}} \mathrm{BiT}$ & $\mathrm{HiD}^{\circ}{ }^{-\mathrm{H}^{\circ} \mathrm{T}}$ \\
\hline & $\mathrm{RT}$ & $\mathrm{RT}$ & $\mathrm{RT}$ & $\mathrm{RT}$ & $\mathrm{RT}$ & $\mathrm{RT}$ \\
\hline 100 & 4.16797 & 6.12531 & 1.95734 & 3.48083 & 5.03085 & 1.55002 \\
\hline 200 & 2.42777 & 3.61085 & 1.18308 & 1.73867 & 2.50873 & .77006 \\
\hline 300 & 1.85069 & 2.78097 & .93028 & 1.14267 & 1.63363 & .49096 \\
\hline 400 & 1.56961 & 2.38144 & .81184 & .82266 & 1.16095 & .33830 \\
\hline 500 & 1.40958 & 2.15684 & .74726 & .61770 & .86165 & .24395 \\
\hline 600 & 1.31014 & 2.01875 & .70861 & .47701 & .65945 & .18244 \\
\hline 700 & 1.24456 & 1.92837 & .68381 & .37690 & .51757 & .14067 \\
\hline 800 & 1.19924 & 1.86630 & .66705 & .30374 & .41508 & .11133 \\
\hline 900 & 1.16678 & 1.82201 & .65523 & .24908 & .33915 & .09007 \\
\hline 1000 & 1.14279 & 1.78939 & .64660 & .20739 & .28163 & .07424 \\
\hline 1100 & 1.12461 & 1.76472 & .64011 & .17503 & .23720 & .06217 \\
\hline 1200 & 1.11050 & 1.74563 & .63513 & .14946 & .20226 & .05280 \\
\hline 1300 & 1.09938 & 1.73059 & .63121 & .12897 & .17436 & .04539 \\
\hline 1400 & 1.09045 & 1.71853 & .62808 & .11235 & .15178 & .03943 \\
\hline 1500 & 1.08319 & 1.70871 & .62552 & .09869 & .13326 & .03457 \\
\hline 2000 & 1.06132 & 1.67917 & .61786 & .05702 & .07706 & .02005 \\
\hline 2500 & 1.05097 & 1.66516 & .61419 & .03718 & .05047 & .01329 \\
\hline & 1.04523 & 1.65735 & .61212 & .02636 & .03605 & .00969 \\
\hline & 1.03933 & 1.64921 & .60987 & .01578 & .02209 & .00630 \\
\hline & 1.03637 & 1.64500 & .60863 & .01116 & .01609 & .00493 \\
\hline
\end{tabular}

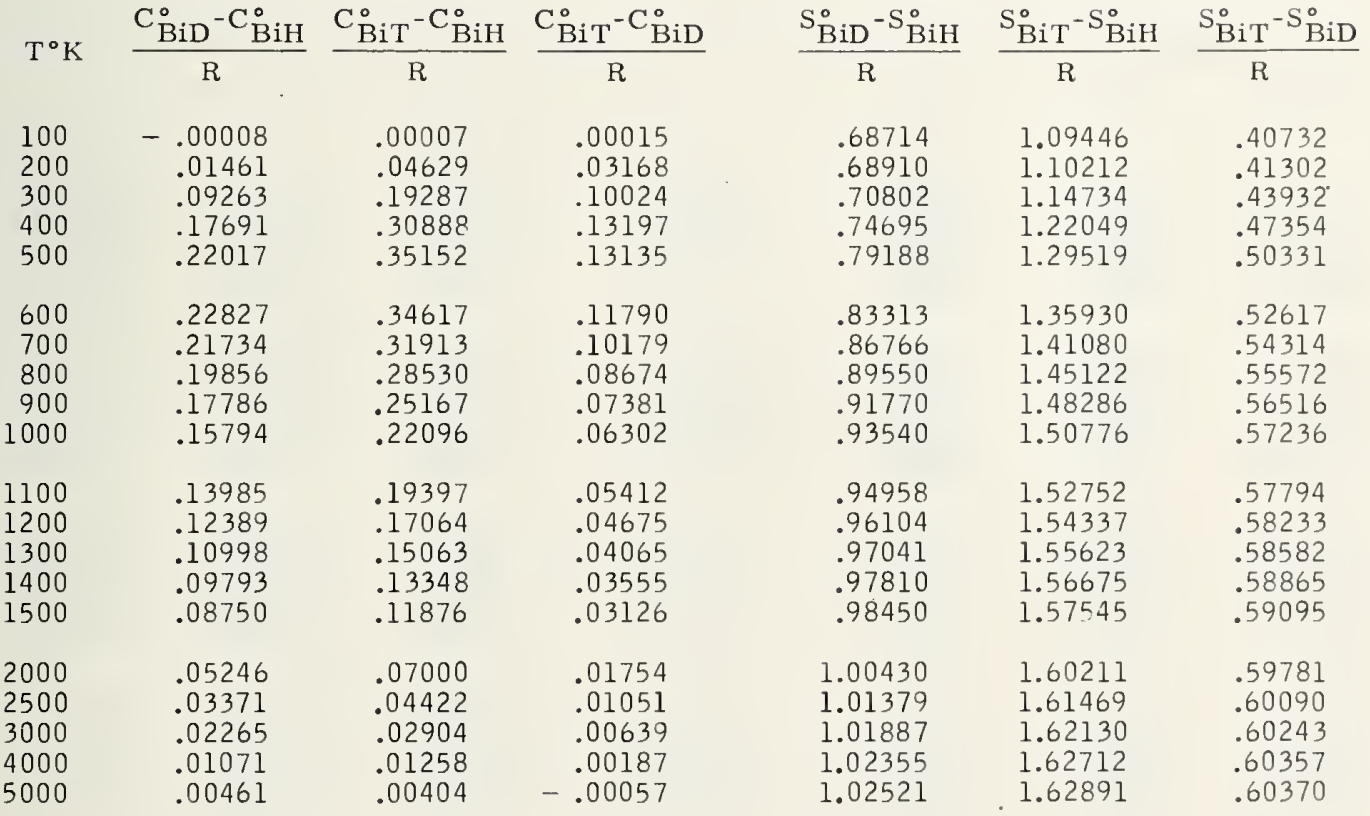




\begin{tabular}{|c|c|c|c|}
\hline \multirow[t]{2}{*}{$\mathrm{T}^{\circ} \mathrm{K}$} & $\mathrm{F}_{\mathrm{OH}}^{\circ}-\mathrm{F}_{\mathrm{OD}}^{\circ}$ & $\mathrm{F}_{\mathrm{OH}}^{\circ}-\mathrm{F}_{\mathrm{OT}}^{\circ}$ & $\mathrm{F}_{\mathrm{OD}}^{\circ}-\mathrm{F}_{\mathrm{OT}}^{\circ}$ \\
\hline & $\mathrm{RT}$ & $\mathrm{RT}$ & $\mathrm{RT}$ \\
\hline $\begin{array}{l}100 \\
200 \\
300 \\
400 \\
500\end{array}$ & $\begin{array}{l}7.85280 \\
4.27679 \\
3.08831 \\
2.49470 \\
2.13905\end{array}$ & $\begin{array}{r}11.37629 \\
6.25094 \\
4.54709 \\
3.69619 \\
3.18699\end{array}$ & $\begin{array}{l}3.52349 \\
1.97415 \\
1.45878 \\
1.20148 \\
1.04794\end{array}$ \\
\hline $\begin{array}{r}600 \\
700 \\
800 \\
900 \\
1000\end{array}$ & $\begin{array}{l}1.90290 \\
1.73568 \\
1.61204 \\
1.51780 \\
1.44425\end{array}$ & $\begin{array}{l}2.84983 \\
2.61202 \\
2.43710 \\
2.30443 \\
2.20141\end{array}$ & $\begin{array}{l}.94693 \\
.87635 \\
.82505 \\
.78663 \\
.75716\end{array}$ \\
\hline $\begin{array}{l}1100 \\
1200 \\
1300 \\
1400 \\
1500\end{array}$ & $\begin{array}{l}1.38581 \\
1.33866 \\
1.30010 \\
1.26822 \\
1.24161\end{array}$ & $\begin{array}{l}2.11990 \\
2.05442 \\
2.00108 \\
1.95710 \\
1.92047\end{array}$ & $\begin{array}{l}.73410 \\
.71578 \\
.70096 \\
.68887 \\
.67885\end{array}$ \\
\hline $\begin{array}{l}2000 \\
2500 \\
3000 \\
4000 \\
5000\end{array}$ & $\begin{array}{l}1.15757 \\
1.11565 \\
1.09206 \\
1.06816 \\
1.05712\end{array}$ & $\begin{array}{l}1.80532 \\
1.74805 \\
1.71573 \\
1.68258 \\
1.66672\end{array}$ & $\begin{array}{l}.64774 \\
.63240 \\
.62368 \\
.61442 \\
.60961\end{array}$ \\
\hline
\end{tabular}

\begin{tabular}{|c|c|c|}
\hline $\mathrm{H}_{\mathrm{OH}}^{\circ}-\mathrm{H}_{\mathrm{OD}}^{\circ}$ & $\mathrm{H}_{\mathrm{OH}}^{\circ}-\mathrm{H}_{\mathrm{O}}^{\circ}$ & $\mathrm{H}_{\mathrm{OD}}^{\circ}-\mathrm{H}_{\mathrm{OT}}^{\circ}$ \\
\hline RT & $\mathrm{RT}$ & $\mathrm{RT}$ \\
\hline $\begin{array}{l}7.16830 \\
3.56790 \\
2.37541 \\
1.77987 \\
1.42042\end{array}$ & $\begin{array}{r}10.27180 \\
5.11481 \\
3.40551 \\
2.55029 \\
2.03127\end{array}$ & $\begin{array}{r}3.10350 \\
1.54691 \\
1.03010 \\
.77041 \\
.61085\end{array}$ \\
\hline $\begin{array}{r}1.17639 \\
.99678 \\
.85722 \\
.74487 \\
.65236\end{array}$ & $\begin{array}{r}1.67635 \\
1.41382 \\
1.20965 \\
1.04578 \\
.91155\end{array}$ & $\begin{array}{l}.49996 \\
.41705 \\
.35242 \\
.30091 \\
.25919\end{array}$ \\
\hline $\begin{array}{r}.57503 \\
.50975 \\
.45416 \\
.40656 \\
.36553\end{array}$ & $\begin{array}{l}.80006 \\
.70657 \\
.62752 \\
.56019 \\
.50250\end{array}$ & $\begin{array}{l}.22504 \\
.19684 \\
.17334 \\
.15362 \\
.13696\end{array}$ \\
\hline $\begin{array}{l}.22734 \\
.15261 \\
.10846 \\
.06165 \\
.03894\end{array}$ & $\begin{array}{l}.31062 \\
.20861 \\
.14906 \\
.08689 \\
.05740\end{array}$ & $\begin{array}{l}.08327 \\
.05600 \\
.04061 \\
.02524 \\
.01847\end{array}$ \\
\hline
\end{tabular}

\begin{tabular}{|c|c|c|c|c|c|c|}
\hline \multirow{2}{*}{$\mathrm{T}^{\circ} \mathrm{K}$} & $\mathrm{C}_{\mathrm{OD}}^{\circ}-\mathrm{C}_{\mathrm{OH}}^{\circ}$ & $\mathrm{C}_{\mathrm{OT}}^{\circ}-\mathrm{C}_{\mathrm{OH}}^{\circ}$ & $\mathrm{C}_{\mathrm{OT}}^{\circ}-\mathrm{C}_{\mathrm{OD}}^{\circ}$ & $\mathrm{S}_{\mathrm{OD}}^{\circ}-\mathrm{S}_{\mathrm{OH}}^{\circ}$ & $\mathrm{S}_{\mathrm{OT}}^{\circ}-\mathrm{S}_{\mathrm{OH}}^{\circ}$ & $\mathrm{S}_{\mathrm{OT}}^{\circ}-\mathrm{S}_{\mathrm{OD}}^{\circ}$ \\
\hline & $\mathrm{R}$ & $\mathrm{R}$ & $\mathrm{R}$ & $\mathrm{R}$ & $\mathrm{R}$ & $\mathrm{R}$ \\
\hline $\begin{array}{l}100 \\
200 \\
300 \\
400 \\
500\end{array}$ & $\begin{array}{l}.06331 \\
.01536 \\
.00619 \\
.00941 \\
.02815\end{array}$ & $\begin{array}{l}.08144 \\
.02004 \\
.01012 \\
.02526 \\
.06858\end{array}$ & $\begin{array}{l}.01813 \\
.00468 \\
.00393 \\
.01585 \\
.04043\end{array}$ & $\begin{array}{l}.68450 \\
.70889 \\
.71290 \\
.71483 \\
.71863\end{array}$ & $\begin{array}{l}1.10449 \\
1.13613 \\
1.14158 \\
1.14590 \\
1.15572\end{array}$ & $\begin{array}{l}.41999 \\
.42724 \\
.42868 \\
.43107 \\
.43709\end{array}$ \\
\hline $\begin{array}{r}600 \\
700 \\
800 \\
900 \\
1000\end{array}$ & $\begin{array}{l}.06124 \\
.10062 \\
.13804 \\
.16856 \\
.19055\end{array}$ & $\begin{array}{l}.12957 \\
.19206 \\
.24479 \\
.28329 \\
.30757\end{array}$ & $\begin{array}{l}.06833 \\
.09144 \\
.10675 \\
.11473 \\
.11702\end{array}$ & $\begin{array}{l}.72651 \\
.73890 \\
.75482 \\
.77293 \\
.79189\end{array}$ & $\begin{array}{l}1.17348 \\
1.19820 \\
1.22745 \\
1.25865 \\
1.28986\end{array}$ & $\begin{array}{l}.44697 \\
.45930 \\
.47263 \\
.48572 \\
.49797\end{array}$ \\
\hline $\begin{array}{l}1100 \\
1200 \\
1300 \\
1400 \\
1500\end{array}$ & $\begin{array}{l}.20441 \\
.21146 \\
.21318 \\
.21105 \\
.20619\end{array}$ & $\begin{array}{r}.31979 \\
.32271 \\
.31891 \\
.31056 \\
.29922\end{array}$ & $\begin{array}{l}.11538 \\
.11125 \\
.10573 \\
.09951 \\
.09303\end{array}$ & $\begin{array}{l}.81078 \\
.82891 \\
.84594 \\
.86166 \\
.87608\end{array}$ & $\begin{array}{l}1.31984 \\
1.34785 \\
1.37356 \\
1.39691 \\
1.41797\end{array}$ & $\begin{array}{l}.50906 \\
.51894 \\
.52762 \\
.53525 \\
.54189\end{array}$ \\
\hline $\begin{array}{l}2000 \\
2500 \\
3000 \\
4000 \\
5000\end{array}$ & $\begin{array}{l}.16633 \\
.12771 \\
.09868 \\
.06258 \\
.04310\end{array}$ & $\begin{array}{l}.23026 \\
.17130 \\
.12859 \\
.07620 \\
.04758\end{array}$ & $\begin{array}{l}.06393 \\
.04359 \\
.02991 \\
.01362 \\
.00448\end{array}$ & $\begin{array}{r}.93023 \\
.96304 \\
.98360 \\
1.00651 \\
1.01818\end{array}$ & $\begin{array}{l}1.49470 \\
1.53944 \\
1.56667 \\
1.59569 \\
1.60932\end{array}$ & $\begin{array}{l}.56447 \\
.57640 \\
.58307 \\
.58918 \\
.59114\end{array}$ \\
\hline
\end{tabular}


Table 3.34

\begin{tabular}{|c|c|c|c|c|c|c|}
\hline \multirow{2}{*}{$\mathrm{T}^{\circ} \mathrm{K}$} & $\begin{array}{ll}\mathrm{F}_{\mathrm{SH}}^{\circ} & \mathrm{F}^{\circ} \\
\mathrm{SD}\end{array}$ & $\begin{array}{ll}\mathrm{F}^{\circ} & \mathrm{F}^{\circ} \\
\mathrm{SH} & \mathrm{ST}^{\prime} \mathrm{T} \\
\end{array}$ & $\begin{array}{ll}\mathrm{F}_{\mathrm{SD}}^{\circ} & \mathrm{F}_{\mathrm{ST}}^{\circ} \\
\end{array}$ & $\begin{array}{ll}\mathrm{H}_{\mathrm{SH}}^{\circ} & \mathrm{H}^{\circ} \\
\mathrm{SD} \\
\end{array}$ & $\begin{array}{ll}\mathrm{H}_{\mathrm{SH}}^{\circ} & \mathrm{H}_{\mathrm{ST}}^{\circ} \\
\end{array}$ & $\begin{array}{ll}\mathrm{H}_{\mathrm{SD}}^{\circ} & \mathrm{II}^{\circ} \\
\mathrm{ST}\end{array}$ \\
\hline & $\mathrm{RT}$ & $\mathrm{RT}$ & $\mathrm{RT}$ & $\mathrm{RT}$ & $\mathrm{RT}$ & $\mathrm{RT}$ \\
\hline $\begin{array}{l}100 \\
200 \\
300 \\
400 \\
500\end{array}$ & $\begin{array}{l}6.19494 \\
3.44366 \\
2.52985 \\
2.07402 \\
1.80302\end{array}$ & $\begin{array}{l}8.98382 \\
5.04057 \\
3.72926 \\
3.07712 \\
2.69149\end{array}$ & $\begin{array}{r}2.78888 \\
1.59691 \\
1.19940 \\
1.00312 \\
.88847\end{array}$ & $\begin{array}{l}5.50713 \\
2.74774 \\
1.82722 \\
1.36263 \\
1.07470\end{array}$ & $\begin{array}{l}7.89358 \\
3.93906 \\
2.61771 \\
1.94472 \\
1.52382\end{array}$ & $\begin{array}{r}2.38645 \\
1.19132 \\
.79048 \\
.58211 \\
.44912\end{array}$ \\
\hline $\begin{array}{r}600 \\
700 \\
800 \\
900 \\
1000\end{array}$ & $\begin{array}{l}1.62584 \\
1.50292 \\
1.41419 \\
1.34810 \\
1.29769\end{array}$ & $\begin{array}{l}2.44113 \\
2.26880 \\
2.14522 \\
2.05376 \\
1.98436\end{array}$ & $\begin{array}{l}.81530 \\
.76588 \\
.73103 \\
.70565 \\
.68667\end{array}$ & $\begin{array}{r}.87368 \\
.72370 \\
.60768 \\
.51596 \\
.44236\end{array}$ & $\begin{array}{r}1.22944 \\
1.01094 \\
.84334 \\
.71202 \\
.60755\end{array}$ & $\begin{array}{l}.35577 \\
.28724 \\
.23566 \\
.19605 \\
.16519\end{array}$ \\
\hline $\begin{array}{l}1100 \\
1200 \\
1300 \\
1400 \\
1500\end{array}$ & $\begin{array}{l}1.25842 \\
1.22731 \\
1.20227 \\
1.18183 \\
1.16498\end{array}$ & $\begin{array}{l}1.93053 \\
1.88805 \\
1.85395 \\
1.82622 \\
1.80337\end{array}$ & $\begin{array}{l}.67209 \\
.66073 \\
.65168 \\
.64439 \\
.63840\end{array}$ & $\begin{array}{l}.38258 \\
.33355 \\
.29298 \\
.25907 \\
.23053\end{array}$ & $\begin{array}{l}.52334 \\
.45472 \\
.39821 \\
.35125 \\
.31185\end{array}$ & $\begin{array}{l}.14074 \\
.12116 \\
.10523 \\
.09218 \\
.08133\end{array}$ \\
\hline $\begin{array}{l}2000 \\
2500 \\
3000 \\
4000 \\
5000\end{array}$ & $\begin{array}{l}1.11279 \\
1.08725 \\
1.07286 \\
1.05800 \\
1.05065\end{array}$ & $\begin{array}{l}1.73306 \\
1.69892 \\
1.67984 \\
1.66033 \\
1.65091\end{array}$ & $\begin{array}{l}.62029 \\
.61167 \\
.60697 \\
.60233 \\
.60025\end{array}$ & $\begin{array}{l}.13909 \\
.09272 \\
.06640 \\
.03956 \\
.02709\end{array}$ & $\begin{array}{l}.18656 \\
.12351 \\
.08780 \\
.05131 \\
.03418\end{array}$ & $\begin{array}{l}.04749 \\
.03079 \\
.02139 \\
.01175 \\
.00708\end{array}$ \\
\hline
\end{tabular}

\begin{tabular}{|c|c|c|c|c|c|c|}
\hline $\mathrm{T}^{\circ} \mathrm{K}$ & $\mathrm{C}_{\mathrm{SD}}^{\circ} \quad \mathrm{C}_{\mathrm{SH}}^{\circ}$ & $\mathrm{C}_{\mathrm{ST}}^{\circ} \quad \mathrm{C}_{\mathrm{SH}}^{\circ}$ & $\mathrm{C}_{\mathrm{ST}}^{\circ} \quad \mathrm{C}_{\mathrm{SD}}^{\circ}$ & $\mathrm{S}_{\mathrm{SD}}^{\circ} \quad \mathrm{S}_{\mathrm{SH}}^{\circ}$ & $\mathrm{S}_{\mathrm{ST}}^{\circ} \mathrm{S}_{\mathrm{SH}}^{\circ}$ & $\mathrm{S}_{\mathrm{ST}}^{\circ} \quad \mathrm{S}_{\mathrm{SD}}^{\circ}$ \\
\hline & $\mathrm{R}$ & $\mathrm{R}$ & $\mathrm{R}$ & $\mathrm{R}$ & $\mathrm{R}$ & $\mathrm{R}$ \\
\hline $\begin{array}{l}100 \\
200 \\
300 \\
400 \\
500\end{array}$ & $\begin{array}{l}.01330 \\
.01154 \\
.01681 \\
.05066 \\
.10473\end{array}$ & $\begin{array}{l}.01679 \\
.01635 \\
.04060 \\
.11436 \\
.20429\end{array}$ & $\begin{array}{l}.00349 \\
.00481 \\
.02379 \\
.06370 \\
.09956\end{array}$ & $\begin{array}{l}.68781 \\
.69592 \\
.70263 \\
.71139 \\
.72832\end{array}$ & $\begin{array}{l}1.09024 \\
1.10151 \\
1.11155 \\
1.13240 \\
1.16767\end{array}$ & $\begin{array}{l}.40243 \\
.40559 \\
.40892 \\
.42101 \\
.43935\end{array}$ \\
\hline $\begin{array}{r}600 \\
700 \\
800 \\
900 \\
1000\end{array}$ & $\begin{array}{l}.15634 \\
.19305 \\
.21336 \\
.22045 \\
.21849\end{array}$ & $\begin{array}{l}.27610 \\
.31922 \\
.33721 \\
.33744 \\
.32666\end{array}$ & $\begin{array}{l}.11976 \\
.12617 \\
.12385 \\
.11699 \\
.10817\end{array}$ & $\begin{array}{l}.75216 \\
.77922 \\
.80651 \\
.83214 \\
.85533\end{array}$ & $\begin{array}{l}1.21169 \\
1.25786 \\
1.30188 \\
1.34174 \\
1.37681\end{array}$ & $\begin{array}{l}.45953 \\
.47864 \\
.49537 \\
.50960 \\
.52148\end{array}$ \\
\hline $\begin{array}{l}1100 \\
1200 \\
1300 \\
1400 \\
1500\end{array}$ & $\begin{array}{l}.21091 \\
.20018 \\
.18796 \\
.17532 \\
.16284\end{array}$ & $\begin{array}{l}.30981 \\
.29010 \\
.26957 \\
.24936 \\
.23013\end{array}$ & $\begin{array}{l}.09890 \\
.08992 \\
.08161 \\
.07404 \\
.06729\end{array}$ & $\begin{array}{l}.87584 \\
.89376 \\
.90929 \\
.92276 \\
.93445\end{array}$ & $\begin{array}{l}1.40719 \\
1.43333 \\
1.45574 \\
1.47497 \\
1.49152\end{array}$ & $\begin{array}{l}.53135 \\
.53957 \\
.54645 \\
.55221 \\
.55707\end{array}$ \\
\hline $\begin{array}{l}2000 \\
2500 \\
3000 \\
4000 \\
5000\end{array}$ & $\begin{array}{l}.11091 \\
.07696 \\
.05500 \\
.02995 \\
.01681\end{array}$ & $\begin{array}{l}.15421 \\
.10685 \\
.07696 \\
.04363 \\
.02662\end{array}$ & $\begin{array}{l}.04330 \\
.02989 \\
.02196 \\
.01368 \\
.00981\end{array}$ & $\begin{array}{r}.97370 \\
.99453 \\
1.00646 \\
1.01844 \\
1.02356\end{array}$ & $\begin{array}{l}1.54650 \\
1.57541 \\
1.59204 \\
1.60902 \\
1.61673\end{array}$ & $\begin{array}{l}.57280 \\
.58088 \\
.58558 \\
.59058 \\
.59317\end{array}$ \\
\hline
\end{tabular}


Table 3.35

\begin{tabular}{|c|c|c|c|c|c|c|}
\hline \multirow{2}{*}{$\mathrm{T}^{\circ} \mathrm{K}$} & $\mathrm{F}_{\mathrm{FH}}^{\circ}{ }^{-\mathrm{F}_{\mathrm{FD}}^{\circ}}$ & $\mathrm{F}_{\mathrm{FH}}^{\circ}{ }^{-\mathrm{F}^{\circ}}$ & $\mathrm{F}_{\mathrm{FD}}^{\circ}{ }^{-\mathrm{F}_{\mathrm{T}}^{\circ}}$ & $\mathrm{H}_{\mathrm{FH}}^{\circ}{ }^{-\mathrm{H}_{\mathrm{FD}}^{\circ}}$ & $\mathrm{H}_{\mathrm{FH}}^{\circ}-\mathrm{H}_{\mathrm{FT}}^{\circ}$ & $\mathrm{H}_{\mathrm{FD}}^{\circ}-\mathrm{H}_{\mathrm{FT}}^{\circ}$ \\
\hline & $\mathrm{RT}$ & $\mathrm{RT}$ & $\mathrm{RT}$ & $\mathrm{RT}$ & $\mathrm{RT}$ & $\mathrm{RT}$ \\
\hline 100 & 8.70772 & 12.58751 & 3.87979 & 7.99543 & 11.44897 & 3.45354 \\
\hline 200 & 4.70981 & 6.86279 & 2.15298 & 3.99811 & 5.72495 & 1.72684 \\
\hline 300 & 3.37708 & 4.95444 & 1.57737 & 2.66548 & 3.81664 & 1.15117 \\
\hline 400 & 2.71073 & 4.00040 & 1.28967 & 1.99886 & 2.86123 & .86237 \\
\hline 500 & 2.31109 & 3.42861 & 1.11752 & 1.59746 & 2.28386 & .68640 \\
\hline 600 & 2.04519 & 3.04889 & 1.00369 & 1.32679 & 1.89200 & .56520 \\
\hline 700 & 1.85622 & 2.77988 & .92366 & 1.12919 & 1.60413 & .47494 \\
\hline 800 & 1.71581 & 2.58082 & .86502 & .97655 & 1.38096 & .40442 \\
\hline 900 & 1.60811 & 2.42888 & .82077 & .85390 & 1.20167 & .34777 \\
\hline 1000 & 1.52354 & 2.31015 & .78661 & .75275 & 1.05422 & .30147 \\
\hline 1100 & 1.45589 & 2.21562 & .75973 & .66783 & .93101 & .26318 \\
\hline 1200 & 1.40095 & 2.13919 & .73824 & .59567 & .82692 & .23125 \\
\hline 1300 & 1.35579 & 2.07660 & .72081 & .53384 & .73823 & .20439 \\
\hline 1400 & 1.31822 & 2.02474 & .70652 & .48049 & .66214 & .18165 \\
\hline 1500 & 1.28668 & 1.98136 & .69467 & .43418 & .59648 & .16229 \\
\hline 2000 & 1.18590 & 1.84365 & .65775 & .27565 & .37439 & .09874 \\
\hline 2500 & 1.13469 & 1.77434 & .63965 & .18803 & .25359 & .06556 \\
\hline 3000 & 1.10542 & 1.73495 & .62954 & .13561 & .18197 & .04637 \\
\hline 4000 & 1.07513 & 1.69444 & .61932 & .07976 & .10620 & .02645 \\
\hline 5000 & 1.06054 & 1.67510 & .61456 & .05276 & .06971 & .01695 \\
\hline
\end{tabular}

\begin{tabular}{|c|c|c|c|c|c|c|}
\hline $\mathrm{T}^{\circ} \mathrm{K}$ & $\mathrm{C}_{\mathrm{FD}}^{\circ}-\mathrm{C}_{\mathrm{FH}}^{\circ}$ & $\mathrm{C}_{\mathrm{FT}}^{\circ}-\mathrm{C}_{\mathrm{FH}}^{\circ}$ & $\mathrm{C}_{\mathrm{FT}}^{\circ}-\mathrm{C}_{\mathrm{FD}}^{\circ}$ & $\mathrm{S}_{\mathrm{FD}}^{\circ}{ }^{-\mathrm{S}_{\mathrm{FH}}^{\circ}}$ & $\mathrm{S}_{\mathrm{FT}}^{\circ}{ }^{-\mathrm{S}_{\mathrm{FH}}^{\circ}}$ & $\mathrm{S}_{\mathrm{FT}}^{\circ}{ }^{-\mathrm{S}_{\mathrm{FD}}^{\circ}}$ \\
\hline $1 \mathrm{~K}$ & $\mathrm{RT}$ & $\mathrm{RT}$ & $\mathrm{RT}$ & $\mathrm{RT}$ & $\mathrm{RT}$ & $\mathrm{RT}$ \\
\hline $\begin{array}{l}100 \\
200 \\
300 \\
400 \\
500\end{array}$ & $\begin{array}{r}-.00161 \\
-.00039 \\
.00001 \\
.00301 \\
.01519\end{array}$ & $\begin{array}{r}-.00192 \\
-.00045 \\
.00094 \\
.01180 \\
.04321\end{array}$ & $\begin{array}{r}-.00031 \\
-.00006 \\
.00093 \\
.00879 \\
.02802\end{array}$ & $\begin{array}{l}.71228 \\
.71169 \\
.71160 \\
.71188 \\
.71362\end{array}$ & $\begin{array}{l}1.13853 \\
1.13783 \\
1.13781 \\
1.13917 \\
1.14475\end{array}$ & $\begin{array}{l}.42625 \\
.42614 \\
.42621 \\
.42729 \\
.43113\end{array}$ \\
\hline $\begin{array}{r}600 \\
700 \\
800 \\
900 \\
1000\end{array}$ & $\begin{array}{l}.03995 \\
.07378 \\
.11013 \\
.14348 \\
.17071\end{array}$ & $\begin{array}{l}.09383 \\
.15265 \\
.20857 \\
.25481 \\
.28892\end{array}$ & $\begin{array}{l}.05388 \\
.07887 \\
.09844 \\
.11133 \\
.11821\end{array}$ & $\begin{array}{l}.71840 \\
.72703 \\
.73926 \\
.75420 \\
.77079\end{array}$ & $\begin{array}{l}1.15689 \\
1.17574 \\
1.19986 \\
1.22720 \\
1.25593\end{array}$ & $\begin{array}{l}.43849 \\
.44871 \\
.46060 \\
.47300 \\
.48514\end{array}$ \\
\hline $\begin{array}{l}1100 \\
1200 \\
1300 \\
1400 \\
1500\end{array}$ & $\begin{array}{l}.19080 \\
.20407 \\
.21148 \\
.21417 \\
.21326\end{array}$ & $\begin{array}{l}.31124 \\
.32342 \\
.32755 \\
.32561 \\
.31933\end{array}$ & $\begin{array}{l}.12044 \\
.11935 \\
.11607 \\
.11144 \\
.10607\end{array}$ & $\begin{array}{l}.78806 \\
.80528 \\
.82195 \\
.83774 \\
.85250\end{array}$ & $\begin{array}{l}1.28460 \\
1.31227 \\
1.33837 \\
1.36261 \\
1.38487\end{array}$ & $\begin{array}{l}.49654 \\
.50699 \\
.51642 \\
.52487 \\
.53237\end{array}$ \\
\hline $\begin{array}{l}2000 \\
2500 \\
3000 \\
4000 \\
5000\end{array}$ & $\begin{array}{l}.18231 \\
.14323 \\
.11110 \\
.06852 \\
.04408\end{array}$ & $\begin{array}{l}.26068 \\
.20049 \\
.15389 \\
.09438 \\
.06112\end{array}$ & $\begin{array}{l}.07837 \\
.05726 \\
.04279 \\
.02586 \\
.01704\end{array}$ & $\begin{array}{r}.91026 \\
.94666 \\
.96982 \\
.99536 \\
1.00779\end{array}$ & $\begin{array}{l}1.46927 \\
1.52075 \\
1.55298 \\
1.58824 \\
1.60539\end{array}$ & $\begin{array}{l}.55901 \\
.57409 \\
.58316 \\
.59288 \\
.59760\end{array}$ \\
\hline
\end{tabular}


Table 3.36

\begin{tabular}{|c|c|c|c|c|c|c|}
\hline $\mathrm{T}^{\circ} \mathrm{K}$ & $\mathrm{F}_{\mathrm{ClH}}^{\circ}{ }^{-\mathrm{F}^{\circ}}$ & $\mathrm{F}_{\mathrm{ClH}}^{\circ}{ }^{-\mathrm{F}^{\circ} \mathrm{ClT}}$ & $\mathrm{F}_{\mathrm{ClD}}^{\circ}{ }^{-\mathrm{F}^{\circ} \mathrm{T}}$ & ${ }_{\mathrm{ClH}}{ }^{-}{ }^{\circ} \mathrm{ClD}$ & $\mathrm{H}_{\mathrm{ClH}}^{\circ}{ }^{-\mathrm{H}^{\circ}} \mathrm{ClT}$ & $\mathrm{H}_{\mathrm{ClD}}^{\circ}{ }^{-\mathrm{H}_{\mathrm{C}}^{\circ} \mathrm{T}}$ \\
\hline & $\mathrm{RT}$ & $\mathrm{RT}$ & RT & $\mathrm{RT}$ & $\mathrm{RT}$ & $\mathrm{RT}$ \\
\hline $\begin{array}{l}100 \\
200 \\
300 \\
400 \\
500\end{array}$ & $\begin{array}{l}6.67090 \\
3.68645 \\
2.69166 \\
2.19471 \\
1.89797\end{array}$ & $\begin{array}{l}9.70017 \\
5.40899 \\
3.97882 \\
3.26529 \\
2.84085\end{array}$ & $\begin{array}{r}3.02927 \\
1.72254 \\
1.28716 \\
1.07058 \\
.94288\end{array}$ & $\begin{array}{l}5.96879 \\
2.98450 \\
1.98925 \\
1.48844 \\
1.18090\end{array}$ & $\begin{array}{l}8.58222 \\
4.29119 \\
2.85883 \\
2.13370 \\
1.68414\end{array}$ & $\begin{array}{r}2.61343 \\
1.30669 \\
.86958 \\
.64526 \\
.50324\end{array}$ \\
\hline $\begin{array}{r}600 \\
700 \\
800 \\
900 \\
1000\end{array}$ & $\begin{array}{l}1.70256 \\
1.56592 \\
1.46641 \\
1.39174 \\
1.33441\end{array}$ & $\begin{array}{l}2.56302 \\
2.37009 \\
2.23058 \\
2.12656 \\
2.04712\end{array}$ & $\begin{array}{l}.86046 \\
.80417 \\
.76417 \\
.73482 \\
.71271\end{array}$ & $\begin{array}{l}.96758 \\
.80829 \\
.68422 \\
.58511 \\
.50471\end{array}$ & $\begin{array}{r}1.37064 \\
1.13693 \\
.95602 \\
.81271 \\
.69746\end{array}$ & $\begin{array}{r}.40306 \\
.32864 \\
.27180 \\
.22760 \\
.19275\end{array}$ \\
\hline $\begin{array}{l}1100 \\
1200 \\
1300 \\
1400 \\
1500\end{array}$ & $\begin{array}{l}1.28949 \\
1.25374 \\
1.22485 \\
1.20122 \\
1.18166\end{array}$ & $\begin{array}{l}1.98519 \\
1.93608 \\
1.89654 \\
1.86430 \\
1.83767\end{array}$ & $\begin{array}{l}.69570 \\
.68234 \\
.67169 \\
.66308 \\
.65601\end{array}$ & $\begin{array}{r}.43872 \\
.38407 \\
.33840 \\
.29997 \\
.26741\end{array}$ & $\begin{array}{l}.60364 \\
.52648 \\
.46242 \\
.40883 \\
.36363\end{array}$ & $\begin{array}{l}.16492 \\
.14241 \\
.12402 \\
.10886 \\
.09622\end{array}$ \\
\hline $\begin{array}{l}2000 \\
2500 \\
3000 \\
4000 \\
5000\end{array}$ & $\begin{array}{l}1.12104 \\
1.09142 \\
1.07487 \\
1.05805 \\
1.05006\end{array}$ & $\begin{array}{l}1.75553 \\
1.71562 \\
1.69338 \\
1.67078 \\
1.66004\end{array}$ & $\begin{array}{l}.63449 \\
.62420 \\
.61851 \\
.61273 \\
.60998\end{array}$ & $\begin{array}{l}.16168 \\
.10721 \\
.07600 \\
.04389 \\
.02881\end{array}$ & $\begin{array}{l}.21824 \\
.14422 \\
.10208 \\
.05897 \\
.03883\end{array}$ & $\begin{array}{l}.05656 \\
.03701 \\
.02608 \\
.01508 \\
.01002\end{array}$ \\
\hline
\end{tabular}

\begin{tabular}{|c|c|c|c|c|c|c|}
\hline \multirow{2}{*}{$\mathrm{T}^{\circ} \mathrm{K}$} & $\mathrm{ClD}_{\mathrm{ClO}}^{\circ}{ }^{-\mathrm{C}_{\mathrm{ClH}}^{\circ}}$ & $\mathrm{ClT}^{\circ}{ }^{-\mathrm{C}_{\mathrm{ClH}}^{\circ}}$ & $\mathrm{ClT}^{\circ}{ }^{-\mathrm{C}_{\mathrm{ClD}}^{\circ}}$ & \multirow{2}{*}{$\frac{\mathrm{S}_{\mathrm{ClD}}^{\circ}{ }^{-\mathrm{S}_{\mathrm{ClH}}^{\circ}}}{\mathrm{R}}$} & $\mathrm{S}_{\mathrm{ClT}}^{\circ}{ }^{-\mathrm{S}_{\mathrm{ClH}}^{\circ}}$ & $\mathrm{SlT}^{-}{ }^{-\mathrm{S}_{\mathrm{ClD}}^{\circ}}$ \\
\hline & $\mathrm{R}$ & $\mathbf{R}$ & $\mathrm{R}$ & & $\mathrm{R}$ & $\mathrm{R}$ \\
\hline $\begin{array}{l}100 \\
200 \\
300 \\
400 \\
500\end{array}$ & $\begin{array}{r}-.00040 \\
-.00003 \\
.00430 \\
.02790 \\
.07312\end{array}$ & $\begin{array}{r}-.00048 \\
.00047 \\
.01671 \\
.07348 \\
.15629\end{array}$ & $\begin{array}{r}-.00008 \\
.00050 \\
.01241 \\
.04558 \\
.08317\end{array}$ & $\begin{array}{l}.70210 \\
.70195 \\
.70240 \\
.70627 \\
.71707\end{array}$ & $\begin{array}{l}1.11794 \\
1.11781 \\
1.11998 \\
1.13160 \\
1.15670\end{array}$ & $\begin{array}{l}.41584 \\
.41586 \\
.41758 \\
.42533 \\
.43963\end{array}$ \\
\hline $\begin{array}{r}600 \\
700 \\
800 \\
900 \\
1000\end{array}$ & $\begin{array}{l}.12459 \\
.16819 \\
.19824 \\
.21503 \\
.22126\end{array}$ & $\begin{array}{l}.23470 \\
.29191 \\
.32531 \\
.33904 \\
.33873\end{array}$ & $\begin{array}{l}.11011 \\
.12372 \\
.12707 \\
.12401 \\
.11747\end{array}$ & $\begin{array}{l}.73498 \\
.75762 \\
.78220 \\
.80663 \\
.82969\end{array}$ & $\begin{array}{l}1.19237 \\
1.23316 \\
1.27457 \\
1.31384 \\
1.34965\end{array}$ & $\begin{array}{l}.45739 \\
.47554 \\
.49237 \\
.50721 \\
.51996\end{array}$ \\
\hline $\begin{array}{l}1100 \\
1200 \\
1300 \\
1400 \\
1500\end{array}$ & $\begin{array}{l}.22001 \\
.21387 \\
.20478 \\
.19413 \\
.18282\end{array}$ & $\begin{array}{l}.32936 \\
.31466 \\
.29717 \\
.27861 \\
.26000\end{array}$ & $\begin{array}{l}.10935 \\
.10079 \\
.09239 \\
.08448 \\
.07718\end{array}$ & $\begin{array}{l}.85077 \\
.86968 \\
.88646 \\
.90124 \\
.91425\end{array}$ & $\begin{array}{l}1.38156 \\
1.40961 \\
1.43412 \\
1.45546 \\
1.47405\end{array}$ & $\begin{array}{l}.53079 \\
.53993 \\
.54766 \\
.55422 \\
.55980\end{array}$ \\
\hline $\begin{array}{l}2000 \\
2500 \\
3000 \\
4000 \\
5000\end{array}$ & $\begin{array}{l}.13045 \\
.09329 \\
.06847 \\
.03977 \\
.02474\end{array}$ & $\begin{array}{l}.18040 \\
.12716 \\
.09248 \\
.05297 \\
.03247\end{array}$ & $\begin{array}{l}.04995 \\
.03387 \\
.02401 \\
.01320 \\
.00773\end{array}$ & $\begin{array}{r}.95937 \\
.98421 \\
.99888 \\
1.01416 \\
1.02125\end{array}$ & $\begin{array}{l}1.53729 \\
1.57140 \\
1.59130 \\
1.61182 \\
1.62120\end{array}$ & $\begin{array}{l}.57792 \\
.58719 \\
.59242 \\
.59766 \\
.59995\end{array}$ \\
\hline
\end{tabular}




\begin{tabular}{|c|c|c|c|c|c|c|}
\hline & $\mathrm{F}_{\mathrm{BrH}}^{\circ}-\mathrm{F}_{\mathrm{BrD}}^{\circ}$ & $\mathrm{F}_{\mathrm{BrH}}^{\circ}-\mathrm{F}_{\mathrm{BrT}}^{\circ}$ & $\mathrm{F}_{\mathrm{BrD}}^{\circ}-\mathrm{F}_{\mathrm{BrT}}^{\circ}$ & $\mathrm{H}_{\mathrm{BrH}}^{\circ}{ }^{-\mathrm{H}_{\mathrm{BrD}}^{\circ}}$ & $\mathrm{H}_{\mathrm{BrH}}^{\circ}{ }^{-\mathrm{H}_{\mathrm{BrT}}^{\circ}}$ & $\mathrm{H}_{\mathrm{BrD}}^{\circ}-\mathrm{H}_{\mathrm{BrT}}^{\mathrm{c}}$ \\
\hline $\mathrm{T}^{\circ} \mathrm{K}$ & $\mathrm{RT}$ & $\mathrm{RT}$ & $\mathrm{RT}$ & $\mathrm{RT}$ & $\mathrm{RT}$ & $\mathrm{RT}$ \\
\hline $\begin{array}{l}100 \\
200 \\
300 \\
400 \\
500\end{array}$ & $\begin{array}{l}6.08559 \\
3.38975 \\
2.49124 \\
2.04303 \\
1.77664\end{array}$ & $\begin{array}{l}8.87556 \\
4.99021 \\
3.69573 \\
3.05165 \\
2.67115\end{array}$ & $\begin{array}{r}2.78997 \\
1.60046 \\
1.20448 \\
1.00863 \\
.89451\end{array}$ & $\begin{array}{l}5.39161 \\
2.69587 \\
1.79604 \\
1.33995 \\
1.05600\end{array}$ & $\begin{array}{l}7.77066 \\
3.88523 \\
2.58533 \\
1.92014 \\
1.50217\end{array}$ & $\begin{array}{r}2.37905 \\
1.18936 \\
.78928 \\
.58020 \\
.44617\end{array}$ \\
\hline $\begin{array}{r}600 \\
700 \\
800 \\
900 \\
1000\end{array}$ & $\begin{array}{l}1.60265 \\
1.48220 \\
1.39542 \\
1.33097 \\
1.28191\end{array}$ & $\begin{array}{l}2.42459 \\
2.25532 \\
2.13425 \\
2.04492 \\
1.97728\end{array}$ & $\begin{array}{l}.82195 \\
.77311 \\
.73883 \\
.71395 \\
.69537\end{array}$ & $\begin{array}{l}.85708 \\
.70845 \\
.59346 \\
.50266 \\
.42991\end{array}$ & $\begin{array}{r}1.20913 \\
.99162 \\
.82495 \\
.69465 \\
.59121\end{array}$ & $\begin{array}{l}.35206 \\
.28316 \\
.23149 \\
.19199 \\
.16130\end{array}$ \\
\hline $\begin{array}{l}1100 \\
1200 \\
1300 \\
1400 \\
1500\end{array}$ & $\begin{array}{l}1.24380 \\
1.21366 \\
1.18945 \\
1.16976 \\
1.15352\end{array}$ & $\begin{array}{l}1.92499 \\
1.88378 \\
1.85077 \\
1.82400 \\
1.80197\end{array}$ & $\begin{array}{l}.68118 \\
.67012 \\
.66133 \\
.65425 \\
.64846\end{array}$ & $\begin{array}{l}.37094 \\
.32267 \\
.28277 \\
.24951 \\
.22155\end{array}$ & $\begin{array}{l}.50806 \\
.44043 \\
.38487 \\
.33879 \\
.30021\end{array}$ & $\begin{array}{l}.13711 \\
.11776 \\
.10211 \\
.08929 \\
.07867\end{array}$ \\
\hline $\begin{array}{l}2000 \\
2500 \\
3000 \\
4000 \\
5000\end{array}$ & $\begin{array}{l}1.10360 \\
1.07939 \\
1.06590 \\
1.05208 \\
1.04537\end{array}$ & $\begin{array}{l}1.73455 \\
1.70204 \\
1.68397 \\
1.66552 \\
1.65661\end{array}$ & $\begin{array}{l}.63096 \\
.62265 \\
.61806 \\
.61344 \\
.61124\end{array}$ & $\begin{array}{l}.13236 \\
.08746 \\
.06209 \\
.03640 \\
.02459\end{array}$ & $\begin{array}{l}.17815 \\
.11726 \\
.08303 \\
.04846 \\
.03257\end{array}$ & $\begin{array}{l}.04580 \\
.02980 \\
.02093 \\
.01206 \\
.00798\end{array}$ \\
\hline
\end{tabular}

\begin{tabular}{|c|c|c|c|c|c|c|}
\hline \multirow{2}{*}{$\mathrm{T}^{\circ} \mathrm{K}$} & $\mathrm{C}_{\mathrm{BrD}}^{\circ}-\mathrm{C}_{\mathrm{BrH}}^{\circ}$ & $\mathrm{C}_{\mathrm{BrT}}^{\circ}-\mathrm{C}_{\mathrm{BrH}}^{\circ}$ & $\mathrm{C}_{\mathrm{BrT}}^{\circ}{ }^{-\mathrm{C}_{\mathrm{BrD}}^{\circ}}$ & $\mathrm{S}_{\mathrm{BrD}}^{\circ}{ }^{-\mathrm{S}_{\mathrm{BrH}}^{\circ}}$ & $\mathrm{S}_{\mathrm{BrT}}^{\circ}{ }^{-\mathrm{S}_{\mathrm{BrH}}^{\circ}}$ & $\mathrm{S}_{\mathrm{BrD}}^{\circ}{ }^{-\mathrm{S}_{\mathrm{BrT}}^{\circ}}$ \\
\hline & $\mathrm{R}$ & $\mathrm{R}$ & $\mathrm{R}$ & $\mathrm{R}$ & $\mathrm{R}$ & $\mathrm{R}$ \\
\hline $\begin{array}{l}100 \\
200 \\
300 \\
400 \\
500\end{array}$ & $\begin{array}{r}.00030 \\
.00017 \\
.01081 \\
.05091 \\
.10958\end{array}$ & $\begin{array}{r}-.00035 \\
.00201 \\
.03611 \\
.12075 \\
.21682\end{array}$ & $\begin{array}{r}-.00005 \\
.00184 \\
.02530 \\
.06984 \\
.10724\end{array}$ & $\begin{array}{l}.69399 \\
.69388 \\
.69520 \\
.70308 \\
.72064\end{array}$ & $\begin{array}{l}1.10491 \\
1.10497 \\
1.11040 \\
1.13152 \\
1.16898\end{array}$ & $\begin{array}{l}.41092 \\
.41109 \\
.41520 \\
.42844 \\
.44834\end{array}$ \\
\hline $\begin{array}{r}600 \\
700 \\
800 \\
900 \\
1000\end{array}$ & $\begin{array}{l}.16331 \\
.20029 \\
.21984 \\
.22582 \\
.22268\end{array}$ & $\begin{array}{l}.29008 \\
.33200 \\
.34772 \\
.34553 \\
.33256\end{array}$ & $\begin{array}{l}.12677 \\
.13171 \\
.12788 \\
.11971 \\
.10988\end{array}$ & $\begin{array}{l}.74557 \\
.77376 \\
.80195 \\
.82831 \\
.85200\end{array}$ & $\begin{array}{l}1.21545 \\
1.26371 \\
1.30930 \\
1.35028 \\
1.38608\end{array}$ & $\begin{array}{l}.46988 \\
.48995 \\
.50735 \\
.52197 \\
.53408\end{array}$ \\
\hline $\begin{array}{l}1100 \\
1200 \\
1300 \\
1400 \\
1500\end{array}$ & $\begin{array}{l}.21405 \\
.20244 \\
.18951 \\
.17630 \\
.16340\end{array}$ & $\begin{array}{l}.31387 \\
.29268 \\
.27097 \\
.24985 \\
.22991\end{array}$ & $\begin{array}{l}.09982 \\
.09024 \\
.08146 \\
.07355 \\
.06651\end{array}$ & $\begin{array}{l}.87285 \\
.89099 \\
.90669 \\
.92025 \\
.93197\end{array}$ & $\begin{array}{l}1.41692 \\
1.44334 \\
1.46590 \\
1.48521 \\
1.50176\end{array}$ & $\begin{array}{l}.54407 \\
.55235 \\
.55921 \\
.56496 \\
.56979\end{array}$ \\
\hline $\begin{array}{l}2000 \\
2500 \\
3000 \\
4000 \\
5000\end{array}$ & $\begin{array}{l}.11052 \\
.07643 \\
.05455 \\
.02977 \\
.01681\end{array}$ & $\begin{array}{l}.15222 \\
.10429 \\
.07414 \\
.04047 \\
.02308\end{array}$ & $\begin{array}{l}.04170 \\
.02786 \\
.01959 \\
.01070 \\
.00627\end{array}$ & $\begin{array}{r}.97123 \\
.99194 \\
1.00381 \\
1.01568 \\
1.02079\end{array}$ & $\begin{array}{l}1.55640 \\
1.58478 \\
1.60094 \\
1.61706 \\
1.62404\end{array}$ & $\begin{array}{l}.58517 \\
.59284 \\
.59713 \\
.60138 \\
.60325\end{array}$ \\
\hline
\end{tabular}


Table 3.38

\begin{tabular}{|c|c|c|c|c|c|c|}
\hline \multirow{2}{*}{$\mathrm{T}^{\circ} \mathrm{K}$} & $\mathrm{F}_{\mathrm{HI}}^{\circ}-\mathrm{F}_{\mathrm{DI}}^{\circ}$ & $\mathrm{F}_{\mathrm{HI}}^{\circ}-\mathrm{F}_{\mathrm{TI}}^{\circ}$ & $\mathrm{F}_{\mathrm{DI}}^{\circ}-\mathrm{F}_{\mathrm{TI}}^{\circ}$ & $\mathrm{H}_{\mathrm{HI}}^{\circ}-\mathrm{H}_{\mathrm{DI}}^{\circ}$ & $\mathrm{H}_{\mathrm{HI}}^{\circ}-\mathrm{H}_{\mathrm{TI}}^{\circ}$ & \multirow{2}{*}{$\frac{\mathrm{H}_{\mathrm{DI}}^{\circ}-\mathrm{H}_{\mathrm{TI}}^{\circ}}{\mathrm{RT}}$} \\
\hline & $\mathrm{RT}$ & $\mathrm{RT}$ & $\mathrm{RT}$ & $\mathrm{RT}$ & $\mathrm{RT}$ & \\
\hline $\begin{array}{l}100 \\
200 \\
300 \\
400 \\
500\end{array}$ & $\begin{array}{l}5.42132 \\
3.05688 \\
2.26918 \\
1.87754 \\
1.64679\end{array}$ & $\begin{array}{l}7.92027 \\
4.51043 \\
3.37552 \\
2.81397 \\
2.48591\end{array}$ & $\begin{array}{r}2.49895 \\
1.45355 \\
1.10634 \\
.93643 \\
.83912\end{array}$ & $\begin{array}{r}4.72884 \\
2.36434 \\
1.57292 \\
1.16627 \\
.90899\end{array}$ & $\begin{array}{l}6.81979 \\
3.40920 \\
2.26198 \\
1.66520 \\
1.28564\end{array}$ & $\begin{array}{r}2.09095 \\
1.04486 \\
.68906 \\
.49893 \\
.37665\end{array}$ \\
\hline $\begin{array}{r}600 \\
700 \\
800 \\
900 \\
1000\end{array}$ & $\begin{array}{l}1.49794 \\
1.39631 \\
1.32404 \\
1.27098 \\
1.23101\end{array}$ & $\begin{array}{l}2.27630 \\
2.13444 \\
2.03431 \\
1.96123 \\
1.90646\end{array}$ & $\begin{array}{l}.77836 \\
.73813 \\
.71027 \\
.69025 \\
.67545\end{array}$ & $\begin{array}{l}.72789 \\
.59357 \\
.49111 \\
.41145 \\
.34863\end{array}$ & $\begin{array}{r}1.01999 \\
.82511 \\
.67815 \\
.56511 \\
.47675\end{array}$ & $\begin{array}{l}.29210 \\
.23154 \\
.18704 \\
.15366 \\
.12812\end{array}$ \\
\hline $\begin{array}{l}1100 \\
1200 \\
1300 \\
1400 \\
1500\end{array}$ & $\begin{array}{l}1.20022 \\
1.17606 \\
1.15678 \\
1.14115 \\
1.12835\end{array}$ & $\begin{array}{l}1.86443 \\
1.83154 \\
1.80538 \\
1.78422 \\
1.76692\end{array}$ & $\begin{array}{l}.66420 \\
.65548 \\
.64860 \\
.64307 \\
.63857\end{array}$ & $\begin{array}{l}.29842 \\
.25784 \\
.22466 \\
.19725 \\
.17441\end{array}$ & $\begin{array}{l}.40666 \\
.35033 \\
.30452 \\
.26684 \\
.23554\end{array}$ & $\begin{array}{l}.10823 \\
.09249 \\
.07986 \\
.06959 \\
.06113\end{array}$ \\
\hline $\begin{array}{l}2000 \\
2500 \\
3000 \\
4000 \\
5000\end{array}$ & $\begin{array}{l}1.08933 \\
1.07062 \\
1.06027 \\
1.04973 \\
1.04466\end{array}$ & $\begin{array}{l}1.71436 \\
1.68928 \\
1.67542 \\
1.66137 \\
1.65463\end{array}$ & $\begin{array}{l}.62503 \\
.61865 \\
.61515 \\
.61164 \\
.60997\end{array}$ & $\begin{array}{l}.10270 \\
.06729 \\
.04752 \\
.02763 \\
.01854\end{array}$ & $\begin{array}{l}.13794 \\
.09011 \\
.06348 \\
.03678 \\
.02458\end{array}$ & $\begin{array}{l}.03524 \\
.02281 \\
.01596 \\
.00915 \\
.00604\end{array}$ \\
\hline
\end{tabular}

\begin{tabular}{|c|c|c|c|c|c|c|}
\hline \multirow{2}{*}{$\mathrm{T}^{\circ} \mathrm{K}$} & $\mathrm{C}_{\mathrm{DI}}^{\circ}-\mathrm{C}_{\mathrm{HI}}^{\circ}$ & $\mathrm{C}_{\mathrm{TI}}^{\circ}-\mathrm{C}_{\mathrm{HI}}^{\circ}$ & $\mathrm{C}_{\mathrm{TI}}^{\circ}-\mathrm{C}_{\mathrm{DI}}^{\circ}$ & $\mathrm{S}_{\mathrm{DI}}^{\circ}-\mathrm{S}_{\mathrm{HI}}^{\circ}$ & $\mathrm{S}_{\mathrm{TI}}^{\circ}-\mathrm{S}_{\mathrm{HI}}^{\circ}$ & $\mathrm{S}_{\mathrm{TI}}^{\circ}-\mathrm{S}_{\mathrm{DI}}^{\circ}$ \\
\hline & $\mathrm{R}$ & $\mathrm{R}$ & $\mathrm{R}$ & $\mathrm{R}$ & $\mathrm{R}$ & $\mathrm{R}$ \\
\hline 100 & -.00014 & -.00017 & -.00003 & .69247 & 1.10048 & .40801 \\
\hline 200 & .00130 & .00690 & .00560 & .69254 & 1.10123 & .40869 \\
\hline 300 & .02530 & .07063 & .04533 & .69625 & 1.11354 & .41729 \\
\hline 400 & .08610 & .18176 & .09566 & .71126 & 1.14877 & .43751 \\
\hline 500 & .15227 & .27736 & .12509 & .73780 & 1.20027 & .46247 \\
\hline 600 & .19890 & .33221 & .13331 & .77005 & 1.25631 & .48626 \\
\hline 700 & .22223 & .35129 & .12906 & .80274 & 1.30933 & .50659 \\
\hline 800 & .22781 & .34700 & .11919 & .83293 & 1.35616 & .52323 \\
\hline 900 & .22225 & .32982 & .10757 & .85953 & 1.39613 & .53660 \\
\hline 1000 & .21064 & .30669 & .09605 & .88238 & 1.42970 & .54732 \\
\hline 1100 & .19625 & .28168 & .08543 & .90180 & 1.45777 & .55597 \\
\hline 1200 & .18105 & .25701 & .07596 & .91822 & 1.48122 & .56300 \\
\hline 1300 & .16611 & .23378 & .06767 & .93212 & 1.50086 & .56874 \\
\hline 1400 & .15200 & .21245 & .06045 & .94391 & 1.51739 & .57348 \\
\hline 1500 & .13897 & .19315 & .05418 & .95394 & 1.53137 & .57743 \\
\hline 2000 & .09009 & .12316 & .03307 & .98662 & 1.57642 & .58980 \\
\hline 2500 & .06105 & .08286 & .02181 & 1.00333 & 1.59917 & .59584 \\
\hline 3000 & .04312 & .05835 & .01523 & 1.01276 & 1.61195 & .59919 \\
\hline 4000 & .02337 & .03165 & .00828 & 1.02210 & 1.62458 & .60248 \\
\hline 5000 & .01329 & .01813 & .00484 & 1.02612 & 1.63005 & .60393 \\
\hline
\end{tabular}




\begin{tabular}{|c|c|c|c|c|c|c|}
\hline $\mathrm{T}^{\circ} \mathrm{K}$ & $\frac{{ }^{\mathcal{F}} \mathrm{H}_{2}-\mathcal{\gamma}_{\mathrm{HD}}}{R T}$ & $\frac{\mathcal{F}_{\mathrm{H}_{2}}-\mathcal{F}_{\mathrm{HT}}}{R T}$ & $\frac{{ }^{F_{H D}}-\mathcal{F}_{D_{2}}}{R T}$ & $\frac{\mathcal{F}_{H D}-\mathcal{F}_{H T}}{R T}$ & $\frac{\mathcal{F}_{H D}-\mathcal{F}_{D T}}{R T}$ & $\frac{{ }^{{ }^{D_{2}}-\mathcal{F}_{D T}}}{R T}$ \\
\hline $\begin{array}{l}100 \\
200 \\
300 \\
400 \\
500\end{array}$ & $\begin{array}{r}4.05871 \\
1.86674 \\
1.18794 \\
.85364 \\
.65344\end{array}$ & $\begin{array}{r}5.45230 \\
2.53418 \\
1.61323 \\
1.15783 \\
.88499\end{array}$ & $\begin{array}{r}4.64281 \\
2.21467 \\
1.40782 \\
1.00460 \\
.76263\end{array}$ & $\begin{array}{r}1.39310 \\
.66720 \\
.42513 \\
.30408 \\
.23146\end{array}$ & $\begin{array}{l}6.39784 \\
3.05069 \\
1.93459 \\
1.37650 \\
1.04190\end{array}$ & $\begin{array}{r}1.75524 \\
.83613 \\
.52685 \\
.37196 \\
.27932\end{array}$ \\
\hline $\begin{array}{r}600 \\
700 \\
800 \\
900 \\
1000\end{array}$ & $\begin{array}{l}.51971 \\
.42467 \\
.35371 \\
.29897 \\
.25568\end{array}$ & $\begin{array}{l}.70291 \\
.57346 \\
.47691 \\
.40251 \\
.34377\end{array}$ & $\begin{array}{l}.60168 \\
.48737 \\
.40255 \\
.33757 \\
.28666\end{array}$ & $\begin{array}{l}.18313 \\
.14873 \\
.12315 \\
.10349 \\
.08805\end{array}$ & $\begin{array}{l}.81953 \\
.66186 \\
.54512 \\
.45594 \\
.38626\end{array}$ & $\begin{array}{l}.21789 \\
.17453 \\
.14261 \\
.11840 \\
.09963\end{array}$ \\
\hline $\begin{array}{l}1100 \\
1200 \\
1300 \\
1400 \\
1500\end{array}$ & $\begin{array}{l}.22081 \\
.19228 \\
.16867 \\
.14890 \\
.13224\end{array}$ & $\begin{array}{l}.29653 \\
.25795 \\
.22609 \\
.19946 \\
.17704\end{array}$ & $\begin{array}{l}.24603 \\
.21310 \\
.18609 \\
.16366 \\
.14487\end{array}$ & $\begin{array}{l}.07568 \\
.06564 \\
.05740 \\
.05053 \\
.04478\end{array}$ & $\begin{array}{l}.33079 \\
.28596 \\
.24926 \\
.21890 \\
.19350\end{array}$ & $\begin{array}{l}.08479 \\
.07289 \\
.06321 \\
.05526 \\
.04865\end{array}$ \\
\hline $\begin{array}{l}2000 \\
2500 \\
3000 \\
4000 \\
5000\end{array}$ & $\begin{array}{l}.07828 \\
.05051 \\
.03457 \\
.01824 \\
.01063\end{array}$ & $\begin{array}{l}.10480 \\
.06794 \\
.04698 \\
.02581 \\
.01625\end{array}$ & $\begin{array}{l}.08516 \\
.05507 \\
.03800 \\
.02047 \\
.01211\end{array}$ & $\begin{array}{l}.02651 \\
.01742 \\
.01240 \\
.00757 \\
.00562\end{array}$ & $\begin{array}{l}.11319 \\
.07306 \\
.05047 \\
.02756 \\
.01692\end{array}$ & $\begin{array}{l}.02805 \\
.01801 \\
.01249 \\
.00711 \\
.00482\end{array}$ \\
\hline
\end{tabular}

\begin{tabular}{|c|c|c|c|}
\hline $\mathrm{T}^{\circ} \mathrm{K}$ & $\frac{\mathcal{F}_{H T}-\mathcal{F}_{D T}}{R T}$ & $\frac{\mathcal{F}_{\mathrm{HT}}-\mathcal{F}_{\mathrm{T}_{2}}}{\mathrm{RT}}$ & $\frac{\mathcal{F}_{\mathrm{DT}}-\mathcal{F}_{T_{2}}}{R T}$ \\
\hline $\begin{array}{l}100 \\
200 \\
300 \\
400 \\
500\end{array}$ & $\begin{array}{r}5.00475 \\
2.38350 \\
1.50946 \\
1.07243 \\
.81045\end{array}$ & $\begin{array}{l}6.97752 \\
3.31402 \\
2.09236 \\
1.48149 \\
1.11553\end{array}$ & $\begin{array}{r}1.97378 \\
.93103 \\
.58324 \\
.40932 \\
.30529\end{array}$ \\
\hline $\begin{array}{r}600 \\
700 \\
800 \\
900 \\
1000\end{array}$ & $\begin{array}{l}.63642 \\
.51314 \\
.42199 \\
.35246 \\
.29822\end{array}$ & $\begin{array}{l}.87281 \\
.70131 \\
.57489 \\
.47881 \\
.40410\end{array}$ & $\begin{array}{l}.23657 \\
.18832 \\
.15304 \\
.12648 \\
.10599\end{array}$ \\
\hline $\begin{array}{l}1100 \\
1200 \\
1300 \\
1400 \\
1500\end{array}$ & $\begin{array}{l}.25511 \\
.22032 \\
.19188 \\
.16838 \\
.14874\end{array}$ & $\begin{array}{l}.34492 \\
.29731 \\
.25848 \\
.22647 \\
.19978\end{array}$ & $\begin{array}{l}.08991 \\
.07708 \\
.06669 \\
.05818 \\
.05112\end{array}$ \\
\hline $\begin{array}{l}2000 \\
2500 \\
3000 \\
4000 \\
5000\end{array}$ & $\begin{array}{l}.08669 \\
.05565 \\
.03807 \\
.02001 \\
.01131\end{array}$ & $\begin{array}{l}.11584 \\
.07408 \\
.05052 \\
.02636 \\
.01477\end{array}$ & $\begin{array}{l}.02921 \\
.01848 \\
.01249 \\
.00649 \\
.00349\end{array}$ \\
\hline
\end{tabular}


Table 4.02

\begin{tabular}{|c|c|c|c|c|c|c|}
\hline \multirow{2}{*}{$\mathrm{T}^{\circ} \mathrm{K}$} & $7_{\mathrm{LiH}}-7_{\mathrm{LiO}}$ & $\mathcal{F}_{\mathrm{LiH}}-\mathcal{F}_{\mathrm{LiT}}$ & $\mathcal{F}_{\text {LiD }}-\boldsymbol{F}_{\text {LiT }}$ & $\mathcal{F}_{\mathrm{NaH}}-\mathcal{F}_{\mathrm{NaD}}$ & $F_{\mathrm{NaH}}-\mathcal{F}_{\mathrm{NaT}}$ & $\mathcal{F}_{\mathrm{NaD}}-\mathcal{F}_{\mathrm{NaT}}$ \\
\hline & RT & RT & RT & RT & RT & RT \\
\hline $\begin{array}{l}100 \\
200 \\
300 \\
400 \\
500\end{array}$ & $\begin{array}{r}2.18032 \\
.94597 \\
.53963 \\
.34579 \\
.23819\end{array}$ & $\begin{array}{r}3.05478 \\
1.30949 \\
.73845 \\
.46920 \\
.32136\end{array}$ & $\begin{array}{l}.87447 \\
.36353 \\
.19884 \\
.12342 \\
.08318\end{array}$ & $\begin{array}{r}1.96607 \\
.82036 \\
.45041 \\
.28020 \\
.18884\end{array}$ & $\begin{array}{r}2.78074 \\
1.13992 \\
.61720 \\
.38056 \\
.25504\end{array}$ & $\begin{array}{l}.81468 \\
.31957 \\
.16680 \\
.10037 \\
.06621\end{array}$ \\
\hline $\begin{array}{r}600 \\
700 \\
800 \\
900 \\
1000\end{array}$ & $\begin{array}{l}.17285 \\
.13054 \\
.10174 \\
.08134 \\
.06639\end{array}$ & $\begin{array}{l}.23232 \\
.17501 \\
.13617 \\
.10874 \\
.08871\end{array}$ & $\begin{array}{l}.05947 \\
.04448 \\
.03444 \\
.02742 \\
.02233\end{array}$ & $\begin{array}{l}.13478 \\
.10041 \\
.07733 \\
.06114 \\
.04936\end{array}$ & $\begin{array}{l}.18133 \\
.13471 \\
.10351 \\
.08166 \\
.06580\end{array}$ & $\begin{array}{l}.04656 \\
.03431 \\
.02619 \\
.02053 \\
.01645\end{array}$ \\
\hline $\begin{array}{l}1100 \\
1200 \\
1300 \\
1400 \\
1500\end{array}$ & $\begin{array}{l}.05516 \\
.04650 \\
.03971 \\
.03429 \\
.02989\end{array}$ & $\begin{array}{l}.07368 \\
.06212 \\
.05306 \\
.04583 \\
.03999\end{array}$ & $\begin{array}{l}.01853 \\
.01564 \\
.01336 \\
.01155 \\
.01010\end{array}$ & $\begin{array}{l}.04055 \\
.03379 \\
.02850 \\
.02429 \\
.02087\end{array}$ & $\begin{array}{r}.05395 \\
.04486 \\
.03775 \\
.03208 \\
.02748\end{array}$ & $\begin{array}{l}.01341 \\
.01108 \\
.00925 \\
.00780 \\
.00662\end{array}$ \\
\hline $\begin{array}{l}2000 \\
2500 \\
3000 \\
4000 \\
5000\end{array}$ & $\begin{array}{l}.01680 \\
.01073 \\
.00744 \\
.00417 \\
.00263\end{array}$ & $\begin{array}{l}.02256 \\
.01446 \\
.01004 \\
.00560 \\
.00343\end{array}$ & $\begin{array}{l}.00577 \\
.00375 \\
.00262 \\
.00144 \\
.00081\end{array}$ & $\begin{array}{l}.01068 \\
.00587 \\
.00318\end{array}$ & $\begin{array}{l}.01376 \\
.00724 \\
.00356\end{array}$ & $\begin{array}{l}.00309 \\
.00137 \\
.00039\end{array}$ \\
\hline
\end{tabular}

\begin{tabular}{|c|c|c|c|c|c|c|}
\hline$T^{\circ} K$ & $\mathcal{F}_{K H}-\mathcal{F}_{K D}$ & $\mathcal{F}_{K H}-\mathcal{F}_{K T}$ & $7_{K D}-7_{K T}$ & $\mathcal{F}_{\mathrm{RbH}}-\mathcal{F}_{\mathrm{RbD}}$ & $\mathcal{F}_{\mathrm{RbH}}-\mathcal{F}_{\mathrm{RbT}}$ & $\mathcal{F}_{R b D}-\mathcal{F}_{R b T}$ \\
\hline $1 \mathrm{~K}$ & RT & $R T$ & $R T$ & RT & $R T$ & RT \\
\hline $\begin{array}{l}100 \\
200 \\
300 \\
400 \\
500\end{array}$ & $\begin{array}{r}1.68552 \\
.70491 \\
.39736 \\
.26117 \\
.19027\end{array}$ & $\begin{array}{r}2.36125 \\
.95637 \\
.52427 \\
.33617 \\
.23925\end{array}$ & $\begin{array}{l}.67574 \\
.25147 \\
.12692 \\
.07502 \\
.04899\end{array}$ & $\begin{array}{r}1.56777 \\
.61967 \\
.32564 \\
.19695 \\
.13041\end{array}$ & $\begin{array}{r}2.21449 \\
.85633 \\
.44420 \\
.26691 \\
.17619\end{array}$ & $\begin{array}{l}.64673 \\
.23667 \\
.11856 \\
.06997 \\
.04579\end{array}$ \\
\hline $\begin{array}{r}600 \\
700 \\
800 \\
900 \\
1000\end{array}$ & $\begin{array}{l}.14921 \\
.12354 \\
.10655 \\
.09478 \\
.08631\end{array}$ & $\begin{array}{l}.18345 \\
.14869 \\
.12569 \\
.10976 \\
.09831\end{array}$ & $\begin{array}{l}.03425 \\
.02516 \\
.01915 \\
.01499 \\
.01201\end{array}$ & $\begin{array}{l}.09201 \\
.06800 \\
.05206 \\
.04095 \\
.03293\end{array}$ & $\begin{array}{l}.12413 \\
.09169 \\
.07018 \\
.05521 \\
.04439\end{array}$ & $\begin{array}{l}.03214 \\
.02370 \\
.01813 \\
.01427 \\
.01147\end{array}$ \\
\hline $\begin{array}{l}1100 \\
1200 \\
1300 \\
1400 \\
1500\end{array}$ & $\begin{array}{l}.08007 \\
.07535 \\
.07171 \\
.06887 \\
.06660\end{array}$ & $\begin{array}{l}.08983 \\
.08342 \\
.07844 \\
.07453 \\
.07140\end{array}$ & $\begin{array}{l}.00978 \\
.00807 \\
.00674 \\
.00567 \\
.00481\end{array}$ & $\begin{array}{l}.02694 \\
.02236 \\
.01876 \\
.01590 \\
.01358\end{array}$ & $\begin{array}{l}.03630 \\
.03010 \\
.02523 \\
.02135 \\
.01816\end{array}$ & $\begin{array}{l}.00937 \\
.00775 \\
.00648 \\
.00545 \\
.00460\end{array}$ \\
\hline $\begin{array}{l}2000 \\
2500 \\
3000\end{array}$ & $\begin{array}{l}.06036 \\
.05802 \\
.05723\end{array}$ & $\begin{array}{l}.06257 \\
.05898 \\
.05745\end{array}$ & $\begin{array}{l}.00223 \\
.00097 \\
.00023\end{array}$ & $\begin{array}{l}.00660 \\
.00322 \\
.00124\end{array}$ & $\begin{array}{l}.00853 \\
.00371 \\
.00077\end{array}$ & $\begin{array}{l}.00194 \\
.00050\end{array}$ \\
\hline
\end{tabular}




\begin{tabular}{|c|c|c|c|}
\hline $\mathrm{T}^{\circ} \mathrm{K}$ & $\frac{{ }^{\beta} \mathrm{CsH}^{-{ }^{7} \mathrm{CsD}}}{R T}$ & $\frac{\mathcal{F}_{\mathrm{CsH}}-\mathcal{F}_{\mathrm{CsT}}}{\mathrm{RT}}$ & $\frac{{ }^{\mathcal{F}} \mathrm{CsD}^{-\mathcal{F}} \mathrm{CsT}}{\mathrm{RT}}$ \\
\hline $\begin{array}{l}100 \\
200 \\
300 \\
400 \\
500\end{array}$ & $\begin{array}{r}1.48311 \\
.57824 \\
.30085 \\
.18081 \\
.11918\end{array}$ & $\begin{array}{r}2.09243 \\
.79753 \\
.40975 \\
.24480 \\
.16097\end{array}$ & $\begin{array}{l}.78543 \\
.30735 \\
.16760 \\
.10803 \\
.07702\end{array}$ \\
\hline $\begin{array}{r}600 \\
700 \\
800 \\
900 \\
1000\end{array}$ & $\begin{array}{l}.08377 \\
.06166 \\
.04700 \\
.03678 \\
.02940\end{array}$ & $\begin{array}{l}.11307 \\
.08330 \\
.06358 \\
.04988 \\
.03997\end{array}$ & $\begin{array}{l}.05867 \\
.04680 \\
.03861 \\
.03267 \\
.02819\end{array}$ \\
\hline $\begin{array}{l}1100 \\
1200 \\
1300 \\
1400 \\
1500\end{array}$ & $\begin{array}{l}.02387 \\
.01964 \\
.01631 \\
.01365 \\
.01148\end{array}$ & $\begin{array}{l}.03257 \\
.02690 \\
.02244 \\
.01888 \\
.01597\end{array}$ & $\begin{array}{l}.02472 \\
.02195 \\
.01969 \\
.01782 \\
.01625\end{array}$ \\
\hline $\begin{array}{l}2000 \\
2500 \\
3000 \\
4000 \\
5000\end{array}$ & $\begin{array}{l}.00486 \\
.00153\end{array}$ & $\begin{array}{l}.00710 \\
.00262\end{array}$ & $\begin{array}{l}.01106 \\
.00815\end{array}$ \\
\hline
\end{tabular}

\begin{tabular}{|c|c|c|}
\hline$\frac{\mathcal{F}_{\mathrm{BeH}}-\mathcal{F}_{\mathrm{BeD}}}{R T}$ & $\frac{\mathcal{F}_{\mathrm{BeH}}-\mathcal{F}_{\mathrm{BeT}}}{R T}$ & $\frac{\mathcal{F}_{\mathrm{BeD}}-\mathcal{F}_{\mathrm{BeT}}}{R T}$ \\
\hline $\begin{array}{r}3.43704 \\
1.56715 \\
.94457 \\
.63624 \\
.45605\end{array}$ & $\begin{array}{r}4.86082 \\
2.19949 \\
1.31440 \\
.87829 \\
.62550\end{array}$ & $\begin{array}{r}1.42379 \\
.63235 \\
.36984 \\
.24206 \\
.16946\end{array}$ \\
\hline $\begin{array}{l}.34105 \\
.26336 \\
.20865 \\
.16880 \\
.13899\end{array}$ & $\begin{array}{l}.46549 \\
.35818 \\
.28305 \\
.22860 \\
.18801\end{array}$ & $\begin{array}{l}.12445 \\
.09483 \\
.07441 \\
.05981 \\
.04903\end{array}$ \\
\hline $\begin{array}{l}.11617 \\
.09835 \\
.08419 \\
.07276 \\
.06340\end{array}$ & $\begin{array}{l}.15703 \\
.13288 \\
.11373 \\
.09831 \\
.08569\end{array}$ & $\begin{array}{l}.04087 \\
.03454 \\
.02956 \\
.02556 \\
.02230\end{array}$ \\
\hline $\begin{array}{l}.03511 \\
.02165 \\
.01423 \\
.00672 \\
.00312\end{array}$ & $\begin{array}{l}.04760 \\
.02949 \\
.01950 \\
.00933 \\
.00439\end{array}$ & $\begin{array}{l}.01250 \\
.00786 \\
.00528 \\
.00262 \\
.00128\end{array}$ \\
\hline
\end{tabular}

\begin{tabular}{|c|c|c|c|}
\hline $\mathrm{T}^{\circ} \mathrm{K}$ & $\frac{\mathcal{F}_{\mathrm{MgH}}-\mathcal{F}_{\mathrm{MgD}}}{R T}$ & $\frac{\mathcal{F}_{\mathrm{MgH}}-\mathcal{F}_{\mathrm{MgT}}}{\mathrm{RT}}$ & $\frac{\mathcal{F}_{M g D}-\mathcal{F}_{M g T}}{R T}$ \\
\hline $\begin{array}{l}100 \\
200 \\
300 \\
400 \\
500\end{array}$ & $\begin{array}{r}2.60497 \\
1.13694 \\
.65264 \\
.42028 \\
.29040\end{array}$ & $\begin{array}{r}3.70039 \\
1.59420 \\
.90409 \\
.57731 \\
.39691\end{array}$ & $\begin{array}{r}1.09543 \\
.45727 \\
.25146 \\
.15704 \\
.10652\end{array}$ \\
\hline $\begin{array}{r}600 \\
700 \\
800 \\
900 \\
1000\end{array}$ & $\begin{array}{l}.21102 \\
.15933 \\
.12398 \\
.09885 \\
.08038\end{array}$ & $\begin{array}{l}.28769 \\
.21705 \\
.16898 \\
.13493 \\
.10997\end{array}$ & $\begin{array}{l}.07668 \\
.05773 \\
.04502 \\
.03609 \\
.02960\end{array}$ \\
\hline $\begin{array}{l}1100 \\
1200 \\
1300 \\
1400 \\
1500\end{array}$ & $\begin{array}{l}.06644 \\
.05568 \\
.04721 \\
.04041 \\
.03489\end{array}$ & $\begin{array}{l}.09118 \\
.07669 \\
.06528 \\
.05615 \\
.04873\end{array}$ & $\begin{array}{l}.02475 \\
.02102 \\
.01808 \\
.01575 \\
.01385\end{array}$ \\
\hline $\begin{array}{l}2000 \\
2500 \\
3000 \\
4000\end{array}$ & $\begin{array}{l}.01828 \\
.01038 \\
.00595 \\
.00126\end{array}$ & $\begin{array}{l}.02644 \\
.01581 \\
.00982 \\
.00342\end{array}$ & $\begin{array}{l}.00817 \\
.00544 \\
.00388 \\
.00218\end{array}$ \\
\hline
\end{tabular}

\begin{tabular}{|c|c|c|}
\hline$\frac{{ }^{{ } \mathrm{CaH}-{ }^{-} \mathrm{CaD}}}{R T}$ & $\frac{\mathcal{F}_{\mathrm{CaH}}-\mathcal{F}_{\mathrm{CoT}}}{\mathrm{RT}}$ & $\frac{{ }^{\mathcal{C}_{\mathrm{CaD}}-\mathcal{F}_{\mathrm{CoT}}}}{\mathrm{RT}}$ \\
\hline $\begin{array}{r}2.27037 \\
.96716 \\
.54157 \\
.34183 \\
.23272\end{array}$ & $\begin{array}{r}3.22421 \\
1.35212 \\
.74744 \\
.46817 \\
.31756\end{array}$ & $\begin{array}{l}.95285 \\
.38447 \\
.20555 \\
.12610 \\
.08465\end{array}$ \\
\hline $\begin{array}{l}.16725 \\
.12522 \\
.09679 \\
.07671 \\
.06205\end{array}$ & $\begin{array}{l}.22800 \\
.17086 \\
.13236 \\
.10526 \\
.08553\end{array}$ & $\begin{array}{l}.06059 \\
.04550 \\
.03546 \\
.02845 \\
.02339\end{array}$ \\
\hline $\begin{array}{l}.05104 \\
.04256 \\
.03590 \\
.03058 \\
.02625\end{array}$ & $\begin{array}{l}.07073 \\
.05936 \\
.05044 \\
.04331 \\
.03753\end{array}$ & $\begin{array}{l}.01961 \\
.01673 \\
.01447 \\
.01267 \\
.01122\end{array}$ \\
\hline $\begin{array}{l}.01327 \\
.00708 \\
.00359\end{array}$ & $\begin{array}{l}.02018 \\
.01195 \\
.00730\end{array}$ & $\begin{array}{l}.00687 \\
.00484 \\
.00369\end{array}$ \\
\hline
\end{tabular}


Table 4.04

\begin{tabular}{|c|c|c|c|c|c|c|}
\hline $\mathrm{T}^{\circ} \mathrm{K}$ & $\frac{\mathcal{F}_{S r H}-\mathcal{F}_{S r D}}{R T}$ & $\frac{\mathcal{F}_{S r H}-\mathcal{F}_{S r T}}{R T}$ & $\frac{\mathcal{F}_{S r D}-\mathcal{F}_{S r T}}{R T}$ & $\frac{\mathcal{F}_{\mathrm{BaH}}-\mathcal{F}_{\mathrm{BaD}}}{R T}$ & $\frac{\mathcal{F}_{\mathrm{BaH}}-\mathcal{\gamma}_{\mathrm{BCT}}}{R T}$ & $\frac{\mathcal{F}_{\mathrm{BaD}}-\mathcal{F}_{\mathrm{BaT}}}{R T}$ \\
\hline $\begin{array}{l}100 \\
200 \\
300 \\
400 \\
500\end{array}$ & $\begin{array}{r}2.12084 \\
.89033 \\
.49174 \\
.30735 \\
.20791\end{array}$ & $\begin{array}{r}3.01224 \\
1.24216 \\
.67666 \\
.41953 \\
.28261\end{array}$ & $\begin{array}{l}.89041 \\
.35134 \\
.18460 \\
.11194 \\
.07451\end{array}$ & $\begin{array}{r}2.06348 \\
.86155 \\
.47364 \\
.29520 \\
.19944\end{array}$ & $\begin{array}{r}2.92971 \\
1.20033 \\
.65053 \\
.40209 \\
.27045\end{array}$ & $\begin{array}{l}.86724 \\
.33929 \\
.17723 \\
.10715 \\
.07122\end{array}$ \\
\hline $\begin{array}{r}600 \\
700 \\
800 \\
900 \\
1000\end{array}$ & $\begin{array}{l}.14883 \\
.11116 \\
.08580 \\
.06796 \\
.05497\end{array}$ & $\begin{array}{l}.20195 \\
.15081 \\
.11651 \\
.09245 \\
.07498\end{array}$ & $\begin{array}{l}.05296 \\
.03951 \\
.03059 \\
.02439 \\
.01992\end{array}$ & $\begin{array}{l}.14272 \\
.10666 \\
.08240 \\
.06537 \\
.05296\end{array}$ & $\begin{array}{l}.19315 \\
.14427 \\
.11153 \\
.08860 \\
.07195\end{array}$ & $\begin{array}{l}.05061 \\
.03776 \\
.02927 \\
.02336 \\
.01910\end{array}$ \\
\hline $\begin{array}{l}1100 \\
1200 \\
1300 \\
1400 \\
1500\end{array}$ & $\begin{array}{l}.04523 \\
.03775 \\
.03188 \\
.02718 \\
.02337\end{array}$ & $\begin{array}{l}.06189 \\
.05185 \\
.04399 \\
.03770 \\
.03262\end{array}$ & $\begin{array}{l}.01658 \\
.01403 \\
.01205 \\
.01046 \\
.00919\end{array}$ & $\begin{array}{l}.04367 \\
.03653 \\
.03092 \\
.02644 \\
.02280\end{array}$ & $\begin{array}{l}.05950 \\
.04995 \\
.04245 \\
.03648 \\
.03162\end{array}$ & $\begin{array}{l}.01593 \\
.01351 \\
.01161 \\
.01012 \\
.00890\end{array}$ \\
\hline $\begin{array}{l}2000 \\
2500 \\
3000 \\
4000\end{array}$ & $\begin{array}{l}.01197 \\
.00653 \\
.00347 \\
.00016\end{array}$ & $\begin{array}{l}.01739 \\
.01017 \\
.00611 \\
.00179\end{array}$ & $\begin{array}{l}.00538 \\
.00361 \\
.00262 \\
.00161\end{array}$ & $\begin{array}{l}.01188 \\
.00667 \\
.00370 \\
.00044\end{array}$ & $\begin{array}{l}.01711 \\
.01020 \\
.00631 \\
.00207\end{array}$ & $\begin{array}{l}.00529 \\
.00358 \\
.00265 \\
.00167\end{array}$ \\
\hline
\end{tabular}

\begin{tabular}{|c|c|c|c|c|c|c|}
\hline $\mathrm{T}^{\circ} \mathrm{K}$ & $\frac{{ }^{{ }_{C C U H}}-\mathcal{F}_{\mathrm{CUD}}}{R T}$ & $\frac{\mathcal{F}_{\mathrm{CuH}}-\mathcal{F}_{\mathrm{CuT}}}{\mathrm{RT}}$ & $\frac{\mathcal{F}_{C U D}-\mathcal{F}_{C U T}}{R T}$ & $\frac{\mathcal{F}_{A g H}-\mathcal{F}_{A g D}}{R T}$ & $\frac{\mathcal{F}_{A g H}-F_{A g T}}{R T}$ & $\frac{\mathcal{F}_{A g D}-\mathcal{F}_{A g T}}{R T}$ \\
\hline $\begin{array}{l}100 \\
200 \\
300 \\
400 \\
500\end{array}$ & $\begin{array}{r}3.57245 \\
1.61431 \\
.96300 \\
.64202 \\
.45607\end{array}$ & $\begin{array}{r}5.11181 \\
2.28654 \\
1.34909 \\
.89089 \\
.62836\end{array}$ & $\begin{array}{r}1.53937 \\
.67224 \\
.38610 \\
.24888 \\
.17230\end{array}$ & $\begin{array}{r}3.23072 \\
1.43701 \\
.84157 \\
.55050 \\
.38387\end{array}$ & $\begin{array}{r}4.62047 \\
2.03483 \\
1.17995 \\
.76648 \\
.53271\end{array}$ & $\begin{array}{r}1.38976 \\
.59783 \\
.33838 \\
.21600 \\
.14885\end{array}$ \\
\hline $\begin{array}{r}600 \\
700 \\
800 \\
900 \\
1000\end{array}$ & $\begin{array}{l}.33850 \\
.25977 \\
.20473 \\
.16491 \\
.13525\end{array}$ & $\begin{array}{l}.46407 \\
.35496 \\
.27918 \\
.22461 \\
.18413\end{array}$ & $\begin{array}{l}.12558 \\
.09519 \\
.07446 \\
.05971 \\
.04889\end{array}$ & $\begin{array}{l}.27973 \\
.21058 \\
.16249 \\
.12775 \\
.10185\end{array}$ & $\begin{array}{r}.38817 \\
.29304 \\
.22734 \\
.18017 \\
.14519\end{array}$ & $\begin{array}{l}.10846 \\
.08247 \\
.06485 \\
.05243 \\
.04335\end{array}$ \\
\hline $\begin{array}{l}1100 \\
1200 \\
1300 \\
1400 \\
1500\end{array}$ & $\begin{array}{l}.11265 \\
.09505 \\
.08111 \\
.06989 \\
.06073\end{array}$ & $\begin{array}{l}.15337 \\
.12947 \\
.11057 \\
.09537 \\
.08298\end{array}$ & $\begin{array}{l}.04073 \\
.03443 \\
.02947 \\
.02550 \\
.02226\end{array}$ & $\begin{array}{l}.08200 \\
.06644 \\
.05397 \\
.04378 \\
.03535\end{array}$ & $\begin{array}{l}.11852 \\
.09772 \\
.08112 \\
.06766 \\
.05655\end{array}$ & $\begin{array}{l}.03653 \\
.03129 \\
.02717 \\
.02389 \\
.02122\end{array}$ \\
\hline $\begin{array}{l}2000 \\
2500 \\
3000 \\
4000 \\
5000\end{array}$ & $\begin{array}{l}.03312 \\
.02007 \\
.01292 \\
.00573 \\
.00232\end{array}$ & $\begin{array}{l}.04564 \\
.02797 \\
.01823 \\
.00829 \\
.00342\end{array}$ & $\begin{array}{l}.01253 \\
.00791 \\
.00532 \\
.00257 \\
.00111\end{array}$ & .00829 & .02155 & .01328 \\
\hline
\end{tabular}


Table 4.05

\begin{tabular}{|c|c|c|c|c|c|c|}
\hline $\mathrm{T}^{\circ} \mathrm{K}$ & $\frac{\mathcal{F}_{A U H}-\mathcal{F}_{A U D}}{R T}$ & $\frac{\mathcal{F}_{A U H}-\mathcal{F}_{A U T}}{R T}$ & $\frac{\mathcal{\gamma}_{A U D}-\mathcal{F}_{A U T}}{R T}$ & $\frac{\mathcal{F}_{\mathrm{ZnH}}-\mathcal{F}_{\mathrm{ZnD}}}{\mathrm{RT}}$ & $\frac{\mathcal{F}_{\mathrm{ZnH}}-\mathcal{F}_{\mathrm{ZnT}}}{\mathrm{RT}}$ & $\frac{\mathcal{F}_{\mathrm{ZnD}}-\mathcal{F}_{\mathrm{ZnT}}}{\mathrm{RT}}$ \\
\hline $\begin{array}{l}100 \\
200 \\
300 \\
400 \\
500\end{array}$ & $\begin{array}{r}4.37693 \\
2.01441 \\
1.22736 \\
.83613 \\
.60568\end{array}$ & $\begin{array}{r}6.32388 \\
2.91308 \\
1.77791 \\
1.21640 \\
.88851\end{array}$ & $\begin{array}{r}1.94706 \\
.89873 \\
.55059 \\
.38031 \\
.28286\end{array}$ & $\begin{array}{r}2.87092 \\
1.26278 \\
.73096 \\
.47402 \\
.32914\end{array}$ & $\begin{array}{r}4.09421 \\
1.77690 \\
1.01505 \\
.65154 \\
.44914\end{array}$ & $\begin{array}{r}1.22330 \\
.51413 \\
.28410 \\
.17754 \\
.12001\end{array}$ \\
\hline $\begin{array}{r}600 \\
700 \\
800 \\
900 \\
1000\end{array}$ & $\begin{array}{l}.45710 \\
.35570 \\
.28361 \\
.23071 \\
.19087\end{array}$ & $\begin{array}{l}.67912 \\
.53747 \\
.43750 \\
.36459 \\
.30993\end{array}$ & $\begin{array}{l}.22205 \\
.18179 \\
.15391 \\
.13390 \\
.11908\end{array}$ & $\begin{array}{l}.23986 \\
.18132 \\
.14105 \\
.11227 \\
.09102\end{array}$ & $\begin{array}{l}.32565 \\
.24527 \\
.19029 \\
.15114 \\
.12234\end{array}$ & $\begin{array}{l}.08580 \\
.06396 \\
.04924 \\
.03888 \\
.03133\end{array}$ \\
\hline $\begin{array}{l}1100 \\
1200 \\
1300 \\
1400 \\
1500\end{array}$ & $\begin{array}{l}.16019 \\
.13610 \\
.11690 \\
.10134 \\
.08859\end{array}$ & $\begin{array}{l}.26800 \\
.23518 \\
.20908 \\
.18797 \\
.17069\end{array}$ & $\begin{array}{l}.10783 \\
.09910 \\
.09220 \\
.08665 \\
.08212\end{array}$ & $\begin{array}{l}.07491 \\
.06241 \\
.05252 \\
.04457 \\
.03805\end{array}$ & $\begin{array}{l}.10055 \\
.08367 \\
.07034 \\
.05962 \\
.05085\end{array}$ & $\begin{array}{l}.02565 \\
.02127 \\
.01783 \\
.01506 \\
.01281\end{array}$ \\
\hline $\begin{array}{l}2000 \\
2500 \\
3000 \\
4000 \\
5000\end{array}$ & $\begin{array}{l}.04970 \\
.03104 \\
.02068 \\
.01009 \\
.00493\end{array}$ & $\begin{array}{l}.11815 \\
.09299 \\
.07898 \\
.06457 \\
.05743\end{array}$ & $\begin{array}{l}.06847 \\
.06196 \\
.05831 \\
.05449 \\
.05251\end{array}$ & $\begin{array}{l}.01810 \\
.00811 \\
.00211\end{array}$ & $\begin{array}{l}.02401 \\
.01059 \\
.00249\end{array}$ & $\begin{array}{l}.00593 \\
.00249 \\
.00039\end{array}$ \\
\hline
\end{tabular}

\begin{tabular}{|c|c|c|c|c|c|c|}
\hline $\mathrm{T}^{\circ} \mathrm{K}$ & $\frac{{ }^{{ }^{7} \mathrm{CdH}}-{ }^{-}{ }_{C d D}}{R T}$ & $\frac{{ }^{{ }^{C} \mathrm{CdH}-{ }^{-} \mathrm{CdT}}}{R T}$ & $\frac{\mathcal{F}_{\mathrm{CdD}}-\mathcal{F}_{\mathrm{CdT}}}{\mathrm{RT}}$ & $\frac{\mathcal{F}_{\mathrm{HgH}^{-}} \mathcal{F}_{\mathrm{HgD}}}{\mathrm{RT}}$ & $\frac{\mathcal{F}_{\mathrm{HgH}^{-}} \mathcal{F}_{\mathrm{HgT}}}{\mathrm{RT}}$ & $\frac{\mathcal{F}_{\mathrm{HgD}}-\mathcal{F}_{\mathrm{HgT}}}{\mathrm{RT}}$ \\
\hline $\begin{array}{l}100 \\
200 \\
300 \\
400 \\
500\end{array}$ & $\begin{array}{r}2.53242 \\
1.09132 \\
.61817 \\
.39373 \\
.26987\end{array}$ & $\begin{array}{r}3.60827 \\
1.52993 \\
.85378 \\
.53779 \\
.36559\end{array}$ & $\begin{array}{r}1.07576 \\
.43857 \\
.23558 \\
.14405 \\
.09571\end{array}$ & $\begin{array}{r}2.32263 \\
.98457 \\
.54779 \\
.34241 \\
.22954\end{array}$ & $\begin{array}{r}3.29968 \\
1.37429 \\
.75237 \\
.46451 \\
.30844\end{array}$ & $\begin{array}{l}.97542 \\
.38890 \\
.20405 \\
.12170 \\
.07858\end{array}$ \\
\hline $\begin{array}{r}600 \\
700 \\
800 \\
900 \\
1000\end{array}$ & $\begin{array}{l}.19504 \\
.14681 \\
.11416 \\
.09114 \\
.07440\end{array}$ & $\begin{array}{l}.26246 \\
.19635 \\
.15174 \\
.12031 \\
.09744\end{array}$ & $\begin{array}{l}.06742 \\
.04954 \\
.03758 \\
.02917 \\
.02304\end{array}$ & $\begin{array}{l}.16115 \\
.11657 \\
.08577 \\
.06339 \\
.04643\end{array}$ & $\begin{array}{l}.21487 \\
.15439 \\
.11288 \\
.08293 \\
.06037\end{array}$ & $\begin{array}{l}.05345 \\
.03759 \\
.02692 \\
.01937 \\
.01379\end{array}$ \\
\hline $\begin{array}{l}1100 \\
1200 \\
1300 \\
1400 \\
1500\end{array}$ & $\begin{array}{l}.06189 \\
.05232 \\
.04489 \\
.03900 \\
.03428\end{array}$ & $\begin{array}{l}.08032 \\
.06719 \\
.05692 \\
.04876 \\
.04215\end{array}$ & $\begin{array}{l}.01844 \\
.01486 \\
.01204 \\
.00976 \\
.00788\end{array}$ & $\begin{array}{l}.03310 \\
.02227 \\
.01319 \\
.00544\end{array}$ & $\begin{array}{l}.04274 \\
.02848 \\
.01665 \\
.00655\end{array}$ & $\begin{array}{l}.00950 \\
.00610 \\
.00333 \\
.00101\end{array}$ \\
\hline $\begin{array}{l}2000 \\
2500 \\
3000 \\
4000 \\
5000\end{array}$ & $\begin{array}{l}.02068 \\
.01493 \\
.01223 \\
.01036 \\
.01021\end{array}$ & $\begin{array}{l}.02264 \\
.01366 \\
.00881 \\
.00393 \\
.00152\end{array}$ & .00197 & & & \\
\hline
\end{tabular}


Table 4.06

\begin{tabular}{|c|c|c|c|c|c|c|}
\hline $\mathrm{T}^{\circ} \mathrm{K}$ & $\frac{\mathcal{F}_{\mathrm{BH}}-\mathcal{F}_{\mathrm{BO}}}{\mathrm{RT}}$ & $\frac{\mathcal{F}_{B H}-\mathcal{F}_{B T}}{R T}$ & $\frac{\mathcal{F}_{\mathrm{BD}}-\mathcal{F}_{\mathrm{BT}}}{R T}$ & $\frac{\mathcal{F}_{A \mid H^{-}} \mathcal{Z}_{A I O}}{R T}$ & $\cdot \frac{\mathcal{Z}_{A I H}-\mathcal{F}_{A I T}}{R T}$ & $\frac{\mathcal{F}_{A I O}-\mathcal{F}_{A I T}}{R T}$ \\
\hline $\begin{array}{l}100 \\
200 \\
300 \\
400 \\
500\end{array}$ & $\begin{array}{r}3.80014 \\
1.74537 \\
1.06064 \\
.71968 \\
.51807\end{array}$ & $\begin{array}{r}5.76045 \\
2.64199 \\
1.60343 \\
1.08812 \\
.78560\end{array}$ & $\begin{array}{r}1.96032 \\
.89663 \\
.54279 \\
.36845 \\
.26754\end{array}$ & $\begin{array}{r}2.98767 \\
1.32756 \\
.77700 \\
.50883 \\
.35615\end{array}$ & $\begin{array}{r}4.25408 \\
1.86813 \\
1.08030 \\
.70070 \\
.48718\end{array}$ & $\begin{array}{r}1.26642 \\
.54058 \\
.30331 \\
.19189 \\
.13104\end{array}$ \\
\hline $\begin{array}{r}600 \\
700 \\
800 \\
900 \\
1000\end{array}$ & $\begin{array}{l}.38742 \\
.29776 \\
.23373 \\
.18654 \\
.15086\end{array}$ & $\begin{array}{l}.59125 \\
.45904 \\
.36530 \\
.29668 \\
.24505\end{array}$ & $\begin{array}{l}.20385 \\
.16129 \\
.13158 \\
.11014 \\
.09420\end{array}$ & $\begin{array}{l}.26131 \\
.19874 \\
.15553 \\
.12456 \\
.10165\end{array}$ & $\begin{array}{l}.35587 \\
.26990 \\
.21088 \\
.16876 \\
.13771\end{array}$ & $\begin{array}{l}.09457 \\
.07117 \\
.05536 \\
.04421 \\
.03607\end{array}$ \\
\hline $\begin{array}{l}1100 \\
1200 \\
1300 \\
1400 \\
1500\end{array}$ & $\begin{array}{l}.12330 \\
.10161 \\
.08426 \\
.07018 \\
.05862\end{array}$ & $\begin{array}{l}.20535 \\
.17423 \\
.14941 \\
.12933 \\
.11287\end{array}$ & $\begin{array}{l}.08206 \\
.07263 \\
.06516 \\
.05916 \\
.05427\end{array}$ & $\begin{array}{l}.08429 \\
.07082 \\
.06019 \\
.05162 \\
.04465\end{array}$ & $\begin{array}{l}.11425 \\
.09609 \\
.08176 \\
.07027 \\
.06091\end{array}$ & $\begin{array}{l}.02997 \\
.02527 \\
.02159 \\
.01866 \\
.01627\end{array}$ \\
\hline $\begin{array}{l}2000 \\
2500 \\
3000 \\
4000\end{array}$ & $\begin{array}{l}.02313 \\
.00592\end{array}$ & $\begin{array}{l}.06272 \\
.03864\end{array}$ & $\begin{array}{l}.03960 \\
.03272\end{array}$ & $\begin{array}{l}.02356 \\
.01342 \\
.00768 \\
.00148\end{array}$ & $\begin{array}{l}.03271 \\
.01925 \\
.01168 \\
.00361\end{array}$ & $\begin{array}{l}.00917 \\
.00584 \\
.00401 \\
.00214\end{array}$ \\
\hline
\end{tabular}

\begin{tabular}{|c|c|c|c|c|c|c|}
\hline $\mathrm{T}^{\circ} \mathrm{K}$ & $\frac{\mathcal{F}_{\operatorname{lnH}}-\mathcal{F}_{\operatorname{lnD}}}{\mathrm{RT}}$ & $\frac{\mathcal{F}_{\mathrm{InH}}-\mathcal{F}_{\operatorname{lnT}}}{R T}$ & $\frac{\mathcal{F}_{\text {InD }}-\mathcal{F}_{\text {inT }}}{R T}$ & $\frac{\mathcal{F}_{T I H^{-}}-\mathcal{F}_{T I D}}{R T}$ & $\frac{\mathcal{F}_{T I H}-\mathcal{F}_{T I T}}{R T}$ & $\frac{\mathcal{F}_{T I D}-\mathcal{F}_{T I T}}{R T}$ \\
\hline $\begin{array}{l}100 \\
200 \\
300 \\
400 \\
500\end{array}$ & $\begin{array}{r}2.67306 \\
1.16647 \\
.66994 \\
.43228 \\
.29980\end{array}$ & $\begin{array}{r}3.80509 \\
1.63387 \\
.92395 \\
.58909 \\
.40489\end{array}$ & $\begin{array}{r}1.13204 \\
.46741 \\
.25402 \\
.15682 \\
.10510\end{array}$ & $\begin{array}{r}2.50998 \\
1.08181 \\
.61302 \\
.39073 \\
.26806\end{array}$ & $\begin{array}{r}3.57375 \\
1.51548 \\
.84638 \\
.53404 \\
.36392\end{array}$ & $\begin{array}{r}1.06388 \\
.43373 \\
.23341 \\
.14335 \\
.09589\end{array}$ \\
\hline $\begin{array}{r}600 \\
700 \\
800 \\
900 \\
1000\end{array}$ & $\begin{array}{l}.21903 \\
.16654 \\
.13071 \\
.10525 \\
.08657\end{array}$ & $\begin{array}{l}.29372 \\
.22199 \\
.17328 \\
.13879 \\
.11357\end{array}$ & $\begin{array}{l}.07470 \\
.05546 \\
.04258 \\
.03355 \\
.02701\end{array}$ & $\begin{array}{l}.19389 \\
.14601 \\
.11349 \\
.09047 \\
.07366\end{array}$ & $\begin{array}{l}.26202 \\
.19663 \\
.15238 \\
.12115 \\
.09834\end{array}$ & $\begin{array}{l}.06815 \\
.05064 \\
.03891 \\
.03070 \\
.02470\end{array}$ \\
\hline $\begin{array}{l}1100 \\
1200 \\
1300 \\
1400 \\
1500\end{array}$ & $\begin{array}{l}.07248 \\
.06160 \\
.05304 \\
.04618 \\
.04062\end{array}$ & $\begin{array}{l}.09459 \\
.07996 \\
.06846 \\
.05926 \\
.05180\end{array}$ & $\begin{array}{l}.02212 \\
.01836 \\
.01544 \\
.01309 \\
.01119\end{array}$ & $\begin{array}{l}.06100 \\
.05127 \\
.04363 \\
.03752 \\
.03259\end{array}$ & $\begin{array}{l}.08118 \\
.06797 \\
.05758 \\
.04925 \\
.04250\end{array}$ & $\begin{array}{l}.02019 \\
.01671 \\
.01397 \\
.01175 \\
.00992\end{array}$ \\
\hline $\begin{array}{l}2000 \\
2500 \\
3000\end{array}$ & $\begin{array}{l}.02389 \\
.01594 \\
.01150\end{array}$ & $\begin{array}{l}.02940 \\
.01878 \\
.01283\end{array}$ & $\begin{array}{l}.00552 \\
.00284 \\
.00134\end{array}$ & $\begin{array}{l}.01785 \\
.01097 \\
.00722\end{array}$ & $\begin{array}{l}.02205 \\
.01214 \\
.00640\end{array}$ & $\begin{array}{l}.00422 \\
.00119\end{array}$ \\
\hline
\end{tabular}




\begin{tabular}{|c|c|c|c|c|c|c|}
\hline $\mathrm{T}^{\circ} \mathrm{K}$ & $\frac{{ }^{7} \mathrm{CH}^{-}{ }^{-} \mathrm{CD}}{\mathrm{RT}}$ & $\frac{{ }^{{ }^{7} \mathrm{CH}}-{ }^{-}{ }_{\mathrm{CT}}}{\mathrm{RT}}$ & $\frac{{ }^{{ }^{C}} \mathrm{CD}-{ }^{\mathcal{F}} \mathrm{CT}}{\mathrm{RT}}$ & $\frac{\mathcal{F}_{\mathrm{SiH}}-\mathcal{F}_{\mathrm{SiD}}}{\mathrm{RT}}$ & $\frac{\mathcal{F}_{\mathrm{SiH}}-\mathcal{F}_{\mathrm{SiT}}}{\mathrm{RT}}$ & $\frac{\mathcal{F}_{S i D}-\mathcal{F}_{S i T}}{R T}$ \\
\hline $\begin{array}{l}100 \\
200 \\
300 \\
400 \\
500\end{array}$ & $\begin{array}{r}5.02265 \\
2.35203 \\
1.46261 \\
1.01852 \\
.75371\end{array}$ & $\begin{array}{l}7.18188 \\
3.34766 \\
2.07069 \\
1.43397 \\
1.05564\end{array}$ & $\begin{array}{r}2.15914 \\
.99559 \\
.60808 \\
.41543 \\
.30192\end{array}$ & $\begin{array}{r}3.71636 \\
1.68889 \\
1.01517 \\
.68222 \\
.48831\end{array}$ & $\begin{array}{r}5.27465 \\
2.37445 \\
1.41245 \\
.94053 \\
.66871\end{array}$ & $\begin{array}{r}1.55830 \\
.67556 \\
.39729 \\
.25832 \\
.18041\end{array}$ \\
\hline $\begin{array}{r}600 \\
700 \\
800 \\
900 \\
1000\end{array}$ & $\begin{array}{l}.57975 \\
.45849 \\
.37047 \\
.30464 \\
.25425\end{array}$ & $\begin{array}{l}.80850 \\
.63733 \\
.51381 \\
.42195 \\
.35194\end{array}$ & $\begin{array}{l}.22875 \\
.17884 \\
.14333 \\
.11731 \\
.09768\end{array}$ & $\begin{array}{l}.36504 \\
.28220 \\
.22411 \\
.18171 \\
.15025\end{array}$ & $\begin{array}{l}.49783 \\
.38396 \\
.30472 \\
.24765 \\
.20532\end{array}$ & $\begin{array}{l}.13179 \\
.10187 \\
.08082 \\
.06594 \\
.05508\end{array}$ \\
\hline $\begin{array}{l}1100 \\
1200 \\
1300 \\
1400 \\
1500\end{array}$ & $\begin{array}{l}.21488 \\
.18363 \\
.15842 \\
.13783 \\
.12085\end{array}$ & $\begin{array}{l}.29748 \\
.25436 \\
.21969 \\
.19145 \\
.16815\end{array}$ & $\begin{array}{l}.08260 \\
.07073 \\
.06127 \\
.05362 \\
.04731\end{array}$ & $\begin{array}{l}.12625 \\
.10634 \\
.09271 \\
.08078 \\
.07106\end{array}$ & $\begin{array}{l}.17323 \\
.14832 \\
.12793 \\
.11292 \\
.10014\end{array}$ & $\begin{array}{l}.04699 \\
.04082 \\
.03597 \\
.03215 \\
.02909\end{array}$ \\
\hline $\begin{array}{l}2000 \\
2500 \\
3000 \\
4000 \\
5000\end{array}$ & $\begin{array}{l}.06829 \\
.04269 \\
.02839 \\
.01380 \\
.00677\end{array}$ & $\begin{array}{l}.09646 \\
.06171 \\
.04238 \\
.02280 \\
.01352\end{array}$ & $\begin{array}{l}.02818 \\
.01903 \\
.01399 \\
.00900 \\
.00675\end{array}$ & $\begin{array}{l}.04185 \\
.02817 \\
.02071 \\
.01331 \\
.00984\end{array}$ & $\begin{array}{l}.06210 \\
.04465 \\
.03540 \\
.02662 \\
.02290\end{array}$ & $\begin{array}{l}.02026 \\
.01648 \\
.01470 \\
.01333 \\
.01308\end{array}$ \\
\hline
\end{tabular}

\begin{tabular}{|c|c|c|c|c|c|c|}
\hline $\mathrm{T}^{\circ} \mathrm{K}$ & $\frac{\mathcal{F}_{\mathrm{SnH}}-\mathcal{F}_{\mathrm{SnD}}}{\mathrm{RT}}$ & $\frac{\mathcal{F}_{\mathrm{SnH}}-\mathcal{F}_{\mathrm{SnT}}}{R T}$ & $\frac{\mathcal{F}_{\mathrm{SnD}}-\mathcal{F}_{\mathrm{SnT}}}{\mathrm{RT}}$ & $\frac{{ }^{7} \mathrm{PbH}-\mathcal{F} \mathrm{PbD}}{\mathrm{RT}}$ & $\frac{\mathcal{F}_{\mathrm{PbH}}-\mathcal{F}_{\mathrm{PbT}}}{\mathrm{RT}}$ & $\frac{\mathcal{F}_{\mathrm{PbD}}-\mathcal{F} \mathrm{PbT}}{\mathrm{RT}}$ \\
\hline $\begin{array}{l}100 \\
200 \\
300 \\
400 \\
500\end{array}$ & $\begin{array}{r}2.93557 \\
1.29512 \\
.75208 \\
.48919 \\
.34082\end{array}$ & $\begin{array}{r}4.18441 \\
1.82069 \\
1.04285 \\
.67118 \\
.46425\end{array}$ & $\begin{array}{r}1.24175 \\
.52203 \\
.28841 \\
.18023 \\
.12202\end{array}$ & $\begin{array}{r}2.85986 \\
1.25648 \\
.72650 \\
.47090 \\
.32715\end{array}$ & $\begin{array}{r}4.07890 \\
1.76649 \\
1.00718 \\
.64588 \\
.44538\end{array}$ & $\begin{array}{r}1.21895 \\
.50997 \\
.28066 \\
.17497 \\
.11822\end{array}$ \\
\hline $\begin{array}{r}600 \\
700 \\
800 \\
900 \\
1000\end{array}$ & $\begin{array}{l}.24954 \\
.18991 \\
.14912 \\
.12015 \\
.09896\end{array}$ & $\begin{array}{l}.33828 \\
.25665 \\
.20111 \\
.16185 \\
.13320\end{array}$ & $\begin{array}{l}.08757 \\
.06574 \\
.05111 \\
.04092 \\
.03354\end{array}$ & $\begin{array}{l}.23885 \\
.18112 \\
.14153 \\
.11331 \\
.09255\end{array}$ & $\begin{array}{l}.32346 \\
.24436 \\
.19041 \\
.15210 \\
.12401\end{array}$ & $\begin{array}{l}.08460 \\
.06324 \\
.04887 \\
.03879 \\
.03146\end{array}$ \\
\hline $\begin{array}{l}1100 \\
1200 \\
1300 \\
1400 \\
1500\end{array}$ & $\begin{array}{l}.08303 \\
.07082 \\
.06125 \\
.05366 \\
.04753\end{array}$ & $\begin{array}{l}.11173 \\
.09527 \\
.08242 \\
.07220 \\
.06397\end{array}$ & $\begin{array}{l}.02806 \\
.02387 \\
.02063 \\
.01804 \\
.01598\end{array}$ & $\begin{array}{l}.07687 \\
.06475 \\
.05522 \\
.04757 \\
.04135\end{array}$ & $\begin{array}{l}.10283 \\
.08650 \\
.07366 \\
.06337 \\
.05502\end{array}$ & $\begin{array}{l}.02596 \\
.02175 \\
.01844 \\
.01580 \\
.01367\end{array}$ \\
\hline $\begin{array}{l}2000 \\
2500 \\
3000 \\
4000 \\
5000\end{array}$ & $\begin{array}{l}.02960 \\
.02157 \\
.01738 \\
.01348 \\
.01181\end{array}$ & $\begin{array}{l}.03986 \\
.02903 \\
.02334 \\
.01791 \\
.01549\end{array}$ & $\begin{array}{l}.00992 \\
.00718 \\
.00573 \\
.00426 \\
.00354\end{array}$ & $\begin{array}{l}.02272 \\
.01393 \\
.00908 \\
.00408 \\
.00159\end{array}$ & $\begin{array}{l}.02997 \\
.01815 \\
.01158 \\
.00476 \\
.00126\end{array}$ & $\begin{array}{l}.00726 \\
.00423 \\
.00251 \\
.00068\end{array}$ \\
\hline
\end{tabular}


Table 4.08

\begin{tabular}{|c|c|c|c|c|c|c|}
\hline $\mathrm{T}^{\circ} \mathrm{K}$ & $\frac{\gamma_{N H}-\gamma_{N D}}{R T}$ & $\frac{\mathcal{F}_{N H}-\mathcal{F}_{N T}}{R T}$ & $\frac{\mathcal{F}_{N D}-\mathcal{F}_{N T}}{R T}$ & $\frac{\mathcal{F}_{\mathrm{PH}}-\mathcal{F} \mathrm{PD}}{\mathrm{RT}}$ & $\frac{\mathcal{F}_{\mathrm{PH}}-\mathcal{F}_{\mathrm{PT}}}{\mathrm{RT}}$ & $\frac{\mathcal{F P D}-\mathcal{F} P T}{R T}$ \\
\hline $\begin{array}{l}100 \\
200 \\
300 \\
400 \\
500\end{array}$ & $\begin{array}{r}5.84218 \\
2.76204 \\
1.73529 \\
1.22212 \\
.91507\end{array}$ & $\begin{array}{l}8.31805 \\
3.91387 \\
2.44583 \\
1.71258 \\
1.27483\end{array}$ & $\begin{array}{r}2.47578 \\
1.15179 \\
.71052 \\
.49044 \\
.35975\end{array}$ & $\begin{array}{r}4.46431 \\
2.06442 \\
1.26477 \\
.86679 \\
.63174\end{array}$ & $\begin{array}{r}6.36080 \\
2.92128 \\
1.77603 \\
1.20832 \\
.87557\end{array}$ & $\begin{array}{r}1.89660 \\
.85692 \\
.51131 \\
.34157 \\
.24386\end{array}$ \\
\hline $\begin{array}{r}600 \\
700 \\
800 \\
900 \\
1000\end{array}$ & $\begin{array}{l}.71201 \\
.56916 \\
.46443 \\
.38532 \\
.32417\end{array}$ & $\begin{array}{l}.98657 \\
.78489 \\
.63789 \\
.52747 \\
.44256\end{array}$ & $\begin{array}{l}.27455 \\
.21573 \\
.17346 \\
.14215 \\
.11839\end{array}$ & $\begin{array}{l}.47966 \\
.37551 \\
.30127 \\
.24667 \\
.20549\end{array}$ & $\begin{array}{l}.66218 \\
.51727 \\
.41471 \\
.33973 \\
.28344\end{array}$ & $\begin{array}{l}.18254 \\
.14178 \\
.11346 \\
.09308 \\
.07797\end{array}$ \\
\hline $\begin{array}{l}1100 \\
1200 \\
1300 \\
1400 \\
1500\end{array}$ & $\begin{array}{l}.27598 \\
.23741 \\
.20610 \\
.18039 \\
.15902\end{array}$ & $\begin{array}{r}.37594 \\
.32283 \\
.27988 \\
.24470 \\
.21554\end{array}$ & $\begin{array}{l}.09996 \\
.08543 \\
.07379 \\
.06432 \\
.05652\end{array}$ & $\begin{array}{l}.17375 \\
.14883 \\
.12895 \\
.11286 \\
.09968\end{array}$ & $\begin{array}{l}.24022 \\
.20639 \\
.17947 \\
.15772 \\
.13992\end{array}$ & $\begin{array}{l}.06649 \\
.05758 \\
.05054 \\
.04488 \\
.04025\end{array}$ \\
\hline $\begin{array}{l}2000 \\
2500 \\
3000 \\
4000 \\
5000\end{array}$ & $\begin{array}{l}.09231 \\
.05940 \\
.04091 \\
.02197 \\
.01288\end{array}$ & $\begin{array}{l}.12496 \\
.08052 \\
.05564 \\
.03019 \\
.01798\end{array}$ & $\begin{array}{l}.03265 \\
.02113 \\
.01474 \\
.00823 \\
.00511\end{array}$ & $\begin{array}{l}.05966 \\
.04067 \\
.03028 \\
.01995 \\
.01518\end{array}$ & $\begin{array}{l}.08595 \\
.06027 \\
.04612 \\
.03173 \\
.02470\end{array}$ & $\begin{array}{r}.02631 \\
.01962 \\
.01586 \\
.01179 \\
.00954\end{array}$ \\
\hline
\end{tabular}

\begin{tabular}{|c|c|c|c|c|c|c|}
\hline $\mathrm{T}^{\circ} \mathrm{K}$ & $\frac{\mathcal{F}_{\mathrm{BiH}}-\mathcal{F}_{\mathrm{BiD}}}{R T}$ & $\frac{\mathcal{F}_{\mathrm{BiH}}-\mathcal{F}_{\mathrm{BiT}}}{R T}$ & $\frac{\mathcal{F}_{\mathrm{BiD}}-\mathcal{F}_{\mathrm{BiT}}}{R T}$ & $\frac{\mathcal{F}_{O H^{-}} \mathcal{F}_{O D}}{R T}$ & $\frac{\mathcal{F}_{O H}-\mathcal{F}_{O T}}{R T}$ & $\frac{\mathcal{F}_{O D}-\mathcal{F}_{O T}}{R T}$ \\
\hline $\begin{array}{l}100 \\
200 \\
300 \\
400 \\
500\end{array}$ & $\begin{array}{r}3.12941 \\
1.38921 \\
.81213 \\
.53105 \\
.37102\end{array}$ & $\begin{array}{r}4.48108 \\
1.96662 \\
1.13674 \\
.73721 \\
.51261\end{array}$ & $\begin{array}{r}1.35168 \\
.57742 \\
.32462 \\
.20618 \\
.14160\end{array}$ & $\begin{array}{l}6.81424 \\
3.23823 \\
2.04975 \\
1.45614 \\
1.10049\end{array}$ & $\begin{array}{l}9.73206 \\
4.60671 \\
2.90286 \\
2.05196 \\
1.54276\end{array}$ & $\begin{array}{r}2.91783 \\
1.36849 \\
.85312 \\
.59582 \\
.44228\end{array}$ \\
\hline $\begin{array}{r}600 \\
700 \\
800 \\
900 \\
1000\end{array}$ & $\begin{array}{l}.27158 \\
.20600 \\
.16068 \\
.12822 \\
.10423\end{array}$ & $\begin{array}{r}.37452 \\
.28414 \\
.22207 \\
.17778 \\
.14516\end{array}$ & $\begin{array}{l}.10295 \\
.07815 \\
.06139 \\
.04957 \\
.04094\end{array}$ & $\begin{array}{l}.86434 \\
.69712 \\
.57348 \\
.47924 \\
.40569\end{array}$ & $\begin{array}{r}1.20560 \\
.96779 \\
.79287 \\
.66020 \\
.55718\end{array}$ & $\begin{array}{l}.34127 \\
.27069 \\
.21939 \\
.18097 \\
.15150\end{array}$ \\
\hline $\begin{array}{l}1100 \\
1200 \\
1300 \\
1400 \\
1500\end{array}$ & $\begin{array}{l}.08605 \\
.07194 \\
.06082 \\
.05189 \\
.04463\end{array}$ & $\begin{array}{l}.12049 \\
.10140 \\
.08636 \\
.07430 \\
.06448\end{array}$ & $\begin{array}{l}.03445 \\
.02947 \\
.02555 \\
.02242 \\
.01986\end{array}$ & $\begin{array}{l}.34725 \\
.30010 \\
.26154 \\
.22966 \\
.20305\end{array}$ & $\begin{array}{r}.47567 \\
.41019 \\
.35685 \\
.31287 \\
.27624\end{array}$ & $\begin{array}{l}.12844 \\
.11012 \\
.09530 \\
.08321 \\
.07319\end{array}$ \\
\hline $\begin{array}{l}2000 \\
2500 \\
3000 \\
4000 \\
5000\end{array}$ & $\begin{array}{l}.02276 \\
.01241 \\
.00667 \\
.00077\end{array}$ & $\begin{array}{l}.03494 \\
.02093 \\
.01312 \\
.00498 \\
.00077\end{array}$ & $\begin{array}{l}.01220 \\
.00853 \\
.00646 \\
.00421 \\
.00297\end{array}$ & $\begin{array}{l}.11901 \\
.07709 \\
.05350 \\
.02960 \\
.01856\end{array}$ & $\begin{array}{l}.16109 \\
.10382 \\
.07170 \\
.03835 \\
.02249\end{array}$ & $\begin{array}{l}.04208 \\
.02674 \\
.01802 \\
.00876 \\
.00395\end{array}$ \\
\hline
\end{tabular}




\begin{tabular}{|c|c|c|c|}
\hline $\mathrm{T}^{\circ} \mathrm{K}$ & $\frac{\mathcal{F}_{S H}-\mathcal{F}_{S D}}{R T}$ & $\frac{\mathcal{F}_{S H}-\mathcal{F}_{S T}}{R T}$ & $\frac{\mathcal{F}_{\mathrm{SD}}-\mathcal{F}_{\mathrm{ST}}}{\mathrm{RT}}$ \\
\hline $\begin{array}{l}100 \\
200 \\
300 \\
400 \\
500\end{array}$ & $\begin{array}{r}5.15638 \\
2.40510 \\
1.49129 \\
1.03546 \\
.76446\end{array}$ & $\begin{array}{l}7.33959 \\
3.39634 \\
2.08503 \\
1.43289 \\
1.04726\end{array}$ & $\begin{array}{r}2.18322 \\
.99125 \\
.59374 \\
.39746 \\
.28281\end{array}$ \\
\hline $\begin{array}{r}600 \\
700 \\
800 \\
900 \\
1000\end{array}$ & $\begin{array}{l}.58728 \\
.46436 \\
.37563 \\
.30954 \\
.25913\end{array}$ & $\begin{array}{l}.79690 \\
.62457 \\
.50099 \\
.40953 \\
.34013\end{array}$ & $\begin{array}{l}.20964 \\
.16022 \\
.12537 \\
.09999 \\
.08101\end{array}$ \\
\hline $\begin{array}{l}1100 \\
1200 \\
1300 \\
1400 \\
1500\end{array}$ & $\begin{array}{l}.21986 \\
.18875 \\
.16371 \\
.14327 \\
.12642\end{array}$ & $\begin{array}{l}.28630 \\
.24382 \\
.20972 \\
.18199 \\
.15914\end{array}$ & $\begin{array}{l}.06643 \\
.05507 \\
.04602 \\
.03873 \\
.03274\end{array}$ \\
\hline $\begin{array}{l}2000 \\
2500 \\
3000 \\
4000 \\
5000\end{array}$ & $\begin{array}{l}.07422 \\
.04869 \\
.03430 \\
.01944 \\
.01209\end{array}$ & $\begin{array}{l}.08883 \\
.05469 \\
.03561 \\
.01610 \\
.00668\end{array}$ & $\begin{array}{l}.01463 \\
.00601 \\
.00131\end{array}$ \\
\hline
\end{tabular}

\begin{tabular}{|c|c|c|}
\hline $\mathcal{F}_{F H}-\mathcal{F}_{F D}$ & $\mathcal{\exists}_{F H}-\mathcal{F}_{F T}$ & $\mathcal{\exists}_{F D}-\mathcal{F}_{F T}$ \\
\hline$R T$ & RT & $\mathrm{RT}$ \\
\hline $\begin{array}{l}7.66916 \\
3.67125 \\
2.33852 \\
1.67217 \\
1.27253\end{array}$ & $\begin{array}{c}10.94328 \\
5.21856 \\
3.31021 \\
2.35617 \\
1.78438\end{array}$ & $\begin{array}{r}3.27413 \\
1.54732 \\
.97171 \\
.68401 \\
.51186\end{array}$ \\
\hline $\begin{array}{r}1.00663 \\
.81766 \\
.67725 \\
.56955 \\
.48498\end{array}$ & $\begin{array}{r}1.40466 \\
1.13565 \\
.93659 \\
.78465 \\
.66592\end{array}$ & $\begin{array}{l}.39803 \\
.31800 \\
.25936 \\
.21511 \\
.18095\end{array}$ \\
\hline $\begin{array}{l}.41733 \\
.36239 \\
.31723 \\
.27966 \\
.24812\end{array}$ & $\begin{array}{l}.57139 \\
.49496 \\
.43237 \\
.38051 \\
.33713\end{array}$ & $\begin{array}{l}.15407 \\
.13258 \\
.11515 \\
.10086 \\
.08901\end{array}$ \\
\hline $\begin{array}{l}.14734 \\
.09613 \\
.06686 \\
.03657 \\
.02198\end{array}$ & $\begin{array}{l}.19942 \\
.13011 \\
.09072 \\
.05021 \\
.03087\end{array}$ & $\begin{array}{l}.05209 \\
.03399 \\
.02388 \\
.01366 \\
.00890\end{array}$ \\
\hline
\end{tabular}

\begin{tabular}{|c|c|c|c|c|c|c|}
\hline $\mathrm{T}^{\circ} \mathrm{K}$ & $\frac{{ }^{{ }^{7} \mathrm{ClH}}-{ }^{7} \mathrm{CID}}{R T}$ & $\frac{\mathcal{F}_{\mathrm{ClH}}-\mathcal{F}_{\mathrm{CIT}}}{\mathrm{RT}}$ & $\frac{\mathcal{F}_{\mathrm{CID}}-\mathcal{F}_{\mathrm{CIT}}}{\mathrm{RT}}$ & $\frac{\mathcal{F}_{\mathrm{BrH}}-\mathcal{F}_{\mathrm{BrD}}}{\mathrm{RT}}$ & $\frac{\mathcal{F}_{\mathrm{BrH}}-\mathcal{F}_{\mathrm{BrT}}}{\mathrm{RT}}$ & $\frac{\mathcal{F}_{\mathrm{BrD}}-\mathcal{F}_{\mathrm{BrT}}}{\mathrm{RT}}$ \\
\hline $\begin{array}{l}100 \\
200 \\
300 \\
400 \\
500\end{array}$ & $\begin{array}{r}5.63234 \\
2.64789 \\
1.65310 \\
1.15615 \\
.85941\end{array}$ & $\begin{array}{l}8.05594 \\
3.76476 \\
2.33459 \\
1.62106 \\
1.19662\end{array}$ & $\begin{array}{r}2.42361 \\
1.11688 \\
.68150 \\
.46492 \\
.33722\end{array}$ & $\begin{array}{r}5.04703 \\
2.35119 \\
1.45268 \\
1.00447 \\
.73808\end{array}$ & $\begin{array}{l}7.23133 \\
3.34598 \\
2.05150 \\
1.40742 \\
1.02692\end{array}$ & $\begin{array}{r}2.18431 \\
.99480 \\
.59882 \\
.40297 \\
.28885\end{array}$ \\
\hline $\begin{array}{r}600 \\
700 \\
800 \\
900 \\
1000\end{array}$ & $\begin{array}{l}.66400 \\
.52736 \\
.42785 \\
.35318 \\
.29585\end{array}$ & $\begin{array}{l}.91879 \\
.72586 \\
.58635 \\
.48233 \\
.40289\end{array}$ & $\begin{array}{l}.25480 \\
.19851 \\
.15851 \\
.12916 \\
.10705\end{array}$ & $\begin{array}{l}.56409 \\
.44364 \\
.35686 \\
.29241 \\
.24335\end{array}$ & $\begin{array}{l}.78036 \\
.61109 \\
.49002 \\
.40069 \\
.33305\end{array}$ & $\begin{array}{l}.21629 \\
.16745 \\
.13317 \\
.10829 \\
.08971\end{array}$ \\
\hline $\begin{array}{l}1100 \\
1200 \\
1300 \\
1400 \\
1500\end{array}$ & $\begin{array}{l}.25093 \\
.21518 \\
.18629 \\
.16266 \\
.14310\end{array}$ & $\begin{array}{l}.34096 \\
.29185 \\
.25231 \\
.22007 \\
.19344\end{array}$ & $\begin{array}{l}.09004 \\
.07668 \\
.06603 \\
.05742 \\
.05035\end{array}$ & $\begin{array}{l}.20524 \\
.17510 \\
.15089 \\
.13120 \\
.11496\end{array}$ & $\begin{array}{l}.28076 \\
.23955 \\
.20654 \\
.17977 \\
.15774\end{array}$ & $\begin{array}{l}.07552 \\
.06446 \\
.05567 \\
.04859 \\
.04280\end{array}$ \\
\hline $\begin{array}{l}2000 \\
2500 \\
3000 \\
4000 \\
5000\end{array}$ & $\begin{array}{l}.08248 \\
.05286 \\
.03631 \\
.01949 \\
.01150\end{array}$ & $\begin{array}{l}.11130 \\
.07139 \\
.04915 \\
.02655 \\
.01581\end{array}$ & $\begin{array}{l}.02883 \\
.01854 \\
.01285 \\
.00707 \\
.00432\end{array}$ & $\begin{array}{l}.06504 \\
.04083 \\
.02734 \\
.01352 \\
.00681\end{array}$ & $\begin{array}{l}.09032 \\
.05781 \\
.03974 \\
.02129 \\
.01238\end{array}$ & $\begin{array}{l}.02530 \\
.01699 \\
.01240 \\
.00778 \\
.00558\end{array}$ \\
\hline
\end{tabular}


Table 4.10

$\begin{array}{rccc}\mathrm{T}^{\circ} \mathrm{K} & \frac{\mathcal{F}_{H I}-\mathcal{F}_{D I}}{R T} & \frac{\mathcal{F}_{H I}-\mathcal{F}_{T I}}{R T} & \frac{\mathcal{F}_{D I}-\mathcal{F}_{T I}}{R T} \\ 100 & 4.38276 & 6.27604 & 1.89329 \\ 200 & 2.01832 & 2.86620 & .84789 \\ 300 & 1.23062 & 1.73129 & .50068 \\ 400 & .83898 & 1.16974 & .33077 \\ 500 & .60823 & .84168 & .23346 \\ 600 & .45938 & .63207 & .17270 \\ 700 & .35775 & .49021 & .13247 \\ 800 & .28548 & .39008 & .10461 \\ 900 & .23242 & .31700 & .08459 \\ 1000 & .19245 & .26223 & .06979 \\ 1100 & .16166 & .22020 & .05854 \\ 1200 & .13750 & .18731 & .04982 \\ 1300 & .11822 & .16115 & .04294 \\ 1400 & .10259 & .13999 & .03741 \\ 1500 & .08979 & .12269 & .03291 \\ 2000 & .05077 & .07013 & .01937 \\ 2500 & .03206 & .04505 & .01299 \\ 3000 & .02171 & .03119 & .00949 \\ 4000 & .01117 & .01714 & .00598 \\ 5000 & .00610 & .01040 & .00431 \\ & & & \end{array}$






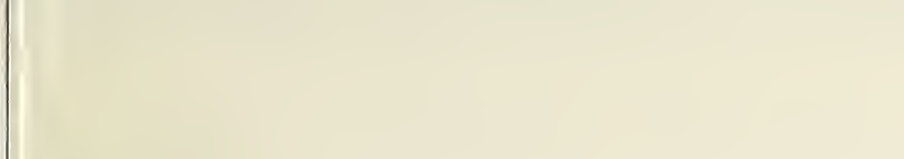




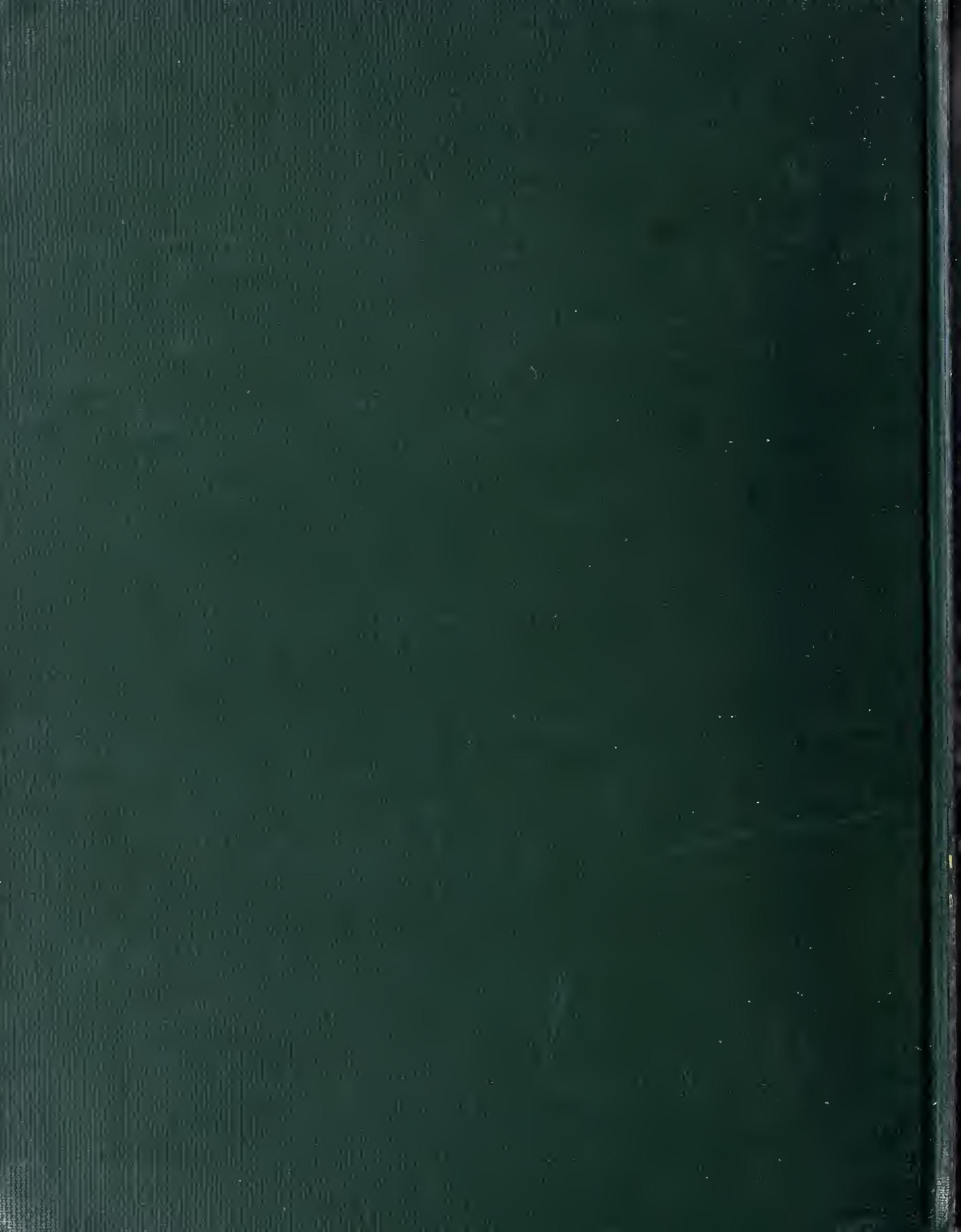

